\title{
Site Observational Work Plan FOR THE UMTRA PROJECT SITE at Ambrosia LaKe, New MeXico
}

\author{
RESEN \\ DEC OS 1994 \\ OSTI
}

September 1994

\section{DISCLAIMER}

This report was prepared as an account of work sponsored by an agency of the United States Government. Neither the United States Government nor any agency thereof, nor any of their employees, makes any warranty, express or implied, or assumes any legal liability or responsibility for the accuracy, completeness, or usefulness of any information, apparatus, product, or process disclosed, or represents that its use would not infringe privately owned rights. Reference herein to any specific commercial product, process, or service by trade name, trademark, manufacturer, or otherwise does not necessarily constitute or imply its endorsement, recommendation, or favoring by the United States Government or any agency thereof. The views and opinions of authors expressed herein do not necessarily state or reflect those of the United States Government or any agency thereof. 


\section{DISCLAIMER}

Portions of this document may be illegible in electronic image products. Images are produced from the best available original document. 


\section{INTENDED FOR PUBLIC RELEASE}

This report has been reproduced from the best available copy. Available in paper copy and microfiche.

Number of pages in this report: 538

DOE and DOE contractors can obtain copies of this report from:

Office of Scientific and Technical Information

P.O. Box 62

Oak Ridge, TN 37831

(615) 576-8401

This report is publicly available from:

National Technical Information Service

Department of Commerce

5285 Port Royal Road

Springfield, VA 22161

(703) $487-4650$ 
Prepared for

U.S. Department of Energy UMTRA Project Office Albuquerque, New Mexico

Prepared by Jacobs Engineering Group Inc. Albuquerque, New Mexico 


\section{EXECUTIVE SUMMARY}

The requirements for ground water compliance for Uranium Mill Tailings Remedial Action (UMTRA) Project sites, including the Ambrosia Lake, New Mexico processing site, are found in the Uranium Mill Tailings Radiation Control Act (UMTRCA) (42 USC $\$ 7901$ et seq.) of 1978 and Subparts A, B, and C of the U.S. Environmental Protection Agency's (EPA) "Health and Environmental Protection Standards for Uranium and Thorium Mill Tailings" codified in 40 CFR Part 192 (1994), and the associated proposed standards published in the Federal Register on September 24, 1987 (52 FR 36000). The EPA proposed standards (52 FR 36000) for Subpart C of 40 CFR Part 192 describe specific conditions for which the U.S. Department of Energy (DOE) may apply for supplemental standards for contaminated ground water rather than meeting background levels or numerical standards.

Residual radioactive materials are currently being consolidated on the site by the DOE in a disposal cell, isolating them from direct human or ecological contact and further dispersion into the environment. Completion of the disposal cell is scheduled for early 1995. An environmental assessment (DOE, 1987a) and a Finding of No Significant Impact (FONSI) (DOE, 1987b) were completed in 1987. Concurrence with the UMTRA Surface Project Ambrosia Lake remedial action plan (RAP) (DOE, 1991) was granted by the U.S. Nuclear Regulatory Commission (NRC) and state of New Mexico in 1990 (NRC, 1990). For the UMTRA Ground Water Project, this site observational work plan (SOWP) is the first document to address ground water compliance for the Ambrosia Lake site.

The Ambrosia Lake UMTRA Project site is within the Grants Mineral Belt and was one of numerous uranium mills supplied by many local mines. Ground water contamination at the site occurred as a result of uranium mill operations. During the period from 1958 to 1963 , approximately 3.0 million tons $(2.7$ million tonnes) of uranium-bearing ore were processed by crushing and grinding, and uranium was removed through a chemical process (DOE, 1991). Contamination of ground water resulted from discharge of waste water, infiltration of water through the tailings pile, hydraulic placement of mill tailings in nearby mines, and water pumped from mine shafts.

The uppermost aquifer at the Ambrosia Lake site is defined as the alluvium and Tres Hermanos-C Sandstones. Saturation of the aquifer was artificially created solely by discharge of mill process water and the extent of saturation is limited in both surface area and vertical extent.

The uppermost aquifer does not represent a ground water resource because of the limited extent of saturation and the inability to consistently sustain a yield of 150 gallons (gal) per day (570 liters [L] per day) to wells, based on a February 1989 aquifer test on DOE Well 675 (DOE, 1991). As demonstrated in this document, and previously in the RAP (DOE, 1991), ground water within the uppermost aquifer meets the qualifications for supplemental standards, Class III ("limited use"), based on limited yield. The major sources of recharge, waste water disposal and infiltration, and water from mine pumping, no longer exist. Therefore, the unit is expected to return to its premilling and mining 
condition of little-to-no saturation, further eliminating the unit as a potential future ground water resource.

The potential for impacts to human health and the environment from contaminated ground water currently does not exist. No domestic or livestock wells accessing ground water from the uppermost aquifer have been identified within a 5 mile (mi) (8 kilometers [km]) radius from the site. Wells located further than $5 \mathrm{mi}(8 \mathrm{~km})$ from the site access ground water from deeper geologic units. Ground water does not discharge to the land surface and the nearest surface water is located approximately $1.5 \mathrm{mi}(2.4 \mathrm{~km})$ southwest of the site. Land use in the area is not expected to change in the future because of the poor quality of the range land, poor soils, and the limited surface or shallow ground water resources available in this semiarid region. Therefore, no current exposure pathways to humans, livestock, or wildlife exist, nor are any foreseen.

The proposed ground water compliance strategy under consideration for application at the Ambrosia Lake site is to perform no remediation, based on the application of supplemental standards because the ground water has "limited use" (40 CFR §192.21(g) (1994)) (52 FR 36000 (1987)). "Limited use" means the ground water is not a current or potential source of drinking water because the quantity of water available is less than $150 \mathrm{gal}(570 \mathrm{~L})$ per day (40 CFR \$192.11(e)) (52 FR 36000). The NRC and state of New Mexico concurred with application of supplemental standards for the UMTRA Surface Project in the NRC's December 31, 1990 final technical evaluation report for the site (NRC, 1990). This compliance strategy should also be applicable for the UMTRA Ground Water Project at the Ambrosia Lake site. 


\section{TABLE OF CONTENTS}

Section

Page

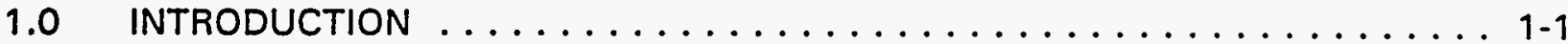

1.1 Purpose and content ......................... 1-1

1.2 Ground water compliance strategy .................... 1-1

1.3 Relationship to other programmatic documents .............1-1

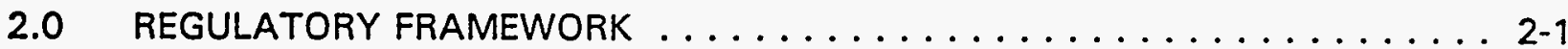

2.1 Uranium mill tailings radiation control act . . . . . . . . . . . . 2-1

2.1.1 EPA ground water standards ................ 2-1

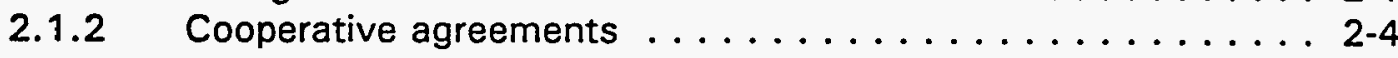

2.2 National environmental policy act . . . . . . . . . . . . . . 2-4

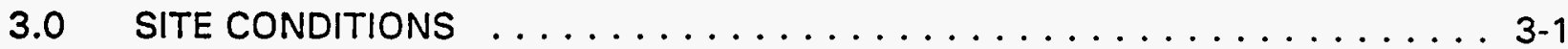

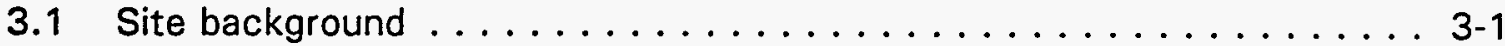

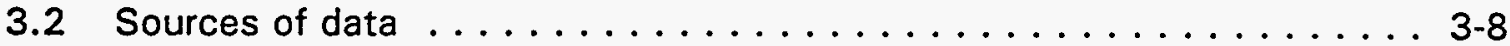

3.3 Conceptual site model . . . . . . . . . . . . . . . . . . . 3-8

3.3.1 Hydrogeologic setting ................... $3-9$

3.3.2 Ground water quality .................... $3-19$

3.3.3 Contaminant fate and transport ............... 3-28

3.3.4 Risk evaluation . . . . . . . . . . . . . . . 3-28

3.4 Assessment of data needs . . . . . . . . . . . . . . . 3-30

3.5 Evaluation of interim remedial action $\ldots \ldots \ldots \ldots \ldots \ldots \ldots \ldots$

4.0 GROUND WATER COMPLIANCE STRATEGY RECOMMENDATION . . . . . . . 4-1

4.1 Ground water compliance strategy evaluation process . . . . . . . . . . . . 4-1

4.2 Site-specific ground water compliance strategr . . . . . . . . . . . . 4-3

4.3 Deviations, contingencies, and rules . . . . . . . . . . . . . 4-4

4.4 Data collection and assessment ................. $4-5$

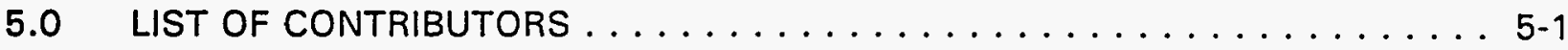

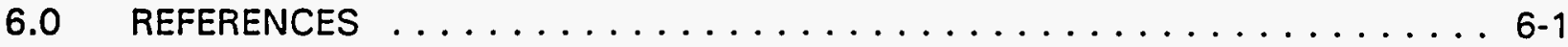

APPENDIX A LITHOLOGIC LOGS, WELL RECORDS, AND WELL COMPLETION LOGS

AMBROSIA LAKE, NEW MEXICO, UMTRA PROJECT SITE

APPENDIX B HYDROGEOLOGIC INFORMATION AMBROSIA LAKE, NEW MEXICO, UMTRA PROJECT SITE

APPENDIX C GROUND WATER QUALITY DATA BY LOCATION AMBROSIA LAKE, NEW MEXICO, UMTRA PROJECT SITE 


\section{LIST OF FIGURES}

Figure

$\underline{\text { Page }}$

3.1 Location of Ambrosia Lake UMTRA site . . . . . . . . . . . . . 3-2

3.2 Ambrosia Lake UMTRA site and surrounding area . . . . . . . . . . . 3-3

3.3 Preremedial action Ambrosia Lake UMTRA site . . . . . . . . . . . . . . . 3-4

3.4 Generalized regional geologic construction for the Ambrosia Lake area,

New Mexico . . . . . . . . . . . . . . . . . . . . . . . . . 3-11

3.5 Locations of geologic cross sections, Ambrosia Lake UMTRA site,

New Mexico ... . . . . . . . . . . . . . . . . . . . . . . . . . 3-12

3.6 Site-specific hydrogeologic cross section (A-A'), Ambrosia Lake UMTRA site,

New Mexico . . . . . . . . . . . . . . . . . . . . . . . . . . 3-13

3.7 Site-specific hydrogeologic cross section (B-B'), Ambrosia Lake UMTRA site,

New Mexico . . . . . . . . . . . . . . . . . . . . . . . . . . . . 3-14

3.8 Sulfate isopleth map for ground water in the alluvium and the weathered

Mancos Shale at the Ambrosia Lake UMTRA site, New Mexico . . . . . . . . 3-21

3.9 Sulfate isopleth map for ground water in the Tres Hermanos $\mathrm{C}_{2}$ Sandstone at the Ambrosia Lake UMTRA site, New Mexico . . . . . . . . . . . . . 3-25

3.10 Nitrate concentrations versus time in monitor Well 678 located the Tres Hermanos-B Sandstone, Ambrosia Lake UMTRA site, New Mexico . . . . . . 3-27

4.1 Ambrosia Lake compliance selection framework . . . . . . . . . . 4-2 


\section{LIST OF TABLES}

Table

$\underline{\text { Page }}$

2.1 Maximum concentration of constituents for ground water protection ..... 2-3

3.1 Water levels for all existing wells, July 13, 1994, Ambrosia Lake UMTRA site ................................ 3-16

3.2 Maximum observed concentrations of EPA MCL constituents in monitor wells located in the alluvium/weathered Mancos Shale at the Ambrosia Lake site during the years from 1980 to 1994

3.3 Maximum observed concentrations of EPA MCL constituents in monitor wells located in the Tres Hermanos- $C_{1}$ Sandstone at the Ambrosia Lake site during the years from 1980 to $1994 \ldots \ldots \ldots \ldots \ldots \ldots$. . . . . . . . . . . . . 3

3.4 Maximum observed concentrations of EPA MCL constituents in monitor wells located in the Tres Hermanos- $\mathrm{C}_{2}$ Sandstone at the Ambrosia Lake site during the years from 1980 to $1994 \ldots \ldots \ldots \ldots \ldots$. . . . . . . . . . . . . . 


\section{LIST OF ACRONYMS AND ABBREVIATIONS}

\section{Acronym}

$A C L$

AEC

$\mathrm{cm} / \mathrm{s}$

$\mathrm{cm}$

DOE

EPA

FONSI

$\mathrm{ft}$

$\mathrm{ft} / \mathrm{day}$

gal

$\mathrm{km}$

L

m

$\mathrm{MCL}$

$\mathrm{mg} / \mathrm{L}$

mi

MSL

NEPA

NRC

PEIS

$\mathrm{pCi} / \mathrm{L}$

RAP

RRM

SOWP

SPEAR

TAGR

TER

UMTRA

UMTRCA

\section{Definition}

alternate concentration limit

Atomic Energy Commission

centimeters per second

centimeter

U.S. Department of Energy

U.S. Environmental Protection Agency

Finding of No Significant Impact

foot

feet per day

gallon

kilometer

liter

meter

Maximum concentration limit

milligrams per liter

mile

mean sea level

National Environmental Policy Act

U.S. Nuclear Regulatory Commission

Programmatic Environmental Impact Statement

picocuries per liter

remedial action plan

residual radioactive material

site observational work plan

Software Program for Environmental Analysis and Reporting

Technical Approach to Ground Water Restoration

Technical Evaluation Report

Uranium Mill Tailings Remedial Action

Uranium Mill Tailings Radiation Control Act 


\subsection{INTRODUCTION}

The "Site Observational Work Plan for Ground Water Investigation at Ambrosia Lake, New Mexico" is a response to requirements for ground water compliance established in the Uranium Mill Tailings Radiation Control Act (UMTRCA) (42 USC \$7901, et. seq. (1978)). This site observational work plan (SOWP) is the first ground water document of the Ambrosia Lake Uranium Mill Tailings Remedial Action (UMTRA) Project to address sitespecific activities necessary to meet compliance with U.S. Environmental Protection Agency (EPA) proposed ground water standards (52 FR 36000 (1987)).

\section{$1.1 \quad$ PURPOSE AND CONTENT}

This SOWP, Revision 0, recommends an appropriate, site-specific ground water compliance strategy for the Ambrosia Lake site.

Section 2.0 describes the requirements for meeting the ground water compliance standards at UMTRA Project sites. Section 3.0 presents a summary of the site's hydrogeological setting, describes the presence and distribution of contaminants in ground water resulting from the milling process, and describes and assesses the potential environmental and human health risks at the site. Section 4.0 presents and provides justifications for the recommended ground water compliance strategy at the Ambrosia Lake site.

\subsection{GROUND WATER COMPLIANCE STRATEGY}

The ground water compliance strategy recommended for the Ambrosia Lake site is to perform no remediation, based on the application of supplemental standards, because the ground water is classified as Class III ("limited use") ground water (40 CFR \$192.21 (g) (1994)) (52 FR 36000 (1987)). "Limited use" means ground water that is not a current or potential source of drinking water because the quantity of water available is less than 150 gallons (gal) 1570 liters [L]) per day (40 CFR §192.11(e)) (52 FR 36000).

\subsection{RELATIONSHIP TO OTHER PROGRAMMATIC DOCUMENTS}

Programmatic documents that provide guidance for this SOWP include the U.S. Department of Energy (DOE) UMTRA Ground Water Program Plan (DOE, 1992a), Programmatic Environmental Impact Statement for the Uranium Mill Tailings Remedial Action Ground Water Project (PEIS) (DOE, 1994) and Technical Approach to Ground Water Restoration (TAGR) (DOE, 1993a). The UMTRA Ground Water Program Plan states the mission needs and objectives for the UMTRA Ground Water Compliance Program and provides an overall technical and management approach for conducting the program. The PEIS provides an objective programmatic decision-making framework for conducting the UMTRA Ground Water Project, assesses the potential programmatic impacts of conducting the project, provides a method for determining the site-specific ground water compliance strategies, and provides data and information that can 
be used to prepare site-specific National Environmental Policy Act (NEPA) (42 USC \$4321 et seq. (1970)) compliance documents more efficiently. The TAGR provides technical guidance for conducting the ground water program.

The environmental impacts from the recommended ground water compliance strategy presented in this SOWP will be assessed in a separate, appropriate, site-specific document that will meet the requirements of the NEPA, (42 USC $\$ 4321$ et seq. (1970)). The site-specific NEPA document will be prepared by the DOE and be based on the framework in the approved PEIS. A record of decision on the PEIS framework is anticipated in 1995. The preferred alternative from the PEIS for conducting the UMTRA Ground Water Project will be published as the record of decision in the Federal Register. 


\subsection{REGULATORY FRAMEWORK}

This section identifies the requirements for recommending a ground water compliance strategy for the Ambrosia Lake site, to achieve compliance with Subpart B of the EPA's "Health and Environmental Protection Standards for Uranium and Thorium Mill Tailings," (40 CFR Part 192 (1994)), the EPA proposed 1987 standards (52 FR 36000), and the EPA's 1994 draft final rule.

\section{$2.1 \quad$ URANIUM MILL TAILINGS RADIATION CONTROL ACT}

The U.S. Congress passed the UMTRCA (42 USC $\$ 7901$ et seq.) in 1978 in response to public concerns about the potential health hazards from exposure to uranium mill tailings over long periods of time. The UMTRCA authorized the DOE to stabilize, dispose of, and control uranium mill tailings and other contaminated materials at uranium mill processing sites.

The UMTRCA has three titles. The UMTRA Project has responsibility for administering only Title I of UMTRCA. Title I of the Act designates 24 inactive processing sites that will undergo remediation; directs the EPA to promulgate standards; requires that the DOE comply with the proposed EPA ground water quality standards in the absence of final standards; mandates remedial action in accordance with standards prescribed by the EPA; directs the U.S. Nuclear Regulatory Commission (NRC), states, and Indian tribes to concur in the remedial actions; directs the NRC to license the disposal sites for long-term disposal; and directs the DOE to enter into cooperative agreements with the affected states and Indian tribes.

In 1988, Congress passed the Uranium Mill Tailings Remedial Action Amendments Act (Amendments Act) (42 USC $\$ 7922$ et seq.) authorizing the DOE to extend without limitation the time needed to complete ground water restoration activities at the processing sites.

\subsubsection{EPA ground water standards}

The UMTRCA requires that the EPA promulgate standards for protecting public health, safety, and the environment from radiological and nonradiological contaminants associated with processing of uranium and the resulting residual radioactive materials (RRM). On January 5, 1983, the EPA promulgated final standards (40 CFR Part 192) for the disposal and cleanup of RRM. On September 3, 1985, the Tenth Circuit Court of Appeals set aside the ground water provisions of the regulations at 40 CFR $\$ 192.20(a)(2)$ and (3) and remanded them to the EPA. The EPA proposed new ground water standards to replace the remanded sections in the Federal Register on September 24, 1987 (52 FR 36000).

The 1987 EPA proposed standards address two ground water contamination scenarios: the first addresses future ground water contamination that may 
occur from tailings piles after disposal (completion of surface remedial action); the second addresses the cleanup of contamination that occurred at the processing sites before disposal of the tailings piles (52 FR 36000 (1987)). Protection of the ground water at the disposal sites is being addressed in the UMTRA Surface Project. The UMTRA Ground Water Project addresses the contamination that occurred at the processing sites and is regulated by Subparts $B$ and $C$ of the proposed 1987 ground water standards. The EPA draft final rule for maximum concentration limits of constituents for ground water protection is presented in Table 2.1 (EPA, 1994).

\section{Subpart B}

Subpart B ("Standards for Cleanup of Land and Buildings Contaminated with Residual Radioactive Materials from Inactive Uranium Processing Sites") requires that remedial action at processing sites be conducted to ensure that the amounts of RRM in ground water meet one of the three following standards presented in 52 FR 36000 (1987):

- Background levels: concentrations of constituents in nearby ground water unaffected by processing activities.

- Maximum concentration limits (MCL): the EPA's maximum concentration limits of certain constituents in ground water, as proposed for the UMTRA Project.

- Alternate concentration limits (ACL): an alternate concentration limit for a constituent that will not pose a substantial present or potential hazard to human health or the environment as long as the alternate concentration limit is not exceeded.

\section{Subpart C}

Under certain specific conditions, the DOE may apply supplemental standards, as described in Subpart $C$, in lieu of the standards of Subparts A or B (52 FR 36000 ) (background levels, MCLs, or ACLs). The supplemental standard applicable at the Ambrosia Lake site for the surface remedial action program, and discussed for further analysis in this SOWP, may be applied because the ground water is classified as having "limited use" (40 CFR \$192.21(g) (1994)) (52 FR 36000 (1987)). Subpart B of the proposed standards defines "limited use" as ground water that 1) is not a current or potential source of drinking water because the concentration of total dissolved solids is in excess of $10,000$ milligrams per liter ( $\mathrm{mg} / \mathrm{L})$; or, 2) because widespread, ambient contamination not due to activities involving RRM from a designated processing site exists, that cannot be cleaned up using treatment methods reasonably employed in public water-supply systems; or, 3) because the quantity of water available is less than $150 \mathrm{gal}(570 \mathrm{~L}$ ) per day (40 CFR $\S 192.11(\mathrm{e})(1994)$ ) (52 FR 36000). 
Table 2.1 Maximum concentration of constituents for ground water protection

\begin{tabular}{|c|c|}
\hline Constituent & $\begin{array}{l}\text { Maximum } \\
\text { concentration }^{a}\end{array}$ \\
\hline Arsenic & 0.05 \\
\hline Barium & 1 \\
\hline Cadmium & 0.01 \\
\hline Chromium & 0.05 \\
\hline Lead & 0.05 \\
\hline Mercury & 0.002 \\
\hline Selenium & 0.01 \\
\hline Silver & 0.05 \\
\hline Nitrate (as N) & 10 \\
\hline Molybdenum & 0.1 \\
\hline Combined radium-226 and radium -228 & $5 \mathrm{pCi} / \mathrm{L}$ \\
\hline Combined uranium-234 and uranium-238 ${ }^{\mathrm{b}}$ & $30 \mathrm{pCi} / \mathrm{L}$ \\
\hline Gross alpha-particle activity (excluding radon and uranium) & $15 \mathrm{pCi} / \mathrm{L}$ \\
\hline $\begin{array}{l}\text { Endrin }(1,2,3,4,10 \text {-hexachloro-6,7-epoxy-1,4,4a,5,6,7,8,8a- } \\
\text { octahydro-1,4-endo,endo-5,8-dimethanonaphthalene) }\end{array}$ & 0.0002 \\
\hline Lindane $(1,2,3,4,5,6$-hexachlorocyclohexane, gamma isomer $)$ & 0.004 \\
\hline Methoxychlor (1,1,1-trichloro-2,2'-bis(p-methoxyphenylethane)) & 0.1 \\
\hline $\begin{array}{l}\text { Toxaphene }\left(\mathrm{C}_{10} \mathrm{H}_{10} \mathrm{Cl}_{6} \text {, technical chlorinated camphene, }\right. \\
67-69 \text { percent chlorine) }\end{array}$ & 0.005 \\
\hline 2,4-D (2,4-dichlorophenoxyacetic acid) & 0.1 \\
\hline 2,4,5-TP Silvex (2,4,5-trichlorophenoxypropionic acid) & 0.01 \\
\hline
\end{tabular}

Source: (EPA, 1994)

a Milligrams per liter, unless stated otherwise.

bWhere secular equilibrium obtains, this criterion will be satisfied by a concentration of $0.044 \mathrm{mg} / \mathrm{L}$. For conditions of other than secular equilibrium, a corresponding value may be derived and applied, based on the measured site-specific ration of the two isotopes of uranium.

pCi/L - picocuries per liter. 
When supplemental standards apply, implementing agencies shall apply any remedial actions for the restoration of ground water contaminated by RRM that are required to assure, at a minimum, protection of human health and the environment. In addition, when limited use ground water applies, supplemental standards shall ensure that current and reasonably projected uses of the affected ground water are preserved (EPA, 1994).

Subpart B also provides for selecting natural flushing as a means to meet the proposed standards. Natural flushing means allowing natural ground water processes to reduce the contamination in ground water to the standards (background levels, MCLs, or ACLs) within 100 years. Institutional controls that protect human health (measures that restrict access to contamination and satisfy beneficial uses of ground water), and an adequate monitoring program must be established and maintained during the period of natural flushing. Ground water also must not be currently, or be projected to become, a source of drinking water during the period of natural flushing (40 CFR §192.12(c)(4)) (52 FR 36000).

Subpart C (Implementation) provides guidance for implementing methods and procedures to provide reasonable assurance that the provisions of Subpart $B$ are satisfied. Subpart $C$ requires the following: that the conditions of Subpart $B$ be met on a site-specific basis using information gathered from site characterization and monitoring; and that the compliance strategy plan or remedial action plan (RAP) contain the proposed compliance strategy, a demonstration of effectiveness, and a characterization and monitoring program, as appropriate to meet the conditions of Subpart $B$.

\subsubsection{Cooperative agreements}

The UMTRCA requires that remedial action be accomplished with the full participation of the affected states (Section 103, State Cooperative Agreements) and Indian tribes (Section 105, Indian Tribe Cooperative Agreements) on whose lands the uranium mill tailings are located. No Indian tribes are affected by the activities at the Ambrosia Lake site. A cooperative agreement for the Surface Project with the State of New Mexico was executed September 11, 1985 (Cooperative Agreement Number DE-FC04-85AL20533, as amended) (DOE, 1985).

\subsection{NATIONAL ENVIRONMENTAL POLICY ACT}

Implementation of the UMTRCA represents a major federal action subject to the requirements of the NEPA (42 USC $\$ 4321$ et seq. (1970). The Council on Environmental Quality's regulations that implement the NEPA are codified in 40 CFR Parts 1500-1508 (1994) and require that each federal agency develop its own implementing procedures (40 CFR \$1507.3). The DOE NEPA regulations are contained in 10 CFR Part 1021 (1994), "National Environmental Policy Act; 
Implementing Procedures." DOE guidance is provided in Recommendations for the Preparation of Environmental Assessments and Environmental Impact Statements (DOE, 1993b).

Pursuant to the NEPA, the DOE drafted a PEIS in 1994 to analyze the potential impacts of implementing the programmatic alternatives proposed for conducting the Ground Water Project. The preferred alternative for conducting the UMTRA Ground Water Project will be recommended by the DOE following a public participation effort, and the record of decision will be published in the Federal Register. All subsequent actions on the UMTRA Ground Water Project must comply with this record of decision, anticipated in 1995.

The Ambrosia Lake site-specific NEPA documentation will be prepared by the DOE and be based on the framework in the approved PEIS that has been published as the record of decision in the Federal Register. The site-specific NEPA document will analyze the potential impacts of the strategy of no remediation, based on the application of supplemental standards, as the recommended site-specific ground water compliance strategy. 


\subsection{SITE CONDITIONS}

The appropriate ground water compliance strategy can be recommended only after the evaluation of site location, surrounding area land use, site history, and hydrogeologic conditions. Evaluation of site location, surrounding area land use, and hydrogeologic conditions assists in the evaluation of potential pathways for risks to human health and the environment. The site history highlights processing operations that have caused contamination, and hydrogeologic conditions describe the mechanisms that control contaminant distribution.

\subsection{SITE BACKGROUND}

\section{Location}

The Ambrosia Lake site is located in McKinley County, New Mexico, about 25 miles (mi) (40 kilometers [km]) north of Grants, New Mexico, and $1 \mathrm{mi}$ $(1.6 \mathrm{~km}$ ) east of New Mexico Highway 509 (Figure 3.1). The town nearest to the site is San Mateo, about $10 \mathrm{mi}(16 \mathrm{~km})$ to the southeast (Figure 3.2).

The site is within the Ambrosia Lake Mining District, near the center of the Grants Mineral Belt. Decommissioned uranium mills, abandoned underground mines, mine shafts and vents, ore piles, tailings piles, and heap leach piles are in proximity to the site. The former mill, former main holding ponds, and tailings pile were in Section 28, and two former small holding ponds were in Section 33, Township 14 North, Range 9 West. A former heap leaching operation associated with the Ambrosia Lake mill was located in Section 27 (Figure 3.3) (USGS, 1980). The approximate elevation of the site is 7,000 feet $(\mathrm{ft})(2,100$ meters [m] above sea level.

\section{Surrounding land use}

McKinley County is rural and sparsely populated. The town closest to the site is San Mateo, about $10 \mathrm{mi}(16 \mathrm{~km})$ to the southeast. The largest city in the area is Grants (in adjacent Cibola County), about $25 \mathrm{mi}(40 \mathrm{~km})$ south of the site, which had a population of 8626 in 1990 (DOC, 1990).

The sites of former uranium mines and mills, and a limited amount of grazing constitute current land use surrounding the site. The "Trailer Park" shown on the USGS topographic map (Figure 3.3) (USGS, 1980) is within about $1 \mathrm{mi}$ $(1.6 \mathrm{~km})$ of the site, but was abandoned when the demand for uranium declined during the late 1970s. More than one dozen former uranium mines are within a $5-\mathrm{mi}(8-\mathrm{km})$ radius of the site (Figure 3.2$)$, and the closest residence is approximately $5 \mathrm{mi}(8 \mathrm{~km})$ south of the site. Not shown on the figure but present throughout the area are numerous abandoned mine spoil piles, ore piles, tailings piles, and heap leach piles resulting from former mining and milling 


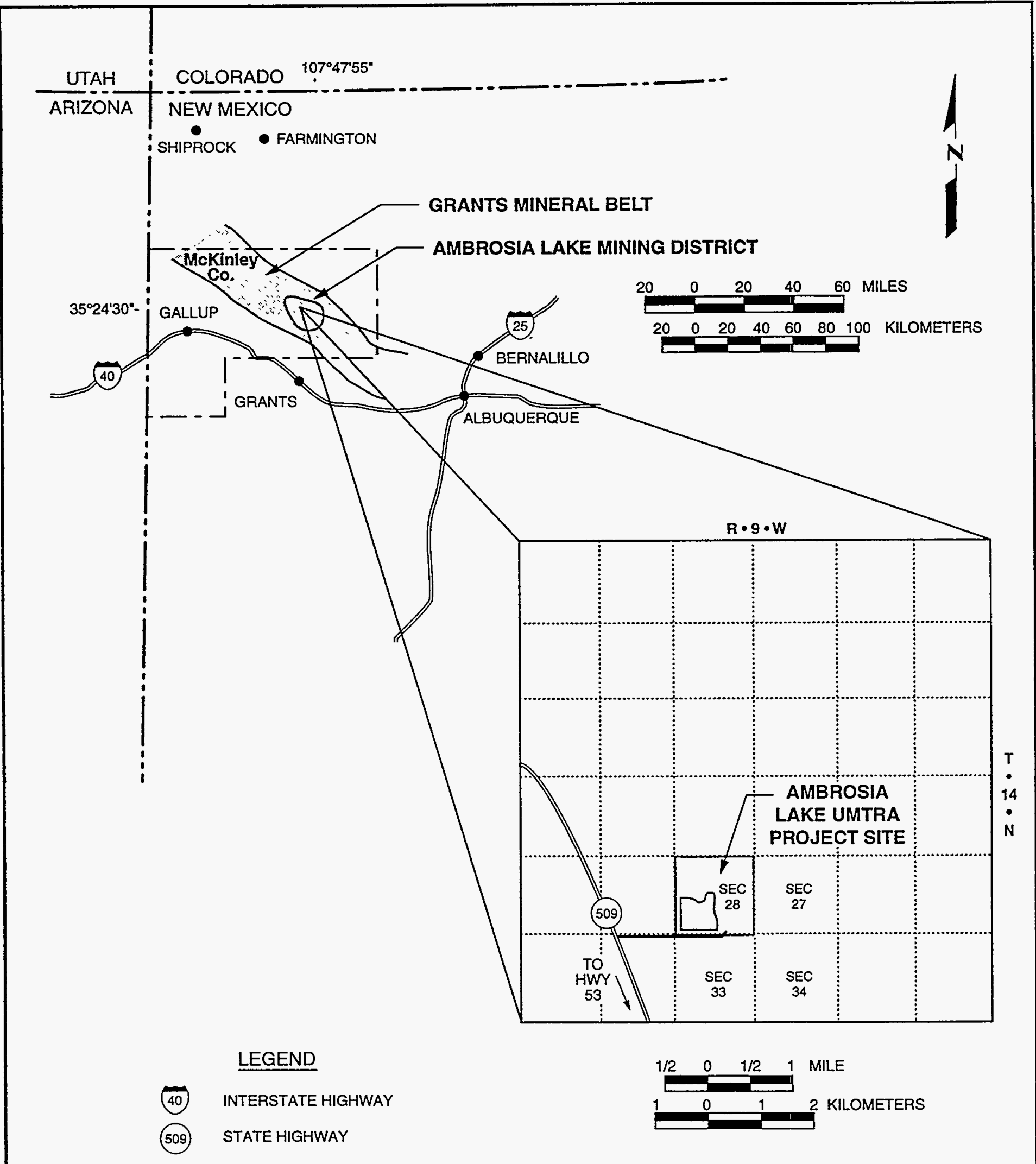

MODIFIED FROM DOE, 1991.

FIGURE 3.1

LOCATION OF AMBROSIA LAKE UMTRA SITE 


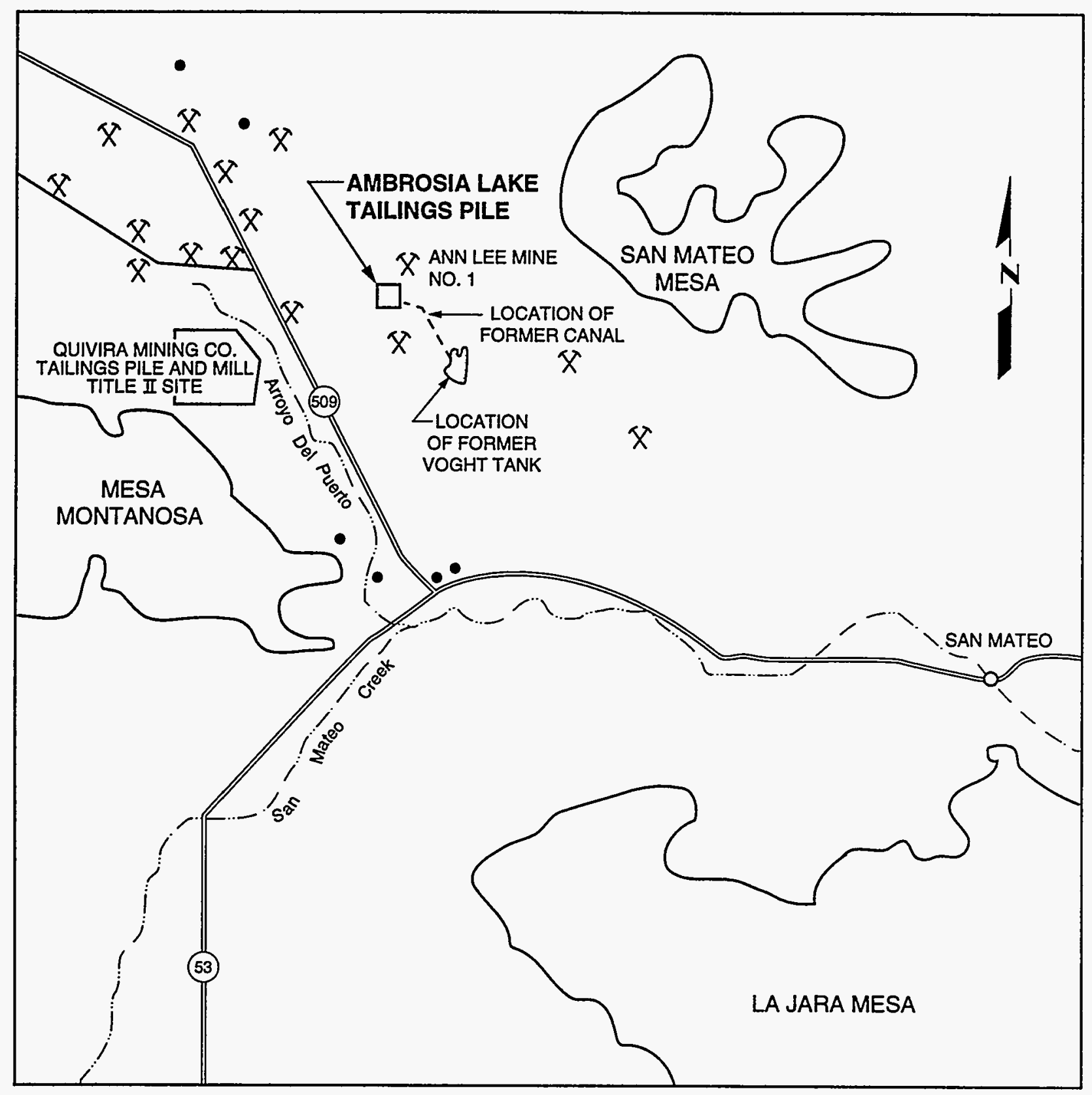

\section{LEGEND}

- DOMESTIC OR STOCK WELL

$X$ UNDERGROUND MINE

-..- EPHEMERAL STREAM

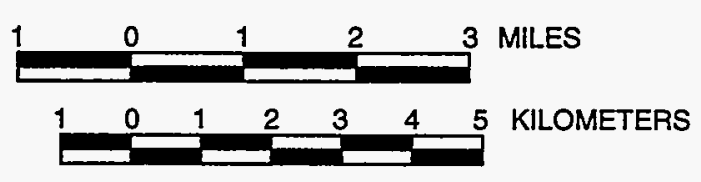

MODIFIED FROM DOE, 1991.

FIGURE 3.2

AMBROSIA LAKE UMTRA SITE AND SURROUNDING AREA 


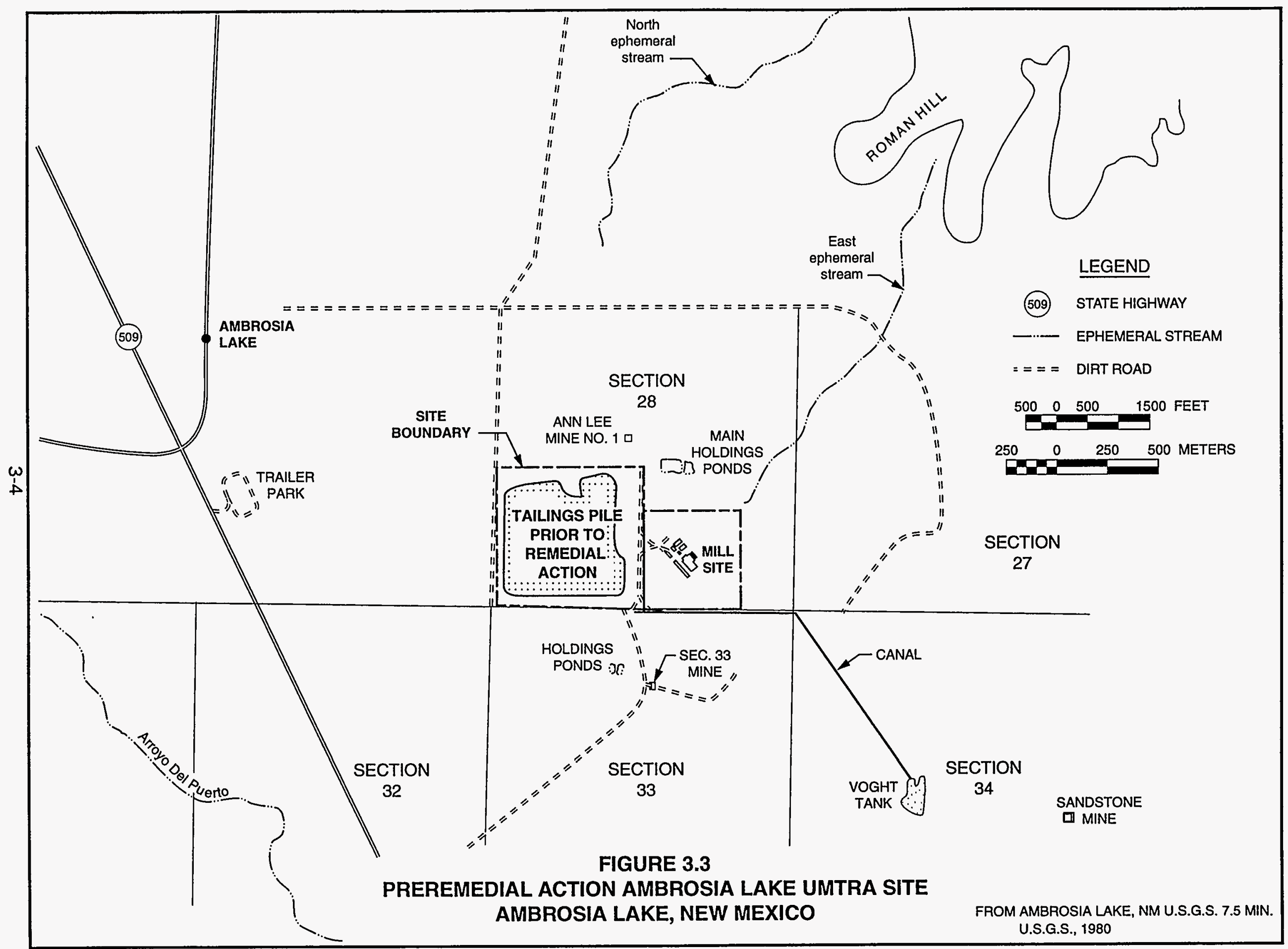


operations (Figure 3.2). Piles associated with the Ambrosia Lake UMTRA Project site have been cleaned up and placed in the disposal cell.

Many of the surrounding uranium mills are undergoing remediation. The former mill closest to the site is Quivira Mining Company, which is about $1 \mathrm{mi}(1.6 \mathrm{~km})$ to the southwest of the Ambrosia Lake site.

\section{Surrounding water use}

Ground water use in the Ambrosia Lake area was dominated by the uranium mining and milling industry, but has declined drastically as a result of the abandonment of mining and milling. The only surrounding ground water uses are for cleanup activities at nearby former mill sites, and for domestic and ranch supplies. The nearest surface water is located approximately $1.5 \mathrm{mi}(2.4 \mathrm{~km})$ southwest of the Ambrosia Lake site.

Water supplies associated with mill cleanup activities are from wells completed in the uranium ore zone (the Westwater Canyon Member of the Morrison Formation) and deeper hydrogeologic units. Domestic and stock wells are also completed in the ore zone or deeper units and obtain water at depths from 300 to greater than $800 \mathrm{ft}(100$ to greater than $240 \mathrm{~m})$. No domestic or livestock wells are completed in any of the shallower zones (alluvium and Tres Hermanos Sandstones) within at least a $5-\mathrm{mi}(8-\mathrm{km})$ radius of the site, with the exception of monitor wells installed by the DOE (DOE, 1991). The nearest municipality operating a public water supply is the town of San Mateo, $10 \mathrm{mi}(16 \mathrm{~km})$ southeast of the Ambrosia Lake site. The water for San Mateo is derived from the Point Lookout Sandstone (Brod, 1979), which is stratigraphically higher than, and not connected with, any of the geologic units at the Ambrosia Lake site.

\section{Contaminant sources}

Contaminant sources described in this section result from two factors: 1) surrounding land uses and 2) the UMTRA Project site. These two factors are significant because of the location of the Ambrosia Lake UMTRA site. A large number of contaminant sources are within the vicinity.

The Ambrosia Lake UMTRA Project site has contributed relatively little contamination within an area that experienced intensive uranium mining and milling activities from the mid-1950s through the late 1970s. Chemical processes for uranium concentration within the area were similar to those employed at the UMTRA Project site; therefore, contaminants from the surrounding mills are similar. For example, the former Quivira Mining Company mill, adjacent to the UMTRA Project site, has produced more than ten times the tailings produced by the UMTRA site, representing a volume of tailings greater than all of the 24 UMTRA Project sites combined. Because similar processing techniques were employed, it is logical to assume that the Quivira Mining Company mill produced at least ten times the volume of waste water as the 
UMTRA Project mill produced. It is unknown how much additional ground water contamination has been produced from the other surrounding mills.

As stated earlier, the area surrounding the site has numerous abandoned mines and vent shafts, and associated spoil piles, ore piles, tailings piles, and heap leach piles. It is important to mention these conditions again, because of their net contribution to widespread ground water contamination. Many of the mines and vent shafts were legally backfilled with RRM, which contain contaminants that can leach into the water-bearing zones adjacent to the mines and vent shafts. Additionally, the mines and vent shafts provide interconnection between water-bearing zones, allowing the mixing of contaminated and noncontaminated ground water. If the piles are located over recharge areas for deeper aquifers, then the contaminants would not be limited to the uppermost aquifer.

The Ambrosia Lake UMTRA site was a former uranium mill that operated as a mechanical and chemical uranium-concentrating plant from 1958 to 1963 . It operated as an ion-exchange plant for uranium concentration from 1971 to 1983. The sources of water for the ion-exchange plant were three mines (the Ann Lee, Sandstone, and Section 27 mines), as well as return water from heap leach piles in Section 27.

Site contamination was caused primarily by the discharge of waste water and tailings from uranium milling during the 1958 to 1963 period. The ion-exchange and heap leach operations contributed little additional contamination when compared to the uranium mill process.

Approximately 3.0 million tons (2.7 million tonnes) of uranium-bearing ore were processed during the period of 1958 to 1963 by crushing and grinding, and then by removing the uranium through a chemical process (DOE, 1991). The operations resulted in the production of a relatively small percentage of uranium concentrate and a relatively large percentage of wastes, consisting of waste water and mill tailings. Contamination of ground water resulted from discharge of waste water, hydraulic placement of mill tailings, and infiltration of water discharged at the surface.

Mill tailings are the fine-grained materials (sand, silt, and clay) resulting from the uranium removal process. Because the uranium represented only a small portion of the ore, the volume of tailings is approximately equal to the amount of ore . processed (i.e. approximately 3 million tons [2.7 million tonnes] of tailings were produced) (DOE, 1991). The tailings were mixed with water from the ore zone (the Westwater Canyon Member of the Morrison Formation) and with waste water from the chemical concentration process and this mixture was piped to the tailings pile.

Waste water was produced from the former mill at a rate of 1 to 5 tons 10.9 to 4.5 tonnes) for every ton of ore processed (Merritt, 1971). Therefore, from 3.0 to 15 million tons ( 2.7 to 13.6 million tonnes) of waste water were produced during the 5 years of ore milling. This is equivalent to between $7.2 \times 10^{8}$ to 
$3.6 \times 10^{9} \mathrm{gal}\left(2.7 \times 10^{9}\right.$ and $\left.1.4 \times 10^{10} \mathrm{~L}\right)$ or 2200 to 11,000 acre-feet of waste water. Waste water was disposed of with the tailings and also collected in two main holding ponds (formerly near the southeast corner of the tailings pile in Section 281; some was possibly discharged to a canal which led to a holding pond called the Voght Tank, previously located in Section 34 (Figure 3.3). Some waste water was also discharged to two holding ponds near the southeast corner of the tailings pile in Section 33 (Figure 3.3).

Approximately 400,000 tons $(360,000$ tonnes) of the total 3 million tons of (2.7 million tonnes) tailings were piped into the Ann Lee Mine using water from the mines and possibly process waste water (DOE, 1991). This was a legal and common practice in the area to protect against subsidence. The Ann Lee Mine was the only mine backfilled with tailings from the Ambrosia Lake site; however, this practice was undertaken at numerous other mines and contributes to widespread ground water contamination in all geologic units from the ore zone to the surface. Section 3.3, conceptual site model, provides additional detail regarding site and surrounding area geology and water quality.

The carbonate process for uranium concentration was used at the Ambrosia Lake site (Merritt, 1971). Process materials contributing to site contamination were uranium ore, caustic soda, soda ash, sulfate salts, and ammonia. Major indicators of contamination resulting from the Ambrosia Lake site process materials and byproducts were uranium, nitrate, sulfate, and sodium.

The ion-exchange process contributed little if any additional contamination to the site. Water that was passed through the ion-exchange columns was returned to its source (the mines), and not discharged to the land surface. Water applied to the heap leach piles was collected with a drain system for recovery of uranium using the ion-exchange columns.

\section{Site Status}

The Phillips Petroleum Company constructed, owned, and operated the former mill from 1957 until 1963, when United Nuclear Corporation purchased the operation. Uranium concentrate was sold to the Atomic Energy Commission (AEC) throughout the mill's operational history. The state of New Mexico owns the site at present; ownership will be transferred to the DOE upon completion of the surface remedial actions.

As part of the UMTRA project surface remediation activities, RRM is being consolidated on the site in a disposal cell that is scheduled for completion in early 1995. Contents of the call include the tailings pile, former mill buildings, wind blown tailings, the heap leach pads (Section 27), the former main holding ponds (Section 28), and materials associated with the two former ponds near the southeast portion of the disposal cell (Section 33), the canal, and Voght Tank (Section 34) (Figure 3.3). An environmental assessment (DOE, 1987a) and a Finding of No Significant Impact (FONSI) (DOE, 1987b) were completed in 
1987. The RAP for surface remediation activities was concurred with by the NRC and state of New Mexico in 1990 (NRC, 1990).

Tailings removal from the Ann Lee or other surrounding mines is technically unfeasible and their removal would pose a threat to personnel. The tailings within these underground mines are currently isolated from contact with human or environmental receptors. The tailings backfilled into the Ann Lee Mine, therefore, are anticipated to qualify for supplemental standards under the surface remediation project and will remain in place. A final application for supplemental surface remediation standards for the backfilled tailings in the Ann Lee Mine will be submitted by the DOE Remedial Action Contractor. Ambrosia Lake site tailings in the Ann Lee Mine have little additional adverse effect on area ground water quality because of the presence of tailings in numerous surrounding mines and the presence of uranium in the ore zone.

In their 1990 technical evaluation of the RAP, the NRC concurred with the finding that the uppermost aquifer at the site (the alluvium, weathered Mancos Shale, and Tres Hermanos-C Sandstones) is incapable of a sustained well yield of 150 gallons per day. Therefore ground water within the uppermost aquifer meets the criteria for Class III, supplemental standards, based on limited use (NRC, 1990). As described in Section 3.3, conceptual site model, the factors controlling well yield will not change over time, with the exception of the saturated thickness of the aquifer, which will decrease. Potential well yield will also decline as the saturated thickness decreases. Therefore, the "limited use" aquifer status should also apply to the ground water portion of this project.

\subsection{SOURCES OF DATA}

Information presented in this SOWP includes observations from site visits and review of data and documentation available at the UMTRA Project Document Control Center in Albuquerque, New Mexico.

Detailed descriptions of uranium concentration, mill byproducts, and process waste streams are provided in Merritt (1971). Site hydrogeologic conditions are described in detail in the RAP (DOE, 1991) and the NRC's final technical evaluation report (TER) (NRC, 1990). Additional site-specific information was obtained through a review of geologic and hydrologic reports cited in the RAP, an examination of monitor well completion reports, and ground water chemistry data contained in the DOE's Software Program for Environmental Analysis and Reporting (SPEAR) Program System (DOE, 1992b). The data contained in the SPEAR Program System are the results from periodic water quality monitoring at the site and encompass the years from 1980 to 1994.

\subsection{CONCEPTUAL SITE MODEL}

The following paragraphs present a summary of the site conceptual model. Additional details regarding the site conceptual model are in the subsections following this summary. 
Surrounding land use was predominantly uranium mining and milling. Mining companies still own the land surrounding the site; however, mining and milling are no longer performed because of economic conditions. Low-density grazing is the current predominant land use.

The tailings in the Ann Lee Mine are isolated from direct contact to human and environmental receptors, and will remain in place. Ground water does not discharge at land surface; therefore a direct exposure pathway to contaminated ground water currently does not exist. No water supply wells are completed in the hydrogeologic zones with contamination attributable to the UMTRA Ambrosia Lake site, and water contaminated by the former uranium mill does not affect other ground water supplies in the area. Also, there are no nearby uses of surface water. Therefore, no current exposure pathways to humans, livestock, or wildlife exist.

As discussed previously, milling operations created a relatively large amount of waste water. Based on geochemical evaluation and the limited amount of natural recharge to the uppermost aquifer, saturation of the uppermost aquifer was created primarily by discharge of the mill waste water. The extent of saturation resulting from Ambrosia Lake site operations is therefore limited in both areal and vertical extent. The aquifer does not represent a ground water resource because of the inability to sustain a yield of $150 \mathrm{gal}(570 \mathrm{~L})$ per day to wells. Therefore, potential future exposure pathways to humans, livestock, or wildlife are also unlikely. Ground water within the uppermost aquifer meets the qualifications for supplemental standards, "limited use," based on limited yield (40 CFR \$192.21(g)) (52 FR 36000). Because the potential for future exposure pathways to humans, livestock, or wildlife is unlikely, the proposed recommended ground water compliance strategy is no remediation based on the application for supplemental standards.

\subsubsection{Hydrogeologic setting}

\section{Physiographic Setting}

The site lies within the Ambrosia Lake valley and west of San Mateo Mesa, a northwest trending ridge. The site is on a relatively flat area at an elevation of approximately $7000 \mathrm{ft}(2100 \mathrm{~m})$. San Mateo Mesa is at an elevation of about $8100 \mathrm{ft}(2500 \mathrm{~m})$.

The site is within the Arroyo del Puerto drainage basin, which slopes downward toward the southeast. The approximate elevation of the drainage channel nearest the site is about $6900 \mathrm{ft}(2100 \mathrm{~m})$. The streams in the area of the site and Ambrosia Lake are primarily ephemeral, containing water only after heavy rainfall. However, a portion of the Arroyo del Puerto approximately $1.5 \mathrm{mi}$ $(2.4 \mathrm{~km})$ southwest of the site (Figure 3.3) appears to contain water for extended periods of time as a result of drainage from the Quivira Mining Company tailings pile. 


\section{Geology}

In descending order, geologic units of interest at the site are as follows (Brod and Stone, 1981):

1. Alluvium: alluvial and eolian deposits of clay, silt, sand, and gravel;

2. Mancos Shale: dark gray, calcareous marine shale containing four silty sandstone interbeds, known as the Tres Hermanos $-C_{1},-C_{2},-B$, and $-A$ Sandstones;

3. Dakota Sandstone: fine- to medium-grained sandstone, probably representative of a marine shore zone; and

4. Morrison Formation: interbedded mudstones, siltstones, and sandstones that have been divided into three members, Brushy Basin Member (mudstones and sandstones that contain uranium ore in some areas), Westwater Canyon Member (sandstones and mudstones that contain the majority of the uranium ore mined in the Ambrosia Lake area and also the primary aquifer), and Recapture Member (interbedded shale, siltstone, and sandstone).

The geologic units beneath the site, with the exception of the alluvium, dip (on a regional basis) toward the northeast at approximately 2 degrees. The alluvium has been deposited nearly horizontally on top of the Mancos Shale. The direction of the dip, relative depths, and relative thicknesses of the units are shown on Figure 3.4, a regional, generalized geologic cross section. The Recapture Member of the Morrison Formation is below the ore zone and is considered an aquitard, retarding the exchange of water between units above it and below it (Brod and Stone, 1981). Site activities should not have impacted deeper units because mining operations did not extend deeper than the Westwater Canyon member (the ore zone) and because the Recapture Member retards the exchange of water between units above and below it. These deeper units are excluded from further description.

The locations of site-specific geologic cross sections constructed from wells installed by the DOE for the UMTRA project are shown on Figure 3.5. Cross sections are shown on Figures 3.6 and 3.7. The cross sections show sitespecific relationships between the shallow geologic units and the thickness of those units. Logs of the Ambrosia Lake UMTRA Project site wells shown on the figures are presented in Appendix A. There are a number of additional UMTRA Project site wells, but because of the large amount of information, logs for those wells are not included with this document. However, well construction details and water levels contained in the SPEAR data base (DOE, 1992b) for all of the wells are in Appendix $B$, Hydrogeologic Information. Additional information is available for review in the UMTRA Project Document Control Center, Albuquerque, New Mexico. Not contained in the SPEAR data base are water 


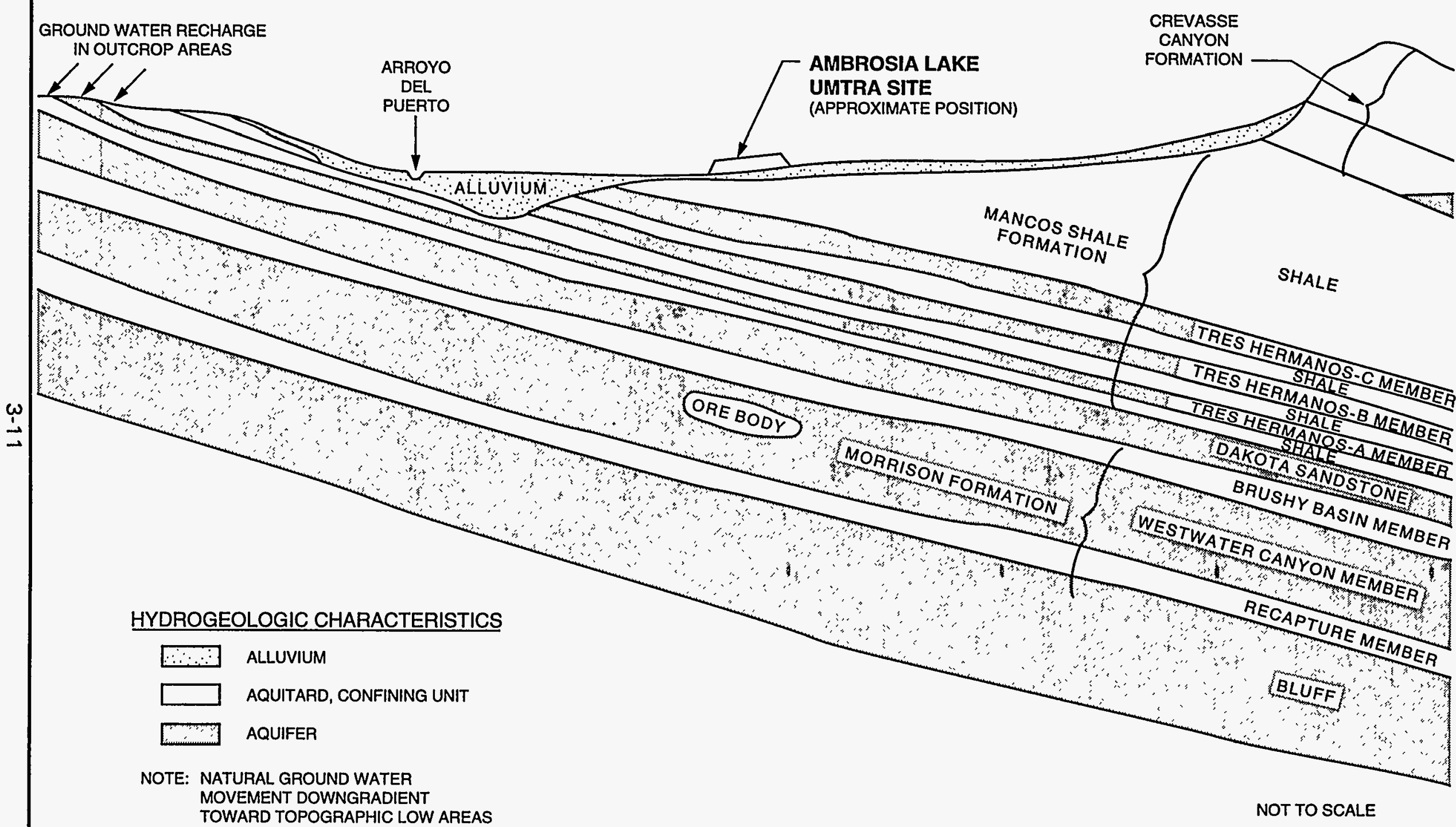

FIGURE 3.4

GENERALIZED REGIONAL GEOLOGIC CROSS SECTION

MODIFIED FROM DOE, 1991. FOR THE AMBROSIA LAKE AREA, NEW MEXICO 


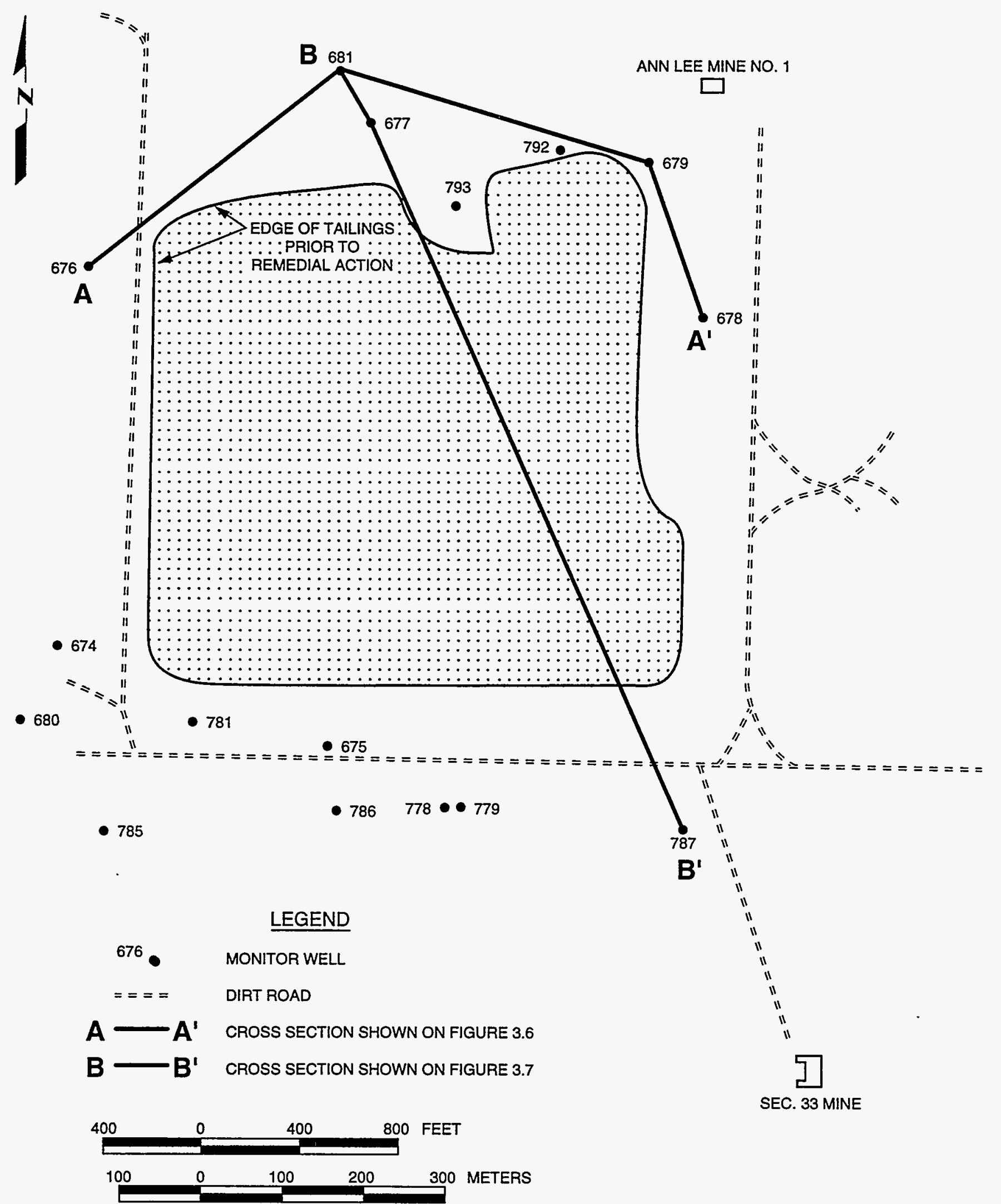

MODIFIED FROM DOE, 1991.

FIGURE 3.5

LOCATIONS OF GEOLOGIC CROSS SECTIONS

AMBROSIA LAKE UMTRA SITE, NEW MEXICO 
A

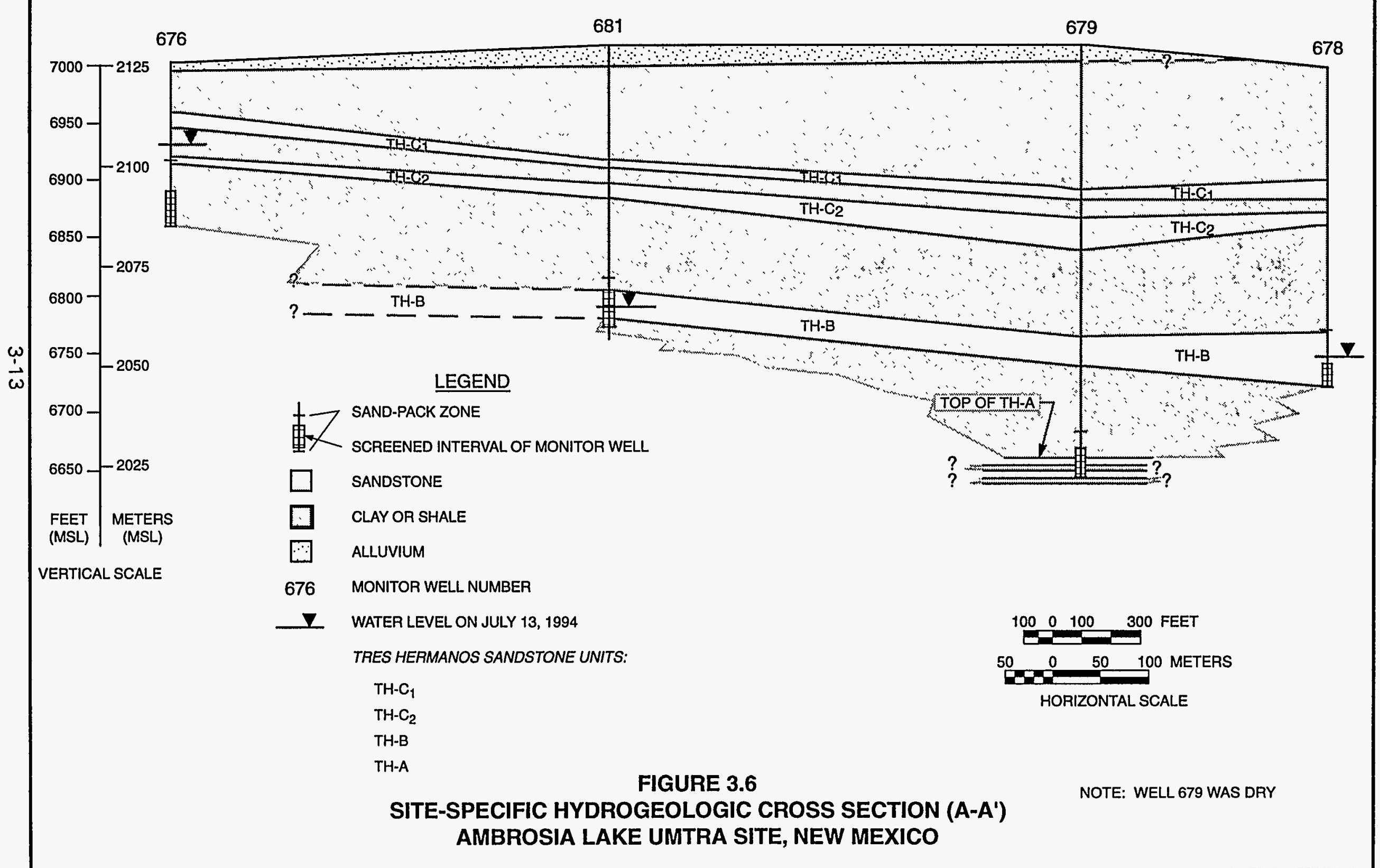




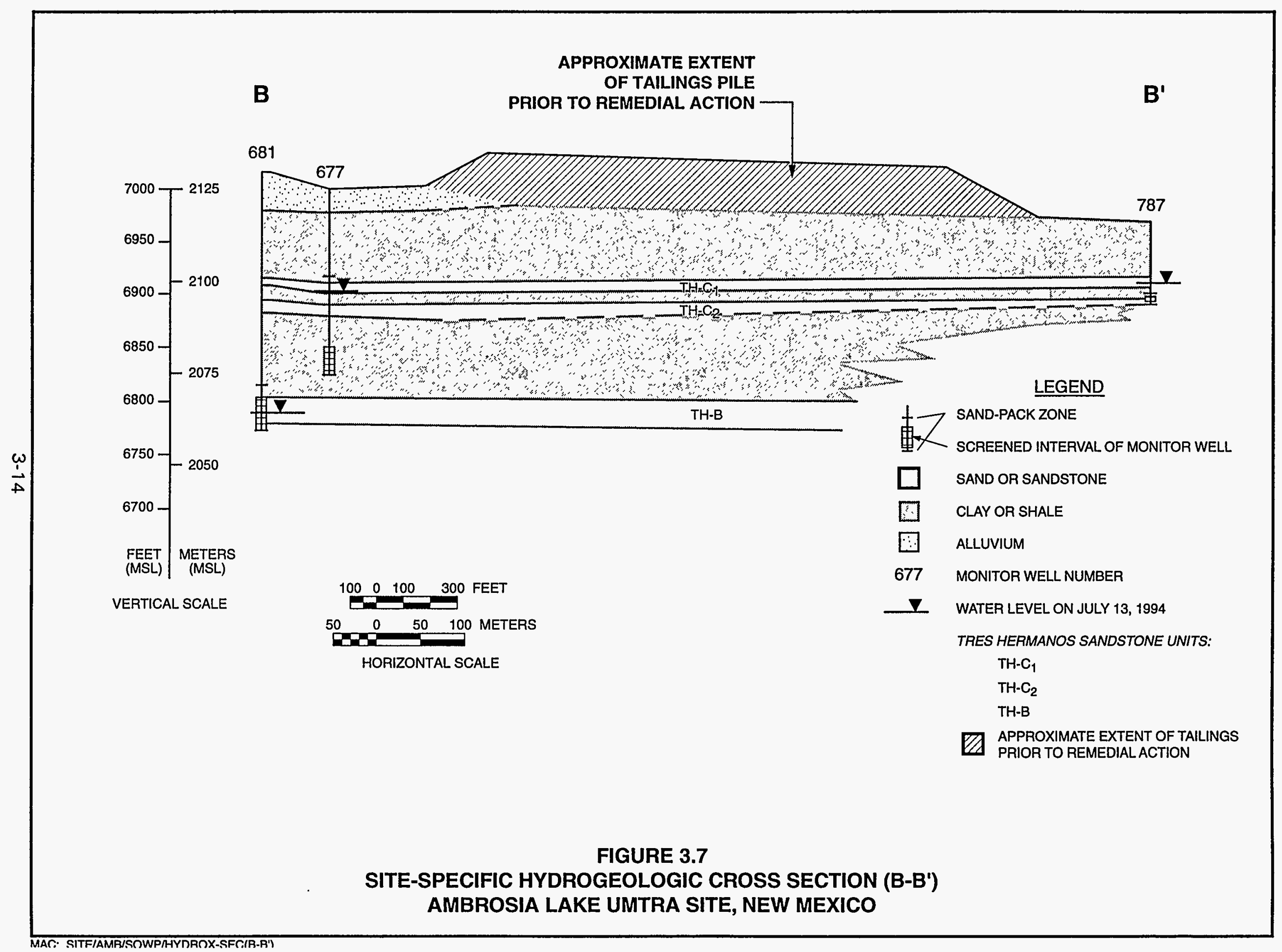


level data from the most recent sampling event, in July 1994. These data are shown in Table 3.1 and on the cross sections (Figures 3.6 and 3.7).

\section{Hydrology}

\section{Ground water}

Hydrogeologic units at the site, in descending order, and the hydraulic properties of each, are as follows:

1. Uppermost aquifer: By definition in the RAP (DOE, 1991), the uppermost aquifer consists of the Tres Hermanos- $C_{1}$ and $-C_{2}$ silty sandstone units, weathered Mancos Shale, and alluvium. The maximum thickness (and depth below grade) of the aquifer is approximately $175 \mathrm{ft}(53 \mathrm{~m})$. However, the maximum saturated thickness is only about $25 \mathrm{ft}(8 \mathrm{~m})$.

To determine the source of water in the uppermost aquifer, it is necessary to evaluate the relationship between recharge and discharge. The major sources of recharge, waste water disposal and water from mine pumping, no longer exist. The only current source of recharge to the uppermost aquifer is rainfall infiltration, which is limited by the semiarid climate.

A primary factor in determining recharge from rainfall is a comparison between rainfall and evapotranspiration. Annual rainfall is low, at 8.8 inches (22 centimeters [cm]) ( $Q M C, 1981$ ), and evapotranspiration is high, at a rate of 54 inches $(140 \mathrm{~cm})$ (NOAA, 1979). Other indicators of the semiarid conditions are the sparse vegetation and poor quality soils. Therefore, the potential for recharge from precipitation is low.

A further indicator of little-to-no recharge is the interaction between surface water and ground water. There have been no uses of ground water in the shallow aquifer, since there are no wells, other than DOE monitor wells, at the site. The Mancos Shale beneath the Tres Hermanos-C Sandstone units acts as a lower confining unit, retarding the infiltration of water into lower units. Therefore, there are no mechanisms for significant reductions or withdrawals of ground water. If there was a significant amount of recharge from rainfall, water in the upper aquifer would build to a level at which the water level would be at ground surface, or it would discharge to surface water bodies. Neither scenario is valid, based on records of water levels and because the surrounding surface water bodies are ephemeral, containing water only after significant rainfall. Therefore, the saturated thickness currently in the unit is expected to either remain about the same or decrease as water spreads laterally and a portion of it migrates upward into the vadose (unsaturated) zone.

An aquifer test conducted February 2-3, 1989 on well 675 and described in the RAP (DOE, 1991), shows that the uppermost aquifer is incapable of a sustained yield of $150 \mathrm{gal}(570 \mathrm{~L})$ per day to a well. Therefore, the unit is 
Table 3.1 Water levels for all existing wells, July 13, 1994, Ambrosia Lake UMTRA site

\begin{tabular}{|c|c|c|c|}
\hline Well No. & TOC elev ${ }^{a}$ & DTW ${ }^{b}$ & WL elev ${ }^{c}$ \\
\hline 674 & 6972.00 & 44.60 & 6927.40 \\
\hline 675 & 6966.00 & 22.35 & 6943.65 \\
\hline 676 & 6991.80 & 64.85 & 6926.95 \\
\hline 677 & 7001.60 & 89.37 & 6912.23 \\
\hline 678 & $\left.69751^{*}\right)$ & 220.80 & 6754.20 \\
\hline 679 & 7011.00 & $<300$ & $(* *)$ \\
\hline 680 & 6972.00 & $<300$ & $\left(*^{*}\right)$ \\
\hline 681 & 7004.00 & 213.41 & 6790.59 \\
\hline 778 & 6962.70 & 27.93 & 6934.77 \\
\hline 779 & 6963.00 & 36.65 & 6926.35 \\
\hline 781 & 6968.40 & 39.64 & 6928.76 \\
\hline 785 & 6961.30 & 43.25 & 6918.05 \\
\hline 786 & 6963.40 & 21.86 & 6941.54 \\
\hline 787 & 6971.30 & 56.33 & 6914.97 \\
\hline 789 & 7003.90 & Dry & $\left(*^{* *}\right)$ \\
\hline 792 & 7001.10 & Dry & $(* *)$ \\
\hline 793 & 6998.60 & 27.55 & 6971.05 \\
\hline 797 & 6972.00 & Dry & $\left(*^{*}\right)$ \\
\hline 798 & 6980.40 & Dry & $(* *)$ \\
\hline
\end{tabular}

aTOC ELEV: Top of casing elevation, in feet, above mean sea level (MSL).

bDTW: Depth to water below top of casing, in feet.

CWL ELEV: Water level elevation, in feet, MSL.

- Estimated for Well 678 because the well head was destroyed.

* * No water level elevation because well was dry or DTW exceeded length of water level indicator. 
considered a "limited use" aquifer, based on limited yield. The calculation set detailing the aquifer test is in Appendix B of this document. The NRC interpreted the test results as follows (NRC, 1990):

As defined in the standards, Class III ground water is not a current or potential source of drinking water for any of three reasons, including inability of the aquifer to provide a sustained yield exceeding $150 \mathrm{gal}$ per day. The DOE determined that the upper aquifer, composed of alluvium, weathered Mancos Shale, and the Tres Hermanos-C Sandstone members, is incapable of sustained yield of 150 gal per day. The DOE's determination is based on the following:

(1) A pump test was performed on Well 675. It produced $0.35 \mathrm{gal}$ per minute $(500 \mathrm{gal}$ per day). The water level was drawn down $13 \mathrm{ft}$ in 12 hours, and the pumping could not be sustained.

(2) Well 675 has the greatest saturated thickness of any wells completed in the uppermost aquifer, and is screened to the base of the saturated zone. Therefore, it probably produces the maximum available water.

(3) The DOE calculated that long-term output of $150 \mathrm{gal}$ per day at Well 675 could not be sustained for more than 1 day. DOE further justifies application of supplemental standards at Ambrosia Lake because water in the uppermost aquifer is of limited extent, and is derived solely from infiltration of mining and milling water sources.

Therefore, there is reasonable assurance that DOE is eligible to apply for supplemental standards because the uppermost aquifer at Ambrosia Lake contains Class III ground water.

Review of hydrogeologic conditions at Ambrosia Lake verifies the supplemental standards are justified for other reasons as well. First, an engineered radon/infiltration barrier is a key component of the design. Infiltration of water through the tailings and recharge of the aquifer will be minimized in this fashion. Also, access to the site by potential ground-water users in the future will be restricted by institutional controls for at least 100 years following completion of the UMTRA Surface Project remedial action.

2. Mancos Shale: The Mancos Shale acts primarily as an aquitard, but by definition in the RAP (DOE, 1991), it contains a portion of the uppermost aquifer, the Tres Hermanos- $C_{1}$ and $-C_{2}$ Sandstones. Also contained within the Mancos Shale are the Tres Hermanos-B and -A Sandstone units which 
are silty sandstone units interbedded within the Mancos Shale. Aquifer tests were not performed on the Tres Hermanos-B and -A Sandstone units. However, based on a lithology similar to the Tres Hermanos-C Sandstone units, the water-bearing capacity of the Tres Hermanos-B and -A Sandstone units is not much greater than the Mancos Shale.

Recharge to the Tres Hermanos-B and -A Sandstone units is from surface water at the locations where the units outcrop, or appear at the surface (Figure 3.4). The Tres Hermanos-B Sandstone unit outcrops about $1.5 \mathrm{mi}$ $(2.4 \mathrm{~km})$ southwest of the Ambrosia Lake site and is recharged by drainage from the nearby Quivira Mining Company uranium mill. Similarly, the Tres Hermanos-A Sandstone unit outcrops slightly further to the southwest and is likely recharged by water affected by the same mill (Bostick, 1985). Recharge to these units has likely decreased since the report by Bostick (1985) was prepared, because the mill is no longer in operation.

Based on water-level data contained in the SPEAR data base (DOE, 1992b), there is an indication that the Tres-Hermanos-B Sandstone unit is only partially saturated. The information is shown graphically on Figures 3.6 and 3.7. Because the unit is relatively thin and only partially saturated, its use as a potential aquifer is limited.

The Mancos Shale units that separate the Tres Hermanos Sandstones and the units that are above the Dakota Sandstone are continuous and act as aquitards because of their thickness and low hydraulic conductivity. According to the RAP (DOE, 1991), "...a hydraulic conductivity of $4.3 \times 10^{-8}$ centimeter per second $(\mathrm{cm} / \mathrm{s})\left(1.2 \times 10^{-4}\right.$ feet per day) was measured in undisturbed Mancos Shale and is probably representative of the vertical hydraulic conductivity...." The low hydraulic conductivity of the Mancos Shale also makes it suitable as a source of low-permeability cover material for UMTRA Project disposal cells, and is used for several Project cells, including the Ambrosia Lake site.

3. Dakota Sandstone: The Dakota Sandstone is considered a secondary aquifer because of the relatively low yield (less than 10 gal [ $40 \mathrm{~L}$ ] per minute) and poor water quality, when compared to the Westwater Canyon Member of the Morrison Formation (DOE, 1987a). Bostick (1985) reports that the Dakota Sandstone is present at land surface near the Quivira Mill and that . surface discharge of water related to the Quivira Mill onto the outcrop of the Dakota Sandstone has caused contamination of the unit. The discharge of contaminated water at the outcrop and the discharge of water possibly from mill tailings placed in surrounding mines are the sources of contaminants found in the Dakota Sandstone beneath the Ambrosia Lake site.

4. Morrison Formation:

a. The Brushy Basin Member acts as an aquitard between the Dakota Sandstone and Westwater Canyon Member; 
b. The Westwater Canyon Member is the principal aquifer in the Ambrosia Lake Mining District, but it is also the major source of uranium ore. Mine pumping began in the mid-1950s to facilitate ore removal from the Westwater Canyon Member. The ground water was discharged to surface drainages where it evaporated, and some infiltrated into the shallower zones. A portion of the water was used for mill process water. Because of the regional mine pumping, a large ground water depression has been created and ground water flow is probably toward the northeast (DOE, 1987a).

c. The Recapture Member acts as an aquitard beneath the Westwater Canyon Member because of its thickness of $165 \mathrm{ft}(50 \mathrm{~m})$ and low permeability (DOE, 1987a).

\section{Surface Water}

The Ambrosia Lake tailings site lies in the drainage basin of the Arroyo del Puerto, an ephemeral stream. Surface water flow occurs only after heavy rain; however, there are ponds approximately $1.5 \mathrm{mi}(2.4 \mathrm{~km})$ southwest of the site. Based on the presence of cattails and marsh grasses, it appears that the ponds contain water throughout the year. The ponds are located in the recharge area for the Tres Hermanos-B Sandstone unit and are fed by water from the Quivira Mining Company uranium tailings pile.

Two unnamed drainage channels originate to the west (north ephemeral stream) and east (east ephemeral stream) of the site (Figure 3.3). There are no perennial streams close to the site.

\subsubsection{Ground water quality}

In order to define the extent of ground water contamination caused by the former uranium milling at the Ambrosia Lake site, water samples were collected from a DOE monitor well network from 1980 through 1994. Figure 3.5 shows a portion of the DOE monitor well network which is representative of current site conditions. Ground water samples collected from the monitor well network have been analyzed to determine the quality of ground water in the alluvium and the weathered Mancos Shale, the Tres Hermanos-C, -B and -A Sandstones, and the Westwater Canyon Member of the Morrison Formation. Ground water quality data by location for all Ambrosia Lake UMTRA Project site wells, including all sampling events, are presented in Appendix $C$ of this document.

\section{Background water quality}

Background ground water quality in the alluvium and the weathered Mancos Shale and Tres Hermanos-C Sandstone at the Ambrosia Lake site is considered to be the same as existing water quality because former mining and milling activities have created the saturated conditions (Bostick, 1985). It has been demonstrated, using geochemical modeling, that the ground water within the 
alluvium and the weathered Mancos Shale is derived entirely from tailings seepage and mill-makeup water (DOE, 1991). Mill-makeup water was generated from mine pumping discharge. Details of the modeling results are presented in Table D.8.25 of the RAP (DOE, 1991).

\section{Extent of contamination}

Because all water contained in the alluvium and the weathered Mancos Shale and Tres Hermanos-C Sandstone in the area of the milling site is primarily a mixture of tailings seepage and mine discharge, the extent of contamination is the same as the extent of ground water. Because of the large ground water depression created by mine pumping, all potential pathways for contamination from the Ambrosia Lake UMTRA site to enter units between the Tres Hermanos-C Sandstone and the Morrison Formation are points of discharge. Thus, there is no UMTRA site-related contamination in those units. Discharge of water from the Quivira Mining Company Mill to the outcrop of the Tres Hermanos-A Sandstone, Tres Hermanos-B Sandstone, and the Dakota Formation caused contamination of these units (Bostick, 1985). The Westwater Canyon Member is potentially contaminated by regional mining and milling processes because water from all overlying units drains into this unit through mine shafts and vent holes.

\section{Alluvium and the weathered Mancos Shale}

Tailings pore water at the site contained as much as $11,000 \mathrm{mg} / \mathrm{L}$ sulfate (DOE, 1991). The sulfate isopleth map (Figure 3.8) is used to define the extent of tailings-related contamination and, very likely, the extent of ground water in the alluvium and the weathered Mancos Shale at the site. Monitor well 620 is more than $1200 \mathrm{ft}(370 \mathrm{~m})$ southeast of the primary ground water mound. Sulfate concentrations and ground water in well 620 are assumed to be derived from the former holding ponds to the east (Figure 3.8). During the period of sampling (1980-1994), concentrations of arsenic, cadmium, chromium, molybdenum, nitrate, selenium, silver, uranium, and the activities of gross alpha and radium226 plus radium-228 exceeded the proposed EPA ground water standards in the alluvium and the weathered Mancos Shale (Table 3.2).

\section{Tres Hermanos-C Sandstone}

The Tres Hermanos- $C_{1}$ Sandstone is separated from the underlying Tres Hermanos- $\mathrm{C}_{2}$ Sandstone by a 10 -to- $15 \mathrm{ft}$-(3-to- $4.5 \mathrm{~m}$-)thick shale unit. This shale unit retards downward migration of contaminants, resulting in slightly higher levels of contamination in the Tres Hermanos- $C_{1}$ Sandstone (Table 3.3), compared to values in the Tres Hermanos $-\mathrm{C}_{2}$ Sandstone (Table 3.4). There are insufficient data to draw a sulfate isopleth map for the Tres Hermanos- $\mathrm{C}_{1}$ Sandstone. The sulfate isopleth map (Figure 3.9) shows the approximate extent of tailings-related contamination in the Tres Hermanos- $\mathrm{C}_{2}$ Sandstone. This map indicates that contamination has moved farther downgradient to the northeast (as compared to Figure D.8.24 in DOE, 1991). In sulfate data from the January, 


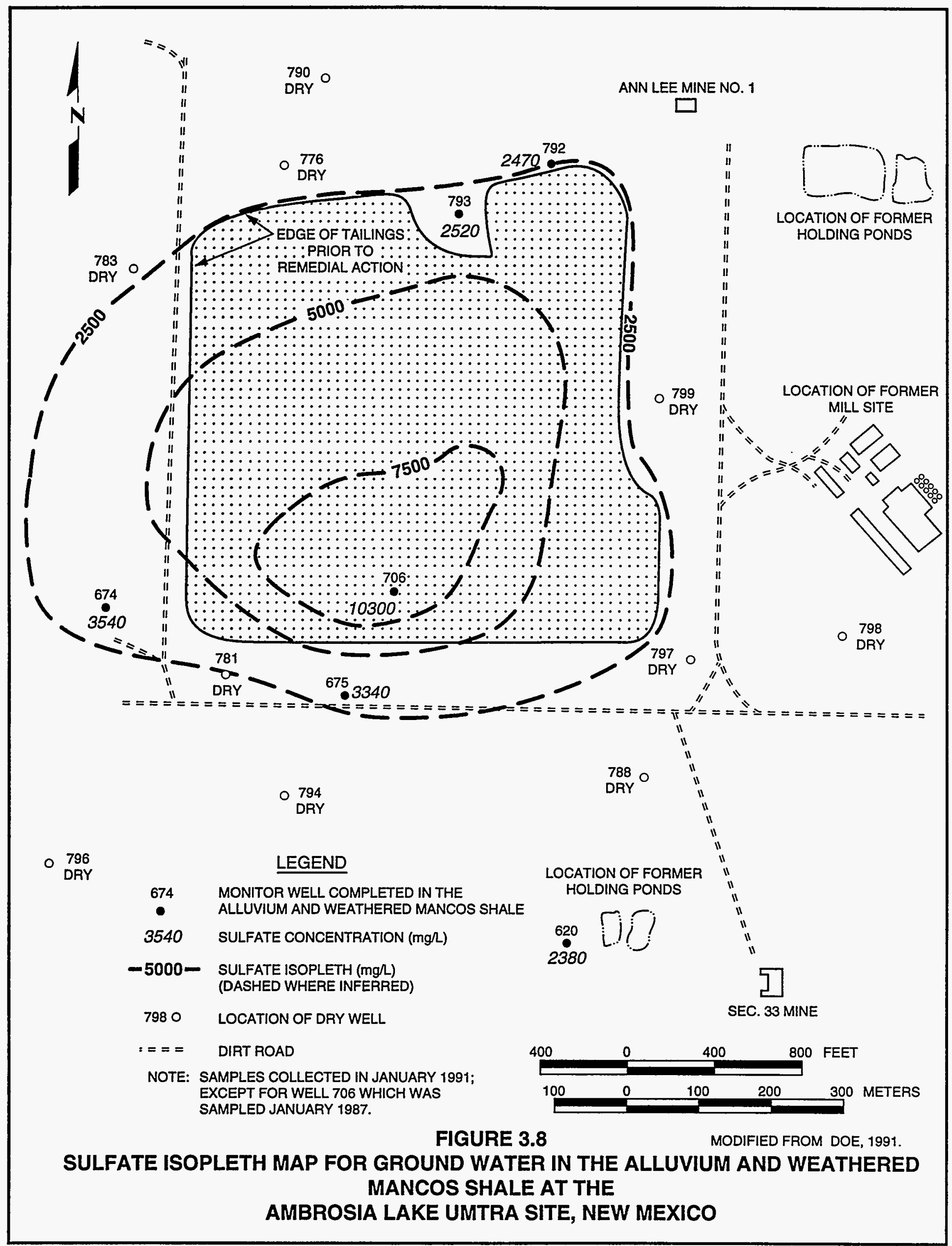


Table 3.2 Maximum observed concentrations of EPA MCL constituents in monitor wells located in the alluvium/weathered Mancos Shale at the Ambrosia Lake site during the years from 1980 to 1994

\begin{tabular}{|c|c|c|c|c|c|c|c|}
\hline \multirow[b]{2}{*}{ Constituent $^{a}$} & \multirow{2}{*}{$\begin{array}{l}\mathrm{MCL}^{\mathrm{b}} \\
(\mathrm{mg} / \mathrm{L})\end{array}$} & \multicolumn{6}{|c|}{ Monitor wells } \\
\hline & & 620 & 674 & 675 & 706 & 792 & 793 \\
\hline Arsenic & 0.05 & 0.01 & 0.02 & 0.02 & $0.33^{c}$ & 0.016 & 0.016 \\
\hline Barium & 1 & 0.1 & $<0.1$ & 0.01 & $<0.1$ & $<0.1$ & $<0.1$ \\
\hline Cadmium & 0.01 & $<0.001$ & 0.003 & 0.003 & $<0.001$ & 0.01 & $0.013^{c}$ \\
\hline Chromium & 0.05 & $0.16^{c}$ & $<0.01$ & $<0.01$ & $0.06^{c}$ & $0.28^{C}$ & $0.28^{c}$ \\
\hline Lead & 0.05 & 0.02 & 0.03 & 0.02 & 0.02 & 0.02 & 0.02 \\
\hline Mercury & 0.002 & $<0.0002$ & $<0.0002$ & $<0.0002$ & $<0.002$ & 0.0007 & 0.0003 \\
\hline Molybdenum & 0.1 & $0.5^{c}$ & $9.81^{c}$ & $2.72^{c}$ & $225^{c}$ & $1.87^{\mathrm{c}}$ & $2.01^{\mathrm{c}}$ \\
\hline Nitrate & $44^{d}$ & 12.1 & $69^{c}$ & $252^{c}$ & 25 & 1.8 & $830^{c}$ \\
\hline Selenium & 0.01 & $0.07^{c}$ & $3.1^{c}$ & $0.51^{\mathrm{c}}$ & $0.088^{c}$ & $2.22^{c}$ & $2.1^{\mathrm{c}}$ \\
\hline Silver & 0.05 & 0.04 & 0.01 & 0.02 & $0.15^{c}$ & $0.11^{\mathrm{c}}$ & $0.11^{c}$ \\
\hline $\begin{array}{l}\text { Combined } \\
\text { Uranium-234 } \\
\text { and }-238\end{array}$ & $0.044^{e}$ & $8.22^{C}$ & $10.7^{c}$ & $2.083^{C}$ & $11.1^{\mathrm{C}}$ & $3.31^{\mathrm{c}}$ & $0.393^{c}$ \\
\hline $\begin{array}{l}\text { Gross alpha } \\
\text { (excluding } \\
\text { radon and } \\
\text { uranium) } \\
\mathrm{pCi} / \mathrm{L}^{f}\end{array}$ & 15 & $5300^{C}$ & $2200^{c}$ & $1700^{c}$ & $15,000^{c}$ & $2400^{C}$ & $320^{c}$ \\
\hline $\begin{array}{l}\text { Combined } \\
\text { Radium-226 } \\
\text { and }-228 \\
\mathrm{pCi} / \mathrm{L}^{\mathrm{f}}\end{array}$ & 5 & $9.13^{C}$ & 0.8 & 4.03 & $131.8^{c}$ & $6.85^{c}$ & 2.3 \\
\hline \multicolumn{8}{|c|}{ 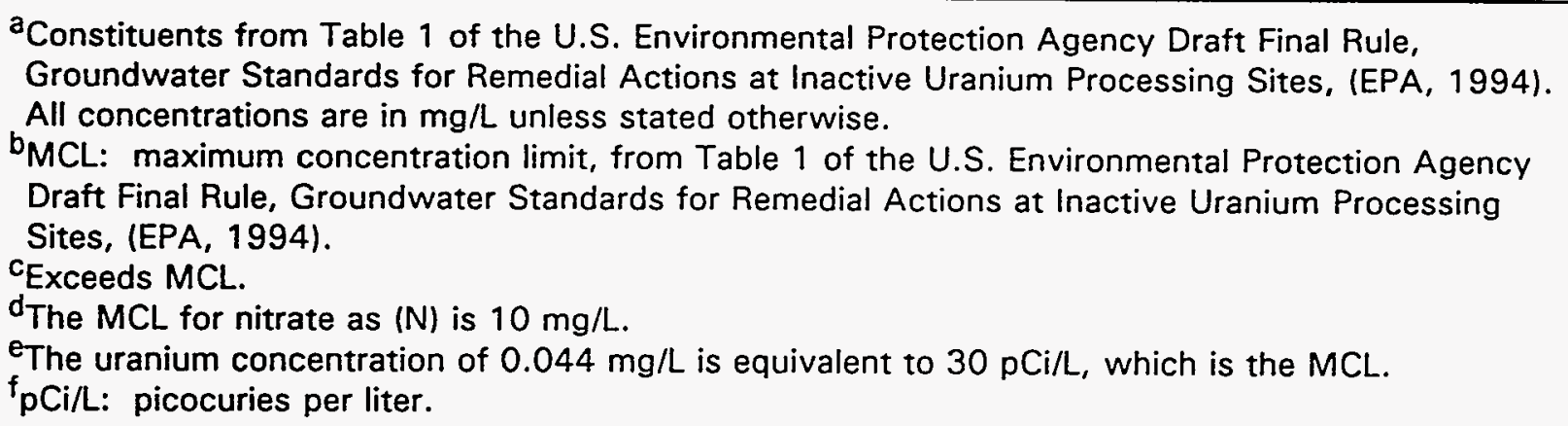 } \\
\hline
\end{tabular}


Table 3.3. Maximum observed concentrations of EPA MCL constituents in monitor wells located in the Tres Hermanos- $\mathrm{C}_{1}$ Sandstone at the Ambrosia Lake site during the years from 1980 to 1994

\begin{tabular}{|c|c|c|c|}
\hline \multirow[b]{2}{*}{ Constituent $^{a}$} & \multirow{2}{*}{$\begin{array}{l}\mathrm{MCL}^{\mathrm{b}} \\
(\mathrm{mg} / \mathrm{L})\end{array}$} & \multicolumn{2}{|c|}{ Monitor wells } \\
\hline & & 778 & 786 \\
\hline Arsenic & 0.05 & 0.022 & 0.02 \\
\hline Barium & 1 & $<0.1$ & 0.3 \\
\hline Cadmium & 0.01 & $0.014^{c}$ & $0.013^{c}$ \\
\hline Chromium & 0.05 & $0.22^{c}$ & $0.14^{c}$ \\
\hline Lead & 0.05 & 0.02 & 0.02 \\
\hline Mercury & 0.002 & 0.0005 & 0.0003 \\
\hline Molybdenum & 0.1 & $0.16^{c}$ & $0.34^{c}$ \\
\hline Nitrate & $44^{d}$ & $430^{c}$ & $55^{c}$ \\
\hline Selenium & 0.01 & $0.28^{c}$ & $0.78^{c}$ \\
\hline Silver & 0.05 & $0.06^{c}$ & 0.02 \\
\hline Combined Uranium-234 and -238 & $0.044^{e}$ & $11.8^{c}$ & $2.88^{c}$ \\
\hline $\begin{array}{l}\text { Gross alpha (excluding radon and } \\
\text { uranium) } \mathrm{pCi} / \mathrm{L}^{f}\end{array}$ & 15 & $9400^{\mathrm{C}}$ & $1300^{c}$ \\
\hline Combined Radium-226 and $-228 \mathrm{pCi} / \mathrm{L}^{f}$ & 5 & $7.93^{\mathrm{C}}$ & $9.92^{\mathrm{c}}$ \\
\hline \multicolumn{4}{|c|}{$\begin{array}{l}\text { aConstituents from Table } 1 \text { of the U.S. Environmental Protection Agency Draft Final } \\
\text { Rule, Groundwater Standards for Remedial Actions at Inactive Uranium Processing } \\
\text { Sites, (EPA, 1994). All concentrations are in mg/L unless stated otherwise. } \\
\mathrm{b}_{\mathrm{MCL}} \text { maximum concentration limit, from Table } 1 \text { of the U.S. Environmental Protection } \\
\text { Agency Draft Final Rule, Groundwater Standards for Remedial Actions at Inactive } \\
\text { Uranium Processing Sites, (EPA, 1994). } \\
\text { Exceeds MCL. } \\
\text { dThe MCL for nitrate as (N) is } 10 \mathrm{mg} / \mathrm{L} \text {. } \\
\text { eThe uranium concentration of } 0.044 \mathrm{mg} / \mathrm{L} \text { is equivalent to } 30 \mathrm{pCi} / \mathrm{L} \text {, which is the MCL. } \\
\mathrm{f}_{\mathrm{pCi} / \mathrm{L} \text { : picocuries per liter. }}\end{array}$} \\
\hline
\end{tabular}


Table 3.4 Maximum observed concentrations of EPA MCL constituents in monitor wells located in the Tres Hermanos $-C_{2}$ Sandstone at the Ambrosia Lake site during the years from 1980 to 1994

\begin{tabular}{|c|c|c|c|c|c|c|}
\hline \multirow[b]{2}{*}{ Constituent $^{a}$} & \multirow{2}{*}{$\begin{array}{l}M \mathrm{MLL}^{\mathrm{b}} \\
(\mathrm{mg} / \mathrm{L})\end{array}$} & \multicolumn{5}{|c|}{ Monitor wells } \\
\hline & & 779 & 785 & 787 & 677 & 676 \\
\hline Arsenic & 0.05 & 0.025 & 0.012 & 0.019 & $<0.01$ & $<0.01$ \\
\hline Barium & 1 & 0.3 & 0.1 & 0.01 & 0.01 & 0.01 \\
\hline Cadmium & 0.01 & $0.016^{c}$ & $0.024^{c}$ & $0.012^{c}$ & 0.004 & 0.003 \\
\hline Chromium & 0.05 & $0.22^{c}$ & $0.24^{c}$ & $0.23^{c}$ & $<0.01$ & $<0.01$ \\
\hline Lead & 0.05 & 0.02 & 0.02 & 0.02 & 0.05 & 0.04 \\
\hline Mercury & 0.002 & 0.0006 & 0.0006 & 0.0004 & $<0.0002$ & $<0.0002$ \\
\hline Molybdenum & 0.1 & $0.14^{c}$ & $0.35^{c}$ & $0.25^{c}$ & 0.024 & $0.595^{c}$ \\
\hline Nitrate & $44^{d}$ & 2.29 & 20 & 29 & 6.9 & 25.6 \\
\hline Selenium & 0.01 & $0.092^{c}$ & $0.324^{c}$ & $0.054^{c}$ & $0.037^{c}$ & $0.091^{c}$ \\
\hline Silver & 0.05 & 0.05 & $0.08^{c}$ & $0.09^{c}$ & $<0.01$ & $<0.01$ \\
\hline $\begin{array}{l}\text { Combined } \\
\text { Uranium-234 and } \\
-238\end{array}$ & $0.044^{e}$ & 0.0238 & $3.30^{\mathrm{c}}$ & 0.018 & 0.016 & $0.207^{c}$ \\
\hline $\begin{array}{l}\text { Gross alpha } \\
\text { (excluding radon and } \\
\text { uranium) } \mathrm{pCi} / \mathrm{L}^{f}\end{array}$ & 15 & $74^{c}$ & $1900^{c}$ & $57.4^{c}$ & $29^{c}$ & $85.9^{c}$ \\
\hline $\begin{array}{l}\text { Combined } \\
\text { Radium }-226 \text { and } \\
-228 \mathrm{pCi} / \mathrm{L}^{f}\end{array}$ & 5 & $5.6^{c}$ & $10.51^{c}$ & 4.2 & $6.45^{c}$ & $24.12^{c}$ \\
\hline \multicolumn{7}{|c|}{ 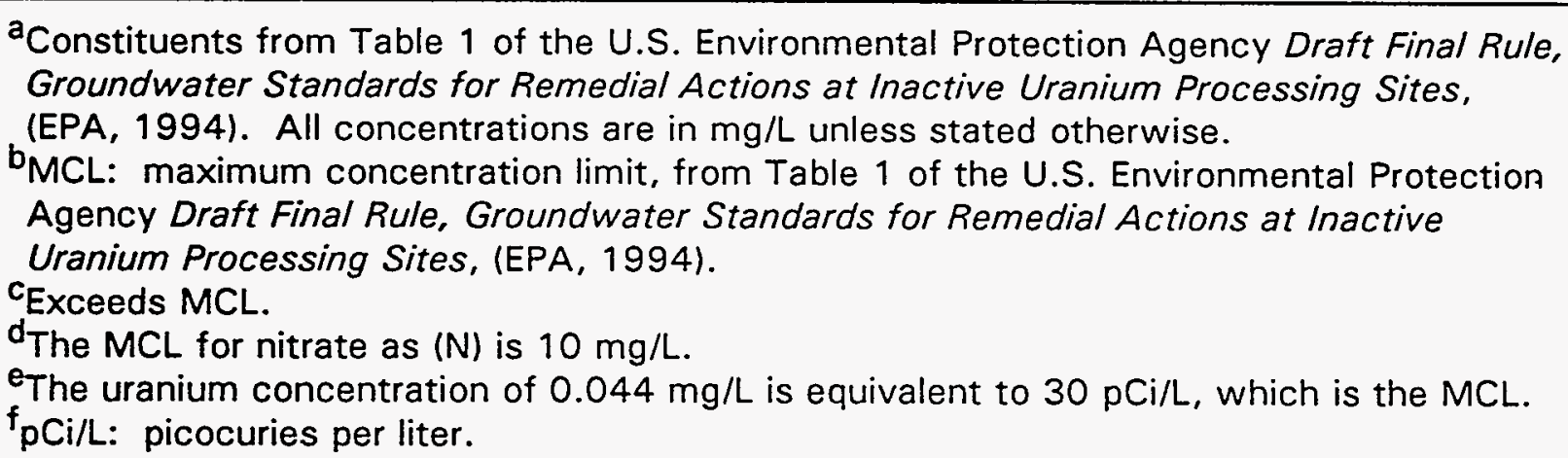 } \\
\hline
\end{tabular}




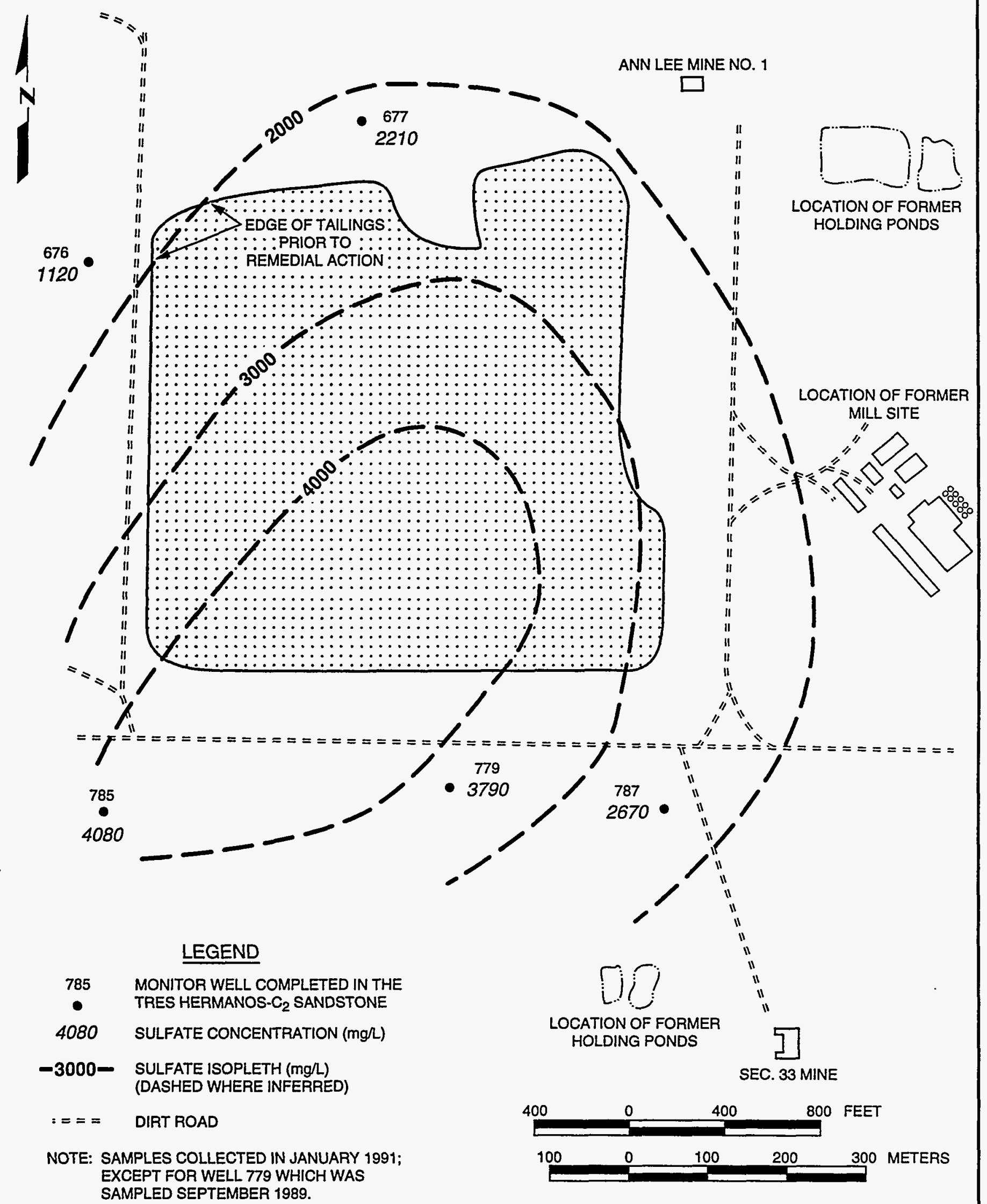

FIGURE 3.9 MODIFIED FROM DOE, 1991.

SULFATE ISOPLETH MAP FOR GROUND WATER IN THE TRES HERMANOS- $C_{2}$ SANDSTONE

AT THE AMBROSIA LAKE UMTRA SITE, NEW MEXICO 
1991 sampling round (used for Figure 3.9), downgradient values are higher and upgradient values are lower, compared to those in the February, 1989 sampling round (used in Figure D.8.24 of DOE, 1991).

Maximum observed concentrations of cadmium, chromium, molybdenum, nitrate, selenium, silver, uranium, and the activities of gross alpha and radium-226 plus radium-228, exceed the EPA MCLs in ground water from wells completed in the Tres Hermanos- $C_{1}$ Sandstone (Table 3.3). Maximum observed concentrations of cadmium, chromium, molybdenum, selenium, silver, uranium and the activities of gross alpha and radium-226 plus radium-228 exceed the EPA MCLs in ground water from wells completed in the Tres Hermanos- $C_{2}$ Sandstone (Table 3.4). Maximum concentrations of several parameters in the Tres Hermanos-C Sandstone have increased over those of previous years (compare Tables 3.3 and 3.4 to Tables D.8.13 and D.8.14, respectively in DOE, 1991), indicating that contamination is moving downgradient.

\section{Units below the Tres Hermanos-C Sandstone}

Units between the bottom of the Tres Hermanos-C Sandstone and the top of the Westwater Canyon Member include, in descending order, the Tres Hermanos-B and -A Sandstones, and the Dakota Formation. Ground water from monitor wells completed in these units does not appear to be affected by siterelated contamination.

Nitrate levels are increasing in monitor well 678, completed in the Tres Hermanos-B Sandstone ( $3410 \mathrm{mg} / \mathrm{L}$ in the December, 1992 sampling round) (Figure 3.10). This increase is not related to uranium processing at the Ambrosia Lake site because: 1) it is not accompanied by increases in other relatively mobile site-related parameters (e.g. molybdenum, uranium, or sulfate), and 2) nitrate concentrations in monitor well 678 are much higher than average concentrations found in tailings pore fluids (approximately $1400 \mathrm{mg} / \mathrm{L}$ ). Further, the Tres Hermanos-B Sandstone is hydrologically isolated from alluvium and the weathered Mancos Shale at the site (see section 3.3.1). Relatively high nitrate levels are most likely related to the nearby Quivira Mining Company tailings pile.

\section{Westwater canyon member}

The Westwater Canyon Member is the primary source of uranium ore in the area and was the focus of intense mining frelated activity. Thus, mining activities not related to UMTRA Project site tailings seepage introduced many other sources of contamination into the Westwater Canyon Member. The DOE made a comparison of concentrations of contaminants in the Tres Hermanos-C Sandstone and the Westwater Canyon Member (Table D.8.26 of DOE, 1991) and concluded that, in general, concentrations in the Tres Hermanos-C Sandstone are lower. This suggests that seepage from the Tres Hermanos- $C$ Sandstone ground water will produce no increases in the concentrations of contaminants in the Westwater Canyon Member. 


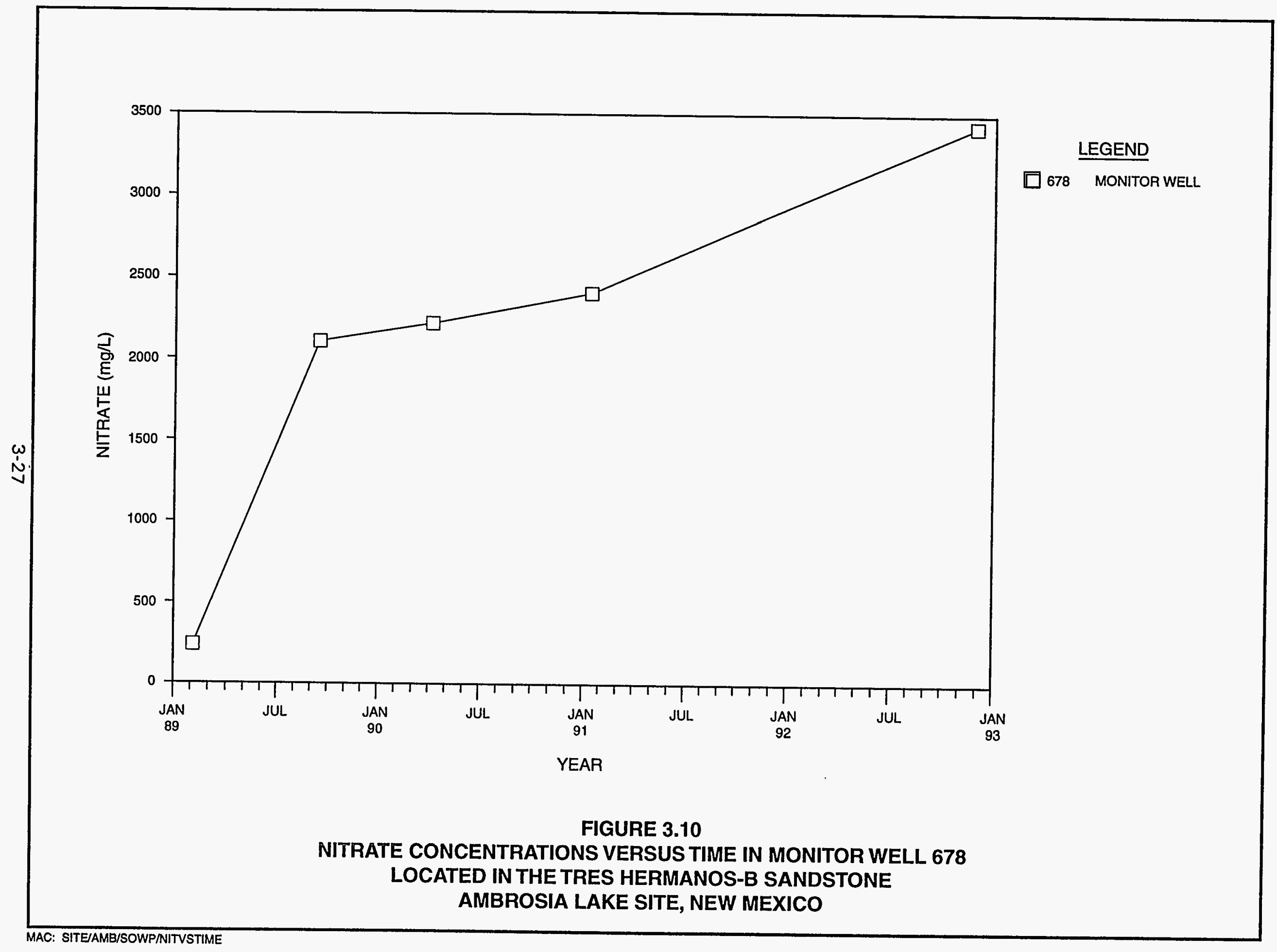




\subsubsection{Contaminant fate and transport}

Percolation of alkaline tailings leachate from the Ambrosia Lake site into ground water has not raised the $\mathrm{pH}$ of ground water in monitor wells at the site. The near-neutral pH (6.84-7.91) measured in the Tres Hermanos- $C$ Sandstone during the November-December 1992 sampling round indicates that the baseneutralization potential of the alluvium and the weathered Mancos Shale and the Tres Hermanos-C Sandstone has not been exhausted. Although an alkaline $\mathrm{pH}$ front has migrated through subsoils beneath the former tailings site, the change in $\mathrm{pH}$ is relatively insignificant and does not affect the solubility of contaminants in tailings seepage (Table D.8.22 of DOE, 1991). The tailings fluid and ground water in the alluvium and the weathered Mancos Shale is oxidizing, but becomes somewhat less oxidizing as it flows from the alluvium and weathered Mancos Shale into the Tres Hermanos-C Sandstone (Table D.8.22 of DOE, 1991). The change, however, does not significantly affect the solubility of most contaminants. The most important EPA MCL-list contaminants in the ground water are nitrate, selenium, molybdenum, and uranium which occur as anions (DOE, 1991). Adsorption by the cation exchange process is not an important mechanism for removal of most of these contaminants at the Ambrosia Lake site. Dilution of tailings seepage by mill makeup water that drained into the alluvium and the weathered Mancos Shale reduced concentrations of these contaminants in the alluvium and the weathered Mancos Shale. Ultimately, contamination at the site will drain through mine shafts and drill holes into the Westwater Canyon Member of the Morrison Formation or be retained with residual moisture in the unsaturated alluvium and the weathered Mancos Shale or Tres Hermanos-C Sandstone.

\subsubsection{Risk evaluation}

Information on site-related risk to human health and the environment is integral to this SOWP to help guide the recommendation of a remediation strategy for the site. A preliminary risk assessment of ground water contamination at the Ambrosia Lake site was published in the RAP (DOE, 1991). This preliminary risk assessment discussed the potential health risks to humans from ingesting contaminated ground water in the uppermost aquifer beneath the former uranium mill processing site.

\section{Potential public health impacts}

The preliminary risk assessment presented in the 1991 RAP (DOE, 1991), qualitatively investigated potential risks to public health from exposure to ground water, assuming the most conservative pathway, exposure through drinking water, from the most contaminated wells at the processing site. The evaluation was based on ground water data available at the time, and followed the hazard index approach proposed by the EPA (EPA, 1989). This preliminary identification of hazards, assessment of toxicity, assessment of potential exposure, and characterization of risk showed that levels of contaminants that would result in potential adverse health effects, if the ground water were 
ingested on a long-term basis, are present in shallow ground water beneath and adjacent to the site. Three indicator chemicals were selected for the evaluation: selenium, radium-226, and natural uranium. Hazard indices for selenium and uranium exceeded an acceptable index of one, and the excess lifetime cancer risks calculated exceeded the EPA acceptable limit of $1 \times 10^{-4}$ (DOE, 1991).

Currently, no domestic wells accessing the contaminated ground water in the uppermost aquifer exist within $5 \mathrm{mi}(8 \mathrm{~km})$ of the site, and surface expression of ground water does not occur. Therefore, with no current exposure pathway present there are no current human health or environmental risks associated with the contaminated ground water attributable to the Ambrosia Lake UMTRA site. To further evaluate overall risks from the site, an evaluation of potential or probable future exposure pathways to contaminated ground water is necessary.

The potential for future use of the most contaminated ground water at the site is not probable. Several site conditions exist that greatly reduce the possibility of future exposure. The NRC and the state of New Mexico concurred with application of supplemental standards for "limited use" ground water at the Ambrosia Lake site for the UMTRA Surface Project (NRC, 1990). The ground water in the saturated zone where contamination occurs has been attributed primarily to previous mill-processing activities. An aquifer test on well 675 performed in February 1989 and described in the RAP (DOE, 1991), shows that the uppermost aquifer is incapable of a sustained yield of $150 \mathrm{gal}(570 \mathrm{~L})$ per day and is considered a "limited use" aquifer based on limited yield. Therefore, the uppermost aquifer lacks both a long-term yield sufficient to provide a continuous drinking water source and threat of chronic exposure for a potential future resident.

Since the major sources of aquifer recharge (waste water disposal and water from mine pumping) no longer exist, the saturated thickness of the aquifer is expected to either remain about the same, or decrease as ground water spreads laterally and a portion of it migrates vertically upward into the vadose zone. Furthermore, ground water contamination attributable to the Ambrosia Lake site has not migrated from the area beneath the site, and no shallow domestic wells have been identified within a $5 \mathrm{mi}(8 \mathrm{~km})$ radius from the site. Land use in the area is not expected to change in the future because of the poor quality of the range land, poor soils, and the limited surface or shallow ground water resources available in this semiarid region. These conditions make it very unlikely that a future resident would install a shallow well immediately adjacent to the fenced disposal site. Therefore, future long-term exposure to ground water is unlikely.

\section{Environmental evaluation}

The potential for impacts to ecological receptors from contaminated ground water from the Ambrosia Lake site currently does not exist. Surface expression of contaminated ground water does not occur, and no other current pathway (such as a well used to water livestock) has been identified. Without a current 
exposure pathway for contaminated ground water to be used by terrestrial and aquatic biota and livestock, current environmental risks do not exist.

Potential future risks to livestock from contaminated ground water used as a drinking water source have not been quantified. However, potential future exposure of contaminated ground water to terrestrial or aquatic biota and livestock would be based on the same site conditions as stated above for the human health risk assessment. Therefore, near the Ambrosia Lake site, the possibility for potential future use of contaminated ground water to water livestock is unlikely.

\subsection{ASSESSMENT OF DATA NEEDS}

The hydrogeologic site description presented is supported by regional and sitespecific data. The information includes hydrogeologic reports prepared by state and federal agencies, site-specific and regional water quality data, site and surrounding area well completion reports, and well hydraulic testing data. Because the data base indicates little variability in the hydrogeologic conditions, no additional data needs have been identified.

\subsection{EVALUATION OF INTERIM REMEDIAL ACTION}

Interim remedial action is not required because no existing risks to human health or livestock result from site contamination, nor can any potential future risks be foreseen. 


\subsection{GROUND WATER COMPLIANCE STRATEGY RECOMMENDATION}

This section explains the application of site-specific data to the PEIS ground water compliance evaluation framework; recommends a ground water compliance strategy for the Ambrosia Lake processing site based on current conditions; analyzes possible deviations from the recommended ground water compliance strategy; and notes contingencies (conceivable reason for recommendation of a different compliance strategy) and rules (criteria) for application of contingencies.

\subsection{GROUND WATER COMPLIANCE STRATEGY EVALUATION PROCESS}

Ground water compliance recommendations at the former Ambrosia Lake processing site were made by using the compliance evaluation framework shown in Figure 4.1. This compliance evaluation framework was developed for analysis in the DOE UMTRA Ground Water PEIS (DOE, 1994). The recommended ground water compliance strategy for the Ambrosia Lake site will be selected by applying site-specific data to the approved PEIS compliance evaluation framework.

The compliance evaluation framework proposed in the PEIS provides for the identification of one or more of three ground water compliance strategies, based upon site-specific data. The three compliance strategies developed in the UMTRA Ground Water PEIS are defined as follows:

- No Remediation: Application of the strategy of no remediation would mean that compliance with the proposed standards would be met without altering the ground water or cleaning it up in any way. This strategy could be applied at sites that have no contamination above MCLs or background levels, or at sites that have contamination above MCLs or background levels but qualify for supplemental standards or ACLs.

- Natural flushing: Natural flushing allows for the natural ground water movement and geochemical processes to decrease the contaminant concentrations to levels within regulatory limits within a given time period. This could be applied at sites where ground water compliance would be achieved with the application of natural flushing within 100 years, where effective monitoring and institutional controls could be maintained, and where the ground water is not currently and is not projected to be a drinking water source.

- Active ground water remediation: Active ground water remediation would require the application of engineered ground water remediation methods such as gradient manipulation, ground water extraction and treatment, or in situ ground water treatment to achieve compliance with the proposed standards. 


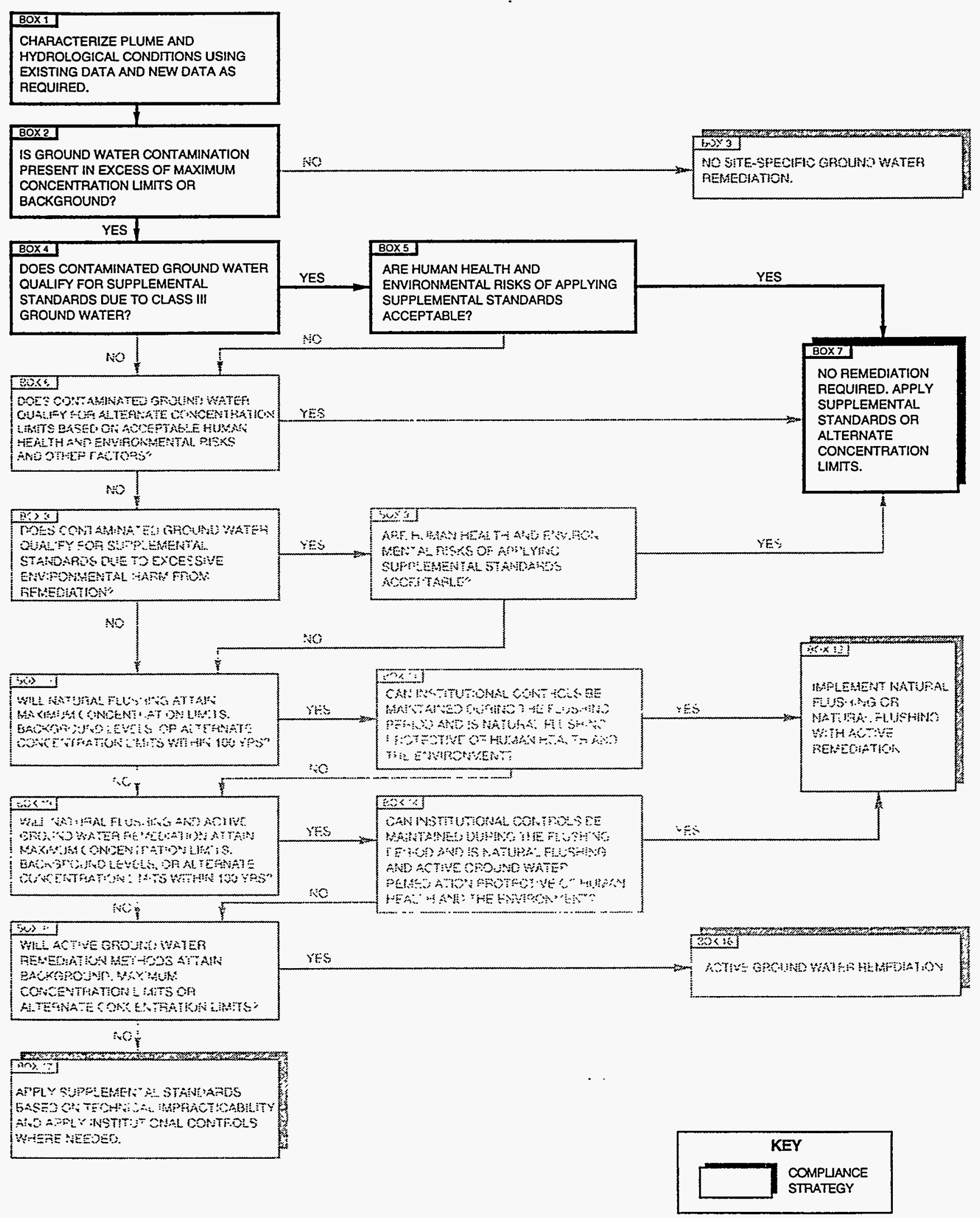

FIGURE 4.1

AMBROSIA LAKE COMPLIANCE SELECTION FRAMEWORK 
The ground water compliance strategy recommended for the Ambrosia Lake site is to perform no remediation, based on the application of supplemental standards to the affected ground water. The recommended compliance strategy is an appropriate compliance strategy to carry forward for analysis in an Ambrosia Lake site-specific NEPA document because the NRC and the state of New Mexico concurred with the RAP and application of supplemental standards for the UMTRA Surface Project (NRC, 1990).

\subsection{SITE-SPECIFIC GROUND WATER COMPLIANCE STRATEGY}

This section describes how the proposed PEIS decision-making framework was used to evaluate and recommend an appropriate ground water compliance strategy for the Ambrosia Lake site.

The ground water compliance strategy for the Ambrosia Lake site was identified through the application of site conditions to the proposed PEIS framework. The evaluation resulted in the identification of the strategy of performing no ground water remediation, based on the application of supplemental standards. The following is a description of the evaluation process, illustrated in Figure 4.1:

\section{First and second steps}

The first two steps (boxes 1 and 2 of Figure 4.1) confirm that ground water quality data obtained from the Ambrosia Lake site have resulted in ground water contamination exceeding background levels or MCLs. The uppermost aquifer was created primarily by discharge of mill process water and therefore the presence of water is primarily artificial. Because all water contained in the alluvium and the weathered Mancos Shale and Tres Hermanos-C Sandstone (uppermost aquifer) in the area of the milling site is primarily a mixture of tailings seepage and mine discharge, the extent of contamination is the same as the extent of background ground water.

\section{Third step}

The third step (box 4 of Figure 4.1 ) determines whether compliance with the proposed standards could be achieved by applying supplemental standards based on the presence of "limited use" ground water. "Limited use" means the ground water is not a current or potential source of drinking water because the quantity of water available is less than $150 \mathrm{gal}(570 \mathrm{~L})$ per day (40 CFR \$192.11(e) (1994)) (52 FR 36000 (1987)). The uppermost aquifer at the Ambrosia Lake site, comprised of the alluvium, upper Mancos Shale, and Tres Hermanos-C Sandstones, is incapable of sustaining a well yield of greater than $150 \mathrm{gal}(570 \mathrm{~L})$ per day based on an aquifer test (DOE, 1991). Therefore, the criterion is met for the Ambrosia Lake site for application of "limited use" ground water (supplemental standards), based on an insufficient yield of ground water. The major sources of recharge, waste water disposal and water from mine pumping, no longer exist. Therefore, the alluvium, upper Mancos Shale, and Tres Hermanos-C Sandstones are expected to return to premilling and 
mining conditions of little-to-no saturation, further eliminating the unit as a potential future ground water resource.

\section{Fourth step}

The fourth step (box 5 of Figure 4.1) determines whether applying supplemental standards results in acceptable human health and environmental risks. Levels of contaminants presenting potential adverse human health effects occur at the site, but would have effect only if the ground water were ingested on a longterm basis. Since there are no domestic wells accessing the contaminated ground water in the uppermost aquifer, and ground water does not discharge to the land surface, there are currently no human health risks associated with the contaminated ground water.

The potential for impacts to the ecological environment from contaminated ground water currently does not exist. Surface expression of ground water contaminated by the UMTRA Project site does not occur, and no other pathway (such as a well used to water livestock) has been identified. Without an exposure pathway for contaminated ground water to be used by terrestrial and aquatic biota and livestock, current environmental risks do not exist.

The potential for future use of the most contaminated ground water at the site is not probable. Ground water data show that the saturated zone at the site has a yield insufficient to provide a continuous drinking water source for a potential future resident. The saturated zone where contamination occurs is primarily attributed to previous processing activities. Since the major sources of aquifer recharge (waste water disposal and water from mine pumping) no longer exist, the saturated thickness of the existing ground water is expected to either remain about the same or decrease, as water spreads laterally and a portion of it migrates upward into the vadose zone. Therefore, future long-term exposure to ground water is unlikely.

\section{Fifth step}

The last step (box 7 of Figure 4.1) is the recommended site-specific compliance strategy. The strategy of performing no remediation, based on the application of supplemental standards to the site ground water is proposed as the recommended compliance strategy for the Ambrosia Lake site. The recommended compliance strategy is appropriate because the proposed PEIS process evaluation criteria and conclusions in the preceding steps are supported by existing site data.

\subsection{DEVIATIONS, CONTINGENCIES, AND RULES}

Elevation of site conditions through the proposed PEIS process supports the preliminary conclusion. The determination of the Ambrosia Lake site-specific hydrogeologic conditions is based on and supported by both regional and sitespecific data. Therefore, deviations that would impact the interpretation of the 
hydrogeologic conditions are not expected. Contingency plans and rules to implement those plans are necessary only if significant deviations are anticipated. Because deviations are not expected, contingency plans will not be required.

\subsection{DATA COLLECTION AND ASSESSMENT}

Additional data collection activities for the Ambrosia Lake site will not be required. Existing regional and site-specific data are adequate to evaluate site hydrologic conditions and site risks. 


\subsection{LIST OF CONTRIBUTORS}

The following individuals contributed to the preparation of this report.

\begin{tabular}{|c|c|}
\hline Name & Contribution \\
\hline M.D. Hansen & $\begin{array}{l}\text { Document coordinator, } \\
\text { National Environmental Policy Act coordinator }\end{array}$ \\
\hline M.B. Leaf & Site manager, document sponsor, document review \\
\hline A. Miller & Primary author, hydrogeology \\
\hline M. Day & Risk evaluation \\
\hline D. Erskine & Geochemistry \\
\hline $\begin{array}{l}\text { D. Bierley, R. Bowen, P. Briggs, } \\
\text { J. Gibb, A. Holm, L. Ulland }\end{array}$ & Document review \\
\hline R. Woodward & Editing \\
\hline J. Martin & Text processing \\
\hline B. Harvey & Graphic design \\
\hline
\end{tabular}




\subsection{REFERENCES}

Bostick, K., 1985. Ground-Water Discharge Plan Analysis For Kerr-McGee Nuclear Corporation, Ambrosia Lake Uranium Mill, Quivira Mining Company, February 1985, Ground Water Section, Ground Water Quality and Hazardous Waste Bureau, New Mexico Environmental Improvement Division, Santa Fe, New Mexico.

Brod, R.C., 1979. Hydrogeology and Water Resources of the Ambrosia Lake-San Mateo area, McKinley and Valencia Counties, New Mexico, June 1979, submitted in partial fulfillment of the requirements for the degree of Master of Science in Geology, New Mexico Institute of Mining and Technology, Socorro, New Mexico.

Brod, R.C. and W. J. Stone, 1981. Hydrogeology of Ambrosia Lake-San Mateo area, McKinley and Cibola Counties, New Mexico, Hydrogeologic Sheet 2, 1981, New Mexico Bureau of Mines \& Mineral Resources, a Division of New Mexico Institute of Mining \& Technology, Socorro, New Mexico.

DOC (U.S. Department of Commerce), 1990. Census of Population: General Population Characteristics, Economics and Statistics Administration, Bureau of the Census, Washington, D.C.

DOE (U.S. Department of Energy), 1994. "Programmatic Environmental Impact Statement for the Uranium Mill Tailings Remedial Action Ground Water Project,"

DOE/EIS-0198, Rev. 3, April 1994, prepared by the U.S. Department of Energy, UMTRA Project Office, Albuquerque Operations Office, Albuquerque, New Mexico.

DOE (U.S. Department of Energy), 1993a. Technical Approach to Groundwater Restoration, DOE/AL/62350-20F, Rev. 1, November 1993, prepared by the U.S. Department of Energy, UMTRA Project Office, Albuquerque Operations Office, Albuquerque, New Mexico.

DOE (U.S. Department of Energy), 1993b. Recommendations for the Preparation of Environmental Assessments and Environmental Impact Statements, May 1993, Office of NEPA Oversight, U.S. Department of Energy, Washington, D.C.

DOE (U.S. Department of Energy), 1992a. UMTRA Groundwater Program Plan, May 1992, prepared by the U.S. Department of Energy, UMTRA Project Office, Albuquerque Operations Office, Albuquerque, New Mexico.

DOE (U.S. Department of Energy), 1992b. Software Program for Environmental Analysis and Reporting (SPEAR) System, U.S. Department of Energy, UMTRA Project Office, Albuquerque Operations Office, Albuquerque, New Mexico. 
DOE (U.S. Department of Energy), 1991. Remedial Action Plan and Site Conceptual Design for Stabilization of the Inactive Uranium Mill Tailings Site at Ambrosia Lake, New Mexico, UMTRA-DOE/AL-050516.0000, November 1991, final, prepared by the U.S. Department of Energy, UMTRA Project Office, Albuquerque Operations Office, Albuquerque, New Mexico.

DOE (U.S. Department of Energy), 1987a. Environmental Assessment, Remedial Action at the Ambrosia Lake Uranium Mill Tailings Site, Ambrosia Lake, New Mexico, DOE/EA-0322, June 1987, prepared by the U.S. Department of Energy, UMTRA Project Office, Albuquerque Operations Office, Albuquerque, New Mexico.

DOE (U.S. Department of Energy), 1987b. Publication of Finding of No Significant Impact, Remedial Action at the Ambrosia Lake Uranium Mill Tailings Site, Ambrosia Lake, New Mexico, UMTRA Project Document Control Center file location number AMB EA, June 17, 1987, prepared by the U.S. Department of Energy, UMTRA Project Office, Albuquerque Operations Office, Albuquerque, New Mexico.

DOE (U.S. Department of Energy), 1985. Cooperative Agreement Between the United States Department of Energy and the State of New Mexico, DOE Cooperative Agreement Number DE-FC04-85AL20533, September 11, 1985, as amended, Uranium Mill Tailings Remedial Action Project, U.S. Department of Energy, Albuquerque Operations Office, Albuquerque, New Mexico.

EPA (U.S. Environmental Protection Agency), 1994. Draft Final Rule, Groundwater Standards for Remedial Actions at Inactive Uranium Processing Sites, UMTRA Project Document Control Center file location number 0.13.5.1, 1994, prepared by the U.S. Environmental Protection Agency, Washington, D.C.

EPA (U.S. Environmental Protection Agency), 1989. Risk Assessment Guidance for Superfund, Volume II, Environmental Evaluation Manual, March 1989. EPA/504/1-89/001, interim final, Office of Emergency and Remedial Response, U.S. Environmental Protection Agency, Washington, D.C.

Merritt, R.C., 1971. The Extractive Metallurgy of Uranium, 1971. Colorado School of Mines Research Institute, Golden, Colorado, prepared for the U.S. Atomic Energy Commission.

NOAA (National Oceanic and Atmospheric Administration), 1979. Climatic Atlas of the United States, prepared by Gale Research Company, Detroit, Michigan, for the National Oceanic and Atmospheric Administration, U.S. Department of Commerce, Washington, D.C.

NRC (U.S. Nuclear Regulatory Commission), 1990. Final Technical Evaluation Report for DOE's Proposed Remedial Action, Ambrosia Lake UMTRA Project Site, New Mexico, December 1990, prepared by the Uranium Recovery Field Office, Region IV, U.S. Nuclear Regulatory Commission, Denver, Colorado. 
QMC (Quivira Mining Company), 1981. "Ambrosia Lake Mill, License Renewal Report," unpublished report, prepared by Quivira Mining Company, Oklahoma City, Oklahoma, for the New Mexico Environmental Improvement Division, Radiation Protection Bureau, Santa Fe, New Mexico.

USGS (U.S. Geological Survey), 1980. U.S. Department of the Interior Geological Survey, Ambrosia Lake Quadrangle, New Mexico-McKinley County, 7.5 minute series (topographic), N3522.5 - W10745/7.5, photorevised 1980.

\section{CODE OF FEDERAL REGULATIONS}

10 CFR Part 1021, "National Environmental Policy Act; Implementation Procedures," U.S. Department of Energy, 1994.

40 CFR Part 192, "Health and Environmental Protection Standards for Uranium and Thorium Mill Tailings," U.S. Environmental Protection Agency, 1994.

40 CFR Part 1500, "Purpose, Policy, and Mandate," (Regulations for Implementing the Procedural Provisions of the National Environmental Policy Act), Council on Environmental Quality, 1994.

\section{FEDERAL REGISTER}

52 FR 36000, "Standards for Remedial Actions at Inactive Uranium Processing Sites; Proposed Rule," September 24, 1987.

\section{UNITED STATES CODE}

42 USC §4321, et seq. National Environmental Policy Act, January 1, 1970.

42 USC $\$ 7901$, et seq. Uranium Mill Tailings Radiation Control Act, November 8, 1978.

42 USC \$7922, et seq. Uranium Mill Tailings Remedial Action Amendments Act, November 51988. 
APPENDIX A

LITHOLOGIC LOGS, WELL RECORDS, AND WELL COMPLETION LOGS AMBROSIA LAKE, NEW MEXICO, UMTRA PROJECT SITE 
AMBROSIA LAKE UMTRA SITE SOWP

WELL LOGS FOR APPENDIX A

RECORD OF DOCUMENTS 
AMBROSIA LAKE UMTRA SITE SOWP

WELL LOGS FOR APPENDIX A

\begin{tabular}{|c|c|c|c|}
\hline WELL NO & LITHLOG & WELL REC & WELL LOG \\
\hline 620 & 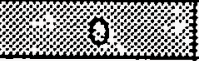 & 2.0. & 0 \\
\hline 674 & $x$ & $x$ & $x$ \\
\hline 675 & $x$ & $x$ & $x$ \\
\hline 676 & $x$ & $x$ & $x$ \\
\hline 677 & $x$ & $x$ & $x$ \\
\hline 678 & $x$ & $x$ & $x$ \\
\hline 679 & $x$ & $x$ & $x$ \\
\hline 680 & $x$ & $x$ & $x$ \\
\hline 681 & $x$ & $\mathrm{X}$ & $x$ \\
\hline 706 & 8 & 6 & 9 \\
\hline 776 & $x$ & $x$ & 0 \\
\hline 777 & $x$ & $x$ & $x$ \\
\hline 778 & $x$ & $x$ & $x$ \\
\hline 779 & $x$ & $x$ & $\mathrm{x}$ \\
\hline 781 & $x$ & $x$ & 8. \\
\hline 782 & $\mathrm{x}$ & $x$ & $x$ \\
\hline 783 & $x$ & $x$ & $x$ \\
\hline 785 & 0 & $x$ & $x$ \\
\hline 786 & $x$ & $x$ & $x$ \\
\hline 787 & $x$ & $x$ & $x$ \\
\hline 790 & $x$ & $x$ & $\mathrm{x}$ \\
\hline 792 & $x$ & $x$ & $x$ \\
\hline 793 & $x$ & $x$ & $x$ \\
\hline 794 & $x$ & $x$ & $x$ \\
\hline 796 & $x$ & $x$ & $x$ \\
\hline 797 & $x$ & $x$ & $x$ \\
\hline 798 & $x$ & $x$ & $x$ \\
\hline 799 & $x$ & $\mathrm{X}$ & $x$ \\
\hline
\end{tabular}

WELL RECORD = DIAGRAM

WELL LOG $=1$ PG FORM

$X=$ RECORD OBTAINED

$0=$ RECORD MISSING 
WELL 674 LOGS 


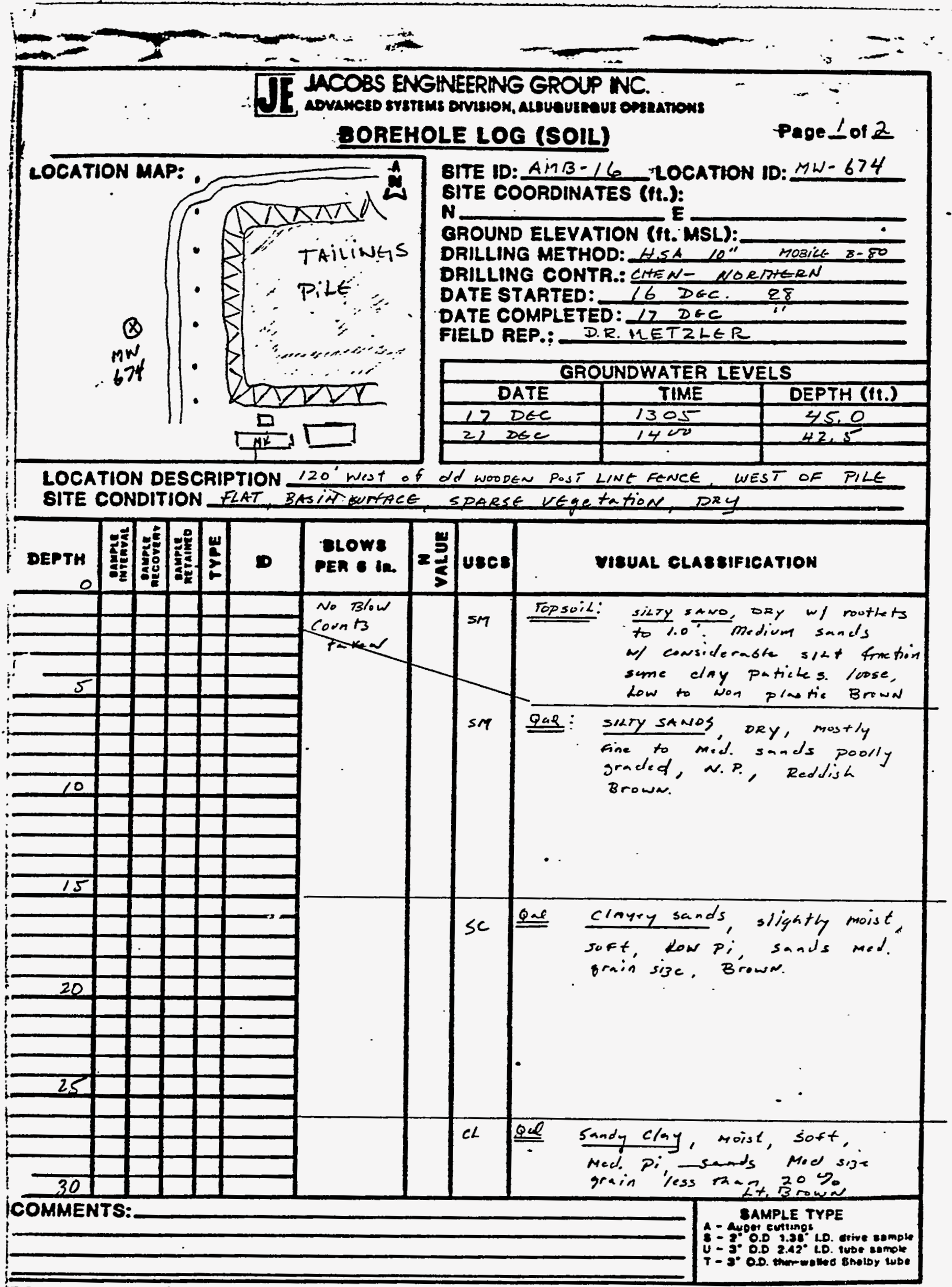

FO-AL-ENG-28 (4185) 


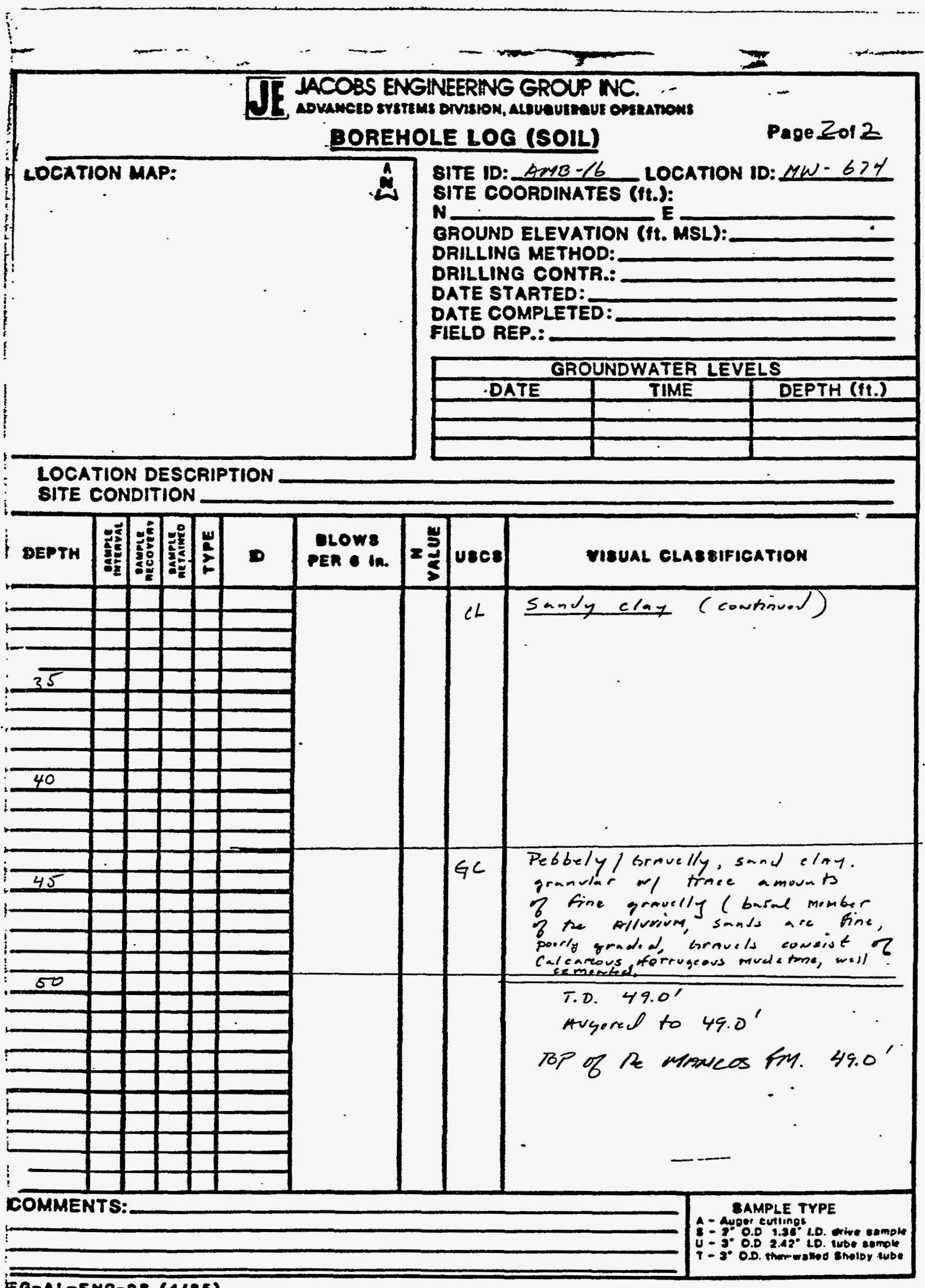

EO-AL-ENO-28 (4185) 


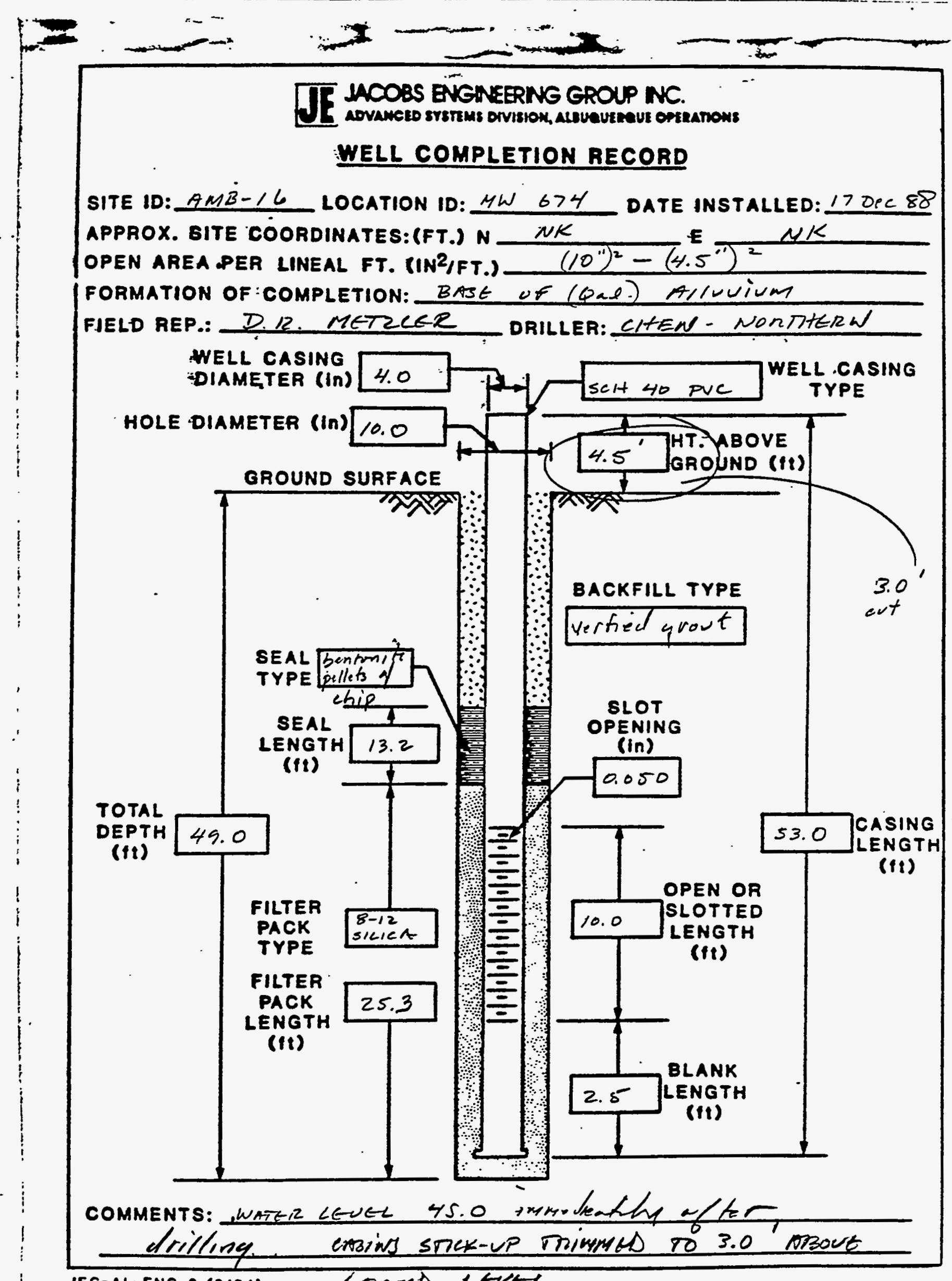

JEG-AL-ENG-3 (3184) GHounD LEVE. 


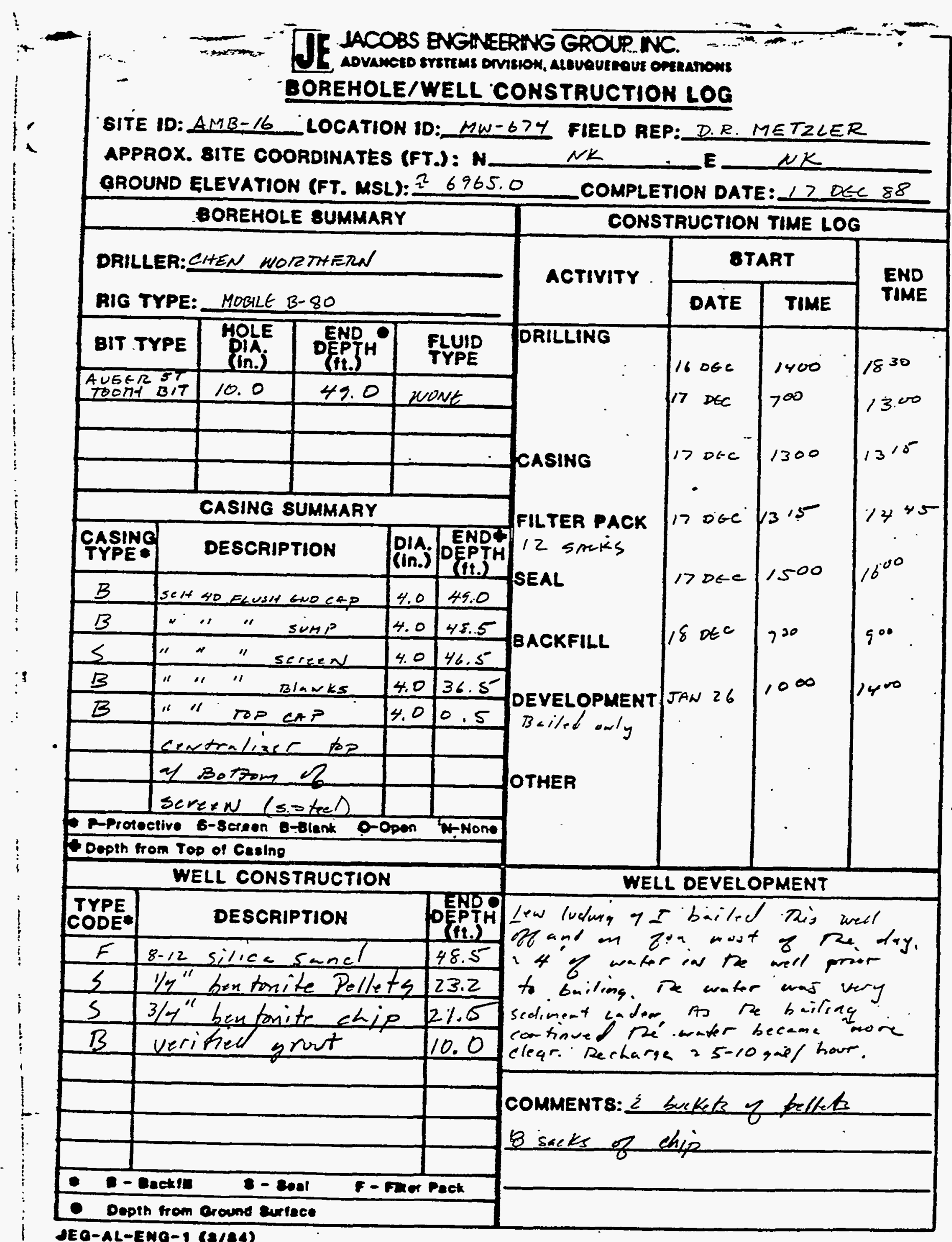


WELL 675 LOGS 


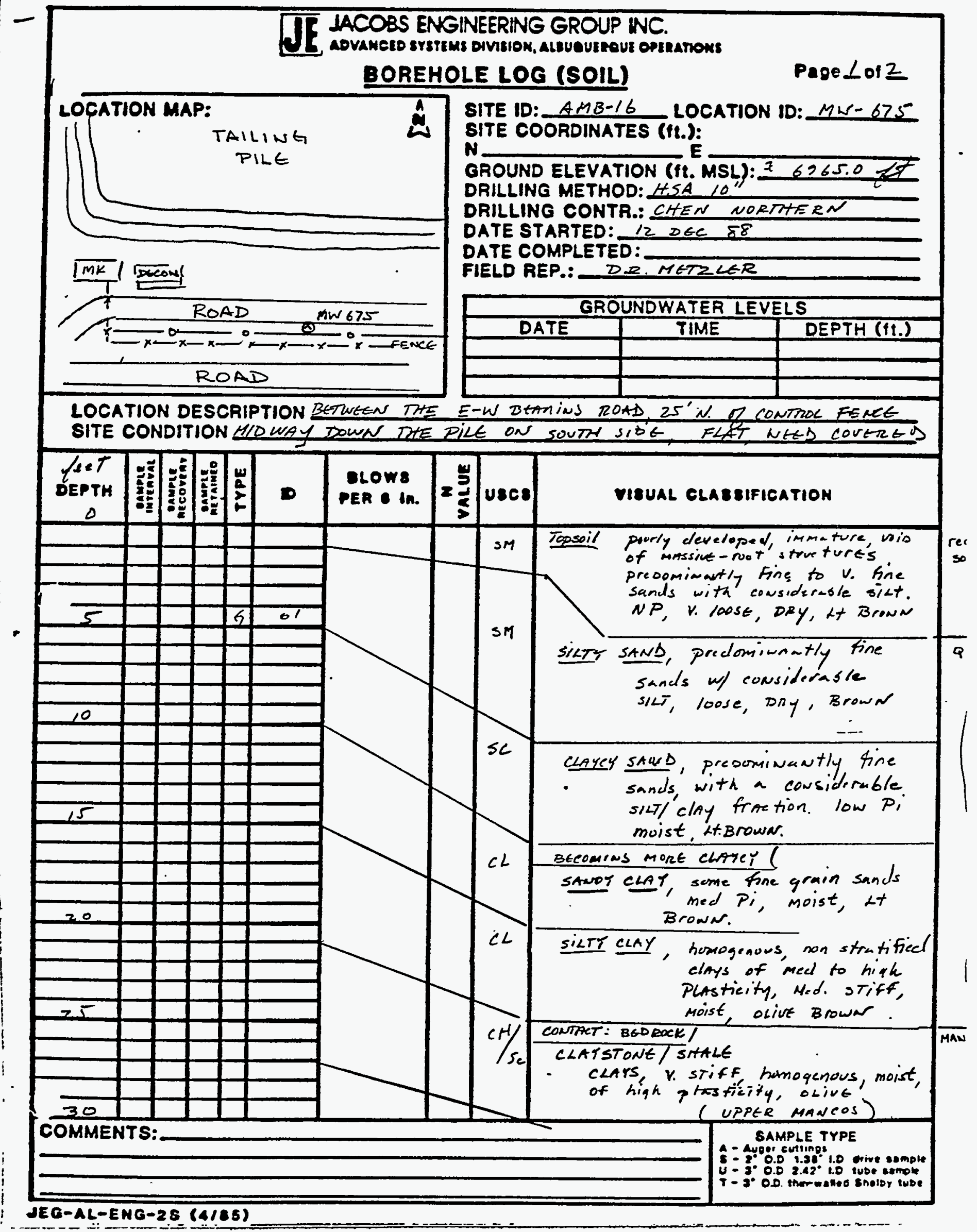




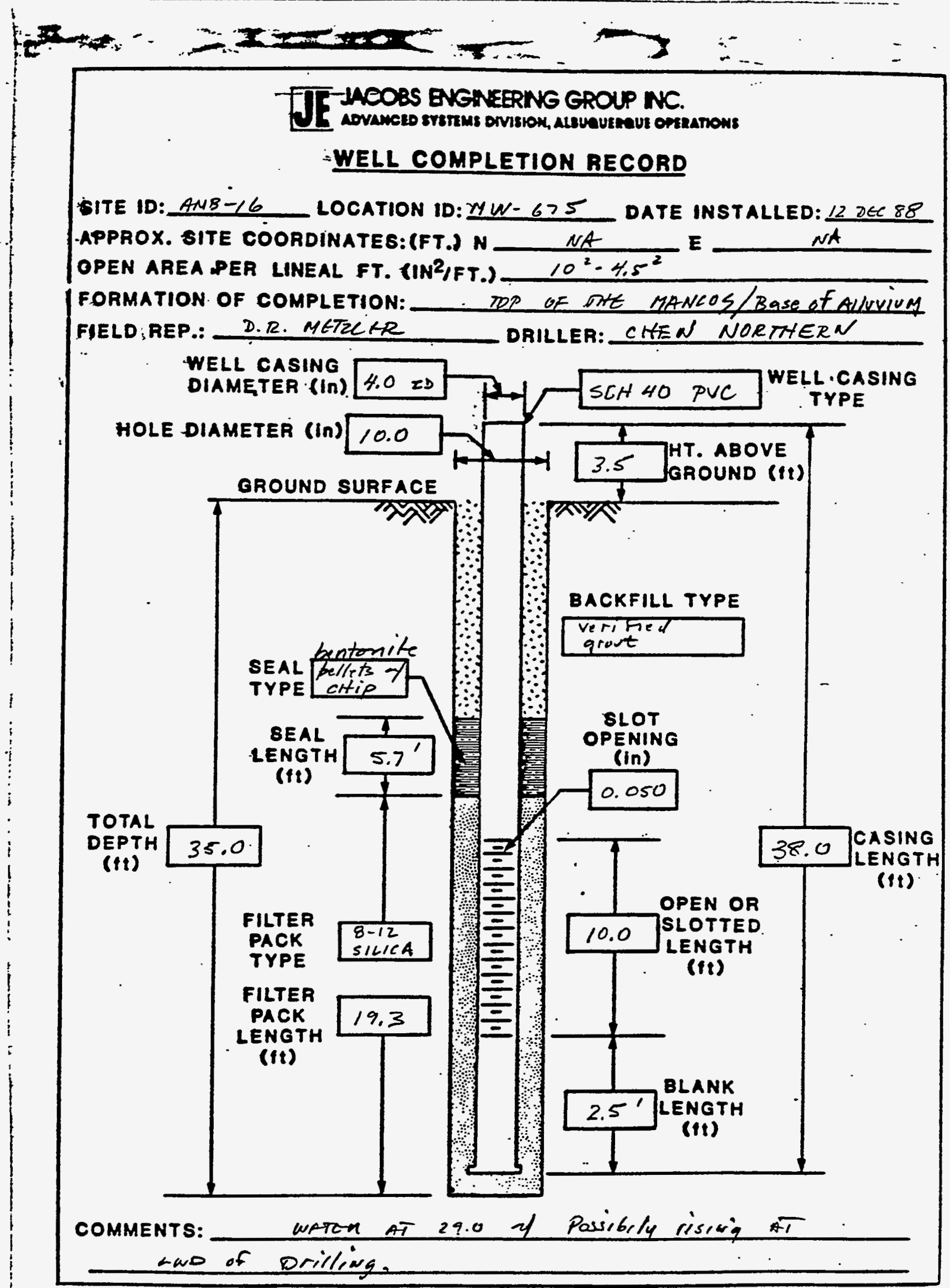

JEG-AL-ENO-3 (3/84) 


\section{IT $2 A C O B S$ ENGMEERING GROUP WNC.

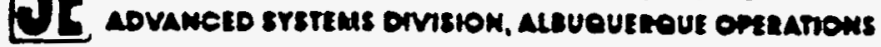 \\ BOREHOLE/WELL CONSTRUCTION LOG}

SITE ID: ANB-16 LOCATION ID:MW-675 FIELD AEP: DR. MEZLCR

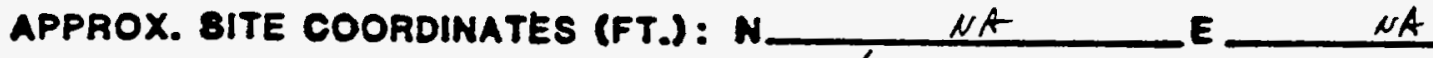

BROUND ELEVATION (FT. MSL): $26965.0^{\prime}$ COMPLETION DATE: 12 DEC 88

BOREHOLE BUMMARY

DRILLER: CHEN NURTAERN

RIG TYPE: MOSILE $80 B$

\begin{tabular}{|c|c|c|c|}
\hline BIT TYPE & $\begin{array}{l}\text { Hole } \\
\text { git: } \\
\text { (in.) }\end{array}$ & $\begin{array}{l}\text { ENP } \\
\text { DEPTH } \\
\text { (ft.) }\end{array}$ & $\begin{array}{l}\text { FLUID } \\
\text { TYPE }\end{array}$ \\
\hline HSA & 10.0 & 35 & Nont.: \\
\hline & & & A Lea \\
\hline & & & Burkes of \\
\hline & & & Chenew woter \\
\hline
\end{tabular}

\begin{tabular}{|c|c|c|c|}
\hline $\begin{array}{l}\text { CASINC } \\
\text { TYPE }\end{array}$ & DESCRIPTION & $\begin{array}{l}\text { Din. } \\
\left.(\operatorname{lin})_{2}\right) \\
\end{array}$ & $\begin{array}{c}\text { END } \\
\text { OEPTH } \\
\text { (ft.) }\end{array}$ \\
\hline$B$ & SCH 40 PUC SUMP + CAP & 4.0 & 35.0 \\
\hline$\leq$ & $"$ " SCREEN & 11 & 32.5 \\
\hline I3 & $" \|$ BLANKS & $" 1$ & 22.5 \\
\hline$P$ & Protective STEAL CrBinS & 858 & 6,0 \\
\hline & & & \\
\hline & & & \\
\hline & & & \\
\hline \multicolumn{3}{|c|}{ E-Protective 8-screen B-biank o-Open } & Depth from Top of Casing \\
\hline \multicolumn{3}{|c|}{ WELL CONSTRUCTION } & \\
\hline $\begin{array}{l}\text { TYPE } \\
\text { CODE }\end{array}$ & \multicolumn{2}{|l|}{ DESCAIPTION } & \\
\hline$F$ & \multicolumn{2}{|l|}{$8-12$ SILICA SAND } & 35.0 \\
\hline 5, & \multicolumn{2}{|l|}{ Bentowite Pellets } & 15.7 \\
\hline$S_{2}$ & \multicolumn{2}{|l|}{ Bentowite chip $3 / 4 "$} & 13.5 \\
\hline$B$ & \multicolumn{2}{|c|}{ bestowite-orust-cement } & 10.0 \\
\hline & \multicolumn{2}{|c|}{ 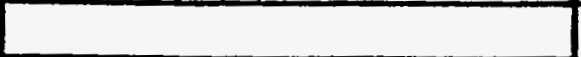 } & \\
\hline & & & \\
\hline & & & \\
\hline & & & \\
\hline \multicolumn{3}{|c|}{ - 0-eackfin - 8onl } & Pack \\
\hline
\end{tabular}

CAsING CONSTRUCTION TIME LOG 
WELL 676 LOGS 


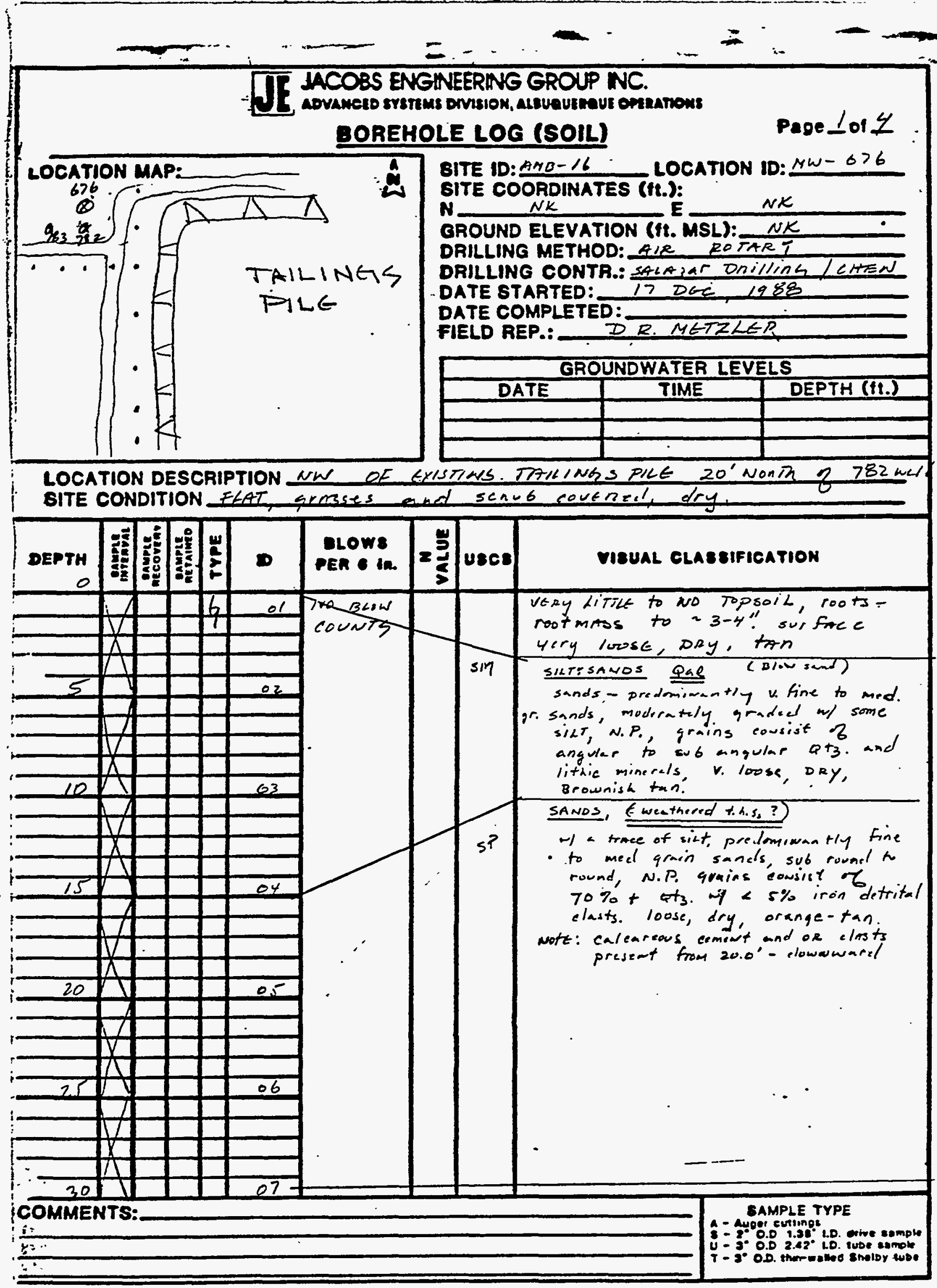

|Eg-AL-EMO-2B (4/EB) 


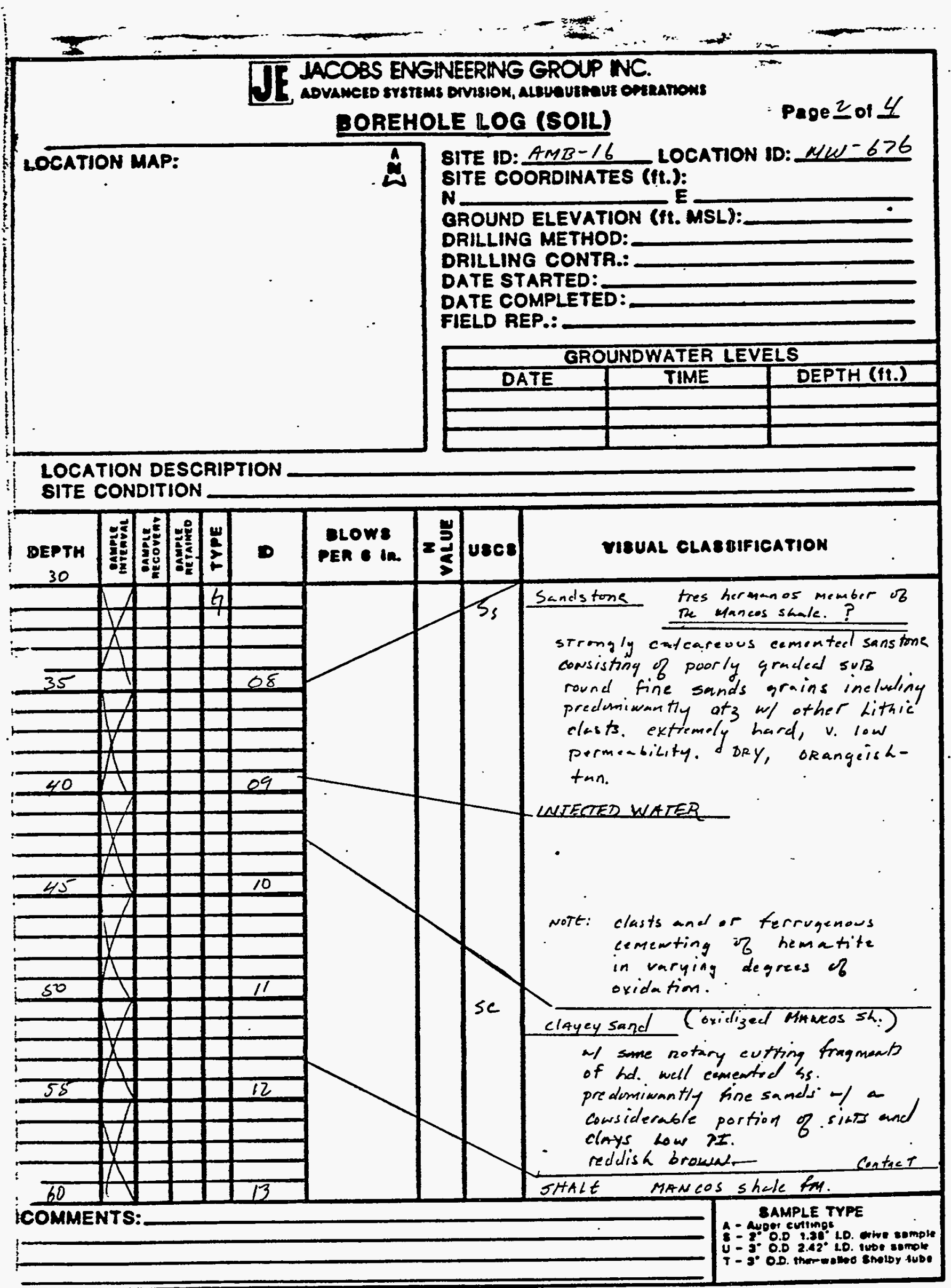

FEO-AL-ENO-28 (4IS5) 


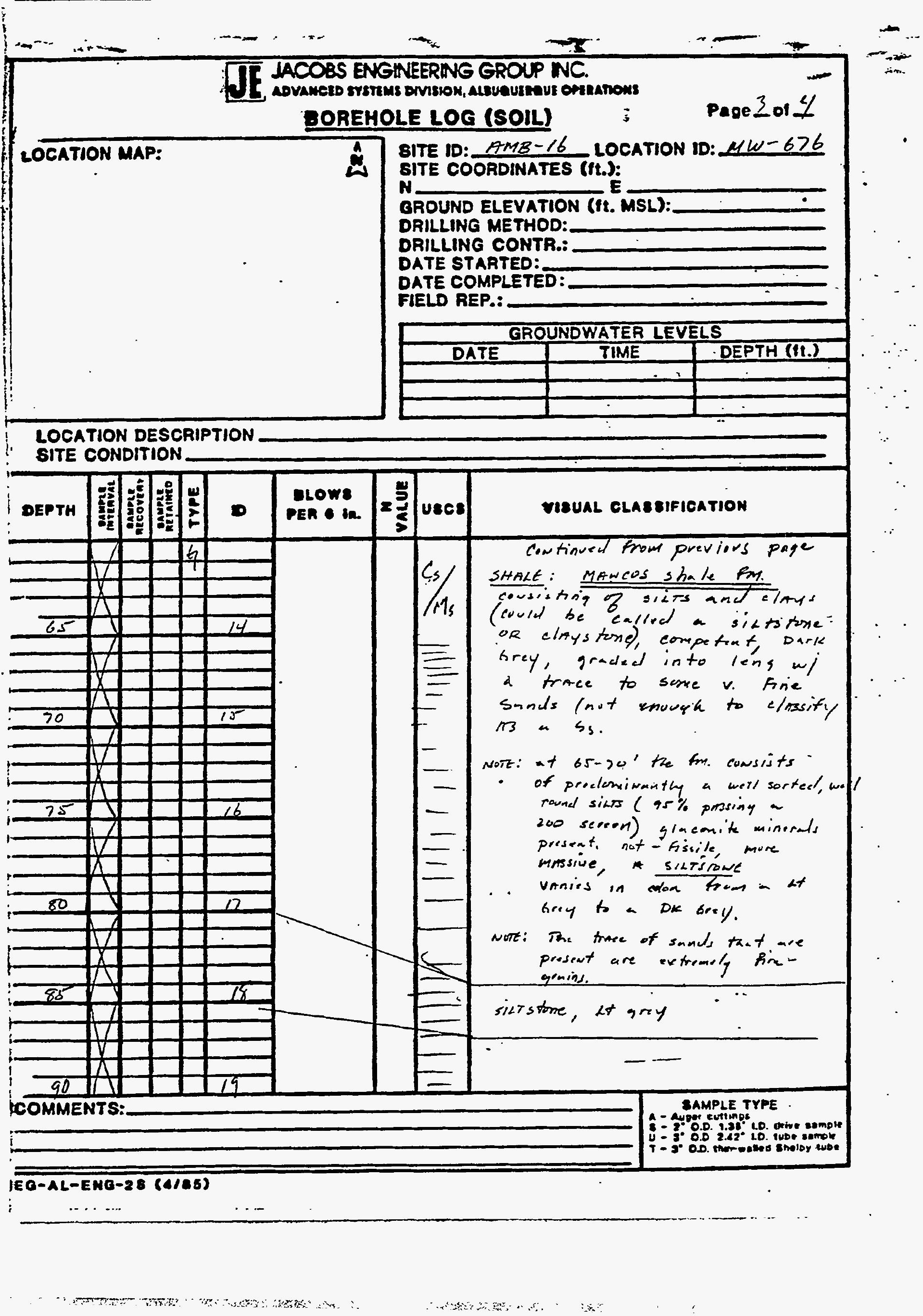




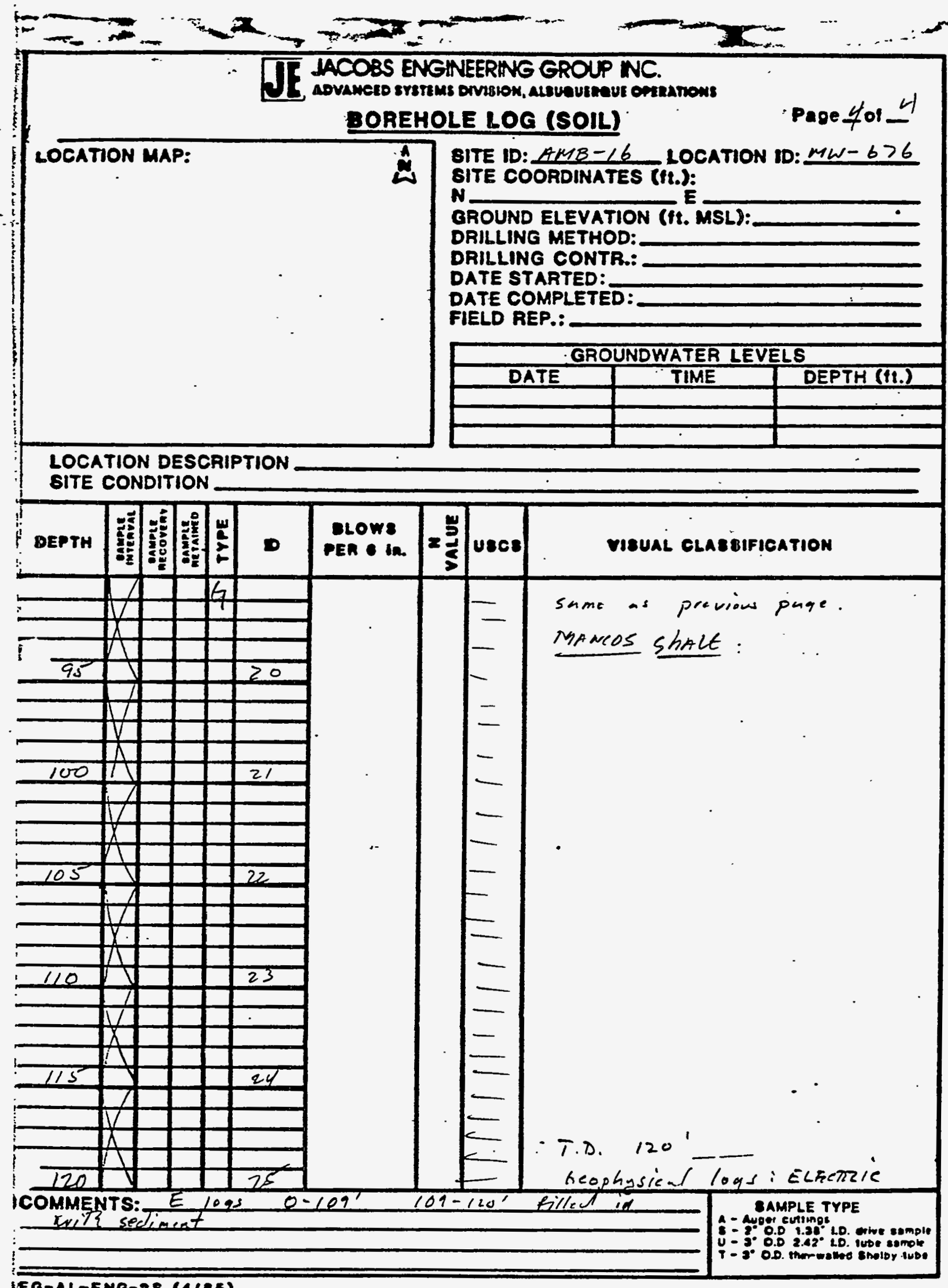

IEO-AL-EMO-28 (4/SB) 


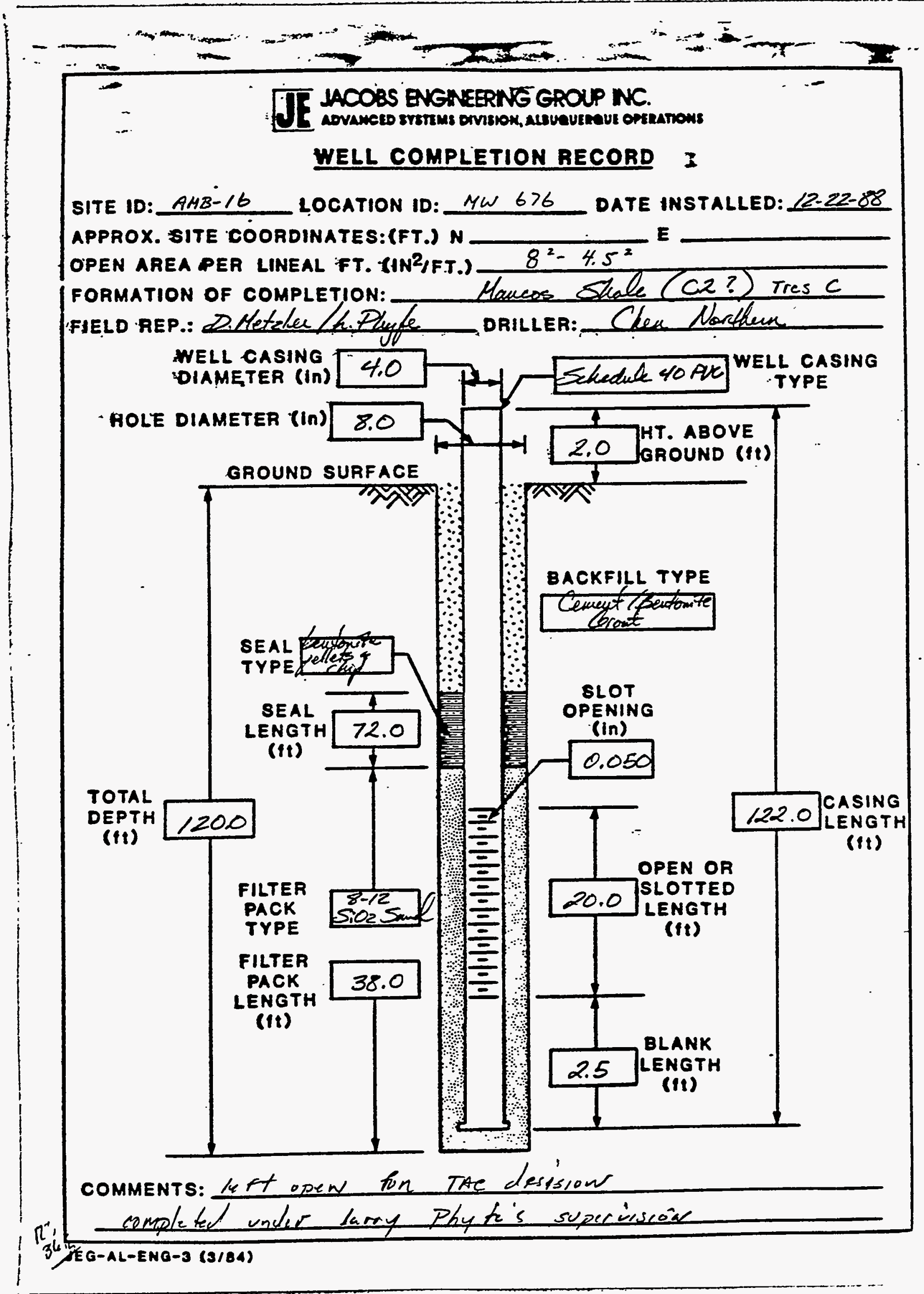




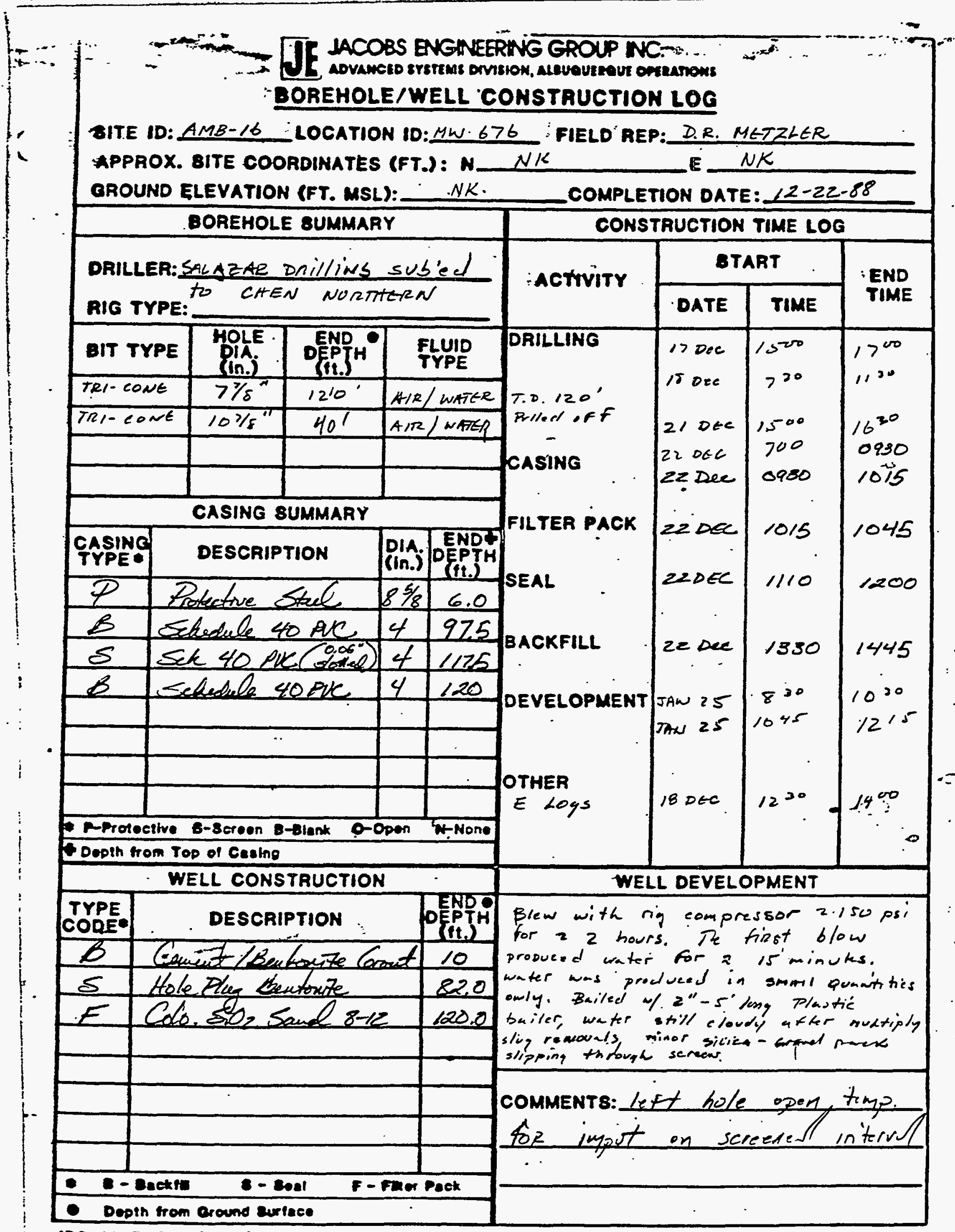

dEQ-AL-ENO-I (a/EA) 
WELL 677 LOGS

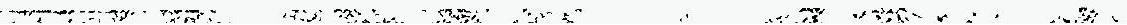




\section{TT JACOBS ENGINEERING GROUP NC.

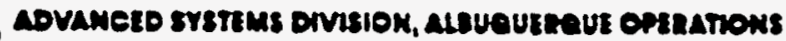

BOREHOLE LOG (SOIL)

Page $<$ of 6

LOCATION MAP:

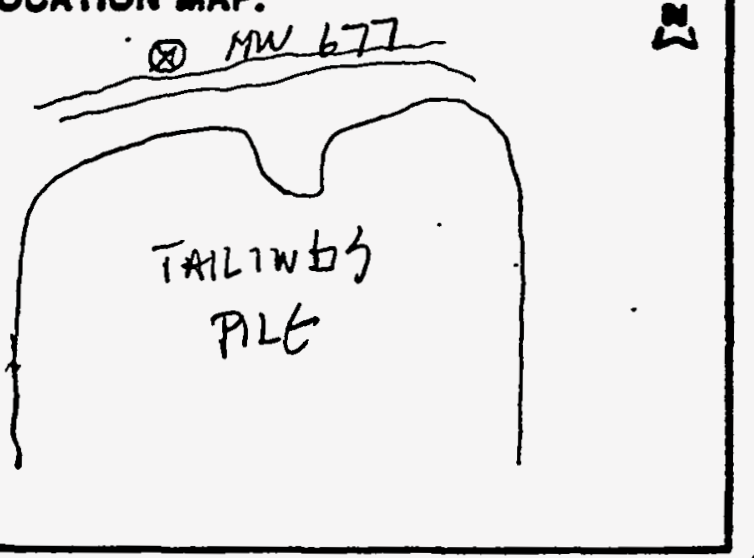

8ITE ID: AMB-16

GITE COORDINATES (tt.)

N NK

GROUND ELEVATION ( 1 . MSL):

DRILLING METHOD: ZUTAA Y

DRILLI

DATE STARTED:

DATE COMPLETED: 20 DEC $8 \delta$

FIELD REP.:

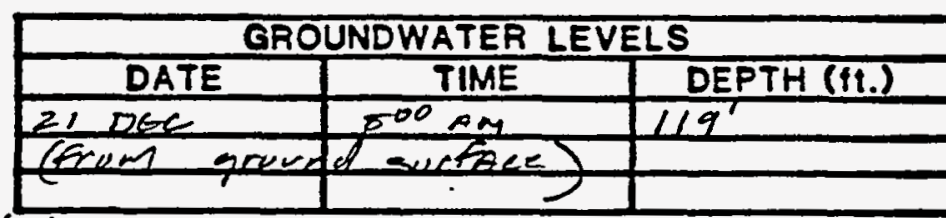

LOCATION DESCRIPTION $N 90^{\circ} \mathrm{W}, 315$

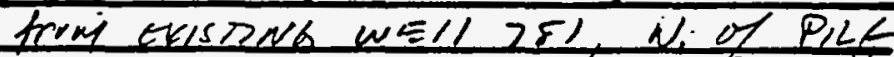

SITE CONDITION FLAT, louse sand w4 sparst werey plante conerewity

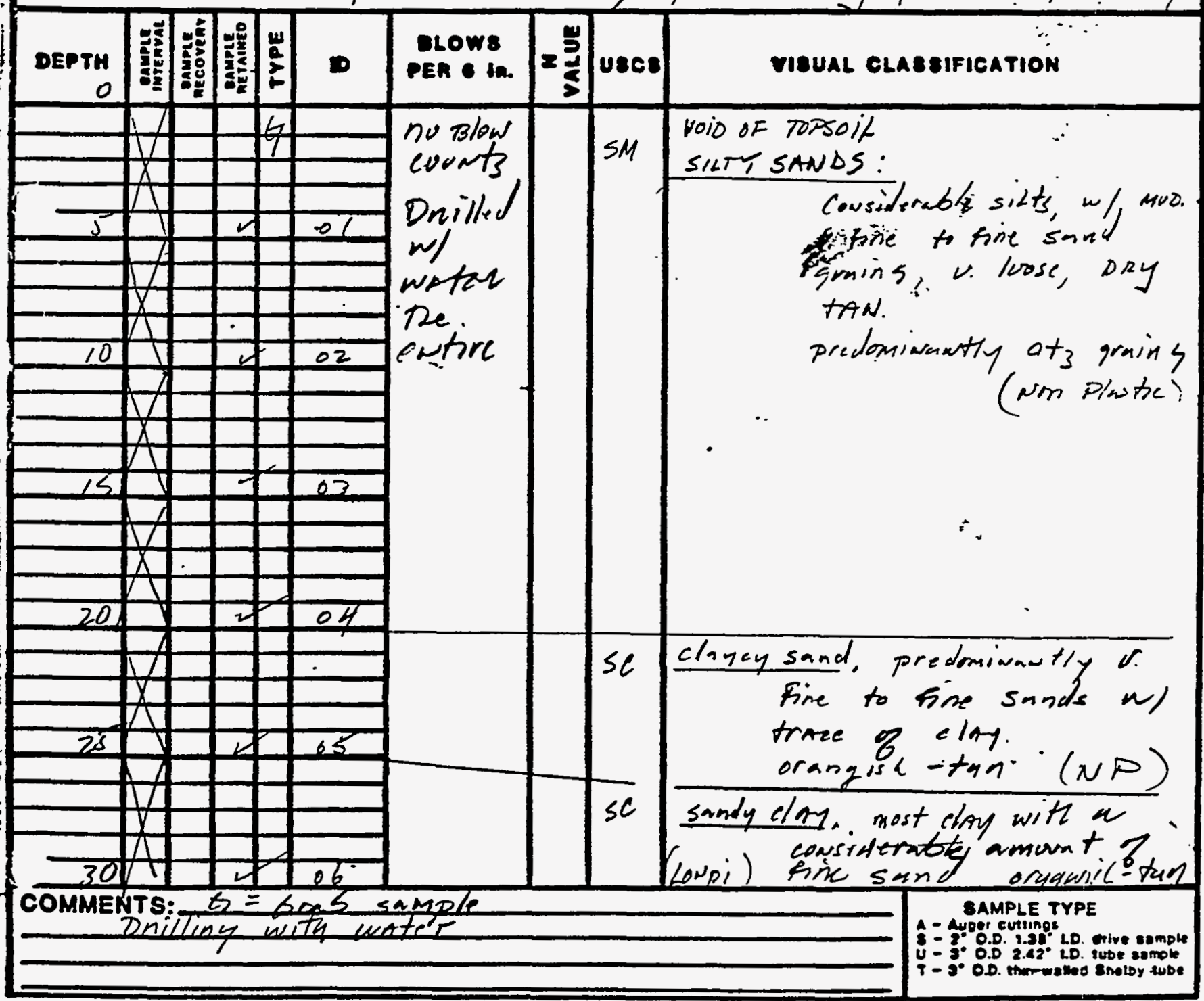

.JEG-AL-ENG-28 (4/ES) 
JI $A$ ACOBS ENGINEERING GROUP INC.

DOREHOLE LOG (SOIL)

Page Zor 6

LOCATION MAP:

SITE ID: AMB-16 LOCATION ID: MN: 677

SITE COORDINATES (ft.):

GROUND ELEVATION (ft. MSL):

DRILLING METHOD:

DRILLING CONTR.:

DATE STARTED:

DATE COMPLETED:

FIELD REP.:

\begin{tabular}{|c|c|c|}
\hline \multicolumn{3}{|c|}{ GROUNDWATER LEVELS } \\
\hline DATE & TIME & DEPTH (fi.) \\
\hline & & \\
\hline & & \\
\hline & & \\
\hline
\end{tabular}

LOCATION DESCRIPTION

SITE CONDITION

\begin{tabular}{|c|c|c|c|c|c|c|c|c|}
\hline DEPTH & 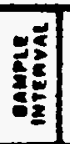 & 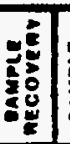 & 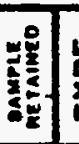 & 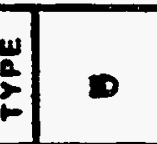 & $\begin{array}{l}\text { OLOW8 } \\
\text { PER }- \text { in. }\end{array}$ & $=\frac{5}{5}$ & Usce & VISUAL CLASBIFICATION \\
\hline & & & & 年 & & & \multirow{21}{*}{$\mathrm{CH}$} & \multirow{21}{*}{ 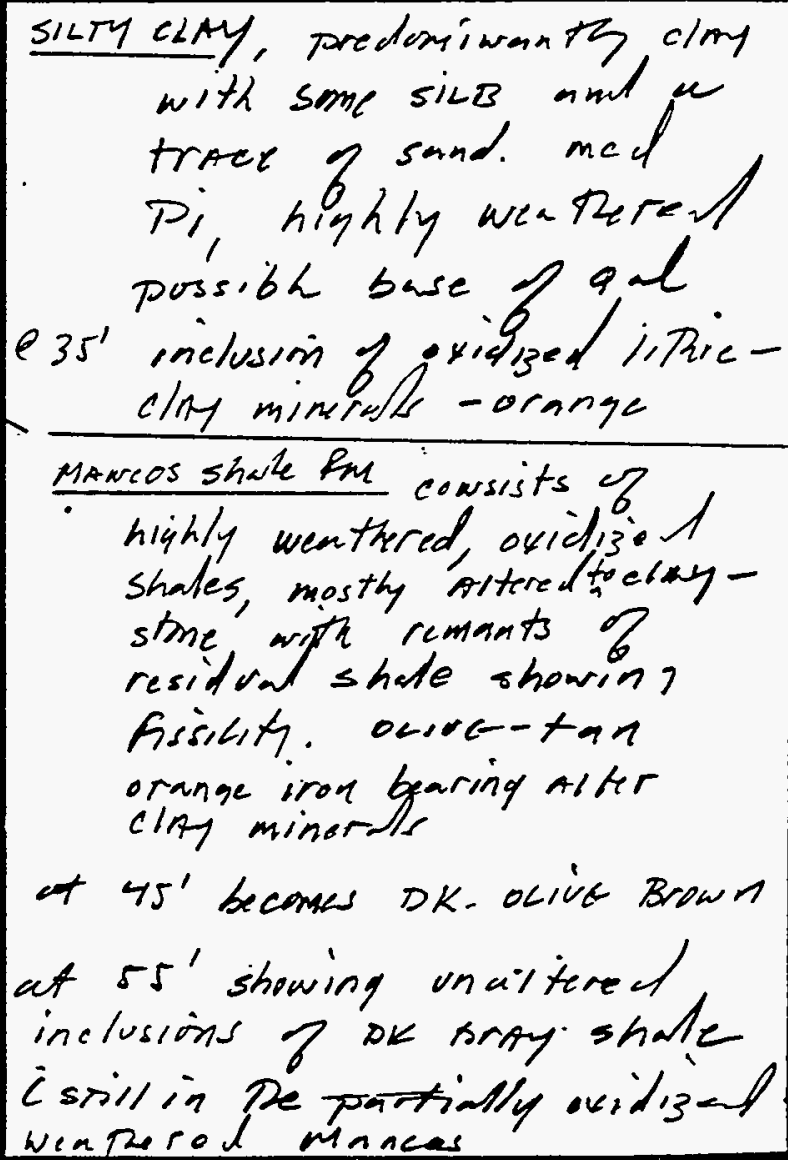 } \\
\hline & & & & & & & & \\
\hline 35 & & & $\pi$ & 67 & & & & \\
\hline & 7 & & & & & & & \\
\hline & & & & & & & & \\
\hline 40 & $y$ & & & 08 & & & & \\
\hline & & & & & & & & \\
\hline & in & & & & & & & \\
\hline & & & & & & & & \\
\hline $4 s$ & & & 4 & 09 & & & & \\
\hline & & & & & & & & \\
\hline & & & & & & & & \\
\hline 50 & & & & 10 & & & & \\
\hline & & & & & & & & \\
\hline & 44 & & & & & & & \\
\hline & & & & & & & & \\
\hline 53 & 1 & & & II & & & & \\
\hline & & & & & & & & \\
\hline & 4 & & & & & & & \\
\hline & & & & & & & & \\
\hline 60 & II & & 7 & 12 & & & & \\
\hline 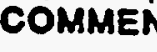 & ITS: & & & & & & & \\
\hline
\end{tabular}

DEG-AL-ENG-28 (4/ES) 


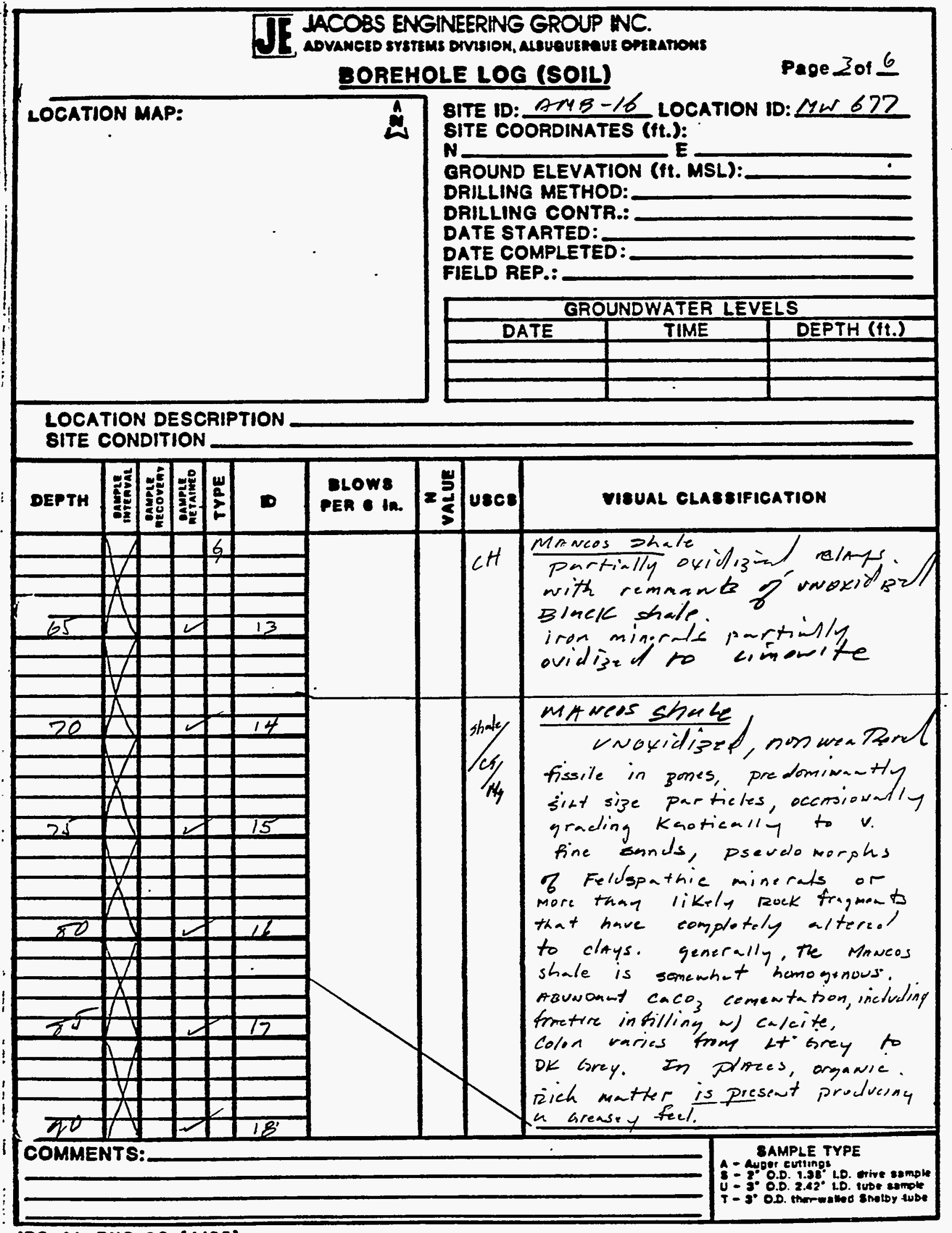




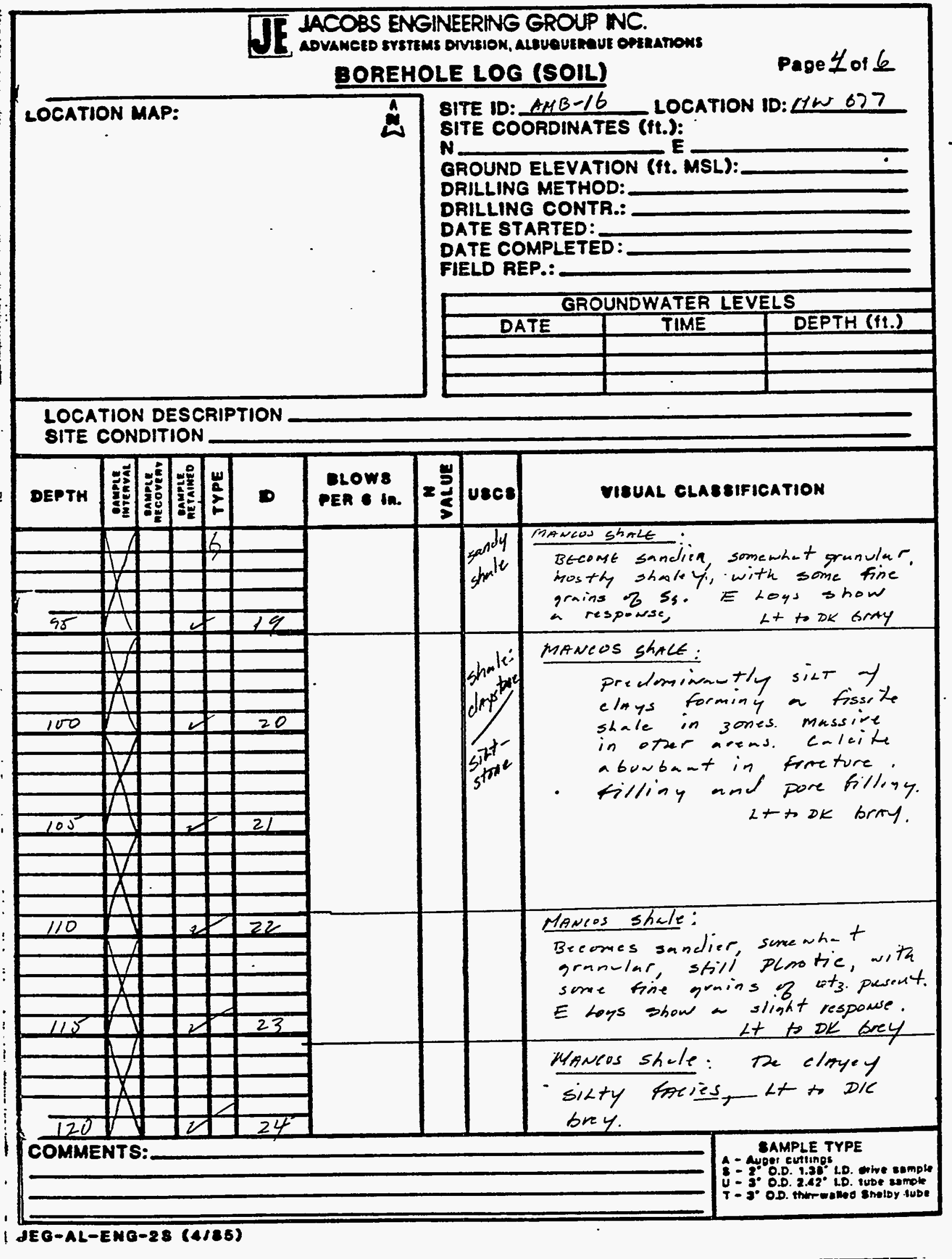




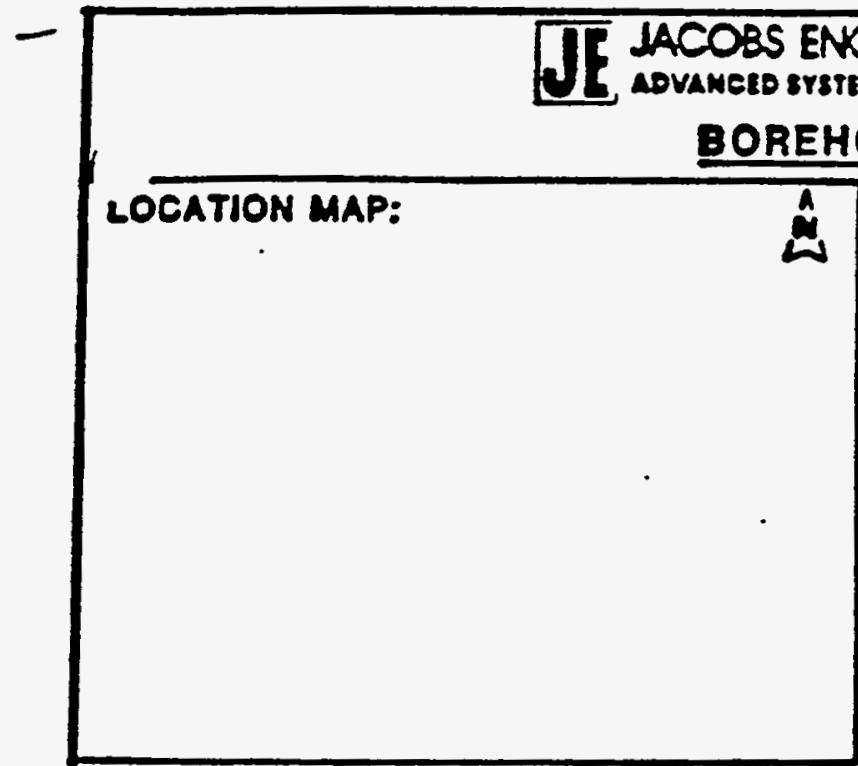

ACOBS ENGINEERING GROUP INC.

A

OOREHOLE LOG (SOIL)

Page 5016

SITE ID: AMB-16 LOCATION ID: $M W-677$ SITE COORDINATES (It.):

N

GROUND ELEVATION (ft. MSL):

DRILLING METHOD:

DRILLING CONTR.:

DATE STARTED:

DATE COMPLETED:

FIELD REP.:

\begin{tabular}{|l|c|c|}
\hline \multicolumn{3}{|c|}{ GROUNDWATER LEVELS } \\
\hline DATE & TIME & DEPTH (I1.) \\
\hline & & \\
\hline & & \\
\hline & & \\
\hline
\end{tabular}

LOCATION DESCRIPTION

SITE CONDITION

\begin{tabular}{|c|c|c|c|c|c|c|c|c|}
\hline DEPTH & 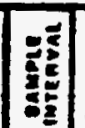 & 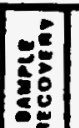 & 㩆 & $\frac{w}{2}$ & 0 & $\begin{array}{l}\text { DLOW } \\
\text { PER } \bullet \text { In. }\end{array}$ & $=\stackrel{s}{s}$ & usce \\
\hline
\end{tabular}

Mancos shale: siety clayey

Pacies, wite abuadu-t

Calcité filling of trectures

and pore spree. horeoyenusy

fissite in plues, lat to $D K$

brey

$130-726$

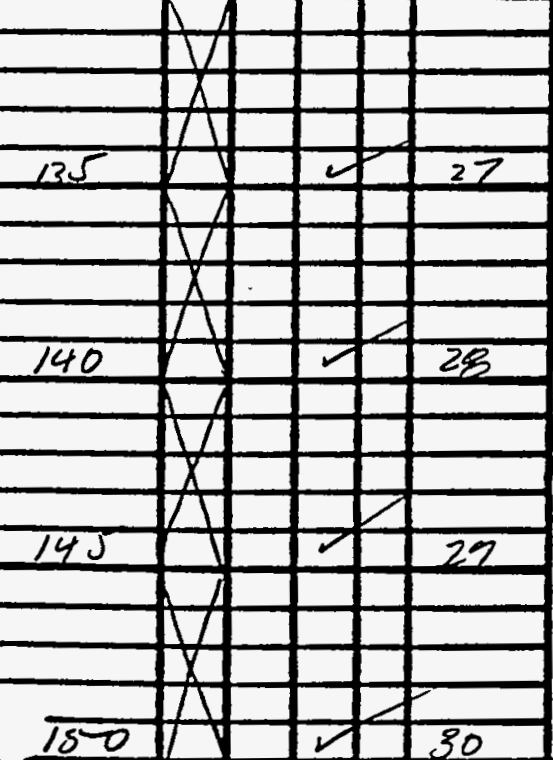

COMMENTS: 


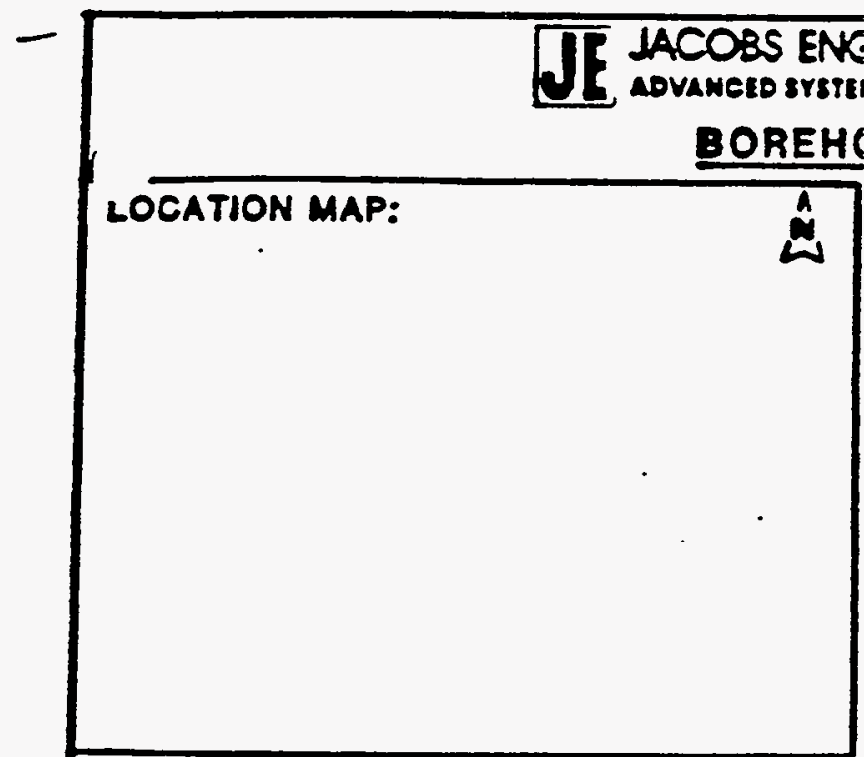

GINEERING GROUP INC.

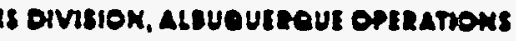

Pape 6 of 6

LOCATION DESCRIPTION

SITE CONDITION

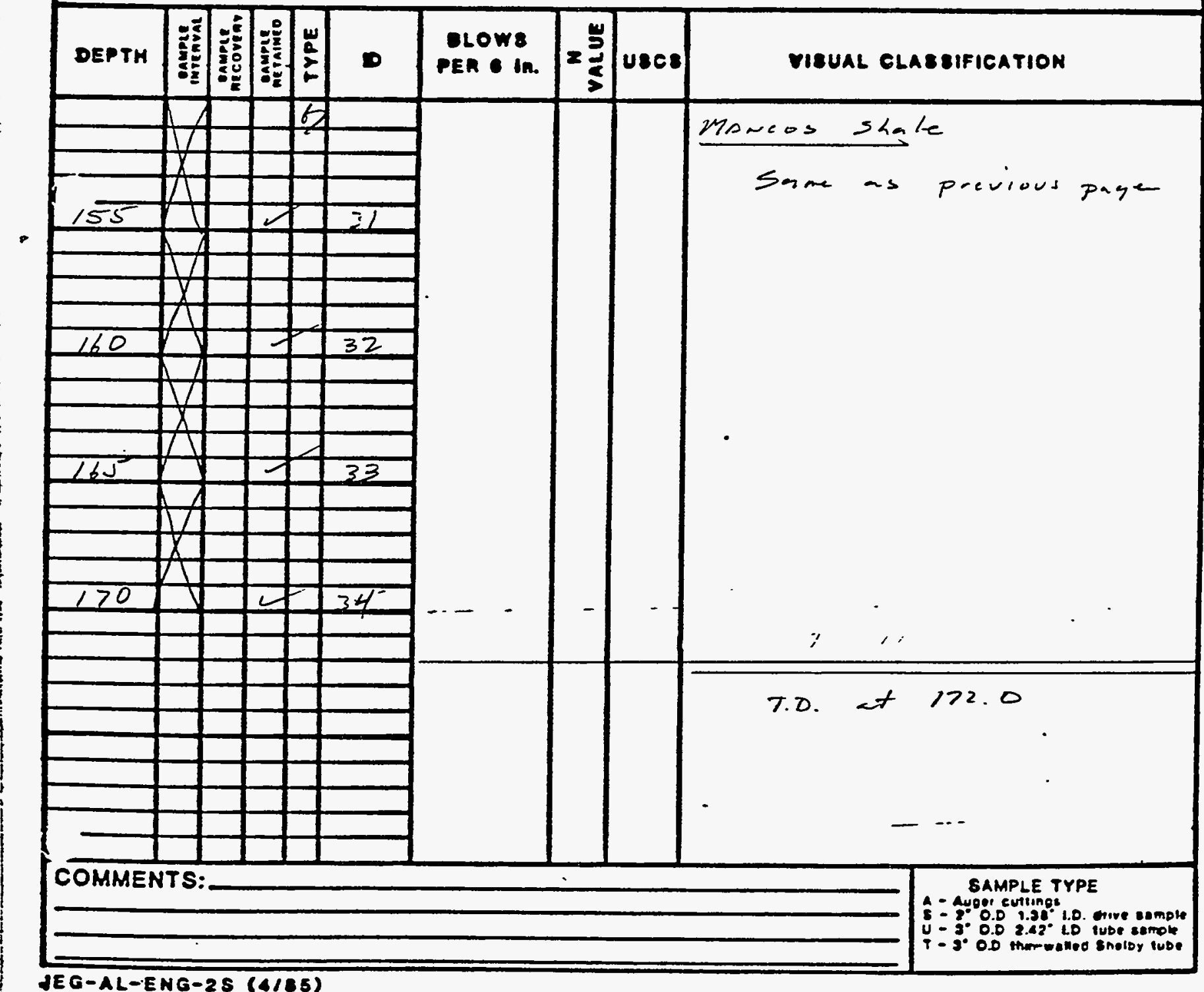

SITE ID: 10 BO-16 LATION ID: $M W-677$

SITE COORDINATES (tt.):

N

E

GROUND ELEVATION (It. MSL):

DRILLING METHOD:

DRILLING CONTR.:

DATE STARTED:

DATE COMPLETED: zo ire के

FIELD REP.:

\begin{tabular}{|c|c|c|}
\hline \multicolumn{3}{|c|}{ GROUNDWATER LEVELS } \\
\hline DATE & TIME & DEPTH (ff.) \\
\hline & & \\
\hline & & \\
\hline & & \\
\hline
\end{tabular}

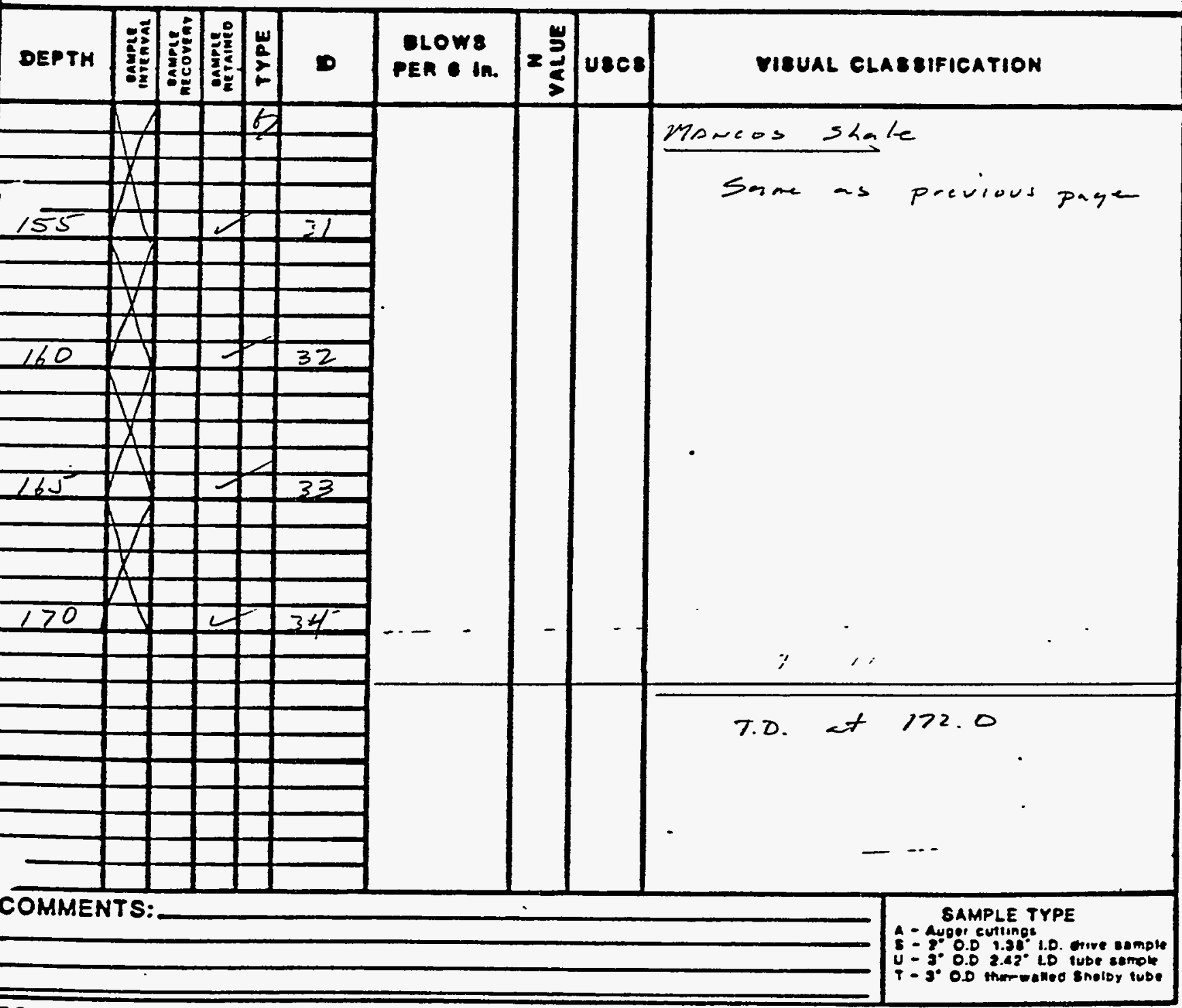




\section{TF JACOBS ENGNEERIG CROUP NC. AOVAMEED STSTEMS ONISHOX, ALSUQUEMQUE OMERATONS}

\section{WELL COMPLETION RECORD}

SITE ID: AMB-16 LOCATION ID: MW 677 DATE INSTALLED: DEC 20,88

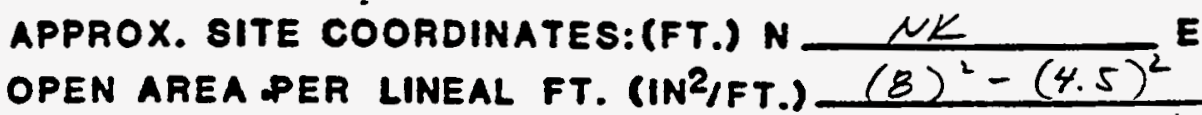

FORMATION OF COMPLETION: MANCOS SATAC; TreS $C$

FIELD REP.: D.R.METZCLER DRILLER: CHFN NORTHERW/ WELL CASING

DIAMETER (In)

4.0 工D

HOLE DIAMETER (in) 8.0

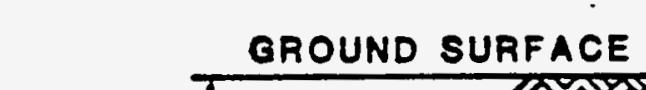

\section{TOTAL \\ DEPTH 172.0
(ft)}

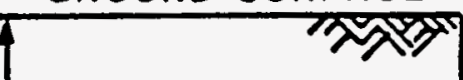

(1)

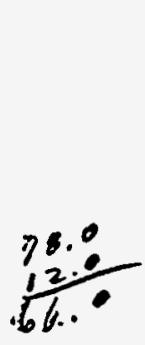

$\frac{v^{16} y^{12}}{38}$
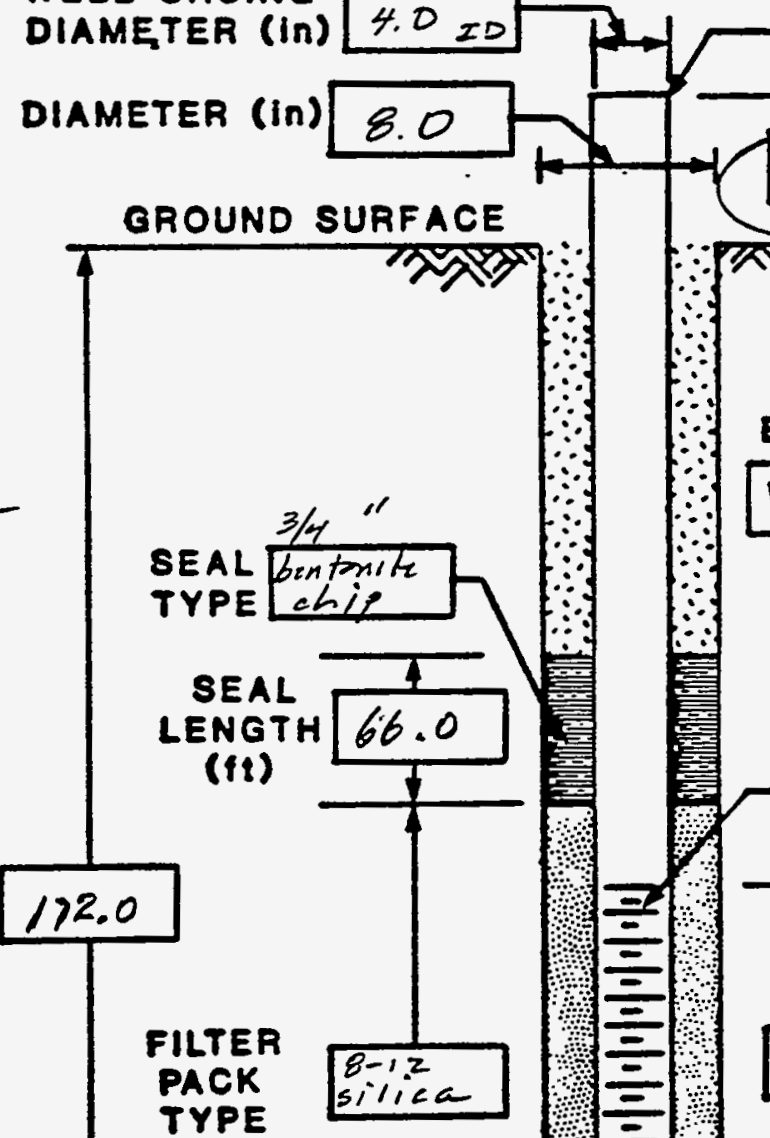
SCH 40 PVC

WELL CASING TYPE
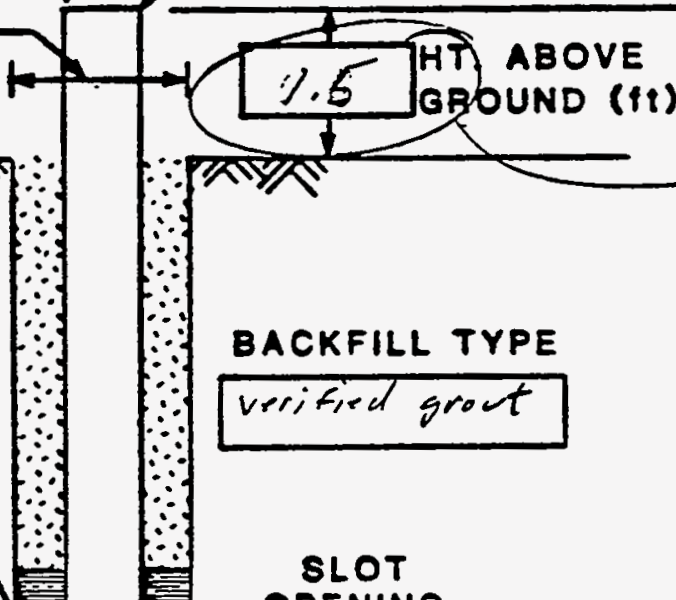


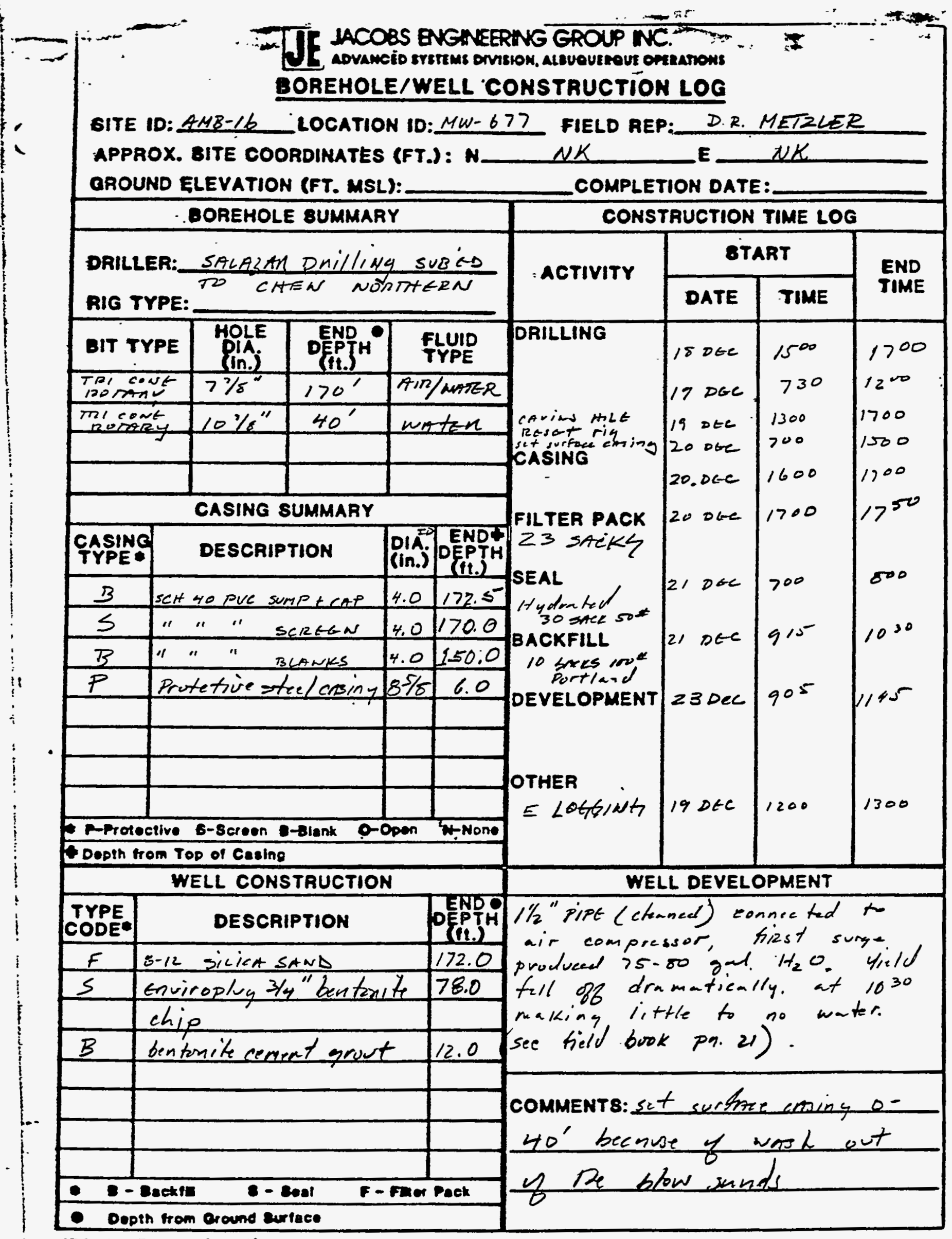


WELL 678 LOGS 


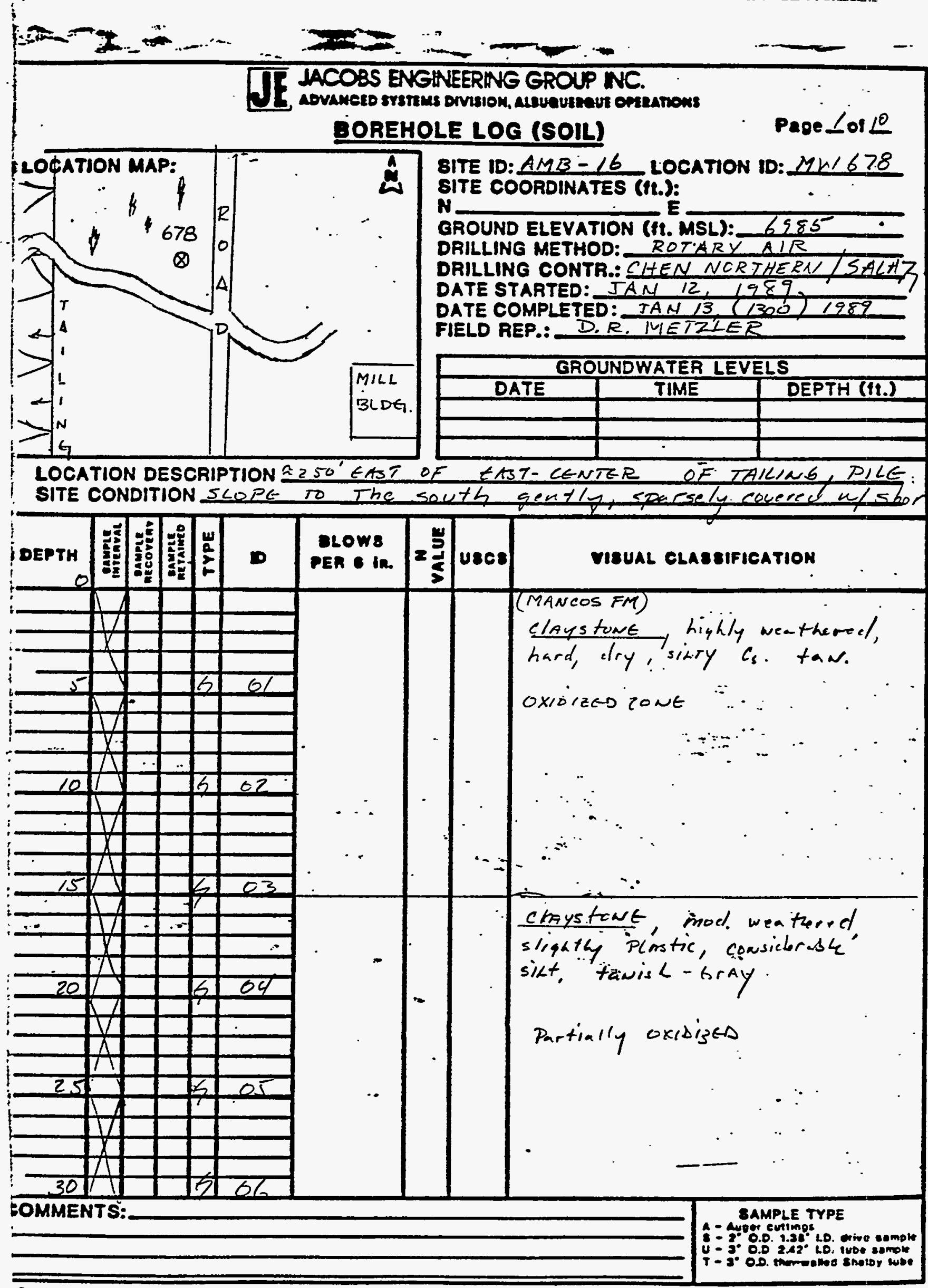




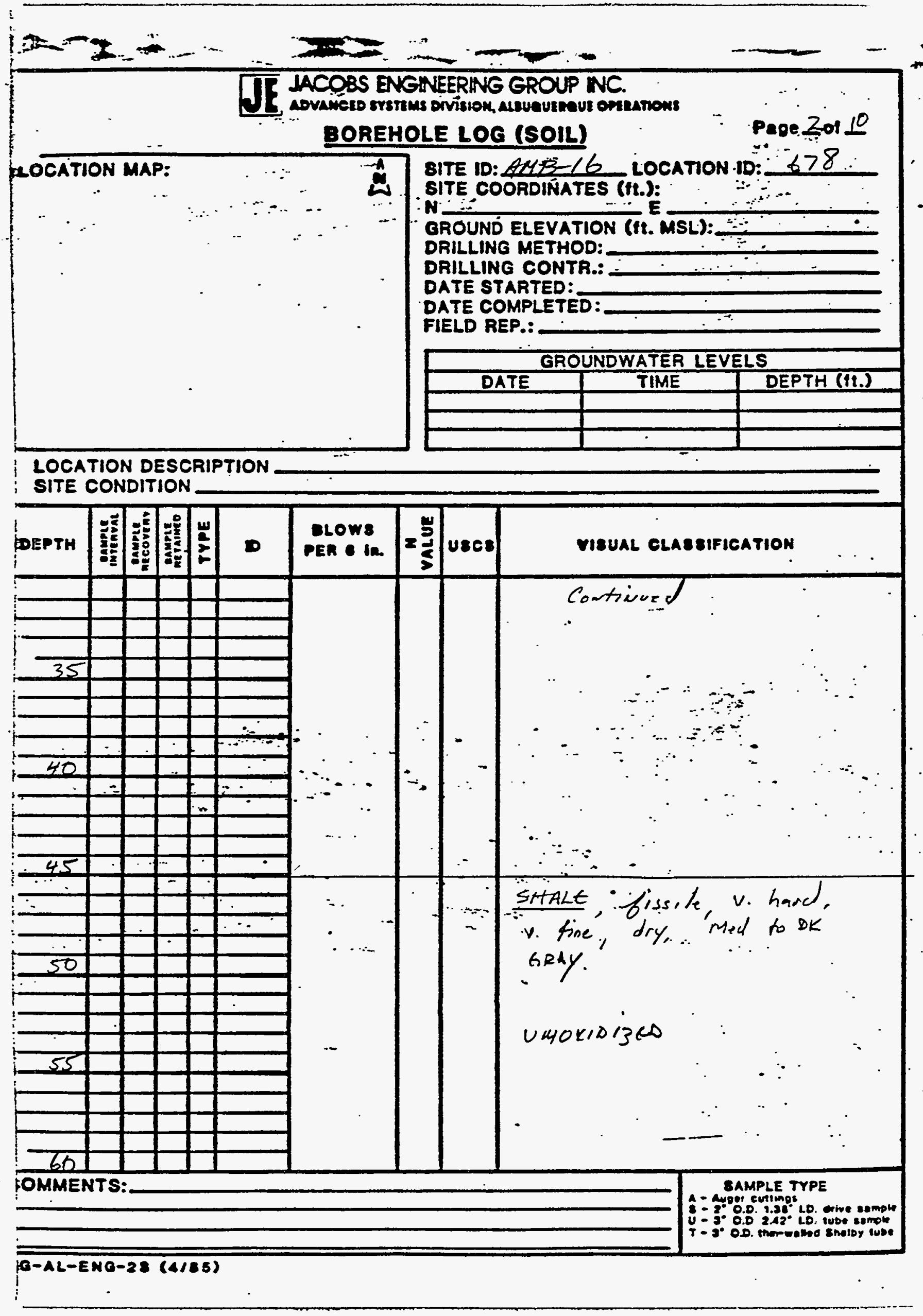




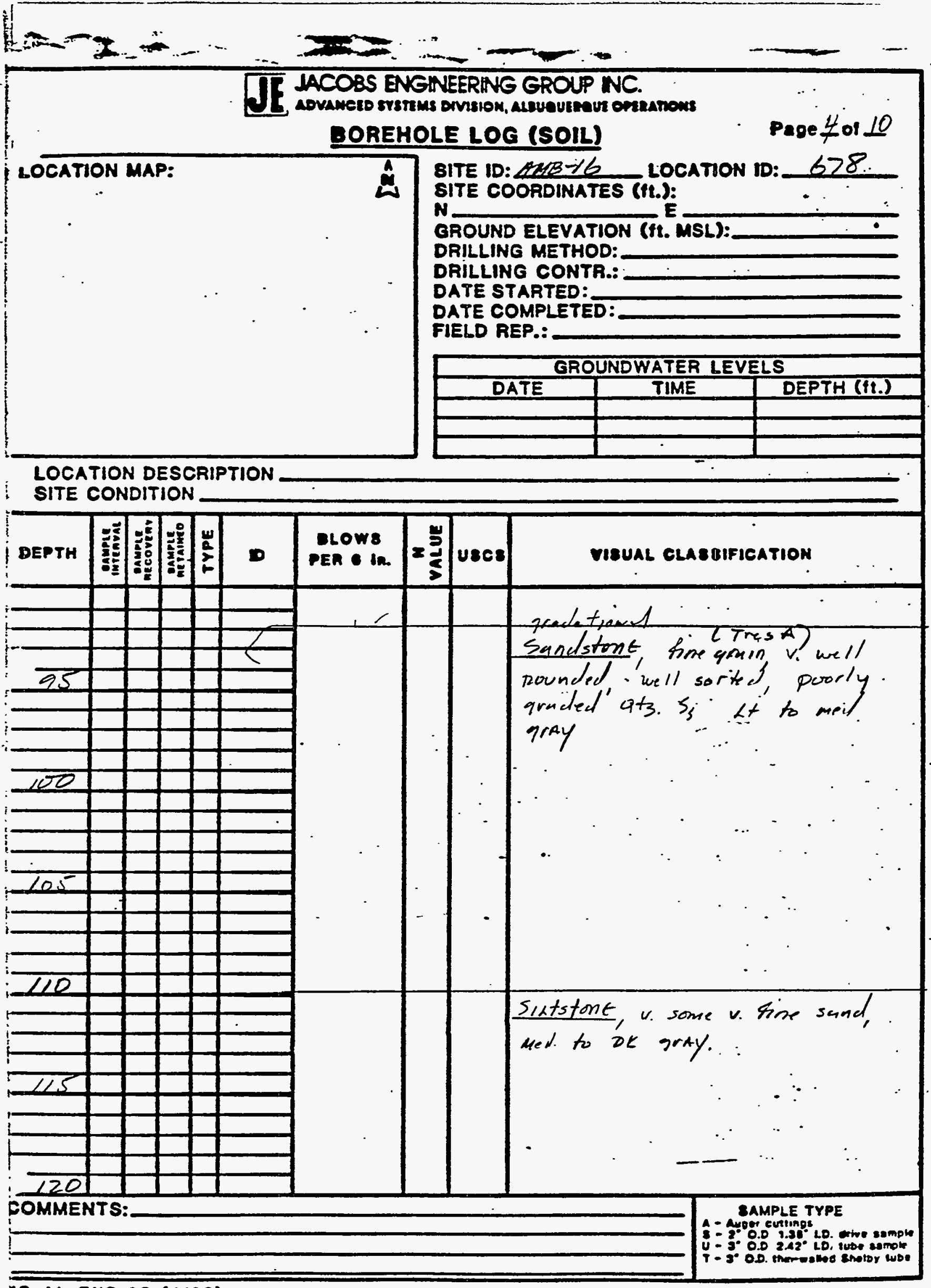

EO-AL-ENO-28 (4ISE) 


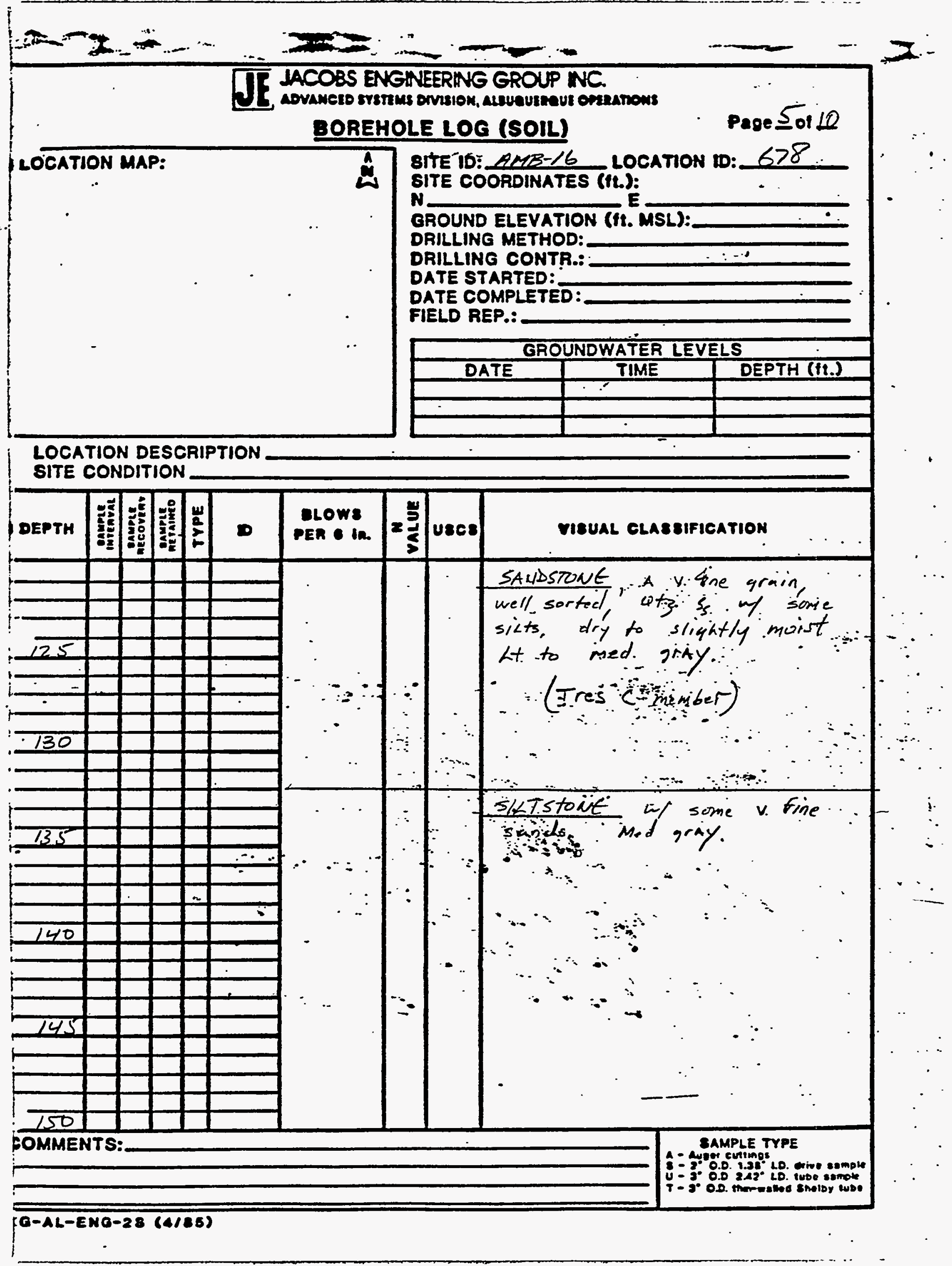




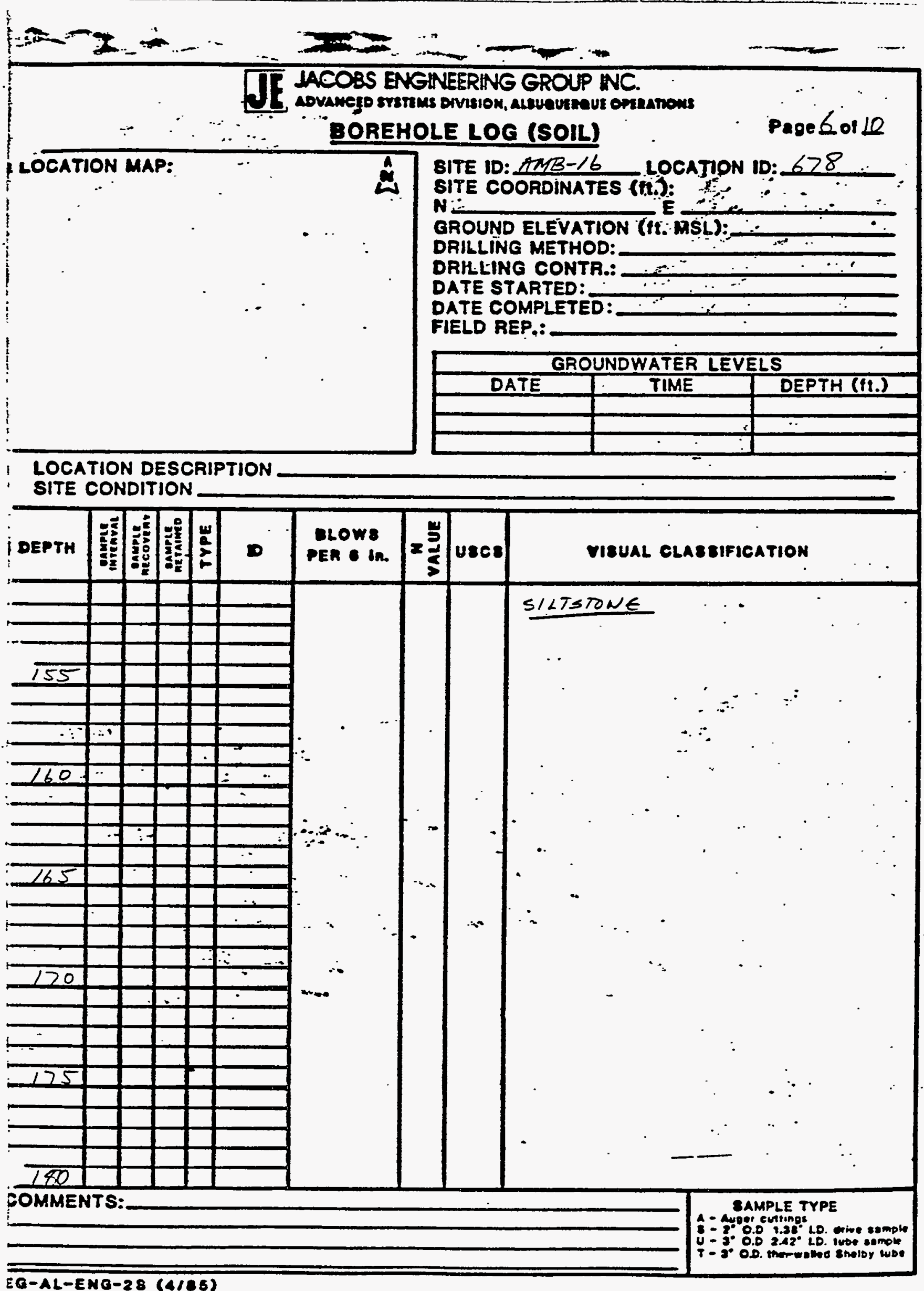




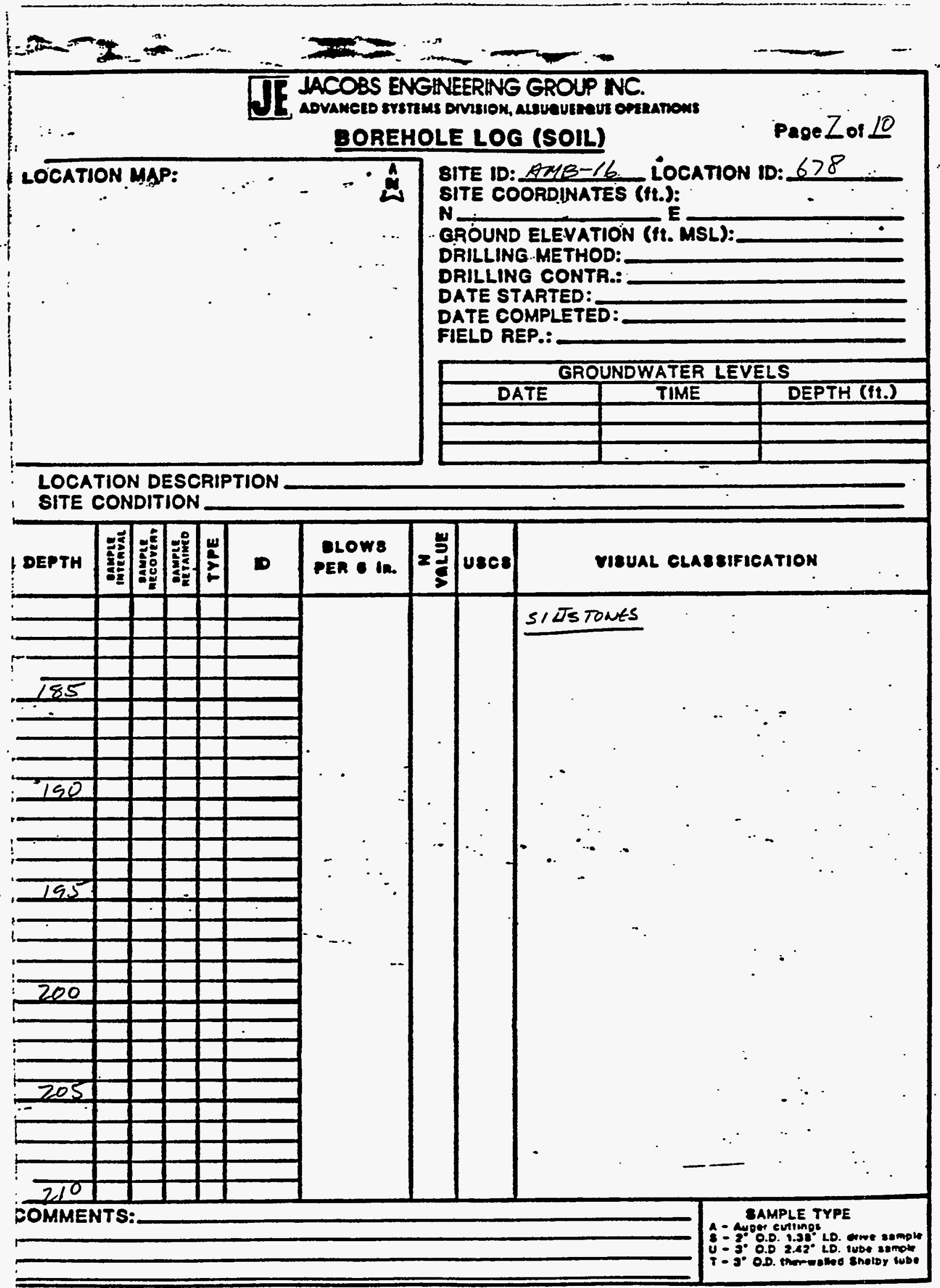

EO-AL-ENO-28 (4/EB) 
$1=-2$

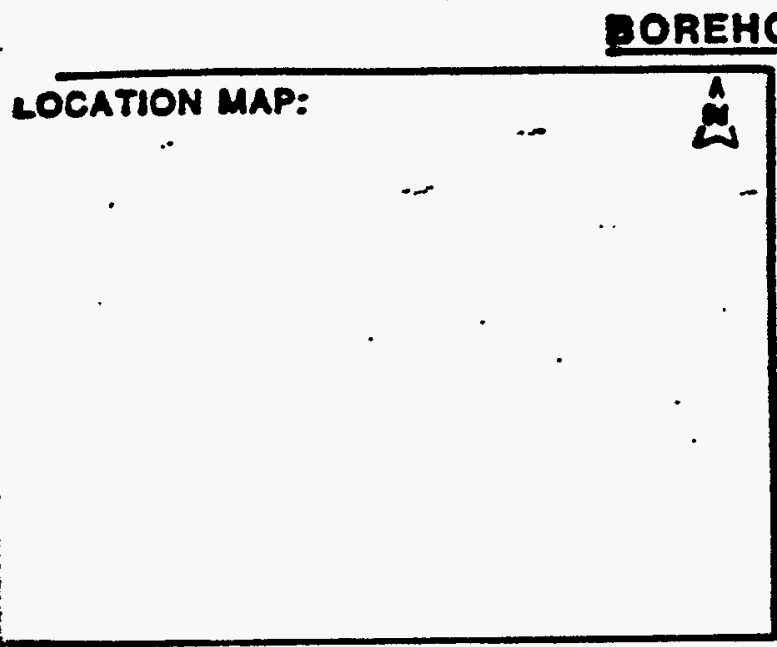

INCOBS ENGNEERING GROUP NC.

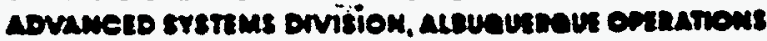

POREHOLE LOG (SOIL)

Page 8 of 10

8ITE ID: AMB-16 LOCATION ID: 678 SITE COORDINATES (fL):

N

GROUND ELEVATION (ft. MSL):

DRILLING METHOD:

DRILLING CONTR.:

DATE STARTED:

DATE COMPLETED:

FIELD REP.:

\begin{tabular}{|c|c|c|}
\hline \multicolumn{3}{|c|}{ GROUNDWATER LEVELS } \\
\hline DATE & TIME & DEPTH (fi.) \\
\hline & & \\
\hline & & \\
\hline
\end{tabular}

LOCATION DESCRIPTION

SITE CONDITION

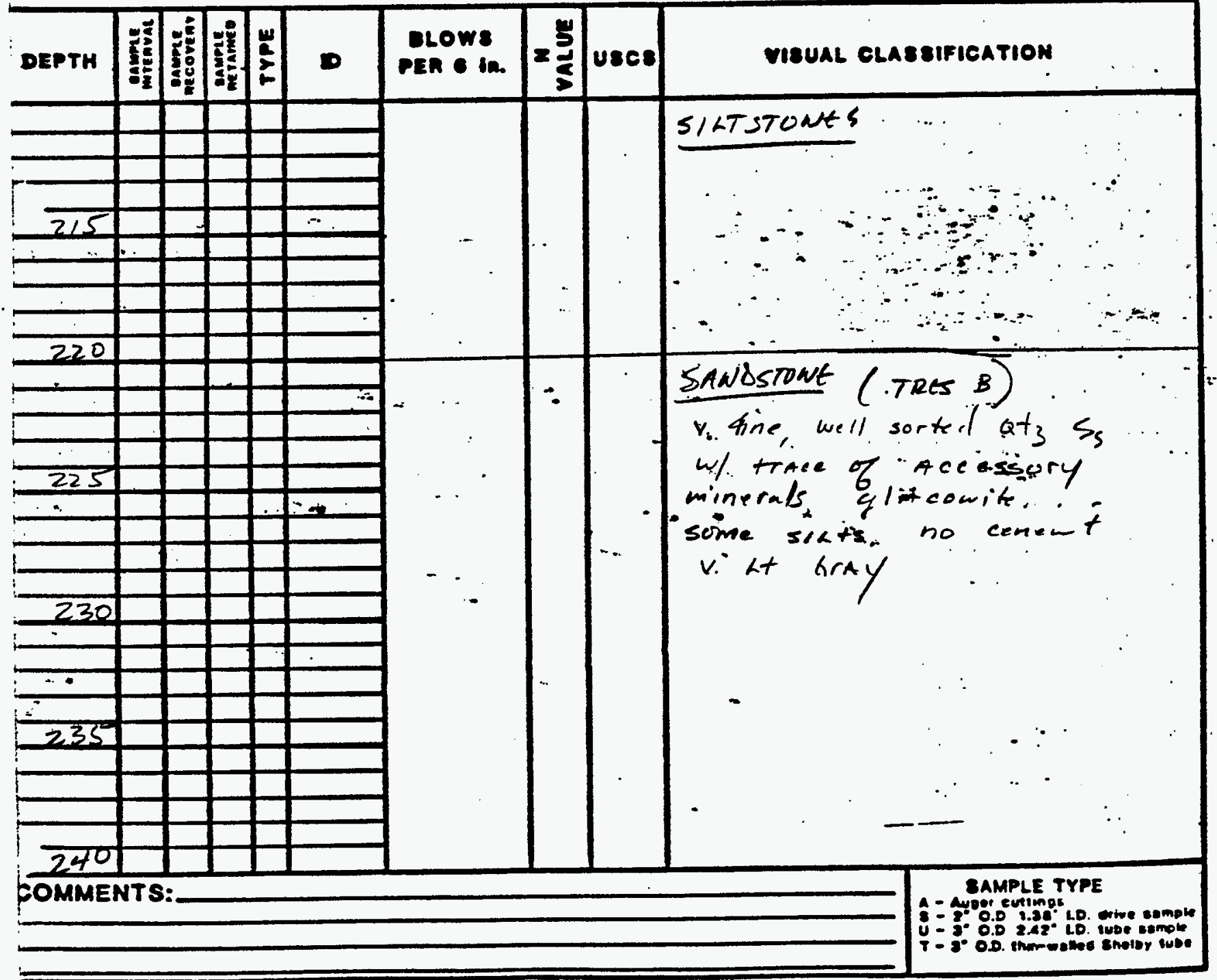

EO-AL-ENO-2B(A1ES) 


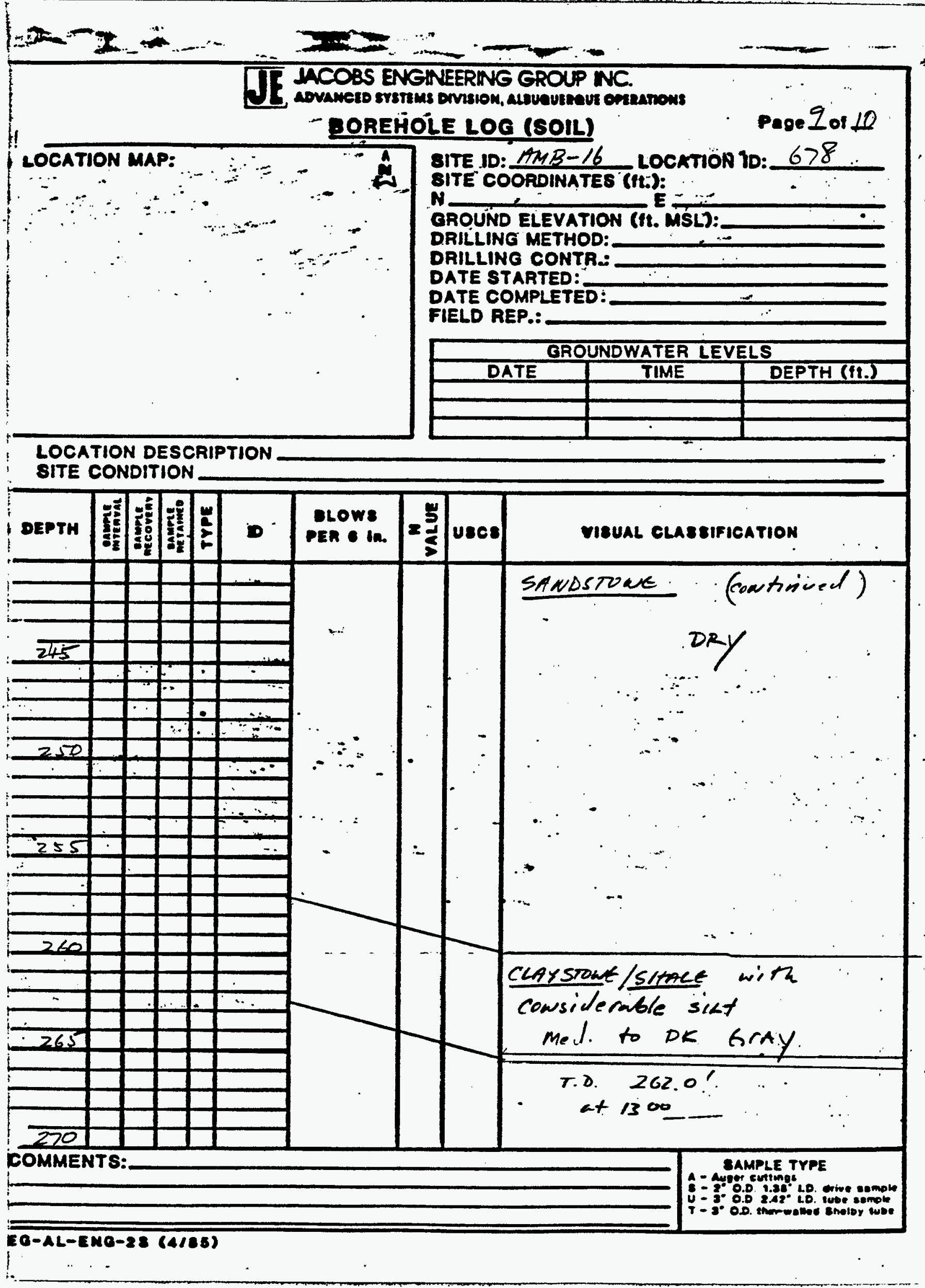




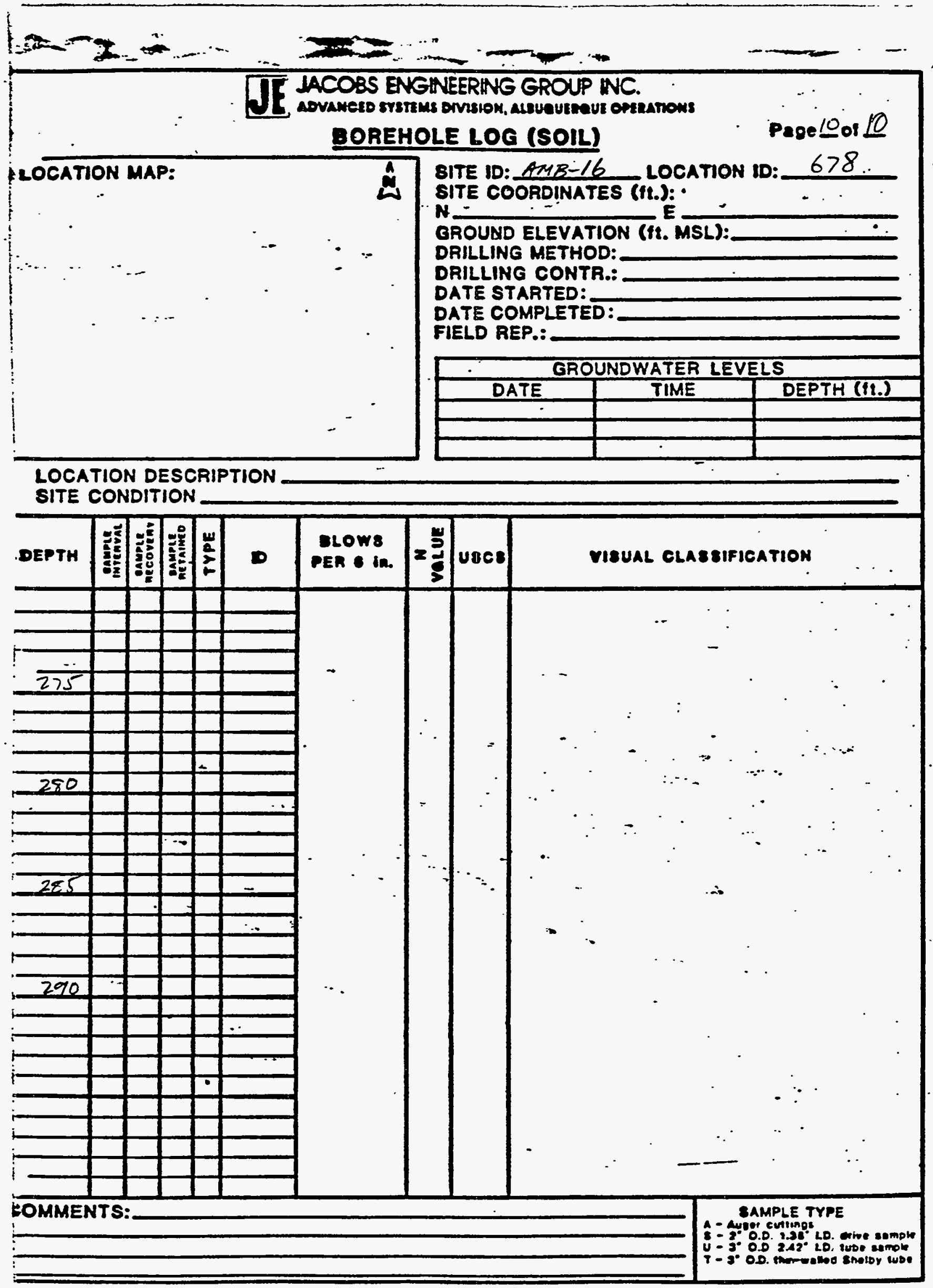




\section{TE"

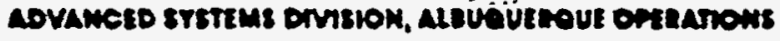

BOREHOLE/WELL CONSTRUCTION LOG

SITE. 10: $4 N B-16$ LOCATION 10: 678 APPROX. BITE COORDINATES (FT.): $N$ OROUND ELEVATION (FT. MSL): 6985 . DOREHOLE BUMMARY

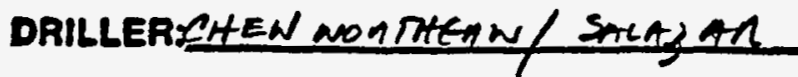
RIG TYPE:

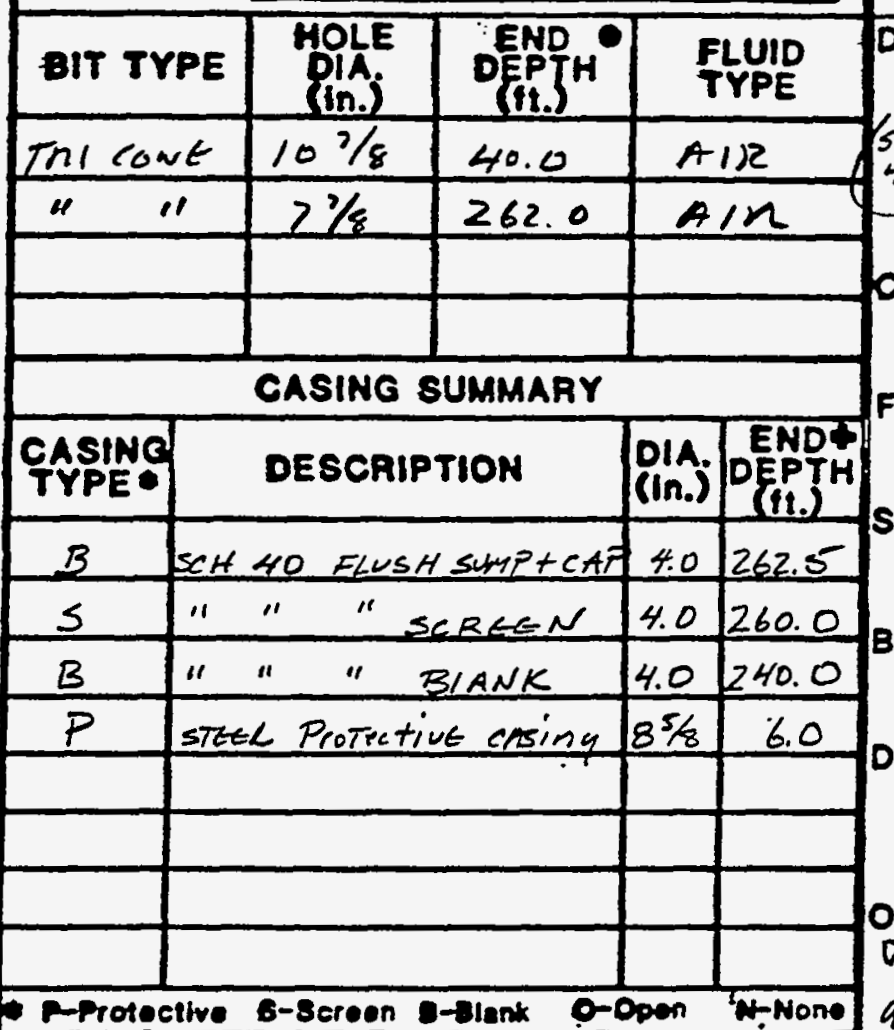
Oepth from Top of Casing

\begin{tabular}{|c|c|c|}
\hline \multicolumn{3}{|c|}{ WELL CONSTRUCTION } \\
\hline $\begin{array}{l}\text { TYPE } \\
\text { CODE }\end{array}$ & DESCRIPTION & $\begin{array}{l}\text { ENDSO } \\
\text { OEPTH }\end{array}$ \\
\hline$F$ & 8-12 SILIEA SAND & 260.0 \\
\hline 5 & $3 / y^{\prime \prime}$ bentonite chip & 217.0 \\
\hline$B$ & bentonite-cement srout & 10.0 \\
\hline & & \\
\hline & & \\
\hline & & \\
\hline & & \\
\hline & & \\
\hline 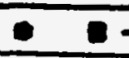 & $8-\cos 1$ & Pect \\
\hline - D. & in from around eurlace & \\
\hline
\end{tabular}

FIELD REP: D.R. METRLER E COMPLETION DATE: $1-13-89$ CONSTRUCTION TIME 1.06

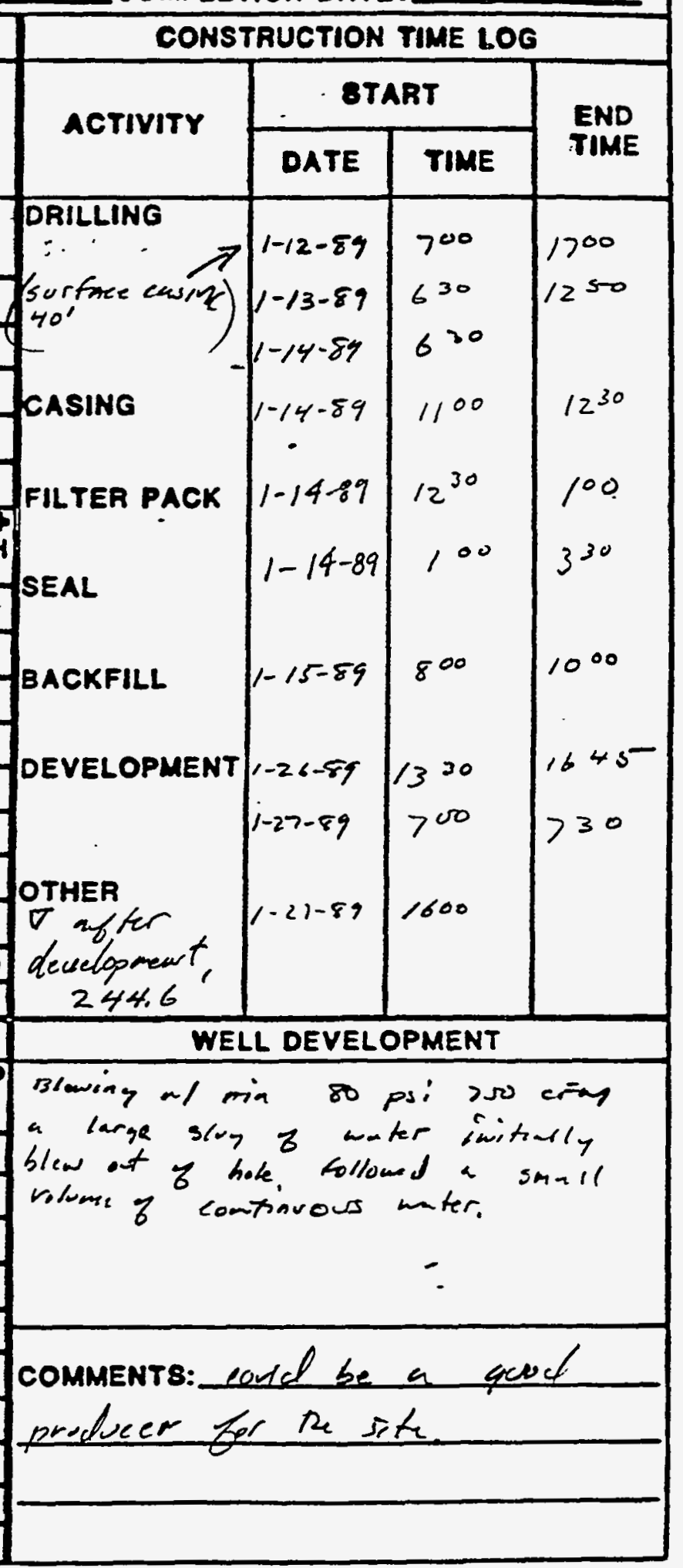


JI LACOBS ENG̈̈IREERNG GROUP NC: :

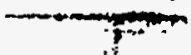

WELL COMPLETION RECORD

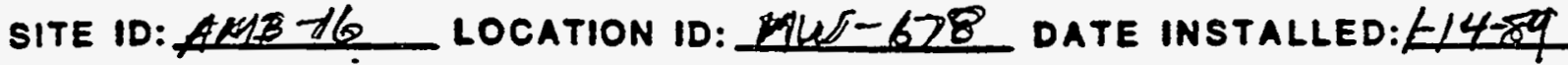

APPROX. SITE COORDINATES:(FT.) N E

OPEN AREA SER LINEAL FT. (IN2/FT.)

IFORMATION OF COMPLETION: MANCOS FM. TZES HEAMMULS B

FIELD REP.: D.R: $M E T Z L E R$

DRILLER:CHEN-NORTHERN

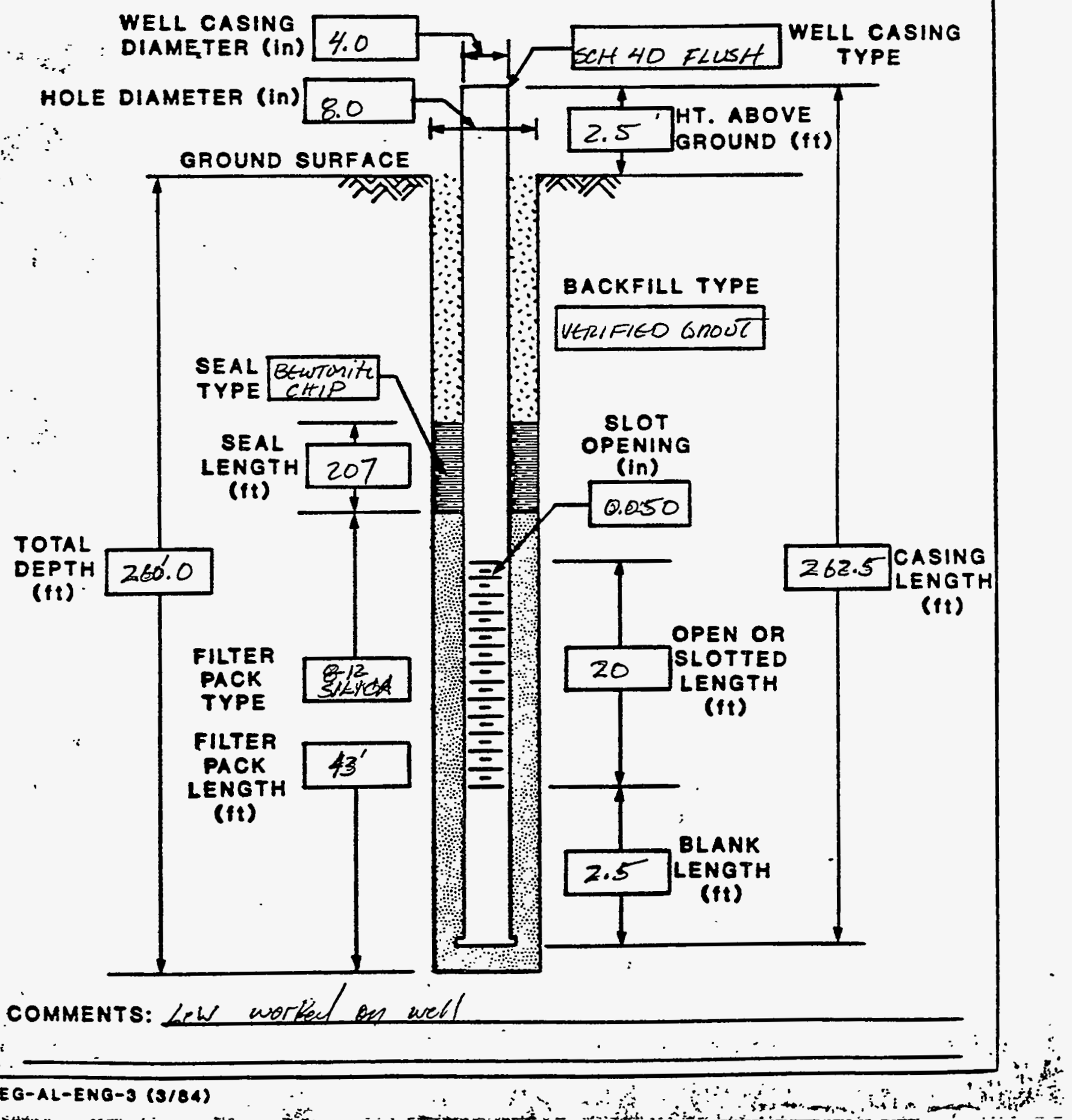


WELL 679 LOGS 


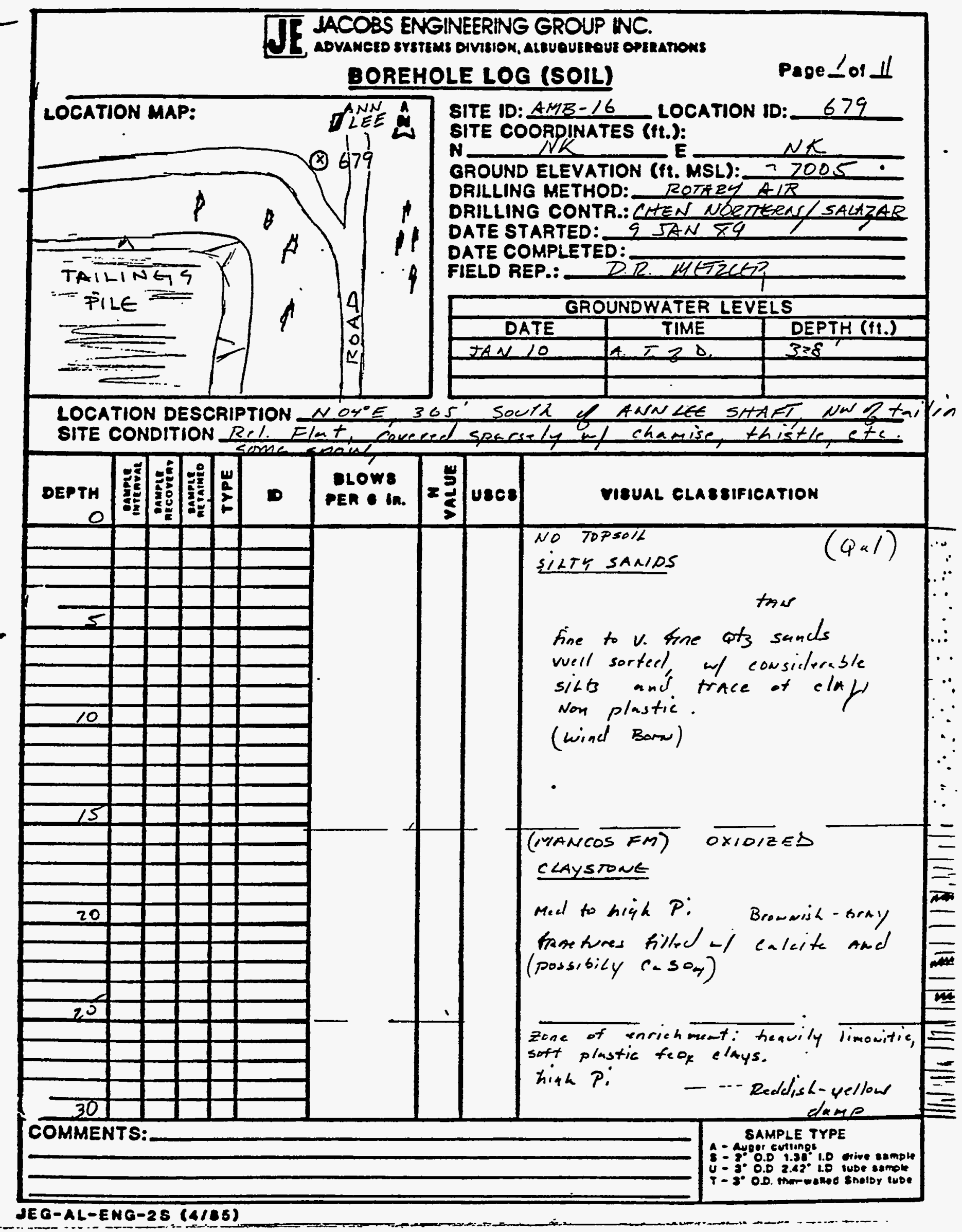




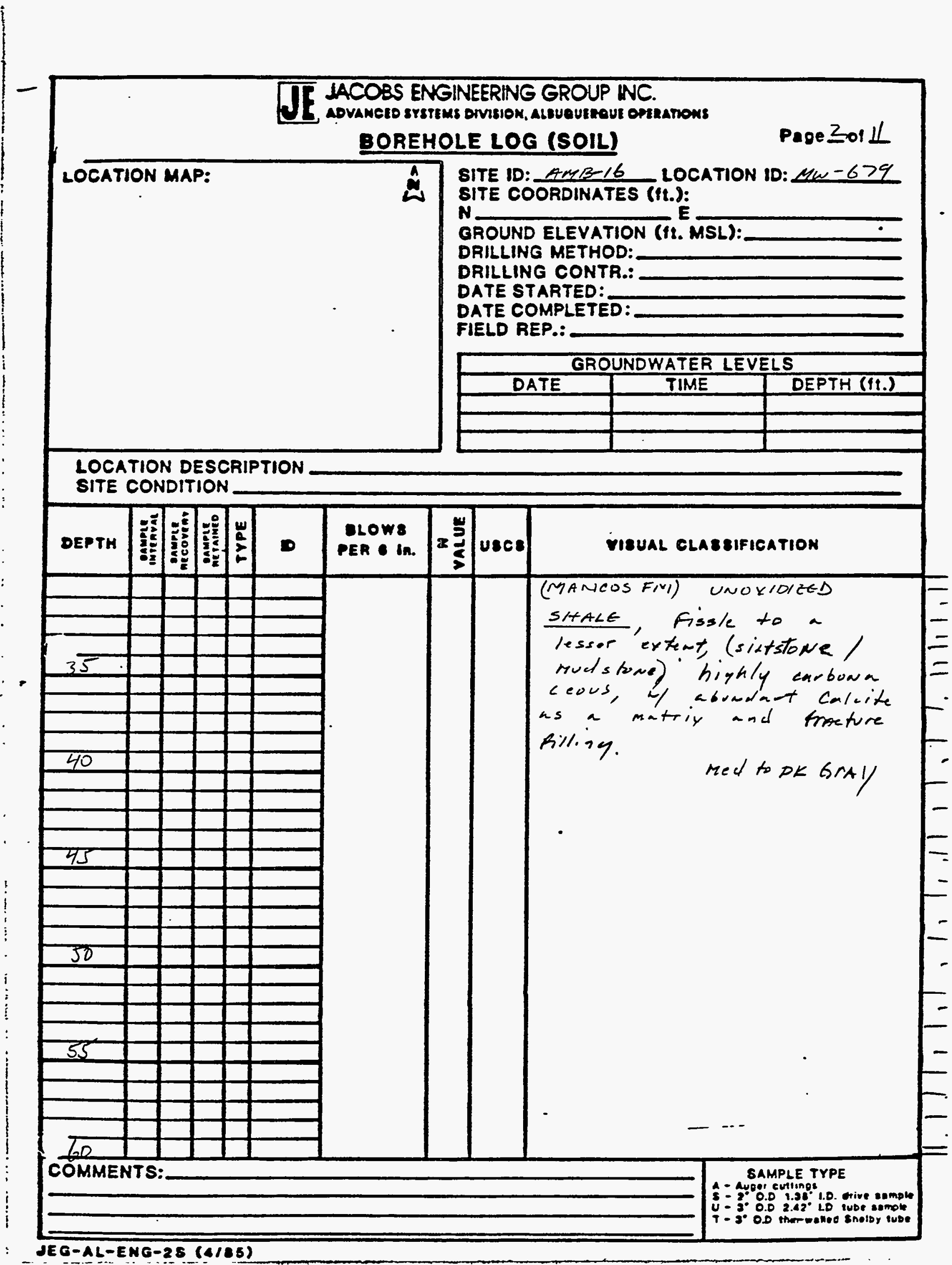


IACOBS ENGINEERING GROUP NC.

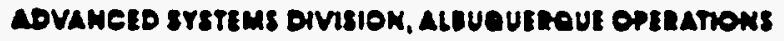

\section{BOREHOLE LOG (SOIL)}

LOCATION MAP:

SITE ID: $A$ ARB-16

LOCATION

10: 2 ww-679

SITE COORDINATES (ft.):

N

GROUND ELEVATION (ft. MSL):

DRILLING METHOD:

DRILLING CONTR.:

DATE STARTED:

DATE COMPLETED:

FIELD REP.:

LOCATION DESCRIPTION

SITE CONDITION

\begin{tabular}{|c|c|c|}
\hline \multicolumn{3}{|c|}{ GROUNDWATER LEVELS } \\
\hline DATE & TIME & DEPTH (fi.) \\
\hline & & \\
\hline & & \\
\hline & & \\
\hline
\end{tabular}

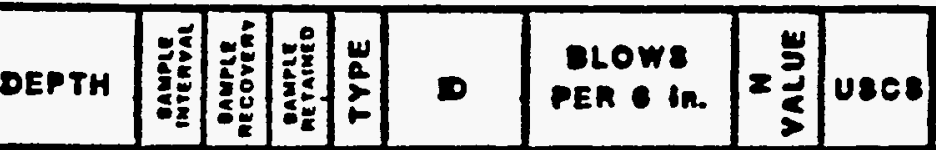

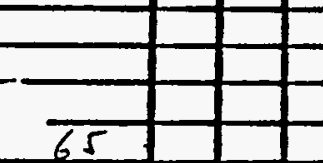

70

75

80

85

SHALE (AMANCOS FM)

(continued) 


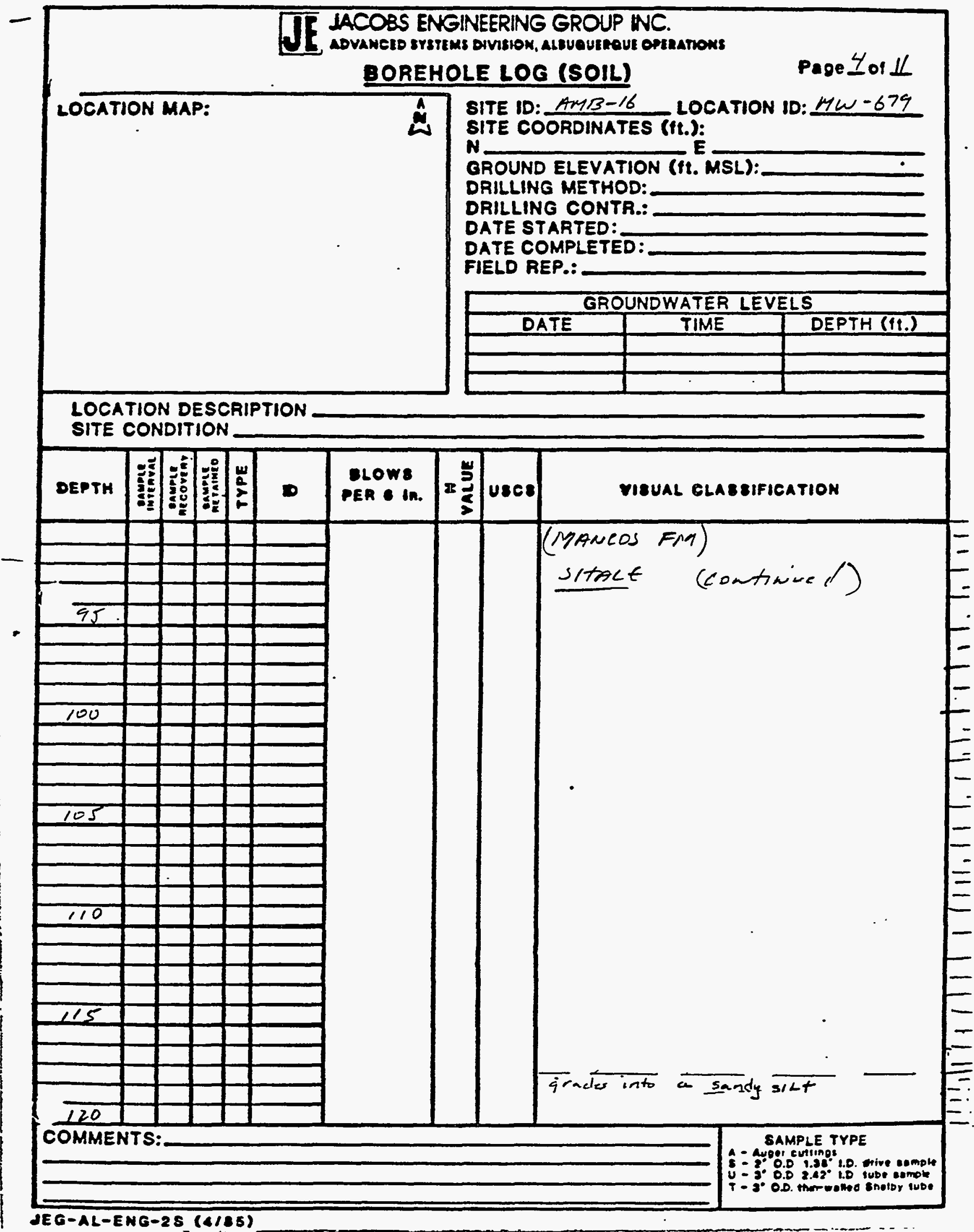


JE JACOBS ENGINEERING GROUP INC.

BOREHOLE LOG (SOIL) Page Sof 11

LOCATION MAP:

A SITE ID: SMB-16 LOCATION ID: Hw - 679

N E

GROUND ELEVATION (ft. MSL):

DRILLING METHOD:

DRILLING CONTR.:

DATE STARTED:

DATE COMPLETED:

FIELD REP.:

\begin{tabular}{|c|c|c|}
\hline \multicolumn{3}{|c|}{ GROUNDWATER LEVELS } \\
\hline DATE & TIME & DEPTH (ft.) \\
\hline & & \\
\hline & & \\
\hline & & \\
\hline
\end{tabular}

LOCATION DESCRIPTION

SITE CONDITION

$\therefore$

\begin{tabular}{|c|c|c|c|c|c|c|c|c|}
\hline DEPTH & 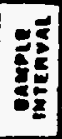 & 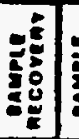 & 势 & 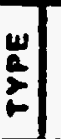 & D & $\begin{array}{l}\text { SLOW8 } \\
\text { PER } 1 \text { in. }\end{array}$ & $=\frac{\omega}{\mathrm{J}}$ & |uses \\
\hline & - & & & & & & & \\
\hline & $\square$ & $E$ & $\rightarrow$ & & & & & \\
\hline & - & ב & - & & & & & \\
\hline 125 & & & & & & & & \\
\hline & & & & & & & & \\
\hline & & - & & & & & & \\
\hline 130 & & & & & & & & \\
\hline & & & & & & & & \\
\hline & & - & - & & & & & \\
\hline & & 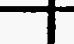 & 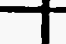 & & & & & \\
\hline 135 & & E & E & & & & & \\
\hline & 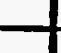 & 4 & - & & & & - & \\
\hline & & $\rightarrow$ & - & & & & & \\
\hline & - & - & & & & & & \\
\hline 140 & & & & & & & & \\
\hline & E & 7 & $\pi$ & & & & & \\
\hline & 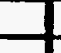 & - & & & & & & \\
\hline 143 & - & - & & & & & & \\
\hline & - & - & - & & & & & \\
\hline & & & 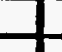 & & & & & \\
\hline & & & 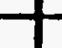 & & & & & \\
\hline 150 & & & & & & & & \\
\hline
\end{tabular}

COMMENTS: 


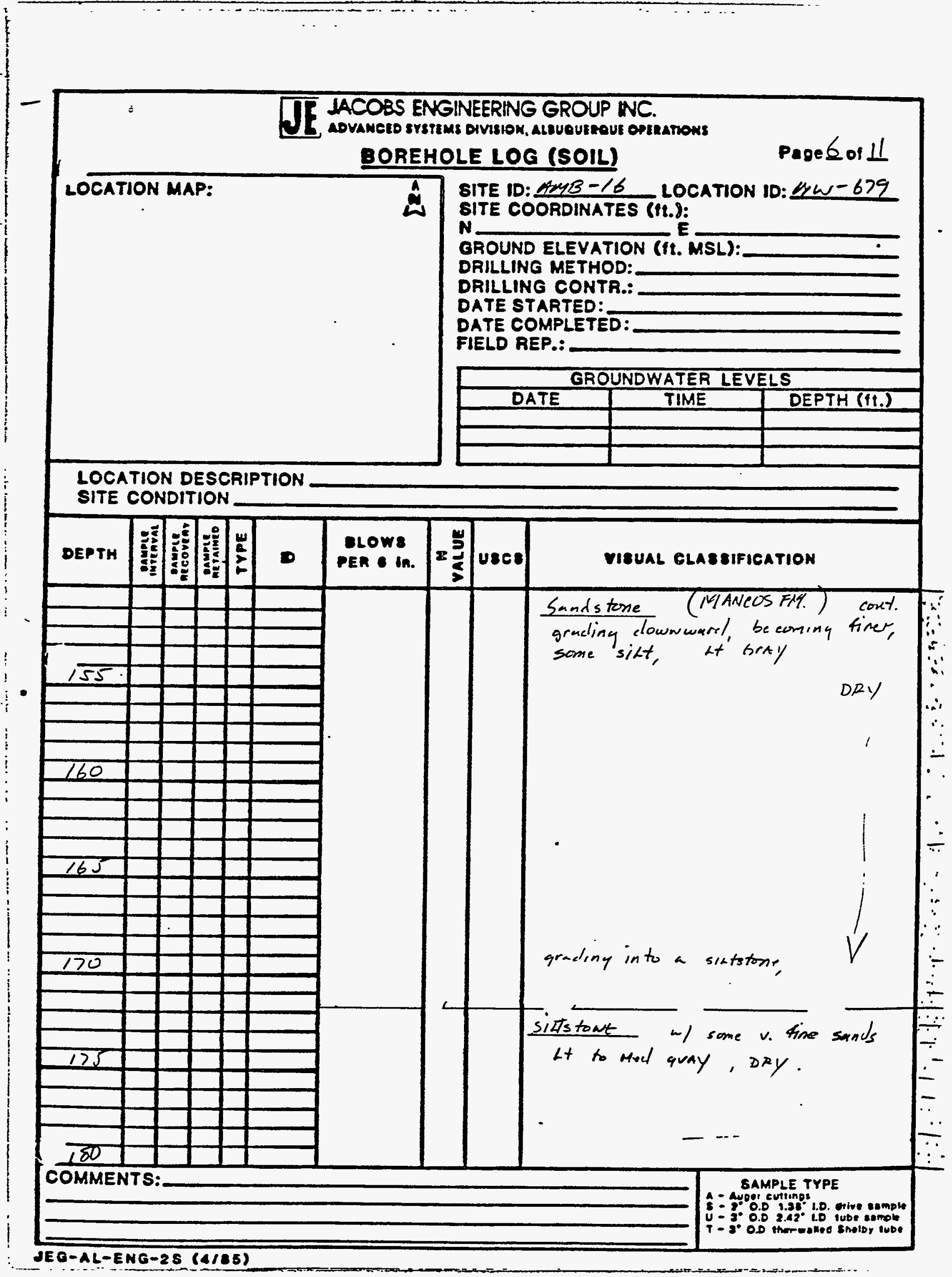




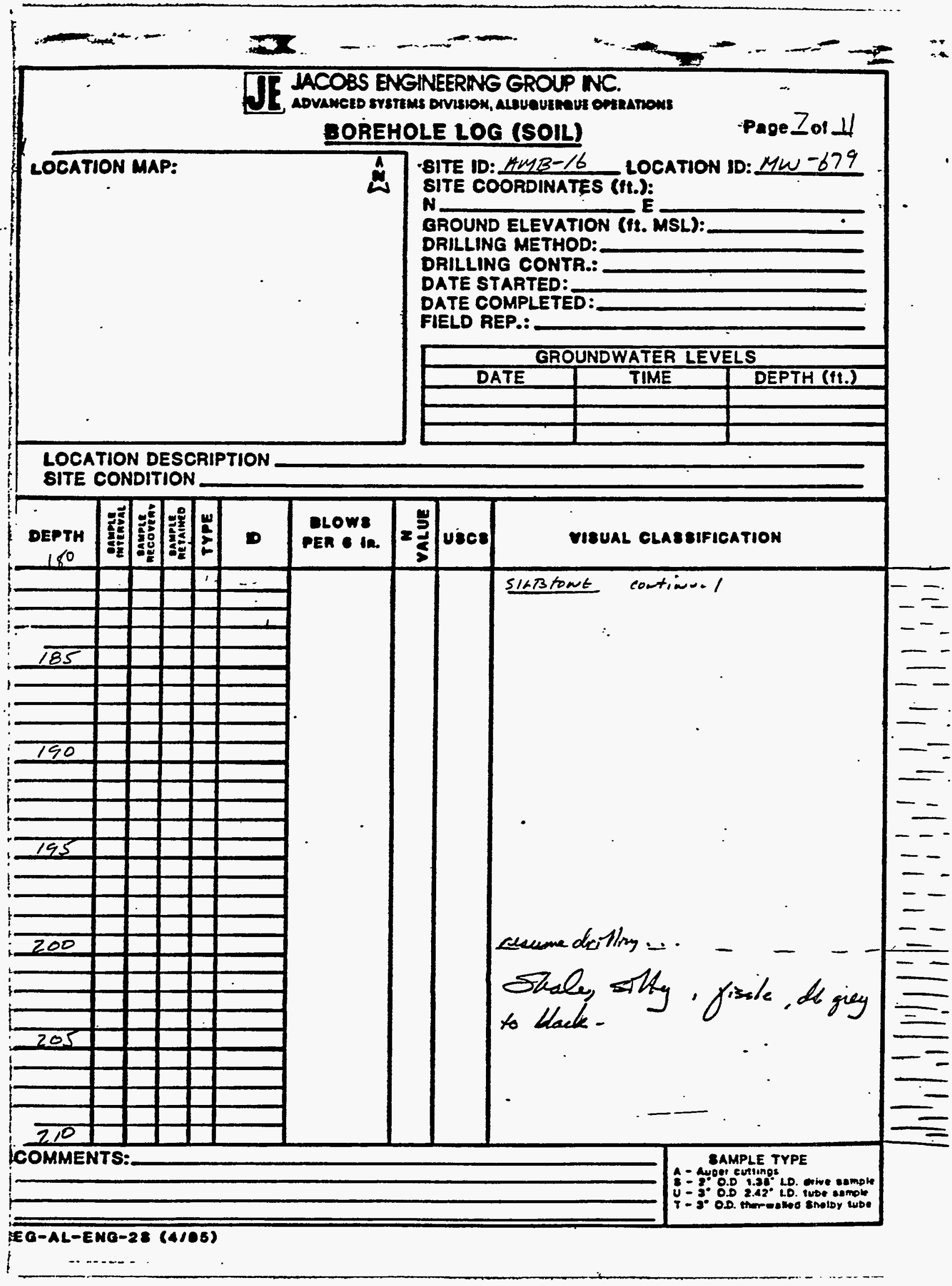




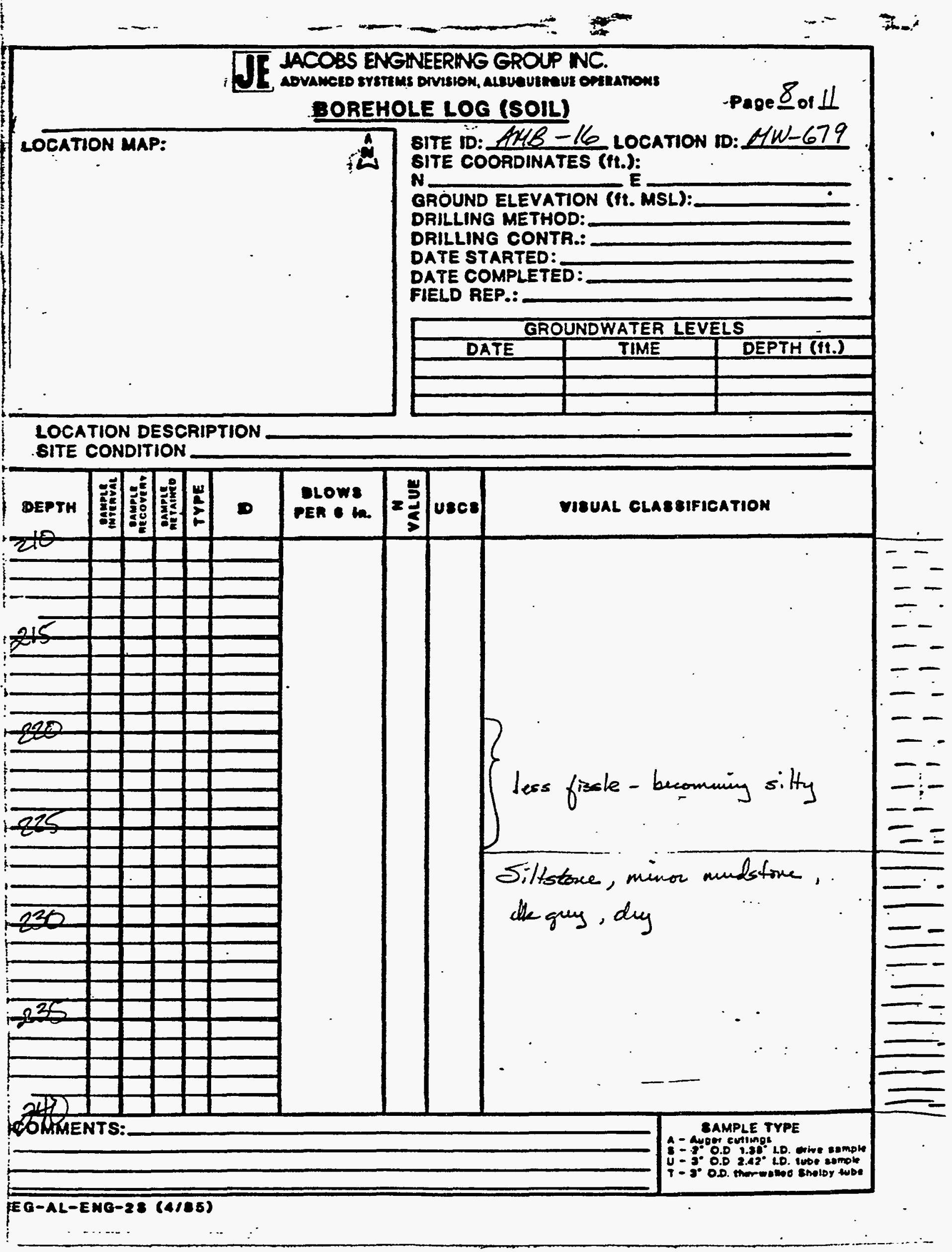




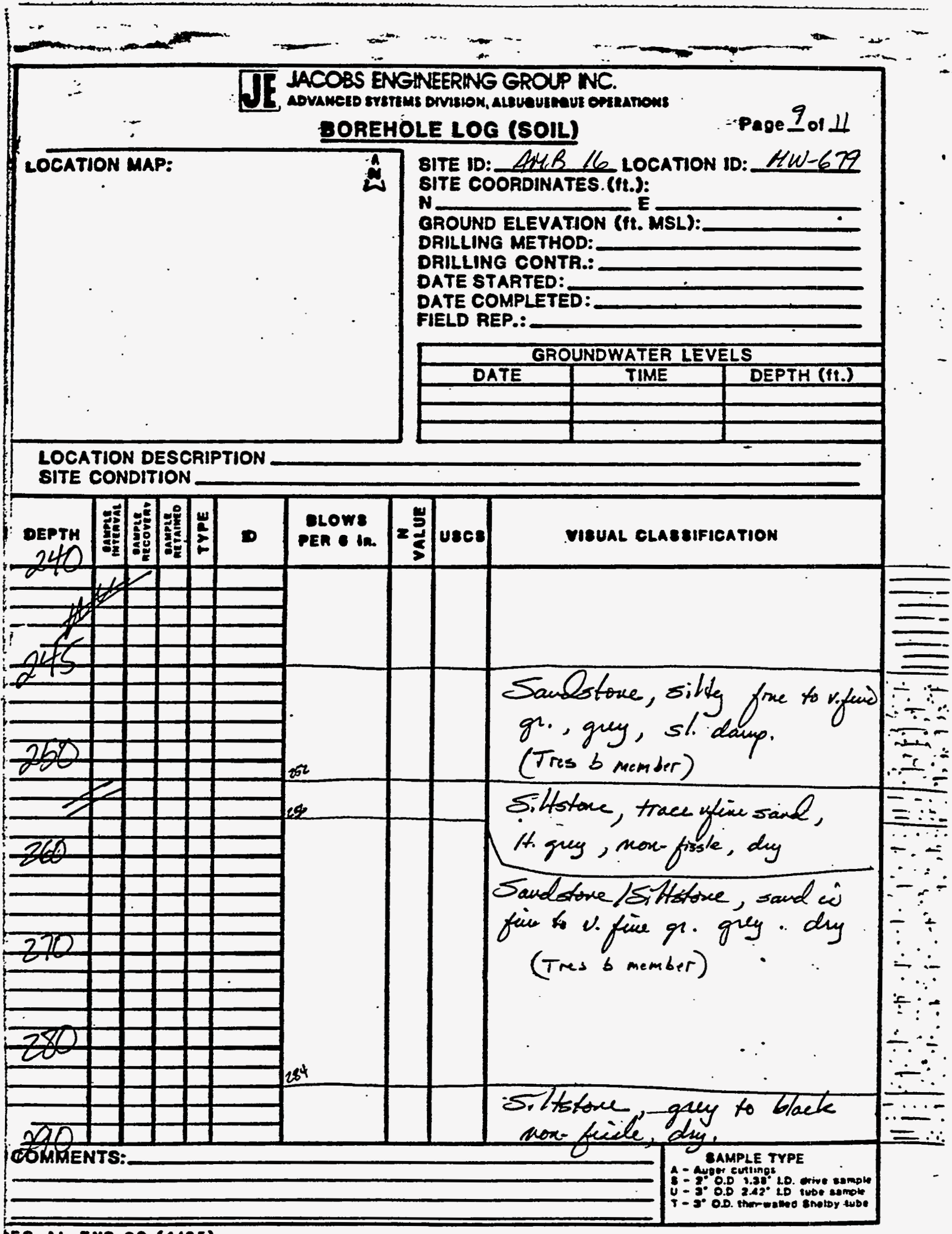

EO-AL-ENO-2) (4/88) 


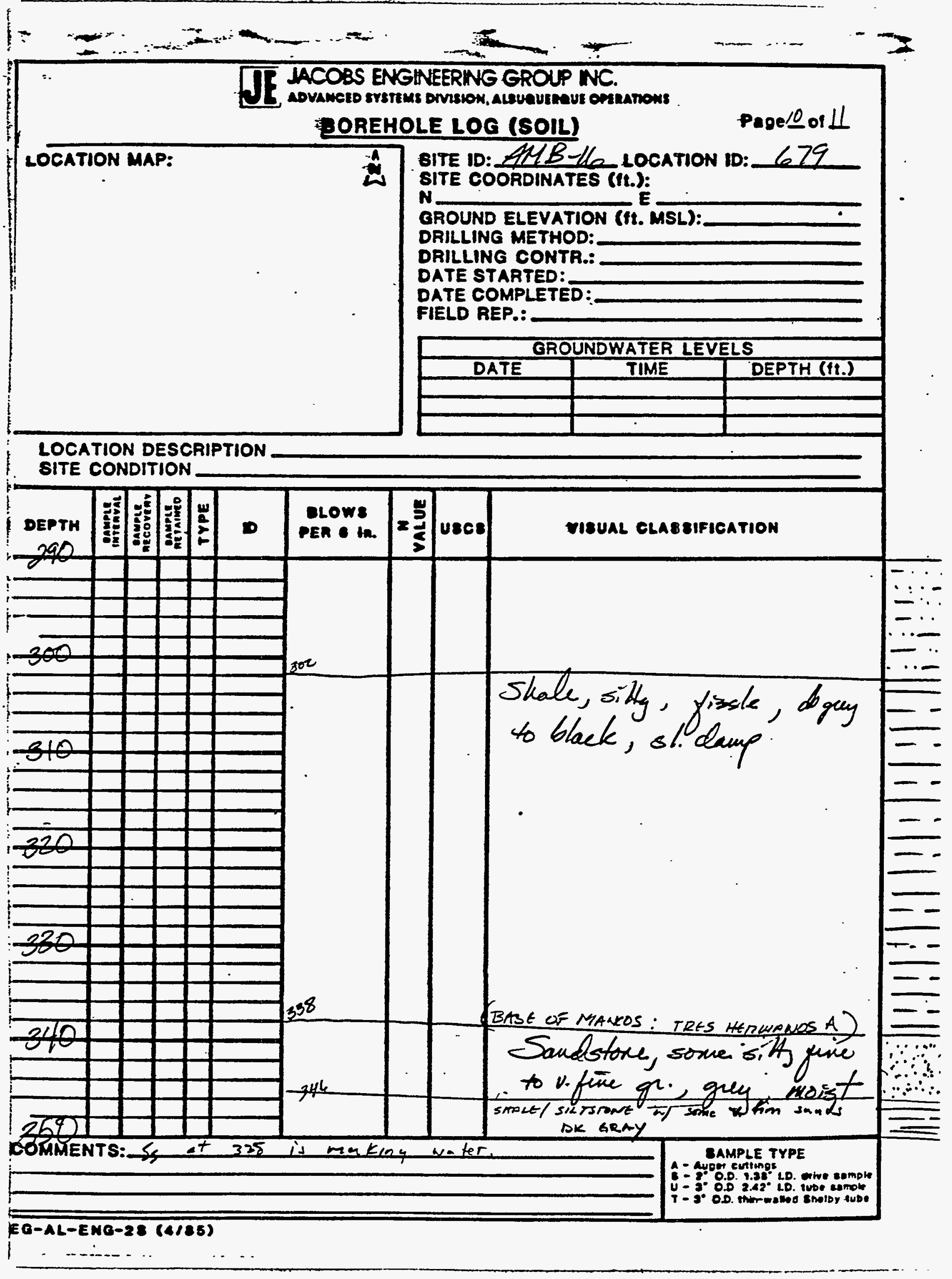




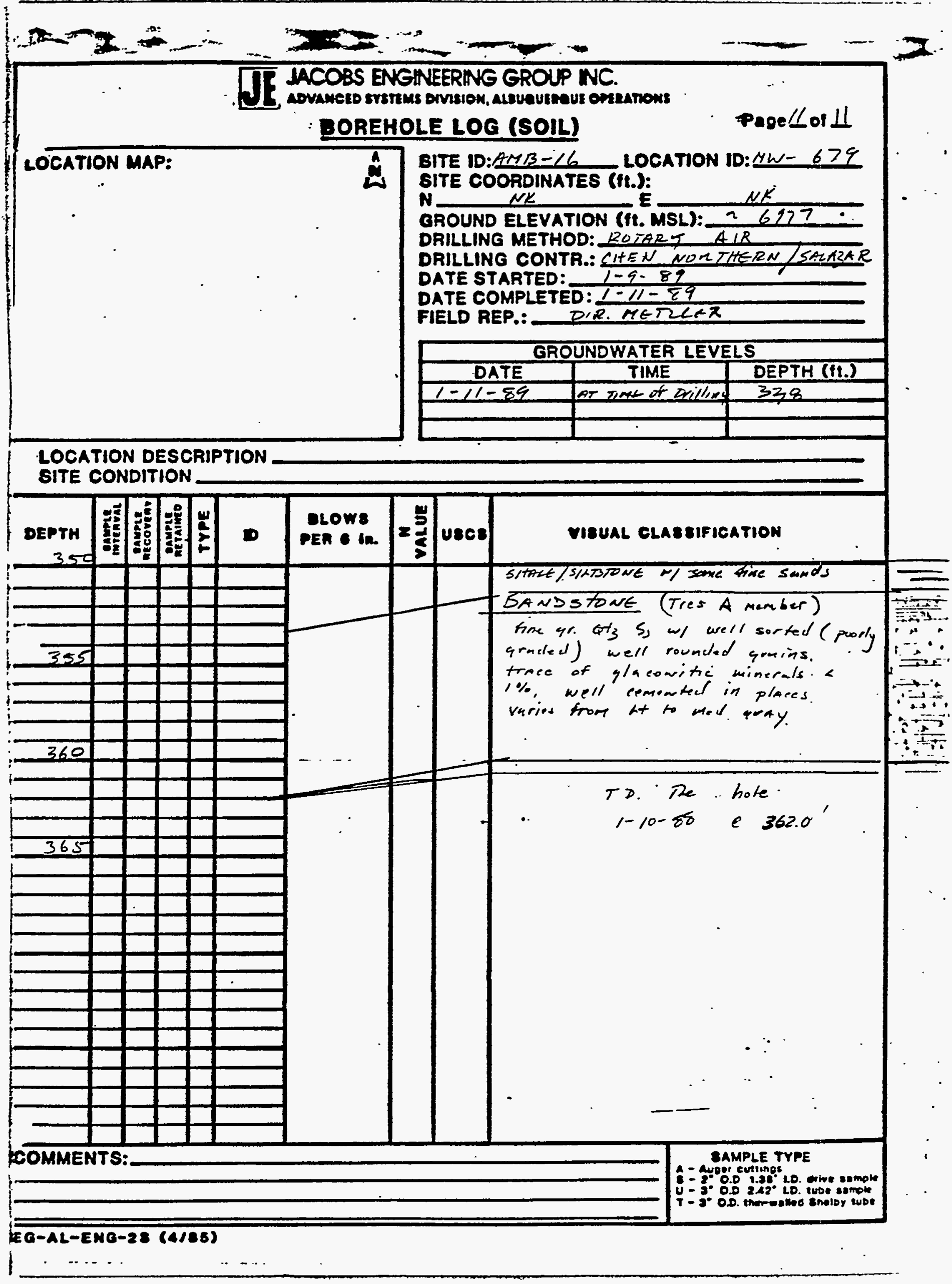




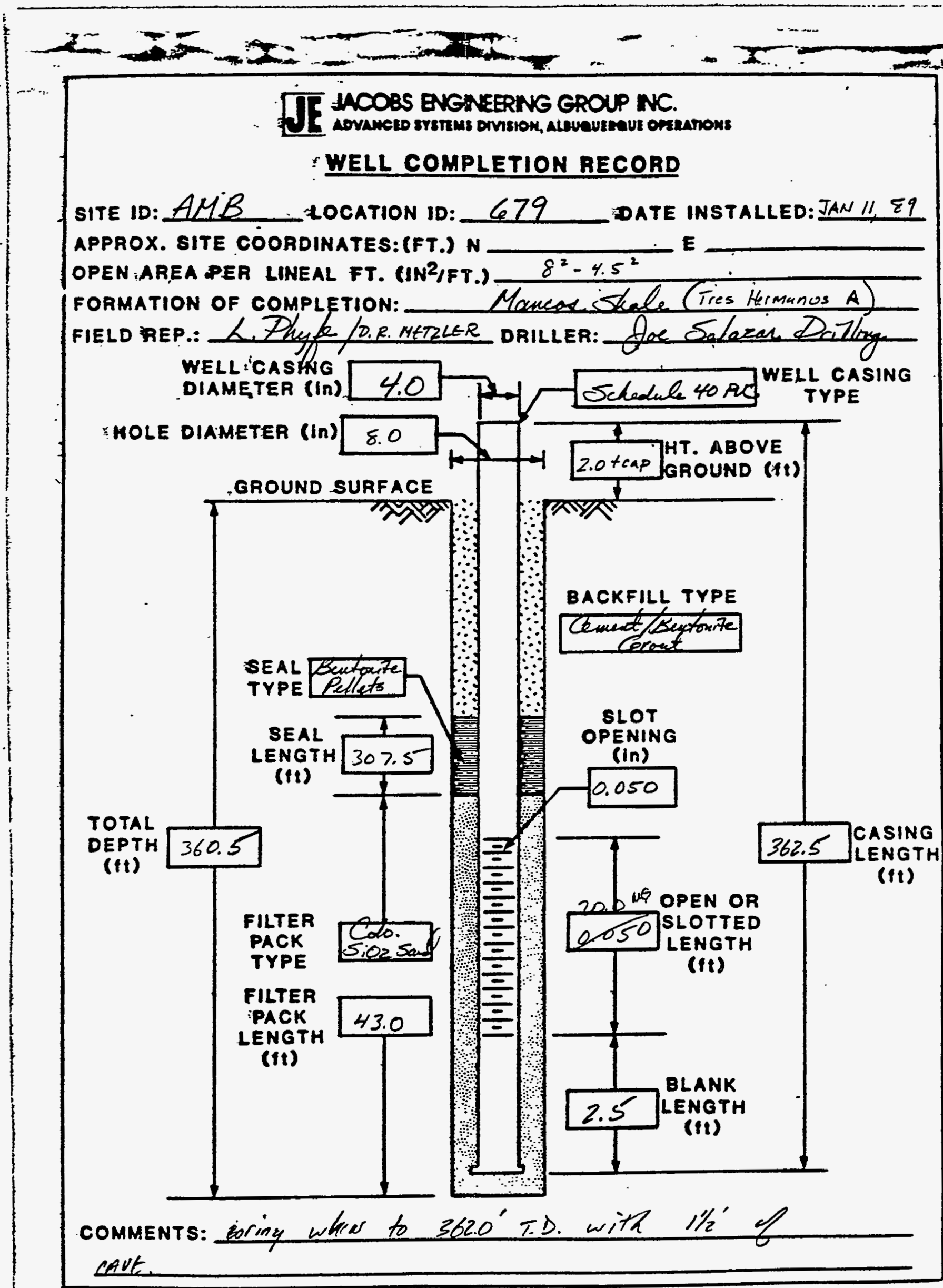

JEG-AL-ENO-3 (3/84) 
(

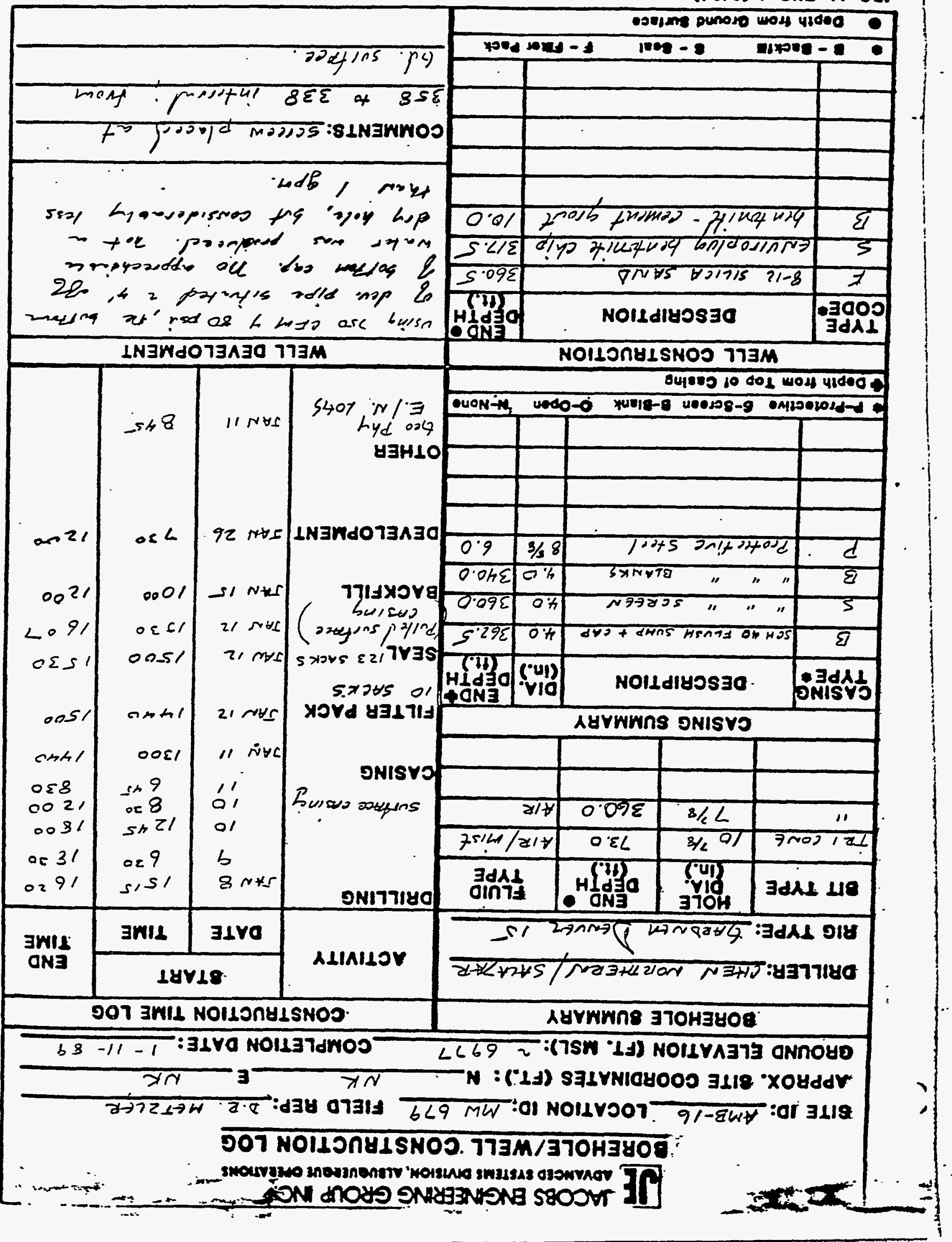


WELL 680 LOGS 


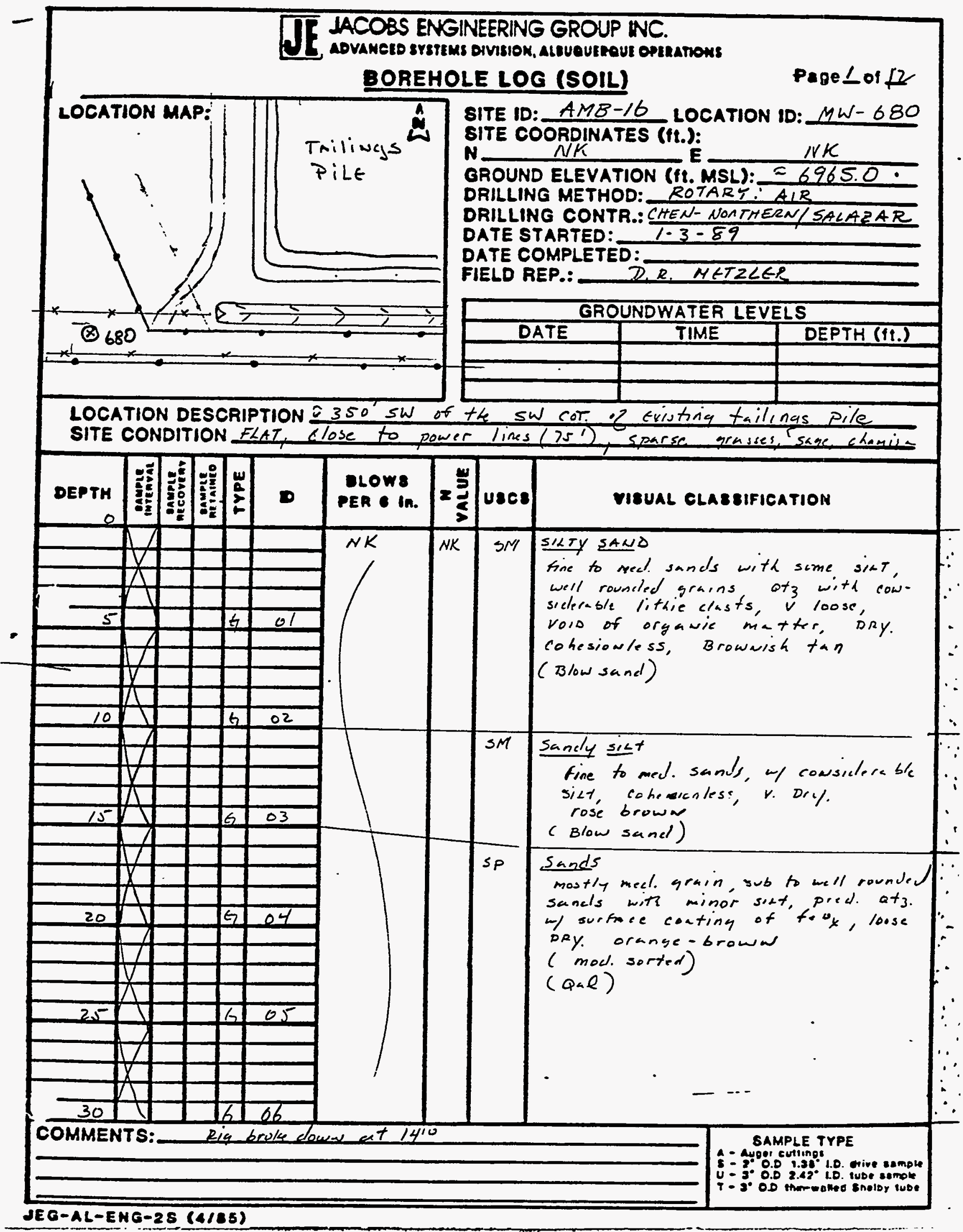




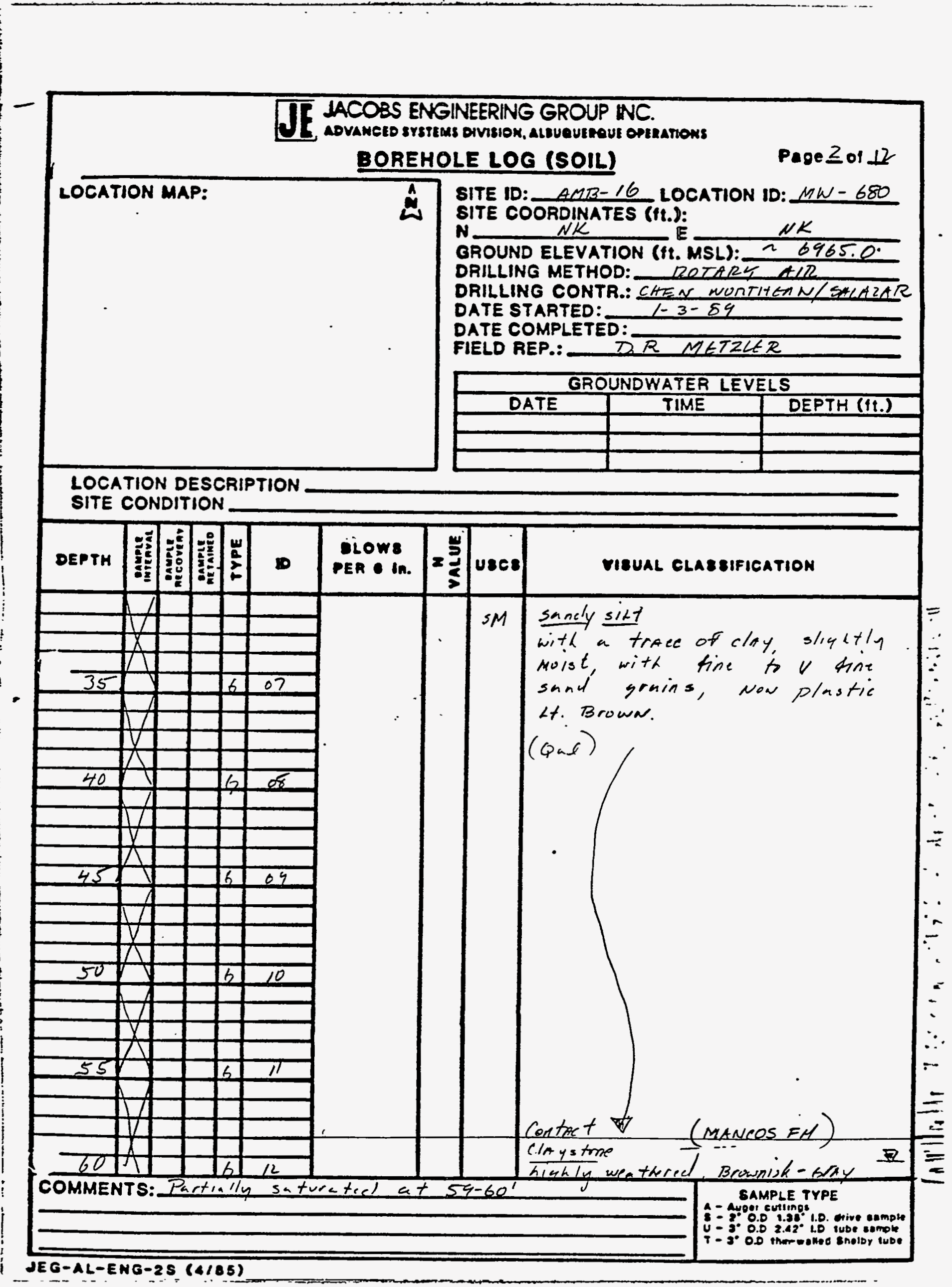


IACOBS ENGINEERING GROUP INC.

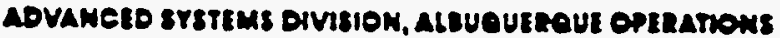

BOREHOLE LOG (SOIL)

Page 3 or 12

LOCATION MAP:

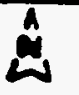

SITE ID: $A M B-16$

LOCATION 1D: 680

SITE COORDINATES (IT.):

N NK

GROUND ELEVATION (ft. MSL): $=6965.0$.

DRILLING METHOD: ROTAR' TIR

DRILLING CONTR.: CHEN-N./SMATar

DATE STARTED:

DATE COMPLETED:

FIELD REP.:

LOCATION DESCRIPTION

SITE CONDITION

\begin{tabular}{|c|c|c|}
\hline \multicolumn{3}{|c|}{ GROUNDWATER LEVELS } \\
\hline DATE & TIME & DEPTH (fi.) \\
\hline & & \\
\hline & & \\
\hline & & \\
\hline
\end{tabular}

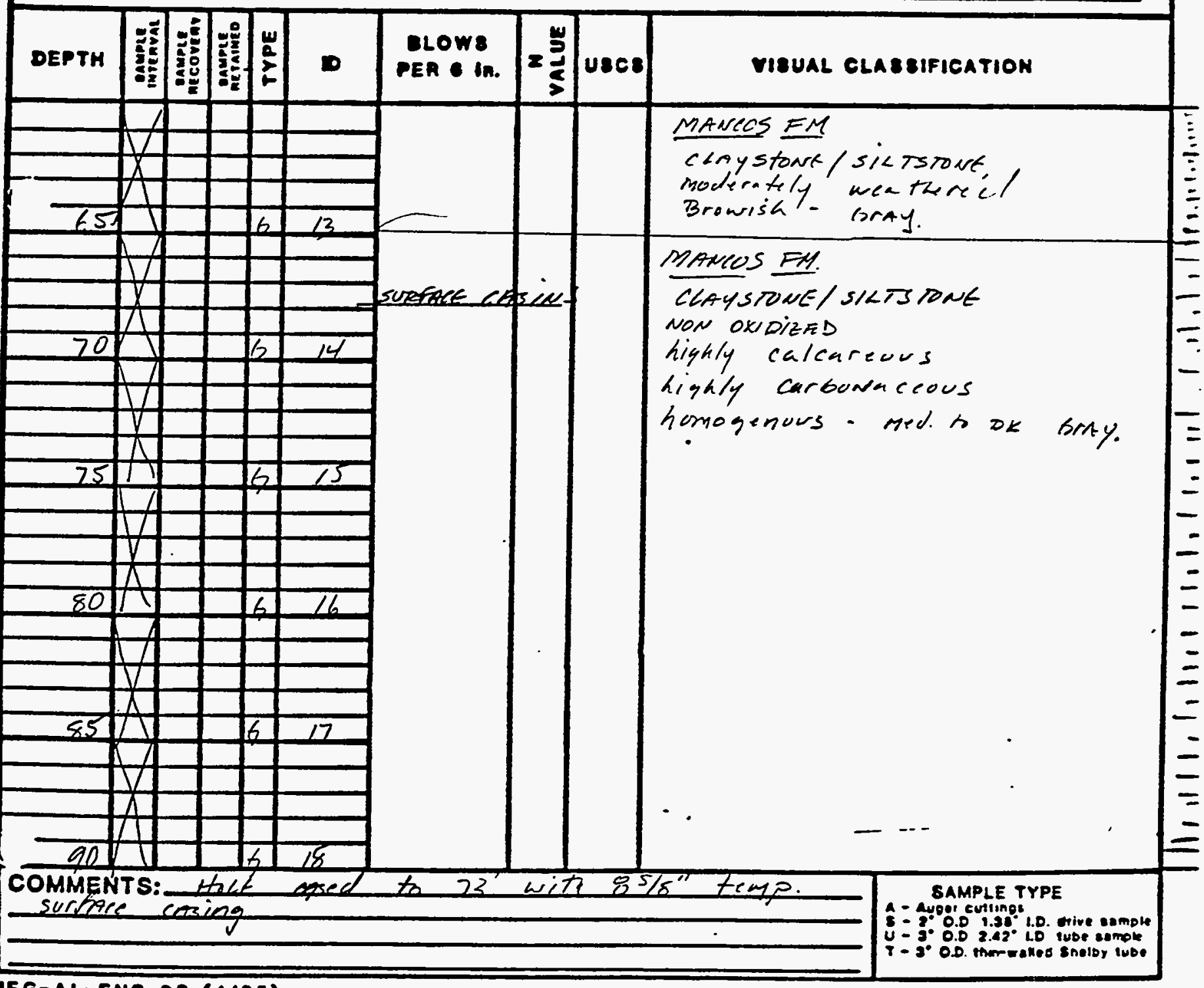




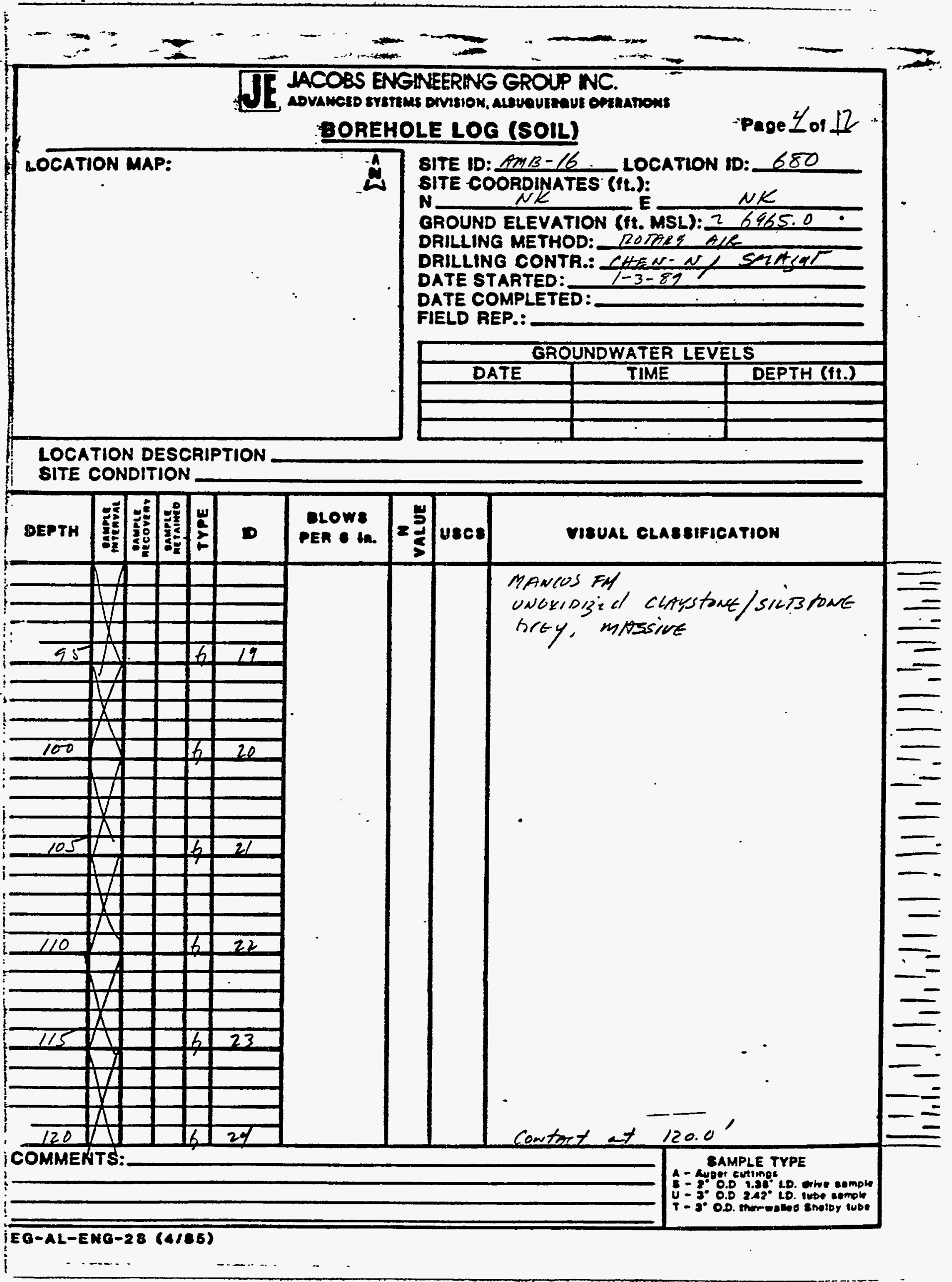


IF JACOBS ENGINEERING GROUP INC.

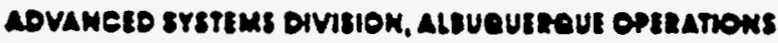

BOREHOLE LOG (SOIL)

Pages of 12

LOCATION MAP:

SITE ID: $A M B-16$

LOCATION ID: 680

SITE COORDINATES (IT.):

N

DRILLING METHOD: RETPRY $A / E$

DRILLING CONTR.: CHEN-N/SALESCR

DATE STARTED:

$1-3-89$

DATE COMPLETED:

FIELD REP.:

\begin{tabular}{|c|c|c|}
\hline \multicolumn{3}{|c|}{ GROUNDWATER LEVELS } \\
\hline DATE & TIME & DEPTH (ft.) \\
\hline & & \\
\hline & & \\
\hline & & \\
\hline
\end{tabular}

LOCATION DESCRIPTION

SITE CONDITION

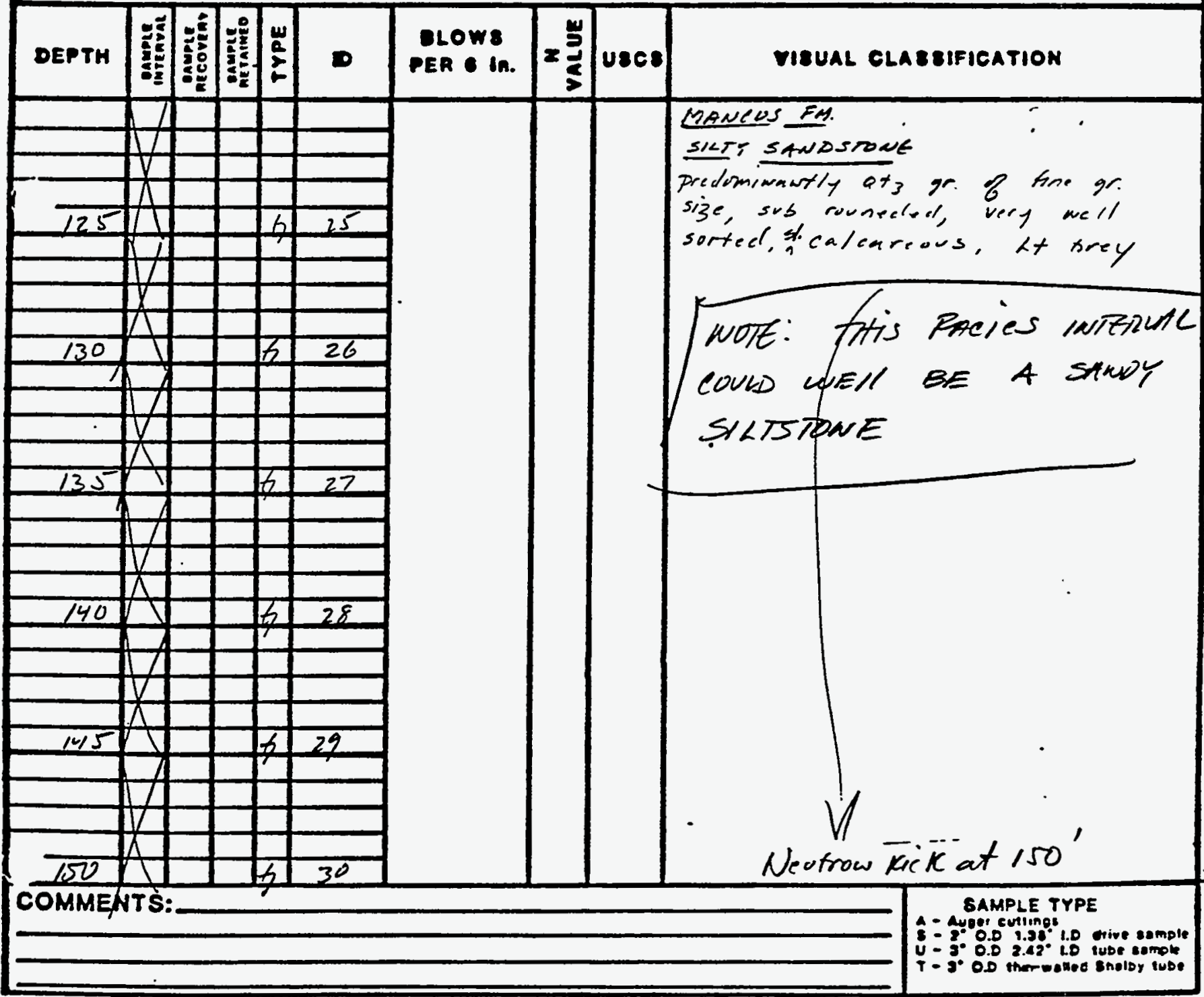




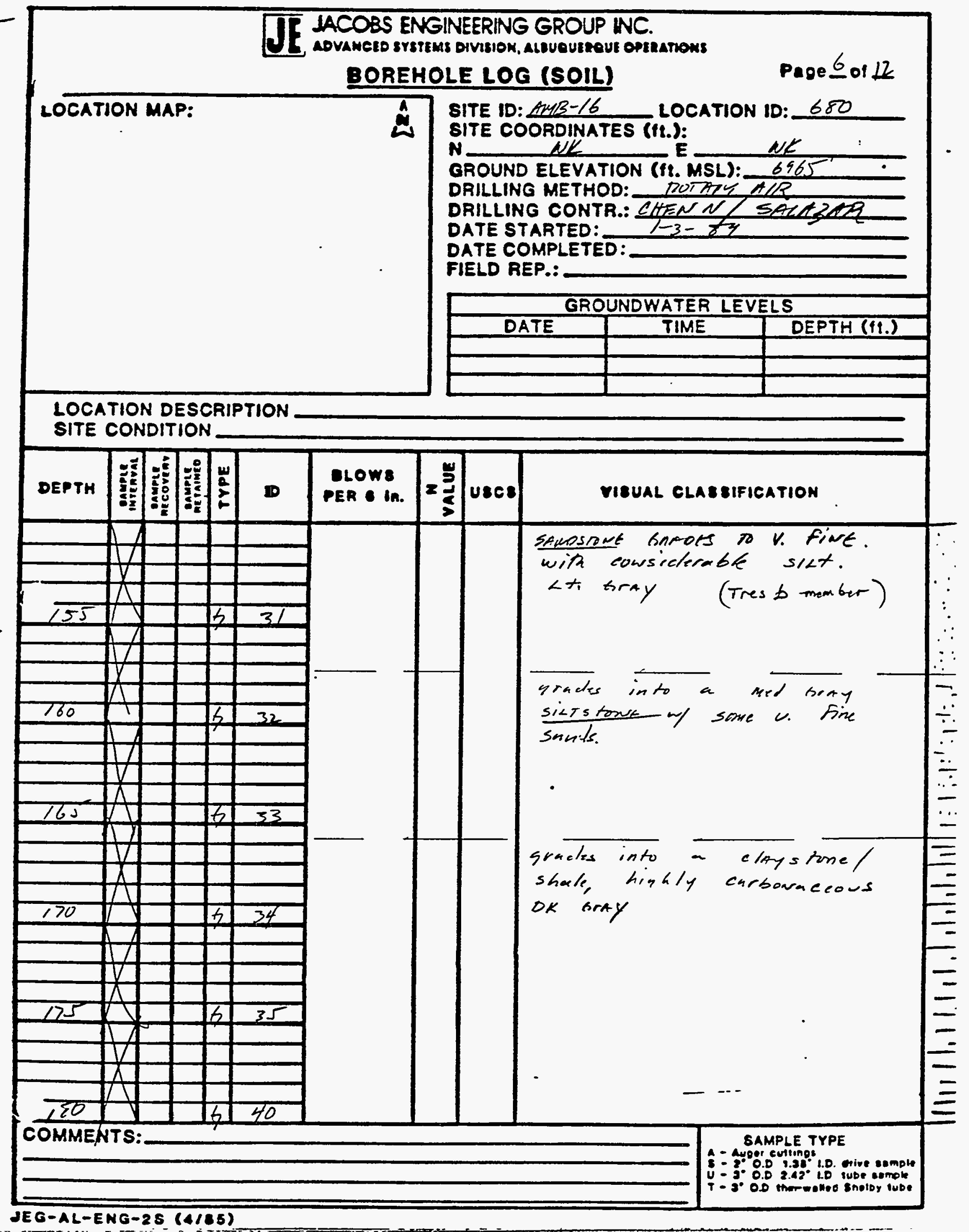




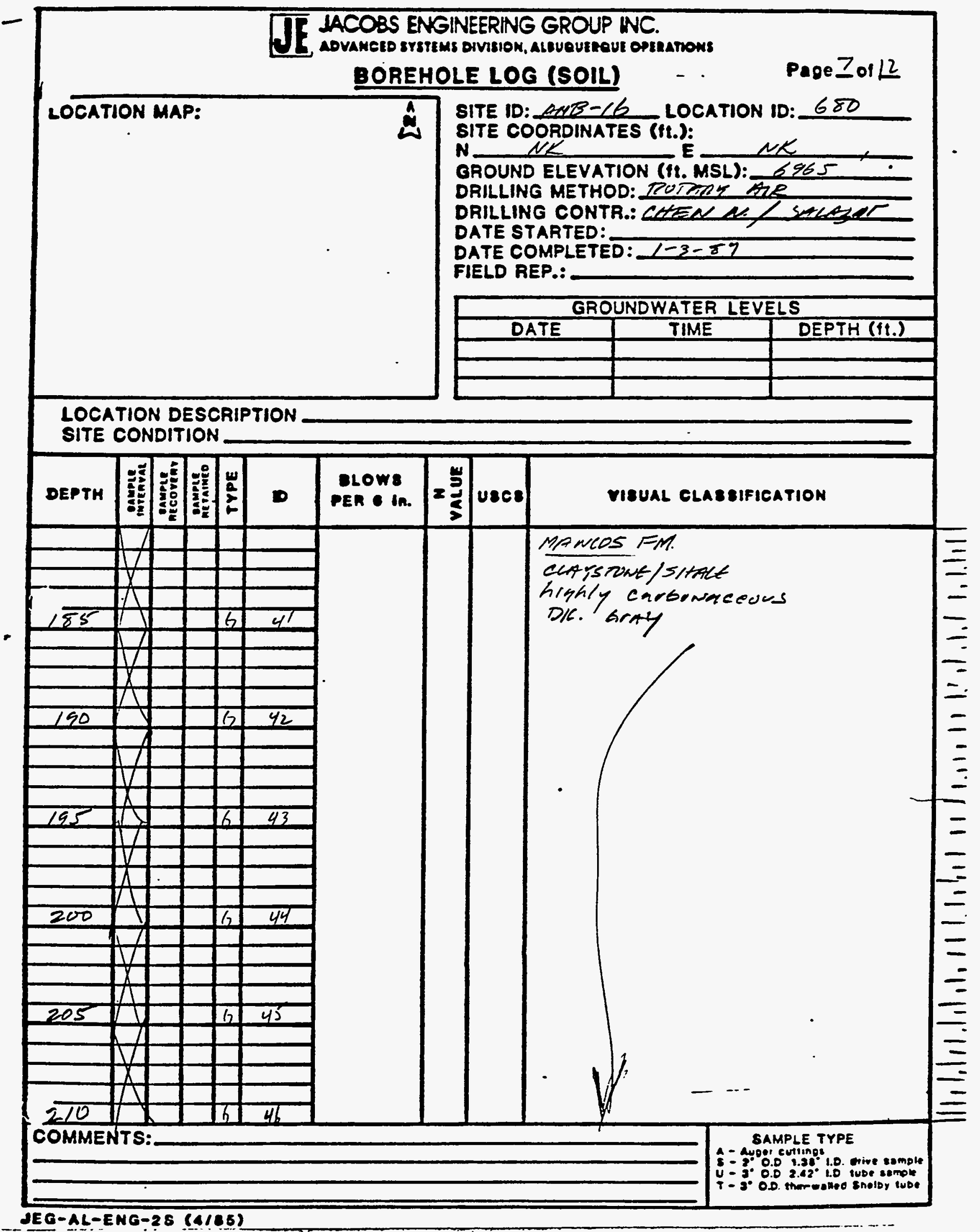




\section{IACOBS ENGINEERING GROUP INC.

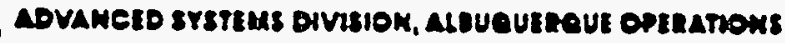

BOREHOLE LOG (SOIL)

Page\& of 12

LOCATION MAP:

ฝे

SITE ID: $A M B-16$

LOCATION ID: 680

SITE COORDINATES (It.):

N NK NATES

GROUND ELEVATION (ft. MSL): 6965

DRILLING METHOD: ROTARY $4 / R$

DRILLING CONTR.: CHEN'N/ SALA2AR

DATE STARTED:

DATE COMPLETED: $1-3-57$

FIELD REP.:

\begin{tabular}{|c|c|c|}
\hline \multicolumn{3}{|c|}{ GROUNDWATER LEVELS } \\
\hline DATE & TIME & DEPTH (ft.) \\
\hline & & \\
\hline & & \\
\hline & & \\
\hline
\end{tabular}

LOCATION DESCRIPTION

SITE CONDITION

\begin{tabular}{|c|c|c|c|c|c|c|c|}
\hline DEPTH & 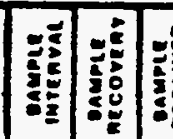 & 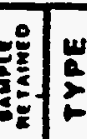 & & $\mathbb{1 0}$ & $\begin{array}{l}\text { BLOWS } \\
\text { PER \& In. }\end{array}$ & $=\frac{5}{5}$ & uses \\
\hline
\end{tabular}

215 7

\begin{tabular}{l|l|l|l|l|l}
\hline & & & & & \\
\hline 25 & & & & & \\
\hline
\end{tabular}

$+1$

H

220

At

(1)

A

225

17

$+$

47

$-2$

Geadeteonel

(MANCOS FM.)

S/LTSTONE: $w /$ some $V$. THE, ine 1 sorted wts. Grains.

DK. GRAY to med. GIAY

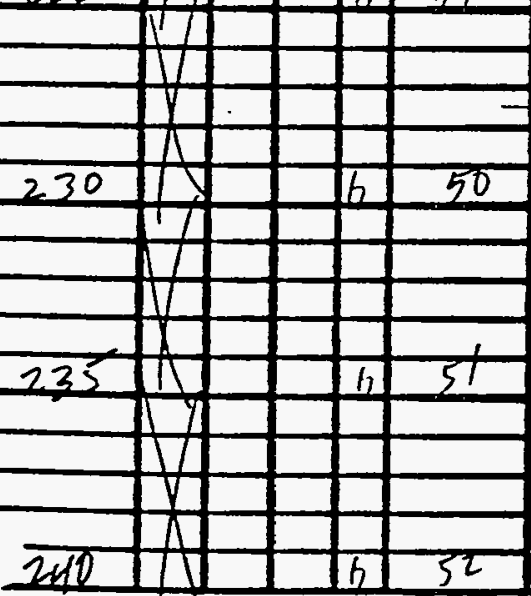

COMMENTS:

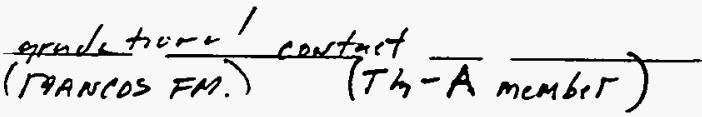

SANbsrowe: $v$. fine srain, well

rounded, well sorted glacowibi at3. sand. V. Lt. GRAY

NoT: Mod: calleariess cemewation.

grades into

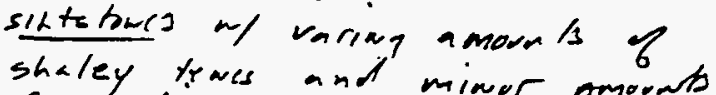

shaley tiowes and
of $r$ tim $\mathrm{sm} n d \mathrm{~s}$. LA toDK DAY
SAMPLE TYPE

A - Aypor cutiong

A =

$u=3^{\circ} 0.0 \quad 2.42^{\circ}$ L.O. Lube semple

$T=3^{\circ} 0.0$ inm-elliod snoloy tube 


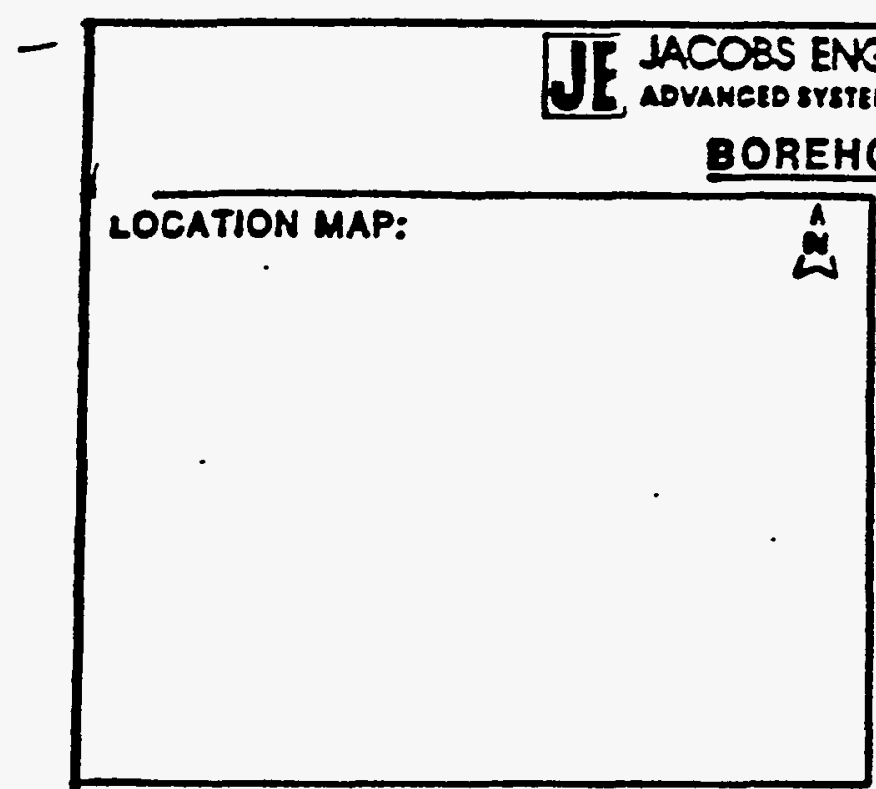

GINEERING GROUP INC.

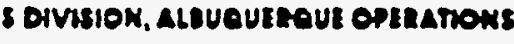

DELE LOG (SOIL)

Page 2 of 12

LOCATION DESCRIPTION

SITE CONDITION

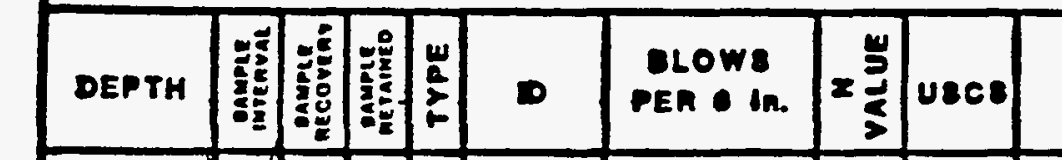

SITE COORDINATES (tt.):

N.

GROUND ELEVATION (ft. MSL):

DRILLING METHOD:

DRILLING CONTR.:

DATE STARTED:

DATE COMPLETED:

FIELD REP.: $R R: M E T Z L R$

\begin{tabular}{|c|c|c|}
\hline \multicolumn{3}{|c|}{ GROUNDWATER LEVELS } \\
\hline DATE & TIME & DEPTH (11.) \\
\hline & & \\
\hline & & \\
\hline & & \\
\hline
\end{tabular}

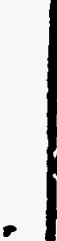

\begin{tabular}{l|l|l|l}
\hline 245 & & 6 & 53
\end{tabular}

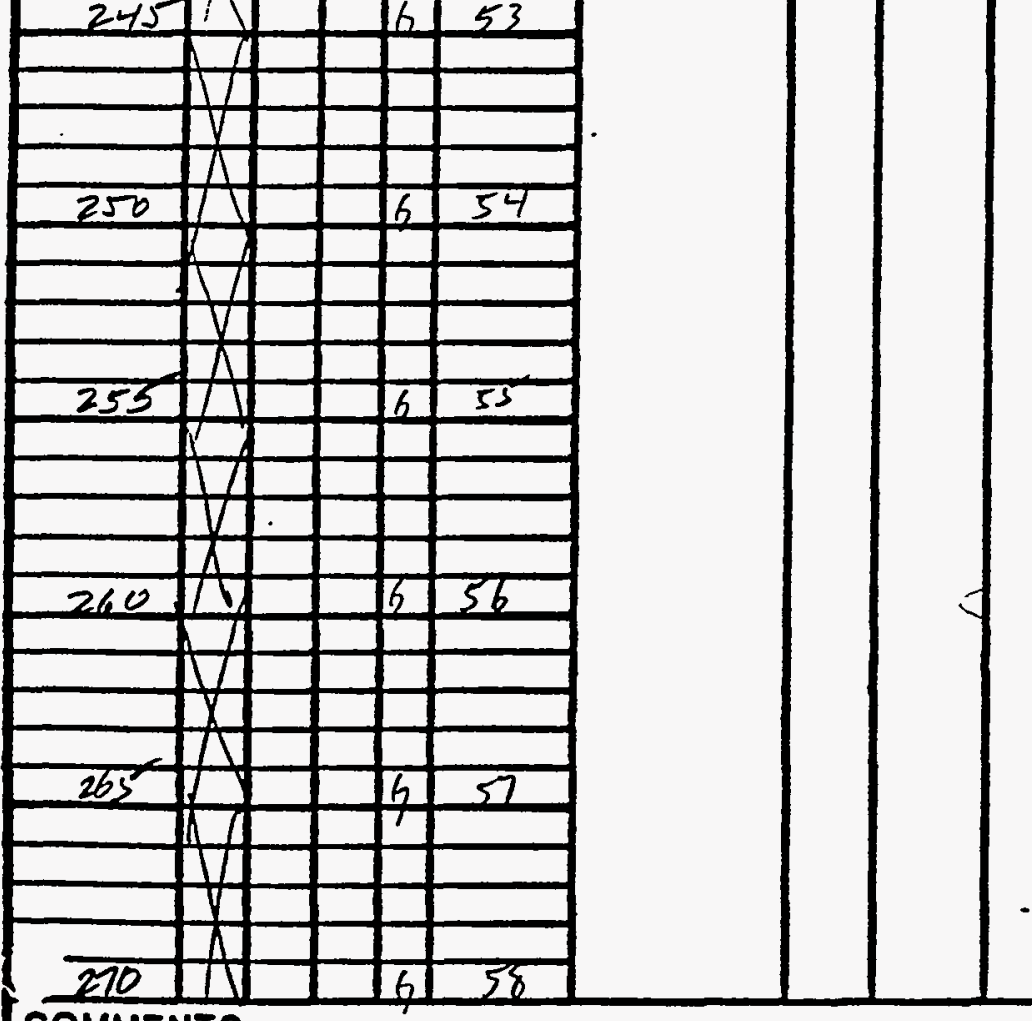
COMMENTS:

SAMPLE TYPE

A - Ayger cutsings U - 3. 0.0 2.42 t.0 tube samole $7-3 \circ$ ob inm warked snotor ind 


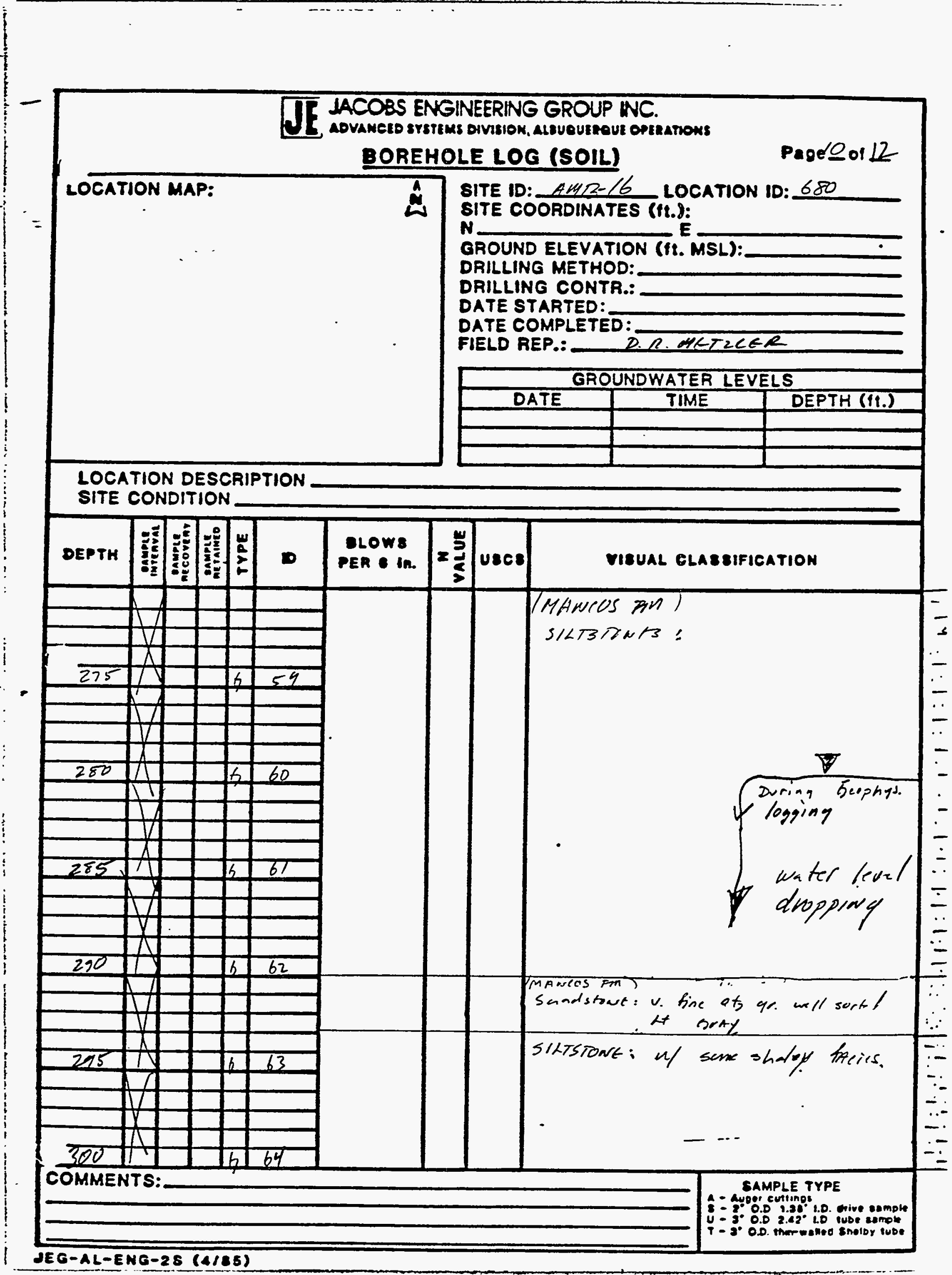




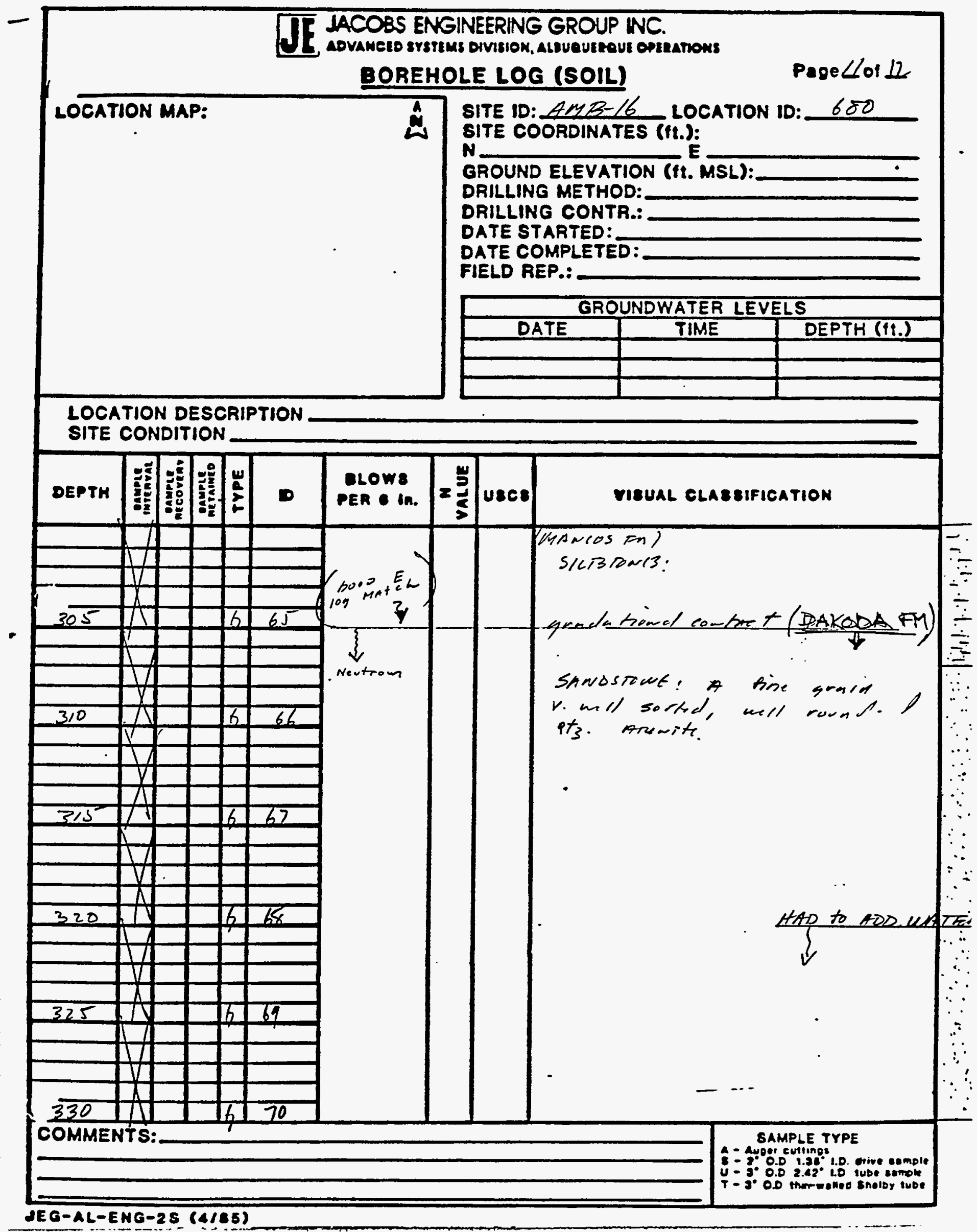




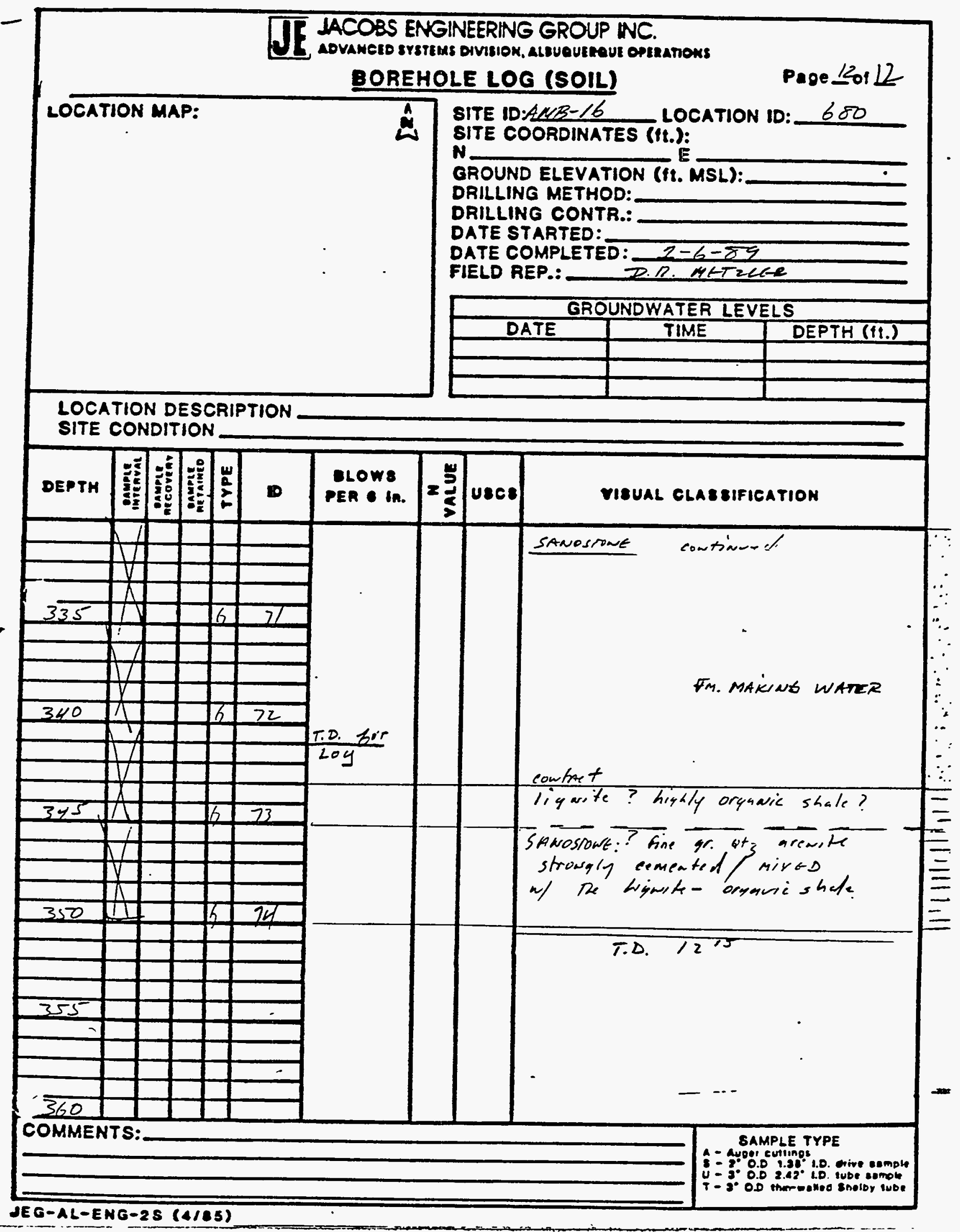




\section{TT IACOBS ENGNEERING GROUP NC. AOVAMEED SYSTIMS ONVISHON, ALSUEUERUE OPERANONB}

\section{WELL COMPLETION RECORD}

SITE ID: AMB- 16 LOCATION ID:

DATE INSTALLED: $1 / 7 / 89$

APPROX. SITE COORDINATES:(FT.) N

OPEN AREA PER LINEAL FT. (IN2/FT.) E NK

|FORMATION OF COMPLETION: Kd DAKODA SS

FIELD REP.: I mackluzie DRILLER: Chex Notherx Selazas

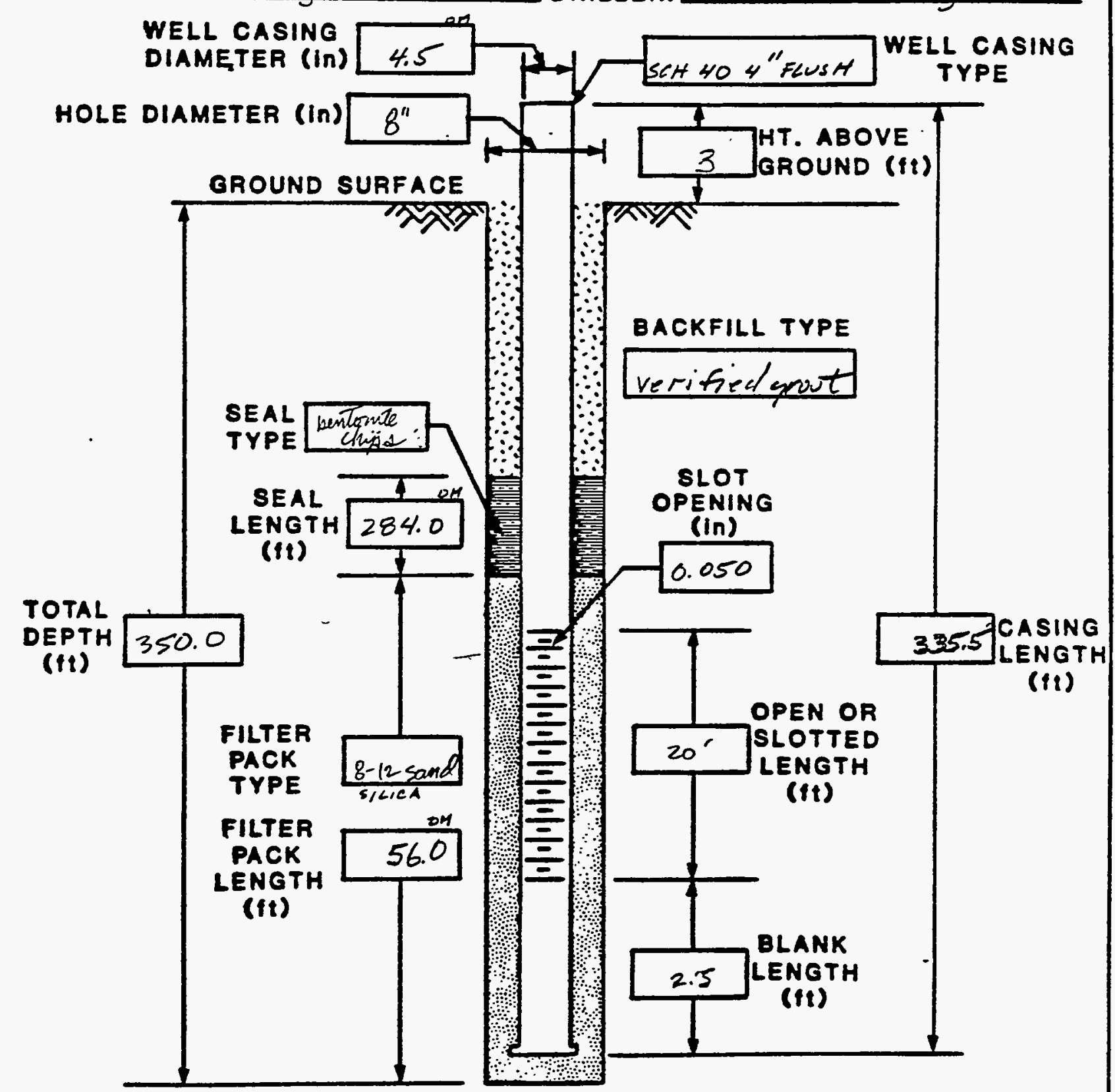

COMMENTS: wed awulas filled w/ siliex from $333.5^{\prime}$ to $350.0^{\prime}$ 


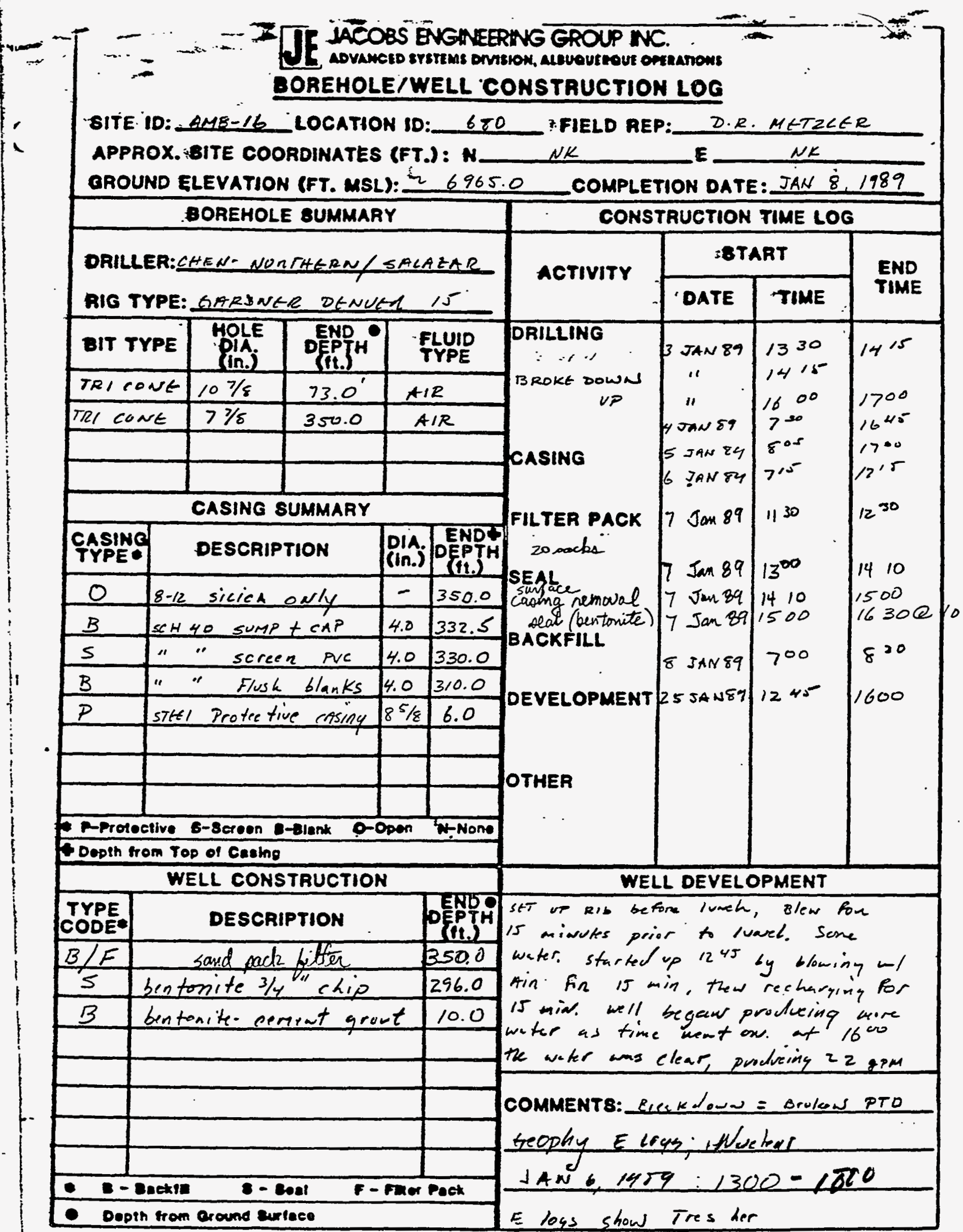

JEO-AL-ENO-S (e/C4) 
WELL 681 LOGS 


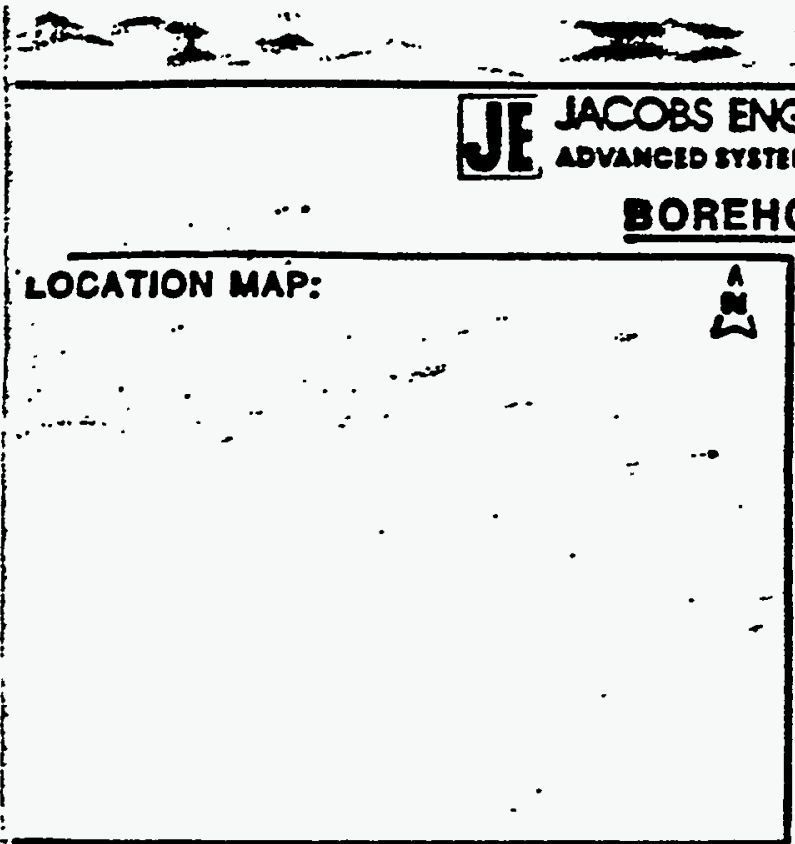

LOCATION DESCRIPTION $N 45^{\circ} \mathrm{W}$ SPARSTY UEGETATRS $\mathrm{N}$ BuneA gras 4 creoscR SITE CONDITION FLAT:

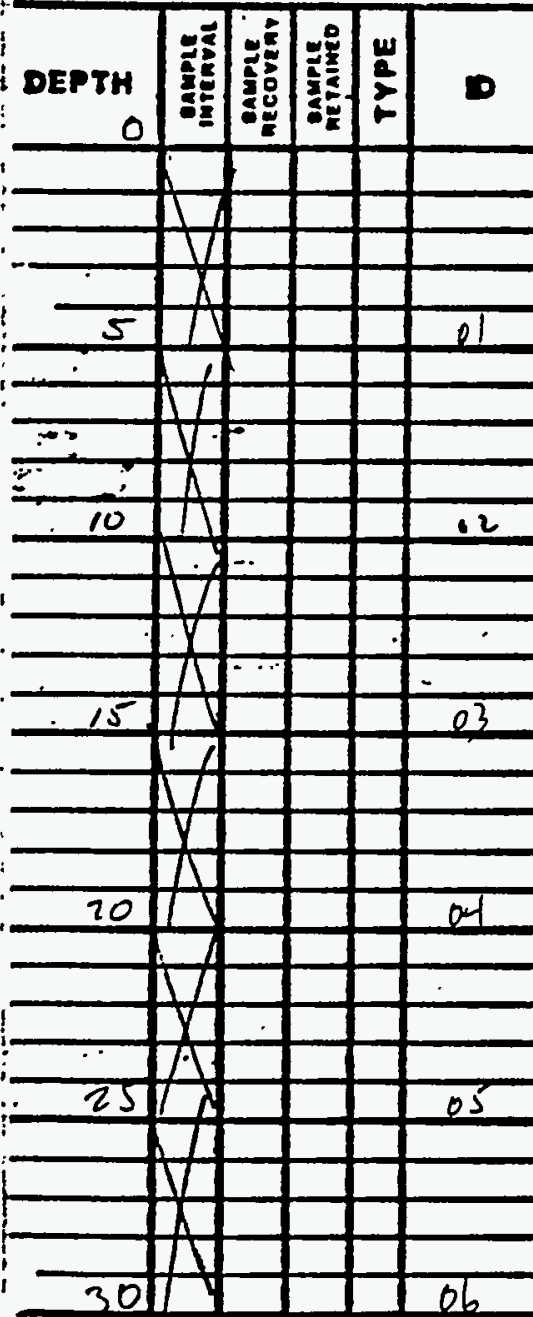
SOMMENTS:

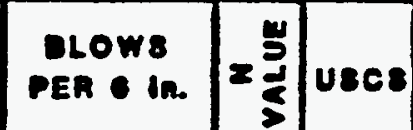

$S M$

SILTY SANDS $(\varphi a /)$

Eolian deriued tine sunds,

DRY, Mod! fracled. angular grains

tan

$-$

Becomís" DARKER

$-$

GROUND ELEVATION (It. MSL):

DRILLING CONTR.: CHEN WUATITIN/SALA2 AR

DATE COMPLETED:

FIELO REP.: DON METZLKR

\begin{tabular}{|c|c|c|}
\hline \multicolumn{3}{|c|}{ GROUNDWATER LEVELS } \\
\hline DATE & TIME & DEPTH (ft.) \\
\hline $1-23-85$ & 1530 & 205 \\
\hline & & \\
\hline & & \\
\hline
\end{tabular}

$200^{\prime}$ trom 677 


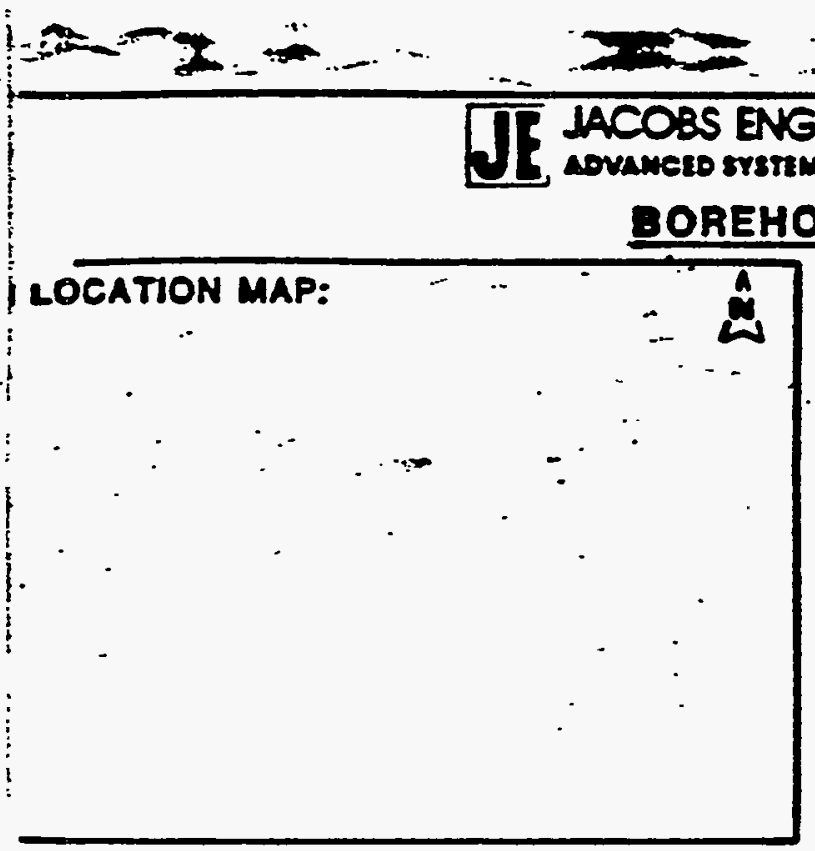

SNEERNG GROUP NC.

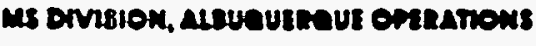

LOG (SOIL)

Page zor 8

BITE ID: OMBB-16 LOCATIOON ID: 681 ORI

SITE COORDINATES (ft.)

GROUND ELEVATION (ft. MSL):

DRILLING METHOD:

DRILLING CONTR.:

DATE STARTED:

DATE COMPLETED:

FIELD REP.:

\begin{tabular}{|c|c|c|}
\hline \multicolumn{3}{|c|}{ GROUNDWATER LEVEL } \\
\hline DATE & TIME & DEPTH (11.) \\
\hline & & \\
\hline & $\ddots$ & \\
\hline & & \\
\hline
\end{tabular}

LOCATION DESCRIPTION

SITE CONDITION

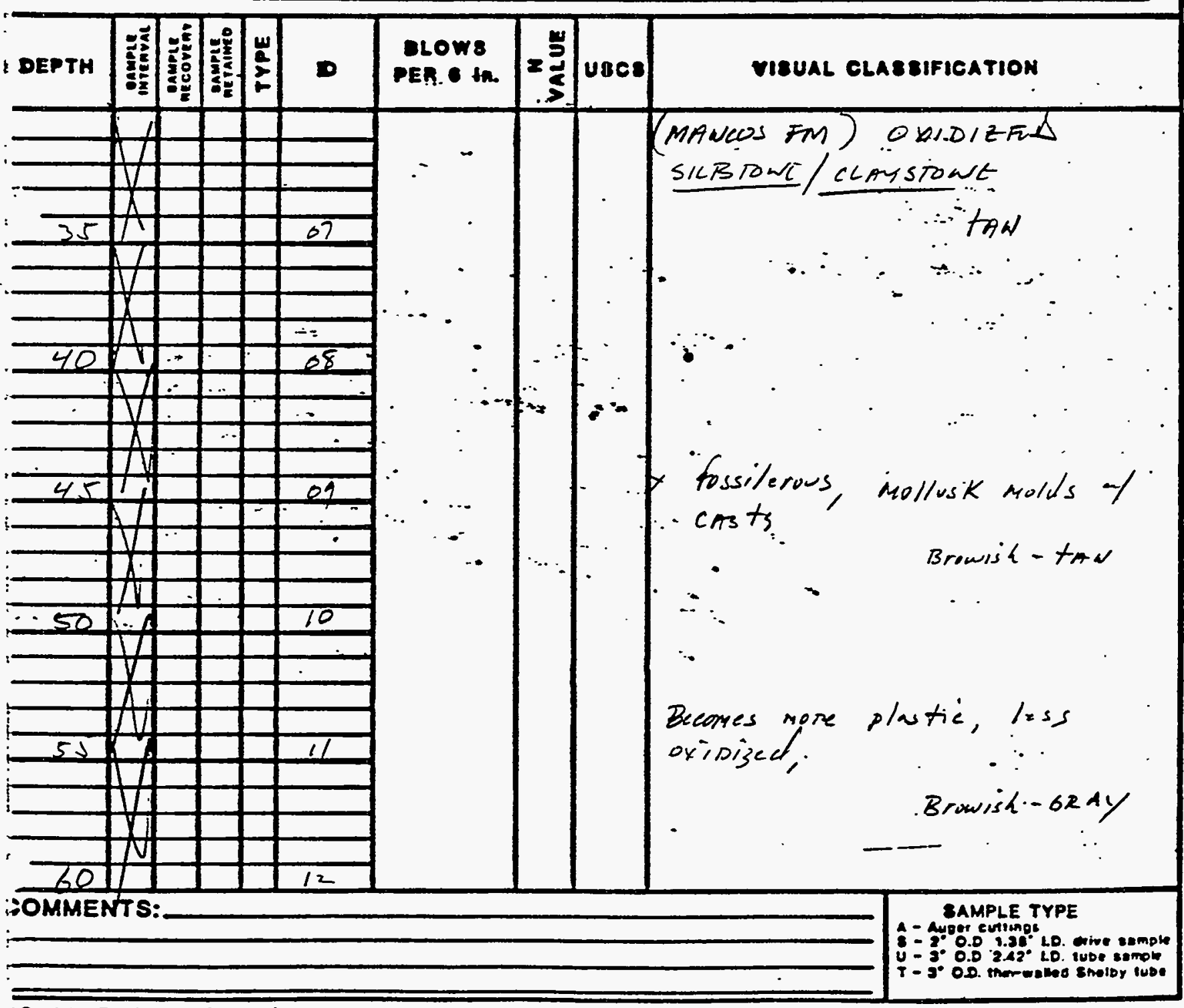




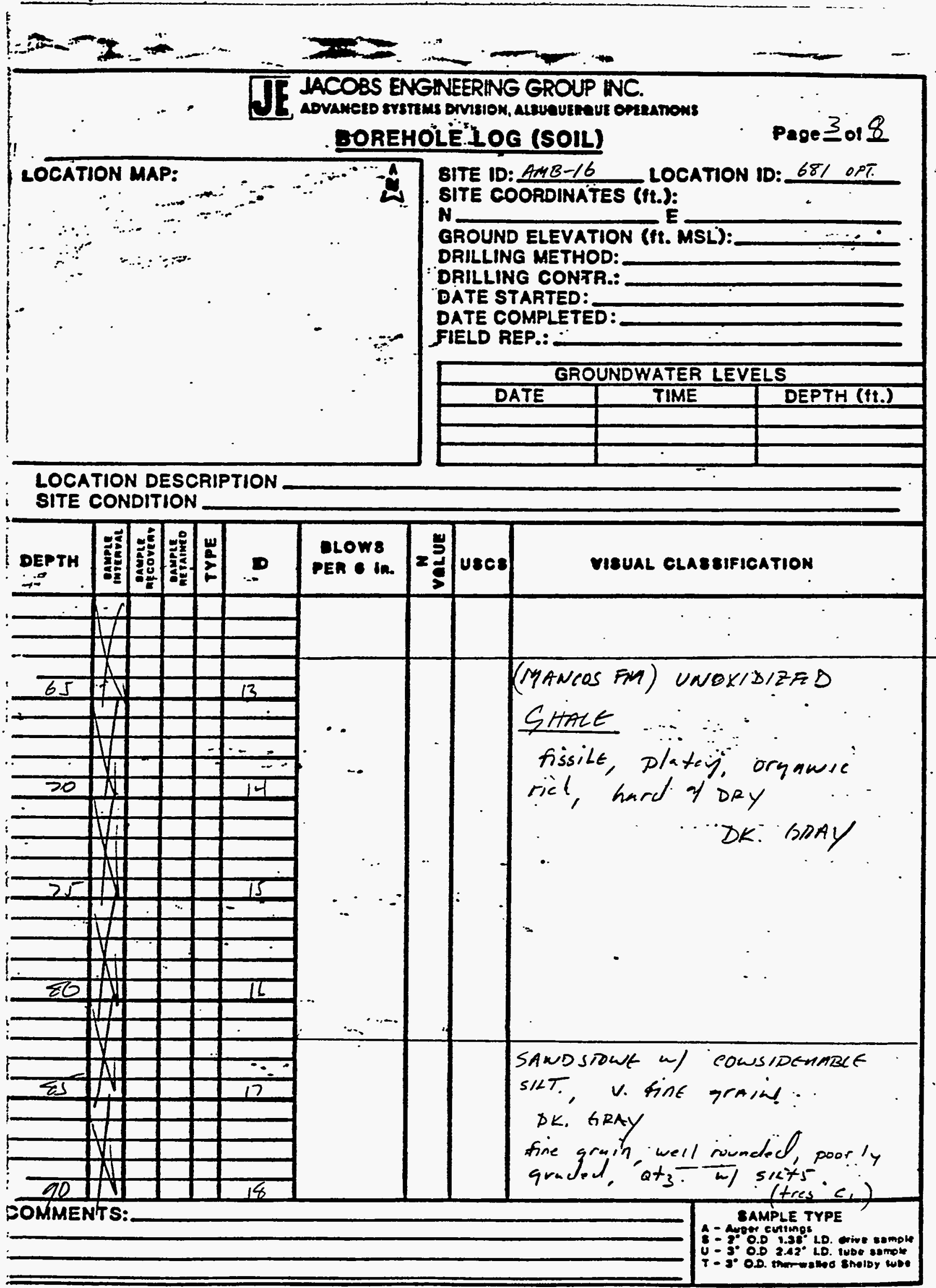


$2=-2-$

JACOBS ENGINEERING GROUP NC.

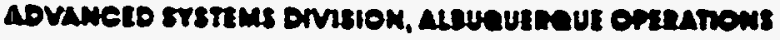

BOREHOLE LOG (SOIL)

Page 4018

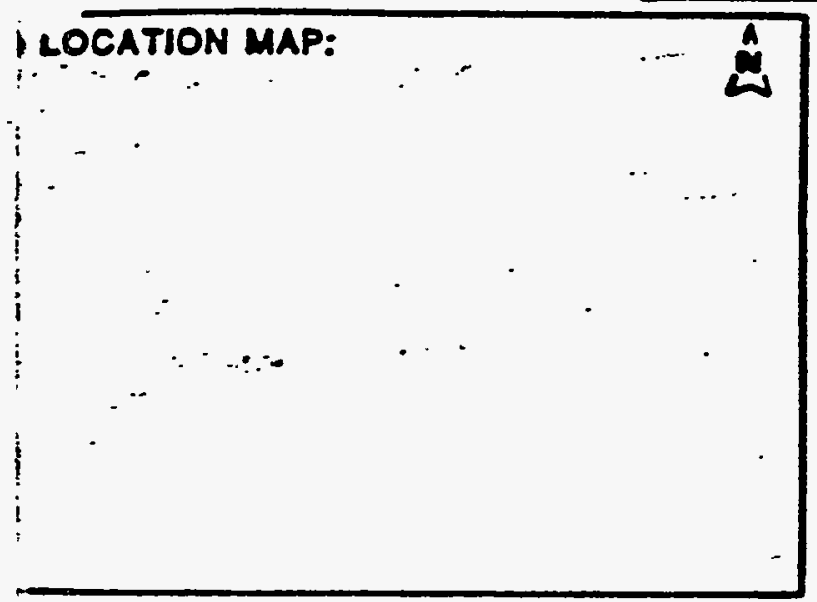

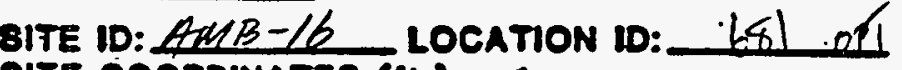

SITE COOADINATES (ti.):

N

GROUND ELEVATION (ft. MSL):

DRILLING METHOD:

DRILLING CONTR.:

DATE STARTED:

DATE COMPLETED:

FIELD REP.:

\begin{tabular}{|c|c|c|}
\hline \multicolumn{3}{|c|}{ GROUNDWATER LEVELS } \\
\hline DATE & TIME & DEPTH (ft.) \\
\hline & & \\
\hline & & \\
\hline & & \\
\hline
\end{tabular}

LOCATION DESCRIPTION

SITE CONDITION

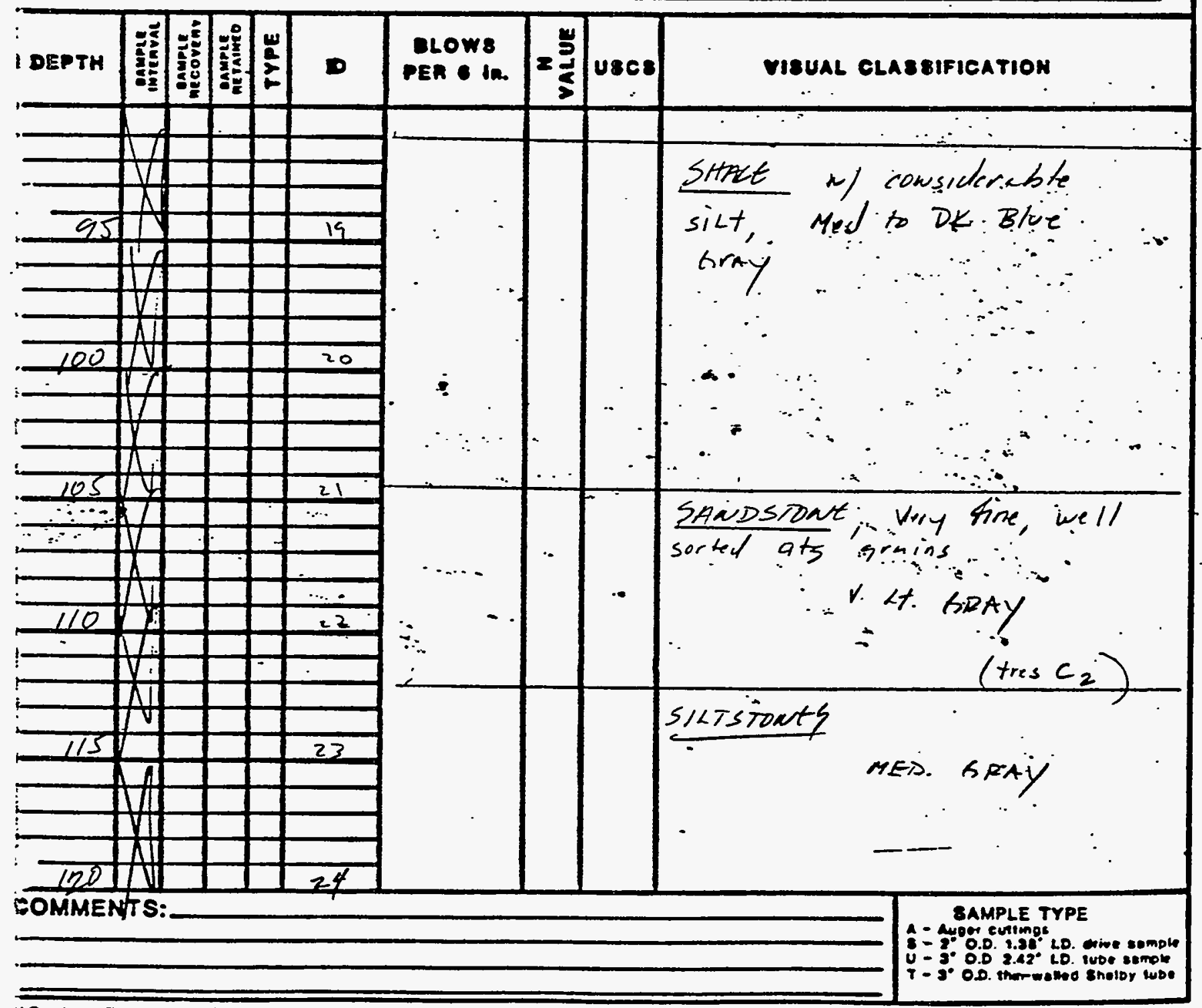




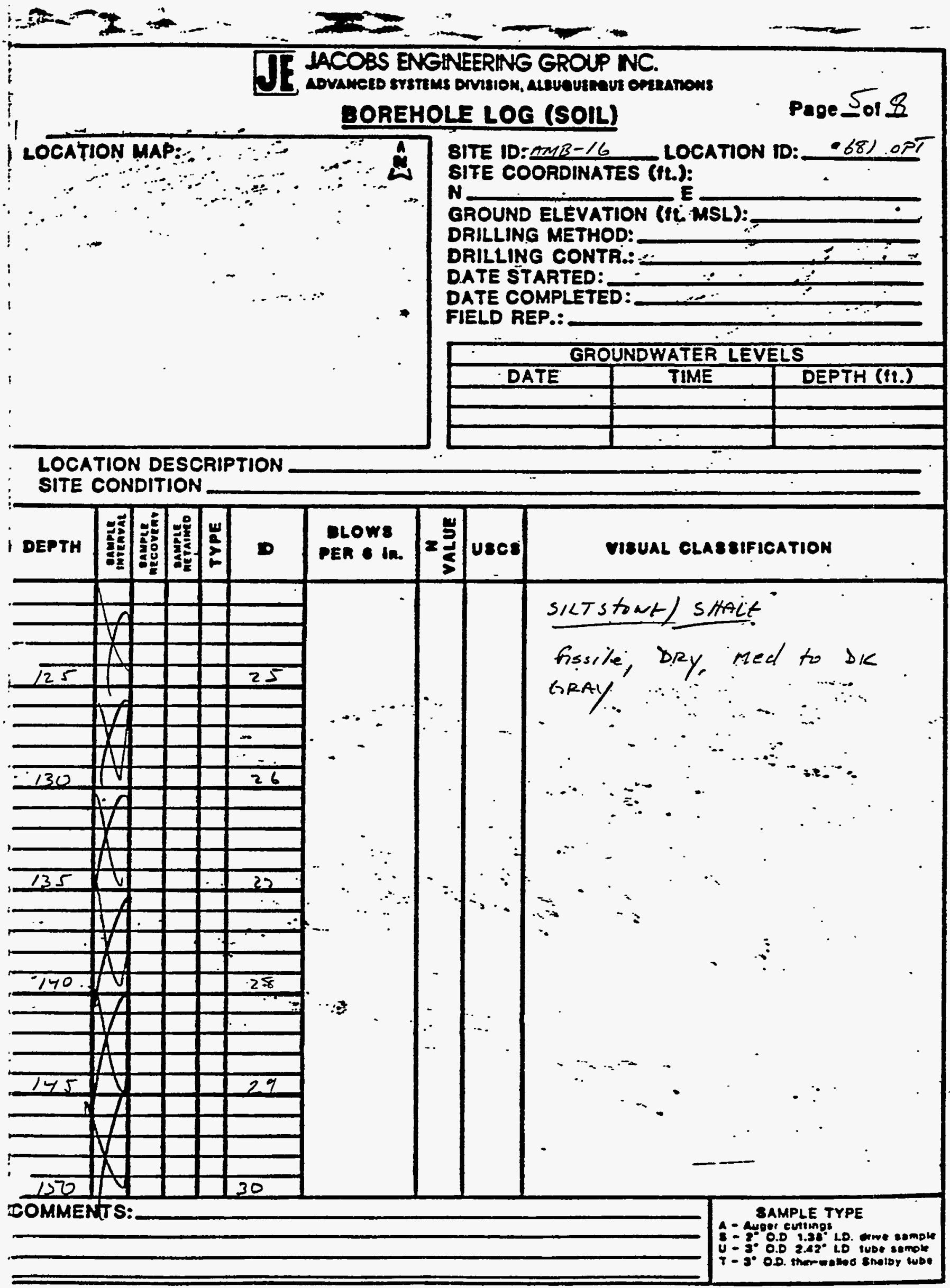




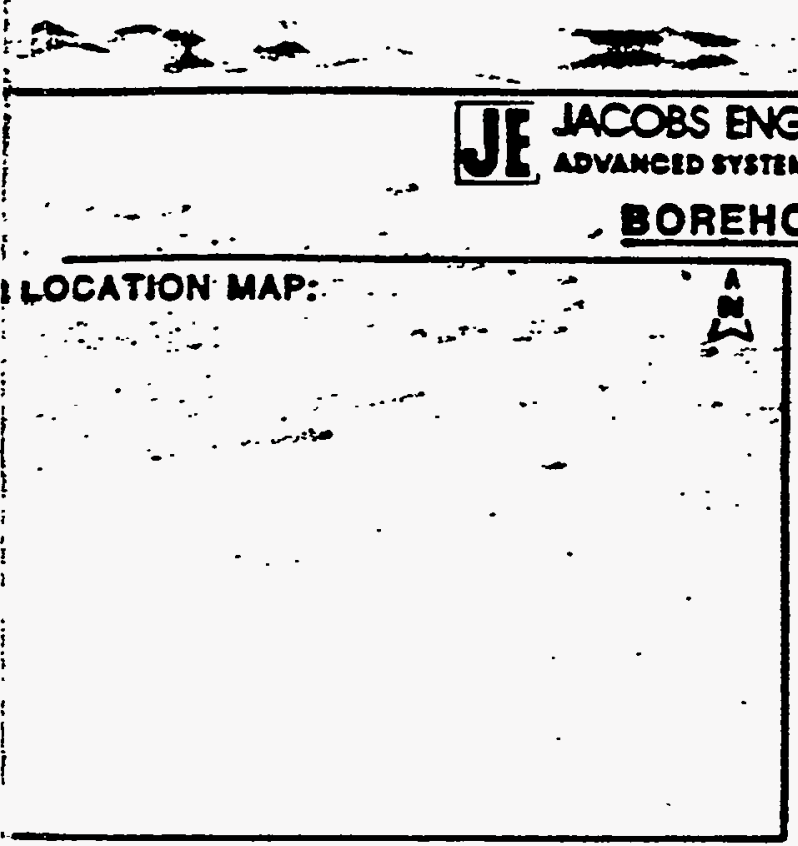

GHEERHE GROUP NC.

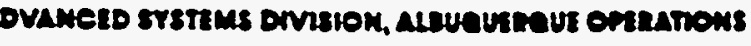

- BOREHOLE LOG (SOIL)

Page 6 or 8 8ITE ID:AMB-16 LOCATION ID: 681 OPT BITE COORDINATES (ft.):

GROUND ELËVATION (fi. MSL):

DRILLING METHOD:

DRILLING CONTR.:

DATE STARTED:

DATE COMPLETED:

FIELD REP.:

\begin{tabular}{|c|c|c|}
\hline \multicolumn{3}{|c|}{ GROUNDWATER LEVELS } \\
\hline DATE & TIME & DEPTH (11.) \\
\hline & & \\
\hline & & \\
\hline & & \\
\hline
\end{tabular}

LOCATION DESCRIPTION

: SITE CONDITION

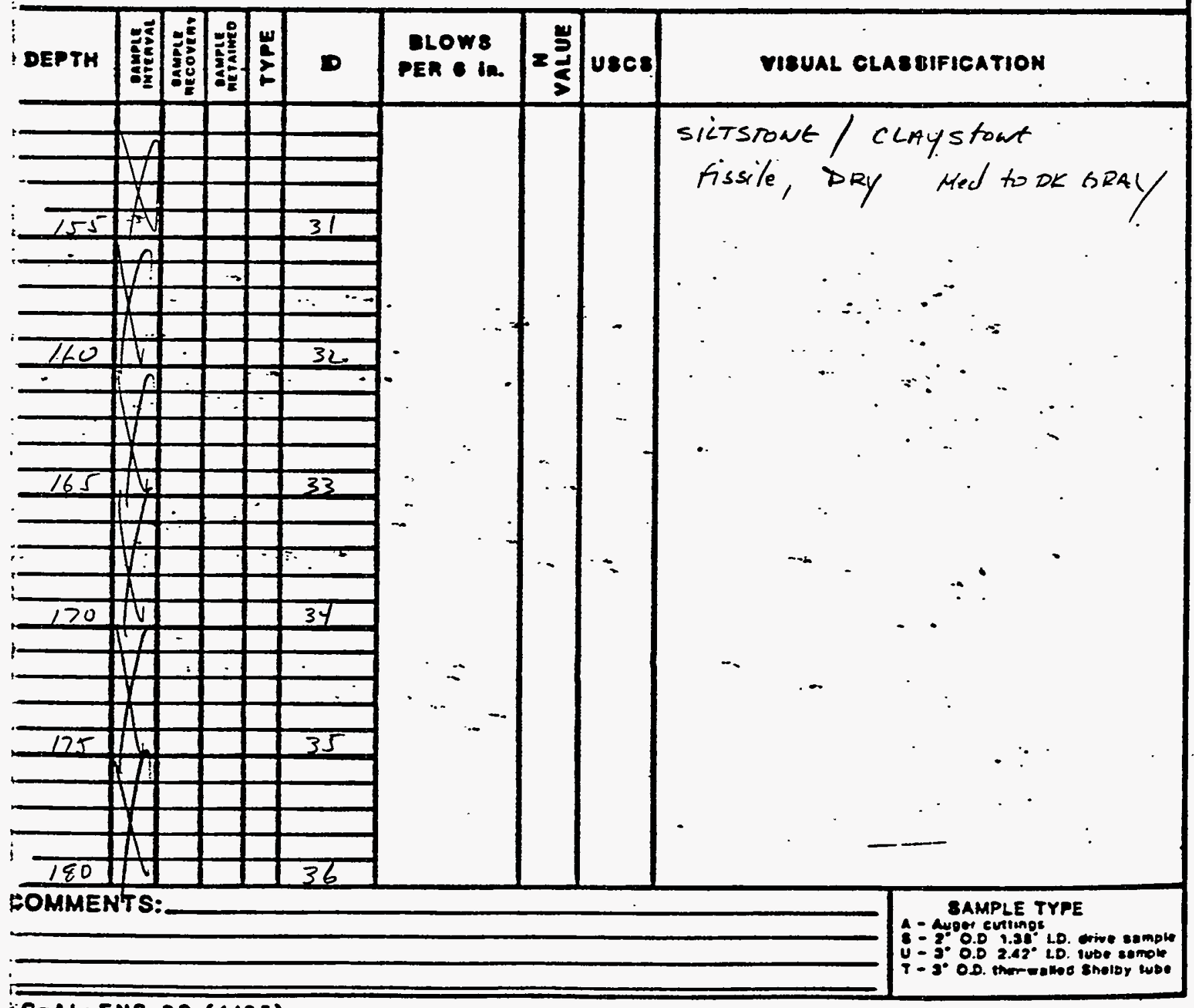

$\because O-A L-E M O-28(4 / E 6)$ 
$12-$

JACOBS ENGINEERANG GROUP NC.

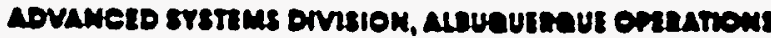

BOREHOLE LOG (SOIL)

Page Zor 8

LOCATION MAP:

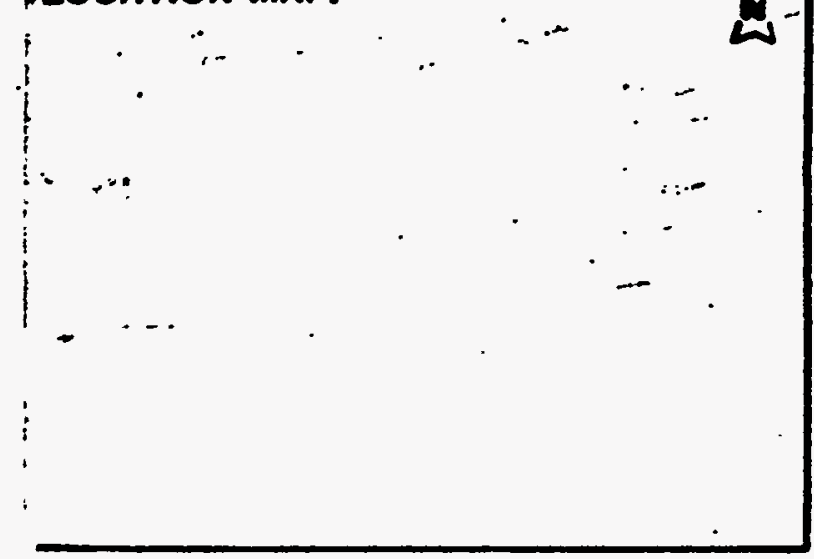

8ITE ID: AMB $\div 16$ - LOCATION ID: $68 /$ OPT

SITE COORDINATES (ft.):

N LOUND ELEVATION (TH, ES

GROUND ELEVATION (fT. MSL):

DRILLING METHOD:

DRILLING CONTR.:

DATE STARTED:

DATE COMPLETED:

FIELD REP.:

\begin{tabular}{|c|c|c|}
\hline \multicolumn{3}{|c|}{ GROUNDWATER LEVELS } \\
\hline DATE & TIME & DEPTH (ft.) \\
\hline & & \\
\hline & & \\
\hline & & \\
\hline
\end{tabular}

LOCATION DESCRIPTION

! SITE CONDITION

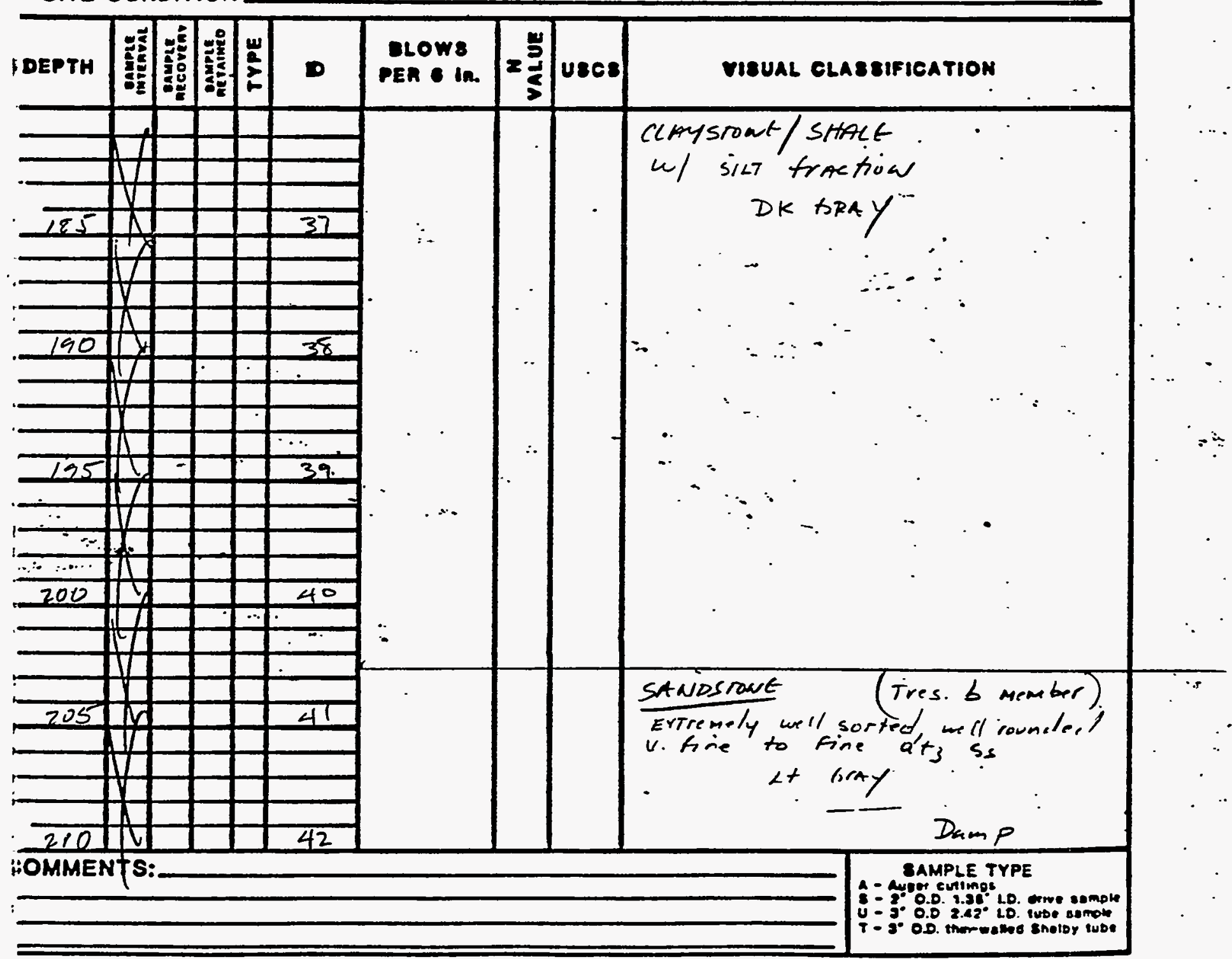

IO-AL-ENO-2B(4/ES) 
$=-2$

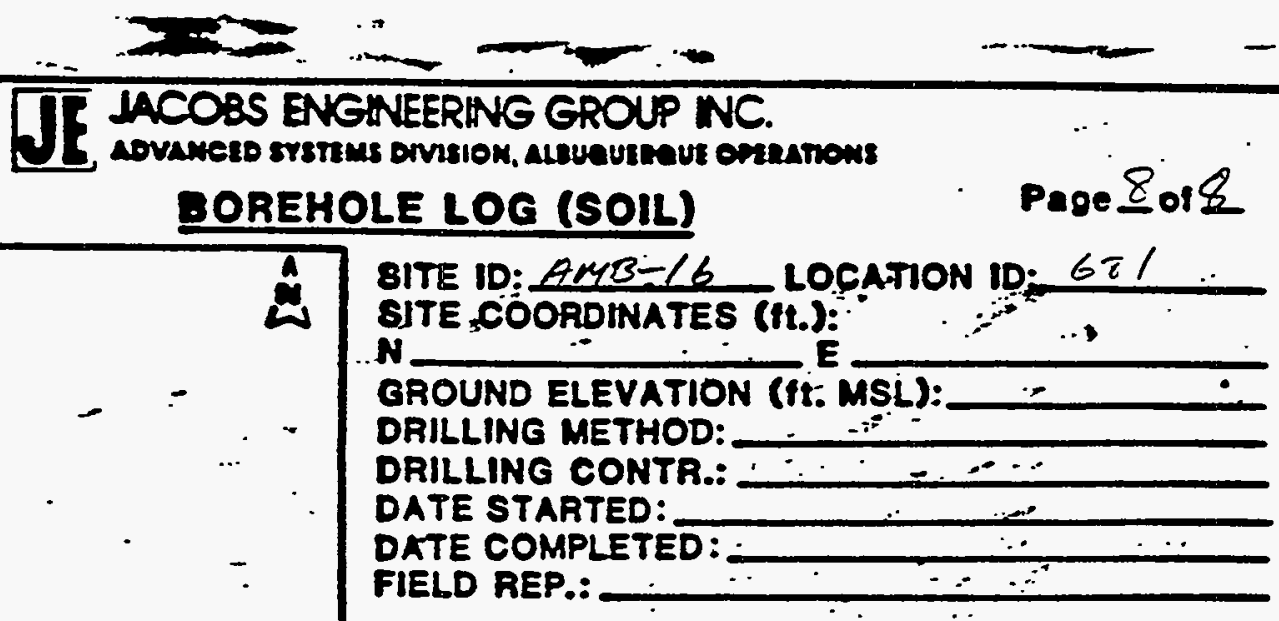

LOCATION MAP:

LOCATION DESCRIPTION

SITE CONDITION

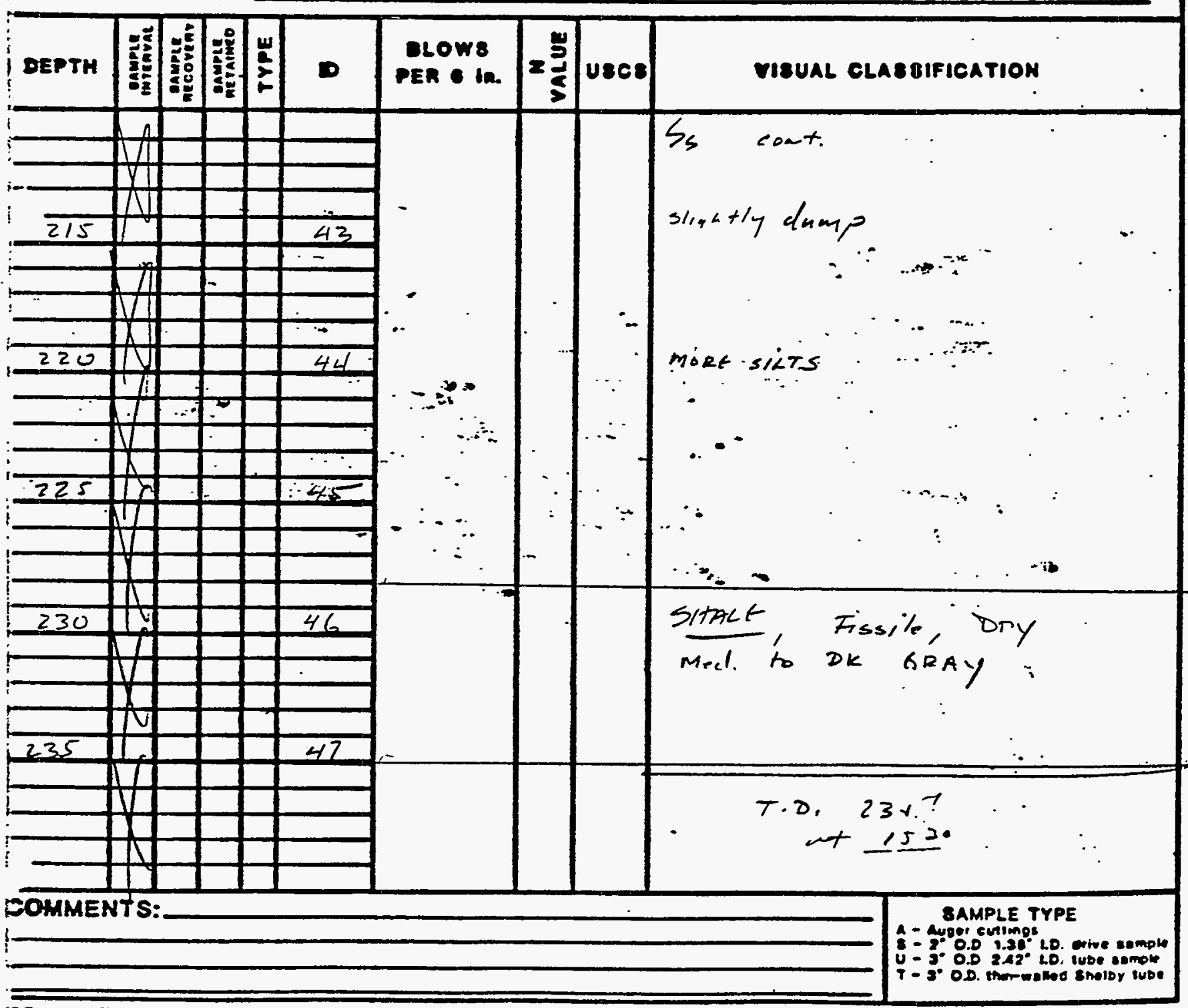




\section{TT JACOBS ENGANERNG GROUP NC.

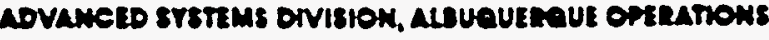

\section{WELL COMPLETION RECORD}

SITE ID: $A M \dot{B}-16$ LOCATION ID: 681 opT. DATE INSTALLED: $1-24-89$ APPROX. SITE COORDINATES:(FT.) $N$ OPEN AREA PER LINEAL FT. (IN2/FT.) $\frac{8^{2}-45^{2}}{2}$ FORMATION OF COMPLETION: IreS $B$ member of the MAWCOS FM FIELD REP.: D.R. METZLER DRILLER: CHEW NOIRTERN

WELL CASING

DIAMETER (in) 4.0 ID WELL CASING HOLE DIAMETER $(\ln ) 8.0$ GROUND SURFACE

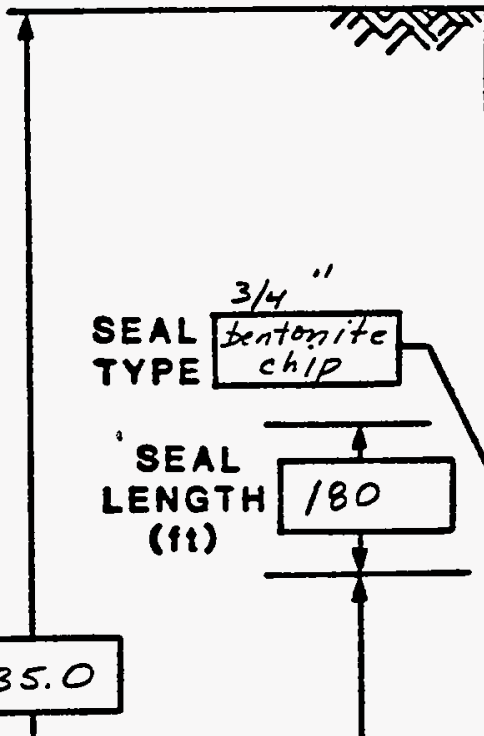

TOTAL DEPTH 235.0 (tt)
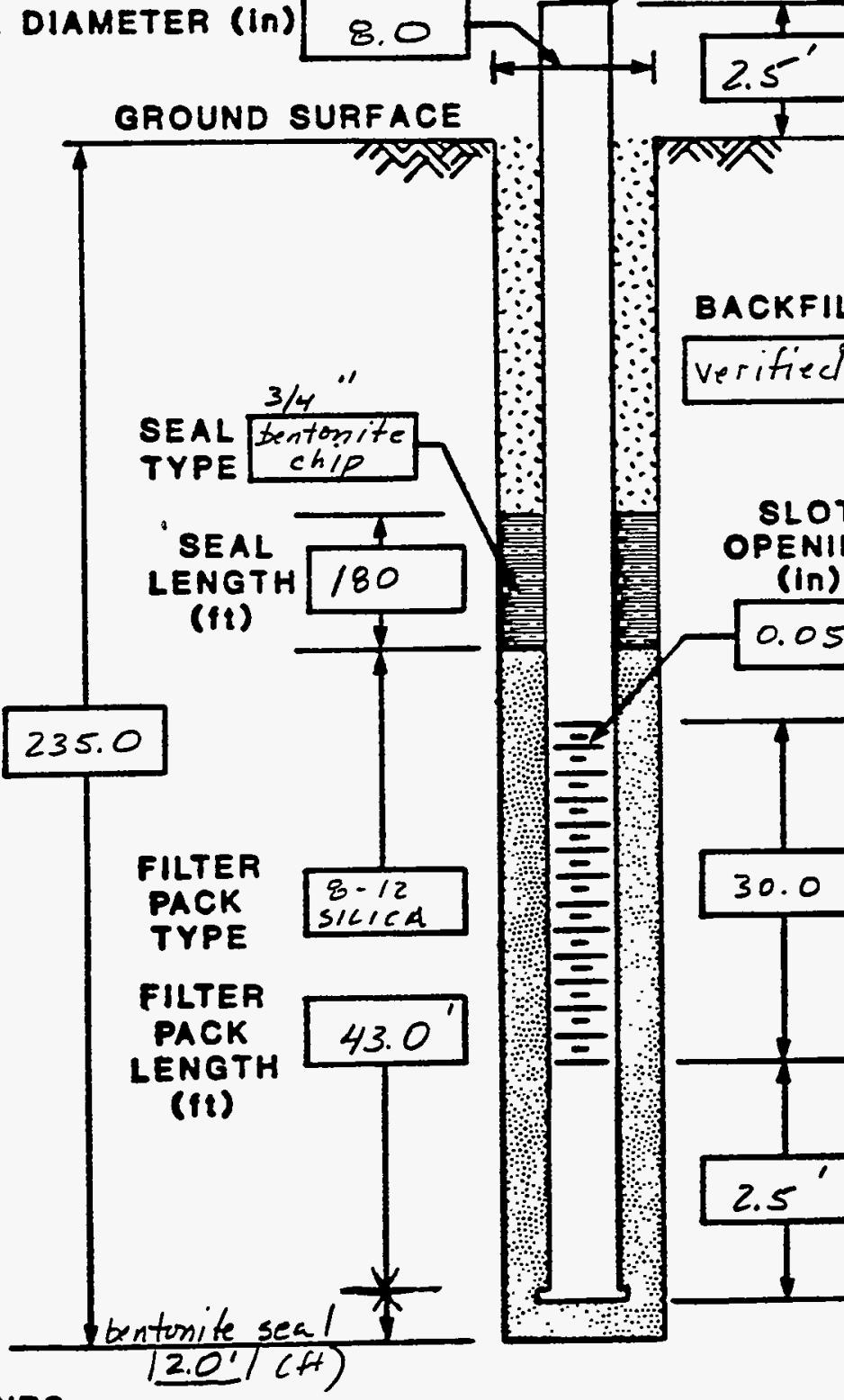
HT. ABOVE GROUND (f1) TYPE

BACKFILL TYPE verified grout

\section{COMMENTS:}




\section{TI JaCOSS ENGWEERT GROUP NC...... -

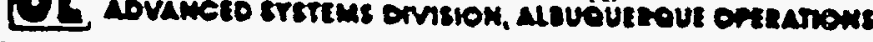 BOREHOLE/WELL CONSTRUCTION LOG}

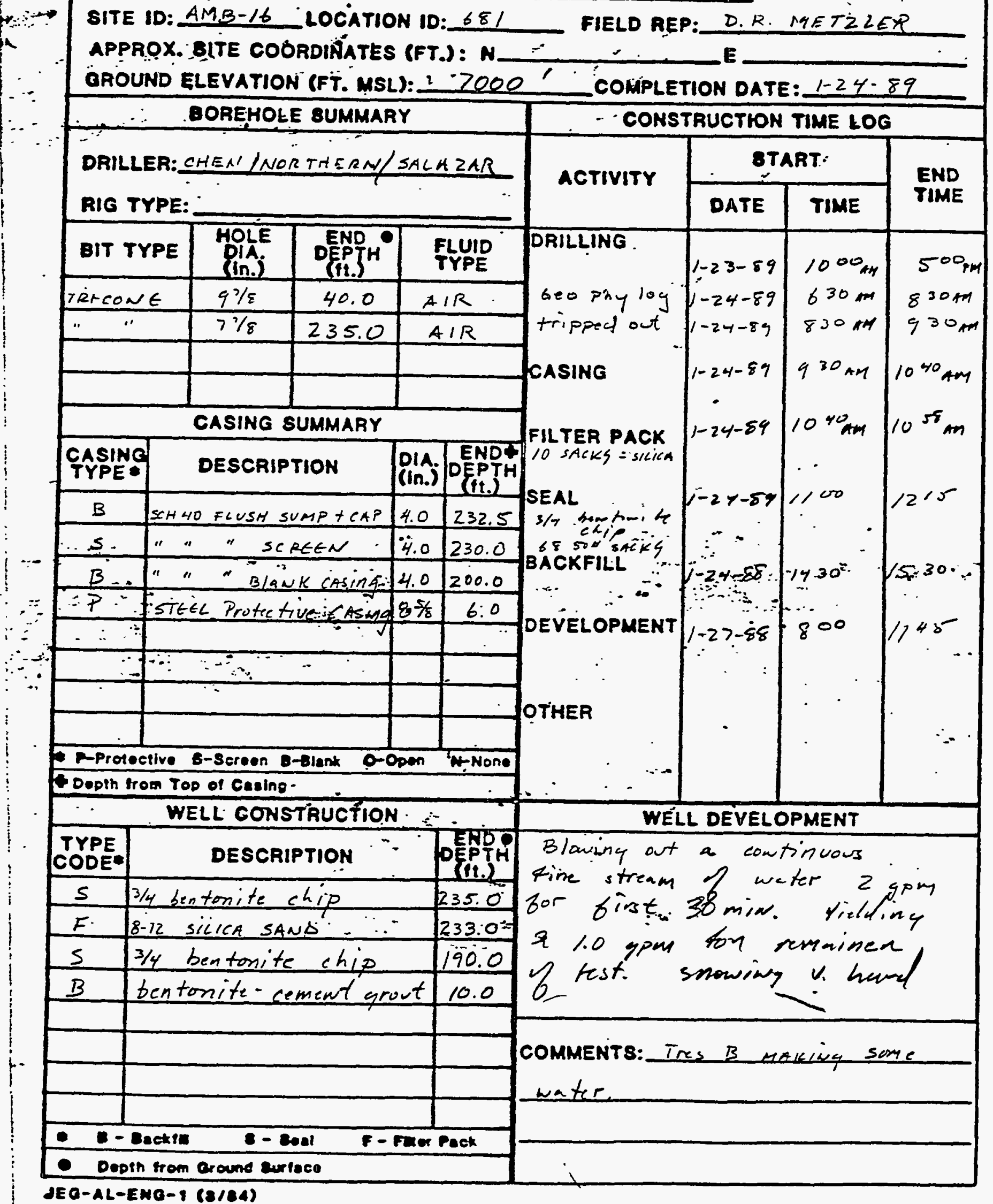


WELL 776 LOGS 


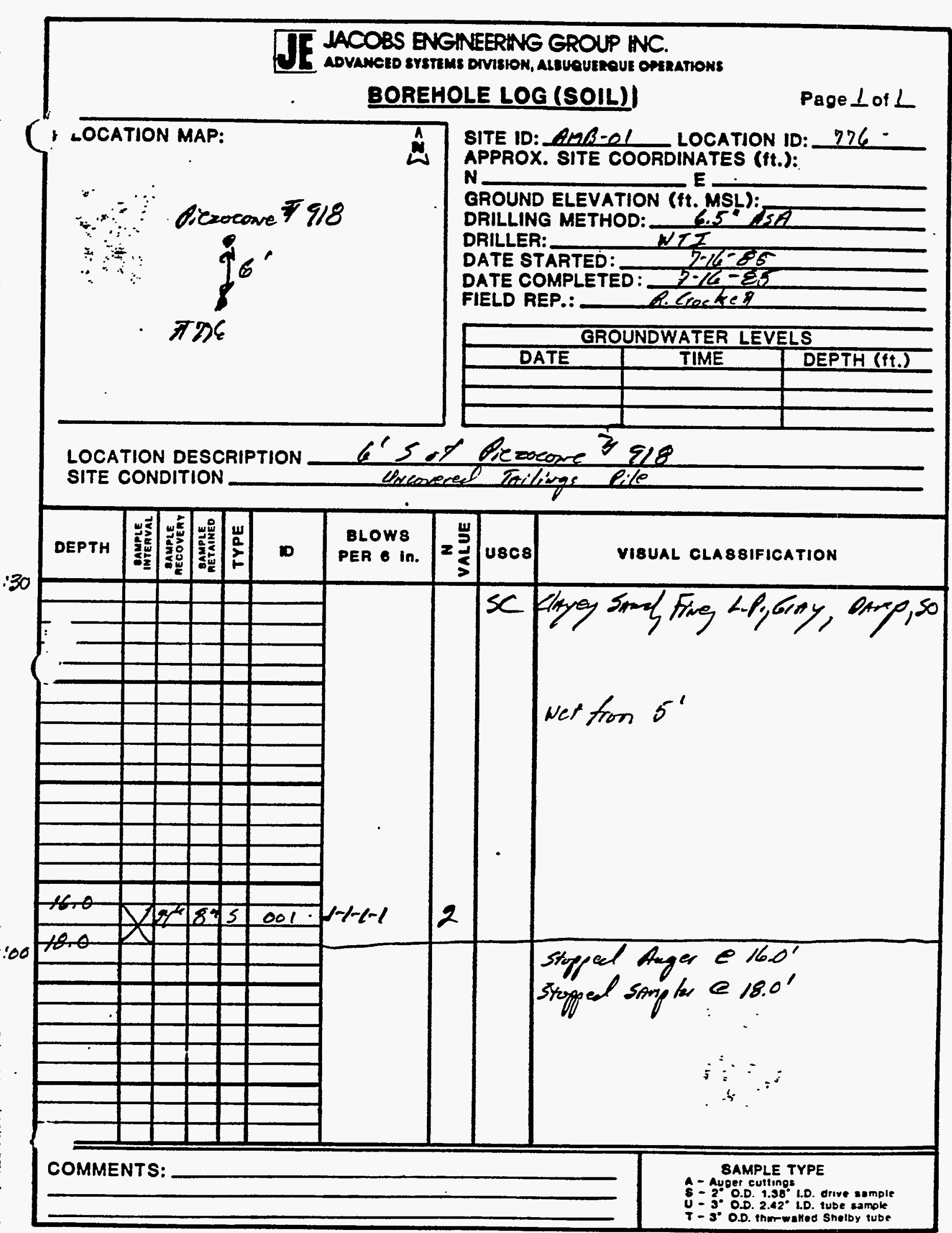

JEG-AL-ENG-2S. (3/84) 


\section{TT MCOBS BNENEERTE GROUP NR.

\section{WELL COMPLETION RECORD}

SITE ID: AMB OL LOCATION ID: 276

DATE INSTALLED: 8/Og/ES

APPROX. SITE COORDINATES:(FT.) N NK E NK OPEN AREA PER LINEAL FT. (IN2/FT.) 150.2 for $4.0^{N} T D$ FORMATION OF COMPLETION:

FIELD -REP.:

$$
\text { COMPLETION: QRaL }
$$
DRILLER: Stewart Bee. (Albert)

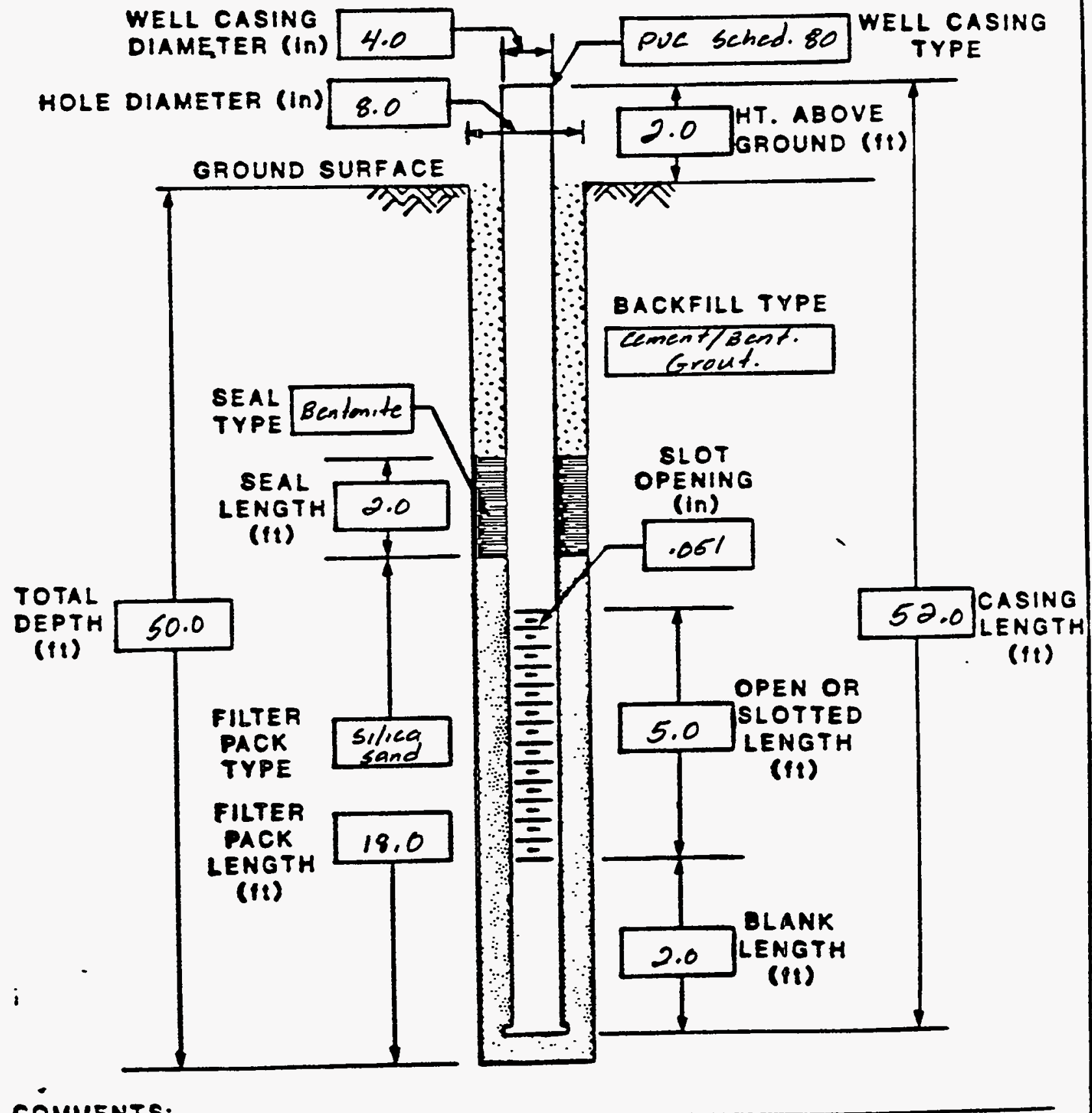

COMMENTS: 
WELL 777 LOGS 


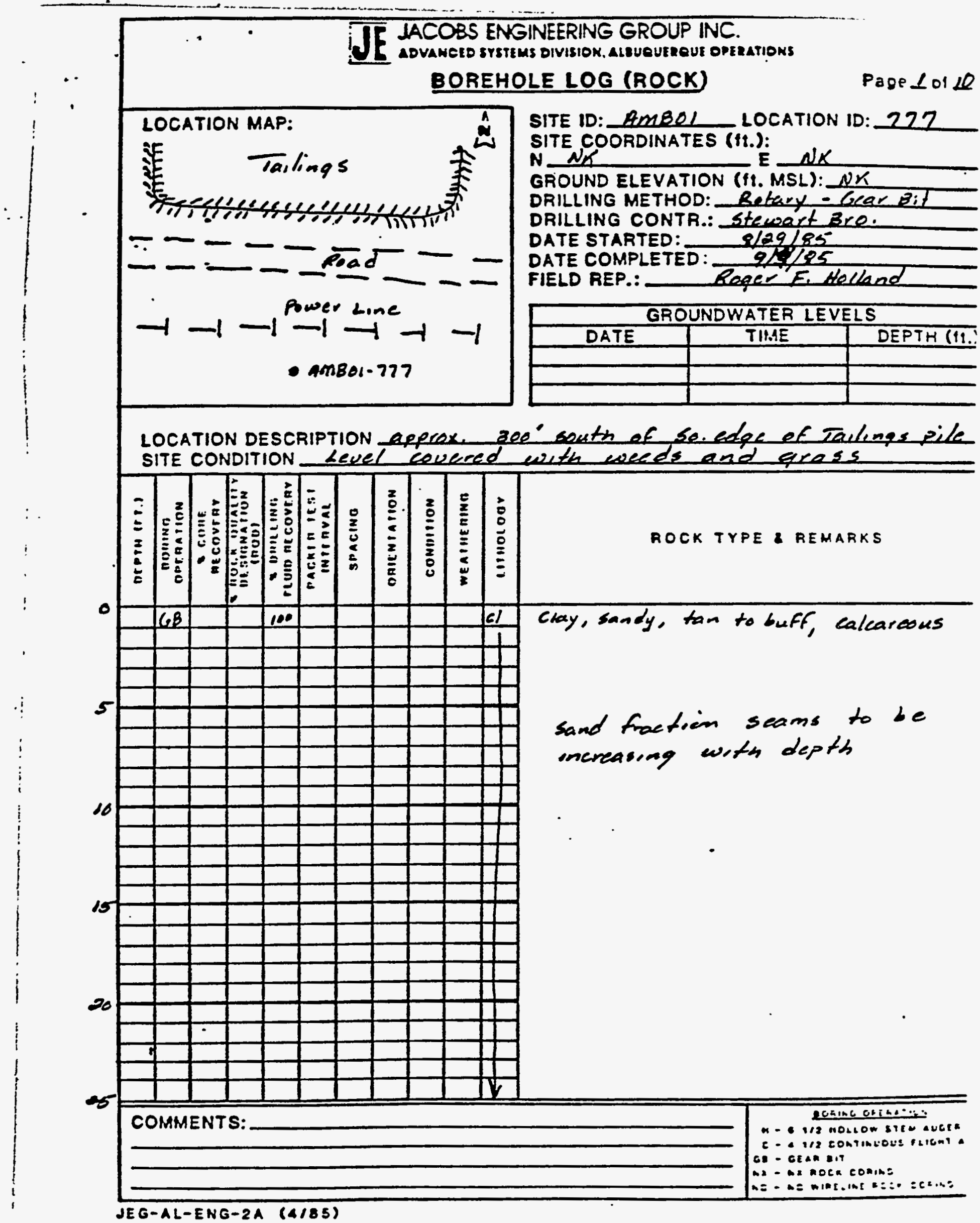




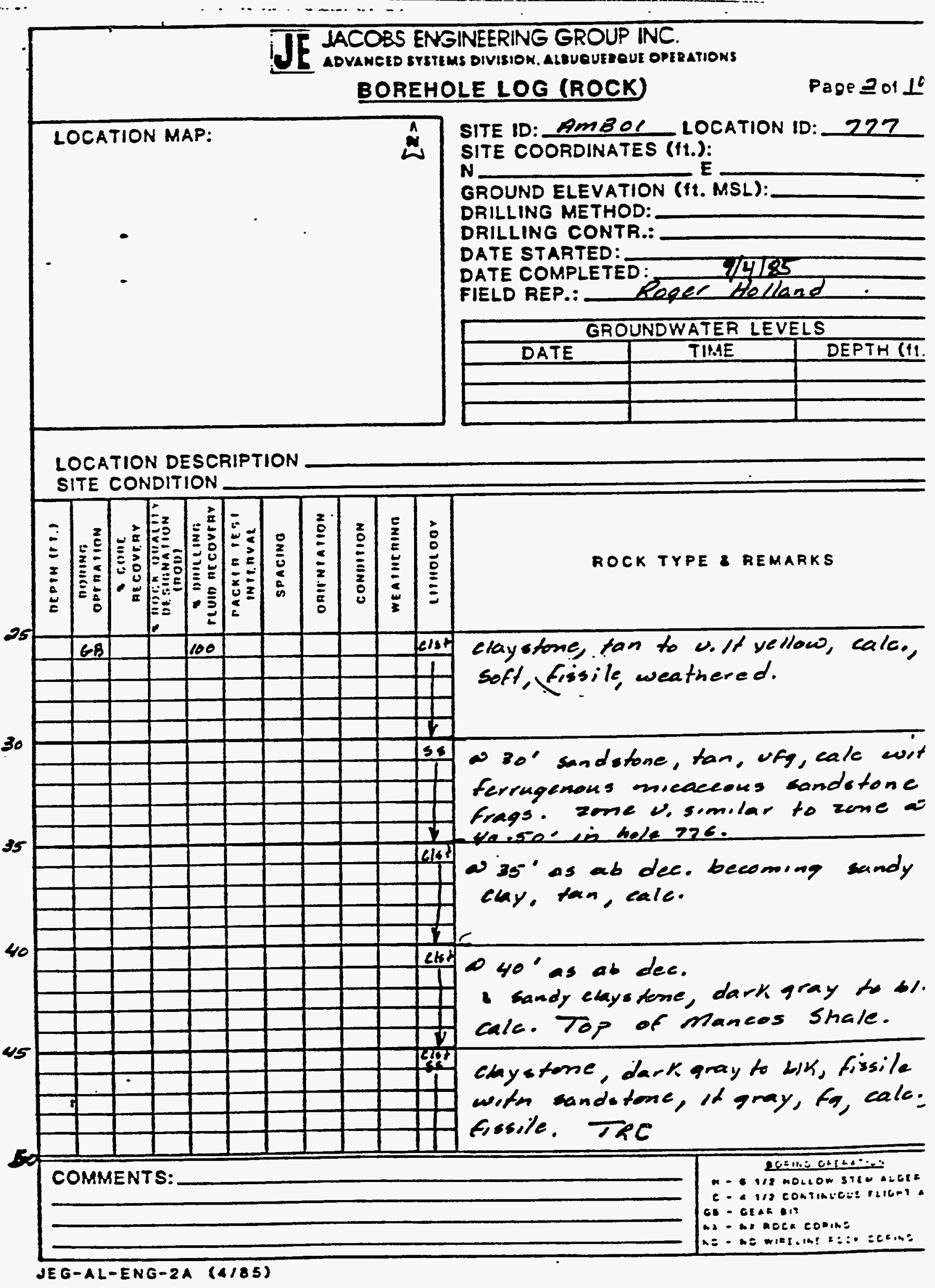


If JACOBS ENGINEERING GROUP INC.

ADVANEED STSTEMS OIVISIOK, ALUQUERQUE OPEATIONS

BOREHOLE LOG (ROCK)

Page 3 or 10

LOCATION MAP:

SITE ID: AMBEI

LOCATION ID: 727

SITE COORDINATES (ft.):

N E

GROUND ELEVATION (11. MSL):

DRILLING METHOD:

DRILLING CONTR.:

DATE STARTED:

DATE COMPLETED:

FIELD REP.:

\begin{tabular}{|c|c|c}
\hline \multicolumn{3}{|c}{ GROUNDWATER LEVELS } \\
\hline DATE & TIR.AE & DEPTH (11.) \\
\hline & & \\
\hline & & \\
\hline & & \\
\hline
\end{tabular}

LOCATION DESCRIPTION

SITE CONDITION

sandstone ene. woth depth

>
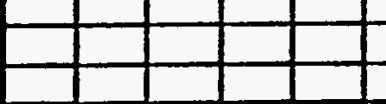

\begin{abstract}
sandobuc aona. anith depth
\end{abstract}
sinictotone, if grey, UF, cale. to noncas frssile

sandstome, med. to dark aray, ufq, calle, fissile becoming clayey.

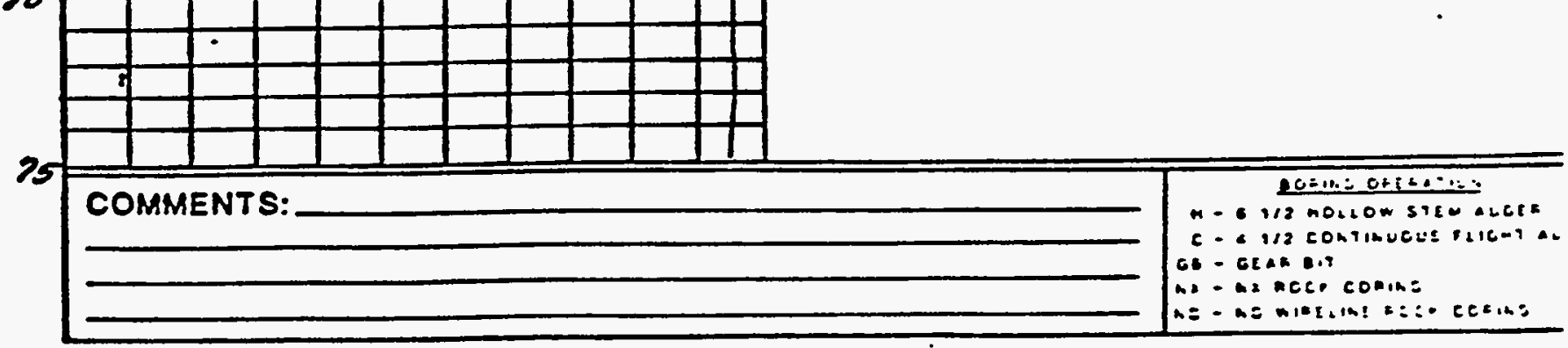




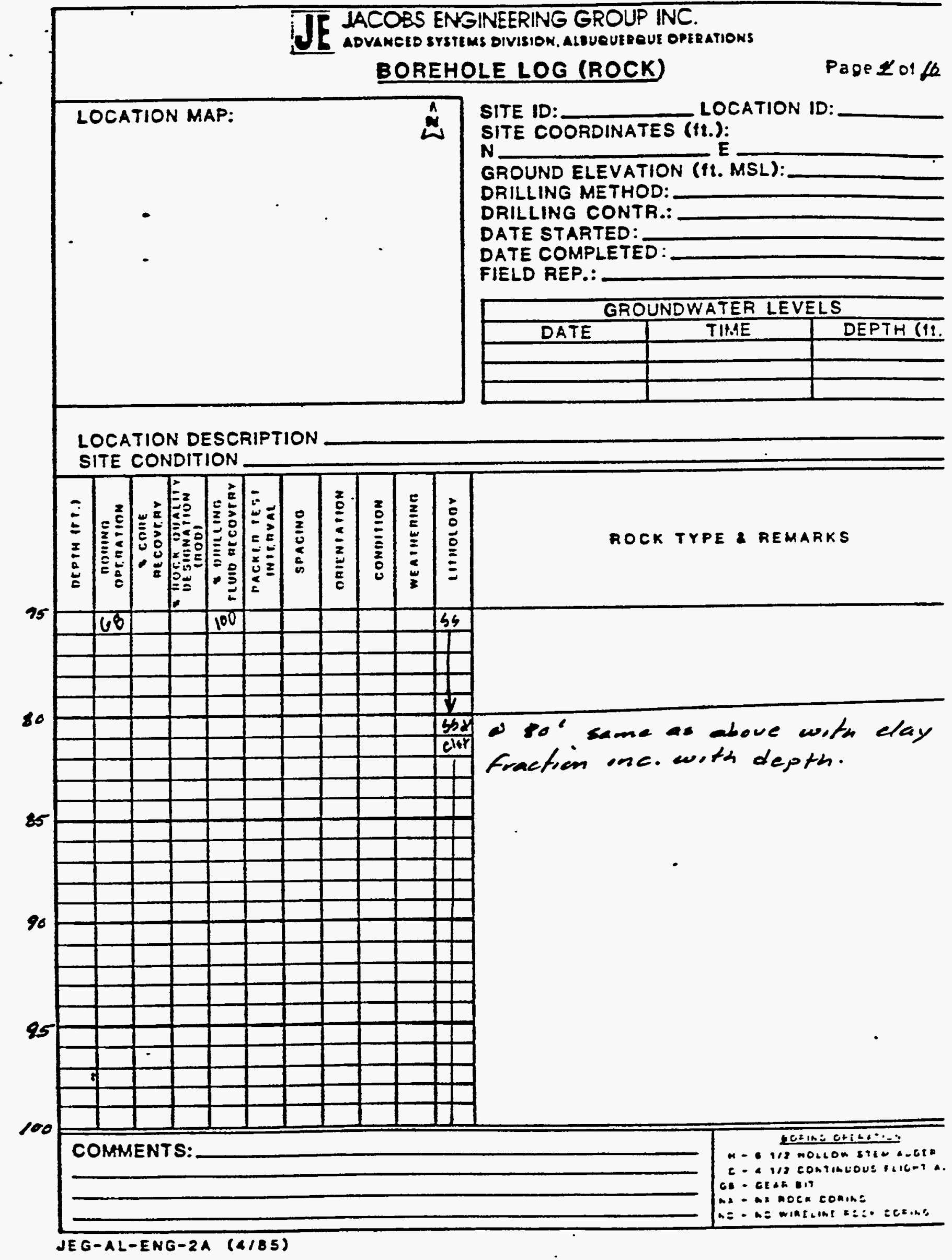




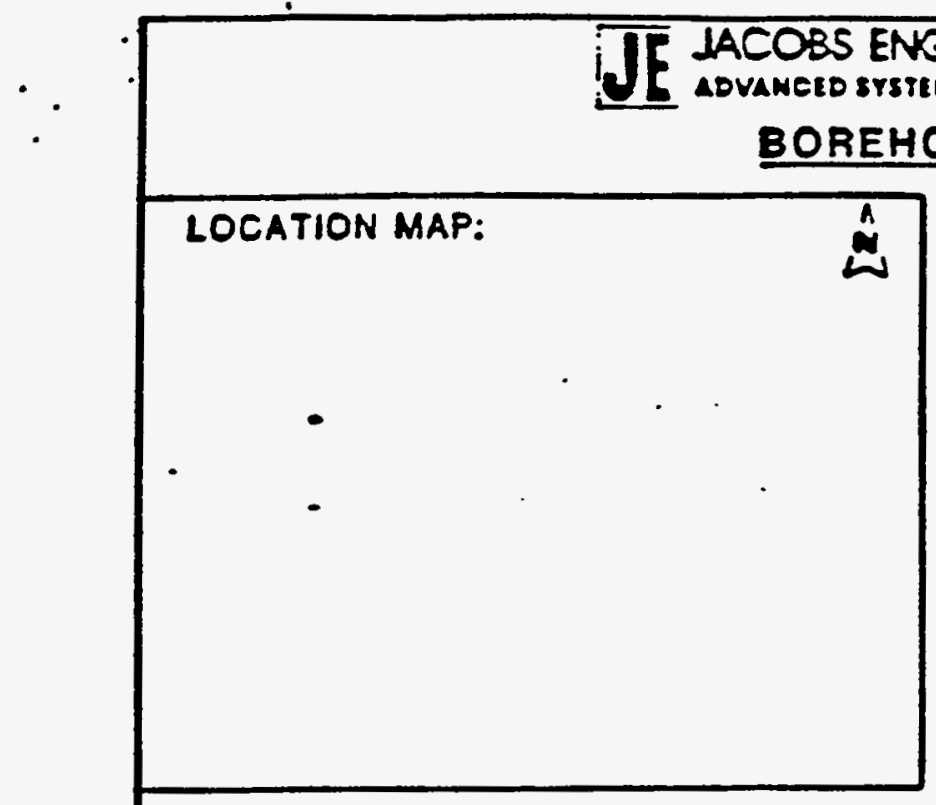

IACOBS ENGINEERING GROUP INC.

DVVANELD STSTEMS DIVISION, ALUUUERQUE OPIRATIONS

BOREHOLE LOG (ROCK)

Page 50116

SITE ID: AMBOI LOCATION ID: 272 SITE COORDINATES (fI.):

$N$ E

GROUND ELEVATION (ft. MSL):

DRILLING METHOD:

DRILLING CONTR.:

DATE STARTED:

DATE COMPLETED:

FIELD REP.:

R.telland

\begin{tabular}{|c|c|c|}
\hline \multicolumn{3}{|c|}{ GROUNDWATER LEVELS } \\
\hline DATE & TIIAE & DEPTH ( 11 \\
\hline & & \\
\hline & & \\
\hline & & \\
\hline
\end{tabular}

LOCATION DESCRIPTION

SITE CONDITION

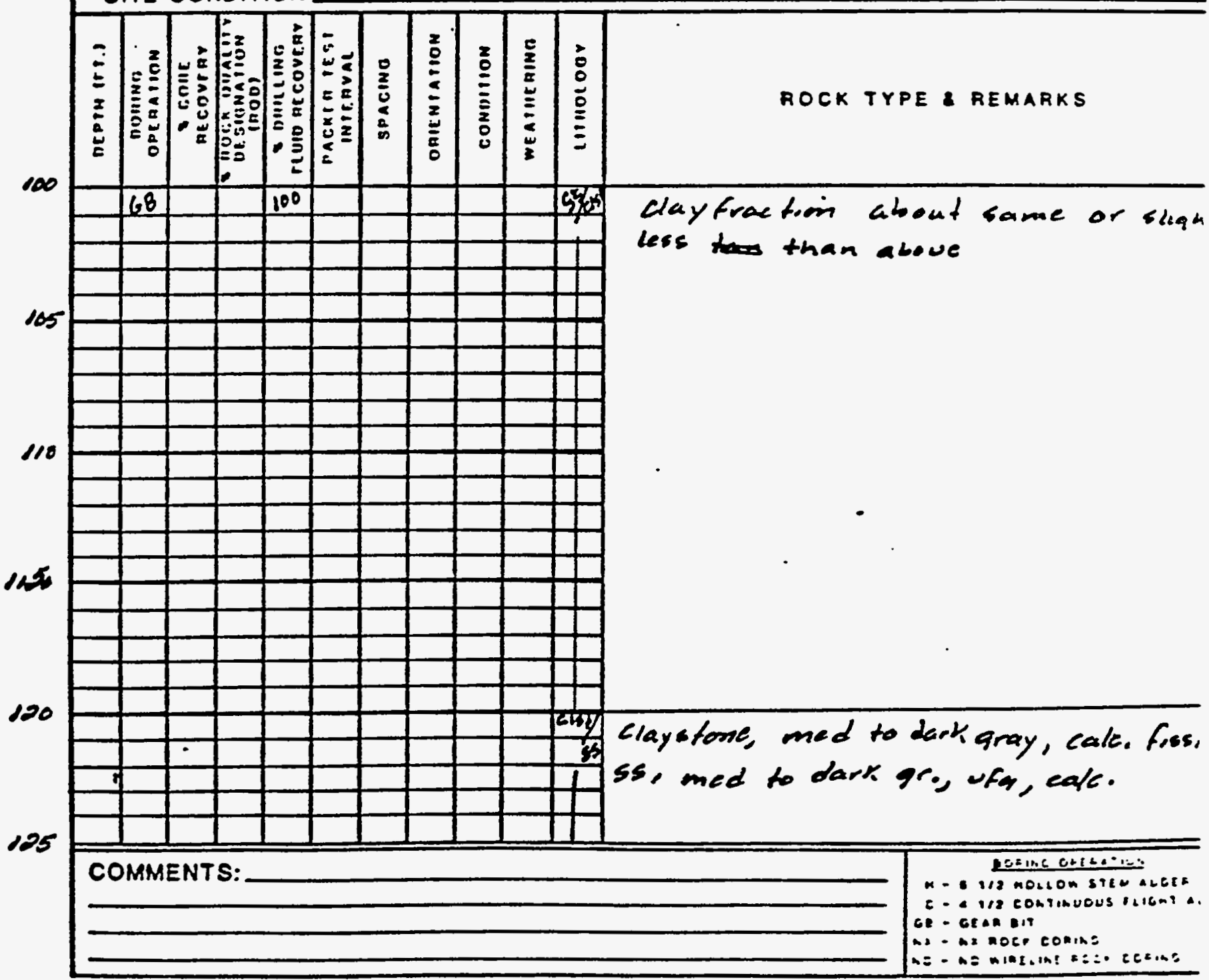

JEG-AL-ENG-2A (4/85) 


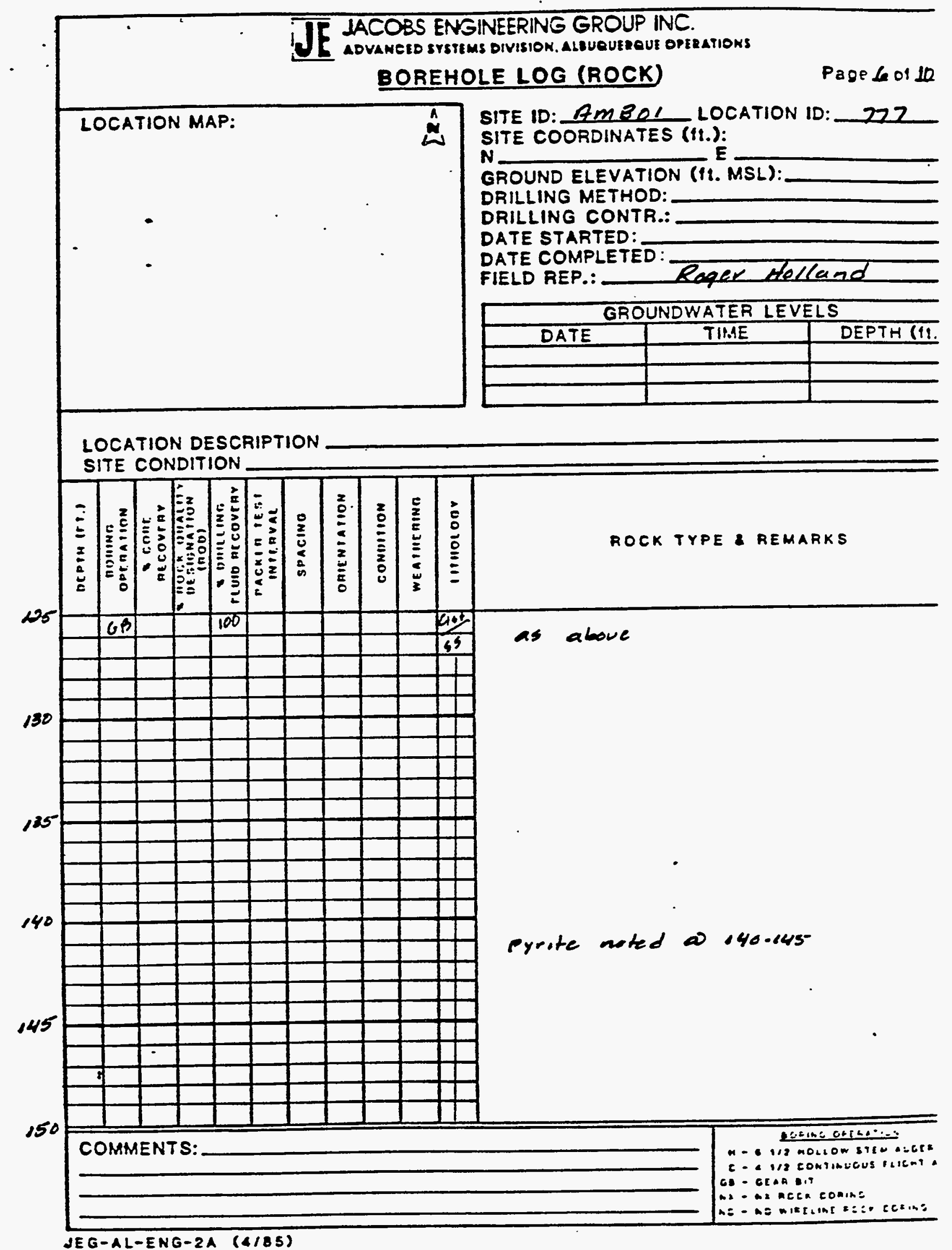


TE IACOBS ENGINEERING GROUP INC. AOVANEED SYSTIMS DIVISIOK, ALQUEUERQUI OPERATIONS

\section{BOREHOLE LOG (ROCK)}

LOCATION MAP:

$\stackrel{\wedge}{\omega}$

SITE ID:AMBOL LOCATION ID: 777

SITE COORDINATES (11.):

$N$

GROUND ELEVATION (ft. MSL):

DRILLING METHOD:

DRILLING CONTR.:

DATE STARTED:

DATE COMPLETED

FIELD REP.: Roger F. Helland

\begin{tabular}{|c|c|c}
\hline \multicolumn{3}{|c}{ GROUNDWATER LEVELS } \\
\hline DATE & TIM.ME & DEPTH (II \\
\hline & & \\
\hline & & \\
\hline & & \\
\hline
\end{tabular}

LOCATION DESCRIPTION

SITE CONDITION

150

0
$\vdots$
$\vdots$
$\vdots$
$\vdots$
$\vdots$

垈

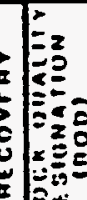

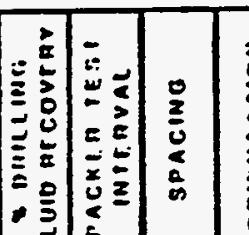

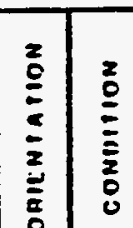

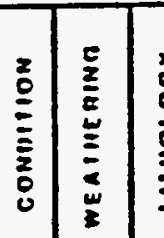

ROCK TYPE 2 REMARKS

155

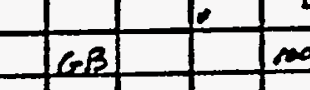

160

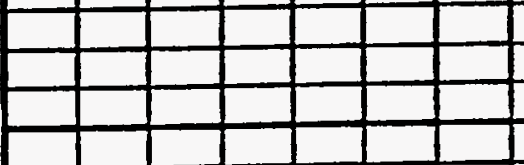

165

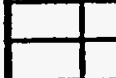

$+$

$+1$

$+$

same as ab woth sandotone fractio me.

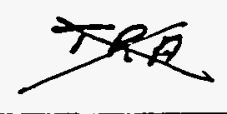

claystone, it greenesh gray, sluntly rale: pyrite soted

mener sandstome

170

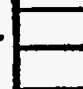

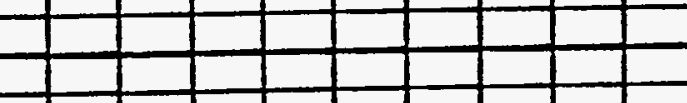

same as ab wothe oute. in sunds tractioin. 
IACOBS ENSINEERING GROUP INC.

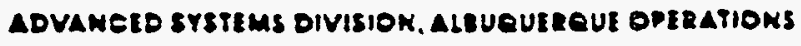

BOREHOLE LOG (ROCK)

Pagesol 10

LOCATION MAP:

$\stackrel{a}{\sim}$

SITE ID: AMBOL

SITE COORDINATES (ti.)

$N$

GROUND ELEVATION (ft. MSL):

DRILLING METHOD:

DRILLING CONTR.:

DATE STARTED:

DATE COMPLETED:

FIELD REP.:

\begin{tabular}{|c|c|c}
\hline \multicolumn{3}{|c|}{ GROUNDWATER LEVELS } \\
\hline DATE & TIRAE & DEPTH (II. \\
\hline & & \\
\hline & & \\
\hline & & \\
\hline
\end{tabular}

LOCATION DESCRIPTION

SITE CONDITION

180
195
190
195
200

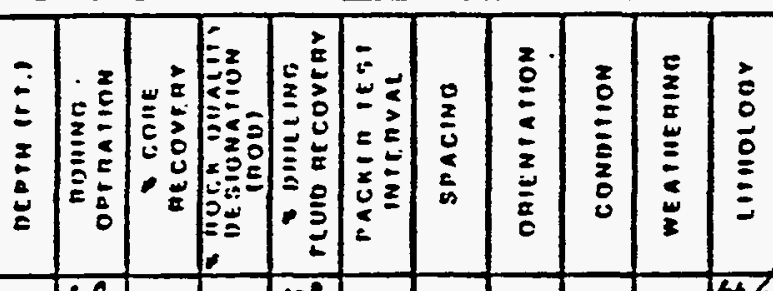

\section{G}

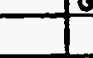
$10^{\circ}$

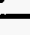

\section{ROCK TYPE AEMARKS}

\section{as ab}

qreenish gray elaystone as ab dec sandy claystaice. med oray to LAT some if geay andotore, ff, calc

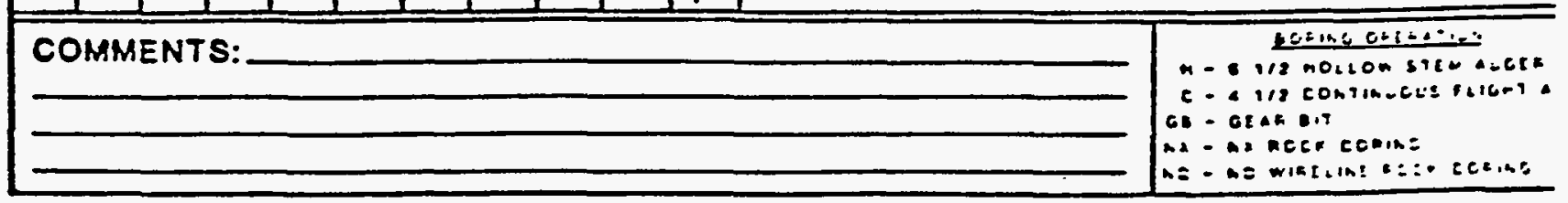

JEG-AL-ENG-2A (4/85) 


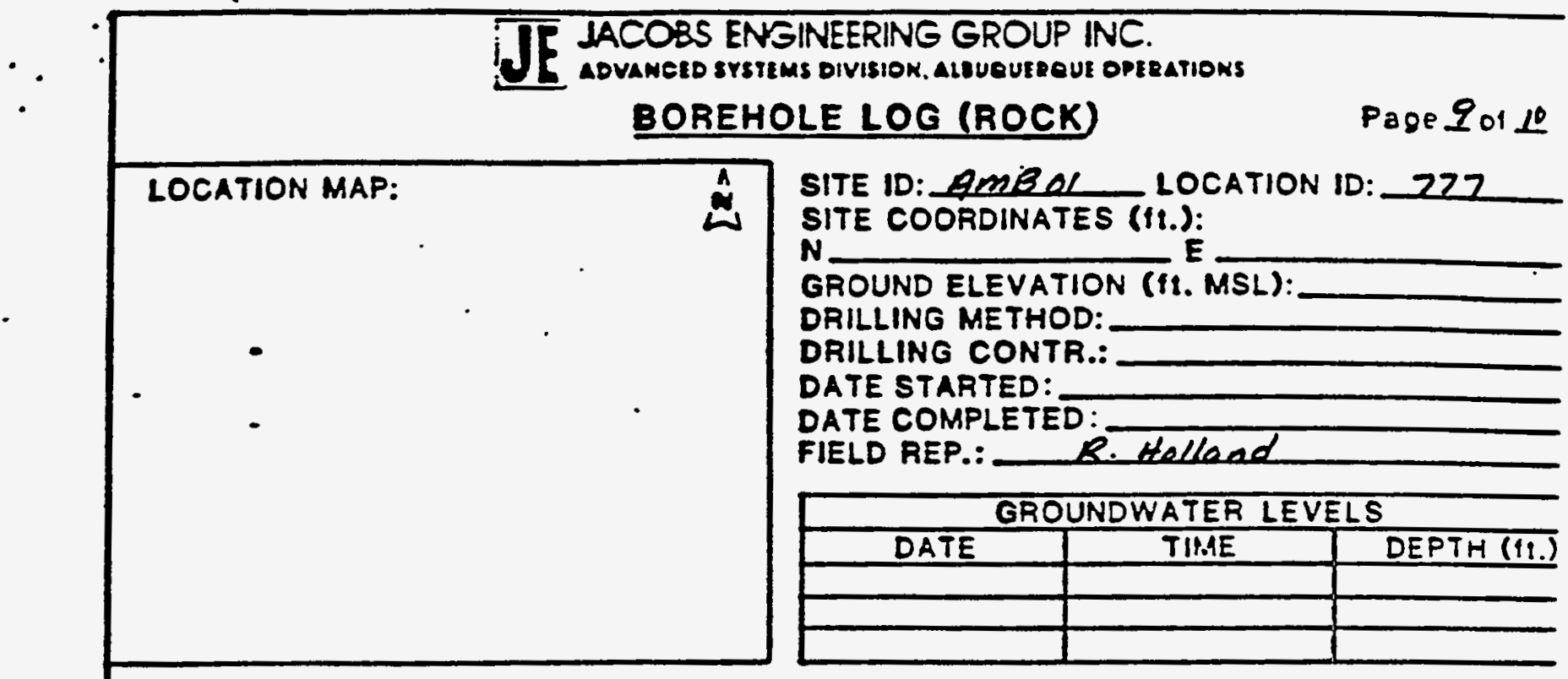

LOCATION DESCRIPTION SITE CONDITION

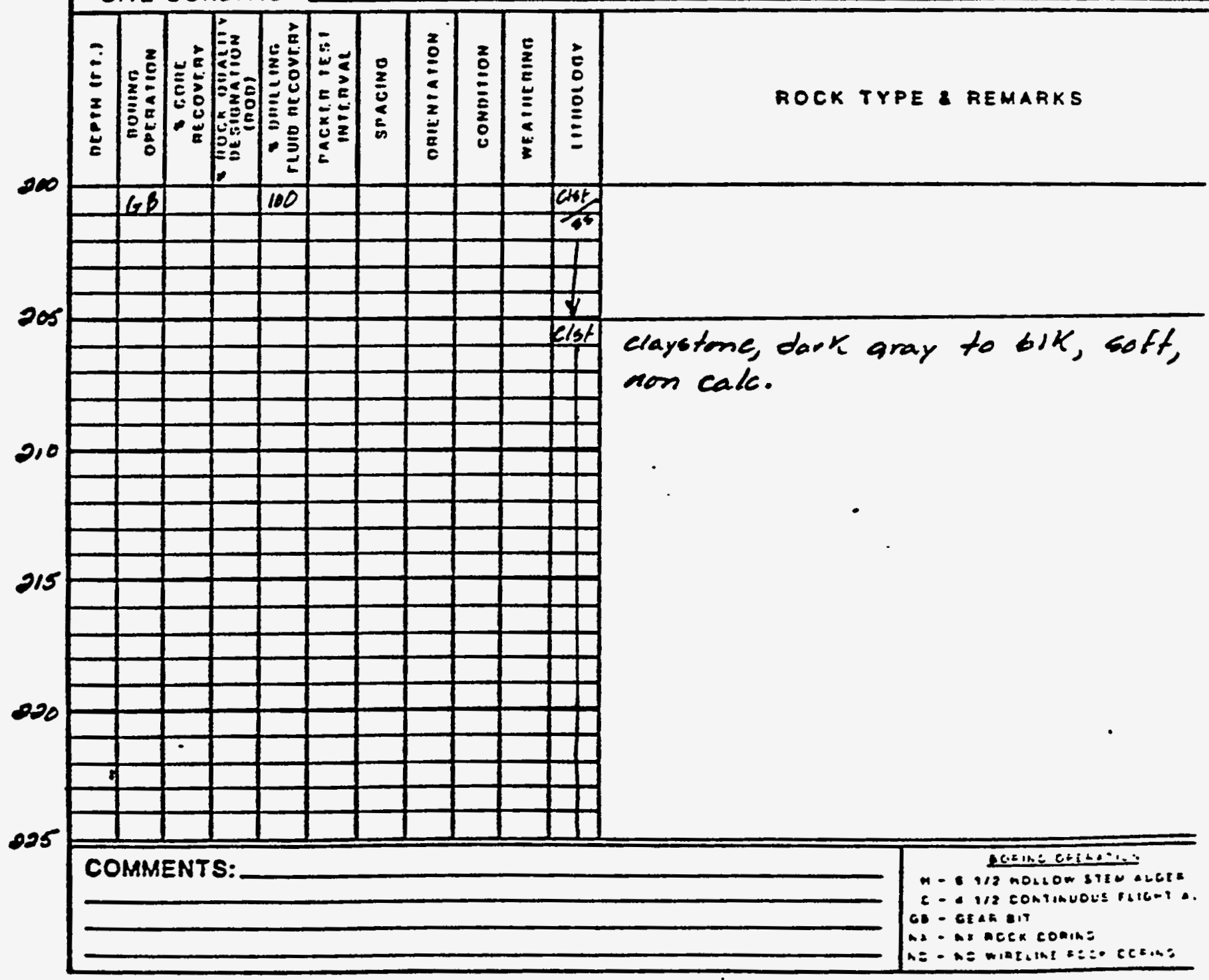

JEG-AL-ENG-2A (4/85) 


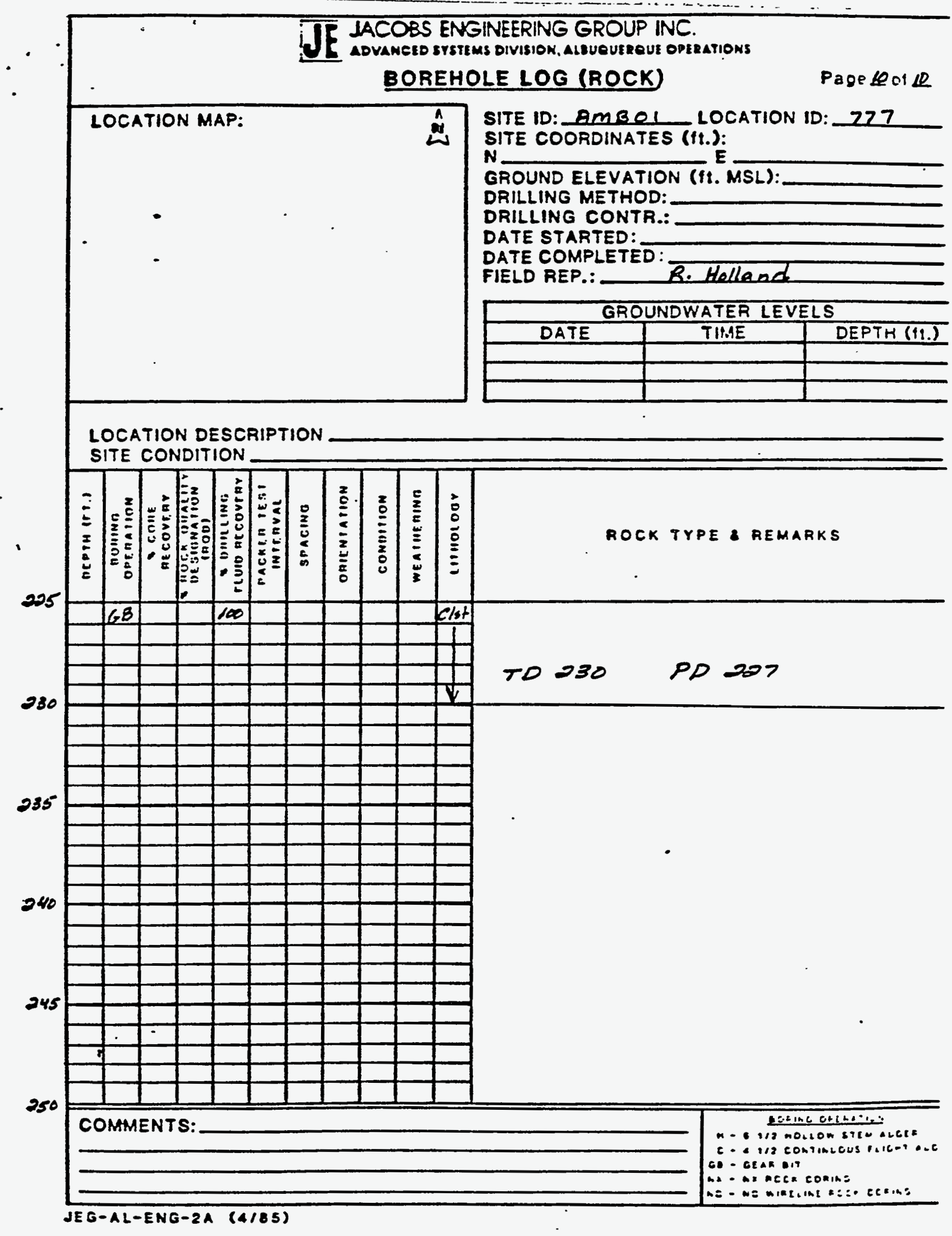




\section{TT MCOSS BRENEERT GROUP NK.

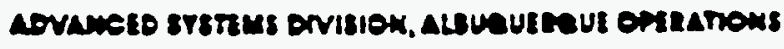

\section{WELL COMPLETION RECORD}

SITE ID:AMBOI LOCATION ID: 277 APPROX. SITE COORDINATES:(FT.) N NK

DATE INSTALLED: $9 / 4 / 85$ OPEN AREA PER LINEAL FT. (IN2/FT.) $P^{\prime \prime}$ hele FORMATION OF COMPLETION: Tres Hermanes $X B$ FIELD-AEP.: Reger follond DRILLER: stewart Bre.

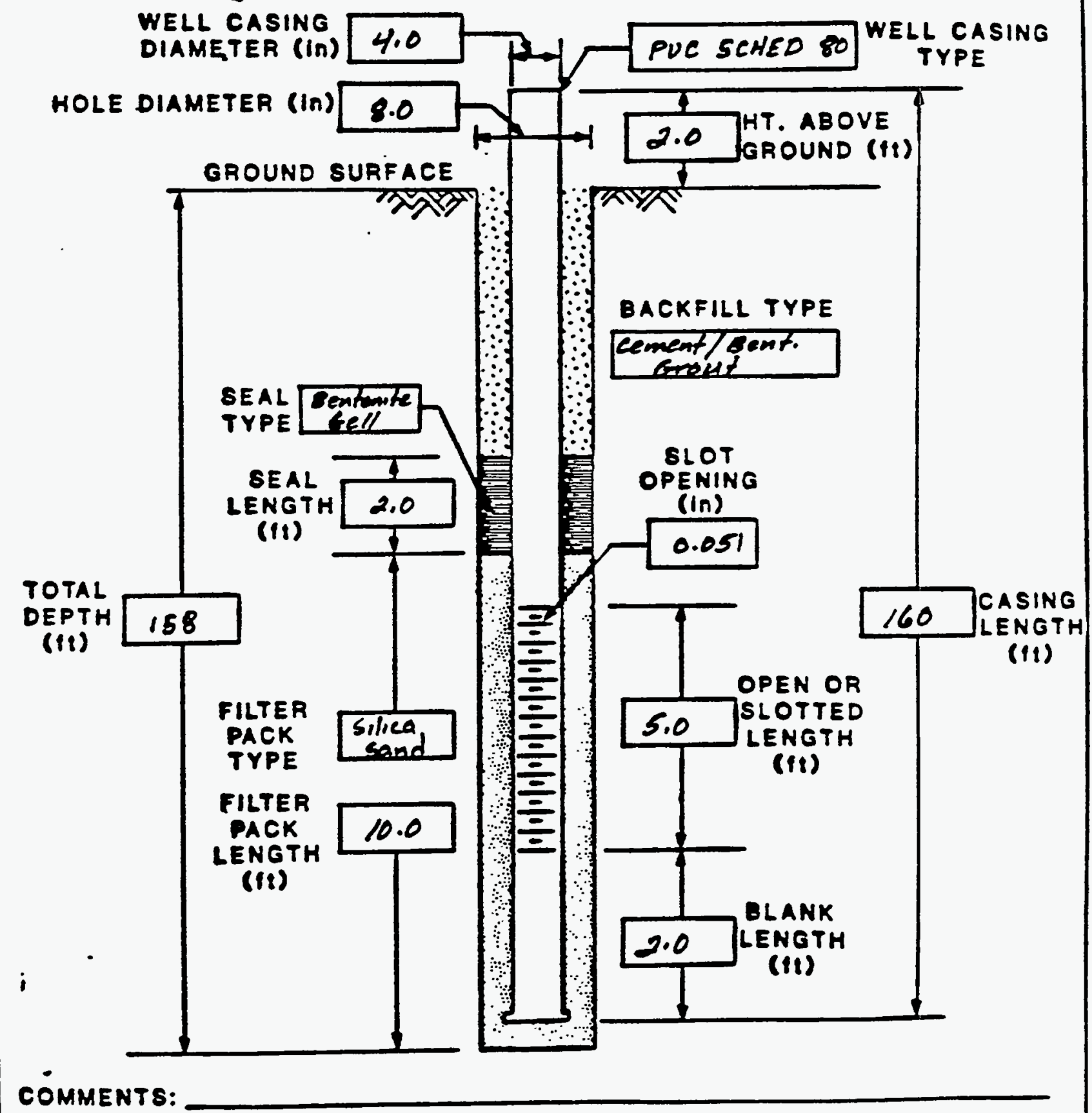




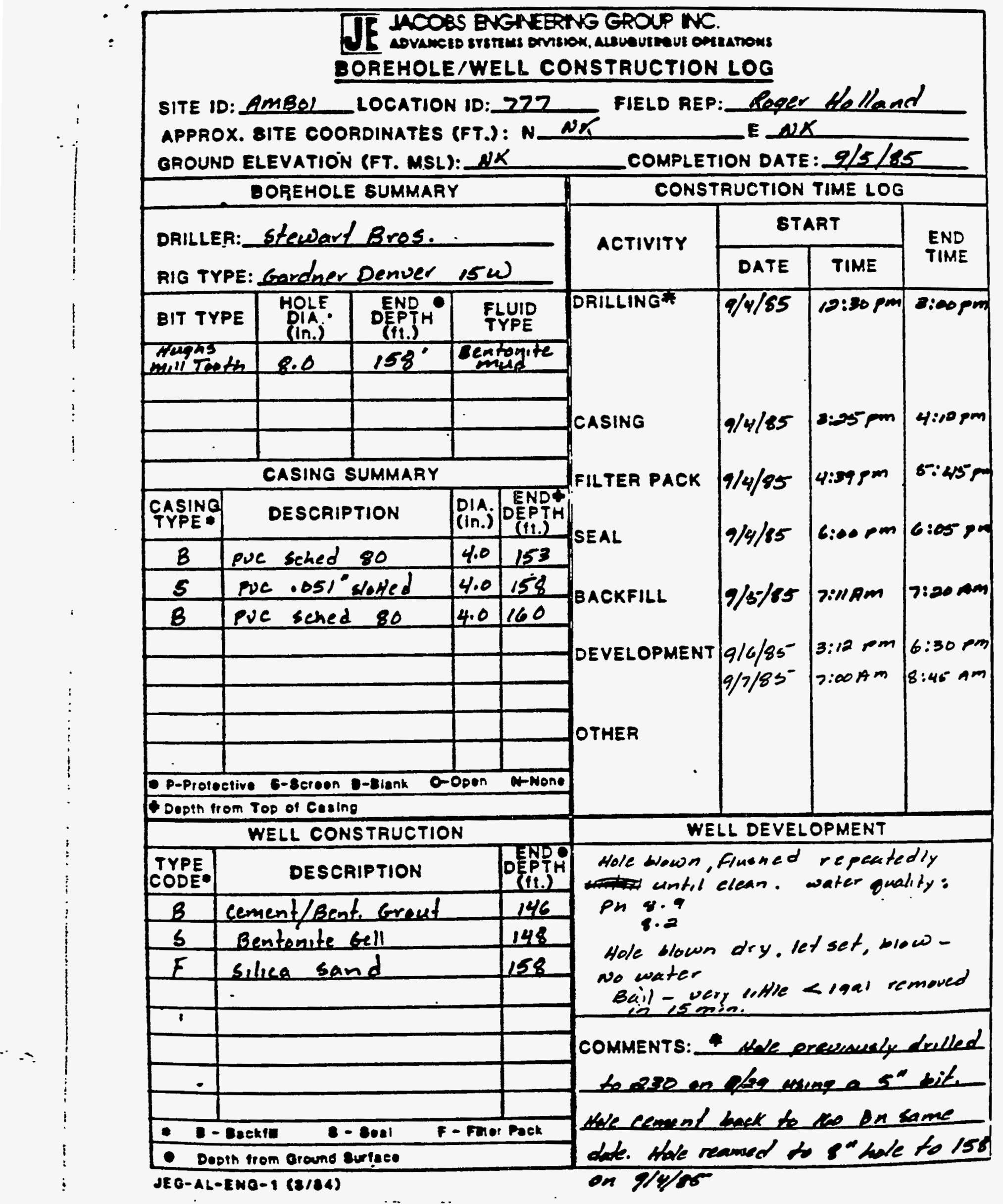


WELL 778 LOGS 


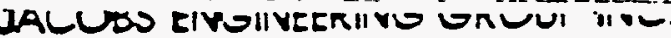

ADVANCED SYSTIMS DIVISIOK. ALUUEURRQUE OPERATIOKS

BOREHOLE LOG (ROCK)

Page 1 or 1

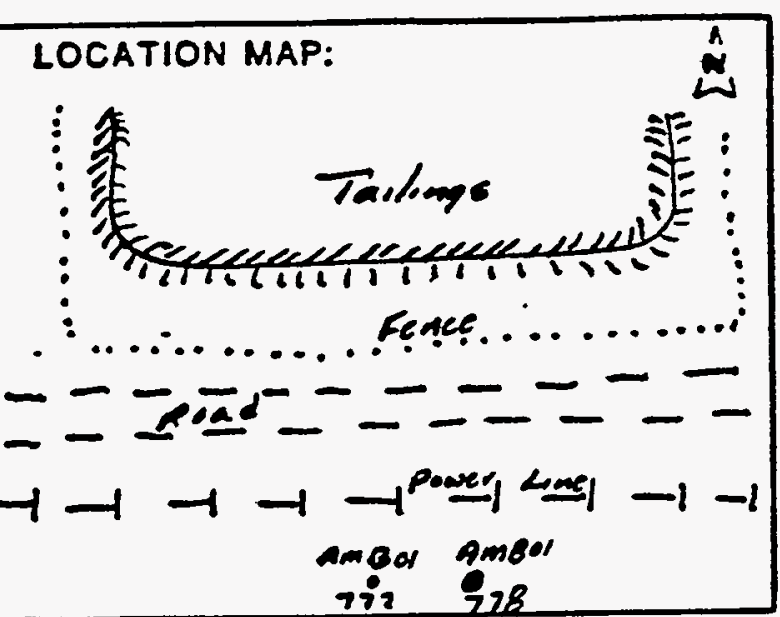

SITE ID:AMBOL LOCATION ID: 778 SITE COORDINATES (ft.):

$N$ NK $E$ NK

GROUND ELEVATION (11. MSL): NK

DRILLING METHOD: Retary - Gear Bit

DRILLING CONTR.: stewart Rre.

DATE STARTED:

$8 / 30 / 85$

DATE COMPLETEP: 8/30/85

FIELD REP.: Peger fowlend

\begin{tabular}{|c|c|c|}
\hline \multicolumn{3}{|c}{ GROUNDWATER LEVELS } \\
\hline DATE & THME & DEPTH (11.) \\
\hline & & \\
\hline & & \\
\hline & & \\
\hline
\end{tabular}

LOCATION DESCRIPTION Approx $300^{\circ}$ sen ef the se. Side of Tailings pile SITE CONDITION hevel losmy soll weth weed and grass cever

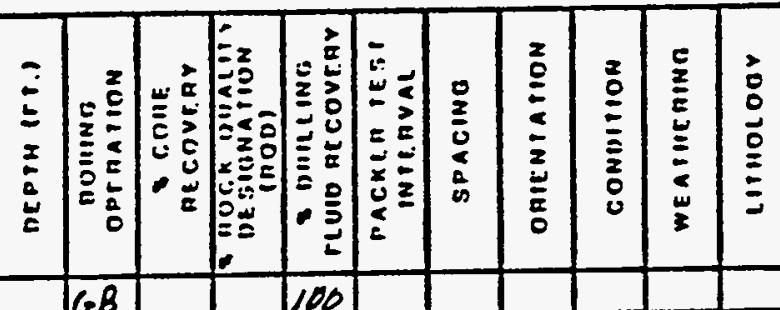

ROCK TYPE \& REMARKS

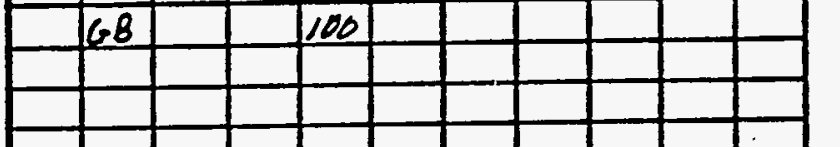

see lithology log for bore hole $7>7$.

Hole drolled to 37 feet.

Screen set in sandy gravel

zone - same zone as suree in 776 .

JEG-AL-ENG-2A (4/85) 


\section{TT MCOBS ENENEERNG GROUP NC.

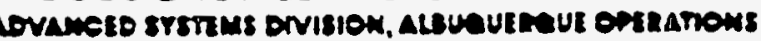

\section{WELL COMPLETION RECORD}

SITE ID: AMBOL LOCATION ID: 728 APPROX. SITE COORDINATES:(FT.) N NK

DATE INSTALLED: $8 / 30 / 85$ OPEN AREA PER LINEAL FT. (IN2/FT.) S.0"bore hole 4.0 "OR casine FORMATION OF COMPLETION: IRCI FIELD-REP.: Peger Helland ORILLER: Stewart Bro.

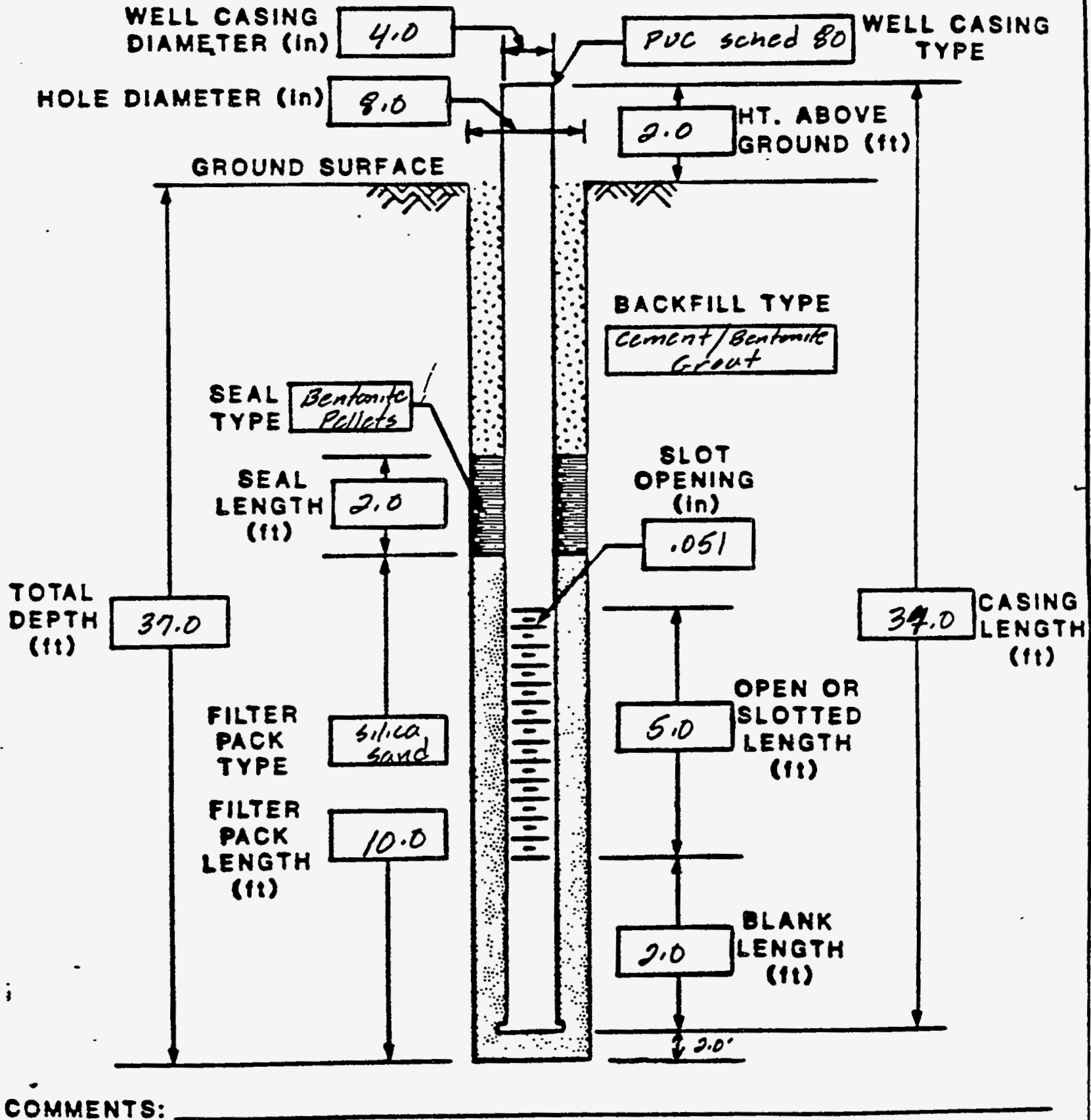




\section{TF JCOBS BNGNEERTE GROUP INC.

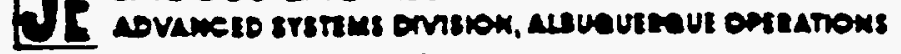 \\ BOREHOLE/WELL CONSTRUCTION LOG}

SITE ID: AmBOL LOCATION ID: 278 FIELD REP: feper Holland APPROX. BITE COORDINATES (FT.): N NK

GROUND ELEVATION (FT. MSL): NK BOREHOLE SUMMARY

DRILLER:_Stewart Bro.(Albertho) RIG TYPE:

\begin{tabular}{|c|c|c|c|}
\hline BIT TYPE & $\begin{array}{c}\text { HODLE } \\
\text { DIA } \\
\text { (In.) }\end{array}$ & $\begin{array}{c}\text { END } \\
\text { DEPTH } \\
\text { (fi.) }\end{array}$ & $\begin{array}{c}\text { FLUID } \\
\text { TYPE }\end{array}$ \\
\hline HughS & 8.0 & 37.0 & Bentonite \\
\hline & & & \\
\hline & & & \\
\hline & & & \\
\hline & & & \\
\hline & \multicolumn{3}{|c|}{ CASING SUMMARY } \\
\hline
\end{tabular}

\begin{tabular}{|c|c|c|c|}
\hline $\begin{array}{c}\text { CASING } \\
\text { TYPEE }\end{array}$ & DESCRIPTION & $\begin{array}{c}\text { DIA. } \\
\text { (In.) }\end{array}$ & $\begin{array}{c}\text { ENDT } \\
\text { DEPTH } \\
\text { (It.) }\end{array}$ \\
\hline$B$ & PUC SChed. 80 & 4.0 & 30.0 \\
\hline$S$ & Well screen PUC .051" & 4.0 & 35.0 \\
\hline & & & \\
\hline$B$ & PUC SChed.80 & 4.0 & 37.0 \\
\hline & & & \\
\hline & & & \\
\hline & & & \\
\hline & & & \\
\hline
\end{tabular}

\begin{tabular}{|c|c|c|}
\hline \multicolumn{3}{|c|}{ WELL CONSTRUCTION } \\
\hline $\begin{array}{l}\text { TYPE } \\
\text { CODE- }\end{array}$ & DESCAIPTION & DEPTH \\
\hline $\bar{B}$ & cement/aenterite Grout & 250 \\
\hline 5 & Bentonite pellets & 27.6 \\
\hline LF & slece sand & 37.0 \\
\hline & - & \\
\hline T & & \\
\hline & & \\
\hline - & & \\
\hline & & \\
\hline .8 & $2-8.0 .1$ & Paek \\
\hline - De & pth from Ground surface & \\
\hline
\end{tabular}

COMPLETION DATE: $8 / 30 / 85$ CONSTRUCTION TIME LOG

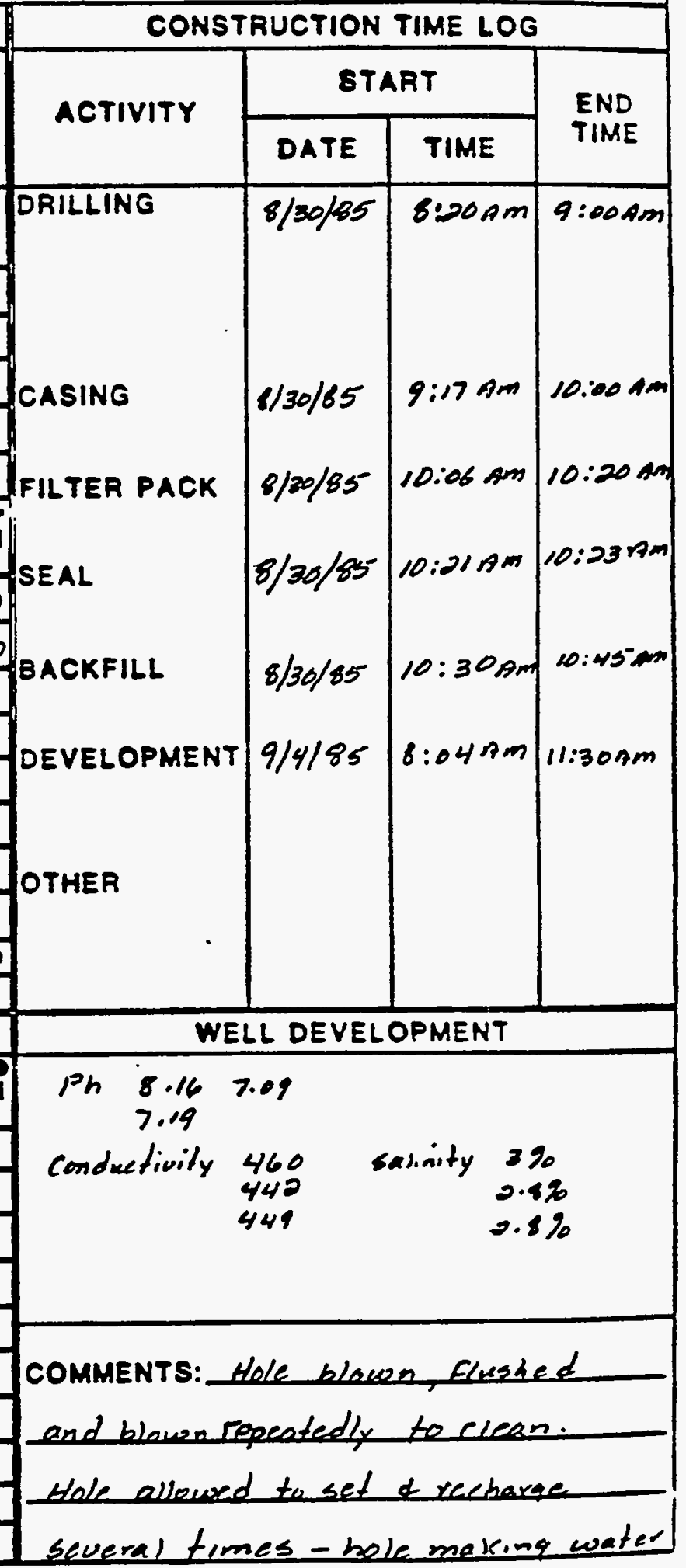


WELL 779 LOGS 


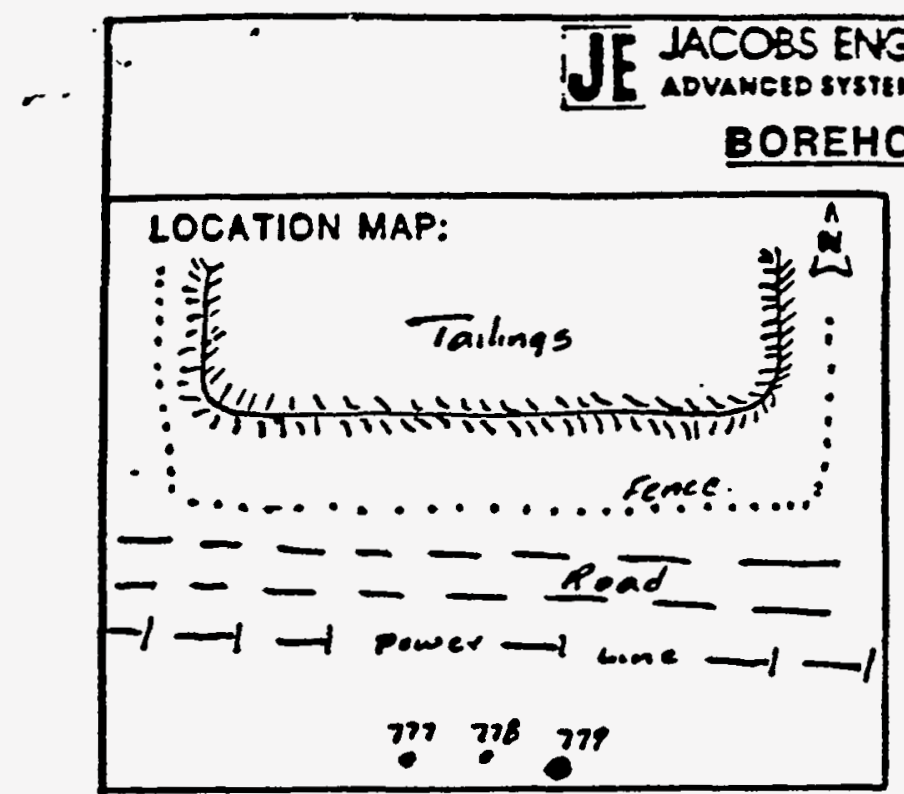

LOCATION DESCRIPTION Rperer 300 'sa. of se. side at Tauhags pile SITE CONDITION Lewel with weed and gcass cauer

\section{DATE COMPLETED}

FIELD REP.: Baper Holland

\begin{tabular}{|c|c|c}
\hline \multicolumn{3}{|c}{ GROUNDWATER LEVELS } \\
\hline DATE & THPAE & DEPTH (11.) \\
\hline & & \\
\hline & & \\
\hline & & \\
\hline
\end{tabular}

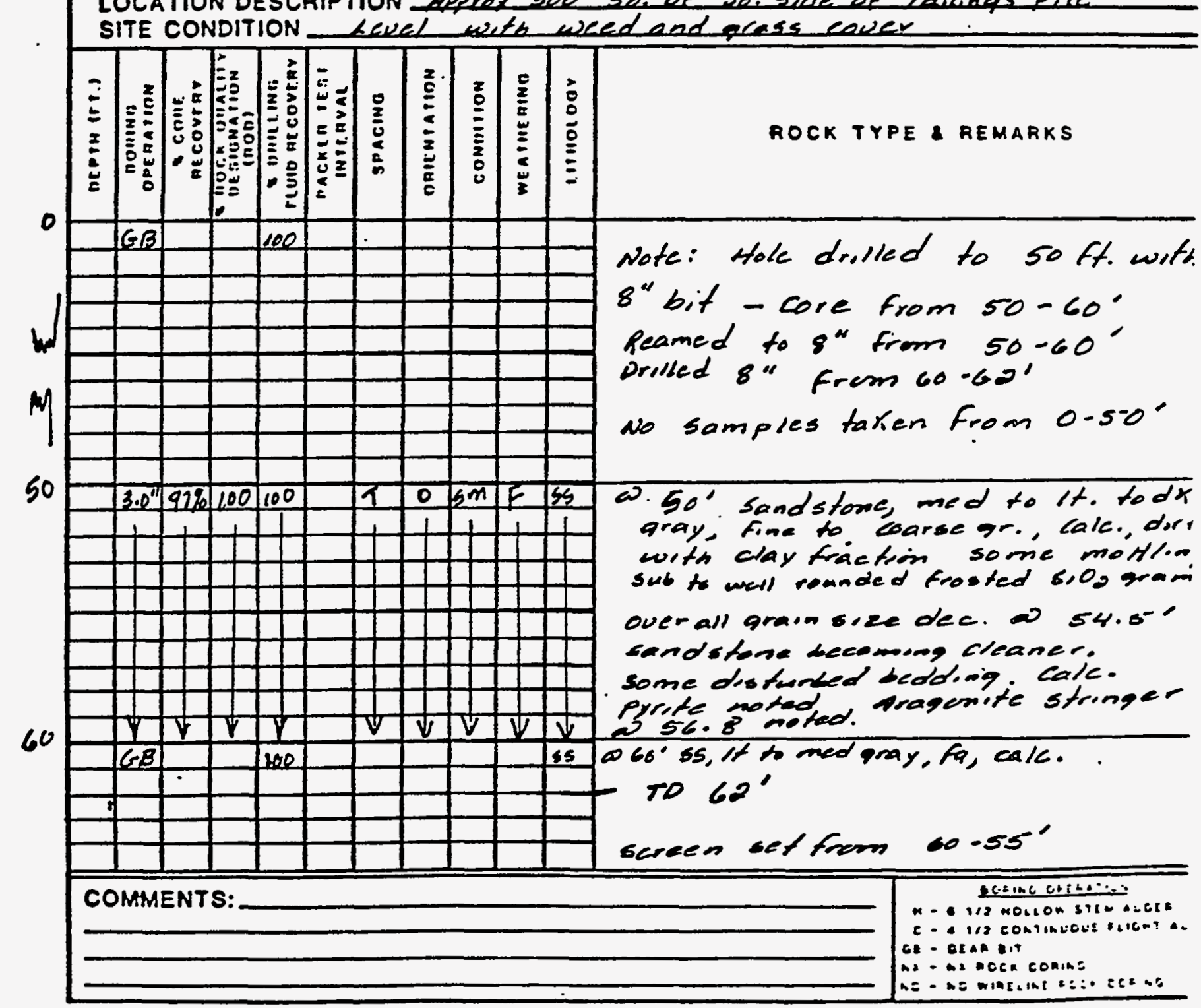

JEG-AL-ENG-2A (A/85) 


\section{TT HCO25 ENGNERIV EROUP NK.

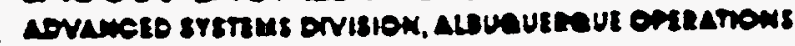

\section{WELL COMPLETION RECORD}

SITE ID:AMBOL LOCATION ID: 729 APPROX. SITE COORDINATES:(FT.) N NK DATE INSTALLED: $2 / 5 / 85$

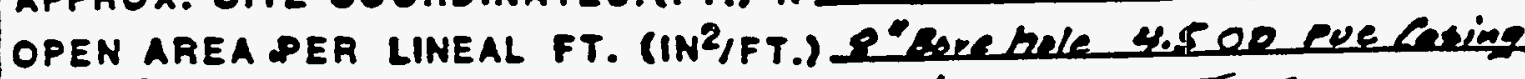
FORMATION OF COMPLETION: TRes termands $C 2$ FIELD-REP.: Paper Hollond DRILLER: Stewbet Bro.

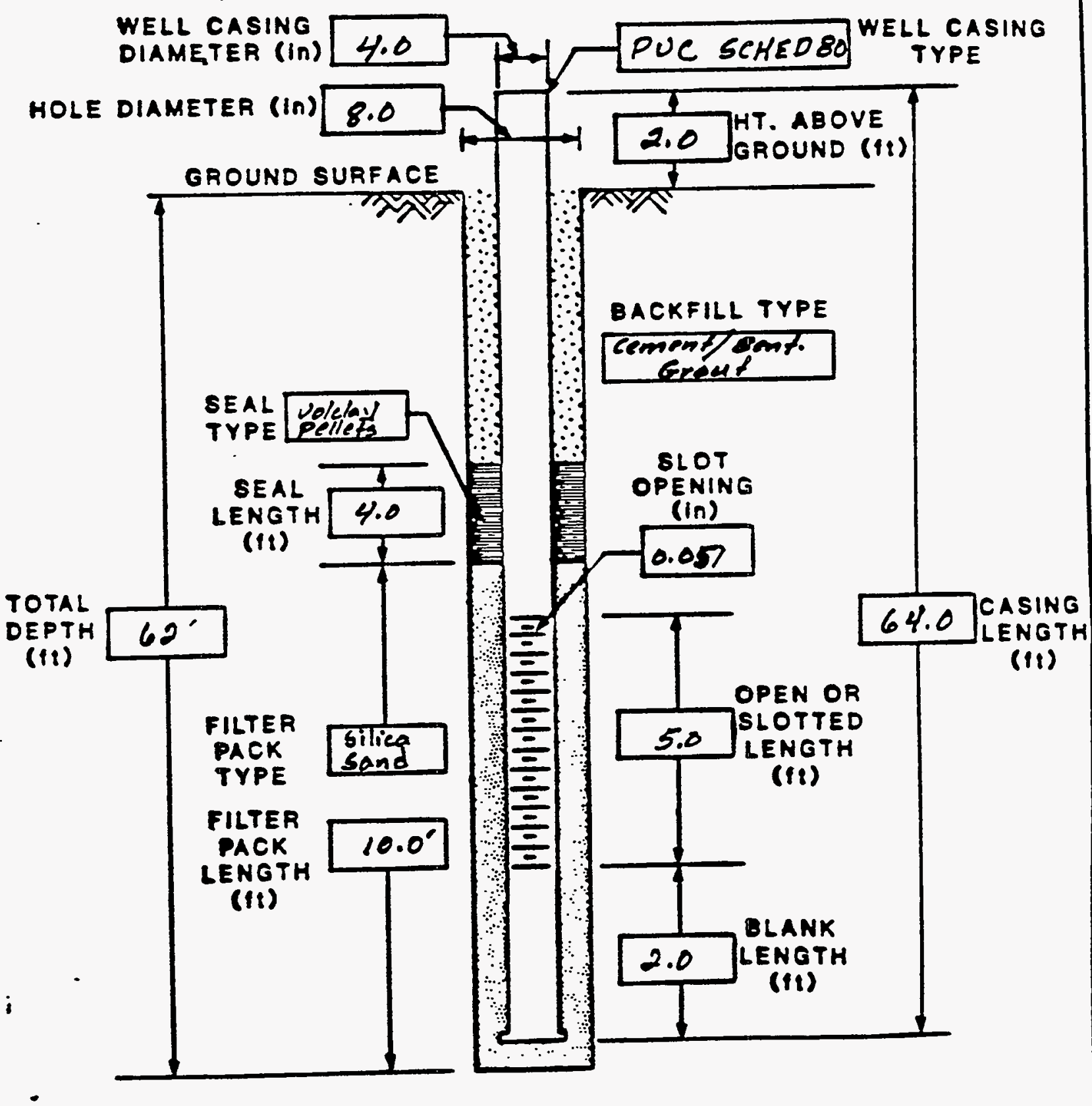

COMMENTS: 


\section{TF JCOSS BNGNEERTE GROUP INC.

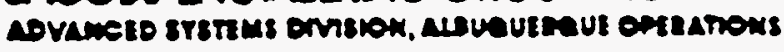 BOREHOLE/WELL CONSTRUCTION LOG}

SITE ID: AmBA LOCATION ID: 279 FIELD REP: Peger flelland APPROX. BITE COORDINATES (FT.): N_NK GROUND ELEVATION (F́T. MSL): NK COMPLETION DATE: $9 / 5 / 85$

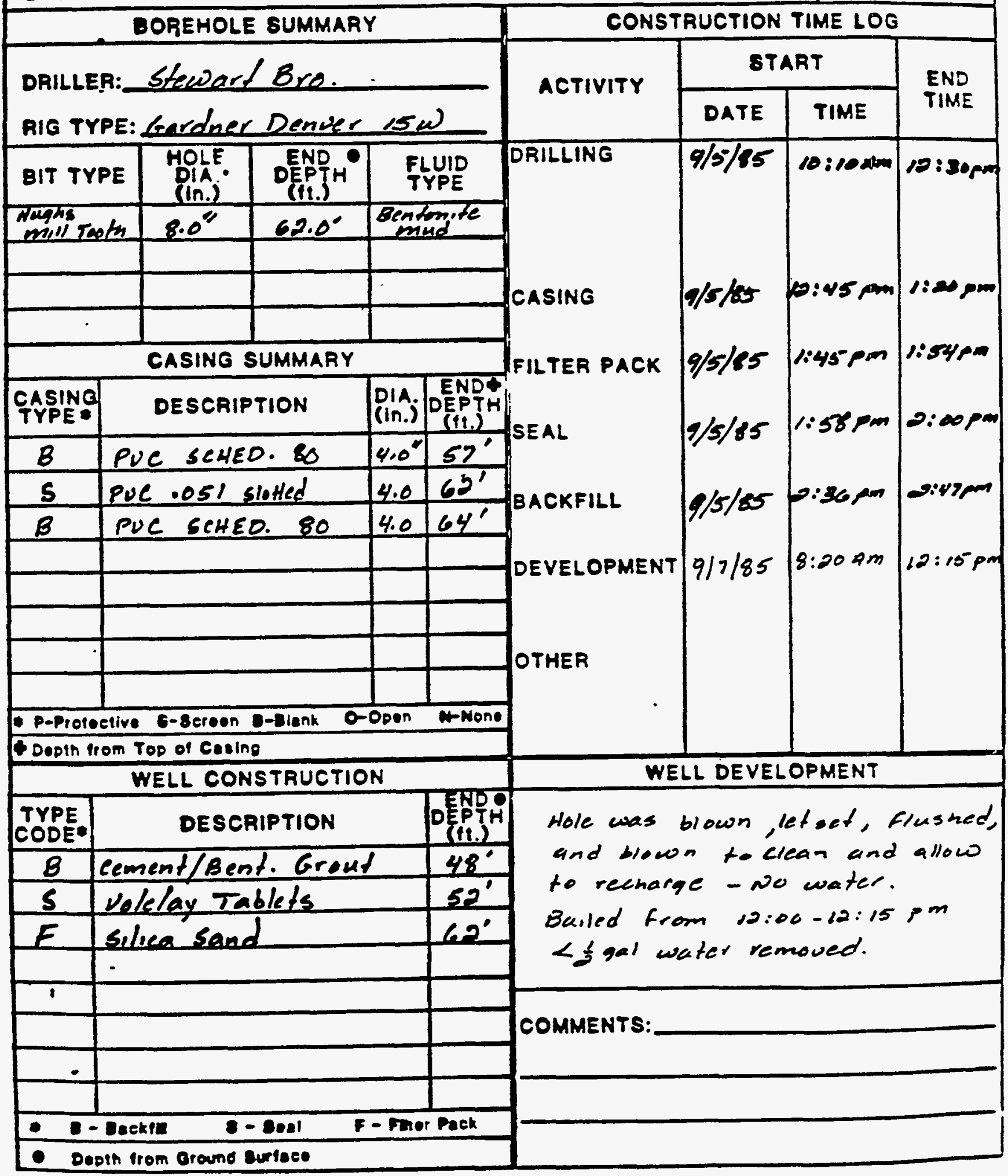


WELL 781 LOGS 


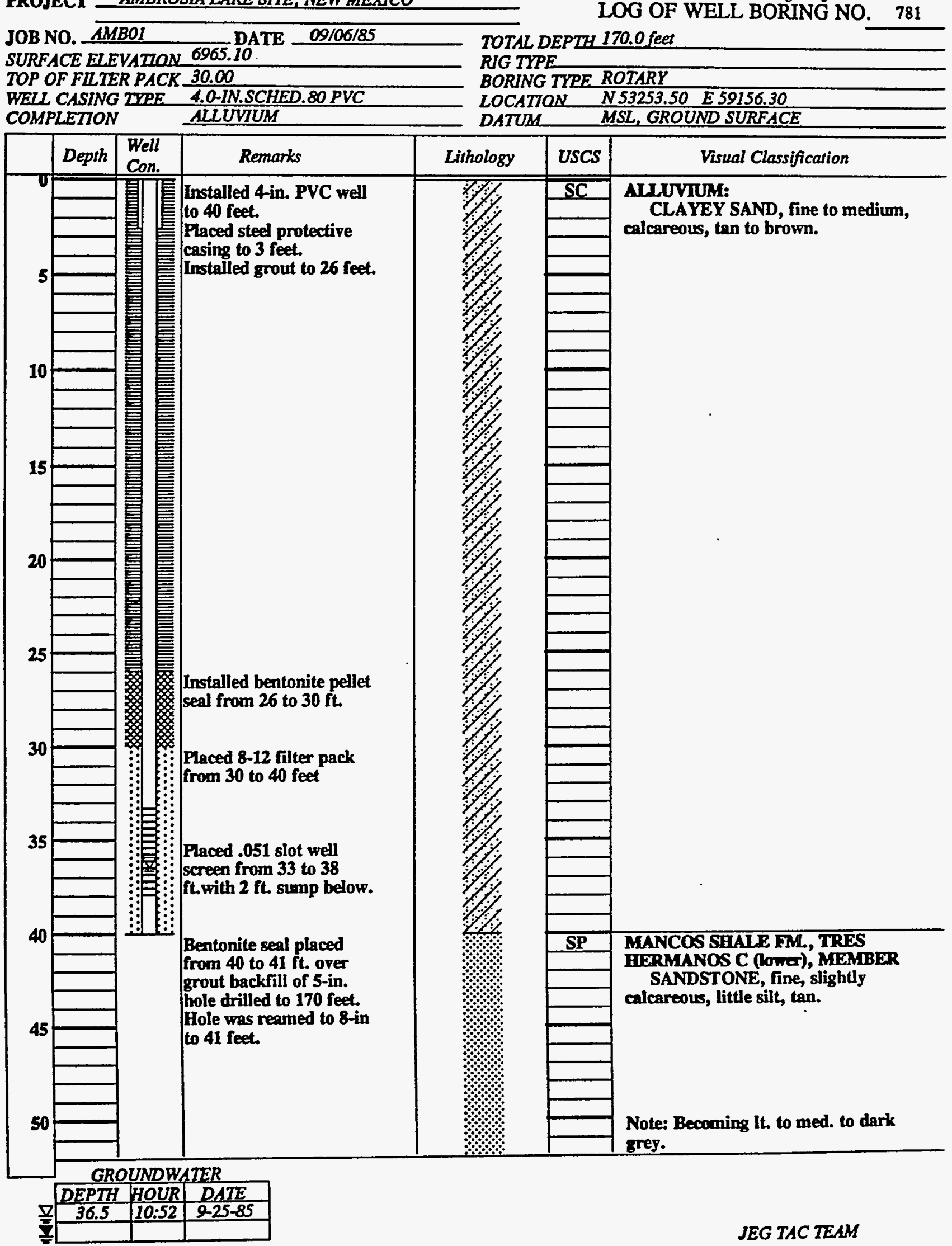




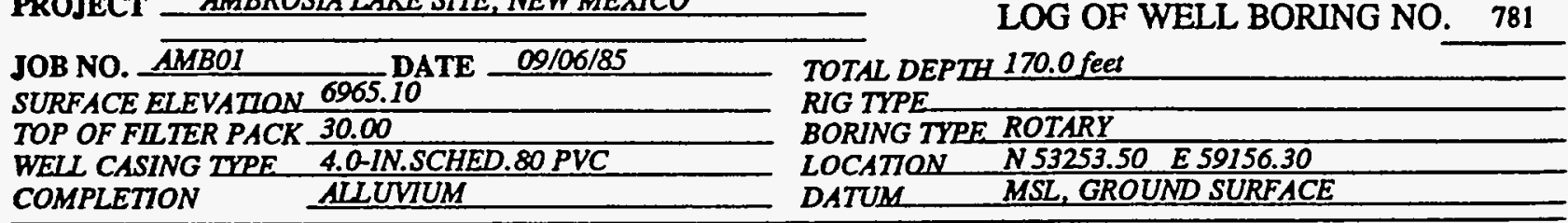

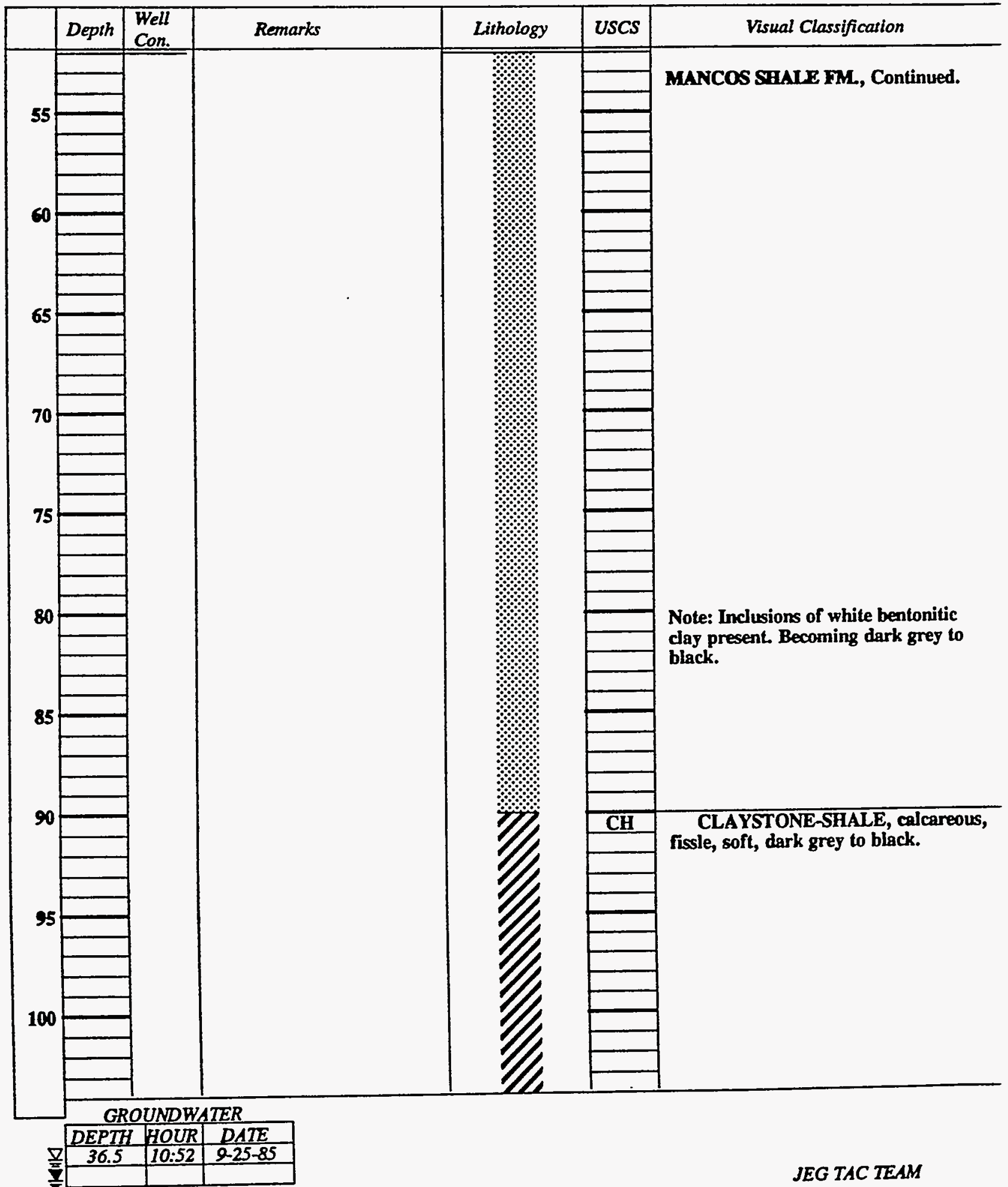




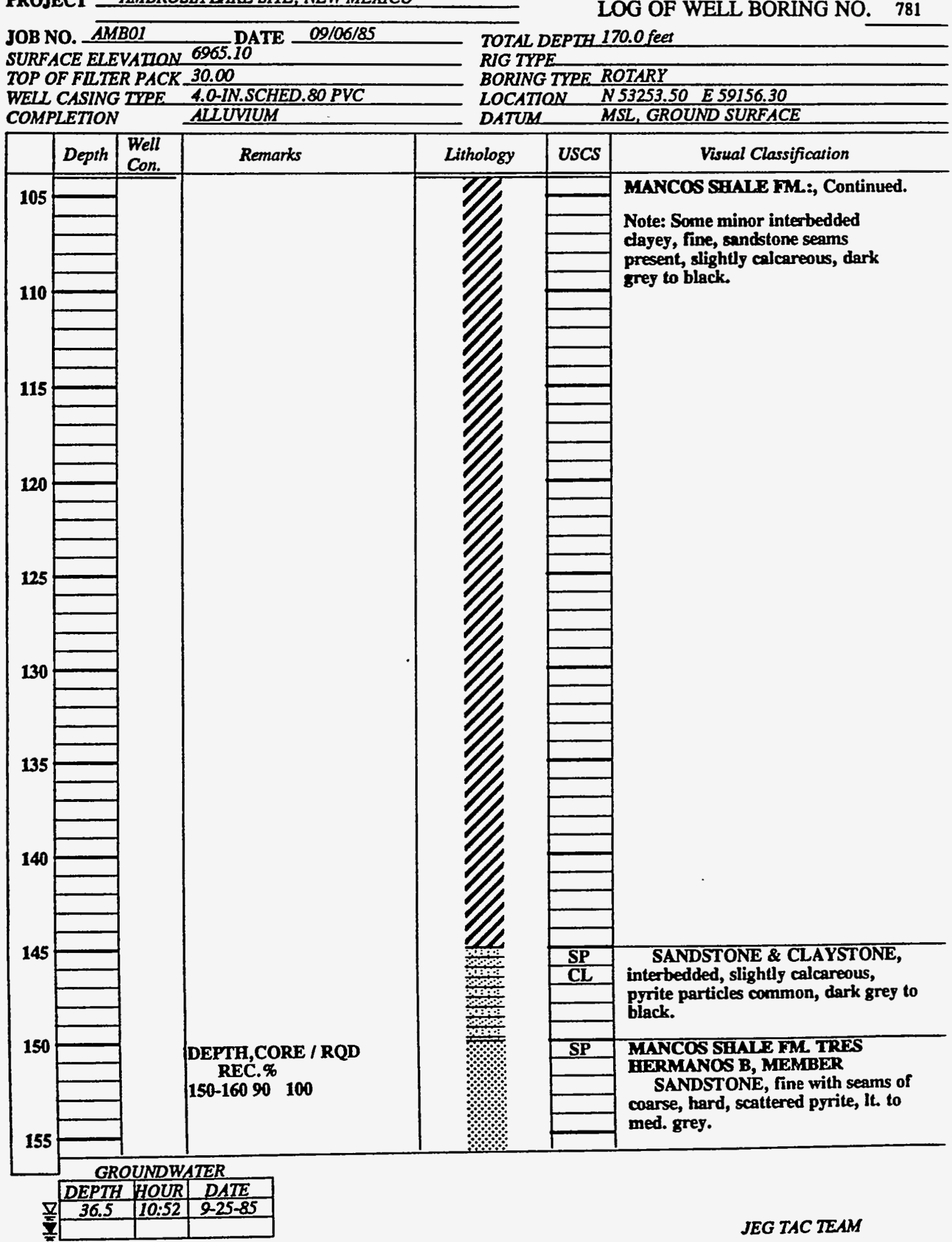



JOB NO. AMBOI DATE 09/06/85
SURFACE ELEVATION 6965.10

TOP OF FILTER PACK 30.00

WELL CASING TYPE 4.0-IN.SCHED.80 PVC COMPLETION ALLUVIUM

LOG OF WELL BORING NO

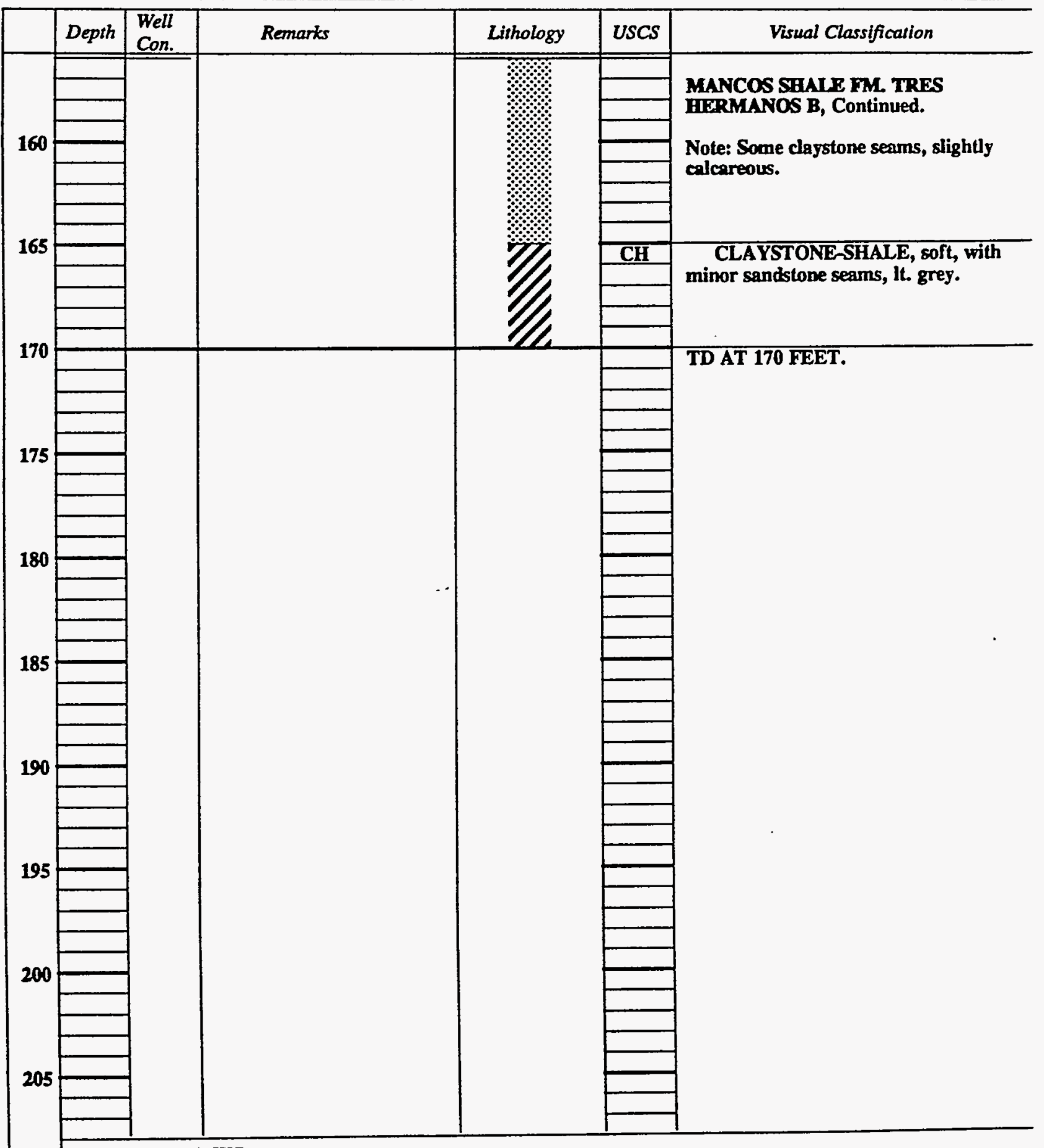

GROUNDWATER

\begin{tabular}{|l|l|}
\hline DEPTH HOUR DATE \\
\hline
\end{tabular}

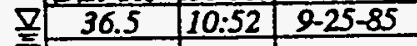




\section{TT HCOSS ENGNERTE GROUP NC.

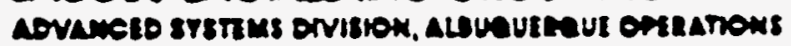

\section{WELL COMPLETION RECORD}

SITE ID: AMBOL LOCATION ID: 781 APPROX. SITE COOROINATES:(FT.) N NK
DATE INSTALLED: $q / 2 / 85$ E NK

OPEN AREA PER LINEAL FT. (IN"2/FT.) \&"Bore hole with 4.5"OD cosing FORMATION OF COMPLETION: FIELD -REP.: Regel $I$ thllond Alluviesm

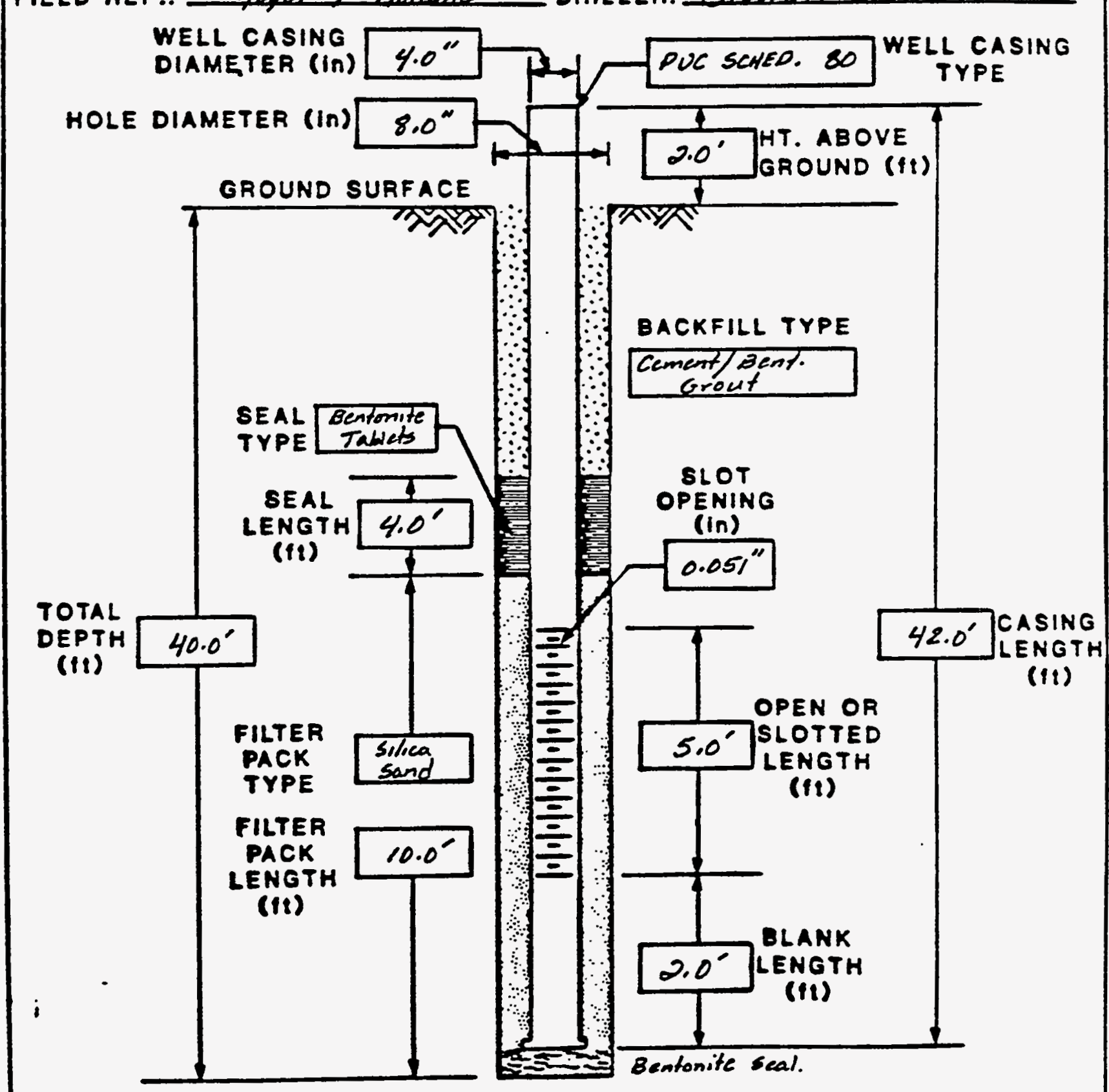

COMMENTS: A small amt of sertenite Tablets was pet in hole Prver to puteng in casing in erder te ised eff cement in botemef hote. 
WELL 782 LOGS 


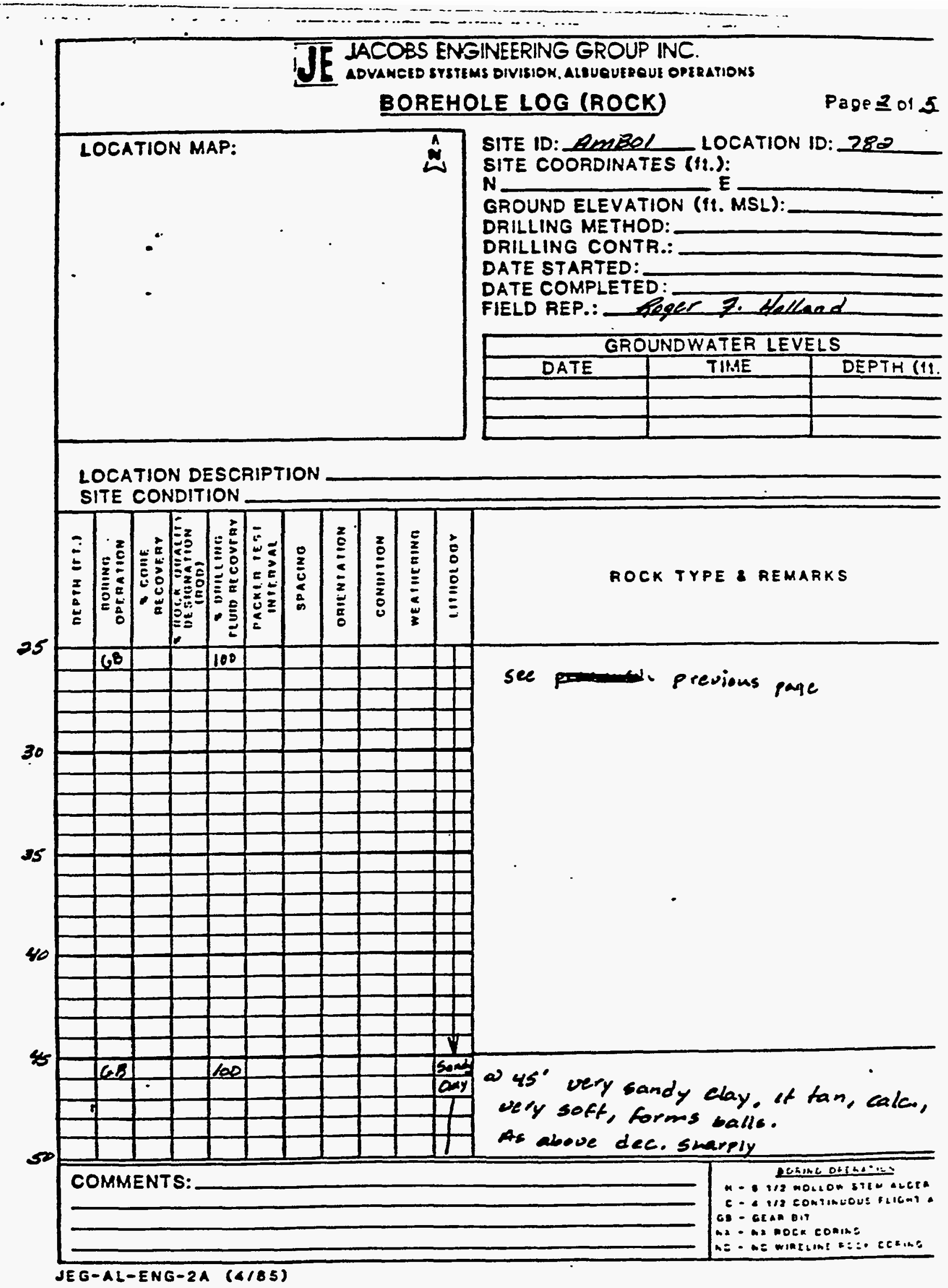




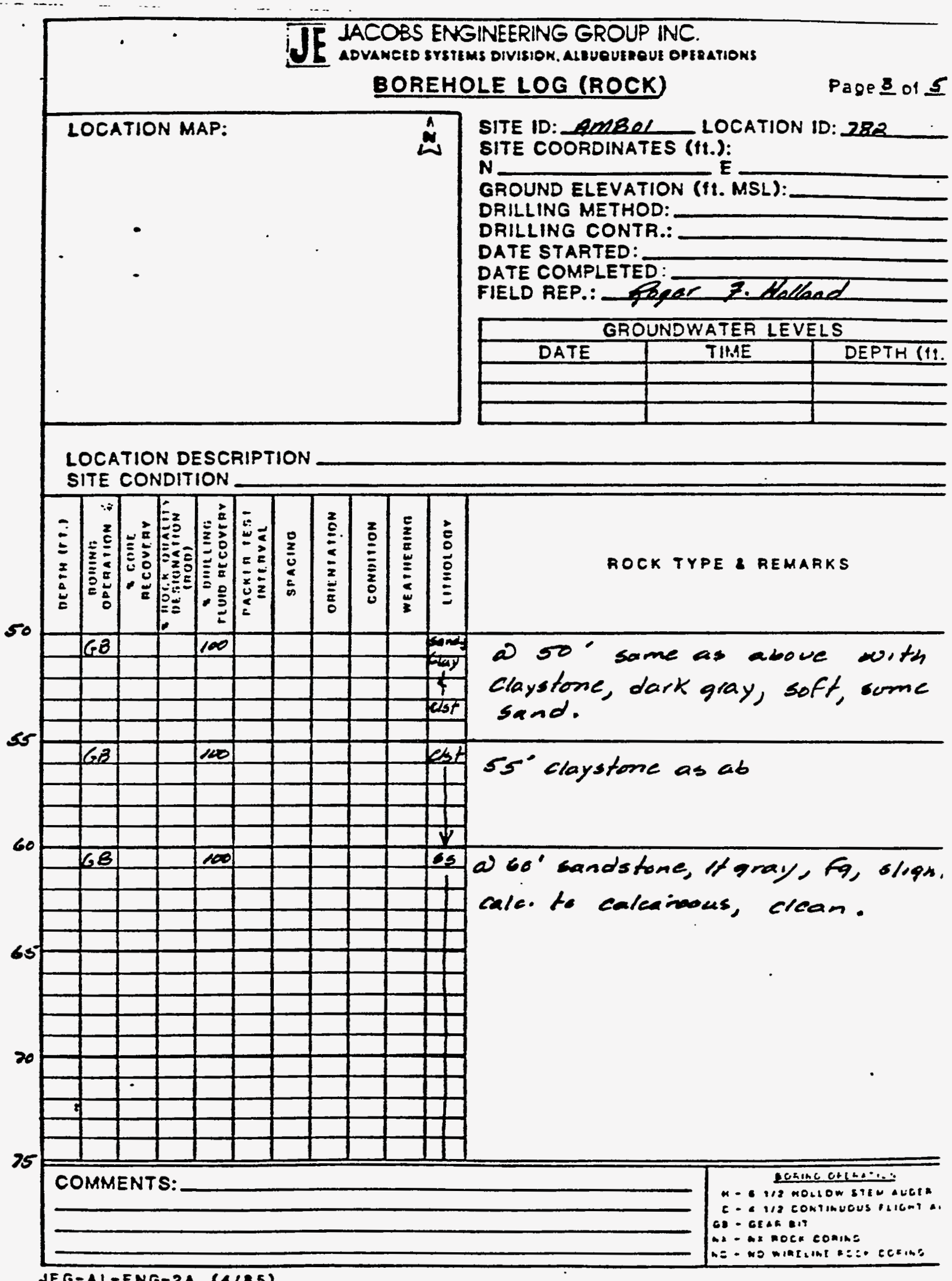




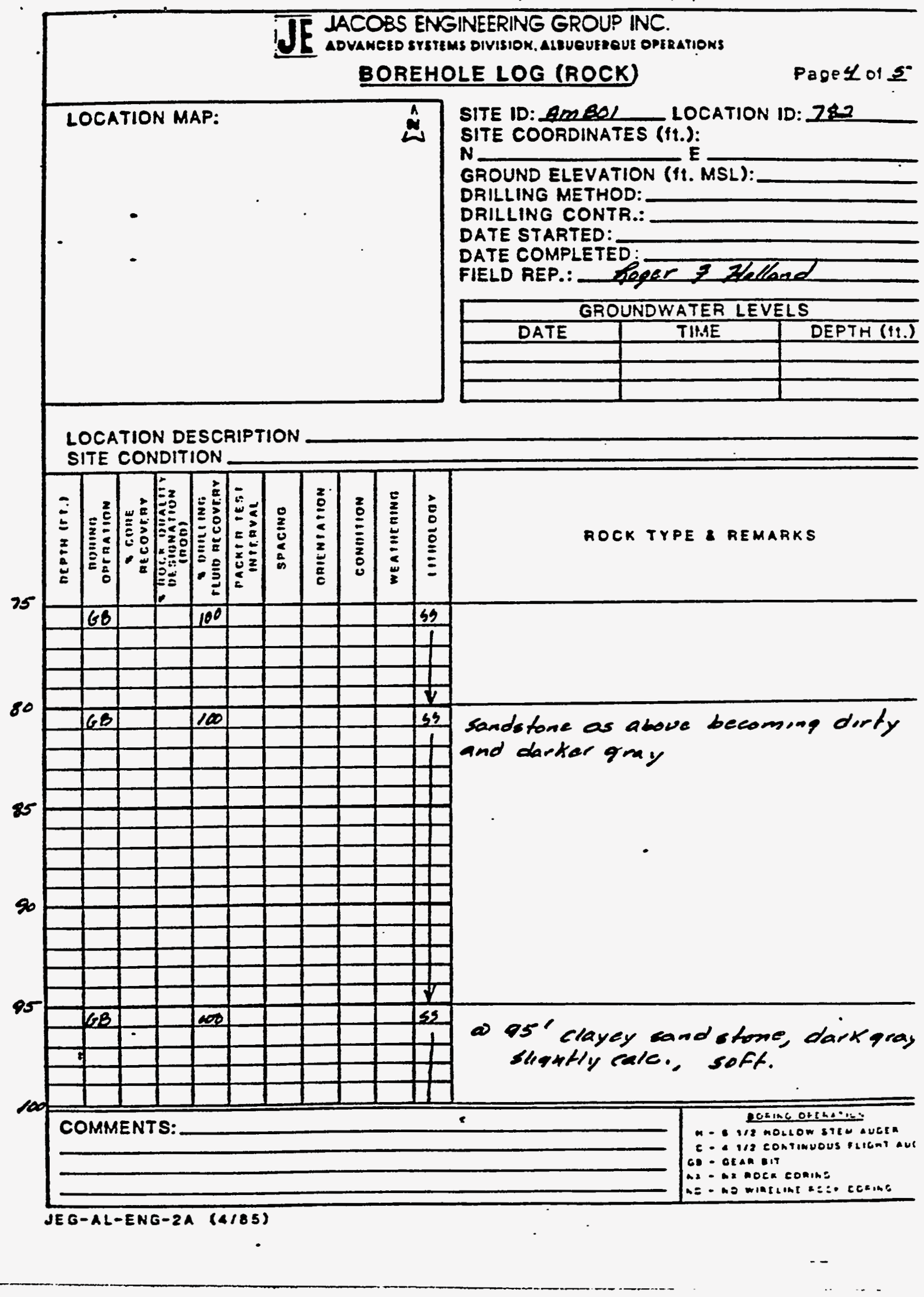




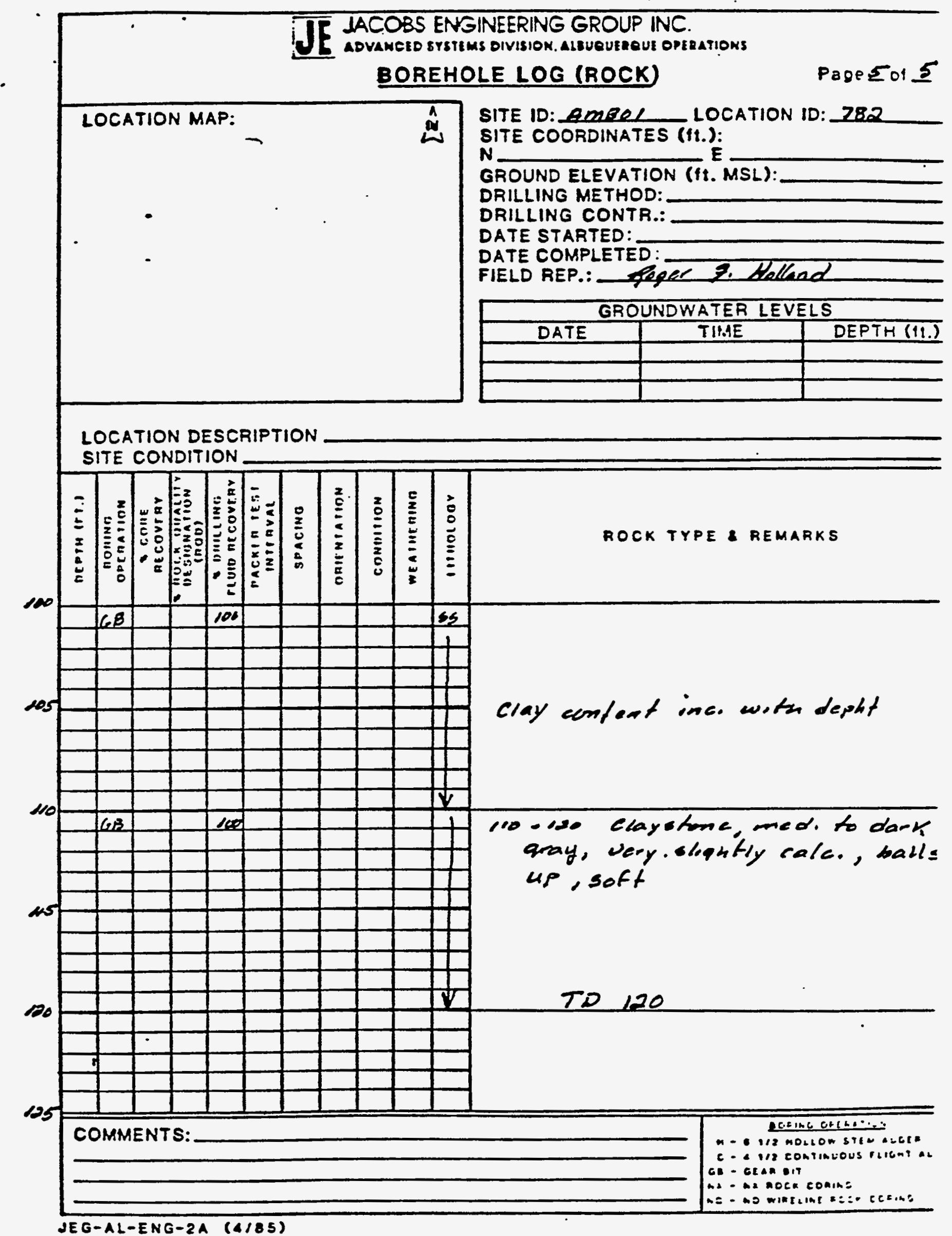




\section{TT HCOSS ENGNERTVG GROUP NK.

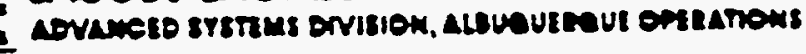

\section{WELL COMPLETION RECORD}

SITE ID: AMBOI LOCATION 10: APPROX. SITE COORDINATES:(FT.) N NK OPEN AREA PER LINEAL FT. (IN2/FT) $O^{*}$ FORMATION OF COMPLETION: Tres Hecenenes $\& C 2$

FIELD-REP.: Pager Halland DRILLER: Steweret Brethers

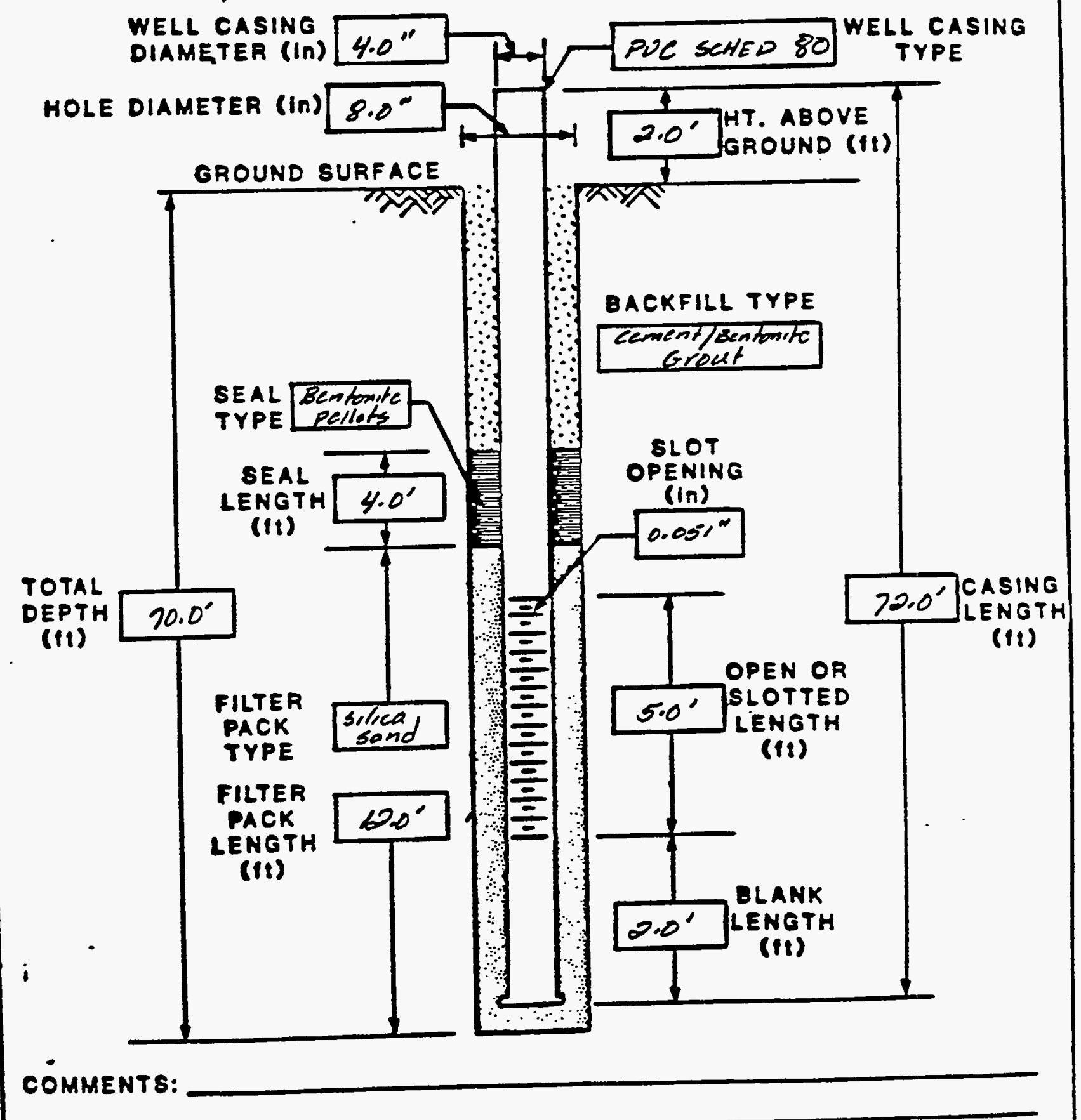

JEG-AL-ENO-S (P/BA)
DATE INSTALLED: $2 / 9 / 85$ E NK 


\section{TT HCOBS BNGN EERTIG GROUP NC.

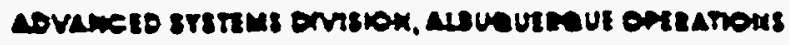 BOREHOLE/WELL CONSTRUCTION LOG}

SITE 10: AMBOI LOCATION ID: 782 FIELD REP: Beqer Helland APPROX. SITE COORDINATES (FT.): N.NK E NK GROUND ELEVATION (FT. MSL): NK COMPLETION DATE: $2 / 9 / 85$

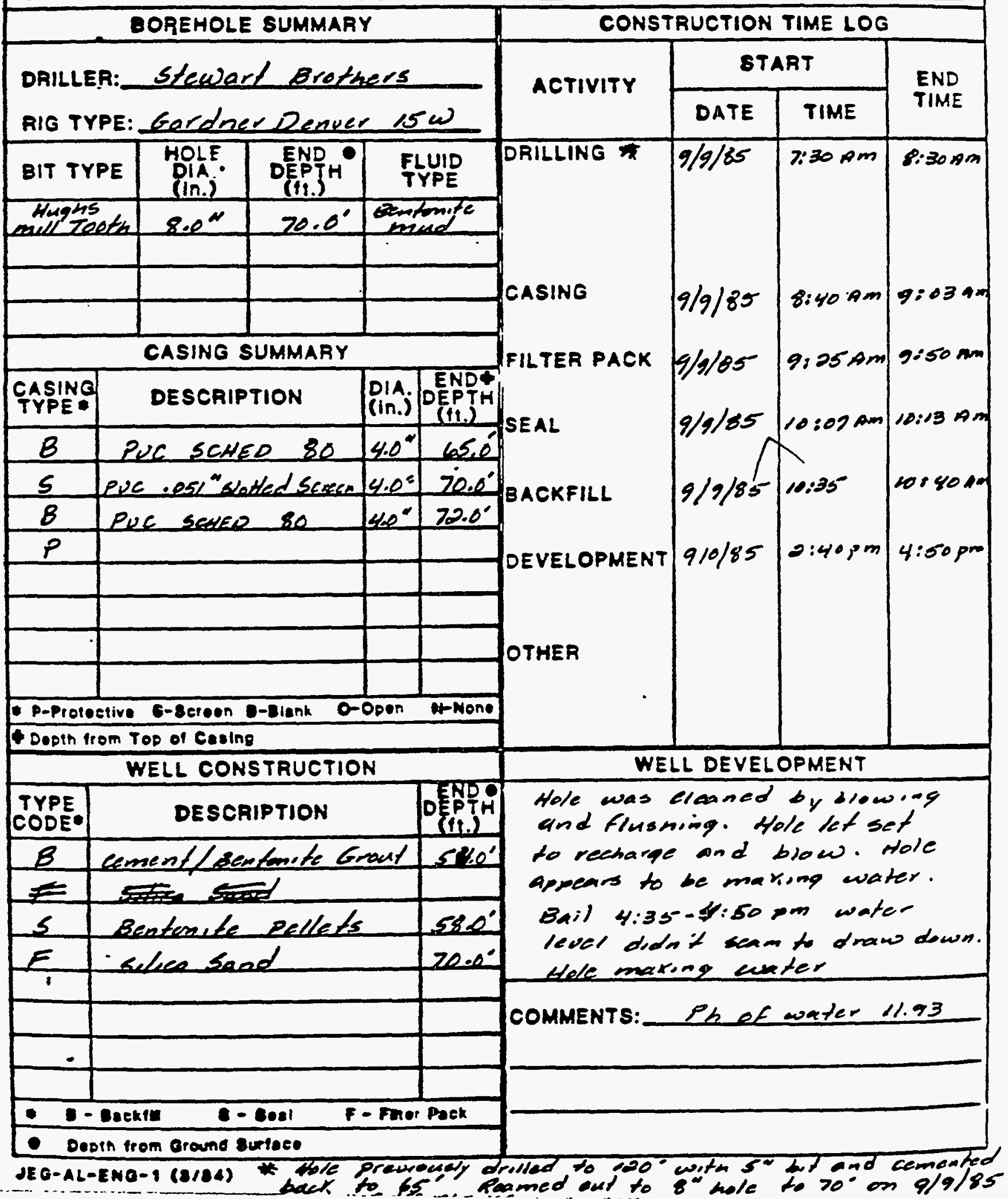


WELL 783 LOGS 


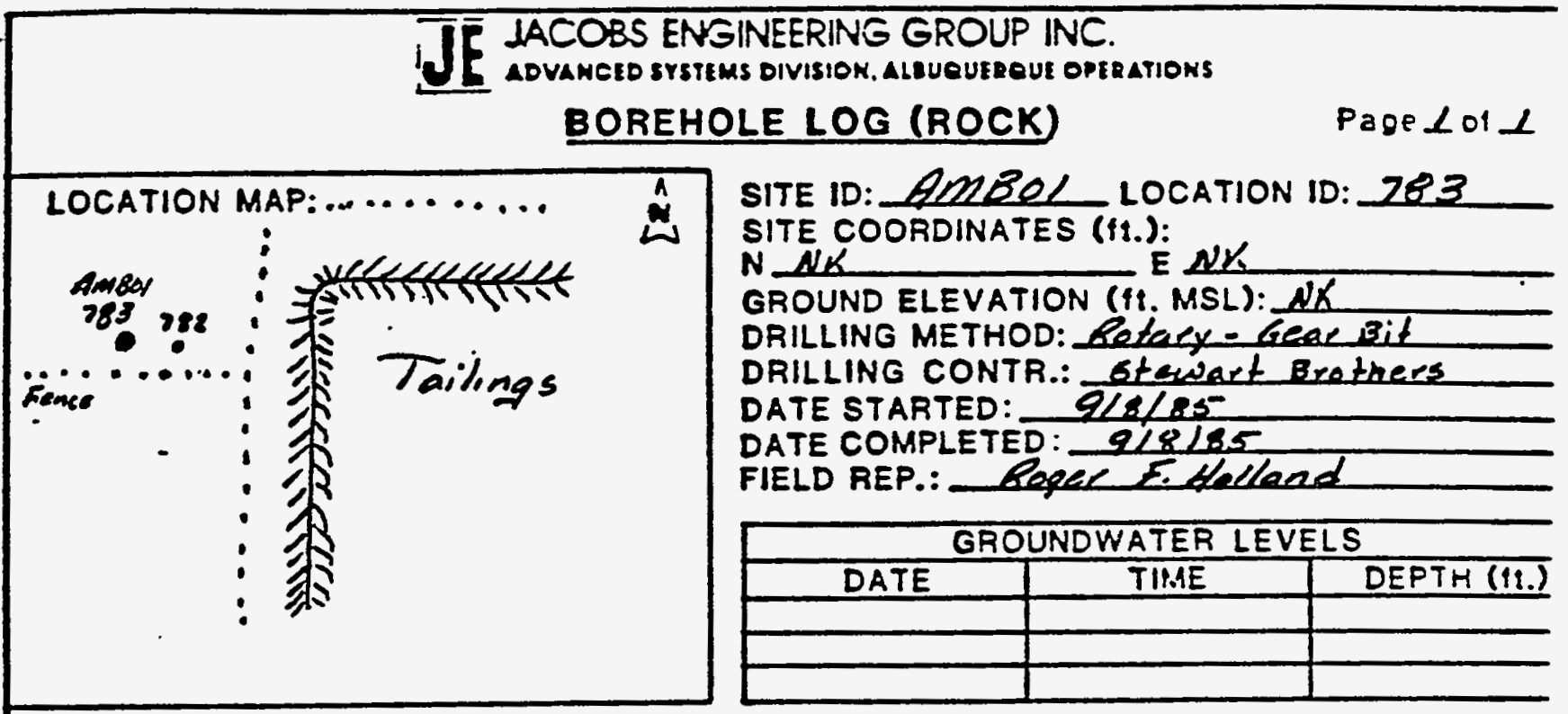

LOCATION DESCRIPTION west of the No west Cor of Touleage Pile SITE CONDITION Level weth arase and weed cover.

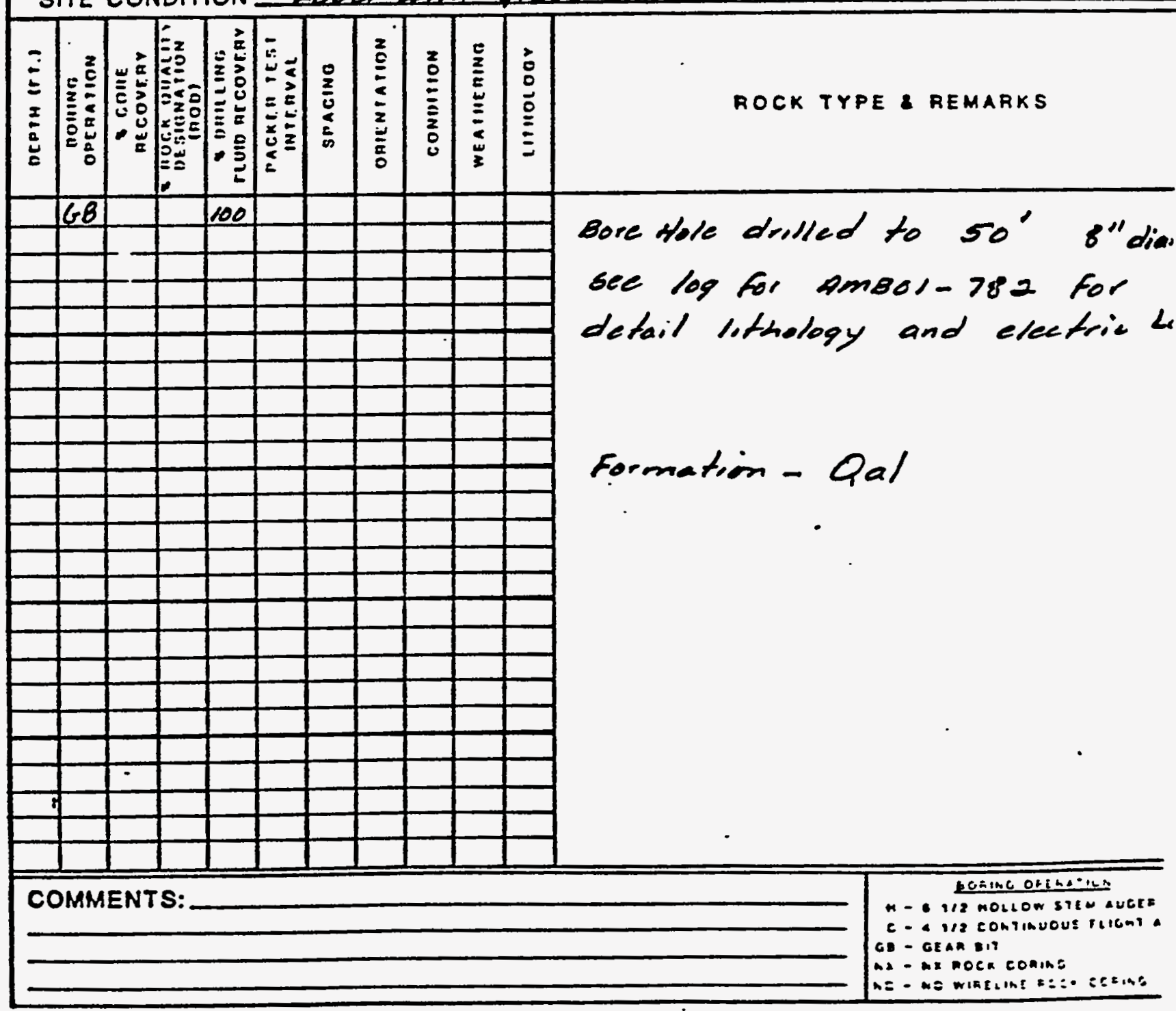

JEG-AL-ENG-2A (4/85) 


\section{TT MAOSS BRENERTIG GROUP NC.

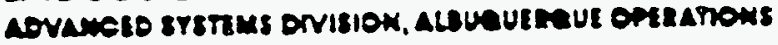

\section{WELL COMPLETION RECORD}

SITE ID: AMBOI LOCATION 1D: 783 DATE INSTALLED: $2 / 8 / 85$ APPROX. SITE COORDINATES:(FT.) N NK E NKT OPEN AREA PER LINEAL FT. (IN2/FT.) $\Omega^{\prime \prime b o r e ~ h a l e ~ w i t h ~} 4.5^{\prime 00}$ cessing FORMATION OF COMPLETION: FIELD-REP.: Roger F. Helland Alluvium

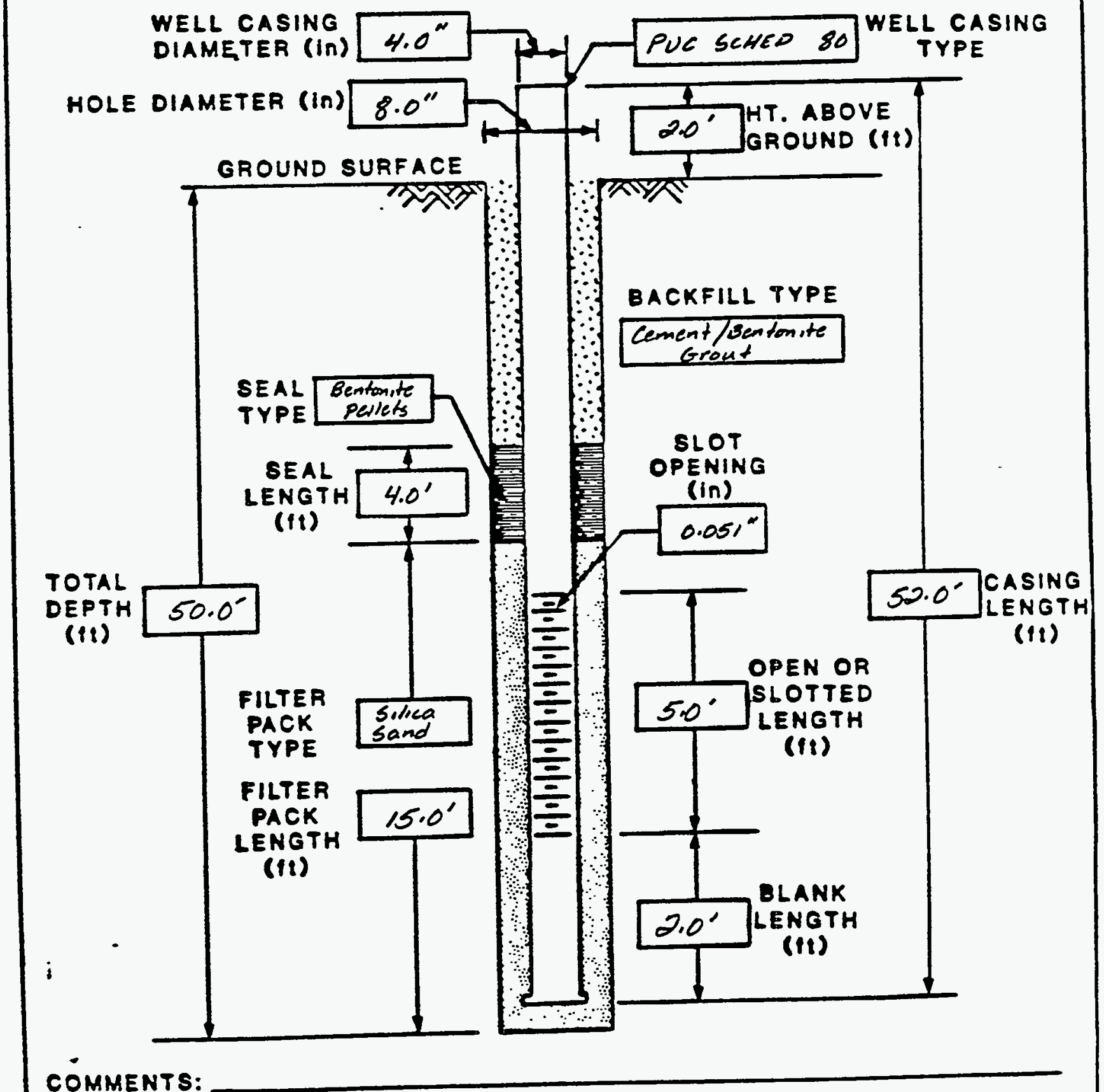

COMMENTS: 


\section{TT HCOSS ENGNEERTG GROUP NC.

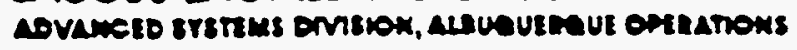 BOREHOLE/WELL CONSTRUCTION LOG}

SITE ID: AMBOI LOCATION ID: 783 APPROX. BITE COORDINATES (FT.): N NK GROUND ELEVATION (FT. MSL): NK

BOREHOLE SUMMARY

DRILLER: Stewart Brothers

RIG TYPE: Gardner Denver 15w

BIT TYPE

Hughs

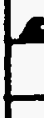

\begin{tabular}{|l|l|}
\hline & \\
\hline & \\
\hline & \\
\hline
\end{tabular}

HOLE. (in.) $8.0^{\prime \prime}$

DEP

END $\bullet$ (11.)

FIUID

-

$50^{\circ}$

$0^{\prime}$

centonite

\begin{tabular}{|c|c|c|}
\hline & CASING SUMMI & \\
\hline $\begin{array}{l}\text { CASING } \\
\text { TYPE: }\end{array}$ & DESCRIPTION & $\begin{array}{l}\text { DiA.j END' } \\
\text { (in.) DEPTH }\end{array}$ \\
\hline
\end{tabular}

\begin{tabular}{|c|c|c|c|}
\hline$B$ & PUC SCHED 80 & $10=0$ & \\
\hline$s$ & Pue .05\%"slorted & $4.0^{\circ}$ & $50.0^{\circ}$ \\
\hline$B$ & PUC SCHED 80 & $4.0^{\circ}$ & $52.0^{\prime}$ \\
\hline$p$ & Steel Casing & $8.0^{\prime \prime}$ & $5.0^{\prime}$ \\
\hline & & & \\
\hline & & & \\
\hline & & & \\
\hline & & & \\
\hline
\end{tabular}

- P-Proteetive S-Bereon D-Blenk O-Open W-Nono Depith from Top of Cesino

\begin{tabular}{|c|c|c|}
\hline \multicolumn{3}{|c|}{ WELL CONSTRUCTION } \\
\hline $\begin{array}{l}\text { TYPE } \\
\text { CODE }\end{array}$ & DESCRIPTION & DEPTH \\
\hline$B$ & cement/Bentericte & $28.0^{\prime}$ \\
\hline 5 & Seatonete rellets & 32.0 \\
\hline$F$ & solica send & $50.0^{\prime}$ \\
\hline & - & \\
\hline 7 & & \\
\hline & & \\
\hline 0 & & \\
\hline & & \\
\hline 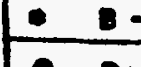 & $8-8.021$ & Pack \\
\hline
\end{tabular}

SEAL

BACKFILL

DEVELOPMENT

OTHER

CASING

FILTER PACK CONSTRUCTION TIME LOG

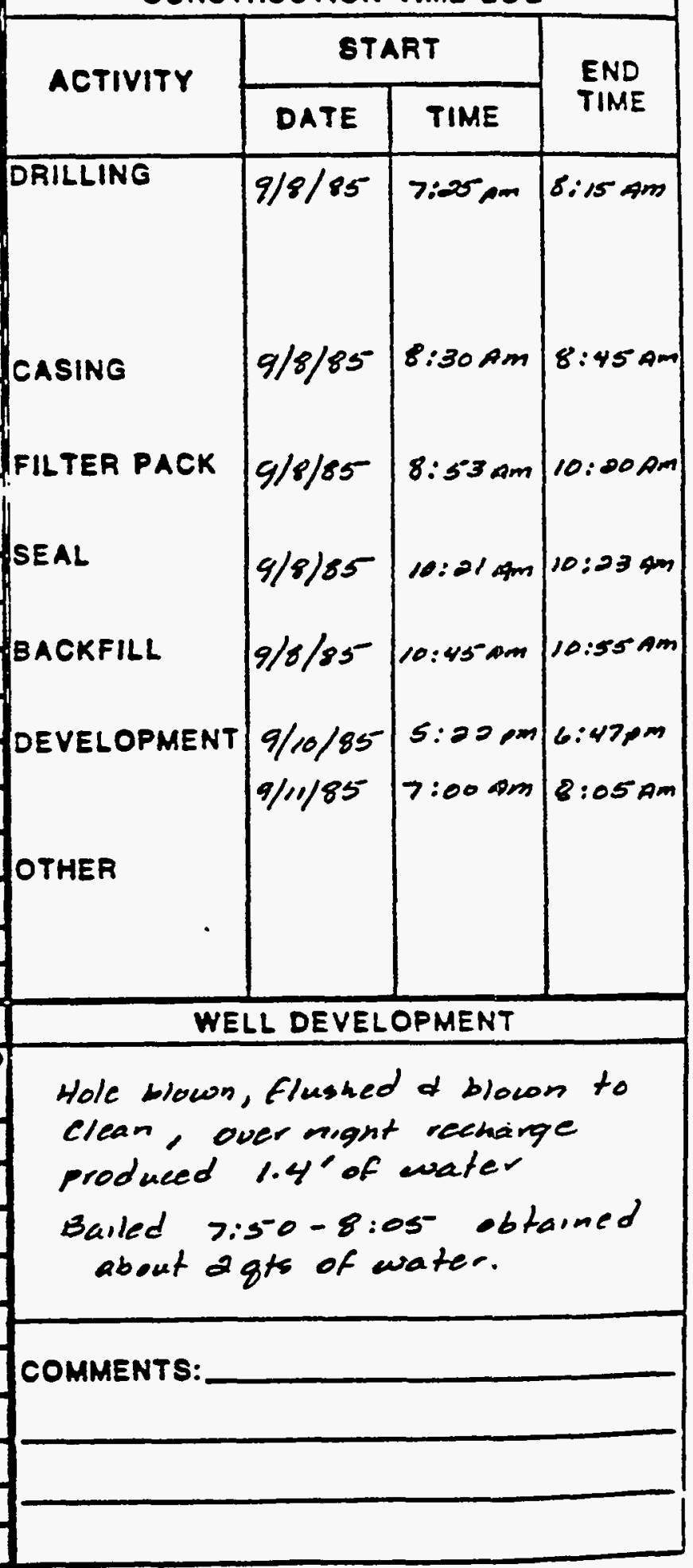

JEG-AB-ENO-1 (8/84) E NK COMPLETION DATE: $9 / 8 / 85$
FIELD REP: Pager F. Holland 
WELL 785 LOGS 


\section{TIE HOOBS BVENEERNG GROUP NC.

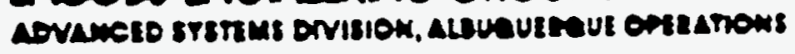

\section{WELL COMPLETION RECORD}

SITE ID: AMBOL LOCATION ID: 285 DATE INSTALLED: $9 / 11 / 85$ APPROX. SITE COORDINATES:(FT.) N NK E NK

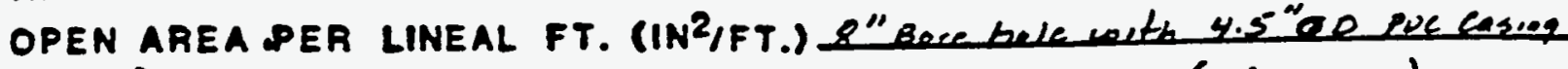
FORMATION OF COMPLETION: Tres Hecosenes $B$ (olancos) FIELD-REP.:

Beger Holland DRILLER: Stewart Brothers WELL CASING
DIAMETER (In) $4.0^{\circ}$
HOLE DIAMETER (In) $8.0^{\prime \prime}$ GROUND SURFACE

TOTAL DEPTH (11)

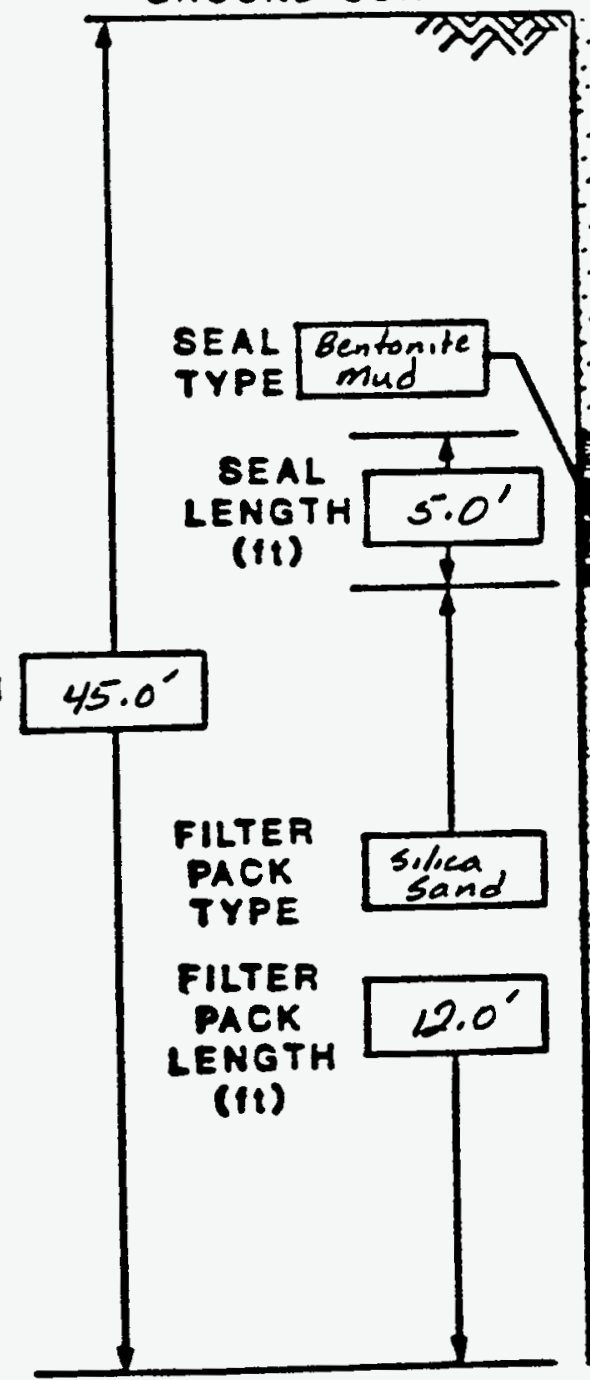

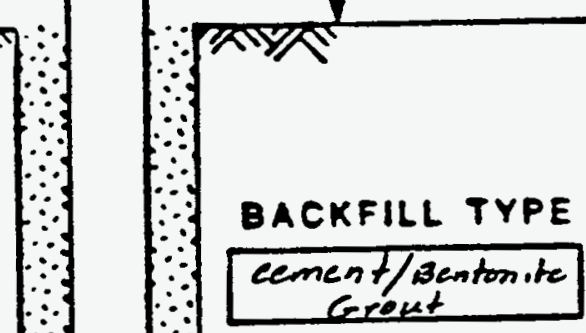

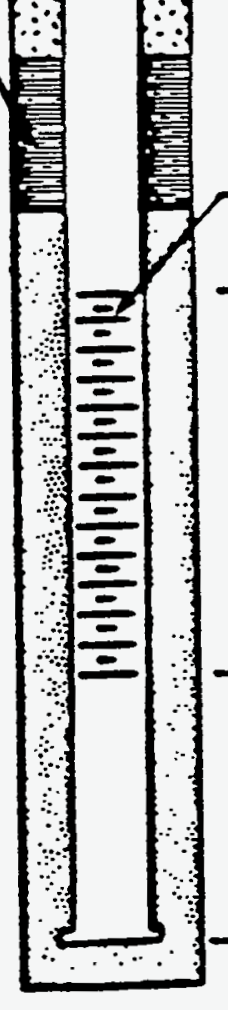

SLOT

OPENING (in)

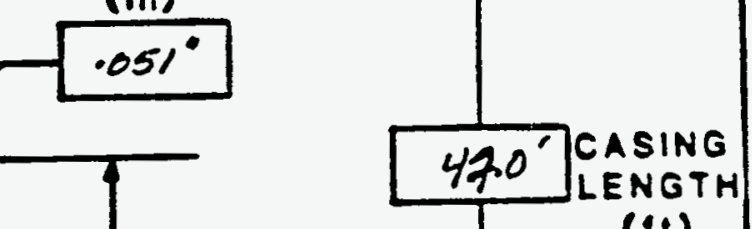
(11)

\section{COMMENTS:}




\section{IT HCOBS ENGANERTIG GROUP NKC.

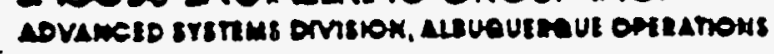 \\ BOREHOLE/WELL CONSTRUCTION LOG}

SITE ID: AMBOI LOCATION ID: 785 FIELD REP: Roger Holland APPROX. SITE COORDINATES (FT.): N NK GROUND ELEVATION (FT. MSL): NK

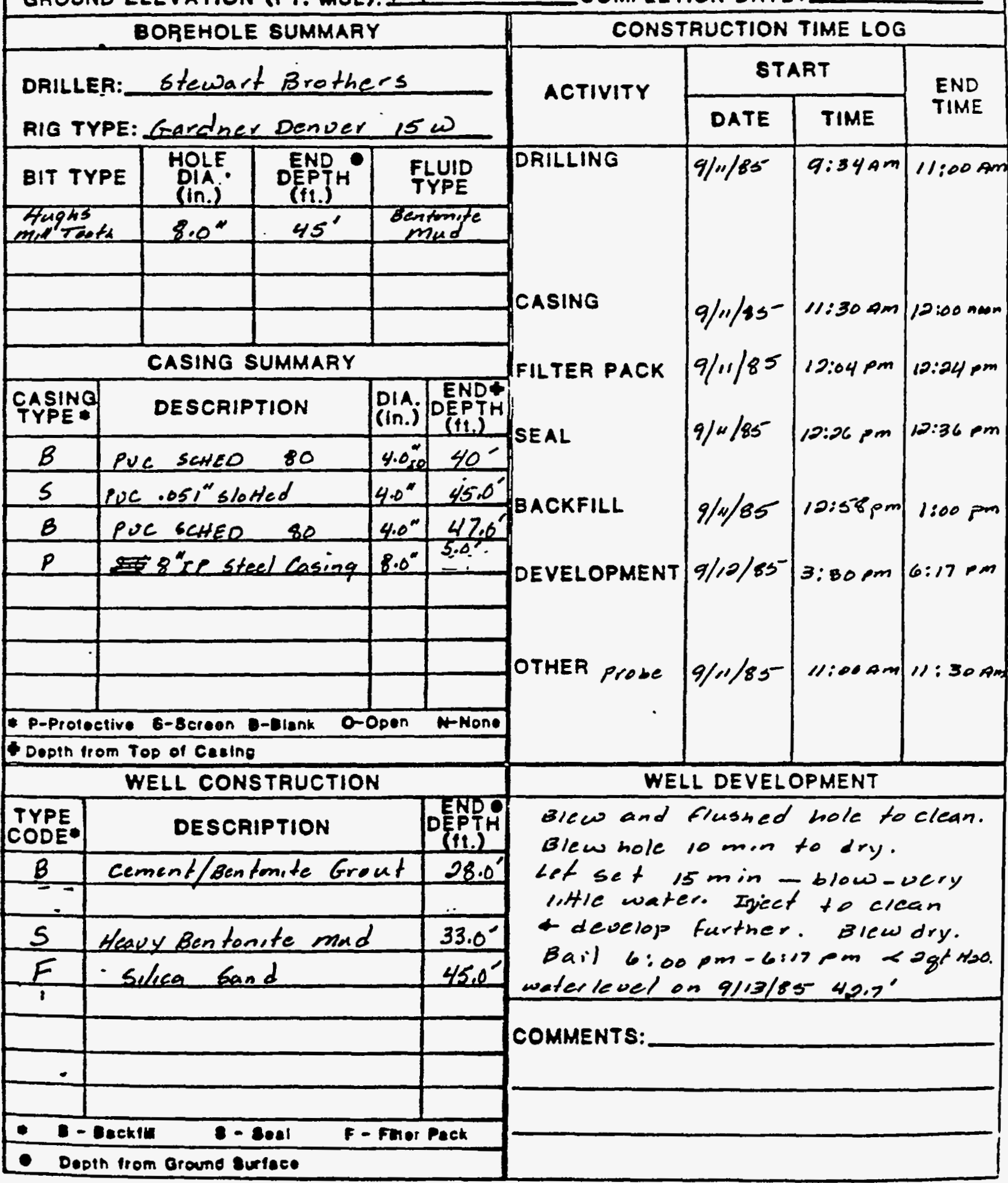

JEG-AL-ENQ-1 (B/OA) 
WELL 786 LOGS 
IACOBS ENGINEERING GROUP INC.

ADVAMELO SYSTEMS DIVISIOM. ALUUEUERQUE ODERATIOKS

BOREHOLE LOG (ROCK) Page 101 ?

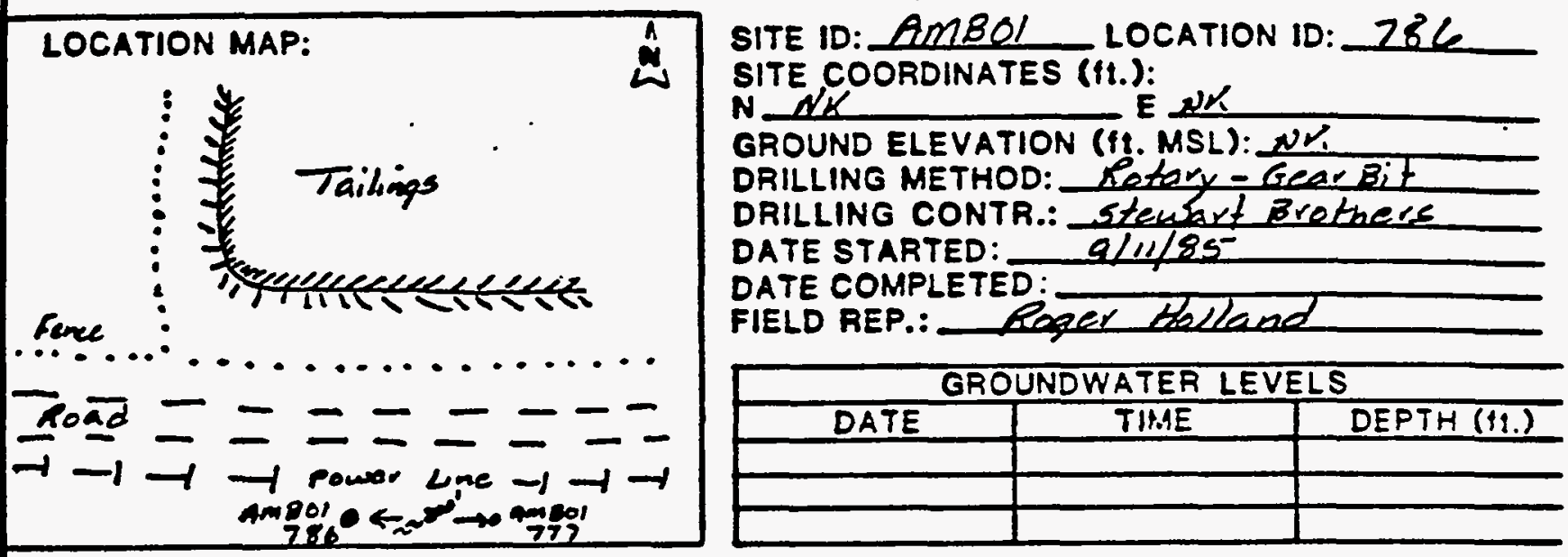

LOCATION DESCRIPTION

SITE CONDITION

0

Sandy clay - clayey sand, tan to buff, calcareus $0-30^{\prime}$

$\omega$

5

10

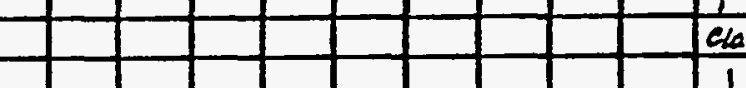

20

\begin{tabular}{llllllllllll}
\hline & & & & & & & & & & \\
\hline
\end{tabular}

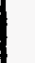

$\infty$

$$
\begin{array}{llllllllllll}
\hline & & & & & & & & & & \\
\hline & & & & & & & & & & \\
\hline
\end{array}
$$
दे

$+$ 


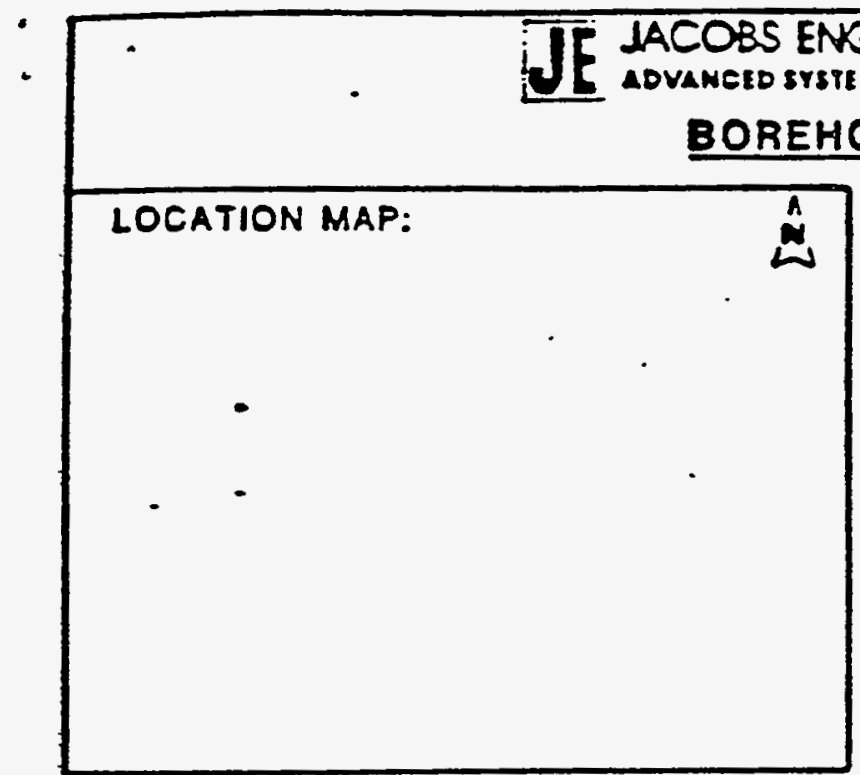

LACOBS ENGINEERING GROUP INC.

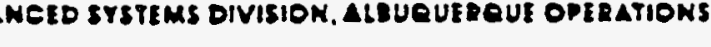

出 SITE ID: AmBOL LOCATION ID: 286

$N$ - NQ

E N

GROUND ELEVATION (ft. MSL):

DRILLING METHOD:

DRILLING CONTR.:

DATE STARTED: YU/QS

DATE COMPLETED

FIELD REP.: Reger blolland

\begin{tabular}{|c|c|c|}
\hline \multicolumn{3}{|c|}{ GROUNDWATER LEVELS } \\
\hline DATE & TIAGE & DEPTH (11.) \\
\hline & & \\
\hline & & \\
\hline & & \\
\hline
\end{tabular}

LOCATION DESCRIPTION

SITE CONDITION

30

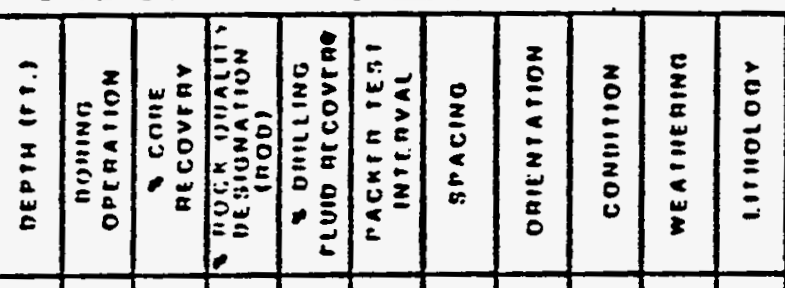

ROCK TYPE REMARKS

as above with sundstene frags. Tan, fine to very fine grained, calc., and numereus well developed clear calcite crystals.

35-40' as above with same sandy claystome, dark aray to b/x, calcareous.

To $35^{\circ}$

35

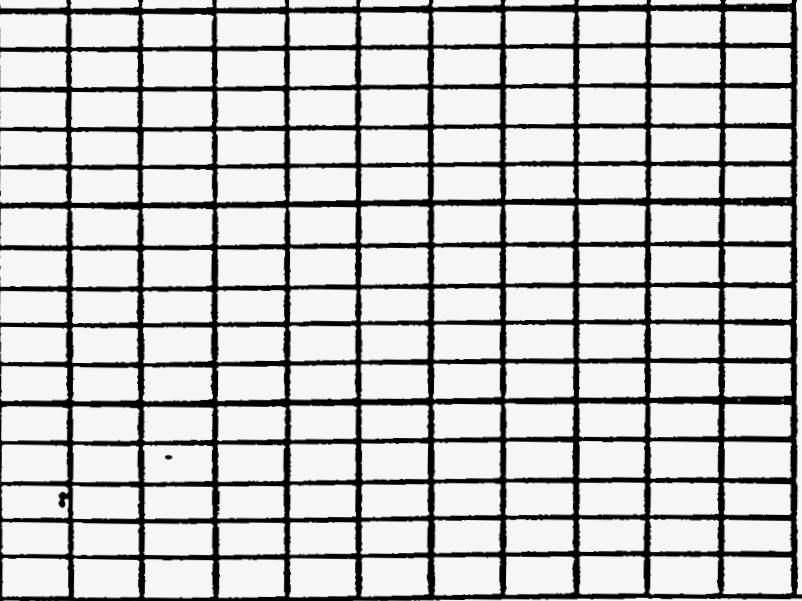




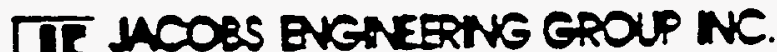

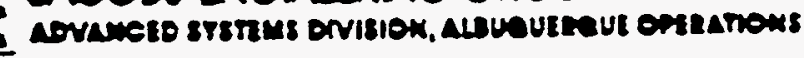

\section{WELL COMPLETION RECORD}

SITE ID: AMBOI LOCATION ID: 786 APPROX. SITE COORDINATES:(FT.) N NK DATE INSTALLED: $q / 11 / 85$

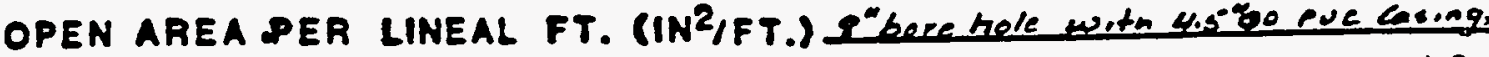
FORMATION OF COMPLETION: Upeer Mnaces/allwid TrCl? FIELD-REP.: Peger Hollend DRILLER: stewart Brothers

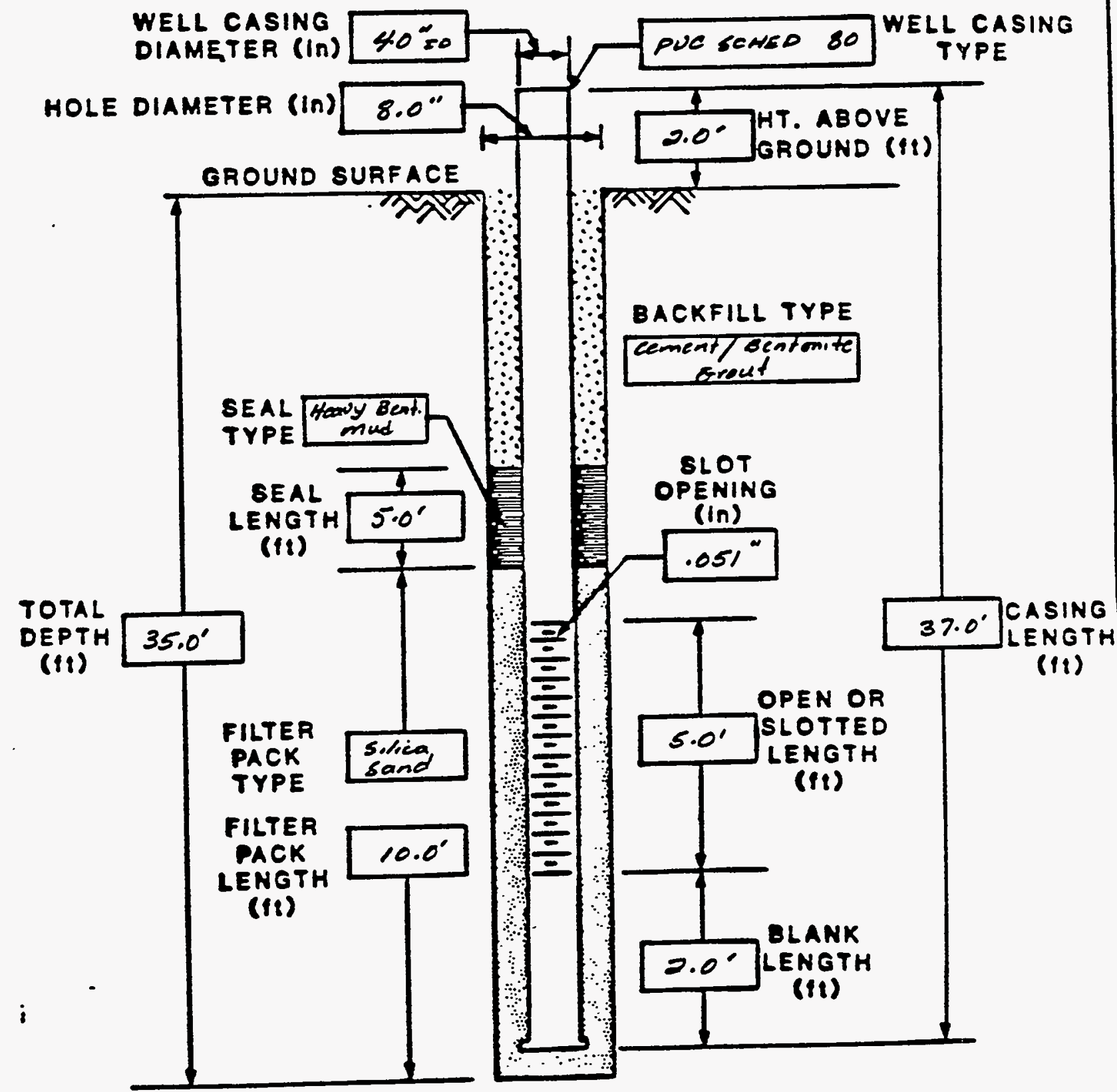

COMMENTS: 


\section{TT MCOBS BNGNEERIG GROUP INC.

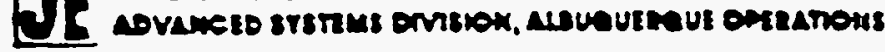 BOREHOLE/WELL CONSTRUCTION LOG}

SITE ID: AMBQL LOCATION ID: 286 APPROX. 8ITE COORDINATES (FT.): N AKK GROUND ELEVATION (FT. MSL): NK BOREHOLE SUMMARY

DRILLER:_stewart Brothers

RIG TYPE: Gardner Denver is $\omega$ (estary)

\begin{tabular}{|c|c|c|c|}
\hline BIT TYPE & $\begin{array}{l}\text { HOLEE. } \\
\text { DIAA. } \\
\text { in.j }\end{array}$ & 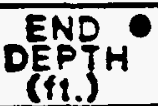 & $\begin{array}{l}\text { FLUID } \\
\text { TYPE }\end{array}$ \\
\hline Haghs Tath & $8.0^{\circ}$ & $35.0^{\prime}$ & $\begin{array}{l}\text { Benpongto } \\
\text { mud }\end{array}$ \\
\hline & & & \\
\hline & & & \\
\hline & & & \\
\hline
\end{tabular}

\begin{tabular}{|c|c|c|c|}
\hline \multicolumn{4}{|c|}{ CASING SUMMARY } \\
\hline $\begin{array}{l}\text { CASING } \\
\text { TYPE }\end{array}$ & DESCRIPTION & $\begin{array}{l}p \mid A \\
(i n . j)\end{array}$ & $\begin{array}{l}\text { END } \\
\text { DEPTH } \\
\text { PET., }\end{array}$ \\
\hline$B$ & RUE SEHED. 80 & $40^{\circ}$ & 30.0 \\
\hline 5 & pue .051" sletted & $4.0^{\circ}$ & $35.0^{\circ}$ \\
\hline$B$ & Pue scmeo. 80 & $4.0^{\circ}$ & $37.0^{\circ}$ \\
\hline$P$ & S'lenates streel lasing & $8.0^{4}$ & 5.0 \\
\hline & & & \\
\hline & & & \\
\hline & & & \\
\hline & & & \\
\hline p-piolo & live 6 -8eroen o-sienk 0 & $\frac{1}{0 p o n}$ & A-None \\
\hline
\end{tabular}

Dopin from Too of Casing

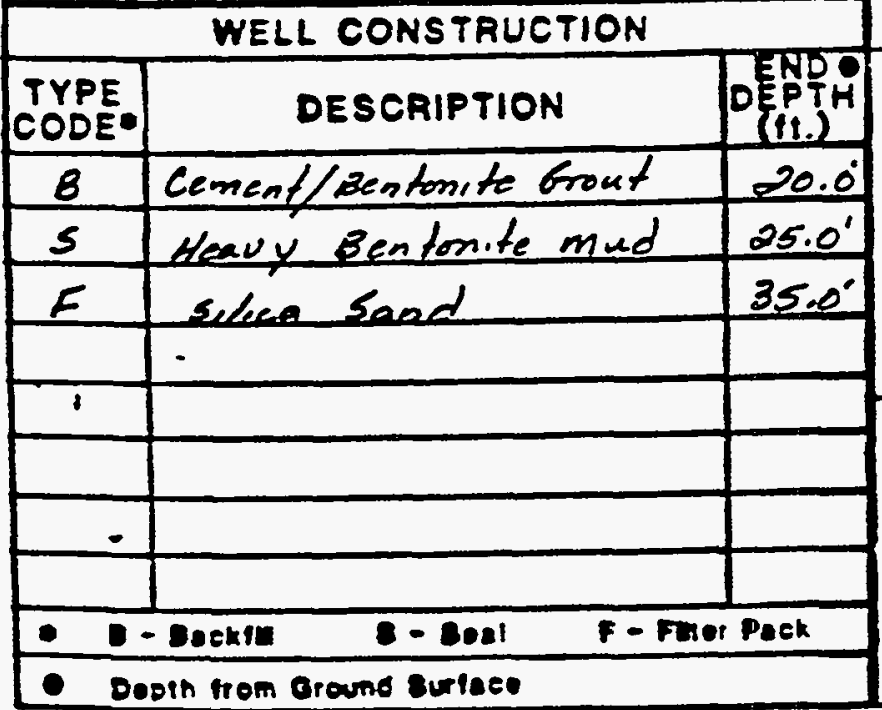

dEO-AL-ENO-I (8184)
FIELD REP: Reqer Holland E NK
CASING

FILTER PACK

SEAL

BACKFILL

DEVELOPMENT

OTHER

Probing

\section{WELL DEVELOPMENT}

Flush a blow hole to clean until 8:33 9 m

Blow: $\cos 3-8: 38$ an

Set: 8:38-8:50 $\mathrm{am}$

Gow: 8:50-9:00 $\mathrm{cm}$ No mater

flush \& sume

310w dry

Letset 9:20-7:30 pm

COMMENTS: Elow 9:30-7:35 Am wery lette water produced cluch of bers tul gi50:0n Exil a:50-11:08 am hble matiog curter semple colleted pn 7.77
END

TIME 
WELL 787 LOGS

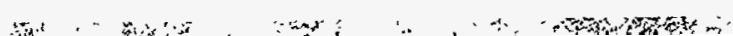

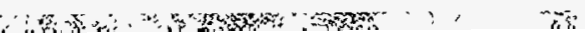




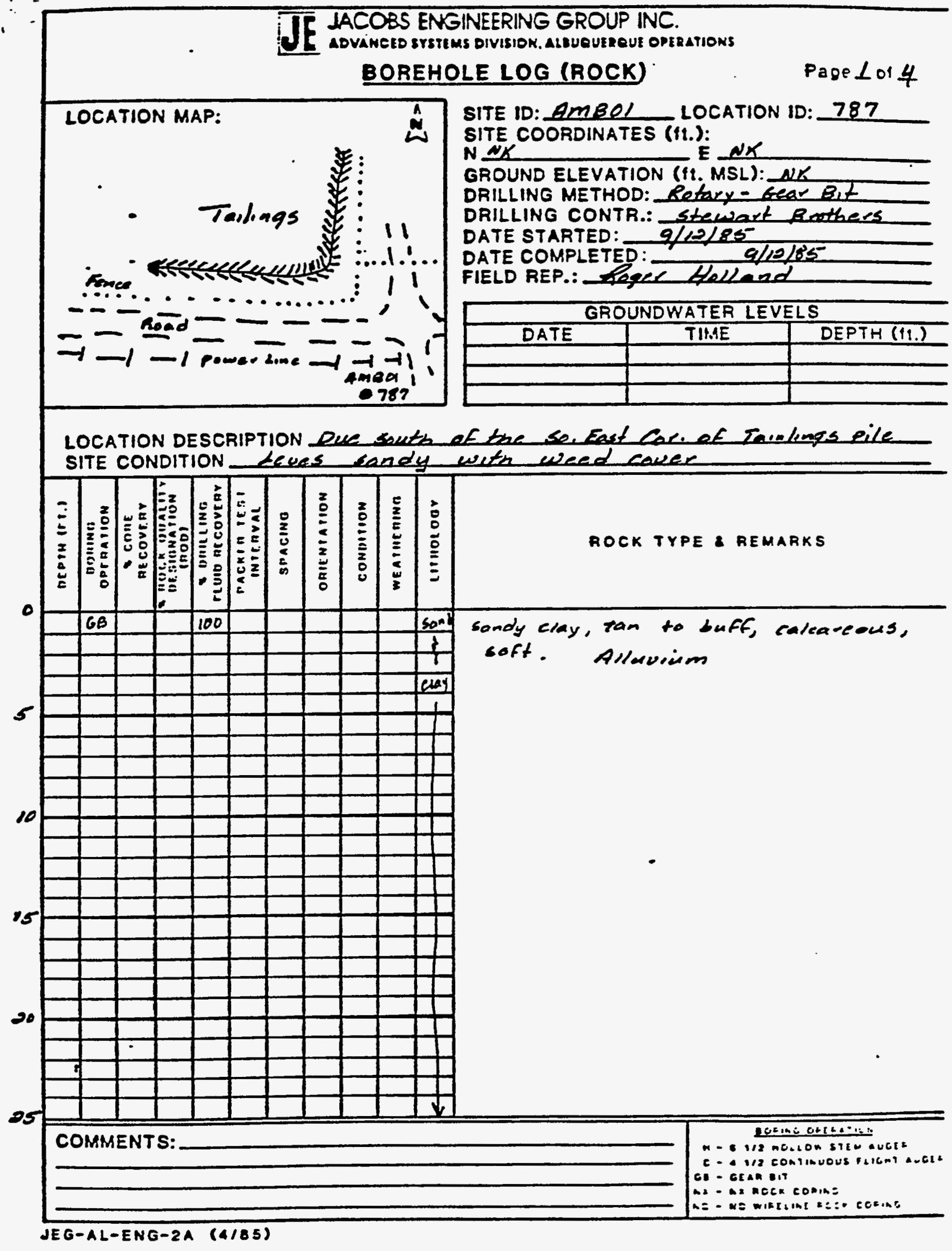




\section{JE IACOBS ENGGINEERING GROUP INC. \\ BOREHOLE LOG (ROCK) \\ Page르 of 4}

LOCATION MAP:

$\stackrel{\leftrightarrow}{\omega}$

SITE ID: AMBQI

SITE COORDINATES (It.):

$N$

GROUND ELEVATION (ft. MSL):

DRILLING METHOD:

DRILLING CONTR.:

DATE STARTED:

DATE COMPLETED:

FIELD REP.:
LOCATION ID: 782

\begin{tabular}{|c|c|c}
\hline \multicolumn{3}{|c|}{ GROUNDWATER LEVELS } \\
\hline DATE & TIMA & DEPTH (11.) \\
\hline & & \\
\hline & & \\
\hline & & \\
\hline
\end{tabular}

LOCATION DESCRIPTION

SITE CONDITION

c

\begin{tabular}{|c|c|c|c|c|c|c|c|c|c|c|c|}
\hline 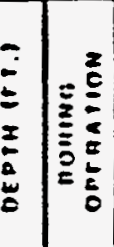 & $\left(\begin{array}{l}0 \\
0\end{array}\right.$ & 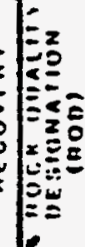 & 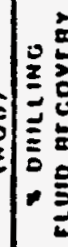 & 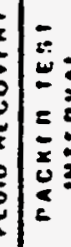 & & 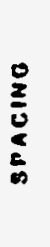 & 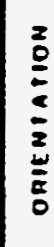 & & & $\bar{E}$ & \\
\hline
\end{tabular}

ROCK TYPE REMARKS

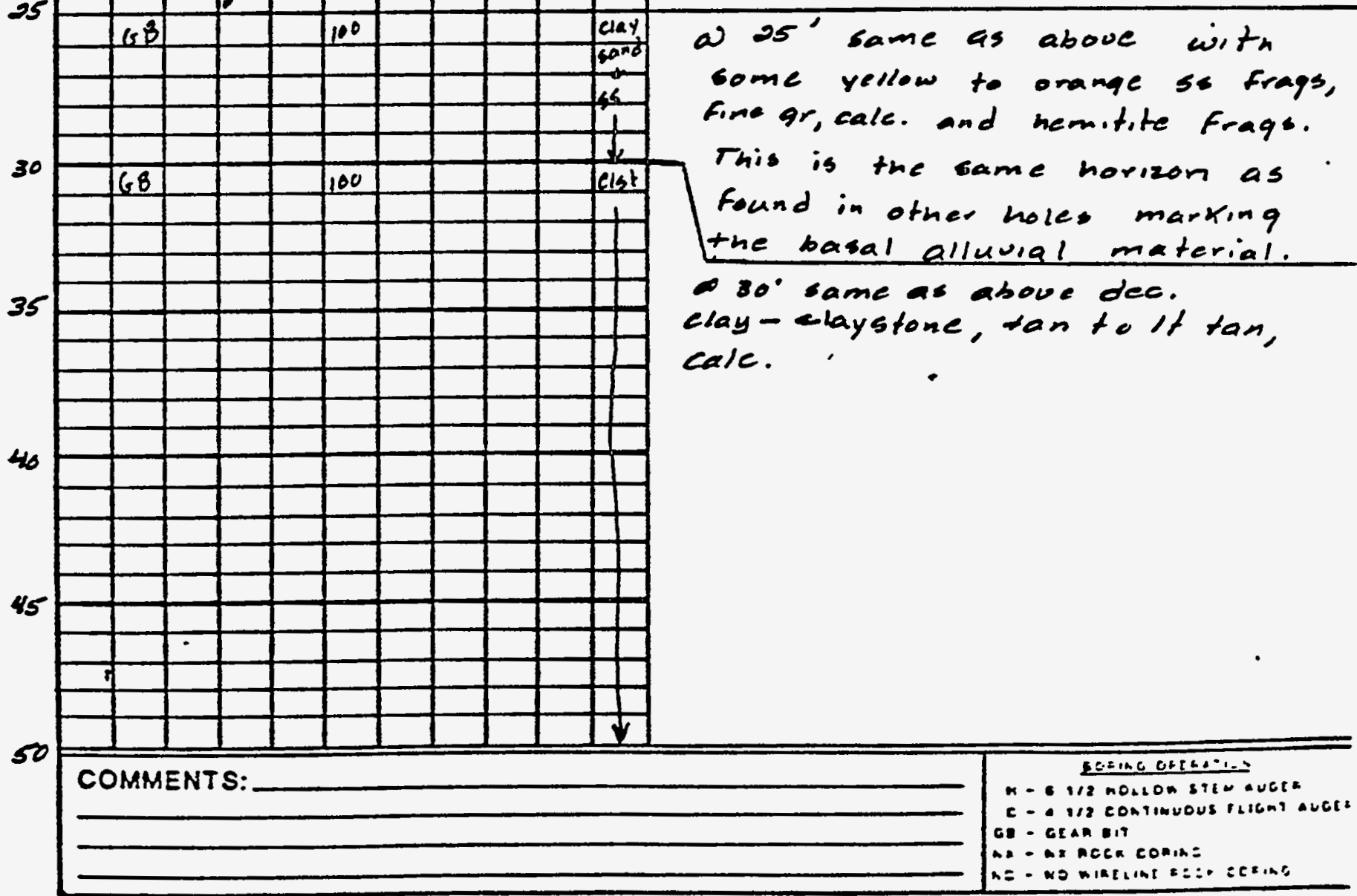

JEG-AL-ENG-2A (A/B5) 
If IACOBS ENGINEERING GROUP INC.

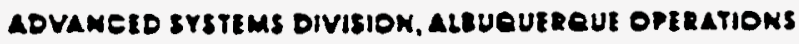

BOREHOLE LOG (ROCK)

Page 3 or 4

LOCATION MAP:

$\stackrel{n}{\omega}$

SITE ID: AMBOI

LOCATION ID: 782

SITE COORDINATES (f:.):

N.

GROUND ELEVATION (ft. MSL):

DRILLING METHOD:

DRILLING CONTR.:

DATE STARTED:

DATE COMPLETED:

FIELD REP.: Reger Helland

\begin{tabular}{|c|c|c|}
\hline \multicolumn{3}{|c|}{ GROUNDWATER LEVELS } \\
\hline DATE & TIP.AE & DEPTH (19.) \\
\hline & & \\
\hline & & \\
\hline & & \\
\hline
\end{tabular}

LOCATION DESCRIPTION

SITE CONDITION

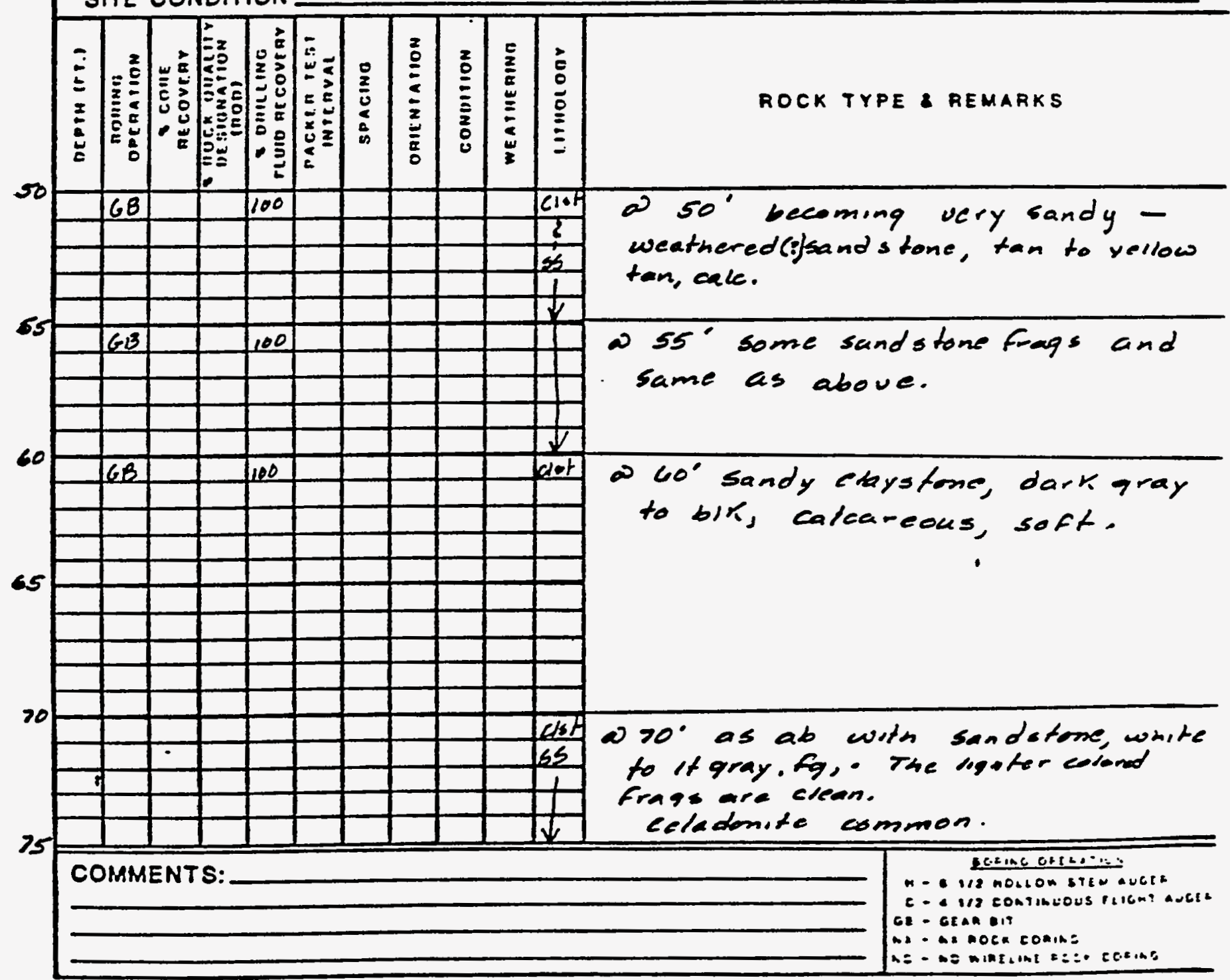

JEG-AL-ENG-2A (4/85) 


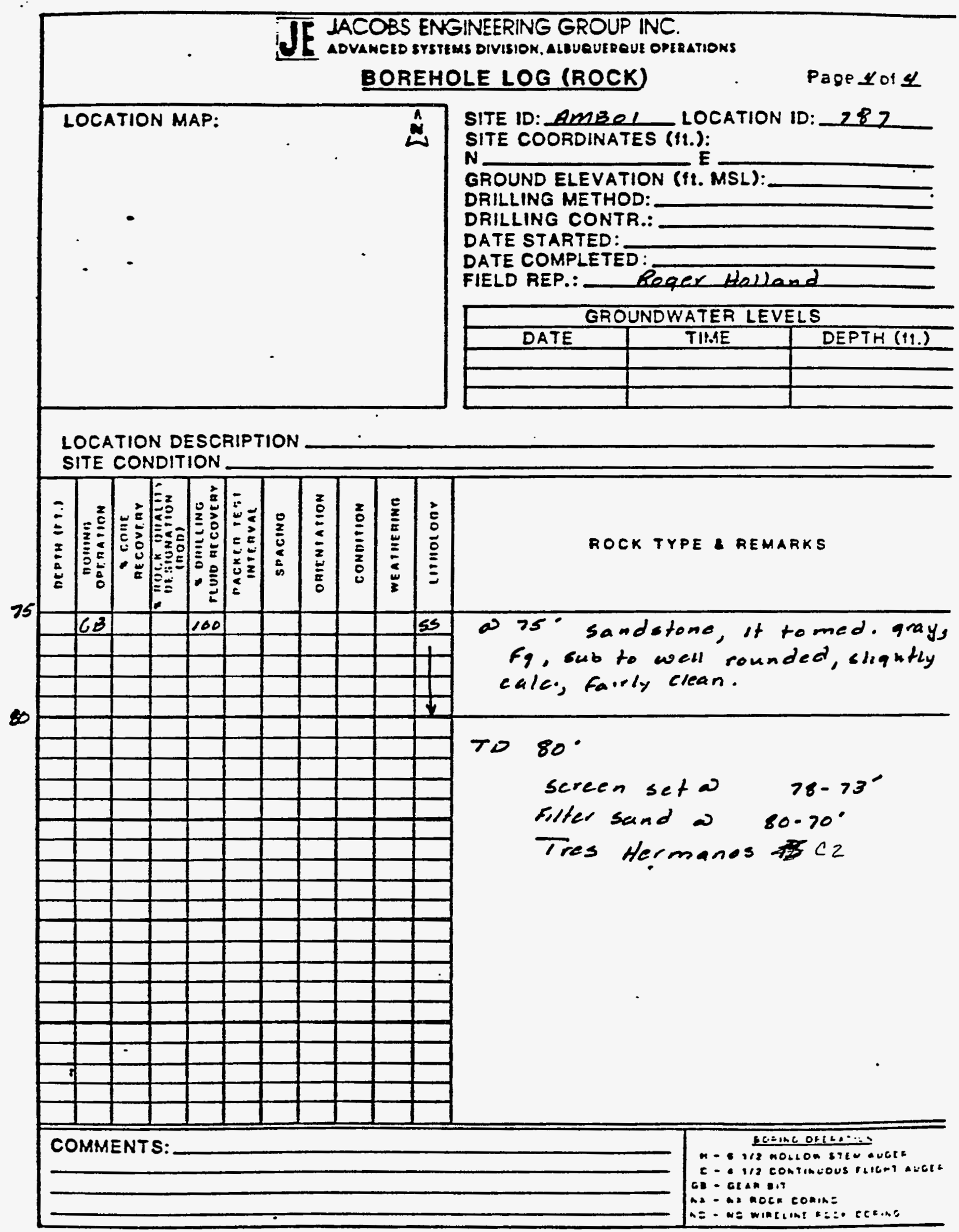




\section{TT MCOBS BNEAERNG GROUP NC.

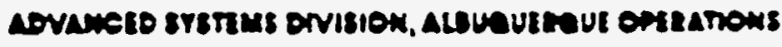

\section{WELL COMPLETION RECORD}

SITE ID: AMBOI LOCATION ID: APPROX. SITE COORDINATES:(FT.) N DK OPEN AREA PER LINEAL FT. (IN2/FT.) \& bere hele wath $4.5^{2}$ "De rue cassone FORMATION OF COMPLETION: Tres termanes $B$ FIELD-REP.: Beger Helland DRILLER: Stewart Brethers

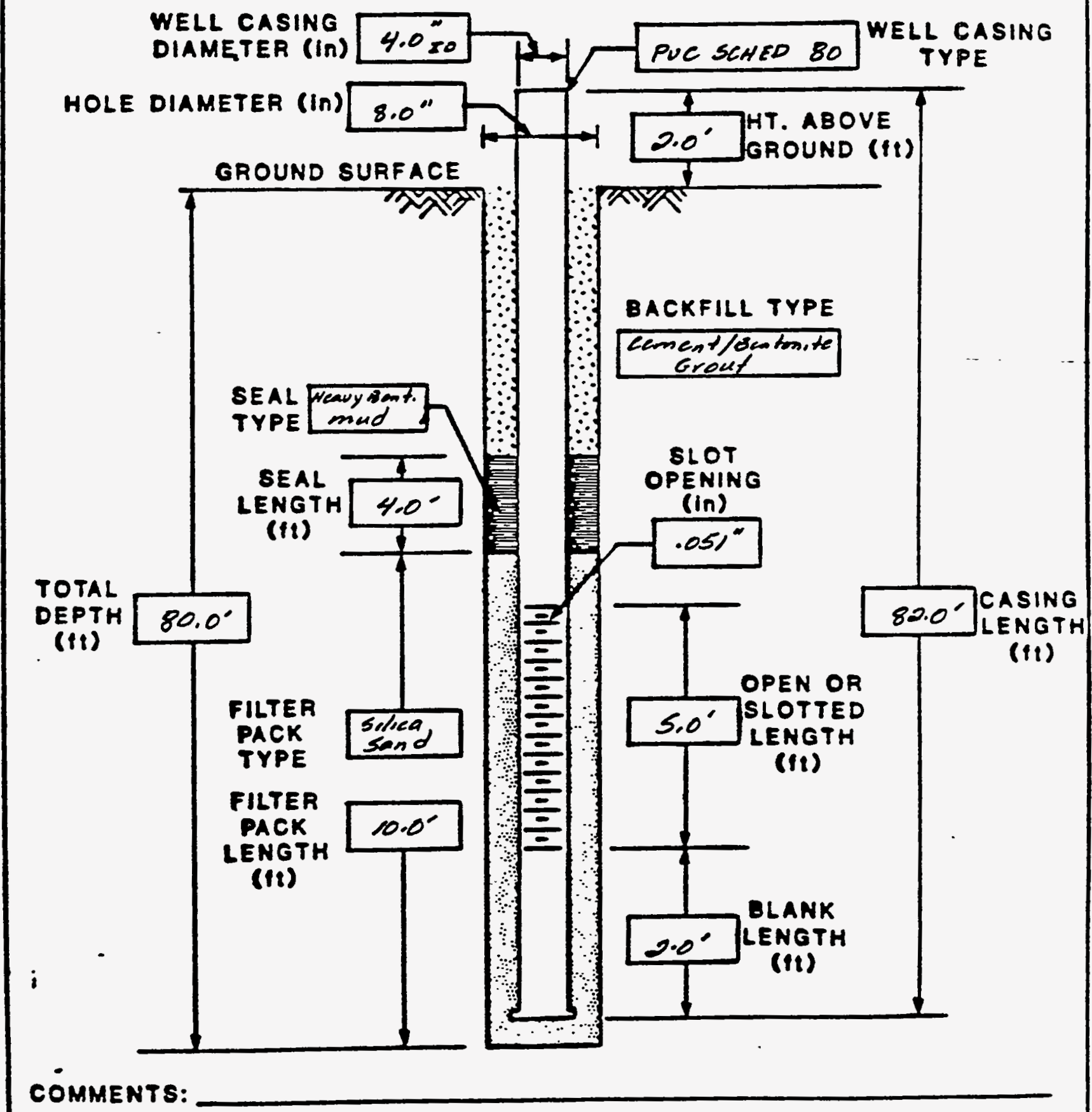

JEG-AL-ENG-S (S/OA)
DATE INSTALLED: $q / 12 / 85-$ E NK 


\section{TT JCOBS BNGNEERTG GROUP INC.

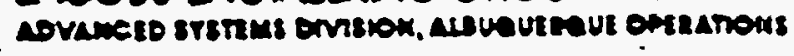 BOREHOLE/WELL CONSTRUCTION LOG}

SITE ID:AMBel LOCATION ID: 787 FIELD REP: Peger Helland APPROX. BITE COORDINATES (FT.): N NK GROUND ELEVATION (FT. MSL): NK. E NK

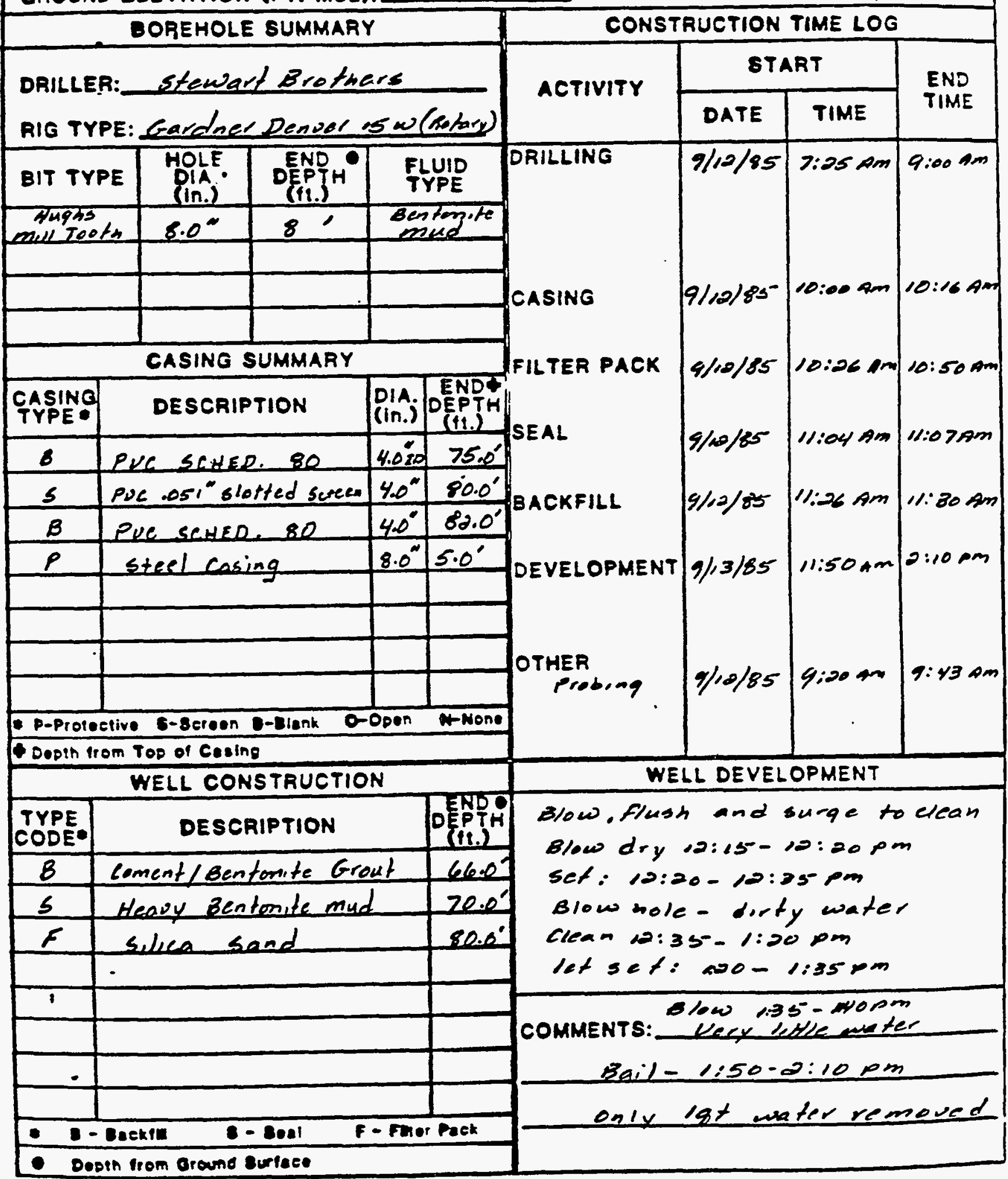


WELL 790 LOGS 
PROJECT AMBROSLA LAKE SITE, NEW MEXICO

Page' of 1

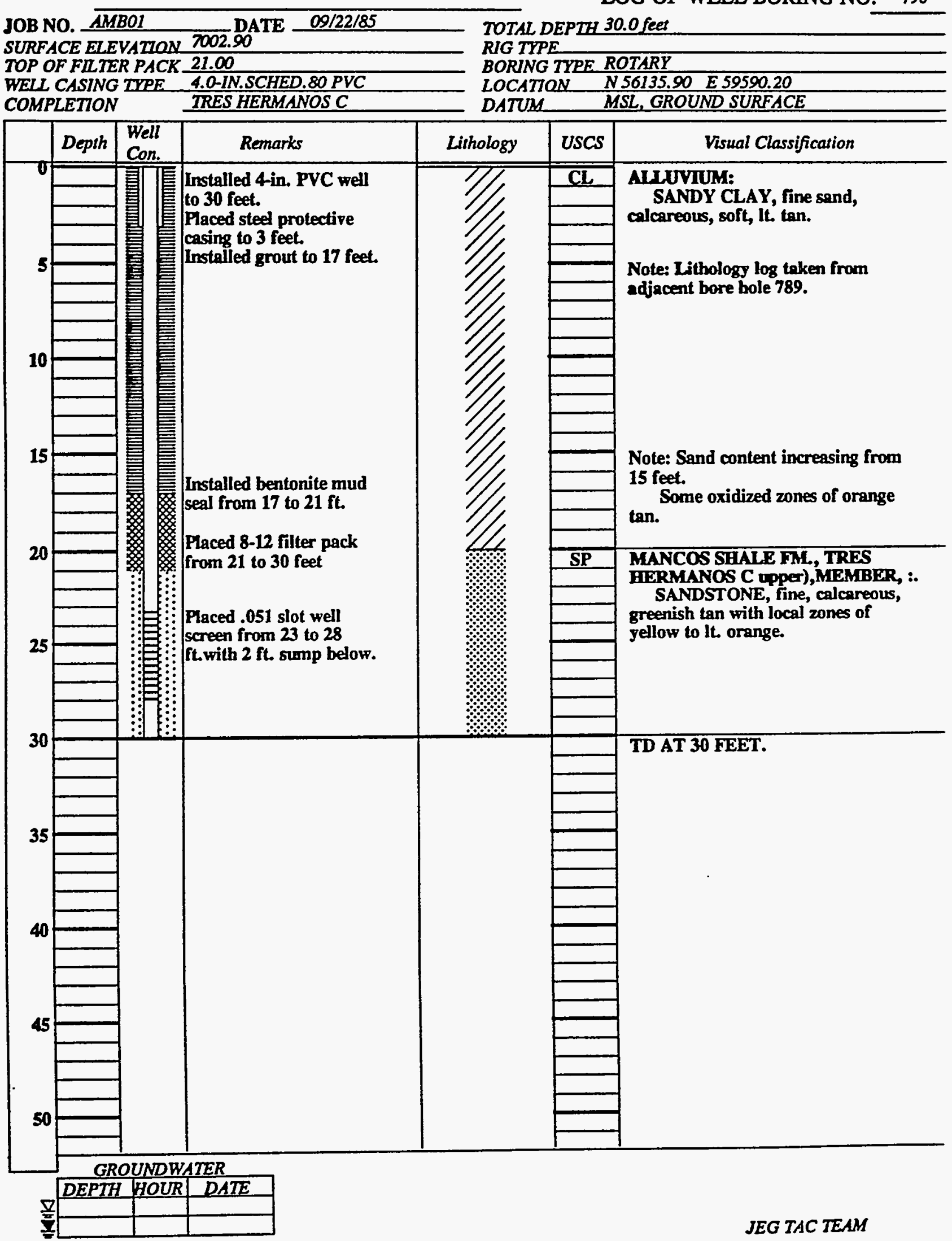




\section{TT HCO3S EVGAERING EROUP NK.

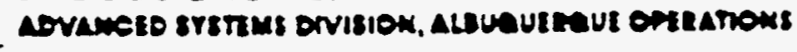

\section{WELL COMPLETION RECORD}

SITE ID: AmBOL LOCATION ID: Z9Q DATE INSTALLED: $9 / 23 / 85$ APPROX. SITE COORDINATES:(FT.) N NK E N

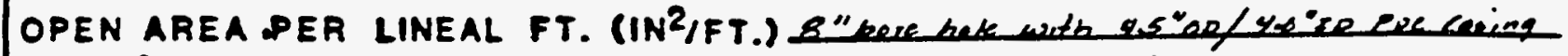
IFORMATION OF COMPLETION: FIELD -REP.: Tees dermanes " $\mathrm{C}$ "

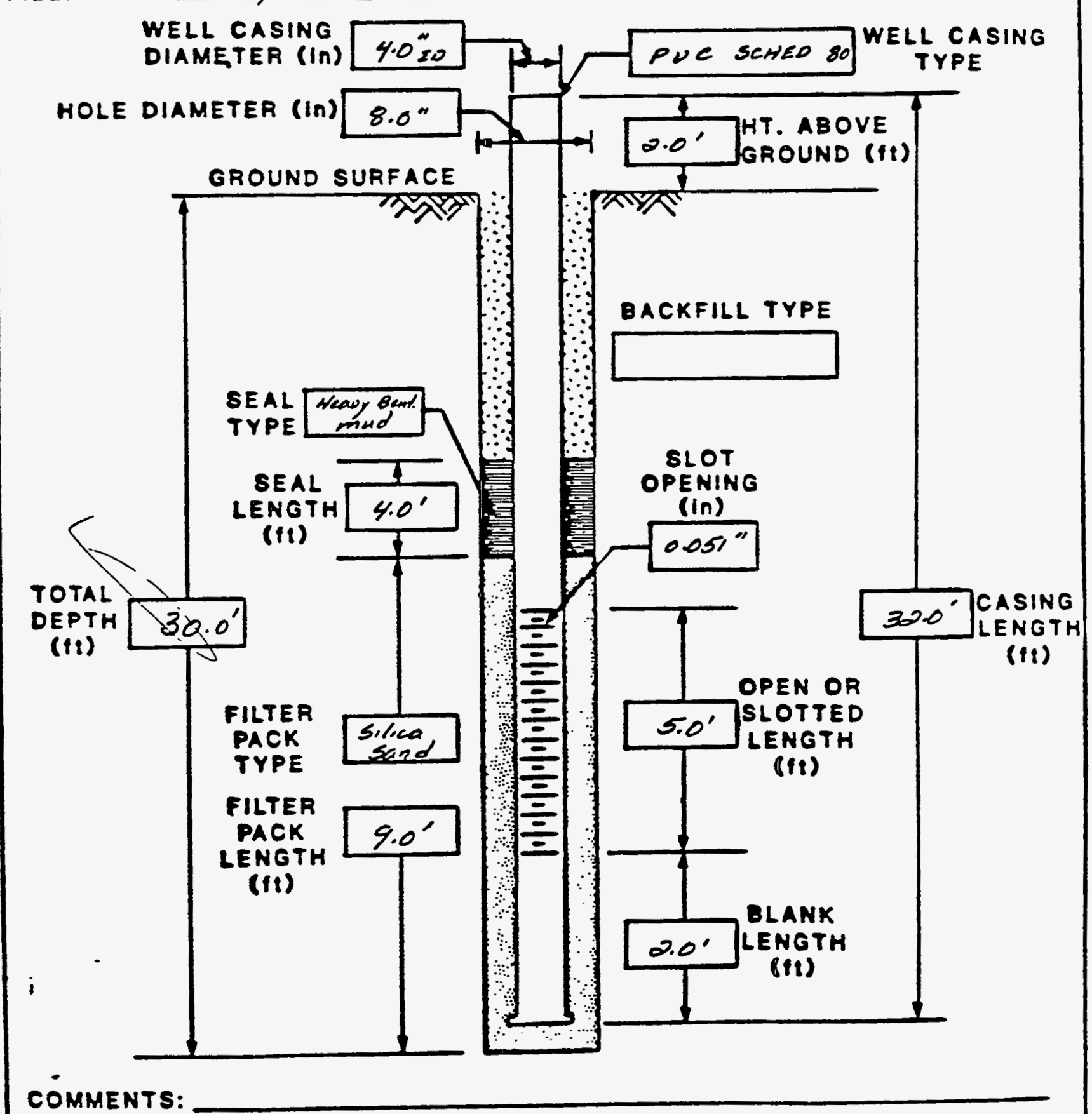
DRILLER: Stewert Brathers 


\section{TT HCOBS ENGNEERIVG GAOUP NK.

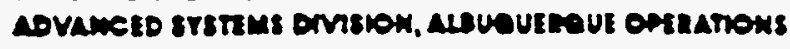 BOREHOLE/WELL CONSTRUCTION LOG}

SITE ID: AmBOL LOCATION ID: 790 FIELD REP: Peger Holland GROUND ELEVATION (FT. MSL): NK COMPLETION DATE: 2/23/85-

\begin{tabular}{|c|}
\hline BOREHOLE SUMMARY \\
\hline DRILLER: Stewart Brothers \\
RIG TYPE: Garduer Denver 15w/Rotary)
\end{tabular}

\begin{tabular}{|c|c|c|c|}
\hline BIT TYPE & $\begin{array}{l}\text { HOLE. } \\
\text { DiA. } \\
\text { (in. }\end{array}$ & DEND ${ }_{\text {(ft. }}{ }^{\circ}$ & $\begin{array}{l}\text { FLUID } \\
\text { TYPE }\end{array}$ \\
\hline $\begin{array}{c}\text { 4ughs } \\
\text { mill Testo }\end{array}$ & $8.0^{\prime \prime}$ & $30.0^{\prime}$ & Bentoricte \\
\hline & & & \\
\hline & & & \\
\hline & & & \\
\hline
\end{tabular}

\begin{tabular}{|c|c|c|c|}
\hline $\begin{array}{l}\text { CASING } \\
\text { TYPE }\end{array}$ & DESCRIPTION & $\begin{array}{l}\text { piA. } \\
\text { (in.) }\end{array}$ & $\begin{array}{c}\text { END } \\
\text { OEPTH } \\
\text { EPT }\end{array}$ \\
\hline$B$ & PUC SCHEO 80 & $4.0^{\prime \prime}$ & 25 \\
\hline 5 & PUC . 05," SloHed & $4.0^{1}$ & $30^{\prime}$ \\
\hline$B$ & PUC SCHED 80 & & $32^{\prime}$ \\
\hline$P$ & sted Carieng & $8.0^{\prime \prime}$ & $50^{\prime}$ \\
\hline & & & \\
\hline & & & \\
\hline & & & \\
\hline & & & \\
\hline
\end{tabular}

CASING CONSTRUCTION TIME LOG

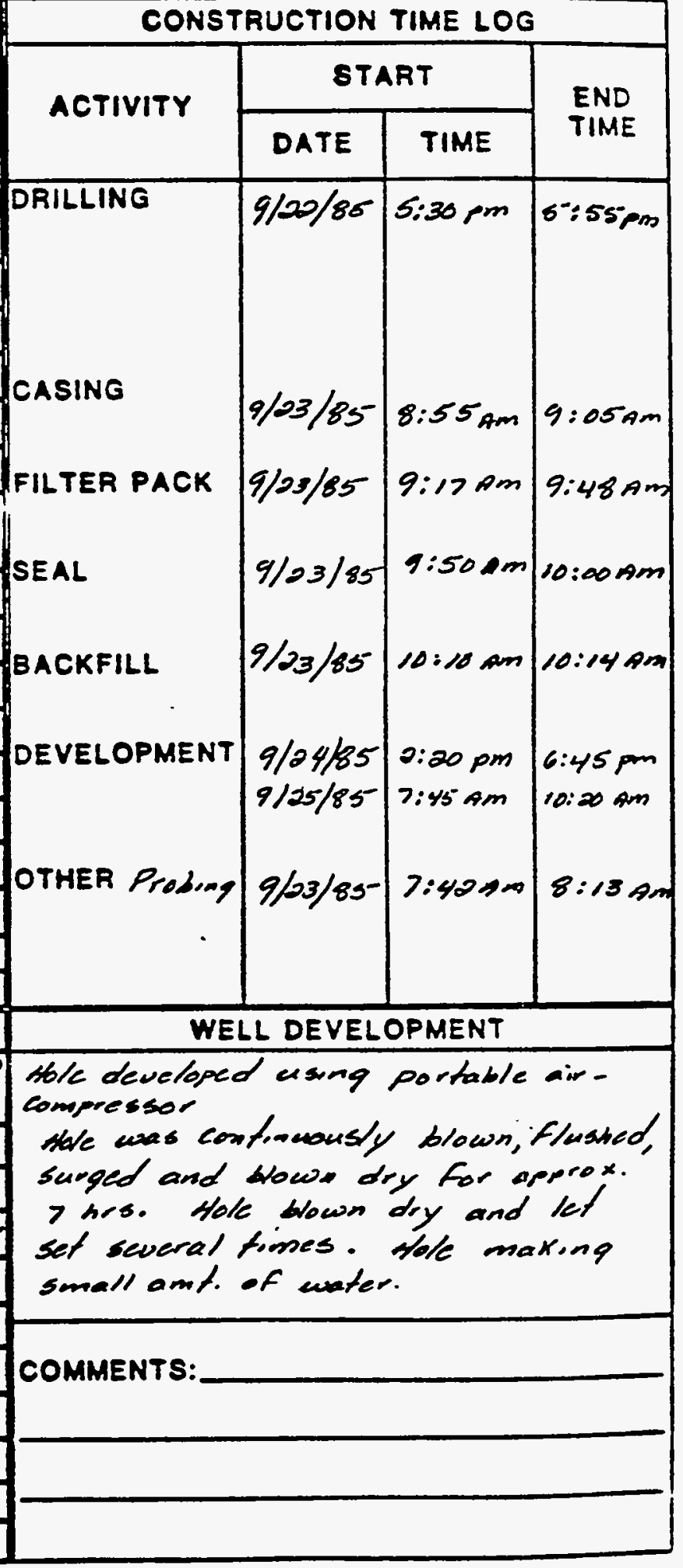

Dopth from Top of Cesing

\begin{tabular}{|c|c|c|}
\hline \multicolumn{3}{|c|}{ WELL CONSTRUCTION } \\
\hline $\begin{array}{l}\text { TYPE } \\
\text { CODE }\end{array}$ & DESCRIPTION & DEPTH \\
\hline$B$ & cement/seraterate Geand & 17.0 \\
\hline$\underline{s}$ & Heave Bentonite mud & 21 \\
\hline$F$ & suleea sand & \\
\hline & - & \\
\hline 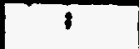 & & \\
\hline & & \\
\hline - & & \\
\hline & & \\
\hline-8 & $8-80.21$ & Poek \\
\hline$-D_{0}$ & oin from Ground Surfaee & \\
\hline
\end{tabular}

\section{WELL DEVELOPMENT}

compressor wele was contincususly blown, fluswed, surged and bown dry for apprex. 7 hrs. Hole bown dry and let set several fimes. Hole maxing small amt. of cuater. 
WELL 792 LOGS 
PROJECT AMBROSLA LAKE SITE, NEW MEXICO

JOB NO. $A M B O 1$ DATE 09/24/85

SURFACE ELEVATION 6999.00

TOP OF FITER PACK

$\begin{array}{ll}\text { WELL CASING TYPE } & \text { 4.0-IN.SCHED.80 PVC } \\ \text { COMPLETION } & \text { ALLUVIUM }\end{array}$

Page $^{1}$ of 1

20.0 feet

TOTAL DE

BORING TYRE ROTARY

COMPLETION

LOCATION N $55724.90 \quad E 60649.10$

DATUM

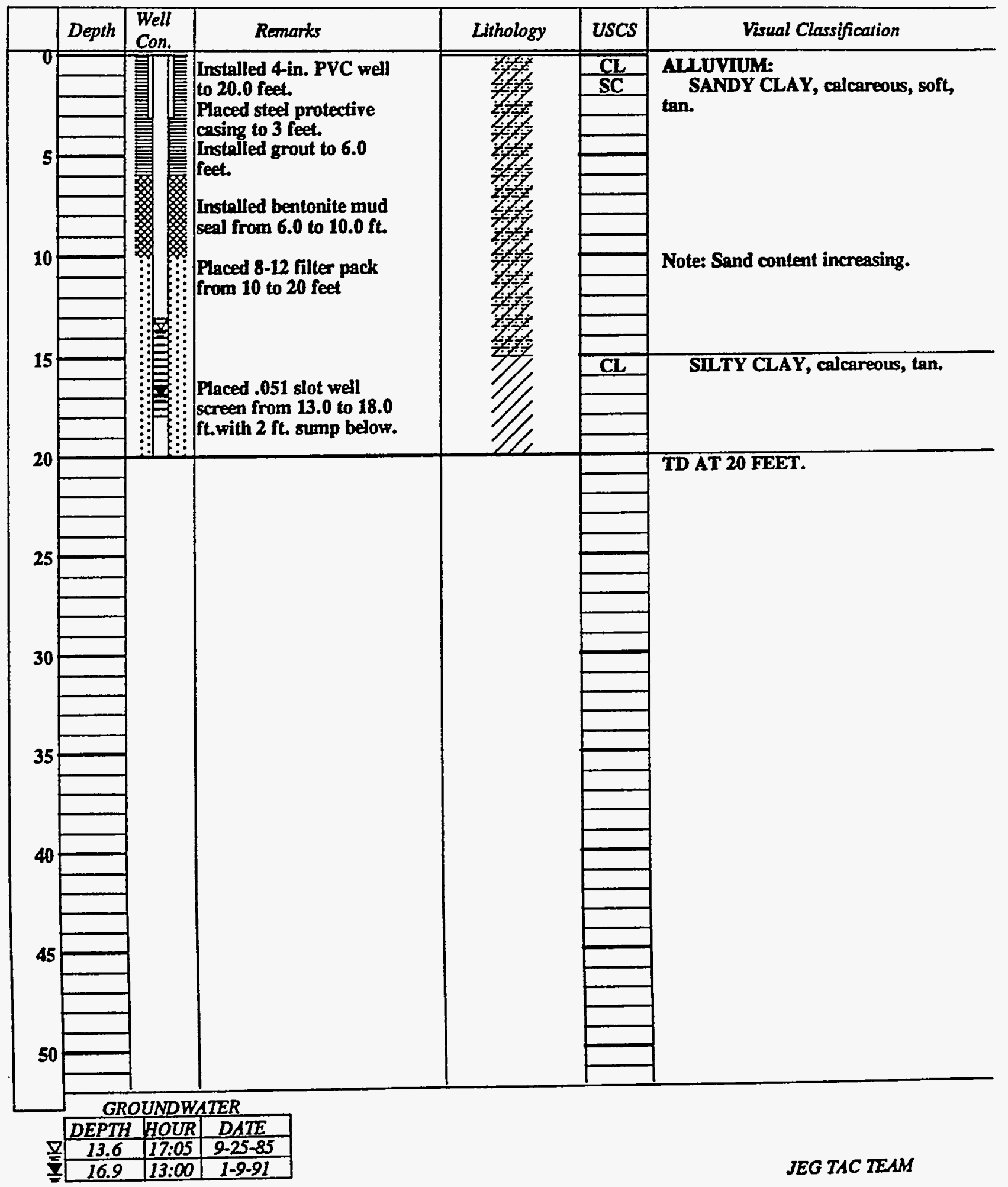




\section{TT UCOBS ENGNERTVG CROUP NR.

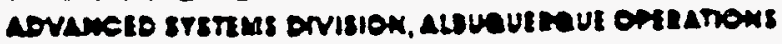

\section{WELL COMPLETION RECORD}

SITE ID: AMBOL LOCATION ID: 792 DATE INSTALLED: $9 / 24 / 85$ APPROX. SITE COORDINATES:(FT.) N NK E NK

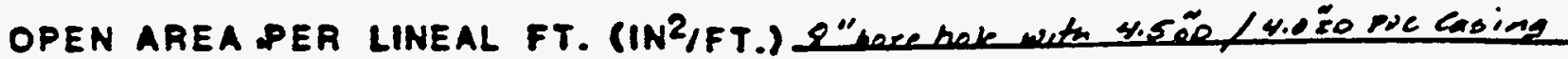
FORMATION OF COMPLETION: FIELD-REP.: Roger Holland (2)

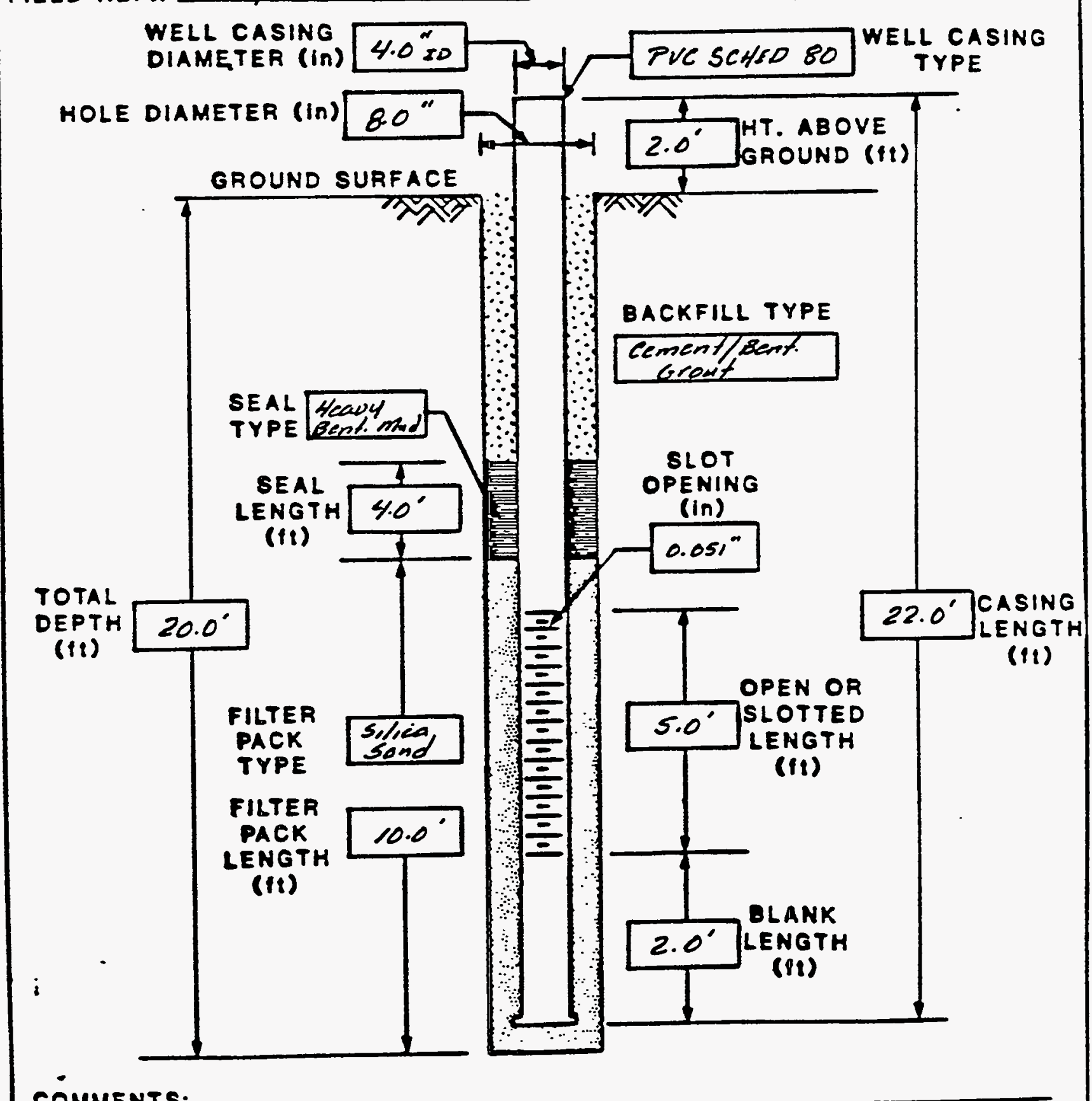

COMMENTS: 


\section{TE HCOBS ENGNEERTG GROUP NC.

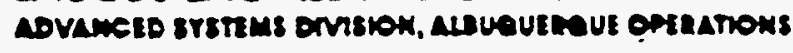 BOREHOLE/WELL CONSTRUCTION LOG}

SITE ID: AmBOL LOCATION ID:_292 FIELD REP: Reger Helland APPROX. BITE COORDINATES (FT.): N NK E NK

GROUND ELEVATIO'N (FT. MSL): NK COMPLETION DATE: $9 / 24 / 85$

\section{BOREHOLE SUMMARY}

DRILLER:_Stewart Brothers

RIG TYPE: Gardner Denver 10w (Rotory)

\begin{tabular}{|c|c|c|c|}
\hline BIT TYPE & $\begin{array}{l}\text { HOLE } \\
\text { DoLA. } \\
\text { (In.) }\end{array}$ & $\begin{array}{c}\text { ENDP } \\
\text { DEPTH } \\
(f \& .)\end{array}$ & $\begin{array}{l}\text { FLUID } \\
\text { TYPE }\end{array}$ \\
\hline $\begin{array}{l}\text { Aughs } \\
\text { mill Toeth }\end{array}$ & $8.0^{11}$ & $20.0^{\prime}$ & $\begin{array}{l}\text { Bentonite } \\
\text { mulat }\end{array}$ \\
\hline & & & \\
\hline & & & \\
\hline & & & \\
\hline
\end{tabular}

\begin{tabular}{|c|c|c|c|}
\hline $\begin{array}{l}\text { CASING } \\
\text { TYPE }\end{array}$ & DESCAIPTION & $\begin{array}{l}\text { DiA. } \\
\text { (in.). }\end{array}$ & $\begin{array}{l}\text { ENDW } \\
\text { DEPTH } \\
(49 .)^{-1}\end{array}$ \\
\hline$B$ & PVE SCHED 80 & $4 \cdot 0_{\text {so }}^{n}$ & $15^{\prime}$ \\
\hline 5 & Pue .05i" slotfed & $4.0^{\circ}$ & $20^{\circ}$ \\
\hline$B$ & PUC SCHED 80 & $4.0^{\prime \prime}$ & $22^{\circ}$ \\
\hline$p$ & Steel casing & $8.0^{4}$ & $5^{\prime}$ \\
\hline & & & \\
\hline & & & \\
\hline & & & \\
\hline & & & \\
\hline paprote & five 6-8eroen B-siank & & A-None \\
\hline
\end{tabular}

Popin from Top of Casing

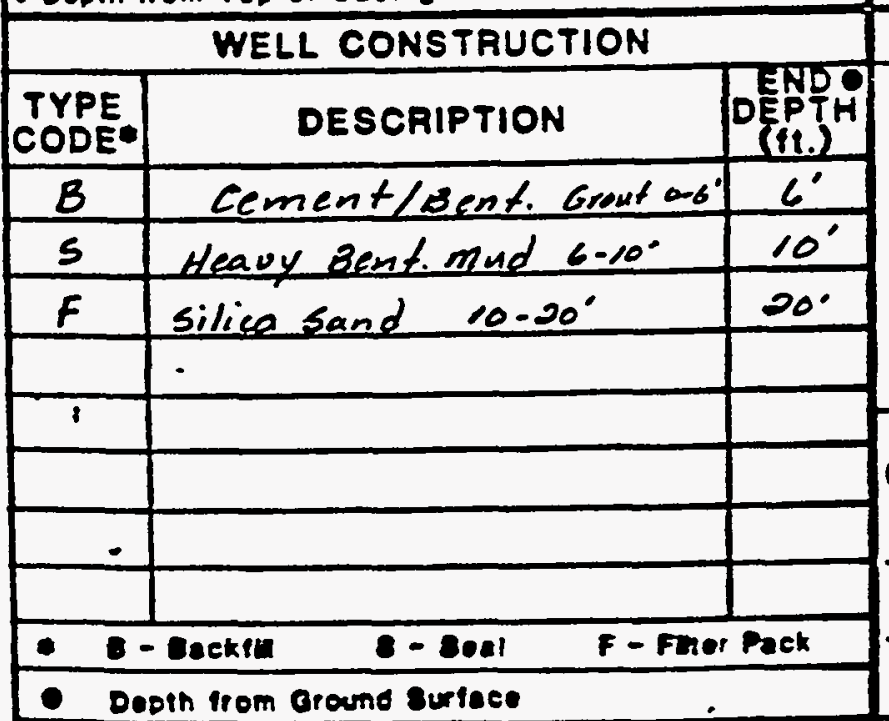

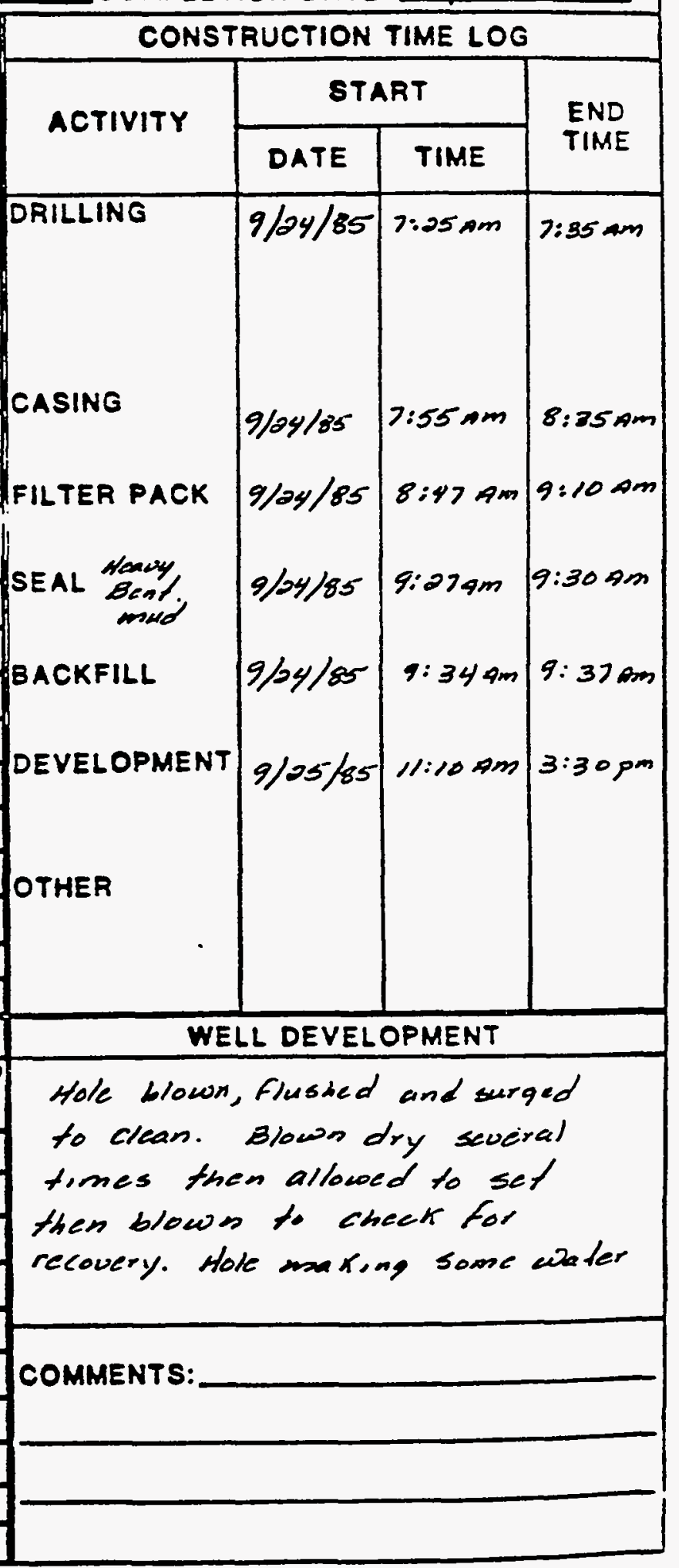


WELL 793 LOGS 
PROJECT AMBROSLA LAKE SITE, NEW MEXICO

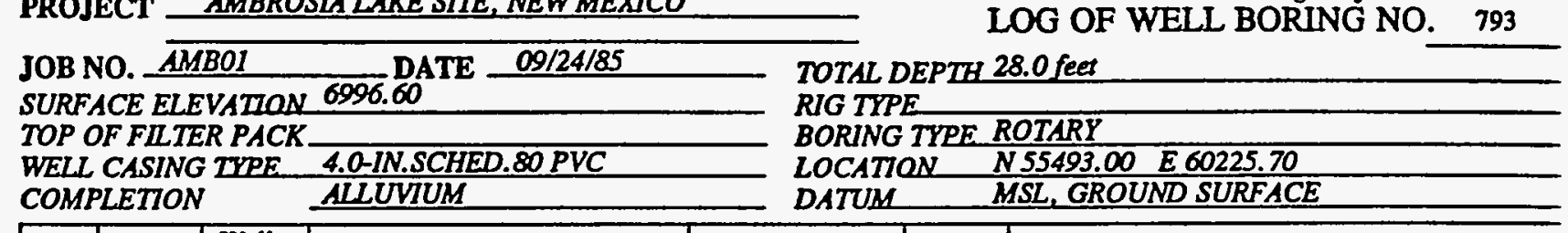

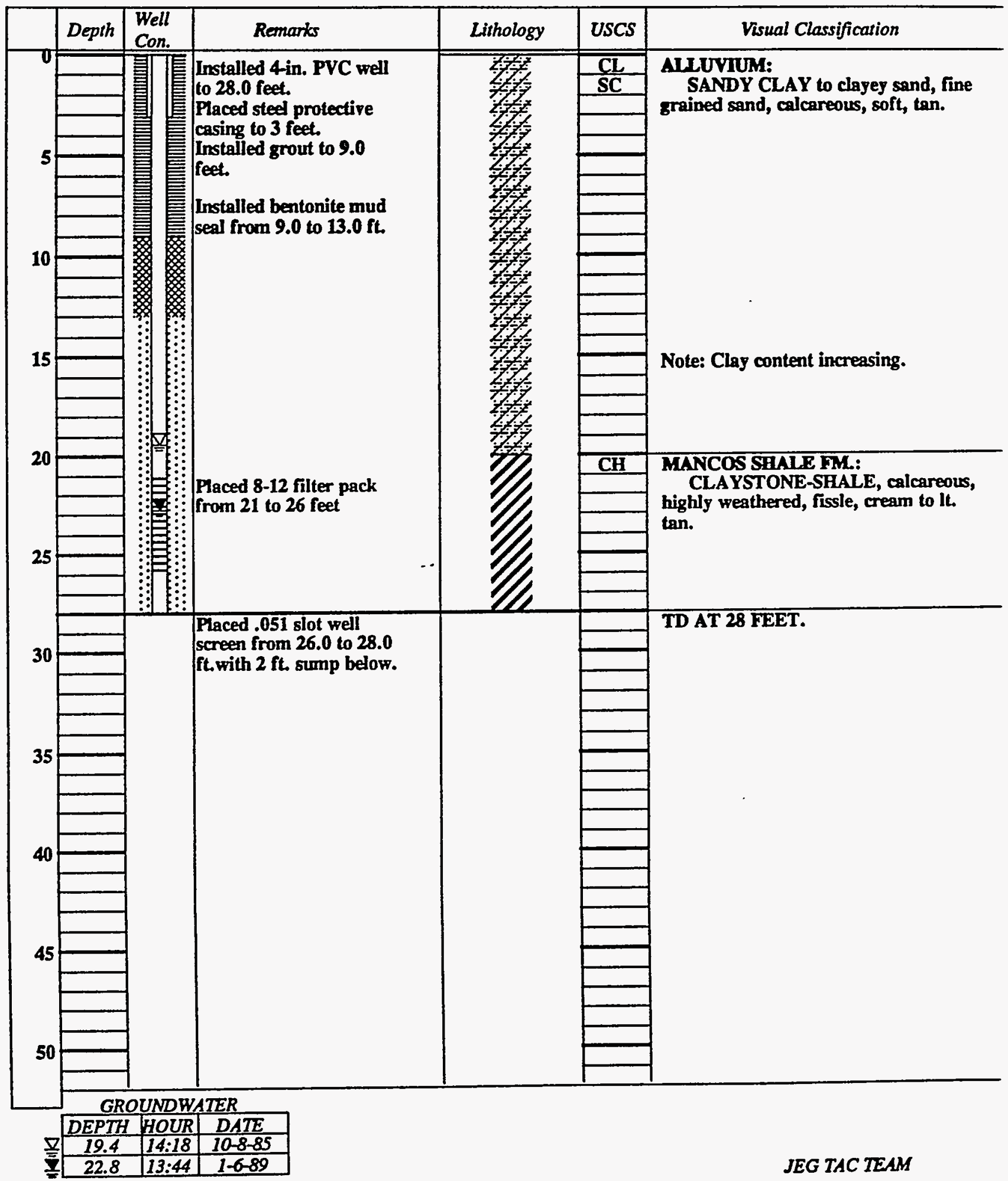




\section{WELL COMPLETION RECORD}

SITE ID: AmBOI LOCATION ID: 793

DATE INSTALLED: $2 / 24 / 85$ APPROX. SITE COORDINATES:(FT.) N NK E $1 K$

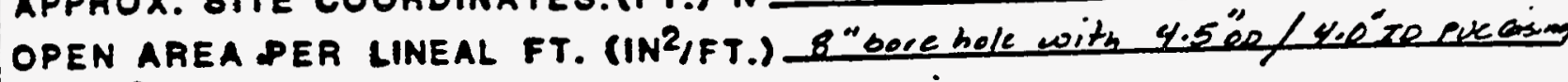
IFORMATION OF COMPLETION:

FIELD-REP.: Reger tolland Alluvium

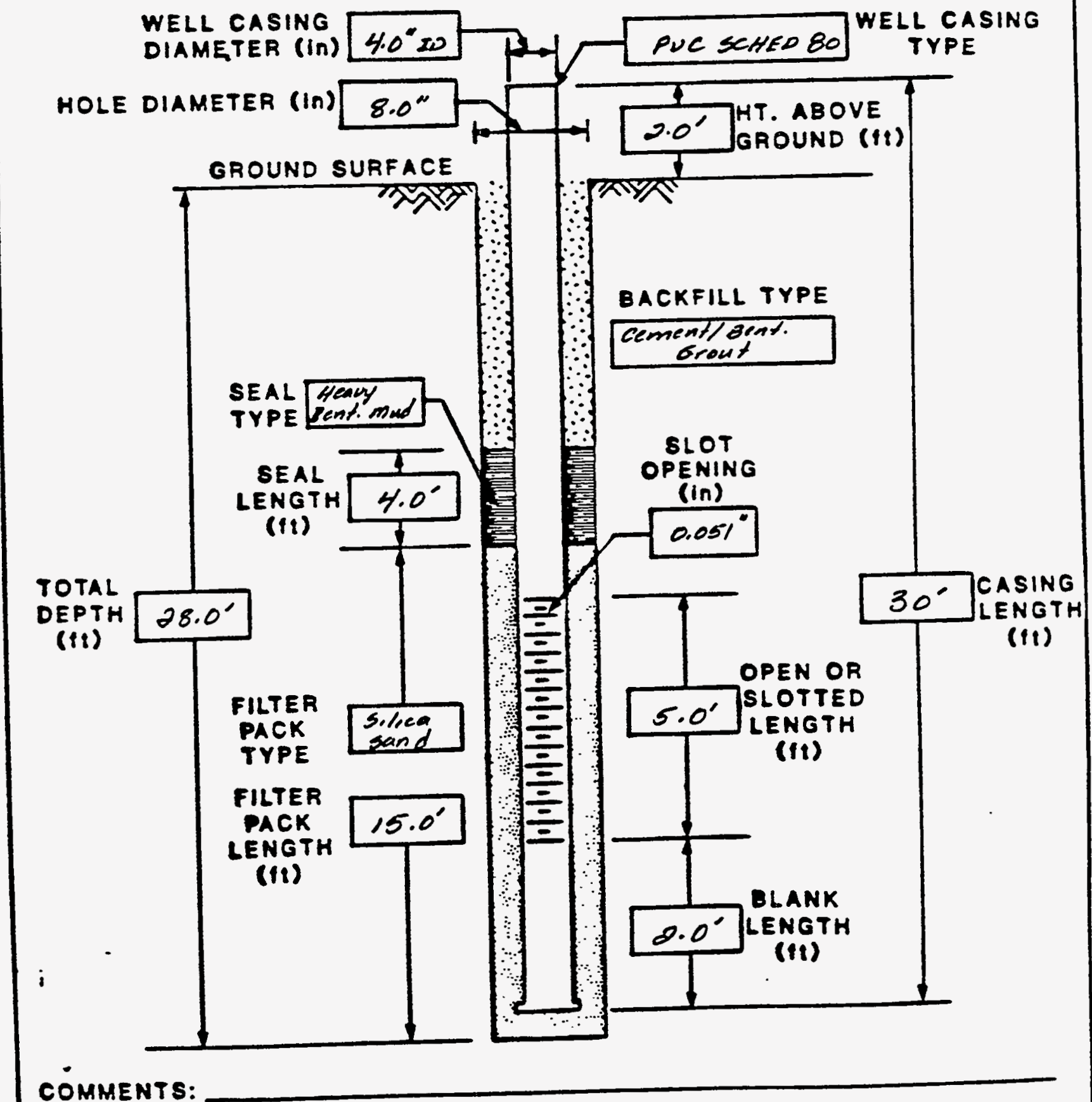


$16 \cdot 19 \cdot 2 \cdot 2 \cdot 3$

\section{WE MOOSS BKGNEERIV CROUP NC. \\ BOREHOLE/WELL CONSTRUCTION LOG}

SITE ID: AMBOI LOCATION ID:_293 FIELD REP:_LRQ_er Helland GROUND ELEVATION (FT. MSL):

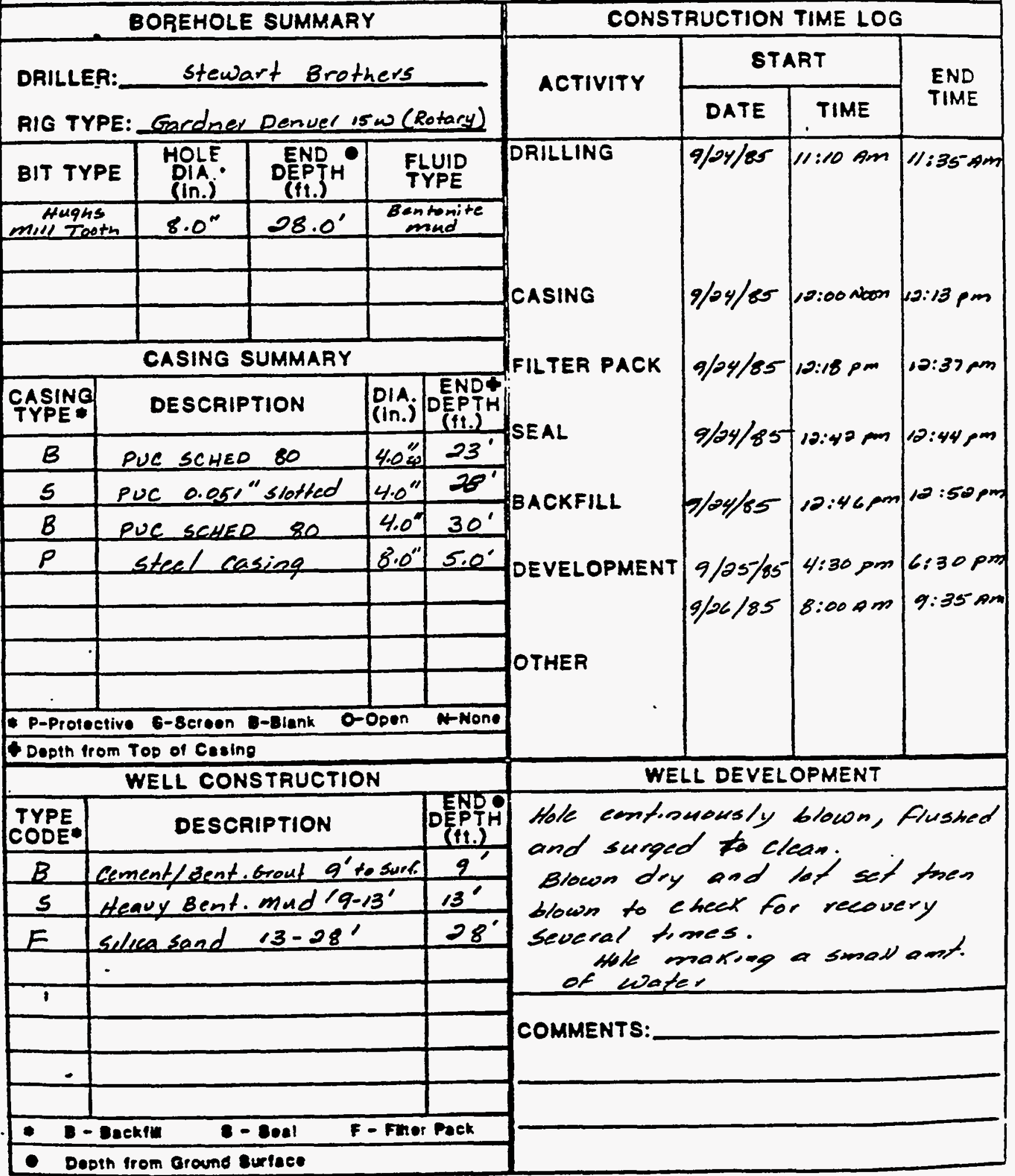


WELL 794 LOGS 


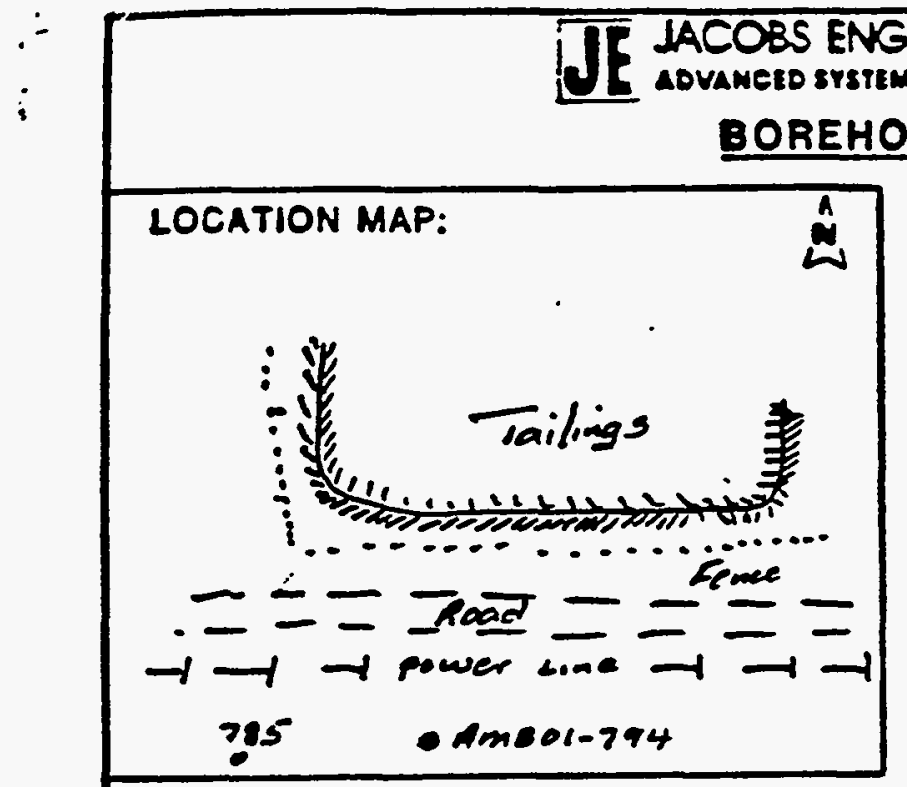

GINEERING GROUP INC.

AOVAMERD SYSTEMS DIVISION. ASQUQUERQUE ORERATIONS

BOREHOLE LOG (ROCK)

Pape L of 1

LOCATION MAP:

SITE ID: AMBOI LOCATION ID: 794

SITE COORDINATES (1t.):

$N$ NK

GROUND ELEVATION ( $f t . M S L): N K$

DRILLING METHOD: Rotory - Gear Bit

DRILLING CONTR.: Stement Brethers

DATE STARTED: $q / 24 / 85$

DATE COMPLETED: Q/24/15

FIELD REP.: Reger Helland

\begin{tabular}{|c|c|c}
\hline \multicolumn{3}{|c}{ GROUNDWATER LEVELS } \\
\hline DATE & TIME & DEPTH (11.) \\
\hline & & \\
\hline & & \\
\hline & & \\
\hline
\end{tabular}

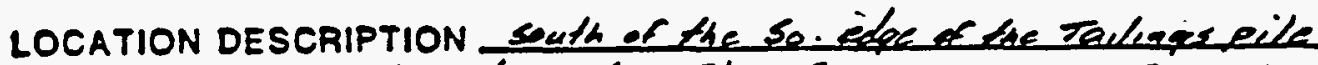

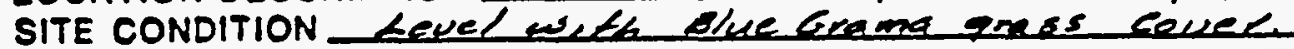

\begin{tabular}{|c|c|c|c|c|c|c|c|}
\hline $\begin{array}{l}0 \\
\vdots \\
z \\
\vdots \\
u\end{array}$ & 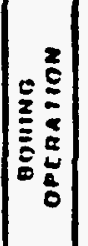 & 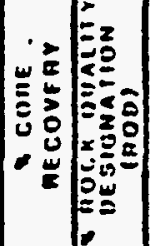 & 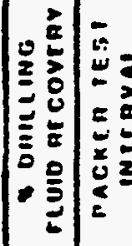 & 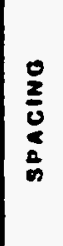 & 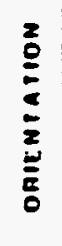 & $\begin{array}{l}0 \\
0 \\
0 \\
0\end{array}$ & 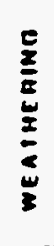 \\
\hline
\end{tabular}

$\circ$ 6

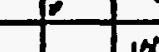

5

\begin{tabular}{lllll|l|l|l|l|l|l|}
\hline & & & & & & & & & & L \\
\hline
\end{tabular}

sandy lay, tan, fa, calc.

\begin{tabular}{|l|l|l|l|l|l|}
\hline & & & & \\
\hline & & & & \\
\hline & & & & \\
\hline
\end{tabular}
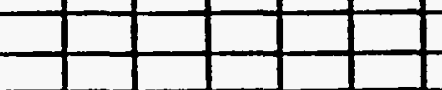

$+$

$1+$

sandetone, fan to yellow to red orango

with hemitite frags - gravely.

correlates with basal alluwium

at ofher bore holes

15

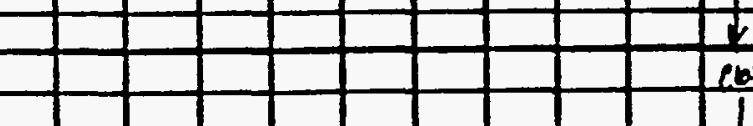

10

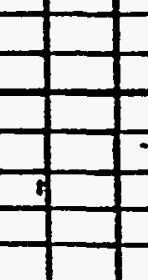

COMMENTS:

15 22' clay, ton, soft, calc. weathere mancos.

$T 20.0^{\circ}$ 
. . TE HOOSS BNGNERNG GROU NK.

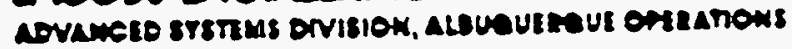

\section{WELL COMPLETION RECORD}

SITE ID: AMBOI LOCATION ID: 794

DATE INSTALLED: g/24/85

APPROX. SITE COORDINATES:(FT.) $N$ D.K E NA

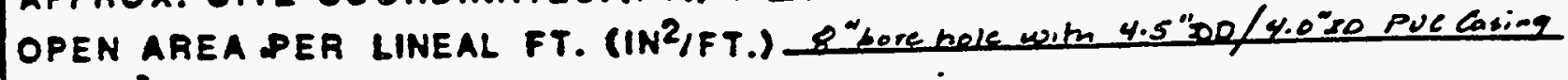
|FORMATION OF COMPLETION:

FIELD -REP.: Reger thlland DRILLER: Alluweren

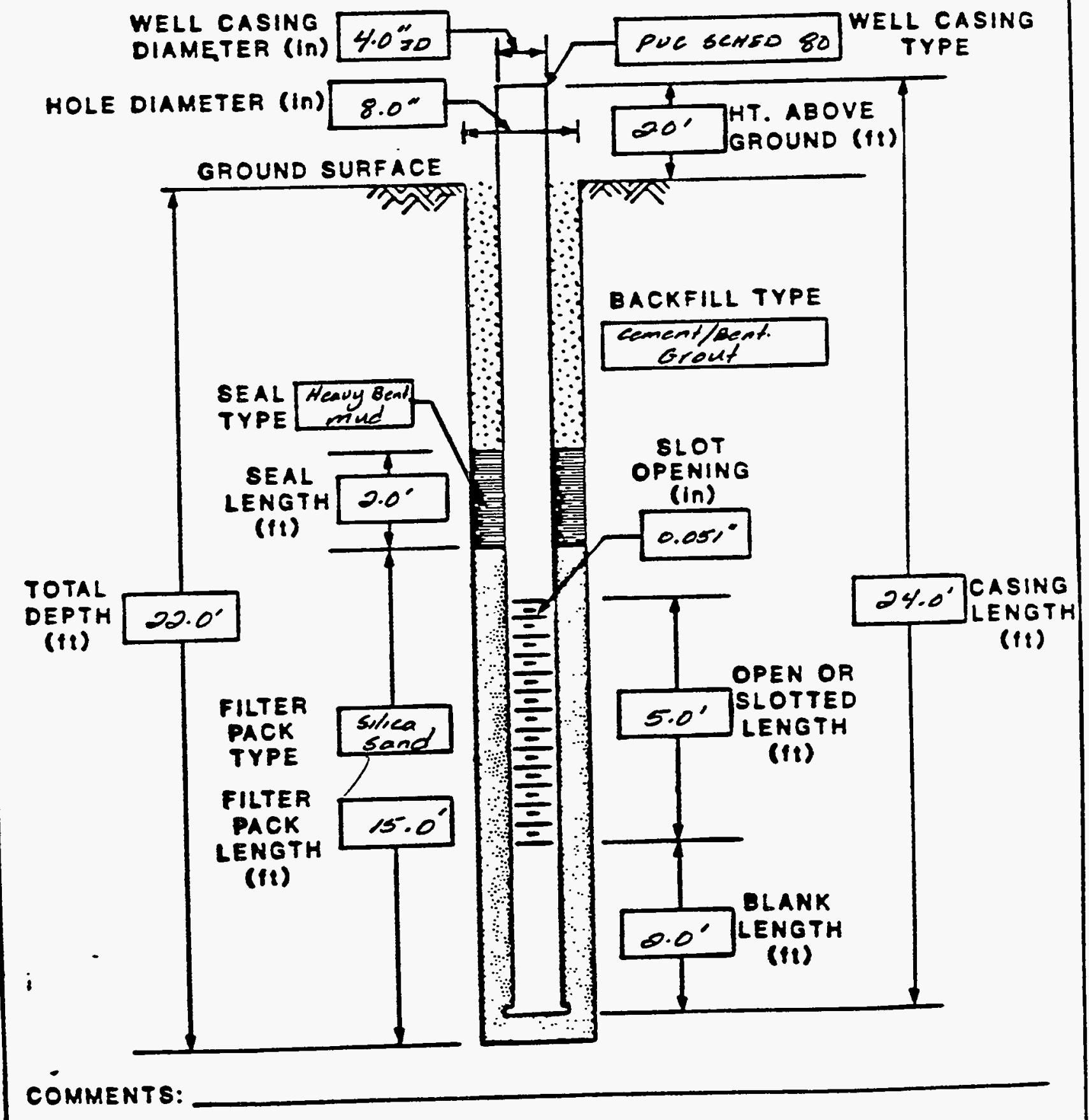

JEG-AL-ENO-S (S/84) 


\section{TT MCOBS BKAN EERTG GROUP NK.

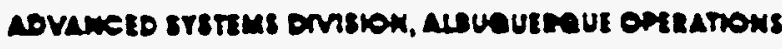 BOREHOLE/WELL CONSTRUCTION LOG}

SITE ID: AmBel LOCATION ID: 294 FIELD REP: Roger Holland APPROX. 8ITE COORDINATES (FT.): $\mathrm{N} N K$ GROUND ELEVATION (FT. MSL): NK COMPLETION DATE: Q/04/SS

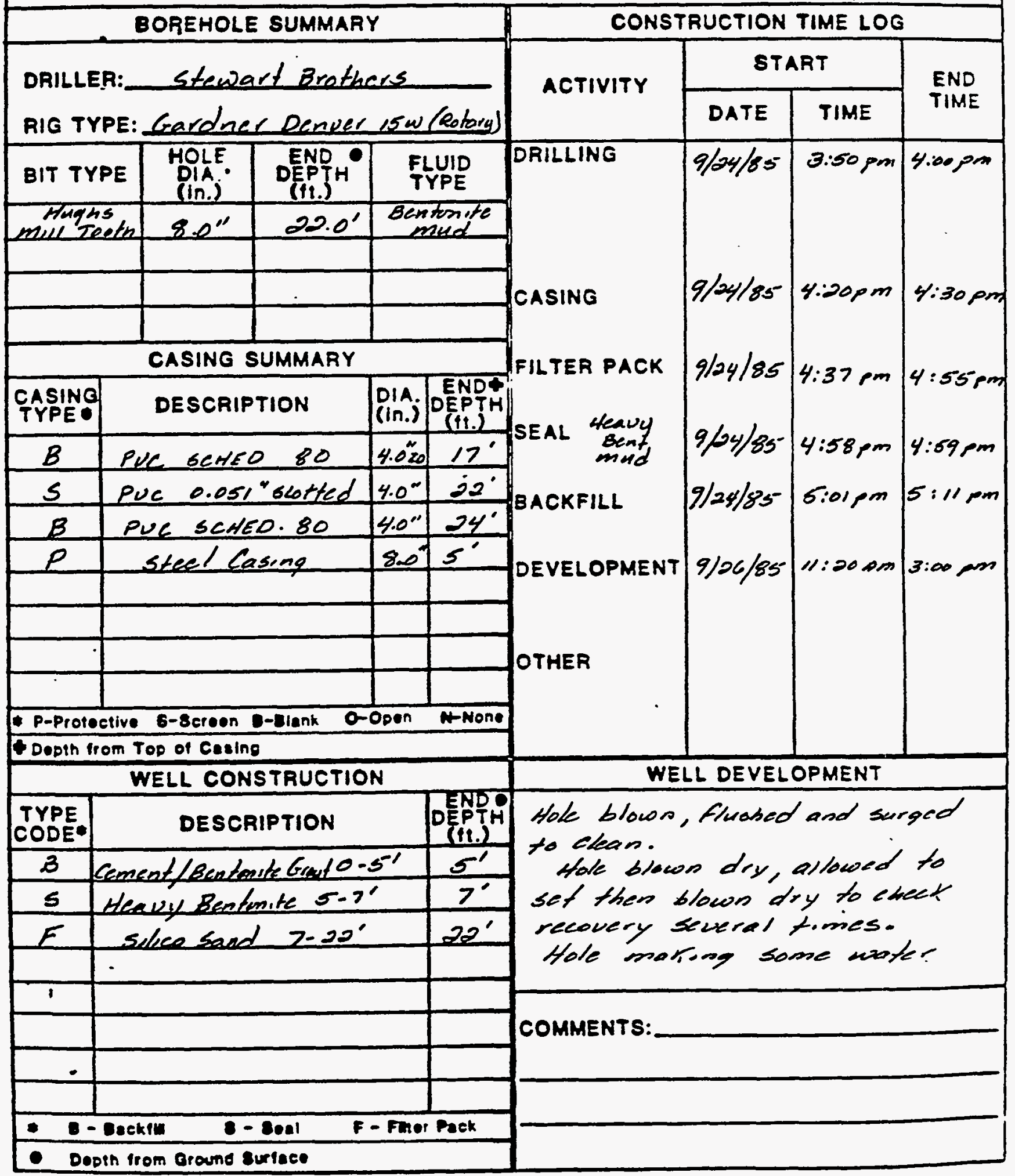


WELL 796 LOGS 
PROJECT AMBROSL LAKE SITE, NEW MEXICO

JOB NO. AMBO1 DATE 09/25/85

SURFACE ELEVATION 6955.10

TOP OF FILTER PACK 10.00

WELL CASING TYPE 4.0-IN.SCHED.80 PVC

COMPLETION ALLUVUM:(dry)
Page I of 1

LOG OF WELL BORING NO.

796

TOTAL DEPTH 23.0 feat

RIG TYPE

BORING TYPE ROTARY

LOCATTON N52337.40 E 58319.60

DATUM MSL, GROUND SURFACE

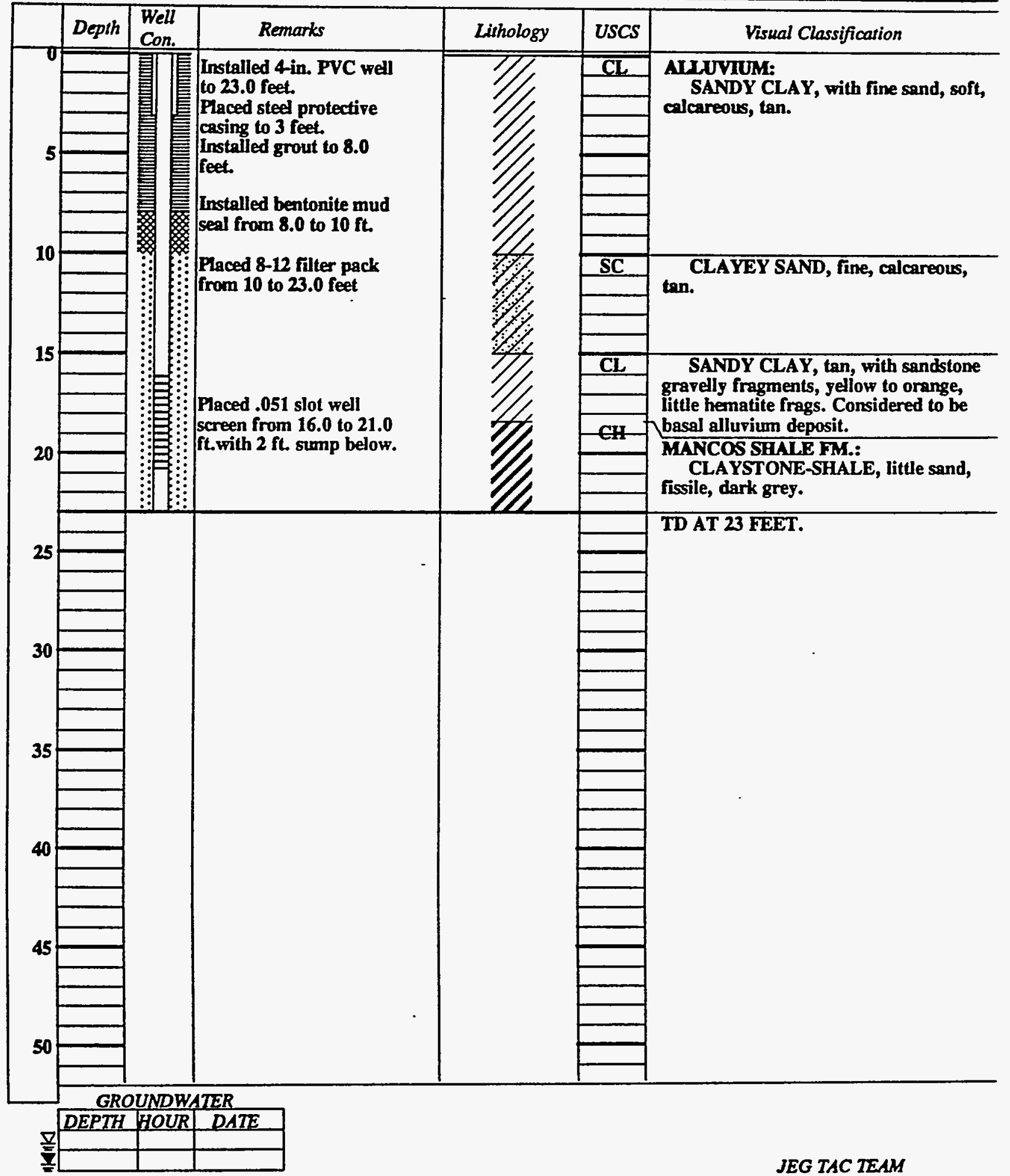




\section{TT UNOBS ANGAEERTG GROUP INC.

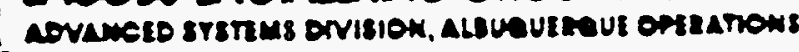

\section{WELL COMPLETION RECORD}

SITE ID: AMBOL LOCATION ID: 796 DATE INSTALLED: $9 / 25 / 85$ APPROX. SITE COORDINATES:(FT.) N NX E N

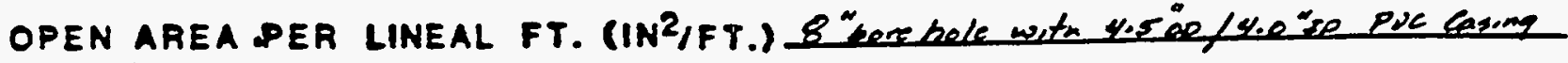
FORMATION OF COMPLETION:

FIELD -REP.: beper Helland Qlluwerim

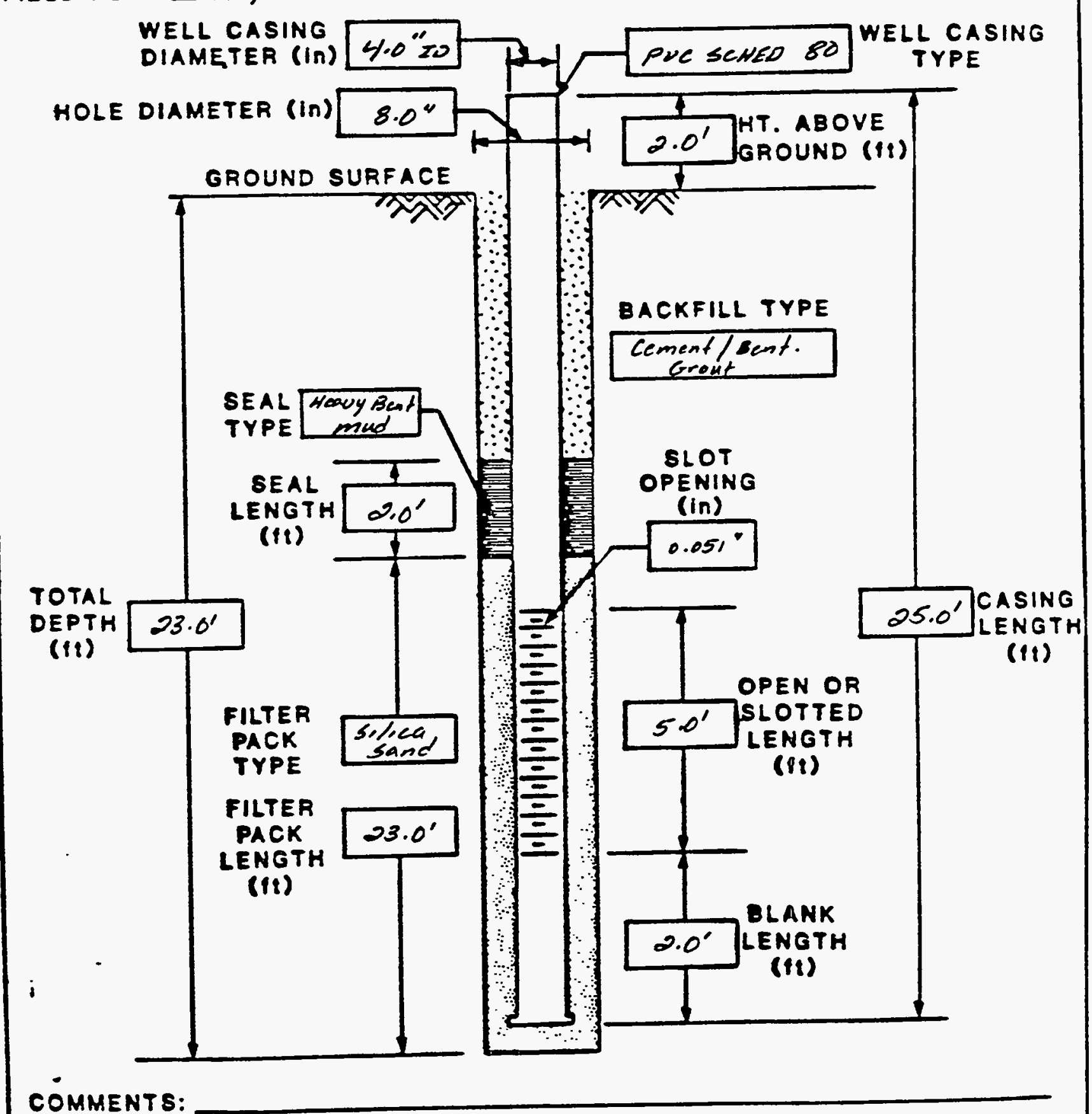

COMMENTS: 


\section{TT HCOBS ENEN EERTE GROUP NK.

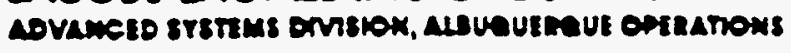 BOREHOLE/WELL CONSTRUCTION LOG}

SITE ID: AmBel LOCATION ID: Z9h FIELD REP: Roger Holland APPROX. BITE COORDINATES (FT.): N NLK GROUND ELEVATION (FT. MSL): NK

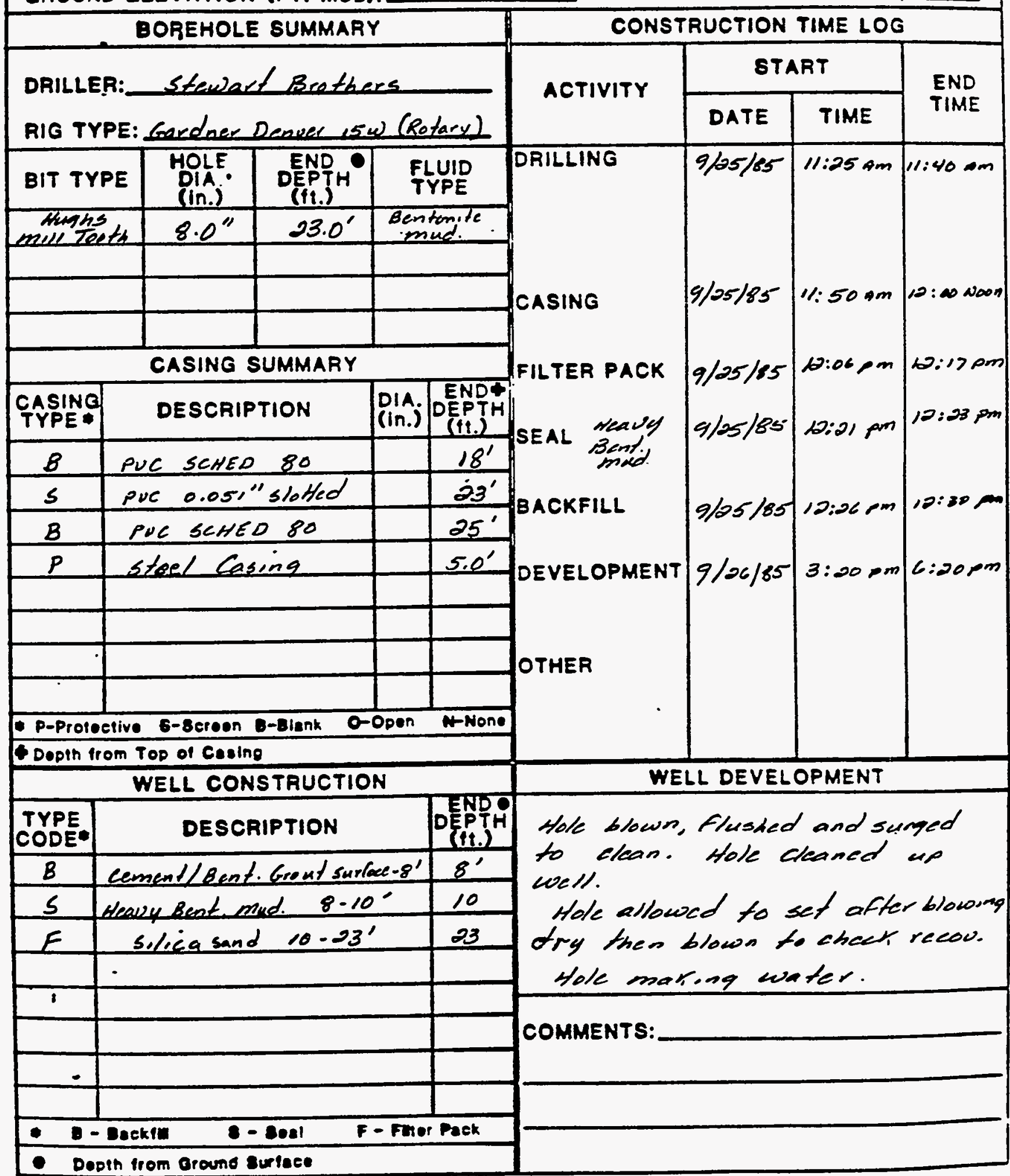


WELL 797 LOGS 
PROJECT AMBROSLA LAKE SITE, NEW MEXICO

JOB NO. $A M B 01$ DATE

SURFACE ELEVATION 6969.70

TOP OF FILTER PACK

WELL CASING TIPE

4.0.IN.SCHED.80 PVC

COMPLETION ALLUVIM:(dry)

TOTAL DEPTH 21.5 feet

LOG OF WELL BORING NO

797

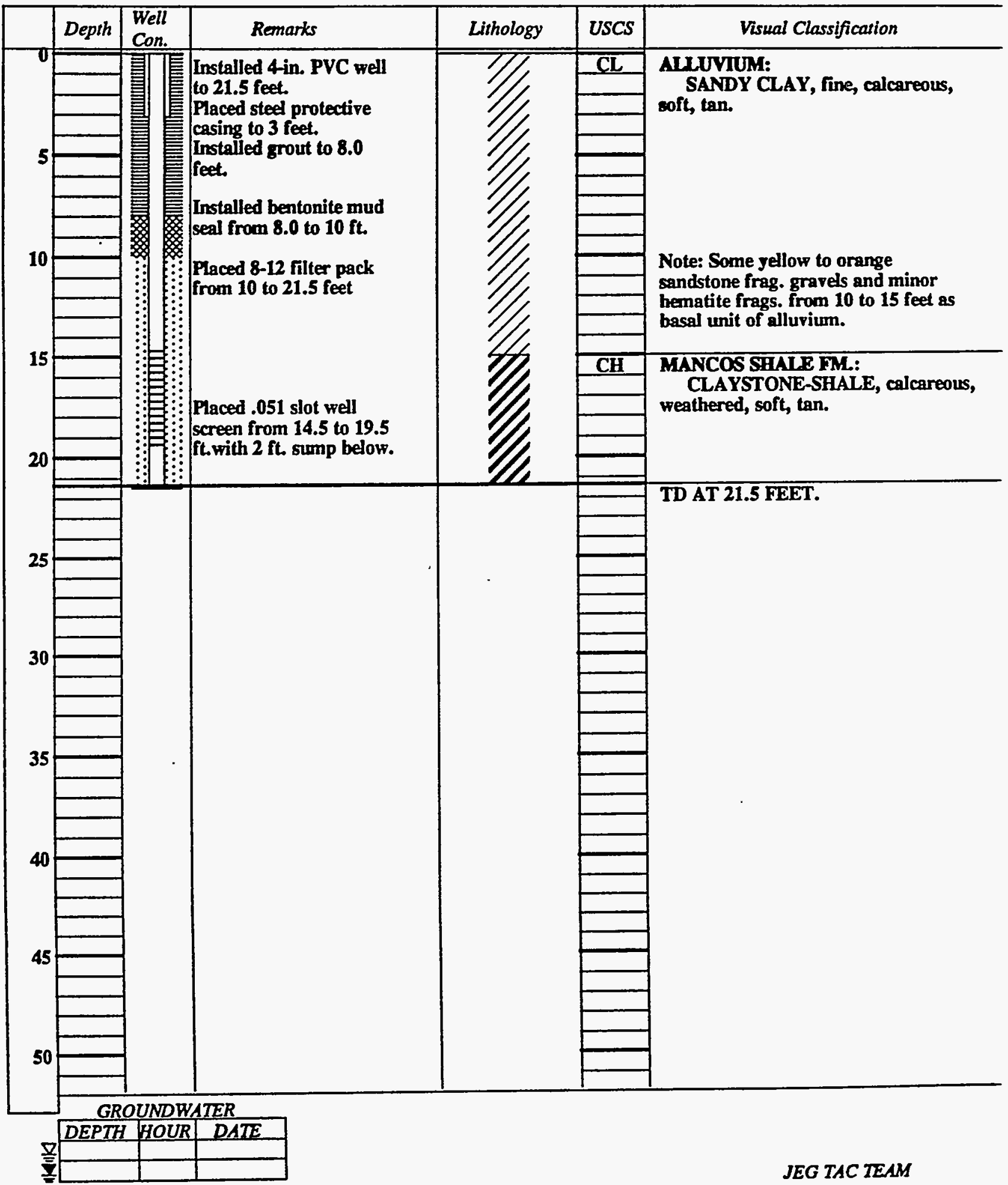




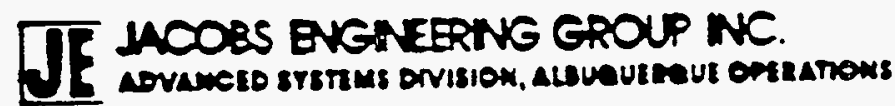

\section{WELL COMPLETION RECORD}

SITE ID:AMBOL LOCATION ID: 797 DATE INSTALLED:7/05/85 APPROX. SITE COORDINATES:(FT.) N Ne E NK

OPEN AREA PER LINEAL FT. (IN2/FT.) \&" pore bole weith $4.5 \% 0 / 4.0^{\circ}$ Fe Pue lesing |FORMATION OF COMPLETION:

FIELD-REP.: Roger Holland Allewiues DRILLER: Stewart Brothers WELL CASING
DIAMETER (IN) $4.0^{*}$ TD PUC SCHED 80 WELL CASING

HOLE DIAMETER (In) $8.0^{\prime \prime}$ GROUND SURFACE
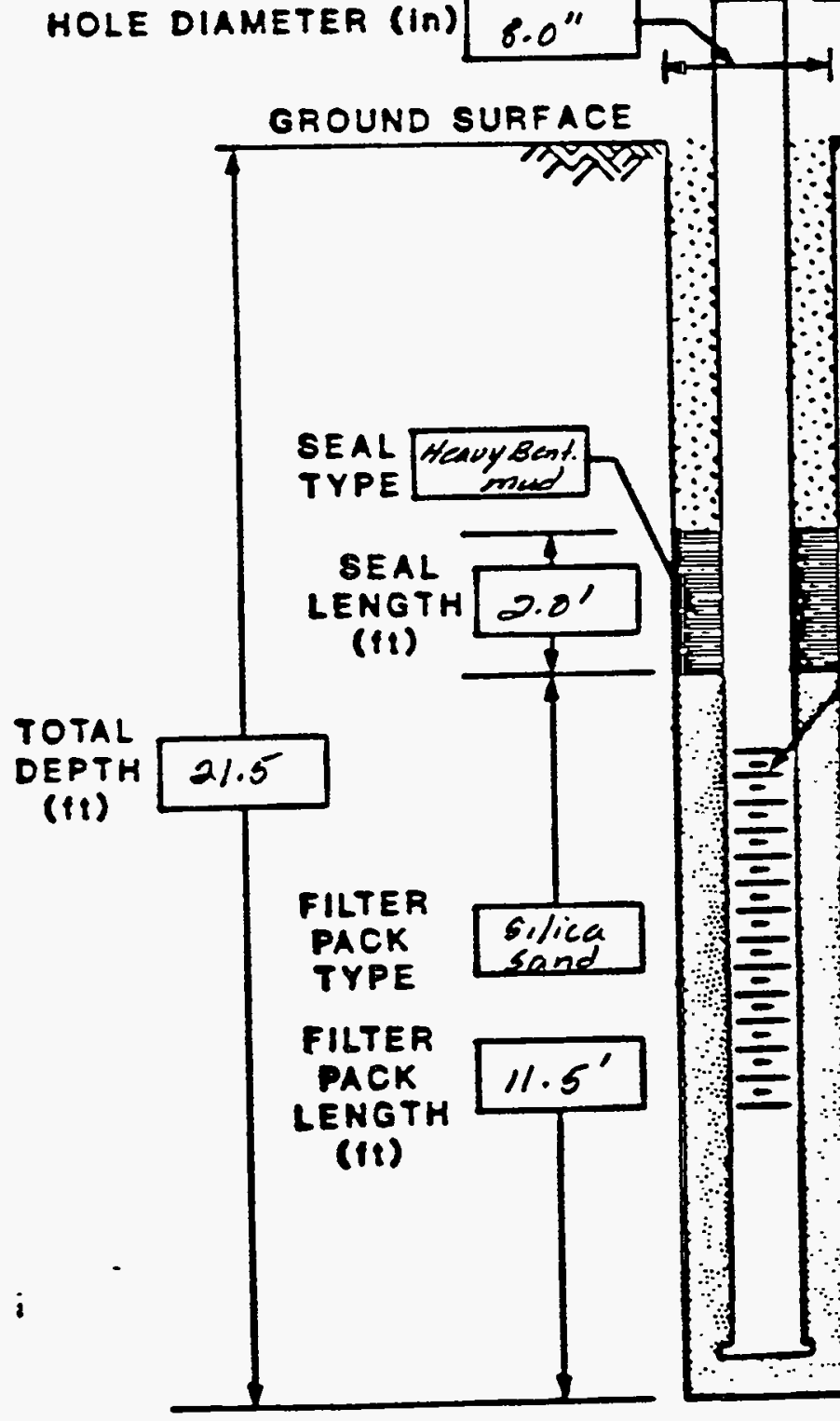

I
$2.0^{\prime}$ GT. ABOVE
GROUND (ti)
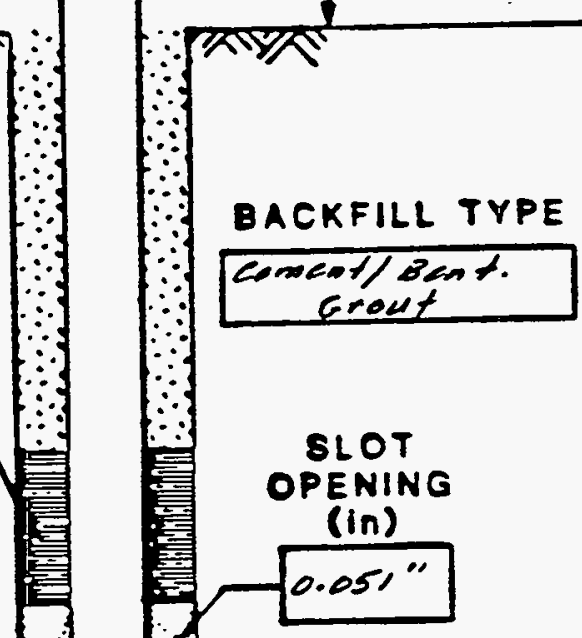

SLOT

OPENING

(In)

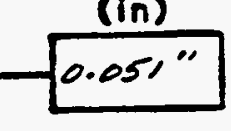


$16 \cdot 19 \cdot 2 \cdot 2 \cdot 3$

\section{TT MCOSS BNENEERIG GROUP NK.

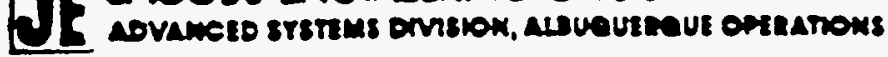 BOREHOLE/WELL CONSTRUCTION LOG}

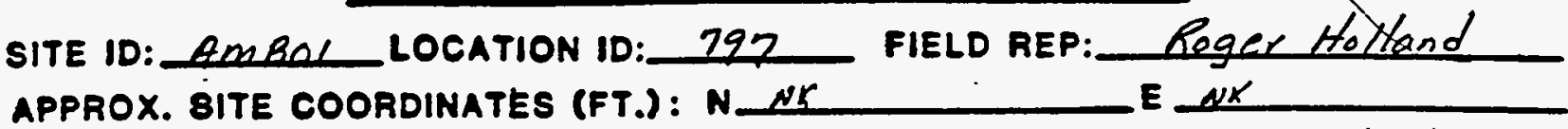
COMPLETION DATE: $9 / 25 / 85$ GAOUND ELEVATION (FT. MSL): NK CONSTRUCTION TIME LOG

DRILLER:_ Stewart Brothers

RIG TYPE: Gardner Denver $15 w$ (Rotary)

\begin{tabular}{|c|c|c|c|}
\hline BIT TYPE & $\begin{array}{l}\text { HOLE } \\
\text { DinA. } \\
\text { (In.) }\end{array}$ & $\begin{array}{l}\text { END } \\
\text { DEPTH } \\
(11 .)\end{array}$ & $\begin{array}{l}\text { FLUID } \\
\text { TYPE }\end{array}$ \\
\hline $\begin{array}{l}\text { Haghs } \\
\text { mill Teeth }\end{array}$ & $8.0^{N}$ & & $\begin{array}{c}\text { Bentonite } \\
\text { mond }\end{array}$ \\
\hline & & & \\
\hline & & & \\
\hline
\end{tabular}

CASING SUMMARY

\begin{tabular}{|c|c|c|c|}
\hline $\begin{array}{l}\text { CASING } \\
\text { TYPE }\end{array}$ & DESCRIPTION & (IIA.) & $\begin{array}{l}\text { END' } \\
\text { DEPTH } \\
\text { (ft.) }\end{array}$ \\
\hline$B$ & PUC SCHED 80 & $4.0^{\prime \prime} \times 0$ & 16.5 \\
\hline$s$ & pue $0.051^{\prime \prime}$ slotted & $4.0^{\prime \prime}$ & 31.5 \\
\hline$B$ & PUC SCHEO 80 & $4.0^{\circ}$ & $23.5^{\circ}$ \\
\hline$P$ & Steel Casing & $8.0^{\prime \prime}$ & 5.0 \\
\hline & & & \\
\hline & & & \\
\hline & & & \\
\hline & & & \\
\hline
\end{tabular}

Dopth from Top of Casing

\begin{tabular}{|c|c|c|}
\hline \multicolumn{3}{|c|}{ WELL CONSTRUCTION } \\
\hline $\begin{array}{l}\text { TYPE } \\
\text { CODE* }\end{array}$ & DESCRIPTION & DEPTH \\
\hline$B$ & Cement/Bent.Grout $0-8^{\prime}$ & $8^{\prime}$ \\
\hline$s$ & Heanysantinnd $8-10$ & $10.0^{-}$ \\
\hline$F$ & Seleca sand $10-21.5$ & 21.5 \\
\hline & - & \\
\hline \multicolumn{3}{|l|}{ 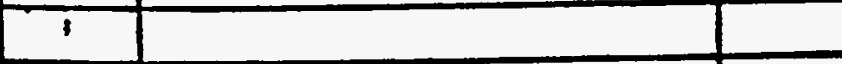 } \\
\hline & & \\
\hline \multicolumn{3}{|l|}{-} \\
\hline \multicolumn{3}{|c|}{ - 8-Beektil 8-8osl F-Fmer Pack } \\
\hline - De & in from Bround surlace & \\
\hline
\end{tabular}

CASING FILTER PACK

OTHER

\section{BACKFILL} DEVELOPMENT

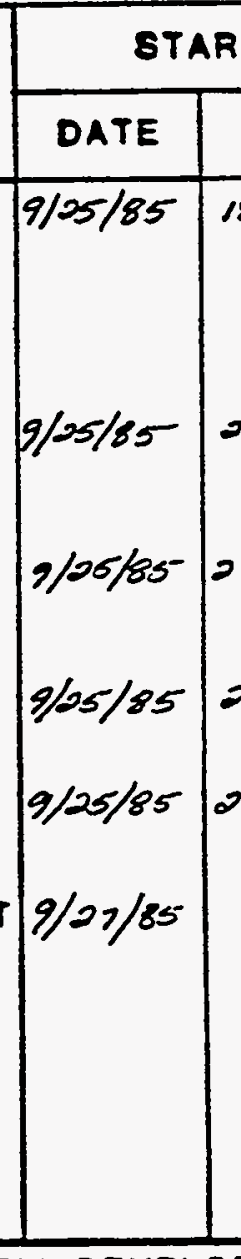

\section{WELL DEVELOPMENT}

Began dewebping o 7:55 am Blew hole, miect and Fiush. to clean Blow dry and tet set 10 min wow - wery little water remaned Flush and clean Let set 10:10 Am - 10:35 Am Blow 10:36 am-11:00 an
END

TIME

$1: 57 \mathrm{pm}$ $7: 558 \mathrm{~m} 11: 00 \mathrm{Am}$

COMMENTS:

Hete opecurs pry 
WELL 798 LOGS

x, 


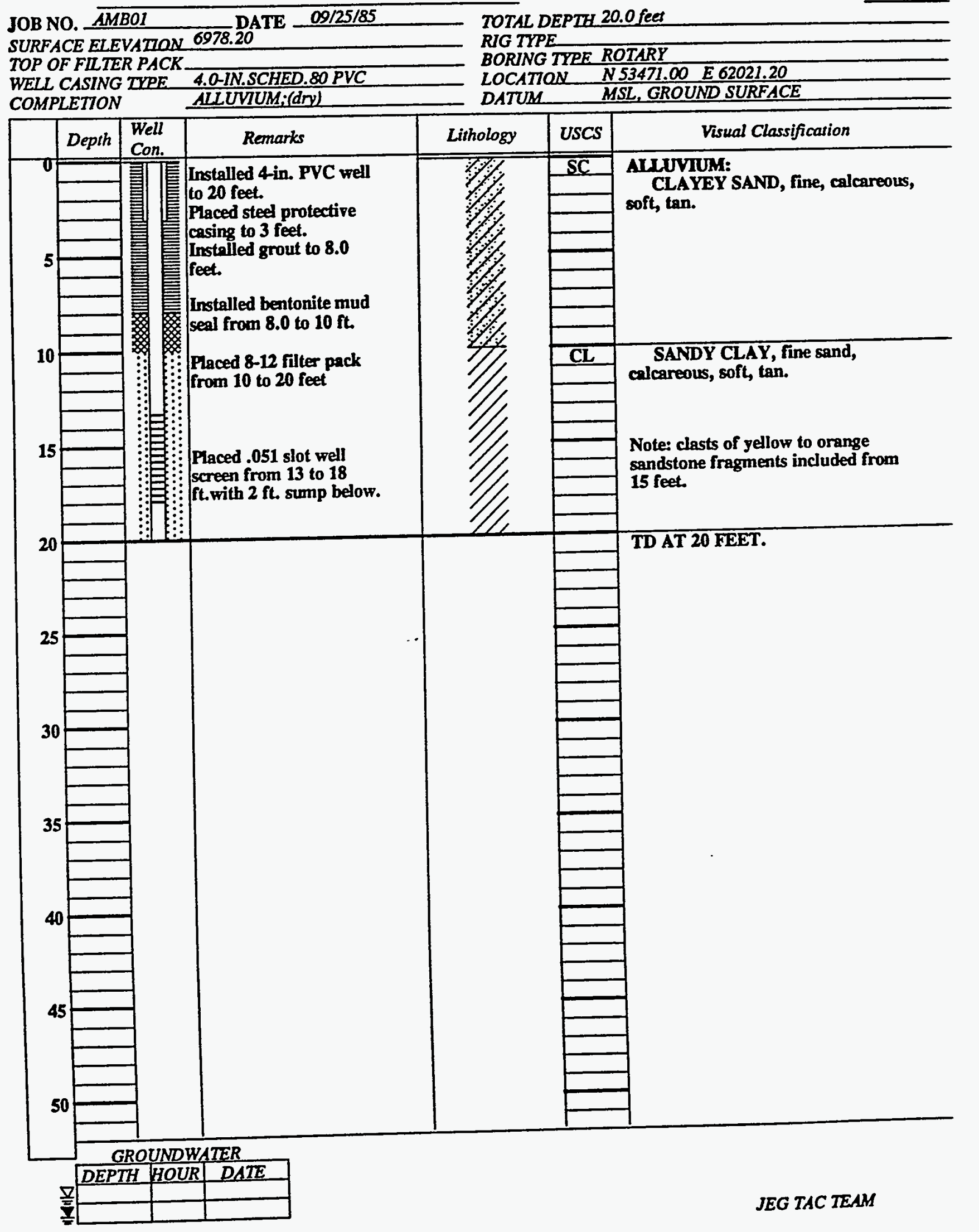

LOG OF WELL BORING NO. 798 


\section{TTE MCOSS ENGNEERTG GROUP NNC.

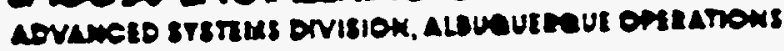

\section{WELL COMPLETION RECORD}

SITE ID: AMBOL LOCATION ID: 798 DATE INSTALLED: $2 / 05 / 85$ APPROX. SITE COORDINATES: (FT.) N NK E NK OPEN AREA PER LINEAL FT. (IN2/FT.) $\Omega^{\prime \prime}$ bare bele wete $45^{20}$ op/4.0"re PUe casing FORMATION OF COMPLETION:

FIELD-REP.: Peger Halland Alluwiunes

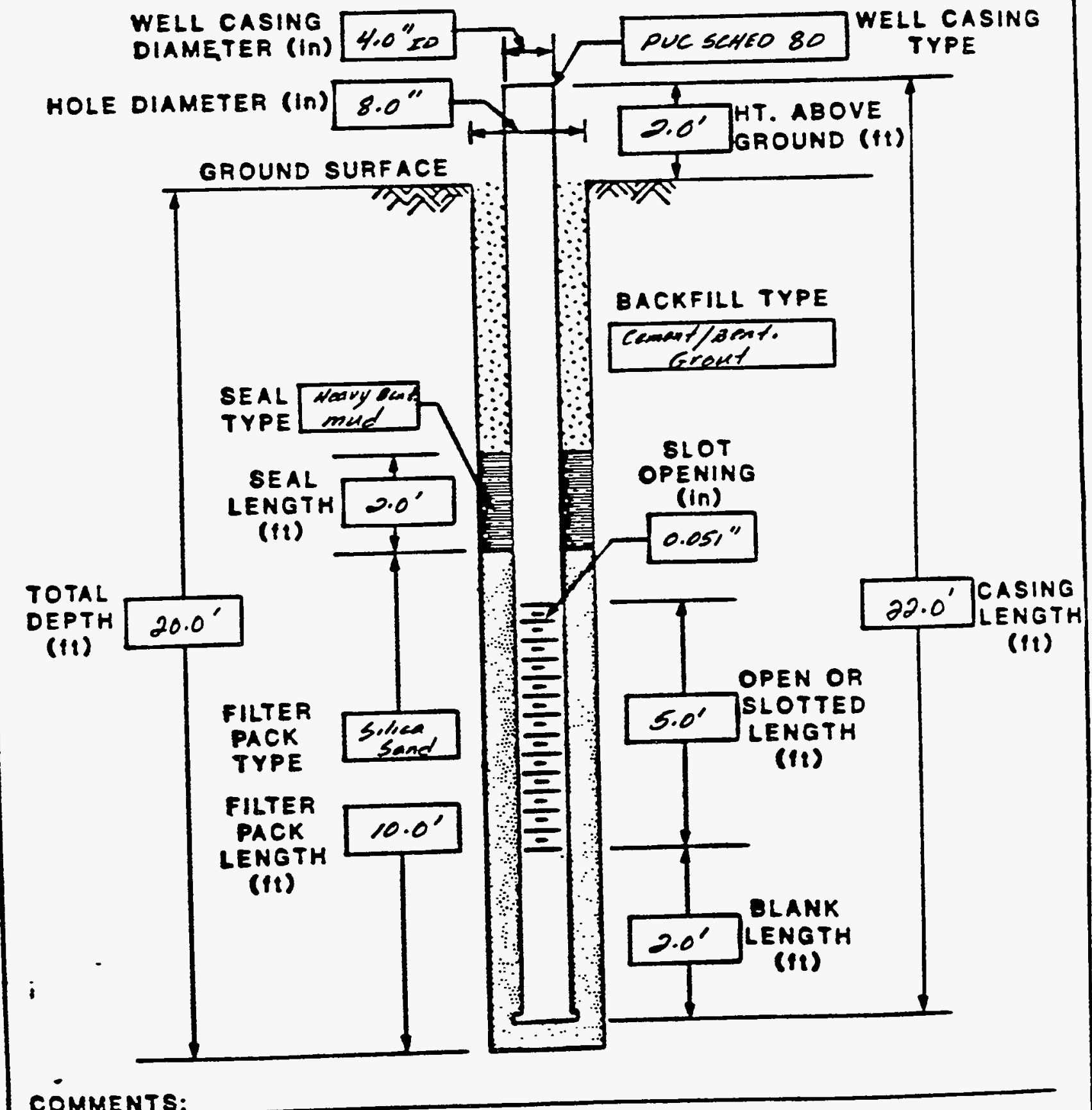


$16.19 \cdot 2 \cdot 2 \cdot 3$

\section{TI HCOBS ENGNEERTE GROUP NC.

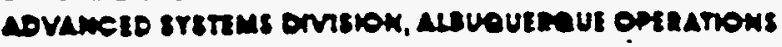 \\ BOREHOLE/WELL CONSTRUCTION LOG}

SITE ID: EmBOL LOCATION 1D: 798 FIELD REP: Roger F. Holland APPROX. BITE COORDINATES (FT.): N NK NK

GROUND ELEVATION (FT. MSL): NK

COMPLETION DATE: $9 / 05 / 85$

\section{BOREHOLE SUMMARY}

DRILLER: Stewart Brothers.

RIG TYPE: Gardner Denver 15w (Rotary)

\begin{tabular}{|c|c|c|c|}
\hline BIT TYPE & $\begin{array}{l}\text { HOLE. } \\
\text { DIA. } \\
\text { (in.) }\end{array}$ & 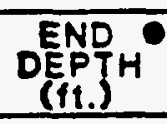 & $\begin{array}{l}\text { FLUID } \\
\text { TYPE }\end{array}$ \\
\hline Harths & $8.0^{\prime \prime}$ & & $\begin{array}{l}\text { Bentonite } \\
\text { mined }\end{array}$ \\
\hline & & & \\
\hline & & & \\
\hline & & & \\
\hline
\end{tabular}

\begin{tabular}{|c|c|c|c|}
\hline \multicolumn{4}{|c|}{ CASING SUMMARY } \\
\hline $\begin{array}{l}\text { CASINO } \\
\text { TYPE }\end{array}$ & DESCRIPTION & $\left.\mid \begin{array}{l}p i A \\
(i n . j) \\
(i n\end{array}\right]$ & $\begin{array}{l}\text { END } \\
\text { DEPTH } \\
\text { (fit.) }\end{array}$ \\
\hline$B$ & PUC SCHED 80 & $4.0_{20}^{\prime \prime}$ & 15 \\
\hline s & puc $0.051^{\prime \prime}$ slofted & $4.0^{\circ}$ & $20^{\prime}$ \\
\hline$B$ & PUE SCHED 80 & $4.0^{\prime \prime}$ & $22^{\prime}$ \\
\hline$p$ & Steel casing & $8.0^{\prime \prime}$ & $5.0^{\prime}$ \\
\hline & & & \\
\hline & & & \\
\hline & & & \\
\hline & five 5 -8ceen $=-8=0$ & Opon & HNono \\
\hline
\end{tabular}

Dopin from TOD of Cesing

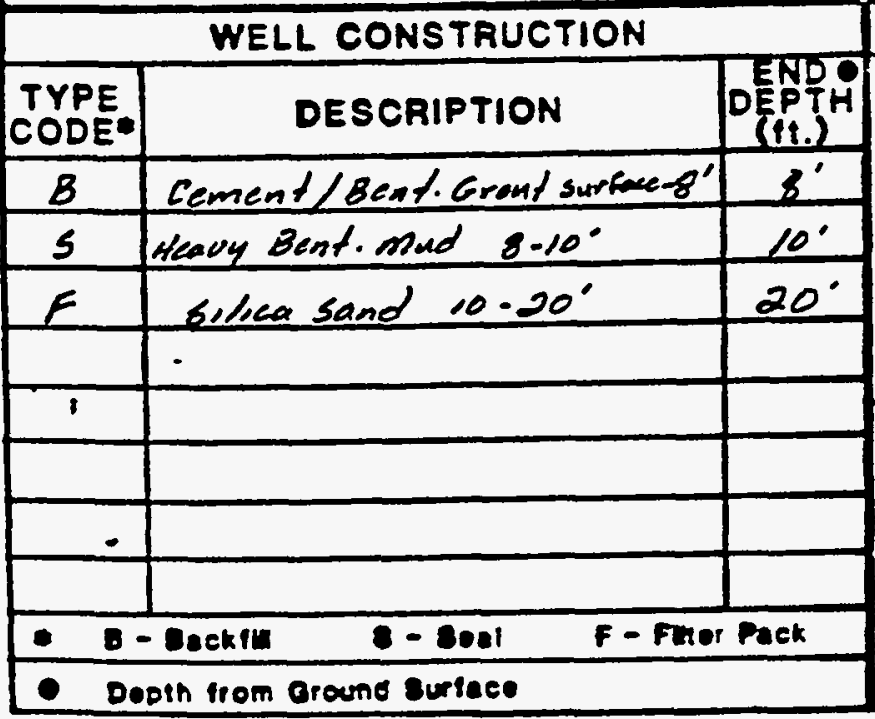

CONSTRUCTION TIME LOG

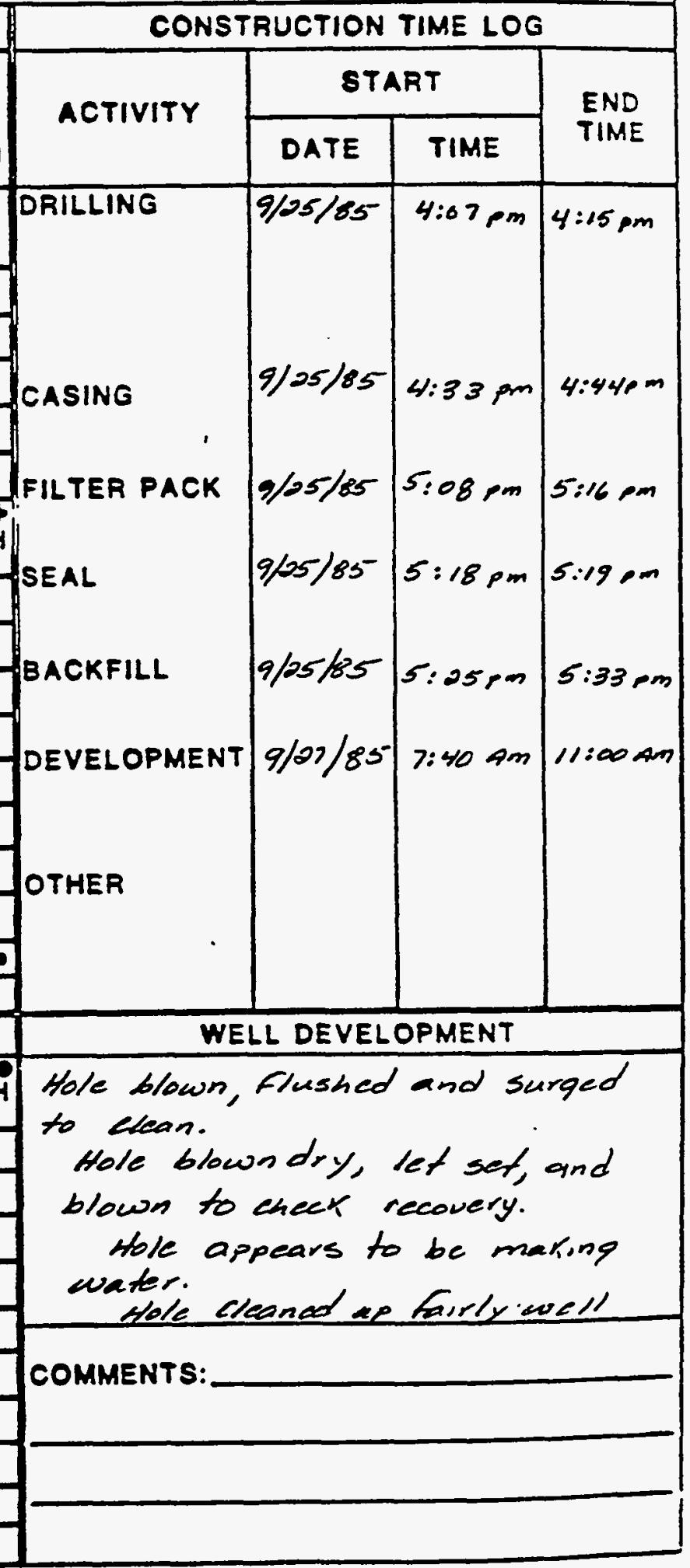


WELL 799 LOGS 


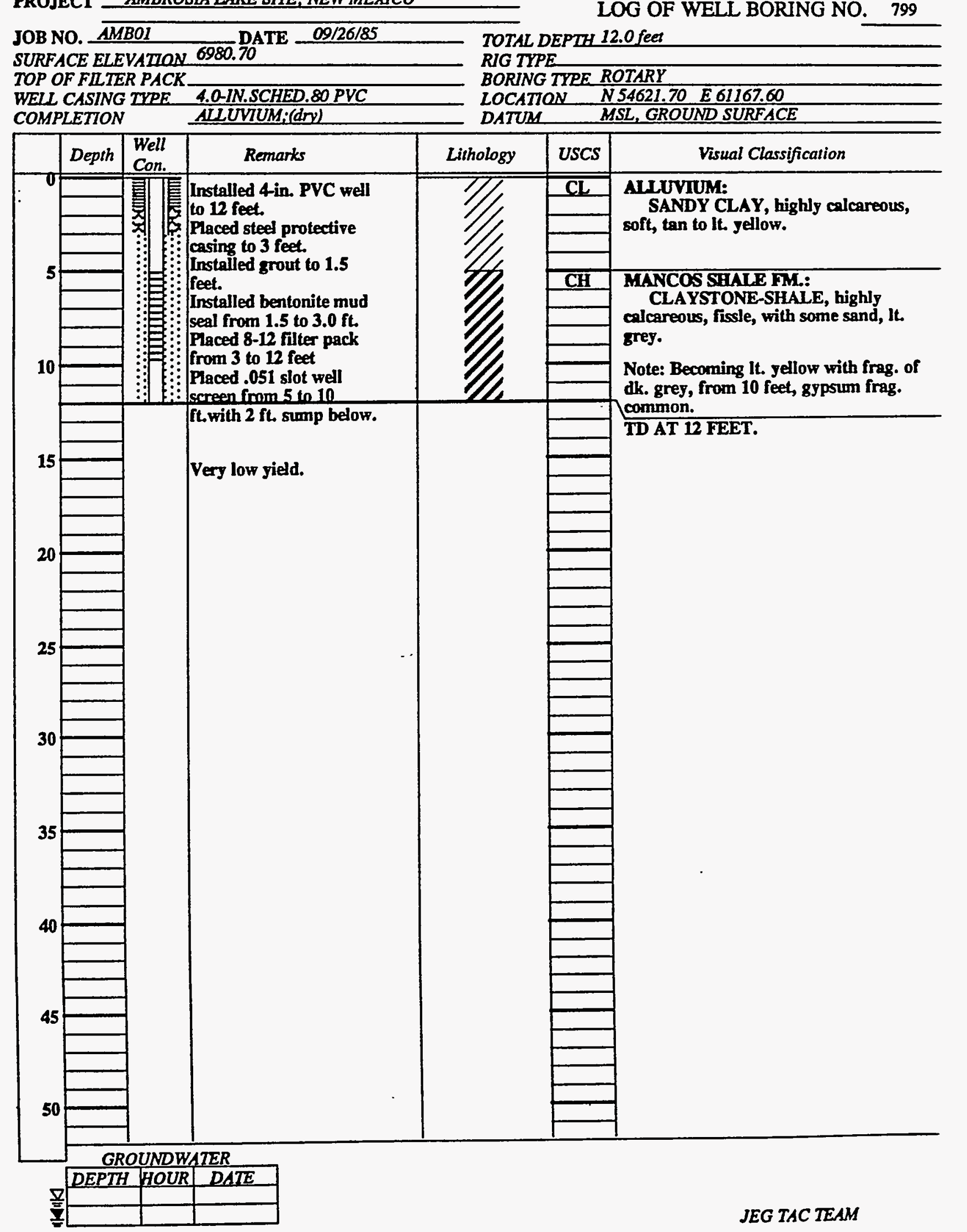




\section{TT MoOss BNeNERING CROUP NC.

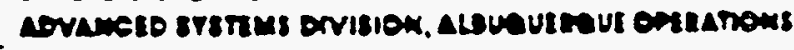

\section{MELL COMPLETION RECORD}

SITE ID: AMBOL LOCATION ID: 799

DATE INSTALLED:

APPROX. SITE COORDINATES:(FT.) N WK

E

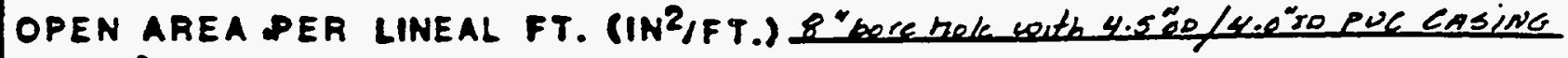
FORMATION OF COMPLETION:

FIELD-REP.: Reger thelland Alluvirion

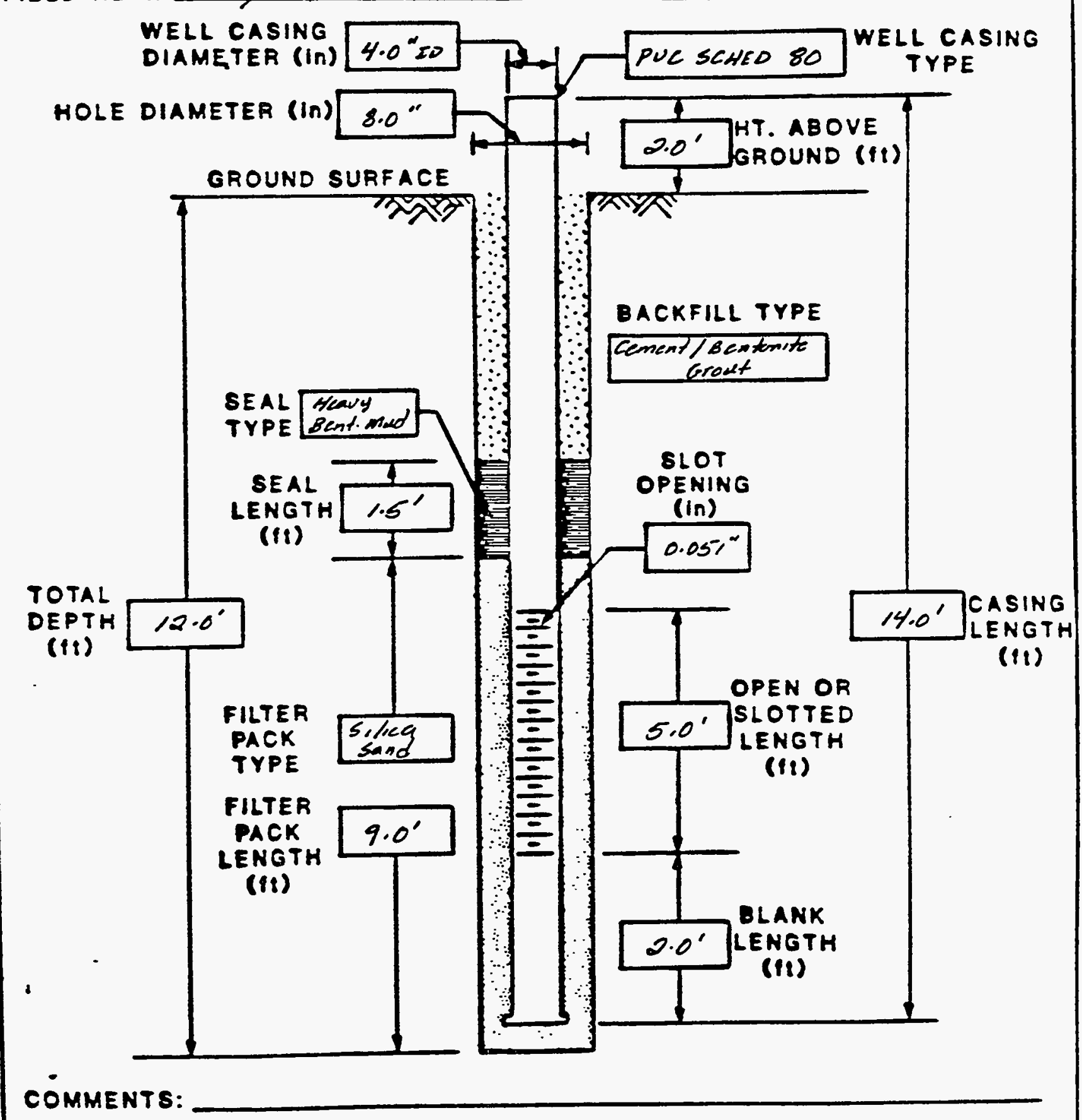

DRILLER: stewert Brothers

WELL CASING DIAMETER (in)

$4 \cdot 0^{\prime \prime 20}$ PUC SCHED 80

HOLE DIAMETER (In) 


\section{TE HCOBS ENGNEERIV GROUP INC.

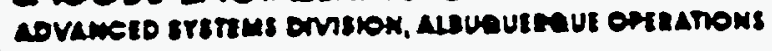 BOREHOLE/WELL CONSTRUCTION LOG}

SITE ID: AMBOL LOCATION ID: 799 FIELD REP: Reaer Helland APPROX. 8ITE COORDINATES (FT.): N WK E NK GROUND ELEVATION (FT. MSL): NK

\section{BOREHOLE SUMMARY}

DRILLER: Steward Brethers

RIG TYPE: Gevedner Denver sow (Rotery)

\begin{tabular}{|c|c|c|c|}
\hline BIT TYPE & $\begin{array}{l}\text { HOLE } \\
\text { OIR } \\
\text { (in.) }\end{array}$ & $\begin{array}{c}\text { END } \\
\text { DeEPTH } \\
\text { (fi.). }\end{array}$ & $\begin{array}{l}\text { FLUID } \\
\text { TYPE } \\
\end{array}$ \\
\hline Musts & $8.0^{11}$ & $10^{\prime}$ & Bentomite \\
\hline & & & \\
\hline & & & \\
\hline & & & \\
\hline
\end{tabular}

CASING SUMMARY

\begin{tabular}{|c|c|c|c|}
\hline $\begin{array}{l}\text { CASING } \\
\text { TYPE }\end{array}$ & DESCRIPTION & \begin{tabular}{|c|} 
Din. \\
$($ in.i)
\end{tabular} & $\begin{array}{l}\text { END } \\
\text { DEPTH } \\
\text { (ti.) }\end{array}$ \\
\hline$B$ & PUC SCHED BD & 4.010 & $7^{\prime}$ \\
\hline 5 & Poc 0.051 "slotted & $4.0^{\prime \prime}$ & 12 \\
\hline$B$ & PUC SCHEP 80 & $4.0^{-1}$ & $14^{\prime}$ \\
\hline$p$ & steel Casing & $8.0^{\circ}$ & 5 \\
\hline & & & \\
\hline & & & \\
\hline & & & \\
\hline & 8 & & \\
\hline
\end{tabular}
Toopin from TOD ol Cosing

\begin{tabular}{|c|c|c|}
\hline \multicolumn{3}{|c|}{ WELL CONSTRUCTION } \\
\hline $\begin{array}{l}\text { TYPE } \\
\text { CODE }\end{array}$ & DESCRIPTION & $\begin{array}{l}\text { EENP } \\
\text { DE } \\
\text { (it.) }\end{array}$ \\
\hline $\bar{B}$ & cement lesent Great surf.-ons & 1.5 \\
\hline$\underline{s}$ & Heary Bent mud 1.5-3' & 3 \\
\hline$E$ & sellua sand $3-12^{\prime}$ & 12 \\
\hline & - & \\
\hline 1 & & \\
\hline & & \\
\hline & & \\
\hline- & $f-\mathrm{rmo}$ & Enet \\
\hline & & \\
\hline
\end{tabular}

\section{CASING} FILTER PACK SEAL BACKFILL DEVELOPMENT

\section{OTHER} COMPLETION DATE: 2106/85CONSTRUCTION TIME LOG

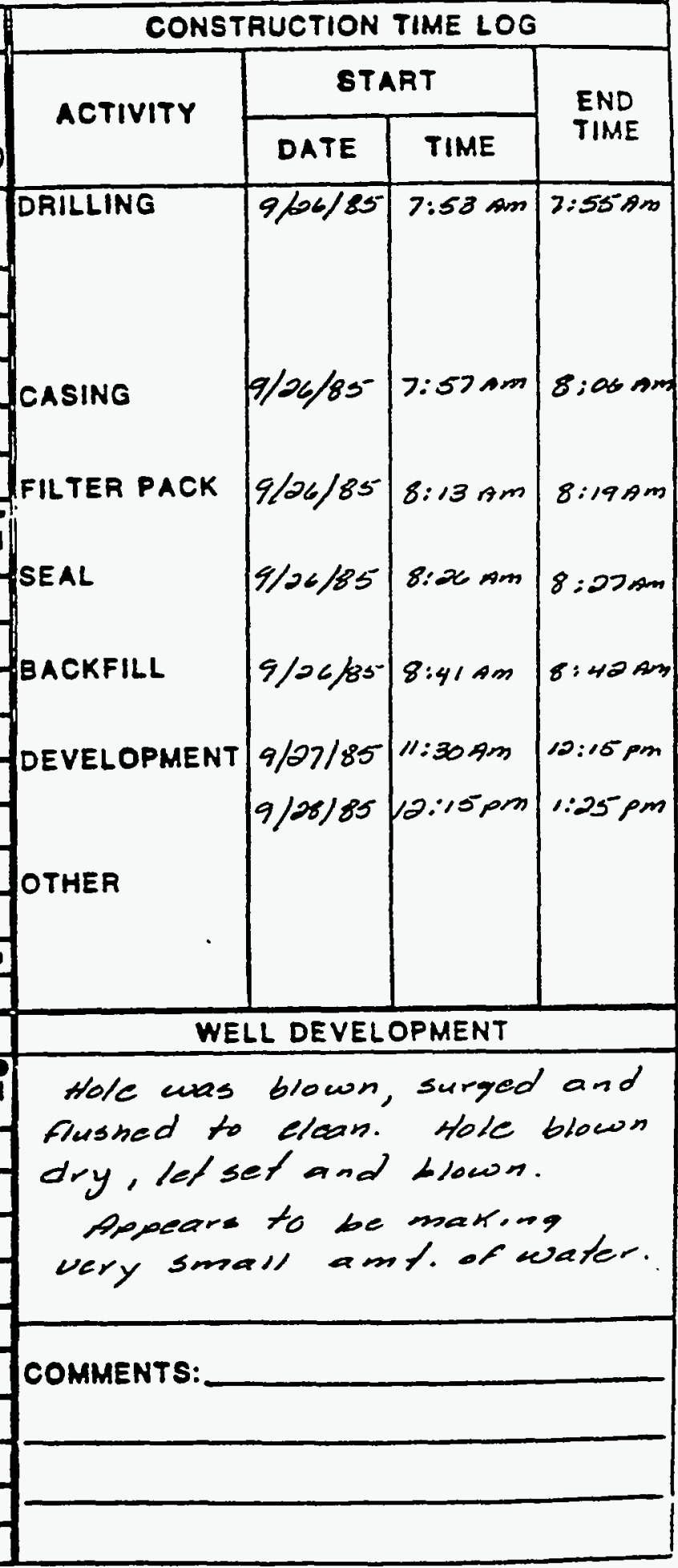

JEG-AL-ENO-I (S/EA) 
APPENDIX B

HYDROGEOLOGIC INFORMATION

AMBROSIA LAKE, NEW MEXICO, UMTRA PROJECT SITE 


\section{TI NACOBS ENGINEERING GROUP NN. .

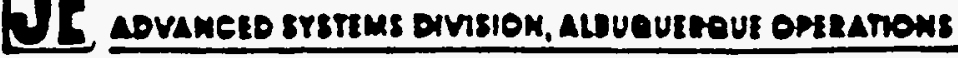

\section{CALCULATION COVER SHEET}

CALC NO.AMB-03-88-15-01-01 DIS CIPLINE HYOROLOGY

NO. OF SHEETS

\section{PROJECT:}

\section{UMTRA}

\section{ITE:}

$$
\text { AMBROSIA LAKE, NEW MEXICO }
$$

FEATURE:

CALCULATION OF TRANSMISSIVITY \& STOFATIUITY

WITHIN ALLUVIUM/WEATHERED MANCOS SHALE

USING JACOB STRAIGHT-LINE METHOD

SOURCES OF DATA:

$$
\text { Aquifer pumping test pATA COLLELTED }
$$

BY TAC - FEB. $2\{3,1989$

SOURCES OF FORMULAE REFERENCES:

GROUNDWATER AND WELLS, 1986, JOHNSON, PP.219257.

prelimimaty calc. $\square$ final CalC. $\square$ supersedes calc. wo.

\begin{tabular}{|l|l|l|l|l|l|l|l}
\hline & & & & & & & \\
\hline & & & & & & & \\
\hline & & & & & & & \\
\hline & & & & & & & \\
\hline $\begin{array}{c}\text { MEV. } \\
\text { mO. }\end{array}$ & GEVIEION & & & & & & \\
\hline
\end{tabular}


BY CHKD. JOB NO.
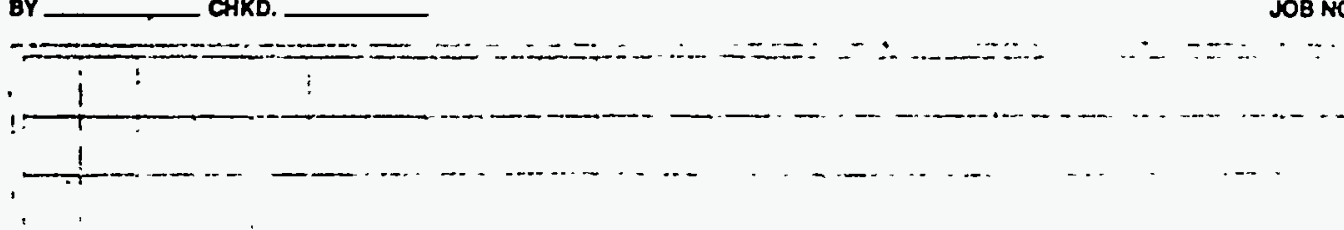

A. PURPOSE OF CALCULATION

To calculate the ". transmissivity and storativity in the :-alluvium/weatherted Mancos-Shale -at -the Ambrosia Lake (AMB-01) - UMTRA

Project Site.

B. - METHOD AND PROCEDURES

An aquifer pumping test was performed on Well 675 with wells OBS-1 and OBS-2 used as observation wells. The pumping test occurred in two -phases:-1)-drawdown - - and 2) recovery. The drawdown phase consisted I of a 12-hour test where Well 675 was pumped at a constant rate of 0.35 gpm before the water level fell below the intake of the pump ending the -drawdown-phase of the test. The recovery phase began immediately after. Water level measurements were recorded electronically and manualiy throughout both phases of the test from the pumping and observation wells. The discharge was routed to a nearby drainage ditch where it could not recharge the alluvial aquifer. Discharge rate was monitored manually throughout the duration of the drawdown -phase.

The Jacob straight-line technique (Johnson, 1986) was used to analyse the drawdown and recovery data to obtain values for transmissivity and storativity. Time-drawdown graphs (Figures 1 through 3) were generated from the drawdown data collected from pumping and

-..- observation wel1s. Residual-drawdown and time-recovery curves were generated from the recovery data collected from observation well OBS-1. The graphs were analysed according to methods described in - "Groundwater and Wells," (Johnson, 1986).

C. -ASSUMPTIONS

1). The aquifer is homogenous and isotropic.

2) The formation has uniform thickness.

3) The formation receives no recharge from any source.

.... 4) The..pumped well penetrates and receives water from the full thickness of the water-bearing formation.

5) The aquifer is infinite in areal extent.

- -6) -The water removed from storage is discharged instantaneously with

- - lowering of the head.

D. - MATERIAL PROPERTIES

Attachment A contains the well log and well construction summary for We11 675. Dbservation points OBS-1 and OBS-2 are located with in 30 ..... feet of Well 675 and penetrated similiar lithologic conditions. 
BY CHKD.

JOB NO.

E. DATA SOURCES

: The -pumping -test was performed and data collected -by the TAC on February 2 and 3, 1989..... The pumping test data and graphs are contained in Attachmet $B$.

F. CALCULATION AND ANALYSIS

The drawdown and recovery igraphs and the calculations are shown in Figures 1 through . 5. Jransmissivity and storativity were calculated using the following equations:

: Iransmissivity

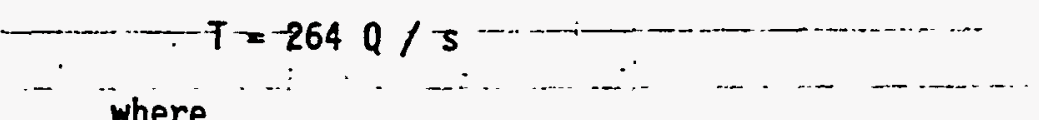

where

$T=$ transmissivity, in $\mathrm{gpd} / \mathrm{ft}$

$Q=$ puming rate, in gpm. The pumping rate was $0.35 \mathrm{gpm}$

$s=$ slope of the time drawdown graph expressed as the change in drawdown between any two times on the log scale whose ratio is 10 (one $\log$ cycle). The time drawdown graphs for the pumping and observation wells, show that effects of casing storage were not exceeded until 200 minutes into the drawdown phase of the test as indicated by the two distinct slopes shown in Figures 1 and 2 . The calculation for the time when casing storage effect becomes negligible is present in calculation number AMB-03-88-15-01-02.

\section{Storativity}

$$
\ddot{s}=0.3 T t_{0} / r^{2}
$$

where

$S=$ storage coefficient

$T=$ transmissivity, in $\mathrm{gpd} / \mathrm{ft}$

$t_{0}=$ intercept of the straight line at zero drawdown

$r$ = distance, in $\mathrm{ft}$, from the pumped well to the observation well. iwhere the drawdown measurements were made. The values of $r$

“G. CONCLUSIONS AND RECOMMENDATIONS

\section{.......................................}

The results of the pumping test analysis are summarized in Table 1.

- Transmisivities and storage coefficient values ranged from 13 to 73

.. gpd/ft and from 0.001 to 0.004 , respectively. The results for $T$ and and $S$. obtained from well. OBS-2. are higher than for the other two wells. The $T$ value of $73 \mathrm{gpd} / \mathrm{ft}$ calculated from drawdown data from

-well-OBS-1 as compared to an average $T$ value of $15.5 \mathrm{gpd} / \mathrm{ft}$ from the other two wells suggests that the aliuvial aquifer is anisotropic and that poor hydraulic connection exists between well OBS-2 and the pumping well. The $T$ values calculated from the drawdown data for wells 675 and OBS-1 -were based on the second slope of the straight-line plot since this is the true aquifer transmissivity if the aquifer is to be pumped for extended periods of time. The results from residual- drawdown and the calculated time-recovery analysis for i well DBS-1 agreed closely to the time -drawdown analysis. (Table 1). 


\section{JE JACOBS ENGINEERING}

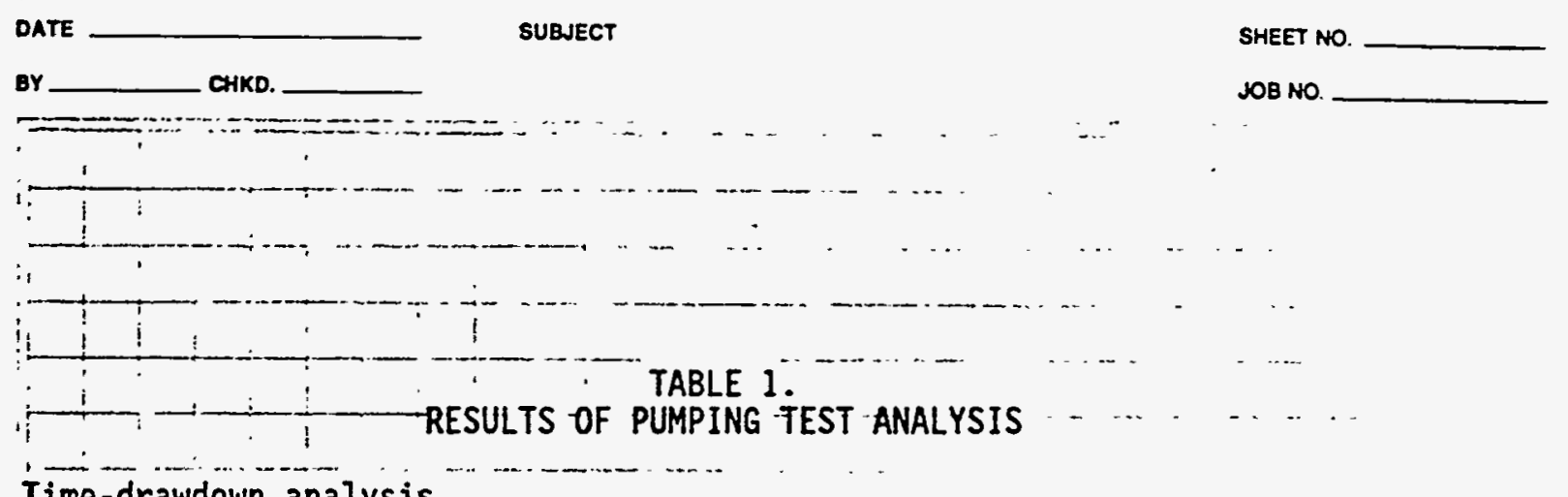

Iime-drawdown analysis

......Hell No. - .. Jransmissivity (gpd/ft) $675 \quad 13$

-.... OBS-1 - .....- 18

$\ldots$ OBS $-2 \ldots \ldots .73$

Storage Coefficient Not applicable

0.001

0.004

Iime-recovery analysis

Hell No. Transmissivity (gpd/ft) OBS-1 19

Storage Coefficient 0.001

Residual-drawdown analysis

Well No. Transmissivity (gpd/ft) OBS-1 22

Storage Coefficient Not applicable 
ATTACHMENT A

WELL LOG AND CONSTRUCTION SUMMARY

WELL 675 


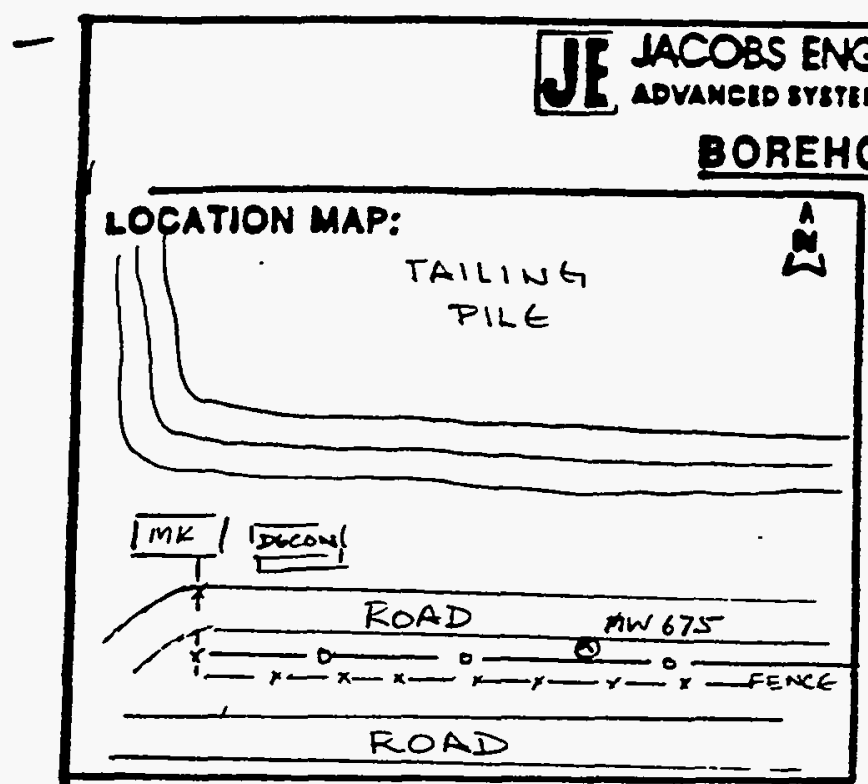

IACOBS ENGINEERING GROUP NC.

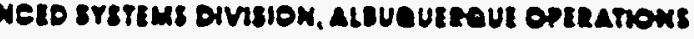

OREHOLE LOG (SOIL)

Page 1 ol 2

LOCATION MAP:

LOCATION DESCRIPTION BETWEEN THE SITE CONDITION MIDWAY DOWN DTE
SITE 1D: AMB-16 LOCATION ID: MN-675 SITE COORDINATES (ft.):

N GROUND ELEVATION (ft. MSL): 26965.0 Lt DRILLING METHOD: $45 A 10 "$

DRILLING CONTR.: CHEN NORTTERN

DATE STARTED: 12 DEC 88

DATE COMPLETED:

FIELD REP.: DE METZLER

\begin{tabular}{|c|c|c|}
\hline \multicolumn{3}{|c|}{ GROUNDWATER LEVELS } \\
\hline DATE & TIME & DEPTH (fi.) \\
\hline & & \\
\hline & & \\
\hline & & \\
\hline
\end{tabular}

-

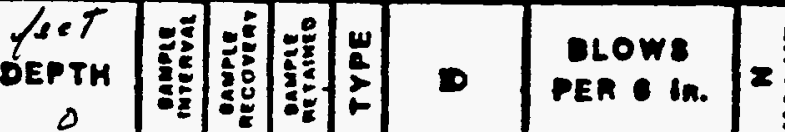

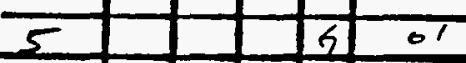

E-W DtAmins ROAD $25^{\prime} \mathrm{N}$. O CONTROC FEMCE PiLE on souTH SIOt, FLAT NESS coverES

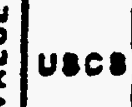

SM

\section{Topsoil}

visual CLABsification

\begin{tabular}{|l|l|l|}
\hline & & $S M$ \\
\hline & & \\
& & \\
\hline
\end{tabular}

10

30 COMMENTS:

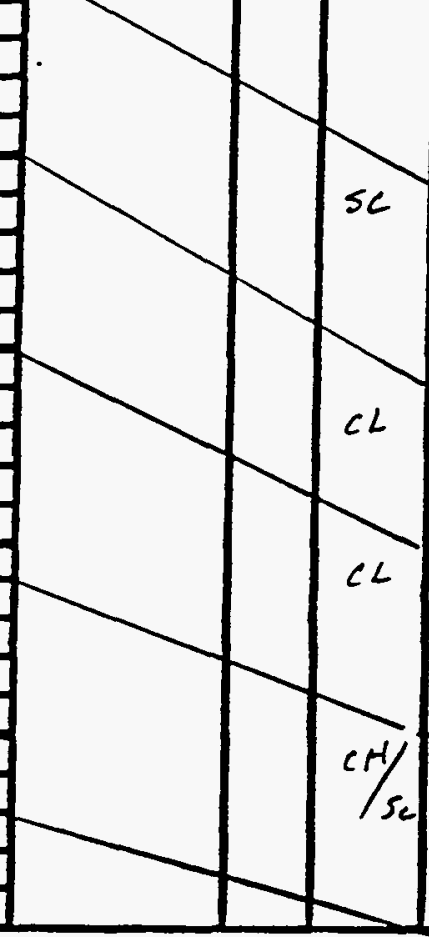

pourly eleveloped, immature, wis of massive - root strue tures predominant/7 fine to $v$. Sine sands with censiderasle silt. NP, V. loose, DRY, Lt Brown

SILT: SAND, predomiunatly fine sands wf considerable

CLAYEY SAuSD, Presominantly irine sands, with a cousidirable. SILT/' Clay fraction low $P$; moist, $2+$ Browar.

BEecmins mont CWATCY (

SAwOY CLAY, some fine grain sands med Pi, moist, Lt Brown.

SiLTY CLAY, hermegenous, non strutified clays of med to high Plasticity, Med. stiff, moist obive Brown. CONTHT: BSD ROCE CLATSTONE / SATALE

CLAYS, $r$ stiff hanogenous, moist, of high 7 tastiaity, olive (UPPER MaNcos)

SAMPLE TYPE A - Aبgon cuting? 1.0 arive cemple

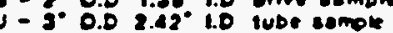
T. $2^{\circ}$ OD inem onod snoloy inde s/LT, loose, Dny, Brown 


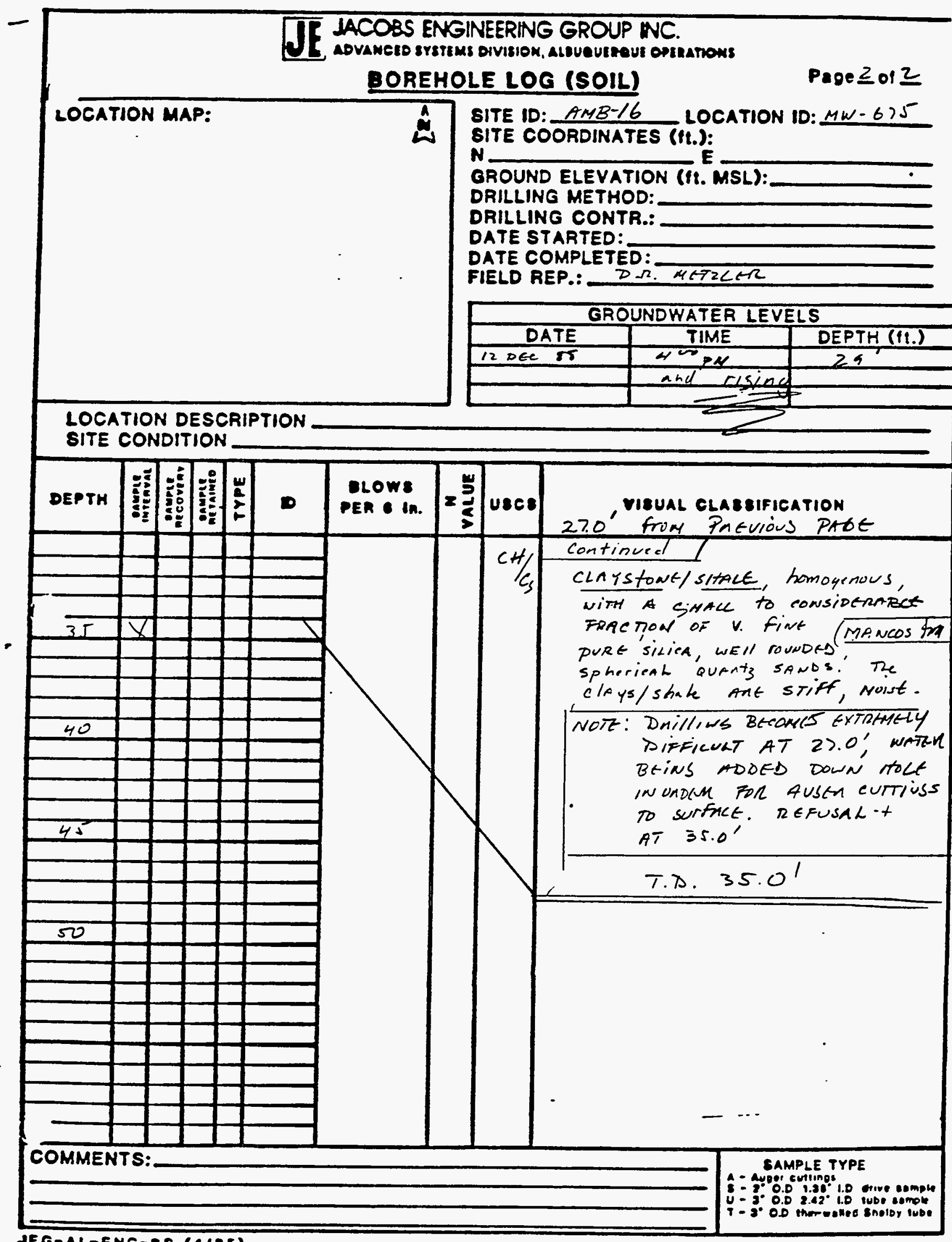



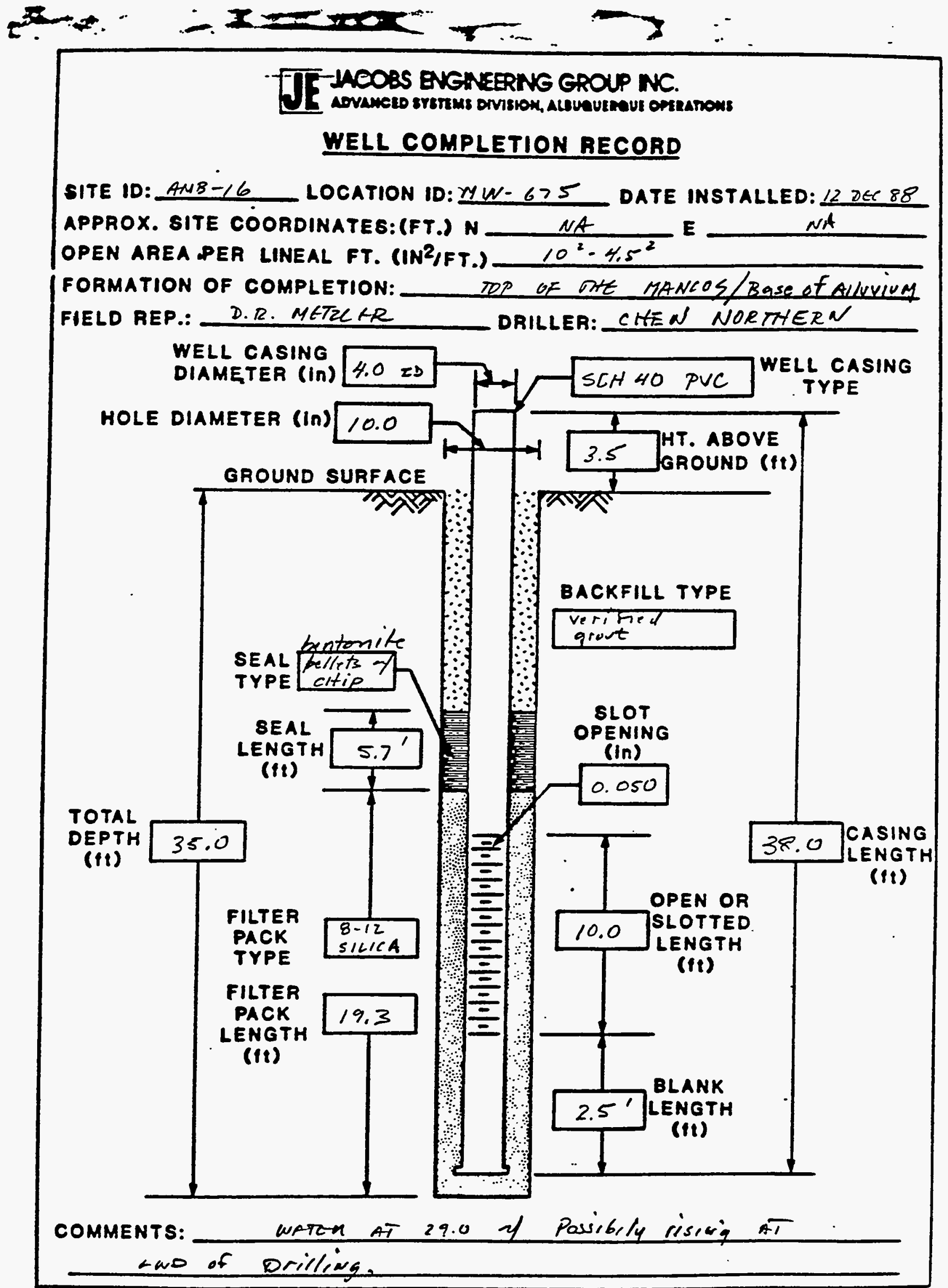

...

2

JEG-AL-ENO-3 (3/84) 


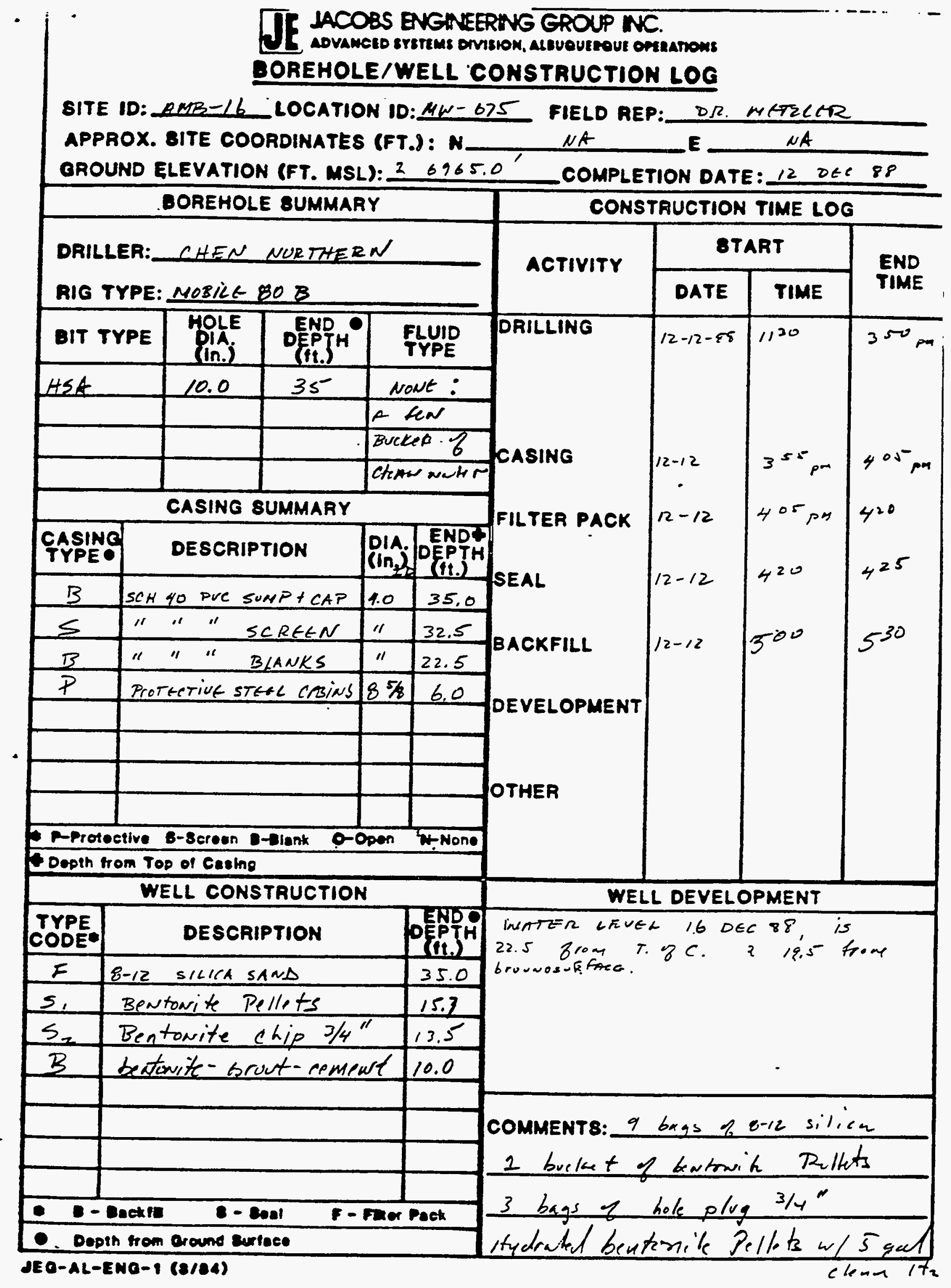




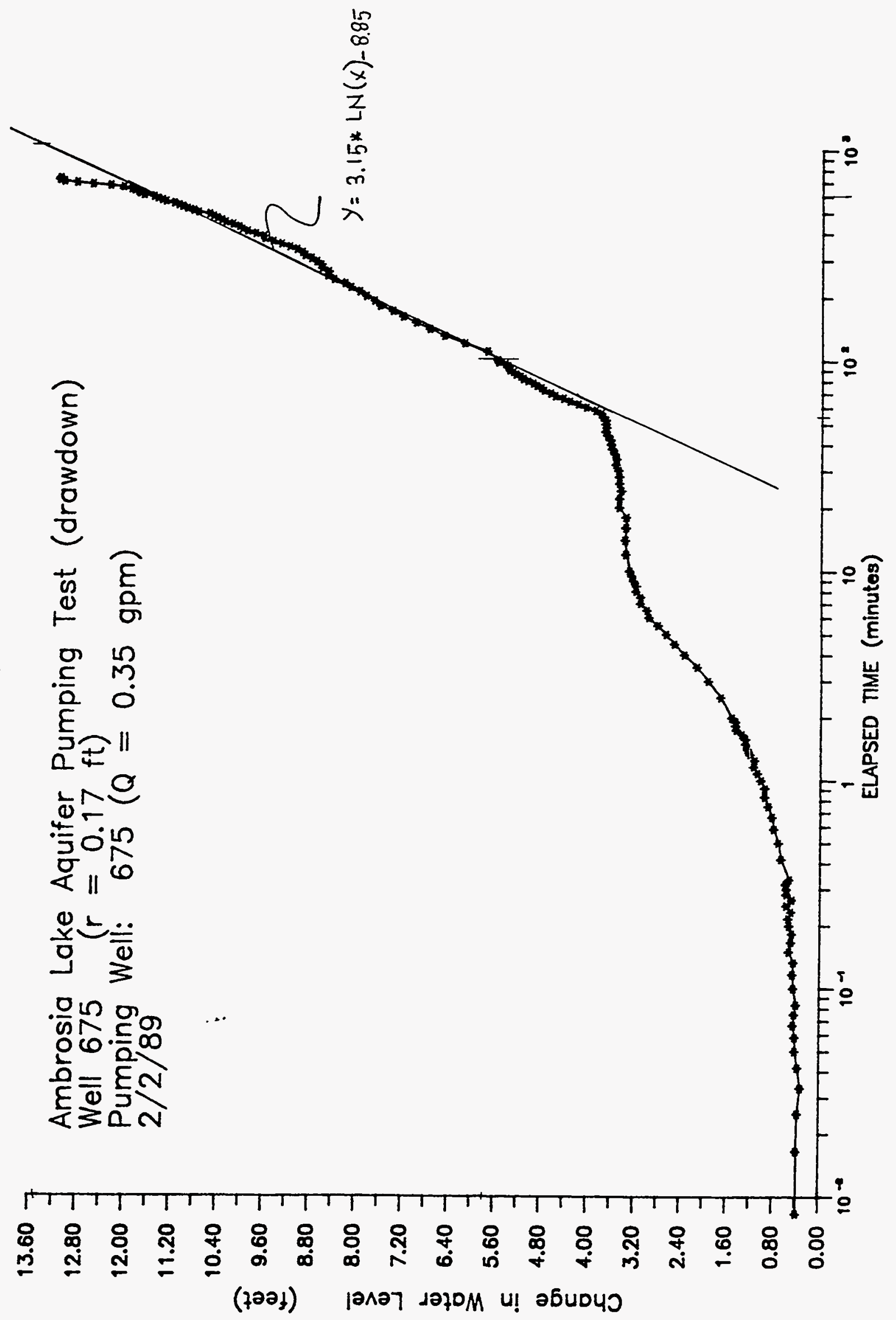


ATTACHMENT B

PUMP TEST DATA AND FIGURES 


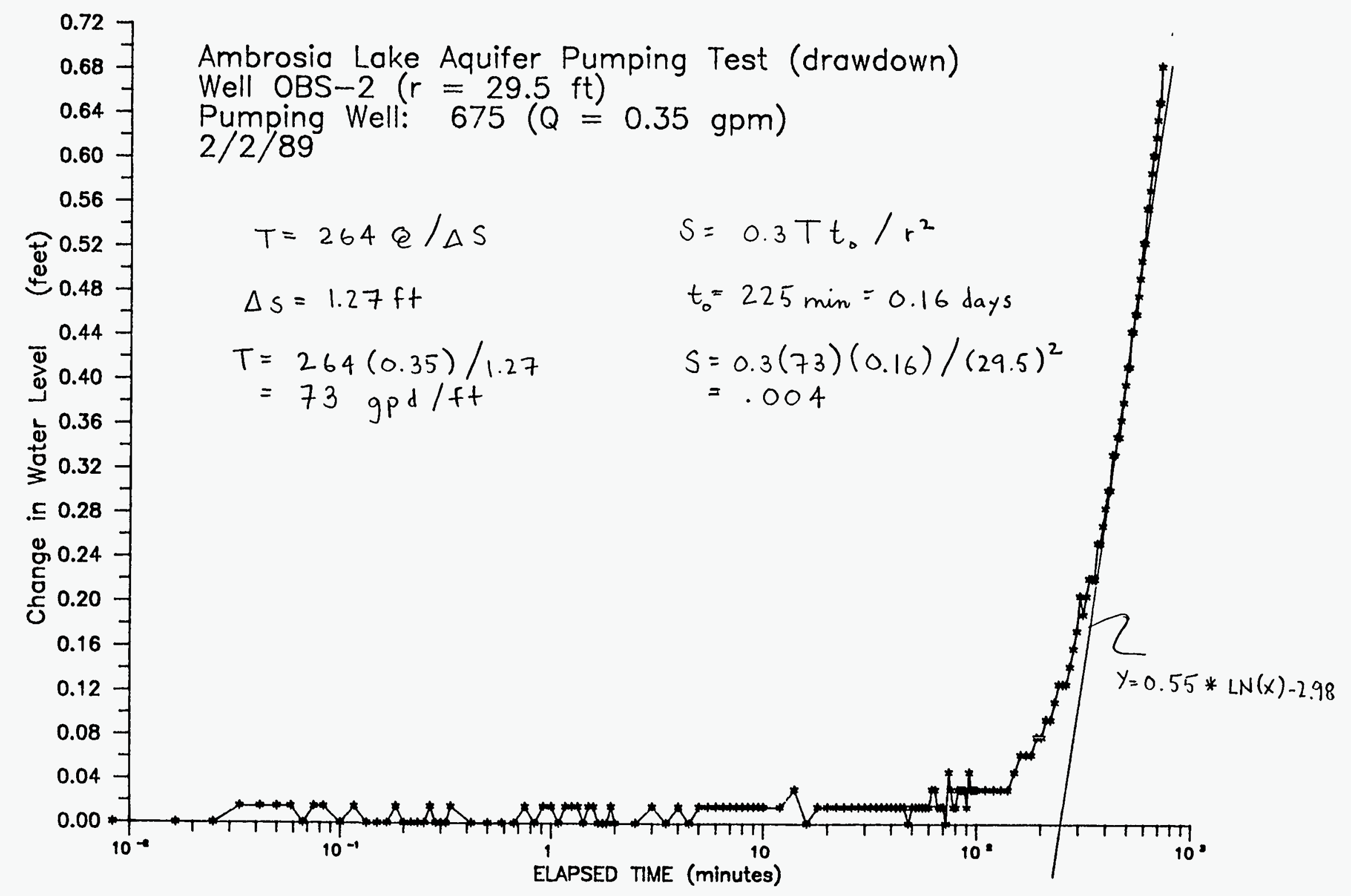




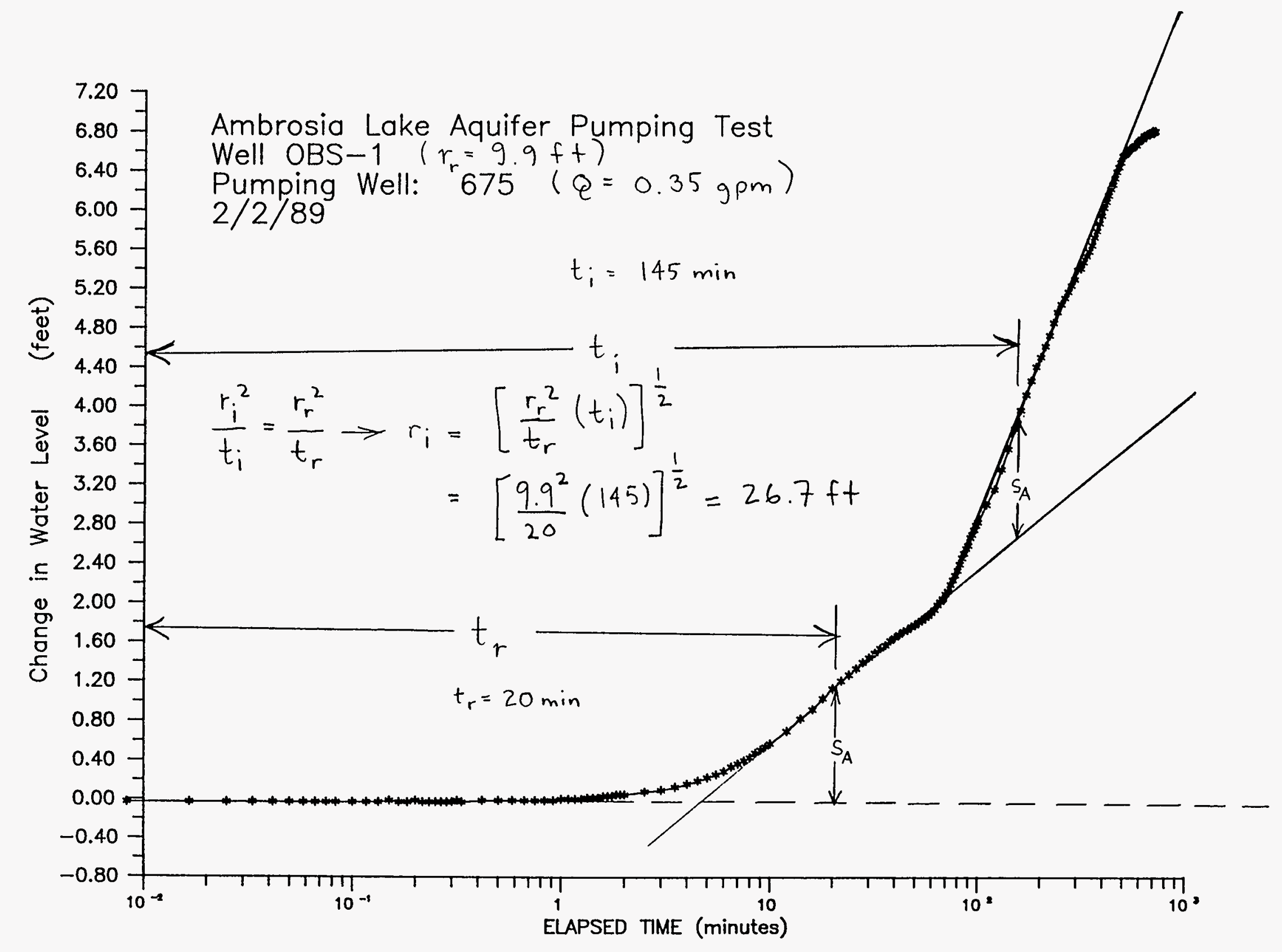




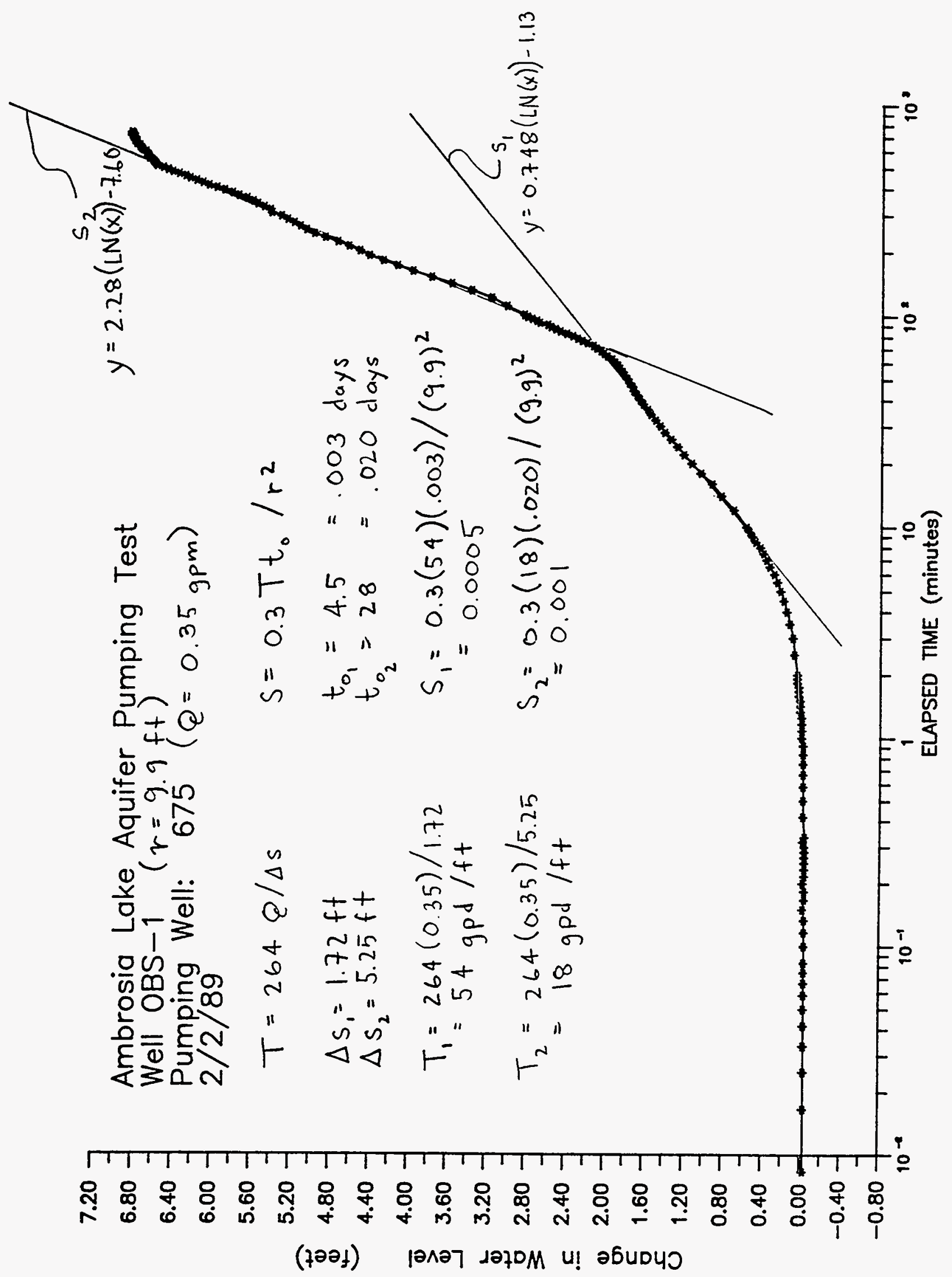




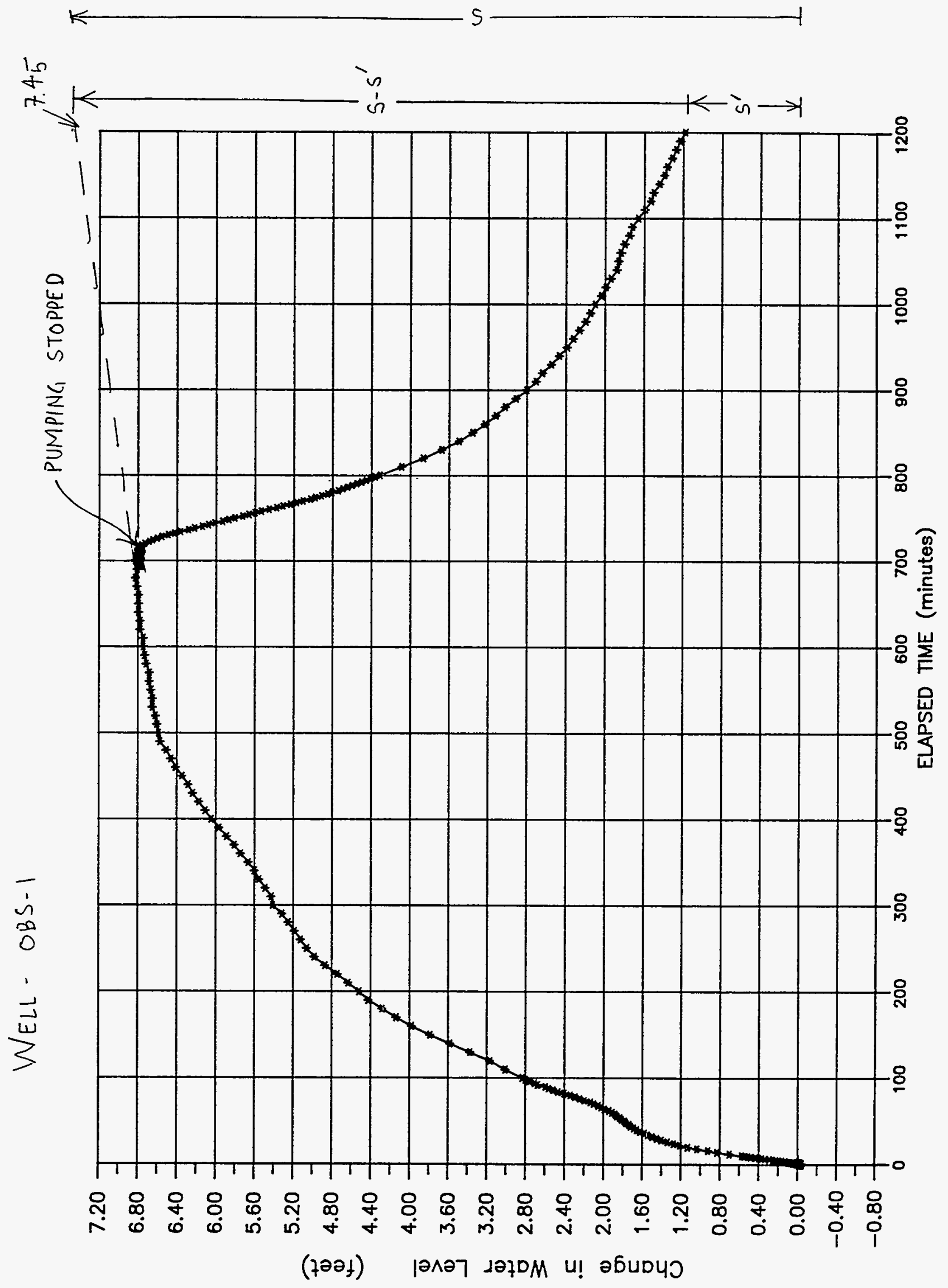




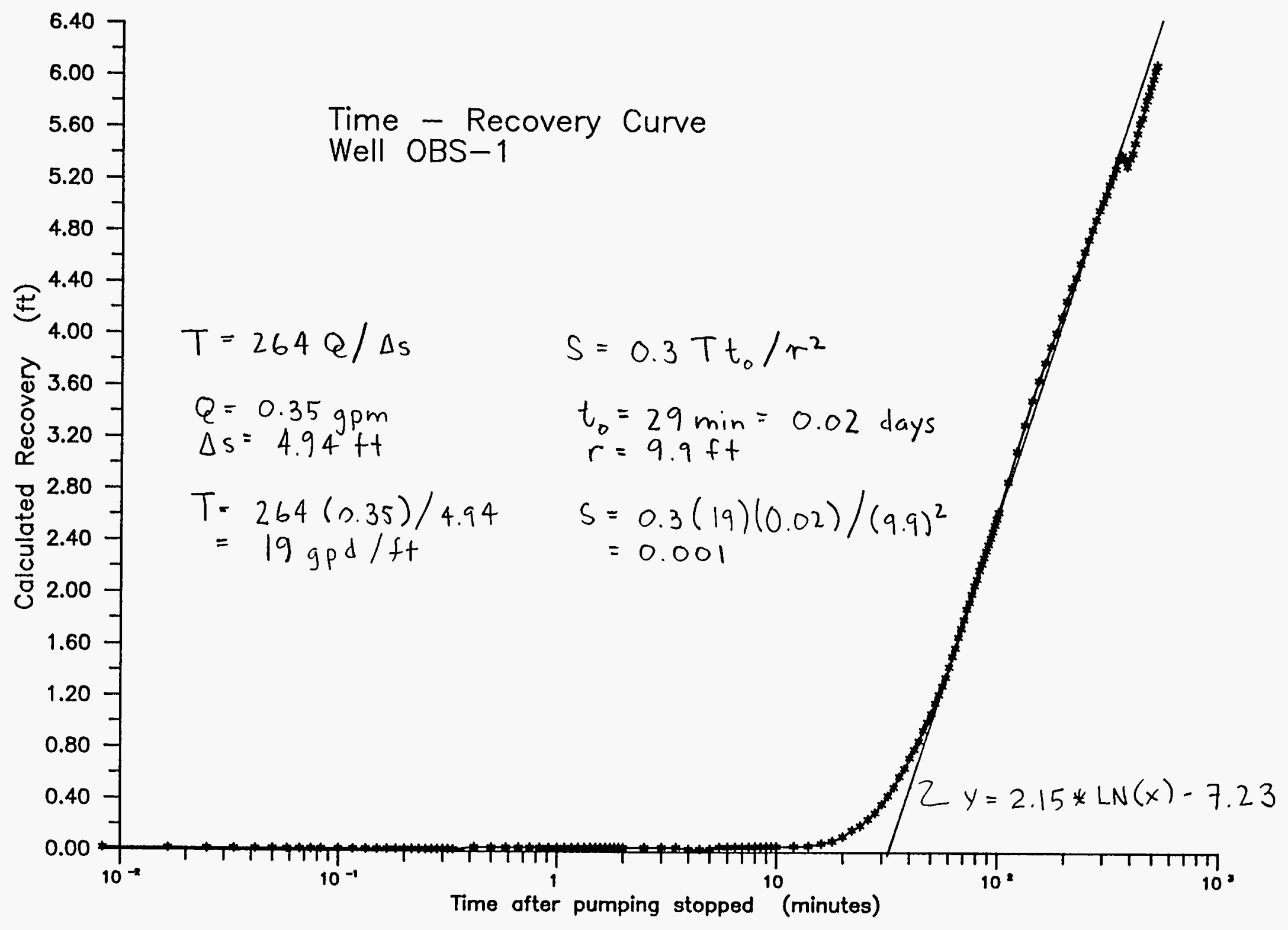




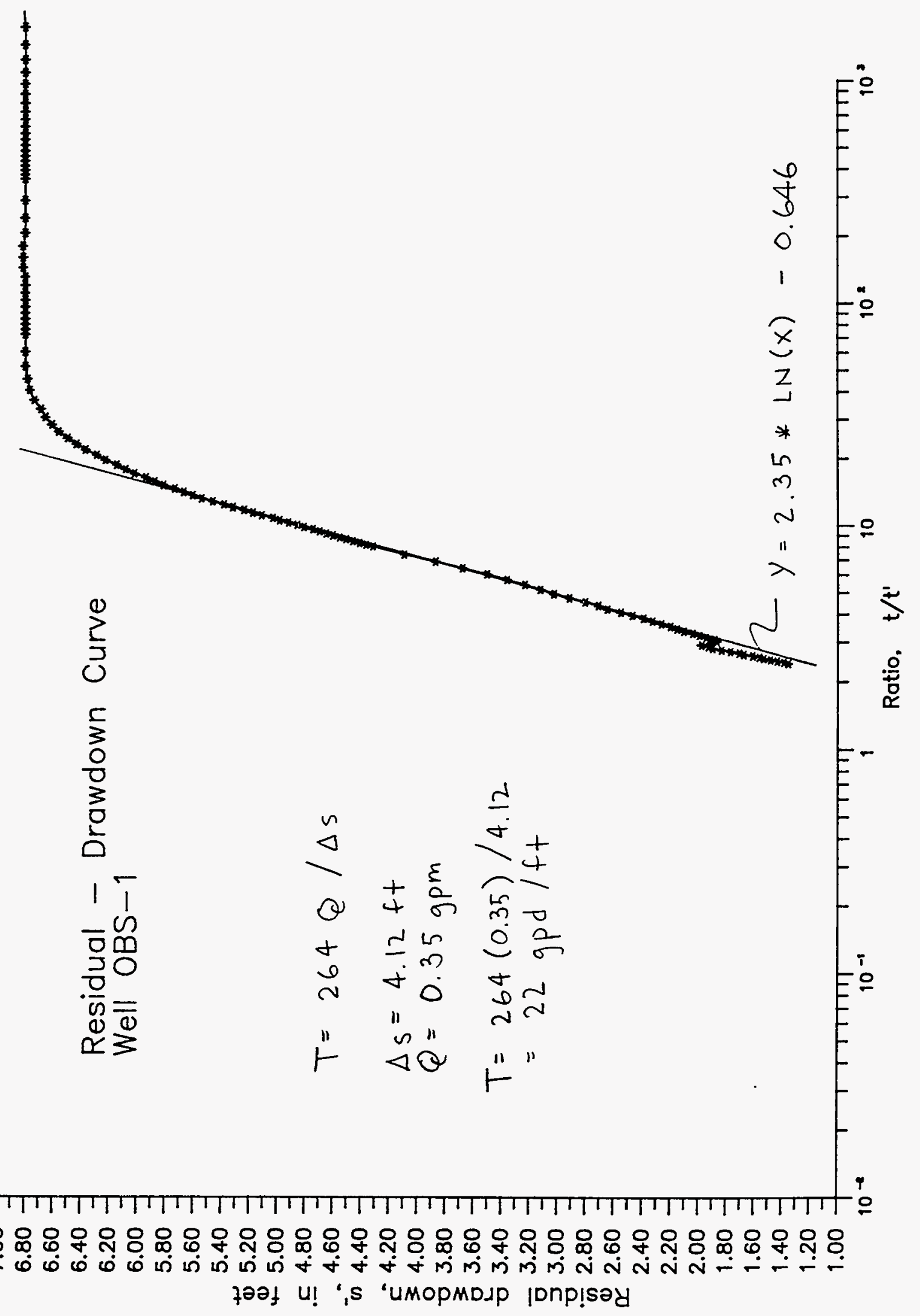




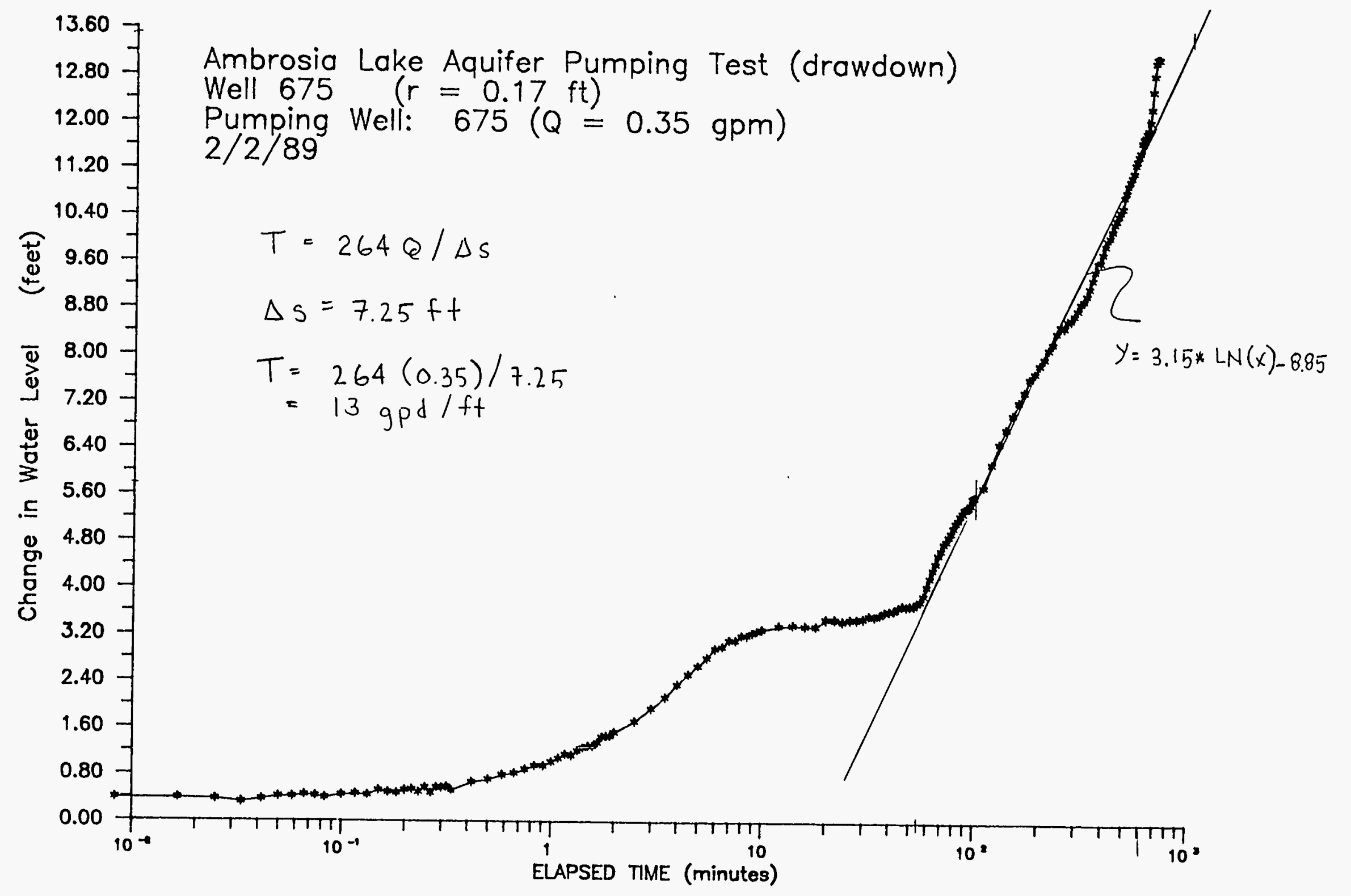




\section{APPENDIX C}

GROUND WATER QUALITY DATA BY LOCATION

AMBROSIA LAKE, NEW MEXICO, UMTRA PROJECT SITE 
GROUNDWATER QUALITY DATA BY LOCATION

SITE: AMBOI AMBROSIA LAKE

LOCATIOH: 0568

NORTH COORDINATE: $\quad 51550.0$ FT

EAST COORDINATE: $\quad 50990.0$ FT

06/10/80 TO $12 / 04 / 92$

REPORT DATE: $08 / 04 / 94$

FORMATION OF COMPLETION: TRES HERMANOS - B SANDSTONE (TB)

HYDRAULIC FLOW RELATIOHSHIP: UHKNOWN (N)

\begin{tabular}{|c|c|c|c|c|c|c|c|}
\hline PARAMETER NAME & LOG DATE & $\begin{array}{c}\text { SAMPLE } \\
\text { ID }\end{array}$ & $\begin{array}{l}\text { UNITS OF } \\
\text { MEASURE }\end{array}$ & PVI & $\begin{array}{l}\text { PARAMETER } \\
\text { VALUE FLAGS }\end{array}$ & $\begin{array}{l}\text { DETECTION } \\
\text { LIMIT }\end{array}$ & $\begin{array}{l}\text { PARAMETER } \\
\text { UNCERTAINTY }\end{array}$ \\
\hline ALKALINITY & $08 / 16 / 83$ & 0001 & $\mathrm{MG} / \mathrm{LACO} 3$ & & 90.20 & $\cdot$ & $\cdot$ \\
\hline ALUMINUMA & $\begin{array}{l}10 / 21 / 80 \\
08 / 16 / 83\end{array}$ & $\begin{array}{l}0001 \\
0001\end{array}$ & $M G / L$ & & $\begin{array}{l}0.89 \\
0.24\end{array}$ & - & $\dot{-}$ \\
\hline ARSENIC & $\begin{array}{l}10 / 21 / 80 \\
08 / 16 / 83\end{array}$ & $\begin{array}{l}0001 \\
0001\end{array}$ & MG/L & & $\begin{array}{l}0.012 \\
0.18\end{array}$ & - & - \\
\hline BARIUN & $\begin{array}{l}10 / 21 / 80 \\
08 / 16 / 83\end{array}$ & $\begin{array}{l}0001 \\
0001\end{array}$ & MG/L & & $\begin{array}{l}0.01 \\
0.014\end{array}$ & 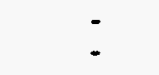 & - \\
\hline BICARBONATE - HCO3 & $\begin{array}{l}10 / 21 / 80 \\
08 / 96 / 83\end{array}$ & $\begin{array}{l}0001 \\
0001\end{array}$ & $M G / L$ & & $\begin{array}{l}152.00 \\
220.00\end{array}$ & - & - \\
\hline BORON & $\begin{array}{l}10 / 21 / 80 \\
08 / 16 / 83\end{array}$ & $\begin{array}{l}0001 \\
0001\end{array}$ & $M G / L$ & & $\begin{array}{l}0.44 \\
0.45\end{array}$ & - & - \\
\hline CADMIUM & $\begin{array}{l}10 / 21 / 80 \\
08 / 16 / 83\end{array}$ & $\begin{array}{l}0001 \\
0001\end{array}$ & $M G / L$ & $<$ & $\begin{array}{l}0.008 \\
0.01\end{array}$ & 0.008 & - \\
\hline CALCIUM & $\begin{array}{l}10 / 21 / 80 \\
08 / 96 / 83\end{array}$ & $\begin{array}{l}0001 \\
0001\end{array}$ & $M G / L$ & & $\begin{array}{l}200.00 \\
210.00\end{array}$ & - & - \\
\hline CARBOHATE & $08 / 16 / 83$ & 0001 & $M G / L$ & $<$ & 10.00 & 10. & - \\
\hline CHLORIDE & $\begin{array}{l}10 / 21 / 80 \\
08 / 16 / 83\end{array}$ & $\begin{array}{l}0001 \\
0001\end{array}$ & $M G / L$ & $<$ & $\begin{array}{l}10.00 \\
18.00\end{array}$ & 10 & - \\
\hline CHROMIUM & $\begin{array}{l}10 / 21 / 80 \\
08 / 16 / 83\end{array}$ & $\begin{array}{l}0001 \\
0001\end{array}$ & $M G / L$ & $<$ & $\begin{array}{l}0.001 \\
0.036\end{array}$ & 0.001 & - \\
\hline COBALT & $\begin{array}{l}10 / 21 / 80 \\
08 / 16 / 83\end{array}$ & $\begin{array}{l}0009 \\
0001\end{array}$ & MG/L & $<$ & $\begin{array}{l}0.006 \\
0.029\end{array}$ & 0.006 & - \\
\hline COPPER & $\begin{array}{l}10 / 21 / 80 \\
08 / 16 / 83\end{array}$ & $\begin{array}{l}0001 \\
0001\end{array}$ & $M G / L$ & & $\begin{array}{l}0.009 \\
0.052\end{array}$ & $\dot{-}$ & - \\
\hline CYANIDE & $08 / 16 / 83$ & 0001 & MG/L & $<$ & 0.01 & 0.01 & - \\
\hline FLUORIDE & $\begin{array}{l}10 / 29 / 80 \\
08 / 16 / 83\end{array}$ & $\begin{array}{l}0001 \\
0001\end{array}$ & $M G / L$ & $<$ & $\begin{array}{l}0.26 \\
1.00\end{array}$ & $i$. & - \\
\hline IRON & $\begin{array}{l}10 / 21 / 80 \\
08 / 16 / 83\end{array}$ & $\begin{array}{l}0001 \\
0001\end{array}$ & $M G / L$ & & $\begin{array}{l}0.18 \\
0.14\end{array}$ & - & : \\
\hline LEAD & $\begin{array}{l}10 / 21 / 80 \\
08 / 16 / 83\end{array}$ & $\begin{array}{l}0001 \\
0001\end{array}$ & $M G / L$ & $<$ & $\begin{array}{l}0.001 \\
0.19\end{array}$ & 0.001 & - \\
\hline MAGKESIUM & $\begin{array}{l}10 / 21 / 80 \\
08 / 96 / 83\end{array}$ & $\begin{array}{l}0001 \\
0001\end{array}$ & $M G / L$ & & $\begin{array}{l}92.00 \\
83.00\end{array}$ & - & - \\
\hline MANGANESE & $\begin{array}{l}10 / 29 / 80 \\
08 / 96 / 83\end{array}$ & $\begin{array}{l}0001 \\
0001\end{array}$ & $M G / L$ & & $\begin{array}{l}0.021 \\
0.11\end{array}$ & - & : \\
\hline MERCURY & $10 / 21 / 80$ & 0001 & $M G / L$ & $<$ & 0.001 & 0.001 & - \\
\hline HOL YBDENUA & $\begin{array}{l}10 / 21 / 80 \\
08 / 16 / 83\end{array}$ & $\begin{array}{l}0001 \\
0001\end{array}$ & $M G / L$ & & $\begin{array}{l}0.035 \\
0.019\end{array}$ & - & - \\
\hline
\end{tabular}

PARAMETER VALUE IMDICATOR (PVI):

< - LESS THAN DETECTION LIMIT

SAMPLE ID COOES:

DOO1 - FILTERED SAMPLE (.45 MICRONS) 
GROUHDWATER QUALITY DATA BY LOCATIOH

SITE: AMBO1 AMBROSIA LAKE

LOCATION: 0568

NORTH COORDIHATE: $\quad 51550.0$ FT

EAST COORDINATE: 50990.0 FT

06/10/80 TO 12/04/92

REPORT DATE: 08/04/94

FORMATIOH OF COMPLETION: TRES HERMANOS - B SAHDSTOHE (TB)

HYDRAULIC FLOH RELATIONSHIP: UHKHOWN (N)

\begin{tabular}{|c|c|c|c|c|c|c|c|}
\hline PARAMETER MAME & LOG DATE & $\underset{\text { SD }}{\text { SAMPLE }}$ & $\begin{array}{l}\text { UNITS OF } \\
\text { MEASURE }\end{array}$ & PVI & $\begin{array}{l}\text { PARAMETER } \\
\text { VALUE FLAGS }\end{array}$ & $\begin{array}{l}\text { DETECTION } \\
\text { LIMIT }\end{array}$ & $\begin{array}{l}\text { PARAMETER } \\
\text { UNCERTAINTY }\end{array}$ \\
\hline HICKEL & $\begin{array}{l}10 / 21 / 80 \\
08 / 16 / 83\end{array}$ & $\begin{array}{l}0001 \\
0001\end{array}$ & $M G / L$ & & $\begin{array}{l}0.038 \\
0.077\end{array}$ & $\dot{-}$ & - \\
\hline HITRATE & $\begin{array}{l}10 / 21 / 80 \\
08 / 16 / 83\end{array}$ & $\begin{array}{l}0001 \\
0001\end{array}$ & MG/L & $<$ & $\begin{array}{l}1.30 \\
1.00\end{array}$ & 1. & - \\
\hline PH & $\begin{array}{l}10 / 21 / 80 \\
08 / 16 / 83\end{array}$ & $\begin{array}{l}0001 \\
0001\end{array}$ & SU & & $\begin{array}{l}7.80 \\
7.30\end{array}$ & - & - \\
\hline POTASSIUM & $\begin{array}{l}10 / 21 / 80 \\
08 / 16 / 83\end{array}$ & $\begin{array}{l}0001 \\
0001\end{array}$ & $M G / L$ & & $\begin{array}{l}6.40 \\
9.00\end{array}$ & - & - \\
\hline RADIUM-226 & $\begin{array}{l}10 / 21 / 80 \\
08 / 16 / 83\end{array}$ & $\begin{array}{l}0001 \\
0001\end{array}$ & $\mathrm{PCI} / \mathrm{L}$ & & $\begin{array}{l}2.45 \\
1.10\end{array}$ & $\dot{-}$ & - \\
\hline RADIUH-226 + RADIUH-228 & $08 / 16 / 83$ & 0001 & $\mathrm{PCI} / \mathrm{L}$ & & 2.20 & - & - \\
\hline RADIUH-228 & $08 / 16 / 83$ & 0001 & $\mathrm{PCl} / \mathrm{L}$ & & 1.10 & - & - \\
\hline SELEHIUM & $\begin{array}{l}10 / 21 / 80 \\
08 / 16 / 83\end{array}$ & $\begin{array}{l}0001 \\
0001\end{array}$ & $M G / L$ & & $\begin{array}{l}0.009 \\
0.21\end{array}$ & : & - \\
\hline SILVER & $\begin{array}{l}10 / 21 / 80 \\
08 / 16 / 83\end{array}$ & $\begin{array}{l}0001 \\
0001\end{array}$ & $M G / L$ & $<$ & $\begin{array}{l}0.002 \\
0.065\end{array}$ & 0.002 & - \\
\hline SCOIUM & $\begin{array}{l}10 / 21 / 80 \\
08 / 16 / 83\end{array}$ & $\begin{array}{l}0001 \\
0001\end{array}$ & $M G / L$ & & $\begin{array}{l}100.00 \\
110.00\end{array}$ & $\dot{-}$ & $\dot{-}$ \\
\hline SPECIFIC CONDUCTANCE & $10 / 21 / 80$ & 0001 & UАНО/СК & & 1860.00 & - & - \\
\hline SULFATE & $\begin{array}{l}10 / 21 / 80 \\
08 / 16 / 83\end{array}$ & $\begin{array}{l}0001 \\
0001\end{array}$ & $M G / L$ & & $\begin{array}{l}990.00 \\
960.00\end{array}$ & $\dot{-}$ & $\begin{array}{l}- \\
-\end{array}$ \\
\hline TOTAL DISSOLVED SOLIDS & $\begin{array}{l}10 / 21 / 80 \\
08 / 16 / 83\end{array}$ & $\begin{array}{l}0001 \\
0001\end{array}$ & MG/L & & $\begin{array}{l}1590.00 \\
1480.00\end{array}$ & $\dot{-}$ & $\dot{-}$ \\
\hline TOTAL SUSPENDED SOLIDS & $10 / 21 / 80$ & 0001 & $M G / L$ & & 124.00 & - & - \\
\hline URAHIUM & $\begin{array}{l}10 / 21 / 80 \\
08 / 16 / 83\end{array}$ & $\begin{array}{l}0001 \\
0001\end{array}$ & $M G / L$ & & $\begin{array}{l}0.027 \\
0.016\end{array}$ & $\dot{-}$ & - \\
\hline VANADIUH & $\begin{array}{l}10 / 21 / 80 \\
08 / 16 / 83\end{array}$ & $\begin{array}{l}0001 \\
0001\end{array}$ & MG/L & $<$ & $\begin{array}{l}0.003 \\
0.046\end{array}$ & 0.003 & - \\
\hline ZINC & $\begin{array}{l}10 / 21 / 80 \\
08 / 16 / 83\end{array}$ & $\begin{array}{l}0001 \\
0001\end{array}$ & $M G / L$ & & $\begin{array}{l}0.17 \\
0.045\end{array}$ & - & - \\
\hline
\end{tabular}

PARAMETER VALUE IMOICATOR (PVI): < - LESS THAK DETECTION LIMIT

SAMPLE ID COOES:

0001 - FILTERED SAMPLE (.45 MICRONS) 
GROUNDWATER QUALITY DATA BY LOCATION

SITE: AMBO1 AMBROSIA LAKE

LOCATION: 0569

MORTH COORDINATE: $\quad 49990.0$ FT

EAST COORDINATE: $\quad 51010.0$ FT

06/10/80 TO 12/04/92

REPORT DATE: $08 / 04 / 94$

FORHATION OF COMPLETION: TRES HERHANOS-A SAHDSTONE (TR) HYDRAULIC FLON RELATIOHSHIP: DOWN GRADIEHT (D)

\begin{tabular}{|c|c|c|c|c|c|c|c|}
\hline PARAMETER NAME & LOG DATE & $\begin{array}{c}\text { SAMPLE } \\
\text { ID }\end{array}$ & $\begin{array}{l}\text { UNITS OF } \\
\text { MEASURE }\end{array}$ & PVI & $\begin{array}{l}\text { PARAMETER } \\
\text { VALUE FLAGS }\end{array}$ & $\begin{array}{l}\text { DETECTION } \\
\text { LIMIT }\end{array}$ & $\begin{array}{l}\text { PARAMETER } \\
\text { UNCERTAINTY }\end{array}$ \\
\hline ALKALINITY & $\begin{array}{l}11 / 14 / 83 \\
10 / 15 / 85\end{array}$ & $\begin{array}{l}0001 \\
0001\end{array}$ & MG/L CACO3 & & $\begin{array}{l}49.20 \\
43 .\end{array}$ & $\dot{-}$ & - \\
\hline ALUMINUM & $\begin{array}{l}11 / 04 / 80 \\
11 / 14 / 83\end{array}$ & $\begin{array}{l}0001 \\
0001\end{array}$ & $M G / L$ & $<$ & $\begin{array}{l}0.72 \\
0.008\end{array}$ & 0.008 & - \\
\hline ARSENIC & $\begin{array}{l}11 / 04 / 80 \\
11 / 14 / 83 \\
10 / 15 / 85\end{array}$ & $\begin{array}{l}0001 \\
0001 \\
0001\end{array}$ & $M G / L$ & $<$ & $\begin{array}{l}0.02 \\
0.032 \\
0.01\end{array}$ & $\frac{-}{0.01}$ & - \\
\hline BARIUM & $\begin{array}{l}11 / 04 / 80 \\
11 / 94 / 83\end{array}$ & $\begin{array}{l}0001 \\
0001\end{array}$ & $\mathrm{HG} / \mathrm{L}$ & $<$ & $\begin{array}{l}0.009 \\
0.013\end{array}$ & 0.013 & - \\
\hline BICARBONATE - HCO3 & $\begin{array}{l}11 / 04 / 80 \\
11 / 14 / 83\end{array}$ & $\begin{array}{l}0001 \\
0001\end{array}$ & $M G / L$ & & $\begin{array}{l}232.00 \\
120.00\end{array}$ & - & - \\
\hline BORON & $\begin{array}{l}11 / 04 / 80 \\
11 / 14 / 83\end{array}$ & $\begin{array}{l}0001 \\
0001\end{array}$ & MG/L & & $\begin{array}{l}0.27 \\
0.26\end{array}$ & $\dot{-}$ & - \\
\hline CADMIUM & $\begin{array}{l}11 / 04 / 80 \\
11 / 14 / 83\end{array}$ & $\begin{array}{l}0001 \\
0001\end{array}$ & MG/L & $<$ & $\begin{array}{l}0.008 \\
0.007\end{array}$ & $\begin{array}{l}0.008 \\
0.007\end{array}$ & - \\
\hline CALCIUM & $\begin{array}{l}11 / 04 / 80 \\
11 / 14 / 83 \\
10 / 15 / 85\end{array}$ & $\begin{array}{l}0001 \\
0001 \\
0001\end{array}$ & $\mathrm{MG} / \mathrm{L}$ & & $\begin{array}{l}98.00 \\
65.00 \\
161 .\end{array}$ & $\begin{array}{c}- \\
- \\
0.01\end{array}$ & $\begin{array}{l}- \\
-\end{array}$ \\
\hline CARBOHATE & $11 / 14 / 83$ & 0001 & MG/L & $<$ & 10.00 & 10. & - \\
\hline CHLORIDE & $\begin{array}{l}11 / 04 / 80 \\
11 / 14 / 83 \\
10 / 15 / 85\end{array}$ & $\begin{array}{l}0001 \\
0001 \\
0001\end{array}$ & $M G / L$ & & $\begin{array}{l}24.00 \\
25.00 \\
35 .\end{array}$ & - & - \\
\hline CHROMIUM & $\begin{array}{l}11 / 04 / 80 \\
11 / 14 / 83\end{array}$ & $\begin{array}{l}0001 \\
0001\end{array}$ & $M G / L$ & $\begin{array}{l}< \\
<\end{array}$ & $\begin{array}{l}0.001 \\
0.026\end{array}$ & $\begin{array}{l}0.001 \\
0.026\end{array}$ & $\dot{-}$ \\
\hline COBALT & $\begin{array}{l}11 / 04 / 80 \\
11 / 14 / 83\end{array}$ & $\begin{array}{l}0001 \\
0001\end{array}$ & $M G / L$ & $\begin{array}{l}< \\
<\end{array}$ & $\begin{array}{l}0.006 \\
0.011\end{array}$ & $\begin{array}{l}0.006 \\
0.011\end{array}$ & - \\
\hline COPPER & $\begin{array}{l}11 / 04 / 80 \\
11 / 14 / 83\end{array}$ & $\begin{array}{l}0001 \\
0001\end{array}$ & $\mathrm{MG} / \mathrm{L}$ & $\begin{array}{l}< \\
<\end{array}$ & $\begin{array}{l}0.001 \\
0.017\end{array}$ & $\begin{array}{l}0.001 \\
0.017\end{array}$ & - \\
\hline CYANIDE & $11 / 14 / 83$ & 0001 & $M G / L$ & $<$ & 1.00 & 1. & - \\
\hline FLUORIDE & $\begin{array}{l}11 / 04 / 80 \\
11 / 14 / 83\end{array}$ & $\begin{array}{l}0001 \\
0001\end{array}$ & $M G / L$ & $\begin{array}{l}< \\
<\end{array}$ & $\begin{array}{l}0.20 \\
1.00\end{array}$ & $\begin{array}{l}0.2 \\
1 .\end{array}$ & - \\
\hline IROA & $\begin{array}{l}11 / 04 / 80 \\
11 / 14 / 83\end{array}$ & $\begin{array}{l}0001 \\
0001\end{array}$ & $M G / L$ & & $\begin{array}{l}0.30 \\
0.14\end{array}$ & - & - \\
\hline LEAD & $\begin{array}{l}11 / 04 / 80 \\
11 / 14 / 83\end{array}$ & $\begin{array}{l}0001 \\
0001\end{array}$ & $M G / L$ & $<$ & $\begin{array}{l}0.001 \\
0.03\end{array}$ & $\begin{array}{c}0.001 \\
-\end{array}$ & - \\
\hline MAGNESIUM & $\begin{array}{l}11 / 04 / 80 \\
11 / 14 / 83 \\
10 / 15 / 85\end{array}$ & $\begin{array}{l}0001 \\
0001 \\
0001\end{array}$ & $M G / L$ & & $\begin{array}{l}92.00 \\
84.00 \\
167 .\end{array}$ & $\begin{array}{c}- \\
0.001\end{array}$ & - \\
\hline MANGANESE & $\begin{array}{l}11 / 04 / 80 \\
11 / 14 / 83\end{array}$ & $\begin{array}{l}0001 \\
0001\end{array}$ & $M G / L$ & & $\begin{array}{l}0.44 \\
0.41\end{array}$ & - & - \\
\hline
\end{tabular}

PARAMETER VALUE INDICATOR (PVI): < - LESS THAN DETECTION LIMIT

SAMPLE ID COOES:

0001 - FILTERED SAMPLE (.45 MICRONS) 
FORMATION OF COMPLETION: TRES HERMANOS-A SANDSTONE (TR) HYDRAULIC FLOW RELATIONSHIP: DOWH GRADIENT (D)

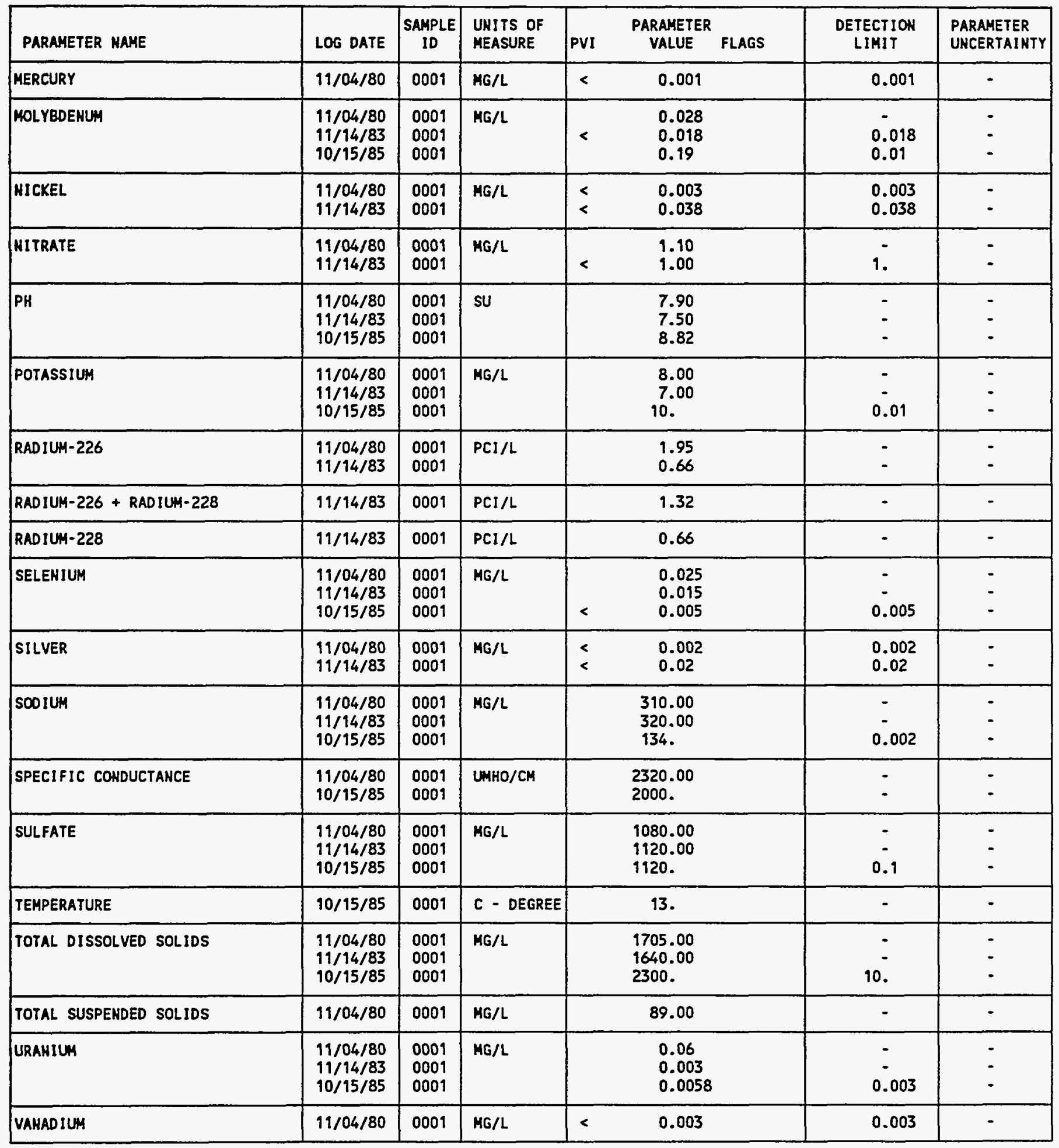

Parameter VAlue IHDICATOR (PVI): < - Less than Detection LIMIT 
GROUHDWATER QUALITY DATA BY LOCATION

SITE: AMBO1 AMBROSIA LAKE

LOCATIOH: 0569

NORTH COORDINATE: $\quad 49990.0$ FT

EAST COORDINATE: $\quad 51010.0$ FT

06/10/80 TO 12/04/92

REPORT OATE: $08 / 04 / 94$

FORMATION OF COMPLETION: TRES HERMANOS-A SAMDSTONE (TR)

HYDRAULIC FLON RELATIONSHIP: DOWN GRADIENT (D)

\begin{tabular}{|c|c|c|c|c|c|c|c|}
\hline PARAMETER NAME & LOG DATE & $\begin{array}{c}\text { SAMPLE } \\
\text { ID }\end{array}$ & $\begin{array}{l}\text { UNITS OF } \\
\text { MEASURE }\end{array}$ & PVI & $\begin{array}{l}\text { PARAMETER } \\
\text { VALUE FLAGS }\end{array}$ & $\begin{array}{l}\text { DETECTIOH } \\
\text { LIMIT }\end{array}$ & $\begin{array}{l}\text { PARAMETER } \\
\text { UNCERTAIHTY }\end{array}$ \\
\hline VAKAD IUM & $19 / 14 / 83$ & 0001 & MG/L & & 0.007 & - & - \\
\hline ZINC & $\begin{array}{l}11 / 04 / 80 \\
11 / 14 / 83\end{array}$ & $\begin{array}{l}0001 \\
0001\end{array}$ & $M G / L$ & & $\begin{array}{l}0.079 \\
0.022\end{array}$ & - & - \\
\hline
\end{tabular}

PARAMETER VALUE INDICATOR (PVI):

$<$ - LESS THAN DETECTION LIMIT

SAMPLE ID COOES

0001 - FILTERED SAMPLE (.45 MICRONS) 
GROUMDHATER OUALITY DATA BY LOCATION

SITE: AMBO1 AMBROSIA LAKE

LOCATION: 0570

HORTH COORDINATE: 51730.0 FT

EAST COORDINATE: $\quad 47650.0$ FT

06/10/80 TO $12 / 04 / 92$

REPORT DATE: 08/04/94

FORMATIOH OF COMPLETION: TRES HERMANOS - B SANDSTOHE (TB)

HYORAULIC FLON RELATIONSHIP: UNKNONN (N)

\begin{tabular}{|c|c|c|c|c|c|c|c|}
\hline PARAHETER NAHE & LOG DATE & $\begin{array}{c}\text { SAMPLE } \\
\text { ID }\end{array}$ & $\begin{array}{l}\text { UNITS OF } \\
\text { MEASURE }\end{array}$ & PVI & $\begin{array}{l}\text { PARAMETER } \\
\text { VALUE FLAGS }\end{array}$ & $\begin{array}{l}\text { DETECTIOH } \\
\text { LIMIT }\end{array}$ & $\begin{array}{l}\text { PARAMETER } \\
\text { UHCERTAINTY }\end{array}$ \\
\hline ALKALINITY & $05 / 24 / 83$ & 0001 & MG/L CACO3 & & 86.10 & - & - \\
\hline ALUMINUM & $\begin{array}{l}10 / 02 / 80 \\
05 / 24 / 83\end{array}$ & $\begin{array}{l}0001 \\
0001\end{array}$ & MG/L & & $\begin{array}{l}0.088 \\
0.22\end{array}$ & - & - \\
\hline ARSEHIC & $\begin{array}{l}10 / 02 / 80 \\
05 / 24 / 83\end{array}$ & $\begin{array}{l}0001 \\
0001\end{array}$ & MG/L & & $\begin{array}{l}0.004 \\
0.21\end{array}$ & $\dot{-}$ & $\dot{-}$ \\
\hline BARIUM & $\begin{array}{l}10 / 02 / 80 \\
05 / 24 / 83\end{array}$ & $\begin{array}{l}0001 \\
0001\end{array}$ & $M G / L$ & $<$ & $\begin{array}{l}0.018 \\
0.02\end{array}$ & 0.02 & - \\
\hline BICARBONATE - HCO3 & $\begin{array}{l}10 / 02 / 80 \\
05 / 24 / 83\end{array}$ & $\begin{array}{l}0001 \\
0001\end{array}$ & $M G / L$ & & $\begin{array}{l}397.00 \\
210.00\end{array}$ & - & - \\
\hline BORON & $\begin{array}{l}10 / 02 / 80 \\
05 / 24 / 83\end{array}$ & $\begin{array}{l}0001 \\
0001\end{array}$ & MG/L & & $\begin{array}{l}0.38 \\
0.25\end{array}$ & - & - \\
\hline CADHIUM & $\begin{array}{l}10 / 02 / 80 \\
05 / 24 / 83\end{array}$ & $\begin{array}{l}0001 \\
0001\end{array}$ & $M G / L$ & $<$ & $\begin{array}{l}0.008 \\
0.017\end{array}$ & 0.008 & $\dot{-}$ \\
\hline CALCIUM & $\begin{array}{l}10 / 02 / 80 \\
05 / 24 / 83\end{array}$ & $\begin{array}{l}0001 \\
0001\end{array}$ & MG/L & & $\begin{array}{l}420.00 \\
430.00\end{array}$ & - & - \\
\hline CARBONATE & $05 / 24 / 83$ & 0001 & HG/L & $<$ & 10.00 & 10. & - \\
\hline CHLORIDE & $\begin{array}{l}10 / 02 / 80 \\
05 / 24 / 83\end{array}$ & $\begin{array}{l}0001 \\
0001\end{array}$ & MG/L & & $\begin{array}{l}2398.00 \\
1840.00\end{array}$ & - & - \\
\hline CHROMIUM & $\begin{array}{l}10 / 02 / 80 \\
05 / 24 / 83\end{array}$ & $\begin{array}{l}0001 \\
0001\end{array}$ & MG/L & & $\begin{array}{l}0.061 \\
0.01\end{array}$ & - & - \\
\hline COBALT & $\begin{array}{l}10 / 02 / 80 \\
05 / 24 / 83\end{array}$ & $\begin{array}{l}0001 \\
0001\end{array}$ & $M G / L$ & $<$ & $\begin{array}{l}0.006 \\
0.04\end{array}$ & $\begin{array}{l}0.006 \\
0.04\end{array}$ & - \\
\hline COPPER & $\begin{array}{l}10 / 02 / 80 \\
05 / 24 / 83\end{array}$ & $\begin{array}{l}0001 \\
0001\end{array}$ & $M G / L$ & $<$ & $\begin{array}{l}0.001 \\
0.03\end{array}$ & 0.03 & - \\
\hline CYAHIDE & $05 / 24 / 83$ & 0001 & MG/L & $<$ & 1.00 & 1. & - \\
\hline FLUORIDE & $\begin{array}{l}10 / 02 / 80 \\
05 / 24 / 83\end{array}$ & $\begin{array}{l}0001 \\
0001\end{array}$ & MG/L & $<$ & $\begin{array}{l}0.10 \\
1.00\end{array}$ & 1. & - \\
\hline IRON & $\begin{array}{l}10 / 02 / 80 \\
05 / 24 / 83\end{array}$ & $\begin{array}{l}0001 \\
0001\end{array}$ & MG/L & & $\begin{array}{r}0.11 \\
28.00\end{array}$ & - & - \\
\hline LEAD & $\begin{array}{l}10 / 02 / 80 \\
05 / 24 / 83\end{array}$ & $\begin{array}{l}0001 \\
0001\end{array}$ & MG/L & & $\begin{array}{l}0.002 \\
0.27\end{array}$ & - & - \\
\hline MAGNESIUH & $\begin{array}{l}10 / 02 / 80 \\
05 / 24 / 83\end{array}$ & $\begin{array}{l}0001 \\
0001\end{array}$ & $M G / L$ & & $\begin{array}{l}1400.00 \\
1900.00\end{array}$ & - & - \\
\hline MAKGANESE & $\begin{array}{l}10 / 02 / 80 \\
05 / 24 / 83\end{array}$ & $\begin{array}{l}0001 \\
0001\end{array}$ & $M G / L$ & & $\begin{array}{l}2.00 \\
1.70\end{array}$ & - & - \\
\hline MERCURY & $10 / 02 / 80$ & 0001 & MG/L & $<$ & 0.001 & 0.001 & - \\
\hline MOL YBDENUM & $\begin{array}{l}10 / 02 / 80 \\
05 / 24 / 83\end{array}$ & $\begin{array}{l}0001 \\
0001\end{array}$ & MG/L & & $\begin{array}{l}0.002 \\
0.031\end{array}$ & - & - \\
\hline
\end{tabular}

PARAMETER VALUE IMDICATOR (PVI): < LESS THAN DETECTION LIMIT

SAMPLE ID COOES:

OOO1 - FILTERED SAMPLE (.45 MICRONS) 
GROUNDWATER QUALITY DATA BY LOCATIOH

SITE: AMBO1 AMBROSIA LAKE

LOCATION: 0570

NORTH COORDINATE: 51730.0 FT

EAST COORDINATE: $\quad 47650.0$ FT

06/10/80 TO $12 / 04 / 92$

REPORT DATE: $08 / 04 / 94$

FORMATION OF COMPLETION: TRES HERMANOS - B SANDSTONE (TB)

HYDRAULIC FLOW RELATIONSHIP: UNKNOWN (N)

\begin{tabular}{|c|c|c|c|c|c|c|c|}
\hline PARAMETER NAME & LOG DATE & $\begin{array}{c}\text { SAMPLE } \\
\text { ID }\end{array}$ & $\begin{array}{l}\text { UNITS OF } \\
\text { MEASURE }\end{array}$ & PVI & $\begin{array}{l}\text { PARAMETER } \\
\text { VALUE FLAGS }\end{array}$ & $\begin{array}{l}\text { DETECTION } \\
\text { LIMIT }\end{array}$ & $\begin{array}{l}\text { PARAMETER } \\
\text { UHCERTAINTY }\end{array}$ \\
\hline HICKEL & $\begin{array}{l}10 / 02 / 80 \\
05 / 24 / 83\end{array}$ & $\begin{array}{l}0001 \\
0001\end{array}$ & $M G / L$ & & $\begin{array}{l}0.01 \\
0.075\end{array}$ & - & - \\
\hline MITRATE & $\begin{array}{l}10 / 02 / 80 \\
05 / 24 / 83\end{array}$ & $\begin{array}{l}0001 \\
0001\end{array}$ & $M G / L$ & & $\begin{array}{r}24.00 \\
6.00\end{array}$ & - & - \\
\hline PH & $\begin{array}{l}10 / 02 / 80 \\
05 / 24 / 83\end{array}$ & $\begin{array}{l}0001 \\
0001\end{array}$ & SU & & $\begin{array}{l}7.00 \\
6.20\end{array}$ & $\dot{-}$ & 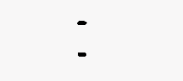 \\
\hline POTASSIUA & $\begin{array}{l}10 / 02 / 80 \\
05 / 24 / 83\end{array}$ & $\begin{array}{l}0001 \\
0001\end{array}$ & MG/L & & $\begin{array}{l}15.00 \\
11.00\end{array}$ & $\dot{-}$ & - \\
\hline RADIUH-226 & $\begin{array}{l}10 / 02 / 80 \\
05 / 24 / 83\end{array}$ & $\begin{array}{l}0001 \\
0001\end{array}$ & $\mathrm{PCI} / \mathrm{L}$ & & $\begin{array}{l}1.41 \\
1.77\end{array}$ & - & 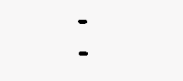 \\
\hline RADIUH-226 + RADIUM-228 & $05 / 24 / 83$ & 0001 & $\mathrm{PCI} / \mathrm{L}$ & & 3.54 & - & - \\
\hline RADIUM-228 & $05 / 24 / 83$ & 0001 & $\mathrm{PCl} / \mathrm{L}$ & & 1.77 & - & - \\
\hline SELENIUM & $\begin{array}{l}10 / 02 / 80 \\
05 / 24 / 83\end{array}$ & $\begin{array}{l}0001 \\
0001\end{array}$ & $M G / L$ & & $\begin{array}{l}0.68 \\
0.24\end{array}$ & $\dot{-}$ & $\dot{-}$ \\
\hline SILVER & $\begin{array}{l}10 / 02 / 80 \\
05 / 24 / 83\end{array}$ & $\begin{array}{l}0001 \\
0001\end{array}$ & $\mathrm{MG} / \mathrm{L}$ & $<$ & $\begin{array}{l}0.002 \\
0.035\end{array}$ & 0.002 & $\dot{-}$ \\
\hline SOOIUM & $\begin{array}{l}10 / 02 / 80 \\
05 / 24 / 83\end{array}$ & $\begin{array}{l}0001 \\
0001\end{array}$ & $M G / L$ & & $\begin{array}{l}800.00 \\
730.00\end{array}$ & $\dot{-}$ & $\dot{-}$ \\
\hline SPECIFIC CONDUCTANCE & $10 / 02 / 80$ & 0001 & UMHO/CM & & 12400.00 & - & $\cdot$ \\
\hline SULFATE & $\begin{array}{l}10 / 02 / 80 \\
05 / 24 / 83\end{array}$ & $\begin{array}{l}0001 \\
0001\end{array}$ & $M G / L$ & & $\begin{array}{l}4780.00 \\
7630.00\end{array}$ & 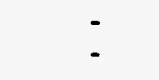 & - \\
\hline TOTAL DISSOLVED SOLIOS & $\begin{array}{l}10 / 02 / 80 \\
05 / 24 / 83\end{array}$ & $\begin{array}{l}0001 \\
0001\end{array}$ & $M G / L$ & & $\begin{array}{r}10710.00 \\
12.70\end{array}$ & - & $\dot{-}$ \\
\hline TOTAL ORGANIC CARBOH & $05 / 24 / 83$ & 0001 & $M G / L$ & & 18.00 & - & - \\
\hline TOTAL SUSPENDED SOLIDS & $10 / 02 / 80$ & 0001 & MG/L & & 36.00 & - & $\cdot$ \\
\hline URANIUM & $\begin{array}{l}10 / 02 / 80 \\
05 / 24 / 83\end{array}$ & $\begin{array}{l}0001 \\
0001\end{array}$ & $M G / L$ & & $\begin{array}{l}0.04 \\
0.08\end{array}$ & - & $\dot{-}$ \\
\hline VANADIUM & $\begin{array}{l}10 / 02 / 80 \\
05 / 24 / 83\end{array}$ & $\begin{array}{l}0001 \\
0001\end{array}$ & $M G / L$ & & $\begin{array}{l}0.028 \\
0.04\end{array}$ & $\dot{-}$ & - \\
\hline ZINC & $\begin{array}{l}10 / 02 / 80 \\
05 / 24 / 83\end{array}$ & $\begin{array}{l}0001 \\
0001\end{array}$ & $M G / L$ & & $\begin{array}{l}0.23 \\
0.70\end{array}$ & - & $\therefore$ \\
\hline
\end{tabular}

PARAMETER VALUE IMDICATOR (PVI): < - LESS THAN DETECTION LIMIT SAMPLE IO COOES:

0001 - FILTERED SAMPLE (.45 MICRONS) 
GROUNDWATER OUALITY DATA BY LOCATIOH

SITE: AMBO1 AMBROSIA LAKE

LOCATION: 0571

MORTH COORDIHATE: $\quad 57190.0$ FT

EAST COORDINATE: $\quad 47500.0$ FT

O6/10/80 TO 12/04/92

REPORT DATE: 08/04/94

FORMATION OF COMPLETION: TRES HERMANOS-A SANDSTONE (TR)

HYDRAULIC FLOW RELATIONSHIP: UNKHOWH (N)

\begin{tabular}{|c|c|c|c|c|c|c|c|}
\hline PARAMETER MAME & LOG DATE & $\begin{array}{c}\text { SAMPLE } \\
\text { ID }\end{array}$ & $\begin{array}{l}\text { UNITS OF } \\
\text { MEASURE }\end{array}$ & PV & $\begin{array}{l}\text { PARAMETER } \\
\text { VALUE FLAGS }\end{array}$ & $\begin{array}{l}\text { DETECTION } \\
\text { LIMIT }\end{array}$ & $\begin{array}{l}\text { PARAMETER } \\
\text { UNCERTAINTY }\end{array}$ \\
\hline ALKALINITY & $08 / 15 / 83$ & 0001 & MG/L CACO3 & & 28.70 & - & - \\
\hline ARSEHIC & $\begin{array}{l}11 / 05 / 80 \\
08 / 15 / 83\end{array}$ & $\begin{array}{l}0001 \\
0001\end{array}$ & MG/L & $<$ & $\begin{array}{l}0.009 \\
0.10\end{array}$ & 0.1 & - \\
\hline BARIUM & $\begin{array}{l}11 / 05 / 80 \\
08 / 15 / 83\end{array}$ & $\begin{array}{l}0001 \\
0001\end{array}$ & MG/L & & $\begin{array}{l}0.063 \\
0.021\end{array}$ & - & - \\
\hline BICARBOHATE - HCO3 & $\begin{array}{l}11 / 05 / 80 \\
08 / 15 / 83\end{array}$ & $\begin{array}{l}0001 \\
0001\end{array}$ & $M G / L$ & & $\begin{array}{l}71.00 \\
70.00\end{array}$ & - & - \\
\hline BORON & $\begin{array}{l}11 / 05 / 80 \\
08 / 15 / 83\end{array}$ & $\begin{array}{l}0001 \\
0001\end{array}$ & MG/L & & $\begin{array}{l}0.27 \\
0.38\end{array}$ & - & - \\
\hline CALCIUM & $\begin{array}{l}11 / 05 / 80 \\
08 / 15 / 83\end{array}$ & $\begin{array}{l}0001 \\
0001\end{array}$ & $M G / L$ & & $\begin{array}{r}100.00 \\
22.00\end{array}$ & - & - \\
\hline CARBOHATE & $08 / 15 / 83$ & 0001 & $M G / L$ & $<$ & 10.00 & 10. & - \\
\hline CHLORIDE & $\begin{array}{l}11 / 05 / 80 \\
08 / 15 / 83\end{array}$ & $\begin{array}{l}0001 \\
0001\end{array}$ & $M G / L$ & & $\begin{array}{r}30.00 \\
2.40\end{array}$ & - & $\dot{-}$ \\
\hline CHROMIUH & $\begin{array}{l}11 / 05 / 80 \\
08 / 15 / 83\end{array}$ & $\begin{array}{l}0001 \\
0001\end{array}$ & $M G / L$ & $<$ & $\begin{array}{l}0.005 \\
0.03\end{array}$ & 0.03 & - \\
\hline COBALT & $\begin{array}{l}11 / 05 / 80 \\
08 / 15 / 83\end{array}$ & $\begin{array}{l}0001 \\
0001\end{array}$ & $M G / L$ & $<$ & $\begin{array}{l}0.006 \\
0.01\end{array}$ & $\begin{array}{l}0.006 \\
0.01\end{array}$ & - \\
\hline LEAD & $\begin{array}{l}11 / 05 / 80 \\
08 / 15 / 83\end{array}$ & $\begin{array}{l}0001 \\
0001\end{array}$ & $M G / L$ & $<$ & $\begin{array}{l}0.001 \\
0.07\end{array}$ & $\begin{array}{l}0.001 \\
0.07\end{array}$ & - \\
\hline MAGHESIUH & $\begin{array}{l}11 / 05 / 80 \\
08 / 15 / 83\end{array}$ & $\begin{array}{l}0001 \\
0001\end{array}$ & $M G / L$ & & $\begin{array}{r}37.00 \\
3.70\end{array}$ & $\dot{-}$ & - \\
\hline MAKGAHESE & $\begin{array}{l}11 / 05 / 80 \\
08 / 15 / 83\end{array}$ & $\begin{array}{l}0001 \\
0001\end{array}$ & $M G / L$ & & $\begin{array}{l}0.071 \\
0.017\end{array}$ & $\dot{-}$ & - \\
\hline HERCURY & $11 / 05 / 80$ & 0001 & MG/L & $<$ & 0.001 & 0.001 & - \\
\hline MOLYBDENUM & $\begin{array}{l}11 / 05 / 80 \\
08 / 15 / 83\end{array}$ & $\begin{array}{l}0001 \\
0001\end{array}$ & MG/L & $<$ & $\begin{array}{l}0.027 \\
0.02\end{array}$ & 0.02 & - \\
\hline
\end{tabular}

PARAMETER VALUE IMDICATOR (PVI): < - LESS THAN DETECTIOH LIMIT 
GROUHDHATER QUALITY DATA BY LOCATION

SITE: AMBOI AMBROSIA LAKE

LOCATION: 0571

MORTH COORDINATE: 57190.0 FT

EAST COORDINATE: $\quad 47500.0$ FT

06/10/80 TO $12 / 04 / 92$

REPORT DATE: $08 / 04 / 94$

FORMATION OF COMPLETION: TRES HERMANOS-A SANDSTONE (TR)

HYDRAULIC FLON RELATIONSHIP: UNKNOWN (N)

\begin{tabular}{|c|c|c|c|c|c|c|c|}
\hline PARAMETER MAME & LOG DATE & $\begin{array}{c}\text { SAMPLE } \\
\text { ID }\end{array}$ & $\begin{array}{l}\text { UNITS OF } \\
\text { MEASURE }\end{array}$ & PVI & $\begin{array}{l}\text { PARAMETER } \\
\text { VALUE FLAGS }\end{array}$ & $\begin{array}{l}\text { DETECTIOH } \\
\text { LIMIT }\end{array}$ & $\begin{array}{l}\text { PARAMETER } \\
\text { UNCERTAIHTY }\end{array}$ \\
\hline NICKEL & $\begin{array}{l}11 / 05 / 80 \\
08 / 15 / 83\end{array}$ & $\begin{array}{l}0001 \\
0001\end{array}$ & MG/L & $<$ & $\begin{array}{l}0.003 \\
0.04\end{array}$ & $\begin{array}{l}0.003 \\
0.04\end{array}$ & - \\
\hline NITRATE & $\begin{array}{l}11 / 05 / 80 \\
08 / 15 / 83\end{array}$ & $\begin{array}{l}0001 \\
0001\end{array}$ & MG/L & $<$ & $\begin{array}{l}1.80 \\
1.00\end{array}$ & 1. & - \\
\hline PH & $\begin{array}{l}11 / 05 / 80 \\
08 / 15 / 83\end{array}$ & $\begin{array}{l}0001 \\
0009\end{array}$ & SU & & $\begin{array}{l}7.80 \\
8.20\end{array}$ & - & - \\
\hline POTASSIUM & $\begin{array}{l}11 / 05 / 80 \\
08 / 95 / 83\end{array}$ & $\begin{array}{l}0001 \\
0001\end{array}$ & $M G / L$ & & $\begin{array}{r}20.00 \\
8.90\end{array}$ & - & - \\
\hline RADIUM-226 & $\begin{array}{l}19 / 05 / 80 \\
08 / 15 / 83\end{array}$ & $\begin{array}{l}0001 \\
0001\end{array}$ & $\mathrm{PCI} / \mathrm{L}$ & & $\begin{array}{l}9.901 \\
2.52\end{array}$ & - & - \\
\hline RADIUM-226 + RADIUM-228 & $08 / 15 / 83$ & 0001 & $\mathrm{PCI/L}$ & & 5.04 & - & - \\
\hline RADIUM-228 & $08 / 15 / 83$ & 0001 & PCI/L & & 2.52 & - & - \\
\hline SELEKIUM & $\begin{array}{l}11 / 05 / 80 \\
08 / 15 / 83\end{array}$ & $\begin{array}{l}0001 \\
0001\end{array}$ & $M G / L$ & $<$ & $\begin{array}{l}0.011 \\
0.10\end{array}$ & 0.1 & - \\
\hline SILVER & $\begin{array}{l}11 / 05 / 80 \\
08 / 15 / 83\end{array}$ & $\begin{array}{l}0001 \\
0001\end{array}$ & $M G / L$ & $<$ & $\begin{array}{l}0.002 \\
0.02\end{array}$ & $\begin{array}{l}0.002 \\
0.02\end{array}$ & - \\
\hline SOOIUM & $\begin{array}{l}19 / 05 / 80 \\
08 / 15 / 83\end{array}$ & $\begin{array}{l}0001 \\
0001\end{array}$ & MG/L & & $\begin{array}{r}170.00 \\
3.30\end{array}$ & $\dot{-}$ & - \\
\hline SPECIFIC CONDUCTANCE & $11 / 05 / 80$ & 0001 & UMHO/CM & & 9530.00 & - & - \\
\hline SULFATE & $\begin{array}{l}11 / 05 / 80 \\
08 / 15 / 83\end{array}$ & $\begin{array}{l}0009 \\
0001\end{array}$ & MG/L & & $\begin{array}{r}760.00 \\
28.00\end{array}$ & 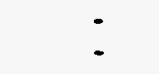 & - \\
\hline TOTAL DISSOLVED SOLIDS & $\begin{array}{l}19 / 05 / 80 \\
08 / 15 / 83\end{array}$ & $\begin{array}{l}0001 \\
0001\end{array}$ & MG/L & & $\begin{array}{r}1130.00 \\
120.00\end{array}$ & 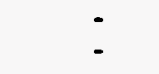 & $\dot{-}$ \\
\hline TOTAL ORGANIC CARBON & $08 / 15 / 83$ & 0001 & $M G / L$ & & 21.00 & - & - \\
\hline TOTAL SUSPENDED SOLIDS & $11 / 05 / 80$ & 0001 & MG/L & & 48.00 & - & - \\
\hline URANIUM & $\begin{array}{l}11 / 05 / 80 \\
08 / 15 / 83\end{array}$ & $\begin{array}{l}0001 \\
0001\end{array}$ & MG/L & & $\begin{array}{l}0.10 \\
0.005\end{array}$ & - & - \\
\hline VANADIUM & $\begin{array}{l}11 / 05 / 80 \\
08 / 15 / 83\end{array}$ & $\begin{array}{l}0001 \\
0001\end{array}$ & MG/L & $<$ & $\begin{array}{l}0.003 \\
0.005\end{array}$ & $\begin{array}{l}0.003 \\
0.005\end{array}$ & - \\
\hline ZINC & $\begin{array}{l}11 / 05 / 80 \\
08 / 15 / 83\end{array}$ & $\begin{array}{l}0001 \\
0001\end{array}$ & MG/L & $<$ & $\begin{array}{l}0.14 \\
0.008\end{array}$ & 0.008 & - \\
\hline
\end{tabular}

PARAMETER VAlUe INDICATOR (PVI): < - LeSS THAN DETECTION LIMIT

SAMPLE ID COOES:

0001 - FILTERED SAMPLE (.45 MICRONS) 
GROUNDWATER OUALITY DATA BY LOCATION

SITE: AMBO1 AMBROSIA LAKE

LOCATION: 0572

NORTH COORDINATE: 52200.0 FT

EAST COORDIHATE: 51100.0 FT

06/10/80 TO $12 / 04 / 92$

REPORT DATE: 08/04/94

FORHATION OF COMPLETION: TRES HERMANOS-A SAHDSTOHE (TR)

HYDRAULIC FLON RELATIONSHIP: UHKNOWN (N)

\begin{tabular}{|c|c|c|c|c|c|c|c|}
\hline PARAMETER NAME & LOG DATE & $\begin{array}{c}\text { SAMPLE } \\
10\end{array}$ & $\begin{array}{l}\text { UNITS OF } \\
\text { MEASURE }\end{array}$ & PVI & $\begin{array}{l}\text { PARAMETER } \\
\text { VALUE FLAGS }\end{array}$ & $\begin{array}{l}\text { DETECTION } \\
\text { LIMIT }\end{array}$ & $\begin{array}{l}\text { PARAMETER } \\
\text { UNCERTAINTY }\end{array}$ \\
\hline ALKALIHITY & $08 / 16 / 83$ & 0001 & $\mathrm{MG} / \mathrm{L} \mathrm{CACO} 3$ & & 14.80 & - & - \\
\hline ALUMIHUM & $08 / 16 / 83$ & 0001 & MG/L & & 0.32 & - & $\cdot$ \\
\hline ARSENIC & $08 / 16 / 83$ & 0001 & $M G / L$ & $<$ & 0.10 & 0.1 & - \\
\hline BARIUM & $08 / 16 / 83$ & 0001 & $M G / L$ & & 0.017 & - & - \\
\hline BICARBONATE - HCO3 & $08 / 16 / 83$ & 0001 & $M G / L$ & & 36.00 & - & - \\
\hline BORON & $08 / 16 / 83$ & 0001 & MG/L & & 0.28 & - & - \\
\hline CADMIUH & $08 / 16 / 83$ & 0001 & $M G / L$ & & 0.008 & - & - \\
\hline CALCIUM & $08 / 16 / 83$ & 0001 & MG/L & & 230.00 & - & - \\
\hline CARBOHATE & $08 / 16 / 83$ & 0001 & $M G / L$ & $<$ & 10.00 & 10. & - \\
\hline CHLORIDE & $08 / 16 / 83$ & 0001 & $M G / L$ & & 510.00 & - & - \\
\hline CHROMIUM & $08 / 16 / 83$ & 0001 & $M G / L$ & & 0.047 & - & - \\
\hline COBALT & $08 / 16 / 83$ & 0001 & MG/L & & 0.023 & - & - \\
\hline COPPER & $08 / 16 / 83$ & 0001 & $M G / L$ & & 0.051 & - & - \\
\hline CYAKIDE & $08 / 16 / 83$ & 0001 & $M G / L$ & $<$ & 0.10 & 0.1 & - \\
\hline FLUORIDE & $08 / 16 / 83$ & 0001 & $M G / L$ & $<$ & 1.00 & 1. & - \\
\hline IRON & $08 / 16 / 83$ & 0001 & $M G / L$ & & 0.26 & - & - \\
\hline LEAD & $08 / 16 / 83$ & 0001 & $M G / L$ & & 0.18 & - & - \\
\hline MAGHESIUH & $08 / 16 / 83$ & 0001 & MG/L & & 230.00 & - & - \\
\hline MANGANESE & $08 / 16 / 83$ & 0001 & $M G / L$ & & 0.83 & - & - \\
\hline MOLYBDENUM & $08 / 16 / 83$ & 0001 & $M G / L$ & & 0.019 & - & - \\
\hline NICKEL & $08 / 16 / 83$ & 0001 & $M G / L$ & & 0.072 & - & - \\
\hline NITRATE & $08 / 16 / 83$ & 0001 & $M G / L$ & $<$ & 1.00 & 1. & - \\
\hline PH & $08 / 16 / 83$ & 0001 & su & & 7.40 & - & - \\
\hline POTASSIUM & $08 / 16 / 83$ & 0001 & $M G / L$ & & 17.00 & - & - \\
\hline RADIUA-226 & $08 / 16 / 83$ & 0001 & $\mathrm{PCl} / \mathrm{L}$ & & 10.65 & - & - \\
\hline RADIUM-226 + RADIUM-228 & $08 / 16 / 83$ & 0001 & $\mathrm{PCI} / \mathrm{L}$ & & 21.30 & - & - \\
\hline RADIUN-228 & $08 / 16 / 83$ & 0001 & $\mathrm{PCI} / \mathrm{L}$ & & 10.65 & - & - \\
\hline SELENIUM & $08 / 16 / 83$ & 0001 & MG/L & & 0.17 & - & - \\
\hline SILVER & $08 / 16 / 83$ & 0001 & $M G / L$ & & 0.06 & $\cdot$ & - \\
\hline SOOIUH & $08 / 16 / 83$ & 0001 & MG/L & & 320.00 & - & - \\
\hline
\end{tabular}

PARAMETER VALUE INDICATOR (PVI): < - LESS THAN DETECTION LIMIT

SAMPLE ID COOES:

0001 - FILTERED SAMPLE (.45 MICRONS) 
GROUNDHATER QUALITY DATA BY LOCATION

SITE: AMBO1 AMBROSIA LAKE

LOCATION: 0572

NORTH COORDINATE: $\quad 52200.0$ FT

EAST COORDINATE: $\quad 51100.0$ FT

06/10/80 TO $12 / 04 / 92$

REPORT DATE: 08/04/94

FORMATION OF COMPLETION: TRES HERMANOS-A SANDSTONE (TR) HYDRAULIC FLOW RELATIONSHIP: UNKNOWN (N)

\begin{tabular}{|c|c|c|c|c|c|c|c|}
\hline PARAMETER MAME & LOG DATE & $\begin{array}{c}\text { SAMPLE } \\
\text { ID }\end{array}$ & $\begin{array}{l}\text { UNITS OF } \\
\text { MEASURE }\end{array}$ & PVI & $\begin{array}{l}\text { PARAMETER } \\
\text { VALUE FLAGS }\end{array}$ & $\begin{array}{l}\text { DETECTION } \\
\text { LIMIT }\end{array}$ & $\begin{array}{l}\text { PARAMETER } \\
\text { UNCERTAIHTY }\end{array}$ \\
\hline SULFATE & $08 / 16 / 83$ & 0001 & $M G / L$ & & 1650.00 & - & - \\
\hline TOTAL DISSOLVED SOLIDS & $08 / 16 / 83$ & 0001 & $M G / L$ & & 2940.00 & - & - \\
\hline TOTAL ORGANIC CARBON & $08 / 16 / 83$ & 0001 & $M G / L$ & & 12.00 & - & - \\
\hline URANIUM & $08 / 16 / 83$ & 0001 & $M G / L$ & & 0.016 & - & - \\
\hline VAKAD IUM & $08 / 16 / 83$ & 0001 & $M G / L$ & & 0.059 & - & - \\
\hline ZIHC & $08 / 16 / 83$ & 0001 & MG/L & & 0.10 & - & - \\
\hline
\end{tabular}

PARAMETER VALUE INDICATOR (PVI):

$<$ - LESS THAN DETECTION LIMIT

SAMPLE ID COOES:

0001 - FILTERED SAMPLE (.45 MICRONS) 
GROUNDHATER QUALITY DATA BY LOCATION

SITE: AMB01 AMBROSIA LAKE

LOCATION: 0583

NORTH COORDIHATE: $\quad 45780.0$ FT

EAST COORDINATE: $\quad 57270.0$ FT

06/10/80 TO 12/04/92

REPORT DATE: $08 / 04 / 94$

FORMATION OF COMPLETIOH: DAKOTA SANDSTONE (KD)

HYDRAULIC FLOW RELATIONSHIP: UNKHOWN (K)

\begin{tabular}{|c|c|c|c|c|c|c|c|}
\hline PARAMETER NAME & LOG DATE & $\begin{array}{c}\text { SAMPLE } \\
\text { ID }\end{array}$ & $\begin{array}{l}\text { UNITS OF } \\
\text { MEASURE }\end{array}$ & PVI & $\begin{array}{l}\text { PARAMETER } \\
\text { VALUE FLAGS }\end{array}$ & $\begin{array}{l}\text { DETECTION } \\
\text { LIMIT }\end{array}$ & $\begin{array}{l}\text { PARAMETER } \\
\text { UNCERTAINTY }\end{array}$ \\
\hline ALKALINITY & $05 / 25 / 83$ & 0001 & MG/L CACO3 & & 172.00 & - & - \\
\hline ALUMINLM & $05 / 25 / 83$ & 0001 & $M G / L$ & $<$ & 0.20 & 0.2 & - \\
\hline ARSEHIC & $05 / 25 / 83$ & 0001 & MG/L & & 0.11 & - & - \\
\hline BARIUH & $05 / 25 / 83$ & 0001 & $M G / L$ & $<$ & 0.02 & 0.02 & $\cdot$ \\
\hline BICARBOHATE - HCO3 & $05 / 25 / 83$ & 0001 & $M G / L$ & & 420.00 & - & - \\
\hline BORON & $05 / 25 / 83$ & 0001 & $M G / L$ & $<$ & 0.05 & 0.05 & $\cdot$ \\
\hline CADHIUH & $05 / 25 / 83$ & 0001 & MG/L & $<$ & 0.01 & 0.09 & - \\
\hline CALCIUM & $05 / 25 / 83$ & 0001 & $H G / L$ & & 4.90 & - & - \\
\hline CARBONATE & $05 / 25 / 83$ & 0001 & $M G / L$ & $<$ & 10.00 & 10. & - \\
\hline CHLORIDE & $05 / 25 / 83$ & 0001 & $M G / L$ & $<$ & 1.00 & 1. & - \\
\hline CHROMIUM & $05 / 25 / 83$ & 0001 & $M G / L$ & $<$ & 0.03 & 0.03 & - \\
\hline COBALT & $05 / 25 / 83$ & 0001 & $M G / L$ & $<$ & 0.04 & 0.04 & - \\
\hline COPPER & $05 / 25 / 83$ & 0001 & $M G / L$ & $<$ & 0.03 & 0.03 & - \\
\hline CYANIDE & $05 / 25 / 83$ & 0009 & MG $/ L$ & $<$ & 1.00 & 1. & - \\
\hline FLUORIDE & $05 / 25 / 83$ & 0001 & $M G / L$ & $<$ & 1.00 & 1. & - \\
\hline IRON & $05 / 25 / 83$ & 0001 & $M G / L$ & $<$ & 0.05 & 0.05 & - \\
\hline LEAD & $05 / 25 / 83$ & 0001 & MG/L & & 0.033 & - & - \\
\hline MAGHESIUM & $05 / 25 / 83$ & 0001 & MG/L & & 29.00 & - & - \\
\hline MAMGAMESE & $05 / 25 / 83$ & 0001 & $M G / L$ & $<$ & 0.02 & 0.02 & - \\
\hline MOLYBDENUH & $05 / 25 / 83$ & 0001 & $M G / L$ & & 0.023 & - & - \\
\hline HICKEL & $05 / 25 / 83$ & 0001 & $M G / L$ & $<$ & 0.08 & 0.08 & $\cdot$ \\
\hline NITRATE & $05 / 25 / 83$ & 0001 & $M G / L$ & $<$ & 1.00 & 1. & - \\
\hline PH & $05 / 25 / 83$ & 0001 & SU & & 8.80 & - & - \\
\hline POTASSIUH & $05 / 25 / 83$ & 0001 & $M G / L$ & & 6.30 & - & - \\
\hline RADIUH-226 & $05 / 25 / 83$ & 0001 & PCI/L & & 0.13 & - & - \\
\hline RADIUH-226 + RADIUMH-228 & $05 / 25 / 83$ & 0004 & $\mathrm{PCI} / \mathrm{L}$ & & 0.26 & $\cdot$ & $\cdot$ \\
\hline RADIUH-228 & $05 / 25 / 83$ & 0001 & PCI/L & & 0.13 & - & $\cdot$ \\
\hline SELERIUN & $05 / 25 / 83$ & 0001 & $M G / L$ & & 0.086 & - & - \\
\hline SILVER & $05 / 25 / 83$ & 0001 & MG/L & $<$ & 0.03 & 0.03 & - \\
\hline SOOIU⿴囗十 & $05 / 25 / 83$ & 0001 & $M G / L$ & & 110.00 & - & - \\
\hline
\end{tabular}

PARAMETER VALUE INDICATOR (PVI):

< - LESS THAN DETECTION LIMIT

SAMPLE ID COOES:

0001 - FILTERED SAMPLE (.45 MICRONS) 
GROUNDWATER QUALITY DATA BY LOCATION

SITE: AMBOI AMBROSIA LAKE

LOCATION: 0583

NORTH COORDINATE: $\quad 45780.0$ FT

EAST COORDINATE: $\quad 57270.0$ FT

O6/10/80 TO $12 / 04 / 92$

REPORT DATE: 08/04/94

FORMATION OF COMPLETION: DAKOTA SANDSTONE (KD)

HYDRAULIC FLON RELATIONSHIP: UNKHOWN (N)

\begin{tabular}{|c|c|c|c|c|c|c|c|}
\hline PARAMETER NAME & LOG DATE & $\underset{\text { SD }}{\text { SAMPLE }}$ & $\begin{array}{l}\text { UNITS OF } \\
\text { MEASURE }\end{array}$ & PVI & $\begin{array}{l}\text { PARAMETER } \\
\text { VALUE FLAGS }\end{array}$ & $\begin{array}{l}\text { DETECTION } \\
\text { LIMIT }\end{array}$ & $\begin{array}{l}\text { PARAMETER } \\
\text { UNCERTAINTY }\end{array}$ \\
\hline SULFATE & $05 / 25 / 83$ & 0001 & MG/L & & 36.00 & - & - \\
\hline TOTAL DISSOLVED SOLIDS & $05 / 25 / 83$ & 0001 & MG/L & & 390.00 & - & - \\
\hline TOTAL DRGANIC CARBOH & $05 / 25 / 83$ & 0001 & $M G / L$ & $<$ & 5.00 & 5. & $\cdot$ \\
\hline VANADIUM & $05 / 25 / 83$ & 0001 & $M G / L$ & $<$ & 0.04 & 0.04 & - \\
\hline ZINC & $05 / 25 / 83$ & 0001 & HG/L & & 0.014 & - & - \\
\hline
\end{tabular}

PARAMETER VALUE INDICATOR (PVI): < - LESS THAN DETECTION LIMIT

SAMPLE ID CODES:

O001 - FILTERED SAMPLE (.45 MICROMS) 
GROUNDWATER OUALITY DATA BY LOCATION

SITE: AMBO1 AMBROSIA LAKE

LOCATION: 0584

NORTH COORDIHATE: $\quad 45290.0$ FT

EAST COOROINATE: $\quad 56310.0$ FT

06/10/80 TO $12 / 04 / 92$

REPORT DATE: 08/04/94

FORMATIOH OF CONPLETION: DAKOTA SANDSTONE (KD)

HYDRAULIC FLOU RELATIONSHIP: UHKNOWN (H)

\begin{tabular}{|c|c|c|c|c|c|c|c|}
\hline PARAMETER NAME & LOG DATE & $\begin{array}{c}\text { SAMPLE } \\
10\end{array}$ & $\begin{array}{l}\text { UNITS OF } \\
\text { MEASURE }\end{array}$ & PVI & $\begin{array}{l}\text { PARAMETER } \\
\text { VALUE FLAGS }\end{array}$ & $\begin{array}{l}\text { DETECTIOH } \\
\text { LIMIT }\end{array}$ & $\begin{array}{l}\text { PARAMETER } \\
\text { UNCERTAINTY }\end{array}$ \\
\hline ALUAINUM & $05 / 26 / 83$ & 0001 & $M G / L$ & $<$ & 0.20 & 0.2 & - \\
\hline ARSENIC & $05 / 26 / 83$ & 0001 & MG/L & $<$ & 0.10 & 0.1 & - \\
\hline BARIUH & $05 / 26 / 83$ & 0001 & $M G / L$ & & 0.05 & - & - \\
\hline BICARBOHATE - HCO3 & $05 / 26 / 83$ & 0001 & MG/L & & 110.00 & - & - \\
\hline BORON & $05 / 26 / 83$ & 0001 & MG/L & $<$ & 0.05 & 0.05 & - \\
\hline CADHIUA & $05 / 26 / 83$ & 0001 & $M G / L$ & $<$ & 0.01 & 0.01 & - \\
\hline CALCIUH & $05 / 26 / 83$ & 0001 & $M G / L$ & & 110.00 & - & - \\
\hline CARBONATE & $05 / 26 / 83$ & 0001 & $M G / L$ & $<$ & 10.00 & 10. & - \\
\hline CHLORIDE & $05 / 26 / 83$ & 0001 & $M G / L$ & & 27.00 & - & - \\
\hline CHROMIUN & $05 / 26 / 83$ & 0001 & $M G / L$ & $<$ & 0.03 & 0.03 & - \\
\hline COBALT & $05 / 26 / 83$ & 0001 & MG/L & $<$ & 0.04 & 0.04 & - \\
\hline COPPER & $05 / 26 / 83$ & 0001 & $M G / L$ & $<$ & 0.03 & 0.03 & - \\
\hline CYAMIDE & $05 / 26 / 83$ & 0001 & MG/L & $<$ & 1.00 & 1. & - \\
\hline IRON & $05 / 26 / 83$ & 0001 & $M G / L$ & $<$ & 0.05 & 0.05 & - \\
\hline LEAD & $05 / 26 / 83$ & 0001 & $M G / L$ & & 0.047 & - & - \\
\hline MAGHESIUM & $05 / 26 / 83$ & 0001 & $\mathrm{HG} / \mathrm{L}$ & & 56.00 & - & - \\
\hline MANGANESE & $05 / 26 / 83$ & 0001 & $M G / L$ & & 0.60 & - & - \\
\hline MOLYBDENUM & $05 / 26 / 83$ & 0001 & $M G / L$ & $<$ & 0.02 & 0.02 & - \\
\hline HICKEL & $05 / 26 / 83$ & 0001 & $\mathrm{MG} / \mathrm{L}$ & $<$ & 0.08 & 0.08 & - \\
\hline HITRATE & $05 / 26 / 83$ & 0001 & $M G / L$ & & 1.00 & - & - \\
\hline POTASSIUH & $05 / 26 / 83$ & 0001 & $M G / L$ & & 10.00 & - & - \\
\hline RADIUA-226 & $05 / 26 / 83$ & 0001 & $\mathrm{PCI} / \mathrm{L}$ & $<$ & 1.30 & 1.3 & - \\
\hline RADIUH-226 + RADIUM-228 & $05 / 26 / 83$ & 0001 & $\mathrm{PCI} / \mathrm{L}$ & & 1.30 & $\cdot$ & - \\
\hline RADIUN-228 & $05 / 26 / 83$ & 0001 & $\mathrm{PCI} / \mathrm{L}$ & $<$ & 1.30 & 1.3 & - \\
\hline SELENIUM & $05 / 26 / 83$ & 0001 & $M G / L$ & & 0.048 & - & - \\
\hline SILVER & $05 / 26 / 83$ & 0001 & $M G / L$ & $<$ & 0.03 & 0.03 & - \\
\hline SOOIUH & $05 / 26 / 83$ & 0001 & MG/L & & 56.00 & - & - \\
\hline SULFATE & $05 / 26 / 83$ & 0001 & MG/L & & 420.00 & - & $\cdot$ \\
\hline URANIUM & $05 / 26 / 83$ & 0001 & MG/L & & 0.004 & - & - \\
\hline VAHADIUM & $05 / 26 / 83$ & 0001 & $M G / L$ & $<$ & 0.04 & 0.04 & - \\
\hline
\end{tabular}

PARAMETER VALUE INDICATOR (PVI)

SAMPLE ID CODES:

0001 - FILTERED SAMPLE (.45 MICRONS) 
GROUNDWATER QUALITY DATA BY LOCATION

SITE: AMB01 AMBROSIA LAKE

LOCATION: 0584

NORTH COORDINATE: $\quad 45290.0$ FT

EAST COORDIHATE: $\quad 56310.0$ FT

06/10/80 TO 12/04/92

REPORT DATE : $08 / 04 / 94$

FORMATION OF COMPLETIOK: DAKOTA SANDSTONE (KD)

HYDRAULIC FLOW RELATIONSHIP: UNKNOWN (N)

\begin{tabular}{|l|c|c|l|c|c|c|c|}
\hline PARAMETER NAME & LOG DATE & $\begin{array}{c}\text { SAMPLE } \\
\text { ID }\end{array}$ & $\begin{array}{l}\text { UNITS OF } \\
\text { MEASURE }\end{array}$ & PVI & $\begin{array}{c}\text { PARAMETER } \\
\text { VALUE FLAGS }\end{array}$ & $\begin{array}{c}\text { DETECTION } \\
\text { LIMIT }\end{array}$ & $\begin{array}{l}\text { PARAMETER } \\
\text { UNCERTAINTY }\end{array}$ \\
\hline ZINC & $05 / 26 / 83$ & 0001 & MG/L & & 0.044 & - & - \\
\hline
\end{tabular}

PARAMETER VALUE IHDICATOR (PVI): < - LESS THAN DETECTION LIMIT

SAMPLE ID COOES:

0001 - FILTERED SAMPLE (.45 MICRONS) 
GROUMDHATER QUALITY DATA BY LOCATION

SITE: AMBO1 AMBROSIA LAKE

LOCATION: 0585

NORTH COORDINATE: $\quad 56520.0$ FT

EAST COORDINATE: $\quad 47090.0$ FT

06/10/80 TO $12 / 04 / 92$

REPORT DATE: 08/04/94

FORMATION OF COMPLETIOH: DAKOTA SAKDSTONE (KD)

HYDRAULIC FLON RELATIONSHIP: UNKHOWN (N)

\begin{tabular}{|c|c|c|c|c|c|c|c|}
\hline PARAMETER MAME & LOG DATE & $\begin{array}{c}\text { SAMPLE } \\
\text { ID }\end{array}$ & $\begin{array}{l}\text { UHITS OF } \\
\text { MEASURE }\end{array}$ & PVI & $\begin{array}{l}\text { PARAMETER } \\
\text { VALUE FLAGS }\end{array}$ & $\begin{array}{l}\text { DETECTION } \\
\text { LIMIT }\end{array}$ & $\begin{array}{l}\text { PARAMETER } \\
\text { UNCERTAINT }\end{array}$ \\
\hline ALKALIHITY & $11 / 14 / 83$ & 0001 & MG/L CACO3 & & 8.20 & - & - \\
\hline ALUMINUM & $\begin{array}{l}11 / 05 / 80 \\
11 / 14 / 83\end{array}$ & $\begin{array}{l}0001 \\
0001\end{array}$ & MG/L & $<$ & $\begin{array}{l}0.05 \\
0.091\end{array}$ & 0.05 & - \\
\hline ARSENIC & $\begin{array}{l}11 / 05 / 80 \\
11 / 14 / 83\end{array}$ & $\begin{array}{l}0001 \\
0001\end{array}$ & $M G / L$ & $<$ & $\begin{array}{l}0.001 \\
0.057\end{array}$ & 0.001 & - \\
\hline BARIUA & $\begin{array}{l}11 / 05 / 80 \\
11 / 14 / 83\end{array}$ & $\begin{array}{l}0001 \\
0001\end{array}$ & $M G / L$ & & $\begin{array}{l}0.03 \\
0.046\end{array}$ & $\dot{-}$ & $\dot{-}$ \\
\hline BICARBOHATE - HCO3 & $\begin{array}{l}11 / 05 / 80 \\
11 / 14 / 83\end{array}$ & $\begin{array}{l}0001 \\
0001\end{array}$ & $M G / L$ & & $\begin{array}{l}24.00 \\
20.00\end{array}$ & : & - \\
\hline BORON & $\begin{array}{l}11 / 05 / 80 \\
11 / 14 / 83\end{array}$ & $\begin{array}{l}0001 \\
0001\end{array}$ & $M G / L$ & & $\begin{array}{l}7.20 \\
4.70\end{array}$ & - & - \\
\hline CADMIUM & $\begin{array}{l}11 / 05 / 80 \\
11 / 14 / 83\end{array}$ & $\begin{array}{l}0001 \\
0001\end{array}$ & $M G / L$ & $<$ & $\begin{array}{l}0.008 \\
0.007\end{array}$ & $\begin{array}{l}0.008 \\
0.007\end{array}$ & - \\
\hline CALCIUAM & $\begin{array}{l}19 / 05 / 80 \\
19 / 14 / 83\end{array}$ & $\begin{array}{l}0009 \\
0001\end{array}$ & $M G / L$ & & $\begin{array}{r}29.00 \\
110.00\end{array}$ & - & - \\
\hline CARBOHATE & $11 / 14 / 83$ & 0001 & $M G / L$ & $<$ & 10.00 & 10. & - \\
\hline CHLORIDE & $\begin{array}{l}19 / 05 / 80 \\
11 / 14 / 83\end{array}$ & $\begin{array}{l}0001 \\
0001\end{array}$ & $M G / L$ & $<$ & $\begin{array}{l}10.00 \\
90.00\end{array}$ & 10. & - \\
\hline CHROMIUM & $\begin{array}{l}11 / 05 / 80 \\
11 / 14 / 83\end{array}$ & $\begin{array}{l}0009 \\
0001\end{array}$ & $M G / L$ & $<$ & $\begin{array}{l}0.001 \\
0.026\end{array}$ & $\begin{array}{l}0.001 \\
0.026\end{array}$ & $\therefore$ \\
\hline COBALT & $\begin{array}{l}11 / 05 / 80 \\
11 / 14 / 83\end{array}$ & $\begin{array}{l}0001 \\
0001\end{array}$ & $M G / L$ & $<$ & $\begin{array}{l}0.006 \\
0.011\end{array}$ & $\begin{array}{l}0.006 \\
0.011\end{array}$ & - \\
\hline COPPER & $\begin{array}{l}11 / 05 / 80 \\
11 / 14 / 83\end{array}$ & $\begin{array}{l}0001 \\
0001\end{array}$ & MG/L & $<$ & $\begin{array}{l}0.19 \\
0.017\end{array}$ & 0.017 & - \\
\hline CYANIDE & $11 / 14 / 83$ & 0009 & MG/L & $<$ & 9.00 & 1. & - \\
\hline FLUORIDE & $\begin{array}{l}11 / 05 / 80 \\
11 / 14 / 83\end{array}$ & $\begin{array}{l}0001 \\
0001\end{array}$ & $M G / L$ & $<$ & $\begin{array}{l}0.20 \\
1.00\end{array}$ & $\begin{array}{l}0.2 \\
1 .\end{array}$ & - \\
\hline IRON & $\begin{array}{l}11 / 05 / 80 \\
11 / 14 / 83\end{array}$ & $\begin{array}{l}0001 \\
0001\end{array}$ & $M G / L$ & $<$ & $\begin{array}{l}0.053 \\
0.024\end{array}$ & 0.024 & - \\
\hline LEAD & $\begin{array}{l}11 / 05 / 80 \\
11 / 14 / 83\end{array}$ & $\begin{array}{l}0001 \\
0001\end{array}$ & MG/L & $<$ & $\begin{array}{l}0.001 \\
0.023\end{array}$ & 0.001 & - \\
\hline MAGNESIUM & $\begin{array}{l}11 / 05 / 80 \\
11 / 14 / 83\end{array}$ & $\begin{array}{l}0001 \\
0001\end{array}$ & $M G / L$ & & $\begin{array}{l}0.27 \\
9.90\end{array}$ & - & - \\
\hline MANGAMESE & $\begin{array}{l}11 / 05 / 80 \\
11 / 14 / 83\end{array}$ & $\begin{array}{l}0001 \\
0001\end{array}$ & MG/L & $<$ & $\begin{array}{l}0.004 \\
0.003\end{array}$ & 0.003 & - \\
\hline MERCURY & $11 / 05 / 80$ & 0001 & MG/L & $<$ & 0.001 & 0.001 & - \\
\hline MOLYBDENUM & $\begin{array}{l}11 / 05 / 80 \\
11 / 14 / 83\end{array}$ & $\begin{array}{l}0001 \\
0001\end{array}$ & $M G / L$ & & $\begin{array}{l}0.066 \\
0.021\end{array}$ & - & - \\
\hline
\end{tabular}

PARAMETER VALUE IHDICATOR (PVI): < - LESS THAN DETECTIOH LIMIT

SAMPLE ID COOES:

0001 - FILTERED SAMPLE (.45 MICRONS) 
GROUNDHATER QUALITY DATA BY LOCATION

SITE: AMBO1 AMBROSIA LAKE

LOCATION: 0585

MORTH COORDINATE: 56520.0 FT

EAST COORDINATE: $\quad 47090.0$ FT

06/10/80 TO 12/04/92

REPORT DATE: 08/04/94

FORMATION OF COMPLETION: DAKOTA SAHDSTONE (KD)

HYDRAULIC FLOW RELATIONSHIP: UNKNOWN (N)

\begin{tabular}{|c|c|c|c|c|c|c|c|}
\hline PARNMETER HAME & LOG DATE & $\begin{array}{c}\text { SAMPLE } \\
\text { ID }\end{array}$ & $\begin{array}{l}\text { UNITS OF } \\
\text { MEASURE }\end{array}$ & PVI & $\begin{array}{l}\text { PARAMETER } \\
\text { VALUE FLAGS }\end{array}$ & $\begin{array}{l}\text { DETECTION } \\
\text { LIMIT }\end{array}$ & $\begin{array}{l}\text { PARAMETER } \\
\text { UNCERTAINTY }\end{array}$ \\
\hline NICKEL & $\begin{array}{l}11 / 05 / 80 \\
11 / 14 / 83\end{array}$ & $\begin{array}{l}0001 \\
0001\end{array}$ & MG/L & $<$ & $\begin{array}{l}0.059 \\
0.038\end{array}$ & 0.038 & - \\
\hline NITRATE & $\begin{array}{l}11 / 05 / 80 \\
11 / 14 / 83\end{array}$ & $\begin{array}{l}0001 \\
0001\end{array}$ & $M G / L$ & & $\begin{array}{l}5.30 \\
1.00\end{array}$ & - & $\dot{-}$ \\
\hline PH & $\begin{array}{l}11 / 05 / 80 \\
11 / 14 / 83\end{array}$ & $\begin{array}{l}0001 \\
0001\end{array}$ & su & & $\begin{array}{l}9.90 \\
9.80\end{array}$ & $\dot{-}$ & $\dot{-}$ \\
\hline POTASSIUH & $\begin{array}{l}11 / 05 / 80 \\
11 / 14 / 83\end{array}$ & $\begin{array}{l}0001 \\
0001\end{array}$ & MG/L & & $\begin{array}{l}11.00 \\
16.00\end{array}$ & - & $\dot{-}$ \\
\hline RADIUM-226 & $\begin{array}{l}11 / 05 / 80 \\
11 / 94 / 83\end{array}$ & $\begin{array}{l}0001 \\
0001\end{array}$ & $\mathrm{PCI} / \mathrm{L}$ & $<$ & $\begin{array}{l}6.60 \\
3.80\end{array}$ & 3.8 & - \\
\hline RADIUM-226 + RADIUM-228 & $19 / 14 / 83$ & 0001 & PCI/L & & 3.80 & - & - \\
\hline RADIUM-228 & $11 / 14 / 83$ & 0001 & $P C 1 / L$ & $<$ & 3.80 & 3.8 & - \\
\hline SELEKIUM & $\begin{array}{l}11 / 05 / 80 \\
11 / 14 / 83\end{array}$ & $\begin{array}{l}0001 \\
0001\end{array}$ & $M G / L$ & & $\begin{array}{l}0.013 \\
0.041\end{array}$ & - & $\dot{-}$ \\
\hline SILVER & $\begin{array}{l}11 / 05 / 80 \\
11 / 14 / 83\end{array}$ & $\begin{array}{l}0001 \\
0001\end{array}$ & MG/L & $<$ & $\begin{array}{l}0.002 \\
0.02\end{array}$ & $\begin{array}{l}0.002 \\
0.02\end{array}$ & - \\
\hline SOO IUM & $\begin{array}{l}11 / 05 / 80 \\
11 / 14 / 83\end{array}$ & $\begin{array}{l}0001 \\
0001\end{array}$ & MG/L & & $\begin{array}{r}75.00 \\
190.00\end{array}$ & - & - \\
\hline SPECIFIC CONDUCTANCE & $11 / 05 / 80$ & 0001 & UMHO/CM & & 530.00 & - & - \\
\hline SULFATE & $\begin{array}{l}11 / 05 / 80 \\
11 / 14 / 83\end{array}$ & $\begin{array}{l}0001 \\
0001\end{array}$ & MG/L & & $\begin{array}{l}170.00 \\
590.00\end{array}$ & 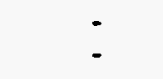 & $\dot{-}$ \\
\hline TOTAL DISSOLVED SOLIDS & $\begin{array}{l}11 / 05 / 80 \\
11 / 14 / 83\end{array}$ & $\begin{array}{l}0001 \\
0001\end{array}$ & MG/L & & $\begin{array}{r}376.00 \\
1120.00\end{array}$ & - & - \\
\hline TOTAL SUSPENDED SOLIDS & $11 / 05 / 80$ & 0001 & MG/L & & 21.00 & - & - \\
\hline URAKIUM & $\begin{array}{l}11 / 05 / 80 \\
11 / 14 / 83\end{array}$ & $\begin{array}{l}0001 \\
0001\end{array}$ & MG/L & & $\begin{array}{l}0.078 \\
0.004\end{array}$ & - & - \\
\hline VANADIUA: & $\begin{array}{l}11 / 05 / 80 \\
11 / 14 / 83\end{array}$ & $\begin{array}{l}0001 \\
0001\end{array}$ & MG/L & $<$ & $\begin{array}{l}0.003 \\
0.005\end{array}$ & $\begin{array}{l}0.003 \\
0.005\end{array}$ & $\dot{-}$ \\
\hline ZINC & $\begin{array}{l}11 / 05 / 80 \\
11 / 14 / 83\end{array}$ & $\begin{array}{l}0001 \\
0001\end{array}$ & MG/L & $<$ & $\begin{array}{l}0.12 \\
0.008\end{array}$ & 0.008 & - \\
\hline
\end{tabular}

PARAMETER VALUE INDICATOR (PVI): < - LESS THAN DETECTION LIMIT

SAMPLE ID CODES:

O001 - FILTERED SAMPLE (.45 MICRONS) 
GROUHDWATER QUALITY DATA BY LOCATIOH

SITE: AMBO1 AMBROSIA LAKE

LOCATIOH: 0586

HORTH COORDIMATE: 53590.0 FT

EAST COORDINATE: $\quad 48410.0$ FT

$06 / 10 / 80$ TO $12 / 04 / 92$

REPORT DATE: 08/04/94

FORMATION OF COMPLETIOH: DAKOTA SANDSTOHE (KD)

HYORAULIC FLOW RELATIONSHIP: UHKHONN (N)

\begin{tabular}{|c|c|c|c|c|c|c|c|}
\hline PARAMETER NAME & LOG DATE & $\begin{array}{l}\text { SAMPLE } \\
\text { ID }\end{array}$ & $\begin{array}{l}\text { UNITS OF } \\
\text { MEASURE }\end{array}$ & PVI & $\begin{array}{l}\text { PARAMETER } \\
\text { VALUE FLAGS }\end{array}$ & $\begin{array}{l}\text { DETECTIOH } \\
\text { LIMIT }\end{array}$ & $\begin{array}{l}\text { PARAMETER } \\
\text { UNCERTAINTY }\end{array}$ \\
\hline ALLMINUM & $\begin{array}{l}10 / 20 / 80 \\
05 / 26 / 83\end{array}$ & $\begin{array}{l}0001 \\
0001\end{array}$ & MG/L & $<$ & $\begin{array}{l}0.05 \\
0.43\end{array}$ & 0.05 & $\dot{-}$ \\
\hline ARSEHIC & $\begin{array}{l}10 / 20 / 80 \\
05 / 26 / 83\end{array}$ & $\begin{array}{l}0001 \\
0001\end{array}$ & MG/L & & $\begin{array}{l}0.033 \\
0.18\end{array}$ & - & - \\
\hline BARIUM & $\begin{array}{l}10 / 20 / 80 \\
05 / 26 / 83\end{array}$ & $\begin{array}{l}0009 \\
0001\end{array}$ & MG/L & & $\begin{array}{l}0.017 \\
0.034\end{array}$ & : & - \\
\hline BICARBONATE - HCOZ & $\begin{array}{l}10 / 20 / 80 \\
05 / 26 / 83\end{array}$ & $\begin{array}{l}0001 \\
0001\end{array}$ & MG/L & $<$ & $\begin{array}{r}279.00 \\
10.00\end{array}$ & 10. & - \\
\hline BORON & $\begin{array}{l}10 / 20 / 80 \\
05 / 26 / 83\end{array}$ & $\begin{array}{l}0001 \\
0001\end{array}$ & MG/L & & $\begin{array}{l}0.58 \\
0.48\end{array}$ & $\dot{-}$ & - \\
\hline CADMIUA & $\begin{array}{l}10 / 20 / 80 \\
05 / 26 / 83\end{array}$ & $\begin{array}{l}0001 \\
0001\end{array}$ & MG/L & $<$ & $\begin{array}{l}0.008 \\
0.029\end{array}$ & 0.008 & - \\
\hline CALCIUM & $\begin{array}{l}10 / 20 / 80 \\
05 / 26 / 83\end{array}$ & $\begin{array}{l}0001 \\
0001\end{array}$ & MG/L & & $\begin{array}{l}450.00 \\
760.00\end{array}$ & 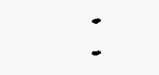 & 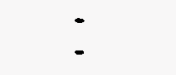 \\
\hline CARBONATE & $05 / 26 / 83$ & 0001 & $M G / L$ & $<$ & 10.00 & 10. & - \\
\hline CHLORIDE & $\begin{array}{l}10 / 20 / 80 \\
05 / 26 / 83\end{array}$ & $\begin{array}{l}0001 \\
0001\end{array}$ & MG/L & & $\begin{array}{r}375.00 \\
1070.00\end{array}$ & 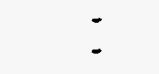 & - \\
\hline CHROMIUA & $\begin{array}{l}10 / 20 / 80 \\
05 / 26 / 83\end{array}$ & $\begin{array}{l}0001 \\
0001\end{array}$ & $M G / L$ & & $\begin{array}{l}0.006 \\
0.096\end{array}$ & - & - \\
\hline COBALT & $\begin{array}{l}10 / 20 / 80 \\
05 / 26 / 83\end{array}$ & $\begin{array}{l}0001 \\
0001\end{array}$ & $M G / L$ & $<$ & $\begin{array}{l}0.006 \\
0.066\end{array}$ & 0.006 & $\dot{-}$ \\
\hline COPPER & $\begin{array}{l}10 / 20 / 80 \\
05 / 26 / 83\end{array}$ & $\begin{array}{l}0001 \\
0001\end{array}$ & MG/L & & $\begin{array}{l}0.011 \\
0.073\end{array}$ & - & $\dot{-}$ \\
\hline CYAHIOE & $05 / 26 / 83$ & 0001 & MG/L & $<$ & 1.00 & 1. & - \\
\hline FLUORIDE & $\begin{array}{l}10 / 20 / 80 \\
05 / 26 / 83\end{array}$ & $\begin{array}{l}0001 \\
0001\end{array}$ & MG/L & $<$ & $\begin{array}{l}0.20 \\
4.00\end{array}$ & 0.2 & - \\
\hline IROH & $\begin{array}{l}10 / 20 / 80 \\
05 / 26 / 83\end{array}$ & $\begin{array}{l}0001 \\
0001\end{array}$ & MG/L & & $\begin{array}{c}0.057 \\
33.00\end{array}$ & - & - \\
\hline LEAD & $\begin{array}{l}10 / 20 / 80 \\
05 / 26 / 83\end{array}$ & $\begin{array}{l}0001 \\
0001\end{array}$ & MG/L & & $\begin{array}{l}0.003 \\
0.46\end{array}$ & - & - \\
\hline MAGHESIUM & $\begin{array}{l}10 / 20 / 80 \\
05 / 26 / 83\end{array}$ & $\begin{array}{l}0001 \\
0001\end{array}$ & MG/L & & $\begin{array}{l}230.00 \\
330.00\end{array}$ & - & - \\
\hline MANGAMESE & $\begin{array}{l}10 / 20 / 80 \\
05 / 26 / 83\end{array}$ & $\begin{array}{l}0009 \\
0009\end{array}$ & MG/L & & $\begin{array}{l}0.74 \\
2.90\end{array}$ & - & - \\
\hline MERCURY & $10 / 20 / 80$ & 0001 & MG/L & $<$ & 0.001 & 0.001 & - \\
\hline MOLYBDENUM & $\begin{array}{l}10 / 20 / 80 \\
05 / 26 / 83\end{array}$ & $\begin{array}{l}0001 \\
0001\end{array}$ & MG/L & & $\begin{array}{l}0.08 \\
0.04\end{array}$ & - & - \\
\hline RICKEL & $10 / 20 / 80$ & 0001 & MG/L & & 0.05 & - & - \\
\hline
\end{tabular}

PARAMETER VALUE IHDICATOR (PVI): < - LESS THAN DETECTION LIMIT

SAMPLE ID COOES:

0001 - FILTERED SAMPLE (.45 MICRONS) 
GROUNDWATER QUALITY DATA BY LOCATION

SITE: AMBOI AMBROSIA LAKE

LOCATION: 0586

NORTH COORDINATE: 53590.0 FT

EAST COORDINATE: $\quad 48410.0$ FT

06/10/80 TO $12 / 04 / 92$

REPORT DATE: $08 / 04 / 94$

FORMATIOH OF COMPLETION: DAKOTA SANDSTOHE (KD)

HYDRAULIC FLON RELATIONSHIP: UNKNOWN (N)

\begin{tabular}{|c|c|c|c|c|c|c|c|}
\hline PARAMETER MAME & LOG DATE & $\begin{array}{c}\text { SAMPLE } \\
\text { ID }\end{array}$ & $\begin{array}{l}\text { UNITS OF } \\
\text { MEASURE }\end{array}$ & PVI & $\begin{array}{l}\text { PARAMETER } \\
\text { VALUE FLAGS }\end{array}$ & $\begin{array}{l}\text { DETECTION } \\
\text { LIMIT }\end{array}$ & $\begin{array}{l}\text { PARAMETER } \\
\text { UNCERTAINTY }\end{array}$ \\
\hline NICKEL & $05 / 26 / 83$ & 0001 & $M G / L$ & & 0.18 & - & - \\
\hline NITRATE & $\begin{array}{l}10 / 20 / 80 \\
05 / 26 / 83\end{array}$ & $\begin{array}{l}0001 \\
0001\end{array}$ & MG/L & & $\begin{array}{l}2.80 \\
2.00\end{array}$ & $\dot{-}$ & - \\
\hline PH & $\begin{array}{l}10 / 20 / 80 \\
05 / 26 / 83\end{array}$ & $\begin{array}{l}0001 \\
0001\end{array}$ & SU & & $\begin{array}{l}7.70 \\
5.00\end{array}$ & - & $\dot{-}$ \\
\hline POTASSIUM & $\begin{array}{l}10 / 20 / 80 \\
05 / 26 / 83\end{array}$ & $\begin{array}{l}0001 \\
0001\end{array}$ & $M G / L$ & & $\begin{array}{l}19.00 \\
26.00\end{array}$ & - & - \\
\hline RADIUM-226 & $\begin{array}{l}10 / 20 / 80 \\
05 / 26 / 83\end{array}$ & $\begin{array}{l}0001 \\
0001\end{array}$ & PCI /L & & $\begin{array}{l}3.07 \\
2.86\end{array}$ & - & - \\
\hline RADIUH-226 + RADIUH-228 & $05 / 26 / 83$ & 0001 & PCI/L & & 5.72 & - & - \\
\hline RADIUH-228 & $05 / 26 / 83$ & 0001 & PCI/L & & 2.86 & - & - \\
\hline SELENIUM & $\begin{array}{l}10 / 20 / 80 \\
05 / 26 / 83\end{array}$ & $\begin{array}{l}0001 \\
0001\end{array}$ & $M G / L$ & & $\begin{array}{l}0.025 \\
0.26\end{array}$ & - & - \\
\hline SILVER & $\begin{array}{l}10 / 20 / 80 \\
05 / 26 / 83\end{array}$ & $\begin{array}{l}0001 \\
0001\end{array}$ & $M G / L$ & $<$ & $\begin{array}{l}0.002 \\
0.10\end{array}$ & 0.002 & - \\
\hline SOOIUH & $\begin{array}{l}10 / 20 / 80 \\
05 / 26 / 83\end{array}$ & $\begin{array}{l}0001 \\
0001\end{array}$ & $M G / L$ & & $\begin{array}{l}210.00 \\
500.00\end{array}$ & - & - \\
\hline SPECIFIC CONDUCTANCE & $10 / 20 / 80$ & 0001 & UМHO/CM & & 4020.00 & - & - \\
\hline SULFATE & $\begin{array}{l}10 / 20 / 80 \\
05 / 26 / 83\end{array}$ & $\begin{array}{l}0001 \\
0001\end{array}$ & $M G / L$ & & $\begin{array}{l}1615.00 \\
2770.00\end{array}$ & - & - \\
\hline TOTAL DISSOLVED SOLIDS & $\begin{array}{l}10 / 20 / 80 \\
05 / 26 / 83\end{array}$ & $\begin{array}{l}0001 \\
0001\end{array}$ & $M G / L$ & & $\begin{array}{l}3280.00 \\
4880.00\end{array}$ & - & - \\
\hline TOTAL SUSPENDED SOLIDS & $10 / 20 / 80$ & 0001 & $M G / L$ & & 124.00 & - & - \\
\hline URANIUA & $\begin{array}{l}10 / 20 / 80 \\
05 / 26 / 83\end{array}$ & $\begin{array}{l}0001 \\
0001\end{array}$ & MG/L & & $\begin{array}{l}0.06 \\
0.004\end{array}$ & - & - \\
\hline VAKADIUH & $\begin{array}{l}10 / 20 / 80 \\
05 / 26 / 83\end{array}$ & $\begin{array}{l}0001 \\
0001\end{array}$ & MG/L & $<$ & $\begin{array}{l}0.003 \\
0.091\end{array}$ & 0.003 & - \\
\hline ZINC & $\begin{array}{l}10 / 20 / 80 \\
05 / 26 / 83\end{array}$ & $\begin{array}{l}0001 \\
0001\end{array}$ & $\mathrm{MG} / \mathrm{L}$ & & $\begin{array}{l}0.21 \\
0.23\end{array}$ & - & - \\
\hline
\end{tabular}

PARAMETER VALUE INDICATOR (PVI): < - LESS THAN DETECTION LIMIT

SAMPLE ID CODES:

0001 - FILTERED SAMPLE (.45 MICROHS) 
GROUNDWATER QUALITY DATA BY LOCATION

SITE: AMBO1 AMBROSIA LAKE

LOCATION: 0587

HORTH COORDIHATE: $\quad 54400.0$ FT

EAST COORDIHATE: $\quad 51480.0$ FT

06/10/80 TO 12/04/92

REPORT DATE: 08/04/94

FORMATION OF COMPLETIOH: DAKOTA SAKDSTOHE (KD)

HYDRAULIC FLOW RELATIONSHIP: UNKKONN (H)

\begin{tabular}{|c|c|c|c|c|c|c|c|}
\hline PARAMETER NAME & LOG DATE & $\underset{10}{\text { SAMPLE }}$ & $\begin{array}{l}\text { UNITS OF } \\
\text { MEASURE }\end{array}$ & PVI & $\begin{array}{l}\text { PARAMETER } \\
\text { VALUE FLAGS }\end{array}$ & $\begin{array}{l}\text { DETECTION } \\
\text { LIMIT }\end{array}$ & $\begin{array}{l}\text { PARAMETER } \\
\text { UNCERTAINTY }\end{array}$ \\
\hline ALKALINITY & $08 / 15 / 83$ & 0001 & HG/L CACO3 & & 258.00 & - & - \\
\hline ALUAIKUM & $08 / 15 / 83$ & 0001 & MG/L & & 0.52 & - & - \\
\hline ARSEHIC & $08 / 15 / 83$ & 0001 & MG/L & & 0.45 & - & - \\
\hline BARIUM & $08 / 15 / 83$ & 0001 & MG/L & & 0.021 & - & - \\
\hline BICARBOHATE - HCO3 & $08 / 15 / 83$ & 0001 & MG/L & & 630.00 & - & - \\
\hline BORON & $08 / 15 / 83$ & 0001 & MG/L & & 0.36 & - & - \\
\hline CADMIUH & $08 / 15 / 83$ & 0001 & $M G / L$ & & 0.02 & - & - \\
\hline CALCIUM & $08 / 15 / 83$ & 0001 & $M G / L$ & & 640.00 & - & - \\
\hline CARBONATE & $08 / 15 / 83$ & 0001 & $M G / L$ & $<$ & 10.00 & 10. & - \\
\hline CHLORIDE & $08 / 15 / 83$ & 0001 & MG/L & & 770.00 & - & - \\
\hline CHROMIUM & $08 / 15 / 83$ & 0001 & $M G / L$ & & 0.096 & $\cdot$ & - \\
\hline COBALT & $08 / 15 / 83$ & 0001 & $M G / L$ & & 0.072 & - & $\cdot$ \\
\hline COPPER & $08 / 15 / 83$ & 0001 & MG/L & & 0.12 & - & - \\
\hline CYAHIDE & $08 / 15 / 83$ & 0001 & $M G / L$ & $<$ & 1.00 & 1. & - \\
\hline FLUORIDE & $08 / 15 / 83$ & 0001 & $M G / L$ & & 1.10 & $\cdot$ & - \\
\hline IRON & $08 / 15 / 83$ & 0001 & $M G / L$ & & 0.82 & - & - \\
\hline LEAD & $08 / 15 / 83$ & 0001 & MG/L & & 0.39 & - & - \\
\hline MAGNESIUM & $08 / 15 / 83$ & 0001 & MG/L & & 440.00 & - & - \\
\hline MAMGAHESE & $08 / 15 / 83$ & 0001 & MG/L & & 2.80 & - & - \\
\hline MOLYBDENUM & $08 / 15 / 83$ & 0001 & $M G / L$ & & 0.055 & $\cdot$ & - \\
\hline NICKEL & $08 / 15 / 83$ & 0001 & $M G / L$ & & 0.14 & - & - \\
\hline NITRATE & $08 / 15 / 83$ & 0001 & $M G / L$ & $<$ & 1.00 & 1. & - \\
\hline $\mathrm{PH}$ & $08 / 15 / 83$ & 0001 & su & & 6.70 & - & - \\
\hline POTASSIUM & $08 / 15 / 83$ & 0001 & $\mathrm{MG} / \mathrm{L}$ & & 15.00 & - & - \\
\hline RADIUH-226 & $08 / 15 / 83$ & 0001 & $\mathrm{PCl} / \mathrm{L}$ & $<$ & 3.30 & 3.3 & $\cdot$ \\
\hline RADIUM-226 + RADIUN-228 & $08 / 15 / 83$ & 0001 & $\mathrm{PCI} / \mathrm{L}$ & & 3.30 & - & - \\
\hline RADIUN-228 & $08 / 15 / 83$ & 0001 & $\mathrm{PCI} / \mathrm{L}$ & $<$ & 3.30 & 3.3 & - \\
\hline SELENIUM & $08 / 15 / 83$ & 0001 & $M G / L$ & & 0.49 & - & - \\
\hline SILVER & $08 / 15 / 83$ & 0001 & $H G / L$ & & 0.14 & - & $\cdot$ \\
\hline SOOIUM & $08 / 15 / 83$ & 0001 & $M G / L$ & & 330.00 & - & - \\
\hline
\end{tabular}

PARAMETER VALUE INDICATOR (PVI):

- LESS than detection Limit
SAMPLE ID COOES:

0001 - FILTERED SAMPLE (.45 MICRONS) 
GROUNDWATER QUALITY DATA BY LOCATION

SITE: AMBOI AMBROSIA LAKE

LOCATION: 0587

MORTH COORDINATE: $\quad 54400.0$ FT

EAST COORDINATE: $\quad 51480.0$ FT

06/10/80 TO $12 / 04 / 92$

REPORT DATE: 08/04/94

FORMATION OF COMPLETION: DAKOTA SAMDSTONE (KD)

HYDRAULIC FLOW RELATIONSHIP: UNKNOWN (N)

\begin{tabular}{|c|c|c|c|c|c|c|c|}
\hline PARAMETER MAME & LOG DATE & $\underset{\text { SAMPLE }}{\text { ID }}$ & $\begin{array}{l}\text { UNITS OF } \\
\text { MEASURE }\end{array}$ & PVI & $\begin{array}{l}\text { PARAMETER } \\
\text { VALUE FLAGS }\end{array}$ & $\begin{array}{l}\text { DETECTION } \\
\text { LIMIT }\end{array}$ & $\begin{array}{l}\text { PARAMETER } \\
\text { UHCERTAINTY }\end{array}$ \\
\hline SULFATE & $08 / 15 / 83$ & 0001 & MG/L & & 2590.00 & - & - \\
\hline TOTAL DISSOLVED SOLIDS & $08 / 15 / 83$ & 0001 & $M G / L$ & & 5380.00 & - & - \\
\hline URANIUM & $08 / 15 / 83$ & 0001 & MG/L & & 0.038 & - & - \\
\hline VANADIUM & $08 / 15 / 83$ & 0001 & MG/L & & 0.098 & - & - \\
\hline $21 \mathrm{kC}$ & $08 / 15 / 83$ & 0001 & $M G / L$ & & 0.14 & - & - \\
\hline
\end{tabular}

PARAMETER VALUE IMDICATOR (PVI): < - LESS THAN DETECTION LIMIT

SAMPLE ID COOES:

0001 - FILTERED SAMPLE (.45 MICRONS) 
GROUNDWATER QUALITY DATA BY LOCATION

SITE: AMBOI AMBROSIA LAKE

LOCATION: 0588

NORTH COORDINATE: $\quad 51630.0$ FT

EAST COORDINATE: $\quad 50720.0$ FT

$06 / 10 / 80$ TO $12 / 04 / 92$

REPORT DATE: 08/04/94

FORHATION OF COMPLETION: DAKOTA SAMDSTONE (KD)

HYDRAULIC FLON RELATIONSHIP: UNKHOWH (N)

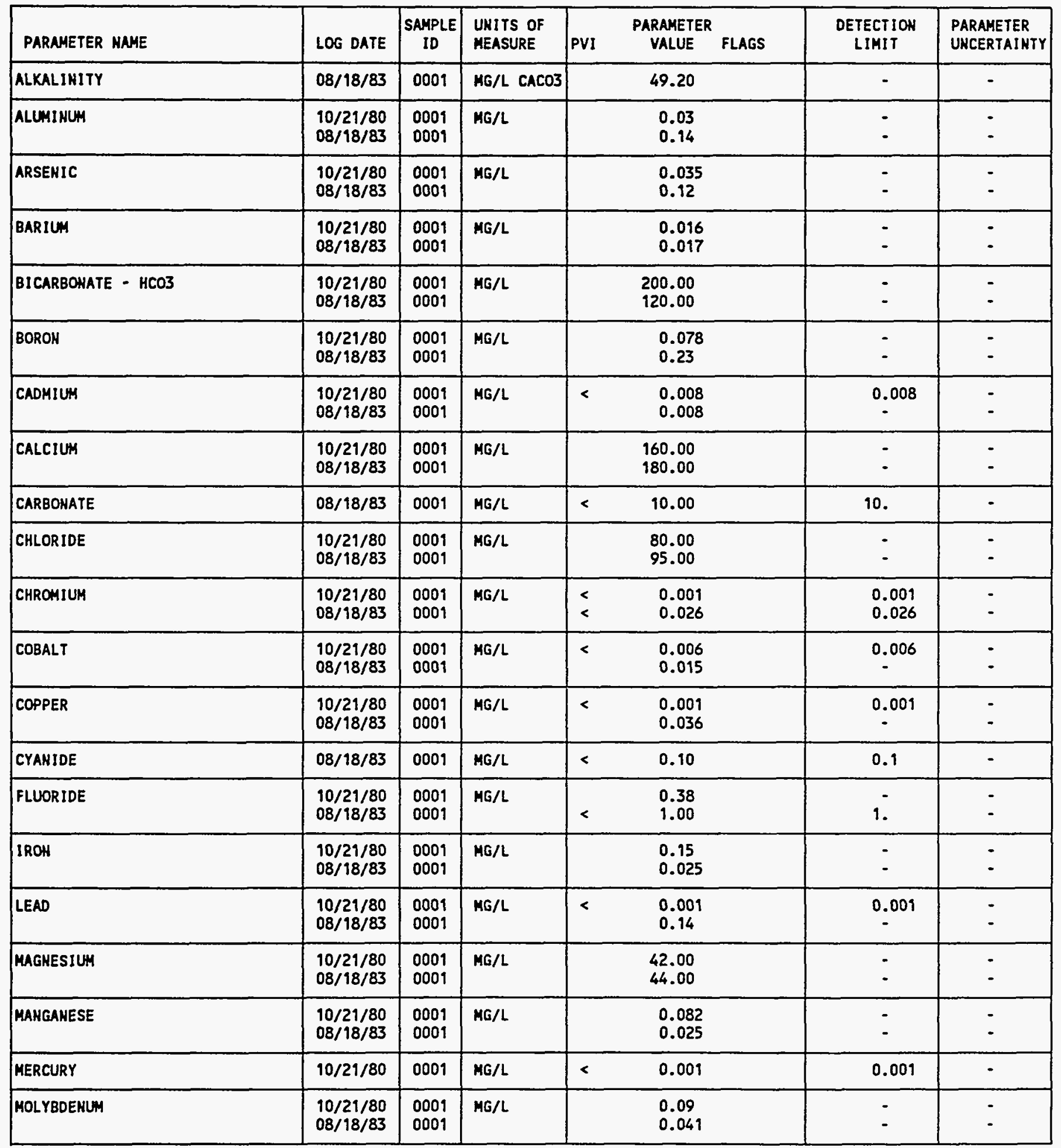

PARAMETER VALUE IMDICATOR (PVI): < - LESS THAN DETECTION LIMIT

SAMPLE ID COOES:

0001 - FILTERED SAMPLE (.45 MICRONS) 
GROUNDHATER QUALITY DATA BY LOCATION

SITE: AMBOI AMBROSIA LAKE

LOCATION: 0588

NORTH COORDINATE: $\quad 51630.0$ FT

EAST COORDIMATE: $\quad 50720.0$ FT

06/10/80 TO $12 / 04 / 92$

REPORT DATE: $08 / 04 / 94$

FORMATION OF COMPLETION: DAKOTA SAMDSTONE (KD)

HYDRAULIC FLON RELATIONSHIP: UNKNOWN (N)

\begin{tabular}{|c|c|c|c|c|c|c|c|}
\hline PARAMETER NAME & LOG DATE & $\underset{\text { ID }}{\text { SAMPLE }}$ & $\begin{array}{l}\text { UNITS OF } \\
\text { MEASURE }\end{array}$ & PVI & $\begin{array}{l}\text { PARAMETER } \\
\text { VALUE FLAGS }\end{array}$ & $\begin{array}{l}\text { DETECTIOH } \\
\text { LIMIT }\end{array}$ & $\begin{array}{l}\text { PARAMETER } \\
\text { UNCERTAINTY }\end{array}$ \\
\hline MICKEL & $\begin{array}{l}10 / 21 / 80 \\
08 / 18 / 83\end{array}$ & $\begin{array}{l}0001 \\
0001\end{array}$ & $M G / L$ & $<$ & $\begin{array}{l}0.003 \\
0.049\end{array}$ & 0.003 & $\dot{-}$ \\
\hline NITRATE & $\begin{array}{l}10 / 21 / 80 \\
08 / 18 / 83\end{array}$ & $\begin{array}{l}0001 \\
0001\end{array}$ & $M G / L$ & $<$ & $\begin{array}{l}1.30 \\
1.00\end{array}$ & 1. & - \\
\hline $\mathrm{PH}$ & $\begin{array}{l}10 / 21 / 80 \\
08 / 18 / 83\end{array}$ & $\begin{array}{l}0001 \\
0001\end{array}$ & SU & & $\begin{array}{l}7.80 \\
7.40\end{array}$ & - & $\dot{-}$ \\
\hline POTASSIUM & $\begin{array}{l}10 / 21 / 80 \\
08 / 18 / 83\end{array}$ & $\begin{array}{l}0001 \\
0001\end{array}$ & $M G / L$ & & $\begin{array}{l}12.00 \\
12.00\end{array}$ & - & - \\
\hline RADIUM-226 & $\begin{array}{l}10 / 21 / 80 \\
08 / 18 / 83\end{array}$ & $\begin{array}{l}0001 \\
0001\end{array}$ & $\mathrm{PCI} / \mathrm{L}$ & & $\begin{array}{r}15.48 \\
2.33\end{array}$ & - & $\dot{-}$ \\
\hline RADIUM-226 + RADIUM-228 & $08 / 18 / 83$ & 0001 & PCI/L & & 4.66 & - & - \\
\hline RADIUM-228 & $08 / 18 / 83$ & 0001 & $\mathrm{PCI} / \mathrm{L}$ & & 2.33 & - & - \\
\hline SELENIUM & $\begin{array}{l}10 / 21 / 80 \\
08 / 18 / 83\end{array}$ & $\begin{array}{l}0001 \\
0001\end{array}$ & $M G / L$ & & $\begin{array}{l}0.11 \\
0.12\end{array}$ & - & - \\
\hline SILVER & $\begin{array}{l}10 / 21 / 80 \\
08 / 18 / 83\end{array}$ & $\begin{array}{l}0001 \\
0001\end{array}$ & $M G / L$ & $<$ & $\begin{array}{l}0.002 \\
0.041\end{array}$ & 0.002 & - \\
\hline SOOIUM & $\begin{array}{l}10 / 21 / 80 \\
08 / 18 / 83\end{array}$ & $\begin{array}{l}0001 \\
0001\end{array}$ & MG/L & & $\begin{array}{l}300.00 \\
280.00\end{array}$ & $\dot{-}$ & - \\
\hline SPECIFIC CONDUCTANCE & $10 / 21 / 80$ & 0001 & UMHO/CM & & 2340.00 & - & - \\
\hline SULFATE & $\begin{array}{l}10 / 21 / 80 \\
08 / 18 / 83\end{array}$ & $\begin{array}{l}0001 \\
0001\end{array}$ & $M G / L$ & & $\begin{array}{l}970.00 \\
900.00\end{array}$ & - & - \\
\hline TOTAL DISSOLVED SOLIDS & $\begin{array}{l}10 / 21 / 80 \\
08 / 18 / 83\end{array}$ & $\begin{array}{l}0001 \\
0001\end{array}$ & $M G / L$ & & $\begin{array}{l}9800.00 \\
1560.00\end{array}$ & $\dot{-}$ & - \\
\hline TOTAL SUSPENDED SOLIDS & $10 / 21 / 80$ & 0001 & $M G / L$ & & 9830.00 & - & - \\
\hline URANIUM & $\begin{array}{l}10 / 21 / 80 \\
08 / 18 / 83\end{array}$ & $\begin{array}{l}0009 \\
0001\end{array}$ & $M G / L$ & & $\begin{array}{l}0.11 \\
0.022\end{array}$ & - & - \\
\hline VAKADIUY & $\begin{array}{l}10 / 21 / 80 \\
08 / 18 / 83\end{array}$ & $\begin{array}{l}0001 \\
0001\end{array}$ & MG/L & & $\begin{array}{l}0.006 \\
0.032\end{array}$ & - & - \\
\hline ZINC & $\begin{array}{l}10 / 21 / 80 \\
08 / 18 / 83\end{array}$ & $\begin{array}{l}0001 \\
0001\end{array}$ & $M G / L$ & & $\begin{array}{l}0.026 \\
0.03\end{array}$ & - & - \\
\hline
\end{tabular}

PARAMETER VALUE INDICATOR (PVI): < - LESS THAN DETECTION LIMIT 
GROUHDWATER QUALITY DATA BY LOCATION

SITE: AMBO1 AMBROSIA LAKE

LOCATION: 0589

NORTH COORDINATE: 50500.0 FT

EAST COORDIHATE: $\quad 54790.0$ FT

06/10/80 TO 12/04/92

REPORT DATE: 08/04/94

FORMATION OF COMPLETION: DAKOTA SANDSTONE (KD)

HYDRAULIC FLON RELATIOWSHIP: UHKHOWN (H)

\begin{tabular}{|c|c|c|c|c|c|c|c|}
\hline PARAMETER NAME & LOG DATE & $\begin{array}{c}\text { SAMPLE } \\
\text { ID }\end{array}$ & $\begin{array}{l}\text { UNITS OF } \\
\text { MEASURE }\end{array}$ & PV! & $\begin{array}{l}\text { PARAMETER } \\
\text { VALUE FLAGS }\end{array}$ & $\begin{array}{l}\text { DETECTION } \\
\text { LIMIT }\end{array}$ & $\begin{array}{l}\text { PARAMETER } \\
\text { UNCERTAINTY }\end{array}$ \\
\hline ALUMIKUM & $\begin{array}{l}10 / 20 / 80 \\
05 / 25 / 83\end{array}$ & $\begin{array}{l}0001 \\
0001\end{array}$ & $M G / L$ & & $\begin{array}{l}0.33 \\
0.51\end{array}$ & 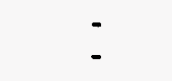 & - \\
\hline ARSEHIC & $\begin{array}{l}10 / 20 / 80 \\
05 / 25 / 83\end{array}$ & $\begin{array}{l}0001 \\
0001\end{array}$ & $M G / L$ & $<$ & $\begin{array}{l}0.015 \\
0.20\end{array}$ & 0.2 & - \\
\hline BARIUM & $\begin{array}{l}10 / 20 / 80 \\
05 / 25 / 83\end{array}$ & $\begin{array}{l}0001 \\
0001\end{array}$ & $M G / L$ & & $\begin{array}{l}0.092 \\
0.06\end{array}$ & - & $\dot{-}$ \\
\hline BICARBONATE - HCO3 & $05 / 25 / 83$ & 0001 & MG/L & $<$ & 10.00 & 10. & - \\
\hline BORON & $\begin{array}{l}10 / 20 / 80 \\
05 / 25 / 83\end{array}$ & $\begin{array}{l}0001 \\
0001\end{array}$ & $M G / L$ & & $\begin{array}{l}0.97 \\
0.54\end{array}$ & - & $\dot{-}$ \\
\hline CADMIUA & $\begin{array}{l}10 / 20 / 80 \\
05 / 25 / 83\end{array}$ & $\begin{array}{l}0001 \\
0001\end{array}$ & $M G / L$ & $<$ & $\begin{array}{l}0.008 \\
0.50\end{array}$ & $\begin{array}{l}0.008 \\
0.5\end{array}$ & $\overline{-}$ \\
\hline CALCIUM & $\begin{array}{l}10 / 20 / 80 \\
05 / 25 / 83\end{array}$ & $\begin{array}{l}0001 \\
0001\end{array}$ & $M G / L$ & & $\begin{array}{l}120.00 \\
110.00\end{array}$ & - & - \\
\hline CARBOHATE & $05 / 25 / 83$ & 0001 & $M G / L$ & $<$ & 10.00 & 10. & - \\
\hline CHLORIDE & $\begin{array}{l}10 / 20 / 80 \\
05 / 25 / 83\end{array}$ & $\begin{array}{l}0001 \\
0001\end{array}$ & MG/L & & $\begin{array}{l}146.00 \\
210.00\end{array}$ & $\dot{-}$ & $\dot{-}$ \\
\hline CHROMIUM & $\begin{array}{l}10 / 20 / 80 \\
05 / 25 / 83\end{array}$ & $\begin{array}{l}0001 \\
0001\end{array}$ & $M G / L$ & $<$ & $\begin{array}{l}0.001 \\
0.50\end{array}$ & $\begin{array}{l}0.001 \\
0.5\end{array}$ & $\dot{-}$ \\
\hline COBALT & $\begin{array}{l}10 / 20 / 80 \\
05 / 25 / 83\end{array}$ & $\begin{array}{l}0001 \\
0001\end{array}$ & MG/L & $<$ & $\begin{array}{l}0.006 \\
0.50\end{array}$ & $\begin{array}{l}0.006 \\
0.5\end{array}$ & $\dot{-}$ \\
\hline COPPER & $\begin{array}{l}10 / 20 / 80 \\
05 / 25 / 83\end{array}$ & $\begin{array}{l}0001 \\
0001\end{array}$ & $M G / L$ & $<$ & $\begin{array}{l}0.005 \\
0.50\end{array}$ & 0.5 & - \\
\hline CYANIDE & $05 / 25 / 83$ & 0001 & $M G / L$ & $<$ & 1.00 & 1. & - \\
\hline FLUORIDE & $\begin{array}{l}10 / 20 / 80 \\
05 / 25 / 83\end{array}$ & $\begin{array}{l}0001 \\
0001\end{array}$ & $M G / L$ & & $\begin{array}{l}0.27 \\
1.00\end{array}$ & $\overline{-}$ & $\dot{-}$ \\
\hline IROH & $\begin{array}{l}10 / 20 / 80 \\
05 / 25 / 83\end{array}$ & $\begin{array}{l}0001 \\
0001\end{array}$ & $M G / L$ & $<$ & $\begin{array}{l}0.093 \\
0.25\end{array}$ & 0.25 & - \\
\hline LEAD & $\begin{array}{l}10 / 20 / 80 \\
05 / 25 / 83\end{array}$ & $\begin{array}{l}0001 \\
0001\end{array}$ & $M G / L$ & $<$ & $\begin{array}{l}0.001 \\
0.027\end{array}$ & 0.001 & - \\
\hline MAGMESIUM & $\begin{array}{l}10 / 20 / 80 \\
05 / 25 / 83\end{array}$ & $\begin{array}{l}0001 \\
0001\end{array}$ & $M G / L$ & $<$ & $\begin{array}{l}0.11 \\
0.50\end{array}$ & 0.5 & - \\
\hline MAMGAHESE & $\begin{array}{l}10 / 20 / 80 \\
05 / 25 / 83\end{array}$ & $\begin{array}{l}0001 \\
0001\end{array}$ & MG/L & $<$ & $\begin{array}{l}0.002 \\
0.05\end{array}$ & $\begin{array}{l}0.002 \\
0.05\end{array}$ & $\dot{-}$ \\
\hline MERCURY & $10 / 20 / 80$ & 0001 & $M G / L$ & $<$ & 0.001 & 0.001 & - \\
\hline MOLYEDENUA & $\begin{array}{l}10 / 20 / 80 \\
05 / 25 / 83\end{array}$ & $\begin{array}{l}0001 \\
0001\end{array}$ & $M G / L$ & $<$ & $\begin{array}{l}0.27 \\
0.011\end{array}$ & 0.011 & : \\
\hline HICKEL & $\begin{array}{l}10 / 20 / 80 \\
05 / 25 / 83\end{array}$ & $\begin{array}{l}0001 \\
0001\end{array}$ & $M G / L$ & $<$ & $\begin{array}{l}0.041 \\
0.01\end{array}$ & 0.01 & - \\
\hline
\end{tabular}

PARAMETER VALUE IHDICATOR (PVI): < - LESS THAN DETECTION LIMIT

SAMPLE ID COOES:

0001 - FILTERED SAMPLE (.45 MICRONS) 
GROUNDHATER QUALITY DATA BY LOCATIOH

SITE: AMBOI AMBROSIA LAKE

LOCATIOH: 0589

HORTH COORDINATE: $\quad 50500.0$ FT

EAST COORDINATE: $\quad 54790.0$ FT

06/10/80 TO 12/04/92

REPORT DATE: 08/04/94

FORMATION OF COMPLETION: DAKOTA SANDSTOHE (KD)

HYDRAULIC FLOW RELATIONSHIP: UNKNOWN (N)

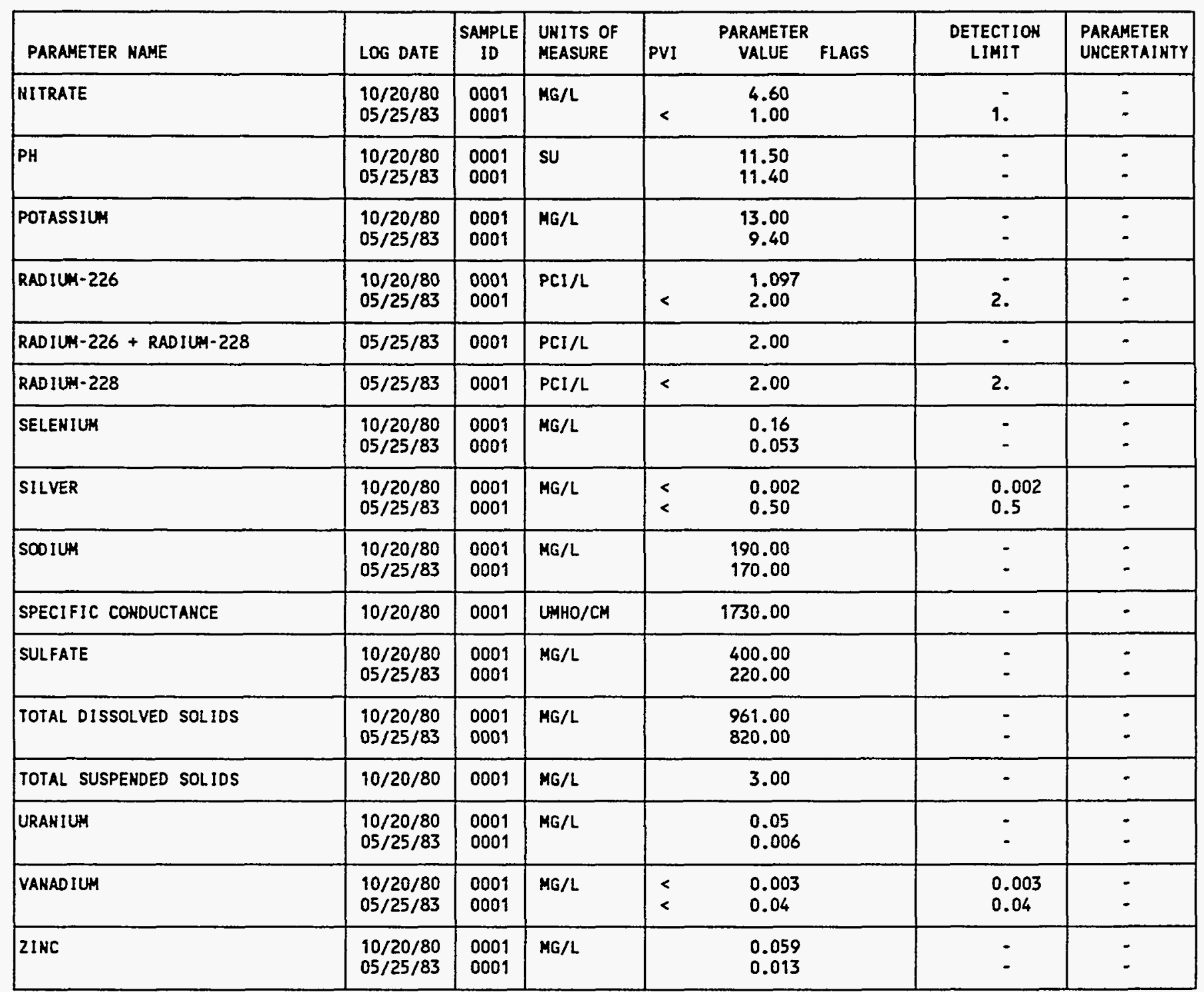

PARAMETER VALUE INOICATOR (PVI): > - LESS THAN DETECTION LIMIT

SAMPLE ID COOES:

0001 - FILTERED SAMPLE (.45 MICRONS) 
GROUNDWATER QUALITY DATA BY LOCATION

SITE: AMBOI AMBROSIA LAKE

LOCATION : 0590

MORTH COOROINATE: 51910.0 FT

EAST COORDINATE: $\quad 54880.0$ FT

06/10/80 TO 12/04/92

REPORT DATE: 08/04/94

FORMATION OF COMPLETION: DAKOTA SAMDSTONE (KD)

HYDRAULIC FLON RELATIONSHIP: UNKNOWN (N)

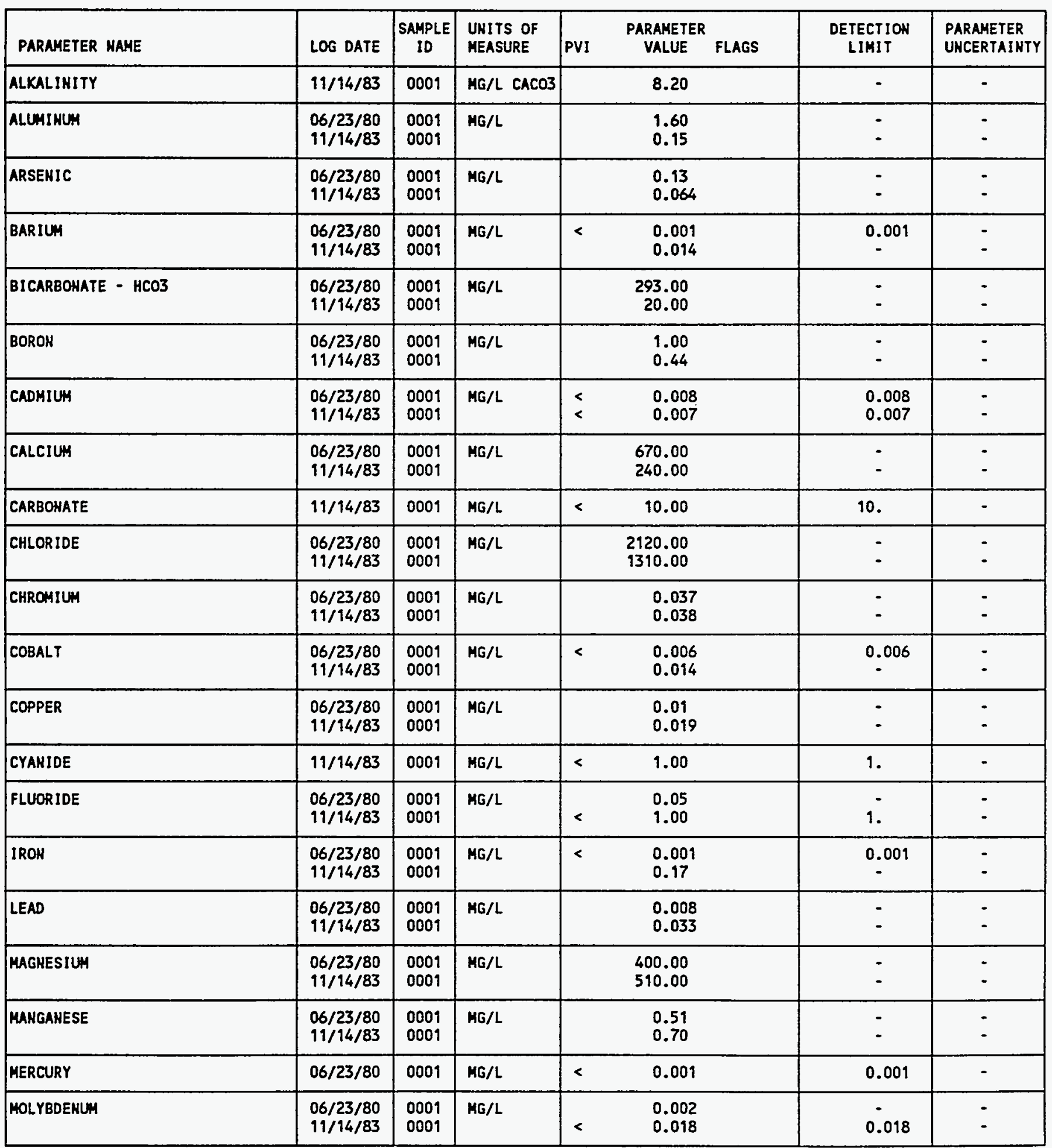

PARAMETER VALUE IMDICATOR (PVI): < - LESS THAN DETECTION LIMIT

SAMPLE ID COOES:

0001 - FILTERED SAMPLE (.45 MICRONS) 
GROUNDHATER QUALITY DATA BY LOCATION

SITE: AMBO1 AMBROSIA LAKE

LOCATION: 0590

MORTH COORDINATE: 51910.0 FT

EAST COORDINATE: $\quad 54880.0$ FT

06/10/80 TO $12 / 04 / 92$

REPORT OATE: $08 / 04 / 94$

FORMATION OF COMPLETION: DAKOTA SANDSTONE (KD)

HYDRAULIC FLOW RELATIONSHIP: UMKHOWN (N)

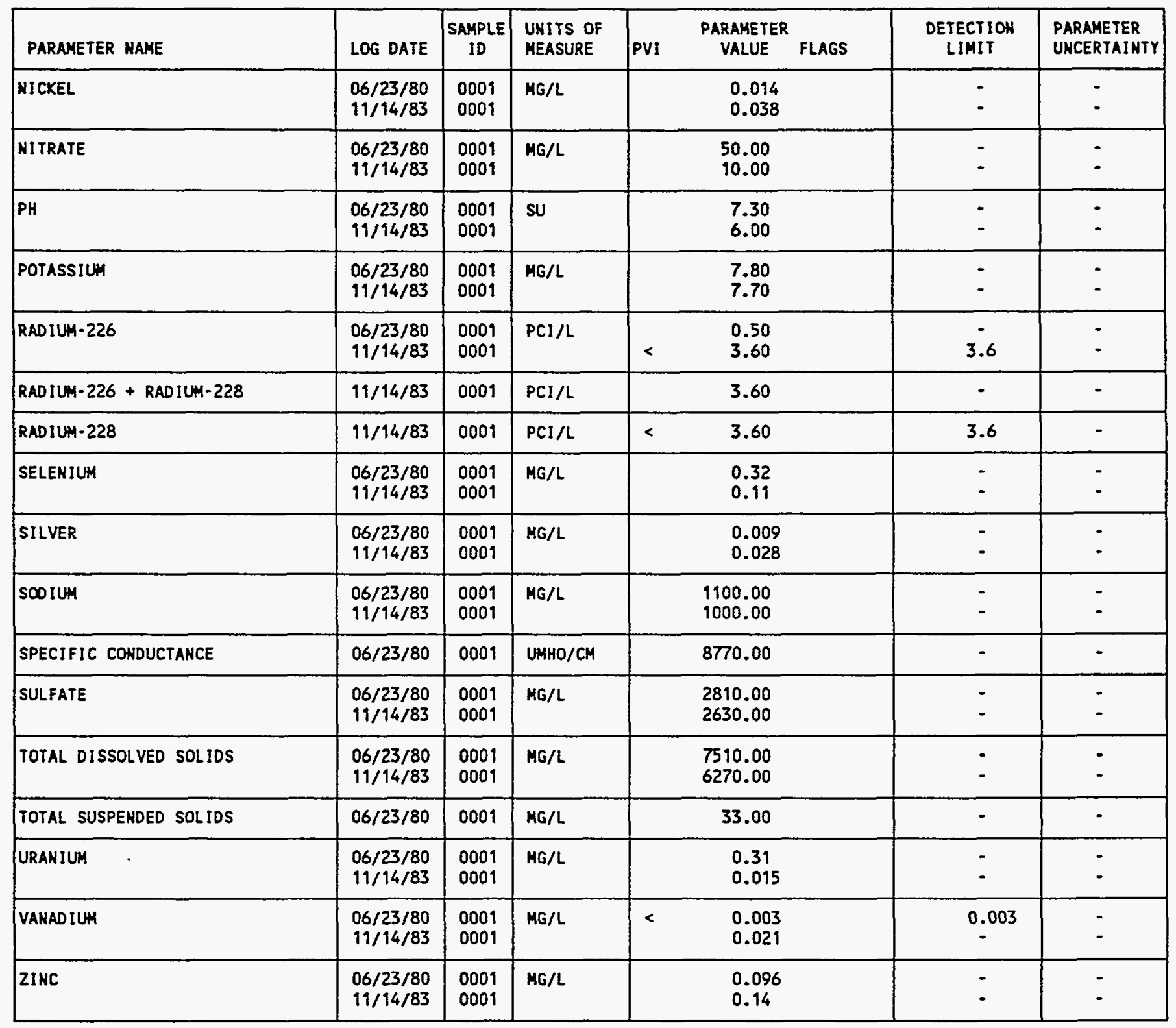

PARAMETER VALUE INDICATOR (PVI): < - LESS THAN DETECTION LIMIT

SAMPLE ID COOES:

0001 - FILTERED SAMPLE (.45 MICRONS) 
GROUHDWATER QUALITY DATA BY LOCATION

SITE: AMBOI AMBROSIA LAKE

LOCATION: 0591

MORTH COORDIHATE: 50580.0 FT

EAST COORDINATE: 55640.0 FT

06/10/80 TO $12 / 04 / 92$

REPORT DATE: 08/04/94

FORMATIOH OF COMPLETION: DAKOTA SAKDSTOHE (KD)

HYDRAULIC FLON RELATIOHSHIP: UHKHOWN (N)

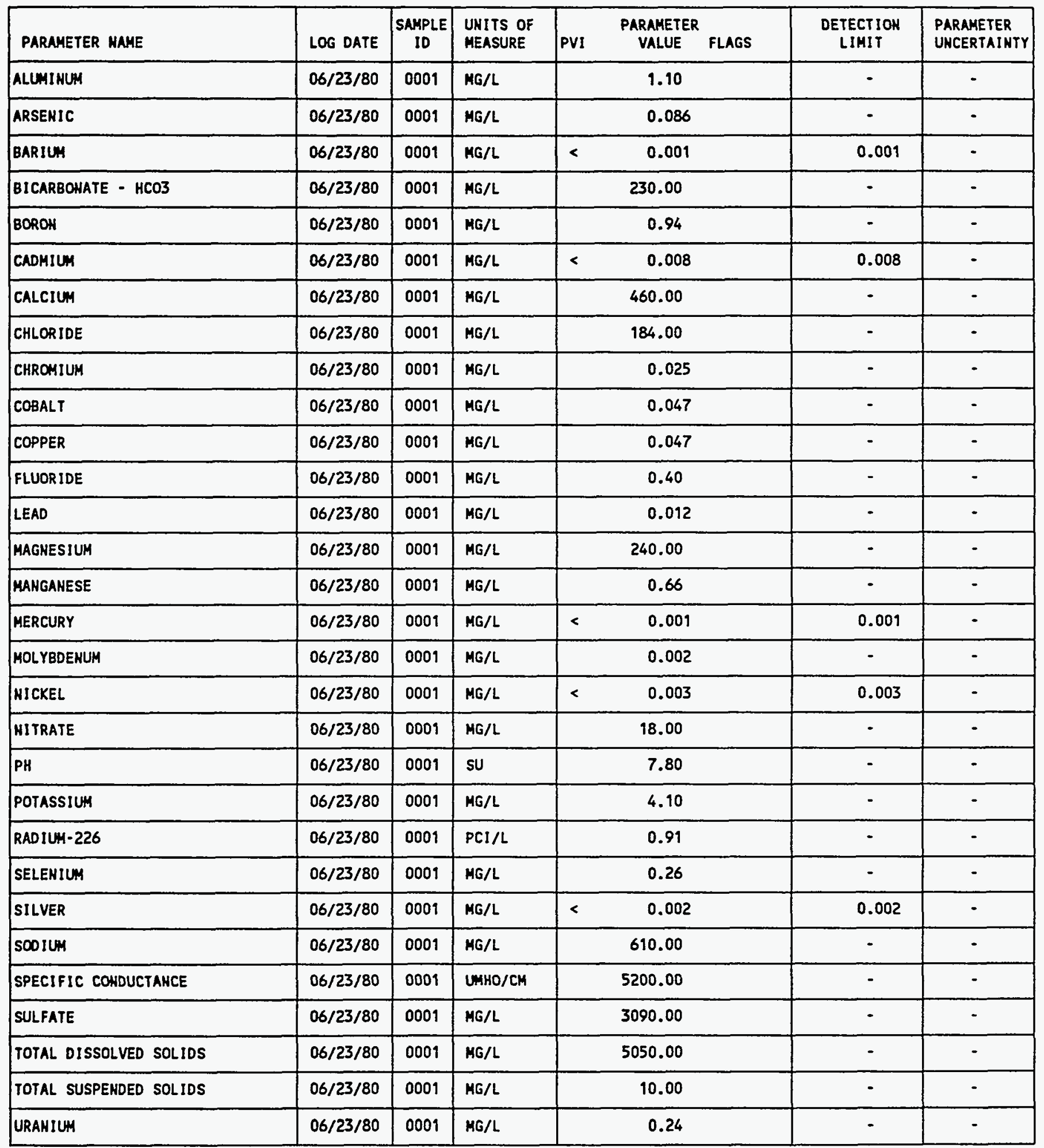

PARAMETER VALUE INDICATOR (PVI): \& - LESS THAN DETECTION LIMIT

SAMPLE ID COOES:

0001 - FILTERED SAMPLE (.45 MICRONS) 


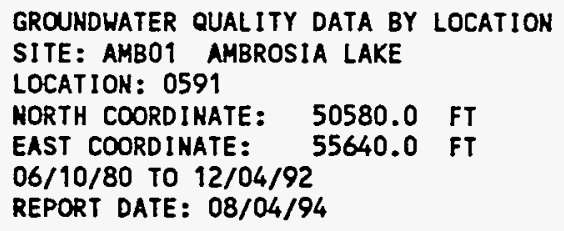

FORMATION OF COMPLETION: DAKOTA SANDSTONE (KD)

HYDRAULIC FLOU RELATIONSHIP: UNKNOWN (N)

\begin{tabular}{|l|c|c|l|l|c|c|c|}
\hline PARAMETER NAME & LOG DATE & $\begin{array}{l}\text { SAMPLE } \\
\text { ID }\end{array}$ & $\begin{array}{l}\text { UNITS OF } \\
\text { MEASURE }\end{array}$ & PVI & $\begin{array}{l}\text { PARAMETER } \\
\text { VALUE } \text { FLAGS }\end{array}$ & $\begin{array}{c}\text { DETECTION } \\
\text { LIMIT }\end{array}$ & $\begin{array}{l}\text { PARAMETER } \\
\text { UNCERTAIMTY }\end{array}$ \\
\hline VANADIUM & $06 / 23 / 80$ & 0001 & MG/L & $<$ & 0.003 & 0.003 & - \\
\hline ZINC & $06 / 23 / 80$ & 0001 & MG/L & & 0.063 & - & - \\
\hline
\end{tabular}

PARAMETER VALUE INDICATOR (PVI):

< - LESS THAN DETECTION LIMIT

SAMPLE IO COOES:

0001 - FILTERED SAMPLE (.45 MICRONS) 
GROUNDHATER QUALITY DATA BY LOCATION

SITE: AMBO1 AMBROSIA LAKE

LOCATIOH: 0592

HORTH COORDIHATE: $\quad 48150.0$ FT

EAST COORDINATE: $\quad 56680.0$ FT

06/10/80 TO 12/04/92

REPORT OATE: 08/04/94

FORMATION OF COMPLETION: DAKOTA SANDSTOHE (KD)

HYDRAULIC FLON RELATIONSHIP: UNKNOWN (N)

\begin{tabular}{|c|c|c|c|c|c|c|c|}
\hline PARAMETER MAME & LOG DATE & $\underset{10}{\text { SAMPLE }}$ & $\begin{array}{l}\text { UHITS OF } \\
\text { MEASURE }\end{array}$ & PVI & $\begin{array}{l}\text { PARAMETER } \\
\text { VALUE FLAGS }\end{array}$ & $\begin{array}{l}\text { DETECTION } \\
\text { LIMIT }\end{array}$ & $\begin{array}{l}\text { PARAMETER } \\
\text { UNCERTAINTY }\end{array}$ \\
\hline ALUMIHUM & $\begin{array}{l}06 / 24 / 80 \\
05 / 24 / 83\end{array}$ & $\begin{array}{l}0001 \\
0001\end{array}$ & MG/L & $<$ & $\begin{array}{l}0.51 \\
0.20\end{array}$ & 0.2 & - \\
\hline ARSENIC & $\begin{array}{l}06 / 24 / 80 \\
05 / 24 / 83\end{array}$ & $\begin{array}{l}0001 \\
0001\end{array}$ & $M G / L$ & $<$ & $\begin{array}{l}0.04 \\
0.10\end{array}$ & 0.1 & $\dot{-}$ \\
\hline BARIUM & $\begin{array}{l}06 / 24 / 80 \\
05 / 24 / 83\end{array}$ & $\begin{array}{l}0001 \\
0001\end{array}$ & MG/L & $<$ & $\begin{array}{l}0.001 \\
0.025\end{array}$ & 0.001 & - \\
\hline BICARBOHATE - HCO3 & $\begin{array}{l}06 / 24 / 80 \\
05 / 24 / 83\end{array}$ & $\begin{array}{l}0001 \\
0001\end{array}$ & $\mathrm{MG} / \mathrm{L}$ & $<$ & $\begin{array}{r}147.00 \\
10.00\end{array}$ & 10. & $\dot{-}$ \\
\hline BOROH & $\begin{array}{l}06 / 24 / 80 \\
05 / 24 / 83\end{array}$ & $\begin{array}{l}0001 \\
0001\end{array}$ & MG/L & $<$ & $\begin{array}{l}1.00 \\
0.05\end{array}$ & 0.05 & - \\
\hline CADMIUM & $\begin{array}{l}06 / 24 / 80 \\
05 / 24 / 83\end{array}$ & $\begin{array}{l}0001 \\
0001\end{array}$ & MG/L & $<$ & $\begin{array}{l}0.008 \\
0.01\end{array}$ & $\begin{array}{l}0.008 \\
0.01\end{array}$ & - \\
\hline CALCIUM & $\begin{array}{l}06 / 24 / 80 \\
05 / 24 / 83\end{array}$ & $\begin{array}{l}0001 \\
0001\end{array}$ & ME/L & & $\begin{array}{l}420.00 \\
140.00\end{array}$ & $\dot{-}$ & - \\
\hline CARBOHATE & $05 / 24 / 83$ & 0001 & $M G / L$ & $<$ & 10.00 & 10. & - \\
\hline CHLORIDE & $\begin{array}{l}06 / 24 / 80 \\
05 / 24 / 83\end{array}$ & $\begin{array}{l}0001 \\
0001\end{array}$ & $M G / L$ & & $\begin{array}{l}44.00 \\
49.00\end{array}$ & $\dot{-}$ & $\dot{-}$ \\
\hline CHROMIUH & $\begin{array}{l}06 / 24 / 80 \\
05 / 24 / 83\end{array}$ & $\begin{array}{l}0001 \\
0001\end{array}$ & MG/L & $<$ & $\begin{array}{l}0.001 \\
0.032\end{array}$ & 0.001 & - \\
\hline COBALT & $\begin{array}{l}06 / 24 / 80 \\
05 / 24 / 83\end{array}$ & $\begin{array}{l}0001 \\
0001\end{array}$ & MG/L & $<$ & $\begin{array}{l}0.006 \\
0.04\end{array}$ & $\begin{array}{l}0.006 \\
0.04\end{array}$ & - \\
\hline COPPER & $\begin{array}{l}06 / 24 / 80 \\
05 / 24 / 83\end{array}$ & $\begin{array}{l}0001 \\
0001\end{array}$ & $M G / L$ & $<$ & $\begin{array}{l}0.001 \\
0.03\end{array}$ & $\begin{array}{l}0.001 \\
0.03\end{array}$ & $\dot{-}$ \\
\hline CYAHIDE & $05 / 24 / 83$ & 0001 & $M G / L$ & & 6.00 & - & - \\
\hline FLUORIDE & $\begin{array}{l}06 / 24 / 80 \\
05 / 24 / 83\end{array}$ & $\begin{array}{l}0001 \\
0001\end{array}$ & MG/L & $<$ & $\begin{array}{l}0.56 \\
1.00\end{array}$ & 1. & - \\
\hline IRON & $\begin{array}{l}06 / 24 / 80 \\
05 / 24 / 83\end{array}$ & $\begin{array}{l}0001 \\
0001\end{array}$ & MG/L & $<$ & $\begin{array}{l}0.008 \\
0.05\end{array}$ & $\begin{array}{l}0.008 \\
0.05\end{array}$ & - \\
\hline LEAD & $\begin{array}{l}06 / 24 / 80 \\
05 / 24 / 83\end{array}$ & $\begin{array}{l}0001 \\
0001\end{array}$ & MG/L & & $\begin{array}{l}0.005 \\
0.024\end{array}$ & : & - \\
\hline MAGNESIUH & $\begin{array}{l}06 / 24 / 80 \\
05 / 24 / 83\end{array}$ & $\begin{array}{l}0001 \\
0001\end{array}$ & MG/L & & $\begin{array}{r}140.00 \\
52.00\end{array}$ & - & : \\
\hline MAKGAKESE & $\begin{array}{l}06 / 24 / 80 \\
05 / 24 / 83\end{array}$ & $\begin{array}{l}0001 \\
0001\end{array}$ & $M G / L$ & $<$ & $\begin{array}{l}0.12 \\
0.02\end{array}$ & 0.02 & : \\
\hline MERCURY & $06 / 24 / 80$ & 0001 & $M G / L$ & $<$ & 0.001 & 0.001 & - \\
\hline MOLYBDENUH & $\begin{array}{l}06 / 24 / 80 \\
05 / 24 / 83\end{array}$ & $\begin{array}{l}0001 \\
0001\end{array}$ & $M G / L$ & $<$ & $\begin{array}{l}0.018 \\
0.02\end{array}$ & 0.02 & - \\
\hline HICKEL & $06 / 24 / 80$ & 0001 & $M G / L$ & $<$ & 0.003 & 0.003 & - \\
\hline
\end{tabular}

PARAMETER VALUE INDICATOR (PVI): < - LESS THAM DETECTION LIMIT

SAMPLE ID COOES:

0001 - FILTERED SAMPLE (.45 MICRONS) 
GROUHDWATER QUALITY DATA BY LOCATION

SITE: AMBO1 AMBROSIA LAKE

LOCATION: 0592

NORTH COORDINATE: $\quad 48150.0$ FT

EAST COORDINATE: $\quad 56680.0$ FT

06/10/80 TO $12 / 04 / 92$

REPORT DATE: 08/04/94

FORMATION OF COMPLETIOH: DAKOTA SANDSTOHE (KD)

HYORAULIC FLOU RELATIOHSHIP: UHKMOWN (N)

\begin{tabular}{|c|c|c|c|c|c|c|c|}
\hline PARAMETER MAKE & LOG DATE & $\underset{\text { SAMPLE }}{\text { ID }}$ & $\begin{array}{l}\text { UNITS OF } \\
\text { MEASURE }\end{array}$ & PVI & $\begin{array}{l}\text { PARAMETER } \\
\text { VALUE FLAGS }\end{array}$ & $\begin{array}{l}\text { DETECTIOH } \\
\text { LIMIT }\end{array}$ & $\begin{array}{l}\text { PARAMETER } \\
\text { UNCERTAINTY }\end{array}$ \\
\hline HICKEL & $05 / 24 / 83$ & 0001 & $M G / L$ & $<$ & 0.08 & 0.08 & - \\
\hline NITRATE & $\begin{array}{l}06 / 24 / 80 \\
05 / 24 / 83\end{array}$ & $\begin{array}{l}0001 \\
0001\end{array}$ & MG/L & & $\begin{array}{r}42.00 \\
1.00\end{array}$ & i. & $\dot{-}$ \\
\hline PH & $\begin{array}{l}06 / 24 / 80 \\
05 / 24 / 83\end{array}$ & $\begin{array}{l}0001 \\
0001\end{array}$ & su & & $\begin{array}{l}7.90 \\
9.00\end{array}$ & - & - \\
\hline POTASSIUA & $\begin{array}{l}06 / 24 / 80 \\
05 / 24 / 83\end{array}$ & $\begin{array}{l}0001 \\
0001\end{array}$ & $M G / L$ & & $\begin{array}{l}4.70 \\
9.20\end{array}$ & - & $\dot{-}$ \\
\hline RADIUH-226 & $\begin{array}{l}06 / 24 / 80 \\
05 / 24 / 83\end{array}$ & $\begin{array}{l}0001 \\
0001\end{array}$ & $\mathrm{PCI} / \mathrm{L}$ & & $\begin{array}{l}1.42 \\
3.16\end{array}$ & - & - \\
\hline RADIUA-226 + RADIUA4-228 & $05 / 24 / 83$ & 0001 & PCI/L & & 6.32 & - & - \\
\hline RADIUN-228 & $05 / 24 / 83$ & 0001 & $\mathrm{PCI} / \mathrm{L}$ & & 3.16 & - & - \\
\hline SELENIUH & $\begin{array}{l}06 / 24 / 80 \\
05 / 24 / 83\end{array}$ & $\begin{array}{l}0001 \\
0001\end{array}$ & MG/L & & $\begin{array}{l}0.26 \\
0.052\end{array}$ & - & - \\
\hline SILVER & $\begin{array}{l}06 / 24 / 80 \\
05 / 24 / 83\end{array}$ & $\begin{array}{l}0001 \\
0001\end{array}$ & $M G / L$ & $<$ & $\begin{array}{l}0.002 \\
0.03\end{array}$ & $\begin{array}{l}0.002 \\
0.03\end{array}$ & - \\
\hline SOOIUM & $\begin{array}{l}06 / 24 / 80 \\
05 / 24 / 83\end{array}$ & $\begin{array}{l}0001 \\
0001\end{array}$ & $M G / L$ & & $\begin{array}{l}290.00 \\
130.00\end{array}$ & - & - \\
\hline SPECIFIC CONDUCTANCE & $06 / 24 / 80$ & 0001 & UнНО/CM & & 3570.00 & - & - \\
\hline SULFATE & $\begin{array}{l}06 / 24 / 80 \\
05 / 24 / 83\end{array}$ & $\begin{array}{l}0001 \\
0001\end{array}$ & $M G / L$ & & $\begin{array}{r}2040.00 \\
760.00\end{array}$ & - & - \\
\hline TOTAL DISSOLVED SOLIDS & $\begin{array}{l}06 / 24 / 80 \\
05 / 24 / 83\end{array}$ & $\begin{array}{l}0001 \\
0001\end{array}$ & MG/L & & $\begin{array}{l}3440.00 \\
1220.00\end{array}$ & - & - \\
\hline TOTAL SUSPENDED SOLIDS & $06 / 24 / 80$ & 0001 & $M G / L$ & & 70.00 & - & - \\
\hline URANIUY & $\begin{array}{l}06 / 24 / 80 \\
05 / 24 / 83\end{array}$ & $\begin{array}{l}0001 \\
0001\end{array}$ & HG/L & & $\begin{array}{l}0.085 \\
0.008\end{array}$ & - & $\dot{-}$ \\
\hline VAKADIUM & $06 / 24 / 80$ & 0001 & $M G / L$ & $<$ & 0.003 & 0.003 & - \\
\hline ZINC & $\begin{array}{l}06 / 24 / 80 \\
05 / 24 / 83\end{array}$ & $\begin{array}{l}0001 \\
0001\end{array}$ & $M G / L$ & & $\begin{array}{l}0.059 \\
0.04\end{array}$ & - & $\dot{-}$ \\
\hline
\end{tabular}

PARAMETER VALUE INDICATOR (PVI):

< - LESS THAN DETECTION LIMIT

SAMPLE ID COOES:

0001 - FILTERED SAMPLE (.45 MICRONS) 
GROUHDHATER QUALITY DATA BY LOCATION

SITE: AMB01 AMBROSIA LAKE

LOCATION: 0593

HORTH COORDINATE: $\quad 52240.0$ FT

EAST COORDINATE: $\quad 45590.0$ FT

06/10/80 TO 12/04/92

REPORT DATE: 08/04/94

FORMATION OF COMPLETION: DAKOTA SAKDSTOHE (KD)

HYDRAULIC FLON RELATIOHSHIP: UNKHOWN (K)

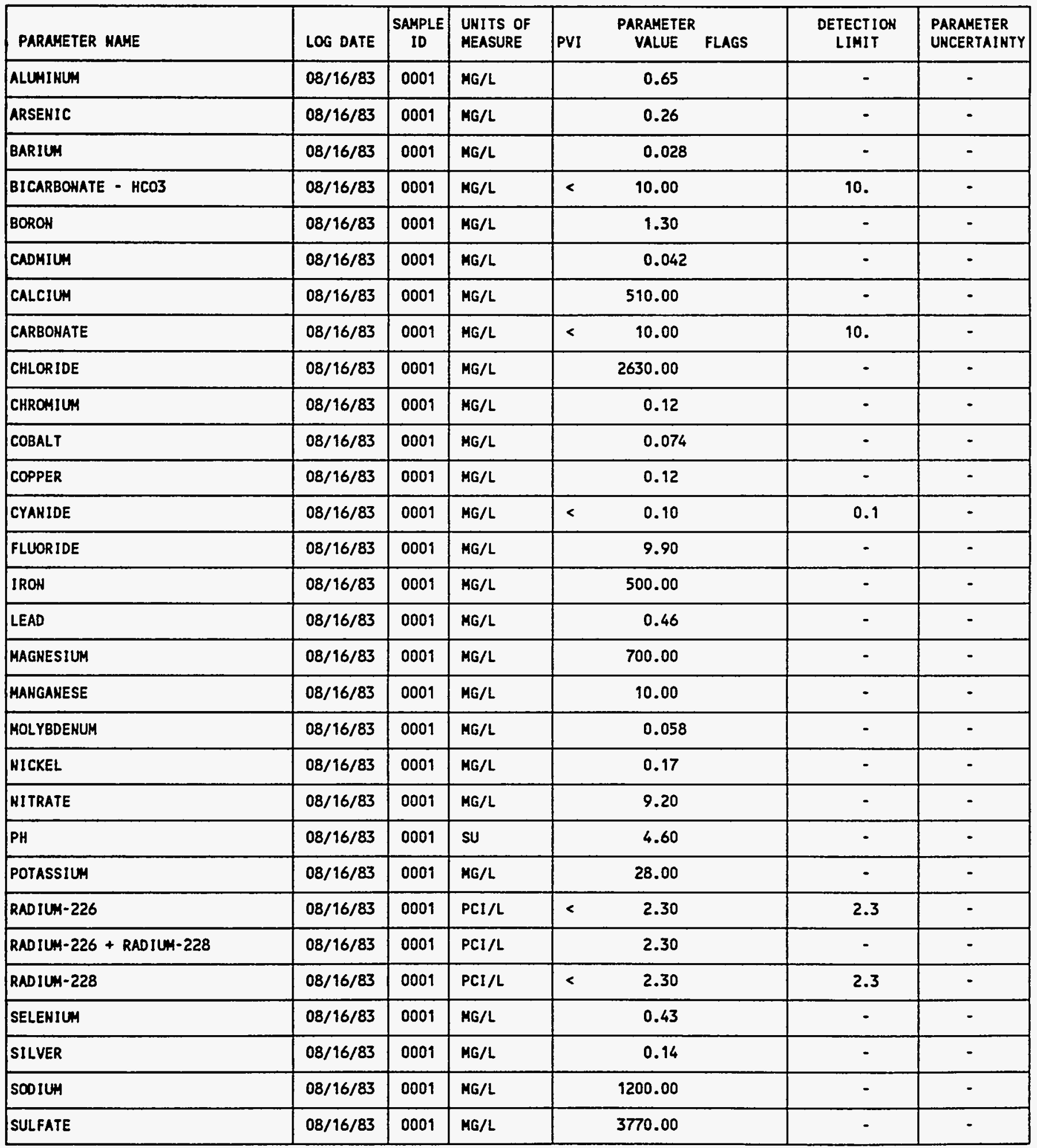

PARAMETER VALUE IMDICATOR (PVI): < - LESS THAN DETECTION LIMIT

SAMPLE ID COOES:

OOO1 - FILTERED SAMPLE (.45 MICRONS) 
GROUNDWATER QUALITY DATA BY LOCATION

SITE: AMBOI AMBROSIA LAKE

LOCATION: 0593

NORTH COORDINATE: $\quad 52240.0$ FT

EAST COORDINATE: $\quad 45590.0$ FT

06/10/80 TO $12 / 04 / 92$

REPORT DATE: $08 / 04 / 94$

FORMATIOH OF COMPLETION: DAKOTA SANDSTONE (KD)

HYDRAULIC FLON RELATIONSHIP: UNKNOWN (N)

\begin{tabular}{|c|c|c|c|c|c|c|c|}
\hline PARAMETER MAME & LOG DATE & $\begin{array}{c}\text { SAMPLE } \\
\text { ID }\end{array}$ & $\begin{array}{l}\text { UNITS OF } \\
\text { MEASURE }\end{array}$ & PVI & $\begin{array}{l}\text { PARAMETER } \\
\text { VALUE FLAGS }\end{array}$ & $\begin{array}{l}\text { DETECTION } \\
\text { LIMIT }\end{array}$ & $\begin{array}{l}\text { PARAMETER } \\
\text { UNCERTAINTY }\end{array}$ \\
\hline TOTAL DISSOLVED SOLIOS & $08 / 16 / 83$ & 0001 & ME/L & & 9320.00 & - & - \\
\hline URAKIUY & $08 / 16 / 83$ & 0001 & $M G / L$ & & 0.003 & - & - \\
\hline VANADIUM & $08 / 16 / 83$ & 0001 & MG/L & & 0.12 & - & - \\
\hline ZINC & $08 / 16 / 83$ & 0001 & $M G / L$ & & 0.25 & - & - \\
\hline
\end{tabular}

PARAMETER VALUE IMDICATOR (PVI): < - LESS THAN DETECTION LIMIT

SAMPLE ID COOES:

0001 - FILTERED SAMPLE (.45 MICRONS) 
GROUNDWATER QUALITY DATA BY LOCATION

SITE: AMBO1 AMBROSIA LAKE

LOCATION: 0594

NORTH COORDINATE: 31650.0 FT

EAST COORDIHATE: 72440.0 FT

06/10/80 TO 12/04/92

REPORT DATE: $08 / 04 / 94$

FORMATION OF COMPLETION: ALLUVIUM (AL)

HYORAULIC FLON RELATIONSHIP: UNKHOWN (N)

\begin{tabular}{|c|c|c|c|c|c|c|}
\hline PARAMETER MAME & LOG DATE & $\begin{array}{c}\text { SAMPLE } \\
\text { ID }\end{array}$ & $\begin{array}{l}\text { UHITS OF } \\
\text { MEASURE }\end{array}$ & \begin{tabular}{l}
\multicolumn{3}{c}{ PARAMETER } \\
PVI \\
VALUE FLAGS
\end{tabular} & $\begin{array}{l}\text { DETECTION } \\
\text { LIMIT }\end{array}$ & $\begin{array}{l}\text { PARAMETER } \\
\text { UNCERTAINTY }\end{array}$ \\
\hline ALKALIHITY & $\begin{array}{l}06 / 10 / 80 \\
07 / 01 / 81\end{array}$ & $\begin{array}{l}0001 \\
0001\end{array}$ & MG/L CACO3 & $\begin{array}{r}101.00 \\
99.20\end{array}$ & $\dot{-}$ & $\dot{-}$ \\
\hline ARSENIC & $\begin{array}{l}06 / 10 / 80 \\
07 / 01 / 81\end{array}$ & $\begin{array}{l}0001 \\
0002\end{array}$ & MG/L & $\begin{array}{l}0.005 \\
0.005\end{array}$ & - & - \\
\hline BICARBOHATE - KCO3 & $\begin{array}{l}06 / 10 / 80 \\
07 / 01 / 81\end{array}$ & $\begin{array}{l}0001 \\
0002\end{array}$ & $M G / L$ & $\begin{array}{l}246.00 \\
242.00\end{array}$ & - & - \\
\hline CADMIUA & $\begin{array}{l}06 / 10 / 80 \\
07 / 01 / 81\end{array}$ & $\begin{array}{l}0001 \\
0002\end{array}$ & MG/L & $\begin{array}{l}0.001 \\
0.001\end{array}$ & $\dot{-}$ & $\dot{-}$ \\
\hline LEAD & $\begin{array}{l}06 / 10 / 80 \\
07 / 01 / 81\end{array}$ & $\begin{array}{l}0001 \\
0002\end{array}$ & $M G / L$ & $\begin{array}{l}0.005 \\
0.005\end{array}$ & - & - \\
\hline MAGNESIUM & $\begin{array}{l}06 / 10 / 80 \\
07 / 01 / 81\end{array}$ & $\begin{array}{l}0001 \\
0002\end{array}$ & $M G / L$ & $\begin{array}{r}10.70 \\
7.60\end{array}$ & - & $\dot{-}$ \\
\hline MOLYBDENUM & $\begin{array}{l}06 / 10 / 80 \\
07 / 01 / 81\end{array}$ & $\begin{array}{l}0001 \\
0002\end{array}$ & MG/L & $\begin{array}{l}0.159 \\
0.155\end{array}$ & $\dot{-}$ & $\dot{-}$ \\
\hline PH & $\begin{array}{l}06 / 10 / 80 \\
07 / 01 / 81\end{array}$ & $\begin{array}{l}0001 \\
0002\end{array}$ & SU & $\begin{array}{l}7.45 \\
7.40\end{array}$ & - & - \\
\hline SULFATE & $\begin{array}{l}06 / 10 / 80 \\
07 / 01 / 81\end{array}$ & $\begin{array}{l}0001 \\
0002\end{array}$ & MG/L & $\begin{array}{l}259.00 \\
265.00\end{array}$ & - & - \\
\hline TOTAL DISSOLVED SOLIDS & $\begin{array}{l}06 / 10 / 80 \\
07 / 01 / 81\end{array}$ & $\begin{array}{l}0001 \\
0002\end{array}$ & MG/L & $\begin{array}{l}656.00 \\
665.00\end{array}$ & - & - \\
\hline URAKILM & $\begin{array}{l}06 / 10 / 80 \\
07 / 01 / 81\end{array}$ & $\begin{array}{l}0001 \\
0002\end{array}$ & MG/L & $\begin{array}{l}0.178 \\
0.22\end{array}$ & - & - \\
\hline VAHADIUM & $\begin{array}{l}06 / 10 / 80 \\
07 / 01 / 81\end{array}$ & $\begin{array}{l}0001 \\
0002\end{array}$ & $M G / L$ & $\begin{array}{l}0.01 \\
0.10\end{array}$ & - & - \\
\hline ZIKC & $06 / 10 / 80$ & 0001 & MG/L & 0.25 & - & - \\
\hline
\end{tabular}

PARAMETER VALUE INDICATOR (PVI):

< - LESS THAN DETECTION LIMIT

SAMPLE ID CODES:

0001 - FILTERED SAMPLE (.45 MICRONS)

0002 - FILTERED REPLICATE SAMPLE (.45 MICRONS) 
GROUNDWATER QUALITY DATA BY LOCATION

SITE: AMBOI AMBROSIA LAKE

LOCATION: 0595

HORTH COORDIHATE: 30640.0 FT

EAST COORDINATE: $\quad 67540.0$ FT

06/10/80 TO $12 / 04 / 92$

REPORT DATE: $08 / 04 / 94$

FORMATION OF COMPLETION: ALLUVIUM (AL)

HYDRAULIC FLON RELATIOHSHIP: UNKNOWN (N)

\begin{tabular}{|c|c|c|c|c|c|c|}
\hline PARAMETER NAME & LOG DATE & $\begin{array}{c}\text { SAMPLE } \\
\text { ID }\end{array}$ & $\begin{array}{l}\text { UNITS OF } \\
\text { MEASURE }\end{array}$ & $\begin{array}{l}\text { PARAMETER } \\
\text { PVI VALUE FLAGS }\end{array}$ & $\begin{array}{l}\text { DETECTION } \\
\text { LIMIT }\end{array}$ & $\begin{array}{l}\text { PARAMETER } \\
\text { UNCERTAIHTY }\end{array}$ \\
\hline ALKALIHITY & $07 / 01 / 81$ & 0001 & $M G / L C A C O 3$ & 108.00 & - & - \\
\hline ARSENIC & $07 / 01 / 81$ & 0001 & $M G / L$ & 0.005 & - & - \\
\hline BARIUH & $07 / 01 / 81$ & 0001 & $M G / L$ & 0.10 & $\cdot$ & - \\
\hline BICARBONATE - HCO3 & $07 / 01 / 81$ & 0001 & $M G / L$ & 264.00 & - & - \\
\hline CADMIUM & $07 / 01 / 81$ & 0001 & $M G / L$ & 0.001 & - & - \\
\hline CALCIUM & $07 / 01 / 81$ & 0001 & $M G / L$ & 58.60 & - & - \\
\hline CHLORIDE & $07 / 01 / 81$ & 0001 & MG/L & 19.00 & - & - \\
\hline LEAD & $07 / 01 / 81$ & 0001 & MG/L & 0.005 & - & $\cdot$ \\
\hline MAGNESIUUM & $07 / 01 / 81$ & 0001 & $M G / L$ & 4.60 & - & $\cdot$ \\
\hline MOLYBDENUM & $07 / 01 / 81$ & 0001 & MG/L & 0.155 & - & - \\
\hline PH & $07 / 01 / 81$ & 0001 & su & 7.50 & - & - \\
\hline POTASSIUM & $07 / 01 / 81$ & 0001 & MG/L & 2.30 & - & - \\
\hline SELENIUM & $07 / 01 / 81$ & 0001 & $M G / L$ & 0.023 & - & - \\
\hline SOOIUM & $07 / 01 / 81$ & 0001 & MG/L & 158.70 & - & - \\
\hline SPECIFIC COHDUCTANCE & $07 / 01 / 81$ & 0001 & UMHO/CM & 879.00 & - & - \\
\hline SULFATE & $07 / 01 / 81$ & 0001 & MG/L & 259.00 & - & - \\
\hline TEMPERATURE & $07 / 01 / 81$ & 0001 & C - DEGREE & 12.20 & - & - \\
\hline TOTAL DISSOLVED SOLIDS & $07 / 01 / 81$ & 0001 & MG/L & 668.00 & - & - \\
\hline URAMIUM-234 & $07 / 01 / 81$ & 0001 & $\mathrm{PCI} / \mathrm{L}$ & 0.18 & - & - \\
\hline VAKADIUM & $07 / 01 / 81$ & 0001 & $M G / L$ & 0.01 & - & - \\
\hline ZINC & $07 / 01 / 81$ & 0001 & MG/L & 0.10 & - & - \\
\hline
\end{tabular}

PARAMETER VALUE INDICATOR (PVI):

< - LESS THAN DETECTION LIMIT

SAMPLE ID CODES:

O001 - FILTERED SAMPLE (.45 MICRONS) 
GROUNDUATER OUALITY DATA BY LOCATION

SITE: AMBOI AMBROSIA LAKE

LOCATION: 0596

NORTH COORDIHATE: 20790.0 FT

EAST COORDINATE: $\quad 64530.0$ FT

06/10/80 TO $12 / 04 / 92$

REPORT DATE: 08/04/94

FORMATIOH OF COMPLETION: ALLUVIUM (AL)

HYDRAULIC FLON RELATIOHSHIP: UHKHOLN (N)

\begin{tabular}{|c|c|c|c|c|c|c|}
\hline PARAMETER HAME & LOG DATE & $\underset{\text { ID }}{\text { SAMPLE }}$ & $\begin{array}{l}\text { UNITS OF } \\
\text { MEASURE }\end{array}$ & $\begin{array}{l}\text { Parameter } \\
\text { PUI } \\
\text { VALUE flags }\end{array}$ & $\begin{array}{l}\text { DETECTIOW } \\
\text { LIMIT }\end{array}$ & $\begin{array}{l}\text { PARAMETER } \\
\text { UNCERTAINTY }\end{array}$ \\
\hline ALKALINITY & $01 / 26 / 82$ & 0001 & MG/L CACO3 & 155.00 & - & - \\
\hline ALCAINUM & $01 / 26 / 82$ & 0001 & $M G / L$ & 0.25 & - & - \\
\hline ARSEKIC & $01 / 26 / 82$ & 0001 & $M G / L$ & 0.005 & - & - \\
\hline BARIUM & $01 / 26 / 82$ & 0001 & $M G / L$ & 0.10 & - & - \\
\hline BICARBONATE - HCO3 & $01 / 26 / 82$ & 0001 & $M G / L$ & 377.00 & - & - \\
\hline CADMIU:M & $01 / 26 / 82$ & 0001 & $M G / L$ & 0.002 & - & - \\
\hline CALCIUA & $01 / 26 / 82$ & 0001 & $M G / L$ & 73.60 & $\cdot$ & - \\
\hline CHLORIDE & $01 / 26 / 82$ & 0001 & $M G / L$ & 20.00 & - & - \\
\hline FLUORIDE & $01 / 26 / 82$ & 0001 & $M G / L$ & 1.15 & - & - \\
\hline IROH & $01 / 26 / 82$ & 0001 & $M G / L$ & 0.10 & - & - \\
\hline LEAD & $01 / 26 / 82$ & 0001 & $M G / L$ & 0.005 & - & - \\
\hline MAGNESIUM & $01 / 26 / 82$ & 0001 & $M G / L$ & 19.50 & $\cdot$ & - \\
\hline MOL YBDENUM & $01 / 26 / 82$ & 0001 & $M G / L$ & 0.01 & - & - \\
\hline PH & $01 / 26 / 82$ & 0001 & SU & 7.40 & - & - \\
\hline POTASSIUM & $01 / 26 / 82$ & 0001 & MG/L & 0.00 & - & - \\
\hline SELENIUM & $01 / 26 / 82$ & 0001 & MG/L & 0.005 & - & - \\
\hline SILICON & $01 / 26 / 82$ & 0001 & $M G / L$ & 13.00 & - & - \\
\hline soolus & $01 / 26 / 82$ & 0001 & $M G / L$ & 101.20 & - & - \\
\hline SPECIFIC CONDUCTANCE & $01 / 26 / 82$ & 0001 & $\mathrm{UMHO} / \mathrm{CM}$ & 638.00 & - & - \\
\hline SULFATE & $01 / 26 / 82$ & 0001 & $M G / L$ & 84.00 & - & - \\
\hline TEMPERATURE & $01 / 26 / 82$ & 0009 & C - DEGREE & 12.50 & - & - \\
\hline TOTAL DISSOLVED SOLIDS & $01 / 26 / 82$ & 0001 & MG/L & 505.00 & - & - \\
\hline URAKIUM-234 & $01 / 26 / 82$ & 0001 & $\mathrm{PCI} / \mathrm{L}$ & 0.005 & - & - \\
\hline VAKADIU: & $01 / 26 / 82$ & 0001 & $M G / L$ & 0.01 & - & - \\
\hline ZINC & $01 / 26 / 82$ & 0001 & MG/L & 0.10 & - & - \\
\hline
\end{tabular}

PARAMETER VALUE IMDICATOR (PVI): < - LESS THAN DETECTION LIMIT

SAMPLE ID COOES:

DOO1 - FILTERED SAMPLE (.45 MICRONS) 
GROUNDWATER QUALITY DATA BY LOCATION

SITE: AMBO1 AMBROSIA LAKE

LOCATION: 0597

MORTH COORDINATE: 19350.0 FT

EAST COORDIHATE: $\quad 65380.0$ FT

$06 / 10 / 80$ TO $12 / 04 / 92$

REPORT DATE: 08/04/94

FORHATION OF COMPLETIOH: ALLUVIUM (AL)

HYDRAULIC FLOW RELATIOHSHIP: UNKHOWN (N)

\begin{tabular}{|c|c|c|c|c|c|c|}
\hline PARAMETER NAME & LOG DATE & $\begin{array}{c}\text { SAMPLE } \\
\text { ID }\end{array}$ & $\begin{array}{l}\text { UNITS OF } \\
\text { MEASURE }\end{array}$ & $\begin{array}{l}\text { PARAMETER } \\
\text { PVI VALUE FLAGS }\end{array}$ & $\begin{array}{l}\text { DETECTION } \\
\text { LIMIT }\end{array}$ & $\begin{array}{l}\text { PARAMETER } \\
\text { UNCERTAIHTY }\end{array}$ \\
\hline ALKALINITY & $01 / 26 / 82$ & 0001 & MG/L CACO3 & 86.50 & - & - \\
\hline ALUMINLM & $01 / 26 / 82$ & 0001 & $M G / L$ & 0.25 & - & - \\
\hline ARSENIC & $01 / 26 / 82$ & 0001 & $M G / L$ & 0.007 & - & - \\
\hline BARIUM & $01 / 26 / 82$ & 0001 & MG/L & 0.15 & - & - \\
\hline BICARBONATE - HCO3 & $01 / 26 / 82$ & 0001 & $M G / L$ & 211.00 & - & - \\
\hline CADMIUM & $01 / 26 / 82$ & 0001 & $\mathrm{MG} / \mathrm{L}$ & 0.001 & - & - \\
\hline CALCIUM & $01 / 26 / 82$ & 0001 & MG/L & 21.20 & - & - \\
\hline CHLORIDE & $01 / 26 / 82$ & 0001 & MG/L & 8.00 & - & - \\
\hline FLUOR IDE & $01 / 26 / 82$ & 0001 & MG/L & 1.90 & - & - \\
\hline IRON & $04 / 26 / 82$ & 0001 & MG/L & 0.10 & - & $\cdot$ \\
\hline LEAD & $01 / 26 / 82$ & 0001 & $M G / L$ & 0.005 & - & - \\
\hline MAGKESIUM & $01 / 26 / 82$ & 0001 & $M G / L$ & 4.00 & - & - \\
\hline MOLYBOENUM & $01 / 26 / 82$ & 0009 & MG/L & 0.012 & - & - \\
\hline PH & $01 / 26 / 82$ & 0001 & su & 7.80 & - & - \\
\hline POTASSIUA & $01 / 26 / 82$ & 0001 & MG/L & 2.30 & - & $\cdot$ \\
\hline SELENIUM & $01 / 26 / 82$ & 0001 & $M G / L$ & 0.005 & - & - \\
\hline SILICOH & $01 / 26 / 82$ & 0001 & MG/L & 22.90 & - & - \\
\hline soolum & $01 / 26 / 82$ & 0001 & $M G / L$ & 126.50 & - & - \\
\hline SPECIFIC CONDUCTANCE & $01 / 26 / 82$ & 0001 & UMHO/CM & 628.00 & - & $\cdot$ \\
\hline SULFATE & $01 / 26 / 82$ & 0001 & MG/L & 31.00 & - & - \\
\hline TEMPERATURE & $01 / 26 / 82$ & 0009 & C - DEGREE & 12.20 & - & - \\
\hline TOTAL DISSOLVED SOLIDS & $01 / 26 / 82$ & 0001 & MG/L & 422.00 & - & - \\
\hline URANIUM-234 & $01 / 26 / 82$ & 0001 & PCI/L & 0.005 & - & $\cdot$ \\
\hline VAHADIUA & $01 / 26 / 82$ & 0009 & MG/L & 0.01 & $\cdot$ & $\because$ \\
\hline ZINC & $01 / 26 / 82$ & 0001 & $M G / L$ & 0.10 & - & - \\
\hline
\end{tabular}

PARAMETER VALUE INDICATOR (PVI): < - LESS THAN DETECTION LIMIT

SAMPLE IO CODES:

OOO1 - FILTERED SAMPLE (.45 MICRONS) 
GROUHDHATER QUALITY DATA BY LOCATIOH

SITE: AMBOI AMBROSIA LAKE

LOCATIOH: 0609

MORTH COORDIHATE: $\quad 55335.0$ FT

EAST COORDIHATE: $\quad 60670.0$ FT

06/10/80 TO $12 / 04 / 92$

REPORT DATE: 08/04/94

FORMATION OF COMPLETION: ALLUVIUM (AL)

HYORAULIC FLON RELATIOHSHIP: ON-SITE (O)

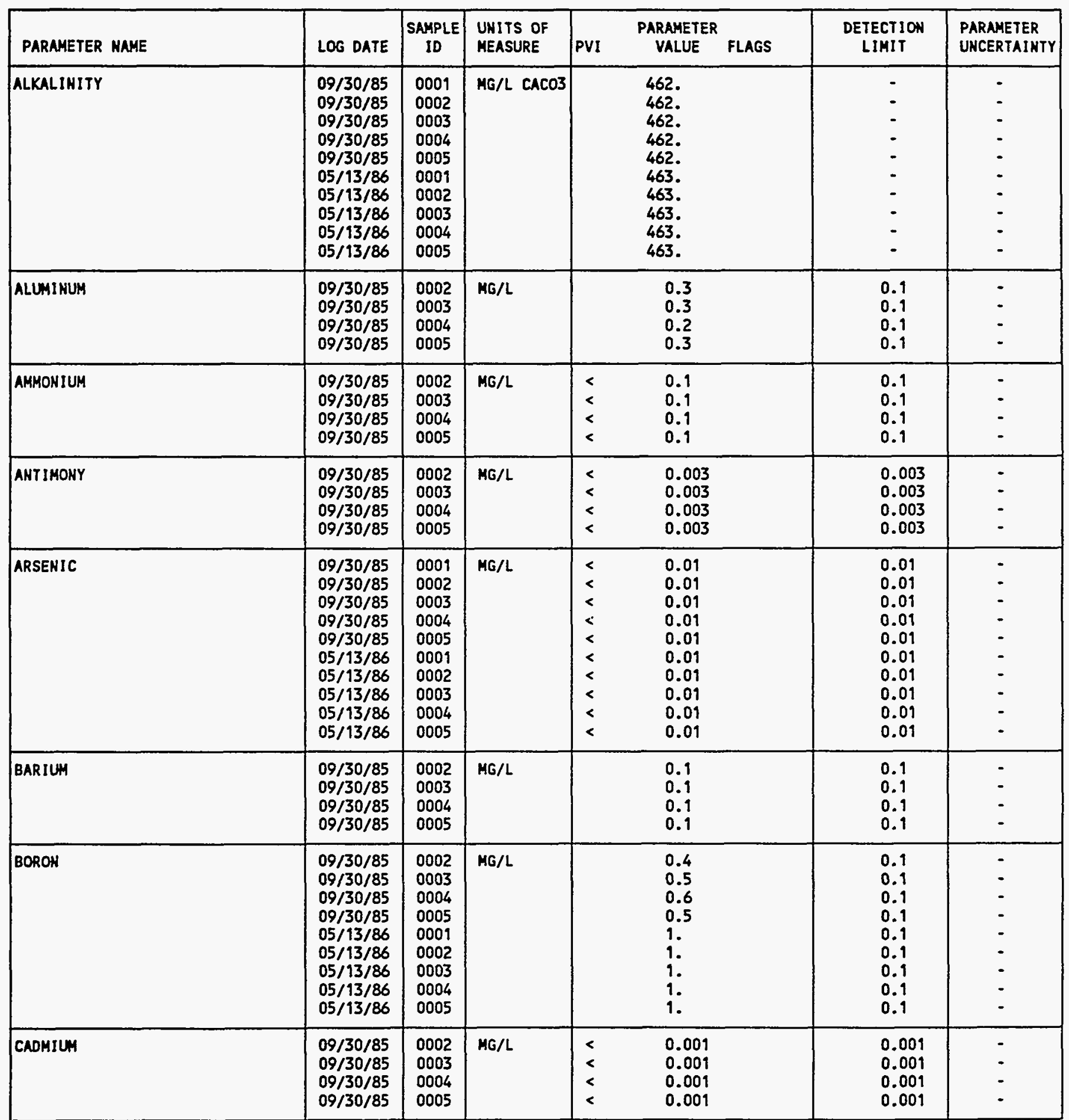

PARAMETER VALUE IKDICATOR (PVI): < - LESS THAK DETECTIOH LIMIT

SAMPLE ID COOES:

0001 - FILTERED SAMPLE (.45 MICRONS)

0002 - FILTERED REPLICATE SAMPLE (.45 MICRONS)

0003 - FILTERED REPLICATE SAMPLE (.45 MICRONS)

0004 - FILTERED REPLICATE SAMPLE (.45 MICRONS) 
GROUNDWATER QUALITY DATA BY LOCATION

SITE: AMBO I AMBROSIA LAKE

LOCATION: 0609

NORTH COORDINATE: $\quad 55335.0$ FT

EAST COORDINATE: $\quad 60670.0$ FT

06/10/80 TO $12 / 04 / 92$

REPORT DATE: 08/04/94

FORMATION OF COMPLETION: ALLUVIUM (AL)

HYDRAULIC FLON RELATIONSHIP: ON-SITE (O)

\begin{tabular}{|c|c|c|c|c|c|c|c|}
\hline PARAMETER MAME & LOG DATE & $\begin{array}{c}\text { SAMPLE } \\
\text { ID }\end{array}$ & $\begin{array}{l}\text { UNITS OF } \\
\text { MEASURE }\end{array}$ & PVI & $\begin{array}{l}\text { PARAMETER } \\
\text { VALUE FLAGS }\end{array}$ & $\begin{array}{l}\text { DETECTIOH } \\
\text { LIMIT }\end{array}$ & $\begin{array}{l}\text { PARAMETER } \\
\text { UHCERTAINTY }\end{array}$ \\
\hline CADMIUM & $\begin{array}{l}05 / 13 / 86 \\
05 / 13 / 86 \\
05 / 13 / 86 \\
05 / 13 / 86 \\
05 / 13 / 86\end{array}$ & $\begin{array}{l}0001 \\
0002 \\
0003 \\
0004 \\
0005\end{array}$ & $M G / L$ & $\begin{array}{l}< \\
< \\
< \\
< \\
<\end{array}$ & $\begin{array}{l}0.001 \\
0.001 \\
0.001 \\
0.001 \\
0.001\end{array}$ & $\begin{array}{l}0.001 \\
0.001 \\
0.001 \\
0.001 \\
0.001\end{array}$ & $\begin{array}{l}- \\
- \\
- \\
-\end{array}$ \\
\hline CALCIUM & $\begin{array}{l}09 / 30 / 85 \\
09 / 30 / 85 \\
09 / 30 / 85 \\
09 / 30 / 85 \\
09 / 30 / 85 \\
05 / 13 / 86 \\
05 / 13 / 86 \\
05 / 13 / 86 \\
05 / 93 / 86 \\
05 / 13 / 86\end{array}$ & $\begin{array}{l}0001 \\
0002 \\
0003 \\
0004 \\
0005 \\
0001 \\
0002 \\
0003 \\
0004 \\
0005\end{array}$ & MG/L & & $\begin{array}{l}562 . \\
610 . \\
536 . \\
540 . \\
492 . \\
566 . \\
566 . \\
566 . \\
566 . \\
566 .\end{array}$ & $\begin{array}{l}0.01 \\
0.01 \\
0.01 \\
0.01 \\
0.01 \\
0.01 \\
0.01 \\
0.01 \\
0.01 \\
0.01\end{array}$ & $\begin{array}{l}- \\
- \\
- \\
- \\
- \\
- \\
-\end{array}$ \\
\hline CHLORIDE & $\begin{array}{l}09 / 30 / 85 \\
09 / 30 / 85 \\
09 / 30 / 85 \\
09 / 30 / 85 \\
09 / 30 / 85 \\
05 / 13 / 86 \\
05 / 13 / 86 \\
05 / 13 / 86 \\
05 / 13 / 86 \\
05 / 13 / 86\end{array}$ & $\begin{array}{l}0001 \\
0002 \\
0003 \\
0004 \\
0005 \\
0001 \\
0002 \\
0003 \\
0004 \\
0005\end{array}$ & $M G / L$ & & $\begin{array}{l}148 . \\
150 . \\
150 . \\
150 . \\
150 . \\
160 . \\
160 . \\
160 . \\
160 . \\
160 .\end{array}$ & $\begin{array}{l}1 . \\
1 . \\
1 . \\
1 . \\
1 . \\
1 . \\
1 . \\
1 . \\
1 .\end{array}$ & $\begin{array}{l}: \\
: \\
- \\
: \\
: \\
-\end{array}$ \\
\hline CHROMIUH & $\begin{array}{l}09 / 30 / 85 \\
09 / 30 / 85 \\
09 / 30 / 85 \\
09 / 30 / 85 \\
05 / 13 / 86 \\
05 / 13 / 86 \\
05 / 13 / 86 \\
05 / 13 / 86 \\
05 / 13 / 86\end{array}$ & $\begin{array}{l}0002 \\
0003 \\
0004 \\
0005 \\
0001 \\
0002 \\
0003 \\
0004 \\
0005\end{array}$ & $M G / L$ & & $\begin{array}{l}0.04 \\
0.03 \\
0.05 \\
0.04 \\
0.2 \\
0.02 \\
0.02 \\
0.02 \\
0.02\end{array}$ & $\begin{array}{l}0.01 \\
0.01 \\
0.01 \\
0.01 \\
0.01 \\
0.01 \\
0.01 \\
0.01 \\
0.01\end{array}$ & $\begin{array}{l}- \\
- \\
- \\
- \\
- \\
- \\
-\end{array}$ \\
\hline COBALT & $\begin{array}{l}09 / 30 / 85 \\
09 / 30 / 85 \\
09 / 30 / 85 \\
09 / 30 / 85 \\
05 / 13 / 86 \\
05 / 13 / 86 \\
05 / 13 / 86 \\
05 / 13 / 86 \\
05 / 13 / 86\end{array}$ & $\begin{array}{l}0002 \\
0003 \\
0004 \\
0005 \\
0001 \\
0002 \\
0003 \\
0004 \\
0005\end{array}$ & $M G / L$ & & $\begin{array}{l}0.07 \\
0.06 \\
0.07 \\
0.09 \\
0.09 \\
0.09 \\
0.09 \\
0.09 \\
0.09\end{array}$ & $\begin{array}{l}0.05 \\
0.05 \\
0.05 \\
0.05 \\
0.05 \\
0.05 \\
0.05 \\
0.05 \\
0.05\end{array}$ & $\begin{array}{l}- \\
- \\
- \\
- \\
- \\
-\end{array}$ \\
\hline COPPER & $\begin{array}{l}09 / 30 / 85 \\
09 / 30 / 85 \\
09 / 30 / 85 \\
09 / 30 / 85\end{array}$ & $\begin{array}{l}0002 \\
0003 \\
0004 \\
0005\end{array}$ & MG/L & & $\begin{array}{l}0.03 \\
0.05 \\
0.04 \\
0.04\end{array}$ & $\begin{array}{l}0.02 \\
0.02 \\
0.02 \\
0.02\end{array}$ & : \\
\hline CYANIDE & $\begin{array}{l}09 / 30 / 85 \\
09 / 30 / 85 \\
09 / 30 / 85\end{array}$ & $\begin{array}{l}0002 \\
0003 \\
0004\end{array}$ & MG/L & $\begin{array}{l}< \\
<\end{array}$ & $\begin{array}{l}0.01 \\
0.01 \\
0.01\end{array}$ & $\begin{array}{l}0.01 \\
0.01 \\
0.01\end{array}$ & : \\
\hline
\end{tabular}

PARAMETER VALUE IMDICATOR (PVI): < - LESS THAN DETECTION LIMIT

SAMPLE ID COOES:

0001 - FILTERED SAMPLE (.45 MICRONS)

O002 - FILTERED REPLICATE SAMPLE (.45 MICROHS)

0003 - FILTERED REPLICATE SAMPLE (. 45 MICRONS)

0004 - FILTERED REPLICATE SAMPLE (.45 MICRONS) 
GROUNDWATER QUALITY DATA BY LOCATION

SITE: AMBOI AMBROSIA LAKE

LOCATION: 0609

NORTH COORDINATE: 55335.0 FT

EAST COORDINATE: $\quad 60670.0$ FT

O6/10/80 TO $12 / 04 / 92$

REPORT DATE: 08/04/94

FORMATIOH OF COMPLETION: ALLUVIUM (AL)

HYDRAULIC FLOH RELATIONSHIP: ON-SITE (O)

\begin{tabular}{|c|c|c|c|c|c|c|c|}
\hline PARAMETER NAME & LOG DATE & $\underset{\text { SDAMLE }}{\text { SD }}$ & $\begin{array}{l}\text { UNITS OF } \\
\text { MEASURE }\end{array}$ & PVI & $\begin{array}{l}\text { PARAMETER } \\
\text { VALUE FLAGS }\end{array}$ & $\begin{array}{l}\text { DETECTION } \\
\text { LIMIT }\end{array}$ & $\begin{array}{l}\text { PARAMETER } \\
\text { UNCERTAINTY }\end{array}$ \\
\hline CYANIDE & $09 / 30 / 85$ & 0005 & $M G / L$ & $<$ & 0.01 & 0.01 & - \\
\hline IRON & $\begin{array}{l}09 / 30 / 85 \\
09 / 30 / 85 \\
09 / 30 / 85 \\
09 / 30 / 85 \\
05 / 13 / 86 \\
05 / 13 / 86 \\
05 / 13 / 86 \\
05 / 13 / 86 \\
05 / 13 / 86\end{array}$ & $\begin{array}{l}0002 \\
0003 \\
0004 \\
0005 \\
0001 \\
0002 \\
0003 \\
0004 \\
0005\end{array}$ & $M G / L$ & $\begin{array}{l}< \\
< \\
<\end{array}$ & $\begin{array}{l}0.03 \\
0.03 \\
0.04 \\
0.03 \\
0.05 \\
0.05 \\
0.05 \\
0.05 \\
0.05\end{array}$ & $\begin{array}{l}0.03 \\
0.03 \\
0.03 \\
0.03 \\
0.03 \\
0.03 \\
0.03 \\
0.03 \\
0.03\end{array}$ & $\begin{array}{l}- \\
- \\
- \\
\dot{-} \\
- \\
- \\
-\end{array}$ \\
\hline LEAD & $\begin{array}{l}09 / 30 / 85 \\
09 / 30 / 85 \\
09 / 30 / 85 \\
09 / 30 / 85\end{array}$ & $\begin{array}{l}0002 \\
0003 \\
0004 \\
0005\end{array}$ & $M G / L$ & $\begin{array}{l}< \\
< \\
<\end{array}$ & $\begin{array}{l}0.01 \\
0.01 \\
0.01 \\
0.01\end{array}$ & $\begin{array}{l}0.01 \\
0.01 \\
0.01 \\
0.01\end{array}$ & $\begin{array}{l}- \\
- \\
-\end{array}$ \\
\hline LEAD-210 & $\begin{array}{l}09 / 30 / 85 \\
09 / 30 / 85 \\
09 / 30 / 85 \\
09 / 30 / 85\end{array}$ & $\begin{array}{l}0002 \\
0003 \\
0004 \\
0005\end{array}$ & $\mathrm{PCI} / \mathrm{L}$ & & $\begin{array}{l}3.2 \\
2.9 \\
2.6 \\
2.7\end{array}$ & $\begin{array}{l}1.5 \\
1.5 \\
1.5 \\
1.5\end{array}$ & $\begin{array}{l}2.5 \\
2.4 \\
2.7 \\
2.8\end{array}$ \\
\hline MAGHESIUM & $\begin{array}{l}09 / 30 / 85 \\
09 / 30 / 85 \\
09 / 30 / 85 \\
09 / 30 / 85 \\
09 / 30 / 85 \\
05 / 13 / 86 \\
05 / 13 / 86 \\
05 / 13 / 86 \\
05 / 13 / 86 \\
05 / 13 / 86\end{array}$ & $\begin{array}{l}0001 \\
0002 \\
0003 \\
0004 \\
0005 \\
0001 \\
0002 \\
0003 \\
0004 \\
0005\end{array}$ & MG/L & & $\begin{array}{l}156 . \\
142 . \\
157 . \\
160 . \\
164 . \\
149 . \\
149 . \\
149 . \\
150 . \\
149 .\end{array}$ & $\begin{array}{l}0.001 \\
0.001 \\
0.001 \\
0.001 \\
0.001 \\
0.001 \\
0.001 \\
0.001 \\
0.001 \\
0.001\end{array}$ & $\begin{array}{l}- \\
: \\
- \\
: \\
- \\
- \\
-\end{array}$ \\
\hline MANGANESE & $\begin{array}{l}09 / 30 / 85 \\
09 / 30 / 85 \\
09 / 30 / 85 \\
09 / 30 / 85 \\
05 / 13 / 86 \\
05 / 13 / 86 \\
05 / 13 / 86 \\
05 / 13 / 86 \\
05 / 13 / 86\end{array}$ & $\begin{array}{l}0002 \\
0003 \\
0004 \\
0005 \\
0001 \\
0002 \\
0003 \\
0004 \\
0005\end{array}$ & $M G / L$ & & $\begin{array}{l}0.47 \\
0.48 \\
0.52 \\
0.49 \\
0.68 \\
0.68 \\
0.68 \\
0.68 \\
0.68\end{array}$ & $\begin{array}{l}0.01 \\
0.01 \\
0.01 \\
0.01 \\
0.01 \\
0.01 \\
0.01 \\
0.01 \\
0.01\end{array}$ & $\begin{array}{l}- \\
- \\
- \\
- \\
- \\
- \\
-\end{array}$ \\
\hline MERCURY & $\begin{array}{l}09 / 30 / 85 \\
09 / 30 / 85 \\
09 / 30 / 85\end{array}$ & $\begin{array}{l}0002 \\
0003 \\
0004\end{array}$ & $M G / L$ & $\begin{array}{l}< \\
<\end{array}$ & $\begin{array}{l}0.0002 \\
0.0002 \\
0.0002\end{array}$ & $\begin{array}{l}0.0002 \\
0.0002 \\
0.0002\end{array}$ & : \\
\hline
\end{tabular}

PARAMETER VALUE IMDICATOR (PVI): < - LESS THAN DETECTION LIMIT

SAMPLE IO COOES:

0001 - FILTERED SAMPLE (.45 MICRONS)

0002 - FILTERED REPLICATE SAMPLE (.45 MICRONS)

0003 - FILTERED REPLICATE SAMPLE (.45 MICRONS)

0004 - FILTERED REPLICATE SAMPLE (.45 MICROHS) 
GROUNDWATER QUALITY DATA BY LOCATION

SITE: AMBO1 AMBROSIA LAKE

LOCATION: 0609

NORTH COORDINATE: $\quad 55335.0$ FT

EAST COORDINATE: 60670.0 FT

06/10/80 TO $12 / 04 / 92$

REPORT DATE: 08/04/94

FORMATION OF COMPLETION: ALLUVIUM (AL)

HYDRAULIC FLOW RELATIONSHIP: ON-SITE (O)

\begin{tabular}{|c|c|c|c|c|c|c|c|}
\hline PARAMETER MAME & LOG DATE & $\begin{array}{c}\text { SAMPLE } \\
\text { ID }\end{array}$ & $\begin{array}{l}\text { UNITS OF } \\
\text { MEASURE }\end{array}$ & PVI & $\begin{array}{l}\text { PARAMETER } \\
\text { VALUE FLAGS }\end{array}$ & $\begin{array}{l}\text { DETECTION } \\
\text { LIMIT }\end{array}$ & $\begin{array}{l}\text { PARAMETER } \\
\text { UNCERTAINTY }\end{array}$ \\
\hline HERCURY & $09 / 30 / 85$ & 0005 & $M G / L$ & $<$ & 0.0002 & 0.0002 & - \\
\hline HOL YBDENUM & $\begin{array}{l}09 / 30 / 85 \\
09 / 30 / 85 \\
09 / 30 / 85 \\
09 / 30 / 85 \\
09 / 30 / 85 \\
05 / 13 / 86 \\
05 / 13 / 86 \\
05 / 13 / 86 \\
05 / 13 / 86 \\
05 / 13 / 86\end{array}$ & $\begin{array}{l}0001 \\
0002 \\
0003 \\
0004 \\
0005 \\
0001 \\
0002 \\
0003 \\
0004 \\
0005\end{array}$ & MG/L & & $\begin{array}{l}1.41 \\
1.47 \\
1.59 \\
1.47 \\
1.47 \\
1.41 \\
1.39 \\
1.4 \\
1.41 \\
1.43\end{array}$ & $\begin{array}{l}0.01 \\
0.01 \\
0.01 \\
0.01 \\
0.01 \\
0.01 \\
0.01 \\
0.01 \\
0.01 \\
0.01\end{array}$ & $\begin{array}{l}- \\
- \\
- \\
- \\
- \\
- \\
-\end{array}$ \\
\hline HICKEL & $\begin{array}{l}09 / 30 / 85 \\
09 / 30 / 85 \\
09 / 30 / 85 \\
09 / 30 / 85\end{array}$ & $\begin{array}{l}0002 \\
0003 \\
0004 \\
0005\end{array}$ & $M G / L$ & & $\begin{array}{l}0.09 \\
0.1 \\
0.09 \\
0.1\end{array}$ & $\begin{array}{l}0.04 \\
0.04 \\
0.04 \\
0.04\end{array}$ & : \\
\hline HITRATE & $\begin{array}{l}09 / 30 / 85 \\
09 / 30 / 85 \\
09 / 30 / 85 \\
09 / 30 / 85 \\
05 / 13 / 86 \\
05 / 13 / 86 \\
05 / 13 / 86 \\
05 / 13 / 86 \\
05 / 13 / 86\end{array}$ & $\begin{array}{l}0002 \\
0003 \\
0004 \\
0005 \\
0001 \\
0002 \\
0003 \\
0004 \\
0005\end{array}$ & MG/L & $\begin{array}{l}< \\
< \\
< \\
<\end{array}$ & $\begin{array}{l}3 . \\
3 . \\
3 . \\
4 . \\
1 . \\
1 . \\
1 . \\
1 . \\
1 .\end{array}$ & $\begin{array}{l}1 . \\
1 . \\
1 . \\
1 . \\
1 . \\
1 . \\
1 . \\
1 .\end{array}$ & $\begin{array}{l}- \\
: \\
- \\
- \\
- \\
-\end{array}$ \\
\hline NITRITE & $\begin{array}{l}09 / 30 / 85 \\
09 / 30 / 85 \\
09 / 30 / 85 \\
09 / 30 / 85\end{array}$ & $\begin{array}{l}0002 \\
0003 \\
0004 \\
0005\end{array}$ & $M G / L$ & $\begin{array}{l}< \\
< \\
<\end{array}$ & $\begin{array}{l}0.1 \\
0.1 \\
0.1 \\
0.1\end{array}$ & $\begin{array}{l}0.1 \\
0.1 \\
0.1 \\
0.1\end{array}$ & $\begin{array}{l}- \\
- \\
-\end{array}$ \\
\hline PH & $\begin{array}{l}09 / 30 / 85 \\
09 / 30 / 85 \\
09 / 30 / 85 \\
09 / 30 / 85 \\
09 / 30 / 85 \\
05 / 13 / 86 \\
05 / 13 / 86 \\
05 / 13 / 86 \\
05 / 13 / 86 \\
05 / 13 / 86\end{array}$ & $\begin{array}{l}0001 \\
0002 \\
0003 \\
0004 \\
0005 \\
0001 \\
0002 \\
0003 \\
0004 \\
0005\end{array}$ & SU & & $\begin{array}{l}7.11 \\
7.11 \\
7.11 \\
7.11 \\
7.11 \\
6.87 \\
6.87 \\
6.87 \\
6.87 \\
6.87\end{array}$ & $\begin{array}{l}- \\
- \\
- \\
- \\
- \\
- \\
-\end{array}$ & $\begin{array}{l}- \\
- \\
- \\
- \\
- \\
- \\
-\end{array}$ \\
\hline PHOSPHATE & $\begin{array}{l}09 / 30 / 85 \\
09 / 30 / 85 \\
09 / 30 / 85 \\
09 / 30 / 85\end{array}$ & $\begin{array}{l}0002 \\
0003 \\
0004 \\
0005\end{array}$ & $M G / L$ & & $\begin{array}{l}0.4 \\
0.4 \\
0.4 \\
0.5\end{array}$ & $\begin{array}{l}0.1 \\
0.1 \\
0.1 \\
0.1\end{array}$ & $\begin{array}{r}- \\
- \\
-\end{array}$ \\
\hline POTASSIUM & $\begin{array}{l}09 / 30 / 85 \\
09 / 30 / 85 \\
09 / 30 / 85 \\
09 / 30 / 85 \\
09 / 30 / 85 \\
05 / 93 / 86 \\
05 / 13 / 86\end{array}$ & $\begin{array}{l}0001 \\
0002 \\
0003 \\
0004 \\
0005 \\
0001 \\
0002\end{array}$ & $M G / L$ & & $\begin{array}{l}1.06 \\
1.14 \\
1.38 \\
1.11 \\
1.1 \\
0.83 \\
0.83\end{array}$ & $\begin{array}{l}0.01 \\
0.01 \\
0.01 \\
0.01 \\
0.01 \\
0.01 \\
0.01\end{array}$ & $\begin{array}{l}- \\
- \\
- \\
. \\
. \\
-\end{array}$ \\
\hline
\end{tabular}

PARAMETER VAlUe INDICATOR (PVI): < - LESS THAN DETECTION LIMIT

SAMPLE ID COOES:

0001 - FILTERED SAMPLE (.45 MICROHS)

0002 - FILTERED REPLICATE SAMPLE (.45 HICRONS)

0003 - FILTERED REPLICATE SAMPLE (.45 MICROHS)

0004 - FILTERED REPLICATE SAMPLE (.45 MICROHS) 
GROUNDWATER QUALITY DATA BY LOCATION

SITE: AMBO1 AMBROSIA LAKE

LOCATION: 0609

NORTH COORDIHATE: 55335.0 FT

EAST COORDINATE: $\quad 60670.0$ FT

06/10/80 TO 12/04/92

REPORT DATE: 08/04/94

FORMATION OF COMPLETION: ALLUVIUN (AL)

HYDRAULIC FLON RELATIONSHIP: OH-SITE (O)

\begin{tabular}{|c|c|c|c|c|c|c|c|}
\hline PARAMETER MAME & LOG DATE & $\begin{array}{c}\text { SAMPLE } \\
\text { ID }\end{array}$ & $\begin{array}{l}\text { UNITS OF } \\
\text { MEASURE }\end{array}$ & PVI & $\begin{array}{l}\text { PARAMETER } \\
\text { VALUE FLAGS }\end{array}$ & $\begin{array}{l}\text { DETECTIOH } \\
\text { LIMIT }\end{array}$ & $\begin{array}{l}\text { PARAMETER } \\
\text { UNCERTAINTY }\end{array}$ \\
\hline POTASSIUM & $\begin{array}{l}05 / 13 / 86 \\
05 / 13 / 86 \\
05 / 13 / 86\end{array}$ & $\begin{array}{l}0003 \\
0004 \\
0005\end{array}$ & MG/L & & $\begin{array}{l}0.83 \\
0.83 \\
0.83\end{array}$ & $\begin{array}{l}0.01 \\
0.01 \\
0.01\end{array}$ & : \\
\hline RADIUH-226 & $\begin{array}{l}09 / 30 / 85 \\
09 / 30 / 85 \\
09 / 30 / 85 \\
09 / 30 / 85\end{array}$ & $\begin{array}{l}0002 \\
0003 \\
0004 \\
0005\end{array}$ & $\mathrm{PCI} / \mathrm{L}$ & & $\begin{array}{r}410 . \\
11 . \\
8.3 \\
7.9\end{array}$ & $\begin{array}{l}1 . \\
1 . \\
1 .\end{array}$ & $\begin{array}{l}20 . \\
3 . \\
2.9 \\
3.2\end{array}$ \\
\hline RADIUH-226 + RADIUH-228 & $\begin{array}{l}09 / 30 / 85 \\
09 / 30 / 85 \\
09 / 30 / 85 \\
09 / 30 / 85\end{array}$ & $\begin{array}{l}0002 \\
0003 \\
0004 \\
0005\end{array}$ & $\mathrm{PCI} / \mathrm{L}$ & & $\begin{array}{r}410.00 \\
11.20 \\
8.60 \\
8.10\end{array}$ & $\begin{array}{l}- \\
- \\
-\end{array}$ & $\begin{array}{l}- \\
- \\
-\end{array}$ \\
\hline RADIUH-228 & $\begin{array}{l}09 / 30 / 85 \\
09 / 30 / 85 \\
09 / 30 / 85 \\
09 / 30 / 85\end{array}$ & $\begin{array}{l}0002 \\
0003 \\
0004 \\
0005\end{array}$ & $\mathrm{PCI} / \mathrm{L}$ & & $\begin{array}{l}0 . \\
0.2 \\
0.3 \\
0.2\end{array}$ & $\begin{array}{l}1 . \\
1 . \\
1 .\end{array}$ & $\begin{array}{l}4 . \\
4.5 \\
5 . \\
3 .\end{array}$ \\
\hline SILICA - SIOZ & $\begin{array}{l}09 / 30 / 85 \\
09 / 30 / 85 \\
09 / 30 / 85 \\
09 / 30 / 85\end{array}$ & $\begin{array}{l}0002 \\
0003 \\
0004 \\
0005\end{array}$ & MG/L & & $\begin{array}{l}11 . \\
11 . \\
10 . \\
12 .\end{array}$ & $\begin{array}{l}2 . \\
2 . \\
2 . \\
2 .\end{array}$ & $\begin{array}{l}- \\
- \\
-\end{array}$ \\
\hline SPECIFIC CONDUCTANCE & $\begin{array}{l}09 / 30 / 85 \\
09 / 30 / 85 \\
09 / 30 / 85 \\
09 / 30 / 85 \\
09 / 30 / 85\end{array}$ & $\begin{array}{l}0001 \\
0002 \\
0003 \\
0004 \\
0005\end{array}$ & UМHO/CH & & $\begin{array}{l}3200 . \\
3200 . \\
3200 \\
3200 \\
3200\end{array}$ & $\begin{array}{l}: \\
: \\
-\end{array}$ & $\begin{array}{l}- \\
- \\
-\end{array}$ \\
\hline
\end{tabular}

PARAMETER VALUE INDICATOR (PVI): < - LESS THAK DETECTIOH LIMIT

SAMPLE ID COOES:

0001 - FILTERED SAMPLE (.45 MICRONS)

0002 - FILTERED REPLICATE SAMPLE (.45 MICRONS)

0003 - FILTERED REPLICATE SAMPLE (.45 MICRONS)

0004 - FILTERED REPLICATE SAMPLE (.45 MICRONS) 
GROUNDWATER QUALITY DATA BY LOCATION

SITE: AMBO1 AMBROSIA LAKE

LOCATION: 0609

KORTH COORDINATE: 55335.0 FT

EAST COORDINATE: $\quad 60670.0$ FT

O6/10/80 TO $12 / 04 / 92$

REPORT DATE: 08/04/94

FORMATION OF COMPLETION: ALLUVIUM (AL)

HYDRAULIC FLOW RELATIONSHIP: OH-SITE (O)

\begin{tabular}{|c|c|c|c|c|c|c|c|}
\hline PARAMETER NAME & LOG DATE & $\mid \begin{array}{c}\text { SAMPLE } \\
\text { ID }\end{array}$ & $\begin{array}{l}\text { UNITS OF } \\
\text { MEASURE }\end{array}$ & PVI & $\begin{array}{l}\text { PARAMETER } \\
\text { VALUE FLAGS }\end{array}$ & $\begin{array}{l}\text { DETECTION } \\
\text { LIMIT }\end{array}$ & $\begin{array}{l}\text { PARAMETER } \\
\text { UNCERTAINTY }\end{array}$ \\
\hline SPECIFIC CONDUCTANCE & $\begin{array}{l}05 / 13 / 86 \\
05 / 13 / 86 \\
05 / 13 / 86 \\
05 / 13 / 86 \\
05 / 13 / 86\end{array}$ & $\begin{array}{l}0001 \\
0002 \\
0003 \\
0004 \\
0005\end{array}$ & LHHO/CM & & $\begin{array}{l}3450 . \\
3450 . \\
3450 . \\
3450 . \\
3450 .\end{array}$ & $\begin{array}{l}- \\
- \\
- \\
-\end{array}$ & $\begin{array}{l}- \\
- \\
- \\
-\end{array}$ \\
\hline STRONTIUM & $\begin{array}{l}09 / 30 / 85 \\
09 / 30 / 85 \\
09 / 30 / 85 \\
09 / 30 / 85 \\
05 / 13 / 86 \\
05 / 13 / 86 \\
05 / 13 / 86 \\
05 / 13 / 86 \\
05 / 13 / 86\end{array}$ & $\begin{array}{l}0002 \\
0003 \\
0004 \\
0005 \\
0001 \\
0002 \\
0003 \\
0004 \\
0005\end{array}$ & $M G / L$ & & $\begin{array}{l}9.8 \\
10 . \\
12 . \\
12 . \\
0.8 \\
0.8 \\
0.8 \\
0.8 \\
0.8\end{array}$ & $\begin{array}{l}0.1 \\
0.1 \\
0.1 \\
0.1 \\
0.1 \\
0.1 \\
0.1 \\
0.1 \\
0.1\end{array}$ & $\begin{array}{l}- \\
- \\
- \\
- \\
- \\
-\end{array}$ \\
\hline SULFATE & $\begin{array}{l}09 / 30 / 85 \\
09 / 30 / 85 \\
09 / 30 / 85 \\
09 / 30 / 85 \\
09 / 30 / 85 \\
05 / 13 / 86 \\
05 / 13 / 86 \\
05 / 13 / 86 \\
05 / 13 / 86 \\
05 / 13 / 86\end{array}$ & $\begin{array}{l}0001 \\
0002 \\
0003 \\
0004 \\
0005 \\
0001 \\
0002 \\
0003 \\
0004 \\
0005\end{array}$ & $M G / L$ & & $\begin{array}{l}2420 . \\
2400 . \\
2400 . \\
2400 . \\
2390 . \\
2540 . \\
2530 . \\
2520 . \\
2540 . \\
2530 .\end{array}$ & $\begin{array}{l}0.1 \\
0.1 \\
0.1 \\
0.1 \\
0.1 \\
0.1 \\
0.1 \\
0.1 \\
0.1 \\
0.1\end{array}$ & $\begin{array}{l}: \\
: \\
: \\
: \\
- \\
-\end{array}$ \\
\hline SULFIDE & $\begin{array}{l}09 / 30 / 85 \\
09 / 30 / 85 \\
09 / 30 / 85 \\
09 / 30 / 85\end{array}$ & $\begin{array}{l}0002 \\
0003 \\
0004 \\
0005\end{array}$ & $M G / L$ & $\begin{array}{l}< \\
< \\
<\end{array}$ & $\begin{array}{l}0.1 \\
0.1 \\
0.1 \\
0.1\end{array}$ & $\begin{array}{l}0.1 \\
0.1 \\
0.1 \\
0.1\end{array}$ & - \\
\hline TEMPERATURE & $\begin{array}{l}09 / 30 / 85 \\
09 / 30 / 85 \\
09 / 30 / 85 \\
09 / 30 / 85 \\
09 / 30 / 85 \\
05 / 13 / 86 \\
05 / 13 / 86 \\
05 / 13 / 86 \\
05 / 13 / 86 \\
05 / 13 / 86\end{array}$ & $\begin{array}{l}0001 \\
0002 \\
0003 \\
0004 \\
0005 \\
0001 \\
0002 \\
0003 \\
0004 \\
0005\end{array}$ & $C$ - DEGREE & & $\begin{array}{l}12 . \\
12 . \\
12 . \\
12 . \\
12 . \\
16 . \\
16 . \\
16 . \\
16 . \\
16 .\end{array}$ & $\begin{array}{l}- \\
- \\
- \\
- \\
- \\
-\end{array}$ & $\begin{array}{l}- \\
: \\
- \\
- \\
- \\
- \\
-\end{array}$ \\
\hline THORIUM-230 & $\begin{array}{l}09 / 30 / 85 \\
09 / 30 / 85 \\
09 / 30 / 85 \\
09 / 30 / 85\end{array}$ & $\begin{array}{l}0002 \\
0003 \\
0004 \\
0005\end{array}$ & $\mathrm{PCI} / \mathrm{L}$ & & $\begin{array}{l}1.4 \\
0.9 \\
0.9 \\
0.5\end{array}$ & $\begin{array}{l}1 . \\
1 . \\
1 .\end{array}$ & $\begin{array}{l}1.1 \\
0.9 \\
0.9 \\
0.8\end{array}$ \\
\hline TIH & $\begin{array}{l}09 / 30 / 85 \\
09 / 30 / 85 \\
09 / 30 / 85 \\
09 / 30 / 85\end{array}$ & $\begin{array}{l}0002 \\
0003 \\
0004 \\
0005\end{array}$ & $M G / L$ & $\begin{array}{l}< \\
< \\
<\end{array}$ & $\begin{array}{l}0.005 \\
0.005 \\
0.005 \\
0.005\end{array}$ & $\begin{array}{l}0.005 \\
0.005 \\
0.005 \\
0.005\end{array}$ & : \\
\hline TOTAL DISSOLVED SOLIDS & $\begin{array}{l}09 / 30 / 85 \\
09 / 30 / 85 \\
09 / 30 / 85\end{array}$ & $\begin{array}{l}0001 \\
0002 \\
0003\end{array}$ & $M G / L$ & & $\begin{array}{l}4310 . \\
4370 . \\
4330 .\end{array}$ & $\begin{array}{l}10 . \\
10 . \\
10 .\end{array}$ & : \\
\hline
\end{tabular}

PARAMETER VALUE IMOICATOR (PVI): < - LESS THAN DETECTION LIMIT

SAMPLE ID COOES:

0001 - FILTERED SAMPLE (.45 MICRONS)

0002 - FILTERED REPLICATE SAMPLE (.45 MICRONS)

0003 - FILTERED REPLICATE SAMPLE (.45 MICRONS)

0004 - FILTERED REPLICATE SAMPLE (.45 MICRONS) 
GROUNDHATER QUALITY DATA BY LOCATION

SITE: AMBO1 AMBROSIA LAKE

LOCATIOH: 0609

NORTH COORDINATE: 55335.0 FT

EAST COORDINATE: 60670.0 FT

06/10/80 TO $12 / 04 / 92$

REPORT DATE: 08/04/94

FORHATION OF COMPLETION: ALLUVIUM (AL)

HYDRAULIC FLOW RELATIONSHIP: OH-SITE (O)

\begin{tabular}{|c|c|c|c|c|c|c|c|}
\hline PARAMETER MAME & LOG DATE & $\underset{\text { SAMPLE }}{\text { SD }}$ & $\begin{array}{l}\text { UNITS OF } \\
\text { MEASURE }\end{array}$ & PVI & $\begin{array}{l}\text { PARAMETER } \\
\text { VALUE FLAGS }\end{array}$ & $\begin{array}{l}\text { DETECTIOH } \\
\text { LIMIT }\end{array}$ & $\begin{array}{l}\text { PARAMETER } \\
\text { UNCERTAINTY }\end{array}$ \\
\hline TOTAL DISSOLVED SOLIDS & $\begin{array}{l}09 / 30 / 85 \\
09 / 30 / 85 \\
05 / 13 / 86 \\
05 / 13 / 86 \\
05 / 13 / 86 \\
05 / 13 / 86 \\
05 / 13 / 86\end{array}$ & $\begin{array}{l}0004 \\
0005 \\
0001 \\
0002 \\
0003 \\
0004 \\
0005\end{array}$ & $M G / L$ & & $\begin{array}{l}4350 . \\
4360 . \\
4360 . \\
4390 . \\
4540 . \\
4370 . \\
4390 .\end{array}$ & $\begin{array}{l}10 . \\
10 . \\
10 . \\
10 . \\
10 . \\
10 . \\
10 .\end{array}$ & $\begin{array}{l}: \\
: \\
: \\
:\end{array}$ \\
\hline URAKIUH & $\begin{array}{l}09 / 30 / 85 \\
09 / 30 / 85 \\
09 / 30 / 85 \\
09 / 30 / 85 \\
09 / 30 / 85 \\
05 / 13 / 86 \\
05 / 13 / 86 \\
05 / 13 / 86 \\
05 / 13 / 86 \\
05 / 13 / 86\end{array}$ & $\begin{array}{l}0001 \\
0002 \\
0003 \\
0004 \\
0005 \\
0001 \\
0002 \\
0003 \\
0004 \\
0005\end{array}$ & MG/L & & $\begin{array}{l}0.0807 \\
0.102 \\
0.0999 \\
0.0848 \\
0.0885 \\
0.0537 \\
0.0534 \\
0.0517 \\
0.05 \\
0.0506\end{array}$ & $\begin{array}{l}0.003 \\
0.003 \\
0.003 \\
0.003 \\
0.003 \\
0.003 \\
0.003 \\
0.003 \\
0.003 \\
0.003\end{array}$ & $\begin{array}{l}: \\
: \\
: \\
: \\
: \\
-\end{array}$ \\
\hline ZINC & $\begin{array}{l}09 / 30 / 85 \\
09 / 30 / 85 \\
09 / 30 / 85 \\
09 / 30 / 85\end{array}$ & $\begin{array}{l}0002 \\
0003 \\
0004 \\
0005\end{array}$ & MG/L & & $\begin{array}{l}0.069 \\
0.063 \\
0.075 \\
0.066\end{array}$ & $\begin{array}{l}0.005 \\
0.005 \\
0.005 \\
0.005\end{array}$ & : \\
\hline
\end{tabular}

PARAMETER VALUE IMDICATOR (PVI): < - LESS THAN DETECTION LIMIT

SAMPLE ID COOES:

0001 - FILTERED SAMPLE (.45 MICRONS)

0002 - FILTERED REPLICATE SAMPLE (.45 MICRONS)

0003 - FILTERED REPLICATE SAMPLE (.45 MICRONS)

0004 - FILTERED REPLICATE SAMPLE (.45 MICRONS) 
GROUNDWATER QUALITY DATA BY LOCATION

SITE: AMBOI AMBROSIA LAKE

LOCATION: 0619

MORTH COORDINATE: $\quad 50910.0$ FT

EAST COORDINATE: $\quad 64040.0$ FT

$06 / 10 / 80$ TO $12 / 04 / 92$

REPORT DATE: $08 / 04 / 94$

FORMATION OF COMPLETION: ALLUVIUM (AL)

HYDRAULIC FLOW RELATIONSHIP: CROSS GRAOIENT (C)

\begin{tabular}{|c|c|c|c|c|c|c|c|}
\hline PARAMETER MAME & LOG DATE & $\underset{\text { ID }}{\text { SAMPLE }}$ & $\begin{array}{l}\text { UNITS OF } \\
\text { MEASURE }\end{array}$ & PVI & $\begin{array}{l}\text { PARAMETER } \\
\text { VALUE FLAGS }\end{array}$ & $\begin{array}{l}\text { DETECTION } \\
\text { LIMIT }\end{array}$ & $\begin{array}{l}\text { PARAMETER } \\
\text { UNCERTAINTY }\end{array}$ \\
\hline ALKALINITY & $10 / 02 / 85$ & 0001 & $\mathrm{MG} / \mathrm{L} \mathrm{CACO}$ & & 597. & - & - \\
\hline ARSENIC & $10 / 02 / 85$ & 0001 & MG/L & $<$ & 0.01 & 0.01 & - \\
\hline CALCIUN & $10 / 02 / 85$ & 0001 & $M G / L$ & & 380. & 0.01 & - \\
\hline CHLORIDE & $10 / 02 / 85$ & 0001 & MG/L & & 165. & 1. & - \\
\hline MAGNESIUA & $10 / 02 / 85$ & 0001 & $M G / L$ & & 110. & 0.001 & - \\
\hline MOLYBDENUM & $10 / 02 / 85$ & 0001 & $M G / L$ & & 0.22 & 0.01 & - \\
\hline $\mathrm{PH}$ & $10 / 02 / 85$ & 0001 & SU & & 7.01 & - & - \\
\hline POTASSIUM & $10 / 02 / 85$ & 0001 & $M G / L$ & & 5.38 & 0.01 & - \\
\hline SELENIUM & $10 / 02 / 85$ & 0001 & MG/L & & 0.033 & 0.005 & - \\
\hline SODIUM & $10 / 02 / 85$ & 0001 & MG/L & & 555. & 0.002 & - \\
\hline SPECIFIC CONDUCTANCE & $10 / 02 / 85$ & 0001 & UMHO/CM & & 2600 . & - & - \\
\hline SULFATE & $10 / 02 / 85$ & 0001 & MG/L & & 1770. & 0.1 & $\cdot$ \\
\hline TEMPERATURE & $10 / 02 / 85$ & 0001 & C - DEGREE & & 12. & - & - \\
\hline TOTAL DISSOLVED SOLIDS & $10 / 02 / 85$ & 0001 & MG/L & & 3200 . & 10. & - \\
\hline URANIUM & $10 / 02 / 85$ & 0001 & MG/L & & 0.95 & 0.003 & - \\
\hline
\end{tabular}

PARAMETER VALUE INDICATOR (PVI):

< - LESS THAN DETECTION LIMIT

SAMPLE ID COOES:

O001 - FILTERED SAMPLE (.45 MICRONS) 
GROUMDWATER QUALITY DATA BY LOCATION

SITE: AMBO1 AMBROSIA LAKE

LOCATION: 0620

MORTH COOROINATE: 52040.0 FT

EAST COORDIHATE: $\quad 60740.0$ FT

D6/10/80 TO $12 / 04 / 92$

REPORT DATE: 08/04/94

FORMATION OF COMPLETION: ALLUVIUM (AL)

HYDRAULIC FLOW RELATIONSHIP: CROSS GRADIENT (C)

\begin{tabular}{|c|c|c|c|c|c|c|c|c|}
\hline PARAMETER NAME & LOG DATE & $\underset{\text { ID }}{\text { SAMPLE }}$ & $\begin{array}{l}\text { UNITS OF } \\
\text { MEASURE }\end{array}$ & PVI & $\begin{array}{l}\text { PARAMETER } \\
\text { VALUE }\end{array}$ & FLAGS & $\begin{array}{l}\text { DETECTION } \\
\text { LIMIT }\end{array}$ & $\begin{array}{l}\text { PARAMETER } \\
\text { UNCERTAINTY }\end{array}$ \\
\hline ALKALINITY & $\begin{array}{l}10 / 02 / 85 \\
01 / 09 / 87 \\
07 / 15 / 88 \\
01 / 12 / 89 \\
09 / 14 / 89 \\
03 / 31 / 90 \\
01 / 09 / 91\end{array}$ & $\begin{array}{l}0001 \\
0001 \\
0001 \\
0001 \\
0001 \\
0001 \\
0001\end{array}$ & $\mathrm{MG} / \mathrm{L}$ CACO3 & & $\begin{array}{l}294 . \\
294 . \\
299 . \\
278 . \\
274 . \\
299 \\
277 .\end{array}$ & & $\begin{array}{l}- \\
- \\
- \\
- \\
-\end{array}$ & $\begin{array}{l}- \\
- \\
- \\
- \\
-\end{array}$ \\
\hline ALUMINUM & $\begin{array}{l}10 / 02 / 85 \\
07 / 15 / 88 \\
01 / 12 / 89 \\
09 / 14 / 89 \\
03 / 31 / 90 \\
01 / 09 / 91\end{array}$ & $\begin{array}{l}0001 \\
0001 \\
0001 \\
0001 \\
0001 \\
0001\end{array}$ & MG/L & $<$ & $\begin{array}{l}0.4 \\
0.3 \\
0.05 \\
0.1 \\
0.1 \\
0.05\end{array}$ & J & $\begin{array}{l}0.1 \\
0.1 \\
0.1 \\
0.1 \\
0.1 \\
0.05\end{array}$ & $\begin{array}{l}- \\
- \\
- \\
- \\
-\end{array}$ \\
\hline AMMON IUM & $\begin{array}{l}10 / 02 / 85 \\
07 / 15 / 88 \\
01 / 12 / 89 \\
09 / 14 / 89 \\
03 / 31 / 90 \\
01 / 09 / 91\end{array}$ & $\begin{array}{l}0001 \\
0001 \\
0001 \\
0001 \\
0001 \\
0001\end{array}$ & $M G / L$ & $\begin{array}{l}< \\
< \\
<\end{array}$ & $\begin{array}{l}0.1 \\
0.1 \\
0.10 \\
0.1 \\
0.1 \\
0.08\end{array}$ & & $\begin{array}{l}0.1 \\
0.1 \\
0.1 \\
0.1 \\
0.1 \\
0.01\end{array}$ & $\begin{array}{l}- \\
- \\
- \\
-\end{array}$ \\
\hline ANT IMONY & $\begin{array}{l}10 / 02 / 85 \\
07 / 15 / 88 \\
01 / 12 / 89 \\
09 / 14 / 89 \\
03 / 31 / 90 \\
01 / 09 / 91\end{array}$ & $\begin{array}{l}0001 \\
0001 \\
0001 \\
0001 \\
0001 \\
0001\end{array}$ & $M G / L$ & $\begin{array}{l}< \\
< \\
<\end{array}$ & $\begin{array}{l}0.003 \\
0.013 \\
0.003 \\
0.003 \\
0.021 \\
0.003\end{array}$ & & $\begin{array}{l}0.003 \\
0.003 \\
0.003 \\
0.003 \\
0.003 \\
0.003\end{array}$ & $\begin{array}{l}- \\
- \\
- \\
-\end{array}$ \\
\hline ARSENIC & $\begin{array}{l}10 / 02 / 85 \\
01 / 09 / 87 \\
07 / 15 / 88 \\
01 / 12 / 89 \\
09 / 14 / 89 \\
03 / 31 / 90 \\
01 / 09 / 91\end{array}$ & $\begin{array}{l}0001 \\
0001 \\
0001 \\
0001 \\
0001 \\
0001 \\
0001\end{array}$ & MG/L & $\begin{array}{l}< \\
< \\
< \\
<\end{array}$ & $\begin{array}{l}0.01 \\
0.003 \\
0.01 \\
0.001 \\
0.01 \\
0.01 \\
0.03\end{array}$ & $\begin{array}{l}J \\
J \\
1\end{array}$ & $\begin{array}{l}0.01 \\
0.01 \\
0.01 \\
0.01 \\
0.01 \\
0.01 \\
0.03\end{array}$ & $\begin{array}{l}- \\
= \\
- \\
- \\
-\end{array}$ \\
\hline BARIUM & $\begin{array}{l}10 / 02 / 85 \\
07 / 15 / 88 \\
01 / 12 / 89 \\
09 / 14 / 89 \\
03 / 31 / 90 \\
01 / 09 / 91\end{array}$ & $\begin{array}{l}0001 \\
0001 \\
0001 \\
0001 \\
0001 \\
0001\end{array}$ & MG/L & $\begin{array}{l}< \\
< \\
<\end{array}$ & $\begin{array}{l}0.1 \\
0.1 \\
0.01 \\
0.1 \\
0.1 \\
0.01\end{array}$ & J & $\begin{array}{l}0.1 \\
0.1 \\
0.1 \\
0.1 \\
0.1 \\
0.01\end{array}$ & $\begin{array}{l}- \\
- \\
- \\
-\end{array}$ \\
\hline BERYLLIUM & $\begin{array}{l}01 / 12 / 89 \\
09 / 14 / 89 \\
03 / 31 / 90 \\
01 / 09 / 91\end{array}$ & $\begin{array}{l}0001 \\
0001 \\
0001 \\
0001\end{array}$ & $M G / L$ & $\begin{array}{l}< \\
< \\
<\end{array}$ & $\begin{array}{l}0.005 \\
0.01 \\
0.01 \\
0.005\end{array}$ & & $\begin{array}{l}0.005 \\
0.01 \\
0.01 \\
0.005\end{array}$ & $\begin{array}{l}- \\
-\end{array}$ \\
\hline BORON & $\begin{array}{l}10 / 02 / 85 \\
01 / 09 / 87 \\
07 / 15 / 88 \\
01 / 12 / 89 \\
09 / 14 / 89 \\
03 / 31 / 90 \\
01 / 09 / 91\end{array}$ & $\begin{array}{l}0001 \\
0001 \\
0001 \\
0001 \\
0001 \\
0001 \\
0001\end{array}$ & MG/L & & $\begin{array}{l}0.4 \\
0.35 \\
0.3 \\
0.37 \\
0.4 \\
0.3 \\
0.35\end{array}$ & & $\begin{array}{l}0.1 \\
0.1 \\
0.1 \\
0.1 \\
0.1 \\
0.1 \\
0.05\end{array}$ & $\begin{array}{l}- \\
. \\
- \\
. \\
-\end{array}$ \\
\hline
\end{tabular}

PARAMETER VAlUe INDICATOR (PVI): \& - LESS thaN DETECTION LIMIT SAMPLE ID COOES:

0001 - FILTERED SAMPLE (.45 MICRONS)

OTHER PARAMETER VALUE FLAGS:

1 - INCREASED DETECTION LIMIT DUE TO REQUIRED DILUTION

J - eSTIMATED VALUE 
GROUNDWATER OUALITY DATA BY LOCATION

SITE: AMBO1 AMBROSIA LAKE

LOCATION: 0620

NORTH COORDINATE: $\quad 52040.0$ FT

EAST COORDINATE: 60740.0 FT

06/10/80 TO 12/04/92

REPORT DATE: $08 / 04 / 94$

FORMATIOH OF COMPLETION: ALLUVIUM (AL)

HYDRAULIC FLON RELATIONSHIP: CROSS GRADIENT (C)

\begin{tabular}{|c|c|c|c|c|c|c|c|c|}
\hline PARAMETER NAME & LOG DATE & $\begin{array}{c}\text { SAMPLE } \\
\text { ID }\end{array}$ & $\begin{array}{l}\text { UNITS OF } \\
\text { MEASURE }\end{array}$ & PVI & $\begin{array}{c}\text { PARAMETER } \\
\text { VALUE }\end{array}$ & FLAGS & $\begin{array}{l}\text { DETECTION } \\
\text { LIMIT }\end{array}$ & $\begin{array}{l}\text { PARAMETER } \\
\text { UNCERTAINTY }\end{array}$ \\
\hline BROMIDE & $\begin{array}{l}07 / 15 / 88 \\
01 / 12 / 89 \\
09 / 14 / 89 \\
03 / 31 / 90 \\
01 / 09 / 91\end{array}$ & $\begin{array}{l}0001 \\
0001 \\
0001 \\
0001 \\
0001\end{array}$ & $M G / L$ & & $\begin{array}{l}0.1 \\
0.42 \\
0.12 \\
0.2 \\
0.4\end{array}$ & & $\begin{array}{l}0.1 \\
0.01 \\
0.1 \\
0.1 \\
0.1\end{array}$ & $\begin{array}{l}- \\
- \\
- \\
-\end{array}$ \\
\hline CADMIUM & $\begin{array}{l}10 / 02 / 85 \\
07 / 15 / 88 \\
01 / 12 / 89 \\
09 / 14 / 89 \\
03 / 31 / 90 \\
01 / 09 / 91\end{array}$ & $\begin{array}{l}0001 \\
0001 \\
0001 \\
0001 \\
0001 \\
0001\end{array}$ & MG/L & $\begin{array}{l}< \\
< \\
< \\
< \\
< \\
<\end{array}$ & $\begin{array}{l}0.001 \\
0.001 \\
0.0001 \\
0.001 \\
0.001 \\
0.001\end{array}$ & $\mathbf{J}$ & $\begin{array}{l}0.001 \\
0.001 \\
0.001 \\
0.001 \\
0.001 \\
0.001\end{array}$ & $\begin{array}{l}- \\
- \\
- \\
- \\
-\end{array}$ \\
\hline CALCIUH & $\begin{array}{l}10 / 02 / 85 \\
01 / 09 / 87 \\
07 / 15 / 88 \\
01 / 12 / 89 \\
09 / 14 / 89 \\
03 / 31 / 90 \\
01 / 09 / 91\end{array}$ & $\begin{array}{l}0001 \\
0001 \\
0001 \\
0001 \\
0001 \\
0001 \\
0001\end{array}$ & $M G / L$ & & $\begin{array}{l}533 . \\
475 . \\
482 . \\
503 . \\
479 . \\
481 \\
535\end{array}$ & & $\begin{array}{l}0.01 \\
0.01 \\
0.01 \\
0.01 \\
0.01 \\
0.01 \\
0.01\end{array}$ & $\begin{array}{l}- \\
- \\
- \\
- \\
-\end{array}$ \\
\hline CHLORIDE & $\begin{array}{l}10 / 02 / 85 \\
01 / 09 / 87 \\
07 / 15 / 88 \\
01 / 12 / 89 \\
09 / 14 / 89 \\
03 / 31 / 90 \\
01 / 09 / 91\end{array}$ & $\begin{array}{l}0001 \\
0001 \\
0001 \\
0001 \\
0001 \\
0001 \\
0001\end{array}$ & $M G / L$ & & $\begin{array}{l}36 . \\
43 . \\
48 . \\
43 . \\
54 . \\
58 . \\
65 .\end{array}$ & & $\begin{array}{l}1 . \\
1 . \\
1 . \\
1 . \\
1 . \\
1 . \\
0.5\end{array}$ & $\begin{array}{l}- \\
- \\
- \\
- \\
-\end{array}$ \\
\hline CHROMIUH & $\begin{array}{l}10 / 02 / 85 \\
01 / 09 / 87 \\
07 / 15 / 88 \\
01 / 12 / 89 \\
09 / 14 / 89 \\
03 / 31 / 90 \\
01 / 09 / 91\end{array}$ & $\begin{array}{l}0001 \\
0001 \\
0001 \\
0001 \\
0001 \\
0001 \\
0001\end{array}$ & $M G / L$ & $\begin{array}{l}< \\
< \\
<\end{array}$ & $\begin{array}{l}0.04 \\
0.02 \\
0.16 \\
0.01 \\
0.01 \\
0.01 \\
0.01\end{array}$ & & $\begin{array}{l}0.01 \\
0.01 \\
0.01 \\
0.01 \\
0.01 \\
0.01 \\
0.01\end{array}$ & $\begin{array}{l}- \\
- \\
- \\
- \\
-\end{array}$ \\
\hline COBALT & $\begin{array}{l}10 / 02 / 85 \\
01 / 09 / 87 \\
07 / 15 / 88 \\
01 / 12 / 89 \\
09 / 14 / 89 \\
03 / 31 / 90 \\
01 / 09 / 91\end{array}$ & $\begin{array}{l}0001 \\
0001 \\
0001 \\
0001 \\
0001 \\
0001 \\
0001\end{array}$ & $M G / L$ & $\begin{array}{l}< \\
< \\
< \\
< \\
<\end{array}$ & $\begin{array}{l}0.06 \\
0.05 \\
0.05 \\
0.01 \\
0.05 \\
0.05 \\
0.03\end{array}$ & $J$ & $\begin{array}{l}0.05 \\
0.05 \\
0.05 \\
0.05 \\
0.05 \\
0.05 \\
0.03\end{array}$ & $\begin{array}{l}- \\
- \\
- \\
- \\
- \\
-\end{array}$ \\
\hline COPPER & $\begin{array}{l}10 / 02 / 85 \\
01 / 09 / 87 \\
07 / 15 / 88 \\
01 / 12 / 89 \\
09 / 14 / 89 \\
03 / 31 / 90 \\
01 / 09 / 91\end{array}$ & $\begin{array}{l}0001 \\
0001 \\
0001 \\
0001 \\
0001 \\
0001 \\
0001\end{array}$ & MG/L & $\begin{array}{l}< \\
< \\
< \\
<\end{array}$ & $\begin{array}{l}0.03 \\
0.01 \\
0.03 \\
0.01 \\
0.02 \\
0.02 \\
0.01\end{array}$ & $\begin{array}{l}\jmath \\
J\end{array}$ & $\begin{array}{l}0.02 \\
0.02 \\
0.02 \\
0.02 \\
0.02 \\
0.02 \\
0.01\end{array}$ & $\begin{array}{l}- \\
- \\
- \\
- \\
- \\
-\end{array}$ \\
\hline CYANIDE & $\begin{array}{l}10 / 02 / 85 \\
01 / 12 / 89 \\
09 / 14 / 89 \\
03 / 31 / 90\end{array}$ & $\begin{array}{l}0001 \\
0001 \\
0001 \\
0001\end{array}$ & MG/L & $\begin{array}{l}< \\
< \\
< \\
<\end{array}$ & $\begin{array}{l}0.01 \\
0.002 \\
0.01 \\
0.01\end{array}$ & $\mathbf{J}$ & $\begin{array}{l}0.01 \\
0.01 \\
0.01 \\
0.01\end{array}$ & - \\
\hline
\end{tabular}

PARAMETER VALUE IMDICATOR (PVI): < - LESS THAN DETECTION LIMIT

SAMPLE ID COOES:

DOO1 - FILTERED SAMPLE (.45 MICRONS)

OTHER PARAMETER VALUE FLAGS:

J. est IMATED ValUe 
GROUNDWATER OUALITY DATA BY LOCATION

SITE: AMBO1 AMBROSIA LAKE

LOCATION: 0620

NORTK COORDINATE: 52040.0 FT

EAST COORDINATE: $\quad 60740.0$ FT

06/10/80 TO 12/04/92

REPORT DATE: $08 / 04 / 94$

FORMATION OF COMPLETION: ALLUVIUM (AL)

HYDRAULIC FLON RELATIOHSHIP: CROSS GRADIENT (C)

\begin{tabular}{|c|c|c|c|c|c|c|c|c|}
\hline PARAMETER MAME & LOG DATE & $\begin{array}{c}\text { SAMPLE } \\
\text { ID }\end{array}$ & $\begin{array}{l}\text { UNITS OF } \\
\text { MEASURE }\end{array}$ & PVI & $\begin{array}{l}\text { PARAMETER } \\
\text { VALUE }\end{array}$ & FLAGS & $\begin{array}{l}\text { DETECTION } \\
\text { LIMIT }\end{array}$ & $\begin{array}{l}\text { PARAMETER } \\
\text { UNCERTAINTY }\end{array}$ \\
\hline CYANIDE & $01 / 09 / 91$ & 0001 & $M G / L$ & $<$ & 0.01 & & 0.01 & $\cdot$ \\
\hline FLUORIDE & $\begin{array}{l}10 / 02 / 85 \\
01 / 09 / 87 \\
07 / 15 / 88 \\
01 / 12 / 89 \\
09 / 14 / 89 \\
03 / 31 / 90 \\
01 / 09 / 91\end{array}$ & $\begin{array}{l}0001 \\
0001 \\
0001 \\
0001 \\
0001 \\
0001 \\
0001\end{array}$ & $M G / L$ & & $\begin{array}{l}0.8 \\
0.51 \\
0.4 \\
0.6 \\
0.5 \\
0.4 \\
0.5\end{array}$ & & $\begin{array}{l}0.1 \\
0.1 \\
0.1 \\
0.1 \\
0.1 \\
0.1 \\
0.1\end{array}$ & $\begin{array}{l}- \\
- \\
- \\
- \\
-\end{array}$ \\
\hline GROSS ALPHA & $\begin{array}{l}01 / 09 / 87 \\
07 / 15 / 88 \\
01 / 12 / 89 \\
09 / 14 / 89 \\
03 / 31 / 90 \\
01 / 09 / 91\end{array}$ & $\begin{array}{l}0001 \\
0001 \\
0001 \\
0001 \\
0001 \\
0001\end{array}$ & $\mathrm{PCl} / \mathrm{L}$ & & $\begin{array}{l}5300 . \\
5200 . \\
852 . \\
3200 . \\
3000 . \\
2178 .\end{array}$ & J & $\begin{array}{l}0.2 \\
0.2 \\
1 . \\
1 . \\
1 . \\
1 .\end{array}$ & $\begin{array}{l}200 . \\
200 . \\
71.4 \\
100 . \\
100 . \\
121 .\end{array}$ \\
\hline GROSS BETA & $\begin{array}{l}01 / 09 / 87 \\
07 / 15 / 88 \\
01 / 12 / 89 \\
09 / 14 / 89 \\
03 / 31 / 90 \\
01 / 09 / 91\end{array}$ & $\begin{array}{l}0001 \\
0001 \\
0001 \\
0001 \\
0001 \\
0001\end{array}$ & $\mathrm{PCI} / \mathrm{L}$ & & $\begin{array}{l}2200 . \\
1510 . \\
1130 . \\
1390 . \\
1500 . \\
1025 .\end{array}$ & & $\begin{array}{l}1 . \\
1 . \\
0.5 \\
0.5 \\
0.5 \\
0.5\end{array}$ & $\begin{array}{c}100 . \\
40 . \\
5.91 \\
40 . \\
100 . \\
44.1\end{array}$ \\
\hline IROH & $\begin{array}{l}10 / 02 / 85 \\
01 / 09 / 87 \\
07 / 15 / 88 \\
01 / 12 / 89 \\
09 / 14 / 89 \\
03 / 31 / 90 \\
01 / 09 / 91\end{array}$ & $\begin{array}{l}0001 \\
0001 \\
0001 \\
0001 \\
0001 \\
0001 \\
0001\end{array}$ & $M G / L$ & $\begin{array}{l}< \\
< \\
<\end{array}$ & $\begin{array}{l}0.03 \\
0.97 \\
0.09 \\
0.02 \\
0.09 \\
0.10 \\
0.03\end{array}$ & $\mathbf{J}$ & $\begin{array}{l}0.03 \\
0.03 \\
0.03 \\
0.03 \\
0.03 \\
0.03 \\
0.03\end{array}$ & $\begin{array}{l}- \\
- \\
- \\
- \\
-\end{array}$ \\
\hline LEAD & $\begin{array}{l}10 / 02 / 85 \\
07 / 15 / 88 \\
01 / 12 / 89 \\
09 / 14 / 89 \\
03 / 31 / 90 \\
01 / 09 / 91\end{array}$ & $\begin{array}{l}0001 \\
0001 \\
0001 \\
0001 \\
0001 \\
0001\end{array}$ & $M G / L$ & $\begin{array}{l}< \\
< \\
< \\
<\end{array}$ & $\begin{array}{l}0.01 \\
0.01 \\
0.001 \\
0.02 \\
0.01 \\
0.03\end{array}$ & $\begin{array}{l}2 \\
I\end{array}$ & $\begin{array}{l}0.01 \\
0.01 \\
0.01 \\
0.01 \\
0.01 \\
0.03\end{array}$ & $\begin{array}{l}- \\
: \\
- \\
-\end{array}$ \\
\hline LEAD-210 & $\begin{array}{l}10 / 02 / 85 \\
07 / 15 / 88 \\
01 / 12 / 89 \\
09 / 14 / 89\end{array}$ & $\begin{array}{l}0001 \\
0001 \\
0001 \\
0001\end{array}$ & $\mathrm{PCI} / \mathrm{L}$ & & $\begin{array}{c}102 . \\
0.7 \\
0.00 \\
0.0\end{array}$ & & $\begin{array}{l}1.5 \\
1.5 \\
1.5 \\
1.5\end{array}$ & $\begin{array}{l}4 . \\
0.9 \\
6.39 \\
0.7\end{array}$ \\
\hline MAGNESIU:A & $\begin{array}{l}10 / 02 / 85 \\
01 / 09 / 87 \\
07 / 15 / 88 \\
01 / 12 / 89 \\
09 / 14 / 89 \\
03 / 31 / 90 \\
01 / 09 / 91\end{array}$ & $\begin{array}{l}0001 \\
0001 \\
0001 \\
0001 \\
0001 \\
0001 \\
0001\end{array}$ & $M G / L$ & & $\begin{array}{l}142 . \\
145 . \\
131 . \\
130 . \\
131 . \\
131 . \\
135 .\end{array}$ & & $\begin{array}{l}0.001 \\
0.001 \\
0.001 \\
0.001 \\
0.001 \\
0.001 \\
0.5\end{array}$ & $\begin{array}{l}- \\
- \\
- \\
- \\
-\end{array}$ \\
\hline MANGANESE & $\begin{array}{l}10 / 02 / 85 \\
01 / 09 / 87 \\
07 / 15 / 88 \\
01 / 12 / 89\end{array}$ & $\begin{array}{l}0001 \\
0001 \\
0001 \\
0001\end{array}$ & HG/L & $<$ & $\begin{array}{l}0.02 \\
0.07 \\
0.03 \\
0.01\end{array}$ & & $\begin{array}{l}0.01 \\
0.01 \\
0.01 \\
0.01\end{array}$ & $\begin{array}{l}- \\
-\end{array}$ \\
\hline
\end{tabular}

PARAMETER VALUE Indicator (PVI): < - LeSS THAN DETECTIOH LIMIT

SAMPLE ID COOES:

0001 - FILTERED SAMPLE (.45 MICRONS)

OTHER PARAMETER VALUE FLAGS:

1 - IMCREASED DETECTION LIMIT DUE TO REQUIRED DILUTION

J - ESTIMATED VALUE 
GROUNDHATER QUALITY DATA BY LOCATION

SITE: AMBOI AMBROSIA LAKE

LOCATION: 0620

NORTH COORDINATE: $\quad 52040.0$ FT

EAST COORDINATE: $\quad 60740.0$ FT

$06 / 10 / 80$ TO $12 / 04 / 92$

REPORT DATE : 08/04/94

FORMATIOM OF COMPLETION: ALLUVIUM (AL)

HYDRAULIC FLOW RELATIONSHIP: CROSS GRADIENT (C)

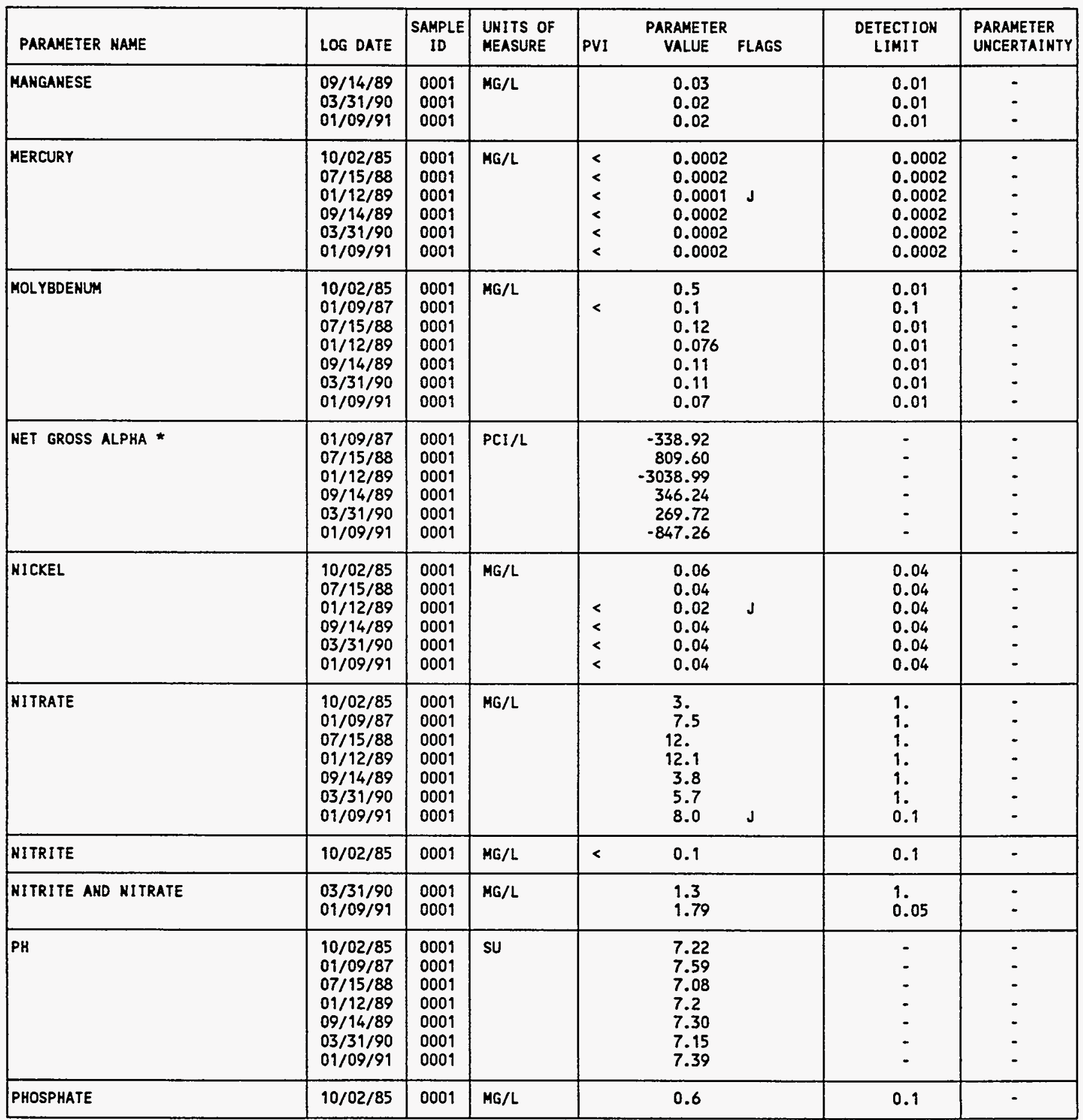

- Net gross alPHa (GROSS ALPHA - URANIUM) WITH 1 Mg URANIUM = 686 PCI

PARAMETER VALUE INDICATOR (PVI): < - LESS THAN DETECTION LIMIT SAMPLE ID COOES:

0001 - FILTERED SAMPLE (.45 MICRONS)

OTHER PARAMETER VALUE FLAGS:

$J$ - estIMATED VALUE 
GROUNDWATER OUALITY DATA BY LOCATION

SITE: AMBO1 AMBROSIA LAKE

LOCATIOH: 0620

NORTH COORDINATE: 52040.0 FT

EAST COORDIHATE: 60740.0 FT

06/10/80 TO $12 / 04 / 92$

REPORT DATE: $08 / 04 / 94$

FORMATION OF COMPLETION: ALLUVIUN (AL)

HYDRAULIC FLON RELATIOHSHIP: CROSS GRADIENT (C)

\begin{tabular}{|c|c|c|c|c|c|c|c|}
\hline PARAMETER MAME & LOG DATE & $\begin{array}{l}\text { SAMPLE } \\
\text { IO }\end{array}$ & $\begin{array}{l}\text { UHITS OF } \\
\text { MEASURE }\end{array}$ & PVI & $\begin{array}{l}\text { PARAMETER } \\
\text { VALUE FLAGS }\end{array}$ & $\begin{array}{l}\text { DETECTION } \\
\text { LIMIT }\end{array}$ & $\begin{array}{l}\text { PARAMETER } \\
\text { UNCERTAINTY }\end{array}$ \\
\hline PHOSPHATE & $\begin{array}{l}07 / 15 / 88 \\
01 / 12 / 89 \\
09 / 14 / 89 \\
03 / 31 / 90 \\
01 / 09 / 91\end{array}$ & $\begin{array}{l}0001 \\
0001 \\
0001 \\
0001 \\
0001\end{array}$ & MG/L & $\begin{array}{l}< \\
< \\
<\end{array}$ & $\begin{array}{l}0.1 \\
0.1 \\
0.1 \\
0.1 \\
0.12\end{array}$ & $\begin{array}{l}0.1 \\
0.1 \\
0.1 \\
0.1 \\
0.05\end{array}$ & $\begin{array}{l}- \\
- \\
- \\
-\end{array}$ \\
\hline POLONIUM-210 & $\begin{array}{l}10 / 02 / 85 \\
07 / 15 / 88 \\
01 / 12 / 89 \\
09 / 14 / 89\end{array}$ & $\begin{array}{l}0001 \\
0001 \\
0001 \\
0001\end{array}$ & $\mathrm{PCI} / \mathrm{L}$ & & $\begin{array}{l}1.9 \\
0.7 \\
0.311 \quad J \\
1.7\end{array}$ & $\begin{array}{l}1 . \\
1 . \\
1 . \\
1 .\end{array}$ & $\begin{array}{l}0.9 \\
0.5 \\
1.22 \\
0.5\end{array}$ \\
\hline POTASSIUA & $\begin{array}{l}10 / 02 / 85 \\
01 / 09 / 87 \\
07 / 15 / 88 \\
01 / 12 / 89 \\
09 / 14 / 89 \\
03 / 31 / 90 \\
01 / 09 / 91\end{array}$ & $\begin{array}{l}0001 \\
0001 \\
0001 \\
0001 \\
0001 \\
0001 \\
0001\end{array}$ & MG/L & & $\begin{array}{l}1.12 \\
0.86 \\
0.92 \\
1.3 \\
1.0 \\
1.5 \\
0.84\end{array}$ & $\begin{array}{l}0.01 \\
0.01 \\
0.01 \\
0.01 \\
0.01 \\
0.01 \\
0.01\end{array}$ & $\begin{array}{l}- \\
- \\
- \\
- \\
- \\
-\end{array}$ \\
\hline RADIUH-226 & $\begin{array}{l}10 / 02 / 85 \\
01 / 09 / 87 \\
07 / 15 / 88 \\
01 / 12 / 89 \\
09 / 14 / 89 \\
03 / 31 / 90 \\
01 / 09 / 91\end{array}$ & $\begin{array}{l}0001 \\
0001 \\
0001 \\
0001 \\
0001 \\
0001 \\
0001\end{array}$ & $\mathrm{PCI} / \mathrm{L}$ & & $\begin{array}{l}2.9 \\
0.2 \\
0.1 \\
9.13 \\
0.0 \\
0.0 \\
0.7\end{array}$ & $\begin{array}{l}1 . \\
1 . \\
1 . \\
1 . \\
1 . \\
1 . \\
1 .\end{array}$ & $\begin{array}{l}0.6 \\
0.3 \\
0.2 \\
1.03 \\
0.1 \\
0.1 \\
0.4\end{array}$ \\
\hline RADIUM-226 + RADIUH- 228 & $\begin{array}{l}10 / 02 / 85 \\
01 / 09 / 87 \\
07 / 15 / 88 \\
01 / 12 / 89 \\
09 / 14 / 89 \\
03 / 31 / 90 \\
01 / 09 / 91\end{array}$ & $\begin{array}{l}0001 \\
0001 \\
0001 \\
0001 \\
0001 \\
0001 \\
0001\end{array}$ & $\mathrm{PCI} / \mathrm{L}$ & & $\begin{array}{l}3.80 \\
0.20 \\
0.40 \\
9.13 \\
0.50 \\
1.00 \\
3.00\end{array}$ & $\begin{array}{l}- \\
- \\
- \\
- \\
- \\
-\end{array}$ & $\begin{array}{l}- \\
- \\
- \\
- \\
- \\
-\end{array}$ \\
\hline RADIUH-228 & $\begin{array}{l}10 / 02 / 85 \\
01 / 09 / 87 \\
07 / 15 / 88 \\
01 / 12 / 89 \\
09 / 14 / 89 \\
03 / 31 / 90 \\
01 / 09 / 91\end{array}$ & $\begin{array}{l}0001 \\
0001 \\
0001 \\
0001 \\
0001 \\
0001 \\
0001\end{array}$ & $\mathrm{PCI} / \mathrm{L}$ & & $\begin{array}{l}0.9 \\
0.0 \\
0.3 \\
0.00 \\
0.5 \\
1.0 \\
2.3\end{array}$ & $\begin{array}{l}1 . \\
1 . \\
1 . \\
1 . \\
1 . \\
1 . \\
1 .\end{array}$ & $\begin{array}{l}1.1 \\
1.1 \\
0.9 \\
6.32 \\
0.8 \\
0.8 \\
1.6\end{array}$ \\
\hline SELENIUM & $\begin{array}{l}10 / 02 / 85 \\
01 / 09 / 87 \\
07 / 15 / 88 \\
01 / 12 / 89 \\
09 / 14 / 89 \\
03 / 31 / 90 \\
01 / 09 / 91\end{array}$ & $\begin{array}{l}0001 \\
0001 \\
0001 \\
0001 \\
0001 \\
0001 \\
0001\end{array}$ & MG/L & & $\begin{array}{l}0.011 \\
0.008 \\
0.058 \\
0.070 \\
0.040 \\
0.024 \\
0.035\end{array}$ & $\begin{array}{l}0.005 \\
0.005 \\
0.005 \\
0.005 \\
0.005 \\
0.005 \\
0.005\end{array}$ & $\begin{array}{l}- \\
- \\
- \\
- \\
-\end{array}$ \\
\hline SILICA - SIOZ & $\begin{array}{l}10 / 02 / 85 \\
07 / 15 / 88 \\
01 / 12 / 89 \\
09 / 14 / 89 \\
03 / 31 / 90 \\
01 / 09 / 91\end{array}$ & $\begin{array}{l}0001 \\
0001 \\
0001 \\
0001 \\
0001 \\
0001\end{array}$ & MG/L & & $\begin{array}{l}11 . \\
13.9 \\
12.0 \\
14 . \\
15 . \\
13.1\end{array}$ & $\begin{array}{l}2 . \\
2 . \\
2 . \\
2 . \\
2 . \\
0.1\end{array}$ & $\begin{array}{l}- \\
- \\
-\end{array}$ \\
\hline
\end{tabular}

PARAMETER VALUE IMDICATOR (PVI): < - LESS THAN DETECTION LIMIT

SAMPLE ID COOES:

0001 - FILTERED SAMPLE (.45 MICRONS)

OTHER PARAMETER VALUE FLAGS:

$J$ - ESTIMATED VALUE 
GROUNDWATER QUALITY DATA BY LOCATION

SITE: AMBOI AMBROSIA LAKE

LOCATION: 0620

NORTH COORDINATE: $\quad 52040.0$ FT

EAST COORDINATE: 60740.0 FT

06/10/80 TO $12 / 04 / 92$

REPORT DATE: 08/04/94

FORMATION OF COMPLETION: ALLUVIUM (AL)

HYDRAULIC FLOW RELATIOHSHIP: CROSS GRADIENT (C)

\begin{tabular}{|c|c|c|c|c|c|c|c|}
\hline PARAMETER NAME & LOG DATE & $\begin{array}{c}\text { SAMPLE } \\
\text { ID }\end{array}$ & $\begin{array}{l}\text { UNITS OF } \\
\text { MEASURE }\end{array}$ & PVI & $\begin{array}{l}\text { PARAMETER } \\
\text { VALUE FLAGS }\end{array}$ & $\begin{array}{l}\text { DETECTIOH } \\
\text { LIMIT }\end{array}$ & $\begin{array}{l}\text { PARAMETER } \\
\text { UNCERTAINTY }\end{array}$ \\
\hline SILVER & $\begin{array}{l}10 / 02 / 85 \\
01 / 09 / 87 \\
07 / 15 / 88 \\
01 / 12 / 89 \\
09 / 14 / 89 \\
03 / 31 / 90 \\
01 / 09 / 91\end{array}$ & $\begin{array}{l}0001 \\
0001 \\
0001 \\
0001 \\
0001 \\
0001 \\
0001\end{array}$ & $M G / L$ & $\begin{array}{l}< \\
< \\
< \\
< \\
<\end{array}$ & $\begin{array}{l}0.01 \\
0.01 \\
0.04 \\
0.01 \\
0.01 \\
0.01 \\
0.01\end{array}$ & $\begin{array}{l}0.01 \\
0.01 \\
0.01 \\
0.01 \\
0.01 \\
0.01 \\
0.01\end{array}$ & $\begin{array}{l}- \\
- \\
- \\
- \\
-\end{array}$ \\
\hline SOOIUM & $\begin{array}{l}10 / 02 / 85 \\
01 / 09 / 87 \\
07 / 15 / 88 \\
01 / 12 / 89 \\
09 / 14 / 89 \\
03 / 31 / 90 \\
01 / 09 / 91\end{array}$ & $\begin{array}{l}0001 \\
0001 \\
0001 \\
0001 \\
0001 \\
0001 \\
0001\end{array}$ & $M G / L$ & & $\begin{array}{l}434 . \\
439 . \\
366 . \\
440 . \\
415 . \\
421 . \\
435\end{array}$ & $\begin{array}{l}0.002 \\
0.002 \\
0.002 \\
0.002 \\
0.002 \\
0.002 \\
5 .\end{array}$ & $\begin{array}{l}- \\
- \\
- \\
- \\
- \\
-\end{array}$ \\
\hline SPECIFIC CONDUCTANCE & $\begin{array}{l}10 / 02 / 85 \\
01 / 09 / 87 \\
07 / 15 / 88 \\
01 / 12 / 89 \\
09 / 14 / 89 \\
03 / 31 / 90 \\
01 / 09 / 91\end{array}$ & $\begin{array}{l}0001 \\
0001 \\
0001 \\
0001 \\
0001 \\
0001 \\
0001\end{array}$ & UМHO/CM & & $\begin{array}{l}3100 . \\
2850 \\
2750 \\
2600 \\
1300 \\
2700 \\
2800\end{array}$ & $\begin{array}{l}- \\
- \\
- \\
- \\
- \\
-\end{array}$ & $\begin{array}{l}- \\
- \\
- \\
- \\
- \\
-\end{array}$ \\
\hline STRONT IUM & $\begin{array}{l}10 / 02 / 85 \\
07 / 15 / 88 \\
01 / 12 / 89 \\
09 / 14 / 89 \\
03 / 31 / 90 \\
01 / 09 / 91\end{array}$ & $\begin{array}{l}0001 \\
0001 \\
0001 \\
0001 \\
0001 \\
0001\end{array}$ & $M G / L$ & & $\begin{array}{l}8.4 \\
6.2 \\
7.40 \\
6.9 \\
6.86 \\
7.15\end{array}$ & $\begin{array}{l}0.1 \\
0.1 \\
0.1 \\
0.1 \\
0.1 \\
0.01\end{array}$ & $\begin{array}{l}- \\
- \\
- \\
- \\
-\end{array}$ \\
\hline SULFATE & $\begin{array}{l}10 / 02 / 85 \\
01 / 09 / 87 \\
07 / 15 / 88 \\
01 / 12 / 89 \\
09 / 14 / 89 \\
03 / 31 / 90 \\
01 / 09 / 91\end{array}$ & $\begin{array}{l}0001 \\
0001 \\
0001 \\
0001 \\
0001 \\
0001 \\
0001\end{array}$ & MG/L & & $\begin{array}{l}2440 \\
2310 \\
2100 \\
2354 \\
2370 \\
2380 \\
2380\end{array}$ & $\begin{array}{l}0.1 \\
0.1 \\
0.1 \\
0.1 \\
0.1 \\
0.1 \\
10 .\end{array}$ & $\begin{array}{l}- \\
- \\
- \\
- \\
- \\
-\end{array}$ \\
\hline SULFIDE & $\begin{array}{l}10 / 02 / 85 \\
07 / 15 / 88 \\
01 / 12 / 89 \\
09 / 14 / 89 \\
03 / 31 / 90 \\
01 / 09 / 91\end{array}$ & $\begin{array}{l}0001 \\
0001 \\
0001 \\
0001 \\
0001 \\
0001\end{array}$ & $M G / L$ & $\begin{array}{l}< \\
< \\
< \\
< \\
< \\
<\end{array}$ & $\begin{array}{l}0.1 \\
0.1 \\
0.01 \\
0.1 \\
0.1 \\
0.1\end{array}$ & $\begin{array}{l}0.1 \\
0.1 \\
0.1 \\
0.1 \\
0.1 \\
0.1\end{array}$ & $\begin{array}{l}- \\
- \\
- \\
-\end{array}$ \\
\hline TEMPERATURE & $\begin{array}{l}10 / 02 / 85 \\
01 / 09 / 87 \\
07 / 15 / 88 \\
01 / 12 / 89 \\
09 / 14 / 89 \\
03 / 31 / 90 \\
01 / 09 / 91\end{array}$ & $\begin{array}{l}0001 \\
0001 \\
0001 \\
0001 \\
0001 \\
0001 \\
0001\end{array}$ & $C \cdot$ DEGREE & & $\begin{array}{l}12 . \\
12 . \\
13 . \\
11.0 \\
13.0 \\
12 . \\
12.5\end{array}$ & $\begin{array}{l}- \\
- \\
- \\
- \\
-\end{array}$ & $\begin{array}{l}- \\
- \\
- \\
- \\
- \\
-\end{array}$ \\
\hline THALLIUM & $\begin{array}{l}01 / 12 / 89 \\
09 / 14 / 89\end{array}$ & $\begin{array}{l}0001 \\
0001\end{array}$ & MG/L & $<$ & $\begin{array}{l}0.005 \quad 1 \\
0.01\end{array}$ & $\begin{array}{l}0.005 \\
0.01\end{array}$ & - \\
\hline
\end{tabular}

PARAMETER VALUE INDICATOR (PVI): < - LESS THAN DETECTION LIMIT

SAMPLE ID COOES:

0001 - FILTERED SAMPLE (.45 MICROHS)

OTHER PARAMETER VALUE FLAGS:

I - INCREASED DETECTION LIMIT DUE TO REQUIRED DILUTION

$J$ - ESTIMATED VALUE 
GROUHDWATER QUALITY DATA BY LOCATION

SITE: AMBOI AMBROSIA LAKE

LOCATION: 0620

HORTH COORDINATE: 52040.0 FT

EAST COORDIHATE: $\quad 60740.0$ FT

$06 / 10 / 80$ TO $12 / 04 / 92$

REPORT DATE: 08/04/94

FORMATION OF COMPLETIOH: ALLUVIUH (AL)

HYDRAULIC FLOH RELATIONSHIP: CROSS GRADIENT (C)

\begin{tabular}{|c|c|c|c|c|c|c|c|c|}
\hline PARAMETER HAME & LOG DATE & $\underset{\text { ID }}{\text { SAMPLE }}$ & $\begin{array}{l}\text { UHITS OF } \\
\text { MEASURE }\end{array}$ & PVI & $\begin{array}{l}\text { PARAMETER } \\
\text { VALUE }\end{array}$ & FLAGS & $\begin{array}{l}\text { DETECTION } \\
\text { LIMIT }\end{array}$ & $\begin{array}{l}\text { PARAMETER } \\
\text { UNCERTAINTY }\end{array}$ \\
\hline THALLILA & $\begin{array}{l}03 / 31 / 90 \\
01 / 09 / 91\end{array}$ & $\begin{array}{l}0001 \\
0001\end{array}$ & $M G / L$ & $<$ & $\begin{array}{l}0.01 \\
0.03\end{array}$ & I & $\begin{array}{l}0.01 \\
0.03\end{array}$ & - \\
\hline THORIUN-230 & $\begin{array}{l}10 / 02 / 85 \\
07 / 15 / 88 \\
01 / 12 / 89 \\
09 / 14 / 89\end{array}$ & $\begin{array}{l}0001 \\
0001 \\
0001 \\
0001\end{array}$ & $\mathrm{PCI} / \mathrm{L}$ & & $\begin{array}{l}4 . \\
0.2 \\
5.14 \\
0.1\end{array}$ & & $\begin{array}{l}1 . \\
1 . \\
1 . \\
1 .\end{array}$ & $\begin{array}{l}1.2 \\
0.4 \\
0.660 \\
0.4\end{array}$ \\
\hline TIH & $\begin{array}{l}10 / 02 / 85 \\
07 / 15 / 88 \\
01 / 12 / 89 \\
09 / 14 / 89 \\
03 / 31 / 90 \\
01 / 09 / 91\end{array}$ & $\begin{array}{l}0001 \\
0001 \\
0001 \\
0001 \\
0001 \\
0001\end{array}$ & $M G / L$ & $\begin{array}{l}< \\
< \\
< \\
<\end{array}$ & $\begin{array}{l}0.005 \\
0.010 \\
0.001 \\
0.1 \\
0.005 \\
0.03\end{array}$ & $\begin{array}{l}d \\
7 \\
7\end{array}$ & $\begin{array}{l}0.005 \\
0.005 \\
0.005 \\
0.1 \\
0.005 \\
0.03\end{array}$ & $\begin{array}{l}- \\
: \\
: \\
:\end{array}$ \\
\hline TOTAL DISSOLVED SOLIDS & $\begin{array}{l}10 / 02 / 85 \\
01 / 09 / 87 \\
07 / 15 / 88 \\
01 / 12 / 89 \\
09 / 14 / 89 \\
03 / 31 / 90 \\
01 / 09 / 91\end{array}$ & $\begin{array}{l}0001 \\
0001 \\
0001 \\
0001 \\
0001 \\
0001 \\
0001\end{array}$ & MG/L & & $\begin{array}{l}4060 . \\
4000 . \\
3940 \\
3782 \\
3680 \\
3550 \\
3860\end{array}$ & & $\begin{array}{l}10 . \\
10 . \\
10 . \\
10 . \\
10 . \\
10 . \\
10 .\end{array}$ & $\begin{array}{l}- \\
- \\
- \\
-\end{array}$ \\
\hline TOTAL KJELDAHL NITROGEN & $01 / 09 / 91$ & 0001 & $M G / L$ & & 2. & & 1. & - \\
\hline TOTAL ORGANIC CARBOH & $\begin{array}{l}10 / 02 / 85 \\
07 / 15 / 88 \\
01 / 12 / 89 \\
09 / 14 / 89 \\
03 / 31 / 90 \\
01 / 09 / 91\end{array}$ & $\begin{array}{l}0001 \\
0001 \\
0001 \\
0001 \\
0001 \\
0001\end{array}$ & $M G / L$ & & $\begin{array}{l}63 . \\
78.6 \\
15 . \\
79.1 \\
5.2 \\
4 .\end{array}$ & $J$ & $\begin{array}{l}1 . \\
1 . \\
1 . \\
1 . \\
1 .\end{array}$ & $\begin{array}{l}- \\
- \\
-\end{array}$ \\
\hline URAKIUM & $\begin{array}{l}10 / 02 / 85 \\
01 / 09 / 87 \\
07 / 15 / 88 \\
01 / 12 / 89 \\
09 / 14 / 89 \\
03 / 31 / 90 \\
01 / 09 / 91\end{array}$ & $\begin{array}{l}0001 \\
0001 \\
0001 \\
0001 \\
0001 \\
0001 \\
0001\end{array}$ & $M G / L$ & & $\begin{array}{l}5.34 \\
8.22 \\
6.40 \\
5.672 \\
4.16 \\
3.98 \\
4.41\end{array}$ & & $\begin{array}{l}0.003 \\
0.003 \\
0.003 \\
0.003 \\
0.003 \\
0.003 \\
0.001\end{array}$ & $\begin{array}{l}: \\
: \\
: \\
:\end{array}$ \\
\hline VANADIUM & $\begin{array}{l}10 / 02 / 85 \\
01 / 09 / 87 \\
07 / 15 / 88 \\
01 / 12 / 89 \\
09 / 14 / 89 \\
03 / 31 / 90 \\
01 / 09 / 91\end{array}$ & $\begin{array}{l}0001 \\
0001 \\
0001 \\
0001 \\
0001 \\
0001 \\
0001\end{array}$ & $M G / L$ & $\begin{array}{l}< \\
< \\
< \\
< \\
<\end{array}$ & $\begin{array}{l}0.01 \\
0.01 \\
0.07 \\
0.01 \\
0.01 \\
0.01 \\
0.01\end{array}$ & & $\begin{array}{l}0.01 \\
0.01 \\
0.01 \\
0.01 \\
0.01 \\
0.01 \\
0.01\end{array}$ & $\begin{array}{l}- \\
- \\
- \\
- \\
-\end{array}$ \\
\hline ZINC & $\begin{array}{l}10 / 02 / 85 \\
07 / 15 / 88 \\
01 / 12 / 89 \\
09 / 14 / 89 \\
03 / 31 / 90 \\
01 / 09 / 91\end{array}$ & $\begin{array}{l}0001 \\
0001 \\
0001 \\
0001 \\
0001 \\
0001\end{array}$ & MG/L & $\begin{array}{l}< \\
< \\
<\end{array}$ & $\begin{array}{l}0.017 \\
0.011 \\
0.01 \\
0.005 \\
0.005 \\
0.005\end{array}$ & & $\begin{array}{l}0.005 \\
0.005 \\
0.01 \\
0.005 \\
0.005 \\
0.005\end{array}$ & : \\
\hline
\end{tabular}

PARAMETER VALUE IMOICATOR (PVI): < - LESS THAN DETECTION LIMIT

SAMPLE ID COOES:

0001 - FILTERED SAMPLE (.45 MICRONS)

OTHER PARAMETER VALUE FLAGS:

1 - INCREASED DETECTIOH LIMIT DUE TO REQUIRED DILUTION

J - ESTIMATED VALUE 
GROUNDHATER QUALITY DATA BY LOCATION

SITE: AMBO1 AMBROSIA LAKE

LOCATION: 0650

NORTH COORDINATE: $\quad 49990.0$ FT

EAST COORDIHATE: $\quad 51010.0$ FT

06/10/80 TO $12 / 04 / 92$

REPORT DATE: 08/04/94

FORMATION OF COMPLETION: ALLUVIUM (AL)

HYDRAULIC FLON RELATIONSHIP: UNKNOWN (N)

\begin{tabular}{|c|c|c|c|c|c|c|c|}
\hline PARAMETER NAME & LOG DATE & $\begin{array}{c}\text { SAMPLE } \\
\text { ID }\end{array}$ & $\begin{array}{l}\text { UNITS OF } \\
\text { MEASURE }\end{array}$ & PVI & $\begin{array}{l}\text { PARAMETER } \\
\text { VALUE FLAGS }\end{array}$ & $\begin{array}{l}\text { DETECTIOH } \\
\text { LIHIT }\end{array}$ & $\begin{array}{l}\text { PARAMETER } \\
\text { UNCERTAINTY }\end{array}$ \\
\hline ALKALIHITY & $\begin{array}{l}05 / 22 / 86 \\
05 / 22 / 86 \\
05 / 22 / 86 \\
05 / 22 / 86 \\
05 / 22 / 86 \\
01 / 09 / 87\end{array}$ & $\begin{array}{l}0001 \\
0002 \\
0003 \\
0004 \\
0005 \\
0001\end{array}$ & MG/L CACO3 & & $\begin{array}{l}260 . \\
260 . \\
260 \\
260 \\
260 \\
283\end{array}$ & $\begin{array}{l}- \\
- \\
- \\
- \\
-\end{array}$ & $\begin{array}{l}- \\
- \\
- \\
- \\
-\end{array}$ \\
\hline ARSENIC & $\begin{array}{l}05 / 22 / 86 \\
05 / 22 / 86 \\
05 / 22 / 86 \\
05 / 22 / 86 \\
05 / 22 / 86 \\
01 / 09 / 87\end{array}$ & $\begin{array}{l}0001 \\
0002 \\
0003 \\
0004 \\
0005 \\
0001\end{array}$ & $M G / L$ & $\begin{array}{l}< \\
< \\
< \\
< \\
<\end{array}$ & $\begin{array}{l}0.01 \\
0.01 \\
0.01 \\
0.01 \\
0.01 \\
0.003\end{array}$ & $\begin{array}{l}0.01 \\
0.01 \\
0.01 \\
0.01 \\
0.01 \\
0.01\end{array}$ & $\begin{array}{l}- \\
- \\
- \\
- \\
-\end{array}$ \\
\hline BOROK & $\begin{array}{l}05 / 22 / 86 \\
05 / 22 / 86 \\
05 / 22 / 86 \\
05 / 22 / 86 \\
05 / 22 / 86 \\
01 / 09 / 87\end{array}$ & $\begin{array}{l}0001 \\
0002 \\
0003 \\
0004 \\
0005 \\
0001\end{array}$ & MG/L & & $\begin{array}{l}0.5 \\
0.6 \\
0.5 \\
0.5 \\
0.5 \\
0.21\end{array}$ & $\begin{array}{l}0.1 \\
0.1 \\
0.1 \\
0.1 \\
0.1 \\
0.1\end{array}$ & $\begin{array}{l}- \\
- \\
- \\
- \\
-\end{array}$ \\
\hline CADMIUM & $\begin{array}{l}05 / 22 / 86 \\
05 / 22 / 86 \\
05 / 22 / 86 \\
05 / 22 / 86 \\
05 / 22 / 86\end{array}$ & $\begin{array}{l}0001 \\
0002 \\
0003 \\
0004 \\
0005\end{array}$ & MG/L & $\begin{array}{l}< \\
< \\
< \\
< \\
<\end{array}$ & $\begin{array}{l}0.001 \\
0.001 \\
0.001 \\
0.001 \\
0.001\end{array}$ & $\begin{array}{l}0.001 \\
0.001 \\
0.001 \\
0.001 \\
0.001\end{array}$ & $\begin{array}{l}- \\
- \\
- \\
-\end{array}$ \\
\hline CALCIUM & $\begin{array}{l}05 / 22 / 86 \\
05 / 22 / 86 \\
05 / 22 / 86 \\
05 / 22 / 86 \\
05 / 22 / 86 \\
01 / 09 / 87\end{array}$ & $\begin{array}{l}0001 \\
0002 \\
0003 \\
0004 \\
0005 \\
0001\end{array}$ & $M G / L$ & & $\begin{array}{l}565 . \\
565 . \\
565 . \\
565 . \\
565 . \\
551 .\end{array}$ & $\begin{array}{l}0.01 \\
0.01 \\
0.01 \\
0.01 \\
0.01 \\
0.01\end{array}$ & $\begin{array}{l}- \\
- \\
- \\
-\end{array}$ \\
\hline CHLORIDE & $\begin{array}{l}05 / 22 / 86 \\
05 / 22 / 86 \\
05 / 22 / 86 \\
05 / 22 / 86 \\
05 / 22 / 86 \\
01 / 09 / 87\end{array}$ & $\begin{array}{l}0001 \\
0002 \\
0003 \\
0004 \\
0005 \\
0001\end{array}$ & $M G / L$ & & $\begin{array}{l}240 \\
240 \\
240 \\
240 \\
240 \\
200\end{array}$ & $\begin{array}{l}1 . \\
1 . \\
1 . \\
1 . \\
1 .\end{array}$ & $\begin{array}{l}- \\
- \\
- \\
- \\
-\end{array}$ \\
\hline CHRONIUH & $\begin{array}{l}05 / 22 / 86 \\
05 / 22 / 86 \\
05 / 22 / 86 \\
05 / 22 / 86 \\
05 / 22 / 86 \\
01 / 09 / 87\end{array}$ & $\begin{array}{l}0001 \\
0002 \\
0003 \\
0004 \\
0005 \\
0001\end{array}$ & $M G / L$ & & $\begin{array}{l}0.04 \\
0.04 \\
0.04 \\
0.04 \\
0.04 \\
0.02\end{array}$ & $\begin{array}{l}0.01 \\
0.01 \\
0.01 \\
0.01 \\
0.01 \\
0.01\end{array}$ & $\begin{array}{l}- \\
- \\
- \\
-\end{array}$ \\
\hline COBALT & $\begin{array}{l}05 / 22 / 86 \\
05 / 22 / 86 \\
05 / 22 / 86 \\
05 / 22 / 86 \\
05 / 22 / 86 \\
01 / 09 / 87\end{array}$ & $\begin{array}{l}0001 \\
0002 \\
0003 \\
0004 \\
0005 \\
0001\end{array}$ & $M G / L$ & & $\begin{array}{l}0.08 \\
0.08 \\
0.08 \\
0.08 \\
0.08 \\
0.05\end{array}$ & $\begin{array}{l}0.05 \\
0.05 \\
0.05 \\
0.05 \\
0.05 \\
0.05\end{array}$ & $\begin{array}{l}- \\
- \\
- \\
- \\
-\end{array}$ \\
\hline COPPER & $01 / 09 / 87$ & 0001 & $M G / L$ & $<$ & 0.01 & 0.02 & - \\
\hline
\end{tabular}

PARAMETER VALUE INDICATOR (PVI): < - LESS THAN DETECTION LIMIT

SAMPLE ID CODES:

OTHER PARAMETER VALUE FLAGS:

0001 - FILTERED SAMPLE (.45 MICRONS)

$d$ - ESTIMATED VALUE 
GROUNDWATER OUALITY DATA BY LOCATION

SITE: AMBOI AMBROSIA LAKE

LOCATION: O650

NORTH COORDINATE: $\quad 49990.0$ FT

EAST COORDINATE: $\quad 59010.0$ FT

$06 / 10 / 80$ TO $12 / 04 / 92$

REPORT DATE: $08 / 04 / 94$

FORMATIOH OF CONPLETION: ALLUVIUM (AL)

HYDRAULIC FLOW RELATIONSHIP: UHKNOWN (H)

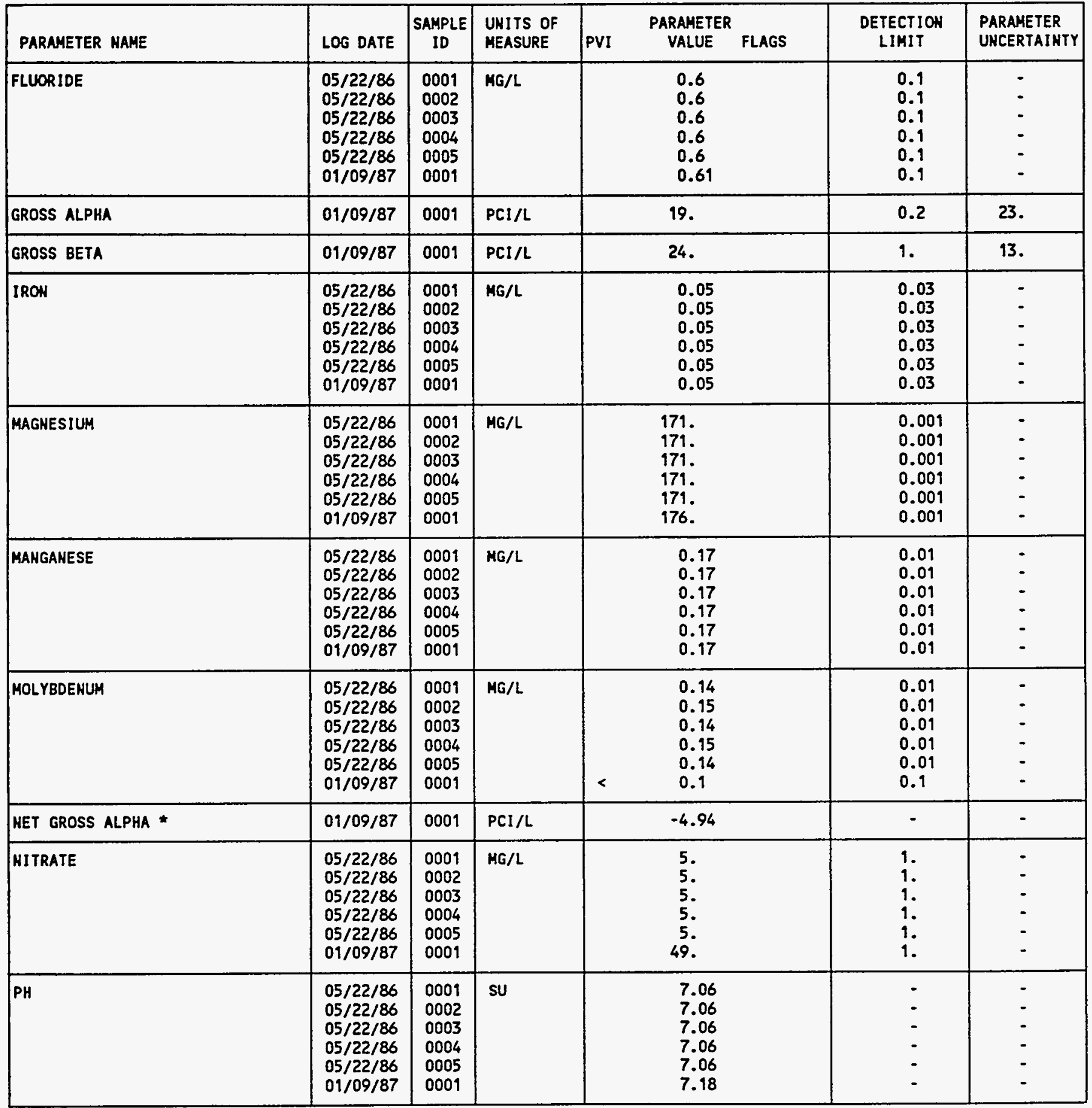

- het GROSS ALPHA (GROSS ALPHA - URANIUA)

WITH 1 MG URANIUA $=686 \mathrm{PCI}$

PARAMETER VALUE IHDICATOR (PVI): < - LESS THAN DETECTION LIMIT
SAMPLE ID CODES:

0001 - FILTERED SAMPLE (.45 MICROHS)

OOO2 - FILTERED REPLICATE SAMPLE (.45 MICRONS)

0003 - FILTERED REPLICATE SAMPLE (.45 MICRONS)

0004 - FILTERED REPLICATE SAMPLE (.45 MICRONS) 
GROUNDWATER QUALITY DATA BY LOCATION

SITE: AMBOI AMBROSIA LAKE

LOCATION: 0650

NORTH COORDINATE: $\quad 49990.0$ FT

EAST COORDINATE: $\quad 51010.0$ FT

06/10/80 TO $12 / 04 / 92$

REPORT DATE: 08/04/94

FORMATION OF COMPLETION: ALLUVIUM (AL)

HYDRAULIC FLOW RELATIONSHIP: UNKNOWN (N)

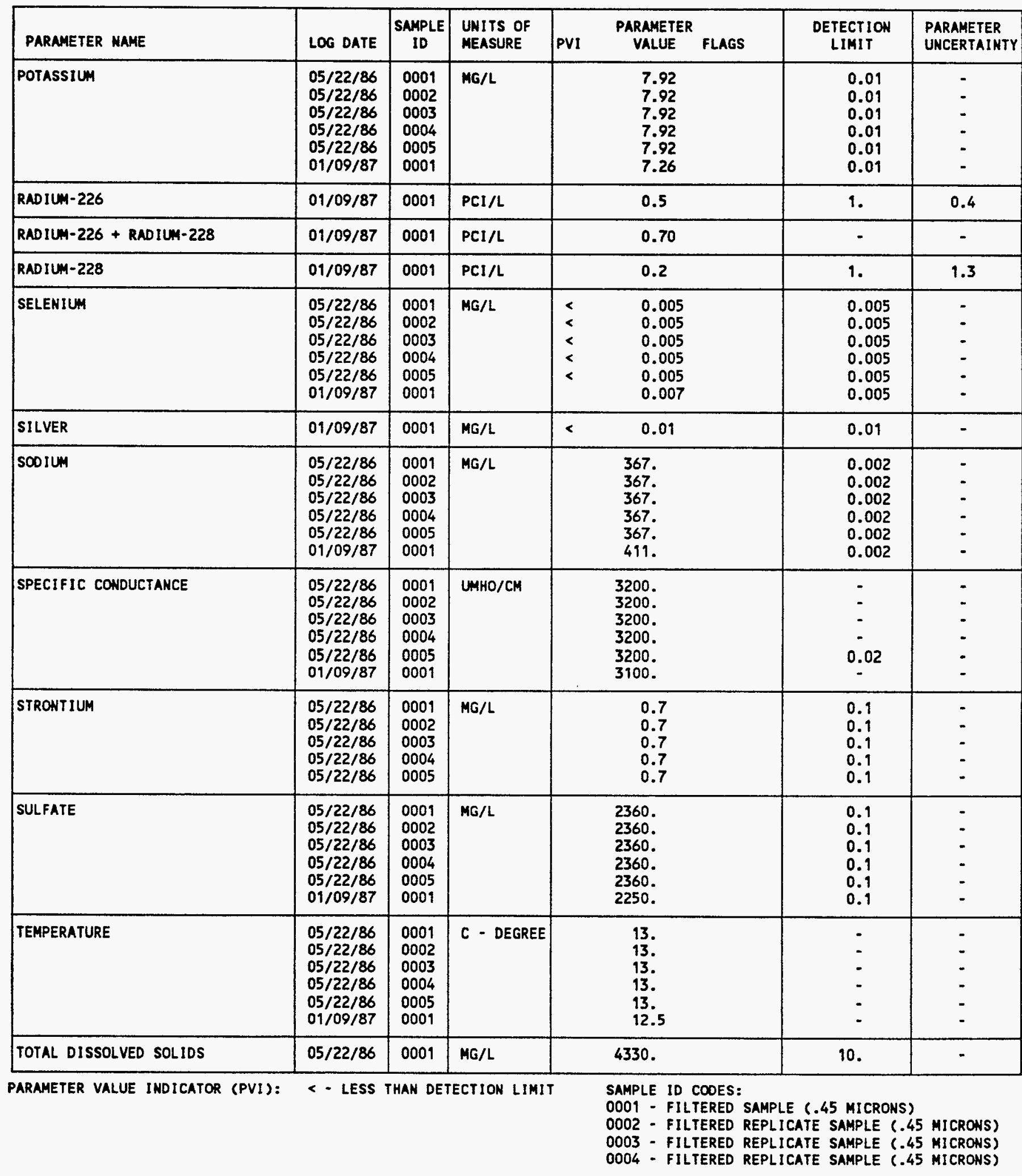


GROUNDWATER QUALITY DATA BY LOCATION

SITE: AMBOI AMBROSIA LAKE

LOCATION: 0650

NORTH COORDINATE: $\quad 49990.0$ FT

EAST COORDINATE: $\quad 51010.0$ FT

O6/10/80 TO $12 / 04 / 92$

REPORT DATE: 08/04/94

FORMATION OF COMPLETION: ALLUVIUM (AL)

HYORAULIC FLON RELATIONSHIP: UHKWOWN (N)

\begin{tabular}{|c|c|c|c|c|c|c|c|}
\hline PARAMETER NAME & LOG DATE & $\begin{array}{c}\text { SAMPLE } \\
10\end{array}$ & $\begin{array}{l}\text { UNITS OF } \\
\text { MEASURE }\end{array}$ & PVI & $\begin{array}{l}\text { PARAMETER } \\
\text { VALUE FLAGS }\end{array}$ & $\begin{array}{l}\text { DETECTION } \\
\text { LIMIT }\end{array}$ & $\begin{array}{l}\text { PARAMETER } \\
\text { UHCERTAIHTY }\end{array}$ \\
\hline TOTAL DISSOLVED SOLIDS & $\begin{array}{l}05 / 22 / 86 \\
05 / 22 / 86 \\
05 / 22 / 86 \\
05 / 22 / 86 \\
01 / 09 / 87\end{array}$ & $\begin{array}{l}0002 \\
0003 \\
0004 \\
0005 \\
0001\end{array}$ & MG/L & & $\begin{array}{l}4310 . \\
4290 \\
4290 \\
4450 \\
4310 .\end{array}$ & $\begin{array}{l}10 . \\
10 . \\
10 . \\
10 . \\
10 .\end{array}$ & $\begin{array}{l}- \\
- \\
-\end{array}$ \\
\hline URANIUM & $\begin{array}{l}05 / 22 / 86 \\
05 / 22 / 86 \\
05 / 22 / 86 \\
05 / 22 / 86 \\
05 / 22 / 86 \\
01 / 09 / 87\end{array}$ & $\begin{array}{l}0001 \\
0002 \\
0003 \\
0004 \\
0005 \\
0001\end{array}$ & $M G / L$ & & $\begin{array}{l}0.0305 \\
0.0299 \\
0.0297 \\
0.0299 \\
0.0351 \\
0.0349\end{array}$ & $\begin{array}{l}0.003 \\
0.003 \\
0.003 \\
0.003 \\
0.003 \\
0.003\end{array}$ & $\begin{array}{l}- \\
- \\
- \\
- \\
-\end{array}$ \\
\hline VANADIUM & $\begin{array}{l}05 / 22 / 86 \\
05 / 22 / 86 \\
05 / 22 / 86 \\
05 / 22 / 86 \\
05 / 22 / 86 \\
01 / 09 / 87\end{array}$ & $\begin{array}{l}0001 \\
0002 \\
0003 \\
0004 \\
0005 \\
0001\end{array}$ & $M G / L$ & & $\begin{array}{l}0.23 \\
0.23 \\
0.23 \\
0.23 \\
0.23 \\
0.01\end{array}$ & $\begin{array}{l}0.01 \\
0.01 \\
0.01 \\
0.01 \\
0.01 \\
0.01\end{array}$ & $\begin{array}{l}- \\
: \\
-\end{array}$ \\
\hline
\end{tabular}

PARAMETER VALUE INDICATOR (PVI):

< - LeSS than deTECTION LIMIT

SAMPLE ID CODES:

0001 - FILTERED SAMPLE (.45 MICRONS)

0002 - EILTERED REPLICATE SAMPLE ( 45 MICRONS)

0003 - FILTERED REPLICATE SAMPLE (.45 MICRONS)

0004 - FILTERED REPLICATE SAMPLE (.45 MICRONS) 


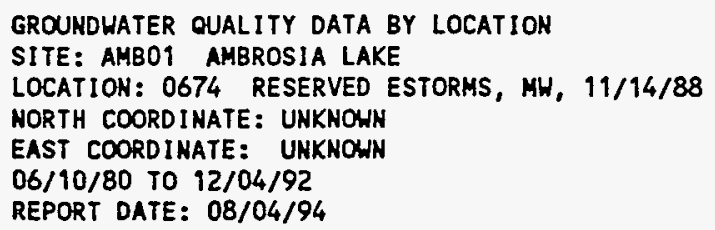

FORMATIOH OF COMPLETION: ALLUVIUM (AL)

HYORAULIC FLON RELATIONSHIP: DOWN GRADIENT (D)

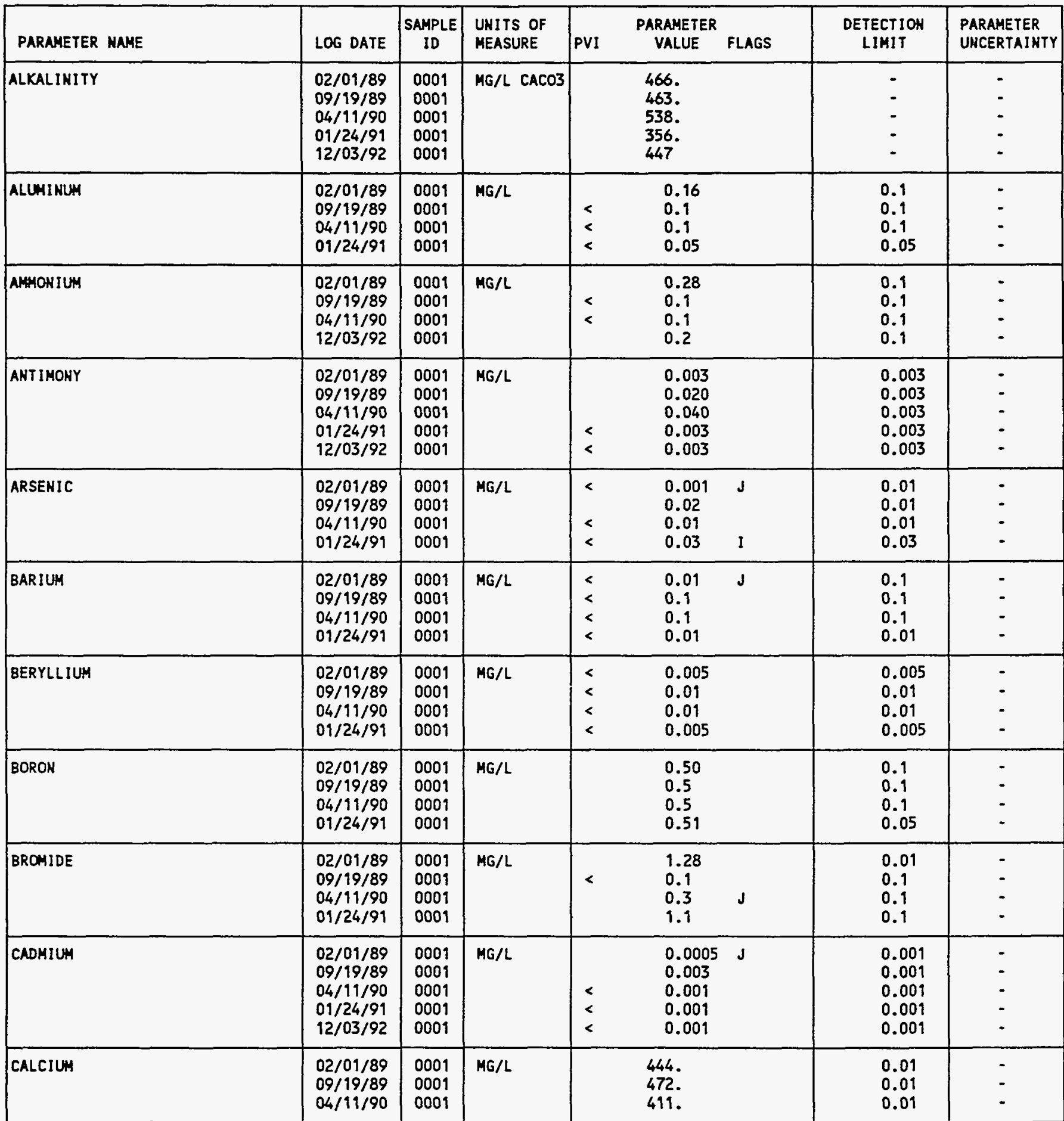

PARAMETER VALUE INDICATOR (PVI): < - LESS THAN DETECTION LIMIT

OTHER PARAMETER VALUE FLAGS:

I - INCREASED DETECTION LIMIT DUE TO REQUIRED DILUTION

$J$ - estimated VALUE 
GROUNDWATER OUALITY DATA BY LOCATION

SITE: AMBO1 AMBROSIA LAKE

LOCATION: 0674 RESERVED ESTORMS, MH, 11/14/88

NORTH COORDIHATE: UNKNOWN

EAST COORDINATE: UNKHOWH

$06 / 10 / 80$ TO $12 / 04 / 92$

REPORT DATE: $08 / 04 / 94$

FORMATIOH OF COMPLETIOH: ALLUVIUM (AL)

HYORAULIC FLON RELATIONSHIP: DOWN GRADIENT (D)

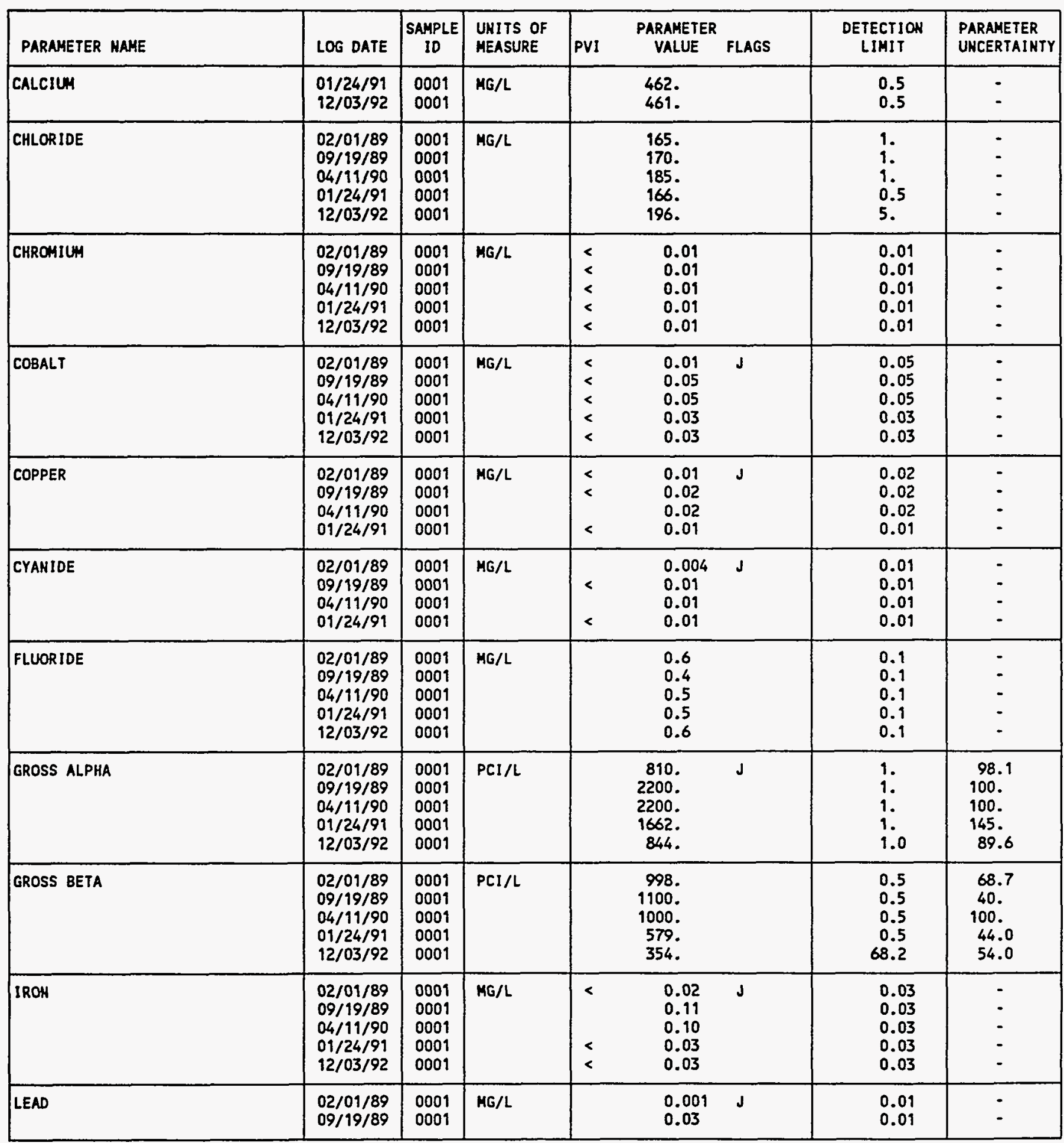

PARAMETER VALUE IHDICATOR (PVI): < - LESS THAN DETECTION LIMIT

SAMPLE ID COOES:

0001 - FILTEREO SAMPLE (.45 MICRONS)

OTHER PARAMETER VALUE FLAGS:

$J$ - ESTIMATEO VALUE 
GROUNDWATER QUALITY DATA BY LOCATION

SITE: AMBOI AMBROSIA LAKE

LOCATION: 0674 RESERVED ESTORMS, MH, 11/14/88

NORTH COORDINATE: UNKNOWN

EAST COORDINATE: UNKMOAH

06/10/80 TO $12 / 04 / 92$

REPORT DATE: 08/04/94

FORMATION OF COMPLETION: ALLUVIUM (AL)

HYDRAULIC FLON RELATIONSHIP: DOWN GRADIENT (D)

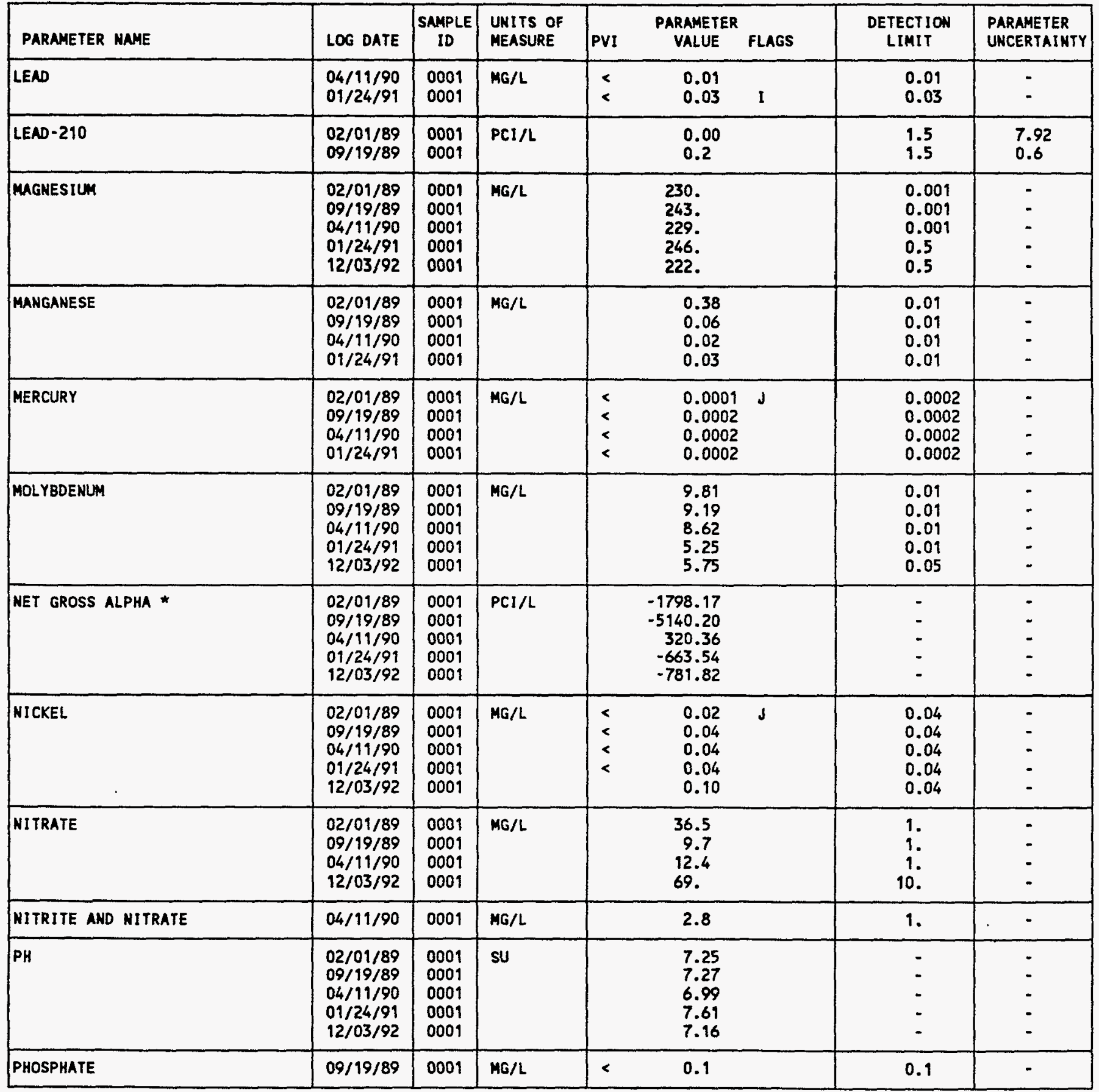

* net gross alpha (gross alpha - uranium) hITh 1 mg URANiUm a 686 PCI

PARAMETER VALUE INDICATOR (PVI): < - LESS THAN DETECTION LIMIT

SAMPLE ID CODES:

0001 - FILTERED SAMPLE (.45 MICRONS)

OTHER PARAMETER VALUE FLAGS:

1 - INCREASED DETECTION LIMIT DUE TO REQUIRED DILUTION

J - ESTIMATED VALUE 
GROUNDWATER QUALITY DATA BY LOCATION

SITE: AMBO1 AMBROSIA LAKE

LOCATION: 0674 RESERVED ESTORMS, MW 11/14/88

MORTH COORDINATE: UNKMOWN

EAST COORDINATE: UNKNOWN

$06 / 10 / 80$ TO $12 / 04 / 92$

REPORT DATE : 08/04/94

FORMATION OF COMPLETION: ALLUVIUM (AL)

HYDRAULIC FLON RELATIONSHIP: DOWN GRADIENT (D)

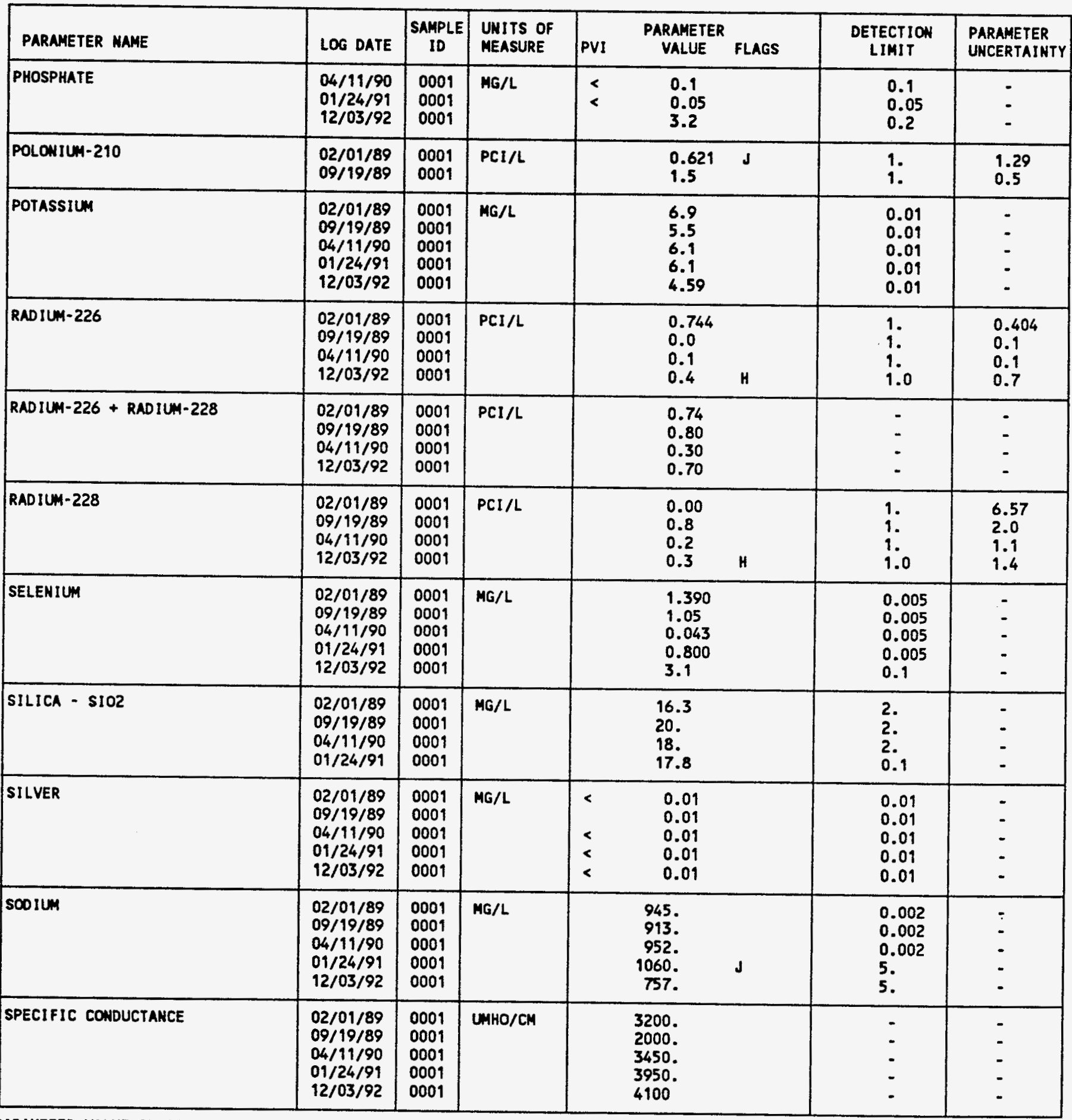

PARAMETER VALUE INDICATOR (PVI): < - LESS THAN DETECTION LIMIT

0001 - FILTERED SAMPLE (.45 MICRONS)

OTHER PARAMETER VALUE FLAGS:

H - HOLD TIME EXPIRED, VALUE SUSPECT

$J$ - ESTIMATED VALUE 
GROUNDHATER QUALITY DATA BY LOCATION

SITE: AMBO1 AMBROSIA LAKE

LOCATION: 0674 RESERVEO ESTORMS, MH, 11/14/88

NORTH COORDINATE: UNKHONH

EAST COORDINATE: UNKNOWN

06/10/80 TO 12/04/92

REPORT DATE: $08 / 04 / 94$

FORHATION OF COHPLETION: ALLUVIUH (AL)

HYORAULIC FLON RELATIONSHIP: DOWN GRADIENT (D)

\begin{tabular}{|c|c|c|c|c|c|c|c|}
\hline PARAMETER NAME & LOG DATE & $\begin{array}{c}\text { SAMPLE } \\
\text { ID }\end{array}$ & $\begin{array}{l}\text { UNITS OF } \\
\text { MEASURE }\end{array}$ & PVI & $\begin{array}{l}\text { PARAMETER } \\
\text { VALUE FLAGS }\end{array}$ & $\begin{array}{l}\text { DETECTION } \\
\text { LIMIT }\end{array}$ & $\begin{array}{l}\text { PARAMETER } \\
\text { UNCERTAINTY }\end{array}$ \\
\hline STRONTIUH & $\begin{array}{l}02 / 01 / 89 \\
09 / 19 / 89 \\
04 / 11 / 90 \\
01 / 24 / 91\end{array}$ & $\begin{array}{l}0001 \\
0001 \\
0001 \\
0001\end{array}$ & $M G / L$ & & $\begin{array}{l}8.70 \\
8.6 \\
8.36 \\
7.05\end{array}$ & $\begin{array}{l}0.1 \\
0.1 \\
0.1 \\
0.01\end{array}$ & $\begin{array}{l}- \\
- \\
-\end{array}$ \\
\hline SULFATE & $\begin{array}{l}02 / 01 / 89 \\
09 / 19 / 89 \\
04 / 11 / 90 \\
01 / 24 / 91 \\
12 / 03 / 92\end{array}$ & $\begin{array}{l}0001 \\
0001 \\
0001 \\
0001 \\
0001\end{array}$ & MG/L & & $\begin{array}{l}3523 . \\
3630 \\
3550 \\
3540 \\
2690\end{array}$ & $\begin{array}{c}0.1 \\
0.1 \\
0.1 \\
10 . \\
10 .\end{array}$ & $\begin{array}{l}- \\
- \\
-\end{array}$ \\
\hline SULFIDE & $\begin{array}{l}02 / 01 / 89 \\
09 / 19 / 89 \\
04 / 11 / 90\end{array}$ & $\begin{array}{l}0001 \\
0001 \\
0001\end{array}$ & MG/L & $\begin{array}{l}< \\
< \\
<\end{array}$ & $\begin{array}{l}0.01 \\
0.1 \\
0.9\end{array}$ & $\begin{array}{l}0.1 \\
0.1 \\
0.1\end{array}$ & - \\
\hline TEMPERATURE & $\begin{array}{l}02 / 01 / 89 \\
09 / 19 / 89 \\
04 / 11 / 90 \\
01 / 24 / 91 \\
12 / 03 / 92\end{array}$ & $\begin{array}{l}0001 \\
0001 \\
0001 \\
0001 \\
0001\end{array}$ & C - DEGREE & & $\begin{array}{l}11.0 \\
13.5 \\
12.5 \\
10.5 \\
11.0\end{array}$ & $\begin{array}{l}- \\
- \\
- \\
-\end{array}$ & $\begin{array}{l}. \\
- \\
. \\
.\end{array}$ \\
\hline THALLIUM & $\begin{array}{l}02 / 01 / 89 \\
09 / 19 / 89 \\
04 / 11 / 90 \\
01 / 24 / 91\end{array}$ & $\begin{array}{l}0001 \\
0001 \\
0001 \\
0001\end{array}$ & MG/L & $\begin{array}{l}< \\
< \\
< \\
<\end{array}$ & $\begin{array}{l}0.005 \\
0.01 \\
0.01 \\
0.03\end{array}$ & $\begin{array}{l}0.005 \\
0.01 \\
0.01 \\
0.03\end{array}$ & $\dot{-}$ \\
\hline THORIUM-230 & $\begin{array}{l}02 / 01 / 89 \\
09 / 19 / 89\end{array}$ & $\begin{array}{l}0001 \\
0001\end{array}$ & $\mathrm{PCl} / \mathrm{L}$ & & $\begin{array}{l}5.25 \\
0.0\end{array}$ & 1. & $\begin{array}{l}1.01 \\
0.3\end{array}$ \\
\hline TIH & $\begin{array}{l}02 / 01 / 89 \\
09 / 19 / 89 \\
04 / 11 / 90 \\
01 / 24 / 91\end{array}$ & $\begin{array}{l}0001 \\
0001 \\
0001 \\
0001\end{array}$ & $M G / L$ & $\begin{array}{l}< \\
<\end{array}$ & $\begin{array}{ll}0.001 & J \\
0.150 & \\
0.047 & \\
0.03 & 1\end{array}$ & $\begin{array}{l}0.005 \\
0.005 \\
0.005 \\
0.03\end{array}$ & $\begin{array}{l}- \\
\text { - } \\
\text { - }\end{array}$ \\
\hline TOTAL DISSOLVED SOLIDS & $\begin{array}{l}02 / 01 / 89 \\
09 / 19 / 89 \\
04 / 11 / 90 \\
01 / 24 / 91 \\
12 / 03 / 92\end{array}$ & $\begin{array}{l}0001 \\
0001 \\
0001 \\
0001 \\
0001\end{array}$ & $M G / L$ & & $\begin{array}{l}5768 . \\
6030 \\
5540 \\
5840 \\
4980\end{array}$ & $\begin{array}{l}10 . \\
10 . \\
10 . \\
10 . \\
10 .\end{array}$ & $\begin{array}{l}- \\
- \\
-\end{array}$ \\
\hline TOTAL KJELDAHL NITROGEN & $\begin{array}{l}01 / 24 / 91 \\
12 / 03 / 92\end{array}$ & $\begin{array}{l}0001 \\
0001\end{array}$ & $\mathrm{MG} / \mathrm{L}$ & & 1. & $\begin{array}{l}1 . \\
1 .\end{array}$ & $\ddot{-}$ \\
\hline TOTAL ORGAHIC CARBOH & $\begin{array}{l}02 / 01 / 89 \\
09 / 19 / 89 \\
04 / 11 / 90 \\
01 / 24 / 91 \\
12 / 03 / 92\end{array}$ & $\begin{array}{l}0001 \\
0001 \\
0001 \\
0001 \\
0001\end{array}$ & $M G / L$ & & $\begin{array}{r}16 . \\
120 \\
45 \\
8 . \\
10\end{array}$ & $\begin{array}{l}1 . \\
1 . \\
1 . \\
1 . \\
1 .\end{array}$ & $\begin{array}{l}- \\
. \\
. \\
.\end{array}$ \\
\hline URAKIUM & $\begin{array}{l}02 / 01 / 89 \\
09 / 19 / 89 \\
04 / 11 / 90 \\
01 / 24 / 91 \\
12 / 03 / 92\end{array}$ & $\begin{array}{l}0001 \\
0001 \\
0001 \\
0001 \\
0001\end{array}$ & $M G / L$ & & $\begin{array}{c}3.802 \\
10.7 \\
2.74 \\
3.39 \\
2.37\end{array}$ & $\begin{array}{l}0.003 \\
0.003 \\
0.003 \\
0.001 \\
0.001\end{array}$ & $\begin{array}{l}- \\
- \\
-\end{array}$ \\
\hline VANADIUM & $02 / 01 / 89$ & 0001 & MG/L & $<$ & 0.01 & 0.01 & - \\
\hline
\end{tabular}

PARAMETER VALUE INDICATOR (PVI): < - LESS THAH DETECTION LIMIT SAMPLE ID COOES:

O001 - FILTERED SAMPLE (.45 MICRONS)

OTHER PARAMETER VALUE FLAGS:

I - INCREASED DETECTION LIMIT DUE TO REQUIRED DILUTION

$J$ - ESTIMATED VALUE 
GROUNDWATER QUALITY DATA BY LOCATION

SITE: AMBO1 AMBROSIA LAKE

LOCATIOH: 0674 RESERVED ESTORHS, MH, 11/14/88

NORTH COORDINATE: UNKNONN

EAST COORDINATE: UNKNOWH

O6/10/80 TO 12/04/92

REPORT DATE: 08/04/94

FORMATION OF COMPLETION: ALLUVIUH (AL)

HYDRAULIC FLOW RELATIOHSHIP: DOWN GRADIENT (D)

\begin{tabular}{|c|c|c|c|c|c|c|c|}
\hline PARAMETER MAME & LOG DATE & $\begin{array}{c}\text { SAMPLE } \\
\text { ID }\end{array}$ & $\begin{array}{l}\text { UHITS OF } \\
\text { MEASURE }\end{array}$ & PVI & $\begin{array}{l}\text { PARAMETER } \\
\text { VALUE FLAGS }\end{array}$ & $\begin{array}{l}\text { DETECTION } \\
\text { LIMIT }\end{array}$ & $\begin{array}{l}\text { PARAMETER } \\
\text { UNCERTAINTY }\end{array}$ \\
\hline VANADIUM & $\begin{array}{l}09 / 19 / 89 \\
04 / 11 / 90 \\
01 / 24 / 91 \\
12 / 03 / 92\end{array}$ & $\begin{array}{l}0001 \\
0001 \\
0001 \\
0001\end{array}$ & $M G / L$ & $\begin{array}{l}< \\
<\end{array}$ & $\begin{array}{l}0.02 \\
0.01 \\
0.01 \\
0.05\end{array}$ & $\begin{array}{l}0.01 \\
0.01 \\
0.01 \\
0.05\end{array}$ & : \\
\hline ZIHC & $\begin{array}{l}02 / 01 / 89 \\
09 / 19 / 89 \\
04 / 11 / 90 \\
01 / 24 / 91\end{array}$ & $\begin{array}{l}0001 \\
0001 \\
0001 \\
0001\end{array}$ & $M G / L$ & $<$ & $\begin{array}{l}0.58 \\
0.005 \\
0.005 \\
0.007\end{array}$ & $\begin{array}{l}0.005 \\
0.005 \\
0.005 \\
0.005\end{array}$ & : \\
\hline
\end{tabular}

PARAMETER VALUE IHDICATOR (PVI):

< - LESS THAM DETECTION LIMIT

SAMPLE ID COOES:

0001 - FILTERED SAMPLE (.45 MICRONS)

OTHER PARAMETER VALUE FLAGS:

1 - IMCREASED DETECTION LIMIT DUE TO REQUIRED DILUTIOH 
GROUNDWATER QUALITY DATA BY LOCATIOH

SITE: AMBOI AMBROSIA LAKE

LOCATION: 0675 RESERVED ESTORHS, MW, 11/14/88

NORTH COORDINATE: UNKNOWN

EAST COORDIHATE: UNKNOWN

$06 / 10 / 80$ TO $12 / 04 / 92$

REPORT DATE: $08 / 04 / 94$

FORHATION OF COMPLETION: MANCOS SHALE (KM)

HYDRAULIC FLON RELATIONSHIP: DOWN GRADIENT (D)

\begin{tabular}{|c|c|c|c|c|c|c|c|}
\hline PARAMETER NAME & LOG DATE & $\begin{array}{c}\text { SAMPLE } \\
\text { ID }\end{array}$ & $\begin{array}{l}\text { UNITS OF } \\
\text { MEASURE }\end{array}$ & PVI & $\begin{array}{l}\text { PARAMETER } \\
\text { VALUE FLAGS }\end{array}$ & $\begin{array}{l}\text { DETECTION } \\
\text { LIMIT }\end{array}$ & $\begin{array}{l}\text { PARAMETER } \\
\text { UNCERTAINTY }\end{array}$ \\
\hline ALKALINITY & $\begin{array}{l}02 / 02 / 89 \\
09 / 14 / 89 \\
04 / 11 / 90 \\
01 / 16 / 91\end{array}$ & $\begin{array}{l}0001 \\
0001 \\
0001 \\
0001\end{array}$ & MG/L CACo3 & & $\begin{array}{l}551 . \\
428 . \\
484 . \\
278 .\end{array}$ & $\begin{array}{l}- \\
- \\
-\end{array}$ & $\begin{array}{l}- \\
- \\
-\end{array}$ \\
\hline ALUNINUM & $\begin{array}{l}02 / 02 / 89 \\
09 / 14 / 89 \\
04 / 11 / 90 \\
01 / 16 / 91\end{array}$ & $\begin{array}{l}0001 \\
0001 \\
0001 \\
0001\end{array}$ & $M G / L$ & $\begin{array}{l}< \\
< \\
< \\
<\end{array}$ & $\begin{array}{l}0.05 \\
0.1 \\
0.1 \\
0.05\end{array}$ & $\begin{array}{l}0.1 \\
0.1 \\
0.1 \\
0.05\end{array}$ & $\begin{array}{l}- \\
\text { - } \\
\text { - }\end{array}$ \\
\hline AMMONIUM & $\begin{array}{l}02 / 02 / 89 \\
09 / 14 / 89 \\
04 / 11 / 90 \\
01 / 16 / 91\end{array}$ & $\begin{array}{l}0001 \\
0001 \\
0001 \\
0001\end{array}$ & MG/L & $\begin{array}{l}< \\
<\end{array}$ & $\begin{array}{l}0.15 \\
0.1 \\
0.1 \\
0.28\end{array}$ & $\begin{array}{l}0.1 \\
0.1 \\
0.1 \\
0.01\end{array}$ & $\begin{array}{l}- \\
\text { - } \\
\text { - }\end{array}$ \\
\hline ANT IMOHY & $\begin{array}{l}02 / 02 / 89 \\
09 / 14 / 89 \\
04 / 11 / 90 \\
01 / 16 / 91\end{array}$ & $\begin{array}{l}0001 \\
0001 \\
0001 \\
0001\end{array}$ & $M G / L$ & $<$ & $\begin{array}{l}0.003 \\
0.021 \\
0.053 \\
0.003\end{array}$ & $\begin{array}{l}0.003 \\
0.003 \\
0.003 \\
0.003\end{array}$ & $\begin{array}{l}- \\
\text { - } \\
\text { - }\end{array}$ \\
\hline ARSENIC & $\begin{array}{l}02 / 02 / 89 \\
09 / 14 / 89 \\
04 / 11 / 90 \\
01 / 16 / 91\end{array}$ & $\begin{array}{l}0001 \\
0001 \\
0001 \\
0001\end{array}$ & $M G / L$ & $\begin{array}{l}< \\
<\end{array}$ & $\begin{array}{l}0.001 \\
0.02 \\
0.01 \\
0.03\end{array}$ & $\begin{array}{l}0.01 \\
0.01 \\
0.01 \\
0.03\end{array}$ & $\begin{array}{l}- \\
\text { - } \\
\text { - }\end{array}$ \\
\hline BARIUH & $\begin{array}{l}02 / 02 / 89 \\
09 / 14 / 89 \\
04 / 11 / 90 \\
01 / 16 / 91\end{array}$ & $\begin{array}{l}0001 \\
0001 \\
0001 \\
0001\end{array}$ & $M G / L$ & $\begin{array}{l}< \\
<\end{array}$ & $\begin{array}{l}0.02 \\
0.1 \\
0.1 \\
0.01\end{array}$ & $\begin{array}{l}0.1 \\
0.1 \\
0.1 \\
0.01\end{array}$ & $\begin{array}{l}- \\
\text { - } \\
\text { - }\end{array}$ \\
\hline BERYLLIUM & $\begin{array}{l}02 / 02 / 89 \\
09 / 14 / 89 \\
04 / 11 / 90 \\
01 / 16 / 91\end{array}$ & $\begin{array}{l}0001 \\
0001 \\
0001 \\
0001\end{array}$ & $M G / L$ & $\begin{array}{l}< \\
< \\
<\end{array}$ & $\begin{array}{l}0.005 \\
0.01 \\
0.01 \\
0.005\end{array}$ & $\begin{array}{l}0.005 \\
0.01 \\
0.01 \\
0.005\end{array}$ & $\begin{array}{l}- \\
- \\
-\end{array}$ \\
\hline BORON & $\begin{array}{l}02 / 02 / 89 \\
09 / 14 / 89 \\
04 / 11 / 90 \\
01 / 16 / 91\end{array}$ & $\begin{array}{l}0001 \\
0001 \\
0001 \\
0001\end{array}$ & $M G / L$ & & $\begin{array}{l}0.91 \\
0.9 \\
0.8 \\
0.73\end{array}$ & $\begin{array}{l}0.1 \\
0.1 \\
0.1 \\
0.05\end{array}$ & $\begin{array}{l}- \\
- \\
-\end{array}$ \\
\hline BROMIDE & $\begin{array}{l}02 / 02 / 89 \\
09 / 14 / 89 \\
04 / 11 / 90 \\
01 / 16 / 91\end{array}$ & $\begin{array}{l}0001 \\
0001 \\
0001 \\
0001\end{array}$ & $M G / L$ & & $\begin{array}{l}2.86 \\
2.2 \\
2.0 \\
7.1\end{array}$ & $\begin{array}{l}0.01 \\
0.1 \\
0.1 \\
0.1\end{array}$ & $\begin{array}{l}- \\
\text { - } \\
\text { - }\end{array}$ \\
\hline CADMIUM & $\begin{array}{l}02 / 02 / 89 \\
09 / 14 / 89 \\
04 / 11 / 90 \\
01 / 16 / 91\end{array}$ & $\begin{array}{l}0001 \\
0001 \\
0001 \\
0001\end{array}$ & $M G / L$ & $<$ & $\begin{array}{l}0.0003 \\
0.003 \\
0.001 \\
0.001\end{array}$ & $\begin{array}{l}0.001 \\
0.001 \\
0.001 \\
0.001\end{array}$ & $\begin{array}{l}- \\
\text { - } \\
\text { - }\end{array}$ \\
\hline CALCIUM & $\begin{array}{l}02 / 02 / 89 \\
09 / 14 / 89 \\
04 / 11 / 90 \\
01 / 16 / 91\end{array}$ & $\begin{array}{l}0001 \\
0001 \\
0001 \\
0001\end{array}$ & $M G / L$ & & $\begin{array}{l}464 . \\
505 . \\
431 \\
496\end{array}$ & $\begin{array}{l}0.01 \\
0.01 \\
0.01 \\
0.01\end{array}$ & $\begin{array}{l}- \\
\text { - } \\
\text { - }\end{array}$ \\
\hline CHLORIDE & $02 / 02 / 89$ & 0001 & $M G / L$ & & 309. & 1. & - \\
\hline
\end{tabular}

PARAMETER VALUE INDICATOR (PVI): < - LESS THAN DETECTION LIHIT

SAMPLE IO COOES:

0001 - FILTERED SAMPLE (.45 HICRONS)

OTHER PARAMETER VALUE FLAGS:

I - INCREASED DETECTION LIMIT DUE TO REQUIRED DILUTION

$\jmath$ - eSTIMATED VALUE 
GROUMDHATER OUALITY DATA BY LOCATION

SITE: AMBOI AMBROSIA LAKE

LOCATIOH: 0675 RESERVED ESTORMS, MH, 11/14/88

HORTH COORDINATE: UHKHOWH

EAST COORDINATE: UNKKOWN

$06 / 10 / 80$ TO $12 / 04 / 92$

REPORT DATE: $08 / 04 / 94$

FORMATIOH OF COMPLETION: MANCOS SHALE (KM)

HYORAULIC FLOW RELATIOHSHIP: DOWN GRADIENT (D)

\begin{tabular}{|c|c|c|c|c|c|c|c|c|}
\hline PARAMETER MANE & LOG DATE & $\underset{\text { SAMPLE }}{\text { ID }}$ & $\begin{array}{l}\text { UNITS OF } \\
\text { MEASURE }\end{array}$ & PVI & $\begin{array}{l}\text { PARAMETER } \\
\text { VALUE }\end{array}$ & FLAGS & $\begin{array}{l}\text { DETECTION } \\
\text { LIMIT }\end{array}$ & $\begin{array}{l}\text { PARAMETER } \\
\text { UNCERTAINTY }\end{array}$ \\
\hline CHLORIDE & $\begin{array}{l}09 / 14 / 89 \\
04 / 11 / 90 \\
01 / 16 / 91\end{array}$ & $\begin{array}{l}0001 \\
0001 \\
0001\end{array}$ & MG/L & & $\begin{array}{l}360 . \\
407 . \\
510 .\end{array}$ & & $\begin{array}{l}1 . \\
1.0 \\
0.5\end{array}$ & : \\
\hline CHROMIUM & $\begin{array}{l}02 / 02 / 89 \\
09 / 14 / 89 \\
04 / 11 / 90 \\
01 / 16 / 91\end{array}$ & $\begin{array}{l}0001 \\
0001 \\
0001 \\
0001\end{array}$ & MG/L & $\begin{array}{l}< \\
< \\
< \\
<\end{array}$ & $\begin{array}{l}0.01 \\
0.01 \\
0.01 \\
0.01\end{array}$ & & $\begin{array}{l}0.01 \\
0.01 \\
0.01 \\
0.01\end{array}$ & - \\
\hline COBALT & $\begin{array}{l}02 / 02 / 89 \\
09 / 14 / 89 \\
04 / 19 / 90 \\
01 / 16 / 91\end{array}$ & $\begin{array}{l}0001 \\
0001 \\
0001 \\
0001\end{array}$ & MG/L & $\begin{array}{l}< \\
< \\
< \\
<\end{array}$ & $\begin{array}{l}0.01 \\
0.05 \\
0.05 \\
0.03\end{array}$ & J & $\begin{array}{l}0.05 \\
0.05 \\
0.05 \\
0.03\end{array}$ & : \\
\hline COPPER & $\begin{array}{l}02 / 02 / 89 \\
09 / 14 / 89 \\
04 / 11 / 90 \\
01 / 16 / 91\end{array}$ & $\begin{array}{l}0001 \\
0001 \\
0001 \\
0001\end{array}$ & $M G / L$ & $\begin{array}{l}< \\
< \\
< \\
<\end{array}$ & $\begin{array}{l}0.01 \\
0.02 \\
0.02 \\
0.01\end{array}$ & J & $\begin{array}{l}0.02 \\
0.02 \\
0.02 \\
0.01\end{array}$ & : \\
\hline CYAHIDE & $\begin{array}{l}02 / 02 / 89 \\
09 / 14 / 89 \\
04 / 11 / 90 \\
01 / 16 / 91\end{array}$ & $\begin{array}{l}0001 \\
0001 \\
0001 \\
0001\end{array}$ & $M G / L$ & & $\begin{array}{l}0.263 \\
0.01 \\
0.36 \\
0.24\end{array}$ & & $\begin{array}{l}0.01 \\
0.01 \\
0.01 \\
0.01\end{array}$ & : \\
\hline FLUORIDE & $\begin{array}{l}02 / 02 / 89 \\
09 / 14 / 89 \\
04 / 11 / 90 \\
01 / 16 / 91\end{array}$ & $\begin{array}{l}0001 \\
0001 \\
0001 \\
0001\end{array}$ & $M G / L$ & & $\begin{array}{l}0.3 \\
0.2 \\
0.3 \\
0.4\end{array}$ & & $\begin{array}{l}0.1 \\
0.1 \\
0.1 \\
0.1\end{array}$ & : \\
\hline GROSS ALPHA & $\begin{array}{l}02 / 02 / 89 \\
09 / 14 / 89 \\
04 / 11 / 90 \\
01 / 16 / 91\end{array}$ & $\begin{array}{l}0001 \\
0001 \\
0001 \\
0001\end{array}$ & $\mathrm{PCI} / \mathrm{L}$ & & $\begin{array}{c}674 . \\
1700 \\
1100 \\
397\end{array}$ & & $\begin{array}{l}1 . \\
1 . \\
1 . \\
1 .\end{array}$ & $\begin{array}{l}103 . \\
100 . \\
100 . \\
74.9\end{array}$ \\
\hline GROSS BETA & $\begin{array}{l}02 / 02 / 89 \\
09 / 14 / 89 \\
04 / 11 / 90 \\
01 / 16 / 91\end{array}$ & $\begin{array}{l}0001 \\
0001 \\
0001 \\
0001\end{array}$ & $\mathrm{PCI} / \mathrm{L}$ & & $\begin{array}{l}0.00 \\
740 . \\
440 \\
201\end{array}$ & & $\begin{array}{l}0.5 \\
0.5 \\
0.5 \\
0.5\end{array}$ & $\begin{array}{l}41.1 \\
40 . \\
30 . \\
33.4\end{array}$ \\
\hline IRON & $\begin{array}{l}02 / 02 / 89 \\
09 / 14 / 89 \\
04 / 11 / 90 \\
01 / 16 / 91\end{array}$ & $\begin{array}{l}0001 \\
0001 \\
0001 \\
0001\end{array}$ & $M G / L$ & & $\begin{array}{l}0.66 \\
0.24 \\
0.21 \\
0.09\end{array}$ & & $\begin{array}{l}0.03 \\
0.03 \\
0.03 \\
0.03\end{array}$ & : \\
\hline LEAD & $\begin{array}{l}02 / 02 / 89 \\
09 / 14 / 89 \\
04 / 19 / 90 \\
01 / 16 / 91\end{array}$ & $\begin{array}{l}0001 \\
0001 \\
0001 \\
0001\end{array}$ & MG/L & $<$ & $\begin{array}{l}0.001 \\
0.02 \\
0.01 \\
0.03\end{array}$ & $\begin{array}{l}\text { J } \\
\text { I }\end{array}$ & $\begin{array}{l}0.01 \\
0.01 \\
0.01 \\
0.03\end{array}$ & $:$ \\
\hline LEAD-210 & $\begin{array}{l}02 / 02 / 89 \\
09 / 14 / 89\end{array}$ & $\begin{array}{l}0001 \\
0001\end{array}$ & $\mathrm{PCI} / \mathrm{L}$ & & $\begin{array}{l}0.00 \\
0.8\end{array}$ & & $\begin{array}{l}1.5 \\
1.5\end{array}$ & $\begin{array}{l}5.28 \\
0.7\end{array}$ \\
\hline MAGHESIUM & $\begin{array}{l}02 / 02 / 89 \\
09 / 14 / 89 \\
04 / 11 / 90 \\
01 / 16 / 91\end{array}$ & $\begin{array}{l}0001 \\
0001 \\
0001 \\
0001\end{array}$ & $M G / L$ & & $\begin{array}{l}340 . \\
338 . \\
292 . \\
288 .\end{array}$ & & $\begin{array}{l}0.001 \\
0.001 \\
0.001 \\
0.5\end{array}$ & : \\
\hline
\end{tabular}

PARAMETER VALUE IMDICATOR (PVI): < - LESS THAN DETECTION LIMIT

SAMPLE ID COOES:

OO01 - FILTERED SAMPLE (.45 MICRONS)

OTHER PARAMETER VALUE FLAGS:

I - INCREASED DETECTION LIMIT DUE TO REQUIRED DILUTION

J - estimated VALUE 
GROUNDWATER QUALITY DATA BY LOCATION

SITE: AMBO1 AMBROSIA LAKE

LOCATION: 0675 RESERVED ESTORMS, MH, 11/14/88

NORTH COORDINATE: UNKNOWN

EAST COORDINATE: UNKNOWN

06/10/80 TO 12/04/92

REPORT DATE : 08/04/94

FORMATIOH OF COMPLETION: MANCOS SHALE (KM)

HYDRAULIC FLON RELATIONSHIP: DOWN GRADIENT (D)

\begin{tabular}{|c|c|c|c|c|c|c|c|}
\hline PARAMETER NAME & LOG DATE & $\underset{\text { ID }}{\text { SAMPLE }}$ & $\begin{array}{l}\text { UHITS OF } \\
\text { MEASURE }\end{array}$ & PVI & $\begin{array}{l}\text { PARAMETER } \\
\text { VALUE FLAGS }\end{array}$ & $\begin{array}{l}\text { DETECTION } \\
\text { LIMIT }\end{array}$ & $\begin{array}{l}\text { PARAMETER } \\
\text { UKCERTAIHTY }\end{array}$ \\
\hline MANGANESE & $\begin{array}{l}02 / 02 / 89 \\
09 / 14 / 89 \\
04 / 11 / 90 \\
01 / 16 / 91\end{array}$ & $\begin{array}{l}0001 \\
0001 \\
0001 \\
0001\end{array}$ & MG/L & $<$ & $\begin{array}{l}0.10 \\
0.04 \\
0.04 \\
0.01\end{array}$ & $\begin{array}{l}0.01 \\
0.01 \\
0.01 \\
0.01\end{array}$ & : \\
\hline MERCURY & $\begin{array}{l}02 / 02 / 89 \\
09 / 14 / 89 \\
04 / 91 / 90 \\
01 / 16 / 99\end{array}$ & $\begin{array}{l}0001 \\
0001 \\
0001 \\
0001\end{array}$ & MG/L & $\begin{array}{l}< \\
< \\
<\end{array}$ & $\begin{array}{l}0.0001 \mathrm{~J} \\
0.0002 \\
0.0002 \\
0.0002\end{array}$ & $\begin{array}{l}0.0002 \\
0.0002 \\
0.0002 \\
0.0002\end{array}$ & $\begin{array}{l}- \\
- \\
-\end{array}$ \\
\hline MOL YBDENUM & $\begin{array}{l}02 / 02 / 89 \\
09 / 14 / 89 \\
04 / 11 / 90 \\
01 / 16 / 91\end{array}$ & $\begin{array}{l}0009 \\
0001 \\
0001 \\
0001\end{array}$ & MG/L & & $\begin{array}{l}2.72 \\
1.77 \\
1.11 \\
0.28\end{array}$ & $\begin{array}{l}0.01 \\
0.01 \\
0.01 \\
0.01\end{array}$ & : \\
\hline MET GROSS ALPHA * & $\begin{array}{l}02 / 02 / 89 \\
09 / 14 / 89 \\
04 / 19 / 90 \\
01 / 16 / 91\end{array}$ & $\begin{array}{l}0001 \\
0001 \\
0001 \\
0001\end{array}$ & $\mathrm{PCI} / \mathrm{L}$ & & $\begin{array}{r}-754.94 \\
993.42 \\
478.48 \\
-302.72\end{array}$ & $:$ & : \\
\hline NICKEL & $\begin{array}{l}02 / 02 / 89 \\
09 / 14 / 89 \\
04 / 11 / 90 \\
01 / 16 / 91\end{array}$ & $\begin{array}{l}0001 \\
0001 \\
0001 \\
0001\end{array}$ & $M G / L$ & $\begin{array}{l}< \\
<\end{array}$ & $\begin{array}{l}0.02 \\
0.04 \\
0.04 \\
0.04\end{array}$ & $\begin{array}{l}0.04 \\
0.04 \\
0.04 \\
0.04\end{array}$ & $\begin{array}{l}- \\
- \\
-\end{array}$ \\
\hline HITRATE & $\begin{array}{l}02 / 02 / 89 \\
09 / 14 / 89 \\
04 / 11 / 90 \\
01 / 16 / 91\end{array}$ & $\begin{array}{l}0001 \\
0001 \\
0001 \\
0001\end{array}$ & MG/L & & $\begin{array}{l}138.6 \\
160 . \\
173 . \\
252 .\end{array}$ & $\begin{array}{l}1 . \\
1 . \\
0.1\end{array}$ & : \\
\hline WITRITE AND NITRATE & $\begin{array}{l}04 / 11 / 90 \\
01 / 16 / 91\end{array}$ & $\begin{array}{l}0001 \\
0001\end{array}$ & MG $/ L$ & & $\begin{array}{l}39.0 \\
54.0\end{array}$ & $\begin{array}{l}1.05 \\
0.05\end{array}$ & - \\
\hline PH & $\begin{array}{l}02 / 02 / 89 \\
09 / 14 / 89 \\
04 / 11 / 90 \\
01 / 16 / 91\end{array}$ & $\begin{array}{l}0001 \\
0001 \\
0001 \\
0001\end{array}$ & SU & & $\begin{array}{l}7.03 \\
6.95 \\
6.81 \\
7.20\end{array}$ & : & : \\
\hline PHOSPHATE . & $\begin{array}{l}02 / 02 / 89 \\
09 / 14 / 89 \\
04 / 11 / 90 \\
01 / 16 / 91\end{array}$ & $\begin{array}{l}0001 \\
0001 \\
0001 \\
0001\end{array}$ & $M G / L$ & $<$ & $\begin{array}{l}0.1 \\
0.1 \\
0.3 \\
0.10\end{array}$ & $\begin{array}{l}0.1 \\
0.1 \\
0.1 \\
0.05\end{array}$ & $\begin{array}{l}- \\
- \\
-\end{array}$ \\
\hline POLONIUM-210 & $\begin{array}{l}02 / 02 / 89 \\
09 / 14 / 89\end{array}$ & $\begin{array}{l}0001 \\
0001\end{array}$ & PCI/L & & $\begin{array}{l}0.00 \\
1.3\end{array}$ & 1. & $\begin{array}{l}1.01 \\
0.5\end{array}$ \\
\hline POTASSIUM & $\begin{array}{l}02 / 02 / 89 \\
09 / 14 / 89 \\
04 / 11 / 90 \\
01 / 16 / 91\end{array}$ & $\begin{array}{l}0001 \\
0001 \\
0001 \\
0001\end{array}$ & $M G / L$ & & $\begin{array}{r}15.6 \\
11.6 \\
1.8 \\
7.6\end{array}$ & $\begin{array}{l}0.01 \\
0.01 \\
0.01 \\
0.01\end{array}$ & : \\
\hline RADIUA-226 & $\begin{array}{l}02 / 02 / 89 \\
09 / 14 / 89 \\
04 / 11 / 90 \\
01 / 16 / 91\end{array}$ & $\begin{array}{l}0001 \\
0001 \\
0001 \\
0001\end{array}$ & $\mathrm{PCI} / \mathrm{L}$ & & $\begin{array}{l}2.05 \\
0.4 \\
0.2 \\
1.6\end{array}$ & $\begin{array}{l}1 . \\
1 . \\
1 .\end{array}$ & $\begin{array}{l}0.271 \\
0.2 \\
0.2 \\
0.6\end{array}$ \\
\hline
\end{tabular}

- HET GROSS ALPHA (GROSS ALPHA - URANIUM) WITH 1 MG URANIUH = 686 PCI

PARAMETER VALUE INDICATOR (PVI): < - LESS THAN DETECTION LIMIT

OTHER PARAMETER VALUE FLAGS:

$J$ - ESTIMATED VALUE 
GROUNDHATER QUALITY DATA BY LOCATION

SITE: AMBOI AMBROSIA LAKE

LOCATION: 0675 RESERVED ESTORHS, MH, 11/14/88

HORTH COORDINATE: UNKNOWN

EAST COORDINATE: UNKHOWN

06/10/80 TO $12 / 04 / 92$

REPORT DATE: 08/04/94

FORMATION OF COMPLETION: MANCOS SHALE (KH)

HYDRAULIC FLOH RELATIOHSHIP: DOWN GRADIENT (D)

\begin{tabular}{|c|c|c|c|c|c|c|c|}
\hline PARAMEETER MAME & LOG DATE & $\begin{array}{c}\text { SAMPLE } \\
10\end{array}$ & $\begin{array}{l}\text { UNITS OF } \\
\text { MEASURE }\end{array}$ & PVI & $\begin{array}{l}\text { PARAMETER } \\
\text { VALUE FLAGS }\end{array}$ & $\begin{array}{l}\text { DETECTION } \\
\text { LIMIT }\end{array}$ & $\begin{array}{l}\text { PARAMETER } \\
\text { UNCERTAINTY }\end{array}$ \\
\hline RADIUH-226 + RADIUH-228 & $\begin{array}{l}02 / 02 / 89 \\
09 / 14 / 89 \\
04 / 11 / 90 \\
01 / 16 / 91\end{array}$ & $\begin{array}{l}0001 \\
0001 \\
0001 \\
0001\end{array}$ & $\mathrm{PCI} / \mathrm{L}$ & & $\begin{array}{l}4.03 \\
1.50 \\
1.50 \\
2.60\end{array}$ & : & : \\
\hline RADIUN-228 & $\begin{array}{l}02 / 02 / 89 \\
09 / 14 / 89 \\
04 / 11 / 90 \\
01 / 16 / 91\end{array}$ & $\begin{array}{l}0001 \\
0001 \\
0001 \\
0001\end{array}$ & $\mathrm{PCI} / \mathrm{L}$ & & $\begin{array}{l}1.98 \\
1.1 \\
1.3 \\
1.0\end{array}$ & $\begin{array}{l}1 . \\
1 . \\
1 .\end{array}$ & $\begin{array}{l}8.10 \\
0.8 \\
0.9 \\
1.6\end{array}$ \\
\hline SELENIUN & $\begin{array}{l}02 / 02 / 89 \\
09 / 14 / 89 \\
04 / 11 / 90 \\
01 / 16 / 91\end{array}$ & $\begin{array}{l}0001 \\
0001 \\
0001 \\
0001\end{array}$ & $M G / L$ & & $\begin{array}{l}0.510 \\
0.421 \\
0.030 \\
0.500\end{array}$ & $\begin{array}{l}0.005 \\
0.005 \\
0.005 \\
0.005\end{array}$ & : \\
\hline SILVER & $\begin{array}{l}02 / 02 / 89 \\
09 / 14 / 89 \\
04 / 11 / 90 \\
01 / 16 / 91\end{array}$ & $\begin{array}{l}0001 \\
0001 \\
0001 \\
0001\end{array}$ & $M G / L$ & $\begin{array}{l}< \\
<\end{array}$ & $\begin{array}{l}0.01 \\
0.02 \\
0.01 \\
0.01\end{array}$ & $\begin{array}{l}0.01 \\
0.01 \\
0.01 \\
0.01\end{array}$ & : \\
\hline SOOIUA & $\begin{array}{l}02 / 02 / 89 \\
09 / 14 / 89 \\
04 / 11 / 90 \\
01 / 16 / 91\end{array}$ & $\begin{array}{l}0001 \\
0001 \\
0001 \\
0001\end{array}$ & $M G / L$ & & $\begin{array}{l}1200 . \\
1090 . \\
1090 \\
1050\end{array}$ & $\begin{array}{l}0.002 \\
0.002 \\
0.002 \\
5 .\end{array}$ & : \\
\hline SPECIFIC CONDUCTAHCE & $\begin{array}{l}02 / 02 / 89 \\
09 / 14 / 89 \\
04 / 11 / 90 \\
01 / 16 / 91\end{array}$ & $\begin{array}{l}0001 \\
0001 \\
0001 \\
0001\end{array}$ & UNHO/CM & & $\begin{array}{l}3600 . \\
2700 . \\
4500 \\
4600 .\end{array}$ & : & $\begin{array}{l}- \\
-\end{array}$ \\
\hline SULFIDE & $\begin{array}{l}02 / 02 / 89 \\
09 / 14 / 89 \\
04 / 11 / 90 \\
01 / 16 / 91\end{array}$ & $\begin{array}{l}0001 \\
0001 \\
0001 \\
0001\end{array}$ & MG/L & $\begin{array}{l}< \\
< \\
<\end{array}$ & $\begin{array}{l}0.01 \\
0.1 \\
0.1 \\
0.1\end{array}$ & $\begin{array}{l}0.1 \\
0.1 \\
0.1 \\
0.1\end{array}$ & : \\
\hline TEMPERATURE & $\begin{array}{l}02 / 02 / 89 \\
09 / 14 / 89 \\
04 / 11 / 90 \\
01 / 16 / 91\end{array}$ & $\begin{array}{l}0001 \\
0001 \\
0001 \\
0001\end{array}$ & C - DEGREE & & $\begin{array}{l}12.0 \\
12.0 \\
12 . \\
11.5\end{array}$ & :- & :- \\
\hline THALLIUM & $\begin{array}{l}02 / 02 / 89 \\
09 / 14 / 89\end{array}$ & $\begin{array}{l}0001 \\
0001\end{array}$ & $M G / L$ & $<$ & $\begin{array}{l}0.005 \\
0.01\end{array}$ & $\begin{array}{l}0.005 \\
0.01\end{array}$ & - \\
\hline
\end{tabular}

PARAMETER VALUE INDICATOR (PVI): < - LESS THAN DETECTION LIMIT

SAMPLE ID COOES:

0001 - FILTERED SAMPLE (.45 MICRONS)

OTHER PARAMETER VALUE fLAGS:

$\mathrm{J}$ - ESTIMATED VALUE 
GROUHDWATER QUALITY DATA BY LOCATIOH

SITE: AMBO1 AMBROSIA LAKE

LOCATION: 0675 RESERVED ESTORMS, MH, 19/14/88

NORTH COORDIHATE: UNKNOWN

EAST COORDIHATE: UNKNOWN

O6/10/80 TO $12 / 04 / 92$

REPORT DATE: 08/04/94

FORMATION OF COMPLETION: MANCOS SHALE (KM)

HYDRAULIC FLON RELATIONSHIP: DOWN GRADIENT (D)

\begin{tabular}{|c|c|c|c|c|c|c|c|}
\hline PARAMETER HAME & LOG DATE & $\begin{array}{l}\text { SAMPLE } \\
\text { ID }\end{array}$ & $\begin{array}{l}\text { UNITS OF } \\
\text { MEASURE }\end{array}$ & PVI & $\begin{array}{l}\text { PARAMETER } \\
\text { VALUE FLAGS }\end{array}$ & $\begin{array}{l}\text { DETECTION } \\
\text { LIMIT }\end{array}$ & $\begin{array}{l}\text { PARAMETER } \\
\text { UNCERTAINTY }\end{array}$ \\
\hline THALLIUM & $\begin{array}{l}04 / 11 / 90 \\
01 / 16 / 91\end{array}$ & $\begin{array}{l}0009 \\
0009\end{array}$ & $M G / L$ & $<$ & $\begin{array}{l}0.01 \\
0.03\end{array}$ & $\begin{array}{l}0.01 \\
0.03\end{array}$ & $\dot{-}$ \\
\hline THORIUH-230 & $\begin{array}{l}02 / 02 / 89 \\
09 / 14 / 89\end{array}$ & $\begin{array}{l}0001 \\
0001\end{array}$ & $\mathrm{PCI} / \mathrm{L}$ & & $\begin{array}{l}1.02 \\
0.1\end{array}$ & 1. & $\begin{array}{l}0.423 \\
0.4\end{array}$ \\
\hline TOTAL DISSOLVED SOLIDS & $\begin{array}{l}02 / 02 / 89 \\
09 / 14 / 89 \\
04 / 11 / 90 \\
01 / 16 / 91\end{array}$ & $\begin{array}{l}0009 \\
0001 \\
0001 \\
0001\end{array}$ & $M G / L$ & & $\begin{array}{l}7208 . \\
6650 . \\
6420 . \\
6350\end{array}$ & $\begin{array}{l}10 . \\
10 . \\
10 . \\
10 .\end{array}$ & : \\
\hline URANIUM & $\begin{array}{l}02 / 02 / 89 \\
09 / 14 / 89 \\
04 / 11 / 90 \\
01 / 16 / 91\end{array}$ & $\begin{array}{l}0001 \\
0001 \\
0001 \\
0001\end{array}$ & $M G / L$ & & $\begin{array}{l}2.083 \\
1.03 \\
0.906 \\
1.02\end{array}$ & $\begin{array}{l}0.003 \\
0.003 \\
0.003 \\
0.001\end{array}$ & $:$ \\
\hline VAHADIUM & $\begin{array}{l}02 / 02 / 89 \\
09 / 14 / 89 \\
04 / 11 / 90 \\
01 / 16 / 91\end{array}$ & $\begin{array}{l}0001 \\
0001 \\
0001 \\
0001\end{array}$ & MG/L & $\begin{array}{l}< \\
< \\
<\end{array}$ & $\begin{array}{l}0.01 \\
0.01 \\
0.01 \\
0.01\end{array}$ & $\begin{array}{l}0.01 \\
0.01 \\
0.01 \\
0.01\end{array}$ & : \\
\hline ZINC & $\begin{array}{l}02 / 02 / 89 \\
09 / 14 / 89 \\
04 / 11 / 90 \\
01 / 16 / 91\end{array}$ & $\begin{array}{l}0001 \\
0001 \\
0001 \\
0001\end{array}$ & MG/L & $\begin{array}{l}< \\
<\end{array}$ & $\begin{array}{l}0.96 \\
0.005 \\
0.005 \\
0.005\end{array}$ & $\begin{array}{l}0.005 \\
0.005 \\
0.005 \\
0.005\end{array}$ & : \\
\hline
\end{tabular}

PARAMETER VALUE INDICATOR (PVI): < - LESS THAN DETECTION LIMIT SAMPLE ID COOES:

0001 - FILTERED SAMPLE (.45 MICRONS)

OTHER PARAMETER VALUE FLAGS:

1 - INCREASED DETECTION LIMIT DUE TO REQUIRED DILUTION

$\mathrm{J}$ - estimated VALUE 
GROUNDWATER QUALITY DATA BY LOCATION

SITE: AMBO1 AMBROSIA LAKE

LOCATION: 0676 RESERVED ESTORMS, MH, 11/14/88

NORTH COORDINATE: UHKHOWN

EAST COORDIMATE: UNKNOWH

06/10/80 TO 12/04/92

REPORT DATE: $08 / 04 / 94$

FORMATIOH OF COMPLETIOH: TRES HERMAMOS - C2 SAMDSTOHE (TT) HYDRAULIC FLOW RELATIOHSHIP: CROSS GRADIENT (C)

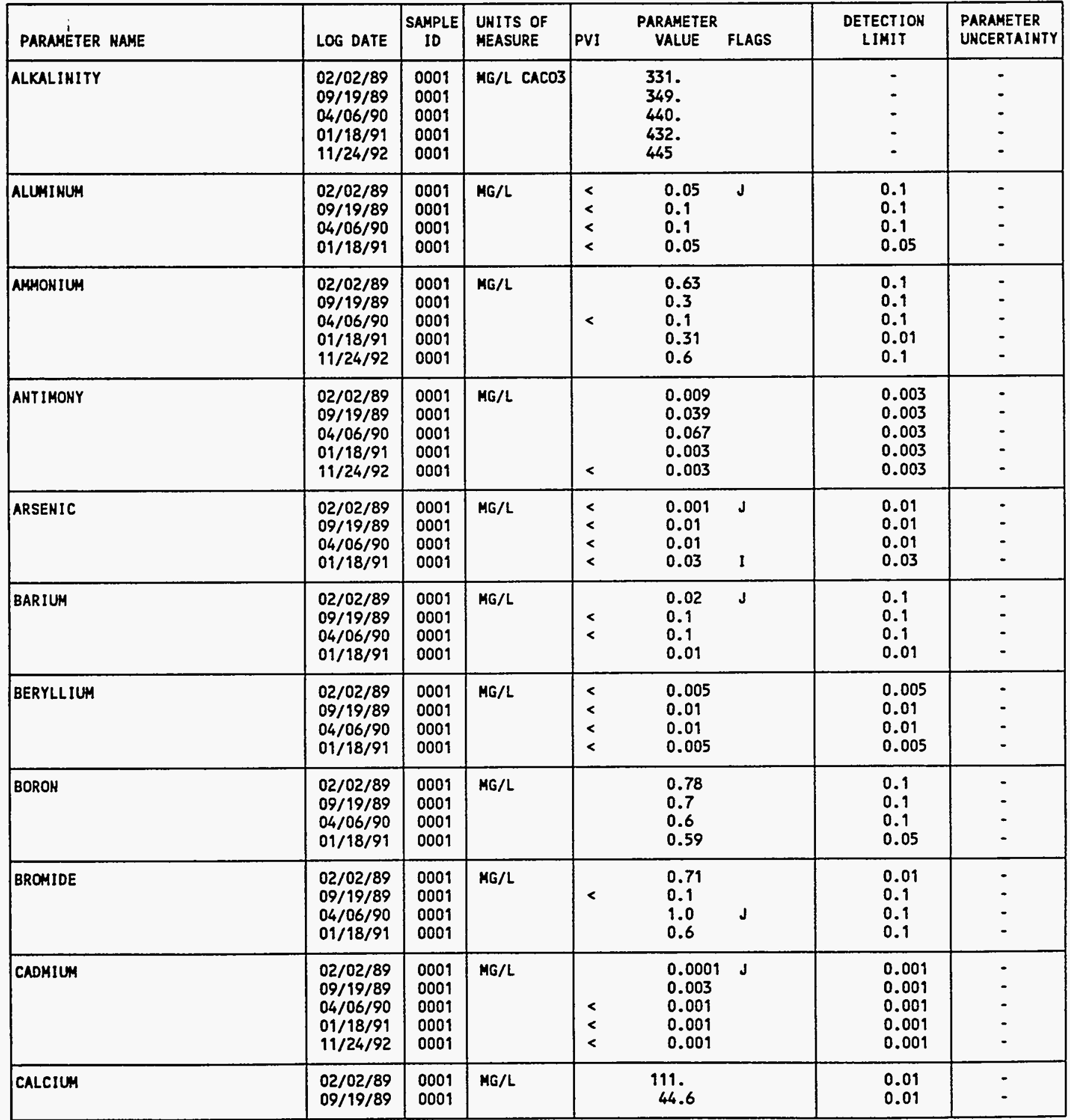

PARAMETER VALUE IMDICATOR (PVI): < - LESS THAN DETECTION LIHIT

OTHER PARAMETER VALUE FLAGS:

I - INCREASED DETECTION LIMIT DUE TO REOUIRED DILUTION

J - ESTIMATED VALUE 
GROUNDWATER OUALITY DATA BY LOCATIOH

SITE: AMBO1 AMBROSIA LAKE

LOCATIOH: 0676 RESERVED ESTORMS, MH, 11/14/88

HORTH COORDINATE: UNKNOWN

EAST COORDINATE: UNKNOWN

O6/10/80 TO $12 / 04 / 92$

REPORT DATE: 08/04/94

FORHATION OF COHPLETION: TRES HERHANOS - C2 SANDSTONE (TT) HYDRAULIC FLON RELATIONSHIP: CROSS GRADIENT (C)

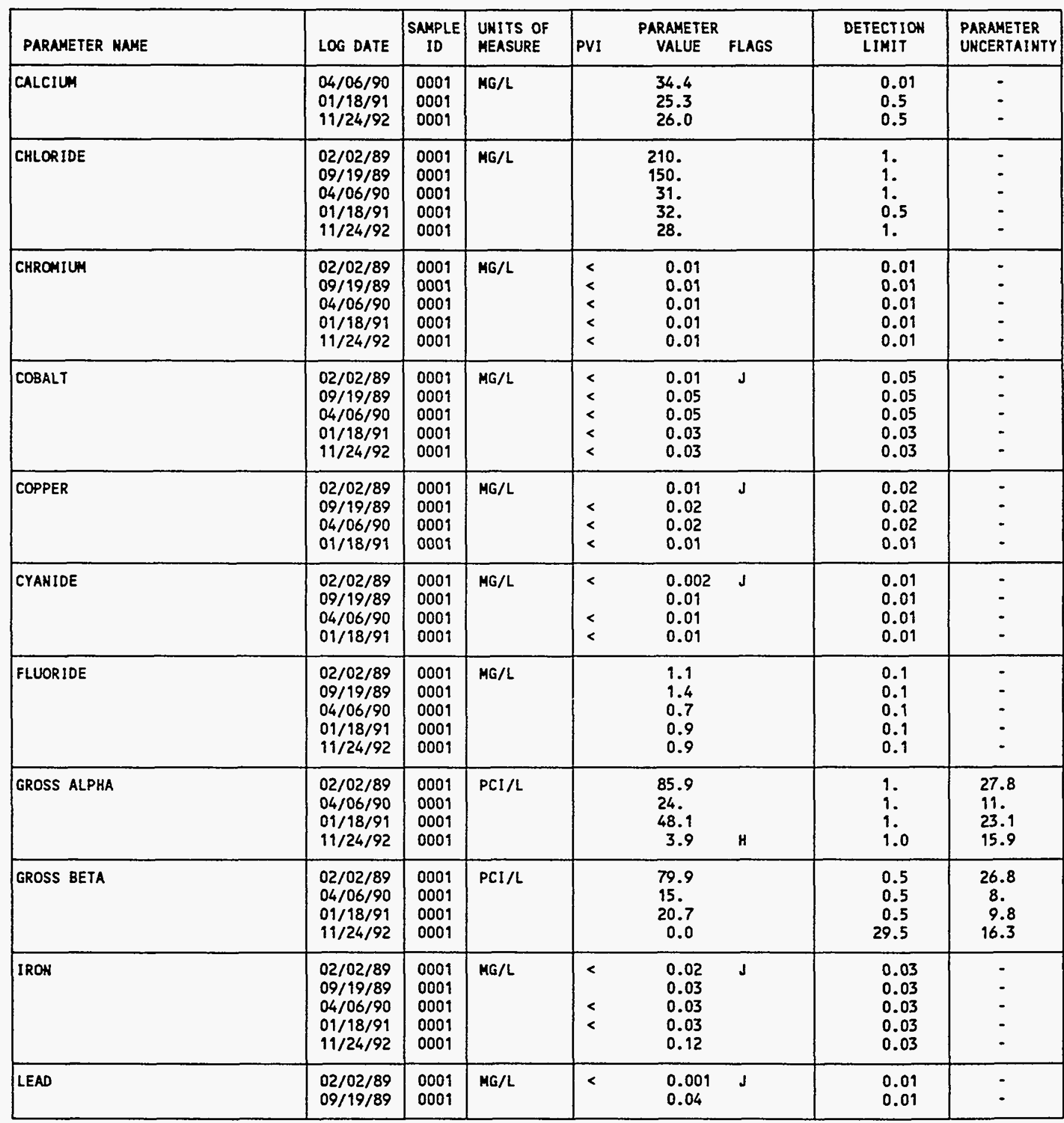

PARAMETER VALUE INDICATOR (PVI): < - LESS THAN DETECTION LIMIT

SAMPLE ID COOES:

0001 - FILTERED SAMPLE (.45 MICRONS)

OTHER PARAMETER VALUE FLAGS:

H - HOLD TIME EXPIRED, VALUE SUSPECT

$J$ - estimated value 
GROUNDHATER QUALITY DATA BY LOCATION

SITE: AMBO1 AMBROSIA LAKE

LOCATION: 0676 RESERVED ESTORHS, MH, 11/14/88

MORTH COORDINATE: UHKNOWN

EAST COORDINATE: UNKNOWN

06/10/80 TO $12 / 04 / 92$

REPORT DATE: 08/04/94

FORMATION OF COMPLETION: TRES HERMANOS - C2 SANDSTONE (TT) HYDRAULIC FLOW RELATIONSHIP: CROSS GRADIEHT (C)

\begin{tabular}{|c|c|c|c|c|c|c|c|c|}
\hline PARAMETER NAME & LOS DATE & $\begin{array}{c}\text { SAMPLE } \\
10\end{array}$ & $\begin{array}{l}\text { UNITS OF } \\
\text { MEASURE }\end{array}$ & PVI & $\begin{array}{l}\text { PARAMETER } \\
\text { VALUE }\end{array}$ & FLAGS & $\begin{array}{l}\text { DETECTION } \\
\text { LIMIT }\end{array}$ & $\begin{array}{l}\text { PARAMETER } \\
\text { UNCERTAINTY }\end{array}$ \\
\hline LEAD & $\begin{array}{l}04 / 06 / 90 \\
01 / 18 / 91\end{array}$ & $\begin{array}{l}0001 \\
0001\end{array}$ & MG/L & $<$ & $\begin{array}{l}0.01 \\
0.03\end{array}$ & 1 & $\begin{array}{l}0.01 \\
0.03\end{array}$ & - \\
\hline LEAD-210 & $02 / 02 / 89$ & 0001 & $\mathrm{PCI} / \mathrm{L}$ & & 0.00 & & 1.5 & 6.21 \\
\hline MAGNESIUM & $\begin{array}{l}02 / 02 / 89 \\
09 / 19 / 89 \\
04 / 06 / 90 \\
01 / 18 / 91 \\
11 / 24 / 92\end{array}$ & $\begin{array}{l}0001 \\
0001 \\
0001 \\
0001 \\
0001\end{array}$ & $M G / L$ & & $\begin{array}{r}33 . \\
15.2 \\
14.3 \\
10.0 \\
9.5\end{array}$ & & $\begin{array}{l}0.001 \\
0.001 \\
0.001 \\
0.1 \\
0.1\end{array}$ & $\begin{array}{l}- \\
- \\
-\end{array}$ \\
\hline MANGANESE & $\begin{array}{l}02 / 02 / 89 \\
09 / 19 / 89 \\
04 / 06 / 90 \\
01 / 18 / 91\end{array}$ & $\begin{array}{l}0001 \\
0001 \\
0001 \\
0001\end{array}$ & MG/L & & $\begin{array}{l}0.13 \\
0.43 \\
0.02 \\
0.03\end{array}$ & & $\begin{array}{l}0.01 \\
0.01 \\
0.01 \\
0.01\end{array}$ & : \\
\hline MOLYBDEENUM & $\begin{array}{l}02 / 02 / 89 \\
09 / 19 / 89 \\
04 / 06 / 90 \\
01 / 18 / 91 \\
11 / 24 / 92\end{array}$ & $\begin{array}{l}0001 \\
0001 \\
0001 \\
0001 \\
0001\end{array}$ & $M G / L$ & & $\begin{array}{l}0.595 \\
0.24 \\
0.02 \\
0.02 \\
0.01\end{array}$ & & $\begin{array}{l}0.01 \\
0.01 \\
0.01 \\
0.01 \\
0.01\end{array}$ & $\begin{array}{l}- \\
- \\
-\end{array}$ \\
\hline NET GROSS ALPHA * & $\begin{array}{l}02 / 02 / 89 \\
04 / 06 / 90 \\
01 / 18 / 91 \\
11 / 24 / 92\end{array}$ & $\begin{array}{l}0001 \\
0001 \\
0001 \\
0001\end{array}$ & $\mathrm{PCI} / \mathrm{L}$ & & $\begin{array}{r}-56.10 \\
18.92 \\
47.76 \\
1.84\end{array}$ & & : & : \\
\hline NICKEL & $\begin{array}{l}02 / 02 / 89 \\
09 / 19 / 89 \\
04 / 06 / 90 \\
01 / 18 / 91 \\
11 / 24 / 92\end{array}$ & $\begin{array}{l}0001 \\
0001 \\
0001 \\
0001 \\
0001\end{array}$ & MG/L & $\begin{array}{l}< \\
< \\
< \\
< \\
<\end{array}$ & $\begin{array}{l}0.02 \\
0.04 \\
0.04 \\
0.04 \\
0.04\end{array}$ & J & $\begin{array}{l}0.04 \\
0.04 \\
0.04 \\
0.04 \\
0.04\end{array}$ & $\begin{array}{l}- \\
- \\
-\end{array}$ \\
\hline MITRITE AMD NITRATE & $\begin{array}{l}04 / 06 / 90 \\
01 / 18 / 91\end{array}$ & $\begin{array}{l}0001 \\
0001\end{array}$ & $M G / L$ & & $\begin{array}{l}5.8 \\
4.74\end{array}$ & & $\begin{array}{l}1 . \\
0.05\end{array}$ & - \\
\hline PH & $\begin{array}{l}02 / 02 / 89 \\
09 / 19 / 89 \\
04 / 06 / 90 \\
01 / 18 / 91 \\
11 / 24 / 92\end{array}$ & $\begin{array}{l}0001 \\
0001 \\
0001 \\
0001 \\
0001\end{array}$ & su & & $\begin{array}{l}8.20 \\
8.02 \\
7.64 \\
8.09 \\
7.91\end{array}$ & & $\begin{array}{l}- \\
- \\
- \\
-\end{array}$ & $\begin{array}{l}- \\
- \\
-\end{array}$ \\
\hline PHOSPHATE & $02 / 02 / 89$ & 0001 & MG/L & $<$ & 0.1 & & 0.1 & - \\
\hline
\end{tabular}

- het gross alPha (GROSS ALPHA - URANIUM) HITH 1 Mg URANIUM = 686 PCI

PARAMETER VALUE IMDICATOR (PVI): < - LESS THAN DETECTION LIMIT SAMPLE ID COOES:

OTHER PARAMETER VALUE FLAGS:

I - IMCREASED DETECTION LIMIT DUE TO REQUIRED DILUTION

J - eStimated VALUE 
GROUNDWATER QUALITY DATA BY LOCATION

SITE: AMBO1 AMBROSIA LAKE

LOCATION: 0676 RESERVED ESTORHS, MH, 11/14/88

NORTH COORDIHATE: UHKNOWN

EAST COORDINATE: UNKNOWN

$06 / 10 / 80$ TO $12 / 04 / 92$

REPORT DATE: 08/04/94

FORMATION OF COHPLETION: TRES HERMAHOS - C2 SAHDSTONE (TT)

HYDRAULIC FLON RELATIONSHIP: CROSS GRADIENT (C)

\begin{tabular}{|c|c|c|c|c|c|c|c|}
\hline PARAMETER NAME & LOG DATE & $\begin{array}{c}\text { SAMPLE } \\
\text { ID }\end{array}$ & $\begin{array}{l}\text { UNITS OF } \\
\text { MEASURE }\end{array}$ & PVI & $\begin{array}{l}\text { PARAMETER } \\
\text { VALUE FLAGS }\end{array}$ & $\begin{array}{l}\text { DETECTION } \\
\text { LIMIT }\end{array}$ & $\begin{array}{l}\text { PARAMETER } \\
\text { UHCERTAINTY }\end{array}$ \\
\hline PHOSPHATE & $\begin{array}{l}09 / 19 / 89 \\
04 / 06 / 90 \\
01 / 18 / 91 \\
11 / 24 / 92\end{array}$ & $\begin{array}{l}0001 \\
0001 \\
0001 \\
0001\end{array}$ & $M G / L$ & $<$ & $\begin{array}{l}0.1 \\
0.3 \\
0.12 \\
0.6\end{array}$ & $\begin{array}{l}0.1 \\
0.1 \\
0.05 \\
0.1\end{array}$ & $\begin{array}{l}- \\
- \\
-\end{array}$ \\
\hline POLONIUM-210 & $02 / 02 / 89$ & 0001 & $\mathrm{PCI} / \mathrm{L}$ & & 0.777 & 1. & 1.33 \\
\hline POTASSIUM & $\begin{array}{l}02 / 02 / 89 \\
09 / 19 / 89 \\
04 / 06 / 90 \\
01 / 18 / 91 \\
11 / 24 / 92\end{array}$ & $\begin{array}{l}0001 \\
0001 \\
0001 \\
0001 \\
0001\end{array}$ & MG/L & & $\begin{array}{c}10.4 \\
5.4 \\
4.8 \\
3.3 \\
3.82\end{array}$ & $\begin{array}{l}0.01 \\
0.01 \\
0.01 \\
0.01 \\
0.01\end{array}$ & $\begin{array}{l}- \\
- \\
- \\
-\end{array}$ \\
\hline RADIUM-226 & $\begin{array}{l}02 / 02 / 89 \\
04 / 06 / 90 \\
01 / 18 / 91 \\
11 / 24 / 92\end{array}$ & $\begin{array}{l}0001 \\
0001 \\
0001 \\
0001\end{array}$ & $\mathrm{PCI} / \mathrm{L}$ & & $\begin{array}{l}1.72 \\
0.4 \\
1.0 \\
0.9\end{array}$ & $\begin{array}{l}1 . \\
1 . \\
1 . \\
0.3\end{array}$ & $\begin{array}{l}0.291 \\
0.3 \\
0.5 \\
0.4\end{array}$ \\
\hline RADIUH-226 + RADIUH-228 & $\begin{array}{l}02 / 02 / 89 \\
04 / 06 / 90 \\
01 / 18 / 91 \\
11 / 24 / 92\end{array}$ & $\begin{array}{l}0001 \\
0001 \\
0001 \\
0001\end{array}$ & $\mathrm{PCI} / \mathrm{L}$ & & $\begin{array}{r}24.12 \\
1.60 \\
4.00 \\
1.30\end{array}$ & $\begin{array}{l}- \\
\dot{ } \\
-\end{array}$ & $\begin{array}{l}- \\
- \\
-\end{array}$ \\
\hline RADIUH -228 & $\begin{array}{l}02 / 02 / 89 \\
04 / 06 / 90 \\
01 / 18 / 91 \\
11 / 24 / 92\end{array}$ & $\begin{array}{l}0001 \\
0001 \\
0001 \\
0001\end{array}$ & $\mathrm{PCI} / \mathrm{L}$ & & $\begin{array}{r}22.4 \\
1.2 \\
3.0 \\
0.4\end{array}$ & $\begin{array}{l}1 . \\
1 . \\
1.0\end{array}$ & $\begin{array}{l}8.63 \\
0.9 \\
1.6 \\
1.5\end{array}$ \\
\hline SELENIUM & $\begin{array}{l}02 / 02 / 89 \\
09 / 19 / 89 \\
04 / 06 / 90 \\
01 / 18 / 91 \\
11 / 24 / 92\end{array}$ & $\begin{array}{l}0001 \\
0001 \\
0001 \\
0001 \\
0001\end{array}$ & $M G / L$ & $\begin{array}{l}< \\
< \\
<\end{array}$ & $\begin{array}{ll}0.091 & \\
0.021 & \\
0.005 & \\
0.03 & 1 \\
0.05 & 1\end{array}$ & $\begin{array}{l}0.005 \\
0.005 \\
0.005 \\
0.03 \\
0.05\end{array}$ & $\begin{array}{l}- \\
- \\
- \\
-\end{array}$ \\
\hline SILICA - SIO2 & $\begin{array}{l}02 / 02 / 89 \\
09 / 19 / 89 \\
04 / 06 / 90 \\
01 / 18 / 91\end{array}$ & $\begin{array}{l}0001 \\
0001 \\
0001 \\
0001\end{array}$ & $M G / L$ & & $\begin{array}{l}13.3 \\
11 . \\
8 . \\
7.1\end{array}$ & $\begin{array}{l}2 . \\
2 . \\
2 . \\
0.1\end{array}$ & $\begin{array}{l}- \\
. \\
.\end{array}$ \\
\hline SILVER & $\begin{array}{l}02 / 02 / 89 \\
09 / 19 / 89 \\
04 / 06 / 90 \\
01 / 18 / 91 \\
11 / 24 / 92\end{array}$ & $\begin{array}{l}0001 \\
0001 \\
0001 \\
0001 \\
0001\end{array}$ & MG $/ L$ & $\begin{array}{l}< \\
< \\
< \\
< \\
<\end{array}$ & $\begin{array}{l}0.01 \\
0.01 \\
0.01 \\
0.01 \\
0.01\end{array}$ & $\begin{array}{l}0.01 \\
0.01 \\
0.01 \\
0.01 \\
0.01\end{array}$ & $\begin{array}{l}- \\
- \\
- \\
-\end{array}$ \\
\hline SOOIUH & $\begin{array}{l}02 / 02 / 89 \\
09 / 19 / 89 \\
04 / 06 / 90 \\
01 / 18 / 91 \\
11 / 24 / 92\end{array}$ & $\begin{array}{l}0001 \\
0001 \\
0001 \\
0001 \\
0001\end{array}$ & $M G / L$ & & $\begin{array}{l}750 . \\
804 . \\
674 . \\
754 . \\
665 .\end{array}$ & $\begin{array}{l}0.002 \\
0.002 \\
0.002 \\
5 . \\
5 .\end{array}$ & $\begin{array}{r}\cdot \\
- \\
- \\
-\end{array}$ \\
\hline SPECIFIC CONDUCTAKCE & $\begin{array}{l}02 / 02 / 89 \\
09 / 19 / 89 \\
04 / 06 / 90 \\
01 / 18 / 91\end{array}$ & $\begin{array}{l}0001 \\
0001 \\
0001 \\
0001\end{array}$ & UHHO/CM & & $\begin{array}{l}2000 . \\
1450 . \\
2000 . \\
2150 .\end{array}$ & $\begin{array}{l}- \\
- \\
-\end{array}$ & $\begin{array}{l}- \\
- \\
-\end{array}$ \\
\hline
\end{tabular}

PARAMETER VALUE INDICATOR (PVI): < - LESS THAN DETECTIOH LIMIT

SAMPLE ID COOES:

OOO1 - FILTERED SAMPLE (.45 MICROAS)

OTHER PARAMETER VALUE FLAGS:

H - HOLD TIME EXPIRED, VALUE SUSPECT

1 - INCREASED DETECTION LIMIT DUE TO REOUIRED DILUTION

J. estIMATED VALUE 
GROUNDWATER QUALITY DATA BY LOCATION

SITE: AMBOI AMBROSIA LAKE

LOCATIOH: 0676 RESERVED ESTORHS, MH, 11/14/88

MORTH COORDIHATE: UNKHOWH

EAST COORDINATE: UNKNOWN

06/10/80 TO 12/04/92

REPORT DATE: 08/04/94

FORMATION OF COMPLETION: TRES HERMANOS - C2 SAKDSTONE (TT) HYDRAULIC FLON RELATIONSHIP: CROSS GRADIENT (C)

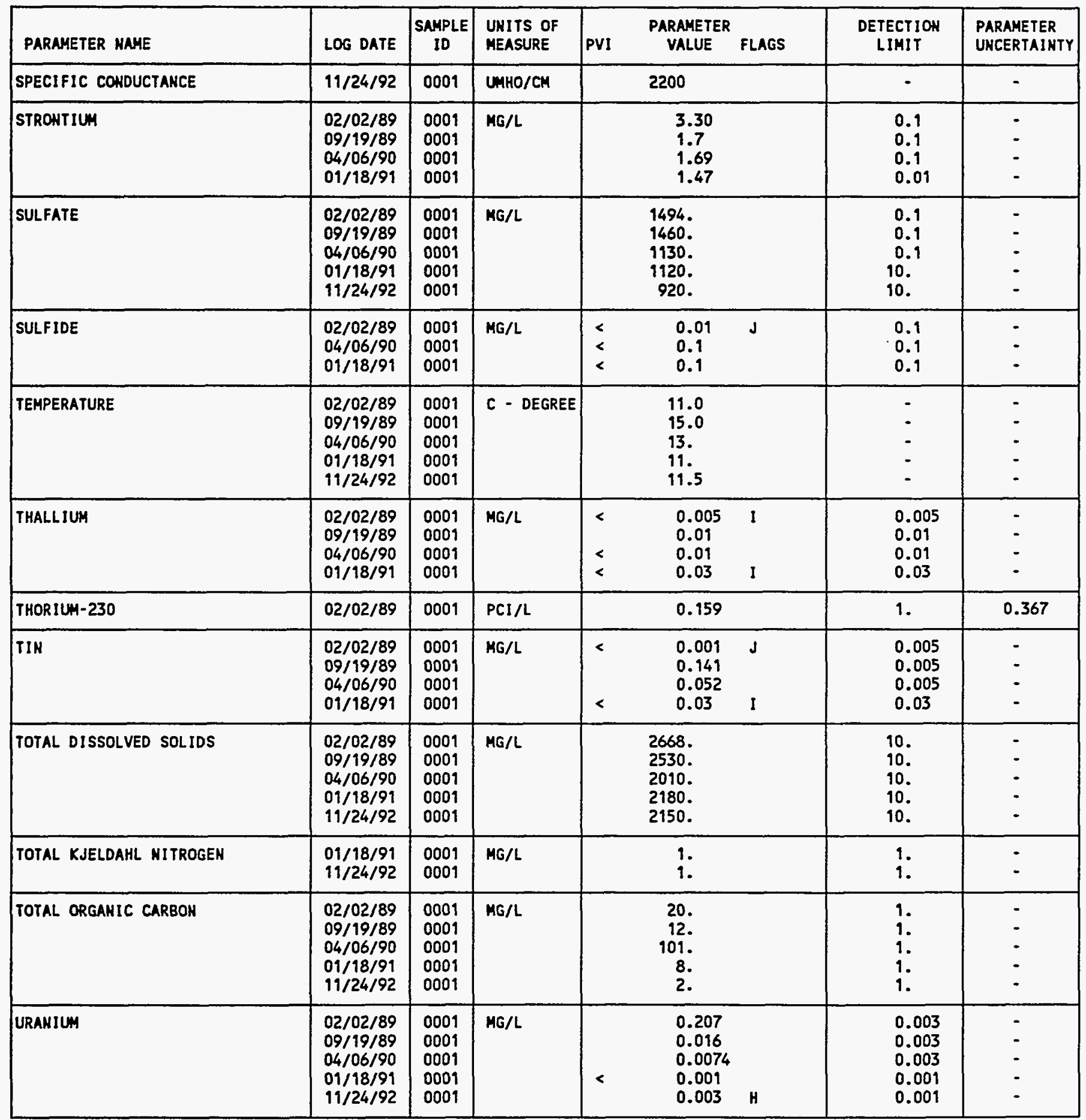

PARAMETER VALUE IMDICATOR (PVI): < - LESS THAK DETECTION LIMIT

SAHPLE ID CODES:

0001 - FILTERED SAMPLE (.45 MICRONS)

OTHER PARAMETER VALUE FLAGS:

H - HOLD TIME EXPIRED, VALUE SUSPECT

1 - INCREASED DETECTION LIMIT DUE TO REOUIRED DILUTION

$\jmath$ - ESTIMATED VALUE 
GROUNDWATER QUALITY DATA BY LOCATION

SITE: AMBOI AMBROSIA LAKE

LOCATION: 0676 RESERVED ESTORMS, MH, 11/14/88

NORTH COORDINATE: UNKNOWN

EAST COORDINATE: UNKNOWH

06/10/80 TO $12 / 04 / 92$

REPORT DATE: 08/04/94

FORMATION OF COMPLETION: TRES HERMANOS - C2 SANDSTONE (TT)

HYDRAULIC FLOW RELATIONSHIP: CROSS GRADIENT (C)

\begin{tabular}{|c|c|c|c|c|c|c|c|}
\hline PARAMETER RAME & LOG DATE & $\begin{array}{c}\text { SAMPLE } \\
\text { ID }\end{array}$ & $\begin{array}{l}\text { UHITS OF } \\
\text { MEASURE }\end{array}$ & PVI & $\begin{array}{l}\text { PARAMETER } \\
\text { VALUE FLAGS }\end{array}$ & $\begin{array}{l}\text { DETECTION } \\
\text { LIMIT }\end{array}$ & $\begin{array}{l}\text { PARAMETER } \\
\text { UHCERTAINTY }\end{array}$ \\
\hline VAKADIUM & $\begin{array}{l}02 / 02 / 89 \\
09 / 19 / 89 \\
04 / 06 / 90 \\
01 / 18 / 91 \\
11 / 24 / 92\end{array}$ & $\begin{array}{l}0001 \\
0001 \\
0001 \\
0001 \\
0001\end{array}$ & $M G / L$ & $\begin{array}{l}< \\
< \\
< \\
<\end{array}$ & $\begin{array}{l}0.01 \\
0.01 \\
0.01 \\
0.01 \\
0.01\end{array}$ & $\begin{array}{l}0.01 \\
0.01 \\
0.01 \\
0.01 \\
0.01\end{array}$ & $\begin{array}{l}- \\
- \\
- \\
-\end{array}$ \\
\hline ZINC & $\begin{array}{l}02 / 02 / 89 \\
09 / 19 / 89 \\
04 / 06 / 90 \\
01 / 18 / 91\end{array}$ & $\begin{array}{l}0001 \\
0001 \\
0001 \\
0001\end{array}$ & $M G / L$ & $<$ & $\begin{array}{l}0.01 \\
0.048 \\
0.005 \\
0.030\end{array}$ & $\begin{array}{l}0.01 \\
0.005 \\
0.005 \\
0.005\end{array}$ & $\begin{array}{l}- \\
- \\
-\end{array}$ \\
\hline
\end{tabular}

PARAMETER VALUE INDICATOR (PVI): < - LESS THAH DETECTION LIMIT

SAMPLE IO COOES:

0001 - FILTERED SAMPLE (.45 MICRONS) 
GROUNDHATER QUALITY DATA BY LOCATION

SITE: AMBO1 AMBROSIA LAKE

LOCATIOH: 0677 RESERVED ESTORMS, MH, 11/14/88

NORTH COOROINATE: UHKNOWN

EAST COOROINATE: UNKNOWN

06/10/80 TO 12/04/92

REPORT DATE: $08 / 04 / 94$

FORMATION OF COMPLETION: TRES HERMANOS - C2 SANDSTOHE (TT) HYDRAULIC FLON RELATIONSHIP: CROSS GRADIENT (C)

\begin{tabular}{|c|c|c|c|c|c|c|c|c|}
\hline PARAMETER HAME & LOG DATE & $\underset{\text { ID }}{\text { SAMPLE }}$ & $\begin{array}{l}\text { UNITS OF } \\
\text { MEASURE }\end{array}$ & PVI & $\begin{array}{l}\text { ARAMETER } \\
\text { VALUE }\end{array}$ & FLAGS & $\begin{array}{l}\text { DETECTION } \\
\text { LIMIT }\end{array}$ & $\begin{array}{l}\text { PARAMETER } \\
\text { UHCERTAINTY }\end{array}$ \\
\hline ALKALIHITY & $\begin{array}{l}02 / 06 / 89 \\
09 / 19 / 89 \\
04 / 11 / 90 \\
01 / 18 / 91\end{array}$ & $\begin{array}{l}0001 \\
0001 \\
0001 \\
0001\end{array}$ & MG/L CACO3 & & $\begin{array}{l}296 . \\
428 . \\
394 . \\
398 .\end{array}$ & & $\begin{array}{l}- \\
- \\
-\end{array}$ & $\begin{array}{l}- \\
- \\
-\end{array}$ \\
\hline ALUMINUM & $\begin{array}{l}02 / 06 / 89 \\
09 / 19 / 89 \\
04 / 11 / 90 \\
01 / 18 / 91\end{array}$ & $\begin{array}{l}0001 \\
0001 \\
0001 \\
0001\end{array}$ & MG/L & $\begin{array}{l}< \\
< \\
< \\
<\end{array}$ & $\begin{array}{l}0.05 \\
0.1 \\
0.1 \\
0.05\end{array}$ & $J$ & $\begin{array}{l}0.1 \\
0.1 \\
0.1 \\
0.05\end{array}$ & $\begin{array}{l}- \\
- \\
-\end{array}$ \\
\hline NMHONIUM & $\begin{array}{l}02 / 06 / 89 \\
09 / 19 / 89 \\
04 / 11 / 90 \\
01 / 18 / 91\end{array}$ & $\begin{array}{l}0001 \\
0001 \\
0001 \\
0001\end{array}$ & MG/L & $<$ & $\begin{array}{l}0.58 \\
0.8 \\
0.1 \\
0.49\end{array}$ & & $\begin{array}{l}0.1 \\
0.1 \\
0.1 \\
0.01\end{array}$ & : \\
\hline ARSENIC & $\begin{array}{l}02 / 06 / 89 \\
09 / 19 / 89 \\
04 / 11 / 90 \\
01 / 18 / 91\end{array}$ & $\begin{array}{l}0001 \\
0001 \\
0001 \\
0001\end{array}$ & $M G / L$ & $\begin{array}{l}< \\
< \\
< \\
<\end{array}$ & $\begin{array}{l}0.001 \\
0.01 \\
0.01 \\
0.05\end{array}$ & $\begin{array}{l}J \\
1\end{array}$ & $\begin{array}{l}0.01 \\
0.01 \\
0.01 \\
0.05\end{array}$ & - \\
\hline BARIUH & $\begin{array}{l}02 / 06 / 89 \\
09 / 19 / 89 \\
04 / 11 / 90 \\
01 / 18 / 91\end{array}$ & $\begin{array}{l}0001 \\
0001 \\
0001 \\
0001\end{array}$ & $M G / L$ & $<$ & $\begin{array}{l}0.02 \\
0.1 \\
0.1 \\
0.01\end{array}$ & J & $\begin{array}{l}0.1 \\
0.1 \\
0.1 \\
0.01\end{array}$ & $\begin{array}{l}- \\
- \\
-\end{array}$ \\
\hline BERYLLIUH & $\begin{array}{l}02 / 06 / 89 \\
09 / 19 / 89 \\
04 / 11 / 90 \\
01 / 18 / 91\end{array}$ & $\begin{array}{l}0001 \\
0001 \\
0001 \\
0001\end{array}$ & $M G / L$ & $\begin{array}{l}< \\
< \\
< \\
<\end{array}$ & $\begin{array}{l}0.005 \\
0.01 \\
0.01 \\
0.005\end{array}$ & & $\begin{array}{l}0.005 \\
0.01 \\
0.01 \\
0.005\end{array}$ & $\begin{array}{l}- \\
- \\
.\end{array}$ \\
\hline CADMIUM & $\begin{array}{l}02 / 06 / 89 \\
09 / 19 / 89 \\
04 / 11 / 90 \\
01 / 18 / 91\end{array}$ & $\begin{array}{l}0001 \\
0001 \\
0001 \\
0001\end{array}$ & MG/L & $<$ & $\begin{array}{l}0.0009 \\
0.004 \\
0.001 \\
0.001\end{array}$ & $J$ & $\begin{array}{l}0.001 \\
0.001 \\
0.001 \\
0.001\end{array}$ & $\begin{array}{l}- \\
- \\
-\end{array}$ \\
\hline CALCIUH & $\begin{array}{l}02 / 06 / 89 \\
09 / 19 / 89 \\
04 / 11 / 90 \\
01 / 18 / 91\end{array}$ & $\begin{array}{l}0001 \\
0001 \\
0001 \\
0001\end{array}$ & $M G / L$ & & $\begin{array}{l}75 . \\
31.9 \\
28.2 \\
30.9\end{array}$ & & $\begin{array}{l}0.01 \\
0.01 \\
0.01 \\
0.5\end{array}$ & $\dot{-}$ \\
\hline CHLORIDE & $02 / 06 / 89$ & 0001 & MG/L & & 73. & & 1. & - \\
\hline
\end{tabular}

PARAMETER VALUE IMDICATOR (PVI): < - LESS THAN DETECTION LIMIT

SAMPLE ID COOES:

0001 - FILTERED SAMPLE (.45 MICRONS)

OTHER PARAMETER VALUE FLAGS:

I - INCREASED DETECTION LIMIT DUE TO REQUIRED DILUTION

J - estimated VALUE 
GROUNDWATER QUALITY DATA BY LOCATION

SITE: AMBOI AMBROSIA LAKE

LOCATION: 06T7 RESERVED ESTORMS, MW, 11/14/88

NORTH COORDINATE: UNKNOWN

EAST COORDINATE: UNKMOWN

06/10/80 TO $12 / 04 / 92$

REPORT DATE: 08/04/94

FORMATION OF COMPLETION: TRES HERMANOS - C2 SANDSTONE (TT)

HYDRAULIC FLON RELATIONSHIP: CROSS GRADIENT (C)

\begin{tabular}{|c|c|c|c|c|c|c|c|c|}
\hline PARAMETER NAME & LOG DATE & $\begin{array}{c}\text { SAMPLE } \\
\text { ID }\end{array}$ & $\begin{array}{l}\text { UNITS OF } \\
\text { MEASURE }\end{array}$ & PVI & $\begin{array}{l}\text { ARAMETER } \\
\text { VALUE }\end{array}$ & FLAGS & $\begin{array}{l}\text { DETECTION } \\
\text { LIMIT }\end{array}$ & $\begin{array}{l}\text { PARAMETER } \\
\text { UNCERTAINTY }\end{array}$ \\
\hline CHLORIDE & $\begin{array}{l}09 / 19 / 89 \\
04 / 11 / 90 \\
01 / 18 / 91\end{array}$ & $\begin{array}{l}0001 \\
0001 \\
0001\end{array}$ & $M G / L$ & & $\begin{array}{l}35 . \\
40 . \\
34 .\end{array}$ & & $\begin{array}{l}1 . \\
1 . \\
0.5\end{array}$ & $\begin{array}{l}- \\
-\end{array}$ \\
\hline CHROMIUM & $\begin{array}{l}02 / 06 / 89 \\
09 / 19 / 89 \\
04 / 11 / 90 \\
01 / 18 / 91\end{array}$ & $\begin{array}{l}0001 \\
0001 \\
0001 \\
0001\end{array}$ & $M G / L$ & $\begin{array}{l}< \\
< \\
<\end{array}$ & $\begin{array}{l}0.01 \\
0.01 \\
0.01 \\
0.01\end{array}$ & & $\begin{array}{l}0.01 \\
0.01 \\
0.01 \\
0.01\end{array}$ & - \\
\hline COBALT & $\begin{array}{l}02 / 06 / 89 \\
09 / 19 / 89 \\
04 / 11 / 90 \\
01 / 18 / 91\end{array}$ & $\begin{array}{l}0001 \\
0001 \\
0001 \\
0001\end{array}$ & $M G / L$ & $\begin{array}{l}< \\
< \\
<\end{array}$ & $\begin{array}{l}0.01 \\
0.05 \\
0.05 \\
0.03\end{array}$ & J & $\begin{array}{l}0.05 \\
0.05 \\
0.05 \\
0.03\end{array}$ & $\begin{array}{l}- \\
- \\
-\end{array}$ \\
\hline COPPER & $\begin{array}{l}02 / 06 / 89 \\
09 / 19 / 89 \\
04 / 11 / 90 \\
01 / 18 / 91\end{array}$ & $\begin{array}{l}0001 \\
0001 \\
0001 \\
0001\end{array}$ & $M G / L$ & $\begin{array}{l}< \\
< \\
<\end{array}$ & $\begin{array}{l}0.01 \\
0.02 \\
0.02 \\
0.01\end{array}$ & J & $\begin{array}{l}0.02 \\
0.02 \\
0.02 \\
0.01\end{array}$ & - \\
\hline CYANIDE & $\begin{array}{l}02 / 06 / 89 \\
09 / 19 / 89 \\
04 / 11 / 90 \\
01 / 18 / 91\end{array}$ & $\begin{array}{l}0001 \\
0001 \\
0001 \\
0001\end{array}$ & $M G / L$ & $\begin{array}{l}< \\
< \\
<\end{array}$ & $\begin{array}{l}0.002 \\
0.01 \\
0.01 \\
0.01\end{array}$ & 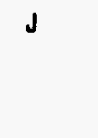 & $\begin{array}{l}0.01 \\
0.01 \\
0.01 \\
0.01\end{array}$ & $\begin{array}{l}- \\
-\end{array}$ \\
\hline FLUORIDE & $\begin{array}{l}02 / 06 / 89 \\
09 / 19 / 89 \\
04 / 11 / 90 \\
01 / 18 / 91\end{array}$ & $\begin{array}{l}0001 \\
0001 \\
0001 \\
0001\end{array}$ & MG/L & & $\begin{array}{l}1.0 \\
0.8 \\
0.8 \\
0.9\end{array}$ & & $\begin{array}{l}0.1 \\
0.1 \\
0.1 \\
0.1\end{array}$ & $\begin{array}{l}- \\
-\end{array}$ \\
\hline GROSS ALPHA & $\begin{array}{l}02 / 06 / 89 \\
09 / 19 / 89 \\
04 / 11 / 90 \\
01 / 18 / 91\end{array}$ & $\begin{array}{l}0001 \\
0001 \\
0001 \\
0001\end{array}$ & $\mathrm{PCI} / \mathrm{L}$ & & $\begin{array}{l}1.48 \\
29 . \\
13 . \\
24.2\end{array}$ & & $\begin{array}{l}1 . \\
1 . \\
1 .\end{array}$ & $\begin{array}{l}17.2 \\
20 . \\
15 . \\
22.9\end{array}$ \\
\hline GROSS BETA & $\begin{array}{l}02 / 06 / 89 \\
09 / 19 / 89 \\
04 / 11 / 90 \\
01 / 18 / 99\end{array}$ & $\begin{array}{l}0001 \\
0001 \\
0001 \\
0001\end{array}$ & $\mathrm{PCl} / \mathrm{L}$ & & $\begin{array}{l}14.0 \\
22 . \\
16 . \\
27.2\end{array}$ & & $\begin{array}{l}0.5 \\
0.5 \\
0.5 \\
0.5\end{array}$ & $\begin{array}{l}23.0 \\
10 . \\
13 . \\
16.8\end{array}$ \\
\hline IRON & $\begin{array}{l}02 / 06 / 89 \\
09 / 19 / 89 \\
04 / 11 / 90 \\
01 / 18 / 91\end{array}$ & $\begin{array}{l}0001 \\
0001 \\
0001 \\
0001\end{array}$ & $M G / L$ & $\begin{array}{l}< \\
< \\
<\end{array}$ & $\begin{array}{l}0.02 \\
0.03 \\
0.03 \\
0.03\end{array}$ & 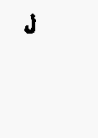 & $\begin{array}{l}0.03 \\
0.03 \\
0.03 \\
0.03\end{array}$ & : \\
\hline LEAD & $\begin{array}{l}02 / 06 / 89 \\
09 / 19 / 89 \\
04 / 11 / 90 \\
01 / 18 / 91\end{array}$ & $\begin{array}{l}0001 \\
0001 \\
0001 \\
0001\end{array}$ & $M G / L$ & $\begin{array}{l}< \\
<\end{array}$ & $\begin{array}{l}0.001 \\
0.05 \\
0.01 \\
0.03\end{array}$ & $\begin{array}{l}J \\
I\end{array}$ & $\begin{array}{l}0.01 \\
0.01 \\
0.01 \\
0.03\end{array}$ & $:$ \\
\hline LEAD-210 & $\begin{array}{l}02 / 06 / 89 \\
09 / 19 / 89\end{array}$ & $\begin{array}{l}0001 \\
0001\end{array}$ & $\mathrm{PCI} / \mathrm{L}$ & & $\begin{array}{l}0.00 \\
0.0\end{array}$ & & $\begin{array}{l}1.5 \\
1.5\end{array}$ & $\begin{array}{l}0.512 \\
0.6\end{array}$ \\
\hline MAGNESIUM & $\begin{array}{l}02 / 06 / 89 \\
09 / 19 / 89 \\
04 / 11 / 90 \\
01 / 18 / 91\end{array}$ & $\begin{array}{l}0001 \\
0001 \\
0001 \\
0001\end{array}$ & $M G / L$ & & $\begin{array}{l}21 . \\
11.8 \\
11.3 \\
11.8\end{array}$ & & $\begin{array}{l}0.001 \\
0.001 \\
0.001 \\
0.1\end{array}$ & : \\
\hline
\end{tabular}

PARAMETER VAlue INDICATOR (PVI): \& LESS THAN DETECTION LIMIT

OTHER PARAMETER VALUE FLAGS:

I - INCREASED DETECTION LIMIT DUE TO REQUIRED DILUTION

J. estimated VAlUe 
GROUNDWATER QUALITY DATA BY LOCATION

SITE: AMBO1 AMBROSIA LAKE

LOCATION: 0677 RESERVED ESTORMS, MH, 11/14/88

MORTH COORDINATE: UNKNOWN

EAST COORDINATE: UNKHOWN

06/10/80 TO 12/04/92

REPORT DATE: 08/04/94

FORMATION OF COMPLETION: TRES HERMANOS - C2 SANDSTONE (TT)

HYDRAULIC FLOW RELATIONSHIP: CROSS GRADIENT (C)

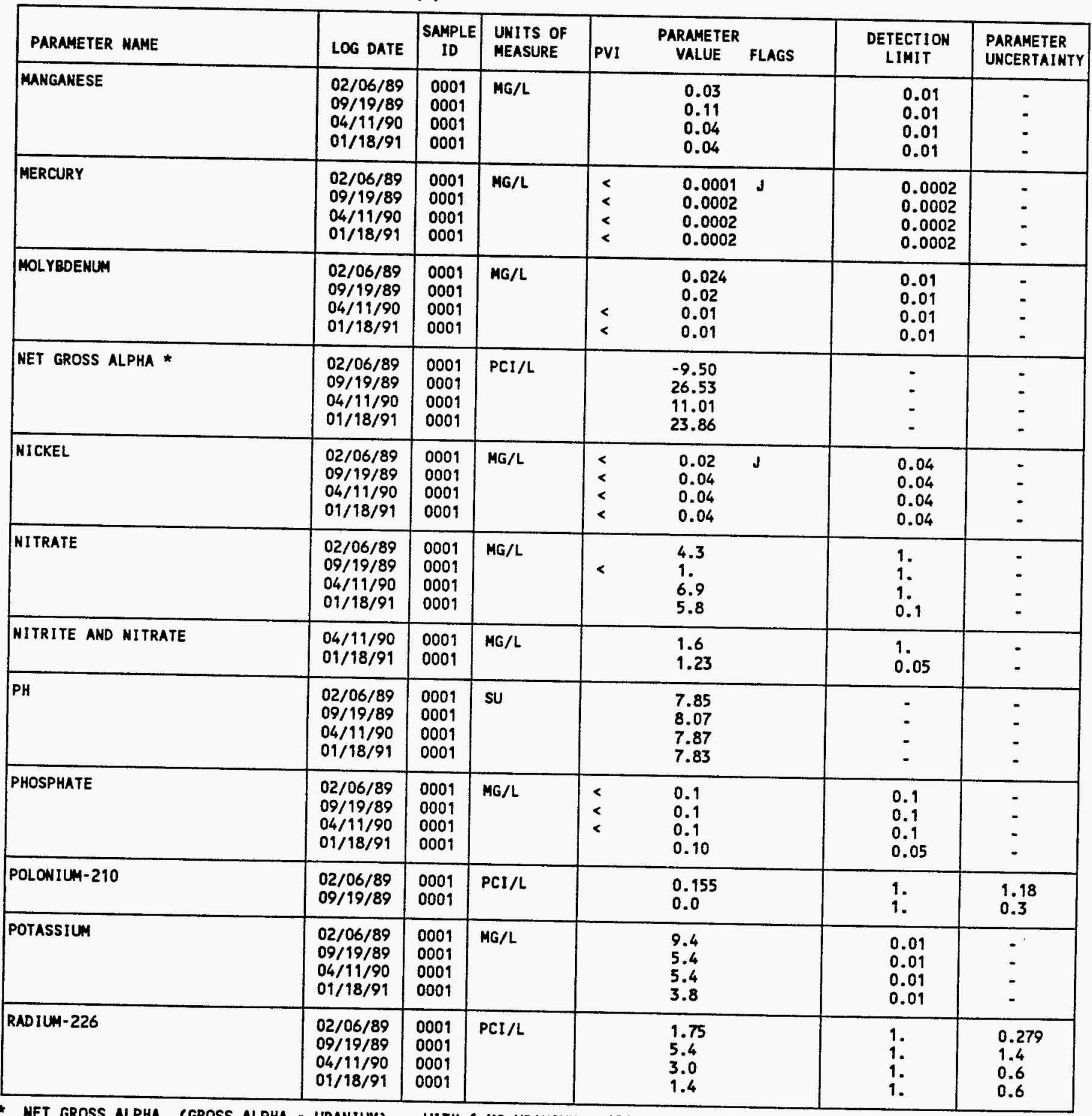

- het gross alPha (gross alpha - URAhiUm)

WITH 1 MG URANIUM $=686 \mathrm{PCI}$

PARAMETER VALUE INDICATOR (PVI): < - LESS THAN DETECTION LIMIT

OTHER PARAMETER VALUE FLAGS:

$J$ - ESTIMATED VALUE 
GROUNDWATER OUALITY DATA BY LOCATION

SITE: AMBO1 AMBROSIA LAKE

LOCATION: 0677 RESERVED ESTORMS, MH, 11/14/88

HORTH COORDINATE: UNKNOWN

EAST COORDINATE: UNKHOHH

$06 / 10 / 80$ TO $12 / 04 / 92$

REPORT DATE: 08/04/94

FORMATION OF COMPLETION: TRES HERMAHOS - C2 SANDSTONE (TT) HYDRAULIC FLOW RELATIONSHIP: CROSS GRADIENT (C)

\begin{tabular}{|c|c|c|c|c|c|c|c|}
\hline PARAMETER NAME & LOG DATE & $\begin{array}{l}\text { SAMPLE } \\
\text { ID }\end{array}$ & $\begin{array}{l}\text { UNITS OF } \\
\text { MEASURE }\end{array}$ & PVI & $\begin{array}{l}\text { PARAMETER } \\
\text { VALUE FLAGS }\end{array}$ & $\begin{array}{l}\text { DETECTION } \\
\text { LIMIT }\end{array}$ & $\begin{array}{l}\text { PARAMETER } \\
\text { UHCERTAINTY }\end{array}$ \\
\hline RADIUM-226 + RADIUM-228 & $\begin{array}{l}02 / 06 / 89 \\
09 / 19 / 89 \\
04 / 11 / 90 \\
01 / 18 / 91\end{array}$ & $\begin{array}{l}0001 \\
0001 \\
0001 \\
0001\end{array}$ & $\mathrm{PCI} / \mathrm{L}$ & & $\begin{array}{l}6.45 \\
6.20 \\
4.50 \\
1.90\end{array}$ & $\begin{array}{l}- \\
- \\
-\end{array}$ & $\begin{array}{l}- \\
- \\
-\end{array}$ \\
\hline RADIUN-228 & $\begin{array}{l}02 / 06 / 89 \\
09 / 19 / 89 \\
04 / 11 / 90 \\
01 / 18 / 91\end{array}$ & $\begin{array}{l}0001 \\
0001 \\
0001 \\
0001\end{array}$ & $\mathrm{PCl} / \mathrm{L}$ & & $\begin{array}{l}4.70 \\
0.8 \\
1.5 \\
0.5\end{array}$ & $\begin{array}{l}1 . \\
1 . \\
1 . \\
1 .\end{array}$ & $\begin{array}{l}9.51 \\
0.8 \\
0.9 \\
1.5\end{array}$ \\
\hline SELENIUM & $\begin{array}{l}02 / 06 / 89 \\
09 / 19 / 89 \\
04 / 11 / 90 \\
01 / 18 / 91\end{array}$ & $\begin{array}{l}0001 \\
0001 \\
0001 \\
0001\end{array}$ & MG/L & $\begin{array}{l}< \\
<\end{array}$ & $\begin{array}{l}0.006 \\
0.037 \\
0.005 \\
0.05\end{array}$ & $\begin{array}{l}0.005 \\
0.005 \\
0.005 \\
0.05\end{array}$ & $\begin{array}{l}- \\
- \\
-\end{array}$ \\
\hline SILICA -5102 & $\begin{array}{l}02 / 06 / 89 \\
09 / 19 / 89 \\
04 / 11 / 90 \\
01 / 18 / 91\end{array}$ & $\begin{array}{l}0001 \\
0001 \\
0001 \\
0001\end{array}$ & $M G / L$ & & $\begin{array}{l}9.3 \\
9.0 \\
8 . \\
8.5\end{array}$ & $\begin{array}{l}2 . \\
2 . \\
2 . \\
0.1\end{array}$ & $\begin{array}{l}- \\
- \\
-\end{array}$ \\
\hline SILVER & $\begin{array}{l}02 / 06 / 89 \\
09 / 19 / 89 \\
04 / 11 / 90 \\
01 / 18 / 91\end{array}$ & $\begin{array}{l}0001 \\
0001 \\
0001 \\
0001\end{array}$ & MG/L & $\begin{array}{l}< \\
< \\
<\end{array}$ & $\begin{array}{l}0.01 \\
0.01 \\
0.01 \\
0.01\end{array}$ & $\begin{array}{l}0.01 \\
0.01 \\
0.01 \\
0.01\end{array}$ & $\begin{array}{l}- \\
- \\
-\end{array}$ \\
\hline SODIUM & $\begin{array}{l}02 / 06 / 89 \\
09 / 19 / 89 \\
04 / 11 / 90 \\
01 / 18 / 91\end{array}$ & $\begin{array}{l}0001 \\
0001 \\
0001 \\
0001\end{array}$ & $M G / L$ & & $\begin{array}{l}1030 . \\
1090 . \\
1140 . \\
1270 .\end{array}$ & $\begin{array}{l}0.002 \\
0.002 \\
0.002 \\
5 .\end{array}$ & $\begin{array}{l}- \\
- \\
-\end{array}$ \\
\hline SPECIFIC CONDUCTANCE & $\begin{array}{l}02 / 06 / 89 \\
09 / 19 / 89 \\
04 / 11 / 90 \\
01 / 18 / 91\end{array}$ & $\begin{array}{l}0001 \\
0001 \\
0001 \\
0001\end{array}$ & UМHO/CM & & $\begin{array}{r}2600 . \\
950 . \\
3380 . \\
4840 .\end{array}$ & $\begin{array}{l}- \\
- \\
-\end{array}$ & - \\
\hline STRONT IUA & $\begin{array}{l}02 / 06 / 89 \\
09 / 19 / 89 \\
04 / 11 / 90 \\
01 / 18 / 91\end{array}$ & $\begin{array}{l}0001 \\
0001 \\
0001 \\
0001\end{array}$ & MG/L & & $\begin{array}{l}2.60 \\
2.0 \\
1.82 \\
1.91\end{array}$ & $\begin{array}{l}0.1 \\
0.1 \\
0.1 \\
0.01\end{array}$ & - \\
\hline SULFATE & $\begin{array}{l}02 / 06 / 89 \\
09 / 19 / 89 \\
04 / 11 / 90 \\
01 / 18 / 91\end{array}$ & $\begin{array}{l}0001 \\
0001 \\
0001 \\
0001\end{array}$ & MG/L & & $\begin{array}{l}2107 . \\
2190 . \\
2160 . \\
2210 .\end{array}$ & $\begin{array}{c}0.1 \\
0.1 \\
0.1 \\
10 .\end{array}$ & - \\
\hline SULFIDE & $\begin{array}{l}02 / 06 / 89 \\
09 / 19 / 89 \\
04 / 11 / 90 \\
01 / 18 / 91\end{array}$ & $\begin{array}{l}0001 \\
0001 \\
0001 \\
0001\end{array}$ & $M G / L$ & $\begin{array}{l}< \\
< \\
< \\
<\end{array}$ & $\begin{array}{l}0.01 \\
0.1 \\
0.1 \\
0.1\end{array}$ & $\begin{array}{l}0.1 \\
0.1 \\
0.1 \\
0.1\end{array}$ & $\begin{array}{l}- \\
- \\
-\end{array}$ \\
\hline TEMPERATURE & $\begin{array}{l}02 / 06 / 89 \\
09 / 19 / 89 \\
04 / 11 / 90 \\
01 / 18 / 91\end{array}$ & $\begin{array}{l}0001 \\
0001 \\
0001 \\
0001\end{array}$ & C - DEGREE & & $\begin{array}{l}12.0 \\
14.0 \\
14 . \\
12.8\end{array}$ & $\begin{array}{l}- \\
- \\
-\end{array}$ & $\begin{array}{l}- \\
- \\
-\end{array}$ \\
\hline THALLIUM & $02 / 06 / 89$ & 0001 & MG/L & $<$ & 0.005 & 0.005 & - \\
\hline
\end{tabular}

PARAMETER VALUE INDICATOR (PVI): < - LESS THAN DETECTION LIMIT

SAMPLE ID COOES:

0001 - FILTERED SAMPLE (.45 MICRONS)

OTHER PARAMETER VALUE FLAGS:

I - INCREASED DETECTION LIMIT DUE TO REQUIRED DILUTIOH

J - estimated value 


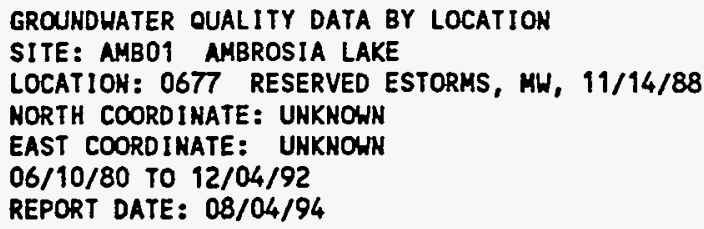

FORMATIOH OF COMPLETIOH: TRES HERMANOS - C2 SAMDSTOHE (TT) HYDRAULIC FLOW RELATIONSHIP: CROSS GRADIENT (C)

\begin{tabular}{|c|c|c|c|c|c|c|c|c|}
\hline PARAMETER MAME & LOG DATE & $\underset{\text { ID }}{\text { SAMPLE }}$ & $\begin{array}{l}\text { UHITS OF } \\
\text { MEASURE }\end{array}$ & PVI & $\begin{array}{l}\text { PARAMETER } \\
\text { VALUE }\end{array}$ & FLAGS & $\begin{array}{l}\text { DETECTIOH } \\
\text { LIMIT }\end{array}$ & $\begin{array}{l}\text { PARAMETER } \\
\text { UNCERTAINTY }\end{array}$ \\
\hline THALLIUM & $\begin{array}{l}09 / 19 / 89 \\
04 / 11 / 90 \\
01 / 18 / 91\end{array}$ & $\begin{array}{l}0001 \\
0001 \\
0001\end{array}$ & MG/L & $<$ & $\begin{array}{l}0.02 \\
0.01 \\
0.03\end{array}$ & I & $\begin{array}{l}0.01 \\
0.09 \\
0.03\end{array}$ & - \\
\hline THORIUA-230 & $\begin{array}{l}02 / 06 / 89 \\
09 / 19 / 89\end{array}$ & $\begin{array}{l}0001 \\
0001\end{array}$ & PCI/L & & $\begin{array}{l}0.285 \\
0.1\end{array}$ & & 1. & $\begin{array}{l}0.463 \\
0.4\end{array}$ \\
\hline TOTAL DISSOLVED SOLIDS & $\begin{array}{l}02 / 06 / 89 \\
09 / 19 / 89 \\
04 / 11 / 90 \\
01 / 18 / 91\end{array}$ & $\begin{array}{l}0001 \\
0001 \\
0001 \\
0001\end{array}$ & $M G / L$ & & $\begin{array}{l}3372 . \\
3470 \\
3500 \\
3600\end{array}$ & & $\begin{array}{l}10 . \\
10 . \\
10 . \\
10 .\end{array}$ & $\begin{array}{l}- \\
- \\
-\end{array}$ \\
\hline URANIUM & $\begin{array}{l}02 / 06 / 89 \\
09 / 19 / 89 \\
04 / 11 / 90 \\
01 / 18 / 91\end{array}$ & $\begin{array}{l}0001 \\
0001 \\
0001 \\
0001\end{array}$ & $M G / L$ & $<$ & $\begin{array}{l}0.016 \\
0.0036 \\
0.0029 \\
0.001\end{array}$ & $\jmath$ & $\begin{array}{l}0.003 \\
0.003 \\
0.003 \\
0.001\end{array}$ & : \\
\hline VAMAOIUM & $\begin{array}{l}02 / 06 / 89 \\
09 / 19 / 89 \\
04 / 11 / 90 \\
01 / 18 / 91\end{array}$ & $\begin{array}{l}0001 \\
0001 \\
0001 \\
0001\end{array}$ & $M G / L$ & $\begin{array}{l}< \\
< \\
<\end{array}$ & $\begin{array}{l}0.01 \\
0.01 \\
0.01 \\
0.01\end{array}$ & & $\begin{array}{l}0.01 \\
0.01 \\
0.01 \\
0.01\end{array}$ & : \\
\hline
\end{tabular}

PARAMETER VALUE INDICATOR (PVI): < - LESS THAN OETECTION LIMIT

SAMPLE ID COOES:

OOO1 - FILTERED SAMPLE (.45 MICRONS)

OTHER PARAMETER VALUE FLAGS:

I - IMCREASED DETECTION LIMIT DUE TO REQUIRED DILUTION

J - ESTIMATED VALUE 
GROUNDWATER QUALITY DATA BY LOCATION

SITE: AMBOI AMBROSIA LAKE

LOCATION: 0678 RESERVED ESTORMS, MW, 11/14/88

NORTH COORDINATE: UNKNOWN

EAST COORDINATE: UNKNOWN

06/10/80 TO $12 / 04 / 92$

REPORT DATE: $08 / 04 / 94$

FORMATION OF COMPLETION: TRES HERMANOS - B SAMDSTONE (TB)

HYDRAULIC FLOW RELATIONSHIP: CROSS GRADIENT (C)

\begin{tabular}{|c|c|c|c|c|c|c|c|c|}
\hline PARAMETER NAME & LOG DATE & $\begin{array}{c}\text { SAMPLE } \\
\text { ID }\end{array}$ & $\begin{array}{l}\text { UNITS OF } \\
\text { MEASURE }\end{array}$ & PVI & $\begin{array}{l}\text { PARAMETER } \\
\text { VALUE }\end{array}$ & FLAGS & $\begin{array}{l}\text { DETECTION } \\
\text { LIMIT }\end{array}$ & $\begin{array}{l}\text { PARAMETER } \\
\text { UNCERTAINTY }\end{array}$ \\
\hline ALKALINITY & $\begin{array}{l}02 / 06 / 89 \\
09 / 19 / 89 \\
04 / 11 / 90 \\
01 / 18 / 91\end{array}$ & $\begin{array}{l}0001 \\
0001 \\
0001 \\
0001\end{array}$ & MG/L CACO3 & & $\begin{array}{l}525 . \\
495 \\
532 \\
535\end{array}$ & & $\ddot{-}$ & $\begin{array}{l}- \\
- \\
-\end{array}$ \\
\hline ALUAINUN & $\begin{array}{l}02 / 06 / 89 \\
09 / 19 / 89 \\
04 / 11 / 90 \\
01 / 18 / 91\end{array}$ & $\begin{array}{l}0001 \\
0001 \\
0001 \\
0001\end{array}$ & $M G / L$ & $<$ & $\begin{array}{l}0.01 \\
0.1 \\
0.1 \\
0.05\end{array}$ & $J$ & $\begin{array}{l}0.1 \\
0.1 \\
0.1 \\
0.05\end{array}$ & $\begin{array}{l}- \\
- \\
-\end{array}$ \\
\hline AMMONIUM & $\begin{array}{l}02 / 06 / 89 \\
09 / 19 / 89 \\
04 / 11 / 90 \\
01 / 18 / 91 \\
12 / 04 / 92\end{array}$ & $\begin{array}{l}0001 \\
0001 \\
0001 \\
0001 \\
0001\end{array}$ & MG/L & & $\begin{array}{l}3.21 \\
0.8 \\
0.6 \\
0.90 \\
1.2\end{array}$ & $J$ & $\begin{array}{l}0.1 \\
0.1 \\
0.1 \\
0.01 \\
0.1\end{array}$ & $\begin{array}{l}- \\
- \\
- \\
-\end{array}$ \\
\hline ANT IMONY & $\begin{array}{l}02 / 06 / 89 \\
09 / 19 / 89 \\
04 / 11 / 90 \\
01 / 18 / 91 \\
12 / 04 / 92\end{array}$ & $\begin{array}{l}0001 \\
0001 \\
0001 \\
0001 \\
0001\end{array}$ & $M G / L$ & $<$ & $\begin{array}{l}0.005 \\
0.095 \\
0.171 \\
0.02 \\
0.02\end{array}$ & I & $\begin{array}{l}0.003 \\
0.003 \\
0.003 \\
0.02 \\
0.02\end{array}$ & $\begin{array}{l}- \\
- \\
- \\
-\end{array}$ \\
\hline ARSENIC & $\begin{array}{l}02 / 06 / 89 \\
09 / 19 / 89 \\
04 / 11 / 90 \\
01 / 18 / 91\end{array}$ & $\begin{array}{l}0001 \\
0001 \\
0001 \\
0001\end{array}$ & $M G / L$ & $<$ & $\begin{array}{l}0.001 \\
0.02 \\
0.02 \\
0.05\end{array}$ & $\begin{array}{l}J \\
I\end{array}$ & $\begin{array}{l}0.01 \\
0.01 \\
0.01 \\
0.05\end{array}$ & $\begin{array}{l}- \\
-\end{array}$ \\
\hline BARIUM & $\begin{array}{l}02 / 06 / 89 \\
09 / 19 / 89 \\
04 / 11 / 90 \\
01 / 18 / 91\end{array}$ & $\begin{array}{l}0001 \\
0001 \\
0001 \\
0001\end{array}$ & MG/L & $<$ & $\begin{array}{l}0.02 \\
0.1 \\
0.1 \\
0.01\end{array}$ & $J$ & $\begin{array}{l}0.1 \\
0.1 \\
0.1 \\
0.01\end{array}$ & $\begin{array}{l}- \\
- \\
-\end{array}$ \\
\hline BERYLLIUM & $\begin{array}{l}02 / 06 / 89 \\
09 / 19 / 89 \\
04 / 11 / 90 \\
01 / 18 / 91\end{array}$ & $\begin{array}{l}0001 \\
0001 \\
0001 \\
0001\end{array}$ & $M G / L$ & $\begin{array}{l}< \\
< \\
<\end{array}$ & $\begin{array}{l}0.005 \\
0.01 \\
0.01 \\
0.005\end{array}$ & & $\begin{array}{l}0.005 \\
0.01 \\
0.01 \\
0.005\end{array}$ & $\begin{array}{l}- \\
- \\
-\end{array}$ \\
\hline BORON & $\begin{array}{l}02 / 06 / 89 \\
09 / 19 / 89 \\
04 / 11 / 90 \\
01 / 18 / 91\end{array}$ & $\begin{array}{l}0001 \\
0001 \\
0001 \\
0001\end{array}$ & $M G / L$ & & $\begin{array}{l}0.88 \\
0.4 \\
0.3 \\
0.37\end{array}$ & & $\begin{array}{l}0.1 \\
0.1 \\
0.1 \\
0.05\end{array}$ & $\begin{array}{l}- \\
- \\
-\end{array}$ \\
\hline BROMIDE & $\begin{array}{l}02 / 06 / 89 \\
09 / 19 / 89 \\
04 / 11 / 90 \\
01 / 18 / 91\end{array}$ & $\begin{array}{l}0001 \\
0001 \\
0001 \\
0001\end{array}$ & $M G / L$ & $\begin{array}{l}< \\
<\end{array}$ & $\begin{array}{l}0.46 \\
0.1 \\
0.1 \\
5.1\end{array}$ & J & $\begin{array}{l}0.01 \\
0.1 \\
0.1 \\
0.1\end{array}$ & $\begin{array}{l}- \\
- \\
-\end{array}$ \\
\hline CAONIUA & $\begin{array}{l}02 / 06 / 89 \\
09 / 19 / 89 \\
04 / 11 / 90 \\
01 / 18 / 91 \\
12 / 04 / 92\end{array}$ & $\begin{array}{l}0001 \\
0001 \\
0001 \\
0001 \\
0001\end{array}$ & $M G / L$ & $\begin{array}{l}< \\
<\end{array}$ & $\begin{array}{l}0.0006 \\
0.010 \\
0.001 \\
0.001 \\
0.0005\end{array}$ & $J$ & $\begin{array}{l}0.001 \\
0.001 \\
0.001 \\
0.001 \\
0.0001\end{array}$ & $\begin{array}{l}- \\
- \\
- \\
-\end{array}$ \\
\hline CALCIUM & $\begin{array}{l}02 / 06 / 89 \\
09 / 19 / 89 \\
04 / 11 / 90\end{array}$ & $\begin{array}{l}0001 \\
0001 \\
0001\end{array}$ & $M G / L$ & & $\begin{array}{l}172 . \\
322 . \\
292 .\end{array}$ & & $\begin{array}{l}0.01 \\
0.01 \\
0.01\end{array}$ & $\begin{array}{l}- \\
-\end{array}$ \\
\hline
\end{tabular}

PARAMETER VALUE INDICATOR (PVI): < - LESS THAN DETECTION LIMIT

OTHER PARAMETER VALUE FLAGS:

I - INCREASED DETECTION LIMIT DUE TO REQUIRED DILUTION

J - ESTIMATED VALUE 
GROUNDWATER QUALITY DATA BY LOCATION

SITE: AMBO1 AMBROSIA LAKE

LOCATION: 0678 RESERVED ESTORMS, MH, 11/14/88

NORTH COORDINATE: UNKNOWN

EAST COORDINATE: UNKNOWN

06/10/80 TO $12 / 04 / 92$

REPORT DATE: 08/04/94

FORMATION OF COMPLETION: TRES HERMANOS - B SANDSTONE (TB)

HYDRAULIC FLOW RELATIONSHIP: CROSS GRADIENT (C)

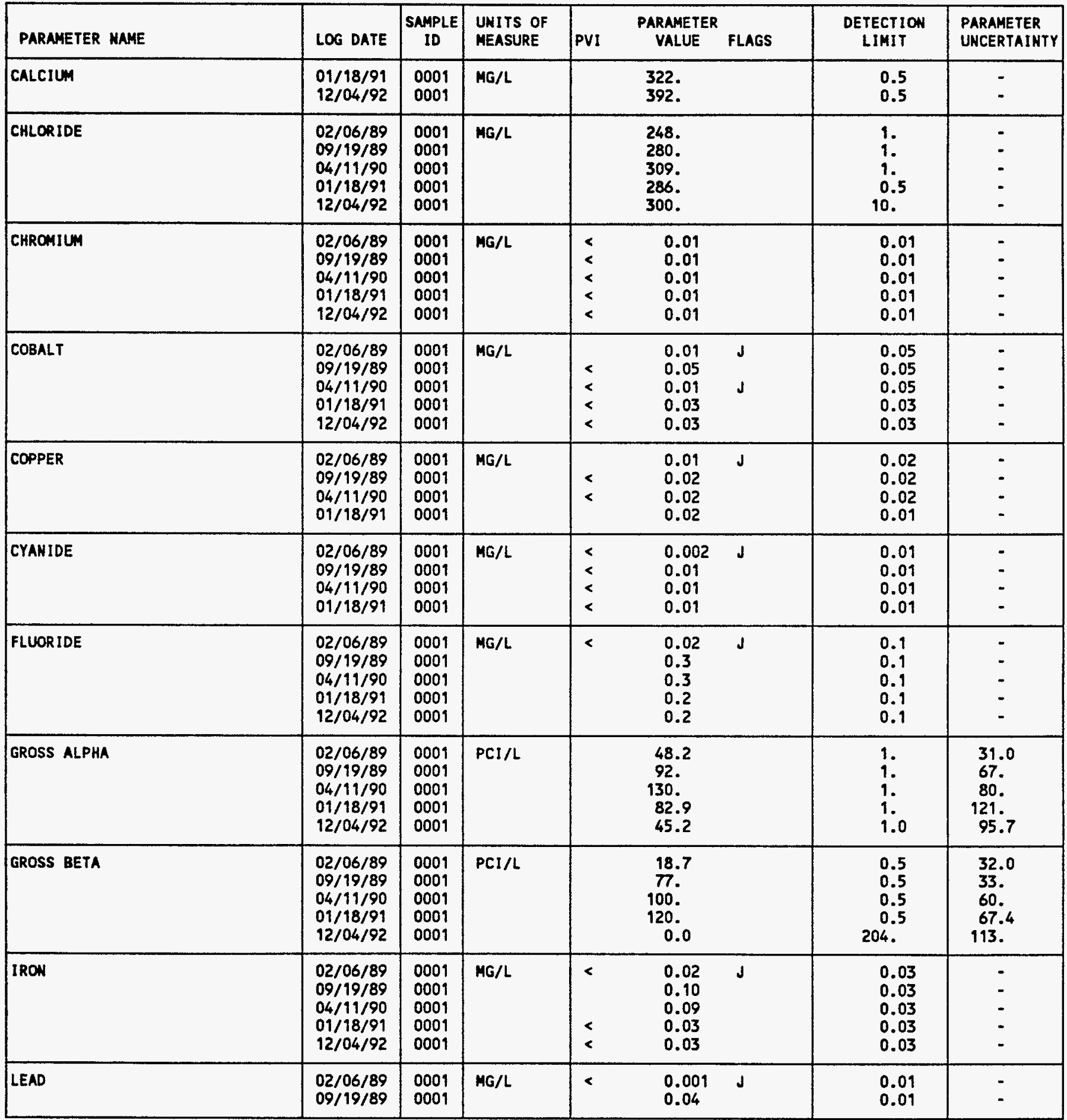

PARAMETER VALUE INDICATOR (PVI): < - LESS THAN DETECTION LIMIT 


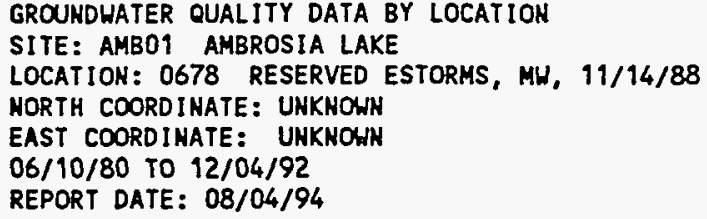

FORMATION OF COMPLETION: TRES HERHANOS - B SANDSTONE (TB) HYORAULIC FLOW RELATIONSHIP: CROSS GRADIENT (C)

\begin{tabular}{|c|c|c|c|c|c|c|c|}
\hline PARAMETER NAME & LOG DATE & $\begin{array}{c}\text { SAMPLE } \\
\text { ID }\end{array}$ & $\begin{array}{l}\text { UNITS OF } \\
\text { MEASURE }\end{array}$ & PVI & $\begin{array}{l}\text { PARAMETER } \\
\text { VALUE FLAGS }\end{array}$ & $\begin{array}{l}\text { DETECTION } \\
\text { LIMIT }\end{array}$ & $\begin{array}{l}\text { PARAMETER } \\
\text { UNCERTAINTY }\end{array}$ \\
\hline LEAD & $\begin{array}{l}04 / 11 / 90 \\
01 / 18 / 91\end{array}$ & $\begin{array}{l}0001 \\
0001\end{array}$ & $M G / L$ & $\begin{array}{l}< \\
<\end{array}$ & $\begin{array}{l}0.01 \\
0.03\end{array}$ & $\begin{array}{l}0.01 \\
0.03\end{array}$ & - \\
\hline LEND-210 & $\begin{array}{l}02 / 06 / 89 \\
09 / 19 / 89\end{array}$ & $\begin{array}{l}0001 \\
0001\end{array}$ & $\mathrm{PCI} / \mathrm{L}$ & & $\begin{array}{l}0.00 \\
0.4\end{array}$ & $\begin{array}{l}1.5 \\
1.5\end{array}$ & $\begin{array}{r}11.0 \\
0.6\end{array}$ \\
\hline MAGNESIUM & $\begin{array}{l}02 / 06 / 89 \\
09 / 19 / 89 \\
04 / 11 / 90 \\
01 / 18 / 91 \\
12 / 04 / 92\end{array}$ & $\begin{array}{l}0001 \\
0001 \\
0001 \\
0001 \\
0001\end{array}$ & $M G / L$ & & $\begin{array}{l}86 . \\
399 . \\
374 . \\
419 . \\
540 .\end{array}$ & $\begin{array}{l}0.001 \\
0.001 \\
0.001 \\
0.5 \\
2 .\end{array}$ & $\begin{array}{l}- \\
- \\
-\end{array}$ \\
\hline MANGANESE & $\begin{array}{l}02 / 06 / 89 \\
09 / 99 / 89 \\
04 / 11 / 90 \\
01 / 18 / 91\end{array}$ & $\begin{array}{l}0001 \\
0001 \\
0001 \\
0001\end{array}$ & $M G / L$ & & $\begin{array}{l}0.12 \\
0.12 \\
0.10 \\
0.08\end{array}$ & $\begin{array}{l}0.01 \\
0.01 \\
0.01 \\
0.01\end{array}$ & $\begin{array}{l}- \\
- \\
-\end{array}$ \\
\hline MERCURY & $\begin{array}{l}02 / 06 / 89 \\
09 / 19 / 89 \\
04 / 11 / 90 \\
01 / 18 / 91\end{array}$ & $\begin{array}{l}0001 \\
0001 \\
0001 \\
0001\end{array}$ & $M G / L$ & $\begin{array}{l}< \\
< \\
< \\
<\end{array}$ & $\begin{array}{l}0.0001 \\
0.0002 \\
0.0002 \\
0.0002\end{array}$ & $\begin{array}{l}0.0002 \\
0.0002 \\
0.0002 \\
0.0002\end{array}$ & $\begin{array}{l}- \\
- \\
-\end{array}$ \\
\hline MOLYBDENUM & $\begin{array}{l}02 / 06 / 89 \\
09 / 19 / 89 \\
04 / 19 / 90 \\
01 / 98 / 99 \\
12 / 04 / 92\end{array}$ & $\begin{array}{l}0001 \\
0001 \\
0001 \\
0001 \\
0001\end{array}$ & $M G / L$ & & $\begin{array}{l}0.016 \\
0.05 \\
0.03 \\
0.02 \\
0.05\end{array}$ & $\begin{array}{l}0.01 \\
0.01 \\
0.01 \\
0.01 \\
0.01\end{array}$ & $\begin{array}{l}- \\
- \\
-\end{array}$ \\
\hline NET GROSS ALPHA & $\begin{array}{l}02 / 06 / 89 \\
09 / 19 / 89 \\
04 / 11 / 90 \\
01 / 18 / 91 \\
12 / 04 / 92\end{array}$ & $\begin{array}{l}0001 \\
0001 \\
0001 \\
0001 \\
0001\end{array}$ & PCI /L & & $\begin{array}{r}28.31 \\
66.62 \\
127.46 \\
57.52 \\
6.10\end{array}$ & $\begin{array}{l}- \\
- \\
- \\
-\end{array}$ & $\begin{array}{l}- \\
- \\
- \\
-\end{array}$ \\
\hline NICKEL & $\begin{array}{l}02 / 06 / 89 \\
09 / 19 / 89 \\
04 / 11 / 90 \\
01 / 18 / 91 \\
12 / 04 / 92\end{array}$ & $\begin{array}{l}0001 \\
0001 \\
0001 \\
0001 \\
0001\end{array}$ & $M G / L$ & & $\begin{array}{l}0.04 \\
0.08 \\
0.05 \\
0.04 \\
0.29\end{array}$ & $\begin{array}{l}0.04 \\
0.04 \\
0.04 \\
0.04 \\
0.04\end{array}$ & $\begin{array}{l}- \\
- \\
- \\
-\end{array}$ \\
\hline NITRATE & $\begin{array}{l}02 / 06 / 89 \\
09 / 19 / 89 \\
04 / 11 / 90 \\
01 / 18 / 91 \\
12 / 04 / 92\end{array}$ & $\begin{array}{l}0001 \\
0001 \\
0001 \\
0001 \\
0001\end{array}$ & $M G / L$ & & $\begin{array}{l}246 . \\
2100 \\
2210 \\
2400 \\
3410\end{array}$ & $\begin{array}{c}1 . \\
1 . \\
1 . \\
0.1 \\
300 .\end{array}$ & $\begin{array}{l}- \\
- \\
- \\
-\end{array}$ \\
\hline NITRITE AND NITRATE & $\begin{array}{l}04 / 11 / 90 \\
01 / 18 / 91\end{array}$ & $\begin{array}{l}0001 \\
0001\end{array}$ & $M G / L$ & & $\begin{array}{l}499 . \\
54.8\end{array}$ & 0.05 & - \\
\hline PH & $\begin{array}{l}02 / 06 / 89 \\
09 / 19 / 89 \\
04 / 11 / 90 \\
01 / 18 / 91 \\
12 / 04 / 92\end{array}$ & $\begin{array}{l}0001 \\
0001 \\
0001 \\
0001 \\
0001\end{array}$ & SU & & $\begin{array}{l}7.71 \\
7.23 \\
7.23 \\
7.57 \\
7.23\end{array}$ & $\begin{array}{l}- \\
- \\
- \\
-\end{array}$ & $\begin{array}{l}- \\
- \\
- \\
-\end{array}$ \\
\hline
\end{tabular}

- NET GROSS ALPHA (GROSS ALPHA - URANIUM) WITH 1 MG URANIUH = 686 PCI

PARAMETER VALUE IMDICATOR (PVI): < - LESS THAN DETECTION LIMIT SAMPLE ID CODES:

0001 - FILTERED SAMPLE (.45 MICROHS)

OTHER PARAMETER VALUE FLAGS:

I - INCREASED DETECTION LIMIT DUE TO REOUIRED DILUTION

J - ESTIMATED VALUE 
GROUHDWATER QUALITY DATA BY LOCATIOH

SITE: AMBO1 AMBROSIA LAKE

LOCATIOH: 0678 RESERVED ESTORMS, MH, 11/14/88

NORTH COORDINATE: UNKNOWN

EAST COOROIHATE: UHKNOUN

O6/10/80 TO 12/04/92

REPORT DATE: $08 / 04 / 94$

FORMATION OF COMPLETIOH: TRES HERMANOS - B SAKDSTONE (TB)

HYDRAULIC FLOU RELATIONSHIP: CROSS GRADIEHT (C)

\begin{tabular}{|c|c|c|c|c|c|c|c|c|}
\hline PARAMETER MAKE & LOG DATE & $\mid \begin{array}{c}\text { SAMPLE } \\
\text { ID }\end{array}$ & $\begin{array}{l}\text { UNITS OF } \\
\text { MEASURE }\end{array}$ & PVI & $\begin{array}{l}\text { PARAMETER } \\
\text { VALUE }\end{array}$ & FLAGS & $\begin{array}{l}\text { DETECTION } \\
\text { LIMIT }\end{array}$ & $\begin{array}{l}\text { PARAMETER } \\
\text { UNCERTAINTY }\end{array}$ \\
\hline PHOSPHATE & $\begin{array}{l}02 / 06 / 89 \\
09 / 19 / 89 \\
04 / 11 / 90 \\
01 / 18 / 91 \\
12 / 04 / 92\end{array}$ & $\begin{array}{l}0001 \\
0001 \\
0001 \\
0001 \\
0001\end{array}$ & MG/L & $\begin{array}{l}< \\
< \\
< \\
<\end{array}$ & $\begin{array}{l}0.1 \\
0.1 \\
0.1 \\
0.08 \\
0.1\end{array}$ & H & $\begin{array}{l}0.1 \\
0.1 \\
0.1 \\
0.05 \\
0.1\end{array}$ & $\begin{array}{l}- \\
- \\
- \\
-\end{array}$ \\
\hline POLONIUA-210 & $\begin{array}{l}02 / 06 / 89 \\
09 / 19 / 89\end{array}$ & $\begin{array}{l}0001 \\
0001\end{array}$ & PCI/L & & $\begin{array}{l}2.17 \\
0.2\end{array}$ & & 1. & $\begin{array}{l}1.61 \\
0.4\end{array}$ \\
\hline POTASSIUM & $\begin{array}{l}02 / 06 / 89 \\
09 / 19 / 89 \\
04 / 11 / 90 \\
01 / 18 / 91 \\
12 / 04 / 92\end{array}$ & $\begin{array}{l}0001 \\
0001 \\
0001 \\
0001 \\
0001\end{array}$ & $M G / L$ & & $\begin{array}{l}23.2 \\
29.3 \\
32.8 \\
21 . \\
24 .\end{array}$ & & $\begin{array}{l}0.01 \\
0.01 \\
0.01 \\
5 . \\
5 .\end{array}$ & $\begin{array}{l}- \\
- \\
- \\
-\end{array}$ \\
\hline RADIUH-226 & $\begin{array}{l}02 / 06 / 89 \\
09 / 19 / 89 \\
04 / 11 / 90 \\
01 / 18 / 91 \\
12 / 04 / 92\end{array}$ & $\begin{array}{l}0001 \\
0001 \\
0001 \\
0001 \\
0001\end{array}$ & $\mathrm{PCI} / \mathrm{L}$ & & $\begin{array}{l}1.71 \\
1.1 \\
0.5 \\
3.0 \\
2.4\end{array}$ & & $\begin{array}{l}1 . \\
1 . \\
1 . \\
1 . \\
0.3\end{array}$ & $\begin{array}{l}0.257 \\
0.4 \\
0.3 \\
0.8 \\
0.6\end{array}$ \\
\hline RADIUH-226 + RADIUM-228 & $\begin{array}{l}02 / 06 / 89 \\
09 / 19 / 89 \\
04 / 11 / 90 \\
01 / 18 / 91 \\
12 / 04 / 92\end{array}$ & $\begin{array}{l}0001 \\
0001 \\
0001 \\
0001 \\
0001\end{array}$ & PCI/L & & $\begin{array}{l}4.63 \\
4.80 \\
6.10 \\
3.90 \\
9.80\end{array}$ & & $\begin{array}{l}- \\
- \\
- \\
-\end{array}$ & $\begin{array}{l}- \\
- \\
- \\
-\end{array}$ \\
\hline RADIUM-228 & $\begin{array}{l}02 / 06 / 89 \\
09 / 19 / 89 \\
04 / 11 / 90 \\
01 / 18 / 91 \\
12 / 04 / 92\end{array}$ & $\begin{array}{l}0001 \\
0001 \\
0001 \\
0001 \\
0001\end{array}$ & $\mathrm{PCI} / \mathrm{L}$ & & $\begin{array}{l}2.92 \\
3.7 \\
5.6 \\
0.9 \\
7.4\end{array}$ & H & $\begin{array}{l}1 . \\
1 . \\
1 . \\
1.0\end{array}$ & $\begin{array}{l}8.08 \\
1.0 \\
1.1 \\
1.6 \\
2.0\end{array}$ \\
\hline SELENIUM & $\begin{array}{l}02 / 06 / 89 \\
09 / 19 / 89 \\
04 / 11 / 90 \\
01 / 18 / 91 \\
12 / 04 / 92\end{array}$ & $\begin{array}{l}0001 \\
0001 \\
0001 \\
0001 \\
0001\end{array}$ & $M G / L$ & & $\begin{array}{l}0.011 \\
0.212 \\
0.100 \\
0.250 \\
0.7\end{array}$ & & $\begin{array}{l}0.005 \\
0.005 \\
0.005 \\
0.005 \\
0.1\end{array}$ & : \\
\hline SILICA - SIOZ & $\begin{array}{l}02 / 06 / 89 \\
09 / 19 / 89 \\
04 / 11 / 90 \\
01 / 18 / 91\end{array}$ & $\begin{array}{l}0001 \\
0001 \\
0001 \\
0001\end{array}$ & $M G / L$ & & $\begin{array}{l}9.4 \\
6 . \\
5 . \\
4.5\end{array}$ & & $\begin{array}{l}2 . \\
2 . \\
2 . \\
0.1\end{array}$ & : \\
\hline SILVER & $\begin{array}{l}02 / 06 / 89 \\
09 / 19 / 89 \\
04 / 11 / 90 \\
01 / 18 / 91 \\
12 / 04 / 92\end{array}$ & $\begin{array}{l}0001 \\
0001 \\
0001 \\
0001 \\
0001\end{array}$ & MG/L & $\begin{array}{l}< \\
< \\
< \\
< \\
<\end{array}$ & $\begin{array}{l}0.01 \\
0.01 \\
0.01 \\
0.01 \\
0.01\end{array}$ & & $\begin{array}{l}0.01 \\
0.01 \\
0.01 \\
0.01 \\
0.01\end{array}$ & $\begin{array}{l}- \\
- \\
-\end{array}$ \\
\hline SODIUN & $\begin{array}{l}02 / 06 / 89 \\
09 / 19 / 89 \\
04 / 11 / 90 \\
01 / 18 / 91 \\
12 / 04 / 92\end{array}$ & $\begin{array}{l}0001 \\
0001 \\
0001 \\
0001 \\
0001\end{array}$ & $\mathrm{MG} / \mathrm{L}$ & & $\begin{array}{l}1320 . \\
2850 \\
2980 \\
3290 \\
3450 .\end{array}$ & J & $\begin{array}{l}0.002 \\
0.002 \\
0.002 \\
5 . \\
20 .\end{array}$ & $\begin{array}{l}- \\
- \\
-\end{array}$ \\
\hline SPECIFIC CONDUCTANCE & $02 / 06 / 89$ & 0001 & UНHO/CM & & 3700. & & - & - \\
\hline
\end{tabular}

PARAMETER VALUE IHDICATOR (PVI): < - LESS THAN DETECTIOH LIMIT SAMPLE ID CODES:

0001 - FILTERED SAMPLE (.45 MICRONS)

OTHER PARAMETER VALUE FLAGS:

H - HOLD TIME EXPIRED, VALUE SUSPECT

$J$ - estimated VALUe 
GROUNDHATER QUALITY DATA BY LOCATION

SITE: AMBOI AMBROSIA LAKE

LOCATION: 0678 RESERVED ESTORMS, MH, 11/14/88

MORTH COORDINATE: UNKNOWN

EAST COORDINATE: UNKHOWN

O6/10/80 TO 12/04/92

REPORT DATE : $08 / 04 / 94$

FORMATION OF COMPLETION: TRES HERMANOS - B SANDSTOWE (TB)

HYDRAULIC FLON RELATIONSHIP: CROSS GRADIENT (C)

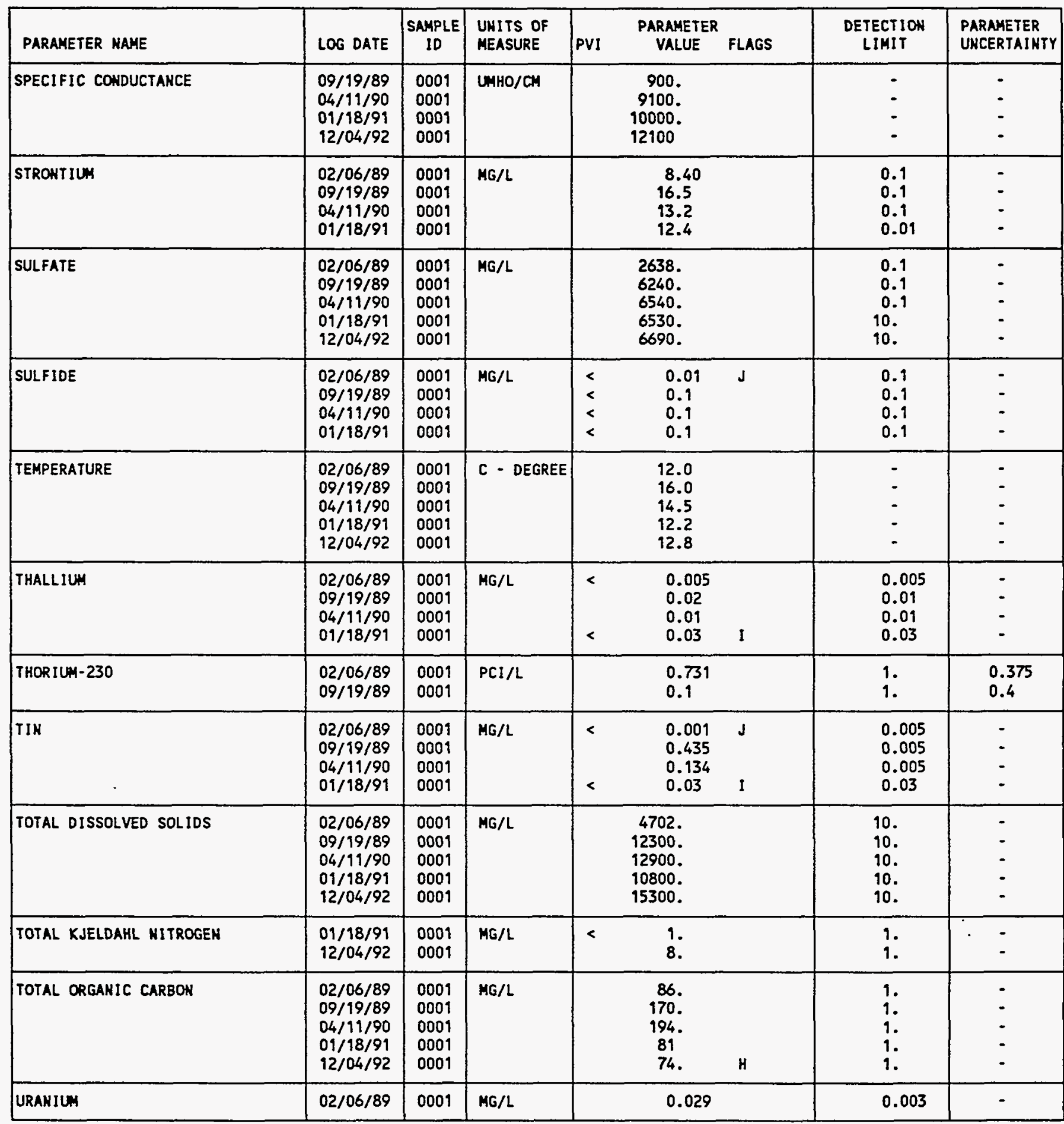

PARAMETER VALUE IMDICATOR (PVI): < - LESS THAN DETECTION LIMIT

SAMPLE ID COOES:

0001 - FILTERED SAMPLE (.45 MICRONS)

OTHER PARAMETER VALUE FLAGS:

H - HOLD TIME EXPIRED, VALUE SUSPECT

I - INCREASED DETECTION LIMIT DUE TO REQUIRED DILUTIOH

J - estiMated VALUE 
GROUMDHATER QUALITY DATA BY LOCATION

SITE: AMBO1 AMBROSIA LAKE

LOCATIOW: 0678 RESERVED ESTORMS, MW, 11/14/88

MORTH COORDINATE: UNKNOWN

EAST COORDINATE: UNKNOWN

06/10/80 TO $12 / 04 / 92$

REPORT DATE: 08/04/94

FORMATION OF COMPLETION: TRES HERMANOS - B SAMDSTONE (TB) HYDRAULIC FLON RELATIONSHIP: CROSS GRADIENT (C)

\begin{tabular}{|c|c|c|c|c|c|c|c|}
\hline PARAMETER NAME & LOG DATE & $\underset{10}{\text { SAMPLE }}$ & $\begin{array}{l}\text { UNITS OF } \\
\text { MEASURE }\end{array}$ & PVI & $\begin{array}{l}\text { PARAMETER } \\
\text { VALUE FLAGS }\end{array}$ & $\begin{array}{l}\text { DETECTION } \\
\text { LIMIT }\end{array}$ & $\begin{array}{l}\text { PARAMETER } \\
\text { UNCERTAINTY }\end{array}$ \\
\hline URAHIUH & $\begin{array}{l}09 / 19 / 89 \\
04 / 11 / 90 \\
01 / 18 / 91 \\
12 / 04 / 92\end{array}$ & $\begin{array}{l}0001 \\
0001 \\
0001 \\
0001\end{array}$ & MG/L & & $\begin{array}{l}0.037 \\
0.0037 \\
0.037 \\
0.057\end{array}$ & $\begin{array}{l}0.003 \\
0.003 \\
0.001 \\
0.001\end{array}$ & : \\
\hline VAHADIUH & $\begin{array}{l}02 / 06 / 89 \\
09 / 19 / 89 \\
04 / 11 / 90 \\
01 / 18 / 91 \\
12 / 04 / 92\end{array}$ & $\begin{array}{l}0001 \\
0001 \\
0001 \\
0001 \\
0001\end{array}$ & MG/L & $\begin{array}{l}< \\
< \\
< \\
< \\
<\end{array}$ & $\begin{array}{l}0.01 \\
0.01 \\
0.01 \\
0.01 \\
0.01\end{array}$ & $\begin{array}{l}0.01 \\
0.01 \\
0.01 \\
0.01 \\
0.01\end{array}$ & : \\
\hline ZINC & $\begin{array}{l}02 / 06 / 89 \\
09 / 19 / 89 \\
04 / 11 / 90 \\
01 / 18 / 91\end{array}$ & $\begin{array}{l}0001 \\
0001 \\
0001 \\
0001\end{array}$ & $M G / L$ & $<$ & $\begin{array}{l}0.02 \\
0.006 \\
3.79 \\
0.005\end{array}$ & $\begin{array}{l}0.005 \\
0.005 \\
0.005 \\
0.005\end{array}$ & $\begin{array}{l}- \\
- \\
-\end{array}$ \\
\hline
\end{tabular}

PARAMETER VALUE INDICATOR (PVI): < - LESS THAN DETECTION LIMIT

SAMPLE ID COOES:

0001 - FILTERED SAMPLE (.45 MICRONS) 
GROUNDWATER QUALITY DATA BY LOCATIOH

SITE: AMBOI AMBROSIA LAKE

LOCATION: 0679 RESERVED ESTORMS, MH, 11/14/88

NORTH COORDINATE: UNKHOWN

EAST COOROINATE: UNKHONM

$06 / 10 / 80$ TO $12 / 04 / 92$

REPORT DATE: $08 / 04 / 94$

FORMATION OF COMPLETION: TRES HERMANOS-A SAHDSTONE (TR)

HYDRAULIC FLOW RELATIONSHIP: DOWN GRADIENT (D)

\begin{tabular}{|c|c|c|c|c|c|c|c|}
\hline PARAMETER NAME & LOE DATE & $\begin{array}{c}\text { SAMPLE } \\
\text { ID }\end{array}$ & $\begin{array}{l}\text { UNITS OF } \\
\text { MEASURE }\end{array}$ & PVI & $\begin{array}{l}\text { PARAMETER } \\
\text { VALUE FLAGS }\end{array}$ & $\begin{array}{l}\text { DETECTIOH } \\
\text { LIMIT }\end{array}$ & $\begin{array}{l}\text { PARAMETER } \\
\text { UNCERTAIKTY }\end{array}$ \\
\hline ALKALINITY & $02 / 03 / 89$ & 0001 & MG/L CACO3 & & 2995. & - & - \\
\hline ALUAINUM & $02 / 03 / 89$ & 0001 & $M G / L$ & & 16.1 & 0.1 & - \\
\hline AMONIUH & $02 / 03 / 89$ & 0001 & MG/L & & 0.40 & 0.1 & - \\
\hline ANTIHONY & $02 / 03 / 89$ & 0001 & MG/L & & 0.003 & 0.003 & - \\
\hline ARSENIC & $02 / 03 / 89$ & 0001 & MG/L & & 0.037 & 0.01 & - \\
\hline BARIUM & $02 / 03 / 89$ & 0001 & $M G / L$ & & 0.09 & 0.1 & - \\
\hline BERYLLIUYM & $02 / 03 / 89$ & 0001 & $M G / L$ & & 0.019 & 0.005 & - \\
\hline BOROH & $02 / 03 / 89$ & 0001 & $M G / L$ & & 0.32 & 0.1 & - \\
\hline BROMIDE & $02 / 03 / 89$ & 0001 & $\mathrm{MG} / \mathrm{L}$ & & 1.04 & 0.01 & - \\
\hline CADMIUH & $02 / 03 / 89$ & 0001 & MG/L & & 0.0019 & 0.001 & - \\
\hline CALCIUM & $02 / 03 / 89$ & 0001 & $M G / L$ & & 129. & 0.01 & $\cdot$ \\
\hline CHLORIDE & $02 / 03 / 89$ & 0001 & MG/L & & 139. & 1. & - \\
\hline CHROMIUM & $02 / 03 / 89$ & 0001 & $M G / L$ & & 0.02 & 0.01 & $\cdot$ \\
\hline COBALT & $02 / 03 / 89$ & 0001 & MG/L & & 0.07 & 0.05 & $\cdot$ \\
\hline COPPER & $02 / 03 / 89$ & 0001 & $M G / L$ & & 0.08 & 0.02 & $\cdot$ \\
\hline CYANIDE & $02 / 03 / 89$ & 0001 & $M G / L$ & & $0.006 \mathrm{~J}$ & 0.01 & - \\
\hline FLUORIDE & $02 / 03 / 89$ & 0001 & $M G / L$ & & 0.6 & 0.1 & $\cdot$ \\
\hline GROSS ALPHA & $02 / 03 / 89$ & 0001 & $\mathrm{PCI} / \mathrm{L}$ & & 74.1 & 1. & 58.9 \\
\hline GROSS BETA & $02 / 03 / 89$ & 0001 & $\mathrm{PCl} / \mathrm{L}$ & & 12.1 & 0.5 & 52.9 \\
\hline IRON & $02 / 03 / 89$ & 0001 & $M G / L$ & & 62. & 0.03 & - \\
\hline LEAD & $02 / 03 / 89$ & 0001 & $M G / L$ & & 0.135 & 0.01 & - \\
\hline LEAD- 210 & $02 / 03 / 89$ & 0001 & $\mathrm{PCl} / \mathrm{L}$ & & 43.9 & 1.5 & 17.9 \\
\hline MAGKESIUY & $02 / 03 / 89$ & 0001 & $M G / L$ & & 520. & 0.001 & $\cdot$ \\
\hline MANGANESE & $02 / 03 / 89$ & 0001 & MG/L & & 6.60 & 0.01 & - \\
\hline MERCURY & $02 / 03 / 89$ & 0001 & $M G / L$ & $<$ & $0.0001 \mathrm{~J}$ & 0.0002 & $\cdot$ \\
\hline MOLYBDEENUH & $02 / 03 / 89$ & 0001 & $\mathrm{MG} / \mathrm{L}$ & & $0.002 \mathrm{~J}$ & 0.01 & - \\
\hline NET GROSS ALPHA * & $02 / 03 / 89$ & 0001 & $\mathrm{PCI} / \mathrm{L}$ & & 45.29 & - & - \\
\hline NICXEL & $02 / 03 / 89$ & 0001 & MG/L & & 0.09 & 0.04 & - \\
\hline
\end{tabular}

- Met gross ALPHA (GROSS ALPHA - URANIUM) WITH 1 MG URAHIUM = 686 PCI

PARAMETER VALUE INDICATOR (PVI): < - LeSS THAN DETECTION LIMIT

SAMPLE ID COOES:

0001 - FILTERED SAMPLE (.45 MICRONS)

OTHER PARAMETER VALUE FLAGS:

\rfloor - ESTIMATED VALUE 
GROUNDHATER QUALITY DATA BY LOCATION

SITE: AMB01 AMBROSIA LAKE

LOCATION: 0679 RESERVED ESTORMS, MH, 11/14/88

NORTH COORDIHATE: UNKNOWN

EAST COORDINATE: UNKNOWN

06/10/80 TO 12/04/92

REPORT DATE: 08/04/94

FORMATION OF COMPLETION: TRES HERMANOS-A SAMDSTONE (TR)

HYDRAULIC FLON RELATIONSHIP: DOWN GRADIENT (D)

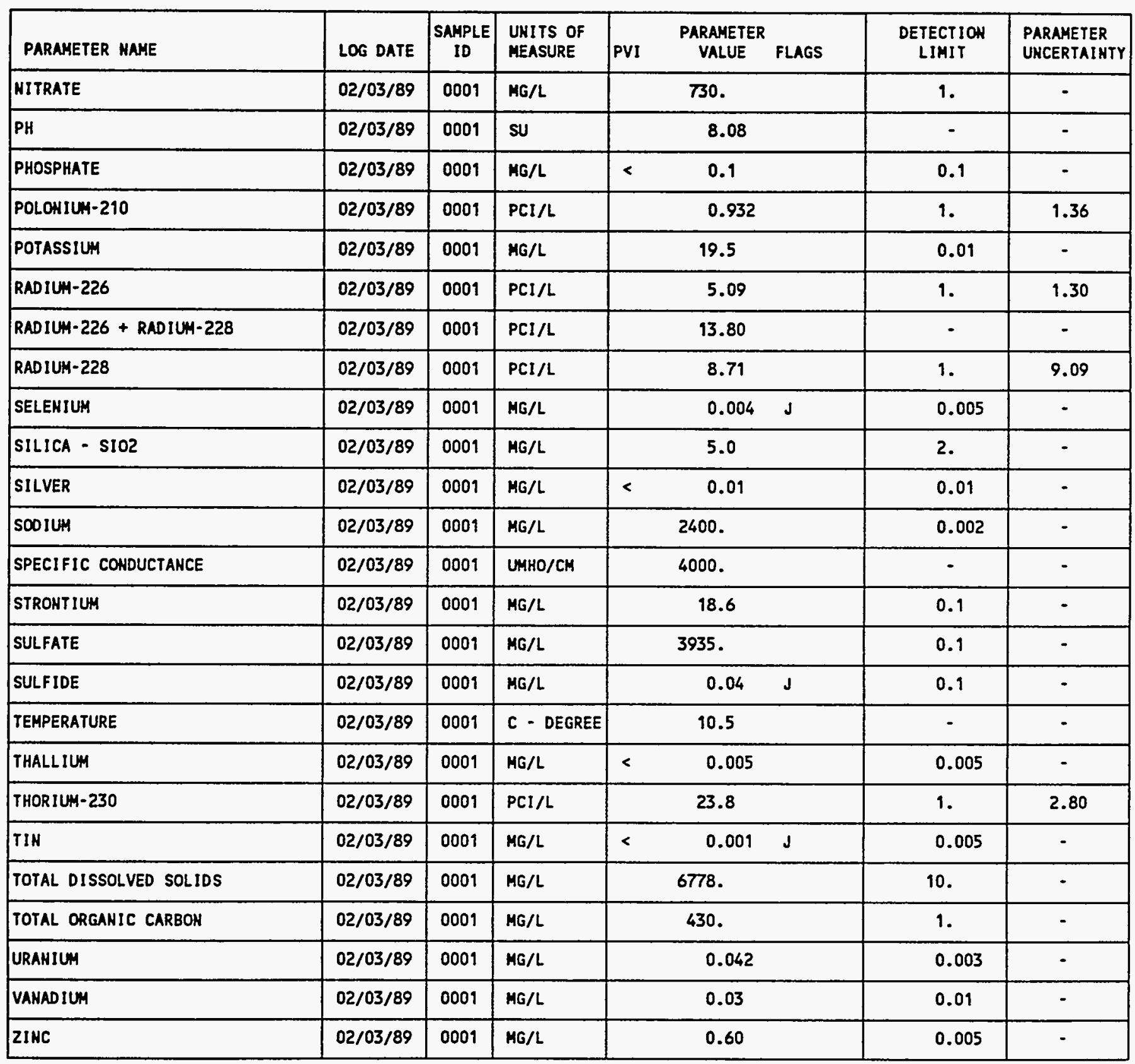

PARAMETER VALUE IHDICATOR (PVI):

< - LESS THAN DETECTION LIMIT

SAMPLE ID COOES:

0001 - FILTERED SAMPLE (.45 MICRONS)

OTHER PARAMETER VALUE FLAGS:

J - ESTIMATED VALUE 
GROUNDWATER QUALITY DATA BY LOCATION

SITE: AMBOI AMBROSIA LAKE

LOCATION: 0680 RESERVED ESTORMS, MH, 11/14/88

MORTH COORDINATE: UNKNOWN

EAST COORDINATE: UNKNOWN

06/10/80 TO $12 / 04 / 92$

REPORT DATE: 08/04/94

FORMATION OF COMPLETION: DAKOTA SANDSTONE (KD)

HYDRAULIC FLOU RELATIONSHIP: UPGRADIENT (U)

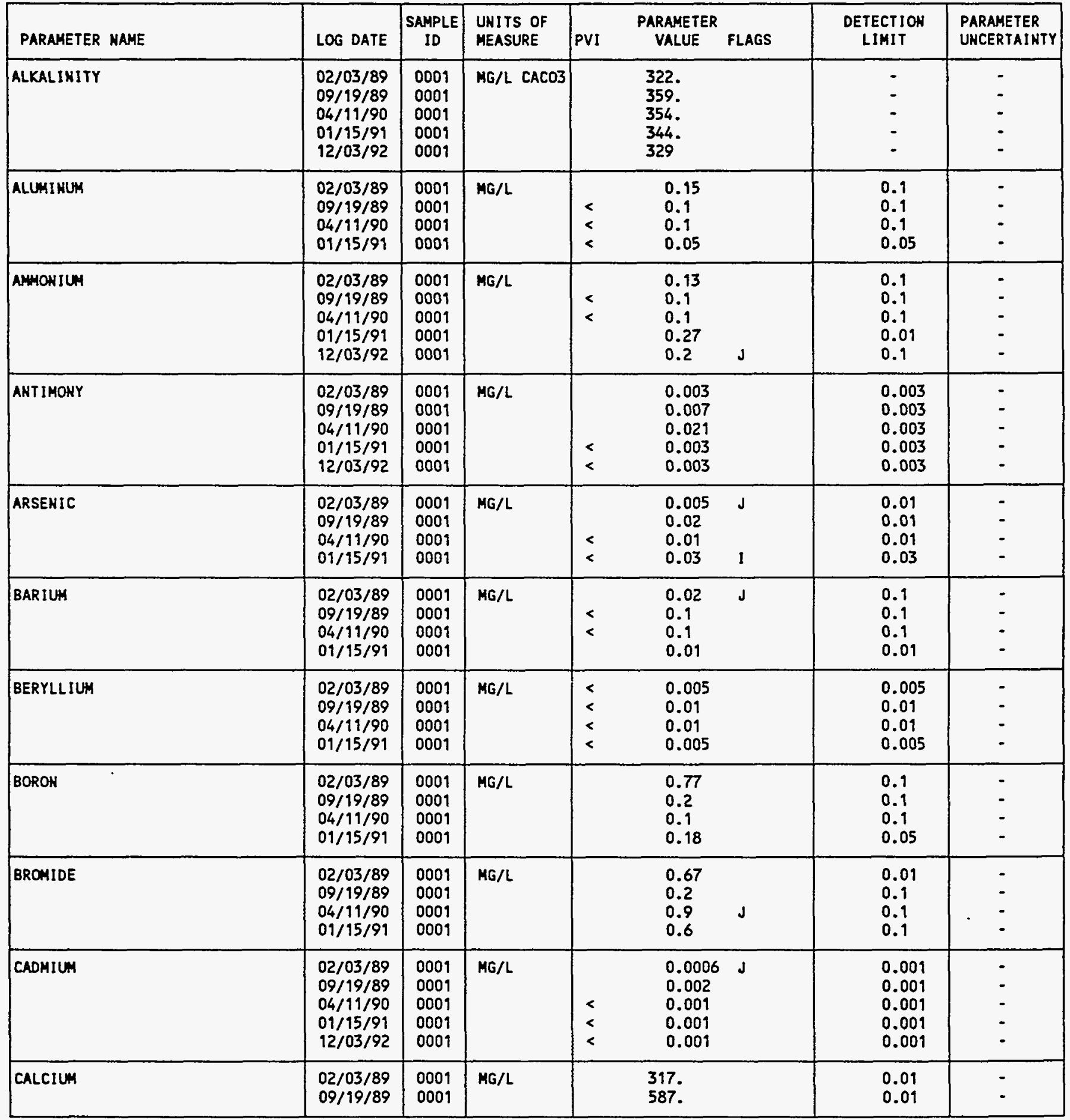

PARAMETER VALUE INDICATOR (PVI): < - LESS THAN DETECTION LIMIT

SAMPLE ID COOES:

0001 - FILTERED SAMPLE (.45 MICRONS)

OTHER PARAMETER VALUE FLAGS:

1 - IHCREASED DETECTION LIMIT OUE TO REQUIRED DILUTION

d - estimated VALUE 
GROUNDWATER OUALITY DATA BY LOCATION

SITE: AMBOI AMBROSIA LAKE

LOCATIOH: 0680 RESERVED ESTORHS, MW, 11/14/88

MORTH COORDINATE: UHKNOWH

EAST COORDINATE: UNKNOUN

06/10/80 TO $12 / 04 / 92$

REPORT DATE: 08/04/94

FORMATION OF COAPLETION: DAKOTA SAMDSTOHE (KD)

HYDRAULIC FLON RELATIONSHIP: UPGRADIENT (U)

\begin{tabular}{|c|c|c|c|c|c|c|c|}
\hline PARAMETER MAHE & LOG DATE & $\underset{10}{\text { SAMPLE }}$ & $\begin{array}{l}\text { UNITS OF } \\
\text { MEASURE }\end{array}$ & PVI & $\begin{array}{l}\text { PARAMETER } \\
\text { VALUE FLAGS }\end{array}$ & $\begin{array}{l}\text { DETECTION } \\
\text { LIHIT }\end{array}$ & $\begin{array}{l}\text { PARAMETER } \\
\text { UNCERTAINTY }\end{array}$ \\
\hline CALCIUM & $\begin{array}{l}04 / 11 / 90 \\
01 / 15 / 91 \\
12 / 03 / 92\end{array}$ & $\begin{array}{l}0001 \\
0001 \\
0001\end{array}$ & $M G / L$ & & $\begin{array}{l}529 . \\
595 \\
600\end{array}$ & $\begin{array}{l}0.01 \\
0.01 \\
2 .\end{array}$ & - \\
\hline CHLORIOE & $\begin{array}{l}02 / 03 / 89 \\
09 / 19 / 89 \\
04 / 11 / 90 \\
01 / 15 / 91 \\
12 / 03 / 92\end{array}$ & $\begin{array}{l}0001 \\
0001 \\
0001 \\
0001 \\
0001\end{array}$ & MG/L & & $\begin{array}{l}178 . \\
61 . \\
73 . \\
69 . \\
80 .\end{array}$ & $\begin{array}{l}1 . \\
1 . \\
1 . \\
0.5 \\
5 .\end{array}$ & $\begin{array}{l}- \\
. \\
. \\
.\end{array}$ \\
\hline CHROHIUH & $\begin{array}{l}02 / 03 / 89 \\
09 / 19 / 89 \\
04 / 11 / 90 \\
01 / 15 / 91 \\
12 / 03 / 92\end{array}$ & $\begin{array}{l}0001 \\
0001 \\
0001 \\
0001 \\
0001\end{array}$ & $\mathrm{MG} / \mathrm{L}$ & $\begin{array}{l}< \\
< \\
< \\
< \\
<\end{array}$ & $\begin{array}{l}0.01 \\
0.01 \\
0.01 \\
0.01 \\
0.01\end{array}$ & $\begin{array}{l}0.01 \\
0.01 \\
0.01 \\
0.01 \\
0.01\end{array}$ & $\begin{array}{l}- \\
- \\
- \\
-\end{array}$ \\
\hline COBALT & $\begin{array}{l}02 / 03 / 89 \\
09 / 19 / 89 \\
04 / 11 / 90 \\
01 / 15 / 91 \\
12 / 03 / 92\end{array}$ & $\begin{array}{l}0001 \\
0001 \\
0001 \\
0001 \\
0001\end{array}$ & $M G / L$ & $\begin{array}{l}< \\
< \\
<\end{array}$ & $\begin{array}{l}0.01 \\
0.05 \\
0.05 \\
0.03 \\
0.03\end{array}$ & $\begin{array}{l}0.05 \\
0.05 \\
0.05 \\
0.03 \\
0.03\end{array}$ & $\begin{array}{l}- \\
- \\
- \\
-\end{array}$ \\
\hline COPPER & $\begin{array}{l}02 / 03 / 89 \\
09 / 19 / 89 \\
04 / 11 / 90 \\
01 / 15 / 91\end{array}$ & $\begin{array}{l}0001 \\
0001 \\
0001 \\
0001\end{array}$ & MG/L & $\begin{array}{l}< \\
<\end{array}$ & $\begin{array}{l}0.02 \\
0.02 \\
0.02 \\
0.01\end{array}$ & $\begin{array}{l}0.02 \\
0.02 \\
0.02 \\
0.01\end{array}$ & $\begin{array}{l}- \\
- \\
-\end{array}$ \\
\hline CYAHIDE & $\begin{array}{l}02 / 03 / 89 \\
09 / 19 / 89 \\
04 / 11 / 90 \\
01 / 15 / 91\end{array}$ & $\begin{array}{l}0001 \\
0001 \\
0001 \\
0001\end{array}$ & $M G / L$ & $\begin{array}{l}< \\
< \\
< \\
<\end{array}$ & $\begin{array}{l}0.002 \\
0.01 \\
0.01 \\
0.01\end{array}$ & $\begin{array}{l}0.01 \\
0.01 \\
0.01 \\
0.01\end{array}$ & $\begin{array}{l}- \\
- \\
-\end{array}$ \\
\hline FLUORIDE & $\begin{array}{l}02 / 03 / 89 \\
09 / 19 / 89 \\
04 / 11 / 90 \\
01 / 15 / 91 \\
12 / 03 / 92\end{array}$ & $\begin{array}{l}0001 \\
0001 \\
0001 \\
0001 \\
0001\end{array}$ & $M G / L$ & & $\begin{array}{l}0.6 \\
0.2 \\
0.2 \\
0.1 \\
0.3\end{array}$ & $\begin{array}{l}0.1 \\
0.1 \\
0.1 \\
0.1 \\
0.1\end{array}$ & $\begin{array}{l}- \\
- \\
- \\
- \\
-\end{array}$ \\
\hline GROSS ALPHA & $\begin{array}{l}02 / 03 / 89 \\
09 / 19 / 89 \\
04 / 11 / 90 \\
01 / 15 / 91 \\
12 / 03 / 92\end{array}$ & $\begin{array}{l}0001 \\
0001 \\
0001 \\
0001 \\
0001\end{array}$ & $\mathrm{PCI} / \mathrm{L}$ & & $\begin{array}{l}15.6 \\
12 . \\
29 . \\
0.0 \\
25.0\end{array}$ & $\begin{array}{l}1 . \\
1 . \\
1 . \\
1.0\end{array}$ & $\begin{array}{l}20.0 \\
20 . \\
18 . \\
28.5 \\
30.5\end{array}$ \\
\hline GROSS BETA & $\begin{array}{l}02 / 03 / 89 \\
09 / 19 / 89 \\
04 / 11 / 90 \\
01 / 15 / 91 \\
12 / 03 / 92\end{array}$ & $\begin{array}{l}0001 \\
0001 \\
0001 \\
0001 \\
0001\end{array}$ & $\mathrm{PCI} / \mathrm{L}$ & & $\begin{array}{l}33.2 \\
33 . \\
30 . \\
22.8 \\
0.0\end{array}$ & $\begin{array}{r}0.5 \\
0.5 \\
0.5 \\
0.5 \\
50.3\end{array}$ & $\begin{array}{l}24.2 \\
11 . \\
14 . \\
18.4 \\
28.4\end{array}$ \\
\hline IRON & $\begin{array}{l}02 / 03 / 89 \\
09 / 19 / 89 \\
04 / 11 / 90 \\
01 / 15 / 91 \\
12 / 03 / 92\end{array}$ & $\begin{array}{l}0001 \\
0001 \\
0001 \\
0001 \\
0001\end{array}$ & $M G / L$ & & $\begin{array}{l}1.61 \\
2.15 \\
2.23 \\
2.31 \\
1.82\end{array}$ & $\begin{array}{l}0.03 \\
0.03 \\
0.03 \\
0.03 \\
0.03\end{array}$ & $\begin{array}{l}- \\
- \\
- \\
-\end{array}$ \\
\hline LEAD & $02 / 03 / 89$ & 0001 & MG $/ L$ & & 0.010 & 0.01 & + \\
\hline
\end{tabular}

PARAMETER VALUE INDICATOR (PVI): < - LESS THAN DETECTION LIMIT

SAHPLE ID COOES:

O001 - FILTERED SAMPLE (.45 MICRONS)

OTHER PARAMETER VALUE FLAGS:

$J$ - ESTIMATED VALUE 
GROUNDWATER QUALITY DATA BY LOCATION

SITE: AMBO1 AMBROSIA LAKE

LOCATION: 0680 RESERVED ESTORMS, HH, 11/14/88

NORTH COORDINATE: UNKHOWN

EAST COORDINATE: UNKHONK

06/10/80 TO $12 / 04 / 92$

REPORT DATE: 08/04/94

FORMATION OF COMPLETIOH: DAKOTA SANDSTONE (KD)

HYORAULIC FLOW RELATIONSHIP: UPGRADIENT (U)

\begin{tabular}{|c|c|c|c|c|c|c|c|c|}
\hline PARAMETER MAME & LOG DATE & $\mid \begin{array}{c}\text { SAMPLE } \\
10\end{array}$ & $\begin{array}{l}\text { UNITS OF } \\
\text { MEASURE }\end{array}$ & PVI & $\begin{array}{l}\text { PARAMETER } \\
\text { VALUEE }\end{array}$ & FLAGS & $\begin{array}{l}\text { DETECTION } \\
\text { LIMIT }\end{array}$ & $\begin{array}{l}\text { PARAMETER } \\
\text { UNCERTAINTY }\end{array}$ \\
\hline LEAD & $\begin{array}{l}09 / 19 / 89 \\
04 / 11 / 90 \\
01 / 15 / 91\end{array}$ & $\begin{array}{l}0001 \\
0001 \\
0001\end{array}$ & $M G / L$ & $<$ & $\begin{array}{l}0.01 \\
0.01 \\
0.03\end{array}$ & 1 & $\begin{array}{l}0.01 \\
0.01 \\
0.03\end{array}$ & - \\
\hline LEAD-290 & $\begin{array}{l}02 / 03 / 89 \\
09 / 19 / 89\end{array}$ & $\begin{array}{l}0001 \\
0001\end{array}$ & $\mathrm{PCl} / \mathrm{L}$ & & $\begin{array}{l}0.00 \\
0.0\end{array}$ & & $\begin{array}{l}1.5 \\
1.5\end{array}$ & $\begin{array}{l}6.25 \\
0.8\end{array}$ \\
\hline MAGNESIUM & $\begin{array}{l}02 / 03 / 89 \\
09 / 19 / 89 \\
04 / 11 / 90 \\
01 / 15 / 91 \\
12 / 03 / 92\end{array}$ & $\begin{array}{l}0001 \\
0001 \\
0001 \\
0001 \\
0001\end{array}$ & $M G / L$ & & $\begin{array}{l}84 . \\
212 . \\
210 . \\
222 . \\
213 .\end{array}$ & & $\begin{array}{l}0.001 \\
0.001 \\
0.001 \\
0.5 \\
0.5\end{array}$ & $\begin{array}{l}- \\
- \\
-\end{array}$ \\
\hline MANGANESE & $\begin{array}{l}02 / 03 / 89 \\
09 / 19 / 89 \\
04 / 11 / 90 \\
01 / 15 / 91\end{array}$ & $\begin{array}{l}0001 \\
0001 \\
0001 \\
0001\end{array}$ & $M G / L$ & & $\begin{array}{l}0.51 \\
1.35 \\
1.35 \\
1.22\end{array}$ & & $\begin{array}{l}0.01 \\
0.01 \\
0.01 \\
0.01\end{array}$ & $\begin{array}{l}- \\
- \\
-\end{array}$ \\
\hline MERCURY & $\begin{array}{l}02 / 03 / 89 \\
09 / 19 / 89 \\
04 / 11 / 90 \\
01 / 15 / 91\end{array}$ & $\begin{array}{l}0001 \\
0001 \\
0001 \\
0001\end{array}$ & MG/L & $\begin{array}{l}< \\
<\end{array}$ & $\begin{array}{l}0.0001 \\
0.0002 \\
0.0002 \\
0.0002\end{array}$ & $J$ & $\begin{array}{l}0.0002 \\
0.0002 \\
0.0002 \\
0.0002\end{array}$ & $\begin{array}{l}- \\
- \\
-\end{array}$ \\
\hline MOLYBDENUM & $\begin{array}{l}02 / 03 / 89 \\
09 / 19 / 89 \\
04 / 11 / 90 \\
01 / 15 / 91 \\
12 / 03 / 92\end{array}$ & $\begin{array}{l}0001 \\
0001 \\
0001 \\
0001 \\
0001\end{array}$ & MG/L & & $\begin{array}{l}0.058 \\
0.12 \\
0.10 \\
0.09 \\
0.08\end{array}$ & & $\begin{array}{l}0.01 \\
0.01 \\
0.01 \\
0.01 \\
0.01\end{array}$ & $\begin{array}{l}- \\
- \\
-\end{array}$ \\
\hline NET GROSS ALPHA * & $\begin{array}{l}02 / 03 / 89 \\
09 / 19 / 89 \\
04 / 11 / 90 \\
01 / 15 / 91 \\
12 / 03 / 92\end{array}$ & $\begin{array}{l}0001 \\
0001 \\
0001 \\
0001 \\
0001\end{array}$ & $\mathrm{PCI} / \mathrm{L}$ & & $\begin{array}{l}-0.18 \\
10.63 \\
27.90 \\
-0.34 \\
24.31\end{array}$ & & $\begin{array}{l}- \\
- \\
-\end{array}$ & $\begin{array}{l}- \\
- \\
-\end{array}$ \\
\hline HICKEL & $\begin{array}{l}02 / 03 / 89 \\
09 / 19 / 89 \\
04 / 11 / 90 \\
01 / 15 / 91 \\
12 / 03 / 92\end{array}$ & $\begin{array}{l}0001 \\
0001 \\
0001 \\
0001 \\
0001\end{array}$ & $M G / L$ & $\begin{array}{l}< \\
< \\
< \\
<\end{array}$ & $\begin{array}{l}0.02 \\
0.04 \\
0.04 \\
0.04 \\
0.10\end{array}$ & J & $\begin{array}{l}0.04 \\
0.04 \\
0.04 \\
0.04 \\
0.04\end{array}$ & $\begin{array}{l}- \\
- \\
- \\
-\end{array}$ \\
\hline NITRATE & $\begin{array}{l}02 / 03 / 89 \\
09 / 19 / 89 \\
04 / 11 / 90 \\
01 / 15 / 91 \\
12 / 03 / 92\end{array}$ & $\begin{array}{l}0001 \\
0001 \\
0001 \\
0001 \\
0001\end{array}$ & $M G / L$ & $<$ & $\begin{array}{l}6.78 \\
1 . \\
0.8 \\
4.4 \\
1.0\end{array}$ & $\mathbf{J}$ & $\begin{array}{l}1 . \\
1 . \\
1 . \\
0.1 \\
1.0\end{array}$ & $\begin{array}{l}- \\
- \\
- \\
-\end{array}$ \\
\hline NITRITE AND NITRATE & $\begin{array}{l}04 / 11 / 90 \\
01 / 15 / 91\end{array}$ & $\begin{array}{l}0001 \\
0001\end{array}$ & $M G / L$ & & $\begin{array}{l}0.2 \\
0.94\end{array}$ & 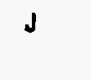 & $\begin{array}{l}1 . \\
0.05\end{array}$ & - \\
\hline PH & $\begin{array}{l}02 / 03 / 89 \\
09 / 19 / 89 \\
04 / 11 / 90 \\
01 / 15 / 91\end{array}$ & $\begin{array}{l}0001 \\
0001 \\
0001 \\
0001\end{array}$ & SU & & $\begin{array}{l}7.12 \\
6.59 \\
6.53 \\
6.9\end{array}$ & & : & $\begin{array}{l}- \\
- \\
-\end{array}$ \\
\hline
\end{tabular}

- MET GROSS ALPHA (GROSS ALPHA - URANIUM) WITH 1 MG URAHIUM = 686 PCI

PARAMETER VALUE IMDICATOR (PVI): < - LESS THAN DETECTION LIMIT

OTHER PARAMETER VALUE FLAGS:

I - IMCREASED DETECTION LIMIT DUE TO REQUIRED DILUTION

$\downarrow$ - ESTIMATED VALUE
SAMPLE ID COOES:

0001 - FILTERED SAMPLE (.45 MICRONS) 
GROUNDWATER QUALITY DATA BY LOCATION

SITE: AMBOI AMBROSIA LAKE

LOCATION: 0680 RESERVED ESTORMS, MH, 11/14/88

NORTH COORDIMATE: UNKHOWH

EAST COORDINATE: UNKNOWH

06/10/80 TO $12 / 04 / 92$

REPORT DATE: 08/04/94

FORMATION OF COMPLETION: DAKOTA SANDSTONE (KD)

HYDRAULIC FLON RELATIONSHIP: UPGRADIENT (U)

\begin{tabular}{|c|c|c|c|c|c|c|c|}
\hline PARAMETER MAME & LOG DATE & $\begin{array}{l}\text { SAMPLE } \\
\text { ID }\end{array}$ & $\begin{array}{l}\text { UNITS OF } \\
\text { MEASURE }\end{array}$ & PVI & $\begin{array}{l}\text { PARAMETER } \\
\text { VALUE FLAGS }\end{array}$ & $\begin{array}{l}\text { DETECTIOH } \\
\text { LIMIT }\end{array}$ & $\begin{array}{l}\text { PARAMETER } \\
\text { UMCERTAINTY }\end{array}$ \\
\hline PH & $12 / 03 / 92$ & 0001 & SU & & 6.74 & - & - \\
\hline PHOSPHATE & $\begin{array}{l}02 / 03 / 89 \\
09 / 19 / 89 \\
04 / 11 / 90 \\
01 / 15 / 91 \\
12 / 03 / 92\end{array}$ & $\begin{array}{l}0001 \\
0001 \\
0001 \\
0001 \\
0001\end{array}$ & $M G / L$ & $\begin{array}{l}< \\
< \\
<\end{array}$ & $\begin{array}{l}0.1 \\
0.1 \\
0.1 \\
0.23 \\
0.3\end{array}$ & $\begin{array}{l}0.1 \\
0.1 \\
0.1 \\
0.05 \\
0.1\end{array}$ & $\begin{array}{l}- \\
- \\
- \\
-\end{array}$ \\
\hline POLOHIUA-210 & $\begin{array}{l}02 / 03 / 89 \\
09 / 19 / 89\end{array}$ & $\begin{array}{l}0001 \\
0001\end{array}$ & $\mathrm{PCI} / \mathrm{L}$ & & $\begin{array}{l}0.466 \\
0.3\end{array}$ & 1. & $\begin{array}{l}1.26 \\
0.4\end{array}$ \\
\hline POTASSIU: & $\begin{array}{l}02 / 03 / 89 \\
09 / 19 / 89 \\
04 / 11 / 90 \\
01 / 15 / 91 \\
12 / 03 / 92\end{array}$ & $\begin{array}{l}0001 \\
0001 \\
0001 \\
0001 \\
0001\end{array}$ & MG/L & & $\begin{array}{c}22.6 \\
9.8 \\
10.9 \\
8.3 \\
8.75\end{array}$ & $\begin{array}{l}0.01 \\
0.01 \\
0.01 \\
0.01 \\
0.01\end{array}$ & $\begin{array}{l}- \\
- \\
- \\
-\end{array}$ \\
\hline RADIUM-226 & $\begin{array}{l}02 / 03 / 89 \\
09 / 19 / 89 \\
04 / 11 / 90 \\
01 / 15 / 91 \\
12 / 03 / 92\end{array}$ & $\begin{array}{l}0001 \\
0001 \\
0001 \\
0001 \\
0001\end{array}$ & PCI/L & & $\begin{array}{l}2.60 \\
1.5 \\
0.9 \\
3.5 \\
2.2\end{array}$ & $\begin{array}{l}1 . \\
1 . \\
1 . \\
1 . \\
0.3\end{array}$ & $\begin{array}{l}0.351 \\
0.4 \\
0.3 \\
0.9 \\
0.6\end{array}$ \\
\hline RADIUM-226 + RADIUM-228 & $\begin{array}{l}02 / 03 / 89 \\
09 / 19 / 89 \\
04 / 11 / 90 \\
01 / 15 / 91 \\
12 / 03 / 92\end{array}$ & $\begin{array}{l}0001 \\
0001 \\
0001 \\
0001 \\
0001\end{array}$ & PCI/L & & $\begin{array}{l}3.82 \\
4.10 \\
4.50 \\
6.40 \\
5.30\end{array}$ & $\begin{array}{l}- \\
- \\
- \\
-\end{array}$ & $\begin{array}{l}- \\
- \\
-\end{array}$ \\
\hline RADILM-228 & $\begin{array}{l}02 / 03 / 89 \\
09 / 19 / 89 \\
04 / 11 / 90 \\
01 / 15 / 91 \\
12 / 03 / 92\end{array}$ & $\begin{array}{l}0001 \\
0001 \\
0001 \\
0001 \\
0001\end{array}$ & PCI/L & & $\begin{array}{l}1.22 \\
2.6 \\
3.6 \\
2.9 \\
3.1\end{array}$ & $\begin{array}{l}1 . \\
1 . \\
1 . \\
1 . \\
1.0\end{array}$ & $\begin{array}{l}8.64 \\
1.2 \\
1.0 \\
1.8 \\
1.7\end{array}$ \\
\hline SELENIUM & $\begin{array}{l}02 / 03 / 89 \\
09 / 19 / 89 \\
04 / 11 / 90 \\
01 / 15 / 91 \\
12 / 03 / 92\end{array}$ & $\begin{array}{l}0001 \\
0001 \\
0001 \\
0001 \\
0001\end{array}$ & $M G / L$ & $\begin{array}{l}< \\
< \\
< \\
<\end{array}$ & $\begin{array}{ll}0.001 & J \\
0.018 & \\
0.005 & \\
0.05 & I \\
0.05 & 1\end{array}$ & $\begin{array}{l}0.005 \\
0.005 \\
0.005 \\
0.05 \\
0.05\end{array}$ & : \\
\hline SILICA - SIOZ & $\begin{array}{l}02 / 03 / 89 \\
09 / 19 / 89 \\
04 / 11 / 90 \\
01 / 95 / 91\end{array}$ & $\begin{array}{l}0001 \\
0001 \\
0001 \\
0001\end{array}$ & $M G / L$ & & $\begin{array}{l}14.2 \\
21 . \\
20 . \\
20.1\end{array}$ & $\begin{array}{l}2 . \\
2 . \\
2 . \\
0.1\end{array}$ & $:$ \\
\hline SILVER & $\begin{array}{l}02 / 03 / 89 \\
09 / 19 / 89 \\
04 / 11 / 90 \\
01 / 15 / 91 \\
12 / 03 / 92\end{array}$ & $\begin{array}{l}0001 \\
0001 \\
0001 \\
0001 \\
0001\end{array}$ & MG/L & $\begin{array}{l}< \\
< \\
< \\
<\end{array}$ & $\begin{array}{l}0.01 \\
0.01 \\
0.01 \\
0.01 \\
0.01\end{array}$ & $\begin{array}{l}0.01 \\
0.01 \\
0.01 \\
0.01 \\
0.01\end{array}$ & : \\
\hline soorum & $\begin{array}{l}02 / 03 / 89 \\
09 / 19 / 89 \\
04 / 11 / 90\end{array}$ & $\begin{array}{l}0009 \\
0001 \\
0001\end{array}$ & MG/L & & $\begin{array}{l}515 . \\
309 . \\
374 .\end{array}$ & $\begin{array}{l}0.002 \\
0.002 \\
0.002\end{array}$ & : \\
\hline
\end{tabular}

parameter Value Ihdicator (PVI): < - Less than Detection LIMIT

SAMPLE ID COOES:

ODO1 - FILTERED SAMPLE (.45 MICRONS)

OTHER PARAMETER VALUE FLAGS:

H - HOLD TIME EXPIRED, VALUE SUSPECT

I - INCREASED DETECTION LIMIT DUE TO REQUIRED DILUTION

J - eSTIMATED VALUE 
GROUNDWATER QUALITY DATA BY LOCATION

SITE: AMB01 AMBROSIA LAKE

LOCATION: 0680 RESERVED ESTORMS, MH, $11 / 14 / 88$

NORTH COORDINATE: UNKNONN

EAST COORDINATE: UNKNOUN

06/10/80 TO $12 / 04 / 92$

REPORT DATE: 08/04/94

FORMATION OF COHPLETION: DAKOTA SAHDSTONE (KD)

HYDRAULIC FLOW RELATIONSHIP: UPGRADIENT (U)

\begin{tabular}{|c|c|c|c|c|c|c|c|}
\hline PARAMETER MAME & LOG DATE & $\begin{array}{c}\text { SAMPLE } \\
\text { ID }\end{array}$ & $\begin{array}{l}\text { UNITS OF } \\
\text { MEASURE }\end{array}$ & PVI & $\begin{array}{l}\text { PARAMETER } \\
\text { VALUE FLAGS }\end{array}$ & $\begin{array}{l}\text { DETECTION } \\
\text { LIMIT }\end{array}$ & $\begin{array}{l}\text { PARAMETER } \\
\text { UNCERTAINTY }\end{array}$ \\
\hline SOOIU: & $\begin{array}{l}01 / 15 / 91 \\
12 / 03 / 92\end{array}$ & $\begin{array}{l}0009 \\
0009\end{array}$ & $M G / L$ & & $\begin{array}{l}332 . \\
308 .\end{array}$ & $\begin{array}{l}5 . \\
5 .\end{array}$ & - \\
\hline SPECIFIC CONDUCTANCE & $\begin{array}{l}02 / 03 / 89 \\
09 / 19 / 89 \\
04 / 11 / 90 \\
01 / 15 / 91 \\
12 / 03 / 92\end{array}$ & $\begin{array}{l}0001 \\
0001 \\
0001 \\
0001 \\
0001\end{array}$ & UMHO/CM & & $\begin{array}{l}2000 . \\
700 \\
2850 \\
3050 \\
3200\end{array}$ & $\begin{array}{l}- \\
- \\
- \\
-\end{array}$ & $\begin{array}{l}- \\
- \\
-\end{array}$ \\
\hline STRONTIUM & $\begin{array}{l}02 / 03 / 89 \\
09 / 19 / 89 \\
04 / 11 / 90 \\
01 / 15 / 91\end{array}$ & $\begin{array}{l}0001 \\
0001 \\
0001 \\
0001\end{array}$ & $M G / L$ & & $\begin{array}{l}5.60 \\
5.7 \\
5.58 \\
5.50\end{array}$ & $\begin{array}{l}0.1 \\
0.1 \\
0.1 \\
0.01\end{array}$ & $\begin{array}{l}- \\
- \\
-\end{array}$ \\
\hline SULFATE & $\begin{array}{l}02 / 03 / 89 \\
09 / 19 / 89 \\
04 / 11 / 90 \\
01 / 15 / 91 \\
12 / 03 / 92\end{array}$ & $\begin{array}{l}0001 \\
0001 \\
0001 \\
0001 \\
0001\end{array}$ & MG/L & & $\begin{array}{l}1862 . \\
2600 . \\
2630 . \\
2430 \\
2390\end{array}$ & $\begin{array}{c}0.1 \\
0.1 \\
0.1 \\
10 . \\
10 .\end{array}$ & $\begin{array}{l}- \\
- \\
- \\
-\end{array}$ \\
\hline SULFIDE & $\begin{array}{l}02 / 03 / 89 \\
09 / 19 / 89 \\
04 / 11 / 90 \\
01 / 15 / 91\end{array}$ & $\begin{array}{l}0001 \\
0001 \\
0001 \\
0001\end{array}$ & MG/L & $\begin{array}{l}< \\
< \\
<\end{array}$ & $\begin{array}{l}0.01 \\
0.1 \\
0.1 \\
0.1\end{array}$ & $\begin{array}{l}0.1 \\
0.1 \\
0.1 \\
0.1\end{array}$ & $:$ \\
\hline TEMPERATURE & $\begin{array}{l}02 / 03 / 89 \\
09 / 19 / 89 \\
04 / 11 / 90 \\
01 / 15 / 91 \\
12 / 03 / 92\end{array}$ & $\begin{array}{l}0001 \\
0001 \\
0001 \\
0001 \\
0001\end{array}$ & $C$ - DEGREE & & $\begin{array}{l}11.0 \\
16.0 \\
14.5 \\
13 . \\
13.4\end{array}$ & $\begin{array}{l}- \\
- \\
-\end{array}$ & $:$ \\
\hline THALLIUM & $\begin{array}{l}02 / 03 / 89 \\
09 / 19 / 89 \\
04 / 11 / 90 \\
01 / 15 / 91\end{array}$ & $\begin{array}{l}0001 \\
0001 \\
0001 \\
0001\end{array}$ & $M G / L$ & $\begin{array}{l}< \\
<\end{array}$ & $\begin{array}{l}0.005 \\
0.01 \\
0.01 \\
0.03\end{array}$ & $\begin{array}{l}0.005 \\
0.01 \\
0.01 \\
0.03\end{array}$ & : \\
\hline THORIUM-230 & $\begin{array}{l}02 / 03 / 89 \\
09 / 19 / 89\end{array}$ & $\begin{array}{l}0001 \\
0001\end{array}$ & $\mathrm{PCI} / \mathrm{L}$ & & $\begin{array}{l}1.96 \\
0.0\end{array}$ & 1. & $\begin{array}{l}0.716 \\
0.2\end{array}$ \\
\hline TIN & $\begin{array}{l}02 / 03 / 89 \\
09 / 19 / 89 \\
04 / 11 / 90 \\
01 / 15 / 91\end{array}$ & $\begin{array}{l}0001 \\
0001 \\
0001 \\
0001\end{array}$ & MG/L & $\begin{array}{l}< \\
< \\
<\end{array}$ & $\begin{array}{ll}0.001 & J \\
0.1 & \\
0.005 & \\
0.03 & I\end{array}$ & $\begin{array}{l}0.005 \\
0.1 \\
0.005 \\
0.03\end{array}$ & : \\
\hline TOTAL DISSOLVED SOLIDS & $\begin{array}{l}02 / 03 / 89 \\
09 / 19 / 89 \\
04 / 11 / 90 \\
01 / 15 / 91 \\
12 / 03 / 92\end{array}$ & $\begin{array}{l}0001 \\
0001 \\
0001 \\
0001 \\
0001\end{array}$ & MG/L & & $\begin{array}{l}3234 . \\
4050 . \\
4060 . \\
5570 . \\
4140 .\end{array}$ & $\begin{array}{l}10 . \\
10 . \\
10 . \\
10 . \\
10 .\end{array}$ & $\begin{array}{l}- \\
: \\
-\end{array}$ \\
\hline TOTAL KJELDAHL NITROGEN & $\begin{array}{l}01 / 15 / 91 \\
12 / 03 / 92\end{array}$ & $\begin{array}{l}0001 \\
0001\end{array}$ & $M G / L$ & & $\begin{array}{l}1 . \\
1 .\end{array}$ & 1. & : \\
\hline TOTAL ORGAHIC CARBOH & $\begin{array}{l}02 / 03 / 89 \\
09 / 19 / 89 \\
04 / 11 / 90\end{array}$ & $\begin{array}{l}0001 \\
0001 \\
0001\end{array}$ & $M G / L$ & & $\begin{array}{l}27 . \\
99.0 \\
49 .\end{array}$ & $\begin{array}{l}1 . \\
1 .\end{array}$ & : \\
\hline
\end{tabular}

PARAMETER VALUE INDICATOR (PVI): < - LESS THAN DETECTION LIMIT

OTHER PARAMETER VALUE FLAGS:

I - INCREASED DETECTIOH LIMIT DUE TO REQUIRED DILUTION

J - ESTIMATED VALUE 
GROUNDWATER OUALITY DATA BY LOCATION

SITE: AMBO1 AMBROSIA LAKE

LOCATION: O680 RESERVED ESTORMS, MH, $11 / 14 / 88$

MORTH COOROIHATE: UNKNOWN

EAST COORDIHATE: UNKHOWN

$06 / 10 / 80$ TO $12 / 04 / 92$

REPORT DATE: 08/04/94

FORMATIOH OF COMPLETION: DAKOTA SANDSTOHE (KD)

HYDRAULIC FLOW RELATIONSHIP: UPGRADIENT (U)

\begin{tabular}{|c|c|c|c|c|c|c|c|}
\hline PARAMETER MAME & LOG DATE & $\begin{array}{c}\text { SAMPLE } \\
\text { I0 }\end{array}$ & $\begin{array}{l}\text { UNITS OF } \\
\text { MEASURE }\end{array}$ & PVI & $\begin{array}{l}\text { PARAMETER } \\
\text { VALUE FLAGS }\end{array}$ & $\begin{array}{l}\text { DETECTION } \\
\text { LIMIT }\end{array}$ & $\begin{array}{l}\text { PARAMETER } \\
\text { UHCERTAINTY }\end{array}$ \\
\hline TOTAL ORGAHIC CARBOH & $\begin{array}{l}01 / 15 / 99 \\
12 / 03 / 92\end{array}$ & $\begin{array}{l}0001 \\
0001\end{array}$ & MG/L & & $\begin{array}{l}4 . \\
8 .\end{array}$ & 1. & - \\
\hline URAKIUM & $\begin{array}{l}02 / 03 / 89 \\
09 / 19 / 89 \\
04 / 11 / 90 \\
01 / 15 / 91 \\
12 / 03 / 92\end{array}$ & $\begin{array}{l}0001 \\
0001 \\
0001 \\
0001 \\
0001\end{array}$ & $M G / L$ & $<$ & $\begin{array}{ll}0.023 & \\
0.0020 & \mathrm{~J} \\
0.0016 \mathrm{~J} & \\
0.001 & \\
0.001 & \end{array}$ & $\begin{array}{l}0.003 \\
0.003 \\
0.003 \\
0.001 \\
0.001\end{array}$ & $\begin{array}{l}- \\
. \\
. \\
-\end{array}$ \\
\hline VAKADIUM & $\begin{array}{l}02 / 03 / 89 \\
09 / 19 / 89 \\
04 / 11 / 90 \\
01 / 15 / 91 \\
12 / 03 / 92\end{array}$ & $\begin{array}{l}0001 \\
0001 \\
0001 \\
0001 \\
0001\end{array}$ & MG/L & $\begin{array}{l}< \\
< \\
<\end{array}$ & $\begin{array}{l}0.01 \\
0.01 \\
0.01 \\
0.01 \\
0.01\end{array}$ & $\begin{array}{l}0.01 \\
0.01 \\
0.01 \\
0.01 \\
0.01\end{array}$ & $\begin{array}{l}: \\
: \\
:\end{array}$ \\
\hline
\end{tabular}

PARAMETER VALUE IHDICATOR (PVI): \& - LESS THAN DETECTION LIMIT

SAMPLE ID COOES:

0001 - FILTERED SAMPLE (.45 MICRONS)

OTHER PARAMETER VALUE FLAGS:

H - HOLD TIME EXPIRED, VALUE SUSPECT

J - estimated VALUE 
GROUHDHATER QUALITY DATA BY LOCATION

SITE: AMBO1 AMBROSIA LAKE

LOCATION: 0681 RESERVED ESTORMS, MH, 11/14/88

MORTH COORDINATE: UNKNOWN

EAST COORDINATE: UMKNOUH

06/10/80 TO $12 / 04 / 92$

REPORT DATE: $08 / 04 / 94$

FORMATION OF COMPLETION: TRES HERMANOS - B SANDSTONE (TB)

HYDRAULIC FLON RELATIONSHIP: CROSS GRADIENT (C)

\begin{tabular}{|c|c|c|c|c|c|c|c|c|}
\hline PARAMETER NAME & LOG DATE & $\begin{array}{c}\text { SAMPLE } \\
\text { ID }\end{array}$ & $\begin{array}{l}\text { UNITS OF } \\
\text { MEASURE }\end{array}$ & PVI & $\begin{array}{l}\text { PARAMETER } \\
\text { VALUE }\end{array}$ & FLAGS & $\begin{array}{l}\text { DETECTIOH } \\
\text { LIMIT }\end{array}$ & $\begin{array}{l}\text { PARAMETER } \\
\text { UNCERTAINTY }\end{array}$ \\
\hline ALKALINITY & $\begin{array}{l}02 / 02 / 89 \\
09 / 19 / 89 \\
04 / 06 / 90 \\
01 / 09 / 91 \\
12 / 04 / 92\end{array}$ & $\begin{array}{l}0001 \\
0001 \\
0001 \\
0001 \\
0001\end{array}$ & MG/L CACO3 & & $\begin{array}{l}363 . \\
329 \\
307 \\
309 \\
283\end{array}$ & & $\begin{array}{l}- \\
- \\
-\end{array}$ & $\begin{array}{l}- \\
- \\
-\end{array}$ \\
\hline ALUMINUM & $\begin{array}{l}02 / 02 / 89 \\
09 / 19 / 89 \\
04 / 06 / 90 \\
01 / 09 / 91\end{array}$ & $\begin{array}{l}0001 \\
0001 \\
0001 \\
0001\end{array}$ & MG/L & $\begin{array}{l}< \\
< \\
<\end{array}$ & $\begin{array}{l}0.05 \\
0.1 \\
0.1 \\
0.05\end{array}$ & 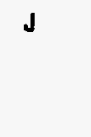 & $\begin{array}{l}0.1 \\
0.1 \\
0.1 \\
0.05\end{array}$ & : \\
\hline AMHONIUM & $\begin{array}{l}02 / 02 / 89 \\
09 / 19 / 89 \\
04 / 06 / 90 \\
01 / 09 / 91 \\
12 / 04 / 92\end{array}$ & $\begin{array}{l}0001 \\
0001 \\
0001 \\
0001 \\
0001\end{array}$ & $M G / L$ & $<$ & $\begin{array}{l}0.50 \\
0.1 \\
0.1 \\
0.40 \\
0.3\end{array}$ & J & $\begin{array}{l}0.1 \\
0.1 \\
0.1 \\
0.01 \\
0.1\end{array}$ & $\begin{array}{l}- \\
- \\
-\end{array}$ \\
\hline ANT IMONY & $\begin{array}{l}02 / 02 / 89 \\
09 / 19 / 89 \\
04 / 06 / 90 \\
01 / 09 / 91 \\
12 / 04 / 92\end{array}$ & $\begin{array}{l}0001 \\
0001 \\
0001 \\
0001 \\
0001\end{array}$ & $M G / L$ & $<$ & $\begin{array}{l}0.019 \\
0.029 \\
0.077 \\
0.005 \\
0.003\end{array}$ & & $\begin{array}{l}0.003 \\
0.003 \\
0.003 \\
0.003 \\
0.003\end{array}$ & $\begin{array}{l}: \\
: \\
-\end{array}$ \\
\hline ARSENIC & $\begin{array}{l}02 / 02 / 89 \\
09 / 19 / 89 \\
04 / 06 / 90 \\
01 / 09 / 91\end{array}$ & $\begin{array}{l}0001 \\
0001 \\
0001 \\
0001\end{array}$ & $M G / L$ & $\begin{array}{l}< \\
<\end{array}$ & $\begin{array}{l}0.003 \\
0.01 \\
0.01 \\
0.03\end{array}$ & $\begin{array}{l}J \\
I\end{array}$ & $\begin{array}{l}0.01 \\
0.01 \\
0.01 \\
0.03\end{array}$ & : \\
\hline BARIUY & $\begin{array}{l}02 / 02 / 89 \\
09 / 19 / 89 \\
04 / 06 / 90 \\
01 / 09 / 91\end{array}$ & $\begin{array}{l}0001 \\
0001 \\
0001 \\
0001\end{array}$ & $M G / L$ & $\begin{array}{l}< \\
<\end{array}$ & $\begin{array}{l}0.02 \\
0.1 \\
0.1 \\
0.01\end{array}$ & J & $\begin{array}{l}0.1 \\
0.1 \\
0.1 \\
0.01\end{array}$ & $\begin{array}{l}- \\
-\end{array}$ \\
\hline BERYLLIUM & $\begin{array}{l}02 / 02 / 89 \\
09 / 19 / 89 \\
04 / 06 / 90 \\
01 / 09 / 91\end{array}$ & $\begin{array}{l}0001 \\
0001 \\
0001 \\
0001\end{array}$ & $M G / L$ & $\begin{array}{l}< \\
< \\
<\end{array}$ & $\begin{array}{l}0.005 \\
0.01 \\
0.01 \\
0.005\end{array}$ & & $\begin{array}{l}0.005 \\
0.01 \\
0.01 \\
0.005\end{array}$ & : \\
\hline BORON & $\begin{array}{l}02 / 02 / 89 \\
09 / 19 / 89 \\
04 / 06 / 90 \\
01 / 09 / 91\end{array}$ & $\begin{array}{l}0001 \\
0001 \\
0001 \\
0001\end{array}$ & $M G / L$ & & $\begin{array}{l}0.47 \\
0.3 \\
0.2 \\
0.25\end{array}$ & & $\begin{array}{l}0.1 \\
0.1 \\
0.1 \\
0.05\end{array}$ & $\begin{array}{l}- \\
- \\
-\end{array}$ \\
\hline BROMIDE & $\begin{array}{l}02 / 02 / 89 \\
09 / 19 / 89 \\
04 / 06 / 90 \\
01 / 09 / 91\end{array}$ & $\begin{array}{l}0001 \\
0001 \\
0001 \\
0001\end{array}$ & $M G / L$ & $<$ & $\begin{array}{l}0.33 \\
0.1 \\
0.4 \\
0.2\end{array}$ & J & $\begin{array}{l}0.01 \\
0.1 \\
0.1 \\
0.1\end{array}$ & $:$ \\
\hline CADMIUM & $\begin{array}{l}02 / 02 / 89 \\
09 / 19 / 89 \\
04 / 06 / 90 \\
01 / 09 / 91 \\
12 / 04 / 92\end{array}$ & $\begin{array}{l}0001 \\
0001 \\
0001 \\
0001 \\
0001\end{array}$ & $M G / L$ & $\begin{array}{l}< \\
<\end{array}$ & $\begin{array}{l}0.0001 \\
0.002 \\
0.001 \\
0.001 \\
0.001\end{array}$ & J & $\begin{array}{l}0.001 \\
0.001 \\
0.001 \\
0.001 \\
0.001\end{array}$ & $\begin{array}{l}- \\
- \\
- \\
-\end{array}$ \\
\hline CALCIUM & $\begin{array}{l}02 / 02 / 89 \\
09 / 19 / 89\end{array}$ & $\begin{array}{l}0001 \\
0001\end{array}$ & MG/L & & $\begin{array}{r}26 . \\
7113 .\end{array}$ & & $\begin{array}{l}0.01 \\
0.01\end{array}$ & - \\
\hline
\end{tabular}

PARAMETER VALUE INDICATOR (PVI): < - LESS THAN DETECTION LIMIT

SAMPLE ID CODES:

0001 - FILTERED SAMPLE (.45 MICROHS)

OTHER PARAMETER VALUE FLAGS:

1 - INCREASED DETECTION LIMIT DUE TO REQUIRED DILUTION

J - estimated VALUe 
GROUNDWATER OUALITY DATA BY LOCATION

SITE: AMBOI AMBROSIA LAKE

LOCATIOH: 0681 RESERVED ESTORMS, MH, 11/14/88

HORTH COORDINATE: UNKNOWH

EAST COORDINATE: UHKHONH

06/10/80 TO 12/04/92

REPORT DATE: 08/04/94

FORMATIOH OF COMPLETIOH: TRES HERMANOS - B SAMDSTONE (TB) HYDRAULIC FLOW RELATIOHSHIP: CROSS GRADIENT (C)

\begin{tabular}{|c|c|c|c|c|c|c|c|c|}
\hline PARAMETER NAME & LOG DATE & $\begin{array}{c}\text { SAMPLE } \\
\text { ID }\end{array}$ & $\begin{array}{l}\text { UNITS OF } \\
\text { MEASURE }\end{array}$ & PVI & $\begin{array}{l}\text { PARAMETER } \\
\text { VALUE }\end{array}$ & FLAGS & $\begin{array}{l}\text { DETECTIOH } \\
\text { LIMIT }\end{array}$ & $\begin{array}{l}\text { PARAMETER } \\
\text { UHCERTAINTY }\end{array}$ \\
\hline CALCIUM & $\begin{array}{l}04 / 06 / 90 \\
01 / 09 / 91 \\
12 / 04 / 92\end{array}$ & $\begin{array}{l}0001 \\
0001 \\
0001\end{array}$ & MG/L & & $\begin{array}{l}6.22 \\
6.1 \\
6.2\end{array}$ & & $\begin{array}{l}0.01 \\
0.1 \\
0.1\end{array}$ & : \\
\hline CHLORIDE & $\begin{array}{l}02 / 02 / 89 \\
09 / 19 / 89 \\
04 / 06 / 90 \\
01 / 09 / 91 \\
12 / 04 / 92\end{array}$ & $\begin{array}{l}0001 \\
0001 \\
0001 \\
0001 \\
0001\end{array}$ & MG/L & & $\begin{array}{l}72 . \\
15 . \\
19 . \\
13.6 \\
12.9\end{array}$ & & $\begin{array}{l}1 . \\
1 . \\
1 . \\
0.5 \\
0.5\end{array}$ & $\begin{array}{l}- \\
\dot{-} \\
\dot{-} \\
\dot{-}\end{array}$ \\
\hline CHROMIUM & $\begin{array}{l}02 / 02 / 89 \\
09 / 19 / 89 \\
04 / 06 / 90 \\
01 / 09 / 91 \\
12 / 04 / 92\end{array}$ & $\begin{array}{l}0001 \\
0001 \\
0001 \\
0001 \\
0001\end{array}$ & MG/L & $\begin{array}{l}< \\
< \\
< \\
<\end{array}$ & $\begin{array}{l}0.01 \\
0.01 \\
0.01 \\
0.01 \\
0.01\end{array}$ & & $\begin{array}{l}0.01 \\
0.01 \\
0.01 \\
0.01 \\
0.01\end{array}$ & $\begin{array}{l}- \\
- \\
- \\
-\end{array}$ \\
\hline COBALT & $\begin{array}{l}02 / 02 / 89 \\
09 / 19 / 89 \\
04 / 06 / 90 \\
01 / 09 / 91 \\
12 / 04 / 92\end{array}$ & $\begin{array}{l}0001 \\
0001 \\
0001 \\
0001 \\
0001\end{array}$ & $M G / L$ & $\begin{array}{l}< \\
< \\
< \\
<\end{array}$ & $\begin{array}{l}0.01 \\
0.05 \\
0.05 \\
0.03 \\
0.03\end{array}$ & J & $\begin{array}{l}0.05 \\
0.05 \\
0.05 \\
0.03 \\
0.03\end{array}$ & :- \\
\hline COPPER & $\begin{array}{l}02 / 02 / 89 \\
09 / 19 / 89 \\
04 / 06 / 90 \\
01 / 09 / 91\end{array}$ & $\begin{array}{l}0001 \\
0001 \\
0001 \\
0001\end{array}$ & $M G / L$ & $<$ & $\begin{array}{l}0.01 \\
0.02 \\
0.02 \\
0.01\end{array}$ & $J$ & $\begin{array}{l}0.02 \\
0.02 \\
0.02 \\
0.01\end{array}$ & $\begin{array}{l}- \\
- \\
-\end{array}$ \\
\hline CYANIDE & $\begin{array}{l}02 / 02 / 89 \\
09 / 19 / 89 \\
04 / 06 / 90 \\
01 / 09 / 91\end{array}$ & $\begin{array}{l}0001 \\
0001 \\
0001 \\
0001\end{array}$ & $M G / L$ & $\begin{array}{l}< \\
< \\
<\end{array}$ & $\begin{array}{l}0.002 \\
0.01 \\
0.01 \\
0.01\end{array}$ & $J$ & $\begin{array}{l}0.01 \\
0.01 \\
0.01 \\
0.01\end{array}$ & $\begin{array}{l}. \\
. \\
.\end{array}$ \\
\hline FLLORIDE & $\begin{array}{l}02 / 02 / 89 \\
09 / 19 / 89 \\
04 / 06 / 90 \\
01 / 09 / 91 \\
12 / 04 / 92\end{array}$ & $\begin{array}{l}0001 \\
0001 \\
0001 \\
0001 \\
0001\end{array}$ & $M G / L$ & & $\begin{array}{l}0.9 \\
0.8 \\
0.8 \\
0.8 \\
0.8\end{array}$ & & $\begin{array}{l}0.1 \\
0.1 \\
0.1 \\
0.1 \\
0.1\end{array}$ & $\begin{array}{l}- \\
\dot{-} \\
- \\
-\end{array}$ \\
\hline GROSS ALPHA & $\begin{array}{l}02 / 02 / 89 \\
09 / 19 / 89 \\
04 / 06 / 90 \\
01 / 09 / 91 \\
12 / 04 / 92\end{array}$ & $\begin{array}{l}0001 \\
0001 \\
0001 \\
0001 \\
0001\end{array}$ & $\mathrm{PCI} / \mathrm{L}$ & & $\begin{array}{c}14.6 \\
9.4 \\
29 . \\
41.6 \\
0.0\end{array}$ & & $\begin{array}{l}1 . \\
1 . \\
1 . \\
1.0\end{array}$ & $\begin{array}{c}16.2 \\
9.7 \\
11 . \\
18.2 \\
10.9\end{array}$ \\
\hline GROSS BETA & $\begin{array}{l}02 / 02 / 89 \\
09 / 19 / 89 \\
04 / 06 / 90 \\
01 / 09 / 91 \\
12 / 04 / 92\end{array}$ & $\begin{array}{l}0001 \\
0001 \\
0001 \\
0001 \\
0001\end{array}$ & $\mathrm{PCl} / \mathrm{L}$ & & $\begin{array}{c}4.37 \\
11 . \\
9.7 \\
32.0 \\
9.5\end{array}$ & & $\begin{array}{r}0.5 \\
0.5 \\
0.5 \\
0.5 \\
23.1\end{array}$ & $\begin{array}{c}21.4 \\
5 . \\
6.5 \\
8.8 \\
14.2\end{array}$ \\
\hline IRON & $\begin{array}{l}02 / 02 / 89 \\
09 / 19 / 89 \\
04 / 06 / 90 \\
01 / 09 / 91 \\
12 / 04 / 92\end{array}$ & $\begin{array}{l}0001 \\
0001 \\
0001 \\
0001 \\
0001\end{array}$ & $M G / L$ & $\begin{array}{l}< \\
< \\
<\end{array}$ & $\begin{array}{l}0.02 \\
0.03 \\
0.04 \\
0.03 \\
0.03\end{array}$ & J & $\begin{array}{l}0.03 \\
0.03 \\
0.03 \\
0.03 \\
0.03\end{array}$ & $\begin{array}{l}- \\
- \\
- \\
-\end{array}$ \\
\hline LEAD & $02 / 02 / 89$ & 0001 & MG/L & $<$ & 0.001 & J & 0.01 & - \\
\hline
\end{tabular}

PARAMETER VALUE IHDICATOR (PVI): < - LESS THAN DETECTION LIMIT

SAMPLE ID COOES:

0001 - FILTERED SAMPLE (.45 MICRONS)

OTHER PARAMETER VALUE FLAGS:

$J$ - ESTIMATED VALUE 


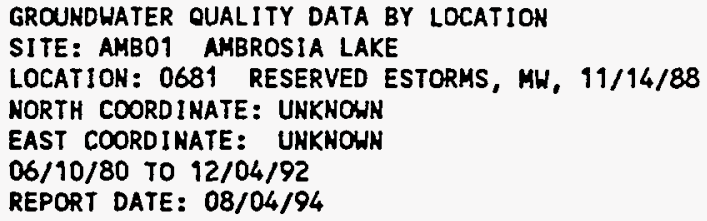

FORMATION OF COMPLETION: TRES HERMANOS - B SANDSTONE (TB) HYDRAULIC FLON RELATIOHSHIP: CROSS GRADIENT (C)

\begin{tabular}{|c|c|c|c|c|c|c|c|}
\hline PARAMETER NAME & LOG DATE & $\begin{array}{c}\text { SAMPLE } \\
10\end{array}$ & $\begin{array}{l}\text { UNITS OF } \\
\text { MEASURE }\end{array}$ & PVI & $\begin{array}{l}\text { PARAMETER } \\
\text { VALUE FLAGS }\end{array}$ & $\begin{array}{l}\text { DETECTION } \\
\text { LIHIT }\end{array}$ & $\begin{array}{l}\text { PARAMETER } \\
\text { UNCERTAINTY }\end{array}$ \\
\hline LEAD & $\begin{array}{l}09 / 19 / 89 \\
04 / 06 / 90 \\
01 / 09 / 91\end{array}$ & $\begin{array}{l}0001 \\
0001 \\
0001\end{array}$ & $M G / L$ & $\begin{array}{l}< \\
<\end{array}$ & $\begin{array}{l}0.03 \\
0.01 \\
0.03\end{array}$ & $\begin{array}{l}0.01 \\
0.01 \\
0.03\end{array}$ & - \\
\hline LEAD-210 & $\begin{array}{l}02 / 02 / 89 \\
09 / 19 / 89\end{array}$ & $\begin{array}{l}0001 \\
0001\end{array}$ & PCI/L & & $\begin{array}{l}0.00 \\
0.4\end{array}$ & $\begin{array}{l}1.5 \\
1.5\end{array}$ & $\begin{array}{l}8.54 \\
0.7\end{array}$ \\
\hline MAGKESIUM & $\begin{array}{l}02 / 02 / 89 \\
09 / 19 / 89 \\
04 / 06 / 90 \\
01 / 09 / 91 \\
12 / 04 / 92\end{array}$ & $\begin{array}{l}0001 \\
0001 \\
0001 \\
0001 \\
0001\end{array}$ & $M G / L$ & & $\begin{array}{l}7 . \\
2.66 \\
2.18 \\
2.0 \\
2.0\end{array}$ & $\begin{array}{l}0.001 \\
0.001 \\
0.001 \\
0.1 \\
0.1\end{array}$ & $\begin{array}{l}- \\
- \\
-\end{array}$ \\
\hline AANGANESE & $\begin{array}{l}02 / 02 / 89 \\
09 / 19 / 89 \\
04 / 06 / 90 \\
01 / 09 / 91\end{array}$ & $\begin{array}{l}0001 \\
0001 \\
0001 \\
0001\end{array}$ & MG/L & & $\begin{array}{l}0.03 \\
0.02 \\
0.02 \\
0.02\end{array}$ & $\begin{array}{l}0.01 \\
0.01 \\
0.01 \\
0.01\end{array}$ & - \\
\hline MERCURY & $\begin{array}{l}02 / 02 / 89 \\
09 / 19 / 89 \\
04 / 06 / 90 \\
01 / 09 / 91\end{array}$ & $\begin{array}{l}0001 \\
0001 \\
0001 \\
0001\end{array}$ & MG/L & $\begin{array}{l}< \\
< \\
< \\
<\end{array}$ & $\begin{array}{l}0.0001 \\
0.0002 \\
0.0002 \\
0.0002\end{array}$ & $\begin{array}{l}0.0002 \\
0.0002 \\
0.0002 \\
0.0002\end{array}$ & $\begin{array}{l}- \\
- \\
-\end{array}$ \\
\hline MOLYBDENUM & $\begin{array}{l}02 / 02 / 89 \\
09 / 19 / 89 \\
04 / 06 / 90 \\
01 / 09 / 91 \\
12 / 04 / 92\end{array}$ & $\begin{array}{l}0001 \\
0001 \\
0001 \\
0001 \\
0001\end{array}$ & $M G / L$ & $\begin{array}{l}< \\
<\end{array}$ & $\begin{array}{l}0.003 \\
0.01 \\
0.01 \\
0.01 \\
0.01\end{array}$ & $\begin{array}{l}0.01 \\
0.01 \\
0.01 \\
0.01 \\
0.01\end{array}$ & $\begin{array}{l}- \\
- \\
- \\
-\end{array}$ \\
\hline HET GROSS ALPHA * & $\begin{array}{l}02 / 02 / 89 \\
09 / 19 / 89 \\
04 / 06 / 90 \\
01 / 09 / 91 \\
12 / 04 / 92\end{array}$ & $\begin{array}{l}0001 \\
0001 \\
0001 \\
0001 \\
0001\end{array}$ & PCI/L & & $\begin{array}{r}7.74 \\
8.79 \\
27.63 \\
41.26 \\
-1.37\end{array}$ & $\begin{array}{l}- \\
- \\
- \\
-\end{array}$ & $\begin{array}{l}- \\
- \\
- \\
-\end{array}$ \\
\hline NICKEL & $\begin{array}{l}02 / 02 / 89 \\
09 / 19 / 89 \\
04 / 06 / 90 \\
01 / 09 / 91 \\
12 / 04 / 92\end{array}$ & $\begin{array}{l}0001 \\
0001 \\
0001 \\
0001 \\
0001\end{array}$ & MG/L & $\begin{array}{l}< \\
< \\
< \\
<\end{array}$ & $\begin{array}{l}0.02 \\
0.04 \\
0.04 \\
0.04 \\
0.04\end{array}$ & $\begin{array}{l}0.04 \\
0.04 \\
0.04 \\
0.04 \\
0.04\end{array}$ & $\begin{array}{l}- \\
- \\
- \\
-\end{array}$ \\
\hline HITRATE & $\begin{array}{l}02 / 02 / 89 \\
09 / 19 / 89 \\
04 / 06 / 90 \\
01 / 09 / 91 \\
12 / 04 / 92\end{array}$ & $\begin{array}{l}0001 \\
0001 \\
0001 \\
0001 \\
0001\end{array}$ & $M G / L$ & $<$ & $\begin{array}{l}1.14 \\
4.4 \\
5.6 \\
1.3 \\
1.0\end{array}$ & $\begin{array}{l}1 . \\
1 . \\
1 . \\
0.1 \\
1.0\end{array}$ & $\begin{array}{l}- \\
- \\
-\end{array}$ \\
\hline NITRITE AND NITRATE & $\begin{array}{l}04 / 06 / 90 \\
01 / 09 / 91\end{array}$ & $\begin{array}{l}0001 \\
0001\end{array}$ & MG/L & & $\begin{array}{l}1.3 \\
0.24\end{array}$ & 0.05 & - \\
\hline PH & $\begin{array}{l}02 / 02 / 89 \\
09 / 19 / 89 \\
04 / 06 / 90 \\
01 / 09 / 91\end{array}$ & $\begin{array}{l}0001 \\
0001 \\
0001 \\
0001\end{array}$ & SU & & $\begin{array}{l}8.44 \\
8.50 \\
8.45 \\
8.81\end{array}$ & $\begin{array}{l}- \\
- \\
-\end{array}$ & $\begin{array}{l}- \\
- \\
-\end{array}$ \\
\hline
\end{tabular}

- NET GROSS ALPHA (GROSS ALPHA - URANIUM) WITH 1 HG URANIUM = 686 PCI

PARAMETER VALUE INDICATOR (PVI): < - LESS THAN DETECTION LIMIT

OTHER PARAMETER VALUE FLAGS:

1 - INCREASED DETECTION LIMIT DUE TO REOUIRED DILUTION

$J$ - ESTIMATED VALUE
SAMPLE ID COOES:

OOO1 - FILTERED SAMPLE (.45 MICRONS) 
GROUNOWATER OUALITY DATA BY LOCATION

SITE: AMBOI AMBROSIA LAKE

LOCATIOH: 0681 RESERVED ESTORMS, MW, 11/14/88

KORTH COORDINATE: UNKNONH

EAST COORDINATE: UHKKOWN

06/10/80 TO 12/04/92

REPORT DATE: 08/04/94

FORHATION OF COMPLETION: TRES HERMANOS - B SAMDSTONE (TB)

HYDRAULIC FLOW RELATIONSHIP: CROSS GRADIENT (C)

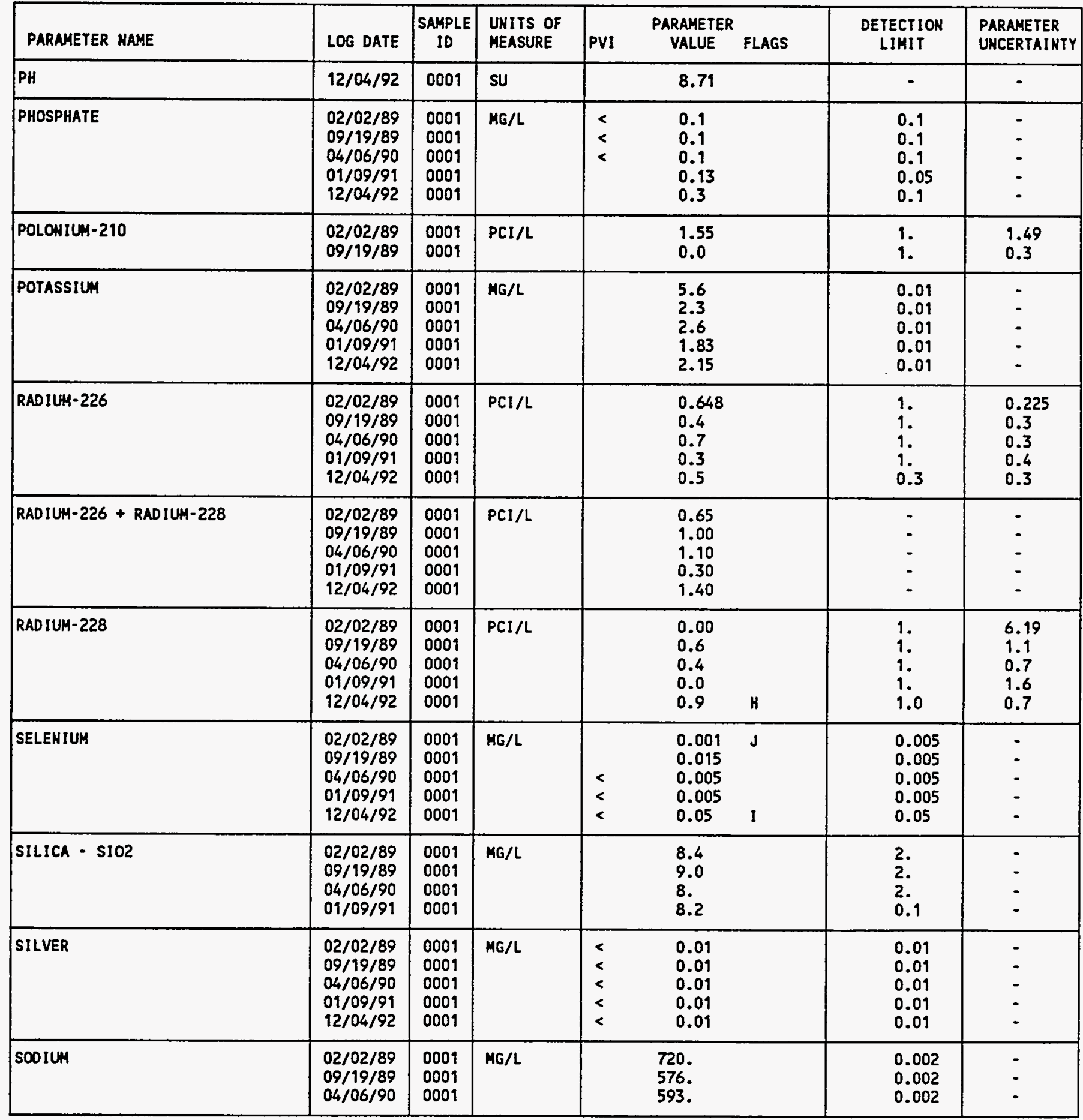

PARAMETER VALUE IndICATOR (PVI): < - LESS THAN DETECTIOH LIMIT

SAMPLE ID COOES:

0001 - FILTERED SAMPLE (.45 MICRONS)

OTHER PARAMETER VALUE FLAGS:

H - HOLD TIME EXPIRED, VALUE SUSPECT

1 - IHCREASED DETECTION LIMIT DUE TO REQUIRED DILUTION

J - estimated VALUE 
GROUNDWATER OUALITY DATA BY LOCATION

SITE: AMBO1 AMBROSIA LAKE

LOCATION: 0681 RESERVED ESTORMS, MW, 11/14/88

NORTH COORDINATE: UNKHOWN

EAST COORDINATE: UNKNOWH

$06 / 10 / 80$ TO $12 / 04 / 92$

REPORT DATE: 08/04/94

FORMATION OF COMPLETION: TRES HERMANOS - B SANDSTONE (TB)

HYDRAULIC FLOW RELATIONSHIP: CROSS GRADIENT (C)

\begin{tabular}{|c|c|c|c|c|c|c|c|}
\hline PARAMETER NAME & LOG DATE & $\underset{\text { ID }}{\text { SAMPLE }}$ & $\begin{array}{l}\text { UNITS OF } \\
\text { MEASURE }\end{array}$ & PVI & $\begin{array}{l}\text { PARAMETER } \\
\text { VALUE FLAGS }\end{array}$ & $\begin{array}{l}\text { DETECTION } \\
\text { LIMIT }\end{array}$ & $\begin{array}{l}\text { PARAMETER } \\
\text { UKCERTAINTY }\end{array}$ \\
\hline soosur & $\begin{array}{l}01 / 09 / 91 \\
12 / 04 / 92\end{array}$ & $\begin{array}{l}0001 \\
0001\end{array}$ & MG/L & & $\begin{array}{l}601 . \\
589 .\end{array}$ & $\begin{array}{l}5 . \\
5 .\end{array}$ & $\dot{-}$ \\
\hline SPECIFIC CONDUCTANCE & $\begin{array}{l}02 / 02 / 89 \\
09 / 19 / 89 \\
04 / 06 / 90 \\
01 / 09 / 91 \\
12 / 04 / 92\end{array}$ & $\begin{array}{l}0001 \\
0001 \\
0001 \\
0001 \\
0001\end{array}$ & Uнно/сн & & $\begin{array}{c}1900 . \\
800 . \\
1790 . \\
1880 \\
2100\end{array}$ & $\begin{array}{l}- \\
- \\
-\end{array}$ & $\begin{array}{l}- \\
- \\
-\end{array}$ \\
\hline STROATIUM & $\begin{array}{l}02 / 02 / 89 \\
09 / 19 / 89 \\
04 / 06 / 90 \\
01 / 09 / 91\end{array}$ & $\begin{array}{l}0001 \\
0001 \\
0001 \\
0001\end{array}$ & $M G / L$ & & $\begin{array}{l}1.00 \\
0.4 \\
0.39 \\
0.41\end{array}$ & $\begin{array}{l}0.1 \\
0.1 \\
0.1 \\
0.01\end{array}$ & $\begin{array}{l}- \\
- \\
-\end{array}$ \\
\hline SULFATE & $\begin{array}{l}02 / 02 / 89 \\
09 / 19 / 89 \\
04 / 06 / 90 \\
01 / 09 / 91 \\
12 / 04 / 92\end{array}$ & $\begin{array}{l}0001 \\
0001 \\
0001 \\
0001 \\
0001\end{array}$ & $M G / L$ & & $\begin{array}{c}1163 . \\
967 . \\
1040 \\
1030 \\
911 .\end{array}$ & $\begin{array}{c}0.1 \\
0.1 \\
0.1 \\
10 . \\
10 .\end{array}$ & $\begin{array}{l}- \\
- \\
- \\
-\end{array}$ \\
\hline SULF IDE & $\begin{array}{l}02 / 02 / 89 \\
09 / 19 / 89 \\
04 / 06 / 90 \\
01 / 09 / 91\end{array}$ & $\begin{array}{l}0001 \\
0001 \\
0001 \\
0001\end{array}$ & MG/L & $\begin{array}{l}< \\
< \\
< \\
<\end{array}$ & $\begin{array}{l}0.01 \\
0.1 \\
0.1 \\
0.1\end{array}$ & $\begin{array}{l}0.1 \\
0.1 \\
0.1 \\
0.1\end{array}$ & - \\
\hline TEMPERATURE & $\begin{array}{l}02 / 02 / 89 \\
09 / 19 / 89 \\
04 / 06 / 90 \\
01 / 09 / 91 \\
12 / 04 / 92\end{array}$ & $\begin{array}{l}0001 \\
0001 \\
0001 \\
0001 \\
0001\end{array}$ & C - DEGREE & & $\begin{array}{l}13.0 \\
14.0 \\
14 . \\
13.8 \\
13.5\end{array}$ & : & : \\
\hline THALLIUH & $\begin{array}{l}02 / 02 / 89 \\
09 / 19 / 89 \\
04 / 06 / 90 \\
01 / 09 / 91\end{array}$ & $\begin{array}{l}0001 \\
0001 \\
0001 \\
0001\end{array}$ & $M G / L$ & $\begin{array}{l}< \\
< \\
<\end{array}$ & $\begin{array}{l}0.005 \\
0.01 \\
0.01 \\
0.03\end{array}$ & $\begin{array}{l}0.005 \\
0.01 \\
0.01 \\
0.03\end{array}$ & : \\
\hline THORIUM-230 & $\begin{array}{l}02 / 02 / 89 \\
09 / 19 / 89\end{array}$ & $\begin{array}{l}0001 \\
0001\end{array}$ & $\mathrm{PCI} / \mathrm{L}$ & \multicolumn{2}{|r|}{$\begin{array}{l}0.00854 \\
0.0\end{array}$} & 1. & $\begin{array}{l}0.245 \\
0.3\end{array}$ \\
\hline TIN & $\begin{array}{l}02 / 02 / 89 \\
09 / 19 / 89 \\
04 / 06 / 90 \\
01 / 09 / 91\end{array}$ & $\begin{array}{l}0001 \\
0001 \\
0001 \\
0001\end{array}$ & $M G / L$ & $\begin{array}{l}< \\
<\end{array}$ & $\begin{array}{l}0.001 \\
0.104 \\
0.056 \\
0.03\end{array}$ & $\begin{array}{l}0.005 \\
0.005 \\
0.005 \\
0.03\end{array}$ & : \\
\hline TOTAL DISSOLVED SOLIDS & $\begin{array}{l}02 / 02 / 89 \\
09 / 19 / 89 \\
04 / 06 / 90 \\
01 / 09 / 91 \\
12 / 04 / 92\end{array}$ & $\begin{array}{l}0001 \\
0001 \\
0001 \\
0001 \\
0001\end{array}$ & HG/L & \multicolumn{2}{|r|}{$\begin{array}{l}2064 . \\
1620 . \\
1710 . \\
1790 . \\
1770 .\end{array}$} & $\begin{array}{l}10 . \\
10 . \\
10 . \\
10 . \\
10 .\end{array}$ & : \\
\hline TOTAL XJELDAHL MITROGEN & $\begin{array}{l}01 / 09 / 91 \\
12 / 04 / 92\end{array}$ & $\begin{array}{l}0001 \\
0001\end{array}$ & $M G / L$ & \multicolumn{2}{|r|}{$\begin{array}{l}1 . \\
1 .\end{array}$} & 1. & $\dot{-}$ \\
\hline TOTAL ORGAKIC CARBON & $\begin{array}{l}02 / 02 / 89 \\
09 / 99 / 89 \\
04 / 06 / 90\end{array}$ & $\begin{array}{l}0001 \\
0001 \\
0001\end{array}$ & MG/L & \multicolumn{2}{|r|}{$\begin{array}{l}44 . \\
67 . \\
45 .\end{array}$} & $\begin{array}{l}1 . \\
1 .\end{array}$ & $\dot{-}$ \\
\hline
\end{tabular}

PARAMETER VALUE INDICATOR (PVI): < - LESS THAN DETECTION LIMIT

SAMPLE ID COOES:

0001 - FILTERED SAMPLE (.45 MICRONS)

OTHER PARAMETER VALUE FLAGS:

1 - IMCREASED DETECTION LIMIT DUE TO REQUIRED DILUTION

J - estimated VALUE 
GROUMDHATER OUALITY DATA BY LOCATION

SITE: AMBO1 AMBROSIA LAKE

LOCATION: 0681 RESERVED ESTORMS, MH, 11/14/88

NORTH COORDINATE: UNKNOWH

EAST COORDINATE: UNKNOWN

06/10/80 TO 12/04/92

REPORT DATE: $08 / 04 / 94$

FORMATION OF COMPLETION: TRES HERHANOS - B SAMDSTONE (TB) HYDRAULIC FLON RELATIONSHIP: CROSS GRADIENT (C)

\begin{tabular}{|c|c|c|c|c|c|c|c|}
\hline PARAMETER MAME & LOG DATE & $\begin{array}{c}\text { SAMPLE } \\
\text { ID }\end{array}$ & $\begin{array}{l}\text { UHITS OF } \\
\text { MEASURE }\end{array}$ & PVI & $\begin{array}{l}\text { PARAMETER } \\
\text { VALUE FLAGS }\end{array}$ & $\begin{array}{l}\text { DETECTION } \\
\text { LIRIT }\end{array}$ & $\begin{array}{l}\text { PARAMETER } \\
\text { UHCERTAINTY }\end{array}$ \\
\hline TOTAL ORGANIC CARBOH & $\begin{array}{l}01 / 09 / 91 \\
12 / 04 / 92\end{array}$ & $\begin{array}{l}0001 \\
0001\end{array}$ & MG/L & & $\begin{array}{l}5 . \\
2 .\end{array}$ & i. & - \\
\hline URANIUM & $\begin{array}{l}02 / 02 / 89 \\
09 / 19 / 89 \\
04 / 06 / 90 \\
01 / 09 / 91 \\
12 / 04 / 92\end{array}$ & $\begin{array}{l}0001 \\
0001 \\
0001 \\
0001 \\
0001\end{array}$ & $M G / L$ & $<$ & $\begin{array}{ll}0.010 & \\
0.0010 & J \\
0.0020 & J \\
0.001 & \\
0.002 & \end{array}$ & $\begin{array}{l}0.003 \\
0.003 \\
0.003 \\
0.001 \\
0.001\end{array}$ & : \\
\hline VANADIUH & $\begin{array}{l}02 / 02 / 89 \\
09 / 19 / 89 \\
04 / 06 / 90 \\
01 / 09 / 91 \\
12 / 04 / 92\end{array}$ & $\begin{array}{l}0001 \\
0001 \\
0001 \\
0001 \\
0001\end{array}$ & MG/L & $\begin{array}{l}< \\
< \\
< \\
<\end{array}$ & $\begin{array}{l}0.01 \\
0.1 \\
0.01 \\
0.01 \\
0.01\end{array}$ & $\begin{array}{l}0.01 \\
0.1 \\
0.01 \\
0.01 \\
0.09\end{array}$ & : \\
\hline
\end{tabular}

PARAMETER VALUE IHOICATOR (PVI): < - LESS THAN DETECTIOH LIHIT

SAMPLE ID COOES:

O001 - FILTERED SAMPLE (.45 MICRONS)

OTHER PARAMETER VALUE FLAGS:

H - HOLD TIME EXPIRED, VALUE SUSPECT

$J$ - estimated VALUE 
GROUNDHATER OUALITY DATA BY LOCATION

SITE: AHBOI AMBROSIA LAKE

LOCATION: 0706

NORTH COORDINATE: 53612.0 FT

EAST COORDINATE: 59942.0 FT

$06 / 10 / 80$ TO $12 / 04 / 92$

REPORT DATE: $08 / 04 / 94$

FORMATION OF CONPLETION: ALLUVIUH (AL)

HYDRAULIC FLON RELATIOHSHIP: ON-SITE (O)

\begin{tabular}{|c|c|c|c|c|c|c|}
\hline PARAMETER NAME & LOG DATE & $\begin{array}{c}\text { SAMPLE } \\
\text { ID }\end{array}$ & $\begin{array}{l}\text { UNITS OF } \\
\text { HEASURE }\end{array}$ & \begin{tabular}{l}
\multicolumn{3}{c}{ PARAMETER } \\
PVI $\quad$ VALUE FLAGS
\end{tabular} & $\begin{array}{l}\text { DETECTIOH } \\
\text { LIMIT }\end{array}$ & $\begin{array}{l}\text { PARAMETER } \\
\text { UNCERTAINTY }\end{array}$ \\
\hline ALKALINITY & $\begin{array}{l}06 / 21 / 82 \\
09 / 30 / 85 \\
05 / 13 / 86 \\
01 / 09 / 87\end{array}$ & $\begin{array}{l}0001 \\
0001 \\
0001 \\
0001\end{array}$ & $M G / L C A C O 3$ & $\begin{array}{l}2300.00 \\
4765 \\
764 \\
5145\end{array}$ & $\begin{array}{l}- \\
- \\
-\end{array}$ & $\begin{array}{l}- \\
- \\
-\end{array}$ \\
\hline ALUMINUM & $06 / 21 / 82$ & 0001 & $M G / L$ & 0.10 & 0.1 & - \\
\hline ARSENIC & $\begin{array}{l}06 / 21 / 82 \\
09 / 30 / 85 \\
05 / 13 / 86 \\
01 / 09 / 87\end{array}$ & $\begin{array}{l}0001 \\
0001 \\
0001 \\
0001\end{array}$ & MG/L & $\begin{array}{l}0.33 \\
0.01 \\
0.01 \\
0.272\end{array}$ & $\begin{array}{l}- \\
0.01 \\
0.01 \\
0.01\end{array}$ & $\begin{array}{l}- \\
- \\
-\end{array}$ \\
\hline BARIUM & $06 / 21 / 82$ & 0001 & $M G / L$ & 0.10 & 0.1 & - \\
\hline BICARBONATE - $\mathrm{HCO} 3$ & $06 / 21 / 82$ & 0001 & $M G / L$ & 5613.00 & - & - \\
\hline BORON & $\begin{array}{l}05 / 13 / 86 \\
01 / 09 / 87\end{array}$ & $\begin{array}{l}0001 \\
0001\end{array}$ & $M G / L$ & $\begin{array}{l}0.7 \\
0.15\end{array}$ & $\begin{array}{l}0.1 \\
0.1\end{array}$ & $\dot{-}$ \\
\hline CADMIUH & $\begin{array}{l}06 / 21 / 82 \\
05 / 13 / 86\end{array}$ & $\begin{array}{l}0001 \\
0001\end{array}$ & $M G / L$ & $\begin{array}{l}0.10 \\
0.001\end{array}$ & $\begin{array}{l}0.1 \\
0.001\end{array}$ & $\dot{-}$ \\
\hline CALCIUM & $\begin{array}{l}06 / 21 / 82 \\
09 / 30 / 85 \\
05 / 13 / 86 \\
01 / 09 / 87\end{array}$ & $\begin{array}{l}0001 \\
0001 \\
0001 \\
0001\end{array}$ & $M G / L$ & $\begin{array}{c}12.00 \\
10.7 \\
520 . \\
9.16\end{array}$ & $\begin{array}{l}- \\
0.01 \\
0.01 \\
0.01\end{array}$ & $\begin{array}{l}- \\
- \\
-\end{array}$ \\
\hline CHLORIDE & $\begin{array}{l}06 / 21 / 82 \\
09 / 30 / 85 \\
05 / 13 / 86 \\
01 / 09 / 87\end{array}$ & $\begin{array}{l}0001 \\
0001 \\
0001 \\
0001\end{array}$ & $M G / L$ & $\begin{array}{l}290.00 \\
300 . \\
460 . \\
489 .\end{array}$ & $\begin{array}{l}- \\
1 . \\
1 .\end{array}$ & $\begin{array}{l}- \\
- \\
-\end{array}$ \\
\hline CHROMIUM & $\begin{array}{l}06 / 21 / 82 \\
05 / 13 / 86 \\
01 / 09 / 87\end{array}$ & $\begin{array}{l}0001 \\
0001 \\
0001\end{array}$ & MG/L & $\begin{array}{l}0.03 \\
0.06 \\
0.02\end{array}$ & $\begin{array}{l}- \\
0.01 \\
0.01\end{array}$ & - \\
\hline COBALT & $\begin{array}{l}05 / 13 / 86 \\
01 / 09 / 87\end{array}$ & $\begin{array}{l}0001 \\
0001\end{array}$ & $M G / L$ & $\begin{array}{l}0.12 \\
0.10\end{array}$ & $\begin{array}{l}0.05 \\
0.05\end{array}$ & - \\
\hline COPPER & $\begin{array}{l}06 / 21 / 82 \\
01 / 09 / 87\end{array}$ & $\begin{array}{l}0001 \\
0001\end{array}$ & $M G / L$ & $\begin{array}{l}0.20 \\
0.07\end{array}$ & 0.02 & - \\
\hline FLUORIDE & $\begin{array}{l}06 / 21 / 82 \\
05 / 13 / 86 \\
01 / 09 / 87\end{array}$ & $\begin{array}{l}0001 \\
0001 \\
0001\end{array}$ & $M G / L$ & $\begin{array}{c}15.00 \\
0.5 \\
14.5\end{array}$ & $\begin{array}{l}- \\
0.1 \\
0.1\end{array}$ & $\begin{array}{l}- \\
-\end{array}$ \\
\hline GROSS ALPHA & $\begin{array}{l}06 / 21 / 82 \\
01 / 09 / 87\end{array}$ & $\begin{array}{l}0001 \\
0001\end{array}$ & $\mathrm{PCI} / \mathrm{L}$ & $\begin{array}{l}15000.00 \\
8000 .\end{array}$ & 0.2 & 400 \\
\hline GROSS BETA & $01 / 09 / 87$ & 0001 & $\mathrm{PCI} / \mathrm{L}$ & 4400 & 1. & 200. \\
\hline IRON & $\begin{array}{l}06 / 21 / 82 \\
05 / 13 / 86 \\
01 / 09 / 87\end{array}$ & $\begin{array}{l}0001 \\
0001 \\
0001\end{array}$ & $M G / L$ & $\begin{array}{l}1.00 \\
0.14 \\
0.75\end{array}$ & $\begin{array}{l}0.03 \\
0.03\end{array}$ & $\begin{array}{l}- \\
-\end{array}$ \\
\hline LEAD & $06 / 21 / 82$ & 0001 & $M G / L$ & 0.02 & - & - \\
\hline MAGHESIUA & $06 / 21 / 82$ & 0001 & $M G / L$ & 55.00 & - & - \\
\hline
\end{tabular}

PARAMETER VALUE INDICATOR (PVI): < - LESS THAN DETECTIOH LIMIT 
GROUNDWATER QUALITY DATA BY LOCATION

SITE: AMBOI AMBROSIA LAKE

LOCATION: 0706

MORTH COORDIHATE: $\quad 53612.0$ FT

EAST COORDINATE: $\quad 59942.0$ FT

06/10/80 TO 12/04/92

REPORT DATE: $08 / 04 / 94$

FORMATION OF COMPLETIOH: ALLUVIUH (AL)

HYDRAULIC FLOW RELATIOHSHIP: OH-SITE (O)

\begin{tabular}{|c|c|c|c|c|c|c|c|}
\hline PARAMETER HAME & LOG DATE & $\begin{array}{c}\text { SAMPLE } \\
10\end{array}$ & $\begin{array}{l}\text { UNITS OF } \\
\text { MEASURE }\end{array}$ & PVI & $\begin{array}{l}\text { PARAMETER } \\
\text { VALUE FLAGS }\end{array}$ & $\begin{array}{l}\text { DETECTION } \\
\text { LIMIT }\end{array}$ & $\begin{array}{l}\text { PARAMETER } \\
\text { UNCERTAINTY }\end{array}$ \\
\hline MAGNESIUM & $\begin{array}{l}09 / 30 / 85 \\
05 / 13 / 86 \\
01 / 09 / 87\end{array}$ & $\begin{array}{l}0001 \\
0001 \\
0001\end{array}$ & MG/L & & $\begin{array}{c}43.2 \\
273 . \\
42.1\end{array}$ & $\begin{array}{l}0.001 \\
0.001 \\
0.001\end{array}$ & : \\
\hline MANGANESE & $\begin{array}{l}05 / 13 / 86 \\
01 / 09 / 87\end{array}$ & $\begin{array}{l}0001 \\
0001\end{array}$ & $M G / L$ & & $\begin{array}{l}0.22 \\
0.09\end{array}$ & $\begin{array}{l}0.01 \\
0.01\end{array}$ & - \\
\hline MERCURY & $06 / 21 / 82$ & 0001 & MG/L & $<$ & 0.002 & 0.002 & - \\
\hline HOLYBDEKUM & $\begin{array}{l}06 / 21 / 82 \\
09 / 30 / 85 \\
05 / 13 / 86 \\
01 / 09 / 87\end{array}$ & $\begin{array}{l}0001 \\
0001 \\
0001 \\
0001\end{array}$ & $M G / L$ & & $\begin{array}{c}225.00 \\
223 . \\
9.37 \\
220 .\end{array}$ & $\begin{array}{l}0.01 \\
0.01 \\
0.01\end{array}$ & : \\
\hline NET GROSS ALPHA * & $01 / 09 / 87$ & 0001 & $\mathrm{PCI} / \mathrm{L}$ & & 2415.96 & - & - \\
\hline HITRATE & $\begin{array}{l}06 / 21 / 82 \\
05 / 13 / 86 \\
01 / 09 / 87\end{array}$ & $\begin{array}{l}0001 \\
0001 \\
0001\end{array}$ & MG/L & $<$ & $\begin{array}{l}1.00 \\
25 . \\
25 .\end{array}$ & $\begin{array}{l}1 . \\
1 .\end{array}$ & : \\
\hline PH & $\begin{array}{l}06 / 21 / 82 \\
09 / 30 / 85 \\
05 / 13 / 86 \\
01 / 09 / 87\end{array}$ & $\begin{array}{l}0001 \\
0001 \\
0001 \\
0001\end{array}$ & SU & & $\begin{array}{l}8.40 \\
9.12 \\
7.25 \\
9.21\end{array}$ & : & - \\
\hline POTASSIUM & $\begin{array}{l}06 / 21 / 82 \\
09 / 30 / 85 \\
05 / 13 / 86 \\
01 / 09 / 87\end{array}$ & $\begin{array}{l}0001 \\
0001 \\
0001 \\
0001\end{array}$ & MG/L & & $\begin{array}{l}9.00 \\
13.3 \\
15.8 \\
0.91\end{array}$ & $\begin{array}{l}- \\
0.01 \\
0.01 \\
0.01\end{array}$ & $\begin{array}{l}- \\
- \\
-\end{array}$ \\
\hline RADIUM-226 & $\begin{array}{l}06 / 21 / 82 \\
01 / 09 / 87\end{array}$ & $\begin{array}{l}0001 \\
0001\end{array}$ & PCI/L & & $\begin{array}{l}4.00 \\
130 .\end{array}$ & 1. & 10. \\
\hline RADIUH-226 + RADIUH-228 & $01 / 09 / 87$ & 0001 & $\mathrm{PCI} / \mathrm{L}$ & & 131.80 & - & - \\
\hline RADIUM-22B & $01 / 09 / 87$ & 0001 & $\mathrm{PCl} / \mathrm{L}$ & & 1.8 & 1. & 6.1 \\
\hline SELENIUM & $\begin{array}{l}06 / 21 / 82 \\
09 / 30 / 85 \\
05 / 13 / 86 \\
01 / 09 / 87\end{array}$ & $\begin{array}{l}0001 \\
0001 \\
0001 \\
0001\end{array}$ & MG/L & & $\begin{array}{l}0.037 \\
0.088 \\
0.01 \\
0.012\end{array}$ & $\begin{array}{l}- \\
0.005 \\
0.005 \\
0.005\end{array}$ & $\begin{array}{l}- \\
-\end{array}$ \\
\hline SILICA - SIO2 & $06 / 21 / 82$ & 0001 & $M G / L$ & & 6.50 & - & - \\
\hline SILVER & $\begin{array}{l}06 / 21 / 82 \\
01 / 09 / 87\end{array}$ & $\begin{array}{l}0001 \\
0001\end{array}$ & $M G / L$ & & $\begin{array}{l}0.15 \\
0.02\end{array}$ & 0.01 & - \\
\hline SOOIUN & $\begin{array}{l}06 / 21 / 82 \\
09 / 30 / 85 \\
05 / 13 / 86 \\
01 / 09 / 87\end{array}$ & $\begin{array}{l}0001 \\
0001 \\
0001 \\
0001\end{array}$ & MG/L & & $\begin{array}{l}7100.00 \\
7020 . \\
1140 . \\
7610 .\end{array}$ & $\begin{array}{l}- \\
0.002 \\
0.002 \\
0.002\end{array}$ & $\begin{array}{l}- \\
- \\
-\end{array}$ \\
\hline SPECIFIC CONDUCTANCE & $\begin{array}{l}06 / 21 / 82 \\
09 / 30 / 85 \\
05 / 13 / 86 \\
01 / 09 / 87\end{array}$ & $\begin{array}{l}0001 \\
0001 \\
0001 \\
0001\end{array}$ & UнК/СM & & $\begin{array}{c}22200.00 \\
17000 \\
4500 \\
14500\end{array}$ & - & : \\
\hline
\end{tabular}

- het GROSS ALPHA (GROSS ALPHA - URANIUM) WITH 1 NG URANIUM = 686 PCI

PARAMETER VALUE INDICATOR (PVI): < - LESS THAN DETECTION LIMIT 
GROUNDWATER QUALITY DATA BY LOCATION

SITE: AMBO1 AMBROSIA LAKE

LOCATION: 0706

NORTH COORDINATE: $\quad 53612.0$ FT

EAST COORDINATE: 59942.0 FT

D6/10/80 TO $12 / 04 / 92$

REPORT DATE: 08/04/94

FORMATION OF COMPLETION: ALLUVIUM (AL)

HYORAULIC FLOW RELATIONSHIP: OW-SITE (O)

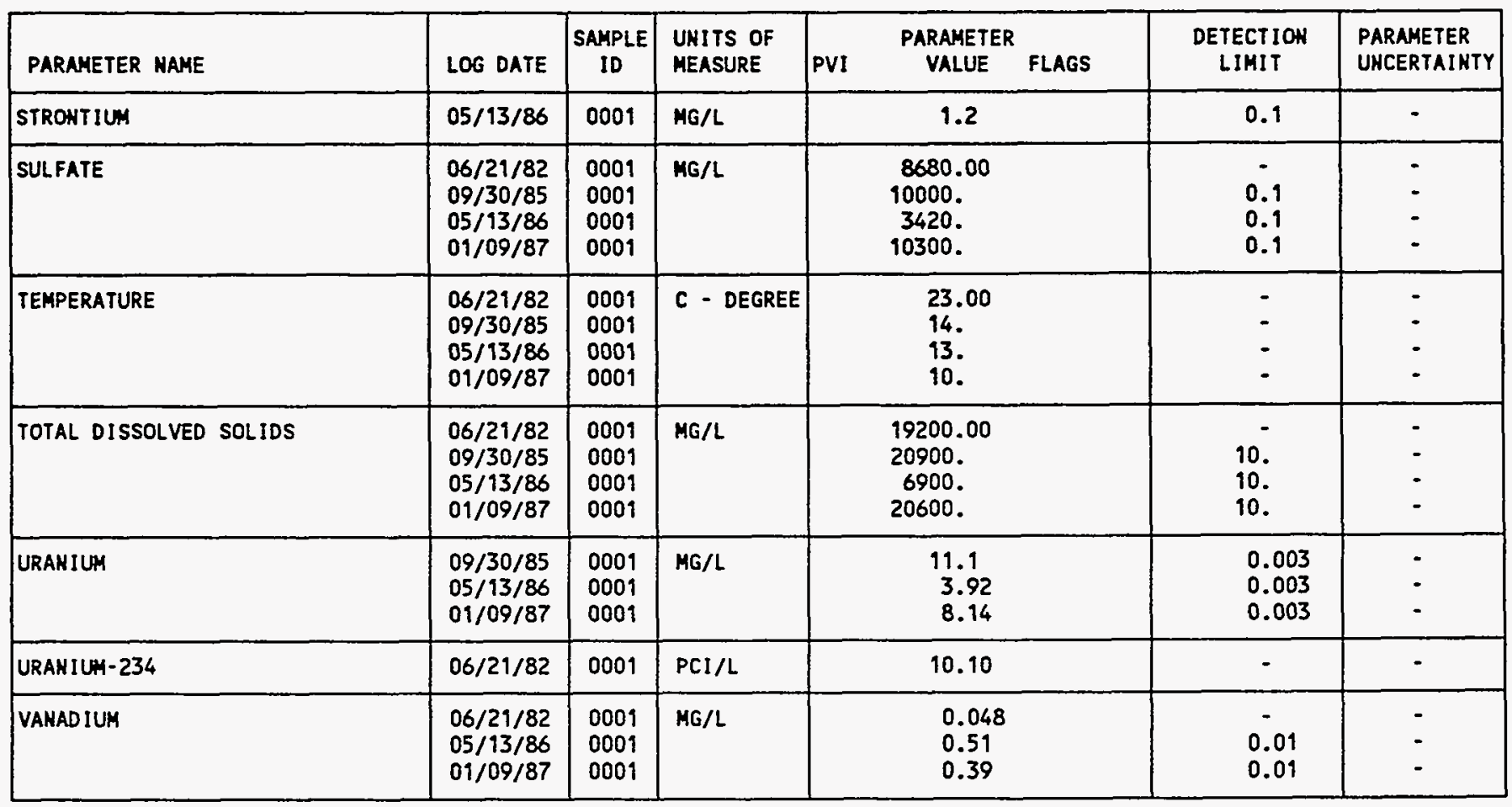

PARAMETER VALUE INDICATOR (PVI):

< - LESS THAN DETECTION LIMIT

SAMPLE ID CODES

0001 - FILTERED SAMPLE (.45 MICRONS) 
GROUNDHATER OUALITY DATA BY LOCATION

SITE: AMBO1 AMBROSIA LAKE

LOCATION: 0707

NORTH COOROINATE: 53788.0 FT

EAST COORDIHATE: 60309.0 FT

$06 / 10 / 80$ TO $12 / 04 / 92$

REPORT DATE: $08 / 04 / 94$

FORMATION OF COMPLETION: URANIUM MILL TAILIHGS (TA)

HYORAULIC FLOU RELATIONSHIP: OH-SITE (O)

\begin{tabular}{|c|c|c|c|c|c|c|c|}
\hline PARAMETER KAME & LOG DATE & $\begin{array}{c}\text { SAMPLE } \\
\text { ID }\end{array}$ & $\begin{array}{l}\text { UNITS OF } \\
\text { MEASURE }\end{array}$ & PV & $\begin{array}{l}\text { PARAMETER } \\
\text { VALUE FLAGS }\end{array}$ & $\begin{array}{l}\text { DETECTION } \\
\text { LIHIT }\end{array}$ & $\begin{array}{l}\text { PARAMETER } \\
\text { UNCERTAINTY }\end{array}$ \\
\hline ALKALINITY & $06 / 21 / 82$ & 0001 & MG/L CACO3 & & 770.00 & - & - \\
\hline ALUMINUM & $0.6 / 21 / 82$ & 0001 & MG/L & & 0.12 & - & - \\
\hline ARSENIC & $06 / 21 / 82$ & 0001 & MG/L & & 0.01 & - & - \\
\hline BARIUM & $06 / 21 / 82$ & 0001 & MG/L & $<$ & 0.10 & 0.1 & - \\
\hline BICARBOHATE - HCO3 & $06 / 21 / 82$ & 0001 & MG/L & & 1879.00 & - & $\cdot$ \\
\hline CADHIUM & $06 / 21 / 82$ & 0001 & MG/L & $<$ & 0.01 & 0.01 & $\cdot$ \\
\hline CALCIUH & $06 / 21 / 82$ & 0001 & $M G / L$ & & 440.00 & - & - \\
\hline CHLORIDE & $06 / 21 / 82$ & 0001 & $M G / L$ & & 213.00 & - & - \\
\hline CHROMIUM & $06 / 21 / 82$ & 0001 & MG/L & & 0.01 & - & - \\
\hline COPPER & $06 / 21 / 82$ & 0001 & MG/L & & 0.063 & - & $\cdot$ \\
\hline FLUORIDE & $06 / 21 / 82$ & 0001 & $\mathrm{MG} / \mathrm{L}$ & & 5.00 & - & - \\
\hline IRON & $06 / 21 / 82$ & 0001 & $M G / L$ & & 1.46 & - & - \\
\hline LEAD & $06 / 21 / 82$ & 0001 & $M G / L$ & & 0.01 & - & - \\
\hline MAGKESIUH & $06 / 21 / 82$ & 0001 & $M G / L$ & & 600.00 & - & - \\
\hline MERCURY & $06 / 21 / 82$ & 0001 & MG/L & $<$ & 0.002 & 0.002 & - \\
\hline MOLYBDENUM & $06 / 21 / 82$ & 0001 & $M G / L$ & & 67.00 & - & - \\
\hline MITRATE & $06 / 21 / 82$ & 0001 & $M G / L$ & $<$ & 1.00 & 1. & - \\
\hline PH & $06 / 21 / 82$ & 0001 & SU & & 6.80 & - & - \\
\hline POTASSIUM & $06 / 21 / 82$ & 0001 & $M G / L$ & & 12.00 & - & - \\
\hline RADIUM-226 & $06 / 21 / 82$ & 0001 & $\mathrm{PCI} / \mathrm{L}$ & & 27.00 & - & - \\
\hline SELENIUH & $06 / 21 / 82$ & 0001 & $M G / L$ & & 0.018 & - & - \\
\hline SILICA - S102 & $06 / 21 / 82$ & 0001 & $M G / L$ & & 9.50 & - & - \\
\hline SILVER & $06 / 21 / 82$ & 0001 & $M G / L$ & & 0.05 & - & - \\
\hline SODIun & $06 / 21 / 82$ & 0001 & $M G / L$ & & 4000.00 & - & - \\
\hline SPECIFIC CONDUCTANCE & $06 / 21 / 82$ & 0001 & $\mathrm{LMHO} / \mathrm{CH}$ & & 13200.00 & $\cdot$ & - \\
\hline SULFATE & $06 / 21 / 82$ & 0001 & MG/L & & 9110.00 & - & - \\
\hline TEMPERATURE & $06 / 21 / 82$ & 0001 & C - DEGREE & & 15.00 & - & - \\
\hline TOTAL DISSOLVED SOLIDS & $06 / 21 / 82$ & 0001 & $M G / L$ & & 15400.00 & - & - \\
\hline URAHIUN-234 & $06 / 21 / 82$ & 0001 & $\mathrm{PCI} / \mathrm{L}$ & & 21.50 & $\cdot$ & $\cdot$ \\
\hline VANADIUM & $06 / 21 / 82$ & 0001 & $M G / L$ & $<$ & 0.09 & 0.01 & - \\
\hline
\end{tabular}

PARAMETER VALUE INDICATOR (PVI): < - LESS THAN DETECTION LIMIT

SAMPLE ID COOES:

0001 - FILTERED SAMPLE (.45 MICRONS) 
GROUNDWATER QUALITY DATA BY LOCATION

SITE: AMBO1 AMBROSIA LAKE

LOCATION: 0707

NORTH COORDINATE: 53788.0 FT

EAST COORDINATE: $\quad 60309.0$ FT

06/10/80 TO $12 / 04 / 92$

REPORT DATE : 08/04/94

FORMATION OF COMPLETION: URAHIUM MILL TAILINGS (TA)

HYDRAULIC FLON RELATIOHSHIP: ON-SITE (O)

\begin{tabular}{|l|l|l|l|l|l|l|l|}
\hline PARAMETER NAME & LOG DATE & $\begin{array}{l}\text { SAMPLE } \\
\text { ID }\end{array}$ & $\begin{array}{l}\text { UNITS OF } \\
\text { MEASURE }\end{array}$ & PVI & $\begin{array}{l}\text { PARAMETER } \\
\text { VALUE }\end{array}$ & $\begin{array}{c}\text { DETECTIOA } \\
\text { LIMIT }\end{array}$ & $\begin{array}{l}\text { PARAMETER } \\
\text { UNCERTAIMTY }\end{array}$ \\
\hline
\end{tabular}

PARAMETER VALUE IMDICATOR (PVI): < - LESS THAN DETECTION LIMIT SAMPLE ID COOES: 
GROUNDHATER QUALITY DATA BY LOCATION

SITE: AMBO1 AMBROSIA LAKE

LOCATION: 0708

HORTH COORDINATE: 53295.0 FT

EAST COORDINATE: $\quad 59872.0$ FT

06/10/80 TO $12 / 04 / 92$

REPORT DATE: $08 / 04 / 94$

FORMATION OF COHPLETION: TRES HERMANOS - C1 SAMDSTOHE (TO) HYDRAULIC FLON RELATIONSHIP: DOWH GRADIENT (D)

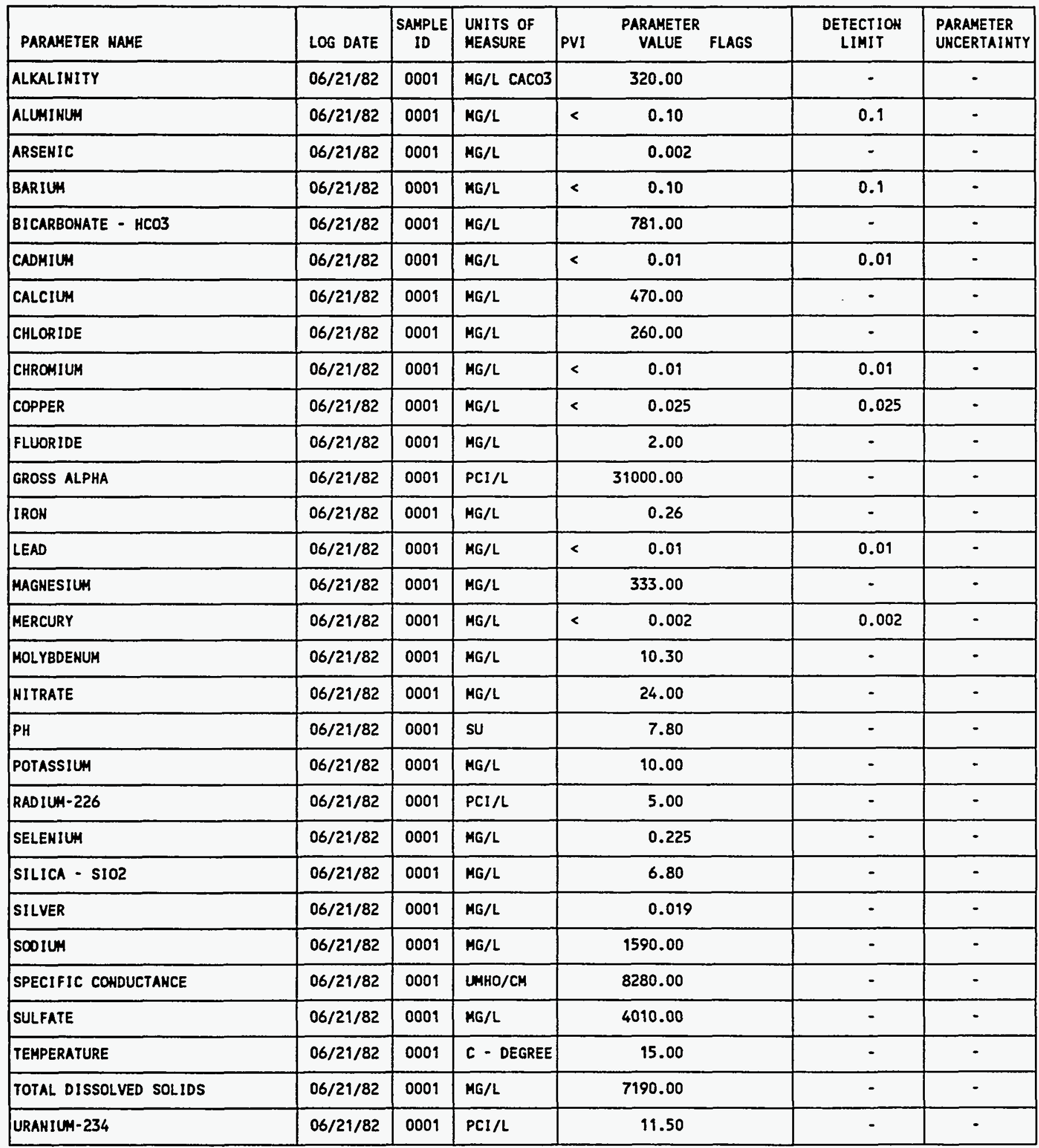

PARAMETER VALUE IMDICATOR (PVI): < - LESS THAM DETECTION LIMIT

SAMPLE ID COOES:

0001 - FILTERED SAMPLE (.45 MICRONS) 
GROUNDWATER QUALITY DATA BY LOCATION

SITE: AMBO1 AMBROSIA LAKE

LOCATIOH: 0708

NORTH COORDINATE: $\quad 53295.0$ FT

EAST COORDIHATE: $\quad 59872.0 \mathrm{FT}$

O6/10/80 TO $12 / 04 / 92$

REPORT DATE: $08 / 04 / 94$

FORMATION OF COMPLETION: TRES HERMAHOS - C1 SAMDSTONE (TO) HYDRAULIC FLOW RELATIOWSHIP: DOWN GRADIENT (D)

\begin{tabular}{|c|c|c|c|c|c|c|c|}
\hline PARAMETER NAME & LOG DATE & $\begin{array}{c}\text { SAMPLE } \\
\text { ID }\end{array}$ & $\begin{array}{l}\text { UNITS OF } \\
\text { MEASURE }\end{array}$ & PVI & $\begin{array}{l}\text { PARAMETER } \\
\text { VALUE FLAGS }\end{array}$ & $\begin{array}{l}\text { DETECTION } \\
\text { LIMIT }\end{array}$ & $\begin{array}{l}\text { PARAMETER } \\
\text { UNCERTAINTY }\end{array}$ \\
\hline VAKADIUA & $06 / 21 / 82$ & 0001 & $M G / L$ & $<$ & 0.01 & 0.01 & - \\
\hline
\end{tabular}

PARAMETER VALUE INDICATOR (PVI): < - LESS THAN DETECTION LIMIT

SAMPLE IO COOES:

0001 - FILTERED SAMPLE (.45 MICRONS) 
GROUNDWATER QUALITY DATA BY LOCATION

SITE: AMBOI AMBROSIA LAKE

LOCATION: 0709

NORTH COORDIHATE: $\quad 55212.0$ FT

EAST COORDIHATE: $\quad 60324.0$ FT

$06 / 10 / 80$ TO $12 / 04 / 92$

REPORT DATE: 08/04/94

FORMATION OF COHPLETIOH: ALLUVIUM (AL)

HYDRAULIC FLOU RELATIONSHIP: UPGRADIENT (U)

\begin{tabular}{|c|c|c|c|c|c|c|}
\hline PARAMETER NAME & LOG DATE & SAMPLE & $\begin{array}{l}\text { UNITS OF } \\
\text { MEASURE }\end{array}$ & $\begin{array}{l}\text { PARAMETER } \\
\text { PALIE }\end{array}$ FLAGS & $\begin{array}{l}\text { DETECTIOH } \\
\text { LIMIT }\end{array}$ & $\begin{array}{l}\text { PARAMETER } \\
\text { UNCERTAINTY }\end{array}$ \\
\hline ALKALINITY & $06 / 21 / 82$ & 0001 & $\mathrm{MG} / \mathrm{L}$ CACO3 & 150.00 & - & - \\
\hline ALUMINUM & $06 / 21 / 82$ & 0001 & $M G / L$ & 0.15 & - & - \\
\hline ARSENIC & $06 / 21 / 82$ & 0001 & MG/L & 0.002 & - & - \\
\hline BARIUA & $06 / 21 / 82$ & 0001 & $M G / L$ & 0.10 & 0.1 & - \\
\hline BICARBONATE - HCO3 & $06 / 21 / 82$ & 0001 & $M G / L$ & 366.00 & - & - \\
\hline CADHILA & $06 / 21 / 82$ & 0001 & $M G / L$ & 0.01 & 0.01 & - \\
\hline CALCIUA & $06 / 21 / 82$ & 0001 & $M G / L$ & 493.00 & - & - \\
\hline CHLORIDE & $06 / 21 / 82$ & 0001 & $M G / L$ & 172.00 & - & - \\
\hline CHROMIUM & $06 / 21 / 82$ & 0001 & $M G / L$ & 0.01 & 0.01 & - \\
\hline COPPER & $06 / 21 / 82$ & 0001 & $M G / L$ & 0.025 & - & - \\
\hline FLUORIDE & $06 / 21 / 82$ & 0001 & MG/L & 2.00 & - & - \\
\hline GROSS ALPHA & $06 / 21 / 82$ & 0001 & $\mathrm{PCI} / \mathrm{L}$ & 158.00 & - & - \\
\hline IRON & $06 / 21 / 82$ & 0001 & $M G / L$ & 0.19 & - & - \\
\hline LEAD & $06 / 21 / 82$ & 0001 & MG/L & 0.01 & 0.01 & - \\
\hline MAGHESIUM & $06 / 21 / 82$ & 0001 & $M G / L$ & 199.00 & - & - \\
\hline MERCURY & $06 / 21 / 82$ & 0001 & $M G / L$ & 0.002 & 0.002 & - \\
\hline MOLYBDENUM & $06 / 21 / 82$ & 0001 & MG/L & 0.025 & 0.025 & - \\
\hline MITRATE & $06 / 21 / 82$ & 0001 & $M G / L$ & 1.00 & - & - \\
\hline PH & $06 / 21 / 82$ & 0001 & SU & 6.88 & - & - \\
\hline POTASSIUM & $06 / 21 / 82$ & 0001 & $M G / L$ & 4.00 & - & - \\
\hline RADIUA-226 & $06 / 21 / 82$ & 0001 & $\mathrm{PCI} / \mathrm{L}$ & 5.00 & - & - \\
\hline SELENIUH & $06 / 21 / 82$ & 0001 & $M G / L$ & 0.15 & - & - \\
\hline SILICA - SIOZ & $06 / 21 / 82$ & 0001 & $M G / L$ & 6.10 & - & - \\
\hline SILVER & $06 / 21 / 82$ & 0001 & $M G / L$ & 0.008 & - & - \\
\hline SOOILA & $06 / 21 / 82$ & 0001 & MG/L & 550.00 & - & - \\
\hline SPECIFIC CONDUCTAKCE & $06 / 21 / 82$ & 0001 & UМно/CM & 4440.00 & - & - \\
\hline SULFATE & $06 / 21 / 82$ & 0001 & $M G / L$ & 2750.00 & - & - \\
\hline TEMPERATURE & $06 / 21 / 82$ & 0001 & C - DEGREE & 14.00 & - & - \\
\hline TOTAL DISSOLVED SOLIDS & $06 / 21 / 82$ & 0001 & $M G / L$ & 4360.00 & - & - \\
\hline URANIUM-234 & $06 / 21 / 82$ & 0001 & $\mathrm{PCI} / \mathrm{L}$ & 0.032 & - & - \\
\hline
\end{tabular}

PARAMETER VALUE INDICATOR (PVI): < - LESS THAN DETECTION LIMIT 
GROUNDWATER OUALITY DATA BY LOCATION

SITE: AMBO1 AMBROSIA LAKE

LOCATION: 0709

NORTH COORDINATE: 55212.0 FT

EAST COORDIHATE: 60324.0 FT

$06 / 10 / 80$ TO $12 / 04 / 92$

REPORT DATE: 08/04/94

FORHATION OF COMPLETIOH: ALLUVIUM (AL)

HYDRAULIC FLOW RELATIOHSHIP: UPGRADIENT (U)

\begin{tabular}{|l|c|c|l|l|l|c|c|}
\hline PARAMETER NAME & LOG DATE & $\begin{array}{c}\text { SAMPLE } \\
\text { ID }\end{array}$ & $\begin{array}{l}\text { UMITS OF } \\
\text { MEASURE }\end{array}$ & PVI & $\begin{array}{c}\text { PARAMETER } \\
\text { VALUE FLAGS }\end{array}$ & $\begin{array}{c}\text { DETECTION } \\
\text { LIHIT }\end{array}$ & $\begin{array}{l}\text { PARAMETER } \\
\text { UNCERTAINTY }\end{array}$ \\
\hline VAMADIUM & $06 / 21 / 82$ & 0001 & MG/L & $<$ & 0.01 & 0.01 & - \\
\hline
\end{tabular}

PARAMETER VALUE IMDICATOR (PVI): < - LESS THAN DETECTION LIMIT

SAMPLE IO COOES:

OOO1 - FILTERED SAMPLE (.45 MICRONS) 
GROUHDWATER QUALITY DATA BY LOCATION

SITE: AMBO1 AMBROSIA LAKE

LOCATION: 0710

NORTH COORDINATE: 55599.0 FT

EAST COORDINATE: 61272.0 FT

06/10/80 TO $12 / 04 / 92$

REPORT DATE: $08 / 04 / 94$

FORMATION OF COMPLETION: ALLUVIUM (AL)

HYDRAULIC FLON RELATIOHSHIP: UPGRADIENT (U)

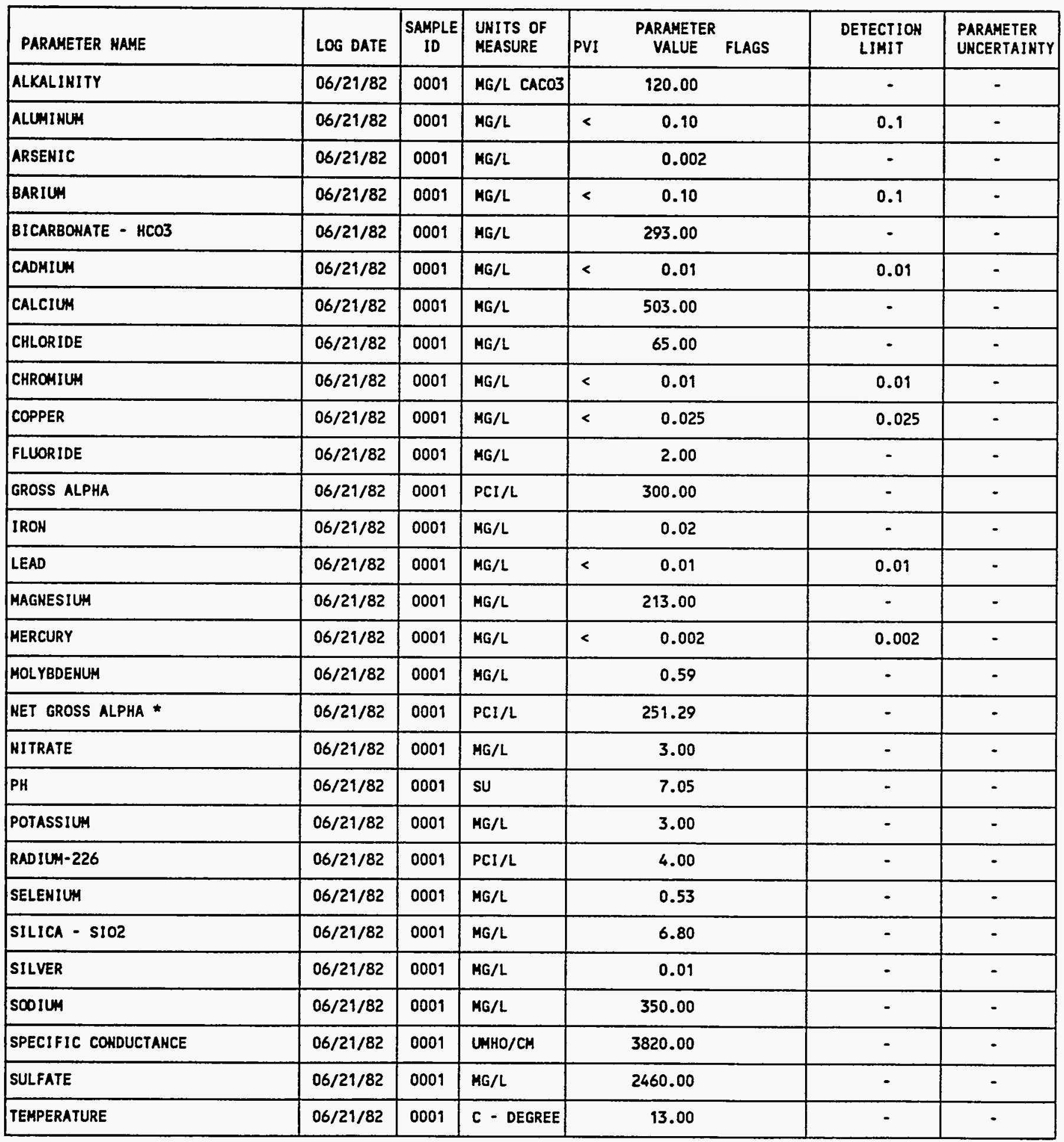

- NET GROSS ALPHA (GROSS ALPHA - URANIUH) HITH 1 MG URANIUH = 686 PCI

PARAMETER VALUE INDICATOR (PVI): < - LESS THAN DETECTION LIMIT 
GROUNDHATER QUALITY DATA BY LOCATION

SITE: AMBO1 AMBROSIA LAKE

LOCATION: 0710

MORTH COORDINATE: $\quad 55599.0$ FT

EAST COORDIHATE: $\quad 61272.0$ FT

06/10/80 TO $12 / 04 / 92$

REPORT DATE: $08 / 04 / 94$

FORMATION OF COMPLETION: ALLUVIUM (AL)

HYDRAULIC FLOW RELATIOHSHIP: UPGRADIENT (U)

\begin{tabular}{|c|c|c|c|c|c|c|c|}
\hline PARAMETER NAME & LOG DATE & $\underset{\text { SAMPLE }}{\text { ID }}$ & $\begin{array}{l}\text { UNITS OF } \\
\text { MEASURE }\end{array}$ & PVI & $\begin{array}{l}\text { PARAMETER } \\
\text { VALUE FLAGS }\end{array}$ & $\begin{array}{l}\text { DETECTIOH } \\
\text { LIMIT }\end{array}$ & $\begin{array}{l}\text { PARAMETER } \\
\text { UMCERTAINTY }\end{array}$ \\
\hline TOTAL DISSOLVED SOLIDS & $06 / 21 / 82$ & 0009 & $M G / L$ & & 3760.00 & - & - \\
\hline URAKIUM & $0.6 / 21 / 82$ & 0001 & $M G / L$ & & 0.071 & - & $\cdot$ \\
\hline VANADIUM & $06 / 21 / 82$ & 0001 & MG/L & $<$ & 0.01 & 0.01 & $\cdot$ \\
\hline
\end{tabular}

PARAMETER VALUE INDICATOR (PVI): < - LESS THAN DETECTION LIMIT

SAMPLE ID COOES:

0009 - FILTERED SAMPLE (.45 MICROHS) 
GROUNDWATER QUALITY DATA BY LOCATION

SITE: AMBO1 AMBROSIA LAKE

LOCATION: 0711

NORTH COORDIHATE: $\quad 44620.0$ FT

EAST COORDINATE: $\quad 58430.0$ FT

06/10/80 TO $12 / 04 / 92$

REPORT DATE: 08/04/94

FORMATION OF COMPLETIOH: TRES HERMANOS - B SAHDSTONE (TB)

HYDRAULIC FLON RELATIONSHIP: UPGRADIENT (U)

\begin{tabular}{|c|c|c|c|c|c|c|c|}
\hline PARAMETER MAME & LOG DATE & $\begin{array}{l}\text { SAMPLE } \\
\text { ID }\end{array}$ & $\begin{array}{l}\text { UNITS OF } \\
\text { MEASURE }\end{array}$ & PVI & $\begin{array}{l}\text { PARAMETER } \\
\text { VALUE FLAGS }\end{array}$ & $\begin{array}{l}\text { DETECTIOH } \\
\text { LIMIT }\end{array}$ & $\begin{array}{l}\text { PARAMETER } \\
\text { UNCERTAINTY }\end{array}$ \\
\hline ALKALINITY & $\begin{array}{l}06 / 20 / 80 \\
10 / 08 / 85 \\
10 / 08 / 85 \\
10 / 08 / 85 \\
10 / 08 / 85 \\
10 / 08 / 85\end{array}$ & $\begin{array}{l}0001 \\
0001 \\
0002 \\
0003 \\
0004 \\
0005\end{array}$ & MG/L CACO3 & & $\begin{array}{l}27.50 \\
268 . \\
268 . \\
268 . \\
268 \text {. } \\
268 \text {. }\end{array}$ & $\begin{array}{l}- \\
- \\
- \\
-\end{array}$ & $\begin{array}{l}- \\
- \\
- \\
-\end{array}$ \\
\hline ALUAINUM & $\begin{array}{c}06 / 20 / 80 \\
10 / 08 / 85 \\
10 / 08 / 85 \\
10 / 08 / 85 \\
10 / 08 / 85\end{array}$ & $\begin{array}{l}0001 \\
0002 \\
0003 \\
0004 \\
0005\end{array}$ & $M G / L$ & & $\begin{array}{l}0.78 \\
0.4 \\
0.5 \\
0.5 \\
0.6\end{array}$ & $\begin{array}{l}- \\
0.1 \\
0.1 \\
0.1 \\
0.1\end{array}$ & $\begin{array}{l}- \\
- \\
-\end{array}$ \\
\hline AMMONIUM & $\begin{array}{l}10 / 08 / 85 \\
10 / 08 / 85 \\
10 / 08 / 85 \\
10 / 08 / 85\end{array}$ & $\begin{array}{l}0002 \\
0003 \\
0004 \\
0005\end{array}$ & $M G / L$ & $<$ & $\begin{array}{l}0.2 \\
0.2 \\
0.2 \\
0.1\end{array}$ & $\begin{array}{l}0.1 \\
0.1 \\
0.1 \\
0.1\end{array}$ & $\begin{array}{l}- \\
-\end{array}$ \\
\hline ANT IMONY & $\begin{array}{l}10 / 08 / 85 \\
10 / 08 / 85 \\
10 / 08 / 85 \\
10 / 08 / 85\end{array}$ & $\begin{array}{l}0002 \\
0003 \\
0004 \\
0005\end{array}$ & MG/L & $\begin{array}{l}< \\
< \\
<\end{array}$ & $\begin{array}{l}0.003 \\
0.003 \\
0.003 \\
0.003\end{array}$ & $\begin{array}{l}0.003 \\
0.003 \\
0.003 \\
0.003\end{array}$ & : \\
\hline ARSENIC & $\begin{array}{l}06 / 20 / 80 \\
10 / 08 / 85 \\
10 / 08 / 85 \\
10 / 08 / 85 \\
10 / 08 / 85 \\
10 / 08 / 85\end{array}$ & $\begin{array}{l}0001 \\
0001 \\
0002 \\
0003 \\
0004 \\
0005\end{array}$ & $M G / L$ & $\begin{array}{l}< \\
< \\
<\end{array}$ & $\begin{array}{l}0.033 \\
0.01 \\
0.01 \\
0.01 \\
0.01 \\
0.01\end{array}$ & $\begin{array}{l}0.01 \\
0.017 \\
0.01 \\
0.01 \\
0.01\end{array}$ & $\begin{array}{l}- \\
- \\
- \\
- \\
-\end{array}$ \\
\hline BARIUM & $\begin{array}{l}06 / 20 / 80 \\
10 / 08 / 85 \\
10 / 08 / 85 \\
10 / 08 / 85 \\
10 / 08 / 85\end{array}$ & $\begin{array}{l}0001 \\
0002 \\
0003 \\
0004 \\
0005\end{array}$ & $M G / L$ & & $\begin{array}{l}0.003 \\
0.2 \\
0.2 \\
0.3 \\
0.1\end{array}$ & $\begin{array}{l}- \\
0.1 \\
0.1 \\
0.1 \\
0.1\end{array}$ & : \\
\hline BICARBOHATE - HCO3 & $06 / 20 / 80$ & 0001 & $M G / L$ & & 67.00 & - & - \\
\hline BORON & $\begin{array}{l}06 / 20 / 80 \\
10 / 08 / 85 \\
10 / 08 / 85 \\
10 / 08 / 85 \\
10 / 08 / 85\end{array}$ & $\begin{array}{l}0001 \\
0002 \\
0003 \\
0004 \\
0005\end{array}$ & $M G / L$ & & $\begin{array}{l}0.50 \\
0.5 \\
0.4 \\
0.4 \\
0.4\end{array}$ & $\begin{array}{l}- \\
0.1 \\
0.1 \\
0.1 \\
0.1\end{array}$ & $\begin{array}{l}- \\
- \\
-\end{array}$ \\
\hline CAOMIUM & $\begin{array}{l}06 / 20 / 80 \\
10 / 08 / 85 \\
10 / 08 / 85 \\
10 / 08 / 85 \\
10 / 08 / 85\end{array}$ & $\begin{array}{l}0001 \\
0002 \\
0003 \\
0004 \\
0005\end{array}$ & MG/L & $\begin{array}{l}< \\
< \\
<\end{array}$ & $\begin{array}{l}0.008 \\
0.001 \\
0.001 \\
0.001 \\
0.001\end{array}$ & $\begin{array}{l}0.008 \\
0.001 \\
0.001 \\
0.001 \\
0.001\end{array}$ & $\begin{array}{l}- \\
\dot{-} \\
\dot{-}\end{array}$ \\
\hline CALCIUM & $\begin{array}{l}06 / 20 / 80 \\
10 / 08 / 85 \\
10 / 08 / 85 \\
10 / 08 / 85 \\
10 / 08 / 85 \\
10 / 08 / 85\end{array}$ & $\begin{array}{l}0001 \\
0001 \\
0002 \\
0003 \\
0004 \\
0005\end{array}$ & MG/L & & $\begin{array}{l}240.00 \\
446 . \\
328 . \\
348 . \\
192 . \\
189 .\end{array}$ & $\begin{array}{l}- \\
0.01 \\
0.01 \\
0.01 \\
0.01 \\
0.01\end{array}$ & $\begin{array}{l}- \\
- \\
- \\
-\end{array}$ \\
\hline
\end{tabular}

PARAMETER VALUE IHOICATOR (PVI): < - LESS THAN DETECTION LIMIT

SAMPLE ID CODES

OTHER PARAMETER VALUE FLAGS:

0001 - FILTERED SAMPLE (.45 MICRONS)

J - ESTIMATED VALUE

0002 - FILTERED REPLICATE SAMPLE (.45 MICRONS)

0003 - FILTERED REPLICATE SAMPLE (.45 MICRONS)

0004 - FILTERED REPLICATE SAMPLE (.45 MICRONS) 
GROUNDWATER QUALITY DATA BY LOCATION

SITE: AMBO1 AMBROSIA LAKE

LOCATION: 0711

NORTH COORDINATE: 44620.0 FT

EAST COORDIKATE: $\quad 58430.0$ FT

D6/10/80 TO $12 / 04 / 92$

REPORT DATE: 08/04/94

FORMATION OF COMPLETION: TRES HERMANOS - B SANDSTOHE (TB)

HYORAULIC FLOW RELATIOHSHIP: UPGRADIENT (U)

\begin{tabular}{|c|c|c|c|c|c|c|c|}
\hline PARAMETER MAME & LOG DATE & $\underset{10}{\text { SAMPLE }}$ & $\begin{array}{l}\text { UNITS OF } \\
\text { MEASURE }\end{array}$ & PVI & $\begin{array}{l}\text { PARAMETER } \\
\text { VALUE FLAGS }\end{array}$ & $\begin{array}{l}\text { DETECTION } \\
\text { LIMIT }\end{array}$ & $\begin{array}{l}\text { PARAMETER } \\
\text { UKCERTAINTY }\end{array}$ \\
\hline CHLORIDE & $\begin{array}{l}06 / 20 / 80 \\
10 / 08 / 85 \\
10 / 08 / 85 \\
10 / 08 / 85 \\
10 / 08 / 85 \\
10 / 08 / 85\end{array}$ & $\begin{array}{l}0001 \\
0001 \\
0002 \\
0003 \\
0004 \\
0005\end{array}$ & MG/L & & $\begin{array}{l}109.00 \\
410 . \\
400 . \\
390 . \\
420 . \\
400 .\end{array}$ & $\begin{array}{l}- \\
1 . \\
1 . \\
1 . \\
1 .\end{array}$ & $\begin{array}{l}- \\
- \\
- \\
-\end{array}$ \\
\hline CHROMIUM & $\begin{array}{l}06 / 20 / 80 \\
10 / 08 / 85 \\
10 / 08 / 85 \\
10 / 08 / 85 \\
10 / 08 / 85\end{array}$ & $\begin{array}{l}0001 \\
0002 \\
0003 \\
0004 \\
0005\end{array}$ & MG/L & & $\begin{array}{l}0.16 \\
0.03 \\
0.05 \\
0.04 \\
0.04\end{array}$ & $\begin{array}{l}- \\
0.01 \\
0.01 \\
0.01 \\
0.01\end{array}$ & $\begin{array}{l}- \\
- \\
-\end{array}$ \\
\hline COBALT & $\begin{array}{l}06 / 20 / 80 \\
10 / 08 / 85 \\
10 / 08 / 85 \\
10 / 08 / 85 \\
10 / 08 / 85\end{array}$ & $\begin{array}{l}0001 \\
0002 \\
0003 \\
0004 \\
0005\end{array}$ & $M G / L$ & $\begin{array}{l}< \\
< \\
< \\
< \\
<\end{array}$ & $\begin{array}{l}0.006 \\
0.05 \\
0.05 \\
0.05 \\
0.05\end{array}$ & $\begin{array}{l}0.006 \\
0.05 \\
0.05 \\
0.05 \\
0.05\end{array}$ & $\begin{array}{l}- \\
- \\
- \\
-\end{array}$ \\
\hline COPPER & $\begin{array}{l}06 / 20 / 80 \\
10 / 08 / 85 \\
10 / 08 / 85 \\
10 / 08 / 85 \\
10 / 08 / 85\end{array}$ & $\begin{array}{l}0009 \\
0002 \\
0003 \\
0004 \\
0005\end{array}$ & $M G / L$ & $\begin{array}{l}< \\
< \\
< \\
<\end{array}$ & $\begin{array}{l}0.001 \\
0.02 \\
0.02 \\
0.02 \\
0.02\end{array}$ & $\begin{array}{l}0.001 \\
0.02 \\
0.02 \\
0.02 \\
0.02\end{array}$ & : \\
\hline CYANIDE & $\begin{array}{l}10 / 08 / 85 \\
10 / 08 / 85 \\
10 / 08 / 85 \\
10 / 08 / 85\end{array}$ & $\begin{array}{l}0002 \\
0003 \\
0004 \\
0005\end{array}$ & $M G / L$ & $\begin{array}{l}< \\
< \\
< \\
<\end{array}$ & $\begin{array}{l}0.01 \\
0.01 \\
0.01 \\
0.01\end{array}$ & $\begin{array}{l}0.01 \\
0.01 \\
0.01 \\
0.01\end{array}$ & $\begin{array}{l}- \\
- \\
-\end{array}$ \\
\hline FLUORIDE & $\begin{array}{l}06 / 20 / 80 \\
10 / 08 / 85 \\
10 / 08 / 85 \\
10 / 08 / 85 \\
10 / 08 / 85\end{array}$ & $\begin{array}{l}0001 \\
0002 \\
0003 \\
0004 \\
0005\end{array}$ & $M G / L$ & & $\begin{array}{l}0.20 \\
0.8 \\
0.7 \\
0.7 \\
0.6\end{array}$ & $\begin{array}{l}. \\
0.1 \\
0.1 \\
0.1 \\
0.1\end{array}$ & $\begin{array}{l}- \\
- \\
- \\
-\end{array}$ \\
\hline IRON & $\begin{array}{l}06 / 20 / 80 \\
10 / 08 / 85 \\
10 / 08 / 85 \\
10 / 08 / 85 \\
10 / 08 / 85\end{array}$ & $\begin{array}{l}0001 \\
0002 \\
0003 \\
0004 \\
0005\end{array}$ & $M G / L$ & $<$ & $\begin{array}{l}0.008 \\
0.37 \\
0.95 \\
0.04 \\
0.07\end{array}$ & $\begin{array}{l}0.008 \\
0.03 \\
0.03 \\
0.03 \\
0.03\end{array}$ & $\begin{array}{l}- \\
- \\
- \\
-\end{array}$ \\
\hline LEAD & $\begin{array}{l}06 / 20 / 80 \\
10 / 08 / 85 \\
10 / 08 / 85 \\
10 / 08 / 85 \\
10 / 08 / 85\end{array}$ & $\begin{array}{l}0001 \\
0002 \\
0003 \\
0004 \\
0005\end{array}$ & MG/L & $\begin{array}{l}< \\
< \\
< \\
< \\
<\end{array}$ & $\begin{array}{l}0.001 \\
0.01 \\
0.01 \\
0.01 \\
0.01\end{array}$ & $\begin{array}{l}0.001 \\
0.01 \\
0.01 \\
0.01 \\
0.01\end{array}$ & $\begin{array}{l}- \\
- \\
- \\
-\end{array}$ \\
\hline LEND-210 & $\begin{array}{l}10 / 08 / 85 \\
10 / 08 / 85 \\
10 / 08 / 85 \\
10 / 08 / 85\end{array}$ & $\begin{array}{l}0002 \\
0003 \\
0004 \\
0005\end{array}$ & $\mathrm{PCI} / \mathrm{L}$ & & $\begin{array}{l}1.4 \\
0.3 \\
0.2 \\
0.4\end{array}$ & $\begin{array}{l}1.5 \\
1.5 \\
1.5 \\
1.5\end{array}$ & $\begin{array}{l}1.1 \\
1.3 \\
1.2 \\
1.1\end{array}$ \\
\hline MAGKESIUM & $\begin{array}{l}06 / 20 / 80 \\
10 / 08 / 85 \\
10 / 08 / 85\end{array}$ & $\begin{array}{l}0001 \\
0001 \\
0002\end{array}$ & $M G / L$ & & $\begin{array}{l}200.00 \\
246 . \\
217 .\end{array}$ & $\begin{array}{l}- \\
0.001 \\
0.001\end{array}$ & $\begin{array}{l}- \\
- \\
-\end{array}$ \\
\hline
\end{tabular}

PARAMETER VALUE INDICATOR (PVI): < - LESS THAN DETECTION LIMIT

SAMPLE 10 CODES:

0001 - FILTERED SAMPLE (.45 HICRONS)

O002 - FILTERED REPLICATE SAMPLE (.45 MICROHS)

0003 - FILTERED REPLICATE SAMPLE (.45 MICRONS)

0004 - FILTERED REPLICATE SAMPLE (.45 MICRONS) 
GROUHDWATER QUALITY DATA BY LOCATION

SITE: AMBO1 AMBROSIA LAKE

LOCATION: 0711

MORTH COORDINATE: $\quad 44620.0$ FT

EAST COORDINATE: $\quad 58430.0$ FT

06/10/80 TO $12 / 04 / 92$

REPORT DATE: 08/04/94

FORMATION OF COMPLETION: TRES HERMANOS - B SANDSTONE (TB)

HYDRAULIC FLOW RELATIONSHIP: UPGRADIEHT (U)

\begin{tabular}{|c|c|c|c|c|c|c|c|}
\hline PARAMETER MAME & LOG DATE & $\begin{array}{c}\text { SAMPLE } \\
10\end{array}$ & $\begin{array}{l}\text { UNITS OF } \\
\text { MEASURE }\end{array}$ & PVI & $\begin{array}{l}\text { PARAMETER } \\
\text { VALUE FLAGS }\end{array}$ & $\begin{array}{l}\text { DETECTION } \\
\text { LIMIT }\end{array}$ & $\begin{array}{l}\text { PARAMETER } \\
\text { UNCERTAINTY }\end{array}$ \\
\hline MAGHESIUH & $\begin{array}{l}10 / 08 / 85 \\
10 / 08 / 85 \\
10 / 08 / 85\end{array}$ & $\begin{array}{l}0003 \\
0004 \\
0005\end{array}$ & MG/L & & $\begin{array}{l}247 . \\
193 . \\
194 .\end{array}$ & $\begin{array}{l}0.001 \\
0.001 \\
0.001\end{array}$ & : \\
\hline MAMGAHESE & $\begin{array}{l}06 / 20 / 80 \\
10 / 08 / 85 \\
10 / 08 / 85 \\
10 / 08 / 85 \\
10 / 08 / 85\end{array}$ & $\begin{array}{l}0001 \\
0002 \\
0003 \\
0004 \\
0005\end{array}$ & MG/L & & $\begin{array}{l}0.74 \\
0.06 \\
0.06 \\
0.03 \\
0.04\end{array}$ & $\begin{array}{l}- \\
0.01 \\
0.01 \\
0.01 \\
0.01\end{array}$ & $\begin{array}{l}: \\
: \\
:\end{array}$ \\
\hline MERCURY & $\begin{array}{l}06 / 20 / 80 \\
10 / 08 / 85 \\
10 / 08 / 85 \\
10 / 08 / 85 \\
10 / 08 / 85\end{array}$ & $\begin{array}{l}0001 \\
0002 \\
0003 \\
0004 \\
0005\end{array}$ & MG/L & $\begin{array}{l}< \\
< \\
< \\
< \\
<\end{array}$ & $\begin{array}{l}0.001 \\
0.0002 \\
0.0002 \\
0.0002 \\
0.0002\end{array}$ & $\begin{array}{l}0.001 \\
0.0002 \\
0.0002 \\
0.0002 \\
0.0002\end{array}$ & $\begin{array}{l}- \\
- \\
-\end{array}$ \\
\hline MOLYYBDENUM & $\begin{array}{l}06 / 20 / 80 \\
10 / 08 / 85 \\
10 / 08 / 85 \\
10 / 08 / 85 \\
10 / 08 / 85 \\
10 / 08 / 85\end{array}$ & $\begin{array}{l}0001 \\
0001 \\
0002 \\
0003 \\
0004 \\
0005\end{array}$ & $M G / L$ & & $\begin{array}{l}0.42 \\
0.26 \\
0.2 \\
0.1 \\
0.3 \\
0.34\end{array}$ & $\begin{array}{l}- \\
0.01 \\
0.01 \\
0.01 \\
0.01 \\
0.01\end{array}$ & $\begin{array}{l}- \\
- \\
- \\
-\end{array}$ \\
\hline NICKEL & $\begin{array}{l}06 / 20 / 80 \\
10 / 08 / 85 \\
10 / 08 / 85 \\
10 / 08 / 85 \\
10 / 08 / 85\end{array}$ & $\begin{array}{l}0001 \\
0002 \\
0003 \\
0004 \\
0005\end{array}$ & MG/L & $<$ & $\begin{array}{l}0.003 \\
0.08 \\
0.09 \\
0.09 \\
0.07\end{array}$ & $\begin{array}{l}0.003 \\
0.04 \\
0.04 \\
0.04 \\
0.04\end{array}$ & $\begin{array}{l}- \\
- \\
-\end{array}$ \\
\hline NITRATE & $\begin{array}{l}06 / 20 / 80 \\
10 / 08 / 85 \\
10 / 08 / 85 \\
10 / 08 / 85 \\
10 / 08 / 85\end{array}$ & $\begin{array}{l}0001 \\
0002 \\
0003 \\
0004 \\
0005\end{array}$ & MG/L & & $\begin{array}{l}0.70 \\
10 . \\
20 . \\
20 . \\
20 .\end{array}$ & $\begin{array}{l}1 . \\
1 . \\
1 .\end{array}$ & $\begin{array}{l}- \\
- \\
-\end{array}$ \\
\hline NITRITE & $\begin{array}{l}10 / 08 / 85 \\
10 / 08 / 85 \\
10 / 08 / 85 \\
10 / 08 / 85\end{array}$ & $\begin{array}{l}0002 \\
0003 \\
0004 \\
0005\end{array}$ & MG/L & $\begin{array}{l}< \\
< \\
<\end{array}$ & $\begin{array}{l}0.1 \\
0.1 \\
0.1 \\
0.1\end{array}$ & $\begin{array}{l}0.1 \\
0.1 \\
0.1 \\
0.1\end{array}$ & : \\
\hline PH & $\begin{array}{l}06 / 20 / 80 \\
10 / 08 / 85 \\
10 / 08 / 85 \\
10 / 08 / 85 \\
10 / 08 / 85 \\
10 / 08 / 85\end{array}$ & $\begin{array}{l}0001 \\
0001 \\
0002 \\
0003 \\
0004 \\
0005\end{array}$ & SU & & $\begin{array}{l}7.70 \\
7.14 \\
7.14 \\
7.14 \\
7.14 \\
7.14\end{array}$ & $\begin{array}{l}- \\
- \\
0.01\end{array}$ & $\begin{array}{l}- \\
- \\
- \\
-\end{array}$ \\
\hline PHOSPHATE & $\begin{array}{l}10 / 08 / 85 \\
10 / 08 / 85 \\
10 / 08 / 85 \\
10 / 08 / 85\end{array}$ & $\begin{array}{l}0002 \\
0003 \\
0004 \\
0005\end{array}$ & MG/L & & $\begin{array}{l}0.4 \\
0.4 \\
0.5 \\
0.4\end{array}$ & $\begin{array}{l}0.1 \\
0.1 \\
0.1 \\
0.1\end{array}$ & $\dot{-}$ \\
\hline POLONIUM-210 & $\begin{array}{l}10 / 08 / 85 \\
10 / 08 / 85 \\
10 / 08 / 85 \\
10 / 08 / 85\end{array}$ & $\begin{array}{l}0002 \\
0003 \\
0004 \\
0005\end{array}$ & $\mathrm{PCI} / \mathrm{L}$ & & $\begin{array}{l}2.7 \\
0 . \\
0 . \\
0 .\end{array}$ & $\begin{array}{l}1 . \\
1 . \\
1 . \\
1 .\end{array}$ & $\begin{array}{l}1 . \\
0.6 \\
2.8 \\
0.5\end{array}$ \\
\hline PARAMETER VALUE IHDICATOR (PVI): & \multicolumn{4}{|c|}{ < - LESS THAN DETECTION LIMIT } & \multicolumn{3}{|l|}{$\begin{array}{l}\text { SAMPLE IO COOES: } \\
0001 \text { - FILTERED } \\
0002 \text { - FILTERED R } \\
0003 \text { - FILTERED R } \\
0004 \text { - FILTERED R }\end{array}$} \\
\hline
\end{tabular}


GROUNDWATER QUALITY DATA BY LOCATION

SITE: AMBOI AMBROSIA LAKE

LOCATION: 0711

NORTH COORDINATE: $\quad 44620.0$ FT

EAST COORDINATE: $\quad 58430.0$ FT

06/10/80 TO $12 / 04 / 92$

REPORT DATE: $08 / 04 / 94$

FORMATION OF CONPLETION: TRES HERMANOS - B SAMDSTONE (TB)

HYDRAULIC FLON RELATIONSHIP: UPGRADIENT (U)

\begin{tabular}{|c|c|c|c|c|c|c|c|}
\hline PARAMETER MAME & LOG DATE & $\underset{\text { ID }}{\text { SAMPLE }}$ & $\begin{array}{l}\text { UHITS OF } \\
\text { MEASURE }\end{array}$ & PVI & $\begin{array}{l}\text { PARAMETER } \\
\text { VALUE FLAGS }\end{array}$ & $\begin{array}{l}\text { DETECTION } \\
\text { LIHIT }\end{array}$ & $\begin{array}{l}\text { PARAMETER } \\
\text { UNCERTAIHTY }\end{array}$ \\
\hline POTASSIUM & $\begin{array}{l}06 / 20 / 80 \\
10 / 08 / 85 \\
10 / 08 / 85 \\
10 / 08 / 85 \\
10 / 08 / 85 \\
10 / 08 / 85\end{array}$ & $\begin{array}{l}0001 \\
0001 \\
0002 \\
0003 \\
0004 \\
0005\end{array}$ & $M G / L$ & & $\begin{array}{l}5.80 \\
5.96 \\
6.21 \\
5.95 \\
6.94 \\
6.58\end{array}$ & $\begin{array}{l}. \\
0.01 \\
0.01 \\
0.01 \\
0.01 \\
0.01\end{array}$ & $\begin{array}{l}- \\
- \\
- \\
- \\
-\end{array}$ \\
\hline RADIUA-226 & $\begin{array}{l}06 / 20 / 80 \\
10 / 08 / 85 \\
10 / 08 / 85 \\
10 / 08 / 85 \\
10 / 08 / 85\end{array}$ & $\begin{array}{l}0001 \\
0002 \\
0003 \\
0004 \\
0005\end{array}$ & $\mathrm{PCI} / \mathrm{L}$ & & $\begin{array}{c}18.00 \\
1 . \\
0.4 \\
17 . \\
1 .\end{array}$ & $\begin{array}{l}. \\
1 . \\
1 . \\
1 .\end{array}$ & $\begin{array}{l}- \\
0.4 \\
0.3 \\
1 . \\
0.4\end{array}$ \\
\hline RADIUH-226 + RADIUM-228 & $\begin{array}{l}10 / 08 / 85 \\
10 / 08 / 85 \\
10 / 08 / 85 \\
10 / 08 / 85\end{array}$ & $\begin{array}{l}0002 \\
0003 \\
0004 \\
0005\end{array}$ & $\mathrm{PCI} / \mathrm{L}$ & & $\begin{array}{r}1.00 \\
0.40 \\
17.00 \\
1.00\end{array}$ & $\begin{array}{l}- \\
- \\
-\end{array}$ & $\begin{array}{l}- \\
- \\
-\end{array}$ \\
\hline RADIUH-228 & $\begin{array}{l}10 / 08 / 85 \\
10 / 08 / 85 \\
10 / 08 / 85 \\
10 / 08 / 85\end{array}$ & $\begin{array}{l}0002 \\
0003 \\
0004 \\
0005\end{array}$ & $\mathrm{PCI} / \mathrm{L}$ & & $\begin{array}{l}0 . \\
0 . \\
0 . \\
0 .\end{array}$ & $\begin{array}{l}1 . \\
1 . \\
1 . \\
1 .\end{array}$ & $\begin{array}{l}1.2 \\
1.3 \\
0.8 \\
0.9\end{array}$ \\
\hline SELENIUM & $\begin{array}{l}06 / 20 / 80 \\
10 / 08 / 85 \\
10 / 08 / 85 \\
10 / 08 / 85 \\
10 / 08 / 85 \\
10 / 08 / 85\end{array}$ & $\begin{array}{l}0001 \\
0001 \\
0002 \\
0003 \\
0004 \\
0005\end{array}$ & $M G / L$ & $\begin{array}{l}< \\
< \\
< \\
< \\
<\end{array}$ & $\begin{array}{l}0.12 \\
0.005 \\
0.005 \\
0.005 \\
0.005 \\
0.005\end{array}$ & $\begin{array}{l}- \\
0.005 \\
0.005 \\
0.005 \\
0.005 \\
0.005\end{array}$ & $\begin{array}{l}- \\
- \\
- \\
-\end{array}$ \\
\hline SILICA - SIOZ & $\begin{array}{l}10 / 08 / 85 \\
10 / 08 / 85 \\
10 / 08 / 85 \\
10 / 08 / 85\end{array}$ & $\begin{array}{l}0002 \\
0003 \\
0004 \\
0005\end{array}$ & $M G / L$ & & $\begin{array}{l}8 . \\
8 . \\
8 . \\
8 .\end{array}$ & $\begin{array}{l}2 . \\
2 . \\
2 . \\
2 .\end{array}$ & $\begin{array}{l}- \\
- \\
-\end{array}$ \\
\hline SILVER & $\begin{array}{l}06 / 20 / 80 \\
10 / 08 / 85 \\
10 / 08 / 85 \\
10 / 08 / 85 \\
10 / 08 / 85\end{array}$ & $\begin{array}{l}0001 \\
0002 \\
0003 \\
0004 \\
0005\end{array}$ & MG/L & $\begin{array}{l}< \\
< \\
< \\
<\end{array}$ & $\begin{array}{l}0.002 \\
0.01 \\
0.01 \\
0.01 \\
0.01\end{array}$ & $\begin{array}{l}0.002 \\
0.01 \\
0.01 \\
0.01 \\
0.01\end{array}$ & $\begin{array}{l}- \\
- \\
-\end{array}$ \\
\hline SOOIUH & $\begin{array}{l}06 / 20 / 80 \\
10 / 08 / 85 \\
10 / 08 / 85 \\
10 / 08 / 85 \\
10 / 08 / 85 \\
10 / 08 / 85\end{array}$ & $\begin{array}{l}0001 \\
0001 \\
0002 \\
0003 \\
0004 \\
0005\end{array}$ & $M G / L$ & & $\begin{array}{l}200.00 \\
286 . \\
216 . \\
467 . \\
780 \\
762 .\end{array}$ & $\begin{array}{l}- \\
0.002 \\
0.002 \\
0.002 \\
0.002 \\
0.002\end{array}$ & $\begin{array}{l}- \\
- \\
- \\
- \\
-\end{array}$ \\
\hline SPECIFIC CONDUCTANCE & $\begin{array}{l}06 / 20 / 80 \\
10 / 08 / 85 \\
10 / 08 / 85 \\
10 / 08 / 85 \\
10 / 08 / 85 \\
10 / 08 / 85\end{array}$ & $\begin{array}{l}0001 \\
0001 \\
0002 \\
0003 \\
0004 \\
0005\end{array}$ & UNHO/CH & & $\begin{array}{l}2800.00 \\
3200 \\
3200 \\
3200 \\
3200 \\
3200\end{array}$ & $\begin{array}{l}- \\
- \\
- \\
-\end{array}$ & $\begin{array}{l}- \\
- \\
- \\
- \\
-\end{array}$ \\
\hline STRONTIUM & $10 / 08 / 85$ & 0002 & $M G / L$ & & 4.3 & 0.1 & - \\
\hline
\end{tabular}

PARAMETER VALUE INDICATOR (PVI): < - LESS THAN DETECTION LIMIT

SAMPLE ID COOES:

0001 - FILTERED SAMPLE (.45 MICROAS)

0002 - FILTERED REPLICATE SAMPLE (.45 MICROHS)

0003 - FILTERED REPLICATE SAMPLE (.45 MICRONS)

0004 - FILTERED REPLICATE SAMPLE (.45 MICRONS) 
GROUMDWATER QUALITY DATA BY LOCATIOH

SITE: AMBO1 AMBROSIA LAKE

LOCATIOH: 0711

MORTH COORDIHATE: $\quad 44620.0$ FT

EAST COORDINATE: $\quad 58430.0$ FT

$06 / 10 / 80$ TO $12 / 04 / 92$

REPORT DATE: 08/04/94

FORMATION OF COMPLETIOH: TRES HERMANOS - B SAMDSTONE (TB)

HYDRAULIC FLON RELATIOHSHIP: UPGRADIENT (U)

\begin{tabular}{|c|c|c|c|c|c|c|c|}
\hline PARAMETER MAME & LOG DATE & $\begin{array}{c}\text { SAMPLE } \\
\text { ID }\end{array}$ & $\begin{array}{l}\text { UHITS OF } \\
\text { MEASURE }\end{array}$ & PVI & $\begin{array}{l}\text { PARAMETER } \\
\text { VALUE FLAGS }\end{array}$ & $\begin{array}{l}\text { DETECTION } \\
\text { LIMIT }\end{array}$ & $\begin{array}{l}\text { PARAMETER } \\
\text { UNCERTAINTY }\end{array}$ \\
\hline STROHTIUM & $\begin{array}{l}10 / 08 / 85 \\
10 / 08 / 85 \\
10 / 08 / 85\end{array}$ & $\begin{array}{l}0003 \\
0004 \\
0005\end{array}$ & MG/L & & $\begin{array}{l}4.9 \\
2 . \\
2 .\end{array}$ & $\begin{array}{l}0.1 \\
0.1 \\
0.1\end{array}$ & - \\
\hline SULFATE & $\begin{array}{l}06 / 20 / 80 \\
10 / 08 / 85 \\
10 / 08 / 85 \\
10 / 08 / 85 \\
10 / 08 / 85 \\
10 / 08 / 85\end{array}$ & $\begin{array}{l}0001 \\
0001 \\
0002 \\
0003 \\
0004 \\
0005\end{array}$ & MG/L & & $\begin{array}{l}1600.00 \\
2070 . \\
1350 . \\
2040 . \\
2060 \text {. } \\
2060 \text {. }\end{array}$ & $\begin{array}{l}- \\
0.1 \\
0.1 \\
0.1 \\
0.1 \\
0.1\end{array}$ & $\begin{array}{l}: \\
: \\
: \\
-\end{array}$ \\
\hline SULFIDE & $\begin{array}{l}10 / 08 / 85 \\
10 / 08 / 85 \\
10 / 08 / 85 \\
10 / 08 / 85\end{array}$ & $\begin{array}{l}0002 \\
0003 \\
0004 \\
0005\end{array}$ & $M G / L$ & $\begin{array}{l}< \\
< \\
<\end{array}$ & $\begin{array}{l}0.1 \\
0.1 \\
0.1 \\
0.1\end{array}$ & $\begin{array}{l}0.1 \\
0.1 \\
0.1 \\
0.1\end{array}$ & $\dot{-}$ \\
\hline TEMPERATURE & $\begin{array}{l}10 / 08 / 85 \\
10 / 08 / 85 \\
10 / 08 / 85 \\
10 / 08 / 85 \\
10 / 08 / 85\end{array}$ & $\begin{array}{l}0001 \\
0002 \\
0003 \\
0004 \\
0005\end{array}$ & C - DEGREE & & $\begin{array}{l}13.5 \\
13.5 \\
13.5 \\
13.5 \\
13.5\end{array}$ & $\begin{array}{l}- \\
- \\
-\end{array}$ & $\begin{array}{l}- \\
- \\
-\end{array}$ \\
\hline THORIUH-230 & $\begin{array}{l}10 / 08 / 85 \\
10 / 08 / 85 \\
10 / 08 / 85 \\
10 / 08 / 85\end{array}$ & $\begin{array}{l}0002 \\
0003 \\
0004 \\
0005\end{array}$ & $\mathrm{PCI} / \mathrm{L}$ & & $\begin{array}{l}0.3 \\
0.1 \\
0.3 \\
0.2\end{array}$ & $\begin{array}{l}1 . \\
1 . \\
1 . \\
1 .\end{array}$ & $\begin{array}{l}0.4 \\
0.3 \\
0.4 \\
0.3\end{array}$ \\
\hline TIN & $\begin{array}{l}10 / 08 / 85 \\
10 / 08 / 85 \\
10 / 08 / 85 \\
10 / 08 / 85\end{array}$ & $\begin{array}{l}0002 \\
0003 \\
0004 \\
0005\end{array}$ & $M G / L$ & $\begin{array}{l}< \\
< \\
< \\
<\end{array}$ & $\begin{array}{l}0.005 \\
0.005 \\
0.005 \\
0.005\end{array}$ & $\begin{array}{l}0.005 \\
0.005 \\
0.005 \\
0.005\end{array}$ & $\begin{array}{l}- \\
-\end{array}$ \\
\hline TOTAL DISSOLVED SOLIDS & $\begin{array}{l}06 / 20 / 80 \\
10 / 08 / 85 \\
10 / 08 / 85 \\
10 / 08 / 85 \\
10 / 08 / 85 \\
10 / 08 / 85\end{array}$ & $\begin{array}{l}0001 \\
0001 \\
0002 \\
0003 \\
0004 \\
0005\end{array}$ & $M G / L$ & & $\begin{array}{l}2540.00 \\
3860 . \\
3910 . \\
3940 . \\
3960 . \\
3980 .\end{array}$ & $\begin{array}{l}. \\
10 . \\
10 . \\
10 . \\
10 . \\
10 .\end{array}$ & $\begin{array}{l}: \\
: \\
:\end{array}$ \\
\hline TOTAL ORGANIC CARBON & $\begin{array}{l}10 / 08 / 85 \\
10 / 08 / 85 \\
10 / 08 / 85\end{array}$ & $\begin{array}{l}0002 \\
0004 \\
0005\end{array}$ & $M G / L$ & & $\begin{array}{l}82 . \\
90 . \\
56 .\end{array}$ & $\begin{array}{l}1 . \\
1 . \\
1 .\end{array}$ & 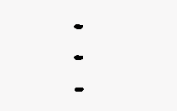 \\
\hline TOTAL SUSPENDED SOLIDS & $06 / 20 / 80$ & 0001 & $M G / L$ & & 46.00 & - & - \\
\hline URAKIUM & $\begin{array}{l}06 / 20 / 80 \\
10 / 08 / 85 \\
10 / 08 / 85 \\
10 / 08 / 85 \\
10 / 08 / 85 \\
10 / 08 / 85\end{array}$ & $\begin{array}{l}0001 \\
0001 \\
0002 \\
0003 \\
0004 \\
0005\end{array}$ & $M G / L$ & & $\begin{array}{l}0.10 \\
0.0069 \\
0.0138 \\
0.0127 \\
0.0028 \quad \mathrm{~J} \\
0.0006 \quad \mathrm{~J}\end{array}$ & $\begin{array}{l}0 . \\
0.003 \\
0.003 \\
0.003 \\
0.003 \\
0.003\end{array}$ & $\begin{array}{l}- \\
- \\
- \\
-\end{array}$ \\
\hline VAKADIUM & $\begin{array}{l}06 / 20 / 80 \\
10 / 08 / 85 \\
10 / 08 / 85 \\
10 / 08 / 85\end{array}$ & $\begin{array}{l}0001 \\
0002 \\
0003 \\
0004\end{array}$ & $M G / L$ & $\begin{array}{l}< \\
< \\
<\end{array}$ & $\begin{array}{l}0.003 \\
0.01 \\
0.01 \\
0.01\end{array}$ & $\begin{array}{l}0.003 \\
0.01 \\
0.01 \\
0.01\end{array}$ & : \\
\hline
\end{tabular}

PARAMETER VALUE IMDICATOR (PVI): < - LESS THAN DETECTION LIMIT

SAMPLE ID COOES:

OTHER PARAMETER VALUE FLAGS:

0001 - FILTERED SAMPLE (.45 MICRONS)

$J$ - ESTIMATED VALUE

0002 - FILTERED REPLICATE SAMPLE (.45 MICRONS)

0003 - FILTERED REPLICATE SAMPLE (.45 MICRONS)

0004 - FILTERED REPLICATE SAMPLE (.45 MICROHS) 
GROUNDWATER QUALITY DATA BY LOCATION

SITE: AMBO1 AMBROSIA LAKE

LOCATIOH: 0711

NORTH COORDINATE: $\quad 44620.0$ FT

EAST COORDINATE: $\quad 58430.0$ FT

O6/10/80 TO 12/04/92

REPORT DATE: 08/04/94

FORMATION OF COMPLETION: TRES KERMANOS - B SANDSTONE (TB)

HYDRAULIC FLOW RELATIOWSHIP: UPGRADIEKT (U)

\begin{tabular}{|c|c|c|c|c|c|c|c|}
\hline PARAMETER MAME & LOG DATE & $\begin{array}{c}\text { SAMPLE } \\
\text { ID }\end{array}$ & $\begin{array}{l}\text { UNITS OF } \\
\text { MEASURE }\end{array}$ & PVI & $\begin{array}{l}\text { PARAMETER } \\
\text { VALUE FLAGS }\end{array}$ & $\begin{array}{l}\text { DETECTIOH } \\
\text { LIMIT }\end{array}$ & $\begin{array}{l}\text { PARAMETER } \\
\text { UNCERTAIHTY }\end{array}$ \\
\hline VAKADIUN & $10 / 08 / 85$ & 0005 & $\mathrm{MG} / \mathrm{L}$ & & 0.1 & 0.01 & - \\
\hline ZINC & $\begin{array}{l}06 / 20 / 80 \\
10 / 08 / 85 \\
10 / 08 / 85 \\
10 / 08 / 85 \\
10 / 08 / 85\end{array}$ & $\begin{array}{l}0001 \\
0002 \\
0003 \\
0004 \\
0005\end{array}$ & $M G / L$ & & $\begin{array}{l}0.15 \\
0.013 \\
0.021 \\
0.011 \\
0.01\end{array}$ & $\begin{array}{l}- \\
0.005 \\
0.005 \\
0.005 \\
0.005\end{array}$ & $\begin{array}{l}- \\
- \\
-\end{array}$ \\
\hline
\end{tabular}

PARAMETER VALUE IMDICATOR (PVI):

< - LESS THAN DETECTION LIMIT

SAMPLE ID COOES:

0001 - FILTERED SAMPLE (.45 MICRONS)

0002 - FILTERED REPLICATE SAMPLE (.45 MICRONS)

0003 - FILTERED REPLICATE SAMPLE (.45 MICRONS)

0004 - FILTERED REPLICATE SAMPLE (.45 MICRONS) 
GROUNDWATER QUALITY DATA BY LOCATIOH

SITE: AMBOI AMBROSIA LAKE

LOCATION: 0712

NORTH COORDINATE: $\quad 58780.0$ FT

EAST COORDINATE: $\quad 53420.0$ FT

06/10/80 TO $12 / 04 / 92$

REPORT DATE: $08 / 04 / 94$

FORMATION OF COMPLETION: ALLUVIUH (AL)

HYDRAULIC FLOW RELATIOHSHIP: UNKNOWN (N)

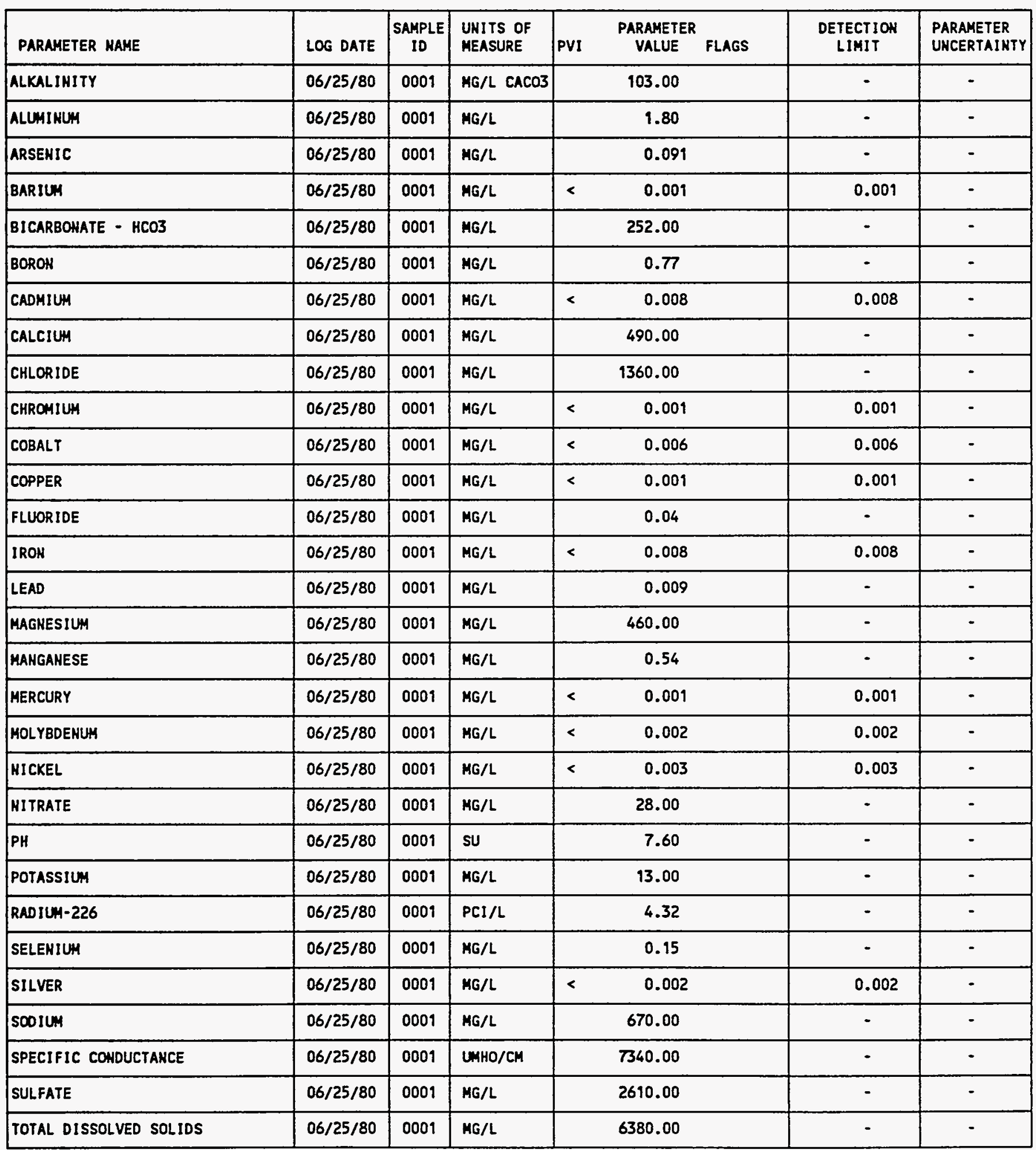

PARAMETER VALUE IMDICATOR (PVI): < - LESS THAN DETECTION LIMIT

SAMPLE ID COOES:

0001 - FILTERED SAMPLE (.45 MICRONS) 
GROUNDWATER QUALITY DATA BY LOCATION

SITE: AMBO1 AMBROSIA LAKE

LOCATION: 0712

NORTH COORDINATE: $\quad 58780.0$ FT

EAST COORDIHATE: $\quad 53420.0$ FT

O6/10/80 TO $12 / 04 / 92$

REPORT DATE: 08/04/94

FORMATION OF COMPLETION: ALLUVIUM (AL)

HYDRAULIC FLOW RELATIONSHIP: UNKNOWN (N)

\begin{tabular}{|l|c|c|l|l|c|c|c|}
\hline PARAMETER HAME & LOG DATE & $\begin{array}{c}\text { SAMPLE } \\
\text { ID }\end{array}$ & $\begin{array}{l}\text { UNITS OF } \\
\text { MEASURE }\end{array}$ & PVI & $\begin{array}{c}\text { PARAMETER } \\
\text { VALUE }\end{array}$ & $\begin{array}{c}\text { DETECTION } \\
\text { LIMIT }\end{array}$ & $\begin{array}{c}\text { PARAMETER } \\
\text { UHCERTAINTY }\end{array}$ \\
\hline TOTAL SUSPENDED SOLIDS & $06 / 25 / 80$ & 0001 & MG/L & 372.00 & - & - \\
\hline URANIUM & $06 / 25 / 80$ & 0001 & MG/L & & 0.044 & - & - \\
\hline VANADIUM & $06 / 25 / 80$ & 0001 & HG/L & $<$ & 0.003 & -0.03 & - \\
\hline ZINC & $06 / 25 / 80$ & 0001 & MG/L & & 0.14 & - & - \\
\hline
\end{tabular}

PARAMETER VALUE INDICATOR (PVI): < - LESS THAN DETECTION LIMIT

SAMPLE ID COOES:

D001 - FILTERED SAMPLE (.45 MICRONS) 
GROUNDWATER QUALITY DATA BY LOCATION

SITE: AMBOI AMBROSIA LAKE

LOCATION: 0713

NORTH COORDINATE: 59395.0 FT

EAST COORDIHATE: $\quad \mathbf{5 0 4 0 0 . 0}$ FT

06/10/80 TO $12 / 04 / 92$

REPORT DATE: 08/04/94

FORMATION OF COMPLETION: ALLUVIUM (AL)

HYDRAULIC FLON RELATIONSHIP: UNKHOWN (N)

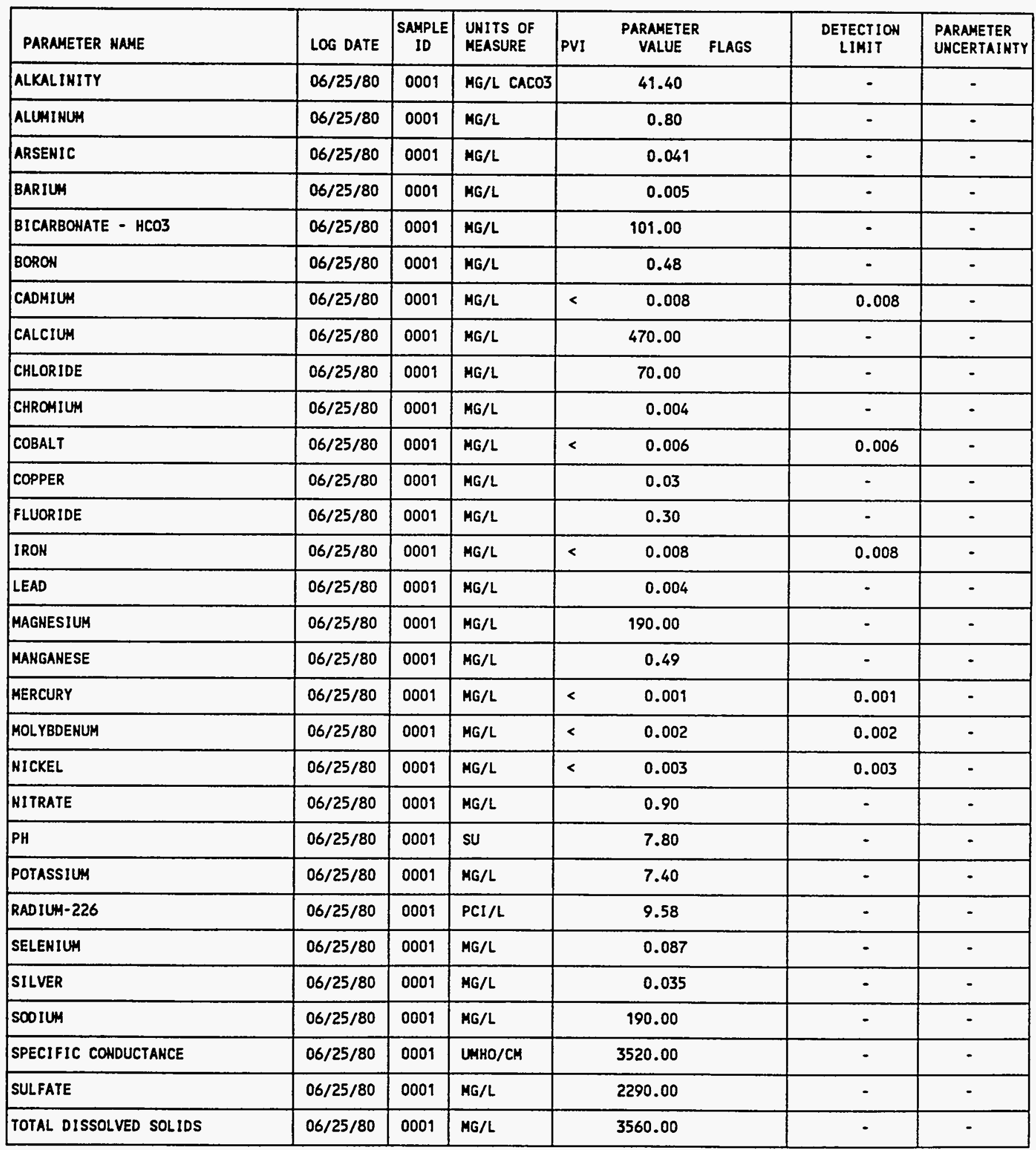

PARAMETER VAlUe IMDICATOR (PVI): < - LESS THAM DETECTION LIMIT 
GROUHDHATER OUALITY DATA BY LOCATION

SITE: AMBO1 AMBROSIA LAKE

LOCATION: 0713

NORTH COORDINATE: 59395.0 FT

EAST COORDINATE: 50400.0 FT

O6/10/80 TO $12 / 04 / 92$

REPORT DATE : 08/04/94

FORMATION OF COHPLETION: ALLUVIUM (AL)

HYDRAULIC FLOW RELATIONSHIP: UNKNOWH (N)

\begin{tabular}{|c|c|c|c|c|c|c|c|}
\hline PARAMETER NAME & LOG DATE & $\underset{\text { SAMPLE }}{\text { ID }}$ & $\begin{array}{l}\text { UNITS OF } \\
\text { MEASURE }\end{array}$ & PVI & $\begin{array}{l}\text { PARAMETER } \\
\text { VALUE FLAGS }\end{array}$ & $\begin{array}{l}\text { DETECTION } \\
\text { LIMIT }\end{array}$ & $\begin{array}{l}\text { PARAMETER } \\
\text { UNCERTAINTY }\end{array}$ \\
\hline TOTAL SUSPENDED SOLIDS & $06 / 25 / 80$ & 0001 & MG/L & & 136.00 & - & - \\
\hline URANIUM & $06 / 25 / 80$ & 0001 & MG/L & & 0.024 & - & - \\
\hline VANADIUM & $06 / 25 / 80$ & 0001 & MG/L & $<$ & 0.003 & 0.003 & - \\
\hline ZINC & $06 / 25 / 80$ & 0001 & $M G / L$ & & 0.049 & - & - \\
\hline
\end{tabular}

PARAMETER VALUE INDICATOR (PVi):

< - LESS THAN DETECTION LIMIT

SAMPLE ID COOES:

0001 - FILTERED SAMPLE (.45 MICRONS) 
GROUNDWATER QUALITY DATA BY LOCATION

SITE: AMBOI AMBROSIA LAKE

LOCATION: 0714

MORTH COORDIHATE: 50050.0 FT

EAST COORDIMATE: $\quad 53110.0$ FT

06/10/80 TO $12 / 04 / 92$

REPORT DATE: 08/04/94

FORMATION OF COMPLETION: ALLUVIUM (AL)

HYDRAULIC FLOW RELATIONSHIP: UHKNOWN (N)

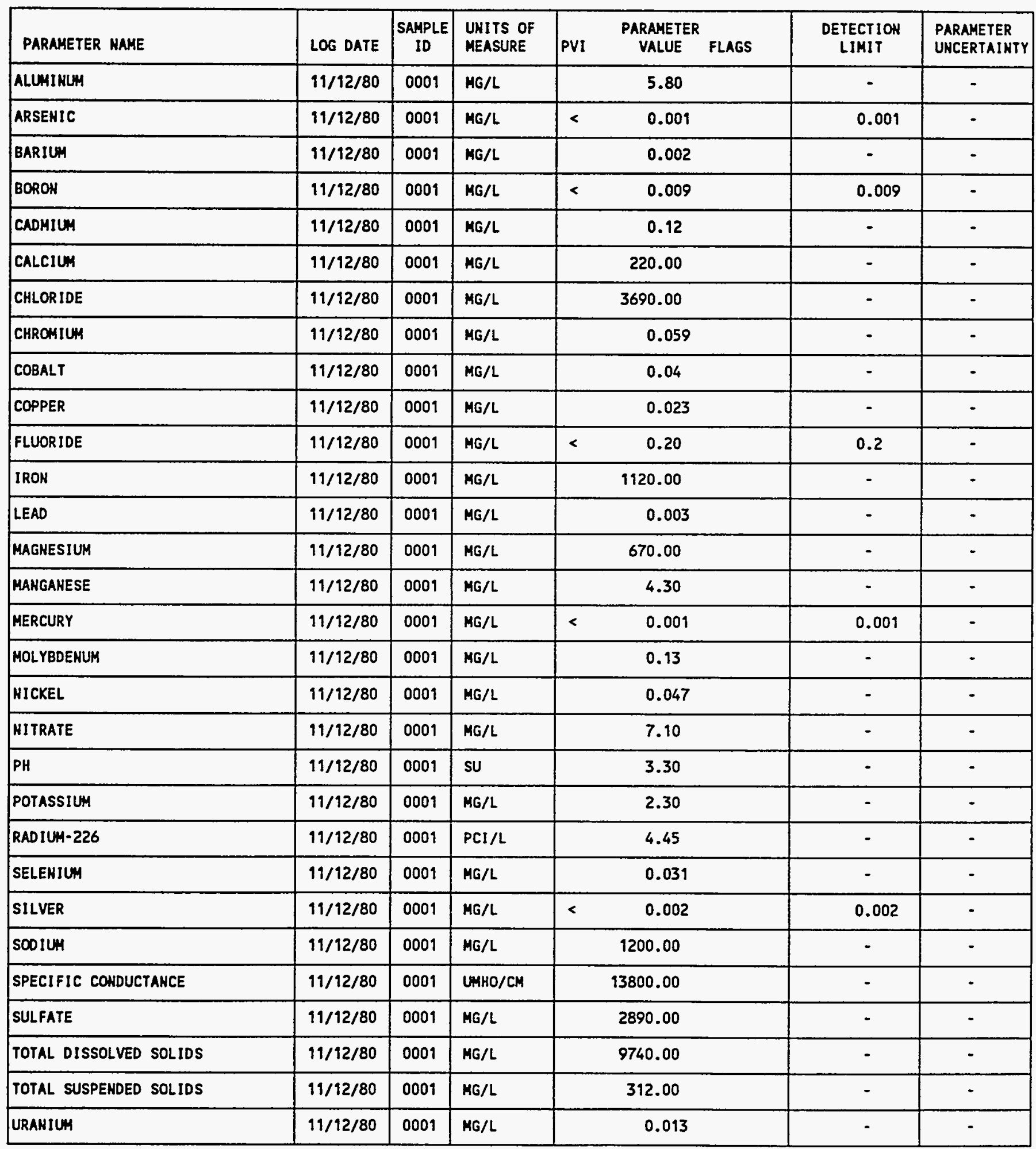

PARAMETER VALUE IHDICATOR (PVI): < - LESS THAN DETECTION LIMIT

SAMPLE ID COOES:

0001 - FILTERED SAMPLE (.45 MICRONS) 
GROUNDHATER QUALITY DATA BY LOCATION

SITE: AMBOI AMBROSIA LAKE

LOCATION: 0714

NORTH COORDINATE: $\quad 50050.0$ FT

EAST COORDINATE: $\quad 53110.0$ FT

06/10/80 TO $12 / 04 / 92$

REPORT DATE: 08/04/94

FORMATION OF COMPLETION: ALLUVIUM (AL)

HYDRAULIC FLON RELATIONSHIP: UNKNOWN (N)

\begin{tabular}{|l|c|c|l|l|c|c|c|}
\hline PARAMETER NAME & LOG DATE & $\begin{array}{c}\text { SAMPLE } \\
\text { ID }\end{array}$ & $\begin{array}{l}\text { UNITS OF } \\
\text { MEASURE }\end{array}$ & PVI & $\begin{array}{c}\text { PARAMETER } \\
\text { VALUE }\end{array}$ & $\begin{array}{c}\text { DETECTIOH } \\
\text { LIMIT }\end{array}$ & $\begin{array}{l}\text { PARAMETER } \\
\text { UNCERTAINTY }\end{array}$ \\
\hline VAKADIUM & $11 / 12 / 80$ & 0001 & MG/L & $<$ & 0.003 & 0.003 & - \\
\hline ZINC & $11 / 12 / 80$ & 0001 & MG/L & & 0.43 & - & - \\
\hline
\end{tabular}

PARAMETER VAlUe IMDICATOR (PVI): < - LESS THAN DETECTION LIMIT

SAMPLE ID COOES:

OOO1 - FILTERED SAMPLE (.45 MICRONS) 
GROUHDHATER QUALITY DATA BY LOCATION

SITE: AMBOI AMBROSIA LAKE

LOCATION: 0715

MORTH COORDIHATE: 50680.0 FT

EAST COORDIHATE: $\quad 52310.0$ FT

06/10/80 TO $12 / 04 / 92$

REPORT DATE: $08 / 04 / 94$

FORMATION OF COMPLETION: ALLUVIUM (AL)

HYDRAULIC FLOW RELATIONSHIP: UMKNONN (N)

\begin{tabular}{|c|c|c|c|c|c|c|c|}
\hline PARAMETER MAME & LOG DATE & $\begin{array}{c}\text { SAMPLE } \\
\text { ID }\end{array}$ & $\begin{array}{l}\text { UNITS OF } \\
\text { MEASURE }\end{array}$ & PVI & $\begin{array}{l}\text { PARAMETER } \\
\text { VALUE FLAGS }\end{array}$ & $\begin{array}{l}\text { DETECTION } \\
\text { LIMIT }\end{array}$ & $\begin{array}{l}\text { PARAMETER } \\
\text { UNCERTAINTY }\end{array}$ \\
\hline ALUMINUM & $11 / 12 / 80$ & 0001 & MG/L & & 5.80 & - & - \\
\hline ARSEHIC & $11 / 12 / 80$ & 0001 & MG/L & $<$ & 0.001 & 0.001 & - \\
\hline BARIUM & $11 / 12 / 80$ & 0001 & $M G / L$ & & 0.016 & - & - \\
\hline BOROH & $11 / 12 / 80$ & 0009 & $M G / L$ & $<$ & 0.009 & 0.009 & - \\
\hline CADMIUM & $11 / 12 / 80$ & 0001 & $M G / L$ & & 0.099 & - & - \\
\hline CALCIUA & $11 / 12 / 80$ & 0009 & $M G / L$ & & 350.00 & - & - \\
\hline CHLORIDE & $11 / 12 / 80$ & 0001 & MG/L & & 3340.00 & - & - \\
\hline CHROMIUM & $11 / 12 / 80$ & 0001 & $M G / L$ & & 0.062 & - & - \\
\hline COBALT & $19 / 12 / 80$ & 0001 & MG/L & & 0.031 & - & - \\
\hline COPPER & $11 / 12 / 80$ & 0001 & $M G / L$ & & 0.086 & - & - \\
\hline FLUORIDE & $11 / 12 / 80$ & 0001 & $M G / L$ & $<$ & 0.20 & 0.2 & - \\
\hline IROH & $11 / 12 / 80$ & 0001 & $M G / L$ & & 990.00 & - & - \\
\hline LEAD & $11 / 12 / 80$ & 0001 & $M G / L$ & & 0.003 & - & - \\
\hline MAGNESIUM & $11 / 12 / 80$ & 0001 & MG/L & & 1300.00 & - & - \\
\hline MANGANESE & $11 / 12 / 80$ & 0001 & MG/L & & 6.80 & - & - \\
\hline MERCURY & $11 / 12 / 80$ & 0001 & $M G / L$ & $<$ & 0.001 & 0.001 & - \\
\hline MOLYBDENUM & $11 / 12 / 80$ & 0001 & MG/L & & 0.12 & - & - \\
\hline HICKEL & $11 / 12 / 80$ & 0001 & $M G / L$ & & 0.041 & - & - \\
\hline HITRATE & $11 / 12 / 80$ & 0001 & $M G / L$ & & 6.30 & - & - \\
\hline $\mathrm{PH}$ & $11 / 12 / 80$ & 0001 & SU & & 3.00 & - & - \\
\hline POTASSIUM & $11 / 12 / 80$ & 0001 & MG/L & & 12.00 & - & - \\
\hline RADIUM-226 & $11 / 12 / 80$ & 0001 & PCI/L & & 14.32 & - & - \\
\hline SELENIUM & $11 / 12 / 80$ & 0001 & $M G / L$ & & 0.12 & - & - \\
\hline SILVER & $11 / 12 / 80$ & 0001 & $M G / L$ & $<$ & 0.002 & 0.002 & - \\
\hline soolum & $11 / 12 / 80$ & 0001 & MG/L & & 1400.00 & - & - \\
\hline SPECIFIC CONDUCTANCE & $11 / 12 / 80$ & 0001 & $\mathrm{UHHO} / \mathrm{CH}$ & & 13200.00 & - & - \\
\hline SULFATE & $11 / 12 / 80$ & 0001 & $M G / L$ & & 3170.00 & - & - \\
\hline TOTAL DISSOLVED SOLIDS & $11 / 12 / 80$ & 0001 & $M G / L$ & & 9090.00 & - & - \\
\hline TOTAL SUSPENDED SOLIDS & $19 / 12 / 80$ & 0001 & $M G / L$ & & 537.00 & - & - \\
\hline URAKIUM & $11 / 12 / 80$ & 0001 & $M G / L$ & & 0.04 & - & - \\
\hline
\end{tabular}

PARAMETER VALUE INDICATOR (PVI):

< - LESS THAN DETECTION LIMIT

SAMPLE 10 COOES:

0001 - FILTERED SAMPLE (.45 MICRONS) 
GROUNDWATER QUALITY DATA BY LOCATION

SITE: AMBO1 AMBROSIA LAKE

LOCATION: 0715

HORTH COORDINATE: $\quad 50680.0$ FT

EAST COORDINATE: $\quad 52310.0$ FT

$06 / 10 / 80$ TO $12 / 04 / 92$

REPORT DATE: $08 / 04 / 94$

FORMATION OF COMPLETION: ALLUVIUM (AL)

HYDRAULIC FLOW RELATIONSHIP: UNKNOWN (N)

\begin{tabular}{|l|c|c|l|l|l|c|c|}
\hline PARAMETER NAME & LOG DATE & $\begin{array}{c}\text { SAMPLE } \\
\text { ID }\end{array}$ & $\begin{array}{l}\text { UNITS OF } \\
\text { MEASURE }\end{array}$ & PVI & $\begin{array}{l}\text { PARAMETER } \\
\text { VALUE }\end{array}$ FLAGS & $\begin{array}{l}\text { DETECTION } \\
\text { LIMIT }\end{array}$ & $\begin{array}{l}\text { PARAMETER } \\
\text { UNCERTAINTY }\end{array}$ \\
\hline VANADIUN & $11 / 12 / 80$ & 0001 & MG/L & $<$ & 0.003 & 0.003 & - \\
\hline ZIMC & $11 / 12 / 80$ & 0001 & MG/L & & 0.52 & - & - \\
\hline
\end{tabular}

PARAMETER VALUE INDICATOR (PVI): < - LESS THAN DETECTION LIMIT

SAMPLE ID COOES:

0001 - FILTERED SAMPLE (.45 MICRONS) 
GROUNDWATER QUALITY DATA BY LOCATION

SITE: AMBOI AMBROSIA LAKE

LOCATION: 0716

NORTH COORDINATE: $\quad 48800.0$ FT

EAST COORDINATE: $\quad 53510.0$ FT

06/10/80 TO 12/04/92

REPORT DATE: 08/04/94

FORMATION OF COMPLETION: ALLUVIUM (AL)

HYDRAULIC FLON RELATIONSHIP: UNKHOWN (H)

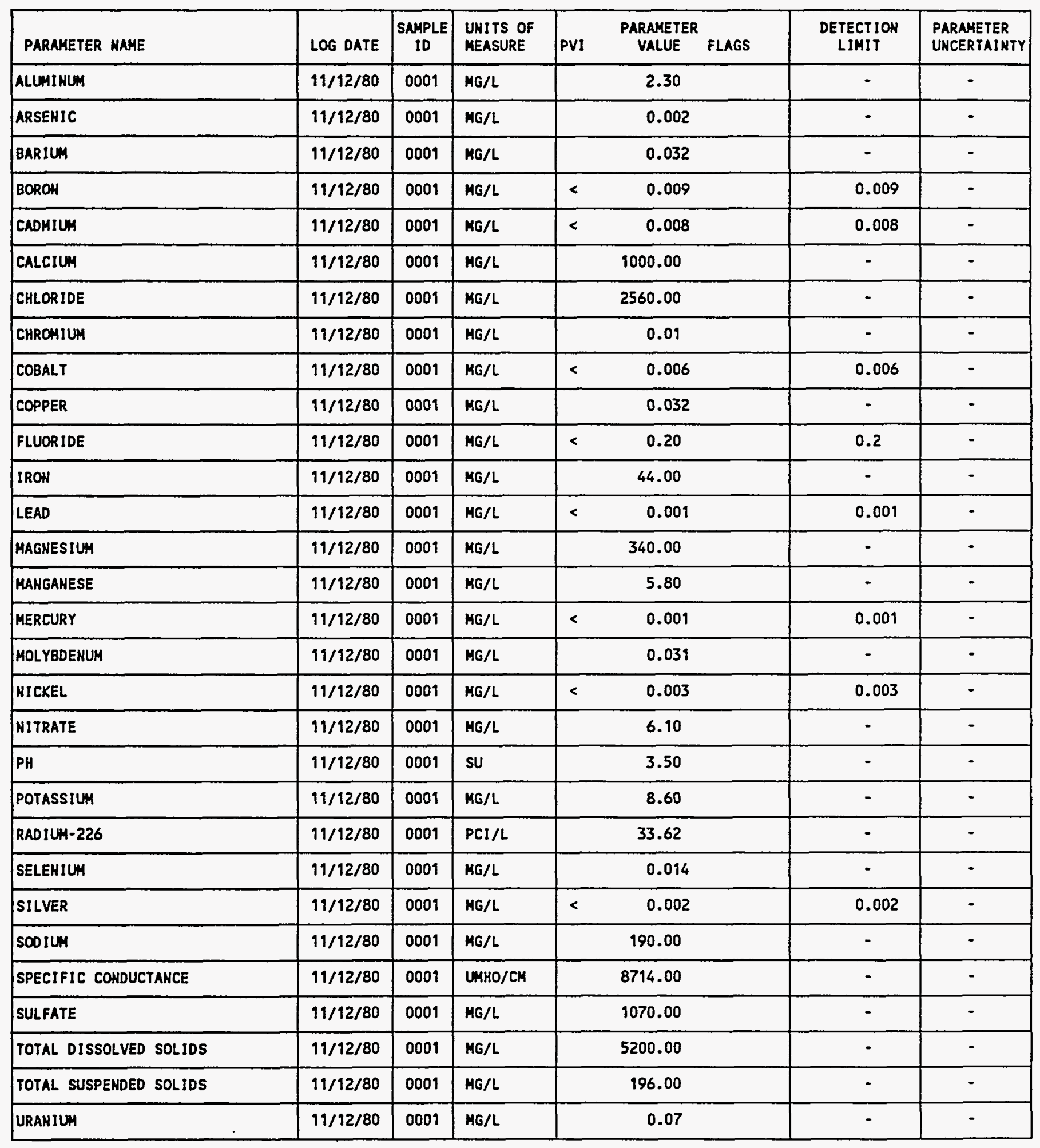

PARAMETER VALUE IMDICATOR (PVI):

< - LESS THAN DETECTION LIMIT

SAMPLE ID COOES:

0001 - FILTERED SAMPLE (.45 HICRONS) 
GROUNDWATER QUALITY DATA BY LOCATION

SITE: AMBOI AMBROSIA LAKE

LOCATION: 0716

NORTH COORDINATE: 48800.0 FT

EAST COORDINATE: $\quad 53510.0$ FT

$06 / 10 / 80$ TO $12 / 04 / 92$

REPORT DATE: $08 / 04 / 94$

FORMATION OF COMPLETIOH: ALLUVIUM (AL)

HYDRAULIC FLON RELATIONSHIP: UNKMOWN (N)

\begin{tabular}{|c|c|c|c|c|c|c|c|}
\hline PARAMETER MAME & LOG DATE & $\begin{array}{c}\text { SAMPLE } \\
\text { ID }\end{array}$ & $\begin{array}{l}\text { UNITS OF } \\
\text { MEASURE }\end{array}$ & PVI & $\begin{array}{l}\text { PARAMETER } \\
\text { VALUE FLAGS }\end{array}$ & $\begin{array}{l}\text { DETECTIOH } \\
\text { LIMIT }\end{array}$ & $\begin{array}{l}\text { PARAMETER } \\
\text { UNCERTAINTY }\end{array}$ \\
\hline VAKADIUH & $11 / 12 / 80$ & 0001 & MG/L & & 0.11 & - & - \\
\hline ZINC & $11 / 12 / 80$ & 0001 & MG/L & & 0.18 & - & - \\
\hline
\end{tabular}

PARAMETER VALUE INDICATOR (PVI): < - LESS THAN DETECTION LIMIT

SAMPLE ID COOES:

O001 - FILTERED SAMPLE (.45 MICRONS) 
GROUNDWATER QUALITY DATA BY LOCATION

SITE: AMBO1 AMBROSIA LAKE

LOCATION: 0717

HORTH COORDINATE: $\quad 48090.0$ FT

EAST COORDIMATE: $\quad 53510.0$ FT

06/10/80 TO 12/04/92

REPORT DATE: 08/04/94

FORMATION OF COMPLETION: MANCOS SHALE (KM)

HYDRAULIC FLON RELATIOHSHIP: UHKHOWN (N)

\begin{tabular}{|c|c|c|c|c|c|c|}
\hline PARAMETER NAME & LOG DATE & $\underset{10}{\text { SAMPLE }}$ & $\begin{array}{l}\text { UNITS OF } \\
\text { MEASURE }\end{array}$ & $\begin{array}{l}\text { PARAMETER } \\
\text { PVI VALUE FLAGS }\end{array}$ & $\begin{array}{l}\text { DETECTION } \\
\text { LIMIT }\end{array}$ & $\begin{array}{l}\text { PARAMETER } \\
\text { UNCERTAINTY }\end{array}$ \\
\hline ALKALINITY & $11 / 12 / 80$ & 0001 & MG/L CACO3 & 381.00 & - & - \\
\hline ALLMINUM & $11 / 12 / 80$ & 0001 & $M G / L$ & 0.05 & 0.05 & - \\
\hline ARSENIC & $11 / 12 / 80$ & 0001 & MG/L & 0.004 & $\cdot$ & - \\
\hline BICARBONATE - HCO3 & $11 / 12 / 80$ & 0001 & MG/L & 930.00 & - & - \\
\hline 8ORON & $11 / 12 / 80$ & 0001 & MG/L & 0.009 & 0.009 & - \\
\hline CADMIUH & $11 / 12 / 80$ & 0001 & MG/L & 0.008 & 0.008 & - \\
\hline CALCIUM & $11 / 12 / 80$ & 0001 & $M G / L$ & 45.00 & - & - \\
\hline COBALT & $11 / 12 / 80$ & 0001 & MG/L & 0.006 & 0.006 & - \\
\hline COPPER & $11 / 12 / 80$ & 0001 & $M G / L$ & 00.001 & 00.001 & $\cdot$ \\
\hline FLUORIDE & $11 / 12 / 80$ & 0001 & $M G / L$ & 0.20 & 0.2 & - \\
\hline IRON & $11 / 12 / 80$ & 0001 & $M G / L$ & 0.34 & - & - \\
\hline LEAD & $11 / 12 / 80$ & 0001 & MG/L & 0.001 & - & - \\
\hline MAGNESIUM & $11 / 12 / 80$ & 0001 & MG/L & 760.00 & - & - \\
\hline MANGAHESE & $11 / 12 / 80$ & 0001 & $M G / L$ & 0.19 & - & - \\
\hline PH & $11 / 12 / 80$ & 0001 & SU & 7.90 & - & - \\
\hline POTASSIUM & $11 / 12 / 80$ & 0001 & MG/L & 22.00 & - & - \\
\hline RADIUH-226 & $11 / 12 / 80$ & 0001 & $\mathrm{PCI} / \mathrm{L}$ & 4.31 & - & - \\
\hline SELEKIUH & $11 / 12 / 80$ & 0001 & $M G / L$ & 0.053 & - & - \\
\hline SILVER & $19 / 12 / 80$ & 0001 & MG/L & 0.002 & 0.002 & - \\
\hline SODIUH & $11 / 12 / 80$ & 0001 & $M G / L$ & 1200.00 & - & - \\
\hline SPECIFIC CONDUCTANCE & $11 / 12 / 80$ & 0001 & UMHO/CH & 10000.00 & - & - \\
\hline SULFATE & $11 / 12 / 80$ & 0001 & $M G / L$ & 2688.00 & - & - \\
\hline TOTAL DISSOLVED SOLIDS & $11 / 12 / 80$ & 0001 & $M G / L$ & 7780.00 & - & - \\
\hline
\end{tabular}

PARAMETER VALUE INDICATOR (PVI):

< - LESS THAN DETECTION LIMIT

SAMPLE ID COOES:

0001 - FILTERED SAMPLE (.45 MICRONS) 
GROUNDWATER QUALITY DATA BY LOCATION

SITE: AMBO1 AMBROSIA LAKE

LOCATION: 0717

NORTH COORDINATE: $\quad 48090.0$ FT

EAST COORDINATE: $\quad 53510.0$ FT

O6/10/80 TO 12/04/92

REPORT DATE: 08/04/94

FORMATION OF COMPLETION: MANCOS SHALE (KM)

HYDRAULIC FLOW RELATIONSHIP: UNKNONN (N)

\begin{tabular}{|c|c|c|c|c|c|c|c|}
\hline PARAMETER NAME & LOG DATE & $\underset{\text { SD }}{\text { SAMPLE }}$ & $\begin{array}{l}\text { UNITS OF } \\
\text { MEASURE }\end{array}$ & PVI & $\begin{array}{l}\text { PARAMETER } \\
\text { VALUE FLAGS }\end{array}$ & $\begin{array}{l}\text { DETECTION } \\
\text { LIMIT }\end{array}$ & $\begin{array}{l}\text { PARAMETER } \\
\text { UHCERTAINTY }\end{array}$ \\
\hline TOTAL SUSPENDED SOLIDS & $11 / 12 / 80$ & 0001 & MG/L & & 81.00 & - & - \\
\hline URANIUM & $11 / 12 / 80$ & 0001 & $M G / L$ & & 0.06 & - & - \\
\hline VANADIUM & $11 / 12 / 80$ & 0001 & $M G / L$ & $<$ & 0.003 & 0.003 & - \\
\hline ZINC & $11 / 12 / 80$ & 0001 & $M G / L$ & & 0.23 & - & - \\
\hline
\end{tabular}

PARAMETER VALUE INDICATOR (PVI):

SAMPLE ID CODES:

0001 - FILTERED SAMPLE (.45 MICROHS) 
GROUNDHATER OUALITY DATA BY LOCATION

SITE: AMBO1 AMBROSIA LAKE

LOCATION: 0718

NORTH COORDINATE: 51470.0 FT

EAST COORDINATE: $\quad 56950.0$ FT

06/10/80 TO $12 / 04 / 92$

REPORT DATE: 08/04/94

FORMATION OF COMPLETION: ALLUVIUM (AL)

HYDRAULIC FLON RELATIONSHIP: UNKHOWN (N)

\begin{tabular}{|c|c|c|c|c|c|c|c|}
\hline PARAKETER MAHE & LOG DATE & $\begin{array}{c}\text { SAMPLE } \\
10\end{array}$ & $\begin{array}{l}\text { UNITS OF } \\
\text { MEASURE }\end{array}$ & PVI & $\begin{array}{l}\text { PARAMETER } \\
\text { VALUE FLAGS }\end{array}$ & $\begin{array}{l}\text { DETECTION } \\
\text { LIMIT }\end{array}$ & $\begin{array}{l}\text { PARAMETER } \\
\text { UMCERTAINTY }\end{array}$ \\
\hline ALKALINITY & $\begin{array}{l}06 / 25 / 80 \\
10 / 16 / 85 \\
05 / 17 / 86\end{array}$ & $\begin{array}{l}0001 \\
0001 \\
0001\end{array}$ & $\mathrm{MG} / \mathrm{L} \mathrm{CACO} 3$ & & $\begin{array}{l}149.00 \\
476 . \\
817 .\end{array}$ & : & : \\
\hline ALUMIRUA & $06 / 25 / 80$ & 0001 & MG/L & & 0.89 & - & - \\
\hline ARSEHIC & $\begin{array}{l}06 / 25 / 80 \\
10 / 16 / 85 \\
05 / 17 / 86\end{array}$ & $\begin{array}{l}0001 \\
0001 \\
0001\end{array}$ & MG/L & $<$ & $\begin{array}{l}0.18 \\
0.01 \\
0.01\end{array}$ & $\begin{array}{l}- \\
0.01 \\
0.01\end{array}$ & : \\
\hline BARIUM & $06 / 25 / 80$ & 0001 & $M G / L$ & $<$ & 0.001 & 0.001 & - \\
\hline BICARBONATE - HCO3 & $06 / 25 / 80$ & 0001 & MG/L & & 363.00 & - & - \\
\hline BORON & $\begin{array}{l}06 / 25 / 80 \\
05 / 17 / 86\end{array}$ & $\begin{array}{l}0001 \\
0001\end{array}$ & $M G / L$ & & $\begin{array}{l}0.91 \\
0.7\end{array}$ & 0.1 & - \\
\hline CADMIUH & $\begin{array}{l}06 / 25 / 80 \\
05 / 17 / 86\end{array}$ & $\begin{array}{l}0001 \\
0001\end{array}$ & $M G / L$ & $<$ & $\begin{array}{l}0.008 \\
0.001\end{array}$ & $\begin{array}{l}0.008 \\
0.001\end{array}$ & - \\
\hline CALCIUM & $\begin{array}{l}06 / 25 / 80 \\
10 / 16 / 85 \\
05 / 17 / 86\end{array}$ & $\begin{array}{l}0001 \\
0001 \\
0001\end{array}$ & $M G / L$ & & $\begin{array}{l}460.00 \\
446 . \\
363 .\end{array}$ & $\begin{array}{l}- \\
0.01 \\
0.01\end{array}$ & $\begin{array}{l}- \\
-\end{array}$ \\
\hline CHLORIDE & $\begin{array}{l}06 / 25 / 80 \\
10 / 16 / 85 \\
05 / 17 / 86\end{array}$ & $\begin{array}{l}0001 \\
0001 \\
0001\end{array}$ & $M G / L$ & & $\begin{array}{l}184.00 \\
190 . \\
180 .\end{array}$ & 1. & : \\
\hline CHROMIUM & $\begin{array}{l}06 / 25 / 80 \\
05 / 17 / 86\end{array}$ & $\begin{array}{l}0001 \\
0001\end{array}$ & MG/L & $<$ & $\begin{array}{l}0.001 \\
0.04\end{array}$ & $\begin{array}{l}0.001 \\
0.01\end{array}$ & $\because$ \\
\hline COBALT & $\begin{array}{l}06 / 25 / 80 \\
05 / 17 / 86\end{array}$ & $\begin{array}{l}0001 \\
0001\end{array}$ & MG/L & $<$ & $\begin{array}{l}0.006 \\
0.09\end{array}$ & $\begin{array}{l}0.006 \\
0.05\end{array}$ & $\dot{-}$ \\
\hline COPPER & $06 / 25 / 80$ & 0001 & MG/L & & 0.015 & - & $\cdot$ \\
\hline FLUORIDE & $\begin{array}{l}06 / 25 / 80 \\
05 / 17 / 86\end{array}$ & $\begin{array}{l}0001 \\
0001\end{array}$ & MG/L & & $\begin{array}{l}0.25 \\
0.4\end{array}$ & 0.1 & - \\
\hline IRON & $\begin{array}{l}06 / 25 / 80 \\
05 / 17 / 86\end{array}$ & $\begin{array}{l}0001 \\
0001\end{array}$ & $M G / L$ & & $\begin{array}{l}0.022 \\
0.61\end{array}$ & 0.03 & - \\
\hline LEAD & $06 / 25 / 80$ & 0001 & $M G / L$ & & 0.01 & - & - \\
\hline MAGNESIUM & $\begin{array}{l}06 / 25 / 80 \\
10 / 16 / 85 \\
05 / 17 / 86\end{array}$ & $\begin{array}{l}0001 \\
0001 \\
0001\end{array}$ & MG/L & & $\begin{array}{l}240.00 \\
395 . \\
246 .\end{array}$ & $\begin{array}{l}0 \\
0.001 \\
0.001\end{array}$ & - \\
\hline MAHGAMESE & $\begin{array}{l}06 / 25 / 80 \\
05 / 17 / 86\end{array}$ & $\begin{array}{l}0001 \\
0001\end{array}$ & MG/L & & $\begin{array}{l}0.012 \\
0.12\end{array}$ & 0.01 & - \\
\hline MERCURY & $06 / 25 / 80$ & 0001 & MG/L & $<$ & 0.001 & 0.001 & - \\
\hline MOLYBDENUH & $\begin{array}{l}06 / 25 / 80 \\
10 / 16 / 85 \\
05 / 17 / 86\end{array}$ & $\begin{array}{l}0001 \\
0001 \\
0001\end{array}$ & MG/L & & $\begin{array}{l}0.046 \\
0.17 \\
0.22\end{array}$ & $\begin{array}{l}- \\
0.01 \\
0.01\end{array}$ & : \\
\hline HICKEL & $06 / 25 / 80$ & 0001 & MG/L & & 0.023 & - & - \\
\hline
\end{tabular}

PARAMETER VALUE IHDICATOR (PVI): < - LESS THAN DETECTION LIMIT

SAMPLE ID CODES:

0001 - FILTERED SAMPLE (.45 MICRONS) 
GROUNDWATER OUALITY DATA BY LOCATION

SITE: AMBO1 AMBROSIA LAKE

LOCATION: 0718

MORTH COORDIMATE: $\quad 51470.0$ FT

EAST COORDINATE: $\quad 56950.0$ FT

06/10/80 TO 12/04/92

REPORT DATE: 08/04/94

FORMATION OF COMPLETION: ALLUVIUM (AL)

HYDRAULIC FLOW RELATIONSHIP: UNKHOWH (K)

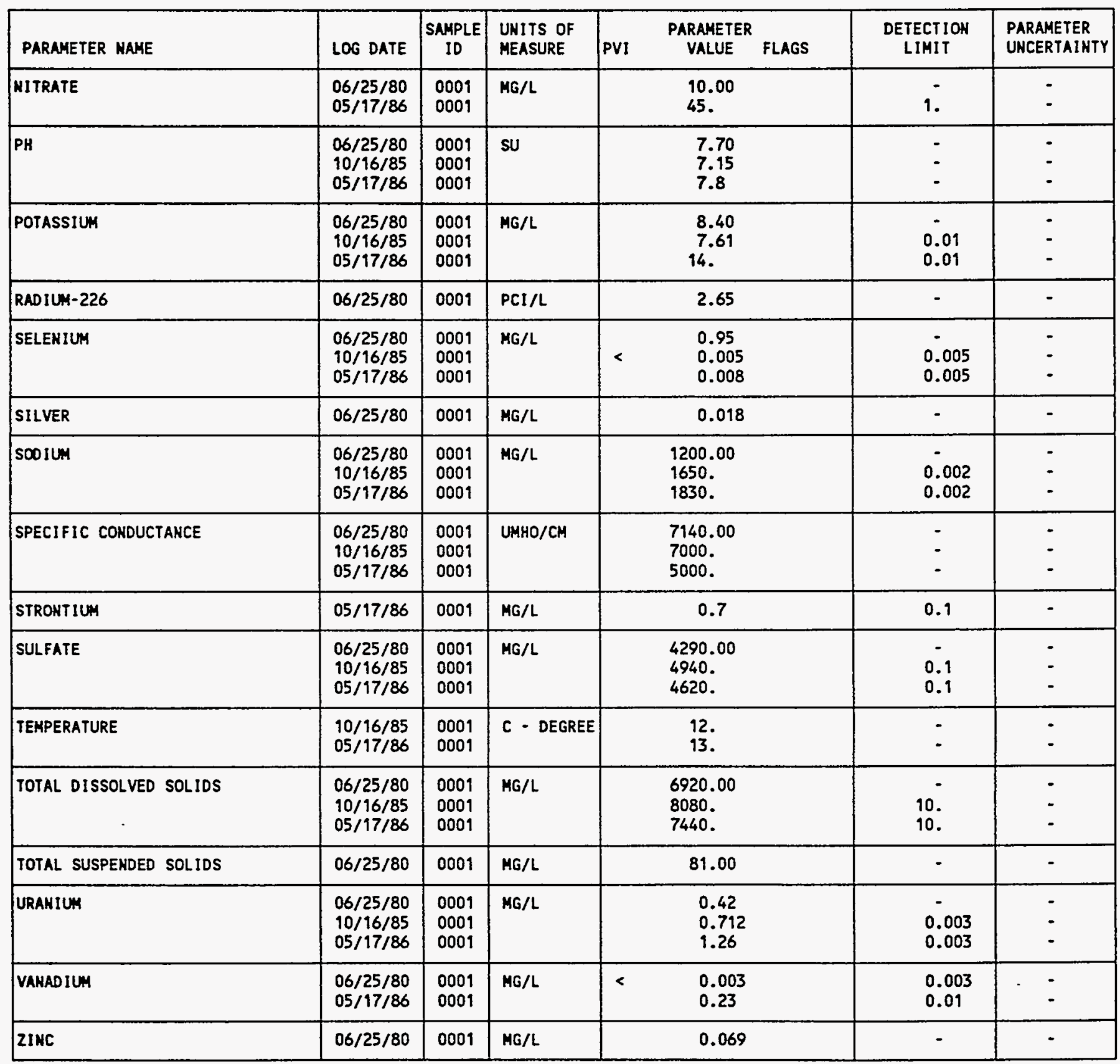

PARAMETER VALUE INDICATOR (PVI):

< - LESS THAN DETECTION LIMIT

SAMPLE ID CODES:

0001 - FILTERED SAMPLE (.45 MICROHS) 
FORMATIOH OF COMPLETIOH: ALLUVIUH (AL)

HYDRAULIC FLON RELATIONSHIP: UNKNOWN (N)

\begin{tabular}{|c|c|c|c|c|c|c|}
\hline PARAMETER NAME & LOG DATE & $\underset{\text { SAMPLE }}{\text { ID }}$ & $\begin{array}{l}\text { UNITS OF } \\
\text { MEASURE }\end{array}$ & $\begin{array}{l}\text { PARAMETER } \\
\text { PVI VALUE FLAGS }\end{array}$ & $\begin{array}{l}\text { DETECTIOH } \\
\text { LIMIT }\end{array}$ & $\begin{array}{l}\text { PARAMETER } \\
\text { UNCERTAINTY }\end{array}$ \\
\hline ALKALINITY & $06 / 25 / 80$ & 0001 & MG/L CACO3 & 266.00 & - & - \\
\hline ALCHINUYH & $06 / 25 / 80$ & 0001 & MG/L & 3.70 & - & - \\
\hline ARSEHIC & $06 / 25 / 80$ & 0001 & $M G / L$ & 0.20 & - & - \\
\hline BARIUN & $06 / 25 / 80$ & 0001 & MG/L & 0.001 & 0.001 & - \\
\hline BICARBOHATE - HCO3 & $06 / 25 / 80$ & 0001 & MG/L & 648.00 & - & - \\
\hline BOROH & $06 / 25 / 80$ & 0001 & MG/L & 0.56 & - & - \\
\hline CADMIUM & $06 / 25 / 80$ & 0001 & $M G / L$ & 0.008 & 0.008 & - \\
\hline CALCIUM & $06 / 25 / 80$ & 0001 & MG/L & 340.00 & - & - \\
\hline CHLORIDE & $06 / 25 / 80$ & 0001 & $M G / L$ & 2350.00 & - & - \\
\hline CHROMIUH & $06 / 25 / 80$ & 0001 & $M G / L$ & 0.048 & - & - \\
\hline COBALT & $06 / 25 / 80$ & 0001 & $M G / L$ & 0.006 & 0.006 & - \\
\hline COPPER & $06 / 25 / 80$ & 0001 & $M G / L$ & 0.001 & 0.001 & - \\
\hline FLUORIDE & $06 / 25 / 80$ & 0001 & $M G / L$ & 0.07 & - & - \\
\hline IRON & $06 / 25 / 80$ & 0001 & $M G / L$ & 0.48 & - & - \\
\hline LEAD & $06 / 25 / 80$ & 0001 & $M G / L$ & 0.033 & - & - \\
\hline MAGNESIUM & $06 / 25 / 80$ & 0001 & $M G / L$ & 990.00 & - & - \\
\hline MANGANESE & $06 / 25 / 80$ & 0001 & $M G / L$ & 0.89 & - & - \\
\hline MERCURY & $06 / 25 / 80$ & 0001 & MG/L & 0.001 & 0.001 & - \\
\hline MOLYBDENUM & $06 / 25 / 80$ & 0001 & $M G / L$ & 0.002 & - & - \\
\hline HICKEL & $06 / 25 / 80$ & 0001 & $M G / L$ & 0.003 & 0.003 & - \\
\hline hitrate & $06 / 25 / 80$ & 0001 & $M G / L$ & 4.70 & - & - \\
\hline PH & $06 / 25 / 80$ & 0001 & su & 7.50 & - & - \\
\hline POTASSIUM & $06 / 25 / 80$ & 0001 & $M G / L$ & 14.00 & - & - \\
\hline RADIUM-226 & $06 / 25 / 80$ & 0001 & $\mathrm{PCI} / \mathrm{L}$ & 3.52 & - & - \\
\hline SELENIUM & $06 / 25 / 80$ & 0001 & MG/L & 0.20 & - & - \\
\hline SILVER & $06 / 25 / 80$ & 0001 & $M G / L$ & 0.002 & 0.002 & - \\
\hline SOOIUM & $06 / 25 / 80$ & 0001 & MG/L & 1500.00 & - & - \\
\hline SPECIFIC CONDUCTANCE & $06 / 25 / 80$ & 0001 & UMHO/CH & 12100.00 & - & - \\
\hline SULFATE & $06 / 25 / 80$ & 0001 & MG/L & 4620.00 & - & - \\
\hline TOTAL DISSOLVED SOLIDS & $06 / 25 / 80$ & 0001 & $M G / L$ & 11300.00 & - & - \\
\hline
\end{tabular}

PARAMETER VALUE IMDICATOR (PVI): < - LESS THAN DETECTION LIMIT

SAMPLE ID COOES:

0001 - FILTERED SAMPLE (.45 MICRONS) 
GROUNDWATER QUALITY DATA BY LOCATIOH

SITE: AMBO1 AMBROSIA LAKE

LOCATION: 0719

NORTH COORDINATE: $\quad 47800.0$ FT

EAST COORDINATE: $\quad 54605.0$ FT

06/10/80 TO $12 / 04 / 92$

REPORT DATE: $08 / 04 / 94$

FORMATION OF COMPLETION: ALLUVIUM (AL)

HYDRAULIC FLOW RELATIONSHIP: UNKNONN (N)

\begin{tabular}{|c|c|c|c|c|c|c|c|}
\hline PARAMETER NAME & LOG DATE & $\begin{array}{c}\text { SAMPLE } \\
\text { ID }\end{array}$ & $\begin{array}{l}\text { UNITS OF } \\
\text { MEASURE }\end{array}$ & PVI & $\begin{array}{l}\text { PARAMETER } \\
\text { VALUE FLAGS }\end{array}$ & $\begin{array}{l}\text { DETECTIOH } \\
\text { LIMIT }\end{array}$ & $\begin{array}{l}\text { PARAMETER } \\
\text { UHCERTAINTY }\end{array}$ \\
\hline TOTAL SUSPENDED SOLIDS & $06 / 25 / 80$ & 0001 & $M G / L$ & & 478.00 & - & - \\
\hline URAKIUM & $06 / 25 / 80$ & 0009 & $M G / L$ & & 0.025 & - & - \\
\hline VAMADIUN & $06 / 25 / 80$ & 0001 & $M G / L$ & $<$ & 0.003 & 0.003 & - \\
\hline ZINC & $06 / 25 / 80$ & 0001 & MG/L & & 0.27 & - & - \\
\hline
\end{tabular}

PARAMETER VALUE INDICATOR (PVI):

< - LESS THAN DETECTION LIMIT

SAMPLE IO COOES:

0009 - FILTERED SAMPLE (.45 MICRONS) 
GROUNDWATER QUALITY DATA BY LOCATIOH

SITE: AMBOI AMBROSIA LAKE

LOCATION: 0720

MORTH COORDIHATE: $\quad 49600.0$ FT

EAST COORDIHATE: $\quad 54110.0$ FT

06/10/80 TO 12/04/92

REPORT DATE: 08/04/94

FORMATION OF COMPLETION: ALLUVIUN (AL)

HYDRAULIC FLON RELATIOHSHIP: UNKNONN (N)

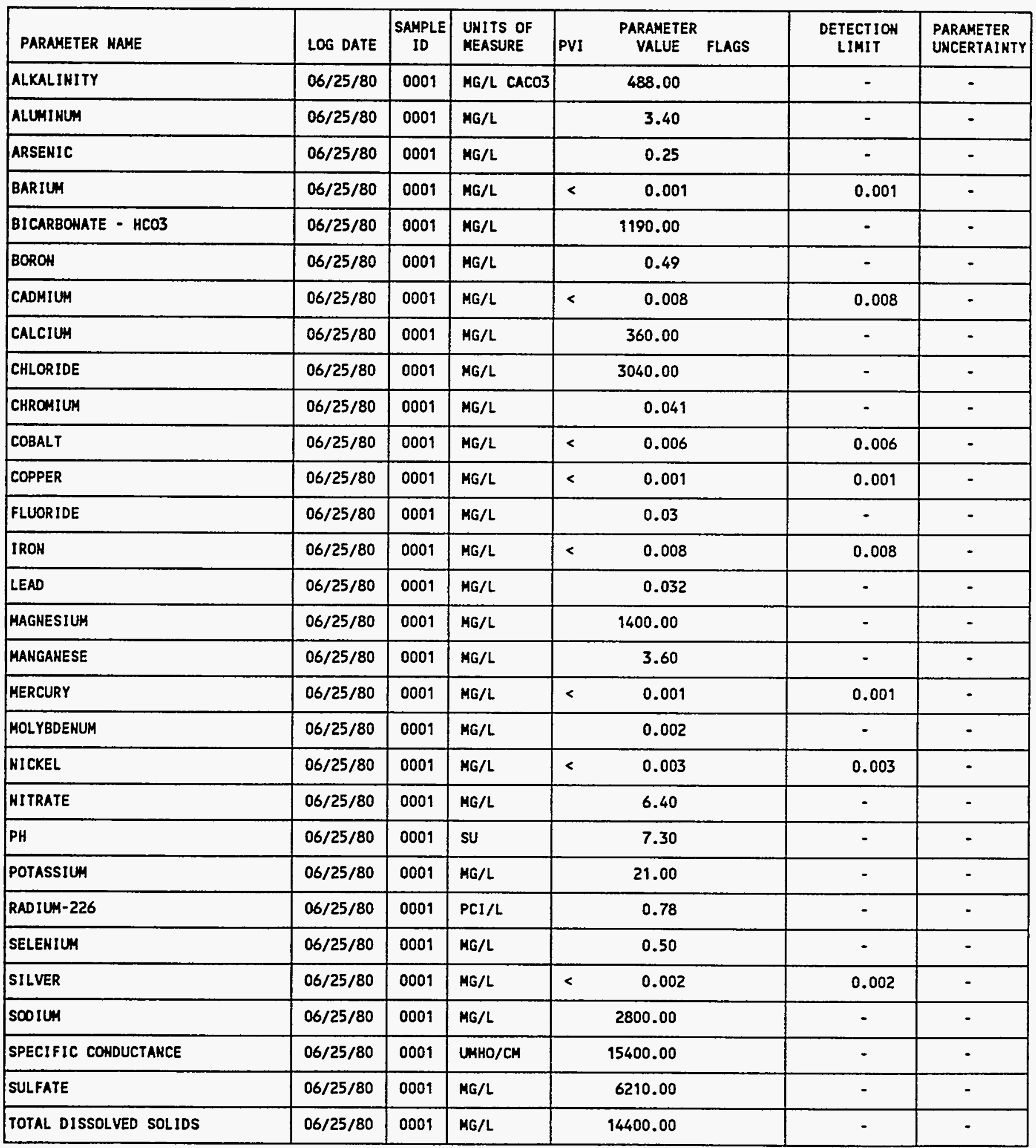

PARAMETER VALUE IHDICATOR (PVI): < - LESS THAN DETECTION LIMIT

SAMPLE ID COOES:

0001 - FILTERED SAMPLE (.45 MICRONS) 
GROUNDWATER QUALITY DATA BY LOCATION

SITE: AMBOI AMBROSIA LAKE

LOCATION: 0720

NORTH COORDINATE: 49600.0 FT

EAST COORDIHATE: $\quad 54110.0$ FT

06/10/80 TO $12 / 04 / 92$

REPORT DATE: 08/04/94

FORHATION OF COMPLETION: ALLUVIUM (AL)

HYDRAULIC FLON RELATIONSHIP: UNKNOWN (N)

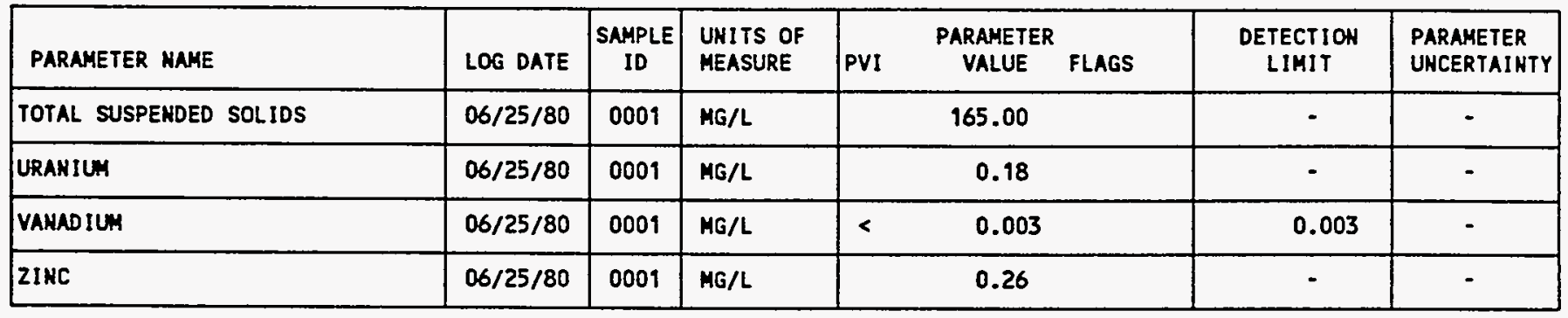

PARAMETER VALUE INDICATOR (PVI): < - LESS THAN DETECTION LIHIT SAMPLE ID COOES:

0001 - FILTERED SAMPLE (.45 MICRONS) 
GROUNDWATER QUALITY DATA BY LOCATION

SITE: AMBO1 AMBROSIA LAKE

LOCATION: 0721

HORTH COORDINATE: 51090.0 FT

EAST COORDINATE: $\quad 48190.0$ FT

06/10/80 TO $12 / 04 / 92$

REPORT DATE: 08/04/94

FORMATIOH OF COMPLETION: TRES HERMANOS-A SAHDSTONE (TR)

HYDRAULIC FLOW RELATIONSHIP: UNKHOWH (H)

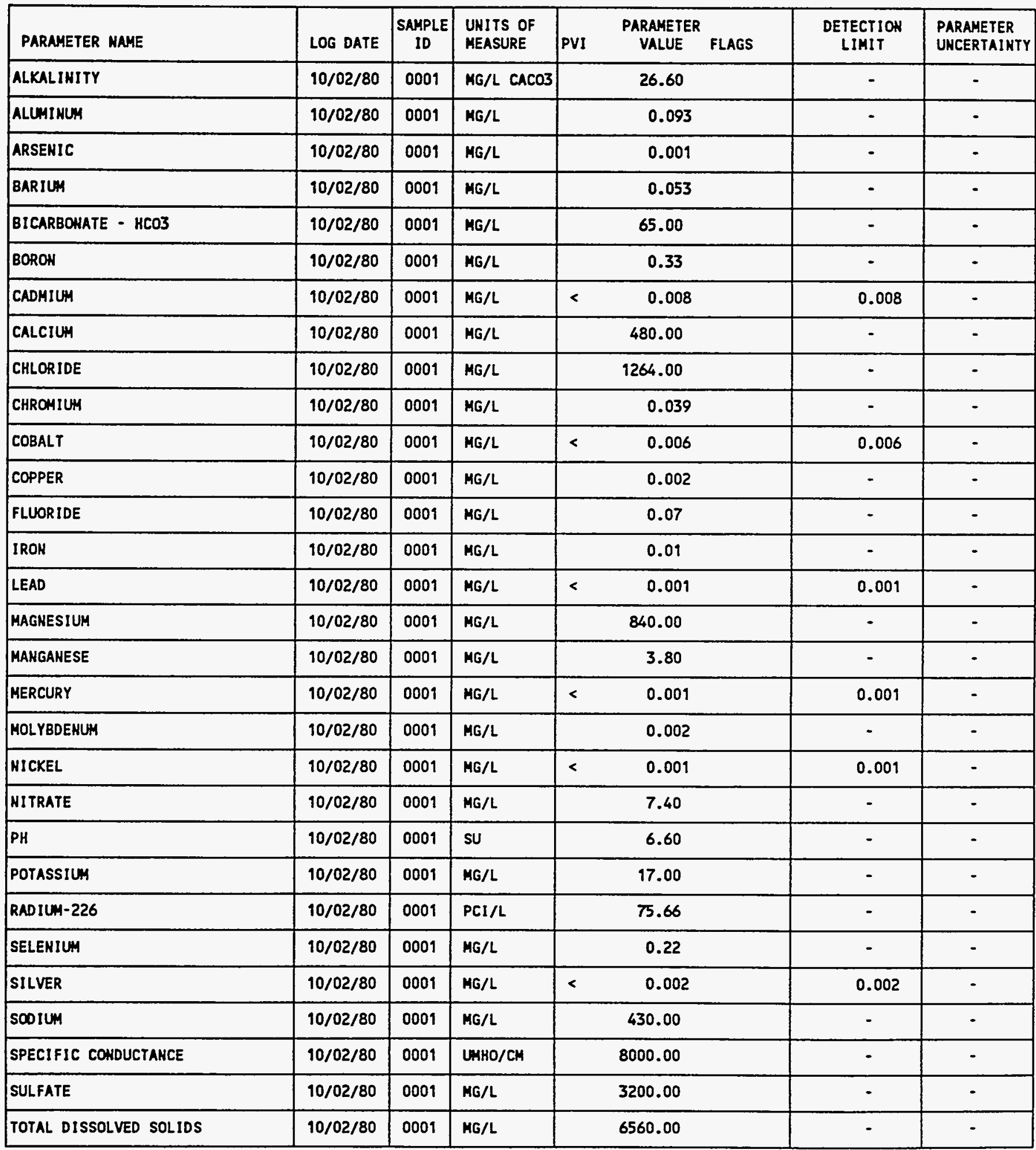

PARAMETER VALUE IMDICATOR (PVI): < - LESS THAH DETECTION LIMIT

SAMPLE ID COOES:

0001 - FILTERED SAMPLE (.45 MICRONS) 
GROUNDHATER QUALITY DATA BY LOCATION

SITE: AMBO1 AMBROSIA LAKE

LOCATIOH: 0721

MORTH COORDINATE: 51090.0 FT

EAST COORDINATE: $\quad 48190.0$ FT

06/10/80 TO $12 / 04 / 92$

REPORT DATE: $08 / 04 / 94$

FORMATION OF COMPLETION: TRES HERHANOS-A SANDSTONE (TR)

HYDRAULIC FLON RELATIONSHIP: UNKNOWN (N)

\begin{tabular}{|c|c|c|c|c|c|c|c|}
\hline PARAMETER NAME & LOG DATE & $\underset{\text { SD }}{\text { SAMPLE }}$ & $\begin{array}{l}\text { UNITS OF } \\
\text { MEASURE }\end{array}$ & PVI & $\begin{array}{l}\text { PARAMETER } \\
\text { VALUE FLAGS }\end{array}$ & $\begin{array}{l}\text { DETECTION } \\
\text { LIMIT }\end{array}$ & $\begin{array}{l}\text { PARAMETER } \\
\text { UHCERTAINTY }\end{array}$ \\
\hline TOTAL SUSPENDED SOLIDS & $10 / 02 / 80$ & 0001 & MG/L & & 46.00 & - & - \\
\hline URAKIUM & $10 / 02 / 80$ & 0001 & MG/L & & 0.02 & - & - \\
\hline VAKADIUM & $10 / 02 / 80$ & 0001 & MG/L & & 0.01 & - & - \\
\hline ZINC & $10 / 02 / 80$ & 0001 & $M G / L$ & & 0.17 & - & - \\
\hline
\end{tabular}

PARAMETER VALUE INDICATOR (PVI):

$<$ - LESS THAN DETECTION LIMIT

SAMPLE ID COOES:

0001 - FILTERED SAMPLE (.45 MICRONS) 
GROUNDWATER QUALITY DATA BY LOCATION

SITE: AMBO1 AMBROSIA LAKE

LOCATION: 0722

MORTH COORDINATE: $\quad 47820.0$ FT

EAST COORDIHATE: $\quad 59070.0$ FT

06/10/80 TO $12 / 04 / 92$

REPORT DATE: $08 / 04 / 94$

FORMATION OF COMPLETION: TRES HERMANOS-A SANDSTONE (TR)

HYDRAULIC FLOW RELATIONSHIP: UPGRADIENT (U)

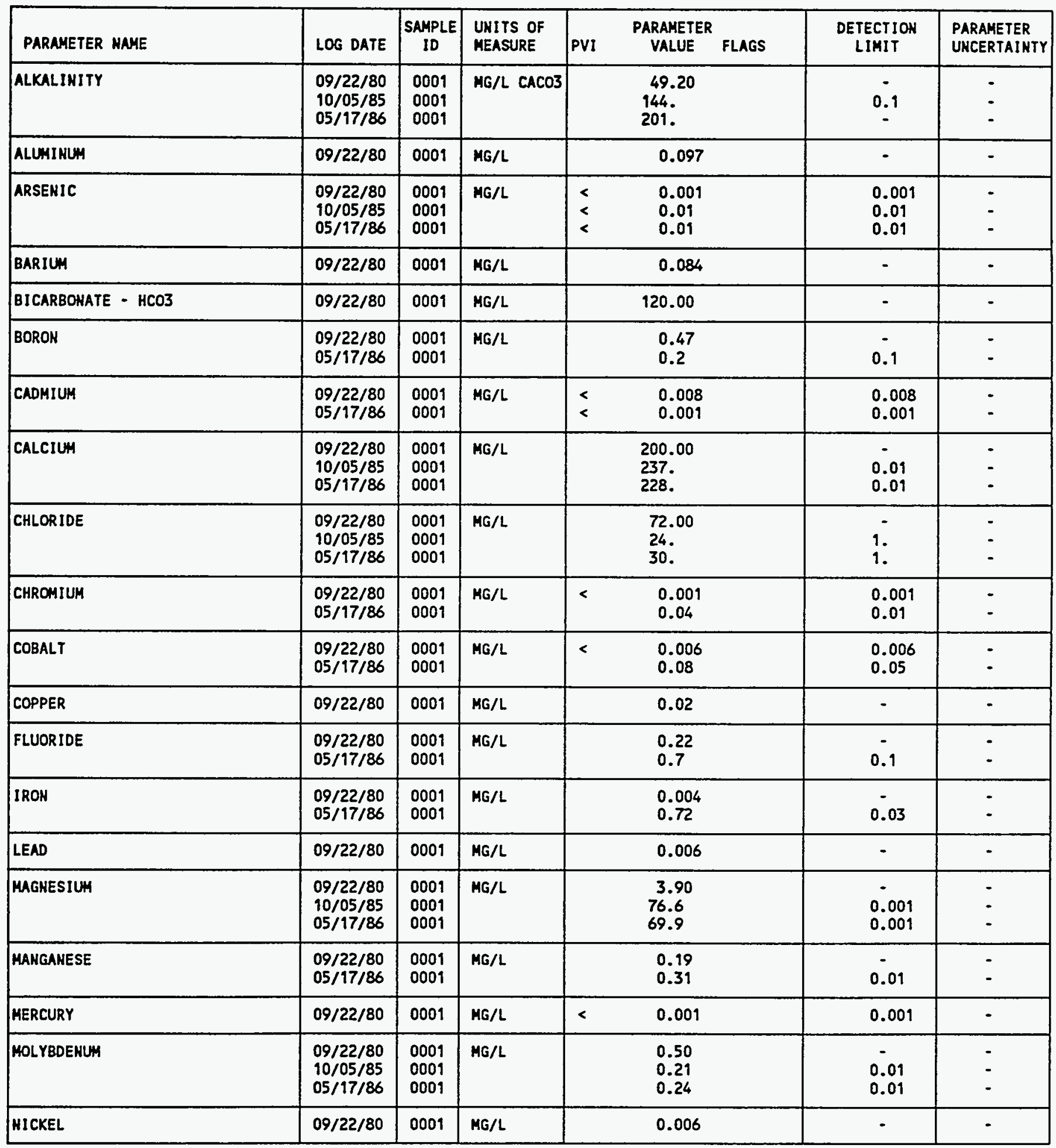

PARAMETER VALUE IMDICATOR (PVI): < - LESS THAM DETECTION LIMIT SAMPLE ID COOES:

0001 - FILTERED SAMPLE (.45 MICRONS) 
GROUNDWATER QUALITY DATA BY LOCATION

SITE: AMBO1 AMBROSIA LAKE

LOCATION: 0722

NORTH COORDINATE: 47820.0 FT

EAST COORDIHATE: 59070.0 FT

06/10/80 TO $12 / 04 / 92$

REPORT DATE: 08/04/94

FORHATION OF COMPLETION: TRES HERMANOS-A SANDSTONE (TR)

HYDRAULIC FLON RELATIONSHIP: UPGRADIENT (U)

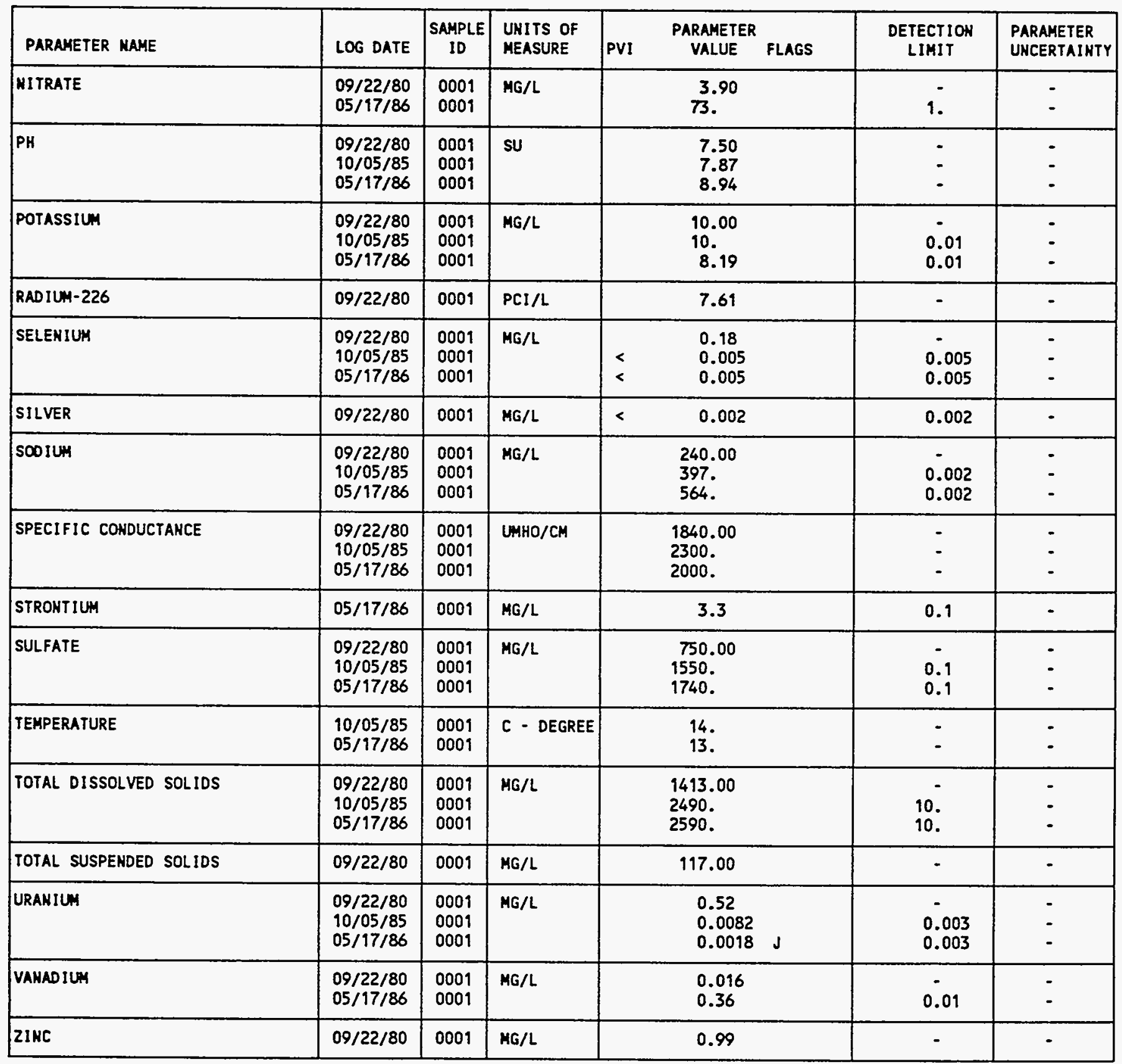

PARAMETER VALUE INDICATOR (PVI): < - LESS THAN DETECTION LIMIT

SAMPLE IO COOES:

O001 - FILTERED SAMPLE (.45 MICRONS)

OTHER PARAMETER VALUE FLAGS:

J - ESTIMATED VALUE 
GROUHDHATER QUALITY DATA BY LOCATION

SITE: AMBOI AMBROSIA LAKE

LOCATION: 0723

NORTH COORDIHATE: $\quad 48190.0$ FT

EAST COORDINATE: $\quad 56540.0$ FT

06/10/80 TO 12/04/92

REPORT DATE: 08/04/94

FORMATIOH OF COMPLETIOH: DAKOTA SANDSTOHE (KD)

HYDRAULIC FLOW RELATIONSHIP: UNKHOWH (H)

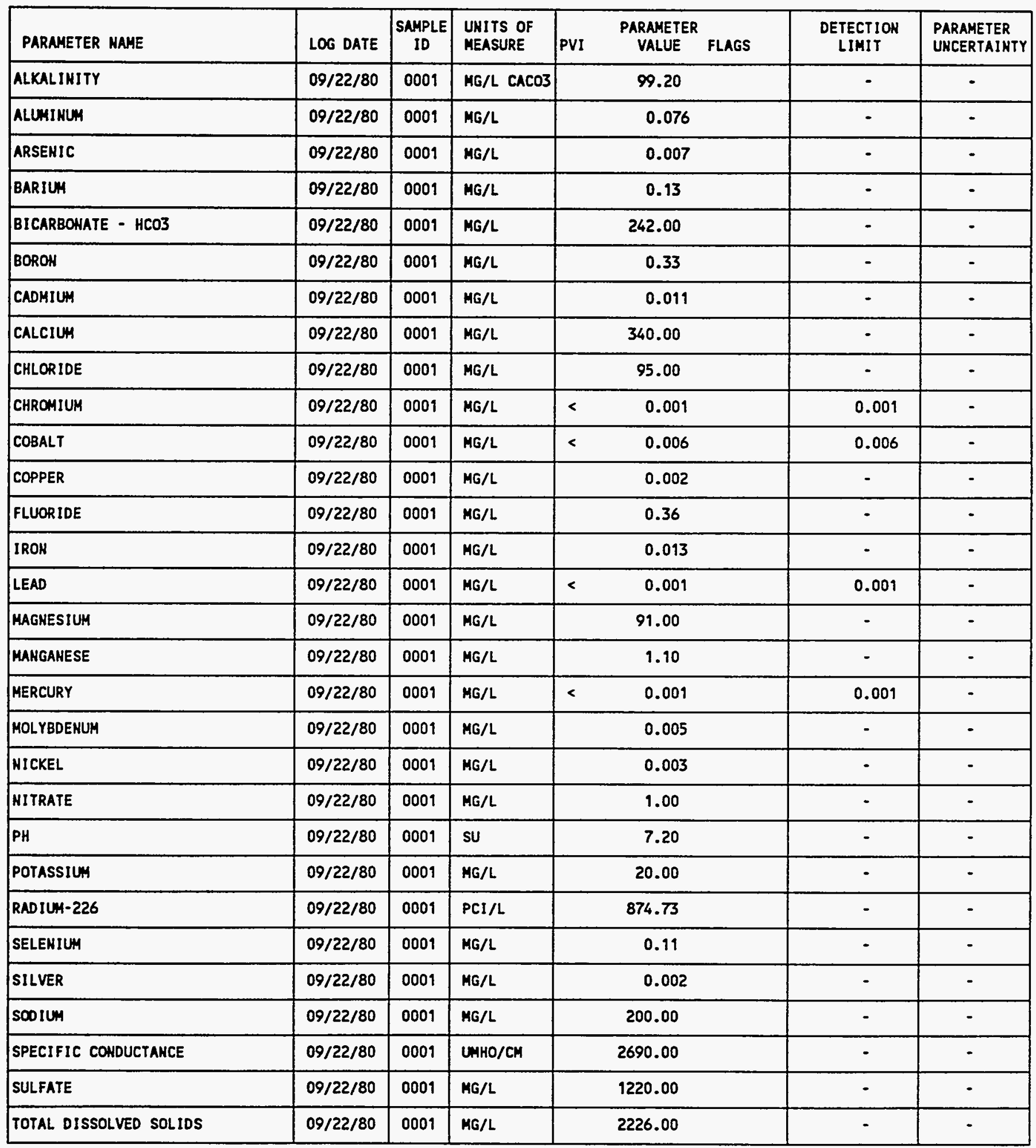

PARAMETER VALUE IHDICATOR (PVI):

< - LESS THAN DETECTIOH LIMIT

SAKPLE ID COOES:

0001 - FILTERED SAMPLE (.45 MICRONS) 
GROUNDWATER QUALITY DATA BY LOCATION

SITE: AMBO1 AMBROSIA LAKE

LOCATION: 0723

NORTH COORDIMATE: $\quad 48190.0 \mathrm{FT}$

EAST COORDINATE: $\quad 56540.0$ FT

06/10/80 TO $12 / 04 / 92$

REPORT DATE: 08/04/94

FORMATION OF COMPLETION: DAKOTA SANDSTONE (KD)

HYDRAULIC FLOW RELATIONSHIP: UNKNOWN (N)

\begin{tabular}{|c|c|c|c|c|c|c|c|}
\hline PARAMETER MAME & LOG DATE & $\underset{\text { SAMPLE }}{\text { ID }}$ & $\begin{array}{l}\text { UNITS OF } \\
\text { MEASURE }\end{array}$ & PVI & $\begin{array}{l}\text { PARAMETER } \\
\text { VALUE FLAGS }\end{array}$ & $\begin{array}{l}\text { DETECTION } \\
\text { LIMIT }\end{array}$ & $\begin{array}{l}\text { PARAMETER } \\
\text { UNCERTAINTY }\end{array}$ \\
\hline TOTAL SUSPENDED SOLIDS & $09 / 22 / 80$ & 0001 & $M G / L$ & & 2180.00 & - & - \\
\hline VANADIUN & $09 / 22 / 80$ & 0001 & $M G / L$ & & 0.015 & - & - \\
\hline
\end{tabular}

Parameter Value indicator (PVI):

< - Less than oetection limit

SAMPLE ID COOES:

0001 - FILTERED SAMPLE (.45 MICRONS) 
GROUNDWATER QUALITY DATA BY LOCATION

SITE: AMBO1 AMBROSIA LAKE

LOCATION: 0724

HORTH COORDINATE: 49715.0 FT

EAST COORDINATE: $\quad 56105.0$ FT

06/10/80 TO 12/04/92

REPORT DATE: 08/04/94

FORMATION OF COMPLETION: DAKOTA SANDSTONE (KD)

HYDRAULIC FLOW RELATIONSHIP: UNKNOWN (N)

\begin{tabular}{|c|c|c|c|c|c|c|c|}
\hline PARAMETER MAME & LOG DATE & $\underset{\text { ID }}{\text { SAMPLE }}$ & $\begin{array}{l}\text { UNITS OF } \\
\text { MEASURE }\end{array}$ & PVI & $\begin{array}{l}\text { PARAMETER } \\
\text { VALUE FLAGS }\end{array}$ & $\begin{array}{l}\text { DETECTION } \\
\text { LIMIT }\end{array}$ & $\begin{array}{l}\text { PARAMETER } \\
\text { UNCERTAINTY }\end{array}$ \\
\hline ALKALINITY & $09 / 23 / 80$ & 0001 & MG/L CACO3 & & 68.90 & - & - \\
\hline ALUMIMUM & $09 / 23 / 80$ & 0001 & $M G / L$ & & 0.09 & - & - \\
\hline ARSENIC & $09 / 23 / 80$ & 0001 & MG/L & & 0.001 & - & - \\
\hline BARIUM & $09 / 23 / 80$ & 0001 & $M G / L$ & & 0.032 & - & - \\
\hline BICARBONATE - HCOZ & $09 / 23 / 80$ & 0001 & MG $/ L$ & & 168.00 & - & - \\
\hline BORON & $09 / 23 / 80$ & 0001 & $M G / L$ & & 0.20 & - & - \\
\hline CADMIUM & $09 / 23 / 80$ & 0001 & MG/L & & 0.02 & - & - \\
\hline CALCIUM & $09 / 23 / 80$ & 0001 & $M G / L$ & & 107.00 & - & - \\
\hline CHLORIDE & $09 / 23 / 80$ & 0001 & MG/L & & 73.00 & - & - \\
\hline CHROMIUM & $09 / 23 / 80$ & 0001 & $M G / L$ & $<$ & 0.001 & 0.001 & - \\
\hline COBALT & $09 / 23 / 80$ & 0001 & $M G / L$ & $<$ & 0.006 & 0.006 & - \\
\hline COPPER & $09 / 23 / 80$ & 0001 & $M G / L$ & & 0.001 & - & - \\
\hline FLUORIDE & $09 / 23 / 80$ & 0001 & $M G / L$ & & 0.34 & - & - \\
\hline IRON & $09 / 23 / 80$ & 0001 & $M G / L$ & & 0.24 & - & - \\
\hline LEAD & $09 / 23 / 80$ & 0001 & $M G / L$ & & 0.002 & - & - \\
\hline MAGKESIUM & $09 / 23 / 80$ & 0001 & $M G / L$ & & 51.00 & - & - \\
\hline MANGANESE & $09 / 23 / 80$ & 0001 & MG/L & & 0.003 & - & - \\
\hline MERCURY & $09 / 23 / 80$ & 0001 & $M G / L$ & $<$ & 0.001 & 0.001 & - \\
\hline MOLYBDENUM & $09 / 23 / 80$ & 0001 & $M G / L$ & & 0.005 & - & - \\
\hline HICKEL & $09 / 23 / 80$ & 0001 & $M G / L$ & & 0.001 & - & - \\
\hline NITRATE & $09 / 23 / 80$ & 0001 & $M G / L$ & & 2.70 & - & - \\
\hline PH & $09 / 23 / 80$ & 0001 & su & & 7.60 & - & - \\
\hline POTASSIUA & $09 / 23 / 80$ & 0001 & MG/L & & 9.30 & - & - \\
\hline RADIUA-226 & $09 / 23 / 80$ & 0001 & $\mathrm{PCI} / \mathrm{L}$ & & 7.75 & - & - \\
\hline SELEKIUM & $09 / 23 / 80$ & 0001 & $M G / L$ & & 0.13 & - & - \\
\hline SILVER & $09 / 23 / 80$ & 0001 & $M G / L$ & $<$ & 0.002 & 0.002 & - \\
\hline soorum & $09 / 23 / 80$ & 0001 & $M G / L$ & & 140.00 & - & - \\
\hline SPECIFIC CONDUCTANCE & $09 / 23 / 80$ & 0001 & ЧМнО/CM & & 1450.00 & - & - \\
\hline SULFATE & $09 / 23 / 80$ & 0001 & $M G / L$ & & 560.00 & - & - \\
\hline TOTAL DISSOLVED SOLIDS & $09 / 23 / 80$ & 0001 & $M G / L$ & & 1110.00 & - & - \\
\hline
\end{tabular}

PARAMETER VALUE INDICATOR (PVI):

< - LESS THAN DETECTION LIMIT

SAMPLE ID CODES:

0001 - FILTERED SAMPLE (.45 MICRONS) 
GROUNDWATER QUALITY DATA BY LOCATION

SITE: AMBOI AMBROSIA LAKE

LOCATION: 0724

NORTH COORDINATE: 49715.0 FT

EAST COORDINATE: $\quad 56105.0$ FT

06/10/80 TO $12 / 04 / 92$

REPORT DATE: $08 / 04 / 94$

FORMATION OF COMPLETION: DAKOTA SANDSTONE (KD)

HYDRAULIC FLON RELATIONSHIP: UNKNOWH (N)

\begin{tabular}{|c|c|c|c|c|c|c|c|}
\hline PARAMETER NAME & LOG DATE & $\begin{array}{c}\text { SAMPLE } \\
\text { ID }\end{array}$ & $\begin{array}{l}\text { UNITS OF } \\
\text { MEASURE }\end{array}$ & PVI & $\begin{array}{l}\text { PARAMETER } \\
\text { VALUE FLAGS }\end{array}$ & $\begin{array}{l}\text { DETECTION } \\
\text { LIMIT }\end{array}$ & $\begin{array}{l}\text { PARAMETER } \\
\text { UKCERTAINTY }\end{array}$ \\
\hline TOTAL SUSPENDED SOLIDS & $09 / 23 / 80$ & 0001 & MG/L & & 56.00 & - & - \\
\hline URAMIUM & $09 / 23 / 80$ & 0001 & $M G / L$ & & 0.08 & - & - \\
\hline VANADIUM & $09 / 23 / 80$ & 0001 & MG/L & & 0.007 & - & $\cdot$ \\
\hline ZINC & $09 / 23 / 80$ & 0001 & MG/L & & 0.091 & - & - \\
\hline
\end{tabular}

PARAMETER VALUE IMDICATOR (PVI): < - LESS THAM DETECTION LIMIT

SAMPLE IO COOES:

0001 - FILTERED SAMPLE (.45 MICRONS) 
GROUNDHATER QUALITY DATA BY LOCATION

SITE: AMBO1 AMBROSIA LAKE

LOCATIOH: 0725

MORTH COORDINATE: $\quad 51880.0$ FT

EAST COORDINATE: $\quad 54950.0$ FT

$06 / 10 / 80$ TO $12 / 04 / 92$

REPORT DATE: 08/04/94

FORMATION OF COHPLETION: DAKOTA SAMDSTONE (KD)

HYDRAULIC FLON RELATIOHSHIP: UNKNOWN (N)

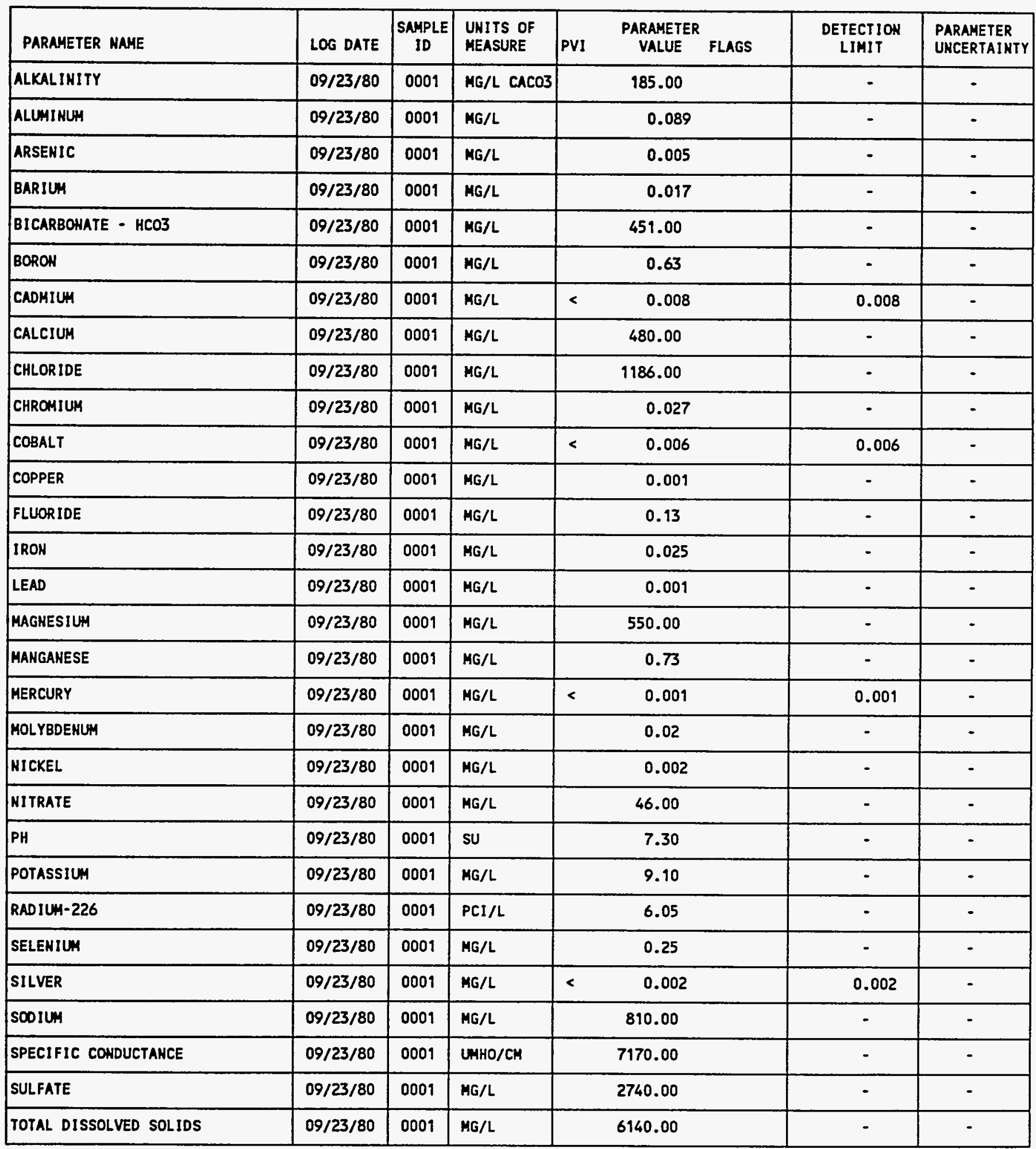

PARAMETER VALUE IMDICATOR (PVI): < - LESS THAN DETECTION LIMIT

SAMPLE ID COOES:

0001 - FILTERED SAMPLE (.45 MICRONS) 
GROUNDHATER QUALITY DATA BY LOCATION

SITE: AMBOI AMBROSIA LAKE

LOCATIOA: 0725

MORTH COORDINATE: $\quad 51880.0$ FT

EAST COORDINATE: $\quad 54950.0$ FT

06/90/80 TO $12 / 04 / 92$

REPORT DATE: 08/04/94

FORMATION OF COMPLETION: DAKOTA SAMDSTONE (KD)

HYORAULIC FLOW RELATIONSHIP: UNKNOWN (N)

\begin{tabular}{|c|c|c|c|c|c|c|c|}
\hline PARAMETER NAME & LOG DATE & $\begin{array}{c}\text { SAMPLE } \\
\text { ID }\end{array}$ & $\begin{array}{l}\text { UNITS OF } \\
\text { MEASURE }\end{array}$ & PVI & $\begin{array}{l}\text { PARAMETER } \\
\text { VALUE FLAGS }\end{array}$ & $\begin{array}{l}\text { DETECTIOH } \\
\text { LIMIT }\end{array}$ & $\begin{array}{l}\text { PARAMETER } \\
\text { UNCERTAINTY }\end{array}$ \\
\hline TOTAL SUSPENDED SOLIDS & $09 / 23 / 80$ & 0001 & $M G / L$ & & 33.00 & - & - \\
\hline URANIUA & $09 / 23 / 80$ & 0001 & $M G / L$ & & 0.11 & - & - \\
\hline VAKADIUM & $09 / 23 / 80$ & 0001 & $M G / L$ & & 0.015 & - & - \\
\hline ZINC & $09 / 23 / 80$ & 0001 & MG/L & & 0.16 & - & - \\
\hline
\end{tabular}

PARAMETER VALUE IHDICATOR (PVI):

- LESS THAN DETECTION LIMIT

SAMPLE ID COOES

0001 - FILTERED SAMPLE (.45 MICRONS) 
GROUNDWATER QUALITY DATA BY LOCATION

SITE: AMBO1 AMBROSIA LAKE

LOCATION: 0726

NORTH COORDINATE: 53980.0 FT

EAST COORDIHATE: $\quad 48560.0$ FT

O6/10/80 TO $12 / 04 / 92$

REPORT DATE: $08 / 04 / 94$

FORMATIOH OF COHPLETIOH: TRES HERMANOS-A SANDSTOHE (TR)

HYDRAULIC FLON RELATIONSHIP: UNKNOWN (H)

\begin{tabular}{|c|c|c|c|c|c|c|c|}
\hline PARAMETER NAME & LOG DATE & SAMPLE & $\begin{array}{l}\text { UNITS OF } \\
\text { MEASURE }\end{array}$ & PVI & $\begin{array}{l}\text { PARAMETER } \\
\text { VALUE FLAGS }\end{array}$ & $\begin{array}{l}\text { DETECTION } \\
\text { LIMIT }\end{array}$ & $\begin{array}{l}\text { PARAMETER } \\
\text { UNCERTAINTY }\end{array}$ \\
\hline ALKALIKITY & $10 / 14 / 80$ & 0001 & MG/L CACO3 & & 4.50 & - & - \\
\hline ALUMINUM & $10 / 14 / 80$ & 0001 & MG/L & & 0.53 & - & - \\
\hline ARSENIC & $10 / 14 / 80$ & 0001 & MG/L & & 0.03 & - & - \\
\hline BICARBONATE - HCO3 & $10 / 14 / 80$ & 0001 & MG/L & & 11.00 & - & - \\
\hline BORON & $10 / 94 / 80$ & 0001 & MG/L & & 60.00 & - & - \\
\hline CADMIUA & $10 / 14 / 80$ & 0001 & $M G / L$ & $<$ & 0.008 & 0.008 & - \\
\hline CALCIUA & $10 / 14 / 80$ & 0001 & MG/L & & 77.00 & - & - \\
\hline COBALT & $10 / 14 / 80$ & 0001 & $M G / L$ & $<$ & 0.006 & 0.006 & - \\
\hline COPPER & $10 / 14 / 80$ & 0001 & $M G / L$ & & 0.32 & - & - \\
\hline FLUORIDE & $10 / 14 / 80$ & 0001 & $M G / L$ & & 0.46 & - & - \\
\hline IRON & $10 / 14 / 80$ & 0001 & $M G / L$ & & 2.20 & - & - \\
\hline LEAD & $10 / 14 / 80$ & 0001 & $M G / L$ & $<$ & 0.001 & 0.001 & - \\
\hline MAGHESIUH & $10 / 14 / 80$ & 0001 & $M G / L$ & & 15.00 & - & - \\
\hline MAKGANESE & $10 / 14 / 80$ & 0001 & $M G / L$ & & 0.15 & - & - \\
\hline PH & $10 / 14 / 80$ & 0001 & SU & & 8.60 & - & - \\
\hline POTASSIUH & $10 / 14 / 80$ & 0001 & MG/L & & 27.00 & - & $\cdot$ \\
\hline RADIUM-226 & $10 / 14 / 80$ & 0001 & $\mathrm{PCI} / \mathrm{L}$ & & 7.13 & - & - \\
\hline SELENIUH & $10 / 14 / 80$ & 0001 & $M G / L$ & & 0.019 & - & - \\
\hline SILVER & $10 / 14 / 80$ & 0001 & $M G / L$ & $<$ & 0.002 & 0.002 & - \\
\hline SOOIUH & $10 / 14 / 80$ & 0001 & $M G / L$ & & 240.00 & - & - \\
\hline SPECIFIC CONDUCTAHCE & $10 / 14 / 80$ & 0001 & $\mathrm{UMHO} / \mathrm{CM}$ & & 1650.00 & - & - \\
\hline SULFATE & $10 / 14 / 80$ & 0001 & MG/L & & 590.00 & - & - \\
\hline TOTAL DISSOLVED SOLIDS & $10 / 14 / 80$ & 0001 & $M G / L$ & & 1230.00 & - & - \\
\hline
\end{tabular}

PARAMETER VALUE IHDICATOR (PVI): < - LESS THAM DETECTION LIMIT

SAMPLE ID CODES:

DOO1 - FILTERED SAMPLE (.45 MICRONS) 
GROUNDWATER QUALITY DATA BY LOCATION

SITE: AMBOI AMBROSIA LAKE

LOCATION: 0726

MORTH COORDINATE: $\quad 53980.0$ FT

EAST COORDINATE: $\quad 48560.0$ FT

O6/10/80 TO $12 / 04 / 92$

REPORT DATE: 08/04/94

FORMATION OF COMPLETION: TRES HERMANOS-A SANDSTONE (TR)

HYDRAULIC FLON RELATIONSHIP: UNKNOWN (N)

\begin{tabular}{|c|c|c|c|c|c|c|c|}
\hline PARAMETER MAME & LOG DATE & $\begin{array}{c}\text { SAMPLE } \\
\text { ID }\end{array}$ & $\begin{array}{l}\text { UNITS OF } \\
\text { MEASURE }\end{array}$ & PVI & $\begin{array}{l}\text { PARAMETER } \\
\text { VALUE FLAGS }\end{array}$ & $\begin{array}{l}\text { DETECTION } \\
\text { LIMIT }\end{array}$ & $\begin{array}{l}\text { PARAMETER } \\
\text { UHCERTAINTY }\end{array}$ \\
\hline TOTAL SUSPEKDED SOLIDS & $10 / 14 / 80$ & 0001 & $M G / L$ & & 248.00 & - & - \\
\hline URANIUM & $10 / 14 / 80$ & 0001 & MG/L & & 0.05 & - & - \\
\hline VANADIUM & $10 / 14 / 80$ & 0001 & MG/L & & 0.051 & $\cdot$ & $\cdot$ \\
\hline ZINC & $10 / 14 / 80$ & 0001 & MG/L & & 1.30 & - & $\cdot$ \\
\hline
\end{tabular}

PARAMETER VALUE INDICATOR (PVI): < - LESS THAN DETECTION LIMIT

SAMPLE ID COOES:

O001 - FILTERED SAMPLE (.45 MICROHS) 
GROUNDWATER OUALITY DATA BY LOCATION

SITE: AMBO1 AMBROSIA LAKE

LOCATION: 0727

NORTH COORDIHATE: 68980.0 FT

EAST COORDIHATE: $\quad \mathbf{5 5 9 6 0 . 0}$ FT

06/10/80 TO 12/04/92

REPORT DATE: $08 / 04 / 94$

FORMATIOH OF COMPLETION: WESTHATER CANYON MEMBER (JH)

HYDRAULIC FLON RELATIOHSHIP: UNKHOWH (N)

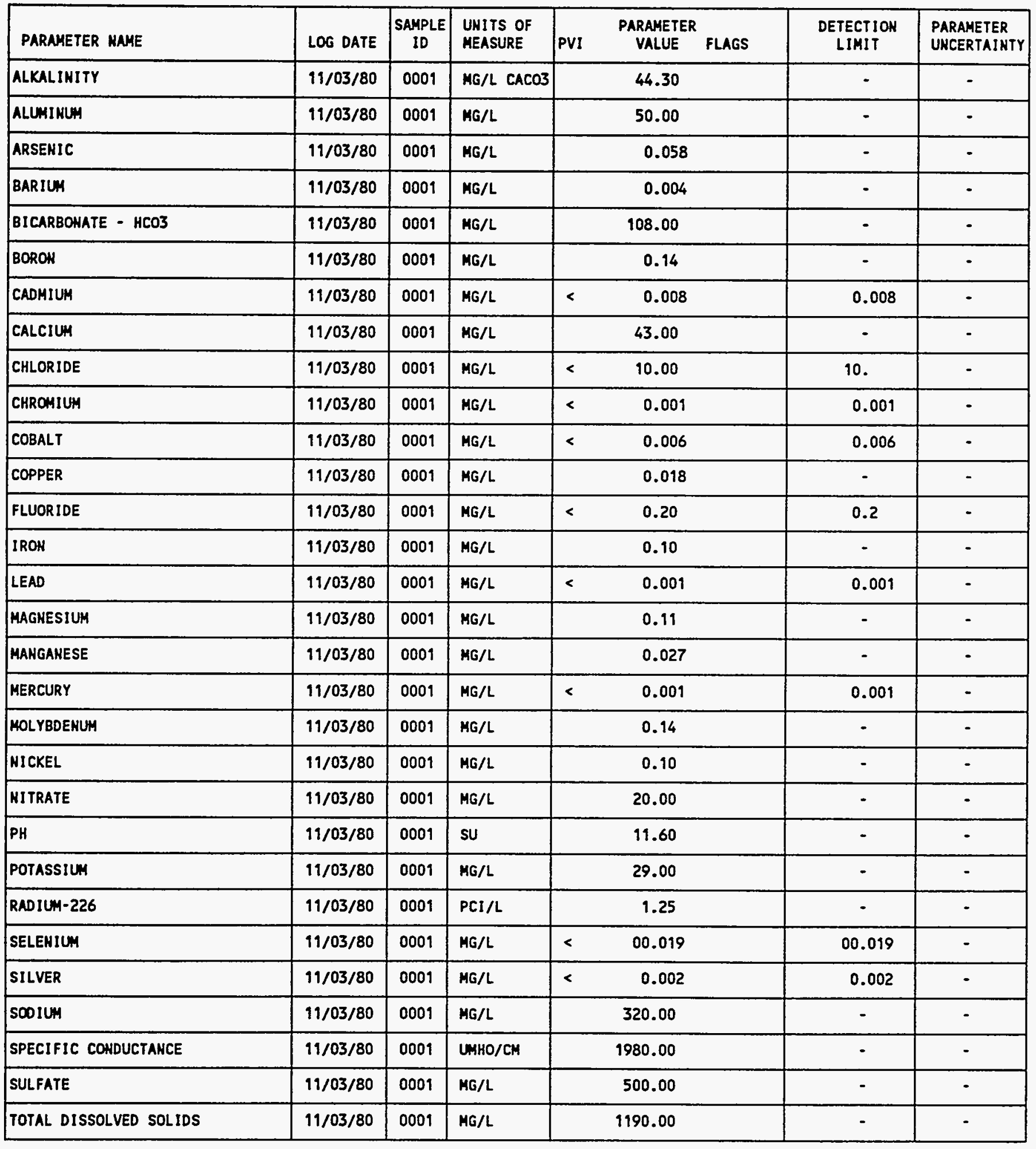

PARAMETER VALUE INDICATOR (PVI): < - LESS THAN DETECTIOH LIMIT 
GROUMDHATER QUALITY DATA BY LOCATION

SITE: AMBOI AMBROSIA LAKE

LOCATION: 0727

NORTH COORDINATE: $\quad 68980.0$ FT

EAST COORDINATE: $\quad 55960.0$ FT

06/10/80 TO $12 / 04 / 92$

REPORT DATE: 08/04/94

FORMATION OF COMPLETION: WESTHATER CANYON MEMBER (JH)

HYDRAULIC FLON RELATIONSHIP: UNKNOWN (N)

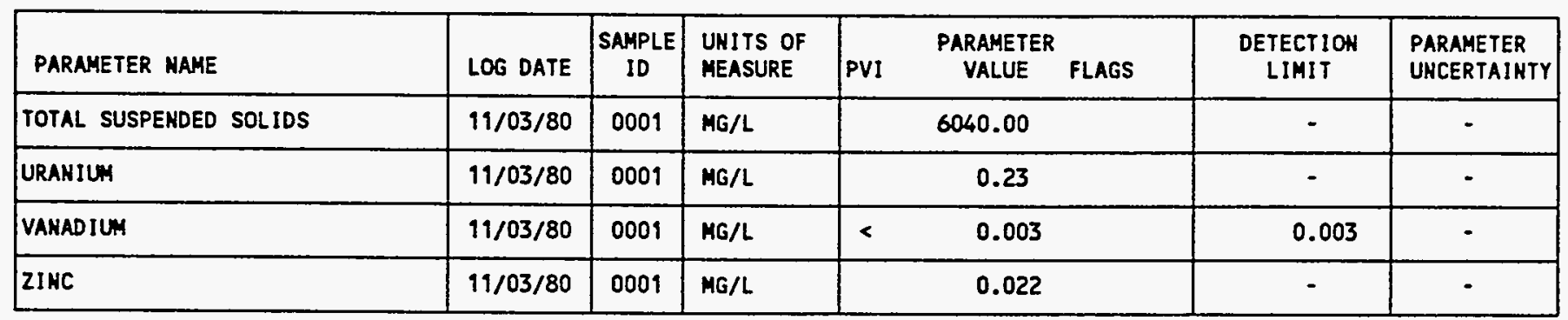

PARAMETER VALUE INDICATOR (PVI): < - LESS THAN DETECTION LIMIT

SAMPLE IO COOES:

O001 - FILTERED SAMPLE (.45 MICRONS) 
GROUNDWATER QUALITY DATA BY LOCATIOH

SITE: AMBOI AMBROSIA LAKE

LOCATION: 0728

HORTH COORDINATE: $\quad 52550.0$ FT

EAST COORDINATE: $\quad 44940.0$ FT

06/10/80 TO $12 / 04 / 92$

REPORT DATE: 08/04/94

FORMATION OF COMPLETION: DAKOTA SAMDSTOHE (KD)

HYDRAULIC FLOW RELATIOHSHIP: UNKNOWN (H)

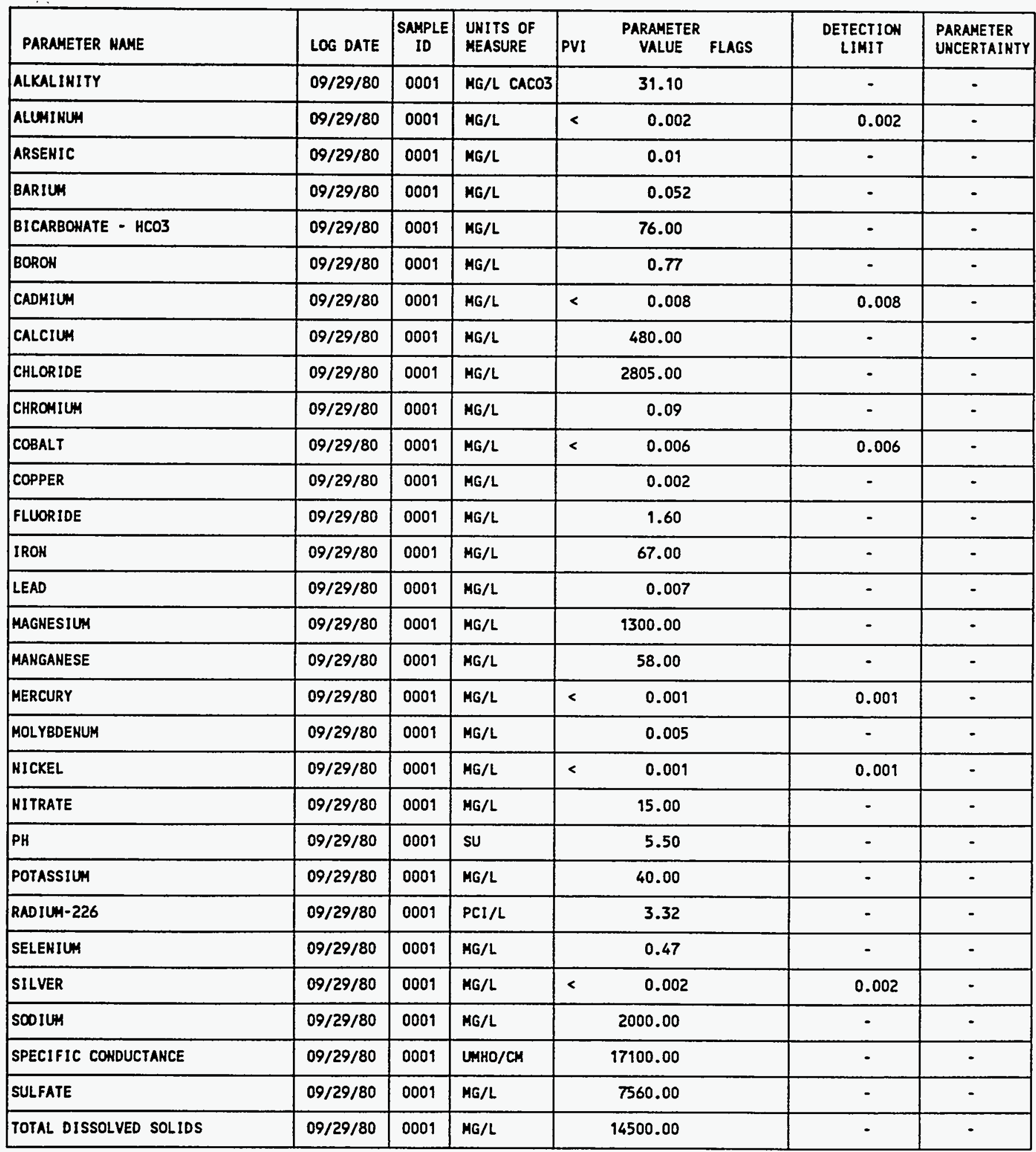

PARAMETER VALUE IMDICATOR (PVI): < - LESS THAN DETECTION LIMIT

SAMPLE ID COOES:

0001 - FILTERED SAMPLE (.45 MICRONS) 
GROUNDWATER OUALITY DATA BY LOCATION

SITE: AMBOI AMBROSIA LAKE

LOCATION: 0728

NORTH COORDINATE: $\quad 52550.0$ FT

EAST COORDINATE: $\quad 44940.0$ FT

O6/10/80 TO $12 / 04 / 92$

REPORT DATE: $08 / 04 / 94$

FORMATION OF COMPLETION: DAKOTA SAMDSTONE (KD)

HYDRAULIC FLON RELATIONSHIP: UNKNOWN (N)

\begin{tabular}{|c|c|c|c|c|c|c|c|}
\hline PARAMETER NAME & LOG DATE & $\begin{array}{c}\text { SAMPLE } \\
\text { ID }\end{array}$ & $\begin{array}{l}\text { UHITS OF } \\
\text { MEASURE }\end{array}$ & PVI & $\begin{array}{l}\text { PARAMETER } \\
\text { VALUE FLAGS }\end{array}$ & $\begin{array}{l}\text { DETECTIOH } \\
\text { LIMIT }\end{array}$ & $\begin{array}{l}\text { PARAMETER } \\
\text { UNCERTAINTY }\end{array}$ \\
\hline TOTAL SUSPENDED SOLIDS & $09 / 29 / 80$ & 0001 & $M G / L$ & & 151.00 & - & - \\
\hline URANIUH & $09 / 29 / 80$ & 0009 & $M G / L$ & & 0.07 & - & - \\
\hline VANADIUM & $09 / 29 / 80$ & 0001 & MG/L & & 0.017 & - & - \\
\hline ZINC & $09 / 29 / 80$ & 0001 & $M G / L$ & & 0.11 & - & - \\
\hline
\end{tabular}

PARAMETER VAlUe INDICATOR (PVI):

< - LESS THAN DETECTION LIMIT

SAMPLE ID COOES:

0001 - FILTERED SAMPLE (.45 MICRONS) 
GROUNDWATER QUALITY DATA BY LOCATION

SITE: AMBO1 AMBROSIA LAKE

LOCATION: 0729

HORTH COORDINATE: 53350.0 FT

EAST COORDINATE: $\quad 53510.0$ FT

06/10/80 TO $12 / 04 / 92$

REPORT DATE: $08 / 04 / 94$

FORMATIOW OF COMPLETIOH: ALLUVIUM (AL)

HYORAULIC FLON RELATIONSHIP: UNKNOWH (H)

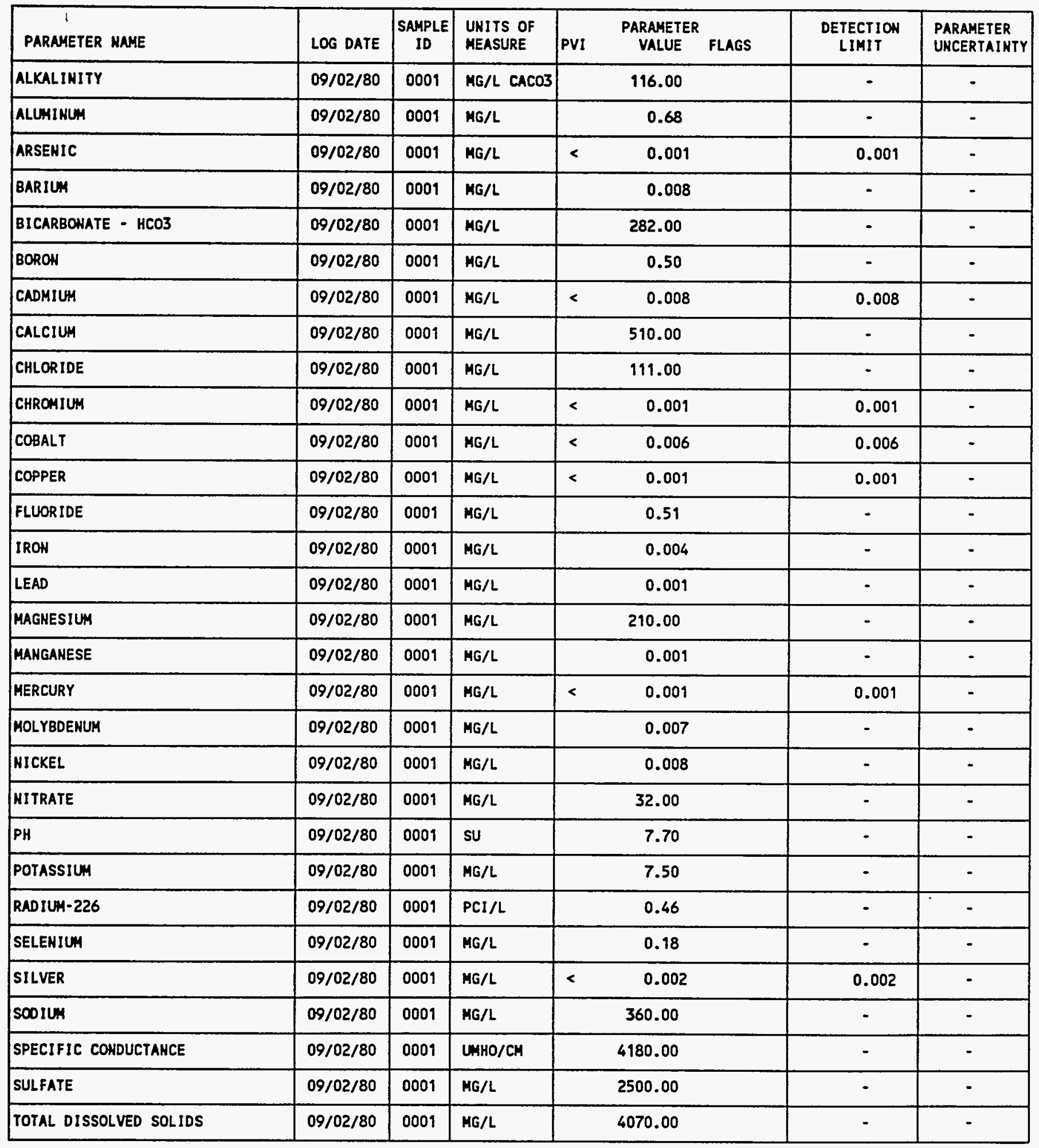

PARAMETER VALUE IMDICATOR (PVI): < - LESS THAH DETECTION LIMIT

SAMPLE ID COOES:

D001 - FILTERED SAMPLE (.45 MICRONS) 
GROUNDWATER QUALITY DATA BY LOCATION

SITE: AMBO1 AMBROSIA LAKE

LOCATION: 0729

MORTH COORDINATE: $\quad 53350.0$ FT

EAST COORDINATE: $\quad 53510.0$ FT

06/10/80 TO $12 / 04 / 92$

REPORT DATE: 08/04/94

FORMATION OF COMPLETION: ALLUVIUM (AL)

HYDRAULIC FLON RELATIONSHIP: UNKNOWN (N)

\begin{tabular}{|c|c|c|c|c|c|c|c|}
\hline PARAMETER MAME & LOG DATE & $\begin{array}{c}\text { SAMPLE } \\
\text { ID }\end{array}$ & $\begin{array}{l}\text { UNITS OF } \\
\text { MEASURE }\end{array}$ & PVI & $\begin{array}{l}\text { PARAMETER } \\
\text { VALUE FLAGS }\end{array}$ & $\begin{array}{l}\text { DETECTION } \\
\text { LIMIT }\end{array}$ & $\begin{array}{l}\text { PARAMETER } \\
\text { UHCERTAIHTY }\end{array}$ \\
\hline TOTAL SUSPENDED SOLIDS & $09 / 02 / 80$ & 0001 & $M G / L$ & & 15.00 & - & - \\
\hline URAKIUM & $09 / 02 / 80$ & 0001 & $M G / L$ & & 0.075 & - & $\cdot$ \\
\hline VAHADIUM & $09 / 02 / 80$ & 0001 & MG/L & & 0.018 & - & - \\
\hline ZINC & $09 / 02 / 80$ & 0001 & $M G / L$ & & 0.014 & - & - \\
\hline
\end{tabular}

PARAMETER VALUE INDICATOR (PVI):

< - LESS THAN DETECTION LIMIT

SAMPLE ID COOES:

0001 - FILTERED SAMPLE (.45 MICRONS) 
GROUNDWATER QUALITY DATA BY LOCATION

SITE: AMBOI AMBROSIA LAKE

LOCATION: 0737

NORTH COORDINATE: 54291.3 FT

EAST COORDINATE: $\quad 60397.3$ FT

06/10/80 TO 12/04/92

REPORT DATE: 08/04/94

FORMATION OF COMPLETIOH: URANIUM MILL TAILINGS (TA)

HYDRAULIC FLON RELATIONSHIP: OW-SITE (O)

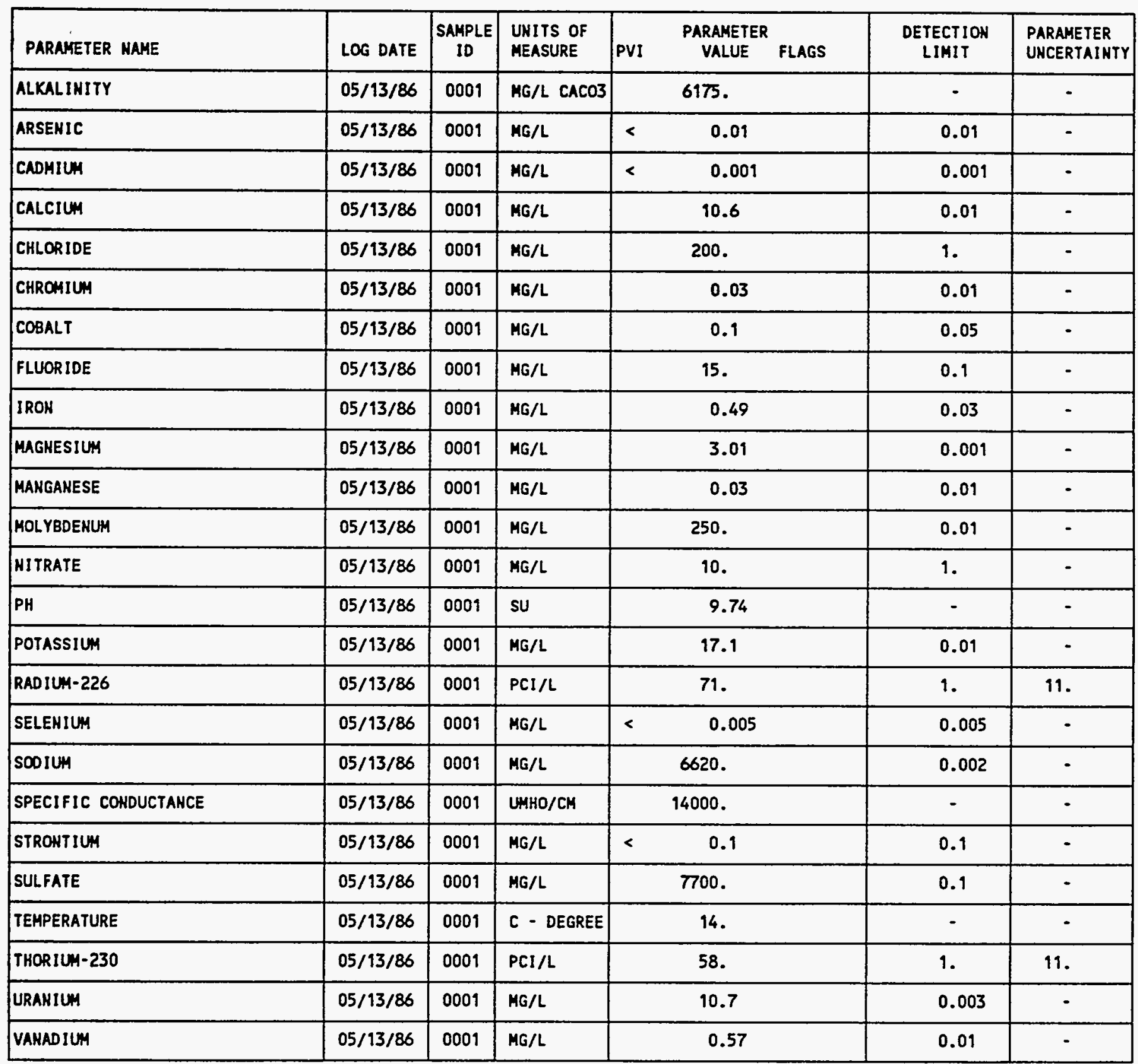

PARAMETER VALUE IHDICATOR (PVI):

< - LESS THAN DETECTION LIMIT

SAMPLE ID COOES:

0001 - FILTERED SAMPLE (.45 MICROHS) 
GROUNDWATER OUALITY DATA BY LOCATION

SITE: AMBO1 AMBROSIA LAKE

LOCATION: 0743

MORTH COORDINATE: $\quad 53782.5$ FT

EAST COORDINATE: 60188.6 FT

06/10/80 TO 12/04/92

REPORT DATE: $08 / 04 / 94$

FORMATION OF COMPLETION: URAHIUM MILL TAILIHGS (TA)

HYDRAULIC FLON RELATIONSHIP: ON-SITE (O)

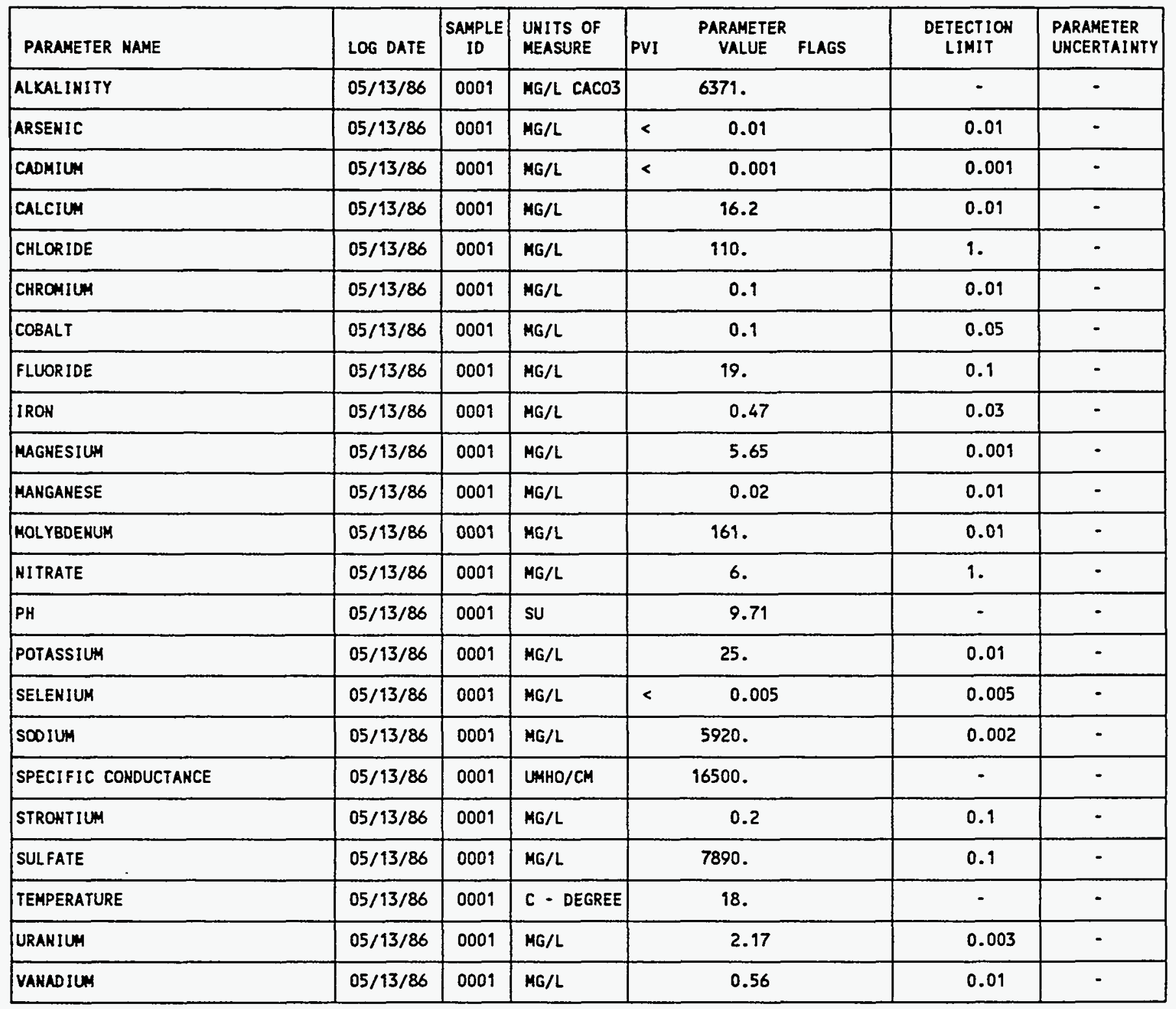

PARAMETER VALUE INDICATOR (PVI):

< - LESS THAM DETECTION LIMIT

SAMPLE ID COOES:

0001 - FILTERED SAMPLE (.45 MICROHS) 
GROUNDWATER QUALITY DATA BY LOCATION

SITE: AMBO1 AMBROSIA LAKE

LOCATION: 0746

MORTH COORDIHATE: $\quad 54193.0$ FT

EAST COORDINATE: $\quad 60806.8$ FT

06/10/80 TO $12 / 04 / 92$

REPORT DATE: $08 / 04 / 94$

FORMATION OF COMPLETIOH: URANIUM MILL TAILINGS (TA)

HYDRAULIC FLOW RELATIONSHIP: ON-SITE (O)

\begin{tabular}{|c|c|c|c|c|c|c|c|}
\hline PARAMETER haMe & LOG DATE & $\begin{array}{c}\text { SAMPLE } \\
\text { ID }\end{array}$ & $\begin{array}{l}\text { UNITS OF } \\
\text { MEASURE }\end{array}$ & PVI & $\begin{array}{l}\text { PARAMETER } \\
\text { VALUE FLAGS }\end{array}$ & $\begin{array}{l}\text { DETECTIOH } \\
\text { LIMIT }\end{array}$ & $\begin{array}{l}\text { PARAMETER } \\
\text { UNCERTAINTY }\end{array}$ \\
\hline ALKALINITY & $09 / 26 / 85$ & 0001 & MG/L CACO3 & & 4341. & - & $\cdot$ \\
\hline ALUMINUM & $09 / 26 / 85$ & 0001 & MG/L & & 0.4 & 0.1 & - \\
\hline AMKONIU: & $09 / 26 / 85$ & 0001 & $M G / L$ & & 0.2 & 0.1 & - \\
\hline ARSENIC & $09 / 26 / 85$ & 0009 & MG/L & $<$ & 0.01 & 0.01 & - \\
\hline BARIUM & $09 / 26 / 85$ & 0009 & $M G / L$ & & 0.3 & 0.1 & $\cdot$ \\
\hline BOROH & $09 / 26 / 85$ & 0001 & MG/L & & 0.7 & 0.1 & - \\
\hline CADMIUH & $09 / 26 / 85$ & 0001 & $M G / L$ & $<$ & 0.001 & 0.001 & - \\
\hline CHROMIUM & $09 / 26 / 85$ & 0001 & MG/L & & 0.03 & 0.01 & - \\
\hline COBALT & $09 / 26 / 85$ & 0001 & $M G / L$ & & 0.08 & 0.05 & - \\
\hline COPPER & $09 / 26 / 85$ & 0001 & MG/L & & 0.04 & 0.02 & - \\
\hline CYANIDE & $09 / 26 / 85$ & 0001 & $M G / L$ & $<$ & 0.01 & 0.01 & - \\
\hline FLUORIDE & $09 / 26 / 85$ & 0001 & MG/L & & 19. & 0.1 & - \\
\hline IRON & $09 / 26 / 85$ & 0001 & MG/L & & 0.21 & 0.03 & - \\
\hline LEAD & $09 / 26 / 85$ & 0001 & $M G / L$ & $<$ & 0.01 & 0.09 & - \\
\hline HERCURY & $09 / 26 / 85$ & 0001 & MG/L & $<$ & 0.0002 & 0.0002 & - \\
\hline MOL YBDENUM & $09 / 26 / 85$ & 0001 & $M G / L$ & & 113. & 0.01 & - \\
\hline KICKEL & $09 / 26 / 85$ & 0001 & $M G / L$ & & 0.14 & 0.04 & - \\
\hline MITRATE & $09 / 26 / 85$ & 0001 & $M G / L$ & & 3. & 1. & $\cdot$ \\
\hline NITRITE & $09 / 26 / 85$ & 0001 & MG/L & $<$ & 0.1 & 0.1 & - \\
\hline PH & $09 / 26 / 85$ & 0001 & su & & 9.97 & - & - \\
\hline PHOSPHATE & $09 / 26 / 85$ & 0001 & $M G / L$ & & 14. & 0.1 & $\cdot$ \\
\hline POTASSIUH & $09 / 26 / 85$ & 0001 & $M G / L$ & & 16.8 & 0.01 & - \\
\hline RADIUM-226 & $09 / 26 / 85$ & 0001 & $\mathrm{PCI} / \mathrm{L}$ & & 140. & 1. & 10. \\
\hline RADIUM-226 + RADIUH-228 & $09 / 26 / 85$ & 0001 & PCI/L & & 140.00 & - & - \\
\hline RADIUM-228 & $09 / 26 / 85$ & 0001 & $\mathrm{PCI} / \mathrm{L}$ & & 0. & 1. & 3.3 \\
\hline
\end{tabular}

PARAMETER VALUE IMDICATOR (PVI): < - LESS THAH DETECTION LIMIT

SAMPLE ID COOES:

0001 - FILTERED SAMPLE (.45 MICRONS) 
GROUHDWATER QUALITY OATA BY LOCATION

SITE: AMBOI AMBROSIA LAKE

LOCATION: 0746

MORTH COORDINATE: $\quad 54193.0$ FT

EAST COORDINATE: $\quad 60806.8$ FT

06/10/80 TO $12 / 04 / 92$

REPORT DATE: 08/04/94

FORMATION OF COMPLETION: URANIUM MILL TAILINGS (TA) HYDRAULIC FLOW RELATIOHSHIP: ON-SITE (O)

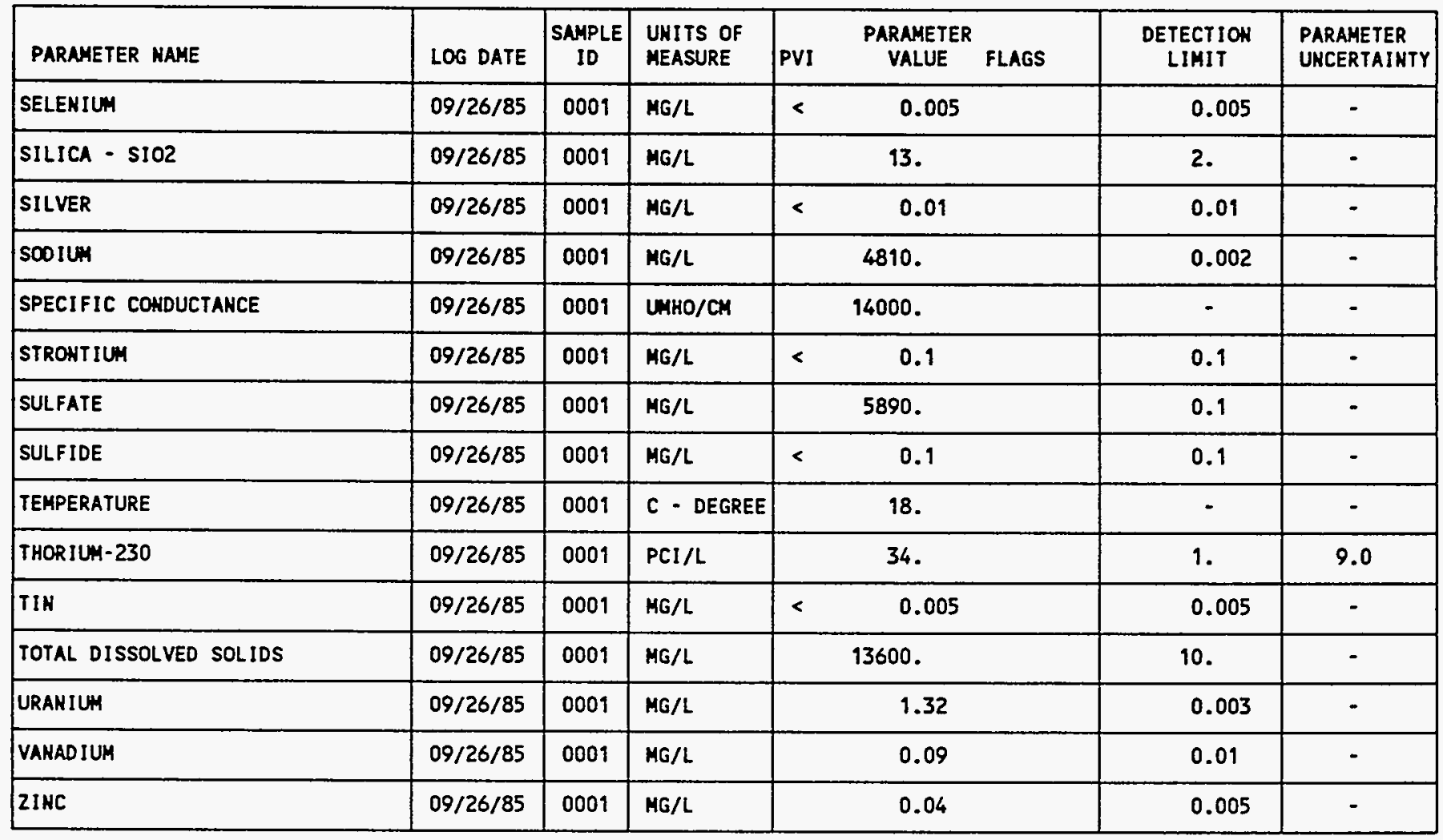

PARAMETER VALUE INDICATOR (PVI):

- LESS THAN DETECTION LIMIT

SAMPLE ID COOES:

0001 - FILTERED SAMPLE (.45 MICRONS) 
GROUNDWATER QUALITY DATA BY LOCATION

SITE: AMBO1 AMBROSIA LAKE

LOCATION: 0747

NORTH COOROINATE: 53990.5 FT

EAST COORDINATE: 60805.1 FT

06/10/80 TO 12/04/92

REPORT DATE: $08 / 04 / 94$

FORMATION OF COMPLETION: ALLUVIUM (AL)

HYDRAULIC FLOU RELATIONSHIP: ON-SITE (O)

\begin{tabular}{|c|c|c|c|c|c|c|c|}
\hline PARAMETER NAME & LOG DATE & $\underset{\text { SAMPLE }}{\text { ID }}$ & $\begin{array}{l}\text { UHITS OF } \\
\text { MEASURE }\end{array}$ & PVI & $\begin{array}{l}\text { PARAMETER } \\
\text { VALUE FLAGS }\end{array}$ & $\begin{array}{l}\text { DETECTION } \\
\text { LIMIT }\end{array}$ & $\begin{array}{l}\text { PARAMETER } \\
\text { UNCERTAINTY }\end{array}$ \\
\hline ALKALINITY & $09 / 26 / 85$ & 0001 & MG/L CACO3 & & 397. & - & - \\
\hline ALUMIKUM & $09 / 26 / 85$ & 0001 & $M G / L$ & & 0.4 & 0.1 & - \\
\hline AMHONIUM & $09 / 26 / 85$ & 0001 & MG/L & & 1.2 & 0.1 & - \\
\hline АКT IMONY & $09 / 26 / 85$ & 0001 & MG/L & $<$ & 0.003 & 0.003 & - \\
\hline ARSEKIC & $09 / 26 / 85$ & 0001 & $M G / L$ & $<$ & 0.01 & 0.01 & - \\
\hline BARIUM & $09 / 26 / 85$ & 0001 & MG/L & & 0.1 & 0.1 & - \\
\hline BOROH & $09 / 26 / 85$ & 0009 & MG/L & & 0.6 & 0.1 & - \\
\hline CADMIUM & $09 / 26 / 85$ & 0001 & $M G / L$ & $<$ & 0.001 & 0.001 & $\cdot$ \\
\hline CALCIUM & $09 / 26 / 85$ & 0001 & MG/L & & 320. & 0.01 & - \\
\hline CHLORIDE & $09 / 26 / 85$ & 0001 & $M G / L$ & & 190. & 1. & - \\
\hline CHROMIUM & $09 / 26 / 85$ & 0001 & MG/L & & 0.04 & 0.01 & - \\
\hline COBALT & $09 / 26 / 85$ & 0001 & $M G / L$ & & 0.09 & 0.05 & - \\
\hline COPPER & $09 / 26 / 85$ & 0001 & $\mathrm{MG} / \mathrm{L}$ & & 0.05 & 0.02 & - \\
\hline CYANIDE & $09 / 26 / 85$ & 0001 & $M G / L$ & $<$ & 0.1 & 0.1 & - \\
\hline FLUORIDE & $09 / 26 / 85$ & 0001 & MG/L & & 2.8 & 0.1 & - \\
\hline IRON & $09 / 26 / 85$ & 0001 & MG/L & & 0.29 & 0.03 & - \\
\hline LEAD & $09 / 26 / 85$ & 0001 & MG/L & $<$ & 0.01 & 0.01 & - \\
\hline LEAD-210 & $09 / 26 / 85$ & 0001 & PCI/L & & 21. & 1.5 & 3. \\
\hline MAGNESIUM & $09 / 26 / 85$ & 0001 & MG/L & & 82.8 & 0.001 & - \\
\hline MANGANESE & $09 / 26 / 85$ & 0001 & MG/L & & 0.1 & 0.01 & - \\
\hline MERCURY & $09 / 26 / 85$ & 0001 & MG/L & $<$ & 0.0002 & 0.0002 & - \\
\hline MOLYBDENUM & $09 / 26 / 85$ & 0001 & $M G / L$ & & 48.7 & 0.01 & - \\
\hline NICKEL & $09 / 26 / 85$ & 0001 & $M G / L$ & & 0.14 & 0.04 & - \\
\hline HITRATE & $09 / 26 / 85$ & 0001 & MG/L & & 3. & 1. & $\cdot$ \\
\hline NITRITE & $09 / 26 / 85$ & 0001 & MG/L & $<$ & 0.1 & 0.1 & - \\
\hline PH & $09 / 26 / 85$ & 0001 & SU & & 8.11 & - & - \\
\hline PHOSPHATE & $09 / 26 / 85$ & 0001 & $M G / L$ & & 0.4 & 0.1 & - \\
\hline POTASSIUM & $09 / 26 / 85$ & 0001 & MG/L & & 24.2 & 0.01 & - \\
\hline RADIUM-226 & $09 / 26 / 85$ & 0001 & $\mathrm{PCl} / \mathrm{L}$ & & 190. & 1. & 20. \\
\hline RADIUH-226 + RADIUM-228 & $09 / 26 / 85$ & 0001 & PCI/L & & 190.00 & - & - \\
\hline
\end{tabular}

PARAMETER VALUE INDICATOR (PVI): < - LESS THAN DETECTION LIMIT 
GROUHDHATER QUALITY DATA BY LOCATION

SITE: AMBO1 AMBROSIA LAKE

LOCATION: 0747

NORTH COORDINATE: 53990.5 FT

EAST COORDINATE: 60805.1 FT

O6/10/80 TO $12 / 04 / 92$

REPORT DATE: $08 / 04 / 94$

FORMATION OF COMPLETION: ALLUVIUM (AL)

HYDRAULIC FLOU RELATIOHSHIP: ON-SITE (O)

\begin{tabular}{|c|c|c|c|c|c|c|c|}
\hline PARAMETER NAME & LOG DATE & $\begin{array}{c}\text { SAMPLE } \\
\text { ID }\end{array}$ & $\begin{array}{l}\text { UNITS OF } \\
\text { MEASURE }\end{array}$ & PVI & $\begin{array}{l}\text { Parameter } \\
\text { VALUE FLAGS }\end{array}$ & $\begin{array}{l}\text { DETECTION } \\
\text { LIMIT }\end{array}$ & $\begin{array}{l}\text { PARAMETER } \\
\text { UKCERTAINTY }\end{array}$ \\
\hline RADIUA-228 & $09 / 26 / 85$ & 0001 & PCI/L & & 0. & 1. & 4.9 \\
\hline SELENIUN & $09 / 26 / 85$ & 0001 & MG/L & $<$ & 0.005 & 0.005 & - \\
\hline SILICA - SIOZ & $09 / 26 / 85$ & 0001 & $M G / L$ & & 15. & 2. & $\cdot$ \\
\hline soolum & $09 / 26 / 85$ & 0001 & MG/L & & 2500. & 0.002 & - \\
\hline SPECIFIC CONDUCTANCE & $09 / 26 / 85$ & 0001 & UнНО/CM & & 10000 & - & - \\
\hline STRONTIUA & $09 / 26 / 85$ & 0001 & MG/L & & 3.9 & 0.1 & - \\
\hline SULFATE & $09 / 26 / 85$ & 0001 & MG/L & & 5940. & 0.1 & - \\
\hline THORIUM-230 & $09 / 26 / 85$ & 0001 & PCI/L & & 0.9 & 1. & 0.9 \\
\hline TIN & $09 / 26 / 85$ & 0001 & $M G / L$ & $<$ & 0.005 & 0.005 & - \\
\hline TOTAL DISSOLVED SOLIDS & $09 / 26 / 85$ & 0001 & $M G / L$ & & 9560. & 10. & - \\
\hline URAHIUN & $09 / 26 / 85$ & 0001 & $M G / L$ & & 1.24 & 0.003 & - \\
\hline VANADIUM & $09 / 26 / 85$ & 0001 & $M G / L$ & $<$ & 0.01 & 0.01 & - \\
\hline ZINC & $09 / 26 / 85$ & 0001 & $M G / L$ & & 1.73 & 0.005 & - \\
\hline
\end{tabular}

PARAMETER VALUE INDICATOR (PVI): < - LESS THAN DETECTION LIMIT

SAMPLE ID COOES:

0001 - FILTERED SAMPLE (.45 MICRONS) 
GROUNDWATER QUALITY DATA BY LOCATIOH

SITE: AMBO1 AMBROSIA LAKE

LOCATION: 0748

MORTH COORDIHATE: 53985.9 FT

EAST COORDINATE: 60803.6 FT

06/10/80 TO 12/04/92

REPORT DATE: $08 / 04 / 94$

FORHATION OF COMPLETIOH: ALLUVIUM (AL)

HYDRAULIC FLON RELATIOHSHIP: ON-SITE (O)

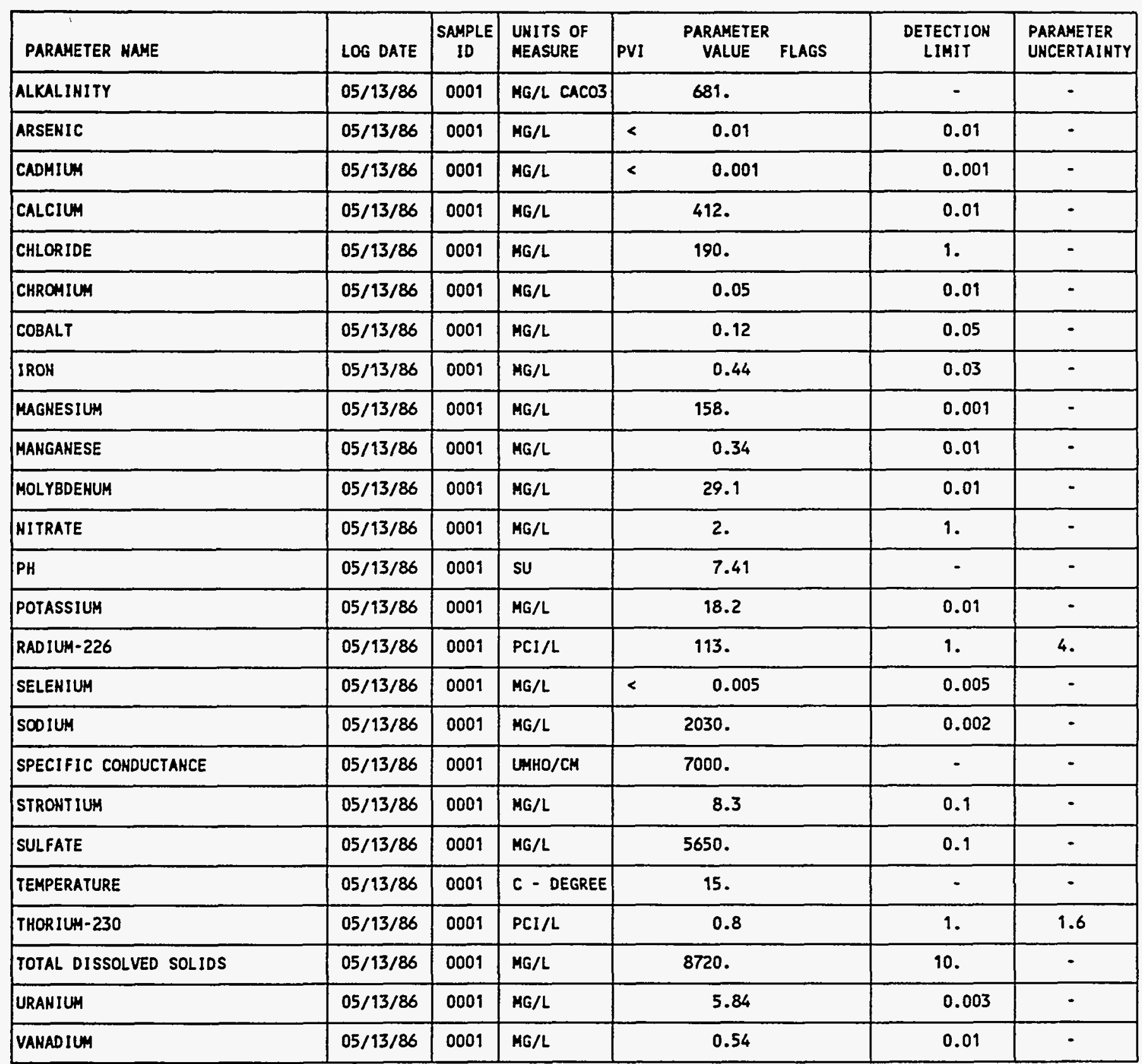

PARAMETER VALUE INDICATOR (PVI): < - LESS THAN DETECTION LIMIT

SAMPLE ID COOES:

0001 - FILTERED SAMPLE (.45 MICRONS) 
GROUNDWATER OUALITY DATA BY LOCATIOH

SITE: AMBO1 AMBROSIA LAKE

LOCATION: 0749

NORTH COORDINATE: $\quad 53981.3$ FT

EAST COORDINATE: 60802.2 FT

06/10/80 TO $12 / 04 / 92$

REPORT DATE: 08/04/94

FORMATION OF COMPLETIOH: ALLUVIUM (AL)

HYDRAULIC FLOW RELATIONSHIP: ON-SITE (O)

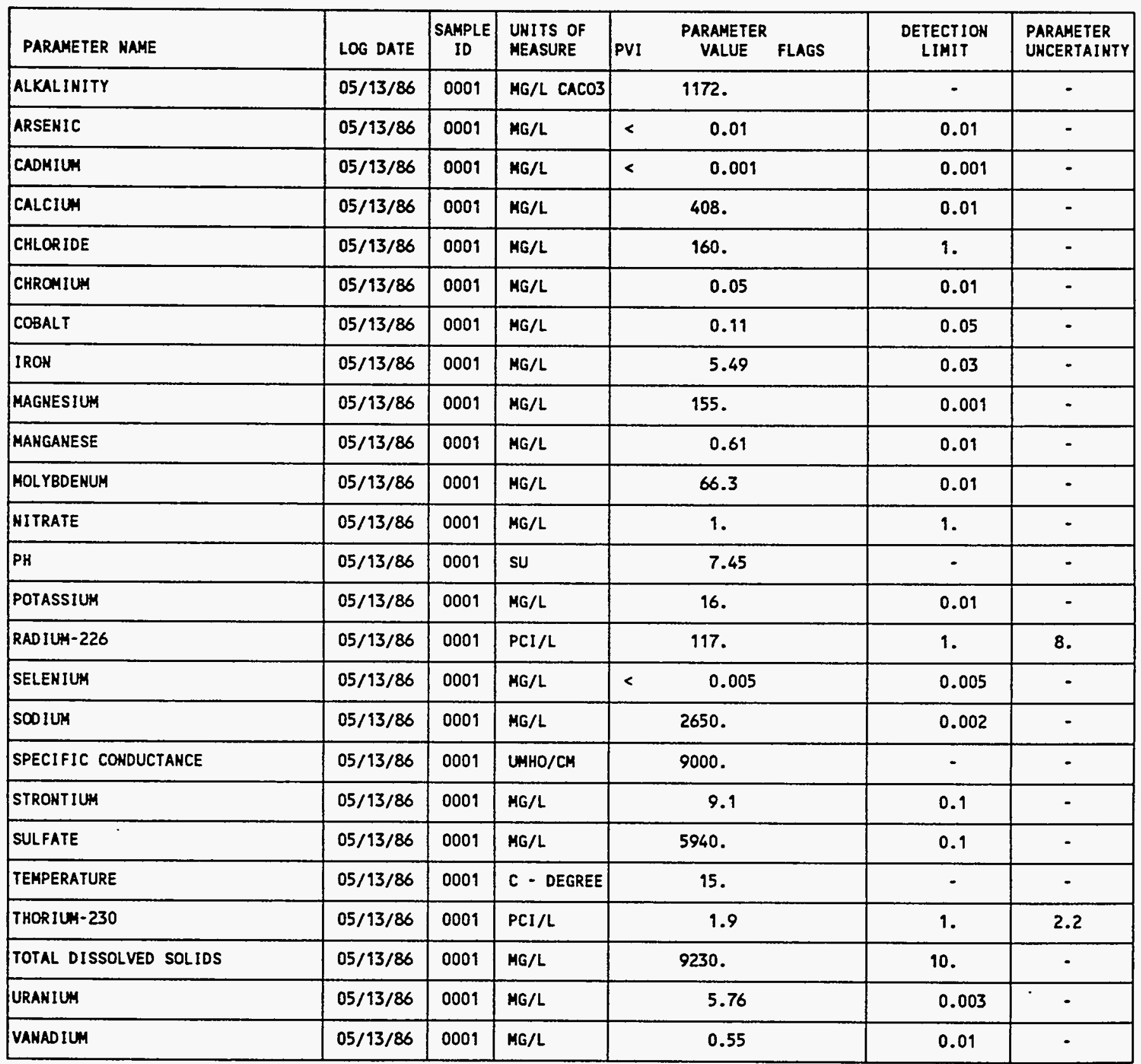

PARAMETER VALUE INDICATOR (PVI): < - LESS THAN DETECTION LIMIT

SAMPLE ID COOES:

0001 - FILTERED SAMPLE (.45 MICRONS) 
GROUMDHATER QUALITY DATA BY LOCATION

SITE: AMBO1 AMBROSIA LAKE

LOCATION: OTSO

HORTH COORDINATE: 53977.5 FT

EAST COOROINATE: 60801.1 FT

$06 / 10 / 80$ TO $12 / 04 / 92$

REPORT DATE: $08 / 04 / 94$

FORMATION OF COHPLETIOH: URANIUM MILL TAILIHGS (TA)

HYDRAULIC FLON RELATIONSHIP: ON-SITE (O)

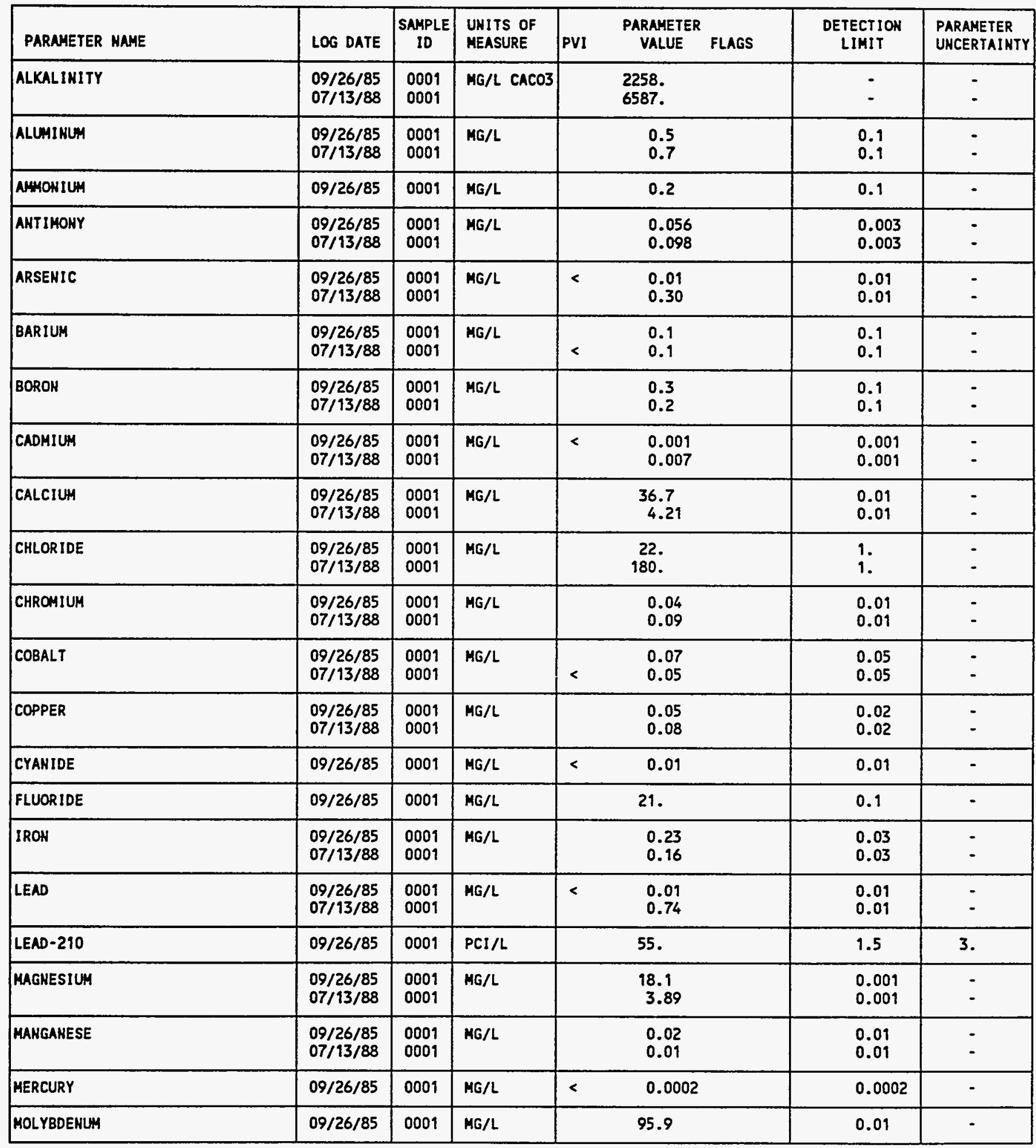

PARAMETER VALUE IMDICATOR (PVI): \& - LESS THAH DETECTION LIMIT

SAMPLE ID COOES:

D001 - FILTERED SAMPLE (.45 MICRONS) 
GROUNDWATER QUALITY DATA BY LOCATION

SITE: AMBO1 AMBROSIA LAKE

LOCATION: 0750

MORTH COORDINATE: 53977.5 FT

EAST COORDINATE: 60809.9 FT

$06 / 10 / 80$ TO $12 / 04 / 92$

REPORT DATE: $08 / 04 / 94$

FORMATION OF COMPLETION: URANIUM MILL TAILINGS (TA)

HYDRAULIC FLON RELATIONSHIP: ON-SITE (O)

\begin{tabular}{|c|c|c|c|c|c|c|c|}
\hline PARAMETER NAME & LOG DATE & $\begin{array}{l}\text { SAMPLE } \\
\text { ID }\end{array}$ & $\begin{array}{l}\text { UNITS OF } \\
\text { MEASURE }\end{array}$ & PV & $\begin{array}{l}\text { PARAMETER } \\
\text { VALUE FLAGS }\end{array}$ & $\begin{array}{l}\text { DETECTION } \\
\text { LIMIT }\end{array}$ & $\begin{array}{l}\text { PARAMETER } \\
\text { UNCERTAINTY }\end{array}$ \\
\hline MOLYBDENUM & $07 / 13 / 88$ & 0001 & MG/L & & 120. & 0.01 & - \\
\hline HICKEL. & $\begin{array}{l}09 / 26 / 85 \\
07 / 13 / 88\end{array}$ & $\begin{array}{l}0001 \\
0001\end{array}$ & MG/L & $<$ & $\begin{array}{l}0.13 \\
0.04\end{array}$ & $\begin{array}{l}0.04 \\
0.04\end{array}$ & - \\
\hline HITRATE & $09 / 26 / 85$ & 0001 & MG/L & & 1. & 1. & - \\
\hline NITRITE & $09 / 26 / 85$ & 0001 & MG/L & $<$ & 0.1 & 0.1 & - \\
\hline PH & $\begin{array}{l}09 / 26 / 85 \\
07 / 13 / 88\end{array}$ & $\begin{array}{l}0001 \\
0001\end{array}$ & SU & & $\begin{array}{r}9.61 \\
10.06\end{array}$ & - & - \\
\hline PHOSPHATE & $\begin{array}{l}09 / 26 / 85 \\
07 / 13 / 88\end{array}$ & $\begin{array}{l}0001 \\
0001\end{array}$ & MG/L & & $\begin{array}{r}4.8 \\
15.9\end{array}$ & $\begin{array}{l}0.1 \\
0.1\end{array}$ & - \\
\hline POTASSIUM & $\begin{array}{l}09 / 26 / 85 \\
07 / 13 / 88\end{array}$ & $\begin{array}{l}0001 \\
0001\end{array}$ & $\mathrm{MG} / \mathrm{L}$ & & $\begin{array}{l}22.3 \\
31 .\end{array}$ & $\begin{array}{l}0.01 \\
0.01\end{array}$ & - \\
\hline RADIUM-226 & $09 / 26 / 85$ & 0001 & $\mathrm{PCI} / \mathrm{L}$ & & 90. & 1. & 10. \\
\hline RADIUA-226 + RADIUA-228 & $09 / 26 / 85$ & 0001 & $\mathrm{PCI} / \mathrm{L}$ & & 90.10 & - & $\cdot$ \\
\hline RADIUH-228 & $09 / 26 / 85$ & 0001 & $\mathrm{PCI} / \mathrm{L}$ & & 0.1 & 1. & 3.2 \\
\hline SELENIUM & $\begin{array}{l}09 / 26 / 85 \\
07 / 13 / 88\end{array}$ & $\begin{array}{l}0001 \\
0001\end{array}$ & $M G / L$ & $<$ & $\begin{array}{l}0.005 \\
0.320\end{array}$ & $\begin{array}{l}0.005 \\
0.005\end{array}$ & - \\
\hline SILICA - SIO2 & $\begin{array}{l}09 / 26 / 85 \\
07 / 13 / 88\end{array}$ & $\begin{array}{l}0001 \\
0001\end{array}$ & MG/L & & $\begin{array}{l}9.0 \\
5.5\end{array}$ & $\begin{array}{l}2 . \\
2 .\end{array}$ & - \\
\hline SILVER & $\begin{array}{l}09 / 26 / 85 \\
07 / 13 / 88\end{array}$ & $\begin{array}{l}0001 \\
0001\end{array}$ & $M G / L$ & $<$ & $\begin{array}{l}0.01 \\
0.01\end{array}$ & $\begin{array}{l}0.01 \\
0.01\end{array}$ & - \\
\hline SOOIUN & $\begin{array}{l}09 / 26 / 85 \\
07 / 13 / 88\end{array}$ & $\begin{array}{l}0001 \\
0001\end{array}$ & $M G / L$ & & $\begin{array}{l}4190 . \\
5880 .\end{array}$ & $\begin{array}{l}0.002 \\
0.002\end{array}$ & - \\
\hline SPECIFIC CONDUCTANCE & $\begin{array}{l}09 / 26 / 85 \\
07 / 13 / 88\end{array}$ & $\begin{array}{l}0001 \\
0001\end{array}$ & UMHO/CM & & $\begin{array}{l}10000 . \\
16000 .\end{array}$ & - & - \\
\hline STROHTIUM & $\begin{array}{l}09 / 26 / 85 \\
07 / 13 / 88\end{array}$ & $\begin{array}{l}0001 \\
0001\end{array}$ & $M G / L$ & & $\begin{array}{l}0.5 \\
0.2\end{array}$ & $\begin{array}{l}0.1 \\
0.1\end{array}$ & - \\
\hline SULFATE & $\begin{array}{l}09 / 26 / 85 \\
07 / 13 / 88\end{array}$ & $\begin{array}{l}0001 \\
0001\end{array}$ & $M G / L$ & & $\begin{array}{l}6550 . \\
6310 .\end{array}$ & $\begin{array}{l}0.1 \\
0.1\end{array}$ & - \\
\hline SULFIDE & $09 / 26 / 85$ & 0001 & $M G / L$ & $<$ & 0.1 & 0.1 & - \\
\hline TEMPERATURE & $\begin{array}{l}09 / 26 / 85 \\
07 / 13 / 88\end{array}$ & $\begin{array}{l}0001 \\
0001\end{array}$ & C - DEGREE & & $\begin{array}{l}17 . \\
18 .\end{array}$ & - & - \\
\hline TIK & $\begin{array}{l}09 / 26 / 85 \\
07 / 13 / 88\end{array}$ & $\begin{array}{l}0001 \\
0001\end{array}$ & $M G / L$ & $<$ & $\begin{array}{l}0.005 \\
0.398\end{array}$ & $\begin{array}{l}0.005 \\
0.005\end{array}$ & - \\
\hline TOTAL DISSOLVED SOLIOS & $\begin{array}{l}09 / 26 / 85 \\
07 / 13 / 88\end{array}$ & $\begin{array}{l}0001 \\
0001\end{array}$ & $M G / L$ & & $\begin{array}{l}13100 . \\
16700 .\end{array}$ & $\begin{array}{l}10 . \\
10 .\end{array}$ & - \\
\hline URAKIUM & $\begin{array}{l}09 / 26 / 85 \\
07 / 13 / 88\end{array}$ & $\begin{array}{l}0001 \\
0001\end{array}$ & $M G / L$ & & $\begin{array}{l}6.27 \\
8.40\end{array}$ & $\begin{array}{l}0.003 \\
0.003\end{array}$ & - \\
\hline
\end{tabular}

PARAMETER VAlUe Indicator (PVI): < - Less than DETECTION LIMIT

SAMPLE ID COOES:

0001 - FILTERED SAMPLE (.45 MICRONS) 
GROUNDWATER OUALITY DATA BY LOCATION

SITE: AMBOI AMBROSIA LAKE

LOCATION: 0750

MORTH COORDINATE: 53977.5 FT

EAST COORDIHATE: 60801.1 FT

$06 / 10 / 80$ TO $12 / 04 / 92$

REPORT DATE: 08/04/94

FORMATIOH OF COMPLETION: URANIUM MILL TAILIHGS (TA)

HYDRAULIC FLON RELATIONSHIP: ON-SITE (O)

\begin{tabular}{|c|c|c|c|c|c|c|c|}
\hline PARAMETER MAME & LOG DATE & $\begin{array}{c}\text { SAHPLE } \\
\text { ID }\end{array}$ & $\begin{array}{l}\text { UMITS OF } \\
\text { MEASURE }\end{array}$ & PVI & $\begin{array}{l}\text { PARAMETER } \\
\text { VALUE FLAGS }\end{array}$ & $\begin{array}{l}\text { DETECTION } \\
\text { LIMIT }\end{array}$ & $\begin{array}{l}\text { PARAMETER } \\
\text { UNCERTAINTY }\end{array}$ \\
\hline VANADIUM & $\begin{array}{l}09 / 26 / 85 \\
07 / 13 / 88\end{array}$ & $\begin{array}{l}0001 \\
0001\end{array}$ & $M G / L$ & $<$ & $\begin{array}{l}0.01 \\
0.30\end{array}$ & $\begin{array}{l}0.01 \\
0.01\end{array}$ & - \\
\hline ZIMC & $\begin{array}{l}09 / 26 / 85 \\
07 / 13 / 88\end{array}$ & $\begin{array}{l}0001 \\
0001\end{array}$ & $M G / L$ & & $\begin{array}{l}0.053 \\
0.030\end{array}$ & $\begin{array}{l}0.005 \\
0.005\end{array}$ & - \\
\hline
\end{tabular}

PARAMETER VALUE IMDICATOR (PVI): < - LESS THAH DETECTION LIMIT

SAMPLE ID COOES:

0001 - FILTERED SAMPLE (.45 MICRONS) 
GROUNDWATER OUALITY DATA BY LOCATION

SITE: AMBOI AMBROSIA LAKE

LOCATION: 0751

NORTH COORDINATE: 53972.8 FT

EAST COORDINATE: 60799.4 FT

06/10/80 TO $12 / 04 / 92$

REPORT DATE: 08/04/94

FORMATION OF COMPLETION: URANIUA RILL TAILINGS (TA)

HYDRAULIC FLON RELATIONSHIP: ON-SITE (O)

\begin{tabular}{|c|c|c|c|c|c|c|c|}
\hline PARAMETER NAME & LOG DATE & $\begin{array}{c}\text { SAMPLE } \\
\text { ID }\end{array}$ & $\begin{array}{l}\text { UNITS OF } \\
\text { MEASURE }\end{array}$ & PVI & $\begin{array}{l}\text { PARAMETER } \\
\text { VALUE FLAGS }\end{array}$ & $\begin{array}{l}\text { DETECTION } \\
\text { LIMIT }\end{array}$ & $\begin{array}{l}\text { PARAMETER } \\
\text { UNCERTAINTY }\end{array}$ \\
\hline ALKALINITY & $\begin{array}{l}10 / 09 / 85 \\
10 / 09 / 85 \\
10 / 09 / 85 \\
10 / 09 / 85 \\
10 / 09 / 85 \\
07 / 13 / 88\end{array}$ & $\begin{array}{l}0001 \\
0002 \\
0003 \\
0004 \\
0005 \\
0001\end{array}$ & MG/L CACO3 & & $\begin{array}{l}4501 . \\
4501 . \\
4501 . \\
4501 . \\
4501 . \\
4512 .\end{array}$ & $\begin{array}{l}- \\
- \\
- \\
-\end{array}$ & $\begin{array}{l}- \\
- \\
- \\
-\end{array}$ \\
\hline ALUMINUM & $\begin{array}{l}10 / 09 / 85 \\
10 / 09 / 85 \\
10 / 09 / 85 \\
10 / 09 / 85 \\
10 / 09 / 85 \\
07 / 13 / 88\end{array}$ & $\begin{array}{l}0001 \\
0002 \\
0003 \\
0004 \\
0005 \\
0001\end{array}$ & $M G / L$ & & $\begin{array}{l}0.2 \\
0.4 \\
0.5 \\
0.4 \\
0.3 \\
0.8\end{array}$ & $\begin{array}{l}0.1 \\
0.1 \\
0.1 \\
0.1 \\
0.1 \\
0.1\end{array}$ & $\begin{array}{l}- \\
- \\
- \\
- \\
-\end{array}$ \\
\hline AMHONIUM & $\begin{array}{l}10 / 09 / 85 \\
10 / 09 / 85 \\
10 / 09 / 85 \\
10 / 09 / 85 \\
10 / 09 / 85\end{array}$ & $\begin{array}{l}0001 \\
0002 \\
0003 \\
0004 \\
0005\end{array}$ & $M G / L$ & & $\begin{array}{l}1.6 \\
1.6 \\
1.8 \\
1.6 \\
1.6\end{array}$ & $\begin{array}{l}0.1 \\
0.1 \\
0.1 \\
0.1 \\
0.1\end{array}$ & $\begin{array}{l}- \\
- \\
- \\
-\end{array}$ \\
\hline ANT IMONY & $\begin{array}{l}10 / 09 / 85 \\
10 / 09 / 85 \\
10 / 09 / 85 \\
10 / 09 / 85 \\
10 / 09 / 85 \\
07 / 13 / 88\end{array}$ & $\begin{array}{l}0001 \\
0002 \\
0003 \\
0004 \\
0005 \\
0001\end{array}$ & $M G / L$ & $\begin{array}{l}< \\
< \\
< \\
< \\
<\end{array}$ & $\begin{array}{l}0.003 \\
0.003 \\
0.003 \\
0.003 \\
0.003 \\
0.070\end{array}$ & $\begin{array}{l}0.003 \\
0.003 \\
0.003 \\
0.003 \\
0.003 \\
0.003\end{array}$ & $\begin{array}{l}- \\
- \\
- \\
-\end{array}$ \\
\hline ARSEHIC & $\begin{array}{l}10 / 09 / 85 \\
10 / 09 / 85 \\
10 / 09 / 85 \\
10 / 09 / 85 \\
10 / 09 / 85 \\
07 / 13 / 88\end{array}$ & $\begin{array}{l}0001 \\
0002 \\
0003 \\
0004 \\
0005 \\
0001\end{array}$ & $M G / L$ & & $\begin{array}{l}0.01 \\
0.01 \\
0.01 \\
0.01 \\
0.01 \\
0.10\end{array}$ & $\begin{array}{l}0.01 \\
0.01 \\
0.01 \\
0.01 \\
0.01 \\
0.01\end{array}$ & $\begin{array}{l}- \\
- \\
- \\
- \\
-\end{array}$ \\
\hline BARIUM & $\begin{array}{l}10 / 09 / 85 \\
10 / 09 / 85 \\
10 / 09 / 85 \\
10 / 09 / 85 \\
10 / 09 / 85 \\
07 / 13 / 88\end{array}$ & $\begin{array}{l}0001 \\
0002 \\
0003 \\
0004 \\
0005 \\
0001\end{array}$ & $M G / L$ & . & $\begin{array}{l}0.1 \\
0.1 \\
0.1 \\
0.1 \\
0.1 \\
0.1\end{array}$ & $\begin{array}{l}0.1 \\
0.1 \\
0.1 \\
0.1 \\
0.1 \\
0.1\end{array}$ & $\begin{array}{l}- \\
- \\
- \\
-\end{array}$ \\
\hline BORON & $\begin{array}{l}10 / 09 / 85 \\
10 / 09 / 85 \\
10 / 09 / 85 \\
10 / 09 / 85 \\
10 / 09 / 85 \\
07 / 13 / 88\end{array}$ & $\begin{array}{l}0001 \\
0002 \\
0003 \\
0004 \\
0005 \\
0001\end{array}$ & $\mathrm{MG} / \mathrm{L}$ & & $\begin{array}{l}0.4 \\
0.4 \\
0.3 \\
0.3 \\
0.4 \\
0.2\end{array}$ & $\begin{array}{l}0.1 \\
0.1 \\
0.1 \\
0.1 \\
0.1 \\
0.1\end{array}$ & $\begin{array}{l}- \\
- \\
- \\
- \\
-\end{array}$ \\
\hline CADHIUM & $\begin{array}{l}10 / 09 / 85 \\
10 / 09 / 85 \\
10 / 09 / 85 \\
10 / 09 / 85 \\
10 / 09 / 85 \\
07 / 13 / 88\end{array}$ & $\begin{array}{l}0001 \\
0002 \\
0003 \\
0004 \\
0005 \\
0001\end{array}$ & MG/L & $\begin{array}{l}< \\
< \\
< \\
<\end{array}$ & $\begin{array}{l}0.001 \\
0.001 \\
0.001 \\
0.001 \\
0.001 \\
0.006\end{array}$ & $\begin{array}{l}0.001 \\
0.001 \\
0.001 \\
0.001 \\
0.001 \\
0.001\end{array}$ & $\begin{array}{l}- \\
- \\
- \\
-\end{array}$ \\
\hline CALCIUM & $10 / 09 / 85$ & 0001 & HG/L & & 1.94 & 0.01 & - \\
\hline
\end{tabular}

PARAMETER VALUE IMDICATOR (PVI): < - LESS THAN DETECTIOH LIMIT

SAMPLE IO COOES:

O001 - FILTERED SAMPLE (.45 MICRONS)

0002 - FILTERED REPLICATE SAMPLE (.45 MICRONS)

0003 - FILTERED REPLICATE SAMPLE (.45 MICROHS)

0004 - FILTERED REPLICATE SAMPLE (.45 MICRONS) 
GROUHDWATER QUALITY DATA BY LOCATION

SITE: AMB01 AMBROSIA LAKE

LOCATION: 0751

HORTH COORDINATE: 53972.8 FT

EAST COORDINATE: 60799.4 FT

06/10/80 TO $12 / 04 / 92$

REPORT DATE: 08/04/94

FORMATIOH OF COMPLETIOH: URAHIUM MILL TAILINGS (TA)

HYDRAULIC FLON RELATIONSHIP: OH-SITE (O)

\begin{tabular}{|c|c|c|c|c|c|c|c|}
\hline PARAMETER NAME & LOG DATE & $\underset{\text { SAMPLE }}{\text { ID }}$ & $\begin{array}{l}\text { UNITS OF } \\
\text { MEASURE }\end{array}$ & PVI & $\begin{array}{l}\text { PARAMETER } \\
\text { VALUE FLAGS }\end{array}$ & $\begin{array}{l}\text { DETECTION } \\
\text { LIMIT }\end{array}$ & $\begin{array}{l}\text { PARAMETER } \\
\text { UNCERTAINTY }\end{array}$ \\
\hline CALCIUM & $\begin{array}{l}10 / 09 / 85 \\
10 / 09 / 85 \\
10 / 09 / 85 \\
10 / 09 / 85 \\
07 / 13 / 88\end{array}$ & $\begin{array}{l}0002 \\
0003 \\
0004 \\
0005 \\
0001\end{array}$ & $M G / L$ & & $\begin{array}{l}1.94 \\
3.63 \\
2.52 \\
1.94 \\
14.2\end{array}$ & $\begin{array}{l}0.01 \\
0.01 \\
0.01 \\
0.01 \\
0.01\end{array}$ & $\begin{array}{l}: \\
: \\
-\end{array}$ \\
\hline CHROMIUH & $\begin{array}{l}10 / 09 / 85 \\
10 / 09 / 85 \\
10 / 09 / 85 \\
10 / 09 / 85 \\
10 / 09 / 85 \\
07 / 13 / 88\end{array}$ & $\begin{array}{l}0001 \\
0002 \\
0003 \\
0004 \\
0005 \\
0001\end{array}$ & MG/L & & $\begin{array}{l}0.03 \\
0.02 \\
0.02 \\
0.04 \\
0.02 \\
0.11\end{array}$ & $\begin{array}{l}0.01 \\
0.01 \\
0.01 \\
0.01 \\
0.01 \\
0.01\end{array}$ & $\begin{array}{l}: \\
: \\
:\end{array}$ \\
\hline COBALT & $\begin{array}{l}10 / 09 / 85 \\
10 / 09 / 85 \\
10 / 09 / 85 \\
10 / 09 / 85 \\
10 / 09 / 85 \\
07 / 13 / 88\end{array}$ & $\begin{array}{l}0001 \\
0002 \\
0003 \\
0004 \\
0005 \\
0001\end{array}$ & $M G / L$ & $<$ & $\begin{array}{l}0.08 \\
0.07 \\
0.07 \\
0.06 \\
0.07 \\
0.05\end{array}$ & $\begin{array}{l}0.05 \\
0.05 \\
0.05 \\
0.05 \\
0.05 \\
0.05\end{array}$ & $\begin{array}{l}- \\
- \\
- \\
-\end{array}$ \\
\hline COPPER & $\begin{array}{l}10 / 09 / 85 \\
10 / 09 / 85 \\
10 / 09 / 85 \\
10 / 09 / 85 \\
10 / 09 / 85 \\
07 / 13 / 88\end{array}$ & $\begin{array}{l}0001 \\
0002 \\
0003 \\
0004 \\
0005 \\
0001\end{array}$ & $M G / L$ & & $\begin{array}{l}0.03 \\
0.03 \\
0.04 \\
0.04 \\
0.03 \\
0.08\end{array}$ & $\begin{array}{l}0.02 \\
0.02 \\
0.02 \\
0.02 \\
0.02 \\
0.02\end{array}$ & $\begin{array}{l}- \\
- \\
- \\
-\end{array}$ \\
\hline IRON & $\begin{array}{l}10 / 09 / 85 \\
10 / 09 / 85 \\
10 / 09 / 85 \\
10 / 09 / 85 \\
10 / 09 / 85 \\
07 / 13 / 88\end{array}$ & $\begin{array}{l}0001 \\
0002 \\
0003 \\
0004 \\
0005 \\
0001\end{array}$ & $M G / L$ & & $\begin{array}{l}0.13 \\
0.1 \\
0.15 \\
0.18 \\
0.12 \\
0.30\end{array}$ & $\begin{array}{l}0.03 \\
0.03 \\
0.03 \\
0.03 \\
0.03 \\
0.03\end{array}$ & $\begin{array}{l}- \\
- \\
- \\
-\end{array}$ \\
\hline LEAD & $\begin{array}{l}10 / 09 / 85 \\
10 / 09 / 85 \\
10 / 09 / 85\end{array}$ & $\begin{array}{l}0001 \\
0002 \\
0003\end{array}$ & $M G / L$ & $\begin{array}{l}< \\
<\end{array}$ & $\begin{array}{l}0.01 \\
0.01 \\
0.01\end{array}$ & $\begin{array}{l}0.01 \\
0.01 \\
0.01\end{array}$ & $:$ \\
\hline
\end{tabular}

PARAMETER VALUE IHDICATOR (PVI): < - LESS THAN DETECTION LIMIT

SAMPLE ID COOES:

0001 - FILTERED SAMPLE (.45 MICRONS)

0002 - FILTERED REPLICATE SAMPLE (.45 MICRONS)

0003 - FILTERED REPLICATE SAMPLE (.45 MICRONS)

0004 - FILTERED REPLICATE SAMPLE (.45 MICROHS) 
GROUNDWATER QUALITY DATA BY LOCATION

SITE: AMBO1 AMBROSIA LAKE

LOCATION: 0751

HORTH COORDINATE: 53972.8 FT

EAST COORDINATE: 60799.4 FT

06/10/80 TO 12/04/92

REPORT DATE : 08/04/94

FORMATION OF COMPLETION: URANIUM MILL TAILINGS (TA)

HYDRAULIC FLOU RELATIONSHIP: OH-SITE (O)

\begin{tabular}{|c|c|c|c|c|c|c|c|}
\hline PARAMETER NAME & LOG DATE & $\begin{array}{c}\text { SAMPLE } \\
\text { ID }\end{array}$ & $\begin{array}{l}\text { UNITS OF } \\
\text { MEASURE }\end{array}$ & PVI & $\begin{array}{l}\text { PARAMETER } \\
\text { VALUE FLAGS }\end{array}$ & $\begin{array}{l}\text { DETECTIOH } \\
\text { LIMIT }\end{array}$ & $\begin{array}{l}\text { PARAMETER } \\
\text { UNCERTAINTY }\end{array}$ \\
\hline LEAD & $\begin{array}{l}10 / 09 / 85 \\
10 / 09 / 85 \\
07 / 13 / 88\end{array}$ & $\begin{array}{l}0004 \\
0005 \\
0001\end{array}$ & $\mathrm{ME} / \mathrm{L}$ & $<$ & $\begin{array}{l}0.01 \\
0.01 \\
0.02\end{array}$ & $\begin{array}{l}0.01 \\
0.01 \\
0.01\end{array}$ & : \\
\hline LEAD-210 & $\begin{array}{l}10 / 09 / 85 \\
10 / 09 / 85 \\
10 / 09 / 85 \\
10 / 09 / 85\end{array}$ & $\begin{array}{l}0001 \\
0002 \\
0003 \\
0004\end{array}$ & $\mathrm{PCI} / \mathrm{L}$ & & $\begin{array}{l}52 . \\
48 . \\
90 . \\
52 .\end{array}$ & $\begin{array}{l}1.5 \\
1.5 \\
1.5 \\
1.5\end{array}$ & $\begin{array}{l}3 . \\
3 . \\
3 .\end{array}$ \\
\hline MAGNESIUM & $\begin{array}{l}10 / 09 / 85 \\
10 / 09 / 85 \\
10 / 09 / 85 \\
10 / 09 / 85 \\
10 / 09 / 85 \\
07 / 13 / 88\end{array}$ & $\begin{array}{l}0001 \\
0002 \\
0003 \\
0004 \\
0005 \\
0001\end{array}$ & $M G / L$ & & $\begin{array}{l}0.225 \\
0.196 \\
0.571 \\
0.282 \\
0.22 \\
31.5\end{array}$ & $\begin{array}{l}0.001 \\
0.001 \\
0.001 \\
0.001 \\
0.001 \\
0.001\end{array}$ & : \\
\hline MERCURY & $\begin{array}{l}10 / 09 / 85 \\
10 / 09 / 85 \\
10 / 09 / 85 \\
10 / 09 / 85 \\
10 / 09 / 85\end{array}$ & $\begin{array}{l}0001 \\
0002 \\
0003 \\
0004 \\
0005\end{array}$ & $M G / L$ & $\begin{array}{l}< \\
< \\
< \\
<\end{array}$ & $\begin{array}{l}0.0002 \\
0.0002 \\
0.0002 \\
0.0002 \\
0.0002\end{array}$ & $\begin{array}{l}0.0002 \\
0.0002 \\
0.0002 \\
0.0002 \\
0.0002\end{array}$ & $:$ \\
\hline KOLYBDEENUM & $\begin{array}{l}10 / 09 / 85 \\
10 / 09 / 85 \\
10 / 09 / 85 \\
10 / 09 / 85 \\
10 / 09 / 85 \\
07 / 13 / 88\end{array}$ & $\begin{array}{l}0001 \\
0002 \\
0003 \\
0004 \\
0005 \\
0001\end{array}$ & $M G / L$ & & $\begin{array}{c}99.0 \\
102 . \\
97.5 \\
102 . \\
104 . \\
118 .\end{array}$ & $\begin{array}{l}0.01 \\
0.01 \\
0.01 \\
0.01 \\
0.01 \\
0.01\end{array}$ & $\begin{array}{l}- \\
: \\
:\end{array}$ \\
\hline HITRATE & $\begin{array}{l}10 / 09 / 85 \\
10 / 09 / 85 \\
10 / 09 / 85 \\
10 / 09 / 85 \\
10 / 09 / 85\end{array}$ & $\begin{array}{l}0001 \\
0002 \\
0003 \\
0004 \\
0005\end{array}$ & $M G / L$ & & $\begin{array}{l}3600 . \\
2600 . \\
4900 . \\
2500 . \\
350 .\end{array}$ & $\begin{array}{l}1 . \\
1 . \\
1 . \\
1 . \\
1 .\end{array}$ & $\begin{array}{l}- \\
- \\
-\end{array}$ \\
\hline NITRITE & $\begin{array}{l}10 / 09 / 85 \\
10 / 09 / 85 \\
10 / 09 / 85 \\
10 / 09 / 85 \\
10 / 09 / 85\end{array}$ & $\begin{array}{l}0001 \\
0002 \\
0003 \\
0004 \\
0005\end{array}$ & $M G / L$ & $\begin{array}{l}< \\
< \\
< \\
<\end{array}$ & $\begin{array}{l}0.1 \\
0.1 \\
0.1 \\
0.1 \\
0.1\end{array}$ & $\begin{array}{l}0.1 \\
0.1 \\
0.1 \\
0.1 \\
0.1\end{array}$ & $\begin{array}{l}- \\
- \\
- \\
-\end{array}$ \\
\hline PH & $10 / 09 / 85$ & 0001 & SU & & 10.13 & - & - \\
\hline
\end{tabular}

PARAMETER VALUE INOICATOR (PVI): < - LESS THAN DETECTION LIMIT

SAMPLE ID CODES:

O001 - FILTERED SAMPLE (.45 MICRONS)

0002 - FILTERED REPLICATE SAMPLE (.45 MICRONS)

0003 - FILTERED REPLICATE SAMPLE (.45 MICRONS)

0004 - FILTERED REPLICATE SAMPLE (.45 MICRONS) 
GROUNDWATER QUALITY DATA BY LOCATION

SITE: AMBOI AMBROSIA LAKE

LOCATION: 0751

NORTH COORDINATE: 53972.8 FT

EAST COORDINATE: 60799.4 FT

06/10/80 TO $12 / 04 / 92$

REPORT DATE: $08 / 04 / 94$

FORMATION OF COMPLETION: URAMIUH MILL TAILIMGS (TA) HYDRAULIC FLON RELATIONSHIP: OH-SITE (O)

\begin{tabular}{|c|c|c|c|c|c|c|c|}
\hline PARANETER NAHE & LOG DATE & $\begin{array}{c}\text { SAMPLE } \\
10\end{array}$ & $\begin{array}{l}\text { UNITS OF } \\
\text { MEASURE }\end{array}$ & PVI & $\begin{array}{l}\text { PARAMETER } \\
\text { VALUE FLAGS }\end{array}$ & $\begin{array}{l}\text { DETECTIOH } \\
\text { LIHIT }\end{array}$ & $\begin{array}{l}\text { PARAMETER } \\
\text { UNCERTAINTY }\end{array}$ \\
\hline PH & $\begin{array}{l}10 / 09 / 85 \\
10 / 09 / 85 \\
10 / 09 / 85 \\
10 / 09 / 85 \\
07 / 13 / 88\end{array}$ & $\begin{array}{l}0002 \\
0003 \\
0004 \\
0005 \\
0001\end{array}$ & SU & & $\begin{array}{r}10.13 \\
10.13 \\
10.13 \\
10.13 \\
9.23\end{array}$ & $\begin{array}{l}- \\
- \\
- \\
-\end{array}$ & $\begin{array}{l}- \\
- \\
. \\
.\end{array}$ \\
\hline PHOSPHATE & $\begin{array}{l}10 / 09 / 85 \\
10 / 09 / 85 \\
10 / 09 / 85 \\
10 / 09 / 85 \\
10 / 09 / 85 \\
07 / 13 / 88\end{array}$ & $\begin{array}{l}0001 \\
0002 \\
0003 \\
0004 \\
0005 \\
0001\end{array}$ & $M G / L$ & & $\begin{array}{l}15 . \\
13 . \\
17 . \\
13 . \\
14 . \\
6.5\end{array}$ & $\begin{array}{l}0.1 \\
0.1 \\
0.1 \\
0.1 \\
0.1 \\
0.1\end{array}$ & $\begin{array}{l}- \\
- \\
- \\
- \\
-\end{array}$ \\
\hline POTASSIUM & $\begin{array}{l}10 / 09 / 85 \\
10 / 09 / 85 \\
10 / 09 / 85 \\
10 / 09 / 85 \\
10 / 09 / 85 \\
07 / 13 / 88\end{array}$ & $\begin{array}{l}0001 \\
0002 \\
0003 \\
0004 \\
0005 \\
0001\end{array}$ & MG/L & & $\begin{array}{l}25.1 \\
24.8 \\
24.7 \\
25.1 \\
25.6 \\
21.5\end{array}$ & $\begin{array}{l}0.01 \\
0.01 \\
0.01 \\
0.01 \\
0.01 \\
0.01\end{array}$ & $\begin{array}{l}- \\
- \\
- \\
.\end{array}$ \\
\hline RADIUH-226 & $\begin{array}{l}10 / 09 / 85 \\
10 / 09 / 85 \\
10 / 09 / 85 \\
10 / 09 / 85 \\
10 / 09 / 85\end{array}$ & $\begin{array}{l}0001 \\
0002 \\
0003 \\
0004 \\
0005\end{array}$ & PCI/L & & $\begin{array}{l}220 . \\
240 \\
190 \\
230 \\
200\end{array}$ & $\begin{array}{l}1 . \\
1 . \\
1 . \\
1 . \\
1 .\end{array}$ & $\begin{array}{l}20 . \\
20 \\
10 . \\
10 . \\
10 .\end{array}$ \\
\hline RADIUH-226 + RADIUH-228 & $\begin{array}{l}10 / 09 / 85 \\
10 / 09 / 85 \\
10 / 09 / 85 \\
10 / 09 / 85 \\
10 / 09 / 85\end{array}$ & $\begin{array}{l}0001 \\
0002 \\
0003 \\
0004 \\
0005\end{array}$ & PCI/L & & $\begin{array}{l}220.00 \\
240.00 \\
190.60 \\
230.00 \\
200.80\end{array}$ & $\begin{array}{l}- \\
- \\
- \\
-\end{array}$ & $\begin{array}{l}- \\
- \\
- \\
-\end{array}$ \\
\hline RADIUM-228 & $\begin{array}{l}10 / 09 / 85 \\
10 / 09 / 85 \\
10 / 09 / 85 \\
10 / 09 / 85 \\
10 / 09 / 85\end{array}$ & $\begin{array}{l}0001 \\
0002 \\
0003 \\
0004 \\
0005\end{array}$ & PCI/L & & $\begin{array}{l}0 . \\
0 . \\
0.6 \\
0 . \\
0.8\end{array}$ & $\begin{array}{l}1 . \\
1 . \\
1 . \\
1 . \\
1 .\end{array}$ & $\begin{array}{l}3.9 \\
4 . \\
4.7 \\
3.1 \\
3.6\end{array}$ \\
\hline SELENIUM & $\begin{array}{l}10 / 09 / 85 \\
10 / 09 / 85 \\
10 / 09 / 85 \\
10 / 09 / 85 \\
10 / 09 / 85 \\
07 / 13 / 88\end{array}$ & $\begin{array}{l}0001 \\
0002 \\
0003 \\
0004 \\
0005 \\
0001\end{array}$ & $M G / L$ & & $\begin{array}{l}0.019 \\
0.015 \\
0.015 \\
0.016 \\
0.018 \\
0.294\end{array}$ & $\begin{array}{l}0.005 \\
0.005 \\
0.005 \\
0.005 \\
0.005 \\
0.005\end{array}$ & $\begin{array}{l}- \\
- \\
- \\
- \\
-\end{array}$ \\
\hline SILICA - SIO2 & $\begin{array}{l}10 / 09 / 85 \\
10 / 09 / 85 \\
10 / 09 / 85 \\
10 / 09 / 85 \\
10 / 09 / 85 \\
07 / 13 / 88\end{array}$ & $\begin{array}{l}0001 \\
0002 \\
0003 \\
0004 \\
0005 \\
0001\end{array}$ & MG/L & & $\begin{array}{l}10 . \\
10 . \\
10 . \\
10 . \\
10 . \\
6.6\end{array}$ & $\begin{array}{l}2 . \\
2 . \\
2 . \\
2 . \\
2 . \\
2 .\end{array}$ & $\begin{array}{l}- \\
- \\
- \\
- \\
-\end{array}$ \\
\hline SILVER & $\begin{array}{l}10 / 09 / 85 \\
10 / 09 / 85 \\
10 / 09 / 85 \\
10 / 09 / 85\end{array}$ & $\begin{array}{l}0001 \\
0002 \\
0003 \\
0004\end{array}$ & $M G / L$ & $\begin{array}{l}< \\
< \\
<\end{array}$ & $\begin{array}{l}0.01 \\
0.01 \\
0.01 \\
0.01\end{array}$ & $\begin{array}{l}0.01 \\
0.01 \\
0.01 \\
0.01\end{array}$ & $\begin{array}{l}- \\
- \\
-\end{array}$ \\
\hline
\end{tabular}

PARAMETER VALUE IHDICATOR (PVI): < - LESS THAN DETECTIOH LIMIT

SAMPLE ID COOES:

0001 - FILTERED SAMPLE (.45 MICRONS)

0002 - FILTERED REPLICATE SAMPLE (.45 MICROWS)

0003 - FILTERED REPLICATE SAMPLE (.45 MICRONS)

0004 - FILTERED REPLICATE SAMPLE (.45 MICRONS) 


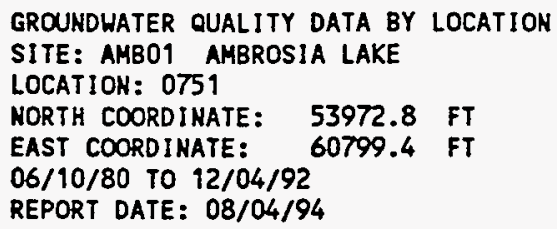

FORMATION OF COMPLETION: URAHIUM MILL TAILINGS (TA) HYDRAULIC FLON RELATIONSHIP: OW-SITE (O)

\begin{tabular}{|c|c|c|c|c|c|c|c|}
\hline PARAMETER NAME & LOG DATE & $\begin{array}{c}\text { SAMPLE } \\
\text { ID }\end{array}$ & $\begin{array}{l}\text { UNITS OF } \\
\text { MEASURE }\end{array}$ & PVI & $\begin{array}{l}\text { PARAMETER } \\
\text { VALUE FLAGS }\end{array}$ & $\begin{array}{l}\text { DETECTIOH } \\
\text { LIMIT }\end{array}$ & $\begin{array}{l}\text { PARAMETER } \\
\text { UNCERTAINTY }\end{array}$ \\
\hline SILVER & $\begin{array}{l}10 / 09 / 85 \\
07 / 13 / 88\end{array}$ & $\begin{array}{l}0005 \\
0001\end{array}$ & HG/L & $<$ & $\begin{array}{l}0.01 \\
0.02\end{array}$ & $\begin{array}{l}0.01 \\
0.01\end{array}$ & $\dot{-}$ \\
\hline Scoivm & $\begin{array}{l}10 / 09 / 85 \\
10 / 09 / 85 \\
10 / 09 / 85 \\
10 / 09 / 85 \\
10 / 09 / 85 \\
07 / 13 / 88\end{array}$ & $\begin{array}{l}0001 \\
0002 \\
0003 \\
0004 \\
0005 \\
0001\end{array}$ & MG/L & & $\begin{array}{l}5240 . \\
4870 . \\
5860 \\
4870 \\
4840 \\
4600\end{array}$ & $\begin{array}{l}0.002 \\
0.002 \\
0.002 \\
0.002 \\
0.002 \\
0.002\end{array}$ & $\begin{array}{l}- \\
- \\
- \\
-\end{array}$ \\
\hline SPECIFIC CONDUCTANCE & $\begin{array}{l}10 / 09 / 85 \\
10 / 09 / 85 \\
10 / 09 / 85 \\
10 / 09 / 85 \\
10 / 09 / 85 \\
07 / 13 / 88\end{array}$ & $\begin{array}{l}0001 \\
0002 \\
0003 \\
0004 \\
0005 \\
0001\end{array}$ & LMHO/CM & & $\begin{array}{l}12000 . \\
12000 . \\
12000 . \\
12000 \\
12000 \\
11000 .\end{array}$ & $\begin{array}{l}- \\
- \\
- \\
-\end{array}$ & $\begin{array}{l}- \\
- \\
- \\
-\end{array}$ \\
\hline SULFATE & $\begin{array}{l}10 / 09 / 85 \\
10 / 09 / 85 \\
10 / 09 / 85 \\
10 / 09 / 85 \\
10 / 09 / 85 \\
07 / 93 / 88\end{array}$ & $\begin{array}{l}0001 \\
0002 \\
0003 \\
0004 \\
0005 \\
0001\end{array}$ & MG/L & & $\begin{array}{l}5416 . \\
5130 . \\
5400 . \\
5320 . \\
5290 . \\
6130 .\end{array}$ & $\begin{array}{l}0.1 \\
0.1 \\
0.1 \\
0.1 \\
0.1 \\
0.1\end{array}$ & $\begin{array}{l}- \\
- \\
- \\
- \\
-\end{array}$ \\
\hline SULFIDE & $\begin{array}{l}10 / 09 / 85 \\
10 / 09 / 85 \\
10 / 09 / 85 \\
10 / 09 / 85 \\
10 / 09 / 85\end{array}$ & $\begin{array}{l}0001 \\
0002 \\
0003 \\
0004 \\
0005\end{array}$ & MG $/ L$ & $\begin{array}{l}< \\
< \\
< \\
< \\
<\end{array}$ & $\begin{array}{l}0.1 \\
0.1 \\
0.1 \\
0.1 \\
0.1\end{array}$ & $\begin{array}{l}0.1 \\
0.1 \\
0.1 \\
0.1 \\
0.1\end{array}$ & $\begin{array}{l}- \\
- \\
-\end{array}$ \\
\hline THORIUH-230 & $\begin{array}{l}10 / 09 / 85 \\
10 / 09 / 85 \\
10 / 09 / 85 \\
10 / 09 / 85 \\
10 / 09 / 85\end{array}$ & $\begin{array}{l}0001 \\
0002 \\
0003 \\
0004 \\
0005\end{array}$ & $\mathrm{PCl} / \mathrm{L}$ & & $\begin{array}{l}70 . \\
60 . \\
50 . \\
50 . \\
60 .\end{array}$ & $\begin{array}{l}1 . \\
1 . \\
1 . \\
1 .\end{array}$ & $\begin{array}{l}10 . \\
10 . \\
10 . \\
10 . \\
10 .\end{array}$ \\
\hline TIN & $\begin{array}{l}10 / 09 / 85 \\
10 / 09 / 85 \\
10 / 09 / 85 \\
10 / 09 / 85 \\
10 / 09 / 85 \\
07 / 13 / 88\end{array}$ & $\begin{array}{l}0001 \\
0002 \\
0003 \\
0004 \\
0005 \\
0001\end{array}$ & $M G / L$ & $\begin{array}{l}< \\
< \\
< \\
< \\
<\end{array}$ & $\begin{array}{l}0.005 \\
0.005 \\
0.005 \\
0.005 \\
0.005 \\
0.338\end{array}$ & $\begin{array}{l}0.005 \\
0.005 \\
0.005 \\
0.005 \\
0.005 \\
0.005\end{array}$ & $\begin{array}{l}- \\
- \\
- \\
-\end{array}$ \\
\hline
\end{tabular}


GROUNDWATER QUALITY DATA BY LOCATION

SITE: AMBOI AMBROSIA LAKE

LOCATION: 0751

MORTH COORDINATE: 53972.8 FT

EAST COORDINATE: $\quad 60799.4$ FT

06/10/80 TO $12 / 04 / 92$

REPORT DATE: $08 / 04 / 94$

FORMATION OF COMPLETIOH: URANIUM MILL TAILIMGS (TA) HYDRAULIC FLOH RELATIONSHIP: OH-SITE (O)

\begin{tabular}{|c|c|c|c|c|c|c|c|}
\hline PARAMETER MAME & LOG DATE & $\begin{array}{c}\text { SAMPLE } \\
\text { I0 }\end{array}$ & $\begin{array}{l}\text { UNITS OF } \\
\text { MEASURE }\end{array}$ & PVI & $\begin{array}{l}\text { PARAMETER } \\
\text { VALUE FLAGS }\end{array}$ & $\begin{array}{l}\text { DETECTION } \\
\text { LIMIT }\end{array}$ & $\begin{array}{l}\text { PARAMETER } \\
\text { UNCERTAINTY }\end{array}$ \\
\hline TOTAL DISSOLVED SOLIDS & $\begin{array}{l}10 / 09 / 85 \\
10 / 09 / 85 \\
10 / 09 / 85 \\
10 / 09 / 85 \\
10 / 09 / 85 \\
07 / 13 / 88\end{array}$ & $\begin{array}{l}0001 \\
0002 \\
0003 \\
0004 \\
0005 \\
0001\end{array}$ & $M G / L$ & & $\begin{array}{l}13300 . \\
12700 . \\
13100 . \\
13000 \\
13000 \\
14300\end{array}$ & $\begin{array}{l}10 . \\
10 . \\
10 . \\
10 . \\
10 . \\
10 .\end{array}$ & $\begin{array}{l}- \\
: \\
:\end{array}$ \\
\hline URAHIUM & $\begin{array}{l}10 / 09 / 85 \\
10 / 09 / 85 \\
10 / 09 / 85 \\
10 / 09 / 85 \\
10 / 09 / 85 \\
07 / 13 / 88\end{array}$ & $\begin{array}{l}0001 \\
0002 \\
0003 \\
0004 \\
0005 \\
0001\end{array}$ & MG/L & & $\begin{array}{c}2.49 \\
2.65 \\
5.68 \\
2.46 \\
2.22 \\
14.6\end{array}$ & $\begin{array}{l}0.003 \\
0.003 \\
0.003 \\
0.003 \\
0.003 \\
0.003\end{array}$ & $\begin{array}{l}: \\
: \\
-\end{array}$ \\
\hline VAKADIUH & $\begin{array}{l}10 / 09 / 85 \\
10 / 09 / 85 \\
10 / 09 / 85 \\
10 / 09 / 85 \\
10 / 09 / 85 \\
07 / 13 / 88\end{array}$ & $\begin{array}{l}0001 \\
0002 \\
0003 \\
0004 \\
0005 \\
0001\end{array}$ & $M G / L$ & $\begin{array}{l}< \\
< \\
< \\
<\end{array}$ & $\begin{array}{l}0.01 \\
0.01 \\
0.01 \\
0.01 \\
0.01 \\
0.15\end{array}$ & $\begin{array}{l}0.01 \\
0.01 \\
0.01 \\
0.01 \\
0.01 \\
0.01\end{array}$ & : \\
\hline ZINC & $\begin{array}{l}10 / 09 / 85 \\
10 / 09 / 85 \\
10 / 09 / 85 \\
10 / 09 / 85 \\
10 / 09 / 85 \\
07 / 13 / 88\end{array}$ & $\begin{array}{l}0001 \\
0002 \\
0003 \\
0004 \\
0005 \\
0001\end{array}$ & $M G / L$ & & $\begin{array}{l}0.044 \\
0.017 \\
0.046 \\
0.031 \\
0.025 \\
0.018\end{array}$ & $\begin{array}{l}0.005 \\
0.005 \\
0.005 \\
0.005 \\
0.005 \\
0.005\end{array}$ & $\begin{array}{l}- \\
- \\
- \\
-\end{array}$ \\
\hline
\end{tabular}

PARAMETER VALUE IHDICATOR (PVI): < - LESS THAN DETECTION LIMIT

SAMPLE ID COOES:

0001 - FILTERED SAMPLE (.45 MICRONS)

0002 - FILTERED REPLICATE SAMPLE (.45 MICRONS)

0003 - FILTERED REPLICATE SAMPLE (.45 MICRONS)

0004 - FILTERED REPLICATE SAMPLE (.45 MICRONS) 
GROUNDWATER QUALITY DATA BY LOCATION

SITE: AMBO1 AMBROSIA LAKE

LOCATION: 0752

NORTH COORDINATE: 53802.6 FT

EAST COORDINATE: $\quad 60808.1$ FT

06/10/80 TO 12/04/92

REPORT DATE: $08 / 04 / 94$

FORMATION OF COMPLETION: URANIUM MILL TAILIMGS (TA)

HYDRAULIC FLON RELATIOHSHIP: ON-SITE (O)

\begin{tabular}{|c|c|c|c|c|c|c|c|}
\hline PARAMETER NAME & LOG DATE & $\begin{array}{c}\text { SAMPLE } \\
\text { ID }\end{array}$ & $\begin{array}{l}\text { UNITS OF } \\
\text { MEASURE }\end{array}$ & PVI & $\begin{array}{l}\text { PARAMETER } \\
\text { VALUE FLAGS }\end{array}$ & $\begin{array}{l}\text { DETECTION } \\
\text { LIMIT }\end{array}$ & $\begin{array}{l}\text { PARAMETER } \\
\text { UHCERTAINTY }\end{array}$ \\
\hline ALKALINITY & $\begin{array}{l}09 / 26 / 85 \\
07 / 13 / 88\end{array}$ & $\begin{array}{l}0001 \\
0001\end{array}$ & $M G / L \quad C A C O 3$ & & $\begin{array}{l}6828 . \\
1637 .\end{array}$ & - & - \\
\hline ALUAINUM & $\begin{array}{l}09 / 26 / 85 \\
07 / 13 / 88\end{array}$ & $\begin{array}{l}0001 \\
0001\end{array}$ & $M G / L$ & & $\begin{array}{l}0.4 \\
0.2\end{array}$ & $\begin{array}{l}0.1 \\
0.1\end{array}$ & - \\
\hline NAFONIUM & $09 / 26 / 85$ & 0001 & $M G / L$ & $<$ & 0.1 & 0.1 & - \\
\hline AKTIMONY & $\begin{array}{l}09 / 26 / 85 \\
07 / 13 / 88\end{array}$ & $\begin{array}{l}0001 \\
0001\end{array}$ & $M G / L$ & $<$ & $\begin{array}{l}0.003 \\
0.088\end{array}$ & $\begin{array}{l}0.003 \\
0.003\end{array}$ & - \\
\hline ARSEKIC & $\begin{array}{l}09 / 26 / 85 \\
07 / 13 / 88\end{array}$ & $\begin{array}{l}0009 \\
0001\end{array}$ & $M G / L$ & $<$ & $\begin{array}{l}0.01 \\
0.11\end{array}$ & $\begin{array}{l}0.01 \\
0.01\end{array}$ & 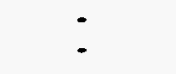 \\
\hline BARIUH & $\begin{array}{l}09 / 26 / 85 \\
07 / 13 / 88\end{array}$ & $\begin{array}{l}0001 \\
0001\end{array}$ & $M G / L$ & $<$ & $\begin{array}{l}0.1 \\
0.1\end{array}$ & $\begin{array}{l}0.1 \\
0.1\end{array}$ & : \\
\hline BORON & $\begin{array}{l}09 / 26 / 85 \\
07 / 13 / 88\end{array}$ & $\begin{array}{l}0001 \\
0001\end{array}$ & $M G / L$ & & $\begin{array}{l}0.4 \\
0.2\end{array}$ & $\begin{array}{l}0.1 \\
0.1\end{array}$ & : \\
\hline BROMIDE & $07 / 13 / 88$ & 0001 & $M G / L$ & $<$ & 0.1 & 0.1 & - \\
\hline CADMIUH & $\begin{array}{l}09 / 26 / 85 \\
07 / 13 / 88\end{array}$ & $\begin{array}{l}0001 \\
0001\end{array}$ & MG/L & $<$ & $\begin{array}{l}0.001 \\
0.006\end{array}$ & $\begin{array}{l}0.001 \\
0.001\end{array}$ & - \\
\hline CALCIUM & $\begin{array}{l}09 / 26 / 85 \\
07 / 13 / 88\end{array}$ & $\begin{array}{l}0001 \\
0001\end{array}$ & $M G / L$ & & $\begin{array}{l}4.28 \\
4.05\end{array}$ & $\begin{array}{l}0.01 \\
0.01\end{array}$ & - \\
\hline CHLORIDE & $\begin{array}{l}09 / 26 / 85 \\
07 / 13 / 88\end{array}$ & $\begin{array}{l}0001 \\
0001\end{array}$ & $M G / L$ & & $\begin{array}{r}6 . \\
280 .\end{array}$ & $\begin{array}{l}1 . \\
1 .\end{array}$ & - \\
\hline CHRONIUM & $\begin{array}{l}09 / 26 / 85 \\
07 / 13 / 88\end{array}$ & $\begin{array}{l}0001 \\
0001\end{array}$ & $M G / L$ & & $\begin{array}{l}0.02 \\
0.04\end{array}$ & $\begin{array}{l}0.01 \\
0.01\end{array}$ & 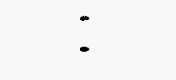 \\
\hline COBALT & $\begin{array}{l}09 / 26 / 85 \\
07 / 13 / 88\end{array}$ & $\begin{array}{l}0001 \\
0001\end{array}$ & ME/L & $<$ & $\begin{array}{l}0.07 \\
0.05\end{array}$ & $\begin{array}{l}0.05 \\
0.05\end{array}$ & $\dot{-}$ \\
\hline COPPER & $\begin{array}{l}09 / 26 / 85 \\
07 / 13 / 88\end{array}$ & $\begin{array}{l}0001 \\
0001\end{array}$ & $M G / L$ & & $\begin{array}{l}0.04 \\
0.03\end{array}$ & $\begin{array}{l}0.02 \\
0.02\end{array}$ & 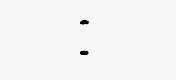 \\
\hline CYANIDE & $09 / 26 / 85$ & 0001 & $M G / L$ & $<$ & 0.01 & 0.01 & - \\
\hline FLUORIDE & $\begin{array}{l}09 / 26 / 85 \\
07 / 13 / 88\end{array}$ & $\begin{array}{l}0001 \\
0001\end{array}$ & $M G / L$ & & $\begin{array}{r}16 . \\
4.3\end{array}$ & $\begin{array}{l}0.1 \\
0.1\end{array}$ & - \\
\hline GROSS ALPHA & $07 / 13 / 88$ & 0001 & $\mathrm{PCI} / \mathrm{L}$ & & 3200 & 0.2 & 300. \\
\hline GROSS BETA & $07 / 13 / 88$ & 0001 & PCI/L & & 1100 & 1. & 100. \\
\hline IRON & $\begin{array}{l}09 / 26 / 85 \\
07 / 13 / 88\end{array}$ & $\begin{array}{l}0001 \\
0001\end{array}$ & $M G / L$ & & $\begin{array}{l}0.18 \\
0.07\end{array}$ & $\begin{array}{l}0.03 \\
0.03\end{array}$ & - \\
\hline LEAD & $\begin{array}{l}09 / 26 / 85 \\
07 / 13 / 88\end{array}$ & $\begin{array}{l}0001 \\
0001\end{array}$ & MG/L & $<$ & $\begin{array}{l}0.01 \\
0.03\end{array}$ & $\begin{array}{l}0.01 \\
0.01\end{array}$ & - \\
\hline LEAD-210 & $09 / 26 / 85$ & 0001 & $\mathrm{PCI} / \mathrm{L}$ & & 66. & 1.5 & 3. \\
\hline MAGNESIUM & $09 / 26 / 85$ & 0001 & $M G / L$ & & 2.3 & 0.001 & - \\
\hline
\end{tabular}

PARAMETER VALUE INDICATOR (PVI): < - LESS THAN DETECTION LIMIT 
GROUNDHATER QUALITY DATA BY LOCATIOH

SITE: AMBO1 AMBROSIA LAKE

LOCATION: 0752

NORTH COORDINATE: $\quad 53802.6$ FT

EAST COORDINATE: 60808.1 FT

$06 / 10 / 80$ TO $12 / 04 / 92$

REPORT DATE: 08/04/94

FORMATIOH OF COMPLETION: URAMIUN MILL TAILIHGS (TA)

HYDRAULIC FLOW RELATIONSHIP: OW-SITE (O)

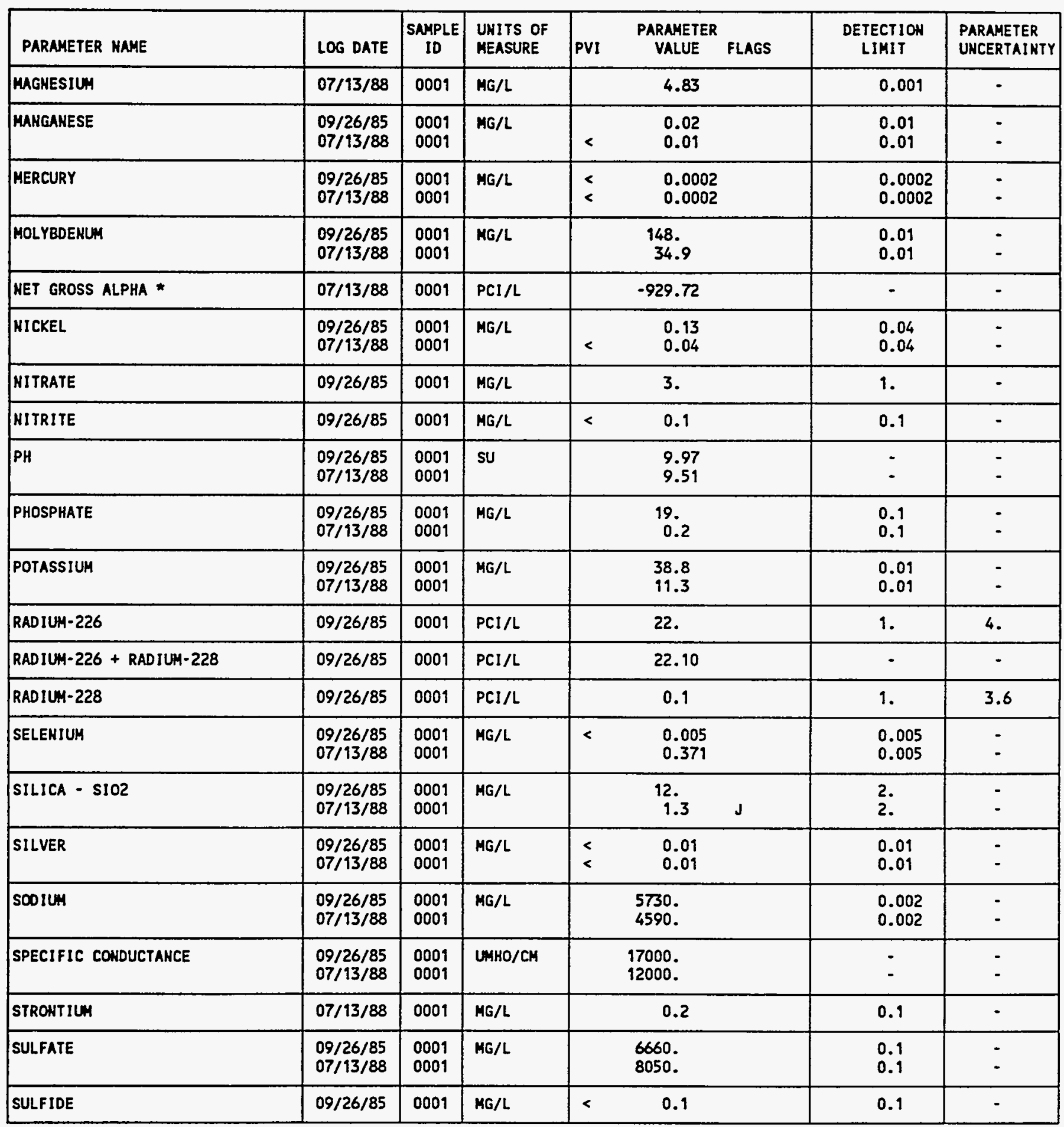

* NET gROSS ALPHA (GROSS ALPHA - URANIUM) WITH 1 Mg URANIUM = G86 PCI

PARAMETER VALUE IndICATOR (PVI): < - LESS THAN DETECTION LIMIT

SAMPLE ID COOES:

0001 - FILTERED SAMPLE (.45 MICROAS)

OTHER PARAMETER VALUE FLAGS:

J - eStIMLTED VALUE 


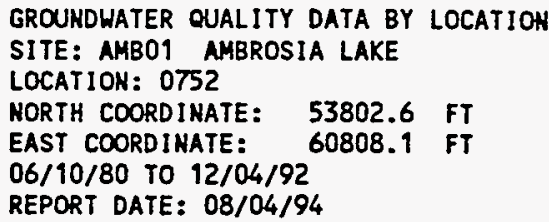

FORMATION OF COMPLETION: URANIUM MILL TAILINGS (TA) HYDRAULIC FLON RELATIONSHIP: ON-SITE (O)

\begin{tabular}{|c|c|c|c|c|c|c|c|}
\hline PARAMETER NAME & LOG DATE & $\underset{\text { SAMPLE }}{\text { ID }}$ & $\begin{array}{l}\text { UNITS OF } \\
\text { MEASURE }\end{array}$ & PVI & $\begin{array}{l}\text { PARAMETER } \\
\text { VALUE FLAGS }\end{array}$ & $\begin{array}{l}\text { DETECTION } \\
\text { LIMIT }\end{array}$ & $\begin{array}{l}\text { PARAMETER } \\
\text { UNCERTAINTY }\end{array}$ \\
\hline TEMPERATURE & $\begin{array}{l}09 / 26 / 85 \\
07 / 13 / 88\end{array}$ & $\begin{array}{l}0001 \\
0001\end{array}$ & C - DEGREE & & $\begin{array}{l}16 . \\
96 .\end{array}$ & - & - \\
\hline THORIUA-230 & $09 / 26 / 85$ & 0001 & $\mathrm{PCI} / \mathrm{L}$ & & 420. & 1. & 30. \\
\hline TIN & $\begin{array}{l}09 / 26 / 85 \\
07 / 13 / 88\end{array}$ & $\begin{array}{l}0001 \\
0001\end{array}$ & MG/L & $<$ & $\begin{array}{l}0.005 \\
0.323\end{array}$ & $\begin{array}{l}0.005 \\
0.005\end{array}$ & - \\
\hline URANIU:M & $\begin{array}{l}09 / 26 / 85 \\
07 / 13 / 88\end{array}$ & $\begin{array}{l}0009 \\
0001\end{array}$ & MG/L & & $\begin{array}{l}8.65 \\
6.02\end{array}$ & $\begin{array}{l}0.003 \\
0.003\end{array}$ & - \\
\hline VANADIUM & $\begin{array}{l}09 / 26 / 85 \\
07 / 13 / 88\end{array}$ & $\begin{array}{l}0001 \\
0001\end{array}$ & MG/L & $<$ & $\begin{array}{l}0.01 \\
0.04\end{array}$ & $\begin{array}{l}0.01 \\
0.01\end{array}$ & - \\
\hline
\end{tabular}

PARAMETER VALUE INDICATOR (PVI): < - LESS THAN DETECTION LIMIT

SAMPLE ID COOES:

0001 - FILTERED SAMPLE (.45 MICRONS) 
GROUNDWATER OUALITY DATA BY LOCATION

SITE: AMBO1 AMBROSIA LAKE

LOCATION: 0759

NORTH COOROINATE: 53623.2 FT

EAST COORDIHATE: $\quad 60219.3$ FT

$06 / 10 / 80$ TO $12 / 04 / 92$

REPORT DATE: $08 / 04 / 94$

FORMATIOH OF COMPLETION: URANIUH MILL TAILIHGS (TA)

HYDRAULIC FLOW RELATIOHSHIP: ON-SITE (O)

\begin{tabular}{|c|c|c|c|c|c|c|c|}
\hline PARAMETER HAME & LOG DATE & $\begin{array}{c}\text { SAMPLE } \\
\text { ID }\end{array}$ & $\begin{array}{l}\text { UNITS OF } \\
\text { MEASURE }\end{array}$ & PVI & $\begin{array}{l}\text { PARAMETER } \\
\text { VALUE FLAGS }\end{array}$ & $\begin{array}{l}\text { DETECTIOH } \\
\text { LIMIT }\end{array}$ & $\begin{array}{l}\text { PARAMETER } \\
\text { UNCERTAINTY }\end{array}$ \\
\hline ALUMIRUM & $\begin{array}{l}10 / 16 / 85 \\
07 / 15 / 88\end{array}$ & $\begin{array}{l}0001 \\
0001\end{array}$ & MG/L & & $\begin{array}{l}0.2 \\
0.9\end{array}$ & $\begin{array}{l}0.1 \\
0.1\end{array}$ & $\dot{-}$ \\
\hline AMAOHIUM & $10 / 16 / 85$ & 0001 & $M G / L$ & & 3.1 & 0.1 & - \\
\hline ANTIMONY & $\begin{array}{l}10 / 16 / 85 \\
07 / 15 / 88\end{array}$ & $\begin{array}{l}0001 \\
0001\end{array}$ & MG/L & $<$ & $\begin{array}{l}0.003 \\
0.083\end{array}$ & $\begin{array}{l}0.003 \\
0.003\end{array}$ & - \\
\hline ARSEHIC & $\begin{array}{l}10 / 16 / 85 \\
07 / 15 / 88\end{array}$ & $\begin{array}{l}0001 \\
0001\end{array}$ & MG/L & $<$ & $\begin{array}{l}0.01 \\
0.17\end{array}$ & $\begin{array}{l}0.01 \\
0.01\end{array}$ & - \\
\hline BARIUM & $\begin{array}{l}10 / 16 / 85 \\
07 / 15 / 88\end{array}$ & $\begin{array}{l}0001 \\
0001\end{array}$ & MG/L & $<$ & $\begin{array}{l}0.1 \\
0.1\end{array}$ & $\begin{array}{l}0.1 \\
0.1\end{array}$ & $\dot{-}$ \\
\hline BORON & $\begin{array}{l}10 / 16 / 85 \\
07 / 15 / 88\end{array}$ & $\begin{array}{l}0001 \\
0001\end{array}$ & $M G / L$ & & $\begin{array}{l}0.3 \\
0.2\end{array}$ & $\begin{array}{l}0.1 \\
0.1\end{array}$ & $\dot{-}$ \\
\hline CAOMIUM & $\begin{array}{l}10 / 16 / 85 \\
07 / 15 / 88\end{array}$ & $\begin{array}{l}0001 \\
0001\end{array}$ & $M G / L$ & $<$ & $\begin{array}{l}0.001 \\
0.008\end{array}$ & $\begin{array}{l}0.001 \\
0.001\end{array}$ & - \\
\hline CALCIUM & $\begin{array}{l}10 / 96 / 85 \\
07 / 15 / 88\end{array}$ & $\begin{array}{l}0001 \\
0001\end{array}$ & MG/L & & $\begin{array}{l}8.38 \\
1.70\end{array}$ & $\begin{array}{l}0.01 \\
0.01\end{array}$ & $\dot{-}$ \\
\hline CHLORIOE & $07 / 15 / 88$ & 0001 & $\mathrm{MG} / \mathrm{L}$ & & 240. & 1. & - \\
\hline CHROMIUM & $\begin{array}{l}10 / 16 / 85 \\
07 / 15 / 88\end{array}$ & $\begin{array}{l}0001 \\
0001\end{array}$ & MG/L & & $\begin{array}{l}0.02 \\
0.11\end{array}$ & $\begin{array}{l}0.01 \\
0.01\end{array}$ & $\dot{-}$ \\
\hline COBALT & $\begin{array}{l}10 / 16 / 85 \\
07 / 15 / 88\end{array}$ & $\begin{array}{l}0001 \\
0001\end{array}$ & MG/L & $<$ & $\begin{array}{l}0.05 \\
0.05\end{array}$ & $\begin{array}{l}0.05 \\
0.05\end{array}$ & $\dot{-}$ \\
\hline COPPER & $\begin{array}{l}10 / 16 / 85 \\
07 / 15 / 88\end{array}$ & $\begin{array}{l}0001 \\
0001\end{array}$ & $M G / L$ & & $\begin{array}{l}0.03 \\
0.09\end{array}$ & $\begin{array}{l}0.02 \\
0.02\end{array}$ & : \\
\hline CYAHIDE & $10 / 16 / 85$ & 0001 & $M G / L$ & $<$ & 0.01 & 0.01 & - \\
\hline IRON & $07 / 15 / 88$ & 0001 & MG/L & & 0.18 & 0.03 & - \\
\hline LEAD & $\begin{array}{l}10 / 16 / 85 \\
07 / 15 / 88\end{array}$ & $\begin{array}{l}0001 \\
0001\end{array}$ & MG/L & $<$ & $\begin{array}{l}0.01 \\
0.02\end{array}$ & $\begin{array}{l}0.01 \\
0.01\end{array}$ & $\dot{-}$ \\
\hline MAGNESIUM & $\begin{array}{l}10 / 16 / 85 \\
07 / 15 / 88\end{array}$ & $\begin{array}{l}0001 \\
0001\end{array}$ & $M G / L$ & & $\begin{array}{l}2.63 \\
0.47\end{array}$ & $\begin{array}{l}0.001 \\
0.001\end{array}$ & - \\
\hline MAKGANESE & $\begin{array}{l}10 / 16 / 85 \\
07 / 15 / 88\end{array}$ & $\begin{array}{l}0001 \\
0001\end{array}$ & $M G / L$ & $<$ & $\begin{array}{l}0.03 \\
0.01\end{array}$ & $\begin{array}{l}0.01 \\
0.01\end{array}$ & - \\
\hline MOLYBDENUH & $\begin{array}{l}10 / 16 / 85 \\
07 / 15 / 88\end{array}$ & $\begin{array}{l}0001 \\
0001\end{array}$ & $M G / L$ & & $\begin{array}{l}247 . \\
135 .\end{array}$ & $\begin{array}{l}0.01 \\
0.01\end{array}$ & - \\
\hline HICKEL & $07 / 15 / 88$ & 0001 & $M G / L$ & $<$ & 0.04 & 0.04 & - \\
\hline NITRITE & $10 / 16 / 85$ & 0001 & MG/L & $<$ & 0.1 & 0.1 & - \\
\hline PHOSPHATE & $07 / 15 / 88$ & 0001 & MG/L & & 9.8 & 0.1 & - \\
\hline POTASSIUM & $\begin{array}{l}10 / 16 / 85 \\
07 / 15 / 88\end{array}$ & $\begin{array}{l}0001 \\
0001\end{array}$ & $M G / L$ & & $\begin{array}{l}46.7 \\
51.9\end{array}$ & $\begin{array}{l}0.01 \\
0.01\end{array}$ & - \\
\hline
\end{tabular}

PARAMETER VALUE INDICATOR (PVI): < - LESS THAN DETECTION LIMIT

SAMPLE ID COOES:

0001 - FILTERED SAMPLE (.45 MICRONS) 
GROUHDWATER QUALITY DATA BY LOCATIOH

SITE: AMBOI AMBROSIA LAKE

LOCATION: 0759

NORTH COORDINATE: 53623.2 FT

EAST COORDIMATE: 60219.3 FT

06/10/80 TO $12 / 04 / 92$

REPORT DATE : 08/04/94

FORMATION OF COMPLETIOH: URAKIUH MILL TAILINGS (TA)

HYDRAULIC FLON RELATIONSHIP: OH-SITE (O)

\begin{tabular}{|c|c|c|c|c|c|c|c|}
\hline PARAMETER MAME & LOG DATE & $\underset{\text { SAMPLE }}{\text { ID }}$ & $\begin{array}{l}\text { UNITS OF } \\
\text { MEASURE }\end{array}$ & PVI & $\begin{array}{l}\text { PARAMETER } \\
\text { VALUE FLAGS }\end{array}$ & $\begin{array}{l}\text { DETECTIOH } \\
\text { LIMIT }\end{array}$ & $\begin{array}{l}\text { PARAMETER } \\
\text { UNCERTAINTY }\end{array}$ \\
\hline SELENIUM & $\begin{array}{l}10 / 16 / 85 \\
07 / 15 / 88\end{array}$ & $\begin{array}{l}0001 \\
0001\end{array}$ & $M G / L$ & & $\begin{array}{l}0.017 \\
0.403\end{array}$ & $\begin{array}{l}0.005 \\
0.005\end{array}$ & - \\
\hline SILICA - SIO2 & $07 / 15 / 88$ & 0001 & $M G / L$ & & 10.7 & 2. & - \\
\hline SILVER & $\begin{array}{l}10 / 16 / 85 \\
07 / 15 / 88\end{array}$ & $\begin{array}{l}0001 \\
0001\end{array}$ & MG/L & $<$ & $\begin{array}{l}0.01 \\
0.02\end{array}$ & $\begin{array}{l}0.01 \\
0.01\end{array}$ & - \\
\hline scoilum & $\begin{array}{l}10 / 16 / 85 \\
07 / 15 / 88\end{array}$ & $\begin{array}{l}0001 \\
0001\end{array}$ & MG/L & & $\begin{array}{l}9880 . \\
5780 .\end{array}$ & $\begin{array}{l}0.002 \\
0.002\end{array}$ & $\dot{-}$ \\
\hline STRONTIU:M & $07 / 15 / 88$ & 0001 & $M G / L$ & & 0.2 & 0.1 & - \\
\hline SULFATE & $\begin{array}{l}10 / 16 / 85 \\
07 / 15 / 88\end{array}$ & $\begin{array}{l}0001 \\
0001\end{array}$ & MG/L & & $\begin{array}{r}11000 \\
8200\end{array}$ & $\begin{array}{l}0.1 \\
0.1\end{array}$ & - \\
\hline SULFIDE & $10 / 16 / 85$ & 0001 & MG/L & $<$ & 0.1 & 0.1 & - \\
\hline TIN & $\begin{array}{l}10 / 16 / 85 \\
07 / 15 / 88\end{array}$ & $\begin{array}{l}0001 \\
0001\end{array}$ & $M G / L$ & $<$ & $\begin{array}{l}0.005 \\
0.400\end{array}$ & $\begin{array}{l}0.005 \\
0.005\end{array}$ & - \\
\hline TOTAL DISSOLVED SOLIDS & $\begin{array}{l}10 / 16 / 85 \\
07 / 15 / 88\end{array}$ & $\begin{array}{l}0001 \\
0001\end{array}$ & $M G / L$ & & $\begin{array}{l}25800 \text {. } \\
18800 \text {. }\end{array}$ & $\begin{array}{l}10 . \\
10 .\end{array}$ & - \\
\hline URANIUM & $07 / 15 / 88$ & 0001 & $M G / L$ & & 12.6 & 0.003 & - \\
\hline VANADIUM & $\begin{array}{l}10 / 16 / 85 \\
07 / 15 / 88\end{array}$ & $\begin{array}{l}0001 \\
0001\end{array}$ & $M G / L$ & $<$ & $\begin{array}{l}0.01 \\
0.17\end{array}$ & $\begin{array}{l}0.01 \\
0.01\end{array}$ & - \\
\hline ZINC & $\begin{array}{l}10 / 16 / 85 \\
07 / 15 / 88\end{array}$ & $\begin{array}{l}0001 \\
0001\end{array}$ & $M G / L$ & & $\begin{array}{l}0.025 \\
0.014\end{array}$ & $\begin{array}{l}0.005 \\
0.005\end{array}$ & $\overline{-}$ \\
\hline
\end{tabular}

PARAMETER VALUE INDICATOR (PVI):

< - Less than Detection limit

SAMPLE ID COOES:

0001 - FILTERED SAMPLE (.45 MICRONS) 
GROUNDWATER QUALITY DATA BY LOCATION

SITE: AMBO1 AMBROSIA LAKE

LOCATION: OTT3

HORTH COORDINATE: 55311.5 FT

EAST COORDIMATE: 61688.9 FT

$06 / 10 / 80$ TO $12 / 04 / 92$

REPORT DATE: 08/04/94

FORMATIOH OF COMPLETIOH: TRES HERMANOS - CI SAMDSTONE (TO) HYDRAULIC FLON RELATIONSHIP: DOWN GRADIENT (D)

\begin{tabular}{|c|c|c|c|c|c|c|c|}
\hline PARAMETER NAME & LOG DATE & $\underset{10}{\text { SAMPLE }}$ & $\begin{array}{l}\text { UNITS OF } \\
\text { MEASURE }\end{array}$ & PVI & $\begin{array}{l}\text { PARAMETER } \\
\text { VALUE FLAGS }\end{array}$ & $\begin{array}{l}\text { DETECTION } \\
\text { LIMIT }\end{array}$ & $\begin{array}{l}\text { PARAMETER } \\
\text { UHCERTAINTY }\end{array}$ \\
\hline ALKALIKITY & $05 / 14 / 86$ & 0001 & MG/L CACO3 & & 2735 & - & - \\
\hline ARSENIC & $05 / 14 / 86$ & 0001 & $M G / L$ & $<$ & 0.01 & 0.01 & - \\
\hline BORON & $05 / 14 / 86$ & 0001 & $M G / L$ & & 0.1 & 0.1 & - \\
\hline CADHIUA & $05 / 14 / 86$ & 0001 & MG/L & $<$ & 0.001 & 0.001 & - \\
\hline CALCIUA & $05 / 14 / 86$ & 0001 & MG/L & & 168. & 0.01 & - \\
\hline CHLORIDE & $05 / 14 / 86$ & 0001 & $M G / L$ & & 90. & 1. & - \\
\hline CHROMIUM & $05 / 14 / 86$ & 0001 & $M G / L$ & & 0.05 & 0.01 & - \\
\hline COBALT & $05 / 14 / 86$ & 0001 & $M G / L$ & & 0.07 & 0.05 & - \\
\hline FLUORIDE & $05 / 14 / 86$ & 0001 & $M G / L$ & & 1.1 & 0.1 & - \\
\hline IRON & $05 / 14 / 86$ & 0001 & $M G / L$ & & 0.05 & 0.03 & - \\
\hline MAGNESIUH & $05 / 14 / 86$ & 0001 & $M G / L$ & & 0.022 & 0.001 & - \\
\hline MANGANESE & $05 / 14 / 86$ & 0001 & $M G / L$ & & 0.03 & 0.01 & - \\
\hline MOLYBDENUM & $05 / 14 / 86$ & 0001 & $H G / L$ & & 0.32 & 0.01 & - \\
\hline HITRATE & $05 / 14 / 86$ & 0001 & $M G / L$ & & 2. & 1. & - \\
\hline PH & $05 / 14 / 86$ & 0001 & su & & 12.41 & - & - \\
\hline POTASSIUM & $05 / 14 / 86$ & 0001 & $M G / L$ & & 56.5 & 0.01 & $\cdot$ \\
\hline RADIUM-226 & $05 / 14 / 86$ & 0001 & $\mathrm{PCL} / \mathrm{L}$ & & 22. & 1. & 2. \\
\hline SELENIUM & $05 / 14 / 86$ & 0001 & $M G / L$ & $<$ & 0.005 & 0.005 & - \\
\hline SOOIUM & $05 / 14 / 86$ & 0001 & $M G / L$ & & 1140 & 0.002 & - \\
\hline SPECIFIC CONDUCTANCE & $05 / 14 / 86$ & 0001 & UHHO/CM & & 10000 . & - & - \\
\hline STRONTIUA & $05 / 14 / 86$ & 0001 & $M G / L$ & & 4.7 & 0.1 & - \\
\hline SULFATE & $05 / 14 / 86$ & 0001 & $M G / L$ & & 135. & 0.1 & - \\
\hline TEMPERATURE & $05 / 14 / 86$ & 0001 & C - DEGREE & & 17. & - & - \\
\hline THORIUA-230 & $05 / 14 / 86$ & 0001 & PCI $/ L$ & & 0. & 1. & 0.1 \\
\hline TOTAL DISSOLVED SOLIDS & $05 / 14 / 86$ & 0001 & $M G / L$ & & 3040 & 10. & - \\
\hline URAKIUM & $05 / 14 / 86$ & 0001 & $M G / L$ & $<$ & $0.0003 \mathrm{~J}$ & 0.003 & - \\
\hline VANADIUN & $05 / 14 / 86$ & 0001 & $M G / L$ & & 0.21 & 0.01 & - \\
\hline
\end{tabular}

PARAMETER VALUE IMDICATOR (PVI):

< - LESS THAN DETECTION LIMIT

SAMPLE ID COOES:

DOO1 - FILTERED SAMPLE (.45 MICRONS)

OTHER PARAMETER VALUE FLAGS:

J- estimated VALUE 
GROUNDWATER OUALITY DATA BY LOCATION

SITE: AMBOI AMBROSIA LAKE

LOCATIOH: 0777

NORTH COORDIHATE: 52881.7 FT

EAST COORDINATE: 60119.8 FT

O6/10/80 TO $12 / 04 / 92$

REPORT DATE: 08/04/94

FORHATION OF COMPLETION: TRES HERMANOS - B SANDSTONE (TB)

HYDRAULIC FLON RELATIONSHIP: UPGRADIENT (U)

\begin{tabular}{|c|c|c|c|c|c|c|c|c|}
\hline PARAMETER MAME & LOG DATE & $\begin{array}{c}\text { SAMPLE } \\
\text { I0 }\end{array}$ & $\begin{array}{l}\text { UNITS OF } \\
\text { MEASURE }\end{array}$ & PVI & $\begin{array}{l}\text { PARAMETER } \\
\text { VALUE }\end{array}$ & FLAGS & $\begin{array}{l}\text { DETECTION } \\
\text { LIMIT }\end{array}$ & $\begin{array}{l}\text { PARAMETER } \\
\text { UNCERTAINTY }\end{array}$ \\
\hline ALKALINITY & $\begin{array}{l}04 / 06 / 88 \\
01 / 12 / 89 \\
09 / 19 / 89 \\
04 / 11 / 90 \\
01 / 22 / 91\end{array}$ & $\begin{array}{l}0001 \\
0001 \\
0001 \\
0001 \\
0001\end{array}$ & MG/L CACO3 & & $\begin{array}{l}337 . \\
256 . \\
105 \\
69 \\
47 .\end{array}$ & $\mathbf{G}$ & $\begin{array}{l}- \\
- \\
- \\
-\end{array}$ & $\begin{array}{l}- \\
- \\
- \\
-\end{array}$ \\
\hline ALUMINUM & $\begin{array}{l}04 / 06 / 88 \\
01 / 12 / 89 \\
09 / 19 / 89 \\
04 / 11 / 90 \\
01 / 22 / 91\end{array}$ & $\begin{array}{l}0001 \\
0001 \\
0001 \\
0001 \\
0001\end{array}$ & $\mathrm{HG} / \mathrm{L}$ & $\begin{array}{l}< \\
< \\
< \\
<\end{array}$ & $\begin{array}{l}0.28 \\
0.05 \\
0.1 \\
0.1 \\
0.05\end{array}$ & $\begin{array}{l}\mathbf{J} \\
\mathbf{G}\end{array}$ & $\begin{array}{l}0.1 \\
0.1 \\
0.1 \\
0.1 \\
0.05\end{array}$ & $\begin{array}{l}- \\
- \\
- \\
-\end{array}$ \\
\hline AMAONIUA & $\begin{array}{l}04 / 06 / 88 \\
01 / 12 / 89 \\
09 / 19 / 89 \\
04 / 11 / 90 \\
01 / 22 / 91\end{array}$ & $\begin{array}{l}0001 \\
0001 \\
0001 \\
0001 \\
0001\end{array}$ & $M G / L$ & & $\begin{array}{l}7.6 \\
5.09 \\
2.2 \\
1.6 \\
1.56\end{array}$ & G & $\begin{array}{l}0.1 \\
0.1 \\
0.1 \\
0.1 \\
0.01\end{array}$ & $\begin{array}{l}- \\
- \\
-\end{array}$ \\
\hline ANT IMONY & $\begin{array}{l}04 / 06 / 88 \\
01 / 12 / 89 \\
09 / 19 / 89 \\
04 / 11 / 90 \\
01 / 22 / 91\end{array}$ & $\begin{array}{l}0001 \\
0001 \\
0001 \\
0001 \\
0001\end{array}$ & $M G / L$ & & $\begin{array}{l}0.009 \\
0.007 \\
0.047 \\
0.099 \\
0.003\end{array}$ & $\mathbf{G}$ & $\begin{array}{l}0.003 \\
0.003 \\
0.003 \\
0.003 \\
0.003\end{array}$ & $\begin{array}{l}- \\
- \\
- \\
-\end{array}$ \\
\hline ARSENIC & $\begin{array}{l}04 / 06 / 88 \\
01 / 12 / 89 \\
09 / 19 / 89 \\
04 / 11 / 90 \\
01 / 22 / 91\end{array}$ & $\begin{array}{l}0001 \\
0001 \\
0001 \\
0001 \\
0001\end{array}$ & $M G / L$ & $<$ & $\begin{array}{l}0.012 \\
0.005 \\
0.01 \\
0.01 \\
0.03\end{array}$ & $\begin{array}{l}\text { J } \\
\text { GI }\end{array}$ & $\begin{array}{l}0.01 \\
0.01 \\
0.01 \\
0.01 \\
0.03\end{array}$ & $\begin{array}{l}- \\
- \\
- \\
-\end{array}$ \\
\hline BARIUM & $\begin{array}{l}04 / 06 / 88 \\
01 / 12 / 89 \\
09 / 19 / 89 \\
04 / 11 / 90 \\
01 / 22 / 91\end{array}$ & $\begin{array}{l}0001 \\
0001 \\
0001 \\
0001 \\
0001\end{array}$ & $\mathrm{MG} / \mathrm{L}$ & $\begin{array}{l}< \\
<\end{array}$ & $\begin{array}{l}0.03 \\
0.02 \\
0.1 \\
0.1 \\
0.04\end{array}$ & $\begin{array}{l}\mathrm{J} \\
\mathrm{J} \\
\mathrm{G}\end{array}$ & $\begin{array}{l}0.1 \\
0.1 \\
0.1 \\
0.1 \\
0.01\end{array}$ & $\begin{array}{l}. \\
. \\
.\end{array}$ \\
\hline BERYLLIUM & $\begin{array}{l}01 / 12 / 89 \\
09 / 19 / 89 \\
04 / 11 / 90 \\
01 / 22 / 91\end{array}$ & $\begin{array}{l}0001 \\
0001 \\
0001 \\
0001\end{array}$ & $M G / L$ & $\begin{array}{l}< \\
< \\
< \\
<\end{array}$ & $\begin{array}{l}0.005 \\
0.01 \\
0.01 \\
0.005\end{array}$ & $\mathbf{G}$ & $\begin{array}{l}0.005 \\
0.01 \\
0.01 \\
0.005\end{array}$ & $\begin{array}{l}- \\
- \\
-\end{array}$ \\
\hline BORON & $\begin{array}{l}04 / 06 / 88 \\
01 / 12 / 89 \\
09 / 19 / 89 \\
04 / 11 / 90 \\
01 / 22 / 91\end{array}$ & $\begin{array}{l}0001 \\
0001 \\
0001 \\
0001 \\
0001\end{array}$ & $M G / L$ & & $\begin{array}{l}0.11 \\
0.16 \\
0.3 \\
0.2 \\
0.29\end{array}$ & $\mathbf{G}$ & $\begin{array}{l}0.1 \\
0.1 \\
0.1 \\
0.1 \\
0.05\end{array}$ & $\begin{array}{l}- \\
- \\
-\end{array}$ \\
\hline BROMIDE & $\begin{array}{l}04 / 06 / 88 \\
01 / 12 / 89 \\
09 / 19 / 89 \\
04 / 11 / 90 \\
01 / 22 / 91\end{array}$ & $\begin{array}{l}0001 \\
0001 \\
0001 \\
0001 \\
0001\end{array}$ & $M G / L$ & $\begin{array}{l}< \\
<\end{array}$ & $\begin{array}{l}0.1 \\
0.19 \\
0.1 \\
0.5 \\
0.4\end{array}$ & $\mathfrak{J}$ & $\begin{array}{l}0.1 \\
0.01 \\
0.1 \\
0.1 \\
0.1\end{array}$ & $\begin{array}{l}- \\
- \\
- \\
-\end{array}$ \\
\hline CADMIUA & $\begin{array}{l}04 / 06 / 88 \\
01 / 12 / 89\end{array}$ & $\begin{array}{l}0001 \\
0001\end{array}$ & $M G / L$ & $<$ & $\begin{array}{l}0.006 \\
0.0001\end{array}$ & $\mathbf{J}$ & $\begin{array}{l}0.001 \\
0.001\end{array}$ & - \\
\hline
\end{tabular}

PARAMETER VALUE INDICATOR (PVI): < - LESS THAN DETECTION LIMIT

SAMPLE IO COOES:

0001 - FILTERED SAMPLE (.45 MICRONS)

OTHER PARAMETER VALUE FLAGS:

G - PH > 9, POSSIBLE GROUT CONTAMINATION

1 - IMCREASED DETECTION LIMIT DUE TO REQUIRED DILUTIOH

$J$ - ESTIMATED VALUE 
FORMATIOH OF COMPLETIOH: TRES HERMANOS - B SANDSTOHE (TB) HYDRAULIC FLON RELATIOHSHIP: UPGRADIENT (U)

\begin{tabular}{|c|c|c|c|c|c|c|c|c|}
\hline PARAMETER MAME & LOG DATE & $\begin{array}{c}\text { SAMPLE } \\
10\end{array}$ & $\begin{array}{l}\text { UNITS OF } \\
\text { MEASURE }\end{array}$ & PVI & $\begin{array}{l}\text { PARAMETER } \\
\text { VALUE }\end{array}$ & FLAGS & $\begin{array}{l}\text { DETECTION } \\
\text { LIMIT }\end{array}$ & $\begin{array}{l}\text { PARAMETER } \\
\text { UMCERTAINTY }\end{array}$ \\
\hline CADKIUM & $\begin{array}{l}09 / 19 / 89 \\
04 / 11 / 90 \\
01 / 22 / 91\end{array}$ & $\begin{array}{l}0001 \\
0001 \\
0001\end{array}$ & $M G / L$ & $<$ & $\begin{array}{l}0.002 \\
0.001 \\
0.001\end{array}$ & G & $\begin{array}{l}0.001 \\
0.001 \\
0.001\end{array}$ & : \\
\hline CALCIUM & $\begin{array}{l}04 / 06 / 88 \\
01 / 12 / 89 \\
09 / 19 / 89 \\
04 / 11 / 90 \\
01 / 22 / 91\end{array}$ & $\begin{array}{l}0001 \\
0001 \\
0001 \\
0001 \\
0001\end{array}$ & MG/L & & $\begin{array}{c}57.2 \\
42 . \\
133 . \\
83.7 \\
134 .\end{array}$ & G & $\begin{array}{l}0.01 \\
0.01 \\
0.01 \\
0.01 \\
0.5\end{array}$ & $\begin{array}{l}- \\
- \\
-\end{array}$ \\
\hline CHLORIDE & $\begin{array}{l}04 / 06 / 88 \\
01 / 12 / 89 \\
09 / 19 / 89 \\
04 / 11 / 90 \\
01 / 22 / 91\end{array}$ & $\begin{array}{l}0001 \\
0001 \\
0001 \\
0001 \\
0001\end{array}$ & $M G / L$ & & $\begin{array}{l}32 . \\
30 . \\
33 . \\
39 . \\
38 .\end{array}$ & G & $\begin{array}{l}1 . \\
1 . \\
1 . \\
1 . \\
0.5\end{array}$ & $\begin{array}{l}- \\
- \\
-\end{array}$ \\
\hline CHROMIUM & $\begin{array}{l}04 / 06 / 88 \\
01 / 12 / 89 \\
09 / 19 / 89 \\
04 / 11 / 90 \\
01 / 22 / 91\end{array}$ & $\begin{array}{l}0001 \\
0001 \\
0001 \\
0001 \\
0001\end{array}$ & $\mathrm{HG} / \mathrm{L}$ & $\begin{array}{l}< \\
< \\
<\end{array}$ & $\begin{array}{l}0.04 \\
0.01 \\
0.01 \\
0.01 \\
0.01\end{array}$ & G & $\begin{array}{l}0.01 \\
0.01 \\
0.01 \\
0.01 \\
0.01\end{array}$ & $\begin{array}{l}- \\
- \\
-\end{array}$ \\
\hline COBALT & $\begin{array}{l}04 / 06 / 88 \\
01 / 12 / 89 \\
09 / 19 / 89 \\
04 / 11 / 90 \\
01 / 22 / 91\end{array}$ & $\begin{array}{l}0001 \\
0001 \\
0001 \\
0001 \\
0001\end{array}$ & MG/L & $\begin{array}{l}< \\
< \\
< \\
<\end{array}$ & $\begin{array}{l}0.01 \\
0.01 \\
0.05 \\
0.05 \\
0.03\end{array}$ & $\begin{array}{l}J \\
J \\
G\end{array}$ & $\begin{array}{l}0.05 \\
0.05 \\
0.05 \\
0.05 \\
0.03\end{array}$ & $\begin{array}{l}- \\
- \\
- \\
-\end{array}$ \\
\hline COPPER & $\begin{array}{l}04 / 06 / 88 \\
01 / 12 / 89 \\
09 / 19 / 89 \\
04 / 11 / 90 \\
01 / 22 / 91\end{array}$ & $\begin{array}{l}0001 \\
0001 \\
0001 \\
0001 \\
0001\end{array}$ & $M G / L$ & $\begin{array}{l}< \\
< \\
< \\
<\end{array}$ & $\begin{array}{l}0.02 \\
0.01 \\
0.02 \\
0.02 \\
0.01\end{array}$ & $\begin{array}{l}J \\
G\end{array}$ & $\begin{array}{l}0.02 \\
0.02 \\
0.02 \\
0.02 \\
0.01\end{array}$ & $\begin{array}{l}- \\
- \\
-\end{array}$ \\
\hline CYAHIDE & $\begin{array}{l}01 / 12 / 89 \\
09 / 19 / 89 \\
04 / 11 / 90 \\
01 / 22 / 91\end{array}$ & $\begin{array}{l}0001 \\
0001 \\
0001 \\
0001\end{array}$ & MG/L & $\begin{array}{l}< \\
<\end{array}$ & $\begin{array}{l}0.004 \\
0.01 \\
0.01 \\
0.01\end{array}$ & $\begin{array}{l}\text { J } \\
\text { G }\end{array}$ & $\begin{array}{l}0.01 \\
0.01 \\
0.01 \\
0.01\end{array}$ & : \\
\hline FLUORIDE & $\begin{array}{l}04 / 06 / 88 \\
01 / 12 / 89 \\
09 / 19 / 89 \\
04 / 19 / 90 \\
01 / 22 / 91\end{array}$ & $\begin{array}{l}0001 \\
0001 \\
0001 \\
0001 \\
0001\end{array}$ & $M G / L$ & & $\begin{array}{l}0.95 \\
0.9 \\
0.4 \\
0.4 \\
0.2\end{array}$ & G & $\begin{array}{l}0.1 \\
0.1 \\
0.1 \\
0.1 \\
0.1\end{array}$ & $\begin{array}{l}: \\
: \\
-\end{array}$ \\
\hline GROSS ALPHA & $\begin{array}{l}04 / 06 / 88 \\
01 / 12 / 89 \\
09 / 19 / 89 \\
04 / 11 / 90 \\
01 / 22 / 91\end{array}$ & $\begin{array}{l}0001 \\
0001 \\
0001 \\
0001 \\
0001\end{array}$ & $P C I / L$ & & $\begin{array}{l}6 . \\
18.8 \\
11 . \\
17 . \\
39.7\end{array}$ & $\begin{array}{l}\mathbf{J} \\
\mathbf{G}\end{array}$ & $\begin{array}{l}0.2 \\
1 . \\
1 . \\
1 . \\
1 .\end{array}$ & $\begin{array}{l}15 . \\
18.6 \\
17 . \\
13 . \\
36.5\end{array}$ \\
\hline GROSS BETA & $\begin{array}{l}04 / 06 / 88 \\
01 / 12 / 89 \\
09 / 19 / 89 \\
04 / 11 / 90 \\
01 / 22 / 91\end{array}$ & $\begin{array}{l}0001 \\
0001 \\
0001 \\
0001 \\
0001\end{array}$ & $\mathrm{PCI} / \mathrm{L}$ & & $\begin{array}{l}19 . \\
32.8 \\
21 . \\
28 . \\
36.8\end{array}$ & G & $\begin{array}{l}1 . \\
0.5 \\
0.5 \\
0.5 \\
0.5\end{array}$ & $\begin{array}{r}9.0 \\
24.2 \\
9.0 \\
11.0 \\
17.9\end{array}$ \\
\hline
\end{tabular}

PARAMETER VALUE INDICATOR (PVI): < - LESS THAN DETECTION LIMIT

SAMPLE ID COOES:

0001 - FILTERED SAMPLE (.45 MICRONS)

OTHER PARAMETER VALUE FLAGS:

G - PH > 9, POSSIBLE GROUT CONTAMIHATION

J - eSTIMATED VALUE 
GROUNDWATER QUALITY DATA BY LOCATIOH

SITE: AMBO1 AMBROSIA LAKE

LOCATION: 0777

MORTH COORDINATE: $\quad 52881.7$ FT

EAST COORDINATE: $\quad 60119.8$ FT

$06 / 10 / 80$ TO $12 / 04 / 92$

REPORT DATE: 08/04/94

FORMATIOH OF COMPLETION: TRES HERMANOS - B SANDSTONE (TB) HYDRAULIC FLON RELATIONSHIP: UPGRADIENT (U)

\begin{tabular}{|c|c|c|c|c|c|c|c|c|}
\hline PARAMETER NAME & LOG DATE & $\begin{array}{c}\text { SAMPLE } \\
\text { ID }\end{array}$ & $\begin{array}{l}\text { UNITS OF } \\
\text { MEASURE }\end{array}$ & PVI & $\begin{array}{l}\text { PARAMETER } \\
\text { VALUE }\end{array}$ & FLAGS & $\begin{array}{l}\text { DETECTION } \\
\text { LIMIT }\end{array}$ & $\begin{array}{l}\text { PARAMETER } \\
\text { UNCERTAINTY }\end{array}$ \\
\hline IRON & $\begin{array}{l}04 / 06 / 88 \\
01 / 12 / 89 \\
09 / 19 / 89 \\
04 / 11 / 90 \\
01 / 22 / 91\end{array}$ & $\begin{array}{l}0001 \\
0001 \\
0001 \\
0001 \\
0001\end{array}$ & $M G / L$ & $\begin{array}{l}< \\
< \\
<\end{array}$ & $\begin{array}{l}0.03 \\
0.02 \\
0.05 \\
0.03 \\
0.03\end{array}$ & $\begin{array}{l}\mathbf{J} \\
\mathbf{G}\end{array}$ & $\begin{array}{l}0.03 \\
0.03 \\
0.03 \\
0.03 \\
0.03\end{array}$ & $\begin{array}{l}- \\
- \\
- \\
-\end{array}$ \\
\hline LEAD & $\begin{array}{l}04 / 06 / 88 \\
01 / 12 / 89 \\
09 / 19 / 89 \\
04 / 11 / 90 \\
01 / 22 / 91\end{array}$ & $\begin{array}{l}0001 \\
0001 \\
0001 \\
0001 \\
0001\end{array}$ & $M G / L$ & $\begin{array}{l}< \\
<\end{array}$ & $\begin{array}{l}0.01 \\
0.001 \\
0.05 \\
0.01 \\
0.03\end{array}$ & $\begin{array}{l}J \\
G I\end{array}$ & $\begin{array}{l}0.01 \\
0.01 \\
0.01 \\
0.01 \\
0.03\end{array}$ & $\begin{array}{l}- \\
- \\
- \\
-\end{array}$ \\
\hline LEAD-210 & $\begin{array}{l}04 / 06 / 88 \\
01 / 12 / 89 \\
09 / 19 / 89\end{array}$ & $\begin{array}{l}0001 \\
0001 \\
0001\end{array}$ & $\mathrm{PCI} / \mathrm{L}$ & & $\begin{array}{l}0.4 \\
0.00 \\
0.1\end{array}$ & & $\begin{array}{l}1.5 \\
1.5 \\
1.5\end{array}$ & $\begin{array}{l}1.1 \\
5.35 \\
0.7\end{array}$ \\
\hline MAGNESIUM & $\begin{array}{l}04 / 06 / 88 \\
01 / 12 / 89 \\
09 / 19 / 89 \\
04 / 11 / 90 \\
01 / 22 / 91\end{array}$ & $\begin{array}{l}0001 \\
0001 \\
0001 \\
0001 \\
0001\end{array}$ & $M G / L$ & $<$ & $\begin{array}{c}0.27 \\
1 . \\
20.5 \\
12.9 \\
22.5\end{array}$ & G & $\begin{array}{l}0.001 \\
1 . \\
0.001 \\
0.001 \\
0.1\end{array}$ & $\begin{array}{l}- \\
- \\
- \\
-\end{array}$ \\
\hline MANGANESE & $\begin{array}{l}04 / 06 / 88 \\
01 / 12 / 89 \\
09 / 19 / 89 \\
04 / 11 / 90 \\
01 / 22 / 91\end{array}$ & $\begin{array}{l}0001 \\
0001 \\
0001 \\
0001 \\
0001\end{array}$ & MG/L & $\begin{array}{l}< \\
< \\
< \\
<\end{array}$ & $\begin{array}{l}0.01 \\
0.01 \\
0.01 \\
0.01 \\
0.01\end{array}$ & $\mathbf{G}$ & $\begin{array}{l}0.01 \\
0.01 \\
0.01 \\
0.01 \\
0.01\end{array}$ & $\begin{array}{l}- \\
- \\
- \\
-\end{array}$ \\
\hline MERCURY & $\begin{array}{l}04 / 06 / 88 \\
01 / 12 / 89 \\
09 / 19 / 89 \\
04 / 11 / 90 \\
01 / 22 / 91\end{array}$ & $\begin{array}{l}0001 \\
0001 \\
0001 \\
0001 \\
0001\end{array}$ & $\mathrm{MG} / \mathrm{L}$ & $\begin{array}{l}< \\
< \\
< \\
<\end{array}$ & $\begin{array}{l}0.0002 \\
0.0001 \\
0.0002 \\
0.0002 \\
0.0002\end{array}$ & J & $\begin{array}{l}0.0002 \\
0.0002 \\
0.0002 \\
0.0002 \\
0.0002\end{array}$ & $\begin{array}{l}- \\
- \\
- \\
-\end{array}$ \\
\hline HOLYBDENUM & $\begin{array}{l}04 / 06 / 88 \\
01 / 12 / 89 \\
09 / 19 / 89 \\
04 / 11 / 90 \\
01 / 22 / 91\end{array}$ & $\begin{array}{l}0001 \\
0001 \\
0001 \\
0001 \\
0001\end{array}$ & $M G / L$ & & $\begin{array}{l}0.10 \\
0.028 \\
0.02 \\
0.06 \\
0.01\end{array}$ & $\mathbf{G}$ & $\begin{array}{l}0.01 \\
0.01 \\
0.01 \\
0.01 \\
0.01\end{array}$ & $\begin{array}{l}- \\
- \\
- \\
-\end{array}$ \\
\hline NET GROSS ALPHA * & $\begin{array}{l}04 / 06 / 88 \\
01 / 12 / 89 \\
09 / 19 / 89 \\
04 / 11 / 90 \\
01 / 22 / 91\end{array}$ & $\begin{array}{l}0001 \\
0001 \\
0001 \\
0001 \\
0001\end{array}$ & $\mathrm{PCl} / \mathrm{L}$ & & $\begin{array}{r}4.97 \\
12.63 \\
9.63 \\
16.31 \\
39.36\end{array}$ & & $\begin{array}{l}- \\
- \\
- \\
-\end{array}$ & $\begin{array}{l}- \\
- \\
- \\
-\end{array}$ \\
\hline NICKEL & $\begin{array}{l}04 / 06 / 88 \\
01 / 12 / 89 \\
09 / 19 / 89 \\
04 / 11 / 90 \\
01 / 22 / 91\end{array}$ & $\begin{array}{l}0001 \\
0001 \\
0001 \\
0001 \\
0001\end{array}$ & $M G / L$ & $\begin{array}{l}< \\
< \\
<\end{array}$ & $\begin{array}{l}0.02 \\
0.02 \\
0.04 \\
0.04 \\
0.04\end{array}$ & $\begin{array}{l}J \\
J\end{array}$ & $\begin{array}{l}0.04 \\
0.04 \\
0.04 \\
0.04 \\
0.04\end{array}$ & $\begin{array}{l}- \\
- \\
- \\
-\end{array}$ \\
\hline NITRATE & $04 / 06 / 88$ & 0001 & $M G / L$ & & 0.9 & $\mathbf{J}$ & 1. & - \\
\hline
\end{tabular}

- NET GROSS ALPHA (GROSS ALPHA - URANIUM) HITH 1 MG URANIUM = 686 PCI

PARAMETER VALUE INDICATOR (PVI): < - LESS THAN DETECTION LIHIT

OTHER PARAMETER VALUE FLAGS:

G - PH > 9, POSSIBLE GROUT COHTAMIHATIOH

1 - INCREASED DETECTION LIMIT DUE TO REQUIRED DILUTION

$J$ - ESTIMATED VALUE 
GROUNDWATER QUALITY DATA BY LOCATION

SITE: AKBO1 AMBROSIA LAKE

LOCATION: 0777

MORTH COORDINATE: $\quad 52881.7$ FT

EAST COORDIHATE: $\quad 60119.8$ FT

$06 / 10 / 80$ TO $12 / 04 / 92$

REPORT DATE: $08 / 04 / 94$

FORMATION OF COMPLETION: TRES HERMANOS - B SANDSTONE (TB)

HYDRAULIC FLOW RELATIOHSHIP: UPGRADIENT (U)

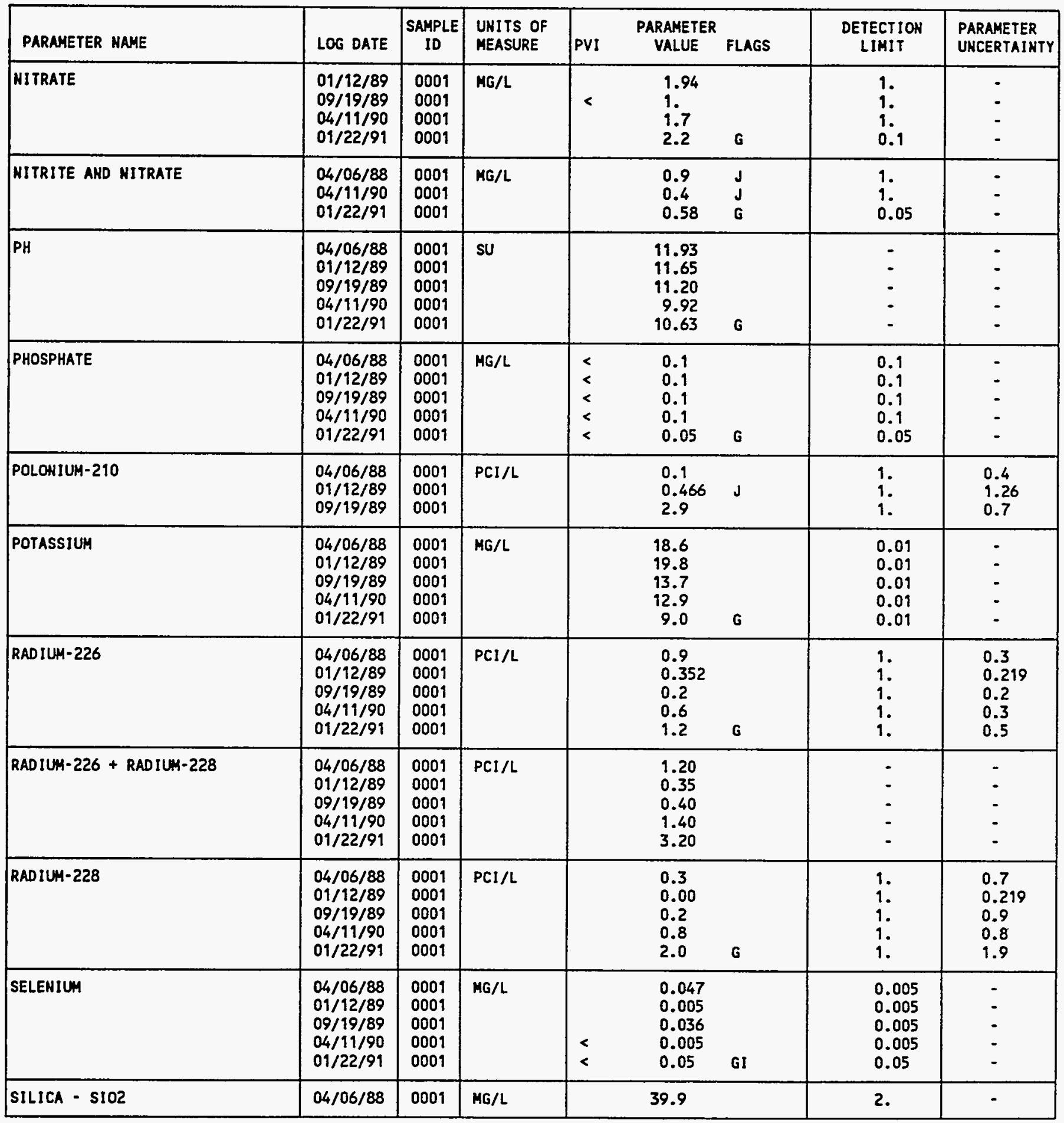

PARAMETER VALLE IMDICATOR (PVI): < - LESS THAN DETECTION LIMIT

SAMPLE 10 COOES:

0001 - FILTERED SAMPLE (.45 MICRONS)

OTHER PARAMETER VALUE FLAGS:

G - PH > 9, POSSIBLE GROUT CONTAMINATIOH

1 - INCREASED DETECTION LIMIT DUE TO REQUIRED DILUTION

$\downarrow$ - ESTIMATED VALUE 
GROUNDWATER QUALITY DATA BY LOCATION

SITE: AMBO1 AMBROSIA LAKE

LOCATION: 0777

NORTH COORDINATE: 52881.7 FT

EAST COORDINATE: $\quad 60119.8$ FT

06/10/80 TO 12/04/92

REPORT DATE: 08/04/94

FORMATION OF COMPLETION: TRES HERMANOS - B SANDSTONE (TB)

HYDRAULIC FLOW RELATIONSHIP: UPGRADIENT (U)

\begin{tabular}{|c|c|c|c|c|c|c|c|c|}
\hline PARAMETER MAME & LOG DATE & $\mid \begin{array}{c}\text { SAMPLE } \\
\text { ID }\end{array}$ & $\begin{array}{l}\text { UNITS OF } \\
\text { MEASURE }\end{array}$ & PVI & $\begin{array}{l}\text { PARAMETER } \\
\text { VALUE }\end{array}$ & FLAGS & $\begin{array}{l}\text { DETECTIOH } \\
\text { LIMIT }\end{array}$ & $\begin{array}{l}\text { PARAMETER } \\
\text { UHCERTAINTY }\end{array}$ \\
\hline SILICA - SIO2 & $\begin{array}{l}01 / 12 / 89 \\
09 / 19 / 89 \\
04 / 11 / 90 \\
01 / 22 / 91\end{array}$ & $\begin{array}{l}0001 \\
0001 \\
0001 \\
0001\end{array}$ & $M G / L$ & & $\begin{array}{l}30.2 \\
24 . \\
22 . \\
24.2\end{array}$ & G & $\begin{array}{l}2 . \\
2 . \\
2 . \\
0.1\end{array}$ & : \\
\hline SILVER & $\begin{array}{l}04 / 06 / 88 \\
01 / 12 / 89 \\
09 / 19 / 89 \\
04 / 19 / 90 \\
01 / 22 / 91\end{array}$ & $\begin{array}{l}0001 \\
0001 \\
0001 \\
0001 \\
0001\end{array}$ & MG/L & $\begin{array}{l}< \\
< \\
< \\
<\end{array}$ & $\begin{array}{l}0.01 \\
0.01 \\
0.01 \\
0.01 \\
0.01\end{array}$ & G & $\begin{array}{l}0.01 \\
0.01 \\
0.01 \\
0.01 \\
0.01\end{array}$ & $\begin{array}{l}- \\
- \\
-\end{array}$ \\
\hline soorum & $\begin{array}{l}04 / 06 / 88 \\
01 / 12 / 89 \\
09 / 19 / 89 \\
04 / 11 / 90 \\
01 / 22 / 91\end{array}$ & $\begin{array}{l}0001 \\
0001 \\
0001 \\
0001 \\
0001\end{array}$ & $M G / L$ & & $\begin{array}{c}599 . \\
840 . \\
896 . \\
1074 . \\
990 .\end{array}$ & GJ & $\begin{array}{l}0.002 \\
0.002 \\
0.002 \\
0.002 \\
5 .\end{array}$ & $\begin{array}{l}- \\
- \\
- \\
-\end{array}$ \\
\hline SPECIFIC CONDUCTANCE & $\begin{array}{l}04 / 06 / 88 \\
01 / 12 / 89 \\
09 / 19 / 89 \\
04 / 11 / 90 \\
01 / 22 / 91\end{array}$ & $\begin{array}{l}0009 \\
0001 \\
0001 \\
0001 \\
0001\end{array}$ & UМно/CH & & $\begin{array}{l}2700 . \\
2525 . \\
1980 . \\
2100 \\
2900 .\end{array}$ & G & $\begin{array}{l}- \\
- \\
- \\
-\end{array}$ & : \\
\hline STRONTIUNA & $\begin{array}{l}04 / 06 / 88 \\
01 / 12 / 89 \\
09 / 19 / 89 \\
04 / 11 / 90 \\
01 / 22 / 91\end{array}$ & $\begin{array}{l}0001 \\
0001 \\
0001 \\
0001 \\
0001\end{array}$ & $M G / L$ & & $\begin{array}{l}0.70 \\
1.51 \\
4.9 \\
3.67 \\
4.90\end{array}$ & G & $\begin{array}{l}0.1 \\
0.1 \\
0.1 \\
0.1 \\
0.01\end{array}$ & $\begin{array}{l}- \\
- \\
- \\
-\end{array}$ \\
\hline SULFATE & $\begin{array}{l}04 / 06 / 88 \\
01 / 12 / 89 \\
09 / 19 / 89 \\
04 / 11 / 90 \\
01 / 22 / 91\end{array}$ & $\begin{array}{l}0001 \\
0001 \\
0001 \\
0001 \\
0001\end{array}$ & MG/L & & $\begin{array}{l}1210 . \\
1632 . \\
2030 . \\
2180 \\
2250\end{array}$ & G & $\begin{array}{c}0.1 \\
0.1 \\
0.1 \\
0.1 \\
10 .\end{array}$ & $\begin{array}{l}- \\
- \\
- \\
-\end{array}$ \\
\hline SULF IDE & $\begin{array}{l}04 / 06 / 88 \\
01 / 12 / 89 \\
09 / 19 / 89 \\
04 / 11 / 90 \\
01 / 22 / 91\end{array}$ & $\begin{array}{l}0001 \\
0001 \\
0001 \\
0001 \\
0001\end{array}$ & $M G / L$ & $<$ & $\begin{array}{l}0.1 \\
0.01 \\
0.1 \\
0.7 \\
8.4\end{array}$ & J & $\begin{array}{l}0.1 \\
0.1 \\
0.1 \\
0.1 \\
0.1\end{array}$ & $\begin{array}{l}- \\
- \\
- \\
-\end{array}$ \\
\hline TEMPERATURE & $\begin{array}{l}04 / 06 / 88 \\
01 / 12 / 89 \\
09 / 19 / 89 \\
04 / 11 / 90 \\
01 / 22 / 91\end{array}$ & $\begin{array}{l}0001 \\
0001 \\
0001 \\
0001 \\
0001\end{array}$ & $C \cdot$ DEGREE & & $\begin{array}{l}12.5 \\
11.0 \\
14.0 \\
13 . \\
11 .\end{array}$ & $\mathbf{G}$ & $\begin{array}{l}- \\
- \\
-\end{array}$ & $\begin{array}{l}- \\
- \\
-\end{array}$ \\
\hline THALLIU: & $\begin{array}{l}01 / 12 / 89 \\
09 / 19 / 89 \\
04 / 11 / 90 \\
01 / 22 / 91\end{array}$ & $\begin{array}{l}0001 \\
0001 \\
0001 \\
0001\end{array}$ & $M G / L$ & $\begin{array}{l}< \\
< \\
<\end{array}$ & $\begin{array}{l}0.005 \\
0.01 \\
0.01 \\
0.03\end{array}$ & $\begin{array}{l}1 \\
\text { GI }\end{array}$ & $\begin{array}{l}0.005 \\
0.01 \\
0.01 \\
0.03\end{array}$ & $\begin{array}{l}- \\
- \\
-\end{array}$ \\
\hline THORIUM-230 & $\begin{array}{l}04 / 06 / 88 \\
01 / 12 / 89 \\
09 / 19 / 89\end{array}$ & $\begin{array}{l}0001 \\
0001 \\
0001\end{array}$ & PCI/L & & $\begin{array}{l}0.0 \\
0.684 \\
0.0\end{array}$ & & $\begin{array}{l}1 . \\
1 . \\
1 .\end{array}$ & $\begin{array}{l}0.4 \\
0.166 \\
0.3\end{array}$ \\
\hline
\end{tabular}

PARAMETER VALUE INDICATOR (PVI): < - LESS THAN DETECTION LIMIT

SAMPLE ID COOES:

0001 - FILTERED SAMPLE (.45 MICRONS)

OTHER PARAMETER VALUE FLAGS:

G - PH > 9, POSSIBLE GROUT CONTAMINATION

I - INCREASED DETECTION LIMIT DUE TO REQUIRED DILUTION

$\downarrow$ - estimated value 
GROUNDWATER QUALITY DATA BY LOCATIOH

SITE: AMBOI AMBROSIA LAKE

LOCATIOH: 0777

NORTH COORDINATE: $\quad 52881.7$ FT

EAST COORDINATE: $\quad 60119.8$ FT

06/10/80 TO $12 / 04 / 92$

REPORT DATE: $08 / 04 / 94$

FORHATION OF COMPLETION: TRES HERMANOS - B SAMDSTOHE (TB)

HYDRAULIC FLOW RELATIOHSHIP: UPGRADIENT (U)

\begin{tabular}{|c|c|c|c|c|c|c|c|c|}
\hline PARAMETER MAME & LOG DATE & $\begin{array}{c}\text { SAMPLE } \\
\text { ID }\end{array}$ & $\begin{array}{l}\text { UNITS OF } \\
\text { MEASURE }\end{array}$ & PVI & $\begin{array}{l}\text { PARAMETER } \\
\text { VALUE }\end{array}$ & FLAGS & $\begin{array}{l}\text { DETECTIOW } \\
\text { LIMIT }\end{array}$ & $\begin{array}{l}\text { PARAMETER } \\
\text { UNCERTAINTY }\end{array}$ \\
\hline TIK & $\begin{array}{l}04 / 06 / 88 \\
01 / 12 / 89 \\
09 / 19 / 89 \\
04 / 11 / 90 \\
01 / 22 / 91\end{array}$ & $\begin{array}{l}0001 \\
0001 \\
0001 \\
0001 \\
0001\end{array}$ & MG/L & $<$ & $\begin{array}{l}0.037 \\
0.002 \\
0.169 \\
0.099 \\
0.03\end{array}$ & $\begin{array}{l}\text { J } \\
\text { GI }\end{array}$ & $\begin{array}{l}0.005 \\
0.005 \\
0.005 \\
0.005 \\
0.03\end{array}$ & $\begin{array}{l}- \\
- \\
.\end{array}$ \\
\hline TOTAL DISSOLVED SOLIDS & $\begin{array}{l}04 / 06 / 88 \\
01 / 12 / 89 \\
09 / 19 / 89 \\
04 / 11 / 90 \\
01 / 22 / 91\end{array}$ & $\begin{array}{l}0001 \\
0001 \\
0001 \\
0001 \\
0001\end{array}$ & $M G / L$ & & $\begin{array}{l}2120 . \\
2544 . \\
2950 . \\
3160 . \\
3320 .\end{array}$ & G & $\begin{array}{l}10 . \\
10 . \\
10 . \\
10 . \\
10 .\end{array}$ & : \\
\hline TOTAL KJELDAHL NITROGEN & $01 / 22 / 91$ & 0001 & $M G / L$ & & 2. & G & 1. & - \\
\hline TOTAL ORGANIC CARBON & $\begin{array}{l}04 / 06 / 88 \\
01 / 12 / 89 \\
09 / 19 / 89 \\
04 / 11 / 90 \\
01 / 22 / 91\end{array}$ & $\begin{array}{l}0001 \\
0001 \\
0001 \\
0001 \\
0001\end{array}$ & $M G / L$ & & $\begin{array}{l}26.0 \\
13 . \\
5.3 \\
14 . \\
10 .\end{array}$ & $\mathbf{G}$ & $\begin{array}{l}1 . \\
1 . \\
1 .\end{array}$ & $\begin{array}{l}- \\
: \\
-\end{array}$ \\
\hline URAHIUH & $\begin{array}{l}04 / 06 / 88 \\
01 / 12 / 89 \\
09 / 19 / 89 \\
04 / 11 / 90 \\
01 / 22 / 91\end{array}$ & $\begin{array}{l}0001 \\
0001 \\
0001 \\
0001 \\
0001\end{array}$ & $M G / L$ & $<$ & $\begin{array}{l}0.0003 \\
0.009 \\
0.0020 \\
0.0010 \\
0.001\end{array}$ & $\begin{array}{l}J \\
J \\
J \\
G\end{array}$ & $\begin{array}{l}0.003 \\
0.003 \\
0.003 \\
0.003 \\
0.001\end{array}$ & : \\
\hline VAHADIUH & $\begin{array}{l}04 / 06 / 88 \\
01 / 12 / 89 \\
09 / 19 / 89 \\
04 / 11 / 90 \\
01 / 22 / 91\end{array}$ & $\begin{array}{l}0001 \\
0001 \\
0001 \\
0001 \\
0001\end{array}$ & $M G / L$ & $<$ & $\begin{array}{l}0.06 \\
0.01 \\
0.02 \\
0.01 \\
0.01\end{array}$ & G & $\begin{array}{l}0.01 \\
0.01 \\
0.01 \\
0.01 \\
0.01\end{array}$ & $\begin{array}{l}- \\
- \\
-\end{array}$ \\
\hline ZIKC & $\begin{array}{l}04 / 06 / 88 \\
01 / 12 / 89 \\
09 / 19 / 89 \\
04 / 11 / 90 \\
01 / 22 / 91\end{array}$ & $\begin{array}{l}0001 \\
0001 \\
0001 \\
0001 \\
0001\end{array}$ & $M G / L$ & $\begin{array}{l}< \\
< \\
<\end{array}$ & $\begin{array}{l}0.005 \\
0.01 \\
0.090 \\
0.007 \\
0.005\end{array}$ & G & $\begin{array}{l}0.005 \\
0.01 \\
0.005 \\
0.005 \\
0.005\end{array}$ & $\begin{array}{l}- \\
- \\
-\end{array}$ \\
\hline
\end{tabular}

PARAMETER VALUE IMDICATOR (PVI): < - LESS THAN DETECTION LIMIT

SAMPLE ID COOES:

0001 - FILTERED SAMPLE (.45 HICRONS)

OTHER PARAMETER VALUE FLAGS:

G - PH > 9, POSSIBLE GROUT CONTAMINATION

1 - IMCREASED DETECTION LIMIT DUE TO REQUIRED DILUTION

J - estimated VALUe 
GROUNDWATER QUALITY DATA BY LOCATION

SITE: AMBOI AMBROSIA LAKE

LOCATION: 0778

MORTH COORDINATE: 52911.5 FT

EAST COOROIHATE: $\quad 60205.4 \mathrm{FT}$

$06 / 10 / 80$ TO $12 / 04 / 92$

REPORT DATE: 08/04/94

FORMATION OF COMPLETION: TRES HERMANOS - C1 SANDSTOHE (TO)

HYORAULIC FLON RELATIONSHIP: DOWN GRADIENT (D)

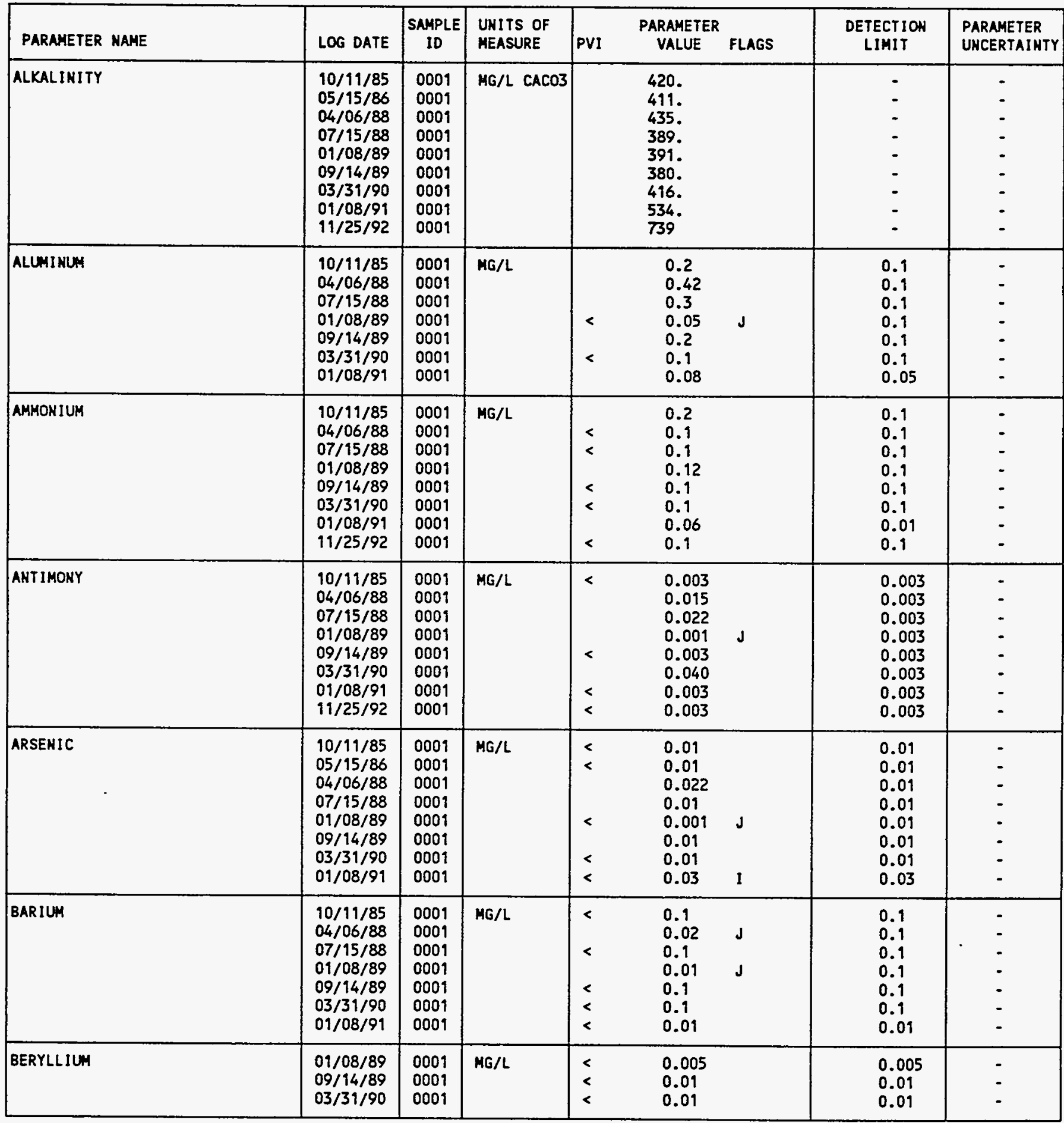

PARAMETER VALUE IHDICATOR (PVI): < - LESS THAN DETECTIOA LIHIT

SAMPLE ID CODES:

0001 - FILTERED SAMPLE (.45 MICRONS)

OTHER PARAMETER VALUE FLAGS:

I - INCREASED DETECTION LIMIT DUE TO REQUIRED DILUTION

J - ESTIMATED VALUE 
GROUNDWATER QUALITY DATA BY LOCATIOH

SITE: AMBO1 AMBROSIA LAKE

LOCATION: 0778

NORTH COORDINATE: 52911.5 FT

EAST COORDIHATE: 60205.4 FT

06/10/80 TO $12 / 04 / 92$

REPORT DATE: 08/04/94

FORMATION OF COMPLETION: TRES HERMANOS - C1 SANDSTONE (TO) HYORAULIC FLOW RELATIONSHIP: DOWH GRADIENT (D)

\begin{tabular}{|c|c|c|c|c|c|c|c|c|}
\hline PARAMETER MAME & LOG DATE & SAMPLE & $\begin{array}{l}\text { UNITS OF } \\
\text { MEASURE }\end{array}$ & PVI & $\begin{array}{l}\text { PARAMETER } \\
\text { VALUE }\end{array}$ & FLAGS & $\begin{array}{l}\text { OETECTIOH } \\
\text { LIMIT }\end{array}$ & $\begin{array}{l}\text { PARAMETER } \\
\text { UNCERTAINTY }\end{array}$ \\
\hline BERYLLILH & $01 / 08 / 91$ & 0001 & MG/L & $<$ & 0.005 & & 0.005 & - \\
\hline BORON & $\begin{array}{l}10 / 11 / 85 \\
05 / 15 / 86 \\
04 / 06 / 88 \\
07 / 15 / 88 \\
01 / 08 / 89 \\
09 / 14 / 89 \\
03 / 31 / 90 \\
01 / 08 / 91\end{array}$ & $\begin{array}{l}0001 \\
0001 \\
0001 \\
0001 \\
0001 \\
0001 \\
0001 \\
0001\end{array}$ & MG/L & & $\begin{array}{l}1.1 \\
0.4 \\
0.63 \\
0.5 \\
0.52 \\
0.5 \\
0.5 \\
0.57\end{array}$ & & $\begin{array}{l}0.1 \\
0.1 \\
0.1 \\
0.1 \\
0.1 \\
0.1 \\
0.1 \\
0.05\end{array}$ & $\begin{array}{l}- \\
- \\
- \\
- \\
-\end{array}$ \\
\hline BROMIDE & $\begin{array}{l}04 / 06 / 88 \\
07 / 15 / 88 \\
01 / 08 / 89 \\
09 / 14 / 89 \\
03 / 31 / 90 \\
01 / 08 / 91\end{array}$ & $\begin{array}{l}0001 \\
0001 \\
0001 \\
0001 \\
0001 \\
0001\end{array}$ & $M G / L$ & & $\begin{array}{l}2.1 \\
2.5 \\
3.10 \\
0.43 \\
1.2 \\
1.8\end{array}$ & & $\begin{array}{l}0.1 \\
0.1 \\
0.01 \\
0.1 \\
0.1 \\
0.1\end{array}$ & $\begin{array}{l}- \\
- \\
- \\
-\end{array}$ \\
\hline CADMIUM & $\begin{array}{l}10 / 11 / 85 \\
05 / 15 / 86 \\
04 / 06 / 88 \\
07 / 15 / 88 \\
01 / 08 / 89 \\
09 / 14 / 89 \\
03 / 31 / 90 \\
01 / 08 / 91 \\
11 / 25 / 92\end{array}$ & $\begin{array}{l}0001 \\
0001 \\
0001 \\
0001 \\
0001 \\
0001 \\
0001 \\
0001 \\
0001\end{array}$ & $M G / L$ & $\begin{array}{l}< \\
< \\
< \\
< \\
< \\
< \\
<\end{array}$ & $\begin{array}{l}0.001 \\
0.001 \\
0.014 \\
0.001 \\
0.0001 \\
0.014 \\
0.001 \\
0.001 \\
0.001\end{array}$ & $J$ & $\begin{array}{l}0.001 \\
0.001 \\
0.001 \\
0.001 \\
0.001 \\
0.001 \\
0.001 \\
0.001 \\
0.001\end{array}$ & $\begin{array}{l}: \\
: \\
: \\
- \\
- \\
-\end{array}$ \\
\hline CALCIUH & $\begin{array}{l}10 / 11 / 85 \\
05 / 15 / 86 \\
04 / 06 / 88 \\
07 / 15 / 88 \\
01 / 08 / 89 \\
09 / 14 / 89 \\
03 / 31 / 90 \\
01 / 08 / 91 \\
11 / 25 / 92\end{array}$ & $\begin{array}{l}0001 \\
0001 \\
0001 \\
0001 \\
0001 \\
0001 \\
0001 \\
0001 \\
0001\end{array}$ & $M G / L$ & & $\begin{array}{l}438 . \\
524 . \\
549 . \\
479 . \\
534 . \\
535 . \\
471 . \\
477 . \\
615 .\end{array}$ & & $\begin{array}{l}0.01 \\
0.01 \\
0.01 \\
0.01 \\
0.01 \\
0.01 \\
0.01 \\
0.01 \\
2 .\end{array}$ & $\begin{array}{l}- \\
- \\
- \\
- \\
- \\
-\end{array}$ \\
\hline CHLORIDE & $\begin{array}{l}10 / 11 / 85 \\
05 / 15 / 86 \\
04 / 06 / 88 \\
07 / 15 / 88 \\
01 / 08 / 89 \\
09 / 14 / 89 \\
03 / 31 / 90 \\
01 / 08 / 91 \\
11 / 25 / 92\end{array}$ & $\begin{array}{l}0001 \\
0001 \\
0001 \\
0001 \\
0001 \\
0001 \\
0001 \\
0001 \\
0001\end{array}$ & $M G / L$ & & $\begin{array}{l}250 . \\
270 . \\
340 \\
300 \\
263 \\
280 \\
308 \\
288 \\
247\end{array}$ & & $\begin{array}{l}1 . \\
1 . \\
1 . \\
1 . \\
1 . \\
1 . \\
1 . \\
0.5 \\
5 .\end{array}$ & $\begin{array}{l}- \\
: \\
: \\
: \\
- \\
-\end{array}$ \\
\hline CHRONIUN & $\begin{array}{l}10 / 11 / 85 \\
05 / 15 / 86 \\
04 / 06 / 88 \\
07 / 15 / 88 \\
01 / 08 / 89 \\
09 / 14 / 89 \\
03 / 31 / 90 \\
01 / 08 / 91 \\
11 / 25 / 92\end{array}$ & $\begin{array}{l}0001 \\
0001 \\
0001 \\
0001 \\
0001 \\
0001 \\
0001 \\
0001 \\
0001\end{array}$ & $M G / L$ & $\begin{array}{l}< \\
< \\
< \\
<\end{array}$ & $\begin{array}{l}0.03 \\
0.05 \\
0.22 \\
0.17 \\
0.01 \\
0.01 \\
0.01 \\
0.01 \\
0.05\end{array}$ & 1 & $\begin{array}{l}0.01 \\
0.01 \\
0.01 \\
0.01 \\
0.01 \\
0.01 \\
0.01 \\
0.01 \\
0.05\end{array}$ & $\begin{array}{l}- \\
- \\
- \\
- \\
- \\
- \\
-\end{array}$ \\
\hline
\end{tabular}

PARAMETER VALUE IHDICATOR (PVI): < - LESS THAN DETECTIOH LIMIT SAMPLE ID COOES:

0009 - FILTERED SAMPLE (.45 MICRONS)

OTHER PARAMETER VALUE FLAGS:

I - INCREASED DETECTION LIMIT DUE TO REQUIRED DILUTION

J - estimated VALUE 
GROUNDWATER QUALITY DATA BY LOCATION

SITE: AMBOI AMBROSIA LAKE

LOCATION: 0778

NORTH COORDINATE: 52911.5 FT

EAST COORDIHATE: 60205.4 FT

06/10/80 TO 12/04/92

REPORT DATE: 08/04/94

FORHATION OF COMPLETION: TRES HERMANOS - C1 SANDSTONE (TO) HYDRAULIC FLOU RELATIOWSHIP: DOWN GRADIENT (D)

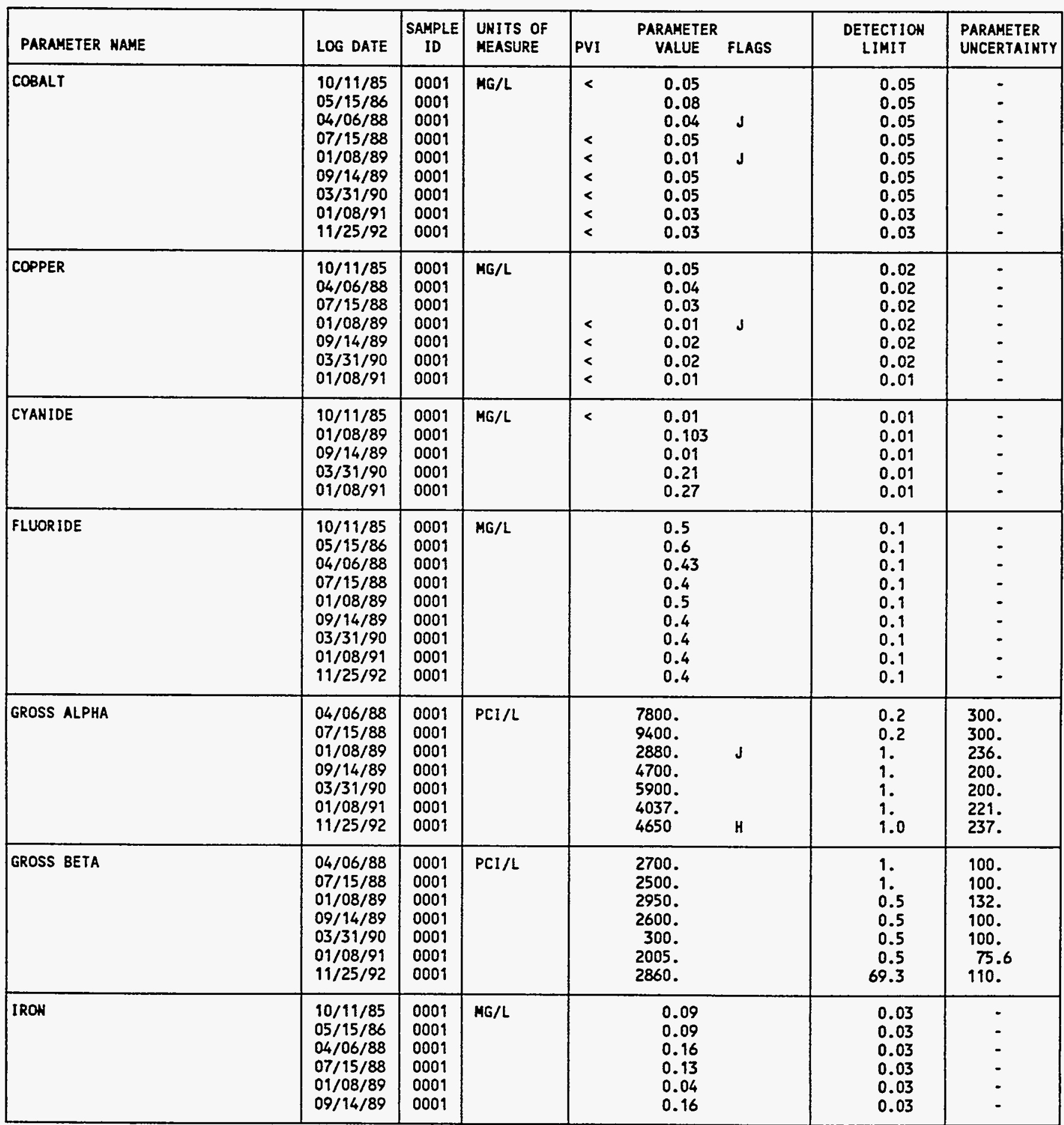

parameter Value indicator (PVI): < - LeSS than DeTECTION limIt SAMPLE ID COOES:

0001 - FILTERED SAMPLE (.45 MICRONS)

OTHER PARAMETER VALUE FLAGS:

H - HOLD TIME EXPIRED, VALUE SUSPECT

J - ESTIMATED VALUE 
GROUNDWATER OUALITY DATA BY LOCATION

SITE: AMBO1 AMBROSIA LAKE

LOCATION: 0778

NORTH COORDINATE: 52911.5 FT

EAST COORDINATE: 60205.4 FT

$06 / 10 / 80$ TO $12 / 04 / 92$

REPORT DATE: 08/04/94

FORMATIOH OF COMPLETIOH: TRES HERMANOS - C1 SAKDSTONE (TO)

HYDRAULIC FLOW RELATIONSHIP: DOWN GRADIENT (D)

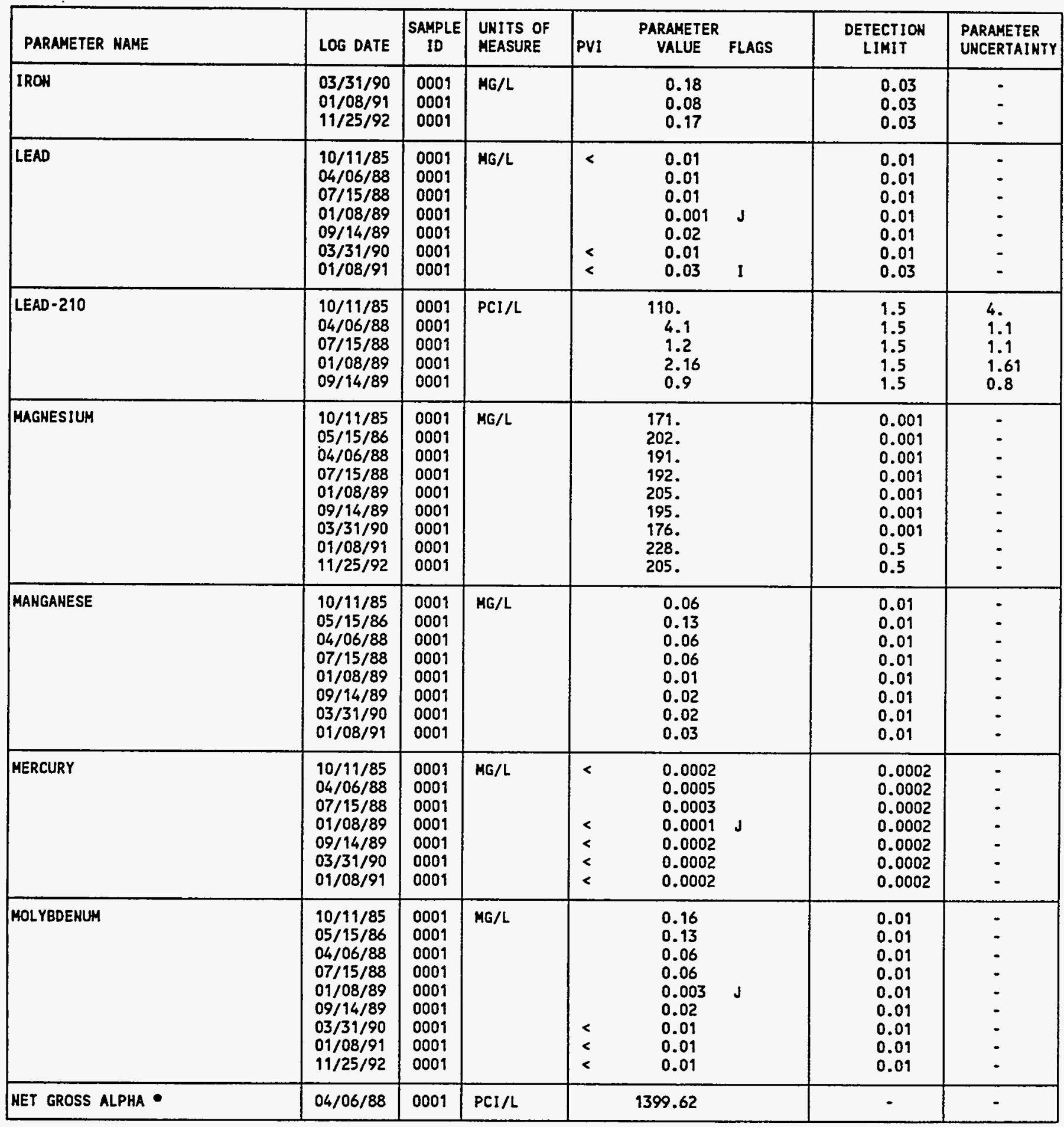

- MET gRoss alPHA (GROSS ALPHA - URAKIUM) HITH 1 MG URANIUM = 686 PCI

PARAMETER VALUE IMDICATOR (PVI): < - LESS THAN DETECTION LIMIT

SAMPLE ID COOES:

0001 - FILTERED SAMPLE (.45 MICRONS)

OTHER PARAMETER VALUE FLAGS:

1 - IHCREASED DETECTION LIMIT DUE TO REQUIRED DILUTION

J - eSTIMATEd VALUe 
GROUNDWATER QUALITY DATA BY LOCATION

SITE: AMBOI AMBROSIA LAKE

LOCATION: 0778

HORTH COORDINATE: 52911.5 FT

EAST COORDINATE: 60205.4 FT

06/10/80 TO 12/04/92

REPORT OATE: $08 / 04 / 94$

FORMATION OF COMPLETION: TRES HERMANOS - C1 SANDSTONE (TO) HYDRAULIC FLON RELATIOHSHIP: DOWN GRADIENT (D)

\begin{tabular}{|c|c|c|c|c|c|c|c|}
\hline PARAMETER NAME & LOG DATE & $\underset{\text { SAMPLE }}{\text { SAMP }}$ & $\begin{array}{l}\text { UNITS OF } \\
\text { MEASURE }\end{array}$ & PVI & $\begin{array}{l}\text { PARAMETER } \\
\text { VALUE FLAGS }\end{array}$ & $\begin{array}{l}\text { DETECTION } \\
\text { LIMIT }\end{array}$ & $\begin{array}{l}\text { PARAMETER } \\
\text { UNCERTAINTY }\end{array}$ \\
\hline HET GROSS ALPHA * & $\begin{array}{l}07 / 15 / 88 \\
01 / 08 / 89 \\
09 / 14 / 89 \\
03 / 31 / 90 \\
01 / 08 / 91 \\
11 / 25 / 92\end{array}$ & $\begin{array}{l}0001 \\
0001 \\
0001 \\
0001 \\
0001 \\
0001\end{array}$ & $\mathrm{PCI} / \mathrm{L}$ & & $\begin{array}{r}3466.10 \\
-2443.36 \\
-801.72 \\
-33.90 \\
-2651.50 \\
-1825.84\end{array}$ & $\begin{array}{l}- \\
- \\
- \\
-\end{array}$ & $\begin{array}{l}- \\
- \\
- \\
-\end{array}$ \\
\hline NICKEL & $\begin{array}{l}10 / 11 / 85 \\
04 / 06 / 88 \\
07 / 15 / 88 \\
01 / 08 / 89 \\
09 / 14 / 89 \\
03 / 31 / 90 \\
01 / 08 / 91 \\
11 / 25 / 92\end{array}$ & $\begin{array}{l}0001 \\
0001 \\
0001 \\
0001 \\
0001 \\
0001 \\
0001 \\
0001\end{array}$ & $M G / L$ & $\begin{array}{l}< \\
< \\
<\end{array}$ & $\begin{array}{l}0.07 \\
0.08 \\
0.07 \\
0.02 \\
0.04 \\
0.04 \\
0.04 \\
0.10\end{array}$ & $\begin{array}{l}0.04 \\
0.04 \\
0.04 \\
0.04 \\
0.04 \\
0.04 \\
0.04 \\
0.04\end{array}$ & $\begin{array}{l}- \\
- \\
- \\
-\end{array}$ \\
\hline NITRATE & $\begin{array}{l}10 / 11 / 85 \\
05 / 15 / 86 \\
04 / 06 / 88 \\
07 / 15 / 88 \\
01 / 08 / 89 \\
09 / 14 / 89 \\
03 / 31 / 90 \\
01 / 08 / 91 \\
11 / 25 / 92\end{array}$ & $\begin{array}{l}0001 \\
0001 \\
0001 \\
0001 \\
0001 \\
0001 \\
0001 \\
0001 \\
0001\end{array}$ & $M G / L$ & & $\begin{array}{l}400 . \\
3 . \\
430 . \\
430 \\
375 . \\
300 . \\
246 . \\
192 . \\
194 .\end{array}$ & $\begin{array}{l}1 . \\
1 . \\
1 . \\
1 . \\
1 . \\
1 . \\
1 . \\
0.1 \\
30 .\end{array}$ & $\begin{array}{l}- \\
- \\
- \\
- \\
- \\
-\end{array}$ \\
\hline NITRITE & $10 / 11 / 85$ & 0009 & MG/L & $<$ & 0.1 & 0.1 & - \\
\hline NITRITE AHD NITRATE & $\begin{array}{l}04 / 06 / 88 \\
03 / 31 / 90 \\
01 / 08 / 91\end{array}$ & $\begin{array}{l}0001 \\
0001 \\
0001\end{array}$ & $M G / L$ & & $\begin{array}{l}430 . \\
55.6 \\
45.6\end{array}$ & $\begin{array}{l}1 . \\
1 . \\
0.05\end{array}$ & $\dot{-}$ \\
\hline $\mathrm{PH}$ & $\begin{array}{l}10 / 11 / 85 \\
05 / 15 / 86 \\
04 / 06 / 88 \\
07 / 15 / 88 \\
01 / 08 / 89 \\
09 / 14 / 89 \\
03 / 31 / 90 \\
01 / 08 / 91 \\
11 / 25 / 92\end{array}$ & $\begin{array}{l}0001 \\
0001 \\
0001 \\
0001 \\
0001 \\
0001 \\
0001 \\
0001 \\
0001\end{array}$ & SU & & $\begin{array}{l}6.9 \\
7.23 \\
7.01 \\
6.92 \\
7.03 \\
7.03 \\
6.83 \\
6.96 \\
6.84\end{array}$ & $\begin{array}{l}- \\
- \\
- \\
- \\
- \\
- \\
-\end{array}$ & $\begin{array}{l}- \\
- \\
- \\
- \\
- \\
-\end{array}$ \\
\hline PHOSPHATE & $\begin{array}{l}10 / 11 / 85 \\
04 / 06 / 88 \\
07 / 15 / 88 \\
01 / 08 / 89 \\
09 / 14 / 89 \\
03 / 31 / 90 \\
01 / 08 / 91 \\
11 / 25 / 92\end{array}$ & $\begin{array}{l}0001 \\
0001 \\
0001 \\
0001 \\
0001 \\
0001 \\
0001 \\
0001\end{array}$ & $M G / L$ & $\begin{array}{l}< \\
< \\
<\end{array}$ & $\begin{array}{l}0.5 \\
0.1 \\
0.1 \\
0.1 \\
0.1 \\
0.1 \\
0.14 \\
0.4\end{array}$ & $\begin{array}{l}0.1 \\
0.1 \\
0.1 \\
0.1 \\
0.1 \\
0.1 \\
0.05 \\
0.1\end{array}$ & $\begin{array}{l}- \\
- \\
- \\
- \\
-\end{array}$ \\
\hline POLONIUAM-210 & $\begin{array}{l}10 / 11 / 85 \\
04 / 06 / 88 \\
07 / 15 / 88 \\
01 / 08 / 89\end{array}$ & $\begin{array}{l}0001 \\
0001 \\
0001 \\
0001\end{array}$ & PCI/L & & $\begin{array}{l}1.1 \\
1.4 \\
1.2 \\
1.18\end{array}$ & $\begin{array}{l}1 . \\
1 . \\
1 . \\
1 .\end{array}$ & $\begin{array}{l}0.8 \\
0.6 \\
0.6 \\
1.72\end{array}$ \\
\hline
\end{tabular}

- NET GROSS ALPHA (GROSS ALPHA - URANIUH) WITH 1 MG URANILM = 686 PCI

PARAMeter VAlUe IMDICATOR (PVI): < - LESS thah Detection limit 
GROUNDWATER OUALITY DATA BY LOCATION

SITE: AMBO1 AMBROSIA LAKE

LOCATION: OTT8

MORTH COORDIHATE: $\quad 52911.5$ FT

EAST COORDIKATE: $\quad 60205.4$ FT

06/10/80 TO 12/04/92

REPORT DATE: 08/04/94

FORHATION OF COMPLETION: TRES HERMANOS - C1 SANDSTONE (TO)

HYDRAULIC FLOW RELATIONSHIP: DOWN GRADIENT (D)

\begin{tabular}{|c|c|c|c|c|c|c|c|}
\hline PARAMETER MAME & LOG DATE & $\underset{\text { SAMPLE }}{\text { SD }}$ & $\begin{array}{l}\text { UNITS OF } \\
\text { MEASURE }\end{array}$ & PVI & $\begin{array}{l}\text { PARAMETER } \\
\text { VALUE FLAGS }\end{array}$ & $\begin{array}{l}\text { DETECTION } \\
\text { LIMIT }\end{array}$ & $\begin{array}{l}\text { PARAMETER } \\
\text { UNCERTAINTY }\end{array}$ \\
\hline POLONIUAM-210 & $09 / 14 / 89$ & 0001 & PCI/L & & 3.5 & 1. & 0.7 \\
\hline POTASSIUH & $\begin{array}{l}10 / 11 / 85 \\
05 / 15 / 86 \\
04 / 06 / 88 \\
07 / 15 / 88 \\
01 / 08 / 89 \\
09 / 14 / 89 \\
03 / 31 / 90 \\
01 / 08 / 91 \\
11 / 25 / 92\end{array}$ & $\begin{array}{l}0001 \\
0001 \\
0001 \\
0001 \\
0001 \\
0001 \\
0001 \\
0001 \\
0001\end{array}$ & $M G / L$ & & $\begin{array}{l}24.5 \\
4.18 \\
2.75 \\
3.6 \\
2.3 \\
2.1 \\
1.9 \\
3.7 \\
3.49\end{array}$ & $\begin{array}{l}0.01 \\
0.01 \\
0.01 \\
0.01 \\
0.01 \\
0.01 \\
0.01 \\
0.01 \\
0.01\end{array}$ & $\begin{array}{l}- \\
- \\
- \\
- \\
- \\
-\end{array}$ \\
\hline RADIUH-226 & $\begin{array}{l}10 / 11 / 85 \\
05 / 15 / 86 \\
04 / 06 / 88 \\
07 / 15 / 88 \\
01 / 08 / 89 \\
09 / 14 / 89 \\
03 / 31 / 90 \\
01 / 08 / 91 \\
11 / 25 / 92\end{array}$ & $\begin{array}{l}0001 \\
0001 \\
0001 \\
0001 \\
0001 \\
0001 \\
0001 \\
0001 \\
0001\end{array}$ & $\mathrm{PCI} / \mathrm{L}$ & & $\begin{array}{l}0.4 \\
2.5 \\
0.1 \\
0.2 \\
1.62 \\
0.4 \\
0.1 \\
0.4 \\
1.0\end{array}$ & $\begin{array}{l}1 . \\
1 . \\
1 . \\
1 . \\
1 . \\
1 . \\
1 . \\
0.3\end{array}$ & $\begin{array}{l}0.2 \\
0.5 \\
0.1 \\
0.2 \\
0.356 \\
0.2 \\
0.2 \\
0.4 \\
0.4\end{array}$ \\
\hline RADIUH-226 + RADIUM-228 & $\begin{array}{l}10 / 11 / 85 \\
04 / 06 / 88 \\
07 / 15 / 88 \\
01 / 08 / 89 \\
09 / 14 / 89 \\
03 / 31 / 90 \\
01 / 08 / 91 \\
11 / 25 / 92\end{array}$ & $\begin{array}{l}0001 \\
0001 \\
0001 \\
0001 \\
0001 \\
0001 \\
0001 \\
0001\end{array}$ & $\mathrm{PCI} / \mathrm{L}$ & & $\begin{array}{l}0.40 \\
0.20 \\
0.30 \\
7.93 \\
0.40 \\
0.90 \\
0.40 \\
1.00\end{array}$ & $\begin{array}{l}- \\
- \\
- \\
- \\
- \\
-\end{array}$ & $\begin{array}{l}- \\
- \\
- \\
- \\
- \\
-\end{array}$ \\
\hline RADIUH-228 & $\begin{array}{l}10 / 11 / 85 \\
04 / 06 / 88 \\
07 / 15 / 88 \\
01 / 08 / 89 \\
09 / 14 / 89 \\
03 / 31 / 90 \\
01 / 08 / 91 \\
11 / 25 / 92\end{array}$ & $\begin{array}{l}0001 \\
0001 \\
0001 \\
0001 \\
0001 \\
0001 \\
0001 \\
0001\end{array}$ & $\mathrm{PCI} / \mathrm{L}$ & & $\begin{array}{l}0 . \\
0.1 \\
0.1 \\
6.31 \\
0.0 \\
0.8 \\
0.0 \\
0.0\end{array}$ & $\begin{array}{l}1 . \\
1 . \\
1 . \\
1 . \\
1 . \\
1 . \\
1.0\end{array}$ & $\begin{array}{l}1.2 \\
0.7 \\
0.7 \\
7.47 \\
0.8 \\
0.8 \\
1.8 \\
1.4\end{array}$ \\
\hline SILICA - SIO2 & $\begin{array}{l}10 / 11 / 85 \\
04 / 06 / 88 \\
07 / 15 / 88 \\
01 / 08 / 89 \\
09 / 14 / 89 \\
03 / 31 / 90 \\
01 / 08 / 91\end{array}$ & $\begin{array}{l}0001 \\
0001 \\
0001 \\
0001 \\
0001 \\
0001 \\
0001\end{array}$ & MG/L & & $\begin{array}{l}15 . \\
15.8 \\
13.9 \\
13.1 \\
15 . \\
15 . \\
14.4\end{array}$ & $\begin{array}{l}2 . \\
2 . \\
2 . \\
2 . \\
2 . \\
2 . \\
0.1\end{array}$ & $\begin{array}{l}- \\
- \\
- \\
- \\
-\end{array}$ \\
\hline
\end{tabular}

PARAMETER VALUE IMDICATOR (PVI): < - LESS THAH DETECTIOH LIMIT

SAMPLE IO COOES:

0001 - FILTERED SAMPLE (.45 MICRONS)

OTHER PARAMETER VALUE FLAGS:

H - HOLD TIME EXPIRED, VALUE SUSPECT 
GROUNDWATER QUALITY DATA BY LOCATION

SITE: AMBO1 AMBROSIA LAKE

LOCATION: 0778

NORTH COORDINATE: 52911.5 FT

EAST COORDINATE: 60205.4 FT

06/10/80 TO $12 / 04 / 92$

REPORT DATE: 08/04/94

FORMATION OF COMPLETION: TRES HERMANOS - CI SANDSTONE (TO) HYDRAULIC FLON RELATIONSHIP: DONH GRADIENT (D)

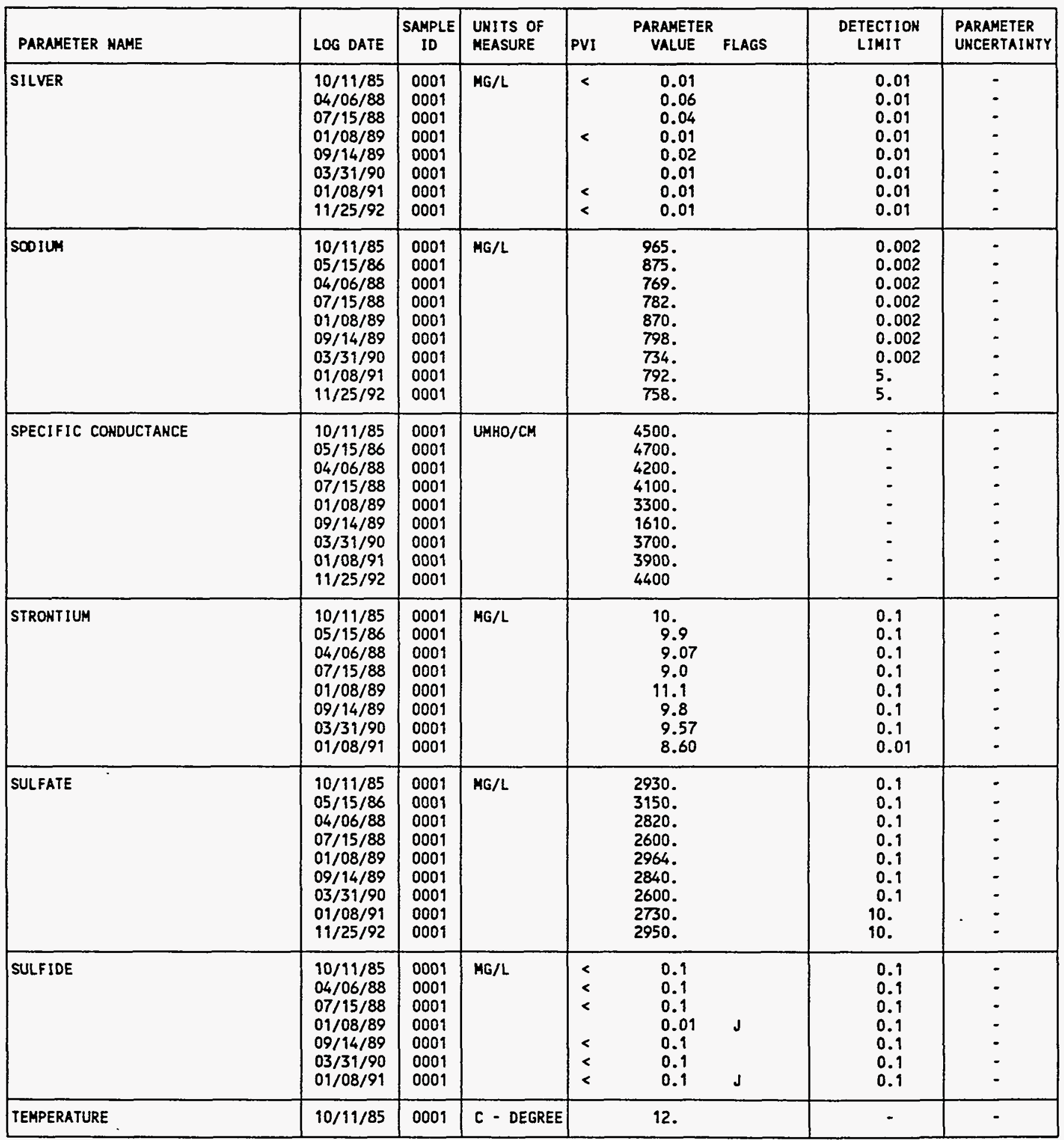

PARAMETER VALUE INDICATOR (PVI): < - LESS THAN DETECTION LIMIT 
GROUNDHATER OUALITY DATA BY LOCATION

SITE: AMBO1 AMBROSIA LAKE

LOCATIOH: 0778

NORTH COORDINATE: 52911.5 FT

EAST COORDIHATE: $\quad 60205.4$ FT

06/10/80 TO $12 / 04 / 92$

REPORT DATE: $08 / 04 / 94$

FORMATION OF COMPLETION: TRES HERMANOS - CI SANDSTONE (TO) HYDRAULIC FLON RELATIOHSHIP: DONH GRADIENT (D)

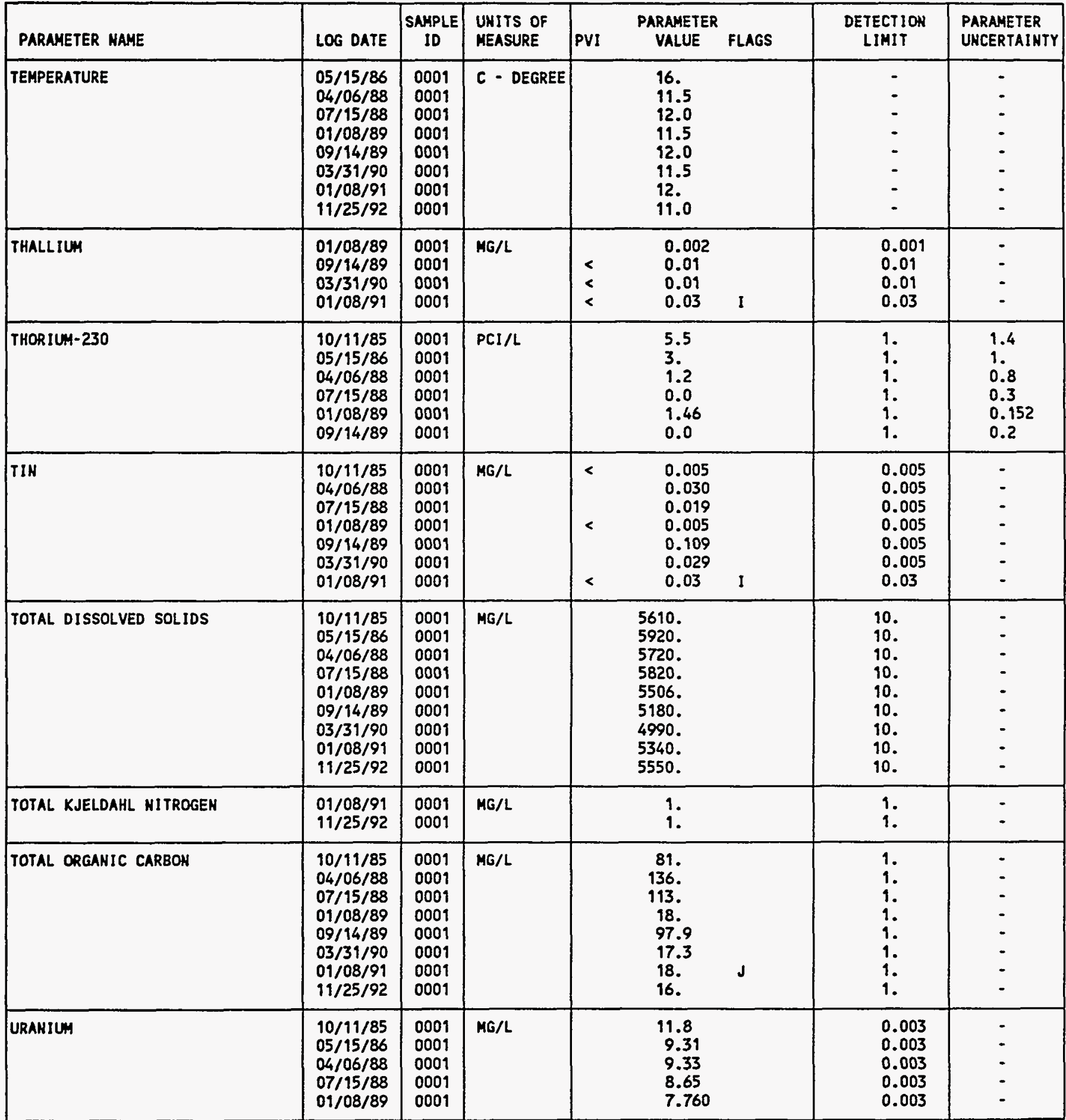

PARAMETER VALUE INDICATOR (PVI): < - LESS THAN DETECTIOH LIMIT SAMPLE IO COOES:

0001 - FILTERED SAMPLE (.45 MICRONS)

OTHER PARAMETER VALUE FLAGS:

I - IKCREASED DETECTION LIMIT DUE TO REQUIRED DILUTION

J - ESTIMATED VALUE 
GROUNDWATER QUALITY DATA BY LOCATION

SITE: AMB01 AMBROSIA LAKE

LOCATION: 0778

NORTH COORDINATE: 52911.5 FT

EAST COORDINATE: $\quad 60205.4$ FT

06/10/80 TO $12 / 04 / 92$

REPORT DATE: 08/04/94

FORMATION OF COMPLETION: TRES HERMANOS - CI SANDSTONE (TO)

HYDRAULIC FLON RELATIONSHIP: DOWN GRADIENT (D)

\begin{tabular}{|c|c|c|c|c|c|c|c|}
\hline PARAMETER MAME & LOG DATE & $\begin{array}{c}\text { SAMPLE } \\
10\end{array}$ & $\begin{array}{l}\text { UNITS OF } \\
\text { MEASURE }\end{array}$ & PVI & $\begin{array}{l}\text { PARAMETER } \\
\text { VALUE FLAGS }\end{array}$ & $\begin{array}{l}\text { DETECTION } \\
\text { LIMIT }\end{array}$ & $\begin{array}{l}\text { PARAMETER } \\
\text { UNCERTAINTY }\end{array}$ \\
\hline URAHIUM & $\begin{array}{l}09 / 14 / 89 \\
03 / 31 / 90 \\
01 / 08 / 91 \\
11 / 25 / 92\end{array}$ & $\begin{array}{l}0001 \\
0001 \\
0001 \\
0001\end{array}$ & MG/L & & $\begin{array}{l}8.02 \\
8.65 \\
9.75 \\
9.44\end{array}$ & $\begin{array}{l}0.003 \\
0.003 \\
0.001 \\
0.001\end{array}$ & $\begin{array}{l}- \\
- \\
-\end{array}$ \\
\hline VAHADIUM & $\begin{array}{l}10 / 11 / 85 \\
05 / 15 / 86 \\
04 / 06 / 88 \\
07 / 15 / 88 \\
01 / 08 / 89 \\
09 / 14 / 89 \\
03 / 31 / 90 \\
01 / 08 / 91 \\
11 / 25 / 92\end{array}$ & $\begin{array}{l}0001 \\
0001 \\
0001 \\
0001 \\
0001 \\
0001 \\
0001 \\
0001 \\
0001\end{array}$ & $M G / L$ & $\begin{array}{l}< \\
< \\
< \\
<\end{array}$ & $\begin{array}{l}0.01 \\
0.23 \\
0.09 \\
0.07 \\
0.01 \\
0.02 \\
0.02 \\
0.01 \\
0.2\end{array}$ & $\begin{array}{l}0.01 \\
0.01 \\
0.01 \\
0.01 \\
0.01 \\
0.01 \\
0.01 \\
0.01 \\
0.2\end{array}$ & $\begin{array}{l}- \\
- \\
- \\
- \\
- \\
- \\
-\end{array}$ \\
\hline ZINC & $\begin{array}{l}10 / 11 / 85 \\
04 / 06 / 88 \\
07 / 15 / 88 \\
01 / 08 / 89 \\
09 / 14 / 89 \\
03 / 31 / 90 \\
01 / 08 / 91\end{array}$ & $\begin{array}{l}0001 \\
0001 \\
0001 \\
0001 \\
0001 \\
0001 \\
0001\end{array}$ & $M G / L$ & $\begin{array}{l}< \\
< \\
<\end{array}$ & $\begin{array}{l}0.039 \\
0.035 \\
0.021 \\
0.01 \\
0.005 \\
0.009 \\
0.005\end{array}$ & $\begin{array}{l}0.005 \\
0.005 \\
0.005 \\
0.01 \\
0.005 \\
0.005 \\
0.005\end{array}$ & $\begin{array}{l}- \\
- \\
- \\
- \\
- \\
-\end{array}$ \\
\hline
\end{tabular}

PARAMETER VALUE INDICATOR (PVI): < - LESS THAN DETECTION LIMIT

SAMPLE ID CODES:

0001 - FILTERED SAMPLE (.45 MICRONS)

OTHER PARAMETER VALUE FLAGS:

H - HOLD TIME EXPIRED, VALUE SUSPECT

I - INCREASED DETECTIÓN LIMIT DUE TO REQUIRED DILUTION 
GROUNDWATER QUALITY DATA BY LOCATION

SITE: AMBO1 AMBROSIA LAKE

LOCATION: 0779

MORTH COORDINATE: 52914.0 FT

EAST COORDINATE: $\quad 60284.7$ FT

06/10/80 TO $12 / 04 / 92$

REPORT OATE: 08/04/94

FORHATION OF COMPLETION: TRES HERMANOS - C2 SANDSTOHE (TT)

HYDRAULIC FLON RELATIOHSHIP: DONH GRADIENT (D)

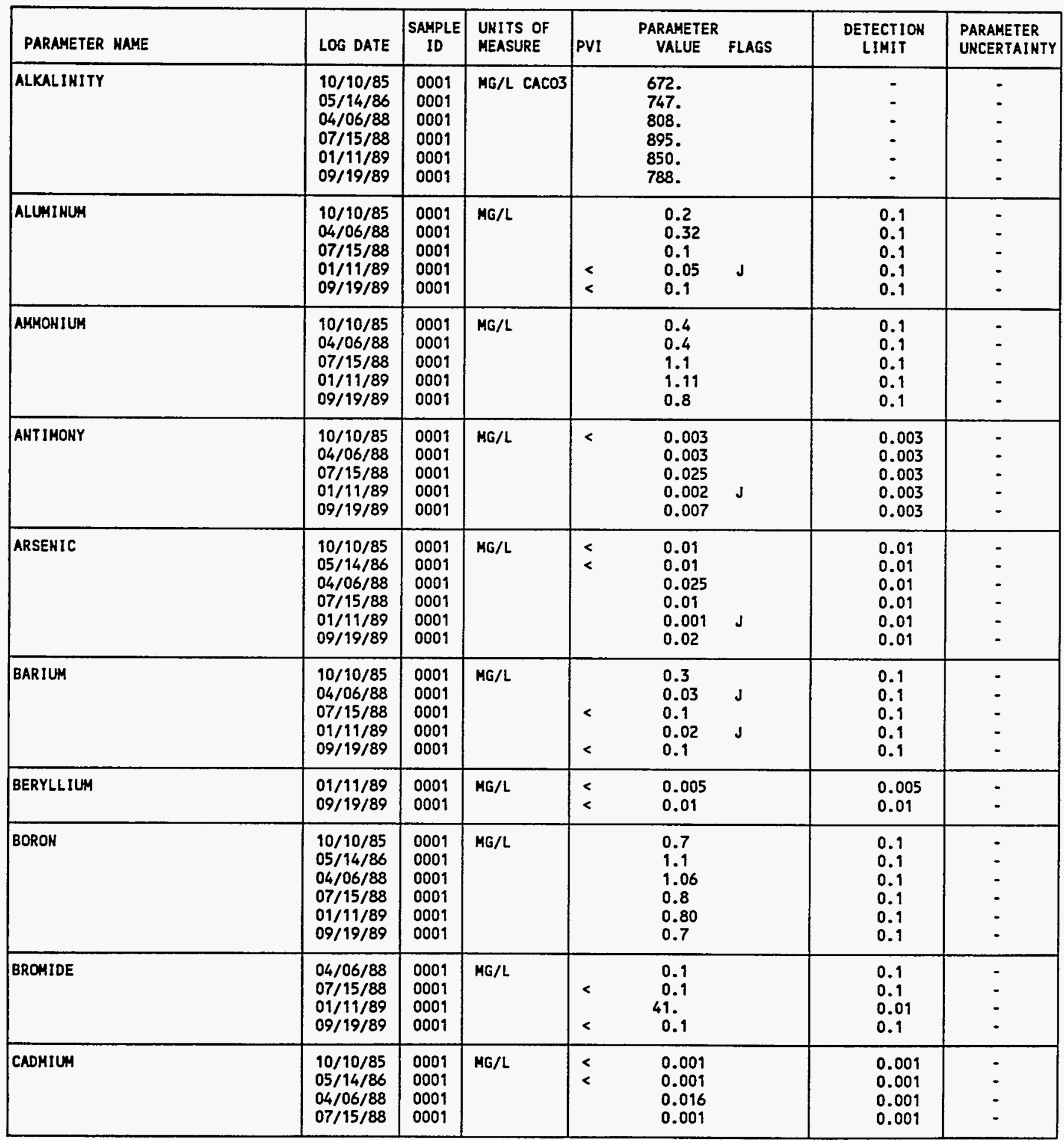

PARAMETER VALUE IMDICATOR (PVI): < - LESS THAN DETECTION LIMIT

SAMPLE ID COOES:

0001 - FILTERED SAMPLE (.45 MICRONS)

OTHER PARAMETER VALUE FLAGS:

J - ESTIMATED VALUE 
GROUNDWATER OUALITY DATA BY LOCATIOH

SITE: AMBOI AMBROSIA LAKE

LOCATION: 0779

HORTH COORDINATE: 52914.0 FT

EAST COORDINATE: 60284.7 FT

$06 / 10 / 80$ TO $12 / 04 / 92$

REPORT DATE: $08 / 04 / 94$

FORMATION OF COHPLETION: TRES HERMANOS - C2 SANDSTONE (TT) HYDRAULIC FLON RELATIOHSHIP: DOWN GRADIENT (D)

\begin{tabular}{|c|c|c|c|c|c|c|c|c|}
\hline PARANETER NAME & LOG DATE & $\underset{\text { ID }}{\text { SAMPLE }}$ & $\begin{array}{l}\text { UNITS OF } \\
\text { MEASURE }\end{array}$ & PVI & $\begin{array}{l}\text { PARAMETER } \\
\text { VALUE }\end{array}$ & FLAGS & $\begin{array}{l}\text { DETECTIOH } \\
\text { LIMIT }\end{array}$ & $\begin{array}{l}\text { PARAMETER } \\
\text { UNCERTAINTY }\end{array}$ \\
\hline CADMIUH & $\begin{array}{l}01 / 11 / 89 \\
09 / 19 / 89\end{array}$ & $\begin{array}{l}0001 \\
0001\end{array}$ & MG/L & $<$ & $\begin{array}{l}0.0001 \\
0.015\end{array}$ & J & $\begin{array}{l}0.001 \\
0.001\end{array}$ & - \\
\hline CALCIUA & $\begin{array}{l}10 / 10 / 85 \\
05 / 14 / 86 \\
04 / 06 / 88 \\
07 / 15 / 88 \\
01 / 11 / 89 \\
09 / 19 / 89\end{array}$ & $\begin{array}{l}0001 \\
0001 \\
0001 \\
0001 \\
0001 \\
0001\end{array}$ & $M G / L$ & & $\begin{array}{l}403 . \\
493 . \\
525 . \\
427 \\
483 \\
407 .\end{array}$ & & $\begin{array}{l}0.01 \\
0.01 \\
0.01 \\
0.01 \\
0.01 \\
0.01\end{array}$ & $\begin{array}{l}- \\
- \\
-\end{array}$ \\
\hline CHLORIDE & $\begin{array}{l}10 / 10 / 85 \\
05 / 14 / 86 \\
04 / 06 / 88 \\
07 / 15 / 88 \\
01 / 11 / 89 \\
09 / 19 / 89\end{array}$ & $\begin{array}{l}0001 \\
0001 \\
0001 \\
0001 \\
0001 \\
0001\end{array}$ & MG/L & & $\begin{array}{l}2 . \\
24 . \\
35 . \\
21 . \\
22 . \\
23 .\end{array}$ & & $\begin{array}{l}1 . \\
1 . \\
1 . \\
1 . \\
1 . \\
1 .\end{array}$ & :- \\
\hline COBALT & $\begin{array}{l}10 / 10 / 85 \\
05 / 14 / 86 \\
04 / 06 / 88 \\
07 / 15 / 88 \\
01 / 11 / 89 \\
09 / 19 / 89\end{array}$ & $\begin{array}{l}0001 \\
0001 \\
0001 \\
0001 \\
0001 \\
0001\end{array}$ & MG/L & $<$ & $\begin{array}{l}0.07 \\
0.1 \\
0.06 \\
0.05 \\
0.01 \\
0.05\end{array}$ & J & $\begin{array}{l}0.05 \\
0.05 \\
0.05 \\
0.05 \\
0.05 \\
0.05\end{array}$ & $\begin{array}{l}- \\
- \\
- \\
-\end{array}$ \\
\hline COPPER & $\begin{array}{l}10 / 10 / 85 \\
04 / 06 / 88 \\
07 / 15 / 88 \\
01 / 11 / 89 \\
09 / 19 / 89\end{array}$ & $\begin{array}{l}0001 \\
0001 \\
0001 \\
0001 \\
0001\end{array}$ & MG/L & $\begin{array}{l}< \\
<\end{array}$ & $\begin{array}{l}0.03 \\
0.03 \\
0.02 \\
0.01 \\
0.02\end{array}$ & J & $\begin{array}{l}0.02 \\
0.02 \\
0.02 \\
0.02 \\
0.02\end{array}$ & $\begin{array}{l}- \\
- \\
. \\
-\end{array}$ \\
\hline GROSS ALPHA & $\begin{array}{l}04 / 06 / 88 \\
07 / 15 / 88 \\
01 / 11 / 89 \\
09 / 19 / 89\end{array}$ & $\begin{array}{l}0001 \\
0001 \\
0001 \\
0001\end{array}$ & $\mathrm{PCI} / \mathrm{L}$ & & $\begin{array}{l}68 . \\
74 . \\
31.6 \\
15 .\end{array}$ & $J$ & $\begin{array}{l}0.2 \\
0.2 \\
1 . \\
1 .\end{array}$ & $\begin{array}{l}46 . \\
50 . \\
44.9 \\
33 .\end{array}$ \\
\hline GROSS BETA & $\begin{array}{l}04 / 06 / 88 \\
07 / 15 / 88 \\
01 / 11 / 89 \\
09 / 19 / 89\end{array}$ & $\begin{array}{l}0001 \\
0001 \\
0001 \\
0001\end{array}$ & PCI/L & & $\begin{array}{l}77 . \\
57 . \\
64.9 \\
16 .\end{array}$ & & $\begin{array}{l}1 . \\
1 . \\
0.5 \\
0.5\end{array}$ & $\begin{array}{l}26 . \\
20 . \\
49.8 \\
18 .\end{array}$ \\
\hline
\end{tabular}

PARAMETER VALUE IMDICATOR (PVI): < - LESS THAN DETECTIOH LIMIT

OTHER PARAMETER VALUE FLAGS:

J. ESTIMATED VALUE 
GROUNDWATER OUALITY DATA BY LOCATION

SITE: AMBOI AMBROSIA LAKE

LOCATION: 0779

NORTH COORDINATE: 52914.0 FT

EAST COORDINATE: 60284.7 FT

06/10/80 TO $12 / 04 / 92$

REPORT DATE: 08/04/94

FORMATION OF COMPLETION: TRES HERMANOS - C2 SANDSTONE (TT) HYDRAULIC FLON RELATIOHSHIP: DOWH GRADIENT (D)

\begin{tabular}{|c|c|c|c|c|c|c|c|c|}
\hline PARAMETER MAME & LOG DATE & $\begin{array}{c}\text { SAMPLE } \\
\text { ID }\end{array}$ & $\begin{array}{l}\text { UHITS OF } \\
\text { MEASURE }\end{array}$ & PVI & $\begin{array}{l}\text { PARAMETER } \\
\text { VALUE }\end{array}$ & FLAGS & $\begin{array}{l}\text { DETECTION } \\
\text { LIMIT }\end{array}$ & $\begin{array}{l}\text { PARAMETER } \\
\text { UNCERTAINTY }\end{array}$ \\
\hline IROW & $\begin{array}{l}10 / 10 / 85 \\
05 / 14 / 86 \\
04 / 06 / 88 \\
07 / 15 / 88 \\
01 / 11 / 89 \\
09 / 19 / 89\end{array}$ & $\begin{array}{l}0001 \\
0001 \\
0001 \\
0001 \\
0001 \\
0001\end{array}$ & MG/L & & $\begin{array}{l}0.07 \\
0.47 \\
2.51 \\
1.44 \\
2.04 \\
1.60\end{array}$ & & $\begin{array}{l}0.03 \\
0.03 \\
0.03 \\
0.03 \\
0.03 \\
0.03\end{array}$ & $\begin{array}{l}- \\
- \\
- \\
- \\
-\end{array}$ \\
\hline LEAD & $\begin{array}{l}10 / 10 / 85 \\
04 / 06 / 88 \\
07 / 15 / 88 \\
01 / 11 / 89 \\
09 / 19 / 89\end{array}$ & $\begin{array}{l}0001 \\
0001 \\
0001 \\
0001 \\
0001\end{array}$ & $M G / L$ & $\begin{array}{l}< \\
<\end{array}$ & $\begin{array}{l}0.01 \\
0.01 \\
0.01 \\
0.002 \\
0.02\end{array}$ & $\mathbf{J}$ & $\begin{array}{l}0.01 \\
0.01 \\
0.01 \\
0.01 \\
0.01\end{array}$ & $\begin{array}{l}- \\
- \\
- \\
-\end{array}$ \\
\hline LEAD-210 & $\begin{array}{l}10 / 10 / 85 \\
04 / 06 / 88 \\
07 / 15 / 88 \\
01 / 11 / 89 \\
09 / 19 / 89\end{array}$ & $\begin{array}{l}0001 \\
0001 \\
0001 \\
0001 \\
0001\end{array}$ & $\mathrm{PCI} / \mathrm{L}$ & & $\begin{array}{l}1.1 \\
0.2 \\
0.0 \\
0.00 \\
0.0\end{array}$ & & $\begin{array}{l}1.5 \\
1.5 \\
1.5 \\
1.5 \\
1.5\end{array}$ & $\begin{array}{l}1.6 \\
1.0 \\
0.9 \\
8.72 \\
0.7\end{array}$ \\
\hline MAGNESIUH & $\begin{array}{l}10 / 10 / 85 \\
05 / 14 / 86 \\
04 / 06 / 88 \\
07 / 15 / 88 \\
01 / 11 / 89 \\
09 / 19 / 89\end{array}$ & $\begin{array}{l}0001 \\
0001 \\
0001 \\
0001 \\
0001 \\
0001\end{array}$ & $M G / L$ & & $\begin{array}{l}303 . \\
388 . \\
406 \\
391 \\
475 \\
392\end{array}$ & & $\begin{array}{l}0.001 \\
0.001 \\
0.001 \\
0.001 \\
0.001 \\
0.001\end{array}$ & $\begin{array}{l}- \\
- \\
- \\
- \\
-\end{array}$ \\
\hline MANGAMESE & $\begin{array}{l}10 / 10 / 85 \\
05 / 14 / 86 \\
04 / 06 / 88 \\
07 / 15 / 88 \\
01 / 11 / 89 \\
09 / 19 / 89\end{array}$ & $\begin{array}{l}0001 \\
0001 \\
0001 \\
0001 \\
0001 \\
0001\end{array}$ & $M G / L$ & & $\begin{array}{l}0.84 \\
1.02 \\
0.96 \\
0.56 \\
0.60 \\
0.54\end{array}$ & & $\begin{array}{l}0.01 \\
0.01 \\
0.01 \\
0.01 \\
0.01 \\
0.01\end{array}$ & $\begin{array}{l}- \\
- \\
- \\
- \\
-\end{array}$ \\
\hline MERCURY & $\begin{array}{l}10 / 10 / 85 \\
04 / 06 / 88 \\
07 / 15 / 88 \\
01 / 11 / 89 \\
09 / 19 / 89\end{array}$ & $\begin{array}{l}0001 \\
0001 \\
0001 \\
0001 \\
0001\end{array}$ & $M G / L$ & $\begin{array}{l}< \\
< \\
<\end{array}$ & $\begin{array}{l}0.0002 \\
0.0006 \\
0.0003 \\
0.0001 \\
0.0002\end{array}$ & $J$ & $\begin{array}{l}0.0002 \\
0.0002 \\
0.0002 \\
0.0002 \\
0.0002\end{array}$ & $\begin{array}{l}- \\
- \\
- \\
-\end{array}$ \\
\hline MOLYBDENUM & $\begin{array}{l}10 / 10 / 85 \\
05 / 14 / 86 \\
04 / 06 / 88 \\
07 / 15 / 88 \\
01 / 11 / 89 \\
09 / 19 / 89\end{array}$ & $\begin{array}{l}0001 \\
0001 \\
0001 \\
0001 \\
0001 \\
0001\end{array}$ & $M G / L$ & $<$ & $\begin{array}{l}0.14 \\
0.08 \\
0.12 \\
0.11 \\
0.001 \\
0.01\end{array}$ & $\downarrow$ & $\begin{array}{l}0.01 \\
0.01 \\
0.01 \\
0.01 \\
0.01 \\
0.01\end{array}$ & $\begin{array}{l}- \\
- \\
- \\
- \\
-\end{array}$ \\
\hline NET GROSS ALPHA * & $\begin{array}{l}04 / 06 / 88 \\
07 / 15 / 88 \\
01 / 11 / 89 \\
09 / 19 / 89\end{array}$ & $\begin{array}{l}0001 \\
0001 \\
0001 \\
0001\end{array}$ & PCI/L & & $\begin{array}{r}62.17 \\
64.33 \\
19.94 \\
7.45\end{array}$ & & $\begin{array}{l}- \\
- \\
-\end{array}$ & $\begin{array}{l}- \\
- \\
-\end{array}$ \\
\hline NICKEL & $\begin{array}{l}10 / 10 / 85 \\
04 / 06 / 88 \\
07 / 15 / 88 \\
01 / 11 / 89\end{array}$ & $\begin{array}{l}0001 \\
0001 \\
0001 \\
0001\end{array}$ & MG/L & $<$ & $\begin{array}{l}0.14 \\
0.14 \\
0.11 \\
0.02\end{array}$ & $J$ & $\begin{array}{l}0.04 \\
0.04 \\
0.04 \\
0.04\end{array}$ & $\begin{array}{l}- \\
- \\
-\end{array}$ \\
\hline
\end{tabular}

- NET GROSS ALPHA (GROSS ALPHA - URAHIUM) HITH 1 MG URAHIUM = 686 PCI

PARAMETER VALUE INDICATOR (PVI): < - LESS THAN DETECTION LIMIT

OTHER PARAMETER VALUE FLAGS:

J - ESTIMATED VALUE
SAMPLE ID COOES:

0001 - FILTERED SAMPLE (.45 MICRONS) 
GROUNDWATER OUALITY DATA BY LOCATIOH

SITE: AMBO1 AMBROSIA LAKE

LOCATION: 0779

NORTH COORDINATE: 52914.0 FT

EAST COORDINATE: $\quad 60284.7$ FT

06/10/80 TO $12 / 04 / 92$

REPORT DATE: 08/04/94

FORMATION OF COHPLETION: TRES HERHANOS - C2 SANDSTONE (TT)

HYDRAULIC FLON RELATIONSKIP: DOWN GRADIENT (D)

\begin{tabular}{|c|c|c|c|c|c|c|c|}
\hline PARAMETER NAME & LOG DATE & $\begin{array}{c}\text { SAMPLE } \\
\text { ID }\end{array}$ & $\begin{array}{l}\text { UNITS OF } \\
\text { MEASURE }\end{array}$ & PVI & $\begin{array}{l}\text { PARAMETER } \\
\text { VALUE FLAGS }\end{array}$ & $\begin{array}{l}\text { DETECTIOH } \\
\text { LIMIT }\end{array}$ & $\begin{array}{l}\text { PARAMETER } \\
\text { UNCERTAINTY }\end{array}$ \\
\hline NICKEL & $09 / 19 / 89$ & 0001 & MG/L & $<$ & 0.04 & 0.04 & - \\
\hline NITRATE & $\begin{array}{l}10 / 10 / 85 \\
05 / 14 / 86 \\
04 / 06 / 88 \\
07 / 15 / 88 \\
01 / 11 / 89 \\
09 / 19 / 89\end{array}$ & $\begin{array}{l}0001 \\
0001 \\
0001 \\
0001 \\
0001 \\
0001\end{array}$ & $M G / L$ & $\begin{array}{l}< \\
<\end{array}$ & $\begin{array}{l}2 . \\
1 . \\
0.2 \\
2.1 \\
2.29 \\
1 .\end{array}$ & $\begin{array}{l}1 . \\
1 . \\
1 . \\
1 . \\
1 .\end{array}$ & $\begin{array}{l}- \\
- \\
- \\
- \\
-\end{array}$ \\
\hline NITRITE & $10 / 10 / 85$ & 0001 & MG/L & $<$ & 0.1 & 0.1 & - \\
\hline NITRITE AND NITRATE & $04 / 06 / 88$ & 0001 & $M G / L$ & & 0.2 & 1. & - \\
\hline PH & $\begin{array}{l}10 / 10 / 85 \\
05 / 14 / 86 \\
04 / 06 / 88 \\
07 / 15 / 88 \\
01 / 11 / 89 \\
09 / 19 / 89\end{array}$ & $\begin{array}{l}0001 \\
0001 \\
0001 \\
0001 \\
0001 \\
0001\end{array}$ & SU & & $\begin{array}{l}6.66 \\
6.62 \\
6.83 \\
6.71 \\
6.72 \\
6.68\end{array}$ & $\begin{array}{l}- \\
- \\
- \\
-\end{array}$ & $\begin{array}{l}- \\
- \\
- \\
- \\
-\end{array}$ \\
\hline PHOSPHATE & $\begin{array}{l}10 / 10 / 85 \\
04 / 06 / 88 \\
07 / 15 / 88 \\
01 / 11 / 89 \\
09 / 19 / 89\end{array}$ & $\begin{array}{l}0001 \\
0001 \\
0001 \\
0001 \\
0001\end{array}$ & $M G / L$ & $\begin{array}{l}< \\
< \\
< \\
<\end{array}$ & $\begin{array}{l}0.4 \\
0.1 \\
0.1 \\
0.1 \\
0.1\end{array}$ & $\begin{array}{l}0.1 \\
0.1 \\
0.1 \\
0.1 \\
0.1\end{array}$ & $\begin{array}{l}- \\
- \\
- \\
-\end{array}$ \\
\hline POLONIUM-210 & $\begin{array}{l}10 / 10 / 85 \\
04 / 06 / 88 \\
07 / 15 / 88 \\
01 / 11 / 89 \\
09 / 19 / 89\end{array}$ & $\begin{array}{l}0001 \\
0001 \\
0001 \\
0001 \\
0001\end{array}$ & $\mathrm{PCI} / \mathrm{L}$ & & $\begin{array}{l}0 . \\
0.3 \\
0.0 \\
1.09 \\
1.3\end{array}$ & $\begin{array}{l}1 . \\
1 . \\
1 . \\
1 . \\
1 .\end{array}$ & $\begin{array}{l}0.6 \\
0.5 \\
0.4 \\
1.40 \\
0.5\end{array}$ \\
\hline POTASSIUM & $\begin{array}{l}10 / 10 / 85 \\
05 / 14 / 86 \\
04 / 06 / 88 \\
07 / 15 / 88 \\
01 / 11 / 89 \\
09 / 19 / 89\end{array}$ & $\begin{array}{l}0001 \\
0001 \\
0001 \\
0001 \\
0001 \\
0001\end{array}$ & $M G / L$ & & $\begin{array}{l}23.9 \\
25.7 \\
27.0 \\
21.1 \\
21.6 \\
20.6\end{array}$ & $\begin{array}{l}0.01 \\
0.01 \\
0.01 \\
0.01 \\
0.01 \\
0.01\end{array}$ & $\begin{array}{l}- \\
- \\
- \\
- \\
-\end{array}$ \\
\hline RADIUM-226 & $\begin{array}{l}10 / 10 / 85 \\
04 / 06 / 88 \\
07 / 15 / 88 \\
01 / 11 / 89 \\
09 / 19 / 89\end{array}$ & $\begin{array}{l}0001 \\
0001 \\
0001 \\
0001 \\
0001\end{array}$ & $\mathrm{PCl} / \mathrm{L}$ & & $\begin{array}{l}4.4 \\
0.4 \\
0.7 \\
2.30 \\
0.0\end{array}$ & $\begin{array}{l}1 . \\
1 . \\
1 . \\
1 .\end{array}$ & $\begin{array}{l}0.7 \\
0.2 \\
0.3 \\
0.584 \\
0.1\end{array}$ \\
\hline RADIUM-226 + RADIUM-228 & $\begin{array}{l}10 / 10 / 85 \\
04 / 06 / 88 \\
07 / 15 / 88 \\
01 / 11 / 89 \\
09 / 19 / 89\end{array}$ & $\begin{array}{l}0001 \\
0001 \\
0001 \\
0001 \\
0001\end{array}$ & $\mathrm{PCI} / \mathrm{L}$ & & $\begin{array}{l}5.60 \\
3.00 \\
3.40 \\
2.30 \\
2.10\end{array}$ & $\begin{array}{l}- \\
- \\
- \\
-\end{array}$ & $\begin{array}{r}- \\
- \\
-\end{array}$ \\
\hline RAD IUM-228 & $\begin{array}{l}10 / 10 / 85 \\
04 / 06 / 88 \\
07 / 15 / 88 \\
01 / 11 / 89 \\
09 / 19 / 89\end{array}$ & $\begin{array}{l}0001 \\
0001 \\
0001 \\
0001 \\
0001\end{array}$ & $\mathrm{PCI} / \mathrm{L}$ & & $\begin{array}{l}1.2 \\
2.6 \\
2.7 \\
0.00 \\
2.1\end{array}$ & $\begin{array}{l}1 . \\
1 . \\
1 . \\
1 .\end{array}$ & $\begin{array}{l}1.1 \\
0.8 \\
1.4 \\
5.64 \\
1.0\end{array}$ \\
\hline SELENIUN & $10 / 10 / 85$ & 0001 & $M G / L$ & $<$ & 0.005 & 0.005 & - \\
\hline
\end{tabular}

PARAMETER VALUE INDICATOR (PVI): < - LESS THAN DETECTIOA LIMIT

SAMPLE ID COOES:

0001 - FILTERED SAMPLE (.45 MICRONS)

OTHER PARAMETER VALUE FLAGS:

$d$ - ESTIMATED VALUE 
GROUNDWATER QUALITY DATA BY LOCATIOH

SITE: AMBOI AMBROSIA LAKE

LOCATION: 0779

MORTH COOROINATE: 52914.0 FT

EAST COORDINATE: 60284.7 FT

06/10/80 TO $12 / 04 / 92$

REPORT DATE: $08 / 04 / 94$

FORMATIOH OF COMPLETIOH: TRES HERMAHOS - C2 SAHDSTOHE (TT)

HYDRAULIC FLON RELATIONSHIP: DONM GRADIENT (D)

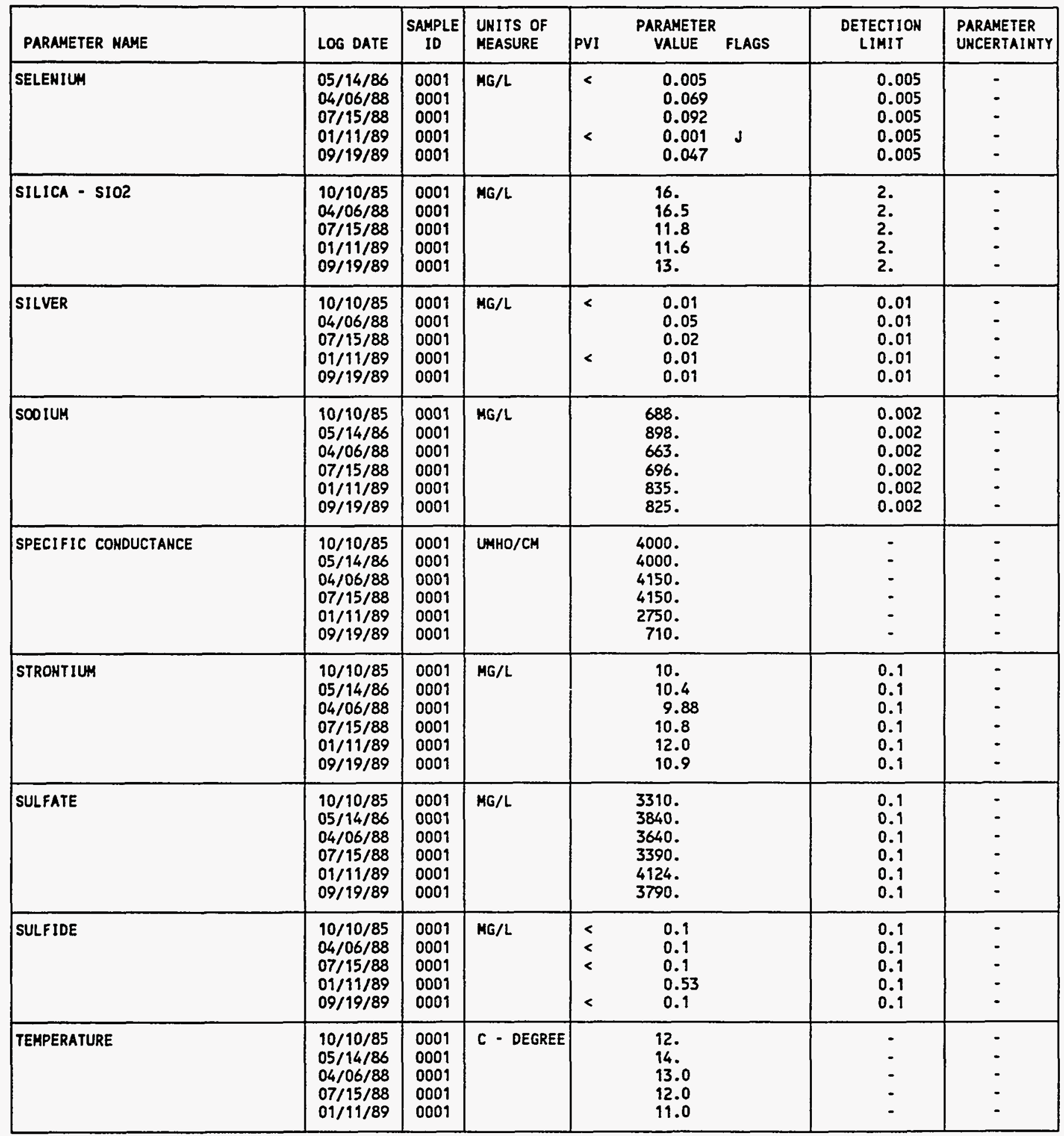

PARAMETER VALUE IHDICATOR (PVI): < - LESS THAN DETECTION LIMIT

SAMPLE IO COOES:

0001 - FILTERED SAMPLE (.45 MICRONS)

OTHER PARAMETER VALUE FLAGS:

$\downarrow$ - estimateo value 
GROUNDHATER QUALITY DATA BY LOCATIOH

SITE: AMBO1 AMBROSIA LAKE

LOCATION: OT79

NORTH COORDINATE: $\quad 52914.0 \mathrm{FT}$

EAST COORDINATE: $\quad 60284.7$ FT

06/10/80 TO $12 / 04 / 92$

REPORT DATE: 08/04/94

FORMATION OF COKPLETION: TRES HERMANOS - C2 SANDSTONE (TT) HYDRAULIC FLON RELATIONSHIP: DOWN GRADIENT (D)

\begin{tabular}{|c|c|c|c|c|c|c|c|}
\hline PARAMETER RAME & LOG DATE & $\begin{array}{c}\text { SAMPLE } \\
\text { ID }\end{array}$ & $\begin{array}{l}\text { UNITS OF } \\
\text { MEASURE }\end{array}$ & PVI & $\begin{array}{l}\text { PARAMETER } \\
\text { VALUE FLAGS }\end{array}$ & $\begin{array}{l}\text { DETECTION } \\
\text { LIMIT }\end{array}$ & $\begin{array}{l}\text { PARAMETER } \\
\text { UNCERTAINTY }\end{array}$ \\
\hline TEMPERATURE & $09 / 19 / 89$ & 0001 & $C \cdot$ DEGREE & & 14.0 & - & - \\
\hline THALLIUH & $\begin{array}{l}01 / 11 / 89 \\
09 / 19 / 89\end{array}$ & $\begin{array}{l}0001 \\
0001\end{array}$ & $M G / L$ & $<$ & $\begin{array}{l}0.005 \\
0.01\end{array}$ & $\begin{array}{l}0.005 \\
0.01\end{array}$ & - \\
\hline THORIUN-230 & $\begin{array}{l}10 / 10 / 85 \\
04 / 06 / 88 \\
07 / 15 / 88 \\
01 / 11 / 89 \\
09 / 19 / 89\end{array}$ & $\begin{array}{l}0001 \\
0001 \\
0001 \\
0001 \\
0001\end{array}$ & $\mathrm{PCI} / \mathrm{L}$ & & $\begin{array}{l}0.2 \\
0.0 \\
0.7 \\
7.04 \\
0.1\end{array}$ & $\begin{array}{l}1 . \\
1 . \\
1 . \\
1 . \\
1 .\end{array}$ & $\begin{array}{l}0.3 \\
0.4 \\
0.6 \\
0.710 \\
0.4\end{array}$ \\
\hline TIN & $\begin{array}{l}10 / 90 / 85 \\
04 / 06 / 88 \\
07 / 15 / 88 \\
01 / 11 / 89 \\
09 / 19 / 89\end{array}$ & $\begin{array}{l}0001 \\
0001 \\
0001 \\
0001 \\
0001\end{array}$ & $M G / L$ & $<$ & $\begin{array}{l}0.005 \\
0.030 \\
0.040 \\
0.001 \\
0.142\end{array}$ & $\begin{array}{l}0.005 \\
0.005 \\
0.005 \\
0.005 \\
0.005\end{array}$ & $\begin{array}{l}- \\
- \\
- \\
-\end{array}$ \\
\hline TOTAL DISSOLVED SOLIOS & $\begin{array}{l}10 / 10 / 85 \\
05 / 14 / 86 \\
04 / 06 / 88 \\
07 / 15 / 88 \\
01 / 11 / 89 \\
09 / 19 / 89\end{array}$ & $\begin{array}{l}0001 \\
0001 \\
0001 \\
0001 \\
0001 \\
0001\end{array}$ & MG/L & & $\begin{array}{l}5900 . \\
6490 . \\
6280 . \\
6730 . \\
6448 . \\
5950 .\end{array}$ & $\begin{array}{l}10 . \\
10 . \\
10 . \\
10 . \\
10 . \\
10 .\end{array}$ & $\begin{array}{l}- \\
- \\
- \\
-\end{array}$ \\
\hline TOTAL ORGANIC CARBON & $\begin{array}{l}04 / 06 / 88 \\
07 / 15 / 88 \\
01 / 11 / 89 \\
09 / 19 / 89\end{array}$ & $\begin{array}{l}0001 \\
0001 \\
0001 \\
0001\end{array}$ & $M G / L$ & & $\begin{array}{l}258 . \\
263 . \\
65 . \\
189 .\end{array}$ & $\begin{array}{l}1 . \\
1 . \\
1 .\end{array}$ & : \\
\hline URANIUM & $\begin{array}{l}10 / 10 / 85 \\
05 / 14 / 86 \\
04 / 06 / 88 \\
07 / 15 / 88 \\
01 / 11 / 89 \\
09 / 19 / 89\end{array}$ & $\begin{array}{l}0001 \\
0001 \\
0001 \\
0001 \\
0001 \\
0001\end{array}$ & MG/L & & $\begin{array}{l}0.0238 \\
0.0066 \\
0.0085 \\
0.0141 \\
0.017 \\
0.011\end{array}$ & $\begin{array}{l}0.003 \\
0.003 \\
0.003 \\
0.003 \\
0.003 \\
0.003\end{array}$ & $\begin{array}{l}- \\
- \\
- \\
-\end{array}$ \\
\hline VANADIUM & $\begin{array}{l}10 / 10 / 85 \\
05 / 14 / 86 \\
04 / 06 / 88 \\
07 / 15 / 88 \\
01 / 11 / 89 \\
09 / 19 / 89\end{array}$ & $\begin{array}{l}0001 \\
0001 \\
0001 \\
0001 \\
0001 \\
0001\end{array}$ & $\mathrm{MG} / \mathrm{L}$ & $<$ & $\begin{array}{l}0.01 \\
0.34 \\
0.11 \\
0.07 \\
0.01 \\
0.01\end{array}$ & $\begin{array}{l}0.01 \\
0.01 \\
0.01 \\
0.01 \\
0.01 \\
0.01\end{array}$ & $\begin{array}{l}- \\
: \\
- \\
-\end{array}$ \\
\hline ZINC & $\begin{array}{l}10 / 10 / 85 \\
04 / 06 / 88 \\
07 / 15 / 88 \\
01 / 11 / 89 \\
09 / 19 / 89\end{array}$ & $\begin{array}{l}0001 \\
0001 \\
0001 \\
0001 \\
0001\end{array}$ & $M G / L$ & $<$ & $\begin{array}{l}0.074 \\
0.019 \\
0.016 \\
0.01 \\
0.007\end{array}$ & $\begin{array}{l}0.005 \\
0.005 \\
0.005 \\
0.01 \\
0.005\end{array}$ & : \\
\hline
\end{tabular}

PARAMETER VALUE IMDICATOR (PVI): < - LESS THAN DETECTION LIMIT

SAMPLE ID COOES:

0001 - FILTERED SAMPLE (.45 MICRONS)

OTHER PARAMETER VALUE FLAGS:

$J$ - ESTIMATED VALUE 
GROUNDWATER OUALITY DATA BY LOCATION

SITE: AMBO1 AMBROSIA LAKE

LOCATION: 0780

HORTH COORDIHATE: $\quad 53252.8$ FT

EAST COORDIHATE: $\quad 59057.6$ FT

06/10/80 TO $12 / 04 / 92$

REPORT DATE: 08/04/94

FORMATION OF COMPLETION: ALLUVIUM (AL)

HYDRAULIC FLOW RELATIONSHIP: DOWN GRADIENT (D)

\begin{tabular}{|c|c|c|c|c|c|c|c|}
\hline PARAMETER MAME & LOG DATE & $\mid \begin{array}{c}\text { SAMPLE } \\
\text { ID }\end{array}$ & $\begin{array}{l}\text { UNITS OF } \\
\text { MEASURE }\end{array}$ & PVI & $\begin{array}{l}\text { PARAMETER } \\
\text { VALUE FLAGS }\end{array}$ & $\begin{array}{l}\text { DETECTION } \\
\text { LIMIT }\end{array}$ & $\begin{array}{l}\text { PARAMETER } \\
\text { UNCERTAINTY }\end{array}$ \\
\hline ALKALINITY & $\begin{array}{l}10 / 05 / 85 \\
05 / 13 / 86 \\
01 / 09 / 87\end{array}$ & $\begin{array}{l}0001 \\
0001 \\
0001\end{array}$ & MG/L CACO3 & & $\begin{array}{r}401 . \\
1917 . \\
609 .\end{array}$ & $\begin{array}{c}0.1 \\
- \\
-\end{array}$ & : \\
\hline ALUMINUM & $10 / 05 / 85$ & 0001 & $M G / L$ & & 0.3 & 0.1 & - \\
\hline ANTIKONY & $10 / 05 / 85$ & 0001 & MG/L & $<$ & 0.003 & 0.003 & - \\
\hline ARSENIC & $\begin{array}{l}10 / 05 / 85 \\
05 / 13 / 86 \\
01 / 09 / 87\end{array}$ & $\begin{array}{l}0001 \\
0001 \\
0001\end{array}$ & MG/L & $<$ & $\begin{array}{l}0.01 \\
0.01 \\
0.005\end{array}$ & $\begin{array}{l}0.01 \\
0.01 \\
0.01\end{array}$ & : \\
\hline BARIUH & $10 / 05 / 85$ & 0001 & MG/L & $<$ & 0.1 & 0.1 & - \\
\hline CADHIUM & $\begin{array}{l}10 / 05 / 85 \\
05 / 13 / 86\end{array}$ & $\begin{array}{l}0001 \\
0001\end{array}$ & $\mathrm{MG} / \mathrm{L}$ & $<$ & $\begin{array}{l}0.001 \\
0.001\end{array}$ & $\begin{array}{l}0.001 \\
0.001\end{array}$ & : \\
\hline CALCIUM & $\begin{array}{l}10 / 05 / 85 \\
05 / 13 / 86 \\
01 / 09 / 87\end{array}$ & $\begin{array}{l}0001 \\
0001 \\
0001\end{array}$ & MG/L & & $\begin{array}{l}404 . \\
746 . \\
457 .\end{array}$ & $\begin{array}{l}0.01 \\
0.01 \\
0.01\end{array}$ & : \\
\hline CHLORIDE & $\begin{array}{l}10 / 05 / 85 \\
05 / 13 / 86 \\
01 / 09 / 87\end{array}$ & $\begin{array}{l}0001 \\
0001 \\
0001\end{array}$ & $M G / L$ & & $\begin{array}{l}210 . \\
280 . \\
300 .\end{array}$ & $\begin{array}{l}1 . \\
1 . \\
1 .\end{array}$ & - \\
\hline CHROMIUM & $\begin{array}{l}10 / 05 / 85 \\
05 / 13 / 86 \\
01 / 09 / 87\end{array}$ & $\begin{array}{l}0001 \\
0001 \\
0001\end{array}$ & MG/L & & $\begin{array}{l}0.03 \\
0.03 \\
0.02\end{array}$ & $\begin{array}{l}0.01 \\
0.01 \\
0.01\end{array}$ & : \\
\hline CYANIDE & $10 / 05 / 85$ & 0001 & $M G / L$ & $<$ & 0.001 & 0.01 & - \\
\hline FLUORIDE & $\begin{array}{l}10 / 05 / 85 \\
05 / 13 / 86 \\
01 / 09 / 87\end{array}$ & $\begin{array}{l}0001 \\
0001 \\
0001\end{array}$ & $M G / L$ & & $\begin{array}{l}2.2 \\
1 . \\
0.69\end{array}$ & $\begin{array}{l}0.1 \\
0.1 \\
0.1\end{array}$ & : \\
\hline GROSS ALPHA & $01 / 09 / 87$ & 0001 & $\mathrm{PCl} / \mathrm{L}$ & & 2300. & 0.2 & 200. \\
\hline GROSS BETA & $01 / 09 / 87$ & 0001 & $\mathrm{PCI} / \mathrm{L}$ & & 980. & 1. & 50. \\
\hline IROH & $\begin{array}{l}10 / 05 / 85 \\
05 / 13 / 86 \\
01 / 09 / 87\end{array}$ & $\begin{array}{l}0001 \\
0001 \\
0001\end{array}$ & $M G / L$ & & $\begin{array}{l}0.49 \\
4.13 \\
0.55\end{array}$ & $\begin{array}{l}0.03 \\
0.03 \\
0.03\end{array}$ & - \\
\hline LEAD & $10 / 05 / 85$ & 0001 & $M G / L$ & $<$ & 0.01 & 0.01 & - \\
\hline
\end{tabular}

PARAMETER VALUE IMDICATOR (PVI): < - LESS THAM DETECTION LIMIT

SAMPLE ID COOES:

0009 - FILTERED SAMPLE (.45 MICRONS)

OTHER PARAMETER VALUE FLAGS:

$J$ - estimateo VALUE 
GROUNDWATER QUALITY DATA BY LOCATION

SITE: AMBOI AMBROSIA LAKE

LOCATIOH: 0780

NORTH COORDINATE: 53252.8 FT

EAST COORDINATE: $\quad 59057.6$ FT

$06 / 10 / 80$ TO $12 / 04 / 92$

REPORT DATE: 08/04/94

FORMATION OF COMPLETIOH: ALLUVIUH (AL)

HYDRAULIC FLON RELATIONSHIP: DOWN GRADIENT (D)

\begin{tabular}{|c|c|c|c|c|c|c|c|}
\hline PARAMETER NAME & LOG DATE & $\begin{array}{c}\text { SAMPLE } \\
\text { ID }\end{array}$ & $\begin{array}{l}\text { UNITS OF } \\
\text { MEASURE }\end{array}$ & PVI & $\begin{array}{l}\text { PARAMETER } \\
\text { VALUE FLAGS }\end{array}$ & $\begin{array}{l}\text { DETECTION } \\
\text { LIMIT }\end{array}$ & $\begin{array}{l}\text { PARAMETER } \\
\text { UNCERTAINTY }\end{array}$ \\
\hline MAGNESIUN & $\begin{array}{l}10 / 05 / 85 \\
05 / 13 / 86 \\
01 / 09 / 87\end{array}$ & $\begin{array}{l}0001 \\
0001 \\
0001\end{array}$ & $M G / L$ & & $\begin{array}{l}222 . \\
442 \\
290\end{array}$ & $\begin{array}{l}0.001 \\
0.001 \\
0.001\end{array}$ & - \\
\hline MANGANESE & $\begin{array}{l}10 / 05 / 85 \\
05 / 13 / 86 \\
01 / 09 / 87\end{array}$ & $\begin{array}{l}0001 \\
0001 \\
0001\end{array}$ & MG/L & & $\begin{array}{l}0.56 \\
4.23 \\
0.33\end{array}$ & $\begin{array}{l}0.01 \\
0.01 \\
0.01\end{array}$ & - \\
\hline HERCURY & $10 / 05 / 85$ & 0001 & $M G / L$ & $<$ & 0.0002 & 0.0002 & - \\
\hline MOLYBDENUM & $\begin{array}{l}10 / 05 / 85 \\
05 / 13 / 86 \\
01 / 09 / 87\end{array}$ & $\begin{array}{l}0001 \\
0001 \\
0001\end{array}$ & $M G / L$ & & $\begin{array}{l}3.17 \\
1.22 \\
3.6\end{array}$ & $\begin{array}{l}0.01 \\
0.01 \\
0.01\end{array}$ & $\begin{array}{l}- \\
-\end{array}$ \\
\hline NET GROSS ALPHA * & $01 / 09 / 87$ & 0001 & $\mathrm{PCI} / \mathrm{L}$ & & -80.42 & - & - \\
\hline NICKEL & $10 / 05 / 85$ & 0001 & MG/L & & 0.1 & 0.04 & - \\
\hline NITRATE & $\begin{array}{l}10 / 05 / 85 \\
05 / 13 / 86 \\
01 / 09 / 87\end{array}$ & $\begin{array}{l}0001 \\
0001 \\
0001\end{array}$ & HG/L & & $\begin{array}{l}9.0 \\
75 . \\
140 .\end{array}$ & $\begin{array}{l}1 . \\
1 .\end{array}$ & $\begin{array}{l}- \\
- \\
-\end{array}$ \\
\hline NITRITE & $10 / 05 / 85$ & 0001 & $M G / L$ & $<$ & 0.1 & 0.1 & - \\
\hline PH & $\begin{array}{l}10 / 05 / 85 \\
05 / 13 / 86 \\
01 / 09 / 87\end{array}$ & $\begin{array}{l}0001 \\
0001 \\
0001\end{array}$ & SU & & $\begin{array}{l}7.64 \\
7.28 \\
7.32\end{array}$ & $\begin{array}{l}- \\
-\end{array}$ & - \\
\hline PHOSPHATE & $10 / 05 / 85$ & 0001 & $\mathrm{MG} / \mathrm{L}$ & & 0.3 & 0.1 & - \\
\hline POTASSIUM & $\begin{array}{l}10 / 05 / 85 \\
05 / 13 / 86 \\
01 / 09 / 87\end{array}$ & $\begin{array}{l}0001 \\
0001 \\
0001\end{array}$ & $M G / L$ & & $\begin{array}{l}9.54 \\
9.67 \\
5.85\end{array}$ & $\begin{array}{l}0.01 \\
0.01 \\
0.01\end{array}$ & $\begin{array}{l}- \\
-\end{array}$ \\
\hline RADILA-226 & $\begin{array}{l}05 / 13 / 86 \\
01 / 09 / 87\end{array}$ & $\begin{array}{l}0001 \\
0001\end{array}$ & PCI/L & & $\begin{array}{l}2.5 \\
2.5\end{array}$ & $\begin{array}{l}1 . \\
1 .\end{array}$ & $\begin{array}{l}0.7 \\
0.8\end{array}$ \\
\hline RADIUM-226 + RADIUM-228 & $01 / 09 / 87$ & 0001 & PCI/L & & 3.10 & - & - \\
\hline RADIUN-228 & $01 / 09 / 87$ & 0001 & $\mathrm{PCI} / \mathrm{L}$ & & 0.6 & 1. & 1.4 \\
\hline SELENIUM & $\begin{array}{l}10 / 05 / 85 \\
05 / 13 / 86 \\
01 / 09 / 87\end{array}$ & $\begin{array}{l}0001 \\
0001 \\
0001\end{array}$ & $M G / L$ & & $\begin{array}{l}0.127 \\
0.009 \\
0.537\end{array}$ & $\begin{array}{l}0.005 \\
0.005 \\
0.005\end{array}$ & - \\
\hline SILICA - S102 & $10 / 05 / 85$ & 0001 & $M G / L$ & & 15. & 2. & - \\
\hline SILVER & $\begin{array}{l}10 / 05 / 85 \\
01 / 09 / 87\end{array}$ & $\begin{array}{l}0001 \\
0001\end{array}$ & MG/L & $\begin{array}{l}< \\
<\end{array}$ & $\begin{array}{l}0.01 \\
0.01\end{array}$ & $\begin{array}{l}0.01 \\
0.01\end{array}$ & - \\
\hline SOOIUM & $\begin{array}{l}10 / 05 / 85 \\
05 / 13 / 86 \\
01 / 09 / 87\end{array}$ & $\begin{array}{l}0001 \\
0001 \\
0001\end{array}$ & MG/L & & $\begin{array}{l}1140 \\
1430 \\
1540\end{array}$ & $\begin{array}{l}0.002 \\
0.002 \\
0.002\end{array}$ & $\begin{array}{l}- \\
-\end{array}$ \\
\hline SPECIFIC CONDUCTANCE & $\begin{array}{l}10 / 05 / 85 \\
05 / 13 / 86\end{array}$ & $\begin{array}{l}0001 \\
0001\end{array}$ & UHHO/CH & & $\begin{array}{l}6000 \\
4400\end{array}$ & - & - \\
\hline
\end{tabular}

- NET GROSS ALPHA (GROSS ALPHA - URANIUH) WITH 1 MG URANIUM = 686 PCI

PARAMETER VALUe INDICATOR (PVI): < - LESS THAN DETECTIOH LIMIT

SAMPLE ID CODES:

0001 - FILTERED SAMPLE (.45 HICRONS) 
GROUMDWATER QUALITY DATA BY LOCATION

SITE: AMBO1 AMBROSIA LAKE

LOCATIOH: 0780

HORTH COORDINATE: 53252.8 FT

EAST COORDIHATE: $\quad 59057.6$ FT

06/10/80 TO 12/04/92

REPORT DATE: $08 / 04 / 94$

FORMATION OF COMPLETION: ALLUVIUM (AL)

HYDRAULIC FLON RELATIOHSHIP: DONN GRADIENT (D)

\begin{tabular}{|c|c|c|c|c|c|c|c|}
\hline PARAMETER MNME & LOG DATE & $\underset{10}{\text { SAMPLE }}$ & $\begin{array}{l}\text { UHITS OF } \\
\text { MEASURE }\end{array}$ & PVI & $\begin{array}{l}\text { PARAMETER } \\
\text { VALUE FLAGS }\end{array}$ & $\begin{array}{l}\text { DETECTION } \\
\text { LIMIT }\end{array}$ & $\begin{array}{l}\text { PARAMETER } \\
\text { UNCERTAINTY }\end{array}$ \\
\hline SPECIFIC CONDUCTAKCE & $01 / 09 / 87$ & 0001 & UNHO/CH & & 5000. & - & - \\
\hline STRONTIUN & $\begin{array}{l}10 / 05 / 85 \\
05 / 13 / 86\end{array}$ & $\begin{array}{l}0001 \\
0001\end{array}$ & MG/L & & $\begin{array}{l}7.8 \\
1.1\end{array}$ & $\begin{array}{l}0.1 \\
0.1\end{array}$ & $\dot{-}$ \\
\hline SULFATE & $\begin{array}{l}10 / 05 / 85 \\
05 / 13 / 86 \\
01 / 09 / 87\end{array}$ & $\begin{array}{l}0001 \\
0001 \\
0001\end{array}$ & MG/L & & $\begin{array}{l}3610 . \\
4300 . \\
4440 .\end{array}$ & $\begin{array}{l}0.1 \\
0.1 \\
0.1\end{array}$ & : \\
\hline SULFIDE & $10 / 05 / 85$ & 0001 & MG/L & $<$ & 0.1 & 0.1 & - \\
\hline TEMPERATURE & $\begin{array}{l}10 / 05 / 85 \\
05 / 13 / 86 \\
01 / 09 / 87\end{array}$ & $\begin{array}{l}0001 \\
0001 \\
0001\end{array}$ & C - DEGREE & & $\begin{array}{l}14 . \\
12 . \\
9.0\end{array}$ & - & : \\
\hline THORIUM-230 & $05 / 13 / 86$ & 0001 & $\mathrm{PCI} / \mathrm{L}$ & & 0.2 & 1. & 0.3 \\
\hline TIH & $10 / 05 / 85$ & 0001 & $M G / L$ & $<$ & 0.005 & 0.005 & - \\
\hline TOTAL DISSOLVED SOLIDS & $\begin{array}{l}10 / 05 / 85 \\
05 / 13 / 86 \\
01 / 09 / 87\end{array}$ & $\begin{array}{l}0001 \\
0001 \\
0001\end{array}$ & $M G / L$ & & $\begin{array}{l}6180 . \\
7250 . \\
8300 .\end{array}$ & $\begin{array}{l}10 . \\
10 . \\
10 .\end{array}$ & - \\
\hline TOTAL ORGANIC CARBON & $10 / 05 / 85$ & 0001 & $M G / L$ & & 25. & 1. & - \\
\hline URANIUM & $\begin{array}{l}10 / 05 / 85 \\
05 / 13 / 86 \\
01 / 09 / 87\end{array}$ & $\begin{array}{l}0001 \\
0001 \\
0001\end{array}$ & MG/L & & $\begin{array}{l}2.8 \\
0.0933 \\
3.47\end{array}$ & $\begin{array}{l}0.003 \\
0.003 \\
0.003\end{array}$ & $\dot{-}$ \\
\hline VAKADIUH & $\begin{array}{l}10 / 05 / 85 \\
05 / 13 / 86 \\
01 / 09 / 87\end{array}$ & $\begin{array}{l}0001 \\
0001 \\
0001\end{array}$ & $M G / L$ & & $\begin{array}{l}0.5 \\
0.5 \\
0.05\end{array}$ & $\begin{array}{l}0.01 \\
0.01 \\
0.01\end{array}$ & $\dot{-}$ \\
\hline ZINC & $10 / 05 / 85$ & 0001 & MG/L & & 0.098 & 0.005 & - \\
\hline
\end{tabular}

PARAMETER VALUE INDICATOR (PVI): < - LESS THAN DETECTION LIMIT

SAMPLE ID COOES:

O001 - FILTERED SAMPLE (.45 MICRONS) 
FORMATION OF COMPLETION: ALLUVIUM (AL)

HYDRAULIC FLON RELATIONSHIP: DOWN GRADIENT (D)

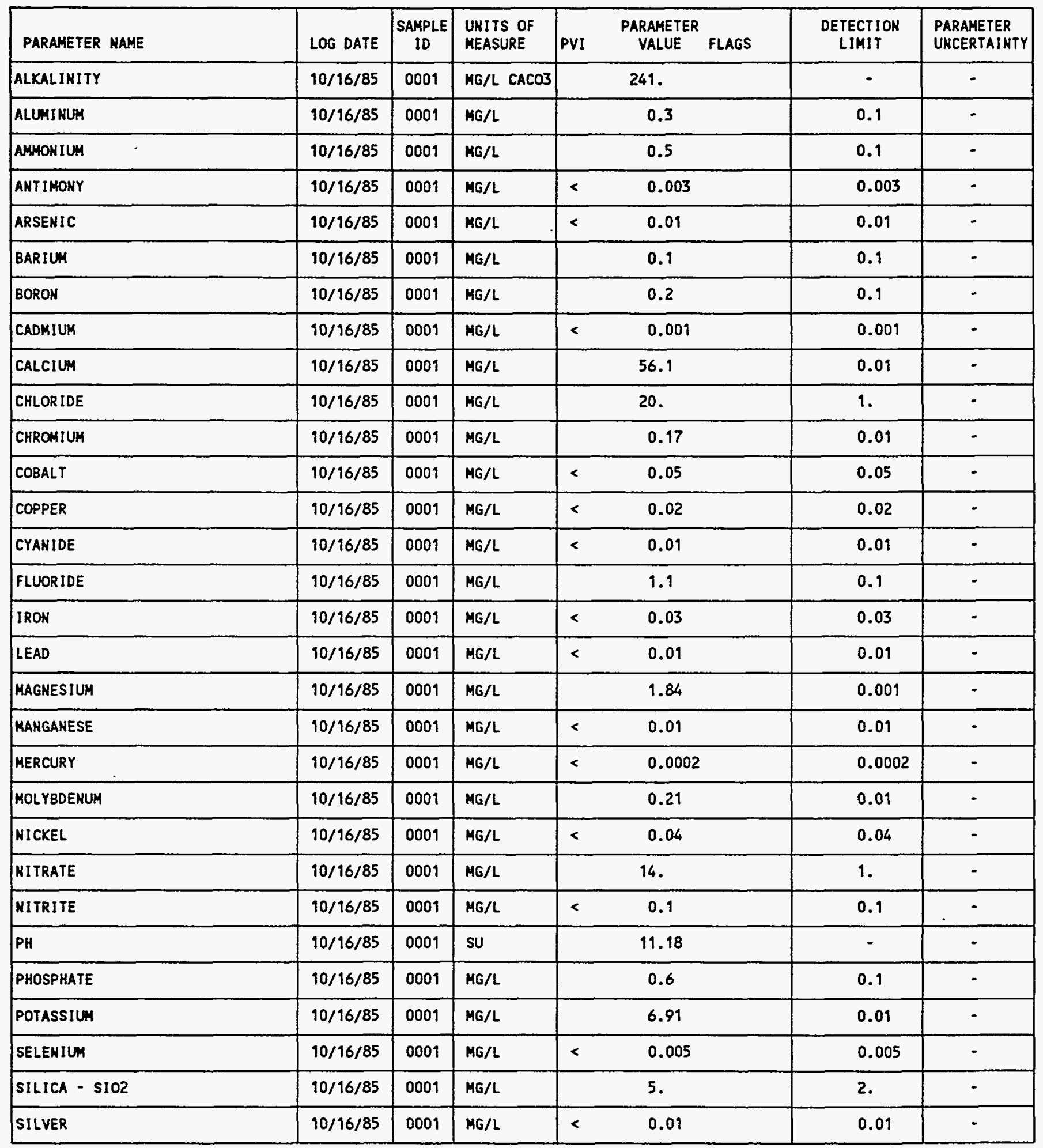


GROUHDWATER QUALITY DATA BY LOCATIOH

SITE: AMBO1 AMBROSIA LAKE

LOCATION: 0781

NORTH COORDINATE: 53253.5 FT

EAST COOROIHATE: $\quad 59156.3$ FT

06/10/80 TO $12 / 04 / 92$

REPORT DATE : 08/04/94

FORMATION OF COMPLETION: ALLUVIUM (AL)

HYDRAULIC FLON RELATIONSHIP: DOWN GRADIENT (D)

\begin{tabular}{|c|c|c|c|c|c|c|c|}
\hline PARAMETER NAME & LOG DATE & $\begin{array}{c}\text { SAMPLE } \\
10\end{array}$ & $\begin{array}{l}\text { UNITS OF } \\
\text { MEASURE }\end{array}$ & PVI & $\begin{array}{l}\text { PARAMETER } \\
\text { VALUE FLAGS }\end{array}$ & $\begin{array}{l}\text { DETECTION } \\
\text { LIMIT }\end{array}$ & $\begin{array}{l}\text { PARAMETER } \\
\text { UNCERTAIKTY }\end{array}$ \\
\hline SOOIUH & $10 / 16 / 85$ & 0001 & $M G / L$ & & 220. & 0.002 & - \\
\hline STRONTIUN & $10 / 16 / 85$ & 0001 & MG/L & & 0.9 & 0.1 & - \\
\hline SULFIDE & $10 / 16 / 85$ & 0001 & MG/L & $<$ & 0.1 & 0.1 & - \\
\hline TEMPERATURE & $10 / 16 / 85$ & 0001 & C - DEGREE & & 10. & - & - \\
\hline THORIUM-230 & $10 / 16 / 85$ & 0001 & $\mathrm{PCl} / \mathrm{L}$ & & 0.6 & 1. & 2. \\
\hline TIN & $10 / 16 / 85$ & 0001 & $M G / L$ & $<$ & 0.005 & 0.005 & - \\
\hline URANIUM & $10 / 16 / 85$ & 0001 & $M G / L$ & & $0.0006 \mathrm{~J}$ & 0.003 & - \\
\hline VAHADIUM & $10 / 16 / 85$ & 0001 & MG/L & $<$ & 0.01 & 0.01 & - \\
\hline ZINC & $10 / 16 / 85$ & 0001 & $M G / L$ & $<$ & 0.005 & 0.005 & - \\
\hline
\end{tabular}

PARAMETER VALUE IMDICATOR (PVI): < - LESS THAN DETECTION LIMIT

SAMPLE ID COOES:

0001 - FILTERED SAMPLE (.45 MICRONS)

OTHER PARAMETER VALUE FLAGS:

$J$ - ESTIMATED VALUE 
GROUNDWATER QUALITY DATA BY LOCATIOH

SITE: AMBO1 AMBROSIA LAKE

LOCATION: 0782

NORTH COORDINATE: 55221.4 FT

EAST COORDINATE: $\quad 58792.8$ FT

06/10/80 TO $12 / 04 / 92$

REPORT DATE: $08 / 04 / 94$

FORMATION OF COMPLETION: TRES HERMANOS - C2 SANDSTONE (TT) HYDRAULIC FLON RELATIONSHIP: CROSS GRADIENT (C)

\begin{tabular}{|c|c|c|c|c|c|c|c|}
\hline PARAMETER NAME & LOG DATE & $\underset{\text { ID }}{\text { SAMPLE }}$ & $\begin{array}{l}\text { UNITS OF } \\
\text { MEASURE }\end{array}$ & PVI & $\begin{array}{l}\text { PARAMETER } \\
\text { VALUE FLAGS }\end{array}$ & $\begin{array}{l}\text { DETECTION } \\
\text { LIMIT }\end{array}$ & $\begin{array}{l}\text { PARAMETER } \\
\text { UHCERTAINTY }\end{array}$ \\
\hline ALKALINITY & $\begin{array}{l}10 / 09 / 85 \\
05 / 16 / 86\end{array}$ & $\begin{array}{l}0001 \\
0001\end{array}$ & MG/L CACO3 & & $\begin{array}{l}1072 . \\
449 .\end{array}$ & - & - \\
\hline ALUMINUM & $10 / 09 / 85$ & 0001 & $M G / L$ & & 0.4 & 0.1 & - \\
\hline AMМОN IUM & $10 / 09 / 85$ & 0001 & MG/L & & 0.8 & 0.1 & - \\
\hline ARSENIC & $\begin{array}{l}10 / 09 / 85 \\
05 / 16 / 86\end{array}$ & $\begin{array}{l}0001 \\
0001\end{array}$ & MG/L & $<$ & $\begin{array}{l}0.01 \\
0.01\end{array}$ & $\begin{array}{l}0.01 \\
0.01\end{array}$ & $\dot{-}$ \\
\hline BARIUM & $10 / 09 / 85$ & 0001 & MG/L & & 0.3 & 0.1 & - \\
\hline BORON & $\begin{array}{l}10 / 09 / 85 \\
05 / 16 / 86\end{array}$ & $\begin{array}{l}0001 \\
0001\end{array}$ & MG/L & & $\begin{array}{l}0.2 \\
0.2\end{array}$ & $\begin{array}{l}0.1 \\
0.1\end{array}$ & - \\
\hline CALCIUM & $\begin{array}{l}10 / 09 / 85 \\
05 / 16 / 86\end{array}$ & $\begin{array}{l}0001 \\
0001\end{array}$ & $M G / L$ & & $\begin{array}{l}258 . \\
192 .\end{array}$ & $\begin{array}{l}0.01 \\
0.01\end{array}$ & $\dot{-}$ \\
\hline CHLORIDE & $\begin{array}{l}10 / 09 / 85 \\
05 / 16 / 86\end{array}$ & $\begin{array}{l}0001 \\
0001\end{array}$ & $M G / L$ & & $\begin{array}{l}20 . \\
12 .\end{array}$ & $\begin{array}{l}1 . \\
1 .\end{array}$ & $\dot{-}$ \\
\hline CHROMIUM & $\begin{array}{l}10 / 09 / 85 \\
05 / 96 / 86\end{array}$ & $\begin{array}{l}0001 \\
0001\end{array}$ & $M G / L$ & & $\begin{array}{l}0.04 \\
0.03\end{array}$ & $\begin{array}{l}0.01 \\
0.01\end{array}$ & - \\
\hline COBALT & $\begin{array}{l}10 / 09 / 85 \\
05 / 16 / 86\end{array}$ & $\begin{array}{l}0001 \\
0001\end{array}$ & $M G / L$ & $<$ & $\begin{array}{l}0.05 \\
0.05\end{array}$ & $\begin{array}{l}0.05 \\
0.05\end{array}$ & - \\
\hline COPPER & $10 / 09 / 85$ & 0001 & $M G / L$ & & 0.02 & 0.02 & - \\
\hline LEAD-210 & $10 / 09 / 85$ & 0001 & $\mathrm{PCl} / \mathrm{L}$ & & 2.9 & 1.5 & 1.5 \\
\hline MAGHESIUM & $\begin{array}{l}10 / 09 / 85 \\
05 / 16 / 86\end{array}$ & $\begin{array}{l}0001 \\
0001\end{array}$ & $M G / L$ & & $\begin{array}{l}0.167 \\
0.161\end{array}$ & $\begin{array}{l}0.001 \\
0.001\end{array}$ & $\dot{-}$ \\
\hline MANGANESE & $\begin{array}{l}10 / 09 / 85 \\
05 / 16 / 86\end{array}$ & $\begin{array}{l}0001 \\
0001\end{array}$ & $M G / L$ & & $\begin{array}{l}0.02 \\
0.01\end{array}$ & $\begin{array}{l}0.01 \\
0.01\end{array}$ & - \\
\hline MERCURY & $10 / 09 / 85$ & 0001 & MG/L & $<$ & 0.0002 & 0.0002 & - \\
\hline MOLYBDENUM & $\begin{array}{l}10 / 09 / 85 \\
05 / 16 / 86\end{array}$ & $\begin{array}{l}0001 \\
0001\end{array}$ & $M G / L$ & & $\begin{array}{l}0.16 \\
0.17\end{array}$ & $\begin{array}{l}0.01 \\
0.01\end{array}$ & $\dot{-}$ \\
\hline NICKEL & $10 / 09 / 85$ & 0001 & MG/L & & 0.06 & 0.04 & - \\
\hline
\end{tabular}

PARAMETER VALUE INDICATOR (PVI): < - LESS THAN DETECTION LIMIT 
REPORT DATE: 08/04/94

FORMATION OF COMPLETION: TRES HERMANOS - C2 SANDSTONE (TT) HYORAULIC FLOH RELATIOHSHIP: CROSS GRADIENT (C)

\begin{tabular}{|c|c|c|c|c|c|c|c|}
\hline PARAMETER MAME & LOG DATE & $\underset{\text { SAMPLE }}{\text { SA }}$ & $\begin{array}{l}\text { UNITS OF } \\
\text { MEASURE }\end{array}$ & PVI & $\begin{array}{l}\text { PARAMETER } \\
\text { VALUE FLAGS }\end{array}$ & $\begin{array}{l}\text { DETECTION } \\
\text { LIMIT }\end{array}$ & $\begin{array}{l}\text { PARAMETER } \\
\text { UNCERTAINTY }\end{array}$ \\
\hline MITRATE & $\begin{array}{l}10 / 09 / 85 \\
05 / 16 / 86\end{array}$ & $\begin{array}{l}0001 \\
0001\end{array}$ & MG/L & $<$ & $\begin{array}{l}4 . \\
1 .\end{array}$ & 1. & - \\
\hline NITRITE & $10 / 09 / 85$ & 0001 & MG/L & $<$ & 0.1 & 0.1 & - \\
\hline PH & $\begin{array}{l}10 / 09 / 85 \\
05 / 16 / 86\end{array}$ & $\begin{array}{l}0001 \\
0001\end{array}$ & SU & & $\begin{array}{l}12.2 \\
11.46\end{array}$ & $\therefore$ & $\therefore$ \\
\hline PHOSPHATE & $10 / 09 / 85$ & 0001 & $M G / L$ & & 0.4 & 0.1 & $\cdot$ \\
\hline POLONIUK-210 & $10 / 09 / 85$ & 0001 & PCI/L & & 0. & 9. & 0.6 \\
\hline POTASSIUH & $\begin{array}{l}10 / 09 / 85 \\
05 / 16 / 86\end{array}$ & $\begin{array}{l}0001 \\
0001\end{array}$ & $M G / L$ & & $\begin{array}{l}24.9 \\
15.3\end{array}$ & $\begin{array}{l}0.01 \\
0.01\end{array}$ & - \\
\hline RADIUHA-226 & $\begin{array}{l}10 / 09 / 85 \\
05 / 16 / 86\end{array}$ & $\begin{array}{l}0001 \\
0001\end{array}$ & $\mathrm{PCI} / \mathrm{L}$ & & $\begin{array}{l}2.4 \\
1 .\end{array}$ & 1. & $\begin{array}{l}0.5 \\
0.4\end{array}$ \\
\hline RADIUM-226 + RADIUH-228 & $10 / 09 / 85$ & 0001 & PCI/L & & 2.80 & - & - \\
\hline RADIUM-228 & $10 / 09 / 85$ & 0001 & $\mathrm{PCI} / \mathrm{L}$ & & 0.4 & 1. & 0.9 \\
\hline SELEMIUM & $\begin{array}{l}10 / 09 / 85 \\
05 / 16 / 86\end{array}$ & $\begin{array}{l}0001 \\
0001\end{array}$ & $M G / L$ & $<$ & $\begin{array}{l}0.005 \\
0.005\end{array}$ & $\begin{array}{l}0.005 \\
0.005\end{array}$ & - \\
\hline SILICA - SIOZ & $90 / 09 / 85$ & 0001 & MG/L & & 4. & 2. & $\cdot$ \\
\hline SILVER & $10 / 09 / 85$ & 0001 & $M G / L$ & $<$ & 0.01 & 0.01 & - \\
\hline SOOIUM & $\begin{array}{l}10 / 09 / 85 \\
05 / 16 / 86\end{array}$ & $\begin{array}{l}0001 \\
0001\end{array}$ & $M G / L$ & & $\begin{array}{l}264 . \\
292 .\end{array}$ & $\begin{array}{l}0.002 \\
0.002\end{array}$ & - \\
\hline SPECIFIC CONDUCTANCE & $\begin{array}{l}10 / 09 / 85 \\
05 / 16 / 86\end{array}$ & $\begin{array}{l}0001 \\
0001\end{array}$ & ᄂMHо/CM & & $\begin{array}{l}4100 . \\
2450 .\end{array}$ & - & - \\
\hline STRONTIUH & $\begin{array}{l}10 / 09 / 85 \\
05 / 16 / 86\end{array}$ & $\begin{array}{l}0001 \\
0001\end{array}$ & $M G / L$ & & $\begin{array}{l}2.6 \\
2.1\end{array}$ & $\begin{array}{l}0.1 \\
0.1\end{array}$ & - \\
\hline SULFATE & $\begin{array}{l}10 / 09 / 85 \\
05 / 16 / 86\end{array}$ & $\begin{array}{l}0001 \\
0001\end{array}$ & $M G / L$ & & $\begin{array}{l}516 . \\
633 .\end{array}$ & $\begin{array}{l}0.1 \\
0.1\end{array}$ & $\dot{-}$ \\
\hline SULFIDE & $10 / 09 / 85$ & 0001 & MG/L & $<$ & 0.1 & 0.1 & - \\
\hline TEMPERATURE & $\begin{array}{l}10 / 09 / 85 \\
05 / 16 / 86\end{array}$ & $\begin{array}{l}0001 \\
0001\end{array}$ & C - DEGREE & & $\begin{array}{l}12.5 \\
16 .\end{array}$ & $\dot{-}$ & $\dot{-}$ \\
\hline THORIUN-230 & $\begin{array}{l}10 / 09 / 85 \\
05 / 16 / 86\end{array}$ & $\begin{array}{l}0001 \\
0001\end{array}$ & $\mathrm{PCI} / \mathrm{L}$ & & $\begin{array}{l}0.5 \\
0.1\end{array}$ & 1. & $\begin{array}{l}0.5 \\
0.2\end{array}$ \\
\hline TIN & $10 / 09 / 85$ & 0001 & $M G / L$ & $<$ & 0.005 & 0.005 & - \\
\hline TOTAL DISSOLVED SOLIOS & $\begin{array}{l}10 / 09 / 85 \\
05 / 16 / 86\end{array}$ & $\begin{array}{l}0001 \\
0001\end{array}$ & MG/L & & $\begin{array}{l}1880 . \\
1340 .\end{array}$ & $\begin{array}{l}10 . \\
10 .\end{array}$ & - \\
\hline TOTAL ORgANIC CARBON & $10 / 09 / 85$ & 0001 & MG/L & & 18. & 1. & - \\
\hline URAKIUM & $\begin{array}{l}10 / 09 / 85 \\
05 / 16 / 86\end{array}$ & $\begin{array}{l}0001 \\
0001\end{array}$ & MG/L & $<$ & $\begin{array}{l}0.0031 \\
0.0003 \quad J\end{array}$ & $\begin{array}{l}0.003 \\
0.003\end{array}$ & - \\
\hline
\end{tabular}

PARAMETER VALUE IMDICATOR (PVI): < - LESS THAN DETECTION LIMIT

SAMPLE ID COOES:

O001 - FILTERED SAMPLE (.45 MICRONS)

OTHER PARAMETER VALUE FLAGS:

J - ESTIMATED VALUE 
GROUNDWATER OUALITY DATA BY LOCATION

SITE: AMBO1 AMBROSIA LAKE

LOCATION: 0782

MORTH COORDIHATE: 55221.4 FT

EAST COORDINATE: $\quad 58792.8$ FT

06/10/80 TO $12 / 04 / 92$

REPORT DATE: 08/04/94

FORMATION OF COMPLETION: TRES HERMANOS - C2 SANDSTONE (TT)

HYDRAULIC FLOW RELATIONSHIP: CROSS GRADIENT (C)

\begin{tabular}{|c|c|c|c|c|c|c|c|}
\hline PARAMETER MAME & LOG DATE & $\begin{array}{c}\text { SAMPLE } \\
10\end{array}$ & $\begin{array}{l}\text { UNITS OF } \\
\text { MEASURE }\end{array}$ & PVI & $\begin{array}{l}\text { PARAMETER } \\
\text { VALUE FLAGS }\end{array}$ & $\begin{array}{l}\text { DETECTIOH } \\
\text { LIMIT }\end{array}$ & $\begin{array}{l}\text { PARAMETER } \\
\text { UNCERTAINTY }\end{array}$ \\
\hline VANADIUA & $\begin{array}{l}10 / 09 / 85 \\
05 / 16 / 86\end{array}$ & $\begin{array}{l}0001 \\
0001\end{array}$ & MG/L & $<$ & $\begin{array}{l}0.01 \\
0.21\end{array}$ & $\begin{array}{l}0.01 \\
0.09\end{array}$ & - \\
\hline ZINC & $10 / 09 / 85$ & 0001 & $M G / L$ & $<$ & 0.005 & 0.005 & - \\
\hline
\end{tabular}

PARAMETER VALUE IMDICATOR (PVI): < - LESS THAN DETECTION LIMIT

SAMPLE ID COOES:

0001 - FILTERED SAMPLE (.45 MICRONS) 
GROUMDHATER OUALITY DATA BY LOCATION

SITE: AMBOI AMBROSIA LAKE

LOCATIOH: 0784

NORTH COORDIHATE: 54129.0 FT

EAST COORDIHATE: $\quad 58763.8$ FT

$06 / 10 / 80$ TO $12 / 04 / 92$

REPORT DATE: 08/04/94

FORMATIOH OF COMPLETIOH: TRES HERMANOS - C2 SANDSTOHE (TT)

HYDRAULIC FLON RELATIONSHIP: DOWN GRADIENT (D)

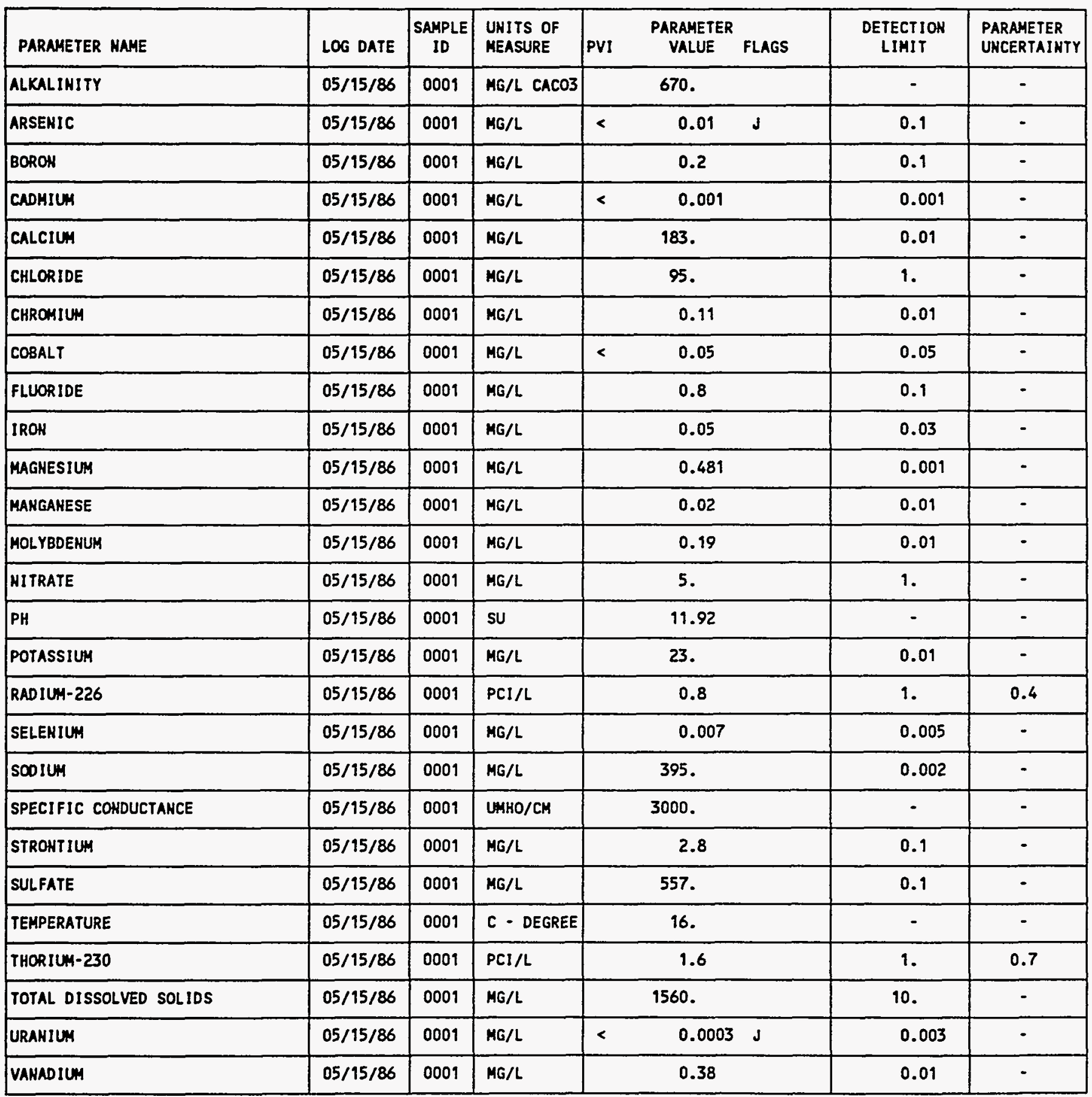

PARAMETER VALUE IMDICATOR (PVI): < - LESS THAN DETECTION LIMIT

SAMPLE ID COOES:

0001 - FILTERED SAMPLE (.45 MICRONS)

OTHER PARAMETER VALUE fLAGS:

J - ESTIMATED VALUE 
GROUNDWATER QUALITY DATA BY LOCATION

SITE: AMBO1 AMBROSIA LAKE

LOCATION: 0785

NORTH COORDINATE: 52770.8 FT

EAST COORDINATE: $\quad 58816.5$ FT

O6/10/80 TO $12 / 04 / 92$

REPORT DATE: $08 / 04 / 94$

FORMATION OF COAPLETION: TRES HERMANOS - C2 SANDSTONE (TT)

HYDRAULIC FLON RELATIOHSHIP: DOWN GRADIENT (D)

\begin{tabular}{|c|c|c|c|c|c|c|c|c|}
\hline PARAMETER NAME & LOG DATE & $\begin{array}{c}\text { SAMPLE } \\
\text { ID }\end{array}$ & $\begin{array}{l}\text { UNITS OF } \\
\text { MEASURE }\end{array}$ & PVI & $\begin{array}{c}\text { PARAMETER } \\
\text { VALUE }\end{array}$ & FLAGS & $\begin{array}{l}\text { DETECTION } \\
\text { LIMIT }\end{array}$ & $\begin{array}{l}\text { PARAMETER } \\
\text { UNCERTAINTY }\end{array}$ \\
\hline ALKALINITY & $\begin{array}{l}10 / 15 / 85 \\
05 / 15 / 86 \\
01 / 09 / 87 \\
04 / 06 / 88 \\
07 / 15 / 88 \\
01 / 08 / 89 \\
09 / 19 / 89 \\
03 / 31 / 90 \\
01 / 15 / 91 \\
11 / 25 / 92\end{array}$ & $\begin{array}{l}0001 \\
0001 \\
0001 \\
0001 \\
0001 \\
0001 \\
0001 \\
0001 \\
0001 \\
0001\end{array}$ & MG/L CACO3 & & $\begin{array}{l}350 . \\
841^{\circ} \\
740 . \\
744 . \\
610 . \\
578 . \\
598 . \\
465 . \\
479 . \\
219\end{array}$ & & $\begin{array}{l}- \\
- \\
- \\
- \\
- \\
- \\
- \\
- \\
-\end{array}$ & $\begin{array}{l}- \\
- \\
- \\
- \\
- \\
- \\
- \\
-\end{array}$ \\
\hline ALLMINUH & $\begin{array}{l}10 / 15 / 85 \\
04 / 06 / 88 \\
07 / 15 / 88 \\
01 / 08 / 89 \\
09 / 19 / 89 \\
03 / 31 / 90 \\
01 / 15 / 91\end{array}$ & $\begin{array}{l}0001 \\
0001 \\
0001 \\
0001 \\
0001 \\
0001 \\
0001\end{array}$ & $M G / L$ & $\begin{array}{l}< \\
< \\
<\end{array}$ & $\begin{array}{l}0.3 \\
0.44 \\
0.1 \\
0.05 \\
0.1 \\
0.1 \\
0.05\end{array}$ & J & $\begin{array}{l}0.1 \\
0.1 \\
0.1 \\
0.1 \\
0.1 \\
0.1 \\
0.05\end{array}$ & $\begin{array}{l}- \\
- \\
- \\
- \\
-\end{array}$ \\
\hline AMMONIUM & $\begin{array}{l}10 / 15 / 85 \\
04 / 06 / 88 \\
07 / 15 / 88 \\
01 / 08 / 89 \\
09 / 19 / 89 \\
03 / 31 / 90 \\
01 / 15 / 91 \\
11 / 25 / 92\end{array}$ & $\begin{array}{l}0001 \\
0001 \\
0001 \\
0001 \\
0001 \\
0001 \\
0001 \\
0001\end{array}$ & $M G / L$ & $\begin{array}{l}< \\
< \\
< \\
<\end{array}$ & $\begin{array}{l}0.2 \\
0.1 \\
0.1 \\
0.18 \\
0.1 \\
0.1 \\
0.28 \\
0.3\end{array}$ & & $\begin{array}{l}0.1 \\
0.1 \\
0.1 \\
0.1 \\
0.1 \\
0.1 \\
0.01 \\
0.1\end{array}$ & $\begin{array}{l}- \\
- \\
- \\
- \\
- \\
-\end{array}$ \\
\hline ANT IHONY & $\begin{array}{l}10 / 15 / 85 \\
04 / 06 / 88 \\
07 / 15 / 88 \\
01 / 08 / 89 \\
09 / 19 / 89 \\
03 / 31 / 90 \\
01 / 15 / 91 \\
11 / 25 / 92\end{array}$ & $\begin{array}{l}0001 \\
0001 \\
0001 \\
0001 \\
0001 \\
0001 \\
0001 \\
0001\end{array}$ & MG/L & $<$ & $\begin{array}{l}0.003 \\
0.032 \\
0.043 \\
0.003 \\
0.014 \\
0.075 \\
0.003 \\
0.003\end{array}$ & & $\begin{array}{l}0.003 \\
0.003 \\
0.003 \\
0.003 \\
0.003 \\
0.003 \\
0.003 \\
0.003\end{array}$ & $\begin{array}{l}- \\
- \\
- \\
- \\
- \\
-\end{array}$ \\
\hline ARSENIC & $\begin{array}{l}10 / 15 / 85 \\
05 / 15 / 86 \\
01 / 09 / 87 \\
04 / 06 / 88 \\
07 / 15 / 88 \\
01 / 08 / 89 \\
09 / 19 / 89 \\
03 / 31 / 90 \\
01 / 15 / 91\end{array}$ & $\begin{array}{l}0001 \\
0001 \\
0001 \\
0001 \\
0001 \\
0001 \\
0001 \\
0001 \\
0001\end{array}$ & $M G / L$ & $\begin{array}{l}< \\
< \\
< \\
<\end{array}$ & $\begin{array}{l}0.01 \\
0.01 \\
0.004 \\
0.026 \\
0.02 \\
0.001 \\
0.02 \\
0.12 \\
0.03\end{array}$ & $\begin{array}{l}J \\
J \\
I\end{array}$ & $\begin{array}{l}0.01 \\
0.01 \\
0.01 \\
0.01 \\
0.01 \\
0.01 \\
0.01 \\
0.01 \\
0.03\end{array}$ & $\begin{array}{l}- \\
- \\
- \\
- \\
- \\
- \\
-\end{array}$ \\
\hline BARIUA & $\begin{array}{l}10 / 15 / 85 \\
04 / 06 / 88 \\
07 / 15 / 88 \\
01 / 08 / 89 \\
09 / 19 / 89 \\
03 / 31 / 90 \\
01 / 15 / 91\end{array}$ & $\begin{array}{l}0001 \\
0001 \\
0001 \\
0001 \\
0001 \\
0001 \\
0001\end{array}$ & $M G / L$ & $\begin{array}{l}< \\
< \\
< \\
<\end{array}$ & $\begin{array}{l}0.1 \\
0.03 \\
0.1 \\
0.02 \\
0.1 \\
0.1 \\
0.01\end{array}$ & $\begin{array}{l}\mathbf{J} \\
\mathbf{J}\end{array}$ & $\begin{array}{l}0.1 \\
0.1 \\
0.1 \\
0.1 \\
0.1 \\
0.1 \\
0.01\end{array}$ & $\begin{array}{l}- \\
- \\
- \\
- \\
-\end{array}$ \\
\hline BERYLLIUM & $01 / 08 / 89$ & 0001 & $M G / L$ & $<$ & 0.005 & & 0.005 & - \\
\hline
\end{tabular}

PARAMETER VALUE IMDICATOR (PVI): < - LESS THAN DETECTION LIMIT

SAMPLE ID COOES:

0001 - FILTERED SAMPLE (.45 MICRONS)

OTHER PARAMETER VALUE FLAGS:

I - INCREASED DETECTION LIMIT DUE TO REQUIRED DILUTION

$\mathrm{J}$ - ESTIMATED VALUE 
GROUNDWATER OUALITY DATA BY LOCATION

SITE: AMBO1 AMBROSIA LAKE

LOCATIOH: 0785

NORTH COORDINATE: 52770.8 FT

EAST COORDINATE: $\quad 58816.5$ FT

06/10/80 TO $12 / 04 / 92$

REPORT DATE: $08 / 04 / 94$

FORMATIOH OF COMPLETIOH: TRES HERMANOS - C2 SAMDSTONE (TT)

HYORAULIC FLOW RELATIONSHIP: DOWN GRADIENT (D)

\begin{tabular}{|c|c|c|c|c|c|c|c|}
\hline PARAMETER MAME & LOG DATE & $\begin{array}{c}\text { SAMPLE } \\
10\end{array}$ & $\begin{array}{l}\text { UNITS OF } \\
\text { MEASURE }\end{array}$ & PVI & $\begin{array}{l}\text { PARAMETER } \\
\text { VALUE FLAGS }\end{array}$ & $\begin{array}{l}\text { DETECTIOH } \\
\text { LIMIT }\end{array}$ & $\begin{array}{l}\text { PARAMETER } \\
\text { UNCERTAINTY }\end{array}$ \\
\hline BERYLLIUM & $\begin{array}{l}09 / 19 / 89 \\
03 / 31 / 90 \\
01 / 15 / 91\end{array}$ & $\begin{array}{l}0001 \\
0001 \\
0001\end{array}$ & $M G / L$ & $\begin{array}{l}< \\
< \\
<\end{array}$ & $\begin{array}{l}0.01 \\
0.01 \\
0.005\end{array}$ & $\begin{array}{l}0.01 \\
0.01 \\
0.005\end{array}$ & - \\
\hline BOROH & $\begin{array}{l}10 / 15 / 85 \\
05 / 15 / 86 \\
01 / 09 / 87 \\
04 / 06 / 88 \\
07 / 15 / 88 \\
01 / 08 / 89 \\
09 / 19 / 89 \\
03 / 31 / 90 \\
01 / 15 / 91\end{array}$ & $\begin{array}{l}0001 \\
0001 \\
0001 \\
0001 \\
0001 \\
0001 \\
0001 \\
0001 \\
0001\end{array}$ & MG/L & & $\begin{array}{l}0.3 \\
0.3 \\
0.30 \\
0.63 \\
0.4 \\
0.42 \\
0.4 \\
0.3 \\
0.28\end{array}$ & $\begin{array}{l}0.1 \\
0.1 \\
0.1 \\
0.1 \\
0.1 \\
0.1 \\
0.1 \\
0.1 \\
0.05\end{array}$ & $\begin{array}{l}- \\
- \\
- \\
- \\
- \\
- \\
-\end{array}$ \\
\hline BROMIDE & $\begin{array}{l}04 / 06 / 88 \\
07 / 15 / 88 \\
01 / 08 / 89 \\
09 / 19 / 89 \\
03 / 31 / 90 \\
01 / 15 / 91\end{array}$ & $\begin{array}{l}0001 \\
0001 \\
0001 \\
0001 \\
0001 \\
0001\end{array}$ & MG/L & & $\begin{array}{l}0.3 \\
1.0 \\
3.25 \\
0.46 \\
1.1 \\
2.3\end{array}$ & $\begin{array}{l}0.1 \\
0.1 \\
0.01 \\
0.1 \\
0.1 \\
0.1\end{array}$ & : \\
\hline CADMIUH & $\begin{array}{l}10 / 15 / 85 \\
05 / 15 / 86 \\
04 / 06 / 88 \\
07 / 15 / 88 \\
01 / 08 / 89 \\
09 / 19 / 89 \\
03 / 31 / 90 \\
01 / 15 / 91 \\
11 / 25 / 92\end{array}$ & $\begin{array}{l}0001 \\
0001 \\
0001 \\
0001 \\
0001 \\
0001 \\
0001 \\
0001 \\
0001\end{array}$ & $M G / L$ & $\begin{array}{l}< \\
< \\
<\end{array}$ & $\begin{array}{l}0.001 \\
0.001 \\
0.009 \\
0.002 \\
0.0011 \\
0.024 \\
0.001 \\
0.001 \\
0.0002 \quad H\end{array}$ & $\begin{array}{l}0.001 \\
0.001 \\
0.001 \\
0.001 \\
0.001 \\
0.001 \\
0.001 \\
0.001 \\
0.0001\end{array}$ & $\begin{array}{l}- \\
. \\
- \\
- \\
- \\
- \\
-\end{array}$ \\
\hline CALCIUH & $\begin{array}{l}10 / 15 / 85 \\
05 / 15 / 86 \\
01 / 09 / 87 \\
04 / 06 / 88 \\
07 / 15 / 88 \\
01 / 08 / 89 \\
09 / 19 / 89 \\
03 / 31 / 90 \\
01 / 15 / 91 \\
11 / 25 / 92\end{array}$ & $\begin{array}{l}0001 \\
0001 \\
0001 \\
0001 \\
0001 \\
0001 \\
0001 \\
0001 \\
0001 \\
0001\end{array}$ & MG/L & & $\begin{array}{l}198 . \\
531 . \\
497 . \\
561 . \\
391 . \\
423 . \\
375 . \\
370 . \\
255 . \\
311 .\end{array}$ & $\begin{array}{l}0.01 \\
0.01 \\
0.01 \\
0.01 \\
0.01 \\
0.01 \\
0.01 \\
0.01 \\
0.5 \\
0.5\end{array}$ & $\begin{array}{l}- \\
- \\
- \\
- \\
- \\
- \\
- \\
-\end{array}$ \\
\hline CHLORIDE & $\begin{array}{l}10 / 15 / 85 \\
05 / 15 / 86 \\
01 / 09 / 87 \\
04 / 06 / 88 \\
07 / 15 / 88 \\
01 / 08 / 89 \\
09 / 19 / 89 \\
03 / 31 / 90 \\
01 / 15 / 91 \\
11 / 25 / 92\end{array}$ & $\begin{array}{l}0001 \\
0001 \\
0001 \\
0001 \\
0001 \\
0001 \\
0001 \\
0001 \\
0001 \\
0001\end{array}$ & $M G / L$ & & $\begin{array}{l}81 . \\
190 . \\
220 . \\
260 . \\
230 . \\
261 . \\
230 . \\
259 . \\
261 . \\
254 .\end{array}$ & $\begin{array}{l}1 . \\
1 . \\
1 . \\
1 . \\
1 . \\
1 . \\
1 . \\
1 . \\
0.5 \\
5 .\end{array}$ & $\begin{array}{l}- \\
- \\
- \\
- \\
- \\
- \\
-\end{array}$ \\
\hline CHROMIUH & $\begin{array}{l}10 / 15 / 85 \\
05 / 15 / 86 \\
01 / 09 / 87 \\
04 / 06 / 88\end{array}$ & $\begin{array}{l}0001 \\
0001 \\
0001 \\
0001\end{array}$ & $M G / L$ & & $\begin{array}{l}0.02 \\
0.07 \\
0.02 \\
0.24\end{array}$ & $\begin{array}{l}0.01 \\
0.01 \\
0.01 \\
0.01\end{array}$ & i \\
\hline
\end{tabular}

PARAMETER VALUE IMDICATOR (PVI): < - LESS THAN DETECTIOH LIMIT SAMPLE ID COOES:

0001 - FILTERED SAMPLE (.45 MICRONS)

OTHER PARAMETER VALUE FLAGS:

H - HOLD TIME EXPIRED, VALUE SUSPECT 
GROUNDWATER QUALITY DATA BY LOCATION

SITE: AMBO1 AMBROSIA LAKE

LOCATION: 0785

HORTH COORDIMATE: $\quad 52770.8$ FT

EAST COORDINATE: $\quad 58816.5$ FT

Q6/10/80 TO 12/04/92

REPORT DATE: $08 / 04 / 94$

FORMATION OF COMPLETIOH: TRES HERMANOS - C2 SANDSTONE (TT) HYDRAULIC FLOW RELATIOHSHIP: DOWN GRADIENT (D)

\begin{tabular}{|c|c|c|c|c|c|c|c|c|}
\hline PARAMETER NAME & LOG DATE & $\begin{array}{c}\text { SAMPLE } \\
\text { ID }\end{array}$ & $\begin{array}{l}\text { UNITS OF } \\
\text { MEASURE }\end{array}$ & PVI & $\begin{array}{l}\text { PARAMETER } \\
\text { VALUE }\end{array}$ & FLAGS & $\begin{array}{l}\text { DETECTION } \\
\text { LIMIT }\end{array}$ & $\begin{array}{l}\text { PARAMETER } \\
\text { UHCERTAIKTY }\end{array}$ \\
\hline CHROHIUH & $\begin{array}{l}07 / 15 / 88 \\
01 / 08 / 89 \\
09 / 19 / 89 \\
03 / 31 / 90 \\
01 / 15 / 91 \\
11 / 25 / 92\end{array}$ & $\begin{array}{l}0001 \\
0001 \\
0001 \\
0001 \\
0001 \\
0001\end{array}$ & $M G / L$ & $<$ & $\begin{array}{l}0.13 \\
0.01 \\
0.01 \\
0.01 \\
0.05 \\
0.02\end{array}$ & & $\begin{array}{l}0.01 \\
0.01 \\
0.01 \\
0.01 \\
0.01 \\
0.01\end{array}$ & $\begin{array}{l}- \\
- \\
- \\
-\end{array}$ \\
\hline COBALT & $\begin{array}{l}10 / 15 / 85 \\
05 / 15 / 86 \\
01 / 09 / 87 \\
04 / 06 / 88 \\
07 / 15 / 88 \\
01 / 08 / 89 \\
09 / 19 / 89 \\
03 / 31 / 90 \\
01 / 15 / 91 \\
11 / 25 / 92\end{array}$ & $\begin{array}{l}0001 \\
0001 \\
0001 \\
0001 \\
0001 \\
0001 \\
0001 \\
0001 \\
0001 \\
0001\end{array}$ & MG/L & $\begin{array}{l}< \\
< \\
< \\
< \\
<\end{array}$ & $\begin{array}{l}0.05 \\
0.1 \\
0.08 \\
0.09 \\
0.05 \\
0.01 \\
0.05 \\
0.05 \\
0.03 \\
0.03\end{array}$ & J & $\begin{array}{l}0.05 \\
0.05 \\
0.05 \\
0.05 \\
0.05 \\
0.05 \\
0.05 \\
0.05 \\
0.03 \\
0.03\end{array}$ & $\begin{array}{l}- \\
- \\
- \\
- \\
- \\
- \\
-\end{array}$ \\
\hline COPPER & $\begin{array}{l}10 / 15 / 85 \\
01 / 09 / 87 \\
04 / 06 / 88 \\
07 / 15 / 88 \\
01 / 08 / 89 \\
09 / 19 / 89 \\
03 / 31 / 90 \\
01 / 15 / 91\end{array}$ & $\begin{array}{l}0001 \\
0001 \\
0001 \\
0001 \\
0001 \\
0001 \\
0001 \\
0001\end{array}$ & $M G / L$ & $\begin{array}{l}< \\
< \\
< \\
<\end{array}$ & $\begin{array}{l}0.03 \\
0.02 \\
0.05 \\
0.03 \\
0.01 \\
0.02 \\
0.02 \\
0.01\end{array}$ & J & $\begin{array}{l}0.02 \\
0.02 \\
0.02 \\
0.02 \\
0.02 \\
0.02 \\
0.02 \\
0.01\end{array}$ & $\begin{array}{l}: \\
: \\
: \\
- \\
-\end{array}$ \\
\hline CYAHIDE & $\begin{array}{l}10 / 15 / 85 \\
01 / 08 / 89 \\
09 / 19 / 89 \\
03 / 31 / 90 \\
01 / 15 / 91\end{array}$ & $\begin{array}{l}0001 \\
0001 \\
0001 \\
0001 \\
0001\end{array}$ & $M G / L$ & $<$ & $\begin{array}{l}0.01 \\
0.350 \\
0.01 \\
0.54 \\
0.28\end{array}$ & & $\begin{array}{l}0.01 \\
0.01 \\
0.01 \\
0.01 \\
0.01\end{array}$ & $\begin{array}{l}- \\
- \\
-\end{array}$ \\
\hline FLUORIDE & $\begin{array}{l}10 / 15 / 85 \\
05 / 15 / 86 \\
01 / 09 / 87 \\
04 / 06 / 88 \\
07 / 15 / 88 \\
01 / 08 / 89 \\
09 / 19 / 89 \\
03 / 31 / 90 \\
01 / 15 / 91 \\
11 / 25 / 92\end{array}$ & $\begin{array}{l}0001 \\
0001 \\
0001 \\
0001 \\
0001 \\
0001 \\
0001 \\
0001 \\
0001 \\
0001\end{array}$ & MG/L & & $\begin{array}{l}1.4 \\
0.6 \\
0.47 \\
0.43 \\
0.2 \\
0.5 \\
0.4 \\
0.4 \\
0.4 \\
0.4\end{array}$ & & $\begin{array}{l}0.1 \\
0.1 \\
0.1 \\
0.1 \\
0.1 \\
0.1 \\
0.1 \\
0.1 \\
0.1 \\
0.1\end{array}$ & $\begin{array}{l}- \\
- \\
- \\
- \\
- \\
- \\
-\end{array}$ \\
\hline GROSS ALPHA & $\begin{array}{l}01 / 09 / 87 \\
04 / 06 / 88 \\
07 / 15 / 88 \\
01 / 08 / 89 \\
09 / 19 / 89 \\
03 / 31 / 90 \\
01 / 15 / 91 \\
11 / 25 / 92\end{array}$ & $\begin{array}{l}0001 \\
0001 \\
0001 \\
0001 \\
0001 \\
0001 \\
0001 \\
0001\end{array}$ & $\mathrm{PCI} / \mathrm{L}$ & & $\begin{array}{c}1900 . \\
1600 . \\
1800 . \\
520 . \\
1200 . \\
1000 . \\
862 . \\
177 .\end{array}$ & H & $\begin{array}{l}0.2 \\
0.2 \\
0.2 \\
1 . \\
1 . \\
1 . \\
1 . \\
1.0\end{array}$ & $\begin{array}{c}100 . \\
10 . \\
100 . \\
81.2 \\
100 . \\
100 . \\
99.2 \\
60.2\end{array}$ \\
\hline GROSS BETA & $\begin{array}{l}01 / 09 / 87 \\
04 / 06 / 88 \\
07 / 15 / 88\end{array}$ & $\begin{array}{l}0001 \\
0001 \\
0001\end{array}$ & $\mathrm{PCI} / \mathrm{L}$ & & $\begin{array}{l}740 . \\
480 \\
450\end{array}$ & & $\begin{array}{l}1 . \\
1 .\end{array}$ & $\begin{array}{l}60 . \\
40 . \\
30 .\end{array}$ \\
\hline
\end{tabular}

PARAMETER VALUE INDICATOR (PVI):

< - LESS THAN DETECTION LIMIT

SAMPLE ID COOES:

0001 - FILTERED SAMPLE (.45 MICRONS)

OTHER PARAMETER VALUE FLAGS:

H - hOLD TIME EXPIRED, VALUE SUSPECT

d - ESTIMATED VALUE 
GROUNDWATER QUALITY DATA BY LOCATIOH

SITE: AMBO1 AMBROSIA LAKE

LOCATION: 0785

NORTH COORDINATE: $52770.8 \mathrm{FT}$

EAST COORDIHATE: $\quad 58816.5$ FT

06/10/80 TO $12 / 04 / 92$

REPORT DATE: 08/04/94

FORMATION OF COMPLETION: TRES HERHANOS - C2 SANDSTOHE (TT)

HYORAULIC FLOH RELATIOHSHIP: DOWH GRADIENT (D)

\begin{tabular}{|c|c|c|c|c|c|c|c|c|}
\hline PARAMETER HAME & LOG DATE & $\begin{array}{c}\text { SAMPLE } \\
\text { ID }\end{array}$ & $\begin{array}{l}\text { UNITS OF } \\
\text { MEASURE }\end{array}$ & PVI & $\begin{array}{l}\text { PARAMETER } \\
\text { VALUE }\end{array}$ & FLAGS & $\begin{array}{c}\text { DETECTION } \\
\text { LIMIT }\end{array}$ & $\begin{array}{l}\text { PARAMETER } \\
\text { UNCERTAINTY }\end{array}$ \\
\hline GROSS BETA & $\begin{array}{l}01 / 08 / 89 \\
09 / 19 / 89 \\
03 / 31 / 90 \\
01 / 15 / 91 \\
11 / 25 / 92\end{array}$ & $\begin{array}{l}0001 \\
0001 \\
0001 \\
0001 \\
0001\end{array}$ & $\mathrm{PCl} / \mathrm{L}$ & & $\begin{array}{l}484 . \\
510 . \\
520 . \\
394 . \\
160 .\end{array}$ & & $\begin{array}{r}0.5 \\
0.5 \\
0.5 \\
0.5 \\
69.9\end{array}$ & $\begin{array}{l}52.6 \\
30 . \\
40 . \\
41.1 \\
48.1\end{array}$ \\
\hline IRON & $\begin{array}{l}10 / 15 / 85 \\
05 / 15 / 86 \\
01 / 09 / 87 \\
04 / 06 / 88 \\
07 / 15 / 88 \\
01 / 08 / 89 \\
09 / 19 / 89 \\
03 / 31 / 90 \\
01 / 15 / 91 \\
11 / 25 / 92\end{array}$ & $\begin{array}{l}0001 \\
0001 \\
0001 \\
0001 \\
0001 \\
0001 \\
0001 \\
0001 \\
0001 \\
0001\end{array}$ & MG/L & $<$ & $\begin{array}{c}0.03 \\
28.8 \\
0.24 \\
0.54 \\
0.27 \\
4.50 \\
1.89 \\
7.10 \\
0.21 \\
13.9\end{array}$ & & $\begin{array}{l}0.03 \\
0.03 \\
0.03 \\
0.03 \\
0.03 \\
0.03 \\
0.03 \\
0.03 \\
0.03 \\
0.03\end{array}$ & $\begin{array}{l}- \\
- \\
- \\
- \\
- \\
- \\
- \\
-\end{array}$ \\
\hline LEAD & $\begin{array}{l}10 / 15 / 85 \\
04 / 06 / 88 \\
07 / 15 / 88 \\
01 / 08 / 89 \\
09 / 19 / 89 \\
03 / 31 / 90 \\
01 / 15 / 91\end{array}$ & $\begin{array}{l}0001 \\
0001 \\
0001 \\
0001 \\
0001 \\
0001 \\
0001\end{array}$ & HG/L & $\begin{array}{l}< \\
< \\
< \\
<\end{array}$ & $\begin{array}{l}0.01 \\
0.01 \\
0.01 \\
0.001 \\
0.02 \\
0.01 \\
0.03\end{array}$ & $\begin{array}{l}J \\
I\end{array}$ & $\begin{array}{l}0.01 \\
0.01 \\
0.01 \\
0.01 \\
0.01 \\
0.01 \\
0.03\end{array}$ & $\begin{array}{l}- \\
- \\
- \\
- \\
- \\
-\end{array}$ \\
\hline LEAD-210 & $\begin{array}{l}10 / 15 / 85 \\
04 / 06 / 88 \\
07 / 15 / 88 \\
01 / 08 / 89 \\
09 / 19 / 89\end{array}$ & $\begin{array}{l}0001 \\
0001 \\
0001 \\
0001 \\
0001\end{array}$ & PCI/L & & $\begin{array}{l}5.5 \\
2.0 \\
0.2 \\
5.72 \\
0.3\end{array}$ & & $\begin{array}{l}1.5 \\
1.5 \\
1.5 \\
1.5 \\
1.5\end{array}$ & $\begin{array}{l}2.9 \\
1.3 \\
1.1 \\
9.07 \\
0.8\end{array}$ \\
\hline MAGNESIUM & $\begin{array}{l}10 / 15 / 85 \\
05 / 15 / 86 \\
01 / 09 / 87 \\
04 / 06 / 88 \\
07 / 15 / 88 \\
01 / 08 / 89 \\
09 / 19 / 89 \\
03 / 31 / 90 \\
01 / 15 / 91 \\
11 / 25 / 92\end{array}$ & $\begin{array}{l}0001 \\
0001 \\
0001 \\
0001 \\
0001 \\
0001 \\
0001 \\
0001 \\
0001 \\
0001\end{array}$ & $M G / L$ & & $\begin{array}{l}80.6 \\
241 . \\
290 . \\
274 . \\
251 . \\
300 . \\
269 . \\
269 . \\
184 . \\
255 .\end{array}$ & & $\begin{array}{l}0.001 \\
0.001 \\
0.001 \\
0.001 \\
0.001 \\
0.001 \\
0.001 \\
0.001 \\
0.5 \\
0.5\end{array}$ & $\begin{array}{l}- \\
- \\
- \\
- \\
- \\
- \\
- \\
-\end{array}$ \\
\hline MANGANESE & $\begin{array}{l}10 / 15 / 85 \\
05 / 15 / 86 \\
01 / 09 / 87 \\
04 / 06 / 88 \\
07 / 15 / 88 \\
01 / 08 / 89 \\
09 / 19 / 89 \\
03 / 31 / 90 \\
01 / 15 / 91\end{array}$ & $\begin{array}{l}0001 \\
0001 \\
0001 \\
0001 \\
0001 \\
0001 \\
0001 \\
0001 \\
0001\end{array}$ & $M G / L$ & & $\begin{array}{l}0.14 \\
1.82 \\
0.76 \\
1.11 \\
0.32 \\
0.42 \\
0.41 \\
4.0 \\
0.27\end{array}$ & & $\begin{array}{l}0.01 \\
0.01 \\
0.01 \\
0.01 \\
0.01 \\
0.01 \\
0.01 \\
0.01 \\
0.01\end{array}$ & $\begin{array}{l}- \\
- \\
- \\
- \\
- \\
- \\
-\end{array}$ \\
\hline MERCURY & $\begin{array}{l}10 / 15 / 85 \\
04 / 06 / 88 \\
07 / 15 / 88 \\
01 / 08 / 89\end{array}$ & $\begin{array}{l}0001 \\
0001 \\
0001 \\
0001\end{array}$ & $M G / L$ & $\begin{array}{l}< \\
<\end{array}$ & $\begin{array}{l}0.0002 \\
0.0006 \\
0.0003 \\
0.0001\end{array}$ & $\mathbf{J}$ & $\begin{array}{l}0.0002 \\
0.0002 \\
0.0002 \\
0.0002\end{array}$ & $\begin{array}{l}- \\
- \\
-\end{array}$ \\
\hline
\end{tabular}

PARAMETER VALUE IHDICATOR (PVI): < - LESS THAN DETECTIOH LIMIT

OTHER PARAMETER VALUE FLAGS:

1 - INCREASED DETECTION LIMIT DUE TO REQUIREO DILUTION

J - ESTIHATED VALUE 
GROUNDWATER QUALITY DATA BY LOCATION

SITE: AMBO1 AMBROSIA LAKE

LOCATION: 0785

NORTH COORDINATE: $\quad 52770.8$ FT

EAST COORDIHATE: $\quad 58816.5$ FT

$06 / 10 / 80$ TO $12 / 04 / 92$

REPORT DATE: 08/04/94

FORMATION OF COMPLETION: TRES HERMANOS - C2 SANDSTONE (TT)

HYDRAULIC FLON RELATIONSHIP: DOWN GRADIENT (D)

\begin{tabular}{|c|c|c|c|c|c|c|c|c|}
\hline PARAMETER NAME & LOG DATE & $\begin{array}{l}\text { SAMPLE } \\
\text { ID }\end{array}$ & $\begin{array}{l}\text { UNITS OF } \\
\text { MEASURE }\end{array}$ & PVI & $\begin{array}{l}\text { PARAMETER } \\
\text { VALUE }\end{array}$ & FLAGS & $\begin{array}{l}\text { DETECTION } \\
\text { LIMIT }\end{array}$ & $\begin{array}{l}\text { PARAMETER } \\
\text { UHCERTAINTY }\end{array}$ \\
\hline MERCURY & $\begin{array}{l}09 / 19 / 89 \\
03 / 31 / 90 \\
01 / 15 / 91\end{array}$ & $\begin{array}{l}0001 \\
0001 \\
0001\end{array}$ & MG/L & $\begin{array}{l}< \\
< \\
<\end{array}$ & $\begin{array}{l}0.0002 \\
0.0002 \\
0.0002\end{array}$ & & $\begin{array}{l}0.0002 \\
0.0002 \\
0.0002\end{array}$ & : \\
\hline MOLYBDENUM & $\begin{array}{l}10 / 15 / 85 \\
05 / 15 / 86 \\
01 / 09 / 87 \\
04 / 06 / 88 \\
07 / 15 / 88 \\
01 / 08 / 89 \\
09 / 19 / 89 \\
03 / 31 / 90 \\
01 / 15 / 91 \\
11 / 25 / 92\end{array}$ & $\begin{array}{l}0001 \\
0001 \\
0001 \\
0001 \\
0001 \\
0001 \\
0001 \\
0001 \\
0001 \\
0001\end{array}$ & $M G / L$ & & $\begin{array}{l}0.35 \\
0.2 \\
0.2 \\
0.14 \\
0.27 \\
0.019 \\
0.07 \\
0.04 \\
0.05 \\
0.04\end{array}$ & & $\begin{array}{l}0.01 \\
0.01 \\
0.01 \\
0.01 \\
0.01 \\
0.01 \\
0.01 \\
0.01 \\
0.01 \\
0.01\end{array}$ & $\begin{array}{l}- \\
- \\
- \\
- \\
- \\
- \\
-\end{array}$ \\
\hline NET GROSS ALPHA • & $\begin{array}{l}01 / 09 / 87 \\
04 / 06 / 88 \\
07 / 15 / 88 \\
01 / 08 / 89 \\
09 / 19 / 89 \\
03 / 31 / 90 \\
01 / 15 / 91 \\
11 / 25 / 92\end{array}$ & $\begin{array}{l}0001 \\
0001 \\
0001 \\
0001 \\
0001 \\
0001 \\
0001 \\
0001\end{array}$ & $\mathrm{PCI} / \mathrm{L}$ & & $\begin{array}{r}-363.80 \\
317.18 \\
654.38 \\
-721.66 \\
191.58 \\
324.29 \\
-359.08 \\
-109.06\end{array}$ & & $\begin{array}{l}- \\
: \\
: \\
- \\
-\end{array}$ & $\begin{array}{l}- \\
- \\
- \\
- \\
- \\
-\end{array}$ \\
\hline HICKEL & $\begin{array}{l}10 / 15 / 85 \\
04 / 06 / 88 \\
07 / 15 / 88 \\
01 / 08 / 89 \\
09 / 19 / 89 \\
03 / 31 / 90 \\
01 / 15 / 91 \\
11 / 25 / 92\end{array}$ & $\begin{array}{l}0001 \\
0001 \\
0001 \\
0001 \\
0001 \\
0001 \\
0001 \\
0001\end{array}$ & $M G / L$ & $\begin{array}{l}< \\
< \\
<\end{array}$ & $\begin{array}{l}0.04 \\
0.15 \\
0.07 \\
0.02 \\
0.04 \\
0.04 \\
0.04 \\
0.13\end{array}$ & J & $\begin{array}{l}0.04 \\
0.04 \\
0.04 \\
0.04 \\
0.04 \\
0.04 \\
0.04 \\
0.04\end{array}$ & $\begin{array}{l}- \\
: \\
- \\
: \\
-\end{array}$ \\
\hline NITRATE & $\begin{array}{l}10 / 15 / 85 \\
05 / 15 / 86 \\
01 / 09 / 87 \\
04 / 06 / 88 \\
07 / 15 / 88 \\
01 / 08 / 89 \\
09 / 19 / 89 \\
03 / 31 / 90 \\
01 / 15 / 91 \\
11 / 25 / 92\end{array}$ & $\begin{array}{l}0001 \\
0001 \\
0001 \\
0001 \\
0001 \\
0001 \\
0001 \\
0001 \\
0001 \\
0001\end{array}$ & $M G / L$ & $\begin{array}{l}< \\
< \\
<\end{array}$ & $\begin{array}{l}20 . \\
1 . \\
5.3 \\
1.8 \\
4.6 \\
0.1 \\
1.4 \\
1.6 \\
3.1 \\
1.0\end{array}$ & $\begin{array}{l}\mathrm{j} \\
\mathrm{j}\end{array}$ & $\begin{array}{l}1 . \\
1 . \\
1 . \\
1 . \\
1 . \\
1 . \\
1 . \\
1 . \\
0.1 \\
1.0\end{array}$ & $\begin{array}{l}- \\
: \\
: \\
: \\
- \\
- \\
-\end{array}$ \\
\hline HITRITE & $10 / 15 / 85$ & 0001 & $M G / L$ & $<$ & 0.1 & & 0.1 & - \\
\hline MITRITE AKD NITRATE & $\begin{array}{l}04 / 06 / 88 \\
03 / 31 / 90 \\
01 / 15 / 91\end{array}$ & $\begin{array}{l}0001 \\
0001 \\
0001\end{array}$ & MG/L & & $\begin{array}{l}1.8 \\
0.4 \\
0.38\end{array}$ & J & $\begin{array}{l}1 . \\
1.05\end{array}$ & : \\
\hline PH & $\begin{array}{l}10 / 15 / 85 \\
05 / 15 / 86 \\
01 / 09 / 87 \\
04 / 06 / 88 \\
07 / 15 / 88\end{array}$ & $\begin{array}{l}0001 \\
0001 \\
0001 \\
0001 \\
0001\end{array}$ & SU & & $\begin{array}{l}7.87 \\
7.35 \\
7.24 \\
7.18 \\
7.08\end{array}$ & & $\begin{array}{l}- \\
- \\
-\end{array}$ & $\begin{array}{l}: \\
- \\
-\end{array}$ \\
\hline
\end{tabular}

- het gross alPha (GROSS ALPha - URANIUH) WITH 1 Hg URANIUM = 686 PCI

PARAMETER VALUE INDICATOR (PVI): < - LESS THAN DETECTION LIMIT SAMPLE ID CODES:

0001 - FILTERED SAMPLE (.45 MICRONS)

OTHER PARAMETER VALUE FLAGS:

$J$ - ESTIMATED VALUE 
GROUHDWATER QUALITY DATA BY LOCATION

SITE: AMBOI AMBROSIA LAKE

LOCATION: 0785

HORTH COORDINATE: 52770.8 FT

EAST COORDINATE: 58816.5 FT

06/10/80 TO $12 / 04 / 92$

REPORT DATE: 08/04/94

FORMATION OF COMPLETIOH: TRES HERMAMOS - C2 SANDSTOHE (TT) HYDRAULIC FLOW RELATIONSHIP: DOWN GRADIENT (D)

\begin{tabular}{|c|c|c|c|c|c|c|c|}
\hline PARAMETER HAME & LOG DATE & $\begin{array}{l}\text { SAMPLE } \\
\text { ID }\end{array}$ & $\begin{array}{l}\text { UNITS OF } \\
\text { MEASURE }\end{array}$ & PVI & $\begin{array}{l}\text { PARAMETER } \\
\text { VALUE FLAGS }\end{array}$ & $\begin{array}{l}\text { DETECTION } \\
\text { LIMIT }\end{array}$ & $\begin{array}{l}\text { PARAMETER } \\
\text { UHCERTAINTY }\end{array}$ \\
\hline PH & $\begin{array}{l}01 / 08 / 89 \\
09 / 19 / 89 \\
03 / 31 / 90 \\
01 / 15 / 91 \\
11 / 25 / 92\end{array}$ & $\begin{array}{l}0001 \\
0001 \\
0001 \\
0001 \\
0001\end{array}$ & SU & & $\begin{array}{l}7.08 \\
6.94 \\
6.80 \\
7.06 \\
7.16\end{array}$ & $\begin{array}{l}- \\
- \\
- \\
-\end{array}$ & $\begin{array}{l}- \\
- \\
- \\
-\end{array}$ \\
\hline PHOSPHATE & $\begin{array}{l}10 / 15 / 85 \\
04 / 06 / 88 \\
07 / 15 / 88 \\
01 / 08 / 89 \\
09 / 19 / 89 \\
03 / 31 / 90 \\
01 / 15 / 91 \\
11 / 25 / 92\end{array}$ & $\begin{array}{l}0001 \\
0001 \\
0001 \\
0001 \\
0001 \\
0001 \\
0001 \\
0001\end{array}$ & $M G / L$ & $\begin{array}{l}< \\
< \\
<\end{array}$ & $\begin{array}{l}0.4 \\
0.1 \\
0.1 \\
0.1 \\
0.1 \\
0.1 \\
0.06 \\
0.1\end{array}$ & $\begin{array}{l}0.1 \\
0.1 \\
0.1 \\
0.1 \\
0.1 \\
0.1 \\
0.05 \\
0.1\end{array}$ & $\begin{array}{l}- \\
- \\
- \\
- \\
- \\
-\end{array}$ \\
\hline POLONIUA-210 & $\begin{array}{l}04 / 06 / 88 \\
07 / 15 / 88 \\
01 / 08 / 89 \\
09 / 19 / 89\end{array}$ & $\begin{array}{l}0001 \\
0001 \\
0001 \\
0001\end{array}$ & $\mathrm{PCI} / \mathrm{L}$ & & $\begin{array}{l}1.2 \\
1.1 \\
0.588 \\
1.3\end{array}$ & $\begin{array}{l}1 . \\
1 . \\
1 .\end{array}$ & $\begin{array}{l}0.6 \\
0.6 \\
1.58 \\
0.5\end{array}$ \\
\hline POTASSIUM & $\begin{array}{l}10 / 15 / 85 \\
05 / 15 / 86 \\
01 / 09 / 87 \\
04 / 06 / 88 \\
07 / 15 / 88 \\
01 / 08 / 89 \\
09 / 19 / 89 \\
03 / 31 / 90 \\
01 / 15 / 91 \\
11 / 25 / 92\end{array}$ & $\begin{array}{l}0001 \\
0001 \\
0001 \\
0001 \\
0001 \\
0001 \\
0001 \\
0001 \\
0001 \\
0001\end{array}$ & $M G / L$ & & $\begin{array}{c}7.73 \\
11.7 \\
8.90 \\
8.25 \\
8.3 \\
8.2 \\
8.1 \\
8.6 \\
6.1 \\
7.57\end{array}$ & $\begin{array}{l}0.01 \\
0.01 \\
0.01 \\
0.01 \\
0.01 \\
0.01 \\
0.01 \\
0.01 \\
0.01 \\
0.01\end{array}$ & $\begin{array}{l}- \\
- \\
- \\
- \\
- \\
- \\
-\end{array}$ \\
\hline RADIUM-226 & $\begin{array}{l}10 / 15 / 85 \\
01 / 09 / 87 \\
04 / 06 / 88 \\
07 / 15 / 88 \\
01 / 08 / 89 \\
09 / 19 / 89 \\
03 / 31 / 90 \\
11 / 25 / 92\end{array}$ & $\begin{array}{l}0001 \\
0001 \\
0001 \\
0001 \\
0001 \\
0001 \\
0001 \\
0001\end{array}$ & $\mathrm{PCI} / \mathrm{L}$ & & $\begin{array}{l}0.4 \\
2.0 \\
0.2 \\
0.6 \\
0.948 \\
0.1 \\
0.4 \\
0.8\end{array}$ & $\begin{array}{l}1 . \\
1 . \\
1 . \\
1 . \\
1 . \\
1 . \\
1 . \\
0.3\end{array}$ & $\begin{array}{l}1.9 \\
0.7 \\
0.2 \\
0.3 \\
0.365 \\
0.2 \\
0.3 \\
0.4\end{array}$ \\
\hline RADIUM-226 + RADIUM-228 & $\begin{array}{l}10 / 15 / 85 \\
01 / 09 / 87 \\
04 / 06 / 88 \\
07 / 15 / 88 \\
01 / 08 / 89 \\
09 / 19 / 89 \\
03 / 31 / 90 \\
11 / 25 / 92\end{array}$ & $\begin{array}{l}0001 \\
0001 \\
0001 \\
0001 \\
0001 \\
0001 \\
0001 \\
0001\end{array}$ & $\mathrm{PCI} / \mathrm{L}$ & & $\begin{array}{r}0.80 \\
2.80 \\
1.00 \\
0.80 \\
10.51 \\
0.50 \\
0.90 \\
1.00\end{array}$ & $\begin{array}{l}- \\
- \\
- \\
- \\
- \\
-\end{array}$ & $\begin{array}{l}- \\
- \\
- \\
- \\
- \\
-\end{array}$ \\
\hline RADIUM-228 & $\begin{array}{l}10 / 15 / 85 \\
01 / 09 / 87 \\
04 / 06 / 88 \\
07 / 15 / 88 \\
01 / 08 / 89 \\
09 / 19 / 89 \\
03 / 31 / 90 \\
11 / 25 / 92\end{array}$ & $\begin{array}{l}0001 \\
0001 \\
0001 \\
0001 \\
0001 \\
0001 \\
0001 \\
0001\end{array}$ & $\mathrm{PCI} / \mathrm{L}$ & & $\begin{array}{l}0.4 \\
0.8 \\
0.8 \\
0.2 \\
9.56 \\
0.4 \\
0.5 \\
0.2\end{array}$ & $\begin{array}{l}1 . \\
1 . \\
1 . \\
1 . \\
1 . \\
1 . \\
1.0\end{array}$ & $\begin{array}{l}4.5 \\
1.1 \\
0.8 \\
0.8 \\
5.26 \\
0.9 \\
0.7 \\
1.5\end{array}$ \\
\hline
\end{tabular}

PARAMETER VALUE IMDICATOR (PVI): < - LESS THAN DETECTION LIMIT

SAMPLE ID CODES:

0001 - FILTERED SAMPLE (.45 MICRONS)

OTHER PARAMETER VALUE FLAGS:

H - HOLD TIME EXPIRED, VALUE SUSPECT 
GROUNDWATER QUALITY DATA BY LOCATION

SITE: AMBO1 AMBROSIA LAKE

LOCATION: 0785

NORTH COORDINATE: 52770.8 FT

EAST COORDINATE: $\quad 58816.5$ FT

06/10/80 TO $12 / 04 / 92$

REPORT DATE: 08/04/94

FORMATIOH OF COMPLETION: TRES HERMANOS - C2 SANDSTOHE (TT)

HYDRAULIC FLON RELATIONSHIP: DOWN GRADIENT (D)

\begin{tabular}{|c|c|c|c|c|c|c|c|}
\hline PARAMETER NAME & LOG DATE & SAMPLE & $\begin{array}{l}\text { UNITS OF } \\
\text { MEASURE }\end{array}$ & PVI & $\begin{array}{l}\text { PARAMETER } \\
\text { VALUE FLAGS }\end{array}$ & $\begin{array}{l}\text { DETECTION } \\
\text { LIMIT }\end{array}$ & $\begin{array}{l}\text { PARAMETER } \\
\text { UNCERTAINTY }\end{array}$ \\
\hline SELENIUA & $\begin{array}{l}10 / 15 / 85 \\
05 / 15 / 86 \\
01 / 09 / 87 \\
04 / 06 / 88 \\
07 / 15 / 88 \\
01 / 08 / 89 \\
09 / 19 / 89 \\
03 / 31 / 90 \\
01 / 15 / 91 \\
11 / 25 / 92\end{array}$ & $\begin{array}{l}0001 \\
0001 \\
0001 \\
0001 \\
0001 \\
0001 \\
0001 \\
0001 \\
0001 \\
0001\end{array}$ & $M G / L$ & $<$ & $\begin{array}{ll}0.003 \\
0.005 \\
0.324 \\
0.123 \\
0.263 \\
0.180 \\
0.264 \\
0.162 \\
0.278 \\
0.06\end{array}$ & $\begin{array}{l}0.005 \\
0.005 \\
0.005 \\
0.005 \\
0.005 \\
0.005 \\
0.005 \\
0.005 \\
0.005 \\
0.03\end{array}$ & $\begin{array}{l}- \\
: \\
: \\
: \\
: \\
- \\
-\end{array}$ \\
\hline SILICA - S102 & $\begin{array}{l}10 / 15 / 85 \\
04 / 06 / 88 \\
07 / 15 / 88 \\
01 / 08 / 89 \\
09 / 19 / 89 \\
03 / 31 / 90 \\
01 / 15 / 91\end{array}$ & $\begin{array}{l}0001 \\
0001 \\
0001 \\
0001 \\
0001 \\
0001 \\
0001\end{array}$ & $M G / L$ & & $\begin{array}{l}19 . \\
20.3 \\
14.5 \\
12.1 \\
11 . \\
9 . \\
7.6\end{array}$ & $\begin{array}{l}2 . \\
2 . \\
2 . \\
2 . \\
2 . \\
2 . \\
0.1\end{array}$ & $\begin{array}{l}- \\
- \\
- \\
- \\
-\end{array}$ \\
\hline SOOIUM & $\begin{array}{l}10 / 15 / 85 \\
05 / 15 / 86 \\
01 / 09 / 87 \\
04 / 06 / 88 \\
07 / 15 / 88 \\
01 / 08 / 89 \\
09 / 19 / 89 \\
03 / 31 / 90 \\
01 / 15 / 91 \\
11 / 25 / 92\end{array}$ & $\begin{array}{l}0001 \\
0001 \\
0001 \\
0001 \\
0001 \\
0001 \\
0001 \\
0001 \\
0001 \\
0001\end{array}$ & $M G / L$ & & $\begin{array}{c}564 . \\
913 . \\
1240 . \\
855 . \\
1090 . \\
1250 . \\
1253 . \\
1270 . \\
1320 . \\
1270 .\end{array}$ & $\begin{array}{l}0.002 \\
0.002 \\
0.002 \\
0.002 \\
0.002 \\
0.002 \\
0.002 \\
0.002 \\
5 . \\
20 .\end{array}$ & $\begin{array}{l}- \\
: \\
- \\
: \\
- \\
-\end{array}$ \\
\hline STRONTIUM & $\begin{array}{l}10 / 15 / 85 \\
05 / 15 / 86 \\
04 / 06 / 88 \\
07 / 15 / 88 \\
01 / 08 / 89\end{array}$ & $\begin{array}{l}0001 \\
0001 \\
0001 \\
0001 \\
0001\end{array}$ & $M G / L$ & & $\begin{array}{l}2.2 \\
4.4 \\
8.26 \\
7.9 \\
9.3\end{array}$ & $\begin{array}{l}0.1 \\
0.1 \\
0.1 \\
0.1 \\
0.1\end{array}$ & $\begin{array}{l}- \\
- \\
-\end{array}$ \\
\hline
\end{tabular}

PARAMETER VALUE INDICATOR (PVI):

< - LESS THAN DETECTION LIMIT

SAMPLE ID COOES:

0001 - FILTERED SAMPLE (.45 MICRONS)

OTHER PARAMETER VALUE fLAGS:

H - HOLD TIHE EXPIRED, VALUE SUSPECT

$\checkmark$ - ESTIMATED VALUE 
GROUMDHATER QUALITY DATA BY LOCATION

SITE: AMBO1 AMBROSIA LAKE

LOCATION: 0785

NORTH COORDINATE: 52770.8 FT

EAST COORDIHATE: $\quad 58816.5$ FT

O6/10/80 TO 12/04/92

REPORT DATE: 08/04/94

FORHATIOH OF COMPLETION: TRES HERMANOS - C2 SAKDSTONE (TT) HYDRAULIC FLON RELATIOHSHIP: DONN GRADIENT (D)

\begin{tabular}{|c|c|c|c|c|c|c|c|c|}
\hline PARAMETER MAME & LOG DATE & $\begin{array}{c}\text { SNMPLE } \\
10\end{array}$ & $\begin{array}{l}\text { UNITS OF } \\
\text { MEASURE }\end{array}$ & PVI & $\begin{array}{l}\text { PARAMETER } \\
\text { VALUE }\end{array}$ & FLAGS & $\begin{array}{l}\text { DETECTION } \\
\text { LIMIT }\end{array}$ & $\begin{array}{l}\text { PARAMETER } \\
\text { UNCERTAINTY }\end{array}$ \\
\hline STRONTIUM & $\begin{array}{l}09 / 19 / 89 \\
03 / 31 / 90 \\
01 / 15 / 91\end{array}$ & $\begin{array}{l}0001 \\
0001 \\
0001\end{array}$ & MG/L & & $\begin{array}{l}8.5 \\
7.63 \\
4.60\end{array}$ & & $\begin{array}{l}0.1 \\
0.1 \\
0.01\end{array}$ & $\begin{array}{l}- \\
-\end{array}$ \\
\hline SULFATE & $\begin{array}{l}10 / 15 / 85 \\
05 / 15 / 86 \\
01 / 09 / 87 \\
04 / 06 / 88 \\
07 / 15 / 88 \\
01 / 08 / 89 \\
09 / 19 / 89 \\
03 / 31 / 90 \\
01 / 15 / 91 \\
11 / 25 / 92\end{array}$ & $\begin{array}{l}0001 \\
0001 \\
0001 \\
0001 \\
0001 \\
0001 \\
0001 \\
0001 \\
0001 \\
0001\end{array}$ & $M G / L$ & & $\begin{array}{l}1560 . \\
3370 . \\
3970 . \\
3510 . \\
3470 . \\
4182 . \\
4100 . \\
3880 . \\
4080 . \\
3700 .\end{array}$ & & $\begin{array}{c}0.1 \\
0.1 \\
0.1 \\
0.1 \\
0.1 \\
0.1 \\
0.1 \\
0.1 \\
10 . \\
10 .\end{array}$ & $\begin{array}{l}- \\
- \\
- \\
- \\
- \\
-\end{array}$ \\
\hline SULFIDE & $\begin{array}{l}10 / 15 / 85 \\
04 / 06 / 88 \\
07 / 15 / 88 \\
01 / 08 / 89 \\
09 / 19 / 89 \\
03 / 31 / 90 \\
01 / 15 / 91\end{array}$ & $\begin{array}{l}0001 \\
0001 \\
0001 \\
0001 \\
0001 \\
0001 \\
0001\end{array}$ & MG/L & $\begin{array}{l}< \\
< \\
< \\
< \\
< \\
< \\
<\end{array}$ & $\begin{array}{l}0.1 \\
0.1 \\
0.1 \\
0.01 \\
0.1 \\
0.1 \\
0.1\end{array}$ & $\begin{array}{l}7 \\
j\end{array}$ & $\begin{array}{l}0.1 \\
0.1 \\
0.1 \\
0.1 \\
0.1 \\
0.1 \\
0.1\end{array}$ & $\begin{array}{l}- \\
- \\
- \\
- \\
-\end{array}$ \\
\hline TEMPERATURE & $\begin{array}{l}10 / 15 / 85 \\
05 / 15 / 86 \\
01 / 09 / 87 \\
04 / 06 / 88 \\
07 / 15 / 88 \\
01 / 08 / 89 \\
09 / 19 / 89 \\
03 / 31 / 90 \\
01 / 15 / 91 \\
11 / 25 / 92\end{array}$ & $\begin{array}{l}0001 \\
0001 \\
0001 \\
0001 \\
0001 \\
0001 \\
0001 \\
0001 \\
0001 \\
0001\end{array}$ & $C$ - DEGREE & & $\begin{array}{l}11 . \\
17 . \\
11 . \\
12.0 \\
13.0 \\
8.0 \\
14.0 \\
13 . \\
11 . \\
10.0\end{array}$ & & $\begin{array}{l}- \\
- \\
- \\
- \\
- \\
-\end{array}$ & $\begin{array}{l}- \\
- \\
- \\
- \\
- \\
-\end{array}$ \\
\hline THALLIUM & $\begin{array}{l}01 / 08 / 89 \\
09 / 19 / 89 \\
03 / 31 / 90 \\
01 / 15 / 91\end{array}$ & $\begin{array}{l}0001 \\
0001 \\
0001 \\
0001\end{array}$ & MG/L & $<$ & $\begin{array}{l}0.003 \\
0.01 \\
0.01 \\
0.03\end{array}$ & 1 & $\begin{array}{l}0.001 \\
0.01 \\
0.01 \\
0.03\end{array}$ & $\dot{-}$ \\
\hline THOR IUM-230 & $\begin{array}{l}10 / 15 / 85 \\
04 / 06 / 88 \\
07 / 15 / 88 \\
01 / 08 / 89\end{array}$ & $\begin{array}{l}0001 \\
0001 \\
0001 \\
0001\end{array}$ & $\mathrm{PCI} / \mathrm{L}$ & & $\begin{array}{r}1.5 \\
0.6 \\
0.2 \\
10.9\end{array}$ & & $\begin{array}{l}1 . \\
1 . \\
1 .\end{array}$ & $\begin{array}{l}2.5 \\
0.6 \\
0.4 \\
0.750\end{array}$ \\
\hline TIN & $\begin{array}{l}10 / 15 / 85 \\
04 / 06 / 88 \\
07 / 15 / 88 \\
01 / 08 / 89 \\
09 / 19 / 89 \\
03 / 31 / 90 \\
01 / 15 / 91\end{array}$ & $\begin{array}{l}0001 \\
0001 \\
0001 \\
0001 \\
0001 \\
0001 \\
0001\end{array}$ & MG/L & $\begin{array}{l}< \\
< \\
<\end{array}$ & $\begin{array}{l}0.005 \\
0.030 \\
0.015 \\
0.005 \\
0.185 \\
0.080 \\
0.03\end{array}$ & 1 & $\begin{array}{l}0.005 \\
0.005 \\
0.005 \\
0.005 \\
0.005 \\
0.005 \\
0.03\end{array}$ & $\begin{array}{l}- \\
- \\
- \\
- \\
-\end{array}$ \\
\hline TOTAL DISSOLVED SOLIDS & $\begin{array}{l}10 / 15 / 85 \\
05 / 15 / 86 \\
01 / 09 / 87 \\
04 / 06 / 88\end{array}$ & $\begin{array}{l}0001 \\
0001 \\
0001 \\
0001\end{array}$ & $M G / L$ & & $\begin{array}{l}2670 . \\
5690 \\
6850 . \\
6950\end{array}$ & & $\begin{array}{l}10 . \\
10 . \\
10 . \\
10 .\end{array}$ & $\begin{array}{l}- \\
- \\
-\end{array}$ \\
\hline
\end{tabular}

PARAMETER VALUE IMDICATOR (PVI): < - LESS THAN DETECTION LIMIT

SAMPLE ID COOES:

0001 - FILTERED SAMPLE (.45 MICROHS)

OTHER PARAMETER VALUE FLAGS:

1 - INCREASED DETECTION LIMIT DUE TO REQUIRED DILUTION

J - estimated value 
GROUNDWATER QUALITY DATA BY LOCATION

SITE: AMBOI AMBROSIA LAKE

LOCATION: 0785

HORTH COORDINATE: 52770.8 FT

EAST COORDINATE: $\quad 58816.5$ FT

06/10/80 TO $12 / 04 / 92$

REPORT DATE: 08/04/94

FORMATION OF COMPLETION: TRES HERMANOS - C2 SAMDSTONE (TT)

HYORAULIC FLOW RELATIONSHIP: DOWH GRADIENT (D)

\begin{tabular}{|c|c|c|c|c|c|c|c|}
\hline PARAMETER NAME & LOG DATE & $\begin{array}{c}\text { SAMPLE } \\
\text { ID }\end{array}$ & $\begin{array}{l}\text { UNITS OF } \\
\text { MEASURE }\end{array}$ & PVI & $\begin{array}{l}\text { PARAMETER } \\
\text { VALUE FLAGS }\end{array}$ & $\begin{array}{l}\text { DETECTION } \\
\text { LIMIT }\end{array}$ & $\begin{array}{l}\text { PARAMETER } \\
\text { UNCERTAINTY }\end{array}$ \\
\hline TOTAL DISSOLVED SOLIDS & $\begin{array}{l}07 / 15 / 88 \\
01 / 08 / 89 \\
09 / 19 / 89 \\
03 / 31 / 90 \\
01 / 15 / 91 \\
11 / 25 / 92\end{array}$ & $\begin{array}{l}0001 \\
0001 \\
0001 \\
0001 \\
0001 \\
0001\end{array}$ & $\mathrm{HG} / \mathrm{L}$ & & $\begin{array}{l}6850 . \\
6842 . \\
6680 \\
6230 \\
6840 \\
6160\end{array}$ & $\begin{array}{l}10 . \\
10 . \\
10 . \\
10 . \\
10 . \\
10 .\end{array}$ & $\begin{array}{l}- \\
- \\
- \\
- \\
-\end{array}$ \\
\hline TOTAL KJELDAHL NITROGEN & $\begin{array}{l}01 / 15 / 91 \\
11 / 25 / 92\end{array}$ & $\begin{array}{l}0001 \\
0001\end{array}$ & $M G / L$ & & 1. & 1. & - \\
\hline TOTAL ORGANIC CARBON & $\begin{array}{l}04 / 06 / 88 \\
07 / 15 / 88 \\
01 / 08 / 89 \\
09 / 19 / 89 \\
03 / 31 / 90 \\
01 / 15 / 91\end{array}$ & $\begin{array}{l}0001 \\
0001 \\
0001 \\
0001 \\
0001 \\
0001\end{array}$ & $M G / L$ & & $\begin{array}{c}207 . \\
161 . \\
13 . \\
162 . \\
22.9 \\
11 .\end{array}$ & $\begin{array}{l}1 . \\
1 . \\
1 . \\
1 . \\
1 . \\
1 .\end{array}$ & $\begin{array}{l}- \\
- \\
- \\
- \\
-\end{array}$ \\
\hline URAKIUN & $\begin{array}{l}10 / 15 / 85 \\
05 / 15 / 86 \\
01 / 09 / 87 \\
04 / 06 / 88 \\
07 / 15 / 88 \\
01 / 08 / 89 \\
09 / 19 / 89 \\
03 / 31 / 90 \\
01 / 15 / 91 \\
11 / 25 / 92\end{array}$ & $\begin{array}{l}0001 \\
0001 \\
0001 \\
0001 \\
0001 \\
0001 \\
0001 \\
0001 \\
0001 \\
0001\end{array}$ & $M G / L$ & & $\begin{array}{l}0.806 \\
1.25 \\
3.30 \\
1.87 \\
1.67 \\
1.810 \\
1.47 \\
0.985 \\
1.78 \\
0.417\end{array}$ & $\begin{array}{l}0.003 \\
0.003 \\
0.003 \\
0.003 \\
0.003 \\
0.003 \\
0.003 \\
0.003 \\
0.001 \\
0.001\end{array}$ & $\begin{array}{l}- \\
- \\
- \\
- \\
- \\
- \\
-\end{array}$ \\
\hline VAHADIUM & $\begin{array}{l}10 / 15 / 85 \\
05 / 15 / 86 \\
01 / 09 / 87 \\
04 / 06 / 88 \\
07 / 15 / 88 \\
01 / 08 / 89 \\
09 / 19 / 89 \\
03 / 31 / 90 \\
01 / 15 / 91 \\
11 / 25 / 92\end{array}$ & $\begin{array}{l}0001 \\
0001 \\
0001 \\
0001 \\
0001 \\
0001 \\
0001 \\
0001 \\
0001 \\
0001\end{array}$ & $M G / L$ & $\begin{array}{l}< \\
< \\
< \\
< \\
<\end{array}$ & $\begin{array}{l}0.01 \\
0.4 \\
0.01 \\
0.12 \\
0.06 \\
0.01 \\
0.01 \\
0.01 \\
0.01 \\
0.01\end{array}$ & $\begin{array}{l}0.01 \\
0.01 \\
0.01 \\
0.01 \\
0.01 \\
0.01 \\
0.01 \\
0.01 \\
0.01 \\
0.01\end{array}$ & $\begin{array}{l}- \\
- \\
- \\
- \\
- \\
- \\
- \\
-\end{array}$ \\
\hline ZINC & $\begin{array}{l}10 / 15 / 85 \\
04 / 06 / 88 \\
07 / 15 / 88 \\
01 / 08 / 89 \\
09 / 19 / 89 \\
03 / 31 / 90 \\
01 / 15 / 91\end{array}$ & $\begin{array}{l}0001 \\
0001 \\
0001 \\
0001 \\
0001 \\
0001 \\
0001\end{array}$ & MG/L & & $\begin{array}{l}0.015 \\
0.315 \\
0.084 \\
0.05 \\
0.005 \\
0.017 \\
0.005\end{array}$ & $\begin{array}{l}0.005 \\
0.005 \\
0.005 \\
0.005 \\
0.005 \\
0.005 \\
0.005\end{array}$ & $\begin{array}{l}- \\
- \\
- \\
- \\
-\end{array}$ \\
\hline
\end{tabular}

PARAMETER VALUE INDICATOR (PVI): < - LESS THAN DETECTION LIMIT

SAMPLE IO CODES:

0001 - FILTERED SAMPLE (.45 MICRONS)

OTHER PARAMETER VALUE FLAGS:

H - HOLD TIHE EXPIRED, VALUE SUSPECT

$J$ - ESTIMATED VALUE 
GROUNDHATER OUALITY DATA BY LOCATION

SITE: AMBOI AMBROSIA LAKE

LOCATION: 0786

MORTH COORDINATE: 52888.5 FT

EAST COORDINATE: $\quad 59760.9$ FT

06/10/80 To $12 / 04 / 92$

REPORT DATE: 08/04/94

FORMATION OF COMPLETION: TRES HERMANOS - C1 SANDSTONE (TO) HYDRAULIC FLOH RELATIOHSHIP: DONN GRADIENT (D)

\begin{tabular}{|c|c|c|c|c|c|c|c|c|}
\hline PARAMETER NAME & LOG DATE & $\begin{array}{c}\text { SAMPLE } \\
\text { ID }\end{array}$ & $\begin{array}{l}\text { UNITS OF } \\
\text { MEASURE }\end{array}$ & PVI & $\begin{array}{l}\text { PARAMETER } \\
\text { VALUE }\end{array}$ & FLAGS & $\begin{array}{l}\text { DETECTION } \\
\text { LIMIT }\end{array}$ & $\begin{array}{l}\text { PARAMETER } \\
\text { UNCERTAINTY }\end{array}$ \\
\hline ALKALINITY & $\begin{array}{l}10 / 03 / 85 \\
10 / 03 / 85 \\
10 / 03 / 85 \\
10 / 03 / 85 \\
10 / 03 / 85 \\
05 / 14 / 86 \\
01 / 09 / 87 \\
07 / 15 / 88 \\
01 / 07 / 89 \\
09 / 19 / 89 \\
03 / 31 / 90 \\
01 / 16 / 91 \\
11 / 24 / 92\end{array}$ & $\begin{array}{l}0001 \\
0002 \\
0003 \\
0004 \\
0005 \\
0001 \\
0001 \\
0001 \\
0001 \\
0001 \\
0001 \\
0001 \\
0001\end{array}$ & $\mathrm{MG} / \mathrm{L} \mathrm{CACO} 3$ & & $\begin{array}{l}381 . \\
381 . \\
381 . \\
381 . \\
381 . \\
349 . \\
415 . \\
431 . \\
222 . \\
497 . \\
494 . \\
471 . \\
524\end{array}$ & & $\begin{array}{l}- \\
- \\
- \\
- \\
- \\
- \\
- \\
- \\
- \\
-\end{array}$ & $\begin{array}{l}- \\
- \\
- \\
- \\
- \\
- \\
- \\
- \\
- \\
- \\
- \\
- \\
-\end{array}$ \\
\hline ALUMIHUM & $\begin{array}{l}10 / 03 / 85 \\
10 / 03 / 85 \\
10 / 03 / 85 \\
10 / 03 / 85 \\
10 / 03 / 85 \\
07 / 15 / 88 \\
01 / 07 / 89 \\
09 / 19 / 89 \\
03 / 31 / 90 \\
01 / 16 / 91\end{array}$ & $\begin{array}{l}0001 \\
0002 \\
0003 \\
0004 \\
0005 \\
0001 \\
0001 \\
0001 \\
0001 \\
0001\end{array}$ & $M G / L$ & $\begin{array}{l}< \\
< \\
<\end{array}$ & $\begin{array}{l}0.4 \\
0.3 \\
0.2 \\
0.2 \\
0.3 \\
0.2 \\
0.05 \\
0.1 \\
0.1 \\
0.05\end{array}$ & $\mathbf{J}$ & $\begin{array}{l}0.1 \\
0.1 \\
0.1 \\
0.1 \\
0.1 \\
0.1 \\
0.1 \\
0.1 \\
0.1 \\
0.05\end{array}$ & $\begin{array}{l}- \\
\text { - } \\
\text { - } \\
\text { - } \\
\text { - } \\
- \\
- \\
-\end{array}$ \\
\hline AMHONIUM & $\begin{array}{l}10 / 03 / 85 \\
10 / 03 / 85 \\
10 / 03 / 85 \\
10 / 03 / 85 \\
10 / 03 / 85 \\
07 / 15 / 88 \\
01 / 07 / 89 \\
09 / 19 / 89 \\
03 / 31 / 90 \\
01 / 16 / 91 \\
11 / 24 / 92\end{array}$ & $\begin{array}{l}0001 \\
0002 \\
0003 \\
0004 \\
0005 \\
0001 \\
0001 \\
0001 \\
0001 \\
0001 \\
0001\end{array}$ & $M G / L$ & $\begin{array}{l}< \\
< \\
< \\
< \\
< \\
< \\
<\end{array}$ & $\begin{array}{l}0.1 \\
0.1 \\
0.2 \\
0.1 \\
0.1 \\
0.1 \\
0.10 \\
0.1 \\
0.1 \\
0.12 \\
0.2\end{array}$ & & $\begin{array}{l}0.1 \\
0.1 \\
0.1 \\
0.1 \\
0.1 \\
0.1 \\
0.1 \\
0.1 \\
0.1 \\
0.01 \\
0.1\end{array}$ & $\begin{array}{l}- \\
- \\
- \\
- \\
- \\
- \\
- \\
- \\
-\end{array}$ \\
\hline AHT IMONY & $\begin{array}{l}10 / 03 / 85 \\
10 / 03 / 85 \\
10 / 03 / 85 \\
10 / 03 / 85 \\
10 / 03 / 85 \\
07 / 15 / 88 \\
01 / 07 / 89 \\
09 / 19 / 89 \\
03 / 31 / 90 \\
01 / 16 / 91 \\
11 / 24 / 92\end{array}$ & $\begin{array}{l}0001 \\
0002 \\
0003 \\
0004 \\
0005 \\
0001 \\
0001 \\
0001 \\
0001 \\
0001 \\
0001\end{array}$ & $M G / L$ & $\begin{array}{l}< \\
< \\
< \\
< \\
< \\
< \\
<\end{array}$ & $\begin{array}{l}0.003 \\
0.003 \\
0.003 \\
0.003 \\
0.003 \\
0.018 \\
0.001 \\
0.004 \\
0.034 \\
0.003 \\
0.003\end{array}$ & $J$ & $\begin{array}{l}0.003 \\
0.003 \\
0.003 \\
0.003 \\
0.003 \\
0.003 \\
0.003 \\
0.003 \\
0.003 \\
0.003 \\
0.003\end{array}$ & $\begin{array}{l}- \\
- \\
- \\
- \\
- \\
- \\
- \\
- \\
- \\
-\end{array}$ \\
\hline ARSEHIC & $\begin{array}{l}10 / 03 / 85 \\
10 / 03 / 85 \\
10 / 03 / 85 \\
10 / 03 / 85 \\
10 / 03 / 85 \\
05 / 14 / 86 \\
01 / 09 / 87\end{array}$ & $\begin{array}{l}0001 \\
0002 \\
0003 \\
0004 \\
0005 \\
0001 \\
0001\end{array}$ & $M G / L$ & $\begin{array}{l}< \\
< \\
< \\
< \\
<\end{array}$ & $\begin{array}{l}0.01 \\
0.01 \\
0.01 \\
0.01 \\
0.01 \\
0.01 \\
0.002\end{array}$ & $\mathbf{J}$ & $\begin{array}{l}0.01 \\
0.01 \\
0.01 \\
0.01 \\
0.01 \\
0.01 \\
0.01\end{array}$ & $\begin{array}{l}- \\
- \\
- \\
- \\
- \\
-\end{array}$ \\
\hline
\end{tabular}

PARAMETER VALUE INDICATOR (PVI): < - LESS THAN DETECTIOH LIMIT

SAMPLE ID COOES:

OTHER PARAMETER VALUE FLAGS:

0001 - FILTERED SAMPLE (.45 MICRONS)

0002 - FILTERED REPLICATE SAMPLE (.45 MICRONS)

0003 - FILTERED REPLICATE SAMPLE (.45 MICRONS)

$J$ - ESTIMATED VALUE

0004 - FILTERED REPLICATE SAMPLE (.45 MICRONS) 
GROUHDHATER QUALITY DATA BY LOCATION

SITE: AMBO1 AMBROSIA LAKE

LOCATION: 0786

NORTH COORDINATE: 52888.5 FT

EAST COORDINATE: 59760.9 FT

06/10/80 TO $12 / 04 / 92$

REPORT DATE: 08/04/94

FORHATION OF COMPLETIOH: TRES HERMANOS - C1 SANDSTONE (TO) HYDRAULIC FLOW RELATIONSHIP: DOWN GRADIENT (D)

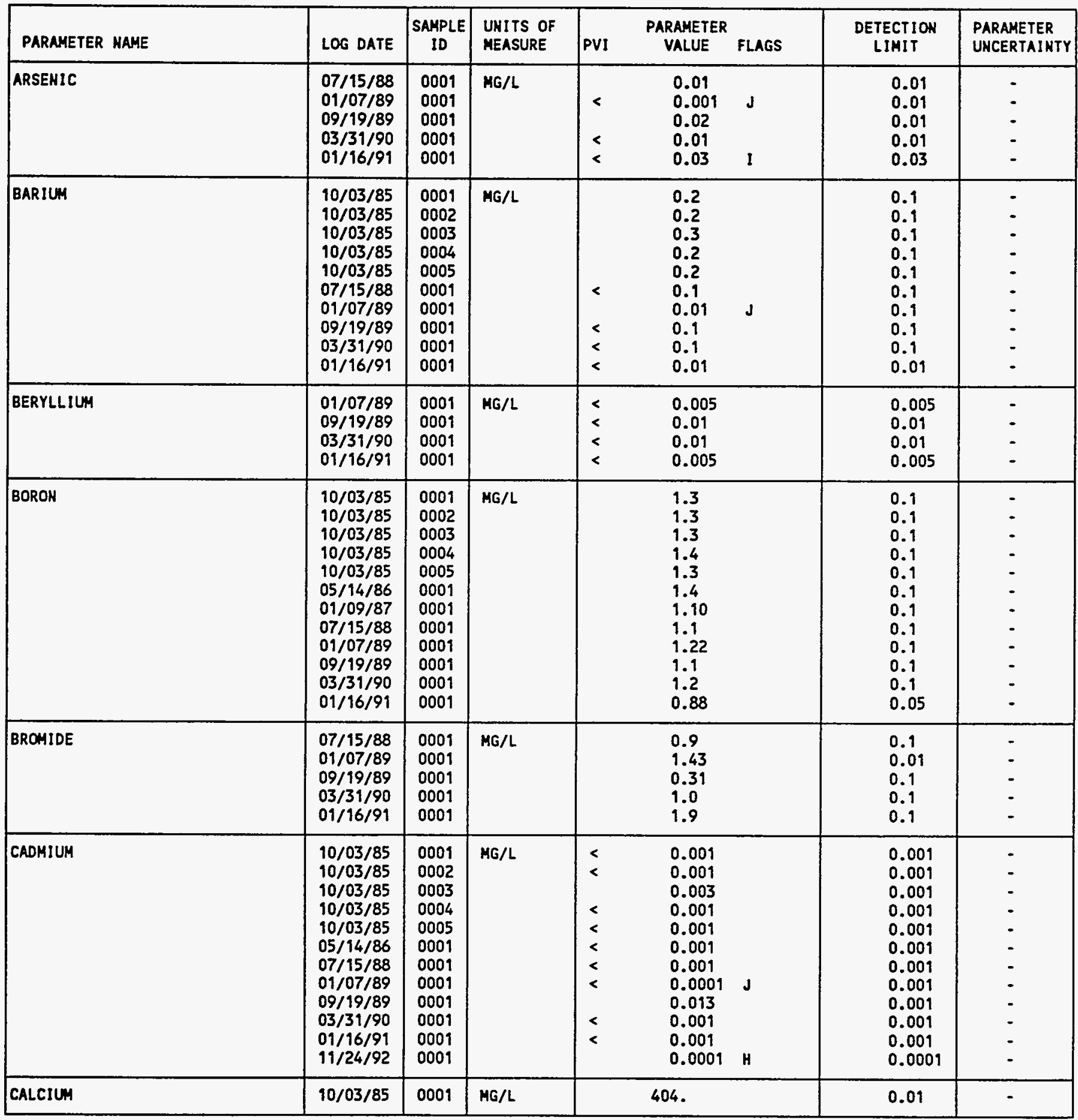

PARAMETER VALUE IMDICATOR (PVI): < - LESS THAN DETECTION LIMIT

SAMPLE ID COOES:

OTHER PARAMETER VALUE FLAGS:

H - HOLD TIME EXPIRED, VALUE SUSPECT

1 - IHCREASED DETECTIOH LIMIT DUE TO REQUIRED DILUTIOH

O001 - FILTERED SAMPLE (.45 MICRONS)

0002 - FILTERED REPLICATE SAMPLE (.45 MICRONS)

0003 - FILTERED REPLICATE SAMPLE (.45 MICRONS)

$\checkmark$ - ESTIMATED VALUE 
GROUNDWATER QUALITY DATA BY LOCATION

SITE: AMBO1 AMBROSIA LAKE

LOCATION: 0786

HORTH COORDINATE: $\quad 52888.5$ FT

EAST COORDINATE: $\quad 59760.9$ FT

$06 / 10 / 80$ TO $12 / 04 / 92$

REPORT DATE: $08 / 04 / 94$

FORMATION OF COMPLETION: TRES HERMANOS - CI SANDSTONE (TO) HYORAULIC FLON RELATIOHSHIP: DOWN GRADIENT (D)

\begin{tabular}{|c|c|c|c|c|c|c|c|c|}
\hline PARAMETER NAME & LOG DATE & $\begin{array}{c}\text { SAMPLE } \\
\text { ID }\end{array}$ & $\begin{array}{l}\text { UHITS OF } \\
\text { MEASURE }\end{array}$ & PVI & $\begin{array}{l}\text { PARAMETEF } \\
\text { VALUE }\end{array}$ & FLAGS & $\begin{array}{l}\text { DETECTION } \\
\text { LIMIT }\end{array}$ & $\begin{array}{l}\text { PARAMETER } \\
\text { UNCERTAINTY }\end{array}$ \\
\hline CALCIUM & $\begin{array}{l}10 / 03 / 85 \\
10 / 03 / 85 \\
10 / 03 / 85 \\
10 / 03 / 85 \\
05 / 14 / 86 \\
01 / 09 / 87 \\
07 / 15 / 88 \\
01 / 07 / 89 \\
09 / 19 / 89 \\
03 / 31 / 90 \\
01 / 16 / 91 \\
11 / 24 / 92\end{array}$ & $\begin{array}{l}0002 \\
0003 \\
0004 \\
0005 \\
0001 \\
0001 \\
0001 \\
0001 \\
0001 \\
0001 \\
0001 \\
0001\end{array}$ & $M G / L$ & & $\begin{array}{l}426 . \\
418 . \\
451 . \\
455 . \\
492 . \\
466 . \\
441 . \\
467 . \\
509 . \\
453 . \\
356 . \\
444 .\end{array}$ & & $\begin{array}{l}0.01 \\
0.01 \\
0.01 \\
0.01 \\
0.01 \\
0.01 \\
0.01 \\
0.01 \\
0.01 \\
0.01 \\
0.5 \\
0.5\end{array}$ & $\begin{array}{l}- \\
- \\
- \\
- \\
- \\
- \\
- \\
-\end{array}$ \\
\hline CHLORIDE & $\begin{array}{l}10 / 03 / 85 \\
10 / 03 / 85 \\
10 / 03 / 85 \\
10 / 03 / 85 \\
10 / 03 / 85 \\
05 / 14 / 86 \\
01 / 09 / 87 \\
07 / 15 / 88 \\
01 / 07 / 89 \\
09 / 19 / 89 \\
03 / 31 / 90 \\
01 / 16 / 91 \\
11 / 24 / 92\end{array}$ & $\begin{array}{l}0001 \\
0002 \\
0003 \\
0004 \\
0005 \\
0001 \\
0001 \\
0001 \\
0001 \\
0001 \\
0001 \\
0001 \\
0001\end{array}$ & MG/L & & $\begin{array}{l}140 . \\
130 . \\
130 . \\
130 . \\
130 . \\
140 . \\
150 . \\
160 . \\
131 . \\
160 . \\
194 . \\
260 . \\
179 .\end{array}$ & & $\begin{array}{l}1 . \\
1 . \\
1 . \\
1 . \\
1 . \\
1 . \\
1 . \\
1 . \\
1 . \\
1 . \\
1 . \\
0.5 \\
1 .\end{array}$ & $\begin{array}{l}- \\
- \\
- \\
- \\
- \\
- \\
- \\
- \\
- \\
-\end{array}$ \\
\hline CHROMIUH & $\begin{array}{l}10 / 03 / 85 \\
10 / 03 / 85 \\
10 / 03 / 85 \\
10 / 03 / 85 \\
10 / 03 / 85 \\
05 / 14 / 86 \\
01 / 09 / 87 \\
07 / 15 / 88 \\
01 / 07 / 89 \\
09 / 19 / 89 \\
03 / 31 / 90 \\
01 / 16 / 91 \\
11 / 24 / 92\end{array}$ & $\begin{array}{l}0001 \\
0002 \\
0003 \\
0004 \\
0005 \\
0001 \\
0001 \\
0001 \\
0001 \\
0001 \\
0001 \\
0001 \\
0001\end{array}$ & MG/L & $\begin{array}{l}< \\
< \\
< \\
< \\
<\end{array}$ & $\begin{array}{l}0.04 \\
0.04 \\
0.04 \\
0.04 \\
0.04 \\
0.03 \\
0.02 \\
0.14 \\
0.01 \\
0.01 \\
0.01 \\
0.01 \\
0.01\end{array}$ & & $\begin{array}{l}0.01 \\
0.01 \\
0.01 \\
0.01 \\
0.01 \\
0.01 \\
0.01 \\
0.01 \\
0.01 \\
0.01 \\
0.01 \\
0.01 \\
0.01\end{array}$ & $\begin{array}{l}- \\
- \\
- \\
- \\
- \\
- \\
- \\
- \\
-\end{array}$ \\
\hline COBALT & $\begin{array}{l}10 / 03 / 85 \\
10 / 03 / 85 \\
10 / 03 / 85 \\
10 / 03 / 85 \\
10 / 03 / 85 \\
05 / 14 / 86 \\
01 / 09 / 87 \\
07 / 15 / 88 \\
01 / 07 / 89 \\
09 / 19 / 89 \\
03 / 31 / 90 \\
01 / 16 / 91 \\
11 / 24 / 92\end{array}$ & $\begin{array}{l}0001 \\
0002 \\
0003 \\
0004 \\
0005 \\
0001 \\
0001 \\
0009 \\
0001 \\
0001 \\
0001 \\
0009 \\
0001\end{array}$ & $M G / L$ & $\begin{array}{l}< \\
< \\
< \\
< \\
<\end{array}$ & $\begin{array}{l}0.07 \\
0.06 \\
0.07 \\
0.08 \\
0.08 \\
0.09 \\
0.05 \\
0.05 \\
0.01 \\
0.05 \\
0.05 \\
0.03 \\
0.03\end{array}$ & J & $\begin{array}{l}0.05 \\
0.05 \\
0.05 \\
0.05 \\
0.05 \\
0.05 \\
0.05 \\
0.05 \\
0.05 \\
0.05 \\
0.05 \\
0.03 \\
0.03\end{array}$ & $\begin{array}{l}- \\
- \\
- \\
- \\
- \\
- \\
- \\
- \\
- \\
- \\
-\end{array}$ \\
\hline COPPER & $10 / 03 / 85$ & 0001 & $M G / L$ & & 0.03 & & 0.02 & - \\
\hline
\end{tabular}

PARAMETER VALUE INDICATOR (PVI): < - LESS THAN DETECTION LIMIT

SAMPLE ID COOES:

OTHER PARAMETER VALUE FLAGS:

0001 - FILTERED SAMPLE (.45 MICRONS)

0002 - FILTERED REPLICATE SAMPLE ( .45 MICROMS)

0003 - FILTERED REPLICATE SAMPLE (.45 MICROHS)

$J$ - estimated VALUE

0004 - FILTERED REPLICATE SAMPLE (.45 MICROMS) 
GROUNDHATER QUALITY DATA BY LOCATION

SITE: AMBO1 AMBROSIA LAKE

LOCATION: 0786

NORTH COORDINATE: 52888.5 FT

EAST COORDINATE: $\quad 59760.9$ FT

06/10/80 TO 12/04/92

REPORT DATE: 08/04/94

FORMATION OF COMPLETION: TRES HERMANOS - CI SAMDSTONE (TO) HYDRAULIC FLON RELATIONSHIP: DONH GRADIENT (D)

\begin{tabular}{|c|c|c|c|c|c|c|c|}
\hline PARAMETER MAME & LOG DATE & $\underset{\text { ID }}{\text { SAMPLE }}$ & $\begin{array}{l}\text { UHITS OF } \\
\text { MEASURE }\end{array}$ & PVI & $\begin{array}{l}\text { PARAMETER } \\
\text { VALUE FLAGS }\end{array}$ & $\begin{array}{l}\text { DETECTION } \\
\text { LIMIT }\end{array}$ & $\begin{array}{l}\text { PARAMETER } \\
\text { UNCERTAINTY }\end{array}$ \\
\hline COPPER & $\begin{array}{l}10 / 03 / 85 \\
10 / 03 / 85 \\
10 / 03 / 85 \\
10 / 03 / 85 \\
01 / 09 / 87 \\
07 / 15 / 88 \\
01 / 07 / 89 \\
09 / 19 / 89 \\
03 / 31 / 90 \\
01 / 16 / 91\end{array}$ & $\begin{array}{l}0002 \\
0003 \\
0004 \\
0005 \\
0001 \\
0001 \\
0009 \\
0001 \\
0001 \\
0001\end{array}$ & MG/L & $\begin{array}{l}< \\
< \\
<\end{array}$ & $\begin{array}{l}0.04 \\
0.04 \\
0.04 \\
0.03 \\
0.02 \\
0.02 \\
0.01 \\
0.02 \\
0.02 \\
0.01\end{array}$ & $\begin{array}{l}0.02 \\
0.02 \\
0.02 \\
0.02 \\
0.02 \\
0.02 \\
0.02 \\
0.02 \\
0.02 \\
0.01\end{array}$ & $\begin{array}{l}- \\
: \\
- \\
- \\
- \\
- \\
-\end{array}$ \\
\hline CYAHIDE & $\begin{array}{l}10 / 03 / 85 \\
10 / 03 / 85 \\
10 / 03 / 85 \\
10 / 03 / 85 \\
10 / 03 / 85 \\
01 / 07 / 89 \\
09 / 19 / 89 \\
03 / 31 / 90 \\
01 / 16 / 91\end{array}$ & $\begin{array}{l}0001 \\
0002 \\
0003 \\
0004 \\
0005 \\
0001 \\
0001 \\
0001 \\
0001\end{array}$ & MG/L & $\begin{array}{l}< \\
< \\
< \\
<\end{array}$ & $\begin{array}{l}0.01 \\
0.01 \\
0.01 \\
0.01 \\
0.01 \\
0.540 \\
0.08 \\
0.96 \\
0.76\end{array}$ & $\begin{array}{l}0.01 \\
0.01 \\
0.01 \\
0.01 \\
0.01 \\
0.01 \\
0.01 \\
0.01 \\
0.01\end{array}$ & $\begin{array}{l}- \\
- \\
- \\
- \\
- \\
- \\
-\end{array}$ \\
\hline FLUORIDE & $\begin{array}{l}10 / 03 / 85 \\
10 / 03 / 85 \\
10 / 03 / 85 \\
10 / 03 / 85 \\
10 / 03 / 85 \\
05 / 14 / 86 \\
01 / 09 / 87 \\
07 / 15 / 88 \\
01 / 07 / 89 \\
09 / 19 / 89 \\
03 / 31 / 90 \\
01 / 16 / 91 \\
11 / 24 / 92\end{array}$ & $\begin{array}{l}0001 \\
0002 \\
0003 \\
0004 \\
0005 \\
0001 \\
0001 \\
0001 \\
0001 \\
0001 \\
0001 \\
0001 \\
0001\end{array}$ & MG/L & & $\begin{array}{l}2.1 \\
2.1 \\
2.1 \\
2.1 \\
2.1 \\
0.6 \\
2.19 \\
1.8 \\
2.2 \\
1.8 \\
1.7 \\
2.4 \\
2.1\end{array}$ & $\begin{array}{l}0.1 \\
0.1 \\
0.1 \\
0.1 \\
0.1 \\
0.1 \\
0.1 \\
0.1 \\
0.1 \\
0.1 \\
0.1 \\
0.1 \\
0.3\end{array}$ & $\begin{array}{l}- \\
- \\
- \\
- \\
- \\
- \\
- \\
- \\
-\end{array}$ \\
\hline GROSS ALPHA & $\begin{array}{l}01 / 09 / 87 \\
07 / 15 / 88 \\
01 / 07 / 89 \\
09 / 19 / 89 \\
03 / 31 / 90 \\
01 / 16 / 91 \\
11 / 24 / 92\end{array}$ & $\begin{array}{l}0001 \\
0001 \\
0001 \\
0001 \\
0009 \\
0001 \\
0001\end{array}$ & $\mathrm{PCI} / \mathrm{L}$ & & $\begin{array}{l}700 . \\
1200 . \\
408 . \\
1300 \\
1200 \\
1278 . \\
1290\end{array}$ & $\begin{array}{l}0.2 \\
0.2 \\
1 . \\
1 . \\
1 . \\
1 . \\
1.0\end{array}$ & $\begin{array}{l}100 . \\
100 . \\
62.1 \\
100 . \\
100 . \\
127 . \\
130 .\end{array}$ \\
\hline GROSS BETA & $\begin{array}{l}01 / 09 / 87 \\
07 / 15 / 88 \\
01 / 07 / 89 \\
09 / 19 / 89 \\
03 / 31 / 90 \\
01 / 16 / 91 \\
11 / 24 / 92\end{array}$ & $\begin{array}{l}0001 \\
0001 \\
0001 \\
0001 \\
0001 \\
0001 \\
0001\end{array}$ & $\mathrm{PCI} / \mathrm{L}$ & & $\begin{array}{l}290 . \\
340 . \\
467 . \\
580 \\
750 \\
627 . \\
590 .\end{array}$ & $\begin{array}{l}1 . \\
1 . \\
0.5 \\
0.5 \\
0.5 \\
0.5 \\
65.8\end{array}$ & $\begin{array}{l}20 . \\
20 . \\
42.9 \\
30 . \\
50 . \\
45.4 \\
59.7\end{array}$ \\
\hline IRON & $\begin{array}{l}10 / 03 / 85 \\
10 / 03 / 85 \\
10 / 03 / 85 \\
10 / 03 / 85 \\
10 / 03 / 85\end{array}$ & $\begin{array}{l}0001 \\
0002 \\
0003 \\
0004 \\
0005\end{array}$ & $M G / L$ & & $\begin{array}{l}0.09 \\
0.1 \\
0.15 \\
0.1 \\
0.15\end{array}$ & $\begin{array}{l}0.03 \\
0.03 \\
0.03 \\
0.03 \\
0.03\end{array}$ & $\begin{array}{l}- \\
- \\
-\end{array}$ \\
\hline $\begin{array}{l}\text { PARAMETER VALUE IMDICATOR (PVI): } \\
\text { OTHER PARAMETER VALUE FLAGS: } \\
J \text { - ESTIMATED VALUE }\end{array}$ & \multicolumn{4}{|c|}{ < - LESS THAN DETECTION LIMIT } & \multicolumn{3}{|l|}{$\begin{array}{l}\text { SAMPLE ID CODES: } \\
\text { O001 - FILTERED } \\
0002 \text { - FILTERED } \\
0003 \text { - FILTERED } \\
0004 \text { - FILTERED }\end{array}$} \\
\hline
\end{tabular}


GROUNDWATER OUALITY DATA BY LOCATION

SITE: AMBO1 AMBROSIA LAKE

LOCATION: 0786

NORTH COORDINATE: $\quad 52888.5$ FT

EAST COORDINATE: $\quad 59760.9$ FT

$06 / 10 / 80$ TO $12 / 04 / 92$

REPORT DATE: $08 / 04 / 94$

FORMATION OF COMPLETION: TRES HERMANOS - CI SANDSTONE (TO) HYDRAULIC FLON RELATIONSHIP: DONN GRADIENT (D)

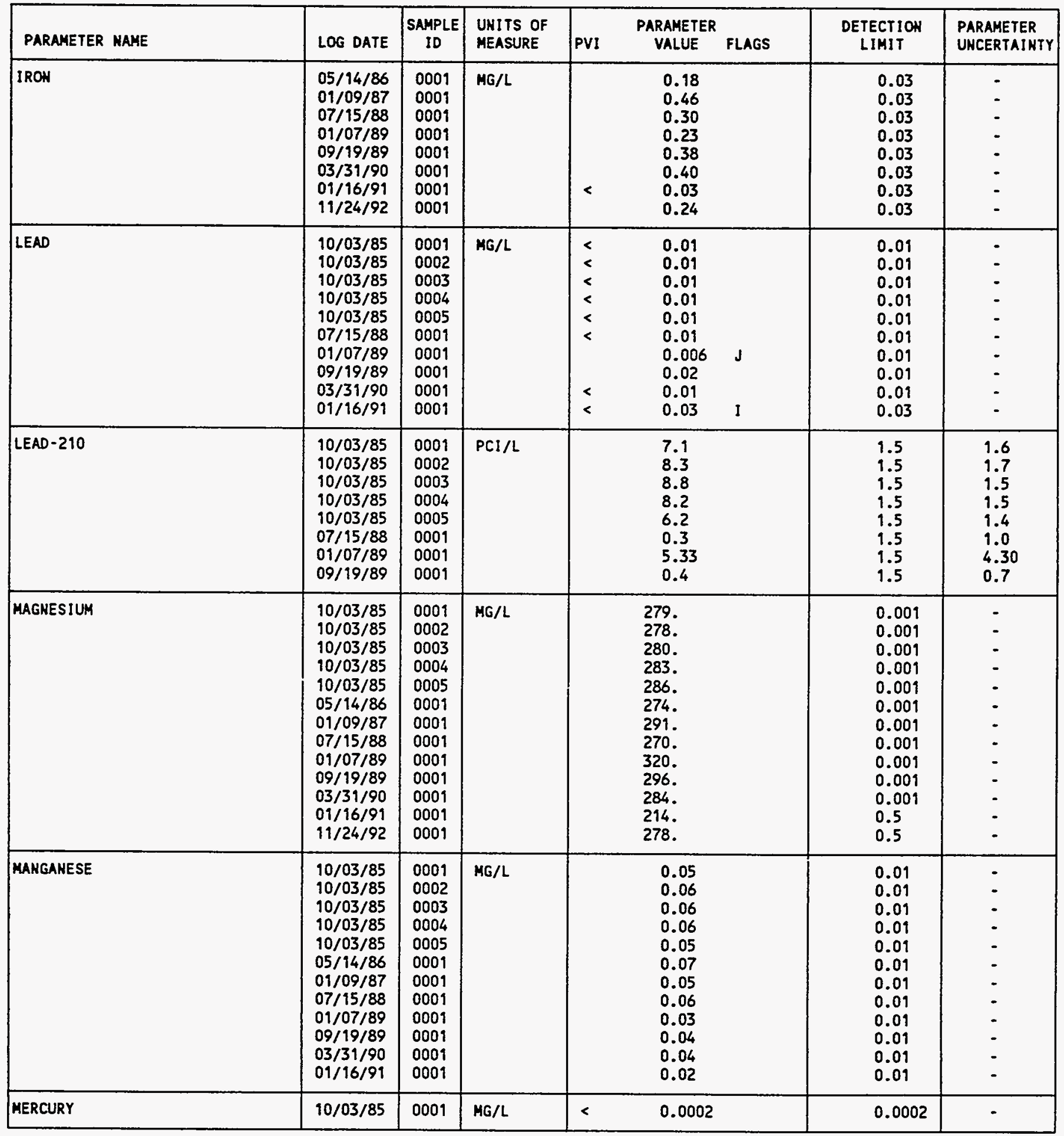

PARAMETER VALUE INDICATOR (PVI): < - LESS THAN DETECTION LIHIT

OTHER PARAMETER VALUE FLAGS:

I - INCREASED DETECTION LIMIT DUE TO-REQUIRED DILUTION

$J$ - estimated VALUE
SAMPLE ID COOES:

0001 - FILTERED SAMPLE (.45 MICROHS)

0002 - FILTERED REPLICATE SAMPLE (.45 MICRONS)

0003 - FILTERED REPLICATE SAMPLE (.45 HICROAS)

0004 - FILTERED REPLICATE SAMPLE (.45 HICRONS) 
GROUMDHATER OUALITY DATA BY LOCATIOH

SITE: AMBO1 AMBROSIA LAKE

LOCATION: 0786

NORTH COORDINATE: $\quad 52888.5$ FT

EAST COORDINATE: $\quad 59760.9$ FT

06/10/80 TO $12 / 04 / 92$

REPORT DATE: 08/04/94

FORMATION OF COMPLETIOH: TRES HERMANOS - C1 SAMDSTONE (TO)

HYDRAULIC FLON RELATIOHSHIP: DOWN GRADIENT (D)

\begin{tabular}{|c|c|c|c|c|c|c|c|c|}
\hline PARAMETER MAME & LOG DATE & $\begin{array}{c}\text { SAMPLE } \\
\text { ID }\end{array}$ & $\begin{array}{l}\text { UNITS OF } \\
\text { MEASURE }\end{array}$ & PVI & $\begin{array}{l}\text { PARAMETER } \\
\text { VALUE }\end{array}$ & FLAGS & $\begin{array}{l}\text { DETECTION } \\
\text { LIMIT }\end{array}$ & $\begin{array}{l}\text { PARANETER } \\
\text { UNCERTAINTY }\end{array}$ \\
\hline MERCURY & $\begin{array}{l}10 / 03 / 85 \\
10 / 03 / 85 \\
10 / 03 / 85 \\
10 / 03 / 85 \\
07 / 15 / 88 \\
01 / 07 / 89 \\
09 / 19 / 89 \\
03 / 31 / 90 \\
01 / 16 / 91\end{array}$ & $\begin{array}{l}0002 \\
0003 \\
0004 \\
0005 \\
0001 \\
0001 \\
0001 \\
0001 \\
0001\end{array}$ & $\mathrm{MG} / \mathrm{L}$ & $\begin{array}{l}< \\
< \\
< \\
< \\
< \\
< \\
<\end{array}$ & $\begin{array}{l}0.0002 \\
0.0002 \\
0.0002 \\
0.0002 \\
0.0003 \\
0.0001 \\
0.0002 \\
0.0002 \\
0.0002\end{array}$ & J & $\begin{array}{l}0.0002 \\
0.0002 \\
0.0002 \\
0.0002 \\
0.0002 \\
0.0002 \\
0.0002 \\
0.0002 \\
0.0002\end{array}$ & $\begin{array}{l}- \\
- \\
- \\
- \\
- \\
-\end{array}$ \\
\hline MOLYBOENUH & $\begin{array}{l}10 / 03 / 85 \\
10 / 03 / 85 \\
10 / 03 / 85 \\
10 / 03 / 85 \\
10 / 03 / 85 \\
05 / 14 / 86 \\
01 / 09 / 87 \\
07 / 15 / 88 \\
01 / 07 / 89 \\
09 / 19 / 89 \\
03 / 31 / 90 \\
01 / 16 / 91 \\
11 / 24 / 92\end{array}$ & $\begin{array}{l}0001 \\
0002 \\
0003 \\
0004 \\
0005 \\
0001 \\
0001 \\
0001 \\
0001 \\
0001 \\
0001 \\
0001 \\
0001\end{array}$ & MG/L & $<$ & $\begin{array}{l}0.2 \\
0.34 \\
0.22 \\
0.1 \\
0.2 \\
0.16 \\
0.1 \\
0.14 \\
0.070 \\
0.15 \\
0.17 \\
0.10 \\
0.18\end{array}$ & & $\begin{array}{l}0.01 \\
0.01 \\
0.01 \\
0.01 \\
0.01 \\
0.01 \\
0.1 \\
0.01 \\
0.01 \\
0.01 \\
0.01 \\
0.01 \\
0.01\end{array}$ & $\begin{array}{l}- \\
- \\
- \\
- \\
- \\
- \\
- \\
- \\
- \\
-\end{array}$ \\
\hline HET GROSS ALPHA - & $\begin{array}{l}01 / 09 / 87 \\
07 / 15 / 88 \\
01 / 07 / 89 \\
09 / 19 / 89 \\
03 / 31 / 90 \\
01 / 16 / 91 \\
11 / 24 / 92\end{array}$ & $\begin{array}{l}0001 \\
0001 \\
0001 \\
0001 \\
0001 \\
0001 \\
0001\end{array}$ & $\mathrm{PCI} / \mathrm{L}$ & & $\begin{array}{r}-109.48 \\
225.88 \\
-518.10 \\
456.22 \\
-89.68 \\
-697.68 \\
-431.86\end{array}$ & & $\begin{array}{l}- \\
- \\
- \\
- \\
-\end{array}$ & $\begin{array}{l}- \\
- \\
- \\
- \\
-\end{array}$ \\
\hline HICKEL & $\begin{array}{l}10 / 03 / 85 \\
10 / 03 / 85 \\
10 / 03 / 85 \\
10 / 03 / 85 \\
10 / 03 / 85 \\
07 / 15 / 88 \\
01 / 07 / 89 \\
09 / 19 / 89 \\
03 / 31 / 90 \\
01 / 16 / 91 \\
11 / 24 / 92\end{array}$ & $\begin{array}{l}0001 \\
0002 \\
0003 \\
0004 \\
0005 \\
0001 \\
0001 \\
0001 \\
0001 \\
0001 \\
0001\end{array}$ & $M G / L$ & $\begin{array}{l}< \\
< \\
< \\
< \\
<\end{array}$ & $\begin{array}{l}0.1 \\
0.1 \\
0.09 \\
0.09 \\
0.04 \\
0.08 \\
0.02 \\
0.04 \\
0.04 \\
0.04 \\
0.13\end{array}$ & $J$ & $\begin{array}{l}0.04 \\
0.04 \\
0.04 \\
0.04 \\
0.04 \\
0.04 \\
0.04 \\
0.04 \\
0.04 \\
0.04 \\
0.04\end{array}$ & $\begin{array}{l}- \\
- \\
- \\
- \\
- \\
- \\
- \\
-\end{array}$ \\
\hline HITRATE & $\begin{array}{l}10 / 03 / 85 \\
10 / 03 / 85 \\
10 / 03 / 85 \\
10 / 03 / 85 \\
10 / 03 / 85 \\
05 / 14 / 86 \\
01 / 09 / 87 \\
07 / 15 / 88 \\
01 / 07 / 89 \\
09 / 19 / 89\end{array}$ & $\begin{array}{l}0001 \\
0002 \\
0003 \\
0004 \\
0005 \\
0001 \\
0001 \\
0001 \\
0001 \\
0001\end{array}$ & $M G / L$ & $<$ & $\begin{array}{l}45 . \\
55 . \\
55 . \\
55 . \\
55 . \\
1 . \\
49 . \\
9.2 \\
24.3 \\
7.5\end{array}$ & & $\begin{array}{l}1 . \\
1 . \\
1 . \\
1 . \\
1 . \\
1 . \\
1 . \\
1 . \\
1 .\end{array}$ & $\begin{array}{l}- \\
- \\
- \\
- \\
- \\
- \\
-\end{array}$ \\
\hline
\end{tabular}

- HET GROSS ALPHA (GROSS ALPHA - URANIUM) WITH 1 MG URAKIUM = 686 PCI

PARAMETER VALUE IMDICATOR (PVI): < - LESS THAN DETECTION LIMIT

OTHER PARAMETER VALUE FLAGS:

J- ESTIMATED VALUE
SAMPLE ID COOES:

0001 - FILTERED SAMPLE (.45 MICRONS)

0002 - FILTERED REPLICATE SAMPLE (.45 MICRONS)

0003 - FILTERED REPLICATE SAMPLE (.45 MICRONS)

0004 - FILTERED REPLICATE SAMPLE (.45 MICRONS) 
GROUNDHATER QUALITY DATA BY LOCATION

SITE: AMBOI AMBROSIA LAKE

LOCATION: 0786

NORTH COORDINATE: 52888.5 FT

EAST COORDINATE: 59760.9 FT

06/10/80 TO $12 / 04 / 92$

REPORT DATE : $08 / 04 / 94$

FORMATION OF COMPLETION: TRES HERMANOS - C1 SANDSTONE (TO)

HYDRAULIC FLOW RELATIONSHIP: DOWN GRADIENT (D)

\begin{tabular}{|c|c|c|c|c|c|c|c|}
\hline PARAMETER NAME & LOG DATE & $\underset{10}{\text { SAMPLE }}$ & $\begin{array}{l}\text { UNITS OF } \\
\text { MEASURE }\end{array}$ & PVI & $\begin{array}{l}\text { PARAMETER } \\
\text { VALUE FLAGS }\end{array}$ & $\begin{array}{l}\text { DETECTIOA } \\
\text { LIMIT }\end{array}$ & $\begin{array}{l}\text { PARAMETER } \\
\text { UNCERTAINTY }\end{array}$ \\
\hline NITRATE & $\begin{array}{l}03 / 31 / 90 \\
01 / 16 / 91 \\
11 / 24 / 92\end{array}$ & $\begin{array}{l}0001 \\
0001 \\
0001\end{array}$ & $M G / L$ & & $\begin{array}{r}6.6 \\
16.4 \\
29.6\end{array}$ & $\begin{array}{l}1 . \\
0.1 \\
4.0\end{array}$ & : \\
\hline NITRITE & $\begin{array}{l}10 / 03 / 85 \\
10 / 03 / 85 \\
10 / 03 / 85 \\
10 / 03 / 85 \\
10 / 03 / 85\end{array}$ & $\begin{array}{l}0001 \\
0002 \\
0003 \\
0004 \\
0005\end{array}$ & $M G / L$ & $\begin{array}{l}< \\
< \\
< \\
<\end{array}$ & $\begin{array}{l}0.1 \\
0.1 \\
0.1 \\
0.1 \\
0.1\end{array}$ & $\begin{array}{l}0.1 \\
0.1 \\
0.1 \\
0.1 \\
0.1\end{array}$ & $\begin{array}{l}- \\
- \\
- \\
-\end{array}$ \\
\hline MITRITE AND MITRATE & $\begin{array}{l}03 / 31 / 90 \\
01 / 16 / 91\end{array}$ & $\begin{array}{l}0001 \\
0001\end{array}$ & MG/L & & $\begin{array}{l}1.5 \\
4.15\end{array}$ & $\begin{array}{l}1.05 \\
0.05\end{array}$ & - \\
\hline $\mathrm{PH}$ & $\begin{array}{l}10 / 03 / 85 \\
10 / 03 / 85 \\
10 / 03 / 85 \\
10 / 03 / 85 \\
10 / 03 / 85 \\
05 / 14 / 86 \\
01 / 09 / 87 \\
07 / 15 / 88 \\
01 / 07 / 89 \\
09 / 19 / 89 \\
03 / 31 / 90 \\
01 / 16 / 91 \\
11 / 24 / 92\end{array}$ & $\begin{array}{l}0001 \\
0002 \\
0003 \\
0004 \\
0005 \\
0001 \\
0001 \\
0001 \\
0001 \\
0001 \\
0001 \\
0001 \\
0001\end{array}$ & SU & & $\begin{array}{l}7 . \\
7 . \\
7 . \\
7 . \\
7 . \\
7.88 \\
7.30 \\
7.00 \\
7.0 \\
7.13 \\
6.80 \\
7.20 \\
7.16\end{array}$ & $\begin{array}{l}- \\
- \\
- \\
- \\
- \\
- \\
- \\
- \\
- \\
-\end{array}$ & $\begin{array}{l}- \\
- \\
- \\
- \\
- \\
- \\
- \\
- \\
-\end{array}$ \\
\hline PHOSPHATE & $\begin{array}{l}10 / 03 / 85 \\
10 / 03 / 85 \\
10 / 03 / 85 \\
10 / 03 / 85 \\
10 / 03 / 85 \\
07 / 15 / 88 \\
01 / 07 / 89 \\
09 / 19 / 89 \\
03 / 31 / 90 \\
01 / 16 / 91 \\
11 / 24 / 92\end{array}$ & $\begin{array}{l}0001 \\
0002 \\
0003 \\
0004 \\
0005 \\
0001 \\
0001 \\
0001 \\
0001 \\
0001 \\
0001\end{array}$ & $M G / L$ & $\begin{array}{l}< \\
<\end{array}$ & $\begin{array}{l}0.4 \\
0.4 \\
0.3 \\
0.4 \\
0.4 \\
0.1 \\
0.9 \\
0.1 \\
0.1 \\
0.20 \\
1.0\end{array}$ & $\begin{array}{l}0.1 \\
0.1 \\
0.1 \\
0.1 \\
0.1 \\
0.1 \\
0.1 \\
0.1 \\
0.1 \\
0.05 \\
0.1\end{array}$ & $\begin{array}{l}- \\
- \\
- \\
- \\
- \\
- \\
- \\
-\end{array}$ \\
\hline POTASSIUM & $\begin{array}{l}10 / 03 / 85 \\
10 / 03 / 85 \\
10 / 03 / 85 \\
10 / 03 / 85 \\
10 / 03 / 85 \\
05 / 14 / 86 \\
01 / 09 / 87 \\
07 / 15 / 88\end{array}$ & $\begin{array}{l}0001 \\
0002 \\
0003 \\
0004 \\
0005 \\
0001 \\
0001 \\
0001\end{array}$ & $M G / L$ & & $\begin{array}{l}5.83 \\
5.65 \\
5.71 \\
5.95 \\
5.72 \\
6.26 \\
5.67 \\
5.1\end{array}$ & $\begin{array}{l}0.01 \\
0.01 \\
0.01 \\
0.01 \\
0.01 \\
0.01 \\
0.01 \\
0.01\end{array}$ & $\begin{array}{l}- \\
: \\
- \\
- \\
-\end{array}$ \\
\hline
\end{tabular}

PARAMETER VALUE INDICATOR (PVI): < - LESS THAN DETECTION LIMIT

OTHER PARAMETER VALUE FLAGS:

$J$ - ESTIMATED VALUE
SAMPLE ID COOES:

0001 - FILTERED SAMPLE (.45 MICRONS)

0002 - FILTERED REPLICATE SAMPLE (.45 MICRONS)

0003 - FILTERED REPLICATE SAMPLE (. 45 MICROHS)

0004 - FILTERED REPLICATE SAMPLE (.45 MICROHS) 
GROUNDWATER QUALITY DATA BY LOCATION

SITE: AMBO1 AMBROSIA LAKE

LOCATION: 0786

HORTH COORDIHATE: $\quad 52888.5$ FT

EAST COOROINATE: $\quad 59760.9$ FT

06/10/80 TO $12 / 04 / 92$

REPORT DATE: 08/04/94

FORMATION OF COHPLETION: TRES HERMANOS - C1 SANDSTONE (TO)

HYDRAULIC FLON RELATIOHSHIP: DOWN GRADIENT (D)

\begin{tabular}{|c|c|c|c|c|c|c|c|c|}
\hline PARAMETER NAME & LOG DATE & $\begin{array}{c}\text { SAMPLE } \\
\text { ID }\end{array}$ & $\begin{array}{l}\text { UNITS OF } \\
\text { MEASURE }\end{array}$ & PVI & $\begin{array}{l}\text { PARAMETEI } \\
\text { VALUE }\end{array}$ & FLAGS & $\begin{array}{l}\text { DETECTION } \\
\text { LIMIT }\end{array}$ & $\begin{array}{l}\text { PARAMETER } \\
\text { UNCERTAINTY }\end{array}$ \\
\hline POTASSIUM & $\begin{array}{l}01 / 07 / 89 \\
09 / 19 / 89 \\
03 / 31 / 90 \\
01 / 16 / 91 \\
11 / 24 / 92\end{array}$ & $\begin{array}{l}0001 \\
0001 \\
0001 \\
0001 \\
0001\end{array}$ & $M G / L$ & & $\begin{array}{l}5.8 \\
5.2 \\
6.2 \\
4.6 \\
6.20\end{array}$ & & $\begin{array}{l}0.01 \\
0.01 \\
0.01 \\
0.01 \\
0.01\end{array}$ & $\begin{array}{l}- \\
\dot{-} \\
\dot{-} \\
-\end{array}$ \\
\hline RADIUM-226 & $\begin{array}{l}10 / 03 / 85 \\
10 / 03 / 85 \\
10 / 03 / 85 \\
10 / 03 / 85 \\
10 / 03 / 85 \\
01 / 09 / 87 \\
07 / 15 / 88 \\
01 / 07 / 89 \\
09 / 19 / 89 \\
03 / 31 / 90 \\
01 / 16 / 91 \\
19 / 24 / 92\end{array}$ & $\begin{array}{l}0001 \\
0002 \\
0003 \\
0004 \\
0005 \\
0001 \\
0001 \\
0001 \\
0001 \\
0001 \\
0001 \\
0001\end{array}$ & $\mathrm{PCI} / \mathrm{L}$ & & $\begin{array}{l}1.7 \\
1.9 \\
1.4 \\
1.9 \\
8.6 \\
0.9 \\
0.7 \\
2.14 \\
0.1 \\
0.1 \\
1.4 \\
1.5\end{array}$ & & $\begin{array}{l}1 . \\
1 . \\
1 . \\
1 . \\
1 . \\
1 . \\
1 . \\
1 . \\
1 . \\
1 . \\
1.0 \\
0.3\end{array}$ & $\begin{array}{l}0.5 \\
0.5 \\
0.4 \\
0.5 \\
1.1 \\
0.5 \\
0.3 \\
0.394 \\
0.2 \\
0.1 \\
0.6 \\
0.5\end{array}$ \\
\hline RADIUM-226 + RADIUM-228 & $\begin{array}{l}10 / 03 / 85 \\
10 / 03 / 85 \\
10 / 03 / 85 \\
10 / 03 / 85 \\
10 / 03 / 85 \\
01 / 09 / 87 \\
07 / 15 / 88 \\
01 / 07 / 89 \\
09 / 19 / 89 \\
03 / 31 / 90 \\
01 / 16 / 91 \\
11 / 24 / 92\end{array}$ & $\begin{array}{l}0001 \\
0002 \\
0003 \\
0004 \\
0005 \\
0001 \\
0001 \\
0001 \\
0001 \\
0001 \\
0001 \\
0001\end{array}$ & $\mathrm{PCl} / \mathrm{L}$ & & $\begin{array}{l}3.70 \\
1.90 \\
1.70 \\
2.00 \\
9.40 \\
1.70 \\
1.40 \\
9.92 \\
0.40 \\
1.40 \\
1.90 \\
1.60\end{array}$ & & $\begin{array}{l}- \\
- \\
- \\
- \\
- \\
- \\
- \\
- \\
-\end{array}$ & $\begin{array}{l}: \\
: \\
: \\
: \\
: \\
: \\
- \\
-\end{array}$ \\
\hline RADIUM-228 & $\begin{array}{l}10 / 03 / 85 \\
10 / 03 / 85 \\
10 / 03 / 85 \\
10 / 03 / 85 \\
10 / 03 / 85 \\
01 / 09 / 87 \\
07 / 15 / 88 \\
01 / 07 / 89 \\
09 / 19 / 89 \\
03 / 31 / 90 \\
01 / 16 / 91 \\
11 / 24 / 92\end{array}$ & $\begin{array}{l}0001 \\
0002 \\
0003 \\
0004 \\
0005 \\
0001 \\
0001 \\
0001 \\
0001 \\
0001 \\
0001 \\
0001\end{array}$ & $\mathrm{PCI} / \mathrm{L}$ & & $\begin{array}{l}2 . \\
0 . \\
0.3 \\
0.1 \\
0.8 \\
0.8 \\
0.7 \\
7.78 \\
0.3 \\
1.3 \\
0.5 \\
0.1\end{array}$ & H & $\begin{array}{l}1 . \\
1 . \\
1 . \\
1 . \\
1 . \\
1 . \\
1 . \\
1 . \\
1 . \\
1 . \\
1.0 \\
1.0\end{array}$ & $\begin{array}{l}2.8 \\
1.3 \\
0.9 \\
1 . \\
1.1 \\
1.2 \\
0.8 \\
5.00 \\
1.0 \\
0.8 \\
1.7 \\
1.5\end{array}$ \\
\hline SELENIUM & $\begin{array}{l}10 / 03 / 85 \\
10 / 03 / 85 \\
10 / 03 / 85 \\
10 / 03 / 85 \\
10 / 03 / 85 \\
05 / 14 / 86 \\
01 / 09 / 87 \\
07 / 15 / 88 \\
01 / 07 / 89 \\
09 / 19 / 89 \\
03 / 31 / 90\end{array}$ & $\begin{array}{l}0001 \\
0002 \\
0003 \\
0004 \\
0005 \\
0001 \\
0001 \\
0001 \\
0001 \\
0001 \\
0001\end{array}$ & $M G / L$ & $\begin{array}{l}< \\
< \\
< \\
<\end{array}$ & $\begin{array}{l}0.014 \\
0.015 \\
0.005 \\
0.005 \\
0.005 \\
0.005 \\
0.544 \\
0.685 \\
0.780 \\
0.519 \\
0.346\end{array}$ & & $\begin{array}{l}0.005 \\
0.005 \\
0.005 \\
0.005 \\
0.005 \\
0.005 \\
0.005 \\
0.005 \\
0.005 \\
0.005 \\
0.005\end{array}$ & $\begin{array}{l}- \\
- \\
- \\
- \\
- \\
- \\
- \\
- \\
-\end{array}$ \\
\hline
\end{tabular}

PARAMETER VALUE IHDICATOR (PVI): < - LESS THAN DETECTIOH LIMIT

SAMPLE ID COOES:

O001 - FILTERED SAMPLE (.45 MICRONS)

OTHER PARAMETER VALUE FLAGS:

0002 - FILTERED REPLICATE SAMPLE (.45 MICRONS)

0003 - FILTERED REPLICATE SAMPLE (.45 MICRONS)

H - hold tIME EXPIREd, VAlUe SUSPECt.

0004 - FILTERED REPLICATE SAMPLE (.45 MICROHS) 
GROUNDWATER QUALITY DATA BY LOCATION

SITE: AMBO1 AMBROSIA LAKE

LOCATION: 0786

HORTH COORDINATE: 52888.5 FT

EAST COORDINATE: $\quad 59760.9$ FT

06/10/80 TO $12 / 04 / 92$

REPORT DATE: $08 / 04 / 94$

FORMATION OF COMPLETION: TRES HERMANOS - C1 SANDSTONE (TO)

HYDRAULIC FLOW RELATIONSHIP: DOWN GRADIENT (D)

\begin{tabular}{|c|c|c|c|c|c|c|c|}
\hline PARAMETER NAME & LOG DATE & $\begin{array}{c}\text { SAMPLE } \\
\text { ID }\end{array}$ & $\begin{array}{l}\text { UNITS OF } \\
\text { MEASURE }\end{array}$ & PVI & $\begin{array}{l}\text { PARAMETER } \\
\text { VALUE FLAGS }\end{array}$ & $\begin{array}{l}\text { DETECTIOH } \\
\text { LIMIT }\end{array}$ & $\begin{array}{l}\text { PARAMETER } \\
\text { UNCERTAIHTY }\end{array}$ \\
\hline SELENIUH & $\begin{array}{l}01 / 16 / 91 \\
11 / 24 / 92\end{array}$ & $\begin{array}{l}0001 \\
0001\end{array}$ & $M G / L$ & & $\begin{array}{l}0.420 \\
0.6\end{array}$ & $\begin{array}{l}0.005 \\
0.1\end{array}$ & $\dot{-}$ \\
\hline SILICA - SIO2 & $\begin{array}{l}10 / 03 / 85 \\
10 / 03 / 85 \\
10 / 03 / 85 \\
10 / 03 / 85 \\
10 / 03 / 85 \\
07 / 15 / 88 \\
01 / 07 / 89 \\
09 / 19 / 89 \\
03 / 31 / 90 \\
01 / 16 / 91\end{array}$ & $\begin{array}{l}0001 \\
0002 \\
0003 \\
0004 \\
0005 \\
0001 \\
0001 \\
0001 \\
0001 \\
0001\end{array}$ & $M G / L$ & & $\begin{array}{c}11 . \\
10 . \\
11 . \\
13 . \\
12 . \\
12.3 \\
11.0 \\
7.5 \\
13 . \\
9.9\end{array}$ & $\begin{array}{l}2 . \\
2 . \\
2 . \\
2 . \\
2 . \\
2 . \\
2 . \\
2 . \\
2 . \\
0.1\end{array}$ & $\begin{array}{l}- \\
- \\
- \\
- \\
- \\
- \\
-\end{array}$ \\
\hline SILVER & $\begin{array}{l}10 / 03 / 85 \\
10 / 03 / 85 \\
10 / 03 / 85 \\
10 / 03 / 85 \\
10 / 03 / 85 \\
01 / 09 / 87 \\
07 / 15 / 88 \\
01 / 07 / 89 \\
09 / 19 / 89 \\
03 / 31 / 90 \\
01 / 16 / 91 \\
11 / 24 / 92\end{array}$ & $\begin{array}{l}0001 \\
0002 \\
0003 \\
0004 \\
0005 \\
0001 \\
0001 \\
0001 \\
0001 \\
0001 \\
0001 \\
0001\end{array}$ & MG/L & $\begin{array}{l}< \\
< \\
< \\
< \\
< \\
< \\
< \\
< \\
<\end{array}$ & $\begin{array}{l}0.01 \\
0.01 \\
0.01 \\
0.01 \\
0.01 \\
0.01 \\
0.02 \\
0.01 \\
0.01 \\
0.01 \\
0.01 \\
0.01\end{array}$ & $\begin{array}{l}0.01 \\
0.01 \\
0.01 \\
0.01 \\
0.01 \\
0.01 \\
0.01 \\
0.01 \\
0.01 \\
0.01 \\
0.01 \\
0.01\end{array}$ & $\begin{array}{l}- \\
: \\
- \\
- \\
- \\
- \\
- \\
-\end{array}$ \\
\hline SOOIU: & $\begin{array}{l}10 / 03 / 85 \\
10 / 03 / 85 \\
10 / 03 / 85 \\
10 / 03 / 85 \\
10 / 03 / 85 \\
05 / 14 / 86 \\
01 / 09 / 87 \\
07 / 15 / 88 \\
01 / 07 / 89 \\
09 / 19 / 89 \\
03 / 31 / 90 \\
01 / 16 / 91 \\
11 / 24 / 92\end{array}$ & $\begin{array}{l}0001 \\
0002 \\
0003 \\
0004 \\
0005 \\
0001 \\
0001 \\
0001 \\
0001 \\
0001 \\
0001 \\
0001 \\
0001\end{array}$ & MG/L & & $\begin{array}{l}883 . \\
663 . \\
668 . \\
660 . \\
600 . \\
688 . \\
645 . \\
547 . \\
615 . \\
581 . \\
613 . \\
659 . \\
604 .\end{array}$ & $\begin{array}{l}0.002 \\
0.002 \\
0.002 \\
0.002 \\
0.002 \\
0.002 \\
0.002 \\
0.002 \\
0.002 \\
0.002 \\
0.002 \\
5 . \\
5 .\end{array}$ & $\begin{array}{l}- \\
- \\
- \\
- \\
- \\
- \\
- \\
- \\
-\end{array}$ \\
\hline SPECIFIC CONDUCTAKCE & $\begin{array}{l}10 / 03 / 85 \\
10 / 03 / 85 \\
10 / 03 / 85 \\
10 / 03 / 85 \\
10 / 03 / 85 \\
05 / 14 / 86 \\
01 / 09 / 87 \\
07 / 15 / 88 \\
01 / 07 / 89 \\
09 / 19 / 89 \\
03 / 31 / 90 \\
01 / 16 / 91 \\
11 / 24 / 92\end{array}$ & $\begin{array}{l}0001 \\
0002 \\
0003 \\
0004 \\
0005 \\
0001 \\
0001 \\
0001 \\
0001 \\
0001 \\
0001 \\
0001 \\
0001\end{array}$ & UMHO/CM & & $\begin{array}{l}3600 . \\
3600 . \\
3600 . \\
3600 . \\
3600 . \\
2950 . \\
3400 . \\
3600 . \\
2550 . \\
1300 . \\
3620 . \\
3650 . \\
4080\end{array}$ & $\begin{array}{l}- \\
: \\
- \\
- \\
- \\
- \\
- \\
-\end{array}$ & $\begin{array}{l}- \\
: \\
- \\
- \\
- \\
- \\
- \\
- \\
-\end{array}$ \\
\hline STRONTIUMA & $10 / 03 / 85$ & 0009 & $M G / L$ & & 10. & 0.1 & - \\
\hline
\end{tabular}

PARAMETER VALUE INDICATOR (PVI): < - LESS THAN DETECTION LIMIT

SAMPLE ID COOES:

0001 - FILTERED SAMPLE (.45 MICRONS)

0002 - FILTERED REPLICATE SAMPLE (.45 MICRONS)

0003 - FILTERED REPLICATE SAMPLE (.45 MICRONS)

0004 - FILTERED REPLICATE SAMPLE (.45 MICRONS) 
GROUNDWATER QUALITY DATA BY LOCATIOH

SITE: AMBO1 AMBROSIA LAKE

LOCATION: 0786

MORTH COORDINATE: 52888.5 FT

EAST COORDINATE: 59760.9 FT

06/10/80 TO $12 / 04 / 92$

REPORT DATE: $08 / 04 / 94$

FORHATION OF COMPLETION: TRES HERMANOS - CI SANDSTOHE (TO) HYDRAULIC FLOW RELATIOWSHIP: DOWN GRADIENT (D)

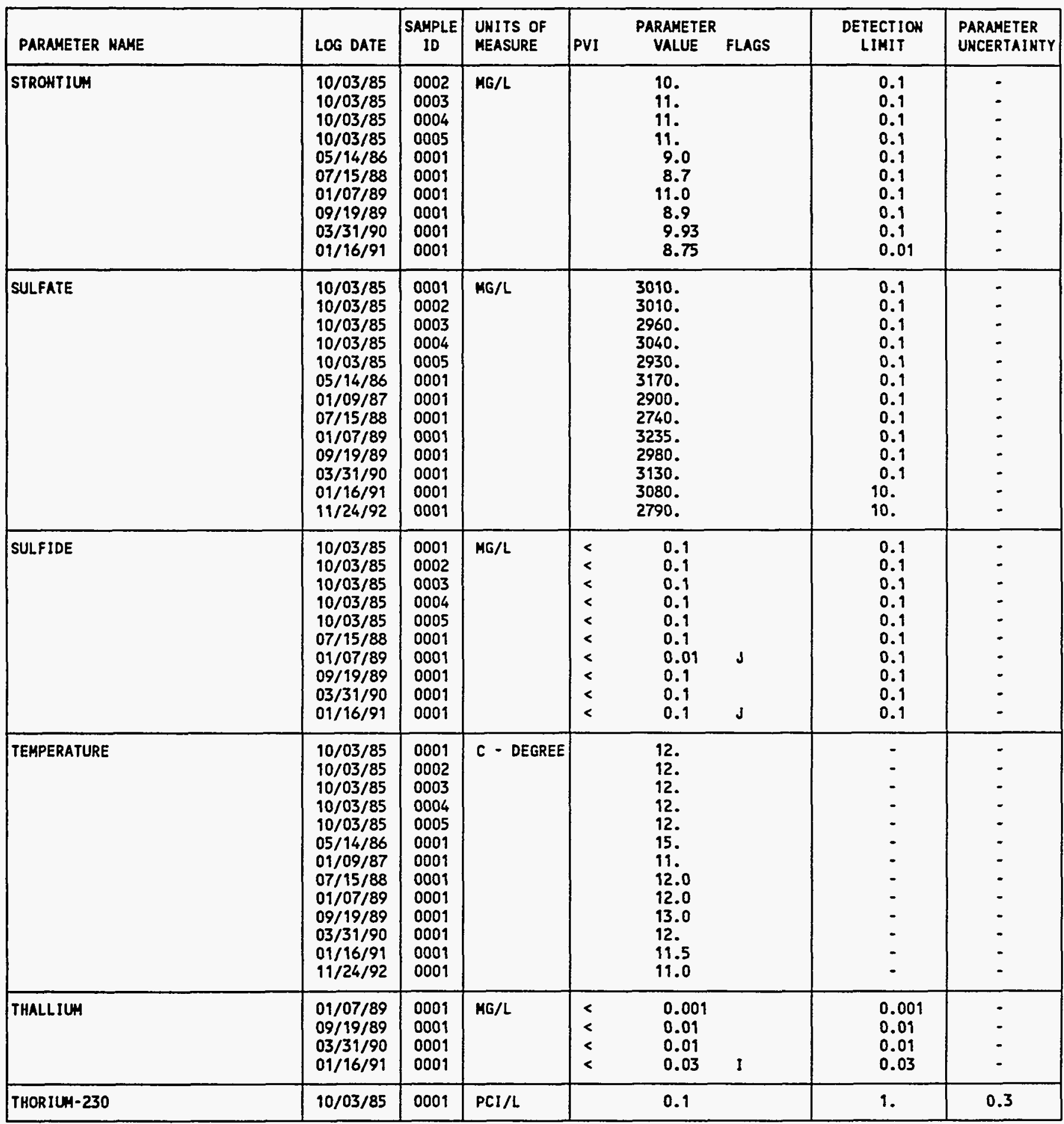

PARAMETER VALUE IMDICATOR (PVI): < - LESS THAN DETECTION LIMIT

SAMPLE IO CODES:

0001 - FILTERED SAMPLE (.45 MICRONS)

OTHER PARAMETER VALUE FLAGS:

I - INCREASED DETECTION LIMIT DUE TO REQUIRED DILUTION

0002 - FILTERED REPLICATE SAMPLE (.45 MICRONS)

$j$ - eSTIMATED VALUE

0004 - FILTERED REPLICATE SAMPLE (.45 MICROHS) 
GROUHDWATER QUALITY DATA BY LOCATION

SITE: AMBO1 AMBROSIA LAKE

LOCATION: 0786

NORTH COORDINATE: 52888.5 FT

EAST COOROINATE: 59760.9 FT

D6/10/80 TO $12 / 04 / 92$

REPORT DATE: 08/04/94

FORMATION OF COMPLETION: TRES HERMAHOS - CI SANDSTONE (TO)

HYDRAULIC FLON RELATIONSHIP: DOWN GRADIENT (D)

\begin{tabular}{|c|c|c|c|c|c|c|c|}
\hline PARAMETER NAME & LOG DATE & $\begin{array}{c}\text { SAMPLE } \\
\text { ID }\end{array}$ & $\begin{array}{l}\text { UNITS OF } \\
\text { MEASURE }\end{array}$ & PVI & $\begin{array}{l}\text { PARAMETER } \\
\text { VALUE FLAGS }\end{array}$ & $\begin{array}{l}\text { DETECTION } \\
\text { LIMIT }\end{array}$ & $\begin{array}{l}\text { PARAMETER } \\
\text { UNCERTAINTY }\end{array}$ \\
\hline THORIUH-230 & $\begin{array}{l}10 / 03 / 85 \\
10 / 03 / 85 \\
10 / 03 / 85 \\
10 / 03 / 85 \\
07 / 15 / 88 \\
01 / 07 / 89 \\
09 / 19 / 89\end{array}$ & $\begin{array}{l}0002 \\
0003 \\
0004 \\
0005 \\
0001 \\
0001 \\
0001\end{array}$ & $\mathrm{PCI} / \mathrm{L}$ & & $\begin{array}{l}0.1 \\
0.4 \\
1.1 \\
2.5 \\
0.4 \\
0.790 \\
0.1\end{array}$ & $\begin{array}{l}1 . \\
1 . \\
1 . \\
1 . \\
1 . \\
1 .\end{array}$ & $\begin{array}{l}0.3 \\
0.4 \\
0.7 \\
1.4 \\
0.5 \\
0.290 \\
0.4\end{array}$ \\
\hline TIN & $\begin{array}{l}10 / 03 / 85 \\
10 / 03 / 85 \\
10 / 03 / 85 \\
10 / 03 / 85 \\
10 / 03 / 85 \\
07 / 15 / 88 \\
01 / 07 / 89 \\
09 / 19 / 89 \\
03 / 31 / 90 \\
01 / 16 / 91\end{array}$ & $\begin{array}{l}0001 \\
0002 \\
0003 \\
0004 \\
0005 \\
0001 \\
0001 \\
0001 \\
0001 \\
0001\end{array}$ & $M G / L$ & $\begin{array}{l}< \\
< \\
< \\
< \\
< \\
< \\
<\end{array}$ & $\begin{array}{l}0.005 \\
0.005 \\
0.005 \\
0.005 \\
0.005 \\
0.013 \\
0.005 \\
0.096 \\
0.007 \\
0.03\end{array}$ & $\begin{array}{l}0.005 \\
0.005 \\
0.005 \\
0.005 \\
0.005 \\
0.005 \\
0.005 \\
0.005 \\
0.005 \\
0.03\end{array}$ & $\begin{array}{l}- \\
- \\
- \\
- \\
- \\
- \\
- \\
- \\
- \\
-\end{array}$ \\
\hline TOTAL DISSOLVED SOLIDS & $\begin{array}{l}10 / 03 / 85 \\
10 / 03 / 85 \\
10 / 03 / 85 \\
10 / 03 / 85 \\
10 / 03 / 85 \\
05 / 14 / 86 \\
01 / 09 / 87 \\
07 / 15 / 88 \\
01 / 07 / 89 \\
09 / 19 / 89 \\
03 / 31 / 90 \\
01 / 16 / 91 \\
11 / 24 / 92\end{array}$ & $\begin{array}{l}0001 \\
0002 \\
0003 \\
0004 \\
0005 \\
0001 \\
0001 \\
0001 \\
0001 \\
0001 \\
0001 \\
0001 \\
0001\end{array}$ & MG/L & & $\begin{array}{l}5250 . \\
5290 . \\
5270 . \\
5240 . \\
5330 . \\
5230 . \\
5440 . \\
5380 . \\
5428 . \\
5060 \\
4880 \\
5390 \\
5540\end{array}$ & $\begin{array}{l}10 . \\
10 . \\
10 . \\
10 . \\
10 . \\
10 . \\
10 . \\
10 . \\
10 . \\
10 . \\
10 . \\
10 . \\
10 .\end{array}$ & $\begin{array}{l}- \\
- \\
- \\
- \\
- \\
- \\
- \\
- \\
- \\
- \\
- \\
- \\
-\end{array}$ \\
\hline TOTAL KJELDAHL NITROGEN & $\begin{array}{l}01 / 16 / 91 \\
11 / 24 / 92\end{array}$ & $\begin{array}{l}0001 \\
0001\end{array}$ & $M G / L$ & & 1. & $\begin{array}{l}1 . \\
1 .\end{array}$ & - \\
\hline TOTAL ORGAHIC CARBON & $\begin{array}{l}10 / 03 / 85 \\
10 / 03 / 85 \\
10 / 03 / 85 \\
07 / 15 / 88 \\
01 / 07 / 89 \\
09 / 19 / 89 \\
03 / 31 / 90 \\
01 / 16 / 91 \\
11 / 24 / 92\end{array}$ & $\begin{array}{l}0002 \\
0003 \\
0005 \\
0001 \\
0001 \\
0001 \\
0001 \\
0001 \\
0001\end{array}$ & MG $/ L$ & & $\begin{array}{c}83 . \\
83 . \\
83 . \\
118 . \\
11 . \\
136 . \\
13.0 \\
10 . \\
14 .\end{array}$ & $\begin{array}{l}1 . \\
1 . \\
1 . \\
1 . \\
1 . \\
1 . \\
1 . \\
1 . \\
1 .\end{array}$ & $\begin{array}{l}- \\
- \\
- \\
- \\
- \\
- \\
-\end{array}$ \\
\hline URANIUM & $\begin{array}{l}10 / 03 / 85 \\
10 / 03 / 85 \\
10 / 03 / 85 \\
10 / 03 / 85 \\
10 / 03 / 85 \\
05 / 14 / 86 \\
01 / 09 / 87 \\
07 / 15 / 88 \\
01 / 07 / 89 \\
09 / 19 / 89\end{array}$ & $\begin{array}{l}0001 \\
0002 \\
0003 \\
0004 \\
0005 \\
0001 \\
0001 \\
0001 \\
0001 \\
0001\end{array}$ & $M G / L$ & & $\begin{array}{l}0.721 \\
0.775 \\
0.721 \\
0.695 \\
0.475 \\
0.699 \\
1.18 \\
1.42 \\
1.350 \\
1.23\end{array}$ & $\begin{array}{l}0.003 \\
0.003 \\
0.003 \\
0.003 \\
0.003 \\
0.003 \\
0.003 \\
0.003 \\
0.003 \\
0.003\end{array}$ & $\begin{array}{l}- \\
- \\
- \\
- \\
- \\
- \\
-\end{array}$ \\
\hline
\end{tabular}

PARAMETER VALUE INDICATOR (PVI): < - LESS THAN DETECTION LIMIT

SAMPLE ID COOES:

OTHER PARAMETER VALUE FLAGS:

I - INCREASED DETECTION LIMIT DUE TO REQUIRED DILUTIOH

0001 - FILTERED SAMPLE (.45 MICRONS)

0002 - FILTERED REPLICATE SAMPLE (.45 MICRONS)

0003 - FILTERED REPLICATE SAMPLE (.45 MICRONS)

$J$ - estimated VALUE

0004 - FILTERED REPLICATE SAMPLE (.45 MICROHS) 
GROUHDUATER QUALITY DATA BY LOCATION

SITE: AMBO1 AMBROSIA LAKE

LOCATION: 0786

NORTH COORDIHATE: 52888.5 FT

EAST COORDINATE: $\quad 59760.9$ FT

$06 / 10 / 80$ TO $12 / 04 / 92$

REPORT DATE: 08/04/94

FORMATION OF COMPLETION: TRES HERMANOS - CI SAMDSTONE (TO)

HYORAULIC FLOW RELATIONSHIP: DONH GRADIENT (D)

\begin{tabular}{|c|c|c|c|c|c|c|c|c|}
\hline PARAMETER NAME & LOG DATE & $\begin{array}{c}\text { SAMPLE } \\
\text { ID }\end{array}$ & $\begin{array}{l}\text { UHITS OF } \\
\text { MEASURE }\end{array}$ & PVI & $\begin{array}{l}\text { PARAMETER } \\
\text { VALUE }\end{array}$ & FLAGS & $\begin{array}{l}\text { DETECTION } \\
\text { LIMIT }\end{array}$ & $\begin{array}{l}\text { PARAMETER } \\
\text { UNCERTAINTY }\end{array}$ \\
\hline URAKIUM & $\begin{array}{l}03 / 31 / 90 \\
01 / 16 / 91 \\
11 / 24 / 92\end{array}$ & $\begin{array}{l}0001 \\
0001 \\
0001\end{array}$ & MG/L & & $\begin{array}{l}1.88 \\
2.88 \\
2.51\end{array}$ & H & $\begin{array}{l}0.003 \\
0.001 \\
0.001\end{array}$ & : \\
\hline VANADIUM & $\begin{array}{l}10 / 03 / 85 \\
10 / 03 / 85 \\
10 / 03 / 85 \\
10 / 03 / 85 \\
10 / 03 / 85 \\
05 / 14 / 86 \\
01 / 09 / 87 \\
07 / 15 / 88 \\
01 / 07 / 89 \\
09 / 19 / 89 \\
03 / 31 / 90 \\
01 / 16 / 91 \\
11 / 24 / 92\end{array}$ & $\begin{array}{l}0001 \\
0002 \\
0003 \\
0004 \\
0005 \\
0001 \\
0001 \\
0001 \\
0001 \\
0001 \\
0001 \\
0001 \\
0001\end{array}$ & $M G / L$ & $\begin{array}{l}< \\
< \\
< \\
< \\
< \\
< \\
< \\
<\end{array}$ & $\begin{array}{l}0.01 \\
0.01 \\
0.01 \\
0.05 \\
0.1 \\
0.42 \\
0.01 \\
0.06 \\
0.01 \\
0.02 \\
0.01 \\
0.01 \\
0.05\end{array}$ & I & $\begin{array}{l}0.01 \\
0.01 \\
0.01 \\
0.01 \\
0.1 \\
0.01 \\
0.01 \\
0.01 \\
0.01 \\
0.01 \\
0.01 \\
0.01 \\
0.05\end{array}$ & $\begin{array}{l}- \\
: \\
: \\
: \\
- \\
- \\
- \\
-\end{array}$ \\
\hline
\end{tabular}

PARAMETER VALUE IHDICATOR (PVI): < - LESS THAN DETECTION LIMIT

SAMPLE ID COOES:

0001 - FILTERED SAMPLE (.45 MICRONS)

OTHER PARAMETER VALUE FLAGS:

0002 - FILTERED REPLICATE SAMPLE (.45 MICRONS)

H - HOLD TIME EXPIRED, VALUE SUSPECT

0003 - FILTERED REPLICATE SAMPLE (.45 MICROHS)

I - IHCREASED DETECTIOH LIMIT DUE TO REQUIRED DILUTIOH

0004 - FILTERED REPLICATE SAMPLE (.45 MICRONS) 
GROUNDHATER QUALITY DATA BY LOCATION

SITE: AMBOI AMBROSIA LAKE

LOCATION: 0787

NORTH COORDINATE: 52842.6 FT

EAST COORDINATE: 61202.9 FT

06/10/80 TO $12 / 04 / 92$

REPORT DATE: $08 / 04 / 94$

FORMATION OF COMPLETION: TRES HERMANOS - C2 SANDSTONE (TT) HYORAULIC FLON RELATIONSHIP: DONN GRADIENT (D)

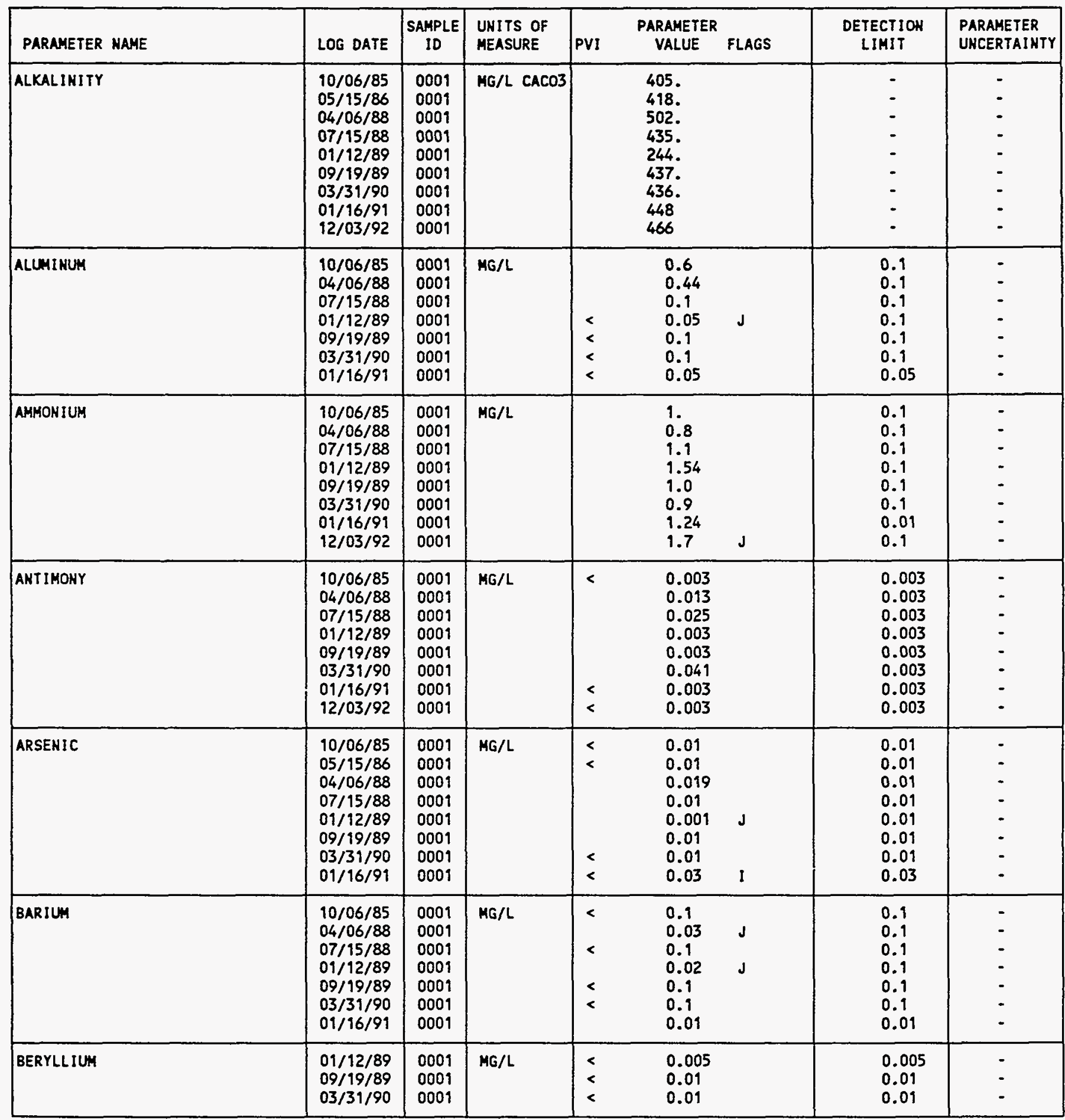

PARAMETER VALUE INDICATOR (PVI): < - LESS THAN DETECTION LIMIT

OTHER PARAMETER VALUE FLAGS:

$I$ - INCREASED DETECTION LIMIT DUE TO. REQUIRED OILUTION

$\downarrow$ - estiMATED VALUE 
GROUHDHATER QUALITY DATA BY LOCATIOH

SITE: AMBO1 AMBROSIA LAKE

LOCATION: 0787

MORTH COORDIHATE: 52842.6 FT

EAST COORDINATE: $\quad 61202.9$ FT

06/10/80 TO 12/04/92

REPORT DATE: 08/04/94

FORMATION OF COMPLETION: TRES HERMANOS - C2 SAMDSTONE (TT) HYORAULIC FLON RELATIONSHIP: DOWN GRADIENT (D)

\begin{tabular}{|c|c|c|c|c|c|c|c|}
\hline PARAMETER hAME & LOG DATE & $\begin{array}{c}\text { SAMPLE } \\
\text { ID }\end{array}$ & $\begin{array}{l}\text { UNITS OF } \\
\text { MEASURE }\end{array}$ & PVI & $\begin{array}{l}\text { PARAMETER } \\
\text { VALUE FLAGS }\end{array}$ & $\begin{array}{l}\text { DETECTIOH } \\
\text { LIMIT }\end{array}$ & $\begin{array}{l}\text { PARAMETER } \\
\text { UNCERTAINTY }\end{array}$ \\
\hline BERYLLIUY & $01 / 16 / 91$ & 0001 & $M G / L$ & $<$ & 0.005 & 0.005 & - \\
\hline BORON & $\begin{array}{l}10 / 06 / 85 \\
05 / 15 / 86 \\
04 / 06 / 88 \\
07 / 15 / 88 \\
01 / 12 / 89 \\
09 / 19 / 89 \\
03 / 31 / 90 \\
01 / 16 / 91\end{array}$ & $\begin{array}{l}0001 \\
0001 \\
0001 \\
0009 \\
0009 \\
0001 \\
0001 \\
0001\end{array}$ & MG/L & & $\begin{array}{l}0.5 \\
0.6 \\
0.78 \\
0.6 \\
0.45 \\
0.6 \\
0.5 \\
0.56\end{array}$ & $\begin{array}{l}0.1 \\
0.1 \\
0.1 \\
0.1 \\
0.1 \\
0.1 \\
0.1 \\
0.05\end{array}$ & $\begin{array}{l}- \\
- \\
: \\
: \\
-\end{array}$ \\
\hline BROAIDE & $\begin{array}{l}04 / 06 / 88 \\
07 / 15 / 88 \\
01 / 12 / 89 \\
09 / 19 / 89 \\
03 / 31 / 90 \\
01 / 16 / 91\end{array}$ & $\begin{array}{l}0001 \\
0001 \\
0001 \\
0001 \\
0001 \\
0001\end{array}$ & MG/L & $<$ & $\begin{array}{l}0.2 \\
0.1 \\
0.14 \\
0.1 \\
0.6 \\
0.2\end{array}$ & $\begin{array}{l}0.1 \\
0.1 \\
0.01 \\
0.1 \\
0.1 \\
0.1\end{array}$ & $\begin{array}{l}- \\
- \\
- \\
-\end{array}$ \\
\hline CADMIU: & $\begin{array}{l}10 / 06 / 85 \\
05 / 15 / 86 \\
04 / 06 / 88 \\
07 / 15 / 88 \\
01 / 12 / 89 \\
09 / 19 / 89 \\
03 / 31 / 90 \\
01 / 16 / 91 \\
12 / 03 / 92\end{array}$ & $\begin{array}{l}0001 \\
0001 \\
0001 \\
0001 \\
0001 \\
0001 \\
0001 \\
0001 \\
0001\end{array}$ & $M G / L$ & $\begin{array}{l}< \\
< \\
< \\
< \\
< \\
< \\
<\end{array}$ & $\begin{array}{l}0.001 \\
0.001 \\
0.007 \\
0.001 \\
0.0001 \quad \mathrm{~J} \\
0.012 \\
0.001 \\
0.001 \\
0.001\end{array}$ & $\begin{array}{l}0.001 \\
0.001 \\
0.001 \\
0.001 \\
0.001 \\
0.001 \\
0.001 \\
0.001 \\
0.001\end{array}$ & $\begin{array}{l}- \\
- \\
- \\
- \\
- \\
- \\
-\end{array}$ \\
\hline CALCIUM & $\begin{array}{l}10 / 06 / 85 \\
05 / 15 / 86 \\
04 / 06 / 88 \\
07 / 15 / 88 \\
01 / 12 / 89 \\
09 / 19 / 89 \\
03 / 31 / 90 \\
01 / 16 / 91 \\
12 / 03 / 92\end{array}$ & $\begin{array}{l}0001 \\
0001 \\
0001 \\
0001 \\
0001 \\
0001 \\
0001 \\
0001 \\
0001\end{array}$ & $M G / L$ & & $\begin{array}{l}351 . \\
308 . \\
423 . \\
290 . \\
129 . \\
311 . \\
296 . \\
335 . \\
331 .\end{array}$ & $\begin{array}{l}0.01 \\
0.01 \\
0.01 \\
0.01 \\
0.01 \\
0.01 \\
0.01 \\
0.5 \\
0.5\end{array}$ & $\begin{array}{l}- \\
- \\
- \\
- \\
- \\
-\end{array}$ \\
\hline CHLORIDE & $\begin{array}{l}10 / 06 / 85 \\
05 / 15 / 86 \\
04 / 06 / 88 \\
07 / 15 / 88 \\
01 / 12 / 89 \\
09 / 19 / 89 \\
03 / 31 / 90 \\
01 / 16 / 91 \\
12 / 03 / 92\end{array}$ & $\begin{array}{l}0001 \\
0001 \\
0001 \\
0001 \\
0001 \\
0001 \\
0001 \\
0001 \\
0001\end{array}$ & $M G / L$ & & $\begin{array}{c}2.8 \\
11 . \\
11 . \\
11 . \\
13 . \\
8.6 \\
12 . \\
12.6 \\
11.2\end{array}$ & $\begin{array}{l}1 . \\
1 . \\
1 . \\
1 . \\
1 . \\
1 . \\
0.5 \\
0.5\end{array}$ & $\begin{array}{l}- \\
- \\
- \\
- \\
- \\
-\end{array}$ \\
\hline CHROMIUM & $\begin{array}{l}10 / 06 / 85 \\
05 / 15 / 86 \\
04 / 06 / 88 \\
07 / 15 / 88 \\
01 / 12 / 89 \\
09 / 19 / 89 \\
03 / 31 / 90 \\
01 / 16 / 91 \\
12 / 03 / 92\end{array}$ & $\begin{array}{l}0001 \\
0001 \\
0001 \\
0001 \\
0001 \\
0001 \\
0001 \\
0001 \\
0001\end{array}$ & MG/L & $\begin{array}{l}< \\
< \\
< \\
<\end{array}$ & $\begin{array}{l}0.04 \\
0.03 \\
0.23 \\
0.10 \\
0.01 \\
0.01 \\
0.01 \\
0.01 \\
0.01\end{array}$ & $\begin{array}{l}0.01 \\
0.01 \\
0.01 \\
0.01 \\
0.01 \\
0.01 \\
0.01 \\
0.01 \\
0.01\end{array}$ & $\begin{array}{l}- \\
- \\
- \\
- \\
- \\
- \\
-\end{array}$ \\
\hline
\end{tabular}

PARAMETER VALUE IHDICATOR (PVI): < - LESS THAN DETECTIOH LIMIT

SAMPLE ID COOES:

0001 - FILTERED SAMPLE (.45 HICRONS)

OTHER PARAMETER VALUE FLAGS:

$J$ - ESTIMATED VALUE 
GROUNDWATER OUALITY DATA BY LOCATION

SITE: AMB01 AMBROSIA LAKE

LOCATION: 0787

MORTH COORDINATE: 52842.6 FT

EAST COORDINATE: $\quad 61202.9$ FT

06/10/80 TO $12 / 04 / 92$

REPORT DATE: $08 / 04 / 94$

FORMATION OF COMPLETION: TRES HERMANOS - C2 SANDSTONE (TT)

HYDRAULIC FLON RELATIONSKIP: DOWN GRADIENT (D)

\begin{tabular}{|c|c|c|c|c|c|c|c|c|}
\hline PARAMETER NAME & LOG DATE & $\begin{array}{c}\text { SAMPLE } \\
\text { ID }\end{array}$ & $\begin{array}{l}\text { UNITS OF } \\
\text { MEASURE }\end{array}$ & PVI & $\begin{array}{c}\text { PARAMETER } \\
\text { VALUE }\end{array}$ & FLAGS & $\begin{array}{c}\text { DETECT ION } \\
\text { LIHIT }\end{array}$ & $\begin{array}{l}\text { PARAMETER } \\
\text { UNCERTAIHTY }\end{array}$ \\
\hline $\cos A L T$ & $\begin{array}{l}10 / 06 / 85 \\
05 / 15 / 86 \\
04 / 06 / 88 \\
07 / 15 / 88 \\
01 / 12 / 89 \\
09 / 19 / 89 \\
03 / 31 / 90 \\
01 / 16 / 91 \\
12 / 03 / 92\end{array}$ & $\begin{array}{l}0001 \\
0001 \\
0001 \\
0001 \\
0001 \\
0001 \\
0001 \\
0001 \\
0001\end{array}$ & $M G / L$ & $\begin{array}{l}< \\
< \\
< \\
< \\
< \\
< \\
< \\
<\end{array}$ & $\begin{array}{l}0.05 \\
0.05 \\
0.10 \\
0.05 \\
0.01 \\
0.05 \\
0.05 \\
0.03 \\
0.03\end{array}$ & $J$ & $\begin{array}{l}0.05 \\
0.05 \\
0.05 \\
0.05 \\
0.05 \\
0.05 \\
0.05 \\
0.03 \\
0.03\end{array}$ & $\begin{array}{l}- \\
- \\
- \\
- \\
- \\
- \\
-\end{array}$ \\
\hline COPPER & $\begin{array}{l}10 / 06 / 85 \\
04 / 06 / 88 \\
07 / 15 / 88 \\
01 / 12 / 89 \\
09 / 19 / 89 \\
03 / 31 / 90 \\
01 / 16 / 91\end{array}$ & $\begin{array}{l}0001 \\
0001 \\
0001 \\
0001 \\
0001 \\
0001 \\
0001\end{array}$ & $M G / L$ & $\begin{array}{l}< \\
< \\
< \\
< \\
<\end{array}$ & $\begin{array}{l}0.03 \\
0.04 \\
0.02 \\
0.01 \\
0.02 \\
0.02 \\
0.01\end{array}$ & $\mathbf{J}$ & $\begin{array}{l}0.02 \\
0.02 \\
0.02 \\
0.02 \\
0.02 \\
0.02 \\
0.01\end{array}$ & $\begin{array}{l}- \\
- \\
- \\
- \\
- \\
-\end{array}$ \\
\hline CYANIDE & $\begin{array}{l}10 / 06 / 85 \\
01 / 12 / 89 \\
09 / 19 / 89 \\
03 / 31 / 90 \\
01 / 16 / 91\end{array}$ & $\begin{array}{l}0001 \\
0001 \\
0001 \\
0001 \\
0001\end{array}$ & $M G / L$ & $\begin{array}{l}< \\
< \\
< \\
<\end{array}$ & $\begin{array}{l}0.01 \\
0.002 \\
0.01 \\
0.01 \\
0.01\end{array}$ & J & $\begin{array}{l}0.01 \\
0.01 \\
0.01 \\
0.01 \\
0.01\end{array}$ & $\begin{array}{l}- \\
- \\
- \\
-\end{array}$ \\
\hline FLUORIDE & $\begin{array}{l}10 / 06 / 85 \\
05 / 15 / 86 \\
04 / 06 / 88 \\
07 / 15 / 88 \\
01 / 12 / 89 \\
09 / 19 / 89 \\
03 / 31 / 90 \\
01 / 16 / 91 \\
12 / 03 / 92\end{array}$ & $\begin{array}{l}0001 \\
0001 \\
0001 \\
0001 \\
0001 \\
0001 \\
0001 \\
0001 \\
0001\end{array}$ & $M G / L$ & & $\begin{array}{l}0.5 \\
0.5 \\
0.30 \\
0.3 \\
0.2 \\
0.2 \\
0.3 \\
0.3 \\
0.3\end{array}$ & & $\begin{array}{l}0.1 \\
0.1 \\
0.1 \\
0.1 \\
0.1 \\
0.1 \\
0.1 \\
0.1 \\
0.1\end{array}$ & $\begin{array}{l}- \\
- \\
- \\
- \\
- \\
- \\
-\end{array}$ \\
\hline GROSS ALPHA & $\begin{array}{l}04 / 06 / 88 \\
07 / 15 / 88 \\
01 / 12 / 89 \\
09 / 19 / 89 \\
03 / 31 / 90 \\
01 / 16 / 91 \\
12 / 03 / 92\end{array}$ & $\begin{array}{l}0001 \\
0001 \\
0001 \\
0001 \\
0001 \\
0001 \\
0001\end{array}$ & PCI /L & & $\begin{array}{c}5 . \\
13 . \\
33.1 \\
0 . \\
8 . \\
57.4 \\
7.0\end{array}$ & $\mathbf{J}$ & $\begin{array}{l}0.2 \\
0.2 \\
1 . \\
1 . \\
1 . \\
1 . \\
1.0\end{array}$ & $\begin{array}{l}26 . \\
28 . \\
40.5 \\
14 . \\
16 . \\
36.6 \\
28.5\end{array}$ \\
\hline GROSS BETA & $\begin{array}{l}04 / 06 / 88 \\
07 / 15 / 88 \\
01 / 12 / 89 \\
09 / 19 / 89 \\
03 / 31 / 90 \\
01 / 16 / 91 \\
12 / 03 / 92\end{array}$ & $\begin{array}{l}0001 \\
0001 \\
0001 \\
0001 \\
0001 \\
0001 \\
0001\end{array}$ & $\mathrm{PCI} / \mathrm{L}$ & & $\begin{array}{r}44 . \\
30 . \\
138 . \\
31 . \\
24 . \\
50.3 \\
1.4\end{array}$ & & $\begin{array}{r}1 . \\
1 . \\
0.5 \\
0.5 \\
0.5 \\
0.5 \\
51.1\end{array}$ & $\begin{array}{l}17 . \\
12 . \\
52.0 \\
12 . \\
14 . \\
18.7 \\
30.4\end{array}$ \\
\hline IRON & $\begin{array}{l}10 / 06 / 85 \\
05 / 15 / 86 \\
04 / 06 / 88 \\
07 / 15 / 88 \\
01 / 12 / 89 \\
09 / 19 / 89 \\
03 / 31 / 90\end{array}$ & $\begin{array}{l}0001 \\
0001 \\
0001 \\
0001 \\
0001 \\
0001 \\
0001\end{array}$ & $M G / L$ & $<$ & $\begin{array}{l}0.18 \\
0.63 \\
1.29 \\
0.32 \\
0.02 \\
0.08 \\
0.23\end{array}$ & $\mathrm{~J}$ & $\begin{array}{l}0.03 \\
0.03 \\
0.03 \\
0.03 \\
0.03 \\
0.03 \\
0.03\end{array}$ & $\begin{array}{l}- \\
- \\
- \\
- \\
-\end{array}$ \\
\hline
\end{tabular}

PARAMETER VALUE INDICATOR (PVI): < - LESS THAN DETECTION LIMIT

SAMPLE 10 CODES:

0001 - FILTERED SAMPLE (.45 MICRONS)

OTHER PARAMETER VALUE FLAGS:

$J$ - ESTIMATED VALUE 
GROUNDHATER QUALITY DATA BY LOCATION

SITE: AMBO1 AMBROSIA LAKE

LOCATION: 0787

MORTH COORDIHATE: 52842.6 FT

EAST COORDINATE: 61202.9 FT

06/10/80 TO $12 / 04 / 92$

REPORT DATE: 08/04/94

FORHATION OF COMPLETION: TRES HERMANOS - C2 SANDSTONE (TT) HYDRAULIC FLON RELATIONSHIP: DOWM GRADIEHT (D)

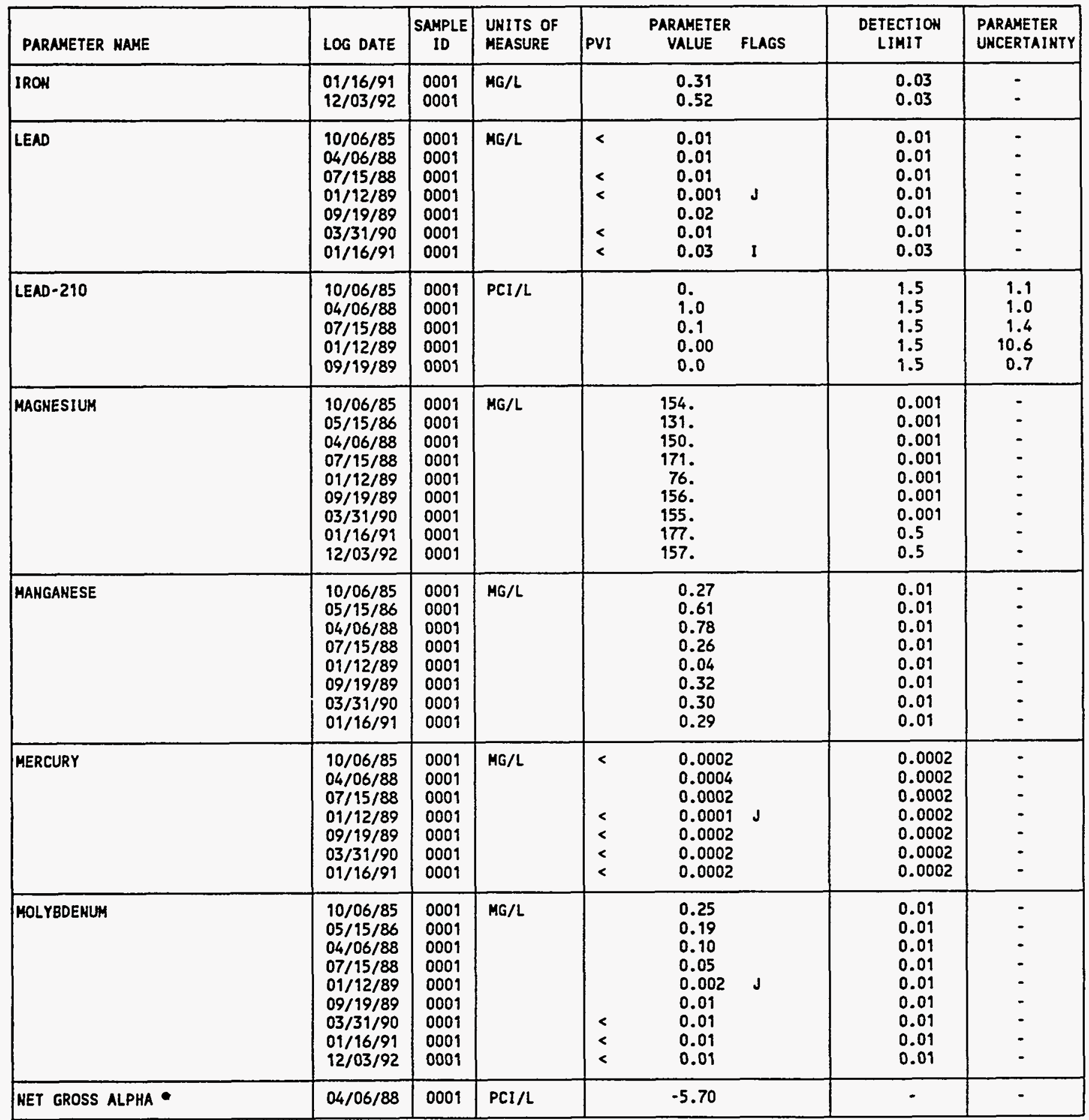

- het gRoss alPHa (GROSS ALPHA - URANIUM) WITH I MG URANIUM = 686 PCI

PARAMETER VALUE IHDICATOR (PVI): < - LESS THAN DETECTION LIMIT

OTHER PARAMETER VALUE FLAGS:

1 - INCREASED DETECTION LIMIT DUE TO REQUIRED DILUTIOH

J - ESTIMATED VALUE
SAMPLE ID COOES:

0001 - FILTERED SAMPLE (.45 MICRONS) 
GROUNDHATER QUALITY DATA BY LOCATION

SITE: AMB01 AMBROSIA LAKE

LOCATION: 0787

NORTH COORDINATE: 52842.6 FT

EAST COORDINATE: $\quad 61202.9$ FT

$06 / 10 / 80$ TO $12 / 04 / 92$

REPORT DATE: $08 / 04 / 94$

FORMATION OF COMPLETION: TRES HERMANOS - C2 SANDSTONE (TT) HYORAULIC FLOW RELATIONSHIP: DOWN GRADIENT (D)

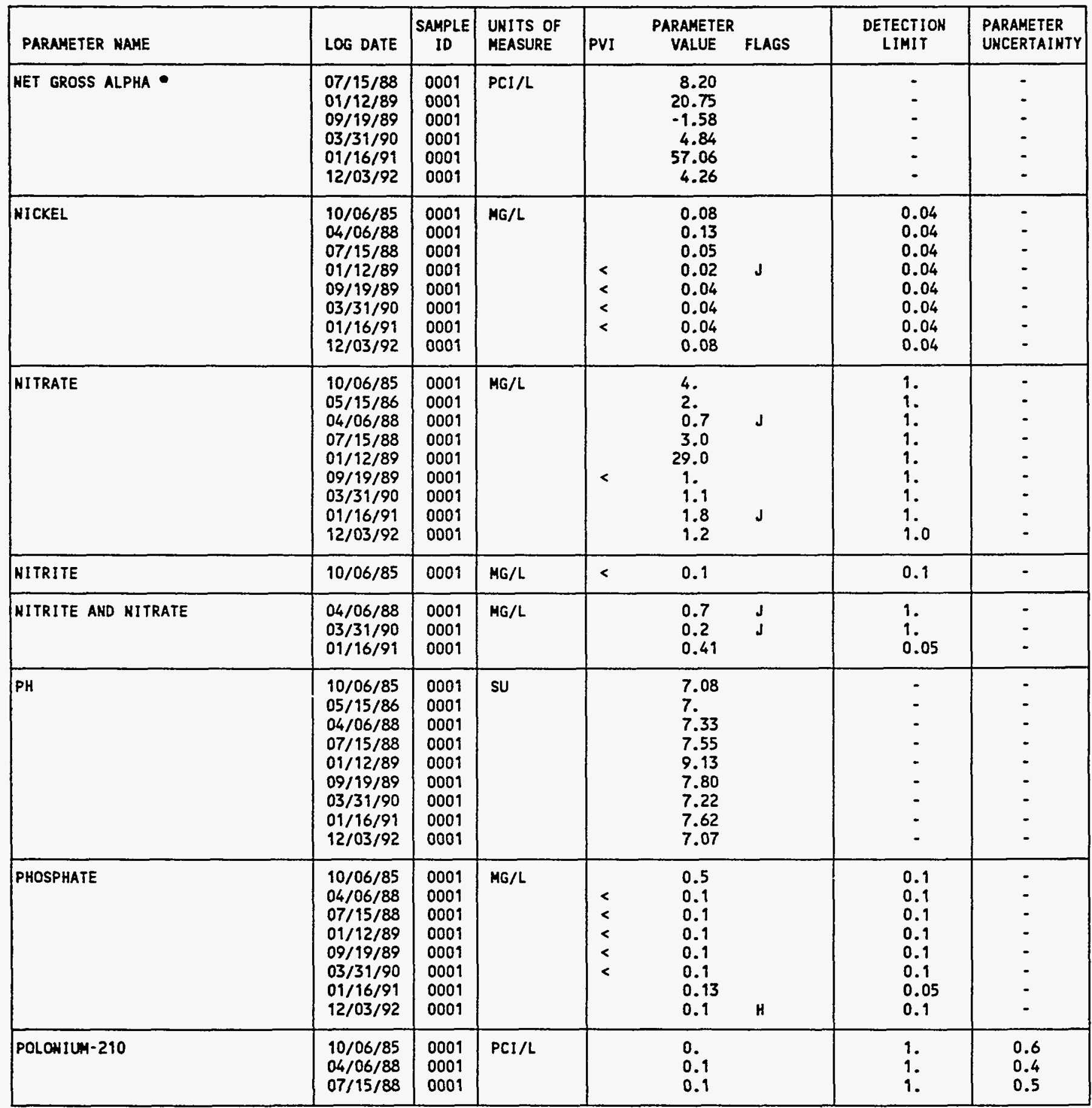

- net gross alpha (Gross alPha - URANIUM) WITH 1 Mg URANIUM = 686 PCI

PARAMETER VALUE INDICATOR (PVI): < - LESS than DETECTION LIMIT

OTHER PARAMETER VALUE FLAGS:

h - hOLD TIME EXPIRED, VALUE SUSPECT'

d - estimated value
SAMPLE ID COOES:

0001 - FILTERED SAMPLE (.45 MICRONS) 
GROUNDHATER QUALITY DATA BY LOCATION

SITE: AMBO1 AMBROSIA LAKE

LOCATIOH: 0787

HORTH COORDIHATE: 52842.6 FT

EAST COORDIHATE: $\quad 61202.9$ FT

06/10/80 TO $12 / 04 / 92$

REPORT DATE: 08/04/94

FORMATIOH OF COMPLETIOH: TRES HERMANOS - C2 SANDSTONE (TT) HYDRAULIC FLOW RELATIONSHIP: DONH GRADIENT (D)

\begin{tabular}{|c|c|c|c|c|c|c|c|c|}
\hline PARAMETER MAME & LOG DATE & $\begin{array}{c}\text { SAMPLE } \\
\text { ID }\end{array}$ & $\begin{array}{l}\text { UNITS OF } \\
\text { MEASURE }\end{array}$ & PVI & $\begin{array}{l}\text { PARAMETER } \\
\text { VALUE }\end{array}$ & FLAGS & $\begin{array}{l}\text { DETECTION } \\
\text { LIMIT }\end{array}$ & $\begin{array}{l}\text { PARAMETER } \\
\text { UNCERTAINTY }\end{array}$ \\
\hline POLONIUN-210 & $\begin{array}{l}01 / 12 / 89 \\
09 / 19 / 89\end{array}$ & $\begin{array}{l}0001 \\
0001\end{array}$ & PCI/L & & $\begin{array}{l}0 . \pi 7 \\
0.3\end{array}$ & J & $\begin{array}{l}1 . \\
1 .\end{array}$ & $\begin{array}{l}1.33 \\
0.4\end{array}$ \\
\hline POTASSIUA & $\begin{array}{l}10 / 06 / 85 \\
05 / 15 / 86 \\
04 / 06 / 88 \\
07 / 15 / 88 \\
01 / 12 / 89 \\
09 / 19 / 89 \\
03 / 31 / 90 \\
01 / 16 / 91 \\
12 / 03 / 92\end{array}$ & $\begin{array}{l}0001 \\
0001 \\
0001 \\
0001 \\
0001 \\
0001 \\
0001 \\
0001 \\
0001\end{array}$ & MG/L & & $\begin{array}{c}22.5 \\
23.1 \\
32.3 \\
17.4 \\
110 . \\
16.5 \\
23.6 \\
15 . \\
12 .\end{array}$ & & $\begin{array}{l}0.01 \\
0.01 \\
0.01 \\
0.01 \\
0.01 \\
0.01 \\
0.01 \\
5 . \\
5 .\end{array}$ & $\begin{array}{l}- \\
- \\
- \\
- \\
- \\
-\end{array}$ \\
\hline RADIUA-226 & $\begin{array}{l}10 / 06 / 85 \\
04 / 06 / 88 \\
07 / 15 / 88 \\
01 / 12 / 89 \\
09 / 19 / 89 \\
03 / 31 / 90 \\
01 / 16 / 91 \\
12 / 03 / 92\end{array}$ & $\begin{array}{l}0001 \\
0001 \\
0001 \\
0001 \\
0001 \\
0001 \\
0001 \\
0001\end{array}$ & PCI/L & & $\begin{array}{l}0.8 \\
0.5 \\
0.7 \\
1.56 \\
0.3 \\
0.3 \\
0.9 \\
1.8\end{array}$ & & $\begin{array}{l}1 . \\
1 . \\
1 . \\
1 . \\
1 . \\
1 . \\
1 . \\
0.3\end{array}$ & $\begin{array}{l}0.3 \\
0.2 \\
0.3 \\
0.250 \\
0.2 \\
0.2 \\
0.5 \\
0.5\end{array}$ \\
\hline RADIUA-226 + RADIUM-228 & $\begin{array}{l}10 / 06 / 85 \\
04 / 06 / 88 \\
07 / 15 / 88 \\
01 / 12 / 89 \\
09 / 19 / 89 \\
03 / 31 / 90 \\
01 / 16 / 91 \\
12 / 03 / 92\end{array}$ & $\begin{array}{l}0001 \\
0001 \\
0001 \\
0001 \\
0001 \\
0001 \\
0001 \\
0001\end{array}$ & PCI/L & & $\begin{array}{l}2.30 \\
1.50 \\
1.50 \\
1.56 \\
2.00 \\
1.20 \\
2.30 \\
4.20\end{array}$ & & $\begin{array}{l}- \\
- \\
- \\
- \\
- \\
-\end{array}$ & $\begin{array}{l}- \\
: \\
: \\
- \\
-\end{array}$ \\
\hline RADIUH-228 & $\begin{array}{l}10 / 06 / 85 \\
04 / 06 / 88 \\
07 / 15 / 88 \\
01 / 12 / 89 \\
09 / 19 / 89 \\
03 / 31 / 90 \\
01 / 16 / 91 \\
12 / 03 / 92\end{array}$ & $\begin{array}{l}0001 \\
0001 \\
0001 \\
0001 \\
0001 \\
0001 \\
0001 \\
0001\end{array}$ & $\mathrm{PCI} / \mathrm{L}$ & & $\begin{array}{l}1.5 \\
1.0 \\
0.8 \\
0.00 \\
1.7 \\
0.9 \\
1.4 \\
2.4\end{array}$ & H & $\begin{array}{l}1 . \\
1 . \\
1 . \\
1 . \\
1 . \\
1 . \\
1 . \\
1.0\end{array}$ & $\begin{array}{l}1.2 \\
0.8 \\
0.8 \\
5.34 \\
0.9 \\
0.9 \\
1.8 \\
0.8\end{array}$ \\
\hline SELEHIUM & $\begin{array}{l}10 / 06 / 85 \\
05 / 15 / 86 \\
04 / 06 / 88 \\
07 / 15 / 88 \\
01 / 12 / 89 \\
09 / 19 / 89 \\
03 / 31 / 90 \\
01 / 16 / 91 \\
12 / 03 / 92\end{array}$ & $\begin{array}{l}0001 \\
0001 \\
0001 \\
0001 \\
0001 \\
0001 \\
0001 \\
0001 \\
0001\end{array}$ & MG/L & $\begin{array}{l}< \\
< \\
< \\
< \\
< \\
<\end{array}$ & $\begin{array}{l}0.005 \\
0.005 \\
0.048 \\
0.054 \\
0.001 \\
0.015 \\
0.005 \\
0.03 \\
0.05\end{array}$ & I & $\begin{array}{l}0.005 \\
0.005 \\
0.005 \\
0.005 \\
0.005 \\
0.005 \\
0.005 \\
0.03 \\
0.05\end{array}$ & $\begin{array}{l}- \\
- \\
- \\
- \\
- \\
- \\
-\end{array}$ \\
\hline SILICA - SIO2 & $\begin{array}{l}10 / 06 / 85 \\
04 / 06 / 88 \\
07 / 15 / 88 \\
01 / 12 / 89 \\
09 / 19 / 89\end{array}$ & $\begin{array}{l}0001 \\
0001 \\
0001 \\
0001 \\
0001\end{array}$ & $M G / L$ & & $\begin{array}{c}16 . \\
17.2 \\
14.4 \\
5.9 \\
12 .\end{array}$ & & $\begin{array}{l}2 . \\
2 . \\
2 . \\
2 . \\
2 .\end{array}$ & : \\
\hline
\end{tabular}

PARAMETer VAlue indicator (PVI): < - LeSS than DETECTION LIMIT

OTHER PARAMETER VALUE FLAGS:

H - hOLD TIME EXPIRED, VALUE SUSPECT

I - INCREASED DETECTIOH LIMIT DUE TO.REQUIRED DILUTION

J - ESTIMATED VALUE 
GROUNDWATER QUALITY DATA BY LOCATION

SITE: AMBO1 AMBROSIA LAKE

LOCATION: 0787

NORTH COORDINATE: 52842.6 FT

EAST COORDINATE: 61202.9 FT

06/10/80 TO 12/04/92

REPORT DATE: 08/04/94

FORMATION OF COMPLETION: TRES HERMANOS - C2 SAKDSTONE (TT)

HYDRAULIC FLON RELATIONSHIP: DOWN GRADIENT (D)

\begin{tabular}{|c|c|c|c|c|c|c|c|}
\hline PARAMETER NAME & LOG DATE & $\begin{array}{c}\text { SAMPLE } \\
\text { ID }\end{array}$ & $\begin{array}{l}\text { UHITS OF } \\
\text { MEASURE }\end{array}$ & PVI & $\begin{array}{l}\text { PARAMETER } \\
\text { VALUE FLAGS }\end{array}$ & $\begin{array}{l}\text { DETECTION } \\
\text { LIMIT }\end{array}$ & $\begin{array}{l}\text { PARAMETER } \\
\text { UNCERTAINTY }\end{array}$ \\
\hline SILICA - SIO2 & $\begin{array}{l}03 / 31 / 90 \\
01 / 16 / 91\end{array}$ & $\begin{array}{l}0001 \\
0001\end{array}$ & MG/L & & 11.6 & 2.1 & - \\
\hline SILVER & $\begin{array}{l}10 / 06 / 85 \\
04 / 06 / 88 \\
07 / 15 / 88 \\
01 / 12 / 89 \\
09 / 19 / 89 \\
03 / 31 / 90 \\
01 / 16 / 91 \\
12 / 03 / 92\end{array}$ & $\begin{array}{l}0001 \\
0001 \\
0001 \\
0001 \\
0001 \\
0001 \\
0001 \\
0001\end{array}$ & MG/L & $\begin{array}{l}< \\
< \\
< \\
< \\
< \\
<\end{array}$ & $\begin{array}{l}0.01 \\
0.09 \\
0.01 \\
0.01 \\
0.01 \\
0.01 \\
0.01 \\
0.01\end{array}$ & $\begin{array}{l}0.01 \\
0.01 \\
0.01 \\
0.01 \\
0.01 \\
0.01 \\
0.01 \\
0.01\end{array}$ & $\begin{array}{l}- \\
- \\
- \\
- \\
- \\
- \\
-\end{array}$ \\
\hline SODIUM & $\begin{array}{l}10 / 06 / 85 \\
05 / 15 / 86 \\
04 / 06 / 88 \\
07 / 15 / 88 \\
01 / 12 / 89 \\
09 / 19 / 89 \\
03 / 31 / 90 \\
01 / 16 / 91 \\
12 / 03 / 92\end{array}$ & $\begin{array}{l}0001 \\
0001 \\
0001 \\
0001 \\
0001 \\
0001 \\
0001 \\
0001 \\
0001\end{array}$ & MG/L & & $\begin{array}{l}463 . \\
755 . \\
497 . \\
599 . \\
735 . \\
681 . \\
665 . \\
761 . \\
702 .\end{array}$ & $\begin{array}{l}0.002 \\
0.002 \\
0.002 \\
0.002 \\
0.002 \\
0.002 \\
0.002 \\
5 . \\
5 .\end{array}$ & $\begin{array}{l}- \\
- \\
- \\
- \\
- \\
- \\
-\end{array}$ \\
\hline SPECIFIC CONDUCTAHCE & $\begin{array}{l}10 / 06 / 85 \\
05 / 15 / 86 \\
04 / 06 / 88 \\
07 / 15 / 88 \\
01 / 12 / 89 \\
09 / 19 / 89 \\
03 / 31 / 90 \\
01 / 16 / 91 \\
12 / 03 / 92\end{array}$ & $\begin{array}{l}0001 \\
0001 \\
0001 \\
0001 \\
0001 \\
0001 \\
0001 \\
0001 \\
0001\end{array}$ & UMHO/CH & & $\begin{array}{c}3300 . \\
3200 \\
3100 \\
3200 \\
2800 \\
150 . \\
2400 . \\
3200 . \\
3570\end{array}$ & $\begin{array}{l}- \\
- \\
- \\
- \\
- \\
- \\
-\end{array}$ & $\begin{array}{l}- \\
- \\
- \\
- \\
. \\
- \\
-\end{array}$ \\
\hline STRONTIUM & $\begin{array}{l}10 / 06 / 85 \\
05 / 15 / 86 \\
04 / 06 / 88 \\
07 / 15 / 88 \\
01 / 12 / 89 \\
09 / 19 / 89 \\
03 / 31 / 90 \\
01 / 16 / 91\end{array}$ & $\begin{array}{l}0001 \\
0001 \\
0001 \\
0001 \\
0001 \\
0001 \\
0001 \\
0001\end{array}$ & $M G / L$ & & $\begin{array}{l}8.6 \\
0.7 \\
5.25 \\
6.9 \\
9.20 \\
8.1 \\
7.62 \\
8.20\end{array}$ & $\begin{array}{l}0.1 \\
0.1 \\
0.1 \\
0.1 \\
0.1 \\
0.1 \\
0.1 \\
0.01\end{array}$ & $\begin{array}{l}- \\
. \\
- \\
- \\
- \\
-\end{array}$ \\
\hline SULFATE & $\begin{array}{l}10 / 06 / 85 \\
05 / 15 / 86 \\
04 / 06 / 88 \\
07 / 15 / 88 \\
01 / 12 / 89 \\
09 / 19 / 89 \\
03 / 31 / 90 \\
01 / 16 / 91 \\
12 / 03 / 92\end{array}$ & $\begin{array}{l}0001 \\
0001 \\
0001 \\
0001 \\
0001 \\
0001 \\
0001 \\
0001 \\
0001\end{array}$ & $M G / L$ & & $\begin{array}{l}2250 . \\
2470 . \\
2240 . \\
2320 . \\
2120 . \\
2670 . \\
2620 . \\
2670 . \\
2530 .\end{array}$ & $\begin{array}{l}0.1 \\
0.1 \\
0.1 \\
0.1 \\
0.1 \\
0.1 \\
0.1 \\
10 . \\
10 .\end{array}$ & $\begin{array}{l}- \\
- \\
- \\
- \\
- \\
- \\
-\end{array}$ \\
\hline SULFIDE & $\begin{array}{l}10 / 06 / 85 \\
04 / 06 / 88 \\
07 / 15 / 88 \\
01 / 12 / 89 \\
09 / 19 / 89 \\
03 / 31 / 90 \\
01 / 16 / 91\end{array}$ & $\begin{array}{l}0001 \\
0001 \\
0001 \\
0001 \\
0001 \\
0001 \\
0001\end{array}$ & MG/L & $\begin{array}{l}< \\
< \\
< \\
< \\
<\end{array}$ & $\begin{array}{l}0.1 \\
0.1 \\
0.1 \\
0.13 \\
0.1 \\
0.1 \\
2.3\end{array}$ & $\begin{array}{l}0.1 \\
0.1 \\
0.1 \\
0.1 \\
0.1 \\
0.1 \\
0.1\end{array}$ & $\begin{array}{l}- \\
- \\
- \\
- \\
- \\
-\end{array}$ \\
\hline
\end{tabular}

PARAMETER VALUE INDICATOR (PVI): < - LESS THAN DETECTIOH LIMIT

SAMPLE IO COOES:

0001 - FILTERED SAMPLE (.45 MICRONS)

OTHER PARAMETER VALUE FLAGS:

J ESTIMATED VALUE 
REPORT DATE: 08/04/94

FORMATIOH OF COMPLETIOH: TRES HERMANOS - C2 SAMDSTOHE (TT) HYDRAULIC FLOW RELATIONSHIP: DOWN GRADIENT (D)

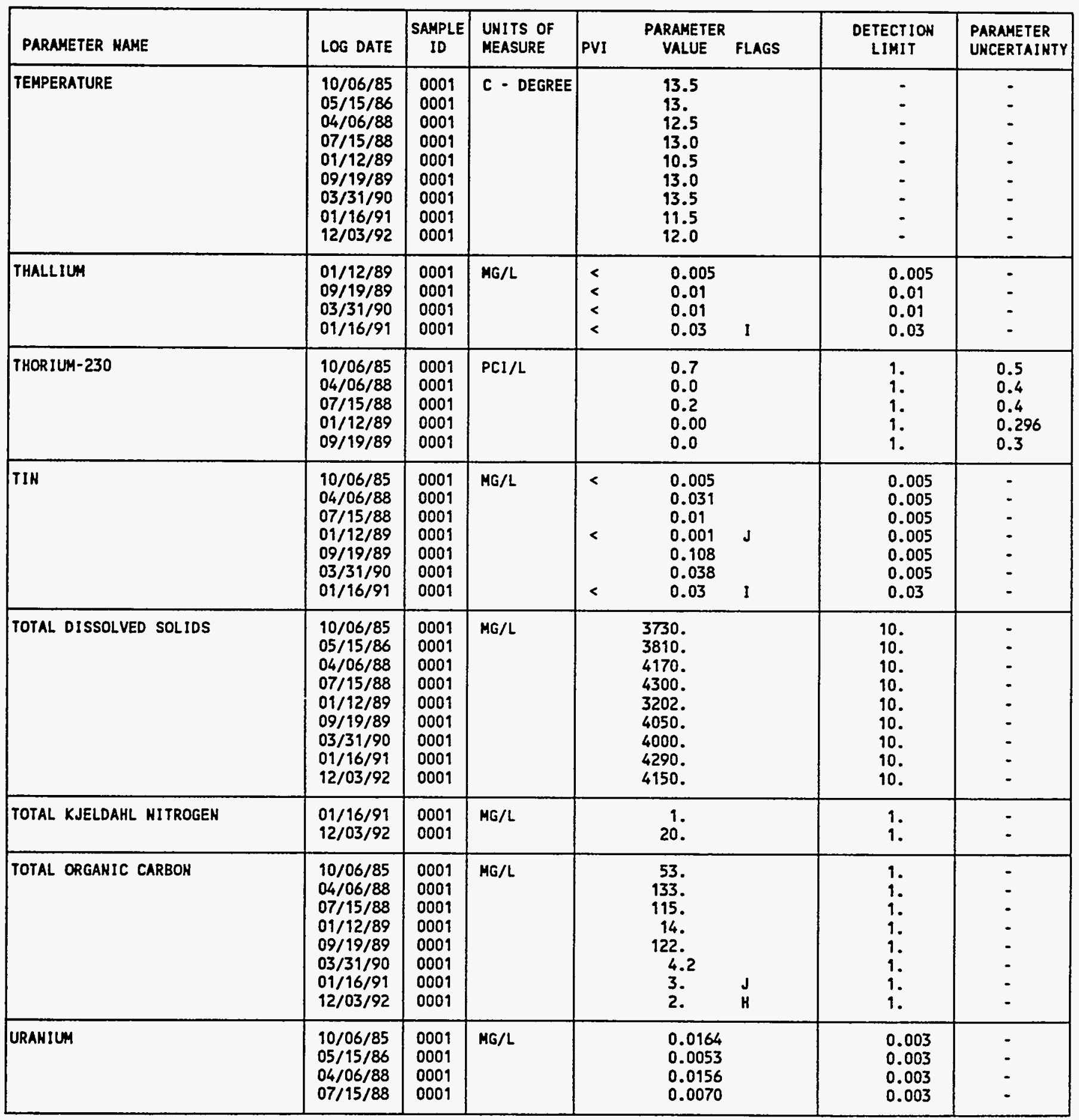

PARAMETER VALUE IMDICATOR (PVI): < - LESS THAN DETECTION LIMIT

SAMPLE ID CODES:

0001 - FILTERED SAMPLE (.45 MICRONS)

OTHER PARAMETER VALUE FLAGS:

H - HOLD TIME EXPIRED, VALUE SUSPECT

1 - INCREASED DETECTION LIMIT DUE TO-REQUIRED DILUTION

$J$ - eSTIMATED VALUE 
GROUNDWATER QUALITY DATA BY LOCATION

SITE: AMBOI AMBROSIA LAKE

LOCATION: 0787

NORTH COOROINATE: 52842.6 FT

EAST COORDINATE: $\quad 61202.9$ FT

06/10/80 TO $12 / 04 / 92$

REPORT DATE: 08/04/94

FORMATIOH OF COMPLETION: TRES HERMANOS - C2 SANDSTONE (TT) HYDRAULIC FLOW RELATIONSHIP: DONN GRADIENT (D)

\begin{tabular}{|c|c|c|c|c|c|c|c|}
\hline PARAMETER NAME & LOG DATE & $\begin{array}{c}\text { SAMPLE } \\
\text { ID }\end{array}$ & $\begin{array}{l}\text { UHITS OF } \\
\text { MEASURE }\end{array}$ & PVI & $\begin{array}{l}\text { PARAMETER } \\
\text { VALUE FLAGS }\end{array}$ & $\begin{array}{l}\text { DETECTION } \\
\text { LIMIT }\end{array}$ & $\begin{array}{l}\text { PARAMETER } \\
\text { UNCERTAINTY }\end{array}$ \\
\hline URAKIUM & $\begin{array}{l}01 / 12 / 89 \\
09 / 19 / 89 \\
03 / 31 / 90 \\
01 / 16 / 91 \\
12 / 03 / 92\end{array}$ & $\begin{array}{l}0001 \\
0001 \\
0001 \\
0001 \\
0001\end{array}$ & $M G / L$ & $<$ & $\begin{array}{l}0.018 \\
0.0023 \quad J \\
0.0046 \\
0.001 \\
0.004\end{array}$ & $\begin{array}{l}0.003 \\
0.003 \\
0.003 \\
0.001 \\
0.001\end{array}$ & $\begin{array}{l}- \\
- \\
- \\
-\end{array}$ \\
\hline VAKADIUM & $\begin{array}{l}10 / 06 / 85 \\
05 / 15 / 86 \\
04 / 06 / 88 \\
07 / 15 / 88 \\
01 / 12 / 89 \\
09 / 19 / 89 \\
03 / 31 / 90 \\
01 / 16 / 91 \\
12 / 03 / 92\end{array}$ & $\begin{array}{l}0001 \\
0001 \\
0001 \\
0001 \\
0001 \\
0001 \\
0001 \\
0001 \\
0001\end{array}$ & $M G / L$ & $\begin{array}{l}< \\
< \\
< \\
< \\
< \\
<\end{array}$ & $\begin{array}{l}0.01 \\
0.29 \\
0.12 \\
0.04 \\
0.01 \\
0.01 \\
0.01 \\
0.01 \\
0.01\end{array}$ & $\begin{array}{l}0.01 \\
0.01 \\
0.01 \\
0.01 \\
0.01 \\
0.01 \\
0.01 \\
0.01 \\
0.01\end{array}$ & $\begin{array}{l}- \\
- \\
- \\
- \\
- \\
- \\
-\end{array}$ \\
\hline ZINC & $\begin{array}{l}10 / 06 / 85 \\
04 / 06 / 88 \\
07 / 15 / 88 \\
01 / 12 / 89 \\
09 / 19 / 89 \\
03 / 31 / 90 \\
01 / 16 / 91\end{array}$ & $\begin{array}{l}0001 \\
0001 \\
0001 \\
0001 \\
0001 \\
0001 \\
0001\end{array}$ & $M G / L$ & $<$ & $\begin{array}{l}0.061 \\
0.034 \\
0.005 \\
0.01 \\
0.028 \\
0.022 \\
0.014\end{array}$ & $\begin{array}{l}0.005 \\
0.005 \\
0.005 \\
0.01 \\
0.005 \\
0.005 \\
0.005\end{array}$ & $\begin{array}{l}- \\
- \\
- \\
- \\
-\end{array}$ \\
\hline
\end{tabular}

PARAMETER VALUE INDICATOR (PVI):

SAMPLE ID COOES:

0001 - FILTERED SAMPLE (.45 MICRONS)

OTHER PARAMETER VALUE FLAGS:

$J$ - ESTIMATED VALUE 
GROUNDWATER QUALITY DATA BY LOCATION

SITE: AMBO1 AMBROSIA LAKE

LOCATION: 0791

NORTH COORDINATE: 55739.9 FT

EAST COORDIHATE: $\quad 60567.0$ FT

$06 / 10 / 80$ TO $12 / 04 / 92$

REPORT DATE: 08/04/94

FORMATION OF COMPLETIOH: TRES HERMANOS - C1 SAMDSTOHE (TO) HYDRAULIC FLOW RELATIONSHIP: DOWH GRADIENT (D)

\begin{tabular}{|c|c|c|c|c|c|c|c|}
\hline PARAMETER KAME & LOG DATE & $\underset{10}{\text { SAMPLE }}$ & $\begin{array}{l}\text { UNITS OF } \\
\text { MEASURE }\end{array}$ & PV & $\begin{array}{l}\text { PARAMETER } \\
\text { VALUE FLAGS }\end{array}$ & $\begin{array}{l}\text { DETECTION } \\
\text { LIMIT }\end{array}$ & $\begin{array}{l}\text { PARAMETER } \\
\text { UNCERTAINTY }\end{array}$ \\
\hline ALKALIKITY & $\begin{array}{l}10 / 11 / 85 \\
05 / 21 / 86\end{array}$ & $\begin{array}{l}0001 \\
0001\end{array}$ & $\mathrm{MG} / \mathrm{L} \quad \mathrm{CACO} 3$ & & $\begin{array}{l}451 . \\
2650 .\end{array}$ & - & $\dot{-}$ \\
\hline ALUAIHUM & $10 / 11 / 85$ & 0001 & $M G / L$ & & 0.6 & 0.1 & - \\
\hline AMHONIUM & $10 / 11 / 85$ & 0001 & MG/L & & 1.6 & 0.1 & - \\
\hline AKTIMONY & $10 / 19 / 85$ & 0001 & MG/L & $<$ & 0.003 & 0.003 & - \\
\hline ARSEKIC & $\begin{array}{l}10 / 11 / 85 \\
05 / 21 / 86\end{array}$ & $\begin{array}{l}0001 \\
0001\end{array}$ & $M G / L$ & $<$ & $\begin{array}{l}0.02 \\
0.01\end{array}$ & $\begin{array}{l}0.01 \\
0.01\end{array}$ & $\dot{-}$ \\
\hline BARIUM & $10 / 11 / 85$ & 0001 & MG/L & & 0.1 & 0.1 & - \\
\hline BORON & $\begin{array}{l}10 / 11 / 85 \\
05 / 21 / 86\end{array}$ & $\begin{array}{l}0001 \\
0001\end{array}$ & $M G / L$ & & $\begin{array}{l}0.3 \\
0.3\end{array}$ & $\begin{array}{l}0.1 \\
0.1\end{array}$ & - \\
\hline CADMILAM & $\begin{array}{l}10 / 19 / 85 \\
05 / 21 / 86\end{array}$ & $\begin{array}{l}0001 \\
0001\end{array}$ & $M G / L$ & $<$ & $\begin{array}{l}0.001 \\
0.001\end{array}$ & $\begin{array}{l}0.001 \\
0.001\end{array}$ & - \\
\hline CALCIUM & $\begin{array}{l}10 / 11 / 85 \\
05 / 21 / 86\end{array}$ & $\begin{array}{l}0001 \\
0001\end{array}$ & MG/L & & $\begin{array}{r}34 . \\
181 .\end{array}$ & $\begin{array}{l}0.01 \\
0.01\end{array}$ & - \\
\hline CHLORIDE & $\begin{array}{l}10 / 11 / 85 \\
05 / 21 / 86\end{array}$ & $\begin{array}{l}0001 \\
0001\end{array}$ & $M G / L$ & & $\begin{array}{l}31 . \\
38 .\end{array}$ & 1. & : \\
\hline CHRONIUM & $\begin{array}{l}10 / 11 / 85 \\
05 / 21 / 86\end{array}$ & $\begin{array}{l}0001 \\
0001\end{array}$ & $M G / L$ & & $\begin{array}{l}0.21 \\
0.04\end{array}$ & $\begin{array}{l}0.01 \\
0.01\end{array}$ & - \\
\hline COBALT & $\begin{array}{l}10 / 11 / 85 \\
05 / 21 / 86\end{array}$ & $\begin{array}{l}0001 \\
0001\end{array}$ & $M G / L$ & $<$ & $\begin{array}{l}0.05 \\
0.06\end{array}$ & $\begin{array}{l}0.05 \\
0.05\end{array}$ & 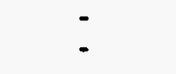 \\
\hline COPPER & $10 / 11 / 85$ & 0001 & $M G / L$ & $<$ & 0.02 & 0.02 & $\cdot$ \\
\hline CYANIDE & $10 / 11 / 85$ & 0001 & MG/L & $<$ & 0.01 & 0.01 & - \\
\hline FLUORIDE & $\begin{array}{l}10 / 11 / 85 \\
05 / 21 / 86\end{array}$ & $\begin{array}{l}0001 \\
0001\end{array}$ & MG/L & & $\begin{array}{l}1.1 \\
0.4\end{array}$ & $\begin{array}{l}0.1 \\
0.1\end{array}$ & $\dot{-}$ \\
\hline IRON & $\begin{array}{l}10 / 11 / 85 \\
05 / 21 / 86\end{array}$ & $\begin{array}{l}0001 \\
0001\end{array}$ & $M G / L$ & $<$ & $\begin{array}{l}0.03 \\
0.03\end{array}$ & $\begin{array}{l}0.03 \\
0.03\end{array}$ & $\dot{-}$ \\
\hline LEAD & $10 / 11 / 85$ & 0001 & $M G / L$ & $<$ & 0.01 & 0.01 & - \\
\hline LEAD-210 & $10 / 11 / 85$ & 0001 & $\mathrm{PCI} / \mathrm{L}$ & & 0.7 & 1.5 & 1.9 \\
\hline MAGHESIUM & $\begin{array}{l}10 / 11 / 85 \\
05 / 21 / 86\end{array}$ & $\begin{array}{l}0001 \\
0001\end{array}$ & MG/L & & $\begin{array}{l}5.19 \\
0.022\end{array}$ & $\begin{array}{l}0.001 \\
0.001\end{array}$ & $\dot{-}$ \\
\hline MANGAHESE & $\begin{array}{l}10 / 11 / 85 \\
05 / 21 / 86\end{array}$ & $\begin{array}{l}0001 \\
0001\end{array}$ & $M G / L$ & $<$ & $\begin{array}{l}0.02 \\
0.01\end{array}$ & $\begin{array}{l}0.01 \\
0.01\end{array}$ & - \\
\hline MERCURY & $10 / 11 / 85$ & 0001 & $M G / L$ & $<$ & 0.0002 & 0.0002 & - \\
\hline HOLYBDEHUM & $\begin{array}{l}10 / 11 / 85 \\
05 / 21 / 86\end{array}$ & $\begin{array}{l}0001 \\
0001\end{array}$ & $M G / L$ & & $\begin{array}{l}0.1 \\
0.21\end{array}$ & $\begin{array}{l}0.01 \\
0.01\end{array}$ & - \\
\hline HICKEL & $10 / 11 / 85$ & 0001 & $M G / L$ & & 0.07 & 0.04 & - \\
\hline
\end{tabular}

PARAMETER VALUE INDICATOR (PVI): < - LESS THAN DETECTIOH LIMIT

SAMPLE ID COOES:

0001 - FILTERED SAMPLE (.45 MICRONS) 
GROUNDWATER QUALITY DATA BY LOCATIOH

SITE: AMBOI AMBROSIA LAKE

LOCATION: 0791

HORTH COORDINATE: 55739.9 FT

EAST COORDIHATE: $\quad 60567.0$ FT

$06 / 10 / 80$ TO $12 / 04 / 92$

REPORT DATE: $08 / 04 / 94$

FORMATIOH OF COMPLETION: TRES HERMANOS - C1 SAMDSTONE (TO)

HYDRAULIC FLON RELATIONSHIP: DOWN GRADIENT (D)

\begin{tabular}{|c|c|c|c|c|c|c|c|}
\hline PARAMETER NAME & LOG DATE & $\underset{\text { SAMPLE }}{\text { ID }}$ & $\begin{array}{l}\text { UNITS OF } \\
\text { MEASURE }\end{array}$ & PVI & $\begin{array}{l}\text { PARAMETER } \\
\text { VALUE FLAGS }\end{array}$ & $\begin{array}{l}\text { DETECTION } \\
\text { LIMIT }\end{array}$ & $\begin{array}{l}\text { PARAMETER } \\
\text { UNCERTAIHTY }\end{array}$ \\
\hline NITRATE & $\begin{array}{l}10 / 11 / 85 \\
05 / 21 / 86\end{array}$ & $\begin{array}{l}0001 \\
0001\end{array}$ & MG/L & & $\begin{array}{l}8 . \\
2 .\end{array}$ & 1. & $\dot{-}$ \\
\hline MITRITE & $10 / 19 / 85$ & 0001 & MG/L & $<$ & 0.1 & 0.1 & - \\
\hline PH & $\begin{array}{l}10 / 11 / 85 \\
05 / 21 / 86\end{array}$ & $\begin{array}{l}0001 \\
0001\end{array}$ & SU & & $\begin{array}{l}12.09 \\
12.46\end{array}$ & - & $\dot{-}$ \\
\hline PHOSPHATE & $10 / 11 / 85$ & 0001 & $M G / L$ & & 0.4 & 0.1 & - \\
\hline POTASSIUAH & $\begin{array}{l}10 / 11 / 85 \\
05 / 21 / 86\end{array}$ & $\begin{array}{l}0001 \\
0001\end{array}$ & MG/L & & $\begin{array}{l}22.3 \\
43.6\end{array}$ & $\begin{array}{l}0.01 \\
0.01\end{array}$ & - \\
\hline RADIUH- 226 & $10 / 11 / 85$ & 0001 & $\mathrm{PCI} / \mathrm{L}$ & & 0.3 & 1. & 1.6 \\
\hline RADIUM-226 + RADIUH-228 & $10 / 11 / 85$ & 0001 & $\mathrm{PCI} / \mathrm{L}$ & & 1.50 & - & - \\
\hline RADIUM-228 & $10 / 11 / 85$ & 0001 & $\mathrm{PCI} / \mathrm{L}$ & & 1.2 & 1. & 3.8 \\
\hline SELENIUM & $\begin{array}{l}10 / 11 / 85 \\
05 / 21 / 86\end{array}$ & $\begin{array}{l}0001 \\
0001\end{array}$ & $\mathrm{MG} / \mathrm{L}$ & $<$ & $\begin{array}{l}0.016 \\
0.005\end{array}$ & $\begin{array}{l}0.005 \\
0.005\end{array}$ & - \\
\hline SILICA - SIOZ & $10 / 11 / 85$ & 0001 & $M G / L$ & & 6. & 2. & - \\
\hline SILVER & $10 / 11 / 85$ & 0001 & $M G / L$ & $<$ & 0.01 & 0.01 & - \\
\hline SOOIUH & $\begin{array}{l}10 / 11 / 85 \\
05 / 21 / 86\end{array}$ & $\begin{array}{l}0001 \\
0001\end{array}$ & $M G / L$ & & $\begin{array}{l}690 . \\
1110 .\end{array}$ & $\begin{array}{l}0.002 \\
0.002\end{array}$ & $\dot{-}$ \\
\hline SPECIFIC CONDUCTANCE & $\begin{array}{l}10 / 11 / 85 \\
05 / 21 / 86\end{array}$ & $\begin{array}{l}0001 \\
0001\end{array}$ & UMHO/CM & & $\begin{array}{l}3000 . \\
8000 .\end{array}$ & - & $\dot{-}$ \\
\hline STRONTIUM & $\begin{array}{l}10 / 11 / 85 \\
05 / 21 / 86\end{array}$ & $\begin{array}{l}0001 \\
0001\end{array}$ & $M G / L$ & & $\begin{array}{l}1.5 \\
8.1\end{array}$ & $\begin{array}{l}0.1 \\
0.1\end{array}$ & $\dot{-}$ \\
\hline SULFATE & $\begin{array}{l}10 / 11 / 85 \\
05 / 21 / 86\end{array}$ & $\begin{array}{l}0001 \\
0001\end{array}$ & MG/L & & $\begin{array}{r}1200 . \\
240 .\end{array}$ & $\begin{array}{l}0.1 \\
0.1\end{array}$ & $\dot{-}$ \\
\hline SULFIDE & $10 / 11 / 85$ & 0001 & MG/L & $<$ & 0.1 & 0.1 & - \\
\hline TEMPERATURE & $\begin{array}{l}10 / 11 / 85 \\
05 / 21 / 86\end{array}$ & $\begin{array}{l}0001 \\
0001\end{array}$ & C - DEGREE & & $\begin{array}{l}11 . \\
15 .\end{array}$ & - & - \\
\hline THOR IUM-230 & $10 / 11 / 85$ & 0001 & $\mathrm{PCI} / \mathrm{L}$ & & 1.1 & 1. & 2.5 \\
\hline TIN & $10 / 11 / 85$ & 0001 & $M G / L$ & $<$ & 0.005 & 0.005 & - \\
\hline TOTAL DISSOLVED SOLIDS & $\begin{array}{l}10 / 11 / 85 \\
05 / 21 / 86\end{array}$ & $\begin{array}{l}0001 \\
0001\end{array}$ & $M G / L$ & & $\begin{array}{l}1950 . \\
3520 .\end{array}$ & $\begin{array}{l}10 . \\
10 .\end{array}$ & $\dot{-}$ \\
\hline URAKIUA & $\begin{array}{l}10 / 11 / 85 \\
05 / 21 / 86\end{array}$ & $\begin{array}{l}0001 \\
0001\end{array}$ & $M G / L$ & $<$ & $\begin{array}{ll}0.0007 & \mathrm{~J} \\
0.0003 & \mathrm{~J}\end{array}$ & $\begin{array}{l}0.003 \\
0.003\end{array}$ & $\dot{-}$ \\
\hline VAKADIUM & $\begin{array}{l}10 / 11 / 85 \\
05 / 21 / 86\end{array}$ & $\begin{array}{l}0001 \\
0001\end{array}$ & $M G / L$ & $<$ & $\begin{array}{l}0.09 \\
0.3\end{array}$ & $\begin{array}{l}0.01 \\
0.01\end{array}$ & - \\
\hline ZINC & $10 / 11 / 85$ & 0001 & $M G / L$ & $<$ & 0.005 & 0.005 & - \\
\hline
\end{tabular}

PARAMETER VALUE INDICATOR (PVI): < - LESS THAN DETECTION LIMIT

SAMPLE ID COOES:

0001 - FILTERED SAMPLE (.45 MICRONS)

OTHER PARAHETER VALUE FLAGS:

$J$ - ESTIMATED VALUE 
GROUNDWATER QUALITY DATA BY LOCATION

SITE: AMBO1 AMBROSIA LAKE

LOCATION: 0791

MORTH COORDIHATE: $\quad 55739.9$ FT

EAST COORDINATE: 60567.0 FT

06/10/80 TO 12/04/92

REPORT DATE: 08/04/94

FORMATIOH OF COMPLETIOH: TRES HERMANOS - C1 SAHDSTONE (TO)

HYDRAULIC FLON RELATIONSHIP: DOWN GRADIENT (D)

\begin{tabular}{|l|l|l|l|l|l|l|l|}
\hline PARAMETER MAME & LOG DATE & $\begin{array}{l}\text { SAMPLE } \\
\text { ID }\end{array}$ & $\begin{array}{l}\text { UMITS OF } \\
\text { MEASURE }\end{array}$ & PVI & $\begin{array}{l}\text { PARAMETER } \\
\text { VALUE }\end{array}$ fLAGS & $\begin{array}{c}\text { DETECTION } \\
\text { LIMIT }\end{array}$ & $\begin{array}{l}\text { PARAMETER } \\
\text { UNCERTAINTY }\end{array}$ \\
\hline
\end{tabular}

PARAMETER VALUE IMDICATOR (PVI): < - LESS THAK DETECTION LIMIT SAMPLE ID COOES: 
GROUNDHATER QUALITY DATA BY LOCATION

SITE: AMBOI AMBROSIA LAKE

LOCATION: 0792

NORTH COORDINATE: 55724.9 FT

EAST COORDINATE: 60649.1 FT

O6/10/80 TO $12 / 04 / 92$

REPORT DATE: $08 / 04 / 94$

FORMATION OF COAPLETIOH: ALLUVIUM (AL)

HYDRAULIC FLON RELATIONSHIP: UPGRADIENT (U)

\begin{tabular}{|c|c|c|c|c|c|c|c|c|}
\hline PARAMETER NAME & LOG DATE & $\begin{array}{c}\text { SAMPLE } \\
\text { ID }\end{array}$ & $\begin{array}{l}\text { UHITS OF } \\
\text { MEASURE }\end{array}$ & PVI & $\begin{array}{l}\text { PARAMETER } \\
\text { VALUE }\end{array}$ & FLAGS & $\begin{array}{l}\text { DETECTION } \\
\text { LIMIT }\end{array}$ & $\begin{array}{l}\text { PARAMETER } \\
\text { UNCERTAINTY }\end{array}$ \\
\hline ALKALINITY & $\begin{array}{l}05 / 15 / 86 \\
04 / 06 / 88 \\
07 / 13 / 88 \\
01 / 06 / 89 \\
09 / 14 / 89 \\
03 / 31 / 90 \\
01 / 16 / 91\end{array}$ & $\begin{array}{l}0001 \\
0001 \\
0001 \\
0001 \\
0001 \\
0001 \\
0001\end{array}$ & MG/L CACO3 & & $\begin{array}{l}329 . \\
334 . \\
294 . \\
323 . \\
292 . \\
302 . \\
240 .\end{array}$ & & $\begin{array}{l}- \\
- \\
- \\
- \\
-\end{array}$ & $\begin{array}{l}- \\
- \\
- \\
- \\
-\end{array}$ \\
\hline ALUAINUA & $\begin{array}{l}04 / 06 / 88 \\
07 / 13 / 88 \\
01 / 06 / 89 \\
09 / 14 / 89 \\
03 / 31 / 90 \\
01 / 16 / 91\end{array}$ & $\begin{array}{l}0001 \\
0001 \\
0001 \\
0001 \\
0001 \\
0001\end{array}$ & $M G / L$ & $\begin{array}{l}< \\
< \\
<\end{array}$ & $\begin{array}{l}0.58 \\
0.2 \\
0.05 \\
0.1 \\
0.1 \\
0.05\end{array}$ & J & $\begin{array}{l}0.1 \\
0.1 \\
0.1 \\
0.1 \\
0.1 \\
0.05\end{array}$ & $\begin{array}{l}- \\
- \\
- \\
-\end{array}$ \\
\hline AMMONIUH & $\begin{array}{l}04 / 06 / 88 \\
07 / 13 / 88 \\
01 / 06 / 89 \\
09 / 14 / 89 \\
03 / 31 / 90 \\
01 / 16 / 91\end{array}$ & $\begin{array}{l}0001 \\
0001 \\
0001 \\
0001 \\
0001 \\
0001\end{array}$ & $M G / L$ & $\begin{array}{l}< \\
< \\
< \\
<\end{array}$ & $\begin{array}{l}0.1 \\
0.1 \\
0.16 \\
0.1 \\
0.1 \\
0.08\end{array}$ & & $\begin{array}{l}0.1 \\
0.1 \\
0.1 \\
0.1 \\
0.1 \\
0.01\end{array}$ & $\begin{array}{l}- \\
- \\
- \\
-\end{array}$ \\
\hline ANT IMOHY & $\begin{array}{l}04 / 06 / 88 \\
07 / 13 / 88 \\
01 / 06 / 89 \\
09 / 14 / 89 \\
03 / 31 / 90 \\
01 / 16 / 91\end{array}$ & $\begin{array}{l}0001 \\
0001 \\
0001 \\
0001 \\
0001 \\
0001\end{array}$ & $M G / L$ & $<$ & $\begin{array}{l}0.013 \\
0.012 \\
0.001 \\
0.003 \\
0.031 \\
0.008\end{array}$ & J & $\begin{array}{l}0.003 \\
0.003 \\
0.003 \\
0.003 \\
0.003 \\
0.003\end{array}$ & $\begin{array}{l}- \\
. \\
- \\
-\end{array}$ \\
\hline ARSENIC & $\begin{array}{l}05 / 15 / 86 \\
04 / 06 / 88 \\
07 / 13 / 88 \\
01 / 06 / 89 \\
09 / 14 / 89 \\
03 / 31 / 90 \\
01 / 16 / 91\end{array}$ & $\begin{array}{l}0001 \\
0001 \\
0001 \\
0001 \\
0001 \\
0001 \\
0001\end{array}$ & HG/L & $\begin{array}{l}< \\
< \\
< \\
<\end{array}$ & $\begin{array}{l}0.01 \\
0.016 \\
0.01 \\
0.001 \\
0.01 \\
0.01 \\
0.03\end{array}$ & $\begin{array}{l}J \\
I\end{array}$ & $\begin{array}{l}0.01 \\
0.01 \\
0.01 \\
0.01 \\
0.01 \\
0.01 \\
0.03\end{array}$ & $\begin{array}{l}- \\
- \\
- \\
-\end{array}$ \\
\hline BARIUM & $\begin{array}{l}04 / 06 / 88 \\
07 / 13 / 88 \\
01 / 06 / 89 \\
09 / 14 / 89 \\
03 / 31 / 90 \\
01 / 16 / 91\end{array}$ & $\begin{array}{l}0001 \\
0001 \\
0001 \\
0001 \\
0001 \\
0001\end{array}$ & $M G / L$ & $\begin{array}{l}< \\
< \\
<\end{array}$ & $\begin{array}{l}0.02 \\
0.1 \\
0.01 \\
0.1 \\
0.1 \\
0.01\end{array}$ & $\begin{array}{l}J \\
J\end{array}$ & $\begin{array}{l}0.1 \\
0.1 \\
0.1 \\
0.1 \\
0.1 \\
0.01\end{array}$ & $\begin{array}{l}- \\
- \\
- \\
-\end{array}$ \\
\hline BERYLLIUM & $\begin{array}{l}01 / 06 / 89 \\
09 / 14 / 89 \\
03 / 31 / 90 \\
01 / 16 / 91\end{array}$ & $\begin{array}{l}0001 \\
0001 \\
0001 \\
0001\end{array}$ & $M G / L$ & $\begin{array}{l}< \\
< \\
<\end{array}$ & $\begin{array}{l}0.005 \\
0.01 \\
0.01 \\
0.005\end{array}$ & & $\begin{array}{l}0.005 \\
0.01 \\
0.01 \\
0.005\end{array}$ & - \\
\hline BOROH & $\begin{array}{l}05 / 15 / 86 \\
04 / 06 / 88 \\
07 / 13 / 88 \\
01 / 06 / 89 \\
09 / 14 / 89 \\
03 / 31 / 90 \\
01 / 16 / 91\end{array}$ & $\begin{array}{l}0001 \\
0001 \\
0001 \\
0001 \\
0009 \\
0001 \\
0001\end{array}$ & $M G / L$ & & $\begin{array}{l}0.4 \\
0.62 \\
0.4 \\
0.53 \\
0.5 \\
0.4 \\
0.50\end{array}$ & & $\begin{array}{l}0.1 \\
0.1 \\
0.1 \\
0.1 \\
0.1 \\
0.1 \\
0.05\end{array}$ & $\begin{array}{l}- \\
- \\
- \\
- \\
-\end{array}$ \\
\hline
\end{tabular}

PARAMETER VALUE INDICATOR (PVI): < - LESS THAN DETECTION LIMIT

SAMPLE ID COOES

0001 - FILTERED SAMPLE (.45 MICRONS)

OTHER PARAMETER VALUE FLAGS:

I - IHCREASED DETECTION LIMIT DUE TO, REQUIRED DILUTION

$J$ - ESTIMATED VALUE 
FORMATION OF COMPLETION: ALLUVIUM (AL)

HYORAULIC FLOU RELATIOHSHIP: UPGRADIEHT (U)

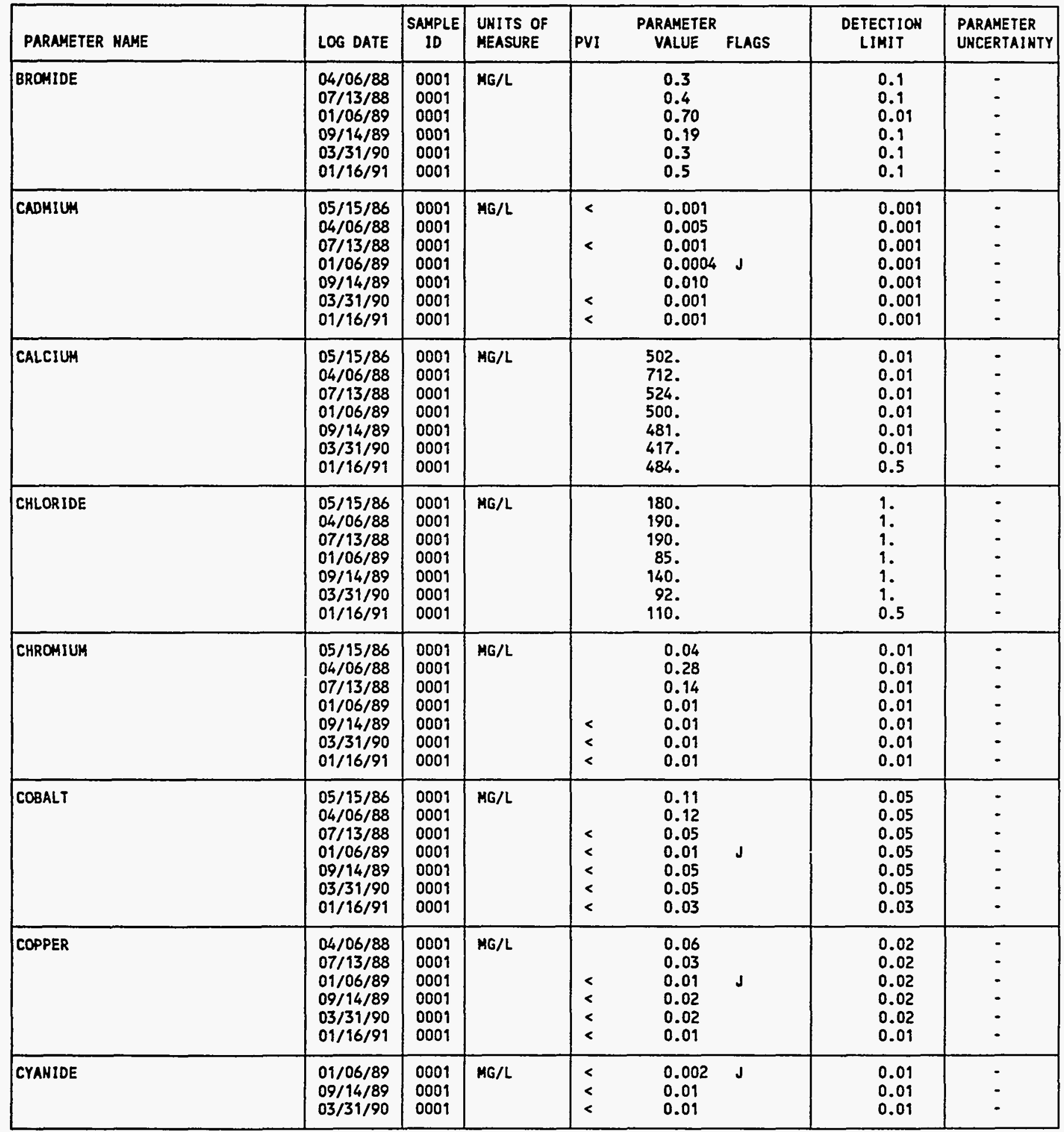

PARAMETER VALUE IHDICATOR (PVI): < - LESS THAN DETECTION LIMIT 


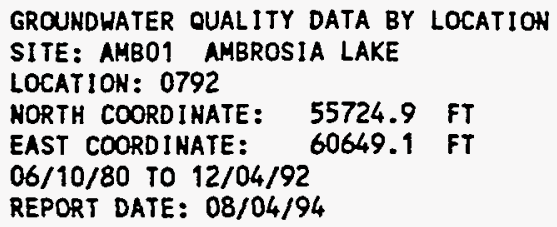

FORMATION OF COMPLETION: ALLUVIUM (AL)

HYORAULIC FLON RELATIONSHIP: UPGRADIENT (U)

\begin{tabular}{|c|c|c|c|c|c|c|c|c|}
\hline PARAMETER KAME & LOG DATE & SAMPLE & $\begin{array}{l}\text { UNITS OF } \\
\text { MEASURE }\end{array}$ & PVI & $\begin{array}{l}\text { PARAMETER } \\
\text { VALUE }\end{array}$ & FLAGS & $\begin{array}{l}\text { DETECTION } \\
\text { LIMIT }\end{array}$ & $\begin{array}{l}\text { PARAMETER } \\
\text { UNCERTAINTY }\end{array}$ \\
\hline CYAKIDE & $01 / 16 / 91$ & 0001 & MG/L & $<$ & 0.01 & & 0.01 & - \\
\hline FLUORIDE & $\begin{array}{l}05 / 15 / 86 \\
04 / 06 / 88 \\
07 / 13 / 88 \\
01 / 06 / 89 \\
09 / 14 / 89 \\
03 / 31 / 90 \\
01 / 16 / 91\end{array}$ & $\begin{array}{l}0001 \\
0001 \\
0001 \\
0001 \\
0001 \\
0001 \\
0001\end{array}$ & MG/L & & $\begin{array}{l}1.1 \\
1.05 \\
0.9 \\
1.0 \\
0.9 \\
0.9 \\
1.2\end{array}$ & & $\begin{array}{l}0.1 \\
0.1 \\
0.1 \\
0.1 \\
0.1 \\
0.1 \\
0.1\end{array}$ & $\begin{array}{l}- \\
. \\
. \\
. \\
.\end{array}$ \\
\hline GROSS ALPHA & $\begin{array}{l}04 / 06 / 88 \\
07 / 13 / 88 \\
01 / 06 / 89 \\
09 / 14 / 89 \\
03 / 31 / 90\end{array}$ & $\begin{array}{l}0001 \\
0001 \\
0001 \\
0001 \\
0001\end{array}$ & $\mathrm{PCI} / \mathrm{L}$ & & $\begin{array}{c}2400 \\
1800 \\
1090 \\
560 \\
1400\end{array}$ & 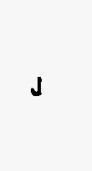 & $\begin{array}{l}0.2 \\
0.2 \\
1 . \\
1 . \\
1 .\end{array}$ & $\begin{array}{c}10 . \\
100 . \\
91.1 \\
40 . \\
100 .\end{array}$ \\
\hline GROSS BETA & $\begin{array}{l}04 / 06 / 88 \\
07 / 13 / 88 \\
01 / 06 / 89 \\
09 / 14 / 89 \\
03 / 31 / 90\end{array}$ & $\begin{array}{l}0001 \\
0001 \\
0001 \\
0001 \\
0001\end{array}$ & $\mathrm{PCI} / \mathrm{L}$ & & $\begin{array}{l}790 . \\
670 . \\
780 . \\
280 . \\
670 .\end{array}$ & & $\begin{array}{l}1 . \\
1 . \\
0.5 \\
0.5 \\
0.5\end{array}$ & $\begin{array}{l}30 . \\
40 . \\
52.0 \\
20 . \\
30 .\end{array}$ \\
\hline IRON & $\begin{array}{l}05 / 15 / 86 \\
04 / 06 / 88 \\
07 / 13 / 88 \\
01 / 06 / 89 \\
09 / 14 / 89 \\
03 / 31 / 90 \\
01 / 16 / 91\end{array}$ & $\begin{array}{l}0001 \\
0001 \\
0001 \\
0001 \\
0001 \\
0001 \\
0001\end{array}$ & MG/L & $<$ & $\begin{array}{l}0.04 \\
0.18 \\
0.08 \\
0.02 \\
0.08 \\
0.25 \\
0.07\end{array}$ & J & $\begin{array}{l}0.03 \\
0.03 \\
0.03 \\
0.03 \\
0.03 \\
0.03 \\
0.03\end{array}$ & $\begin{array}{l}- \\
- \\
- \\
- \\
- \\
-\end{array}$ \\
\hline LEAD & $\begin{array}{l}04 / 06 / 88 \\
07 / 13 / 88 \\
01 / 06 / 89 \\
09 / 14 / 89 \\
03 / 31 / 90 \\
01 / 16 / 91\end{array}$ & $\begin{array}{l}0001 \\
0001 \\
0001 \\
0001 \\
0001 \\
0001\end{array}$ & $M G / L$ & $\begin{array}{l}< \\
< \\
< \\
<\end{array}$ & $\begin{array}{l}0.01 \\
0.01 \\
0.001 \\
0.02 \\
0.01 \\
0.03\end{array}$ & $\begin{array}{l}J \\
1\end{array}$ & $\begin{array}{l}0.01 \\
0.01 \\
0.01 \\
0.01 \\
0.01 \\
0.03\end{array}$ & $\begin{array}{l}- \\
- \\
- \\
-\end{array}$ \\
\hline LEAD-210 & $\begin{array}{l}04 / 06 / 88 \\
07 / 13 / 88 \\
01 / 06 / 89 \\
09 / 14 / 89\end{array}$ & $\begin{array}{l}0001 \\
0001 \\
0001 \\
0001\end{array}$ & $\mathrm{PCI} / \mathrm{L}$ & & $\begin{array}{l}2.8 \\
0.8 \\
0.0818 \\
0.4\end{array}$ & & $\begin{array}{l}1.5 \\
1.5 \\
1.5 \\
1.5\end{array}$ & $\begin{array}{l}1.0 \\
1.2 \\
5.22 \\
0.7\end{array}$ \\
\hline MAGNESIUM & $\begin{array}{l}05 / 15 / 86 \\
04 / 06 / 88 \\
07 / 13 / 88 \\
01 / 06 / 89 \\
09 / 14 / 89 \\
03 / 31 / 90 \\
01 / 16 / 91\end{array}$ & $\begin{array}{l}0001 \\
0001 \\
0001 \\
0001 \\
0001 \\
0001 \\
0001\end{array}$ & $M G / L$ & & $\begin{array}{l}82.5 \\
96.0 \\
103 . \\
102 . \\
105 . \\
101 . \\
143 .\end{array}$ & & $\begin{array}{l}0.001 \\
0.001 \\
0.001 \\
0.001 \\
0.001 \\
0.001 \\
0.5\end{array}$ & $\begin{array}{l}- \\
- \\
- \\
. \\
- \\
-\end{array}$ \\
\hline MAHGANESE & $\begin{array}{l}05 / 15 / 86 \\
04 / 06 / 88 \\
07 / 13 / 88 \\
01 / 06 / 89 \\
09 / 14 / 89 \\
03 / 31 / 90\end{array}$ & $\begin{array}{l}0001 \\
0001 \\
0001 \\
0001 \\
0001 \\
0001\end{array}$ & $\mathrm{MG} / \mathrm{L}$ & $<$ & $\begin{array}{l}0.05 \\
0.04 \\
0.03 \\
0.01 \\
0.01 \\
0.01\end{array}$ & & $\begin{array}{l}0.01 \\
0.01 \\
0.01 \\
0.01 \\
0.01 \\
0.01\end{array}$ & $\begin{array}{l}- \\
: \\
- \\
-\end{array}$ \\
\hline
\end{tabular}

PARAMETER VALUE IHDICATOR (PVI):

< - LESS THAN DETECTION LIMIT

SAMPLE ID CODES:

0001 - FILTERED SAMPLE (.45 MICRONS)

OTHER PARAMETER VALUE FLAGS:

I - INCREASED DETECTION LIMIT DUE TO REQUIRED DILUTION

J - ESTIMATED VALUE 
GROUMDHATER OUALITY DATA BY LOCATION

SITE: AMBOI AMBROSIA LAKE

LOCATION: 0792

MORTH COORDINATE: 55724.9 FT

EAST COORDINATE: 60649.1 FT

$06 / 10 / 80$ TO $12 / 04 / 92$

REPORT DATE: $08 / 04 / 94$

FORMATIOH OF COMPLETION: ALLUVIUM (AL)

HYDRAULIC FLOW RELATIONSHIP: UPGRADIENT (U)

\begin{tabular}{|c|c|c|c|c|c|c|c|c|}
\hline PARAMETER NAME & LOG DATE & $\begin{array}{c}\text { SAMPLE } \\
\text { ID }\end{array}$ & $\begin{array}{l}\text { UNITS OF } \\
\text { MEASURE }\end{array}$ & PVI & $\begin{array}{l}\text { PARAMETER } \\
\text { VALUE }\end{array}$ & FLAGS & $\begin{array}{l}\text { DETECTION } \\
\text { LIHIT }\end{array}$ & $\begin{array}{l}\text { PARAMETER } \\
\text { UNCERTAINTY }\end{array}$ \\
\hline MAKGAHESE & $01 / 16 / 91$ & 0001 & MG/L & & 0.01 & & 0.01 & - \\
\hline MERCURY & $\begin{array}{l}04 / 06 / 88 \\
07 / 13 / 88 \\
01 / 06 / 89 \\
09 / 14 / 89 \\
03 / 31 / 90 \\
01 / 16 / 91\end{array}$ & $\begin{array}{l}0001 \\
0001 \\
0001 \\
0001 \\
0001 \\
0001\end{array}$ & MG/L & $\begin{array}{l}< \\
< \\
< \\
<\end{array}$ & $\begin{array}{l}0.0007 \\
0.0002 \\
0.0001 \\
0.0002 \\
0.0002 \\
0.0002\end{array}$ & J & $\begin{array}{l}0.0002 \\
0.0002 \\
0.0002 \\
0.0002 \\
0.0002 \\
0.0002\end{array}$ & $\begin{array}{l}- \\
- \\
- \\
-\end{array}$ \\
\hline MOLYBDENUM & $\begin{array}{l}05 / 15 / 86 \\
04 / 06 / 88 \\
07 / 13 / 88 \\
01 / 06 / 89 \\
09 / 14 / 89 \\
03 / 31 / 90 \\
01 / 16 / 91\end{array}$ & $\begin{array}{l}0001 \\
0001 \\
0001 \\
0001 \\
0001 \\
0001 \\
0001\end{array}$ & MG/L & & $\begin{array}{l}1.87 \\
1.63 \\
1.26 \\
1.410 \\
1.19 \\
1.25 \\
1.17\end{array}$ & & $\begin{array}{l}0.01 \\
0.01 \\
0.01 \\
0.01 \\
0.01 \\
0.01 \\
0.01\end{array}$ & $\begin{array}{l}: \\
: \\
- \\
-\end{array}$ \\
\hline HET GROSS ALPHA • & $\begin{array}{l}04 / 06 / 88 \\
07 / 13 / 88 \\
01 / 06 / 89 \\
09 / 14 / 89 \\
03 / 31 / 90\end{array}$ & $\begin{array}{l}0001 \\
0001 \\
0001 \\
0001 \\
0001\end{array}$ & PCI/L & & $\begin{array}{r}300.84 \\
-45.34 \\
-590.70 \\
-256.34 \\
199.50\end{array}$ & & $\begin{array}{l}- \\
- \\
-\end{array}$ & : \\
\hline HICKEL & $\begin{array}{l}04 / 06 / 88 \\
07 / 13 / 88 \\
01 / 06 / 89 \\
09 / 14 / 89 \\
03 / 31 / 90 \\
01 / 16 / 91\end{array}$ & $\begin{array}{l}0001 \\
0001 \\
0001 \\
0001 \\
0001 \\
0001\end{array}$ & $M G / L$ & $\begin{array}{l}< \\
< \\
< \\
<\end{array}$ & $\begin{array}{l}0.13 \\
0.04 \\
0.02 \\
0.04 \\
0.04 \\
0.04\end{array}$ & J & $\begin{array}{l}0.04 \\
0.04 \\
0.04 \\
0.04 \\
0.04 \\
0.04\end{array}$ & $:$ \\
\hline HITRATE & $\begin{array}{l}05 / 15 / 86 \\
04 / 06 / 88 \\
07 / 13 / 88 \\
01 / 06 / 89 \\
09 / 14 / 89 \\
03 / 31 / 90 \\
01 / 16 / 91\end{array}$ & $\begin{array}{l}0001 \\
0001 \\
0001 \\
0001 \\
0001 \\
0001 \\
0001\end{array}$ & $M G / L$ & $<$ & $\begin{array}{l}1 . \\
1.6 \\
1.8 \\
0.2 \\
1.2 \\
0.9 \\
1.3\end{array}$ & $\begin{array}{l}J \\
J \\
J\end{array}$ & $\begin{array}{l}1 . \\
1 . \\
1 . \\
1 . \\
1 . \\
0.1\end{array}$ & $\begin{array}{l}- \\
- \\
- \\
-\end{array}$ \\
\hline HITRITE AHD NITRATE & $\begin{array}{l}04 / 06 / 88 \\
03 / 31 / 90 \\
01 / 16 / 91\end{array}$ & $\begin{array}{l}0001 \\
0001 \\
0001\end{array}$ & $M G / L$ & & $\begin{array}{l}1.6 \\
0.2 \\
0.31\end{array}$ & J & $\begin{array}{l}1 . \\
1.05 \\
0.05\end{array}$ & - \\
\hline PH & $\begin{array}{l}05 / 15 / 86 \\
04 / 06 / 88 \\
07 / 13 / 88 \\
01 / 06 / 89 \\
09 / 14 / 89 \\
03 / 31 / 90 \\
01 / 16 / 91\end{array}$ & $\begin{array}{l}0001 \\
0001 \\
0001 \\
0001 \\
0001 \\
0001 \\
0001\end{array}$ & su & & $\begin{array}{l}7.35 \\
7.13 \\
7.08 \\
7.11 \\
7.05 \\
7.05 \\
7.54\end{array}$ & & $\begin{array}{l}- \\
- \\
- \\
- \\
-\end{array}$ & $\begin{array}{l}- \\
- \\
- \\
-\end{array}$ \\
\hline PHOSPHATE & $\begin{array}{l}04 / 06 / 88 \\
07 / 13 / 88 \\
01 / 06 / 89 \\
09 / 14 / 89 \\
03 / 31 / 90\end{array}$ & $\begin{array}{l}0001 \\
0001 \\
0001 \\
0001 \\
0001\end{array}$ & MG/L & $\begin{array}{l}< \\
< \\
< \\
<\end{array}$ & $\begin{array}{l}0.1 \\
0.1 \\
0.1 \\
0.1 \\
0.1\end{array}$ & & $\begin{array}{l}0.1 \\
0.1 \\
0.1 \\
0.1 \\
0.1\end{array}$ & : \\
\hline
\end{tabular}

- het gROSS ALPHA (GROSS ALPHA - URANIUM) HITH 1 HG URANIUH = 686 PCI

PARAMETER VALUE IMDICATOR (PVI): < - LESS THAN DETECTIOH LIMIT SAMPLE ID CODES:

0009 - FILTERED SAMPLE (.45 MICRONS)

OTHER PARAMETER VALUE FLAGS:

J - ESTIMATED VALUE 
GROUNDHATER QUALITY DATA BY LOCATIOW

SITE: AMBO1 AMBROSIA LAKE

LOCATION: 0792

NORTH COORDINATE: 55724.9 FT

EAST COORDINATE: 60649.1 FT

06/10/80 TO $12 / 04 / 92$

REPORT DATE: $08 / 04 / 94$

FORMATION OF COMPLETION: ALLUVIUM (AL)

HYDRAULIC FLOW RELATIONSHIP: UPGRADIENT (U)

\begin{tabular}{|c|c|c|c|c|c|c|c|}
\hline PARAMETER MAME & LOG DATE & $\underset{\text { SD }}{\text { SAMPLE }}$ & $\begin{array}{l}\text { UNITS OF } \\
\text { MEASURE }\end{array}$ & PVI & $\begin{array}{l}\text { PARAMETER } \\
\text { VALLUE FLAGS }\end{array}$ & $\begin{array}{l}\text { DETECTION } \\
\text { LIMIT }\end{array}$ & $\begin{array}{l}\text { PARAMETER } \\
\text { UNCERTAINTY }\end{array}$ \\
\hline PHOSPHATE & $01 / 16 / 91$ & 0001 & $M G / L$ & & 0.05 & 0.05 & - \\
\hline POLONIUA-210 & $\begin{array}{l}04 / 06 / 88 \\
07 / 13 / 88 \\
01 / 06 / 89 \\
09 / 14 / 89\end{array}$ & $\begin{array}{l}0001 \\
0001 \\
0001 \\
0001\end{array}$ & $\mathrm{PCl} / \mathrm{L}$ & & $\begin{array}{l}2.1 \\
1.7 \\
0.196 \\
2.3\end{array}$ & $\begin{array}{l}1 . \\
1 . \\
1 .\end{array}$ & $\begin{array}{l}0.7 \\
0.7 \\
1.49 \\
0.6\end{array}$ \\
\hline POTASSIUM & $\begin{array}{l}05 / 15 / 86 \\
04 / 06 / 88 \\
07 / 13 / 88 \\
01 / 06 / 89 \\
09 / 14 / 89 \\
03 / 31 / 90 \\
01 / 16 / 91\end{array}$ & $\begin{array}{l}0001 \\
0001 \\
0001 \\
0001 \\
0001 \\
0001 \\
0001\end{array}$ & MG/L & & $\begin{array}{l}1.01 \\
0.62 \\
0.61 \\
0.8 \\
0.7 \\
0.8 \\
0.89\end{array}$ & $\begin{array}{l}0.01 \\
0.01 \\
0.01 \\
0.01 \\
0.01 \\
0.01 \\
0.01\end{array}$ & $\begin{array}{l}- \\
- \\
- \\
-\end{array}$ \\
\hline RADIUM-226 & $\begin{array}{l}05 / 15 / 86 \\
04 / 06 / 88 \\
07 / 13 / 88 \\
01 / 06 / 89 \\
09 / 94 / 89 \\
03 / 31 / 90\end{array}$ & $\begin{array}{l}0001 \\
0001 \\
0001 \\
0001 \\
0001 \\
0001\end{array}$ & $\mathrm{PCI} / \mathrm{L}$ & & $\begin{array}{l}0.8 \\
0.2 \\
0.4 \\
0.751 \\
0.1 \\
0.8\end{array}$ & $\begin{array}{l}1 . \\
1 . \\
1 . \\
1 . \\
1 .\end{array}$ & $\begin{array}{l}0.4 \\
0.2 \\
0.2 \\
0.309 \\
0.1 \\
0.3\end{array}$ \\
\hline RADIUM-226 + RADIUM-228 & $\begin{array}{l}04 / 06 / 88 \\
07 / 13 / 88 \\
01 / 06 / 89 \\
09 / 14 / 89 \\
03 / 31 / 90\end{array}$ & $\begin{array}{l}0001 \\
0001 \\
0001 \\
0001 \\
0001\end{array}$ & $\mathrm{PCI} / \mathrm{L}$ & & $\begin{array}{l}0.30 \\
0.50 \\
6.85 \\
0.20 \\
2.50\end{array}$ & $\begin{array}{l}- \\
- \\
- \\
-\end{array}$ & $\begin{array}{l}- \\
- \\
-\end{array}$ \\
\hline RAD IUA-228 & $\begin{array}{l}04 / 06 / 88 \\
07 / 13 / 88 \\
01 / 06 / 89 \\
09 / 14 / 89 \\
03 / 31 / 90\end{array}$ & $\begin{array}{l}0001 \\
0001 \\
0001 \\
0001 \\
0001\end{array}$ & PCI/L & & $\begin{array}{l}0.1 \\
0.1 \\
6.10 \\
0.1 \\
1.7\end{array}$ & $\begin{array}{l}1 . \\
1 . \\
1 . \\
1 .\end{array}$ & $\begin{array}{l}0.8 \\
0.7 \\
4.20 \\
0.9 \\
0.9\end{array}$ \\
\hline SELENIUM & $\begin{array}{l}05 / 15 / 86 \\
04 / 06 / 88 \\
07 / 13 / 88 \\
01 / 06 / 89 \\
09 / 14 / 89 \\
03 / 31 / 90 \\
01 / 16 / 91\end{array}$ & $\begin{array}{l}0001 \\
0001 \\
0001 \\
0001 \\
0001 \\
0001 \\
0001\end{array}$ & $M G / L$ & $<$ & $\begin{array}{l}0.005 \\
0.889 \\
0.767 \\
1.800 \\
1.62 \\
2.22 \\
0.950\end{array}$ & $\begin{array}{l}0.005 \\
0.005 \\
0.005 \\
0.005 \\
0.005 \\
0.005 \\
0.005\end{array}$ & $\begin{array}{l}- \\
- \\
- \\
-\end{array}$ \\
\hline SILICA - SIO2 & $\begin{array}{l}04 / 06 / 88 \\
07 / 13 / 88 \\
01 / 06 / 89 \\
09 / 14 / 89 \\
03 / 31 / 90 \\
01 / 16 / 91\end{array}$ & $\begin{array}{l}0001 \\
0001 \\
0001 \\
0001 \\
0001 \\
0001\end{array}$ & $M G / L$ & & $\begin{array}{l}19.7 \\
15.9 \\
15.5 \\
17 . \\
15 . \\
14.3\end{array}$ & $\begin{array}{l}2 . \\
2 . \\
2 . \\
2 . \\
2 . \\
0.1\end{array}$ & $\begin{array}{l}- \\
- \\
- \\
-\end{array}$ \\
\hline SILVER & $\begin{array}{l}04 / 06 / 88 \\
07 / 13 / 88 \\
01 / 06 / 89 \\
09 / 14 / 89 \\
03 / 31 / 90 \\
01 / 16 / 91\end{array}$ & $\begin{array}{l}0001 \\
0001 \\
0001 \\
0001 \\
0001 \\
0001\end{array}$ & $M G / L$ & $\begin{array}{l}< \\
< \\
<\end{array}$ & $\begin{array}{l}0.11 \\
0.03 \\
0.01 \\
0.01 \\
0.01 \\
0.01\end{array}$ & $\begin{array}{l}0.09 \\
0.01 \\
0.01 \\
0.01 \\
0.01 \\
0.01\end{array}$ & $\begin{array}{l}- \\
- \\
- \\
-\end{array}$ \\
\hline SOOIUN & $\begin{array}{l}05 / 15 / 86 \\
04 / 06 / 88\end{array}$ & $\begin{array}{l}0001 \\
0001\end{array}$ & $M G / L$ & & $\begin{array}{l}730 . \\
369 .\end{array}$ & $\begin{array}{l}0.002 \\
0.002\end{array}$ & - \\
\hline
\end{tabular}

PARAMETER VALUE INDICATOR (PVI): < - LESS THAN DETECTION LIMIT

SAMPLE ID COOES

0001 - FILTERED SAMPLE (.45 MICRONS) 
GROUNDWATER OUALITY DATA BY LOCATION

SITE: AMBO1 AMBROSIA LAKE

LOCATIOH: 0792

NORTH COORDINATE: 55724.9 FT

EAST COORDIHATE: 60649.1 FT

06/10/80 TO $12 / 04 / 92$

REPORT DATE: 08/04/94

FORHATION OF COMPLETION: ALLUVIUM (AL)

HYDRAULIC FLON RELATIOHSHIP: UPGRADIENT (U)

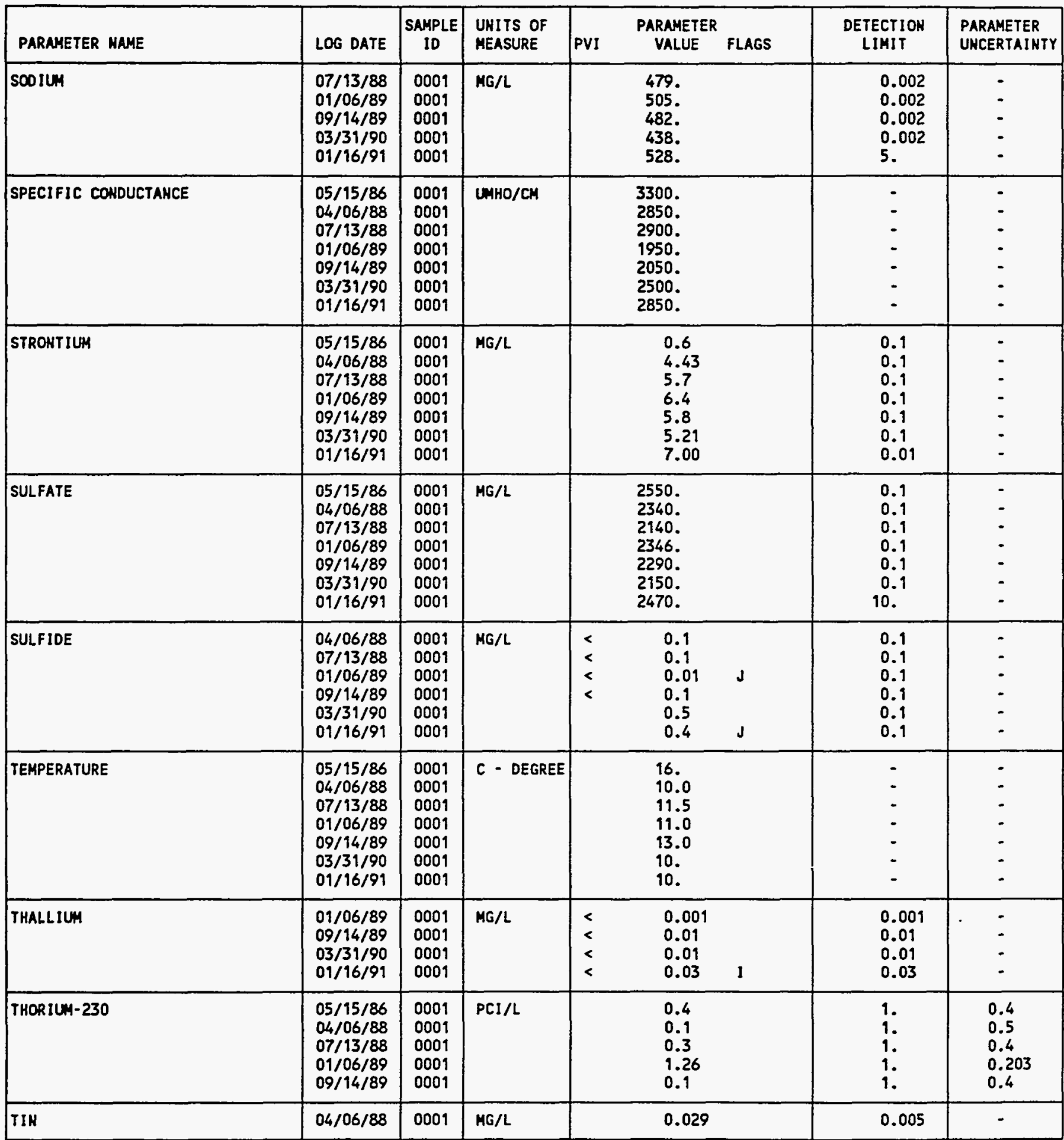

PARAMETER VALUE IMDICATOR (PVI): < - LeSS THAN DETECTION LIMIT

OTHER PARAMETER VALUE FLAGS:

1 - INCREASED DETECTION LIMIT DUE TO REQUIRED DILUTION

$J$ - ESTIMATED VALUE 
GROUNDHATER QUALITY DATA BY LOCATION

SITE: AMBOI AMBROSIA LAKE

LOCATION: 0792

NORTH COORDINATE: 55724.9 FT

EAST COORDINATE: 60649.1 FT

06/10/80 TO $12 / 04 / 92$

REPORT DATE: $08 / 04 / 94$

FORMATION OF COMPLETION: ALLUVIUH (AL)

MYDRAULIC FLON RELATIONSHIP: UPGRADIENT (U)

\begin{tabular}{|c|c|c|c|c|c|c|c|c|}
\hline PARAMETER NAME & LOG DATE & $\mid \begin{array}{c}\text { SAMPLE } \\
\text { ID }\end{array}$ & $\begin{array}{l}\text { UHITS OF } \\
\text { MEASURE }\end{array}$ & PVI & $\begin{array}{l}\text { PARAMETER } \\
\text { VALUE }\end{array}$ & FLAGS & $\begin{array}{l}\text { DETECTIOH } \\
\text { LIMIT }\end{array}$ & $\begin{array}{l}\text { PARAMETER } \\
\text { UNCERTAIHTY }\end{array}$ \\
\hline TIN & $\begin{array}{l}07 / 13 / 88 \\
01 / 06 / 89 \\
09 / 14 / 89 \\
03 / 31 / 90 \\
01 / 16 / 91\end{array}$ & $\begin{array}{l}0001 \\
0001 \\
0001 \\
0009 \\
0009\end{array}$ & MG/L & $\begin{array}{l}< \\
<\end{array}$ & $\begin{array}{l}0.014 \\
0.005 \\
0.9 \\
0.014 \\
0.03\end{array}$ & I & $\begin{array}{l}0.005 \\
0.005 \\
0.1 \\
0.005 \\
0.03\end{array}$ & $\begin{array}{l}- \\
- \\
- \\
-\end{array}$ \\
\hline TOTAL DISSOLVED SOLIDS & $\begin{array}{l}05 / 15 / 86 \\
04 / 06 / 88 \\
07 / 13 / 88 \\
01 / 06 / 89 \\
09 / 14 / 89 \\
03 / 31 / 90 \\
01 / 16 / 91\end{array}$ & $\begin{array}{l}0001 \\
0001 \\
0001 \\
0001 \\
0001 \\
0001 \\
0001\end{array}$ & $M G / L$ & & $\begin{array}{l}4110 . \\
4100 \\
4180 \\
3782 \\
3750 \\
3360 \\
4120\end{array}$ & & $\begin{array}{l}10 . \\
10 . \\
10 . \\
10 . \\
10 . \\
10 . \\
10 .\end{array}$ & $\begin{array}{l}- \\
- \\
- \\
- \\
-\end{array}$ \\
\hline TOTAL KJELDAHL NITROGEN & $01 / 16 / 91$ & 0001 & MG/L & & 1. & & 1. & - \\
\hline TOTAL ORGAHIC CARBON & $\begin{array}{l}04 / 06 / 88 \\
07 / 13 / 88 \\
01 / 06 / 89 \\
09 / 14 / 89 \\
03 / 31 / 90 \\
01 / 16 / 91\end{array}$ & $\begin{array}{l}0001 \\
0001 \\
0001 \\
0001 \\
0001 \\
0001\end{array}$ & MG/L & & $\begin{array}{c}95.7 \\
75.8 \\
7 . \\
79.6 \\
8.2 \\
6 .\end{array}$ & . & $\begin{array}{l}1 . \\
1 . \\
1 . \\
1 . \\
1 .\end{array}$ & $\begin{array}{l}: \\
: \\
:\end{array}$ \\
\hline URANIUH & $\begin{array}{l}05 / 15 / 86 \\
04 / 06 / 88 \\
07 / 13 / 88 \\
01 / 06 / 89 \\
09 / 14 / 89 \\
03 / 31 / 90 \\
01 / 16 / 91\end{array}$ & $\begin{array}{l}0001 \\
0001 \\
0001 \\
0001 \\
0001 \\
0001 \\
0001\end{array}$ & $M G / L$ & & $\begin{array}{l}3.31 \\
3.06 \\
2.69 \\
2.450 \\
1.19 \\
1.75 \\
2.25\end{array}$ & & $\begin{array}{l}0.003 \\
0.003 \\
0.003 \\
0.003 \\
0.003 \\
0.003 \\
0.001\end{array}$ & $\begin{array}{l}- \\
- \\
- \\
- \\
-\end{array}$ \\
\hline VANADIUM & $\begin{array}{l}05 / 15 / 86 \\
04 / 06 / 88 \\
07 / 13 / 88 \\
01 / 06 / 89 \\
09 / 14 / 89 \\
03 / 31 / 90 \\
01 / 16 / 91\end{array}$ & $\begin{array}{l}0001 \\
0001 \\
0001 \\
0001 \\
0001 \\
0001 \\
0001\end{array}$ & MG/L & $\begin{array}{l}< \\
< \\
< \\
<\end{array}$ & $\begin{array}{l}0.26 \\
0.15 \\
0.06 \\
0.01 \\
0.01 \\
0.01 \\
0.01\end{array}$ & & $\begin{array}{l}0.01 \\
0.01 \\
0.01 \\
0.01 \\
0.01 \\
0.01 \\
0.01\end{array}$ & $\begin{array}{l}- \\
- \\
- \\
- \\
-\end{array}$ \\
\hline ZINC & $\begin{array}{l}04 / 06 / 88 \\
07 / 13 / 88 \\
01 / 06 / 89 \\
09 / 14 / 89 \\
03 / 31 / 90 \\
01 / 16 / 91\end{array}$ & $\begin{array}{l}0001 \\
0001 \\
0001 \\
0001 \\
0001 \\
0001\end{array}$ & $M G / L$ & $\begin{array}{l}< \\
<\end{array}$ & $\begin{array}{l}0.070 \\
0.016 \\
0.01 \\
0.005 \\
0.016 \\
0.024\end{array}$ & & $\begin{array}{l}0.005 \\
0.005 \\
0.01 \\
0.005 \\
0.005 \\
0.005\end{array}$ & $\begin{array}{l}- \\
- \\
- \\
-\end{array}$ \\
\hline
\end{tabular}

PARAMETER VALUE INDICATOR (PVI): < - LESS THAN DETECTION LIMIT

SAMPLE ID CODES:

0001 - FILTERED SAMPLE (.45 MICRONS)

OTHER PARAMETER VALUE FLAGS:

1 - INCREASED DETECTION LIMIT DUE TO REQUIRED DILUTION

$J$ - ESTIMATED VALUE 
GROUNDWATER OUALITY DATA BY LOCATION

SITE: AMBOI AKBROSIA LAKE

LOCAT ION: 0793

NORTH COORDINATE: $\quad 55493.0$ FT

EAST COORDINATE: 60225.7 FT

06/10/80 TO 12/04/92

REPORT DATE: 08/04/94

FORMATION OF COMPLETION: ALLUVIUM (AL)

HYDRAULIC FLON RELATIONSHIP: UPGRADIENT (U)

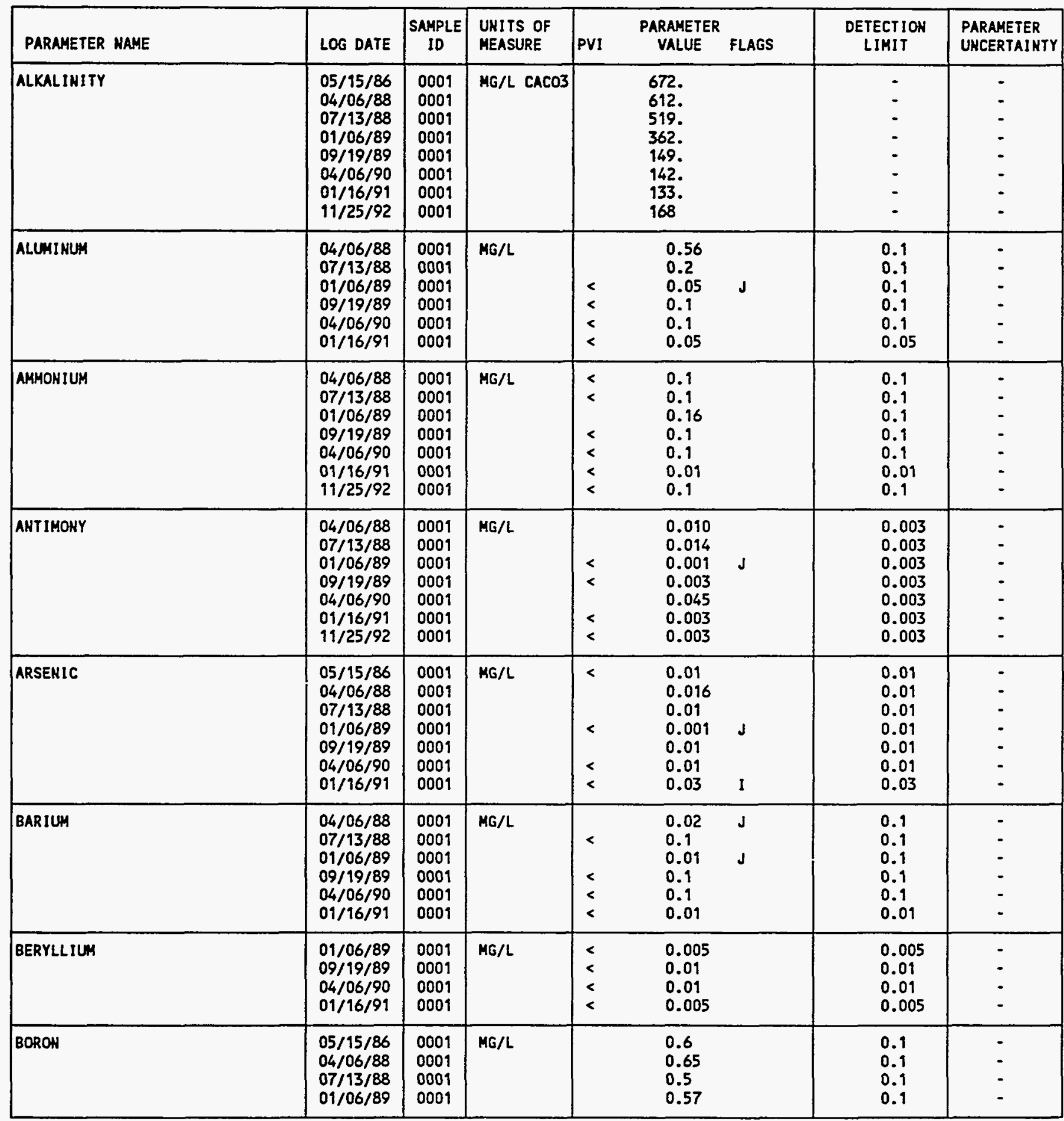

PARAMETER VALUE INDICATOR (PVI): < - LESS THAN DETECTION LIHIT

SAMPLE ID CODES:

0001 - FILTERED SAMPLE (.45 MICRONS)

OTHER PARAMETER VALUE FLAGS:

I - IHCREASED DETECTIOH LIMIT DUE TO'REOUIRED OILUTION

$J$ - estimated VALUe 
GROUNDWATER OUALITY DATA BY LOCATION

SITE: AHBOI AMBROSIA LAKE

LOCATION: 0793

NORTH COORDINATE: 55493.0 FT

EAST COORDINATE: 60225.7 FT

06/10/80 TO $12 / 04 / 92$

REPORT DATE: 08/04/94

FORMATION OF COMPLETION: ALLUVIUM (AL)

HYDRAULIC FLOH RELATIOHSHIP: UPGRADIENT (U)

\begin{tabular}{|c|c|c|c|c|c|c|c|}
\hline PARAMETER NAME & LOG DATE & $\begin{array}{c}\text { SAMPLE } \\
10\end{array}$ & $\begin{array}{l}\text { UNITS OF } \\
\text { NEASURE }\end{array}$ & PVI & $\begin{array}{l}\text { PARAMETER } \\
\text { VALUE FLAGS }\end{array}$ & $\begin{array}{l}\text { DETECTION } \\
\text { LIKIT }\end{array}$ & $\begin{array}{l}\text { PARAMETER } \\
\text { UHCERTAIKTY }\end{array}$ \\
\hline BORON & $\begin{array}{l}09 / 19 / 89 \\
04 / 06 / 90 \\
01 / 16 / 91\end{array}$ & $\begin{array}{l}0001 \\
0001 \\
0001\end{array}$ & $M G / L$ & & $\begin{array}{l}0.6 \\
0.6 \\
0.69\end{array}$ & $\begin{array}{l}0.1 \\
0.1 \\
0.05\end{array}$ & $\begin{array}{l}- \\
-\end{array}$ \\
\hline BROHIDE & $\begin{array}{l}04 / 06 / 88 \\
07 / 13 / 88 \\
01 / 06 / 89 \\
09 / 19 / 89 \\
04 / 06 / 90 \\
01 / 16 / 91\end{array}$ & $\begin{array}{l}0001 \\
0001 \\
0001 \\
0001 \\
0001 \\
0001\end{array}$ & $M G / L$ & & $\begin{array}{l}1.1 \\
1.6 \\
2.42 \\
0.19 \\
3.8 \\
9.1\end{array}$ & $\begin{array}{l}0.1 \\
0.1 \\
0.01 \\
0.1 \\
0.1 \\
0.1\end{array}$ & $\begin{array}{l}- \\
- \\
. \\
- \\
-\end{array}$ \\
\hline CADMIUM & $\begin{array}{l}05 / 15 / 86 \\
04 / 06 / 88 \\
07 / 13 / 88 \\
01 / 06 / 89 \\
09 / 19 / 89 \\
04 / 06 / 90 \\
01 / 16 / 91 \\
11 / 25 / 92\end{array}$ & $\begin{array}{l}0001 \\
0001 \\
0001 \\
0001 \\
0001 \\
0001 \\
0001 \\
0001\end{array}$ & $M G / L$ & $\begin{array}{l}< \\
< \\
< \\
<\end{array}$ & $\begin{array}{l}0.001 \\
0.005 \\
0.001 \\
0.0002 \quad J \\
0.013 \\
0.001 \\
0.002 \\
0.05\end{array}$ & $\begin{array}{l}0.001 \\
0.001 \\
0.001 \\
0.001 \\
0.001 \\
0.001 \\
0.001 \\
0.05\end{array}$ & $\begin{array}{l}- \\
- \\
- \\
- \\
- \\
-\end{array}$ \\
\hline CALCIUM & $\begin{array}{l}05 / 15 / 86 \\
04 / 06 / 88 \\
07 / 13 / 88 \\
01 / 06 / 89 \\
09 / 19 / 89 \\
04 / 06 / 90 \\
01 / 16 / 91 \\
11 / 25 / 92\end{array}$ & $\begin{array}{l}0001 \\
0001 \\
0001 \\
0001 \\
0001 \\
0001 \\
0001 \\
0001\end{array}$ & $M G / L$ & & $\begin{array}{l}510 . \\
610 . \\
500 . \\
600 . \\
590 . \\
507 . \\
600 . \\
615 .\end{array}$ & $\begin{array}{l}0.01 \\
0.01 \\
0.01 \\
0.01 \\
0.01 \\
0.01 \\
0.01 \\
2 .\end{array}$ & $\begin{array}{l}- \\
- \\
- \\
- \\
- \\
-\end{array}$ \\
\hline CHLORIDE & $\begin{array}{l}05 / 15 / 86 \\
04 / 06 / 88 \\
07 / 13 / 88 \\
01 / 06 / 89 \\
09 / 19 / 89 \\
04 / 06 / 90 \\
01 / 16 / 91 \\
11 / 25 / 92\end{array}$ & $\begin{array}{l}0001 \\
0001 \\
0001 \\
0001 \\
0001 \\
0001 \\
0001 \\
0001\end{array}$ & $M G / L$ & & $\begin{array}{l}230 . \\
170 \\
200 \\
208 \\
430 \\
518 \\
643 . \\
77\end{array}$ & $\begin{array}{l}1 . \\
1 . \\
1 . \\
1 . \\
1 . \\
1 . \\
0.5 \\
1 .\end{array}$ & $\begin{array}{l}- \\
- \\
- \\
- \\
- \\
-\end{array}$ \\
\hline CHROMIUH & $\begin{array}{l}05 / 15 / 86 \\
04 / 06 / 88 \\
07 / 13 / 88 \\
01 / 06 / 89 \\
09 / 19 / 89 \\
04 / 06 / 90 \\
01 / 16 / 91 \\
11 / 25 / 92\end{array}$ & $\begin{array}{l}0001 \\
0001 \\
0001 \\
0001 \\
0001 \\
0001 \\
0001 \\
0001\end{array}$ & $M G / L$ & $\begin{array}{l}< \\
< \\
< \\
<\end{array}$ & $\begin{array}{l}0.03 \\
0.28 \\
0.15 \\
0.01 \\
0.01 \\
0.01 \\
0.01 \\
0.01\end{array}$ & $\begin{array}{l}0.01 \\
0.01 \\
0.01 \\
0.01 \\
0.01 \\
0.01 \\
0.01 \\
0.01\end{array}$ & $\begin{array}{l}- \\
- \\
- \\
- \\
- \\
-\end{array}$ \\
\hline CoBALT & $\begin{array}{l}05 / 15 / 86 \\
04 / 06 / 88 \\
07 / 13 / 88 \\
01 / 06 / 89 \\
09 / 19 / 89 \\
04 / 06 / 90 \\
01 / 16 / 91 \\
11 / 25 / 92\end{array}$ & $\begin{array}{l}0001 \\
0001 \\
0001 \\
0001 \\
0001 \\
0001 \\
0001 \\
0001\end{array}$ & MG/L & $\begin{array}{l}< \\
< \\
< \\
< \\
< \\
<\end{array}$ & $\begin{array}{l}0.08 \\
0.13 \\
0.05 \\
0.01 \\
0.05 \\
0.05 \\
0.03 \\
0.03\end{array}$ & $\begin{array}{l}0.05 \\
0.05 \\
0.05 \\
0.05 \\
0.05 \\
0.05 \\
0.03 \\
0.03\end{array}$ & $\begin{array}{l}- \\
- \\
- \\
- \\
- \\
-\end{array}$ \\
\hline COPPER & $04 / 06 / 88$ & 0001 & MG/L & & 0.06 & 0.02 & - \\
\hline
\end{tabular}

PARAMETER VALUE IMDICATOR (PVI): < - LESS THAN DETECTION LIMIT

SAMPLE ID COOES:

0001 - FILTERED SAMPLE (.45 MICROAS)

OTHER PARAMETER VALUE FLAGS:

I - INCREASED DETECTION LIHIT DUE TO REQUIRED DILUTION

$J$ - ESTIMATED VALUE 
GROUHDWATER QUALITY DATA BY LOCATIOH

SITE: AMBOI AMBROSIA LAKE

LOCATION: 0793

NORTH COORDIHATE: 55493.0 FT

EAST COORDIHATE: $\quad 60225.7$ FT

06/10/80 TO $12 / 04 / 92$

REPORT DATE: 08/04/94

FORMATION OF COMPLETION: ALLUVIUM (AL)

HYORAULIC FLOH RELATIOHSHIP: UPGRADIENT (U)

\begin{tabular}{|c|c|c|c|c|c|c|c|c|}
\hline PARAMETER MAME & LOG DATE & $\underset{\text { ID }}{\text { SAMPLE }}$ & $\begin{array}{l}\text { UNITS OF } \\
\text { MEASURE }\end{array}$ & PVI & $\begin{array}{l}\text { PARAMETER } \\
\text { VALUE }\end{array}$ & FLAGS & $\begin{array}{l}\text { DETECTION } \\
\text { LIMIT }\end{array}$ & $\begin{array}{l}\text { PARAMETER } \\
\text { UNCERTAIHTY }\end{array}$ \\
\hline COPPER & $\begin{array}{l}07 / 13 / 88 \\
01 / 06 / 89 \\
09 / 19 / 89 \\
04 / 06 / 90 \\
01 / 16 / 91\end{array}$ & $\begin{array}{l}0001 \\
0001 \\
0001 \\
0001 \\
0001\end{array}$ & $M G / L$ & $\begin{array}{l}< \\
< \\
< \\
<\end{array}$ & $\begin{array}{l}0.03 \\
0.01 \\
0.02 \\
0.02 \\
0.01\end{array}$ & $J$ & $\begin{array}{l}0.02 \\
0.02 \\
0.02 \\
0.02 \\
0.01\end{array}$ & : \\
\hline CYAHIDE & $\begin{array}{l}01 / 06 / 89 \\
09 / 19 / 89 \\
04 / 06 / 90 \\
01 / 16 / 91\end{array}$ & $\begin{array}{l}0001 \\
0001 \\
0001 \\
0001\end{array}$ & MG/L & $\begin{array}{l}< \\
< \\
< \\
<\end{array}$ & $\begin{array}{l}0.002 \\
0.01 \\
0.01 \\
0.01\end{array}$ & $J$ & $\begin{array}{l}0.01 \\
0.01 \\
0.01 \\
0.01\end{array}$ & $\begin{array}{l}- \\
- \\
-\end{array}$ \\
\hline FLUORIDE & $\begin{array}{l}05 / 15 / 86 \\
04 / 06 / 88 \\
07 / 13 / 88 \\
01 / 06 / 89 \\
09 / 19 / 89 \\
04 / 06 / 90 \\
01 / 16 / 91 \\
11 / 25 / 92\end{array}$ & $\begin{array}{l}0001 \\
0001 \\
0001 \\
0001 \\
0001 \\
0001 \\
0001 \\
0001\end{array}$ & $M G / L$ & & $\begin{array}{l}2.2 \\
2.50 \\
2.3 \\
2.8 \\
2.4 \\
2.5 \\
2.9 \\
5.5\end{array}$ & & $\begin{array}{l}0.1 \\
0.1 \\
0.1 \\
0.1 \\
0.1 \\
0.1 \\
0.1 \\
0.2\end{array}$ & $\begin{array}{l}- \\
- \\
- \\
- \\
- \\
-\end{array}$ \\
\hline GROSS ALPHA & $\begin{array}{l}04 / 06 / 88 \\
07 / 13 / 88 \\
01 / 06 / 89 \\
09 / 19 / 89 \\
04 / 06 / 90 \\
01 / 16 / 91 \\
11 / 25 / 92\end{array}$ & $\begin{array}{l}0001 \\
0001 \\
0001 \\
0001 \\
0001 \\
0001 \\
0001\end{array}$ & $\mathrm{PCI} / \mathrm{L}$ & & $\begin{array}{c}320 . \\
170 . \\
84.6 \\
69 . \\
66 . \\
115 . \\
3.2\end{array}$ & H & $\begin{array}{l}0.2 \\
0.2 \\
1 . \\
1 . \\
1 . \\
1.0 \\
1.0\end{array}$ & $\begin{array}{l}50 . \\
40 . \\
31.2 \\
34 . \\
30 . \\
54.3 \\
37.8\end{array}$ \\
\hline GROSS BETA & $\begin{array}{l}04 / 06 / 88 \\
07 / 13 / 88 \\
01 / 06 / 89 \\
09 / 19 / 89 \\
04 / 06 / 90 \\
01 / 16 / 91 \\
11 / 25 / 92\end{array}$ & $\begin{array}{l}0001 \\
0001 \\
0001 \\
0001 \\
0001 \\
0001 \\
0001\end{array}$ & $\mathrm{PCI} / \mathrm{L}$ & & $\begin{array}{c}120 . \\
62 . \\
63.8 \\
58 . \\
18 . \\
67.7 \\
0.0\end{array}$ & & $\begin{array}{l}1 . \\
1 . \\
0.5 \\
0.5 \\
0.5 \\
0.5 \\
98.9\end{array}$ & $\begin{array}{l}20 . \\
13 . \\
25.4 \\
21 . \\
21 . \\
24.0 \\
56.5\end{array}$ \\
\hline IROH & $\begin{array}{l}05 / 15 / 86 \\
04 / 06 / 88 \\
07 / 13 / 88 \\
01 / 06 / 89 \\
09 / 19 / 89 \\
04 / 06 / 90 \\
01 / 16 / 91 \\
11 / 25 / 92\end{array}$ & $\begin{array}{l}0001 \\
0001 \\
0001 \\
0001 \\
0001 \\
0001 \\
0001 \\
0001\end{array}$ & HG/L & $\begin{array}{l}< \\
< \\
<\end{array}$ & $\begin{array}{l}0.04 \\
0.17 \\
0.08 \\
0.02 \\
0.09 \\
0.09 \\
0.03 \\
0.03\end{array}$ & J & $\begin{array}{l}0.03 \\
0.03 \\
0.03 \\
0.03 \\
0.03 \\
0.03 \\
0.03 \\
0.03\end{array}$ & $\begin{array}{l}: \\
: \\
: \\
: \\
-\end{array}$ \\
\hline LEAD & $\begin{array}{l}04 / 06 / 88 \\
07 / 13 / 88 \\
01 / 06 / 89 \\
09 / 19 / 89 \\
04 / 06 / 90 \\
01 / 16 / 91\end{array}$ & $\begin{array}{l}0001 \\
0001 \\
0001 \\
0001 \\
0001 \\
0001\end{array}$ & $M G / L$ & $\begin{array}{l}< \\
< \\
< \\
<\end{array}$ & $\begin{array}{l}0.01 \\
0.01 \\
0.001 \\
0.02 \\
0.01 \\
0.03\end{array}$ & $\begin{array}{l}J \\
I\end{array}$ & $\begin{array}{l}0.01 \\
0.01 \\
0.01 \\
0.01 \\
0.01 \\
0.03\end{array}$ & $\begin{array}{l}- \\
- \\
- \\
-\end{array}$ \\
\hline LEAD-210 & $\begin{array}{l}04 / 06 / 88 \\
07 / 13 / 88 \\
01 / 06 / 89\end{array}$ & $\begin{array}{l}0001 \\
0001 \\
0001\end{array}$ & $\mathrm{PCI} / \mathrm{L}$ & & $\begin{array}{l}1.8 \\
0.5 \\
5.09\end{array}$ & & $\begin{array}{l}1.5 \\
1.5 \\
1.5\end{array}$ & $\begin{array}{l}1.1 \\
1.1 \\
3.71\end{array}$ \\
\hline
\end{tabular}

PARAMETER VALUE INOICATOR (PVI): < - LESS THAN DETECTION LIMIT

SAMPLE ID COOES:

0001 - FILTERED SAMPLE (.45 MICRONS)

OTHER PARAMETER VALUE FLAGS:

H - HOLD TIME EXPIRED, VALUE SUSPECT

I - INCREASED DETECTION LIMIT DUE TO'REQUIRED DILUTIOH

J - estimated VALUE 
GROUNDHATER QUALITY DATA BY LOCATIOH

SITE: AMBO1 AMBROSIA LAKE

LOCATION: 0793

NORTH COORDINATE: 55493.0 FT

EAST COORDINATE: $60225.7 \mathrm{FT}$

06/10/80 TO 12/04/92

REPORT DATE: $08 / 04 / 94$

FORMATION OF COMPLETION: ALLUVIUM (AL)

HYDRAULIC FLON RELATIONSHIP: UPGRADIENT (U)

\begin{tabular}{|c|c|c|c|c|c|c|c|c|}
\hline PARAMETER NAME & LOG DATE & $\begin{array}{c}\text { SAMPLE } \\
\text { ID }\end{array}$ & $\begin{array}{l}\text { UNITS OF } \\
\text { MEASURE }\end{array}$ & PVI & $\begin{array}{l}\text { PARAMETER } \\
\text { VALUE }\end{array}$ & FLAGS & $\begin{array}{l}\text { DETECTION } \\
\text { LIMIT }\end{array}$ & $\begin{array}{l}\text { PARAMETER } \\
\text { UNCERTAINTY }\end{array}$ \\
\hline LEAD-210 & $09 / 19 / 89$ & 0001 & $\mathrm{PCI} / \mathrm{L}$ & \multicolumn{3}{|c|}{0.0} & 1.5 & 0.7 \\
\hline MAGNESIUM & $\begin{array}{l}05 / 15 / 86 \\
04 / 06 / 88 \\
07 / 13 / 88 \\
01 / 06 / 89 \\
09 / 19 / 89 \\
04 / 06 / 90 \\
01 / 16 / 91 \\
11 / 25 / 92\end{array}$ & $\begin{array}{l}0001 \\
0001 \\
0001 \\
0001 \\
0001 \\
0001 \\
0001 \\
0001\end{array}$ & $M G / L$ & \multicolumn{3}{|c|}{$\begin{array}{l}138 . \\
140 . \\
143 . \\
150 . \\
170 . \\
158 . \\
194 . \\
178 .\end{array}$} & $\begin{array}{l}0.001 \\
0.001 \\
0.001 \\
0.001 \\
0.001 \\
0.001 \\
0.5 \\
0.5\end{array}$ & $\begin{array}{l}- \\
- \\
- \\
- \\
- \\
-\end{array}$ \\
\hline MANGANESE & $\begin{array}{l}05 / 15 / 86 \\
04 / 06 / 88 \\
07 / 13 / 88 \\
01 / 06 / 89 \\
09 / 19 / 89 \\
04 / 06 / 90 \\
01 / 16 / 91\end{array}$ & $\begin{array}{l}0001 \\
0001 \\
0001 \\
0001 \\
0001 \\
0001 \\
0001\end{array}$ & $M G / L$ & \multicolumn{3}{|c|}{$\begin{array}{l}0.07 \\
0.04 \\
0.02 \\
0.01 \\
0.01 \\
0.01 \\
0.01\end{array}$} & $\begin{array}{l}0.01 \\
0.01 \\
0.01 \\
0.01 \\
0.01 \\
0.01 \\
0.01\end{array}$ & $\begin{array}{l}- \\
- \\
- \\
- \\
- \\
-\end{array}$ \\
\hline HERCURY & $\begin{array}{l}04 / 06 / 88 \\
07 / 13 / 88 \\
01 / 06 / 89 \\
09 / 19 / 89 \\
04 / 06 / 90 \\
01 / 16 / 91\end{array}$ & $\begin{array}{l}0001 \\
0001 \\
0001 \\
0001 \\
0001 \\
0001\end{array}$ & MG/L & \multicolumn{3}{|c|}{$\begin{array}{l}0.0003 \\
0.0002 \\
0.0001 \\
0.0002 \\
0.0002 \\
0.0002\end{array}$} & $\begin{array}{l}0.0002 \\
0.0002 \\
0.0002 \\
0.0002 \\
0.0002 \\
0.0002\end{array}$ & $\begin{array}{l}- \\
- \\
- \\
- \\
-\end{array}$ \\
\hline MOLYBDENUM & $\begin{array}{l}05 / 15 / 86 \\
04 / 06 / 88 \\
07 / 13 / 88 \\
01 / 06 / 89 \\
09 / 19 / 89 \\
04 / 06 / 90 \\
01 / 16 / 91 \\
11 / 25 / 92\end{array}$ & $\begin{array}{l}0001 \\
0001 \\
0001 \\
0001 \\
0001 \\
0001 \\
0001 \\
0001\end{array}$ & $M G / L$ & \multicolumn{3}{|c|}{$\begin{array}{l}1.59 \\
2.01 \\
1.41 \\
1.450 \\
1.19 \\
0.25 \\
0.07 \\
0.06\end{array}$} & $\begin{array}{l}0.01 \\
0.01 \\
0.01 \\
0.01 \\
0.01 \\
0.01 \\
0.01 \\
0.01\end{array}$ & $\begin{array}{l}- \\
- \\
- \\
- \\
- \\
-\end{array}$ \\
\hline NET GROSS ALPHA - & $\begin{array}{l}04 / 06 / 88 \\
07 / 13 / 88 \\
01 / 06 / 89 \\
09 / 19 / 89 \\
04 / 06 / 90 \\
01 / 16 / 91 \\
11 / 25 / 92\end{array}$ & $\begin{array}{l}0001 \\
0001 \\
0001 \\
0001 \\
0001 \\
0001 \\
0001\end{array}$ & $\mathrm{PCl} / \mathrm{L}$ & \multicolumn{3}{|c|}{$\begin{array}{r}50.40 \\
1.93 \\
-45.74 \\
7.95 \\
37.99 \\
97.85 \\
-18.07\end{array}$} & $\begin{array}{l}- \\
- \\
- \\
- \\
- \\
-\end{array}$ & $\begin{array}{l}- \\
- \\
- \\
- \\
- \\
-\end{array}$ \\
\hline NICKEL & $\begin{array}{l}04 / 06 / 88 \\
07 / 13 / 88 \\
01 / 06 / 89 \\
09 / 19 / 89 \\
04 / 06 / 90 \\
01 / 16 / 91 \\
11 / 25 / 92\end{array}$ & $\begin{array}{l}0001 \\
0001 \\
0001 \\
0001 \\
0001 \\
0001 \\
0001\end{array}$ & $M G / L$ & \multicolumn{3}{|c|}{$\begin{array}{l}0.14 \\
0.05 \\
0.02 \\
0.04 \\
0.04 \\
0.04 \\
0.08\end{array}$} & $\begin{array}{l}0.04 \\
0.04 \\
0.04 \\
0.04 \\
0.04 \\
0.04 \\
0.04\end{array}$ & $\begin{array}{l}- \\
- \\
- \\
- \\
- \\
-\end{array}$ \\
\hline HITRATE & $\begin{array}{l}05 / 15 / 86 \\
04 / 06 / 88 \\
07 / 13 / 88 \\
01 / 06 / 89\end{array}$ & $\begin{array}{l}0001 \\
0001 \\
0001 \\
0001\end{array}$ & MG/L & \multicolumn{3}{|c|}{$\begin{array}{l}55 . \\
130 . \\
210 . \\
252 .\end{array}$} & $\begin{array}{l}1 . \\
1 . \\
1 . \\
1 .\end{array}$ & $\begin{array}{l}- \\
- \\
-\end{array}$ \\
\hline
\end{tabular}

- NET GROSS ALPHA (GROSS ALPHA - URANIUM) WITH 1 HG URANIUM = 686 PCI

PARAMETER VALUE INDICATOR (PVI): < - LESS THAN DETECTION LIMIT

OTHER PARAMETER VALUE FLAGS:

$J$ - eSTIMATED VALUE
SAMPLE IO COOES:

0001 - FILTERED SAMPLE (.45 MICRONS) 
GROUNDWATER QUALITY DATA BY LOCATION

SITE: AMBOI AMBROSIA LAKE

LOCATION: 0793

HORTH COORDINATE: 55493.0 FT

EAST COORDINATE: $\quad 60225.7$ FT

06/10/80 TO $12 / 04 / 92$

REPORT DATE: 08/04/94

FORMATION OF COMPLETIOH: ALLUVIUM (AL)

HYORAULIC FLOW RELATIONSHIP: UPGRADIENT (U)

\begin{tabular}{|c|c|c|c|c|c|c|c|}
\hline PARAMETER HAME & LOG DATE & $\underset{\text { SAMPLE }}{\text { ID }}$ & $\begin{array}{l}\text { UNITS OF } \\
\text { MEASURE }\end{array}$ & PVI & $\begin{array}{l}\text { PARAMETER } \\
\text { VALUE FLAGS }\end{array}$ & $\begin{array}{l}\text { DETECTION } \\
\text { LIMIT }\end{array}$ & $\begin{array}{l}\text { PARAMETER } \\
\text { UNCERTAINTY }\end{array}$ \\
\hline MITRATE & $\begin{array}{l}09 / 19 / 89 \\
04 / 06 / 90 \\
01 / 16 / 91 \\
11 / 25 / 92\end{array}$ & $\begin{array}{l}0001 \\
0001 \\
0001 \\
0001\end{array}$ & $M G / L$ & & $\begin{array}{l}500 . \\
536 . \\
655 . \\
830 .\end{array}$ & $\begin{array}{c}1 . \\
1.0 .1 \\
150 .\end{array}$ & : \\
\hline HITRITE AHD NITRATE & $\begin{array}{l}04 / 06 / 88 \\
04 / 06 / 90 \\
01 / 16 / 91\end{array}$ & $\begin{array}{l}0001 \\
0001 \\
0001\end{array}$ & MG/L & & $\begin{array}{l}130 . \\
121 . \\
140 .\end{array}$ & $\begin{array}{l}1 . \\
1.0 \\
0.05\end{array}$ & : \\
\hline PH & $\begin{array}{l}05 / 15 / 86 \\
04 / 06 / 88 \\
07 / 13 / 88 \\
01 / 06 / 89 \\
09 / 19 / 89 \\
04 / 06 / 90 \\
01 / 16 / 91 \\
11 / 25 / 92\end{array}$ & $\begin{array}{l}0001 \\
0001 \\
0001 \\
0001 \\
0001 \\
0001 \\
0001 \\
0001\end{array}$ & su & & $\begin{array}{l}7.37 \\
7.37 \\
7.28 \\
7.41 \\
7.22 \\
7.15 \\
7.76 \\
7.46\end{array}$ & $\begin{array}{l}- \\
- \\
- \\
- \\
- \\
-\end{array}$ & $\begin{array}{l}- \\
- \\
- \\
- \\
- \\
-\end{array}$ \\
\hline PHOSPHATE & $\begin{array}{l}04 / 06 / 88 \\
07 / 13 / 88 \\
01 / 06 / 89 \\
09 / 19 / 89 \\
04 / 06 / 90 \\
01 / 16 / 91 \\
11 / 25 / 92\end{array}$ & $\begin{array}{l}0001 \\
0001 \\
0001 \\
0001 \\
0001 \\
0001 \\
0001\end{array}$ & $M G / L$ & $\begin{array}{l}< \\
< \\
< \\
< \\
< \\
<\end{array}$ & $\begin{array}{l}0.1 \\
0.1 \\
0.1 \\
0.1 \\
0.1 \\
0.05 \\
0.2\end{array}$ & $\begin{array}{l}0.1 \\
0.1 \\
0.1 \\
0.1 \\
0.1 \\
0.05 \\
0.1\end{array}$ & $\begin{array}{l}: \\
: \\
- \\
-\end{array}$ \\
\hline POLONIUA-210 & $\begin{array}{l}04 / 06 / 88 \\
07 / 13 / 88 \\
01 / 06 / 89 \\
09 / 19 / 89\end{array}$ & $\begin{array}{l}0001 \\
0001 \\
0001 \\
0001\end{array}$ & PCI/L & & $\begin{array}{l}0.0 \\
0.0 \\
0.00 \\
0.0\end{array}$ & $\begin{array}{l}1 . \\
1 . \\
1 .\end{array}$ & $\begin{array}{l}0.4 \\
0.4 \\
1.38 \\
0.3\end{array}$ \\
\hline POTASSIUM & $\begin{array}{l}05 / 15 / 86 \\
04 / 06 / 88 \\
07 / 13 / 88 \\
01 / 06 / 89 \\
09 / 19 / 89 \\
04 / 06 / 90 \\
01 / 16 / 91 \\
11 / 25 / 92\end{array}$ & $\begin{array}{l}0001 \\
0001 \\
0001 \\
0001 \\
0001 \\
0001 \\
0001 \\
0001\end{array}$ & $M G / L$ & & $\begin{array}{l}2.49 \\
1.68 \\
2.2 \\
1.9 \\
2.4 \\
2.8 \\
2.6 \\
4.16\end{array}$ & $\begin{array}{l}0.01 \\
0.01 \\
0.01 \\
0.01 \\
0.01 \\
0.01 \\
0.01 \\
0.01\end{array}$ & $\begin{array}{l}- \\
- \\
- \\
- \\
-\end{array}$ \\
\hline RADIUW-226 & $\begin{array}{l}05 / 15 / 86 \\
04 / 06 / 88 \\
07 / 13 / 88 \\
01 / 06 / 89 \\
09 / 19 / 89 \\
04 / 06 / 90 \\
01 / 16 / 91 \\
11 / 25 / 92\end{array}$ & $\begin{array}{l}0001 \\
0001 \\
0001 \\
0001 \\
0001 \\
0001 \\
0001 \\
0001\end{array}$ & $\mathrm{PCI} / \mathrm{L}$ & & $\begin{array}{l}0.7 \\
0.0 \\
0.4 \\
1.36 \\
0.2 \\
0.3 \\
1.6 \\
0.5\end{array}$ & $\begin{array}{l}1 . \\
1 . \\
1 . \\
1 . \\
1 . \\
1 . \\
1 . \\
0.3\end{array}$ & $\begin{array}{l}0.3 \\
0.1 \\
0.2 \\
0.450 \\
0.2 \\
0.2 \\
0.6 \\
0.3\end{array}$ \\
\hline RADIUH-226 + RADIUH-228 & $\begin{array}{l}04 / 06 / 88 \\
07 / 13 / 88 \\
01 / 06 / 89 \\
09 / 19 / 89 \\
04 / 06 / 90 \\
01 / 16 / 91 \\
11 / 25 / 92\end{array}$ & $\begin{array}{l}0001 \\
0001 \\
0001 \\
0001 \\
0001 \\
0001 \\
0001\end{array}$ & $\mathrm{PCI} / \mathrm{L}$ & & $\begin{array}{l}0.00 \\
0.60 \\
1.36 \\
0.70 \\
1.00 \\
2.30 \\
0.90\end{array}$ & $\begin{array}{l}: \\
: \\
: \\
:\end{array}$ & $\begin{array}{l}- \\
- \\
- \\
- \\
- \\
-\end{array}$ \\
\hline RADIUN-228 & $04 / 06 / 88$ & 0001 & $\mathrm{PCI} / \mathrm{L}$ & & 0.0 & 1. & 0.8 \\
\hline
\end{tabular}

PARAMETER VALUE IHDICATOR (PVI): < - LESS THAN DETECTION LIMIT

SAMPLE ID COOES:

0001 - FILTERED SAMPLE (.45 MICRONS)

OTHER PARAMETER VALUE FLAGS:

J. ESTIMATED VALUE 
GROUNDHATER QUALITY DATA BY LOCATION

SITE: AMBOI AMBROSIA LAKE

LOCATION: 0793

MORTH COORDINATE: 55493.0 FT

EAST COORDINATE: $\quad 60225.7$ FT

06/10/80 TO $12 / 04 / 92$

REPORT DATE: 08/04/94

FORMATION OF COMPLETION: ALLUVIUN (AL)

HYDRAULIC FLOW RELATIONSHIP: UPGRADIENT (U)

\begin{tabular}{|c|c|c|c|c|c|c|c|}
\hline PARAMETER MAME & LOG DATE & $\begin{array}{c}\text { SAMPLE } \\
\text { ID }\end{array} \mid$ & $\begin{array}{l}\text { UNITS OF } \\
\text { MEASURE }\end{array}$ & PVI & $\begin{array}{l}\text { PARAMETER } \\
\text { VALUE FLAGS }\end{array}$ & $\begin{array}{l}\text { DETECTION } \\
\text { LIMIT }\end{array}$ & $\begin{array}{l}\text { PARAMETER } \\
\text { UHCERTAINTY }\end{array}$ \\
\hline RADIUH-228 & $\begin{array}{l}07 / 13 / 88 \\
01 / 06 / 89 \\
09 / 19 / 89 \\
04 / 06 / 90 \\
01 / 16 / 91 \\
11 / 25 / 92\end{array}$ & $\begin{array}{l}0001 \\
0009 \\
0001 \\
0001 \\
0001 \\
0001\end{array}$ & PCI/L & & $\begin{array}{l}0.2 \\
0.00 \\
0.5 \\
0.7 \\
0.7 \\
0.4\end{array}$ & $\begin{array}{l}1 . \\
1 . \\
1 . \\
1 . \\
1.0\end{array}$ & $\begin{array}{r}0.7 \\
18.1 \\
0.9 \\
0.9 \\
1.9 \\
1.3\end{array}$ \\
\hline SILVER & $\begin{array}{l}04 / 06 / 88 \\
07 / 13 / 88 \\
01 / 06 / 89 \\
09 / 19 / 89 \\
04 / 06 / 90 \\
01 / 16 / 91 \\
11 / 25 / 92\end{array}$ & $\begin{array}{l}0001 \\
0001 \\
0001 \\
0001 \\
0001 \\
0001 \\
0001\end{array}$ & MG/L & $\begin{array}{l}< \\
< \\
< \\
<\end{array}$ & $\begin{array}{l}0.11 \\
0.03 \\
0.01 \\
0.01 \\
0.01 \\
0.01 \\
0.01\end{array}$ & $\begin{array}{l}0.01 \\
0.01 \\
0.01 \\
0.01 \\
0.01 \\
0.01 \\
0.01\end{array}$ & $\begin{array}{l}- \\
: \\
- \\
-\end{array}$ \\
\hline SOOIUM & $\begin{array}{l}05 / 15 / 86 \\
04 / 06 / 88 \\
07 / 13 / 88 \\
01 / 06 / 89 \\
09 / 19 / 89 \\
04 / 06 / 90 \\
01 / 16 / 91 \\
11 / 25 / 92\end{array}$ & $\begin{array}{l}0001 \\
0001 \\
0001 \\
0001 \\
0001 \\
0001 \\
0001 \\
0001\end{array}$ & MG/L & & $\begin{array}{l}685 . \\
433 . \\
511 . \\
555 . \\
654 . \\
689 . \\
901 . \\
849 .\end{array}$ & $\begin{array}{l}0.002 \\
0.002 \\
0.002 \\
0.002 \\
0.002 \\
0.002 \\
5 . \\
5 .\end{array}$ & $\begin{array}{l}: \\
: \\
: \\
: \\
-\end{array}$ \\
\hline STRONTIUM & $\begin{array}{l}05 / 15 / 86 \\
04 / 06 / 88 \\
07 / 13 / 88 \\
01 / 06 / 89 \\
09 / 19 / 89 \\
04 / 06 / 90 \\
01 / 16 / 91\end{array}$ & $\begin{array}{l}0001 \\
0001 \\
0001 \\
0001 \\
0001 \\
0001 \\
0001\end{array}$ & $M G / L$ & & $\begin{array}{l}1.1 \\
5.89 \\
8.1 \\
10.3 \\
5.8 \\
11.4 \\
12.5\end{array}$ & $\begin{array}{l}0.1 \\
0.1 \\
0.1 \\
0.1 \\
0.1 \\
0.1 \\
0.01\end{array}$ & $\begin{array}{l}- \\
- \\
- \\
-\end{array}$ \\
\hline
\end{tabular}

PARAMETER VALUE IMDICATOR (PVI): < - LESS THAN DETECTION LIMIT SAMPLE ID COOES:

0001 - FILTERED SAMPLE (.45 MICRONS)

OTHER PARAMETER VALUE FLAGS:

H - hOLd TIME EXPIRED, VALUE SUSPECT -

J - estIMATED VALUE 
GROUNDWATER OUALITY DATA BY LOCATION

SITE: AMBO1 AMBROSIA LAKE

LOCATIOH: 0793

NORTH COORDIHATE: 55493.0 FT

EAST COOROIHATE: $\quad 60225.7$ FT

06/10/80 TO 12/04/92

REPORT DATE: $08 / 04 / 94$

FORHATIOH OF COMPLETION: ALLUVIUH (AL)

HYDRAULIC FLOW RELATIONSHIP: UPGRADIENT (U)

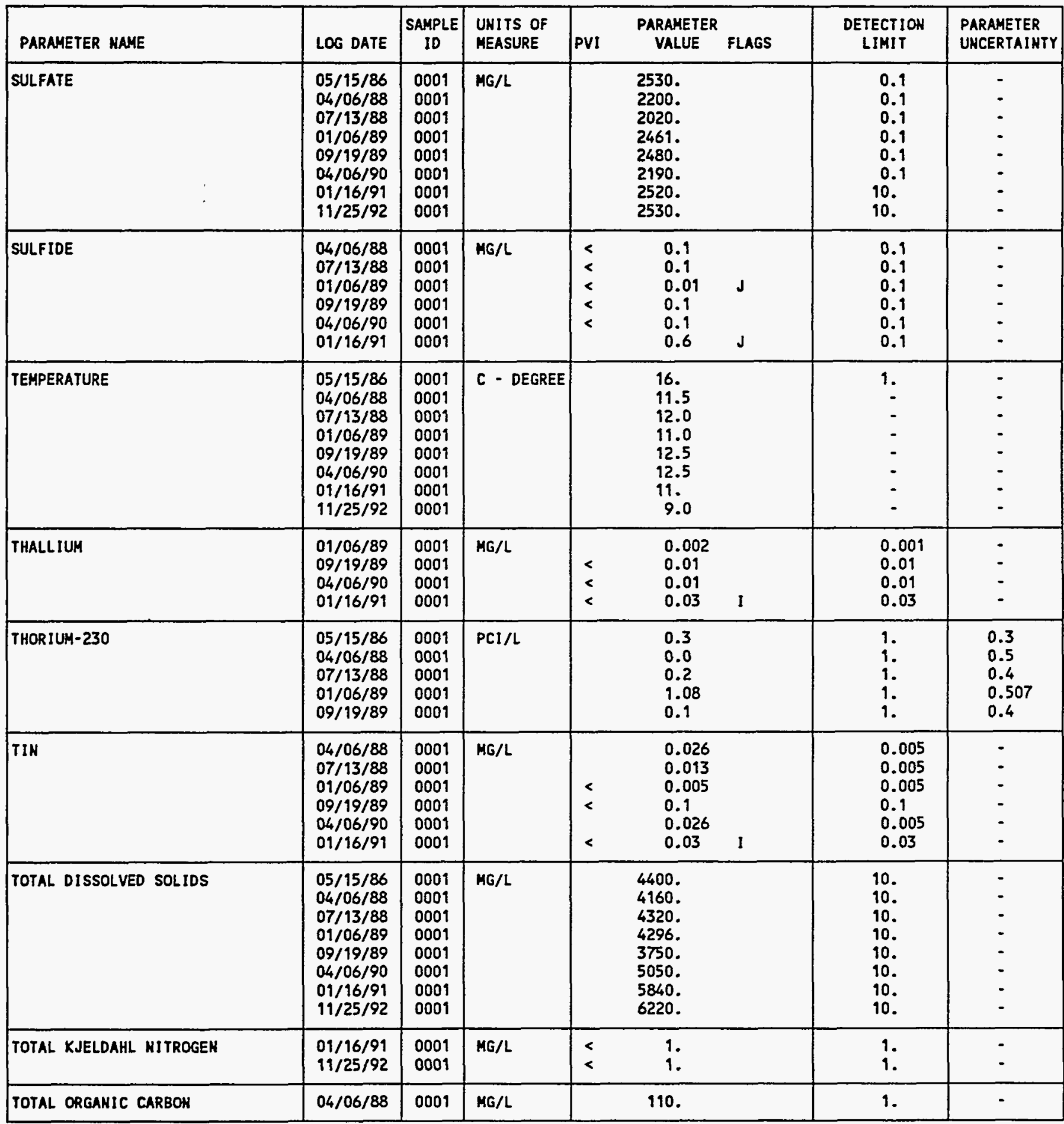

PARAMETER VALLE INDICATOR (PVI): < - LESS THAN DETECTION LIHIT

SAMPLE ID COOES:

0001 - FILTERED SAMPLE (.45 MICRONS)

OTHER PARAMETER VALUE FLAGS:

1 - INCREASED DETECTIOH LIMIT OUE TO REQUIRED DILUTION

s - estimated value 
GROUNDWATER QUALITY DATA BY LOCATION

SITE: AMBO1 AMBROSIA LAKE

LOCATION: 0793

NORTH COORDINATE: 55493.0 FT

EAST COORDINATE: 60225.7 FT

06/10/80 TO $12 / 04 / 92$

REPORT DATE: 08/04/94

FORMATION OF COMPLETIOH: ALLUVIUM (AL)

HYDRAULIC FLON RELATIONSHIP: UPGRADIENT (U)

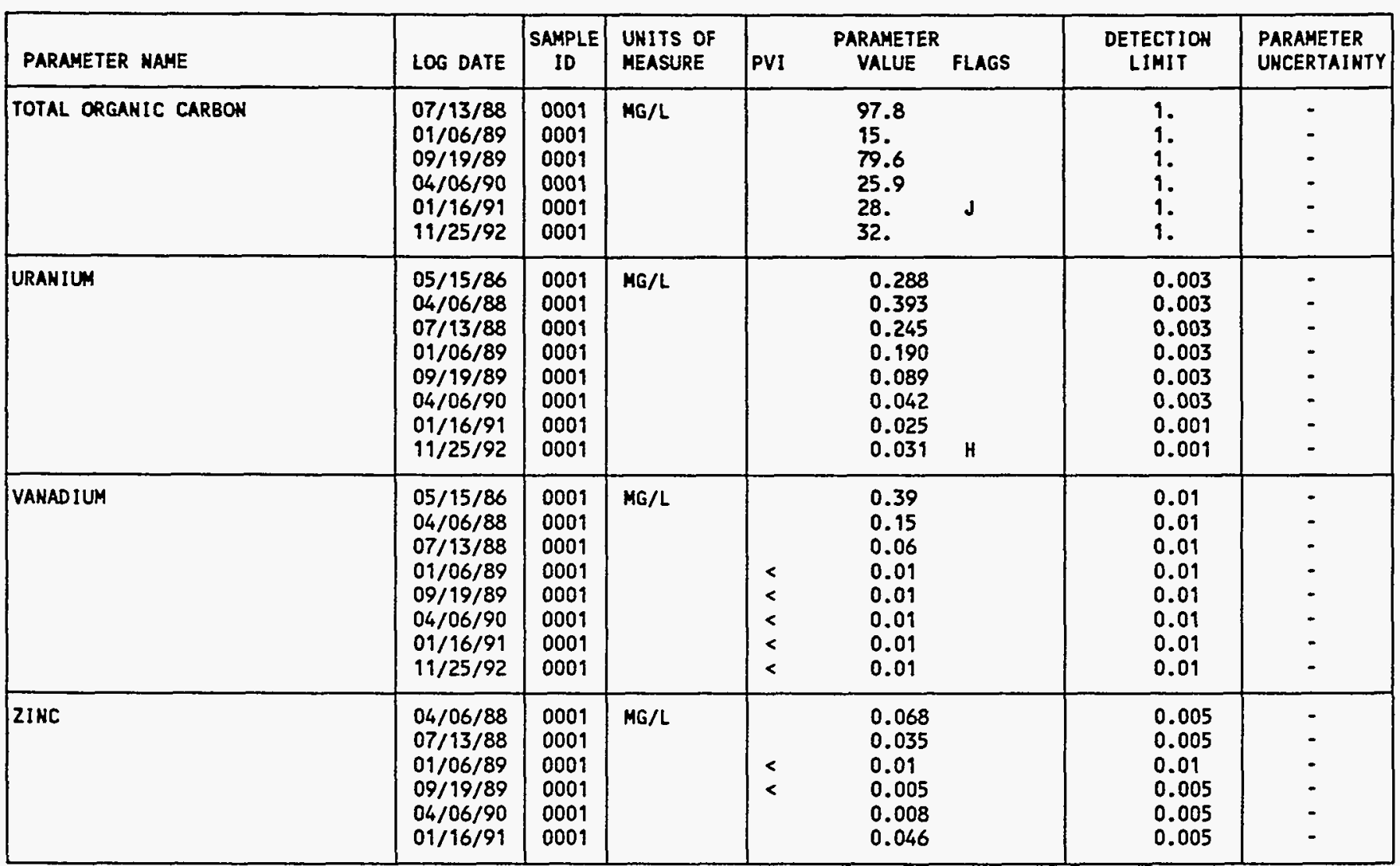

PARAMETER VALUE INDICATOR (PVI):

< - LESS THAN DETECTION LIMIT

SAMPLE ID COOES:

0001 - FILTERED SAMPLE (.45 MICRONS)

OTHER PARAMETER VALUE FLAGS:

H - HOLD TIME EXPIRED, VALUE SUSPECT

J- estimated VALUE

DATA FILE NAME: M: IDART \AMBOIIGWO10012.DAT 